\title{
ID: LB2 \\ Development and validation of an analytical HPLC method to assess chemical and radiochemical purity of [68Ga]Ga-NODAGA-Exendin-4 fully automated produced
}

\author{
Silvia Migliari, Nuclear Medicine and Molecular Imaging Department, University Hospital \\ of Parma, smigliari@ao.pr.it
}

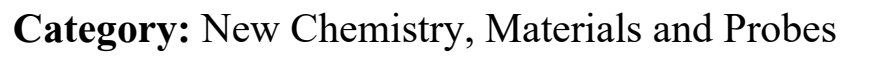

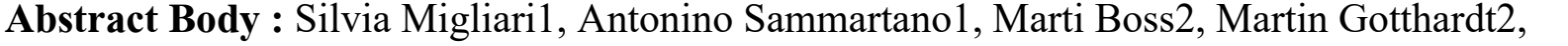

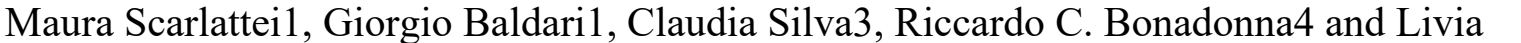

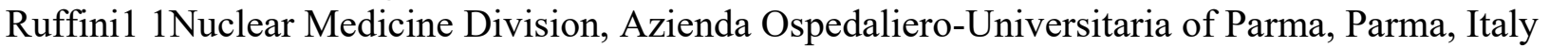

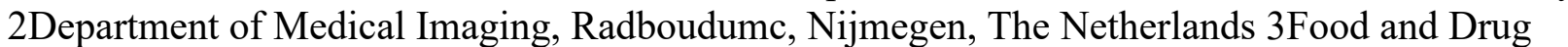

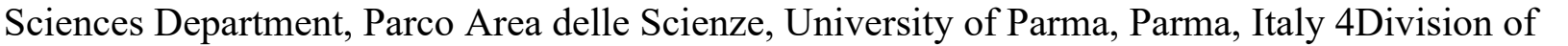

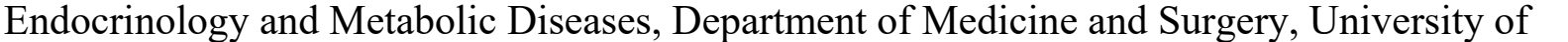

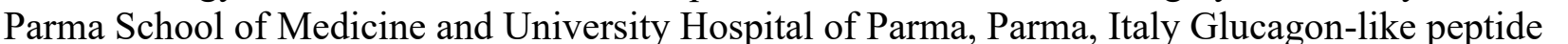

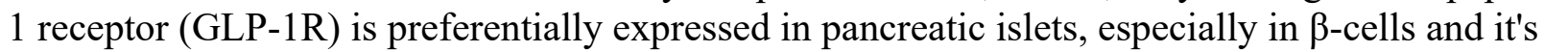

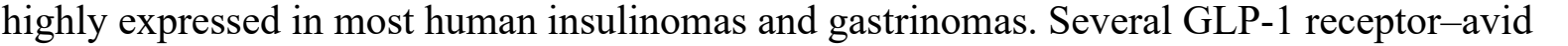

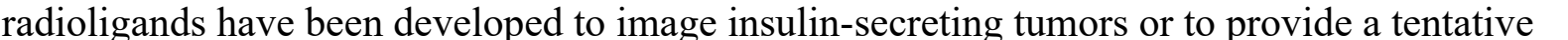

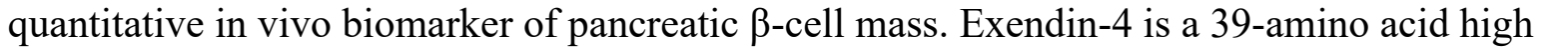

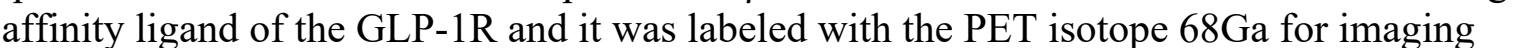

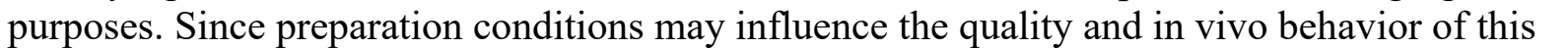

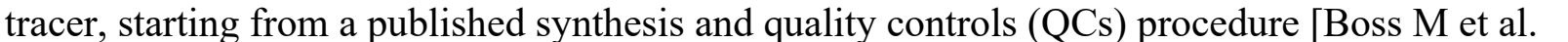

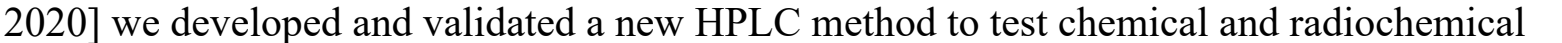
पाm

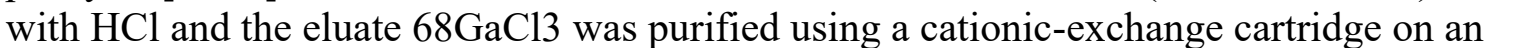

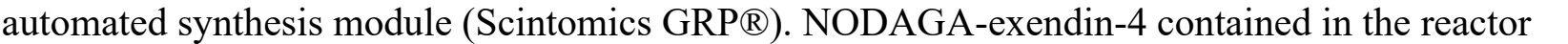

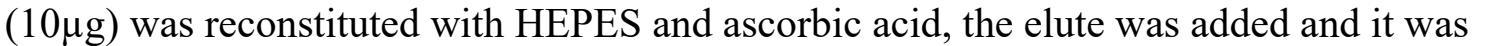

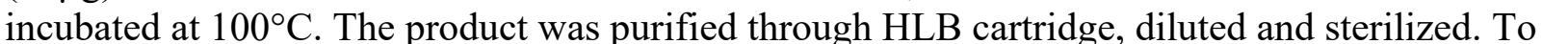

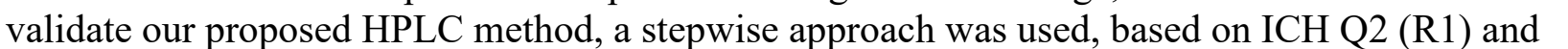

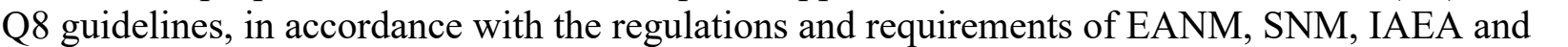

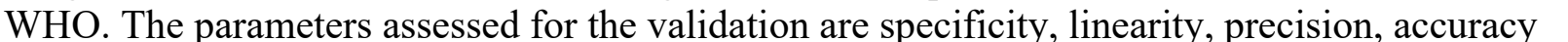

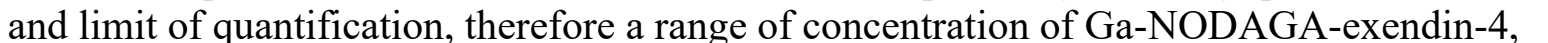

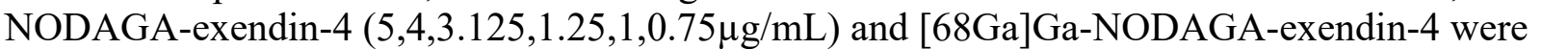

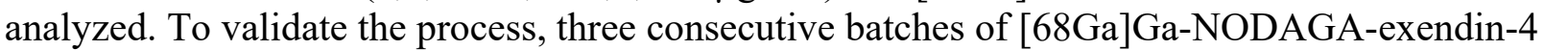

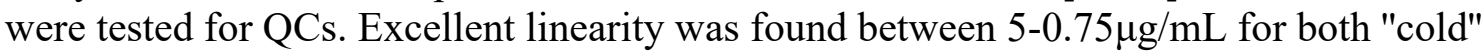

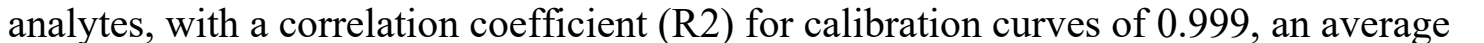

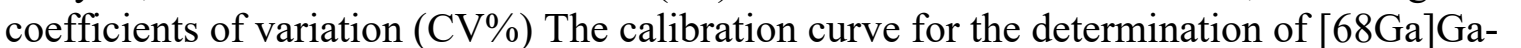

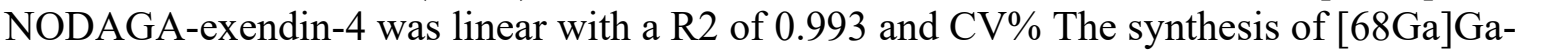

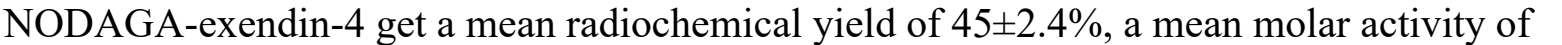

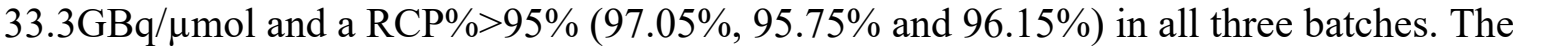

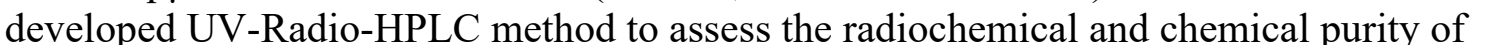
घम
\end{abstract}




\section{References: $\square \square \square \square \square$}

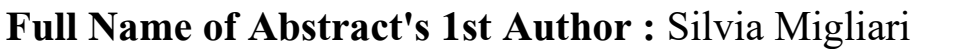

First Name: $\square[\|]\|\|$

Last Name: $\square \square \square \square \square$

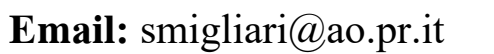

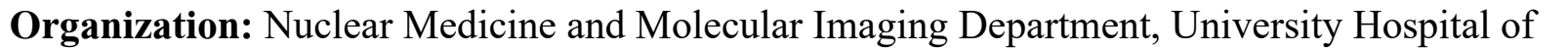
$\square \square \square \square \square$

Country: $\square[\| m$ 


\title{
ID: LB3 \\ Quality Assurance system allows hospital radiopharmacist to select the most appropriate method in determining radiochemical purity of 99mTc-MBrIDA
}

Silvia Migliari, Nuclear Medicine and Molecular Imaging Department, University Hospital of Parma, smigliari@ao.pr.it

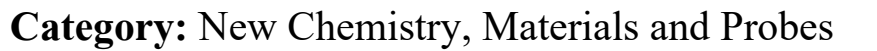

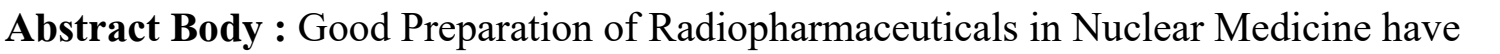

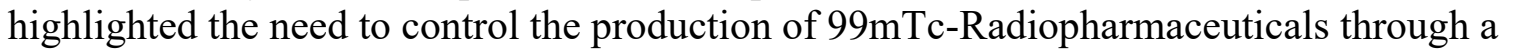

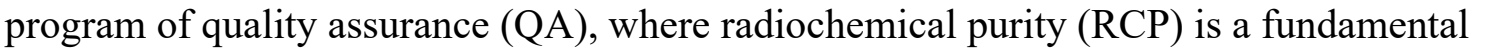

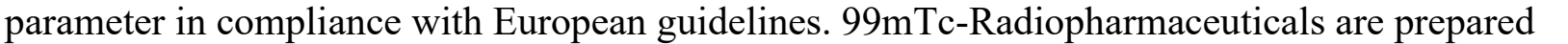

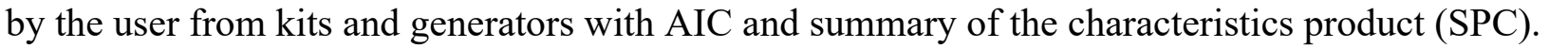

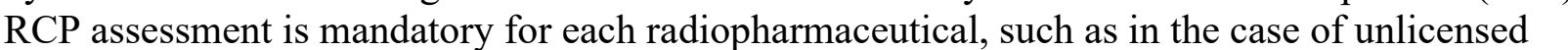

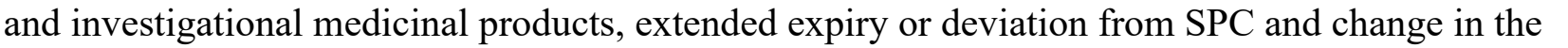

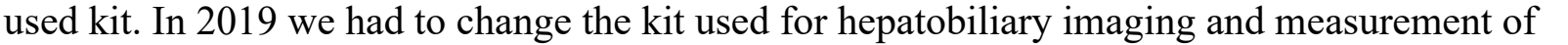

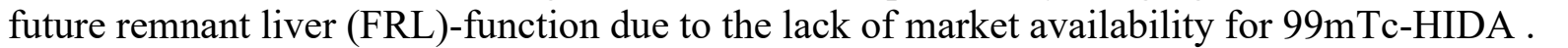

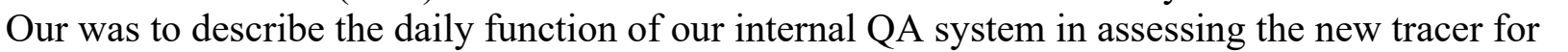

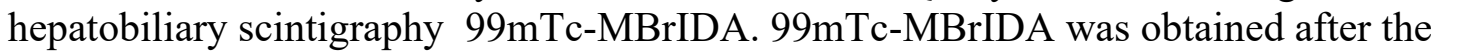

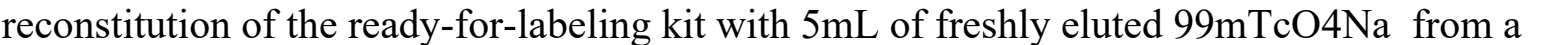

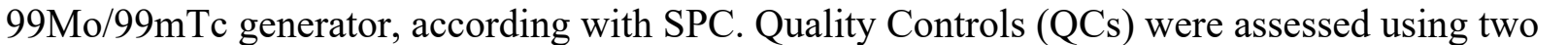

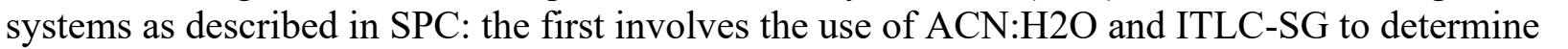

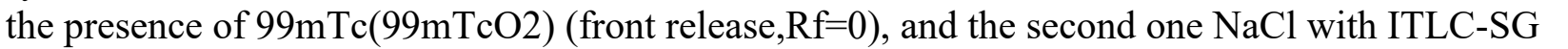
ए

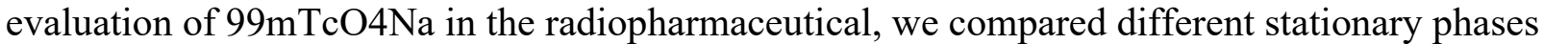

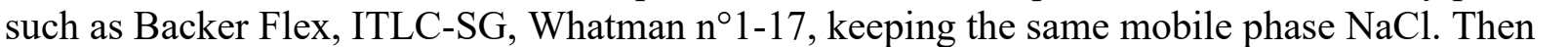

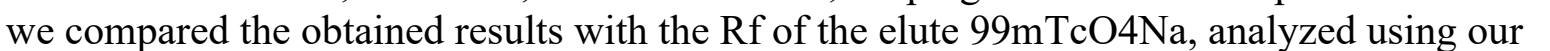

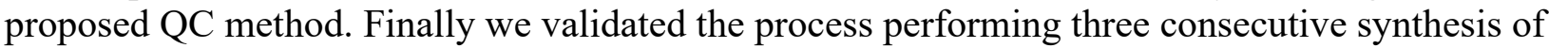

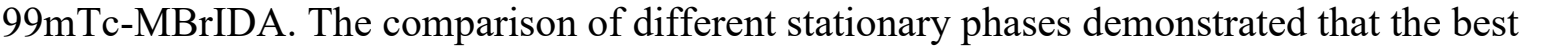

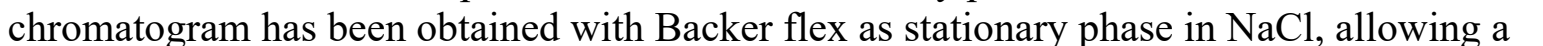
एம1

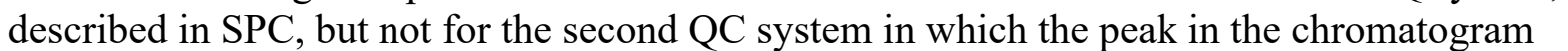

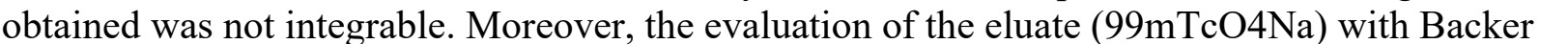

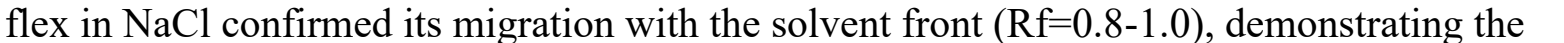

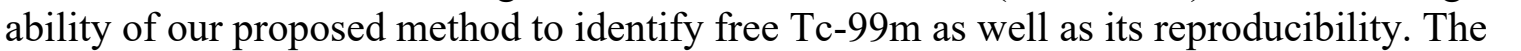

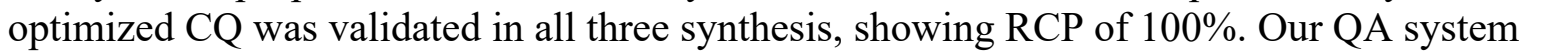

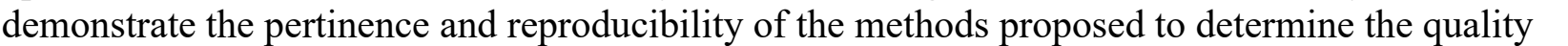

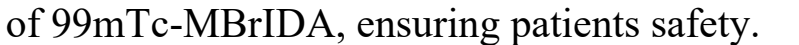

References: $\square \square \| \Pi$

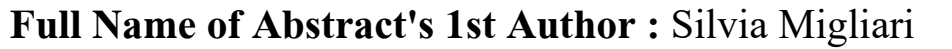


First Name: $\square[\| \square|m| m$

Last Name: $\square \square\|m\| m$

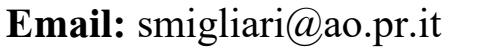

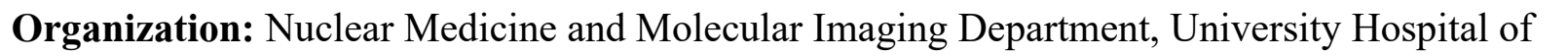

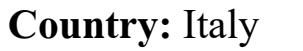




\title{
ID: LB4 \\ Preclinical Development of [89Zr]Zr-Isatuximab for CD38-targeted immunoPET imaging of Multiple Myeloma
}

Natalia Herrero Alvarez, MSKCC, herreron@mskcc.org

\section{Category: $\square \square\|\| \| \square$}

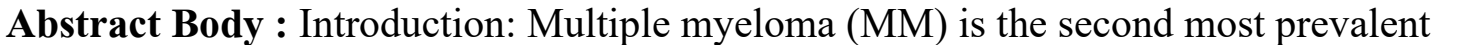

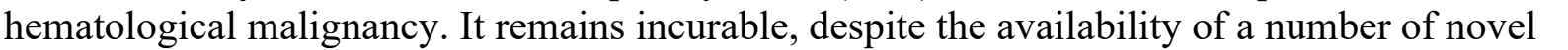
therapeutic approaches.[1] Disease prognosis depends on a clinician's ability to monitor disease

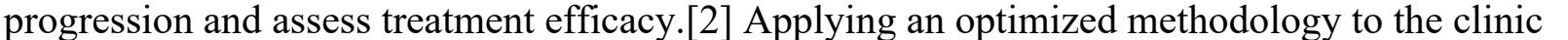

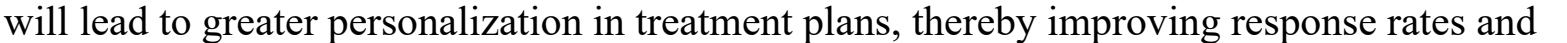

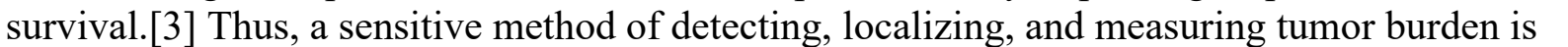

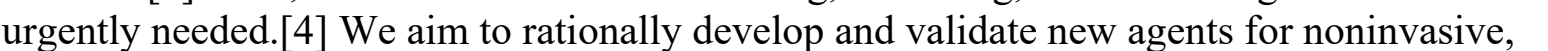

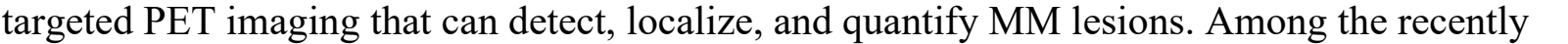

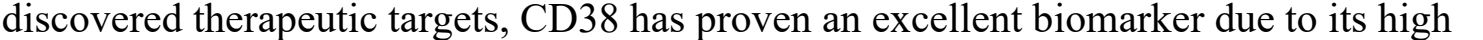

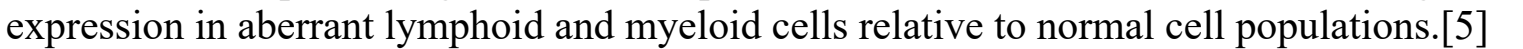

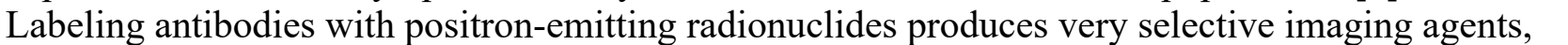

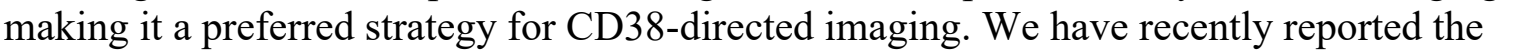

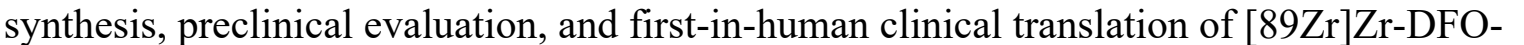

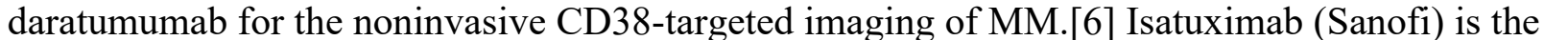

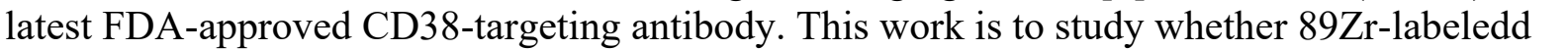

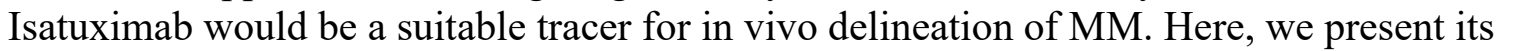

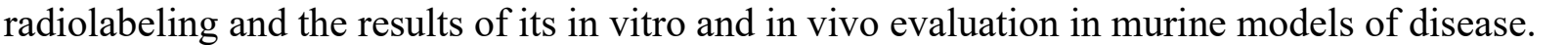

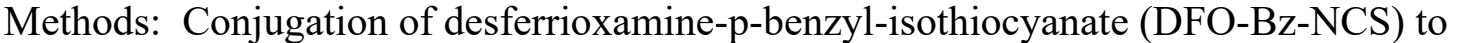
ए

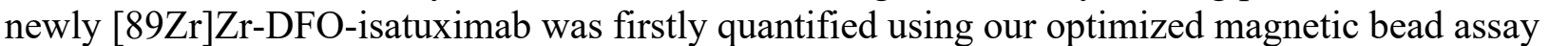

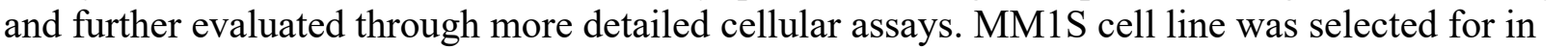

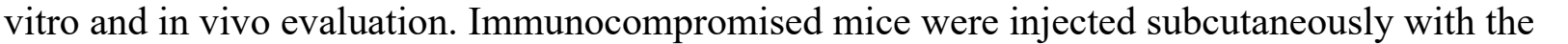

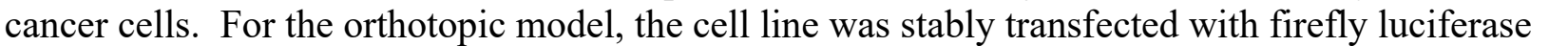

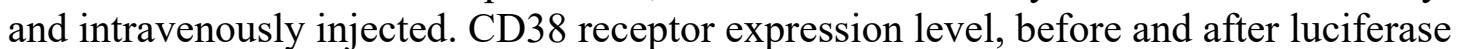

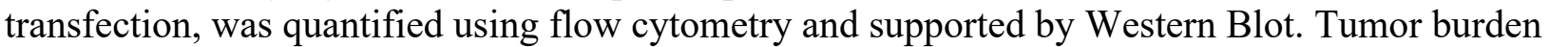

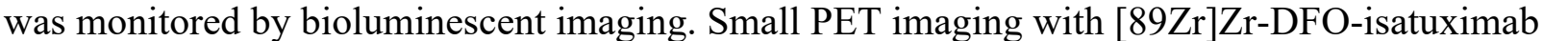

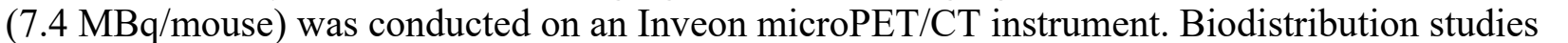

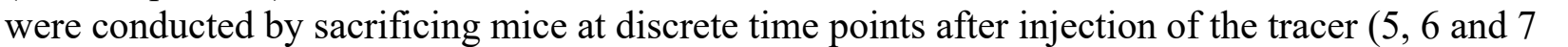

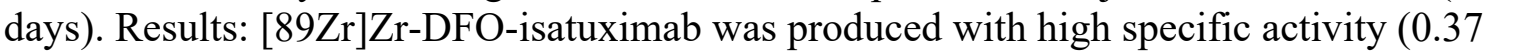

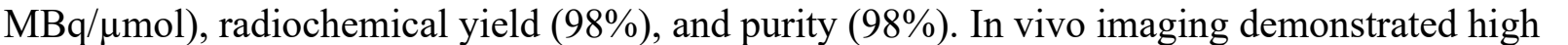

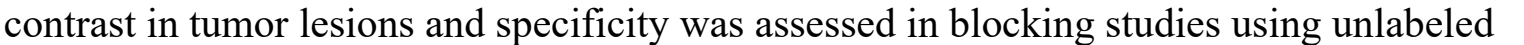

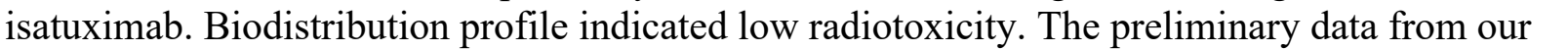

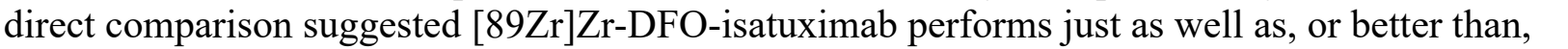
घम ए
\end{abstract}




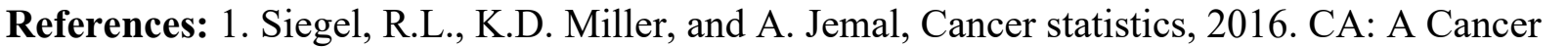

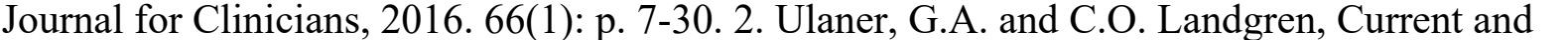

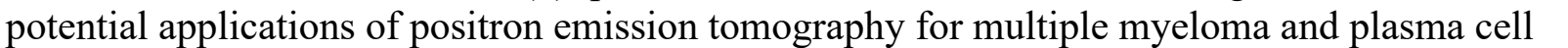

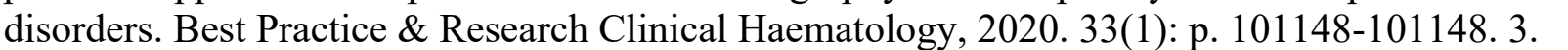

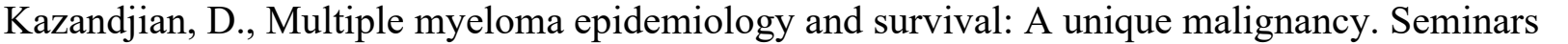
पाm

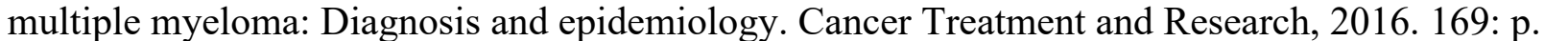

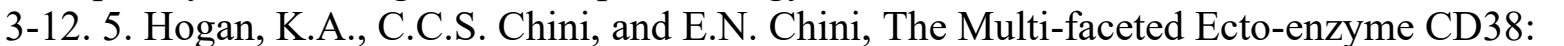

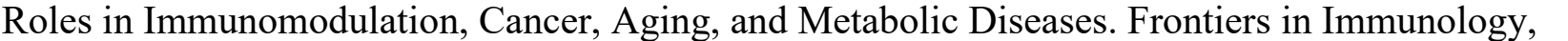

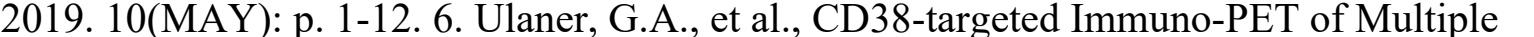

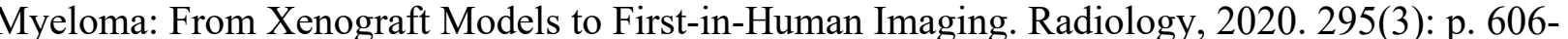
$\square \square \square$

\section{Image/Figure:}

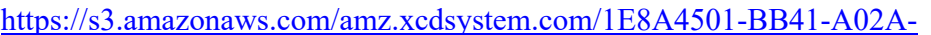

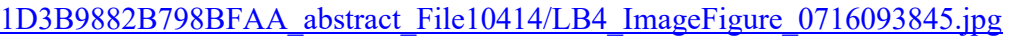

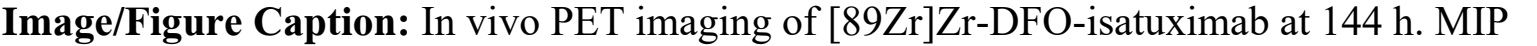

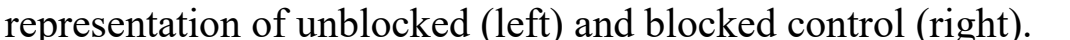

First Name: $\square \square \square\|\|\|\|$

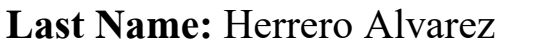

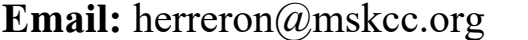

Organization: $\square \square \square \square \square \square$

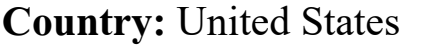




\title{
ID: LB6
}

High-throughput Imaging of Insect Larvae as Replacement for Mammalian Models of Gut Inflammation

\author{
Anton Windfelder, Justus Liebig University of Giessen, Giessen, Germany, \\ Anton.G.Mueller@med.uni-giessen.de
}

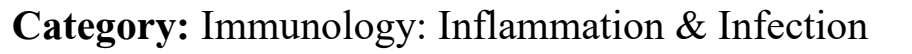

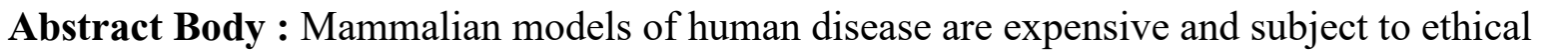

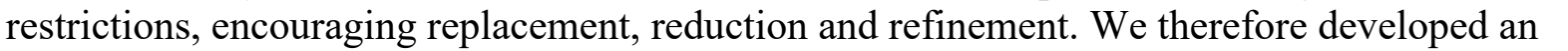

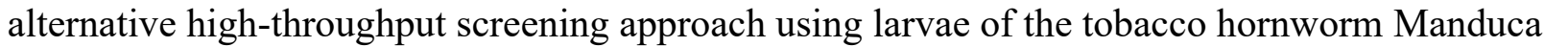

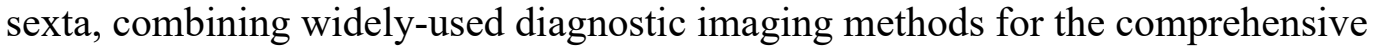

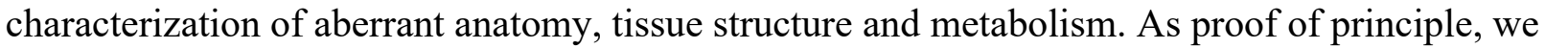
पामा

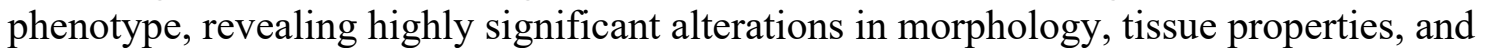

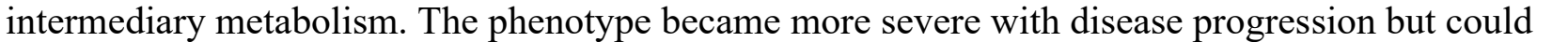

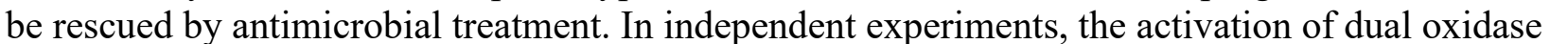

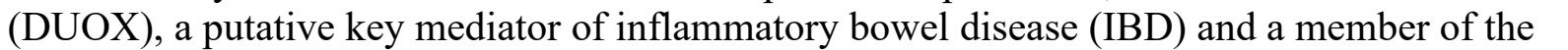

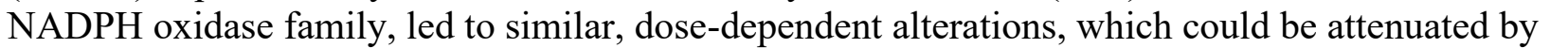

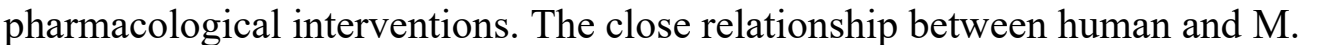

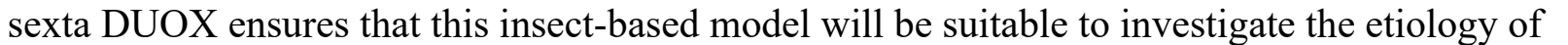

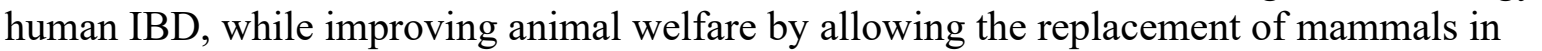

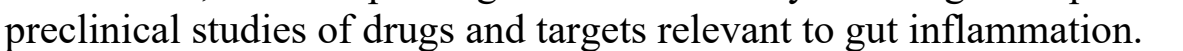

\section{Image/Figure:}

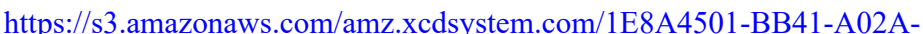

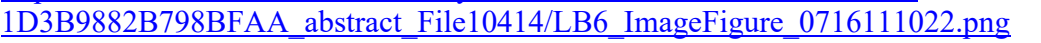

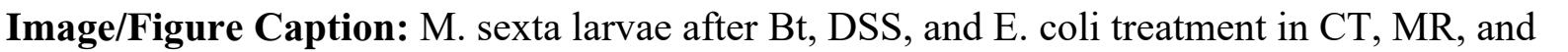

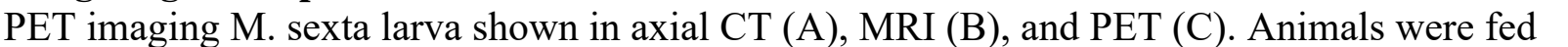

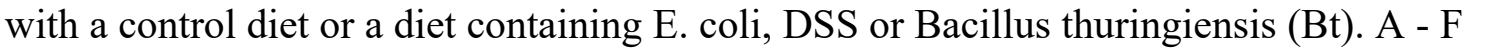

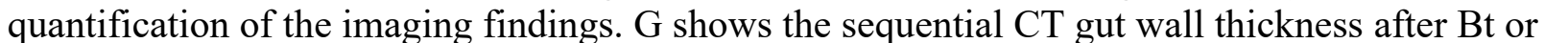

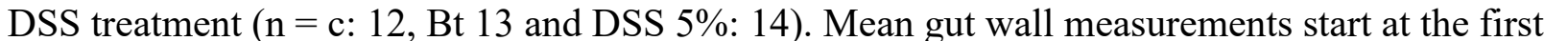

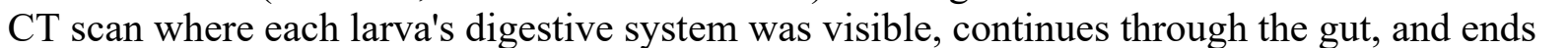

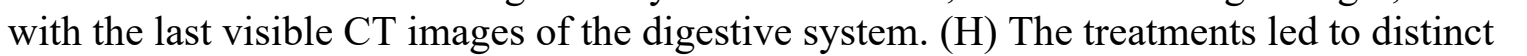

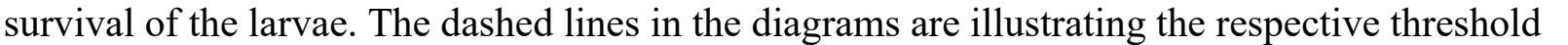

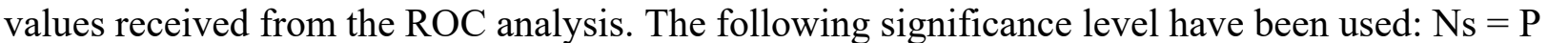
$>0.05, *=\mathrm{P} \leq 0.05, * * \mathrm{P} \leq 0.01, * * *=\mathrm{P} \leq 0.001$ and $* * * *=\mathrm{P} \leq 0.0001$. Error bars represent

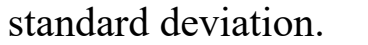

First Name: $\square \square \square \square \mid \square$ 


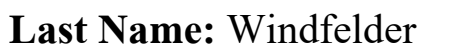

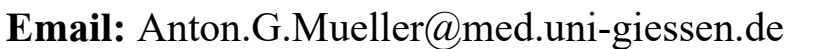

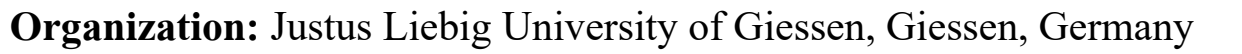

Country: $\square \square \square \square \square \square$ 


\title{
ID: LB7 \\ Novel Development of a Positron Emission Tomography (PET) Imaging Tracer to Detect P2Y12 in vivo
}

Meixiang Yu, Houston Methodist Research Institute, myu@houstonmethodist.org

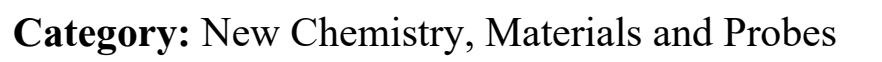

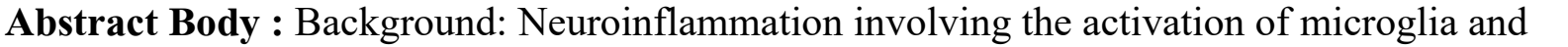

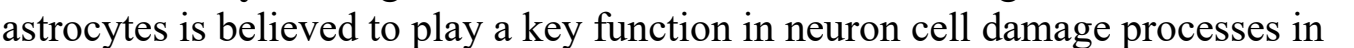
neurodegenerative disorders, including Alzheimer's disease and Parkinson's disease. Currently

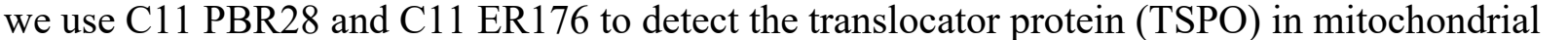

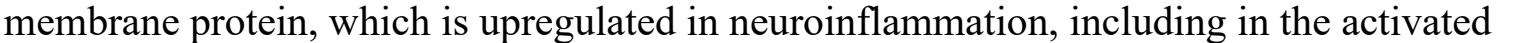

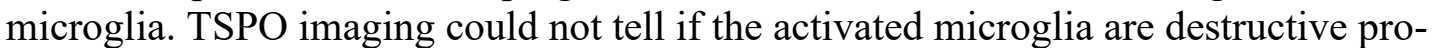

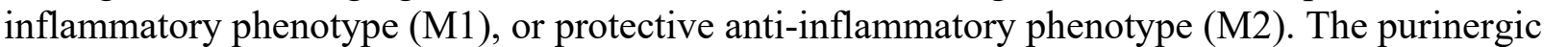

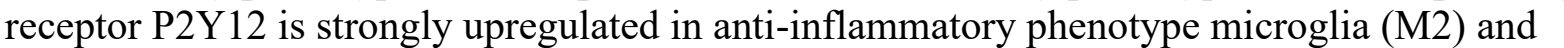

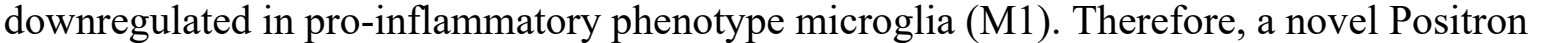

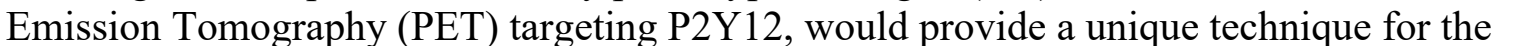

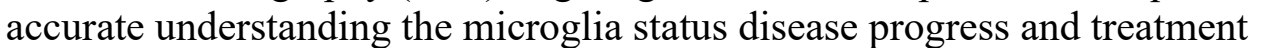

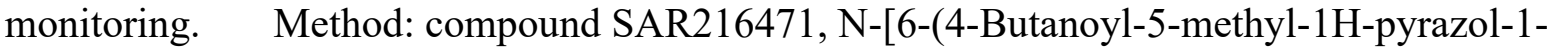

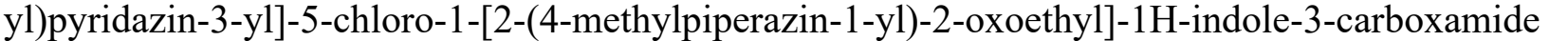

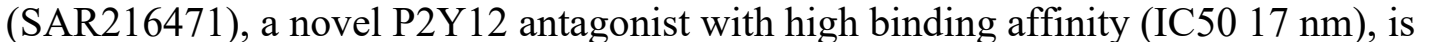

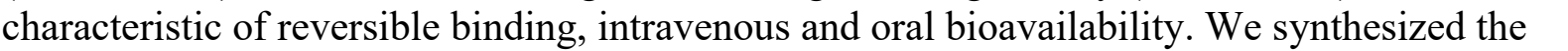

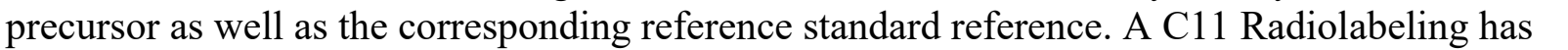

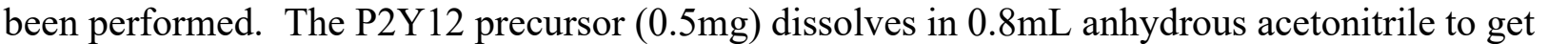
ए

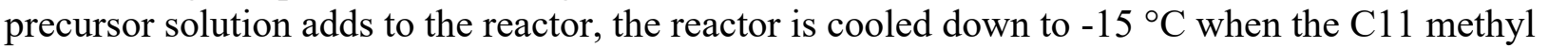

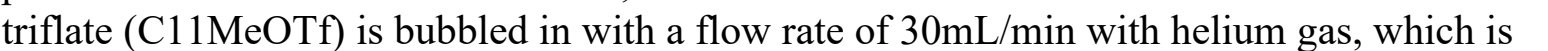

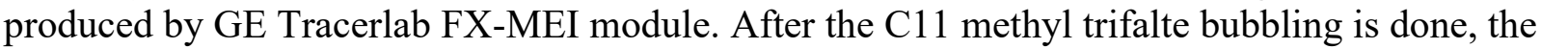

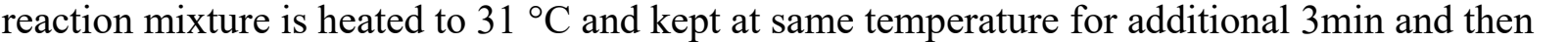

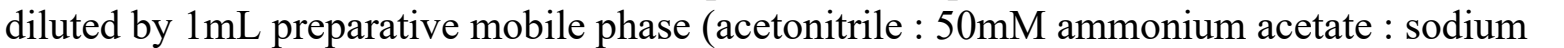

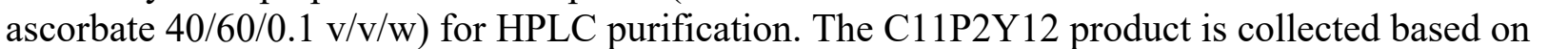

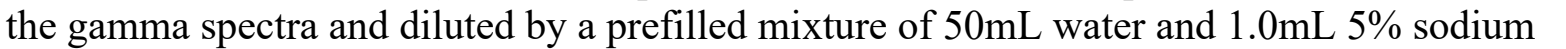

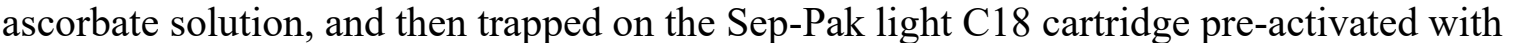

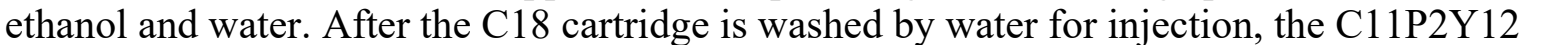

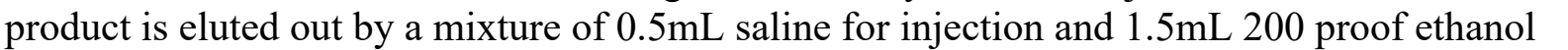

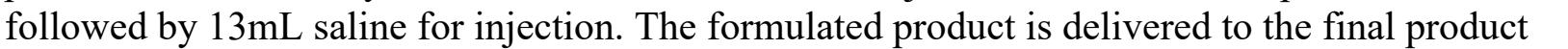

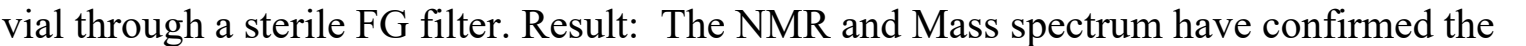

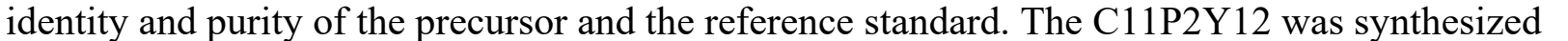

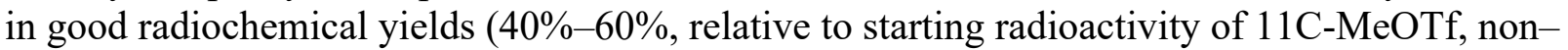

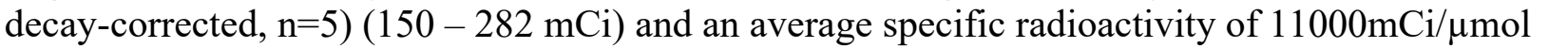

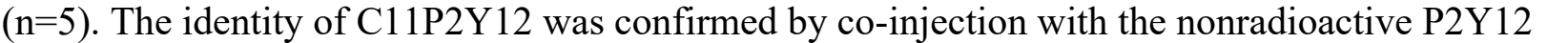

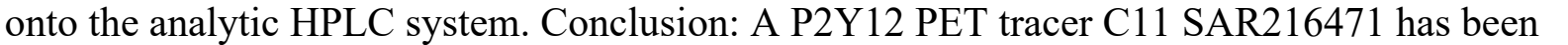

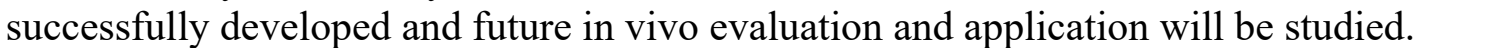




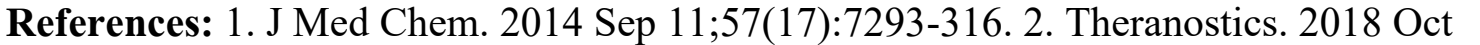

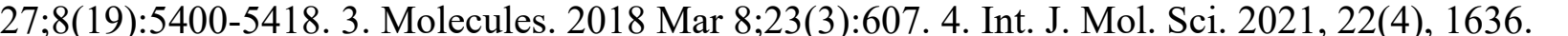

Image/Figure:

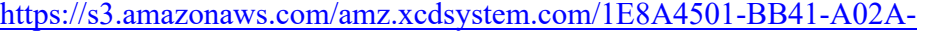

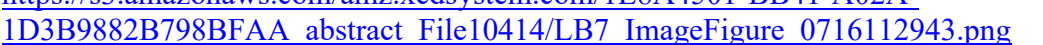

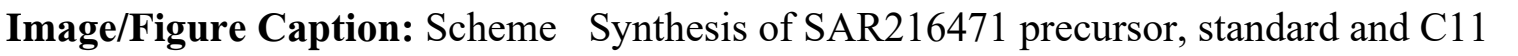

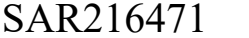

First Name: $\square \square \square\|\| \square \square$

Last Name: $\square \square \square$

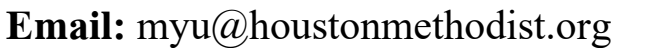

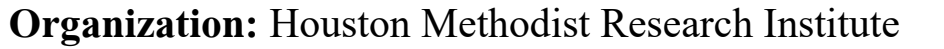

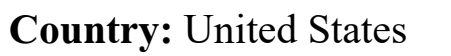




\title{
ID: LB9 \\ Monitoring of the therapeutic process of photodynamic therapy using quantitative vasculature segmentation by photoacoustic microscopy
}

Su Woong Yoo, Chonnam National University Hwasun Hospital, yoosw.md@gmail.com

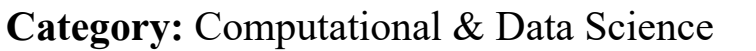

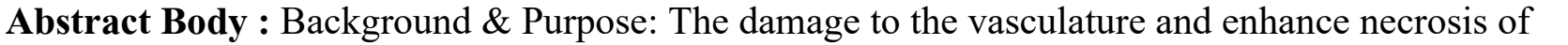

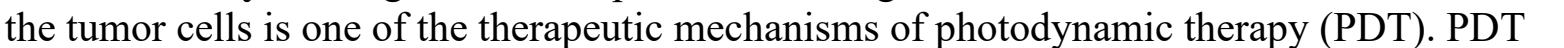

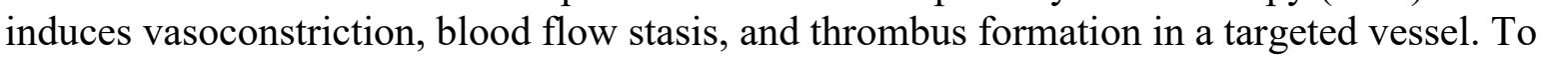

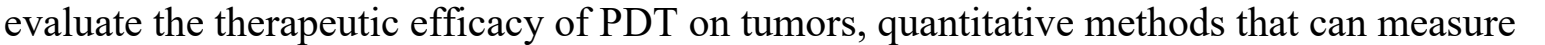

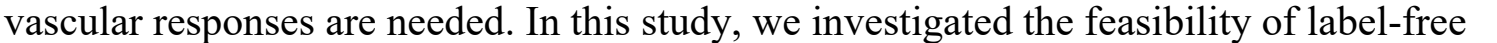

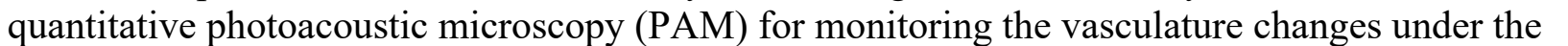

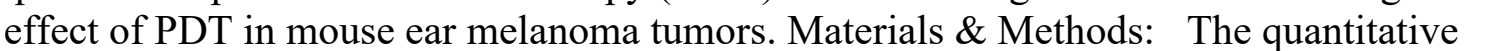

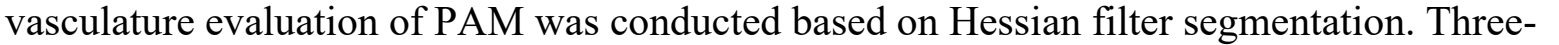

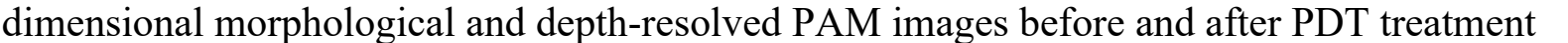

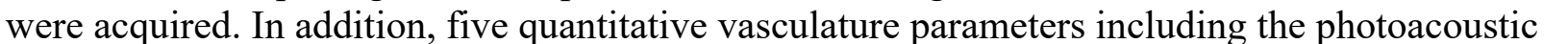

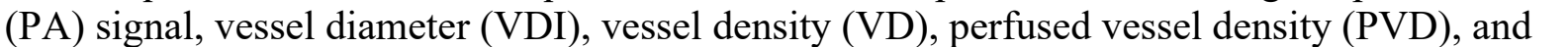

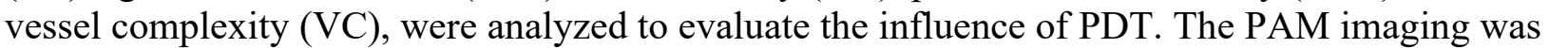

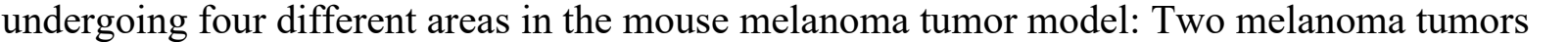

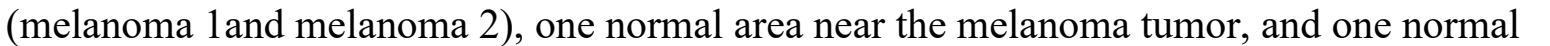

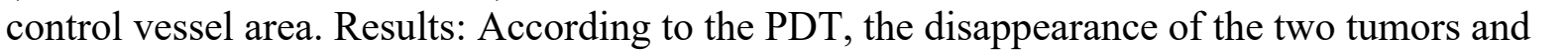
ए

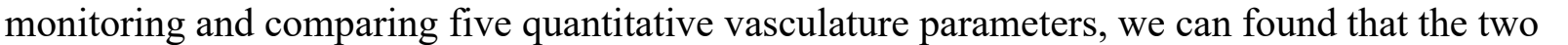

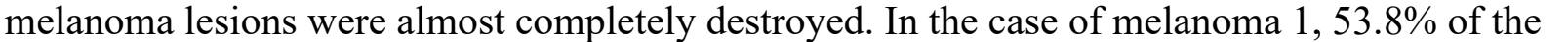

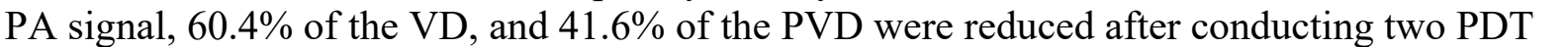

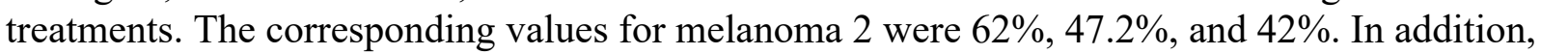

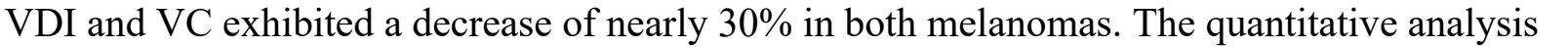

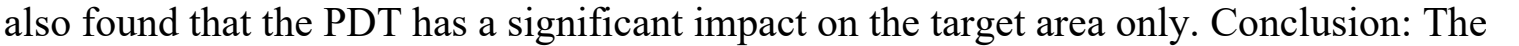

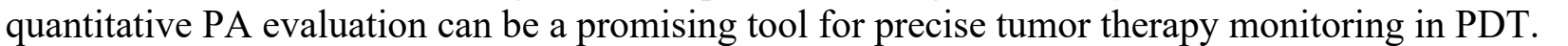

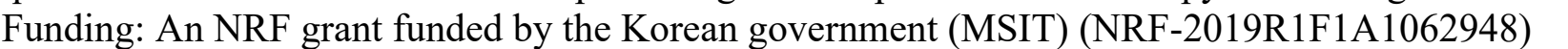

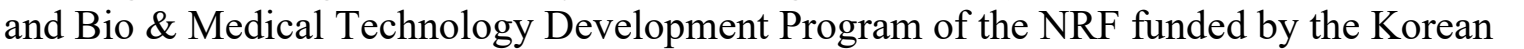

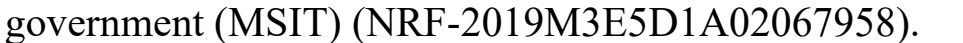

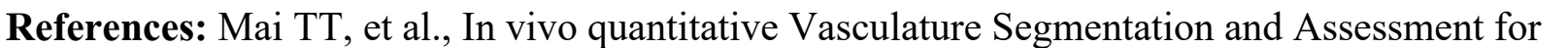

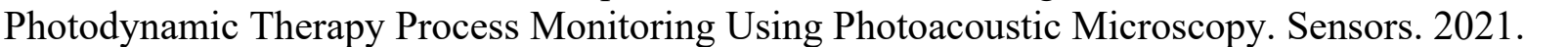

First Name: $\square \square \square \square \square \square \square$

Last Name: $\square \square \square \square$

Email: $\square \square \| \square \square \square \square \square \square \square \square$ 
Organization:

Country: $\square \square \square \square \square \square \square \square \square$ 


\title{
ID: LB10 \\ Pharmacological activation of hypoxia inducible factor $1 \alpha(\mathrm{HIF}-1 \alpha)$ promotes tumor blood vessel formation and enhances tumor-targeted drug delivery
}

Asmaa Elshafei, EXMI, aelshafei@ukaachen.de

Category: $\square \square\|\| \| \square \mid \square$

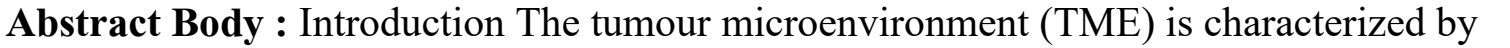

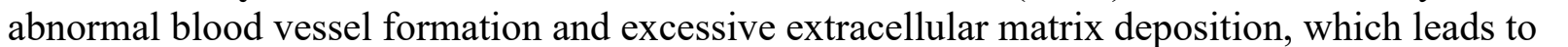

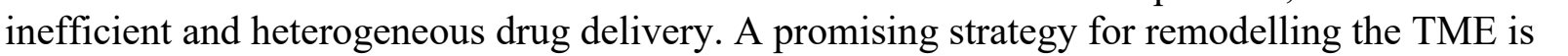

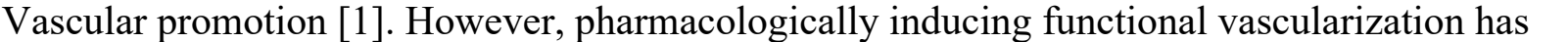

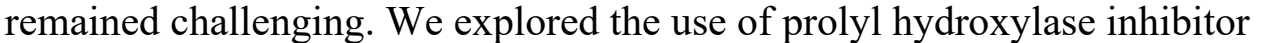

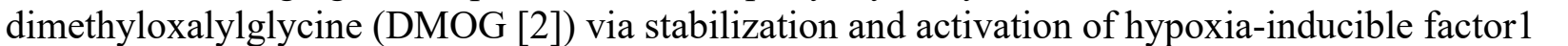

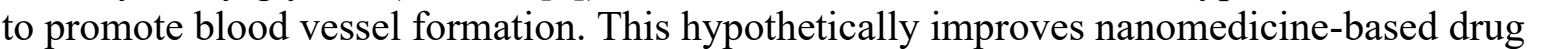

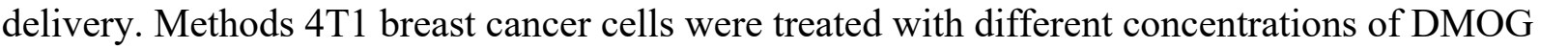

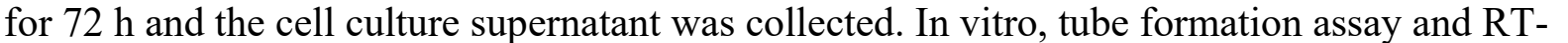

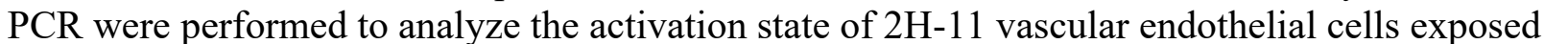

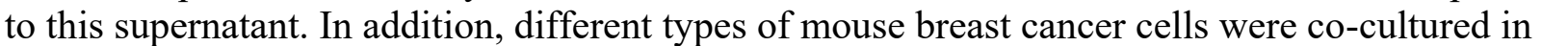

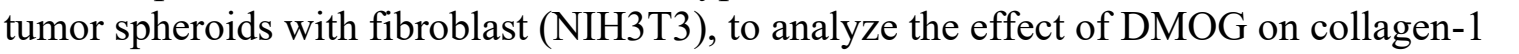

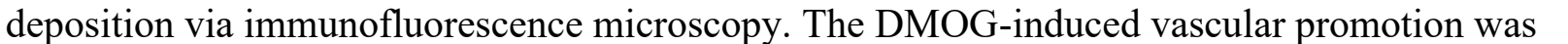

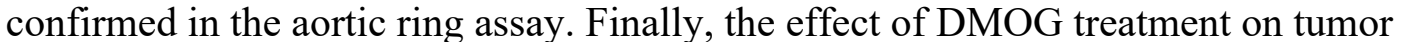

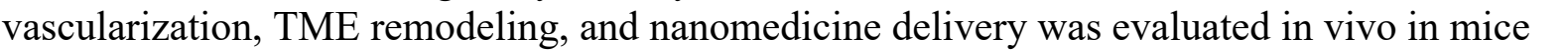

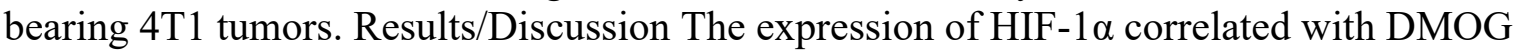

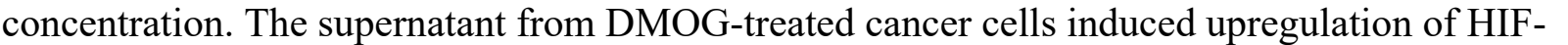

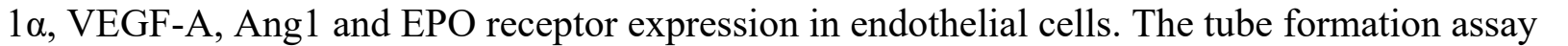

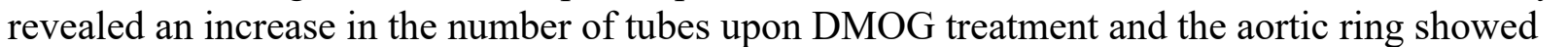

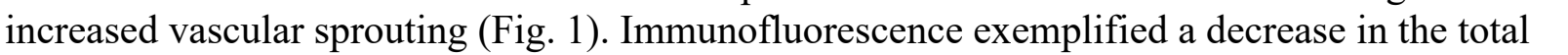

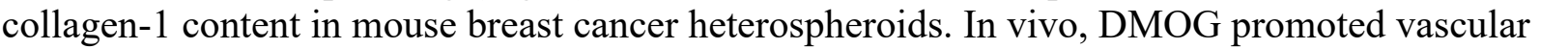

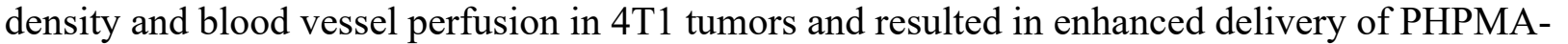

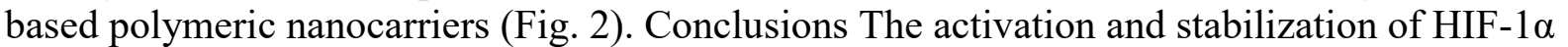

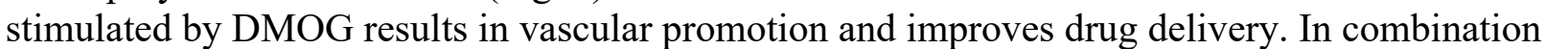

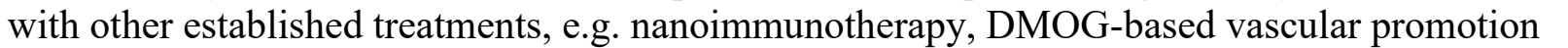

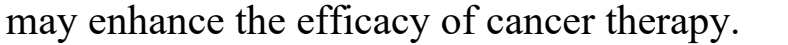

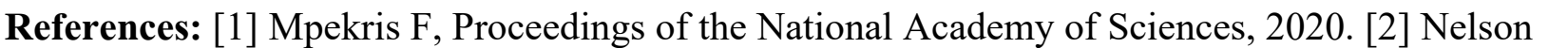

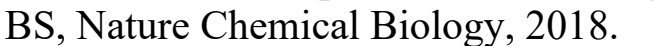

\section{Image/Figure:}

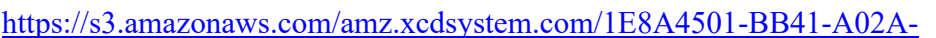

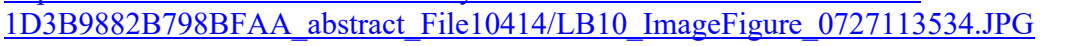




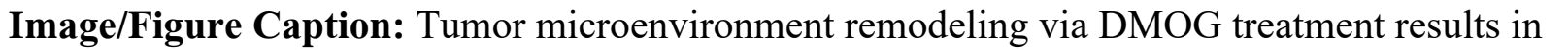

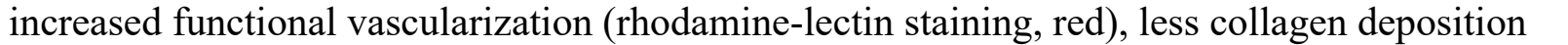

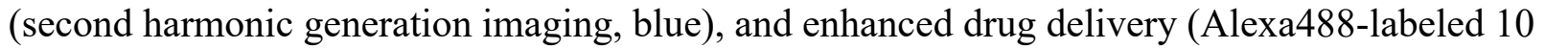

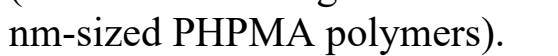

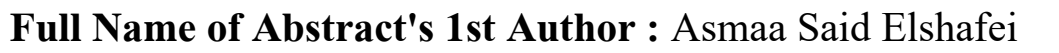

First Name: $\square \square \square \square$

Last Name: $\square[\|\square\|\|\| \|$

Email: $\square\|\|\|\square\| \square \square \square \square \square \square \square \| \square \square \square$

Organization: $\square \square \square \square \square$

Country: $\square \square \square \square \square \square$ 


\title{
ID: LB11 \\ Novel Nanobubbles Covalently Linked to FN3hPD-L1 Nanobody as a Contrast Agent for Ultrasound Molecular Imaging of Human PD-L1 Expression in Living Subjects
}

\author{
Arutselvan Natarajan, Stanford University, anatarajan@stanford.edu
}

Category: $\square \square\|ा\| ा \|$

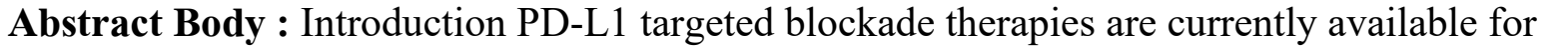

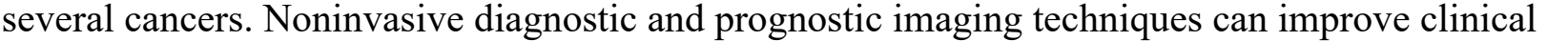

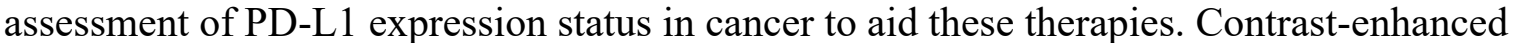

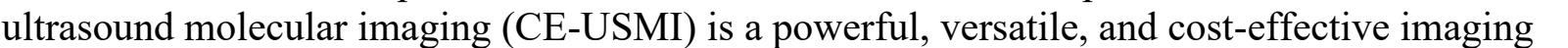

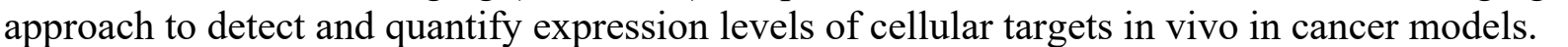

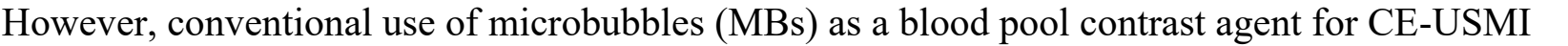

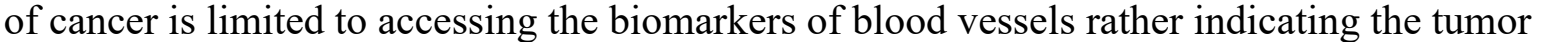

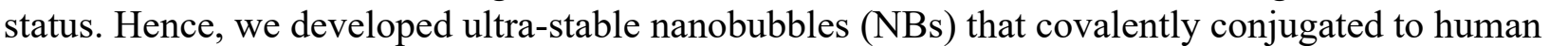

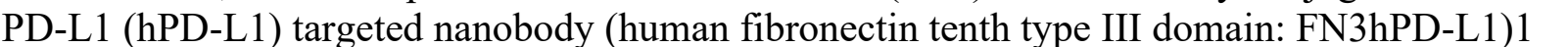

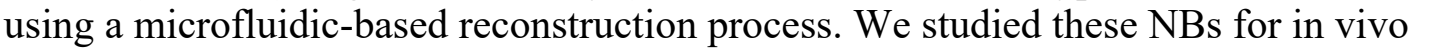

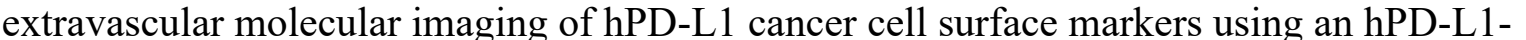

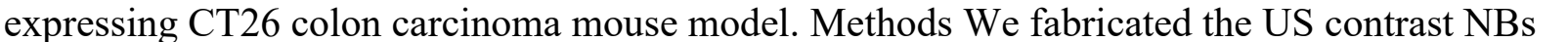

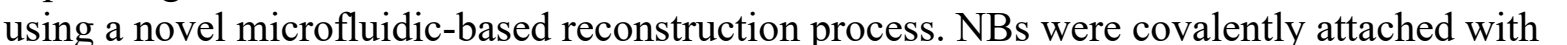

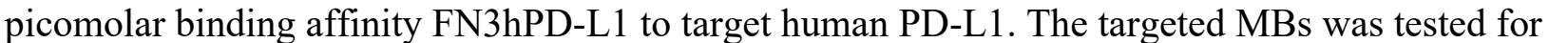

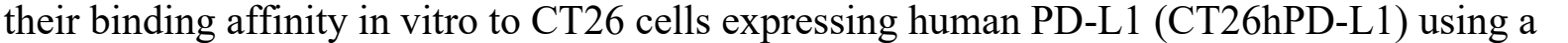

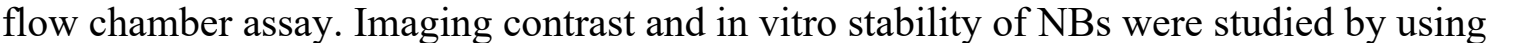

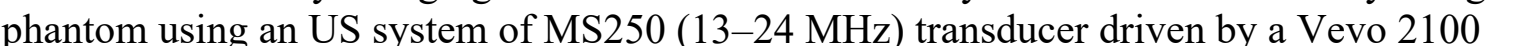

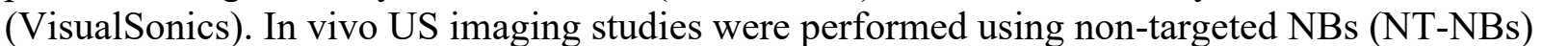

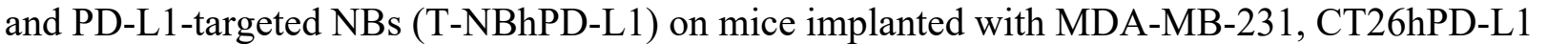

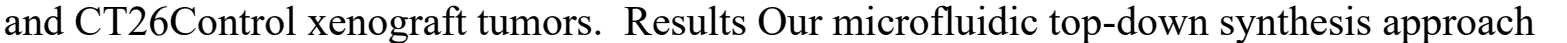

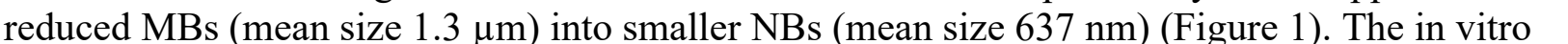

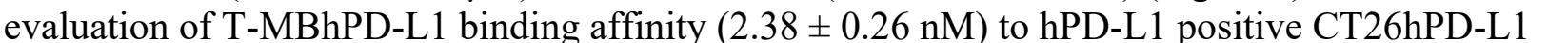

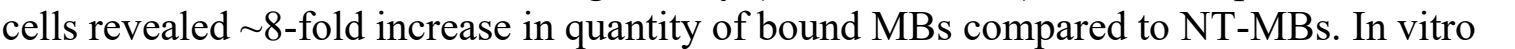

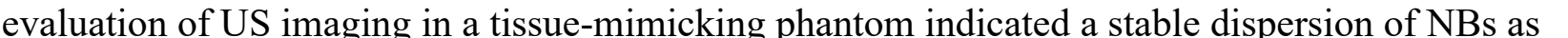

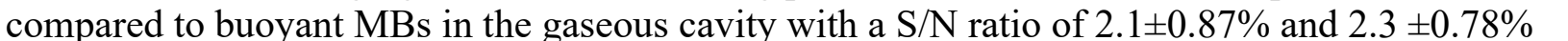

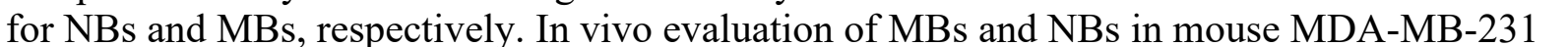

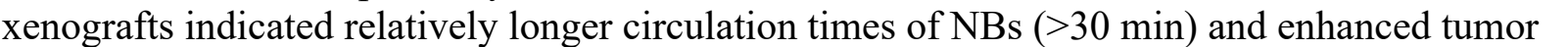

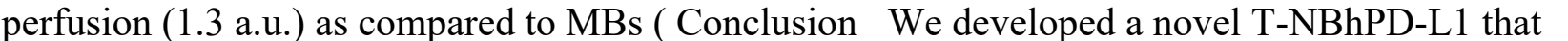

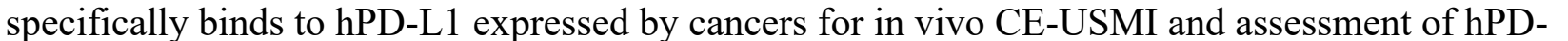

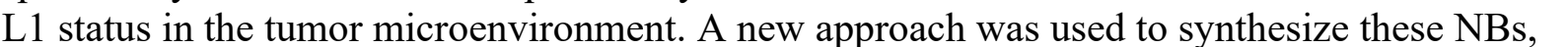

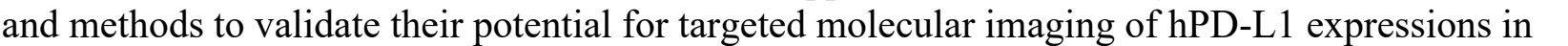

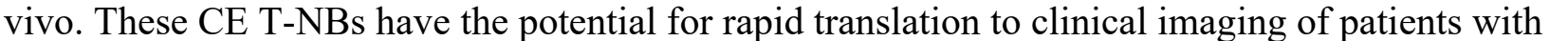

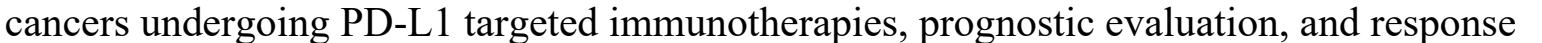

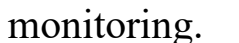




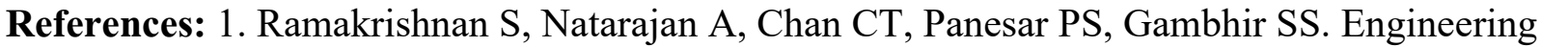

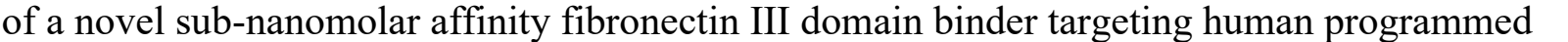

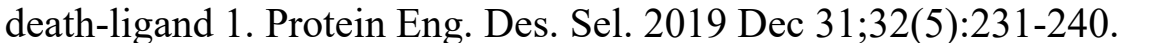

\section{Image/Figure:}

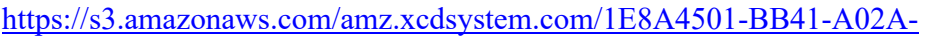

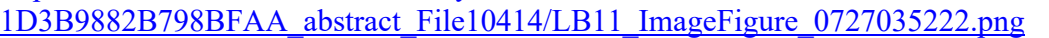

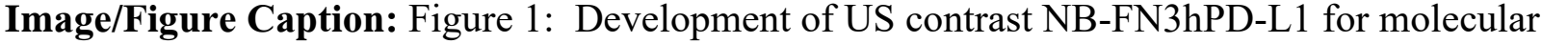

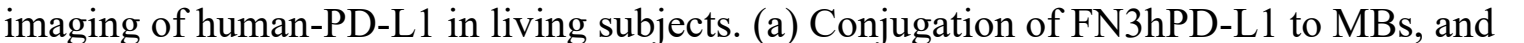

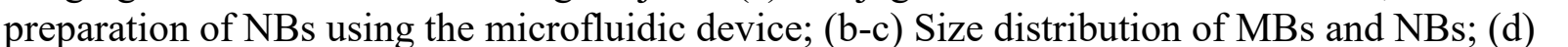

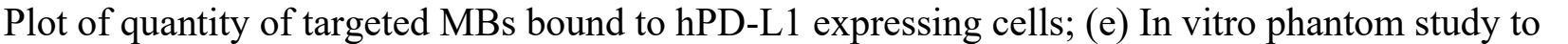

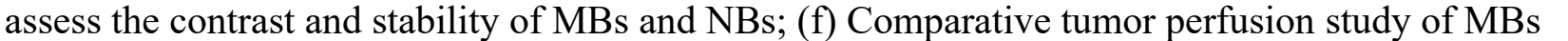

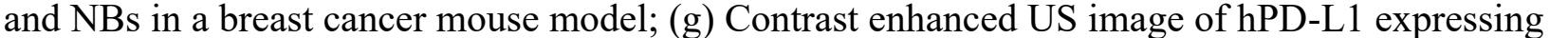

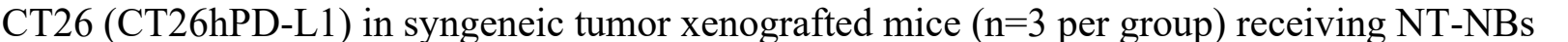
पाm

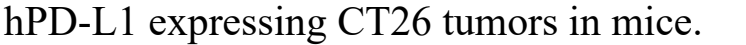

First Name: $\square \square\|\|\|\|\|\| \square$

Last Name: $\square \square\|\|\|\|\|\|$

Email: $\square\|\|\|\|\|\| \square \square \square\|\|\|\|\|\| \square$

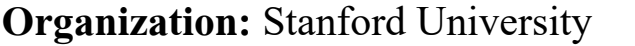

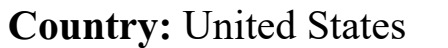




\title{
ID: LB12 \\ Interrogating the Metabolic Evolution of Glioblastoma by Hyperpolarized MRI, NMR Metabolomics and Artificial Intelligence (AI)
}

kang lin hsieh, University of Texas Health Science center at Houston, kang.lin.hsieh@uth.tmc.edu

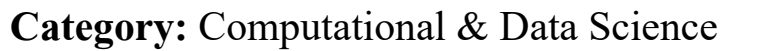

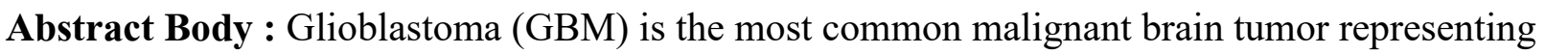

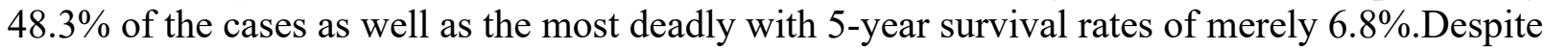

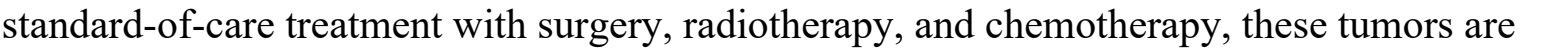

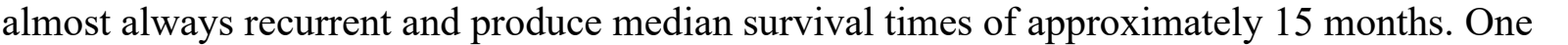

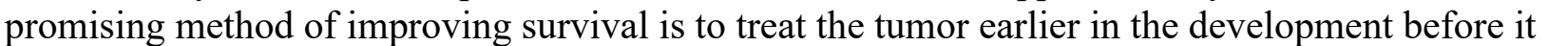

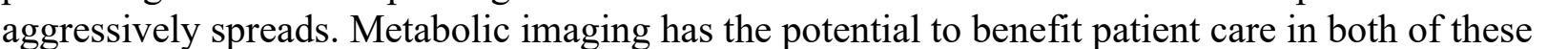

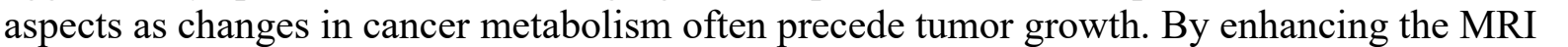

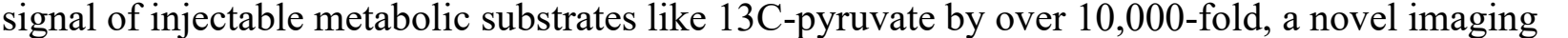

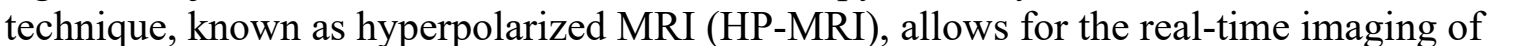

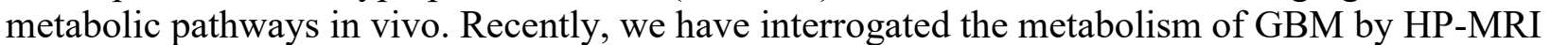

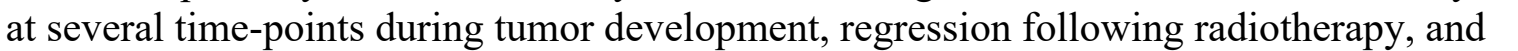

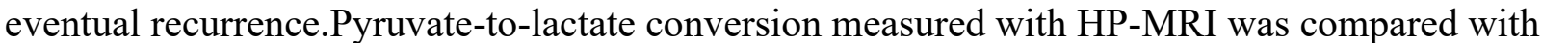

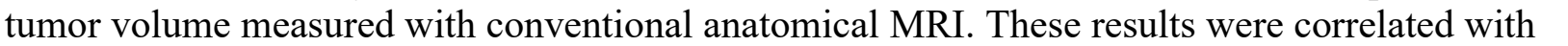

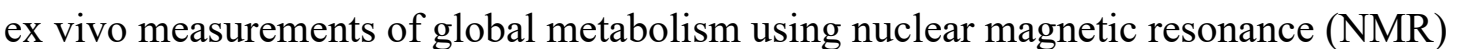

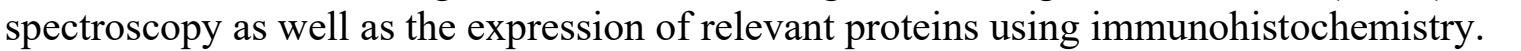

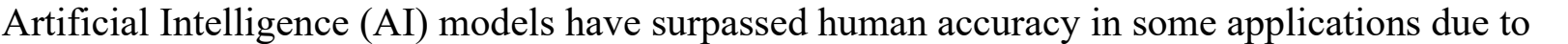

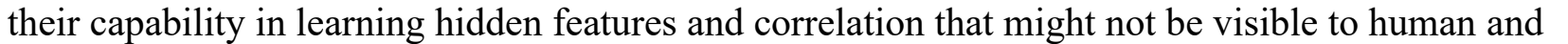

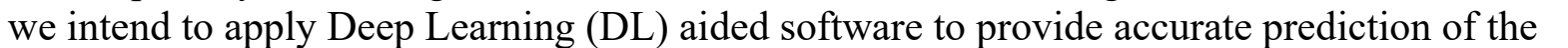

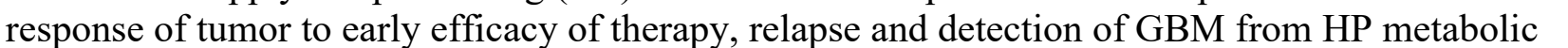

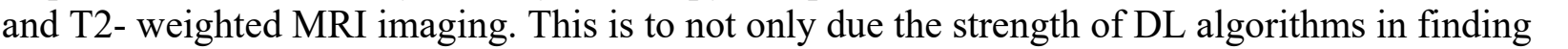

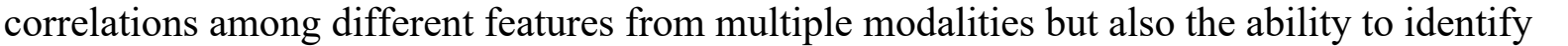

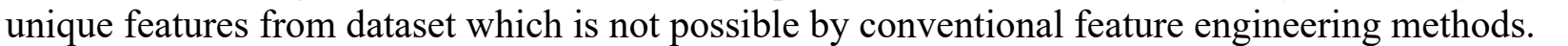

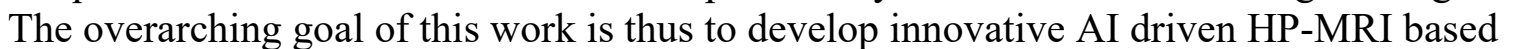

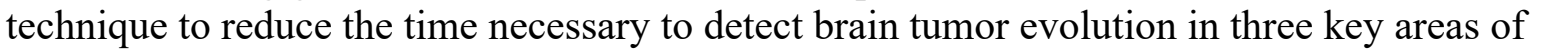

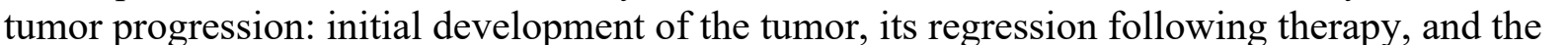

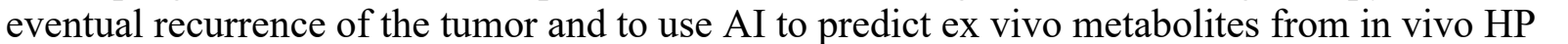

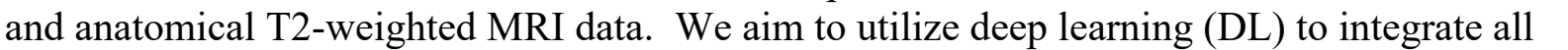

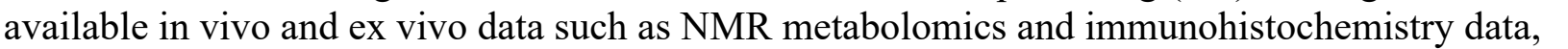

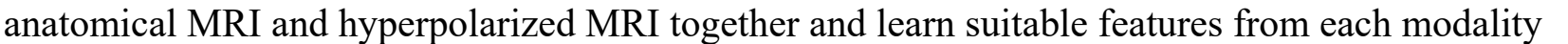

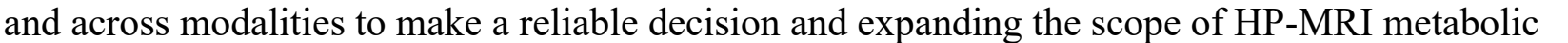

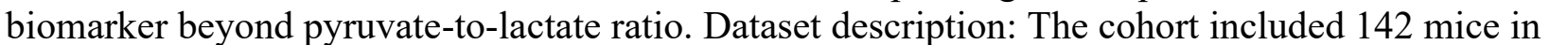

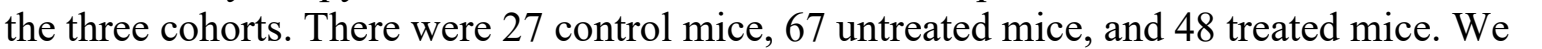

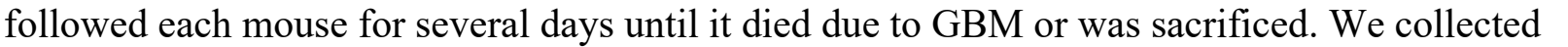

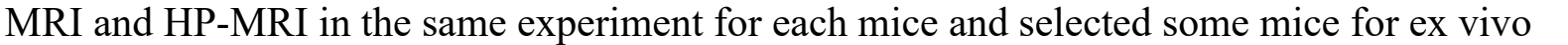

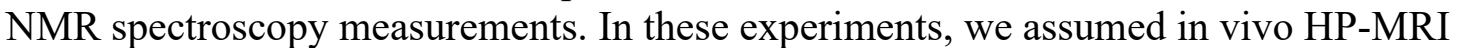




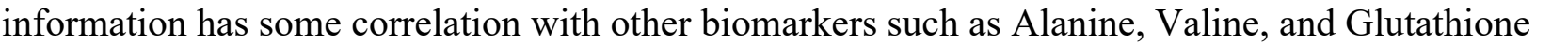

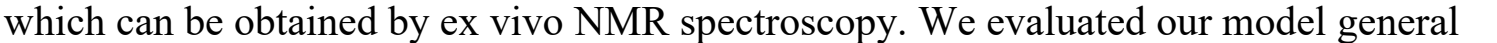

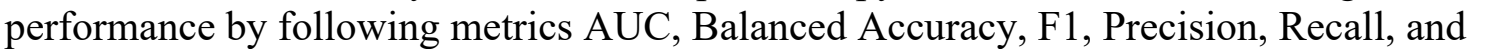

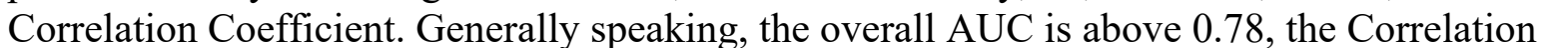

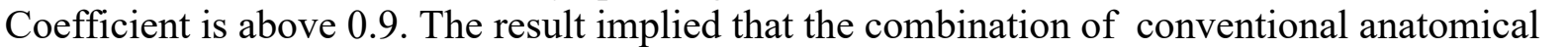

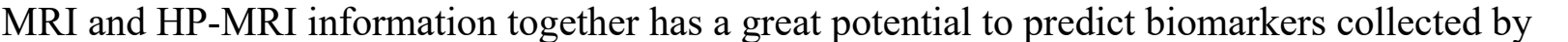

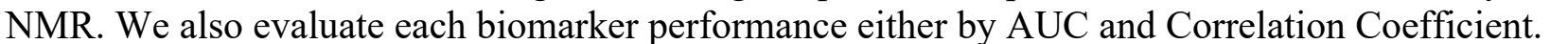

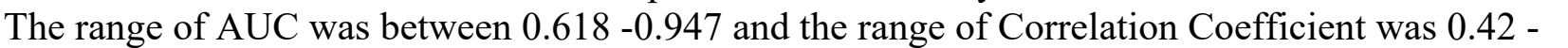

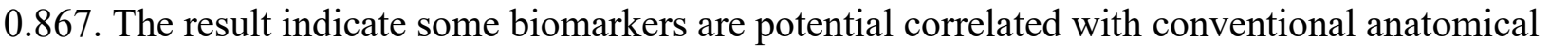

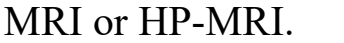

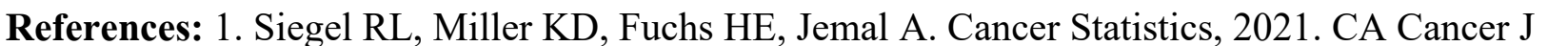

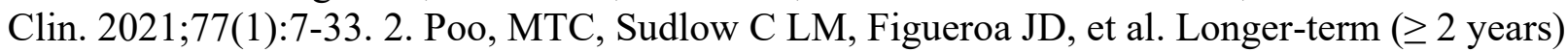

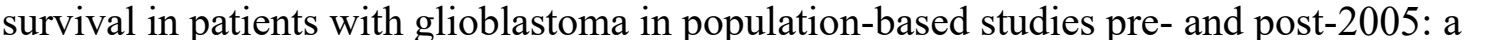

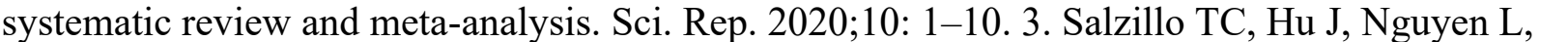

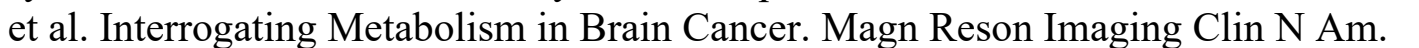

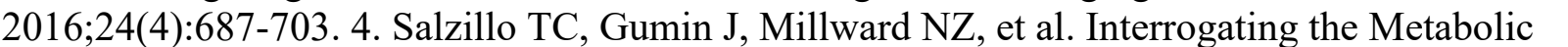

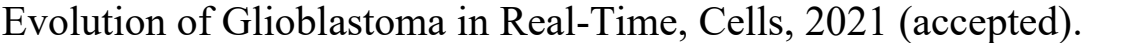

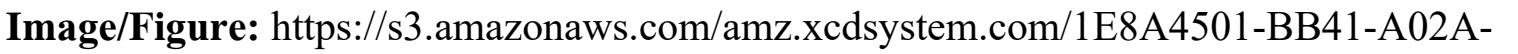

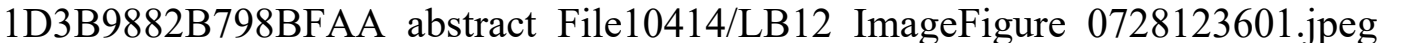

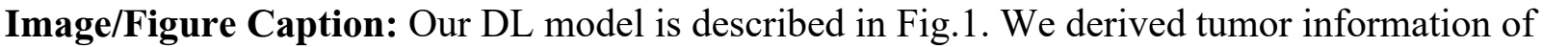

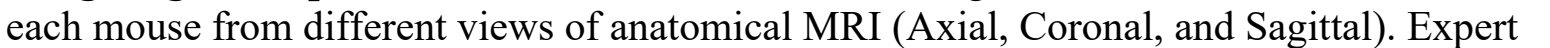

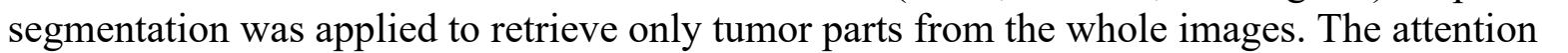
ए

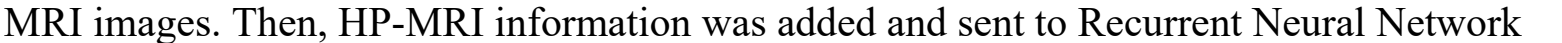

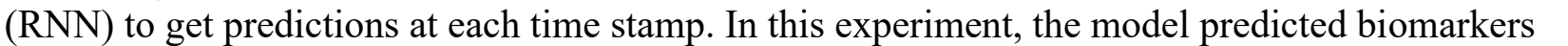

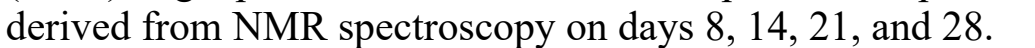

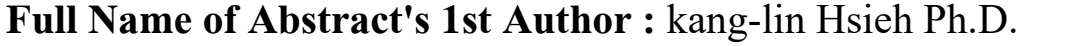

First Name: $\square\|\square\| m \mid m$

Last Name: $\square\|\|\|\|$

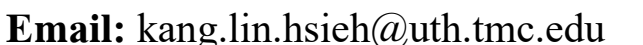

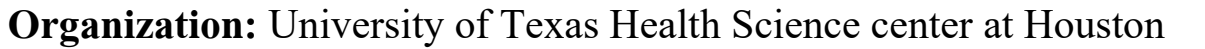

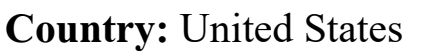




\title{
ID: LB13 \\ Evaluation of Glycans and Mucins as Targets for Molecular Imaging of \\ Pancreatic Ductal Adenocarcinoma: An Immunohistochemical Study
}

\author{
Ruben Houvast, Leiden University Medical Center, r.d.houvast@lumc.nl
}

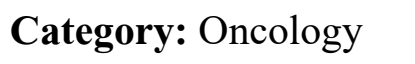

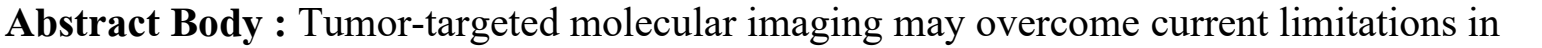

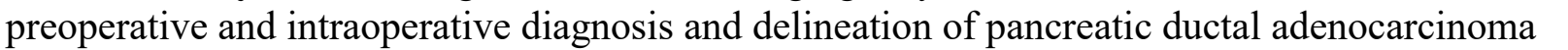

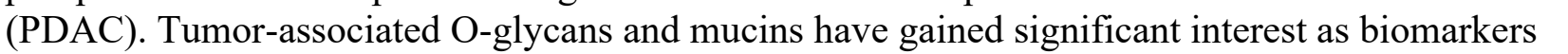

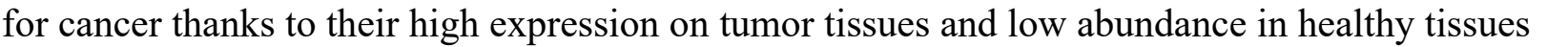

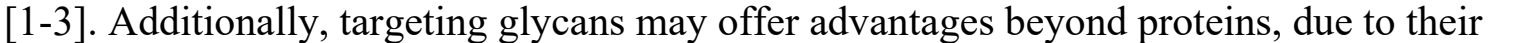

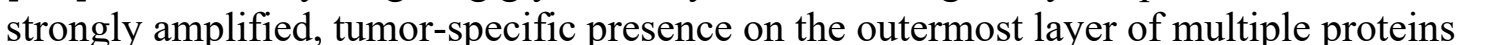

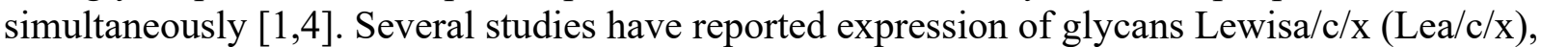

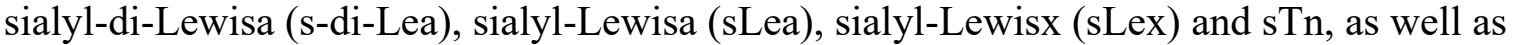

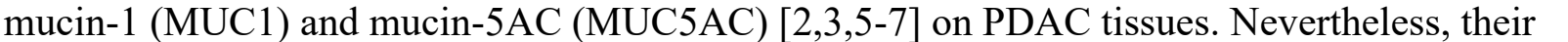

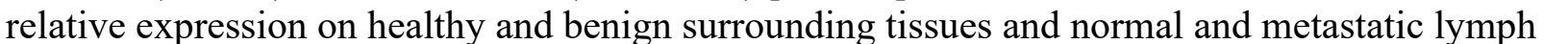

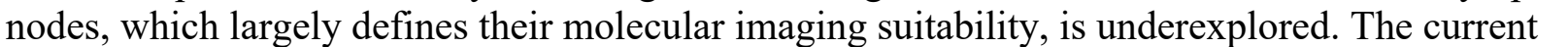

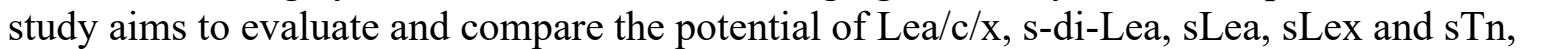

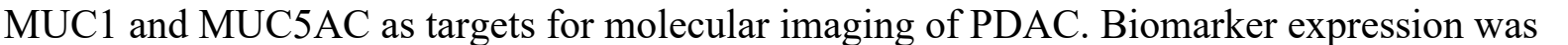

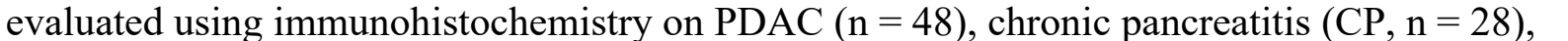

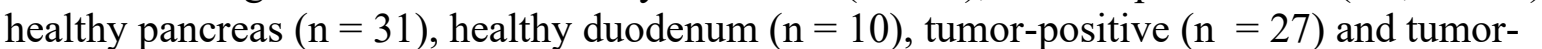

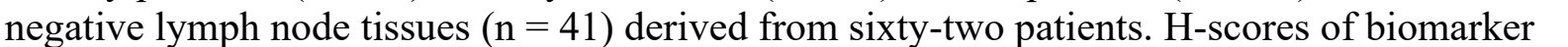

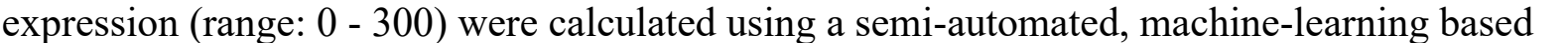

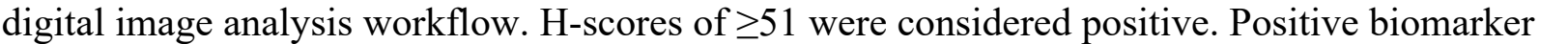

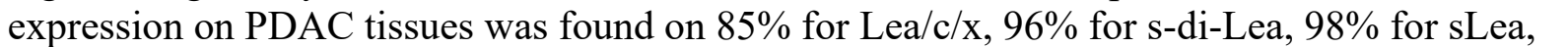

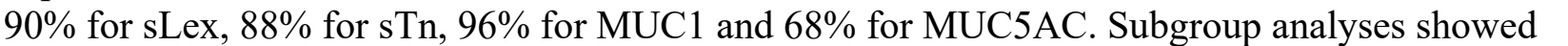
ए

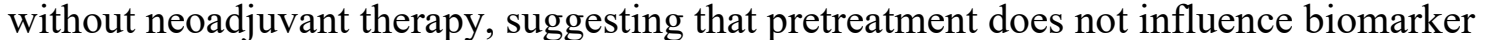

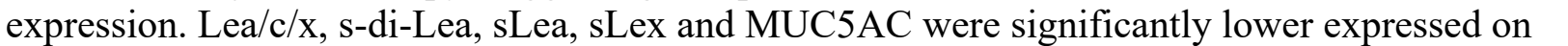

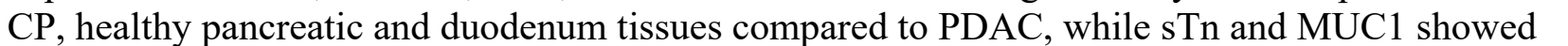

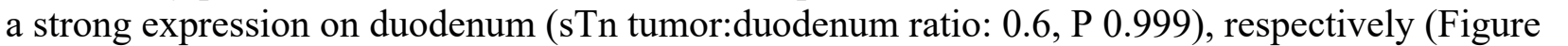

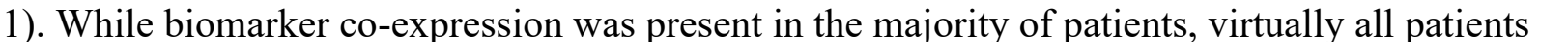

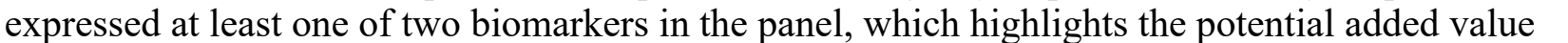

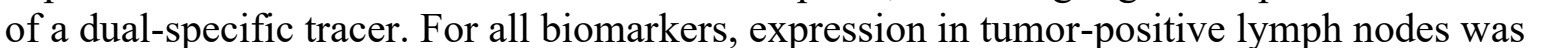

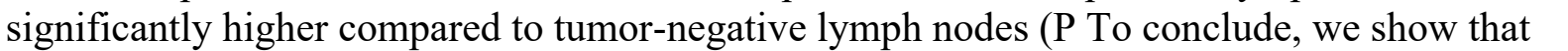

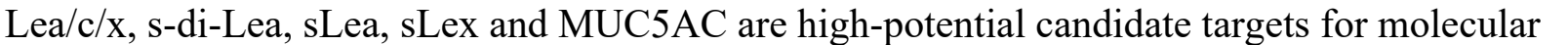

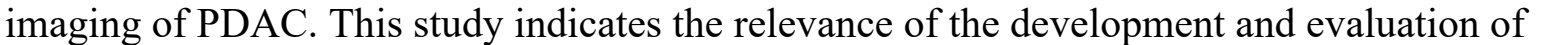

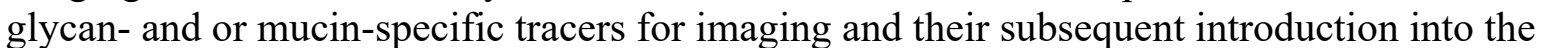
पाणाणाण
\end{abstract}

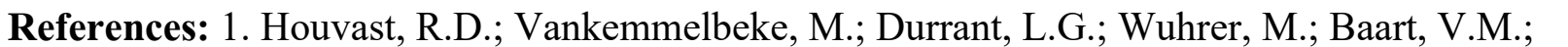

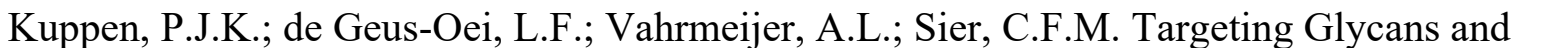




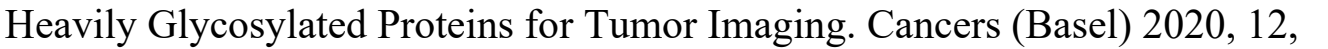

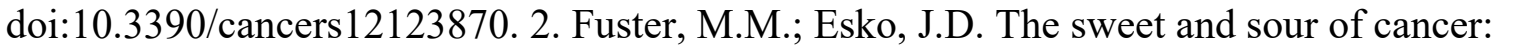

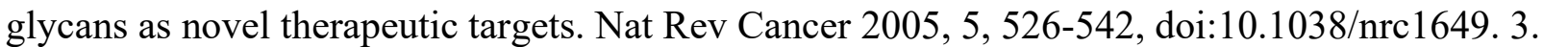

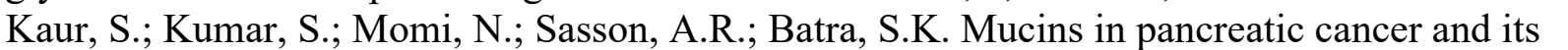

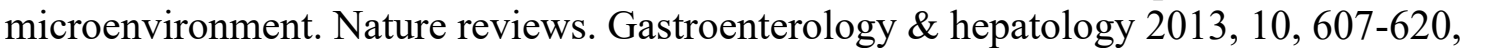

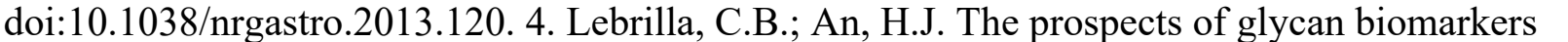

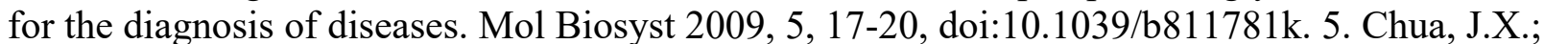

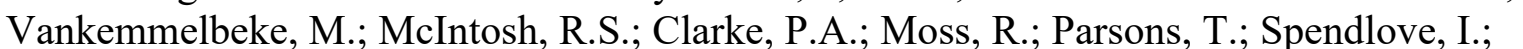

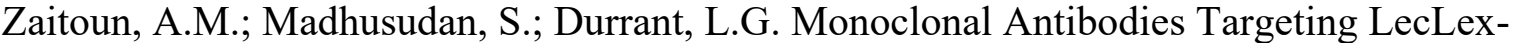

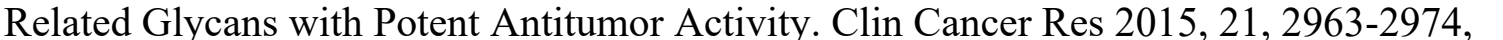

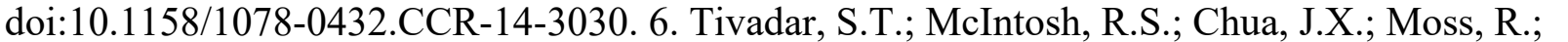

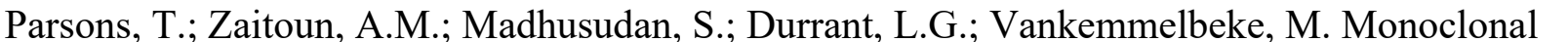

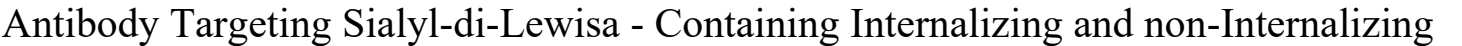

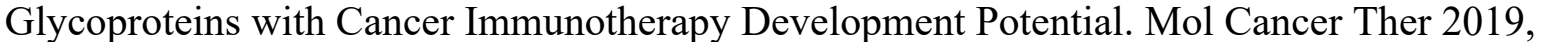

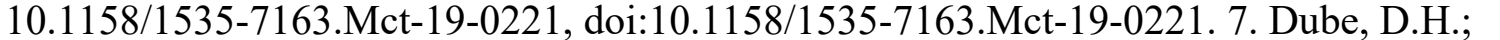

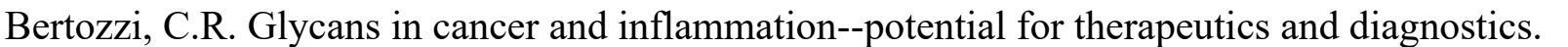

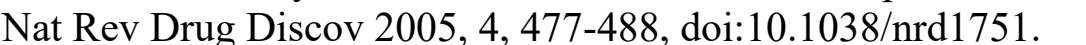

\section{Image/Figure:}

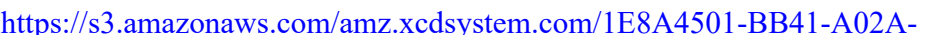

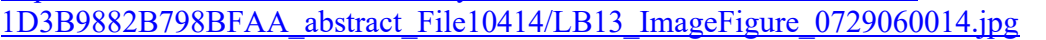

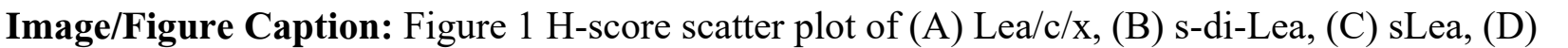

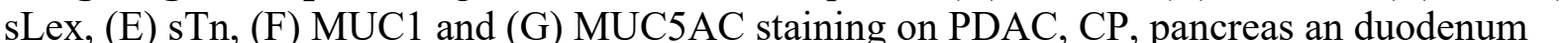

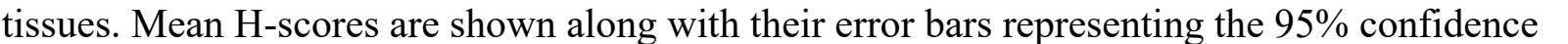

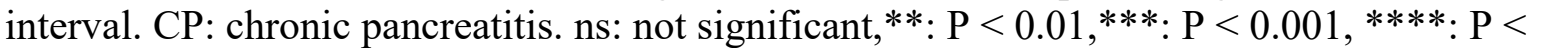

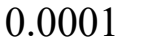

Full Name of Abstract's 1st Author : $\square \square \square\|\square \square \square \square \square\| \square$

First Name: $\square \square \square \| \square$

Last Name: $\square \square \square \square\|\| \Pi$

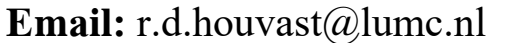

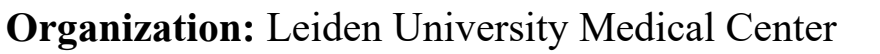

Country: $\square \square\|\square \mid\|\|\| \| \square$ 


\title{
ID: LB14 \\ In vivo multimodal imaging of NASH disease progress in a mouse model \\ Jen-Chieh Tseng, PerkinEImer Inc., Jen-Chieh.Tseng@PERKINELMER.COM
}

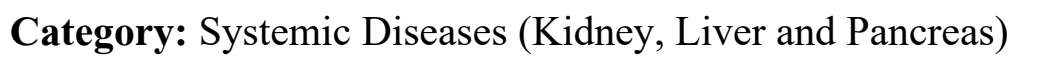

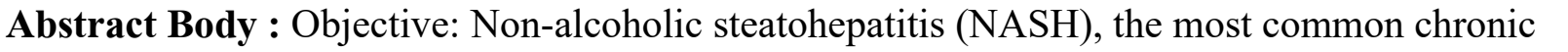

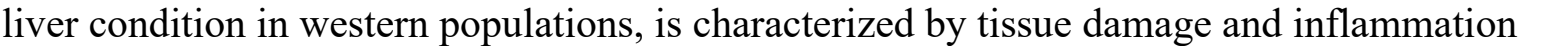

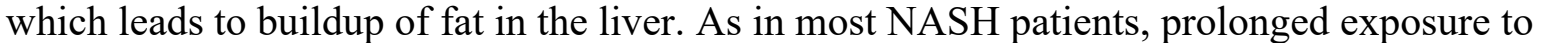

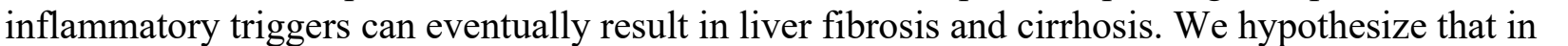

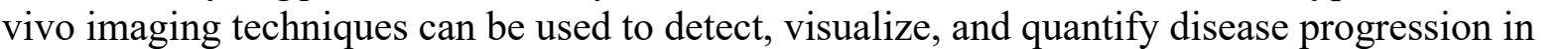

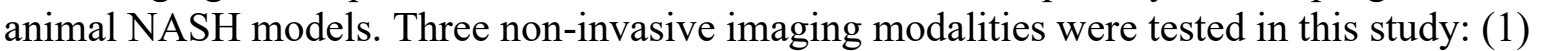

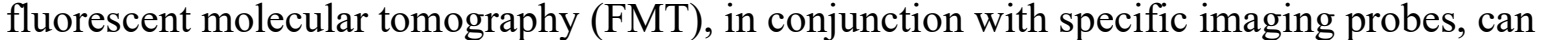

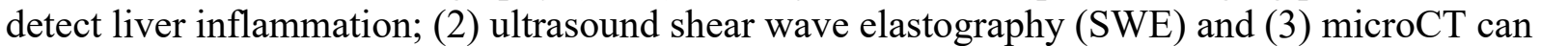

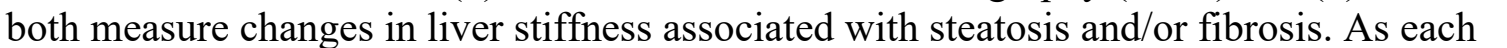
ए

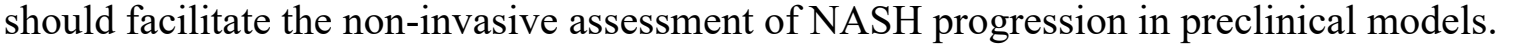

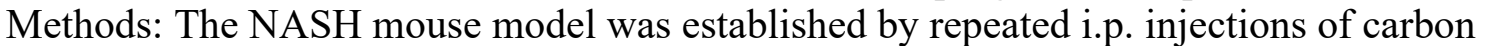

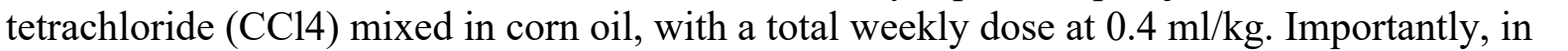

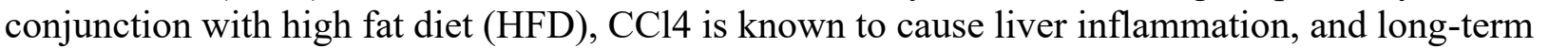

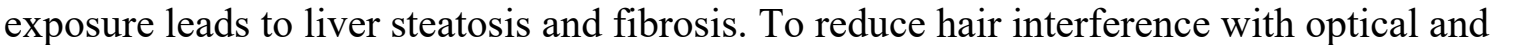

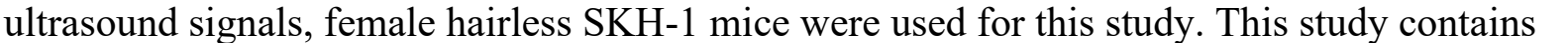

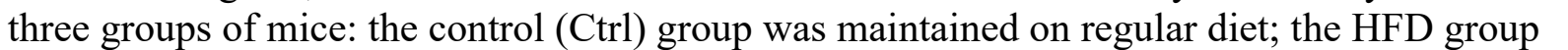

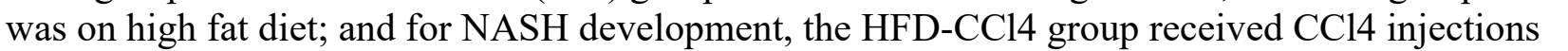

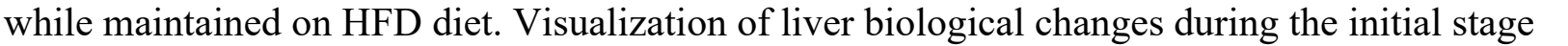

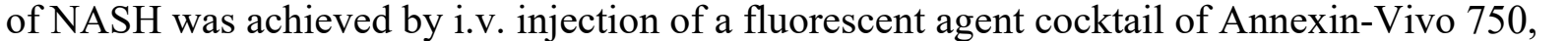

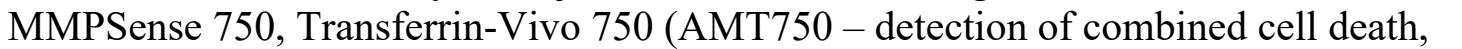

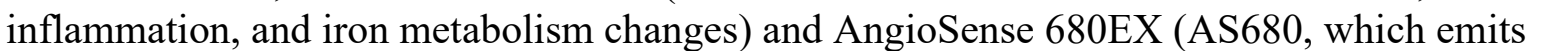

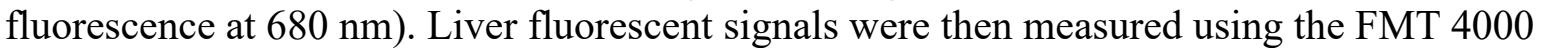

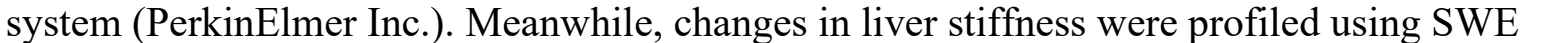

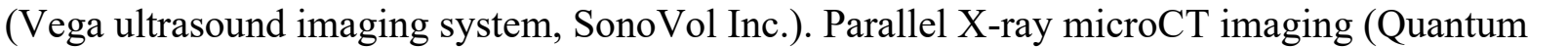

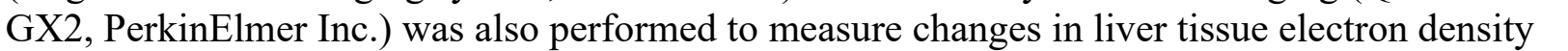

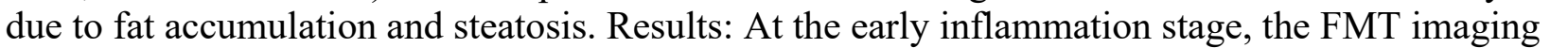

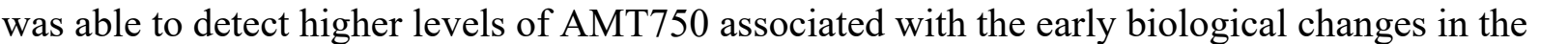

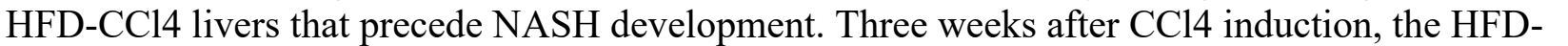

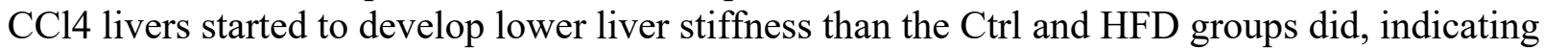

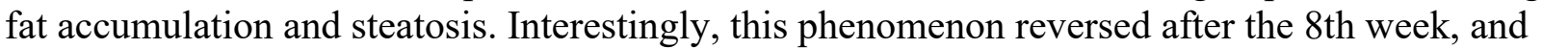

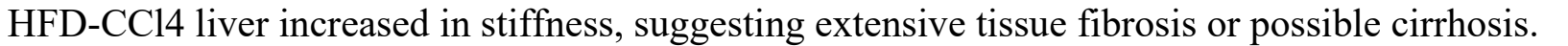

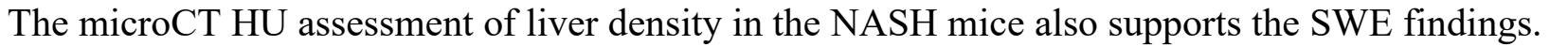

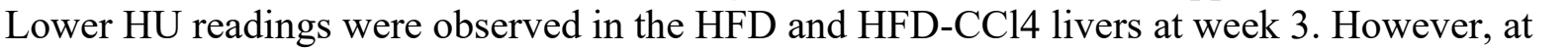

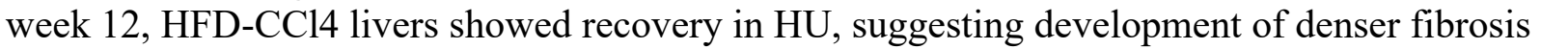

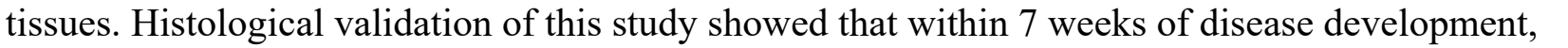

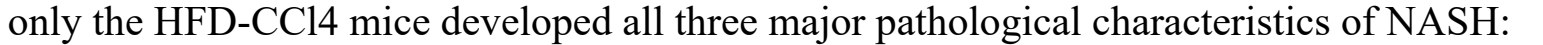




\section{Image/Figure:}

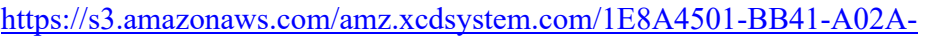

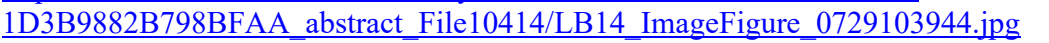

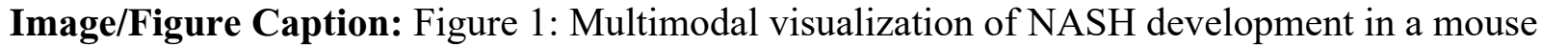

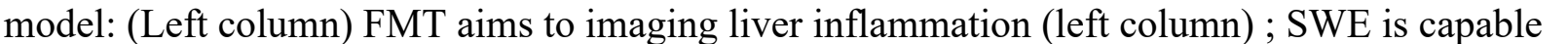

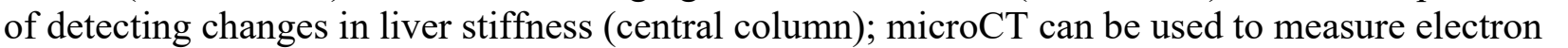

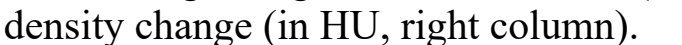

First Name: $\square \square\|\| \square \square \| \square$

Last Name: $\square \square\|\| \square$

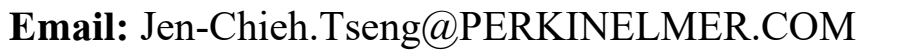

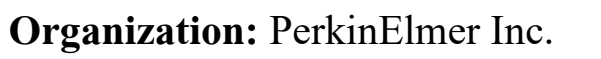

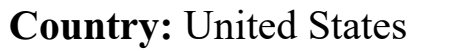




\title{
ID: LB15 \\ Using non-invasive fluorescence and ultrasound imaging to assess the biological and functional cardiotoxic effects of 5-fluorouracil in mice
}

Jen-Chieh Tseng, PerkinEImer Inc., Jen-Chieh.Tseng@PERKINELMER.COM

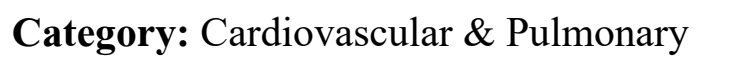

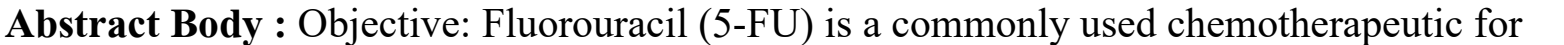

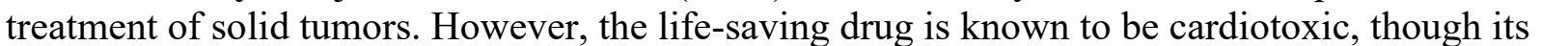

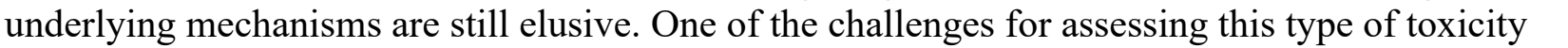

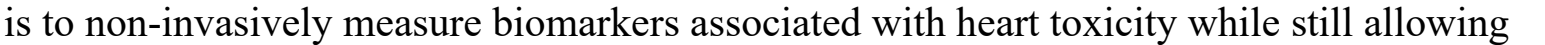

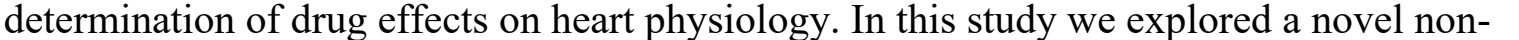

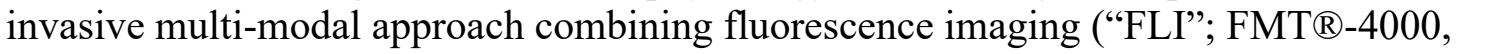

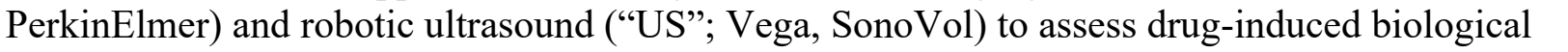

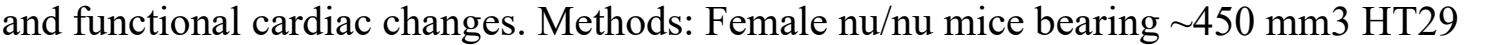

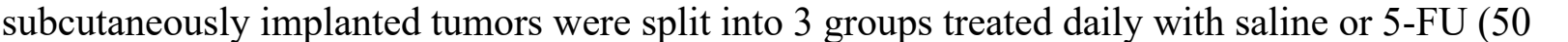

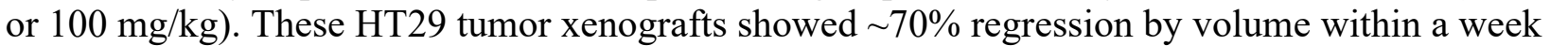

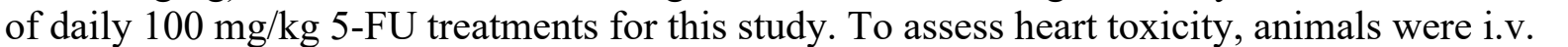

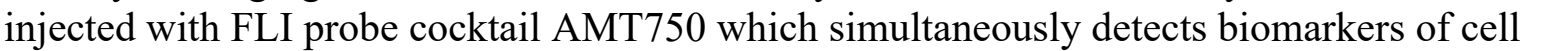

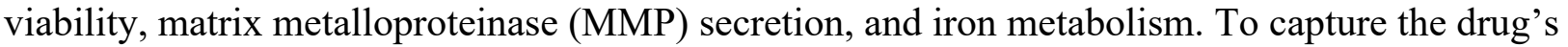

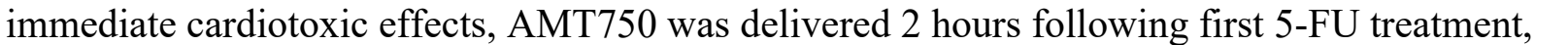

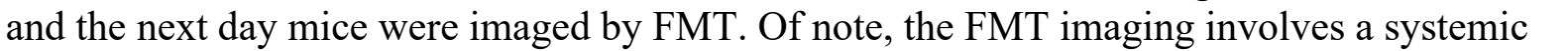

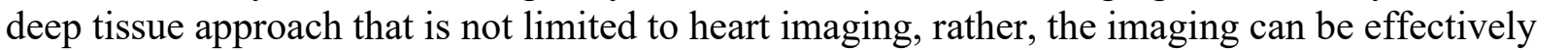

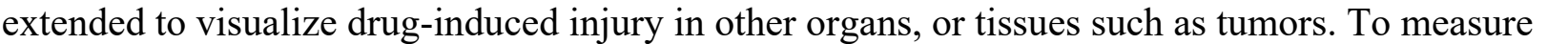
ए।

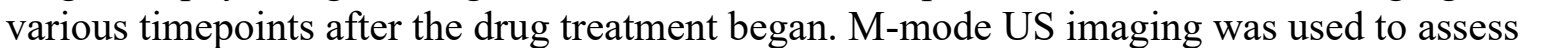

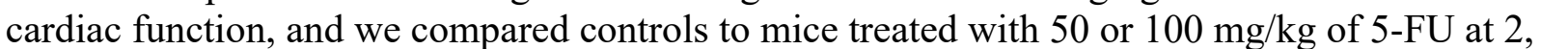

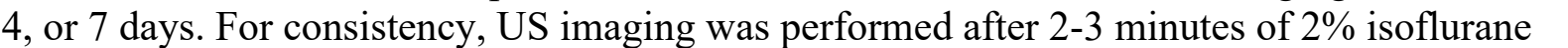

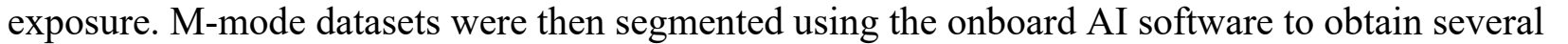

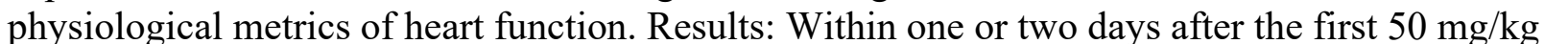

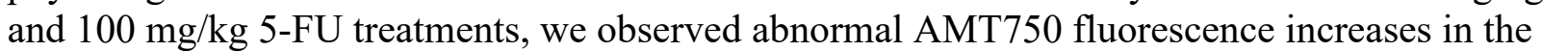
ए

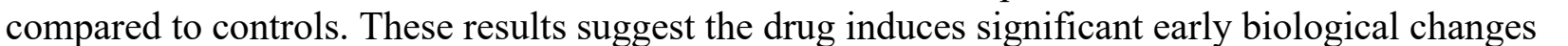

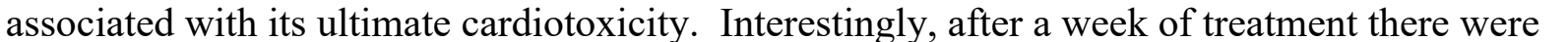

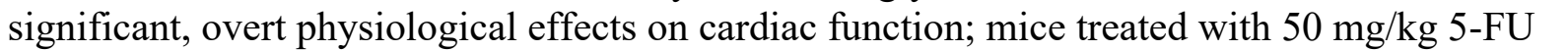

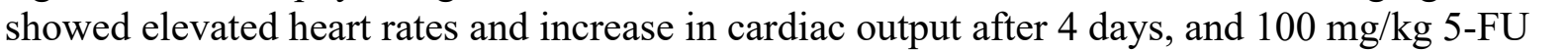

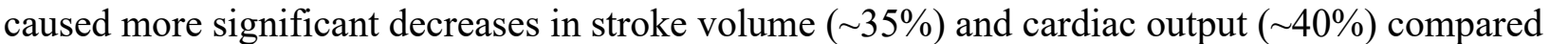

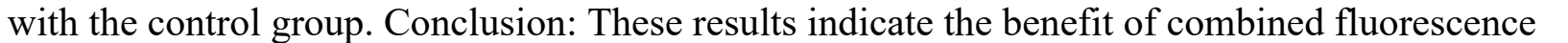

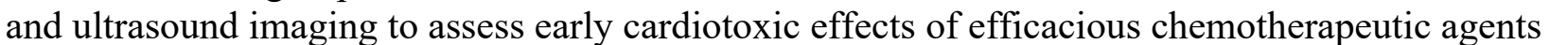

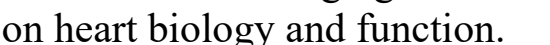

\section{Image/Figure:}




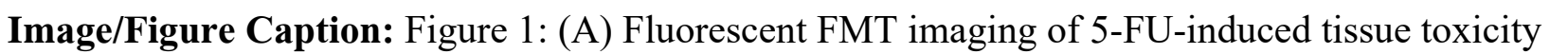

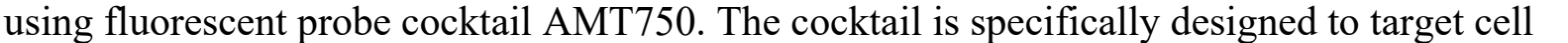

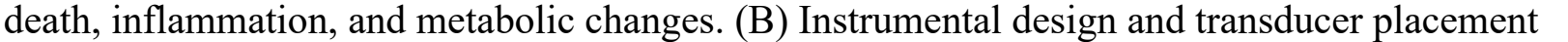

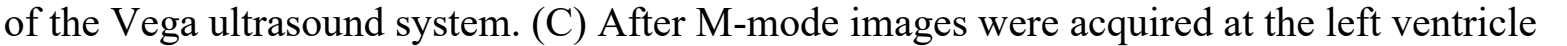

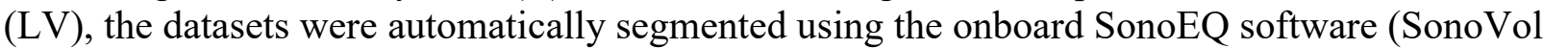

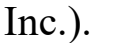

First Name: $\square \square\|\| \square \square \| \square$

Last Name: $\square \square\|\| \| \square$

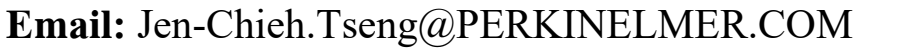

Organization: $\square \square\|\| \| \square \square \square \square \square \square \square \square$

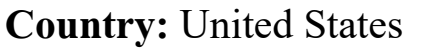




\title{
ID: LB16 \\ A novel SPECT radioimmunoimaging tracer for HER2 expressing Breast \\ Cancer
}

\author{
Sneha Mithun, Tata Memorial Hospital, snehacnair@gmail.com
}

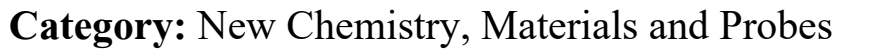

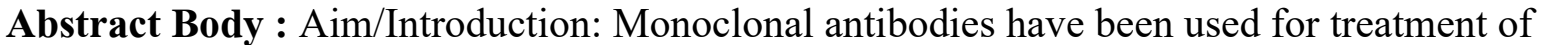

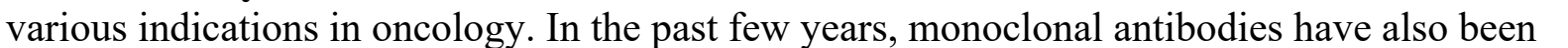
ए

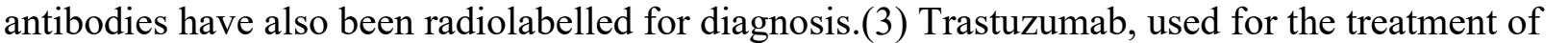

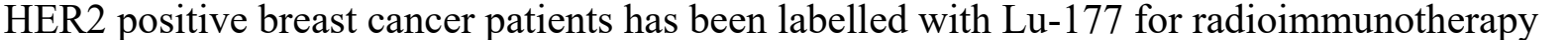

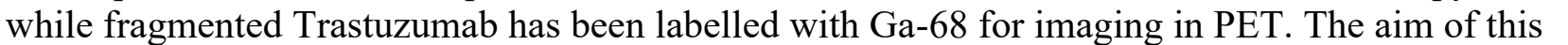

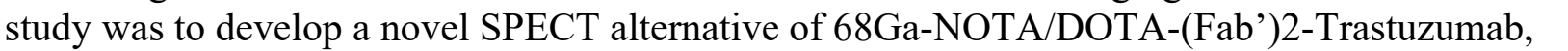

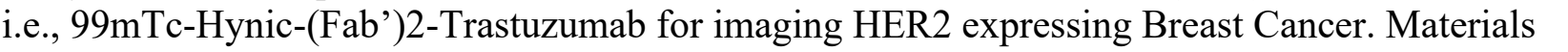

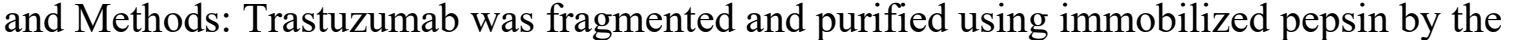

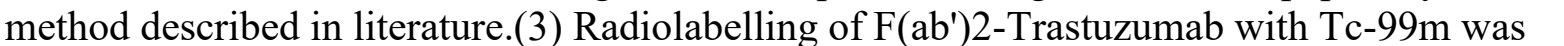

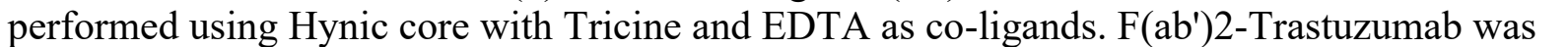

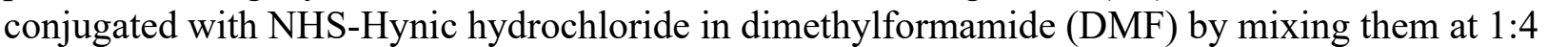

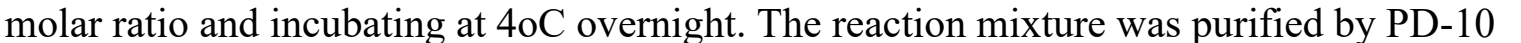

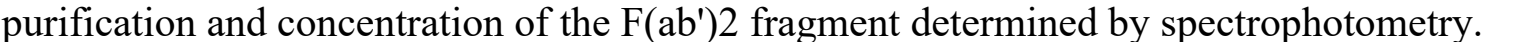

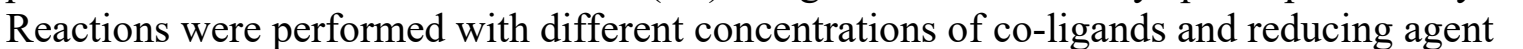

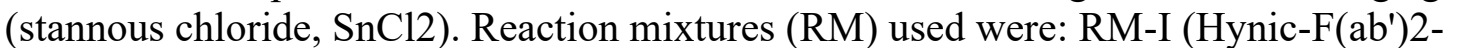

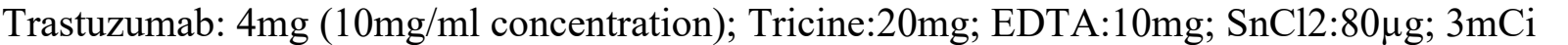

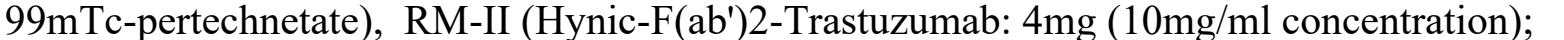

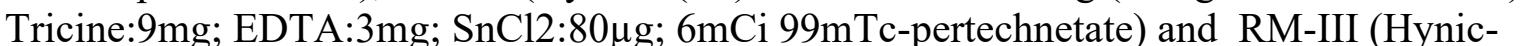

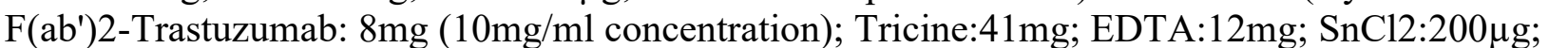

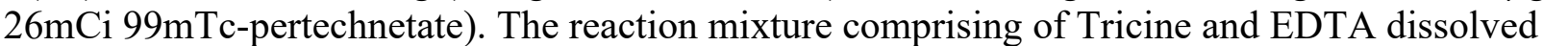
ए

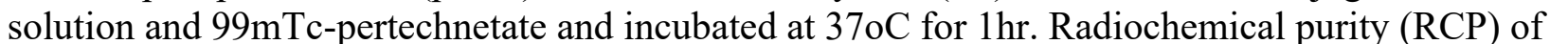
ए

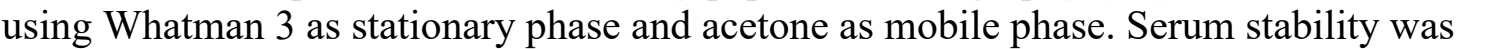

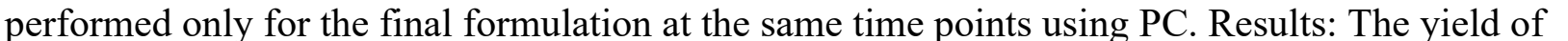

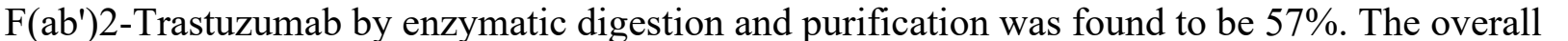

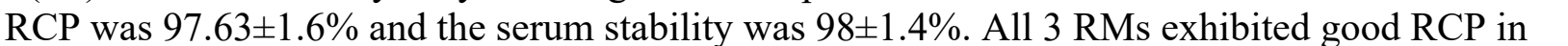

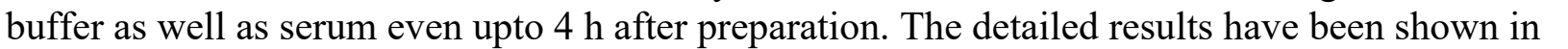

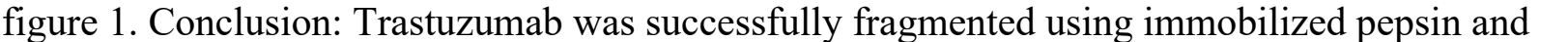

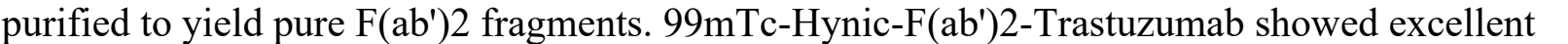

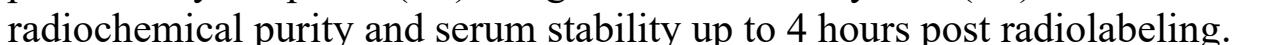

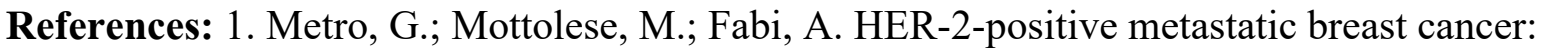

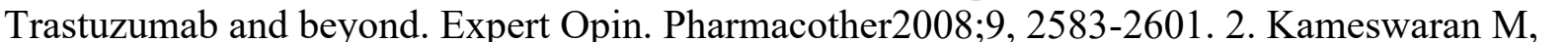

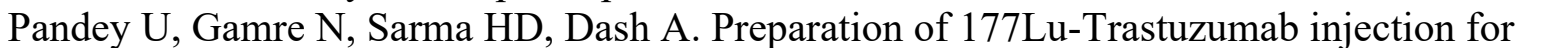




\section{Image/Figure:}

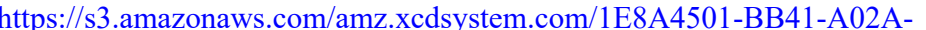

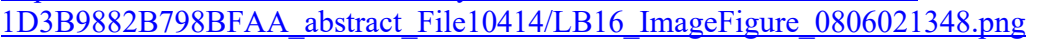

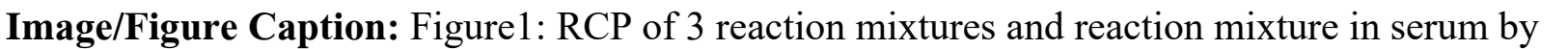

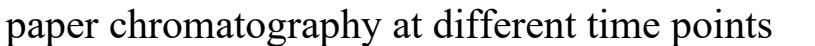

First Name: $\square \square \square \square \square$

Last Name: $\square \square \| \square \mid \square$

Email: $\square\|\|\|\|\|\|\|\square \square \square\| m \square \square$

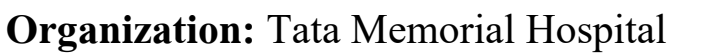

Country: $\square \square \| m$ 


\title{
ID: LB18 \\ [124I]-ATMi for in vivo PET imaging of ataxia telangiectasia mutated (ATM) kinase in mouse models of glioblastoma
}

Sheryl Roberts, Memorial Sloan Kettering Cancer Center, roberts3@mskcc.org

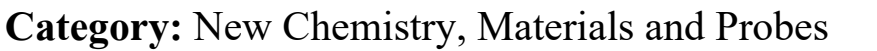

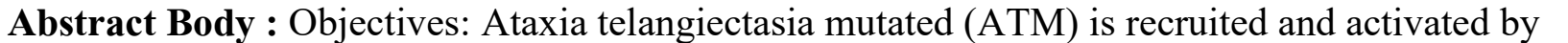

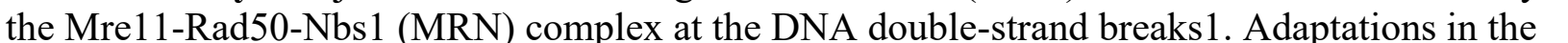
DDR pathway are hallmarks of cancer and ATM's regulatory role in DDR can be advantageous

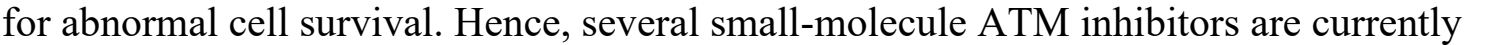

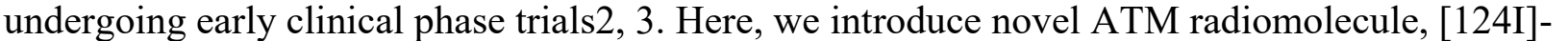

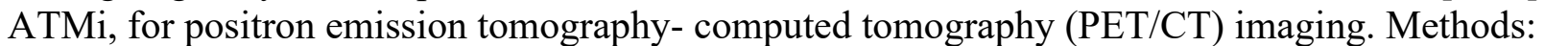

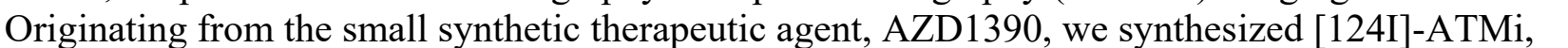

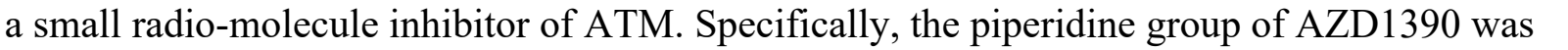

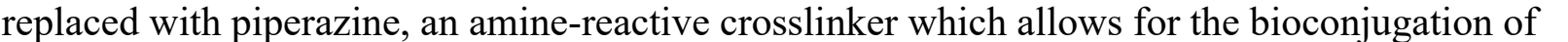

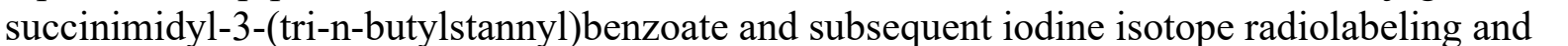

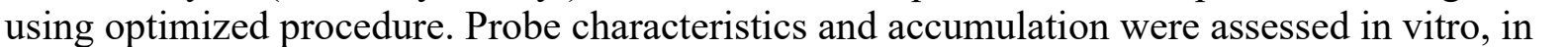

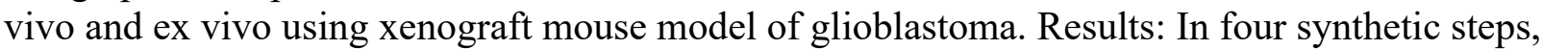

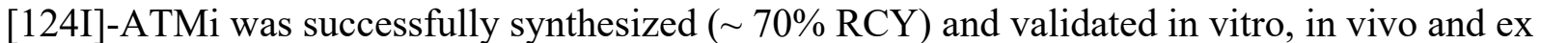

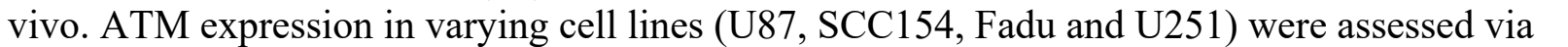

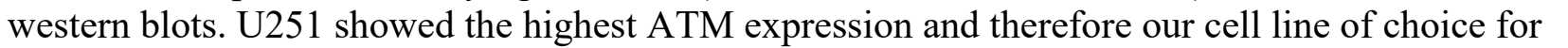

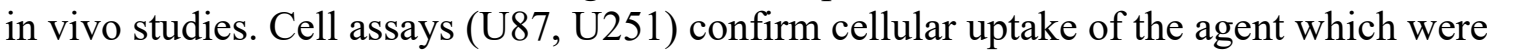
blockable $(20 \times)$. Ex vivo biodistribution, specificity and pharmacokinetics of were determined

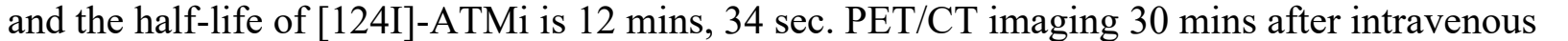
ए

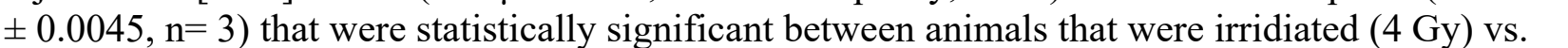

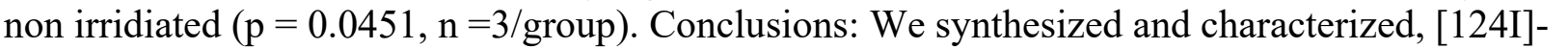

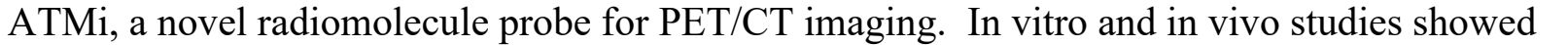
ए

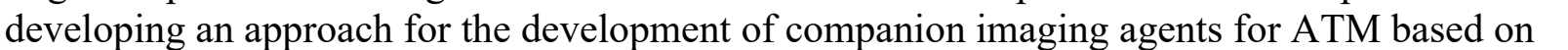

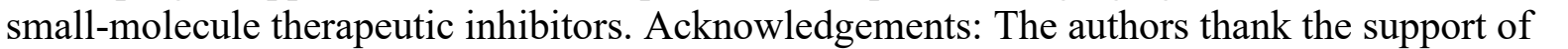

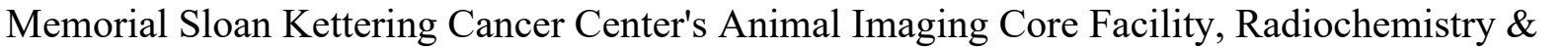

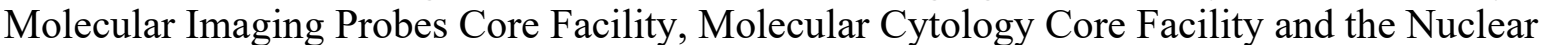

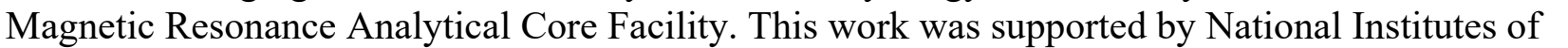

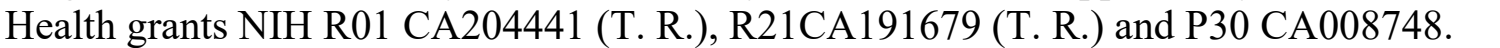

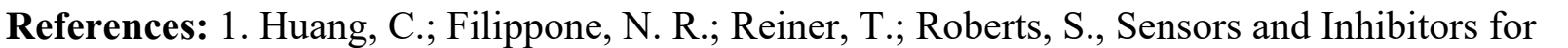

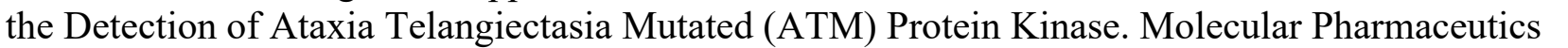

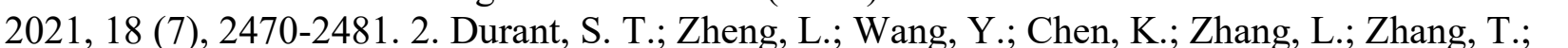

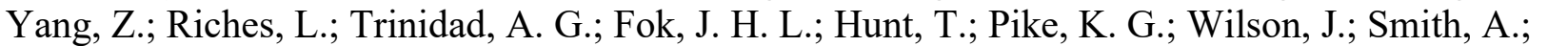

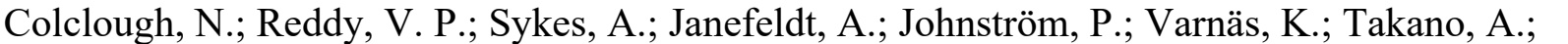

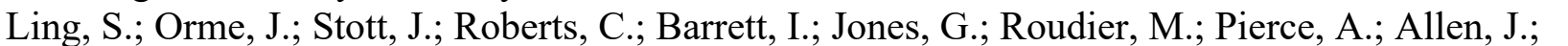




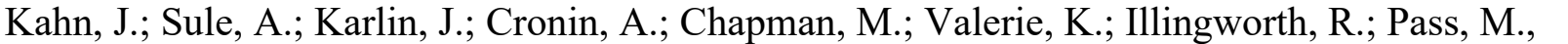

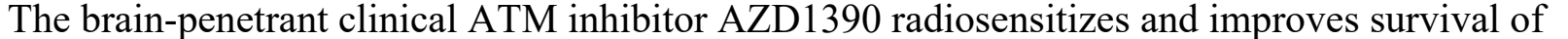

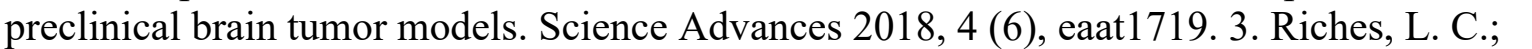

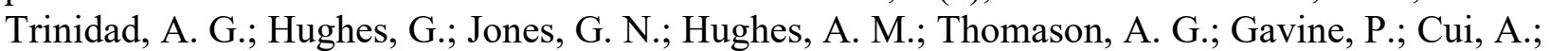

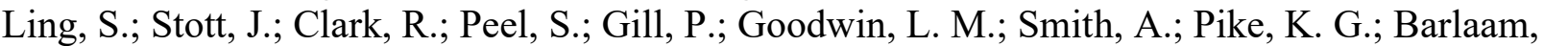

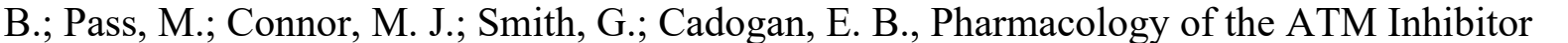

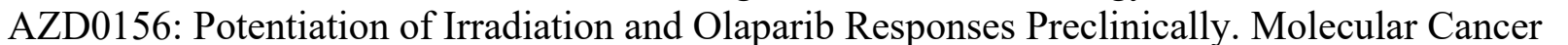

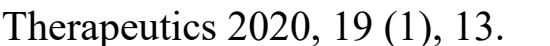

\section{Image/Figure:}

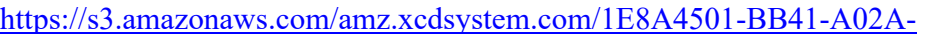

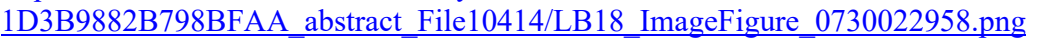

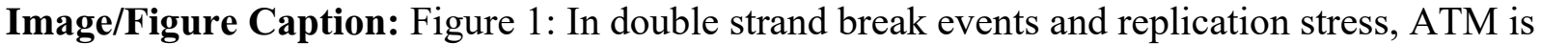

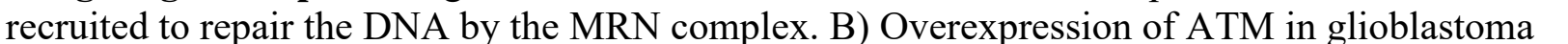

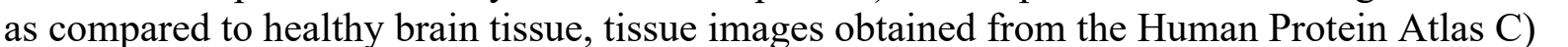

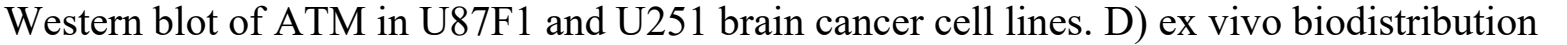

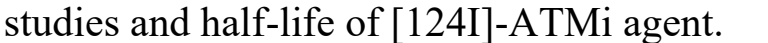

First Name: $\square \square \square \| \square \mid \square$

Last Name: $\square \square \square \square\|\| \|$

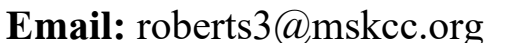

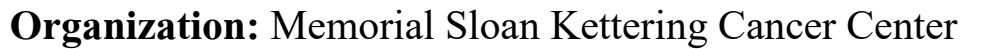

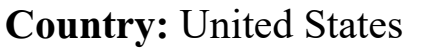




\title{
ID: LB19 \\ Focused ultrasound module for molecular imaging using a G4 Sofie $\mu$ PET system
}

\author{
Michael Campbell, Lakehead University, mike.campbell@LakeheadU.ca
}

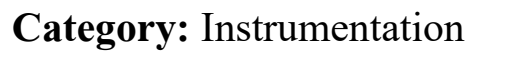

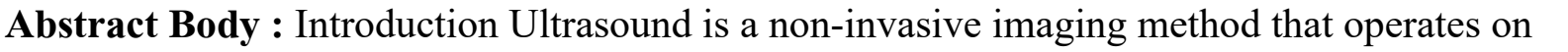

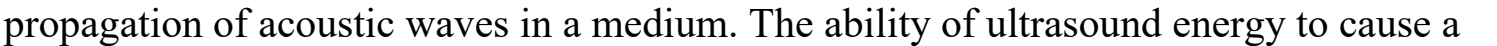

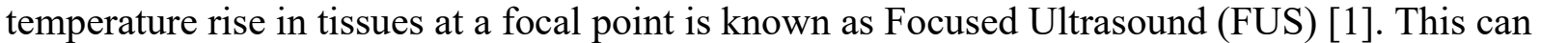

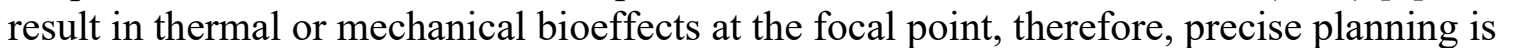

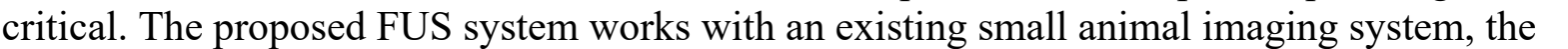

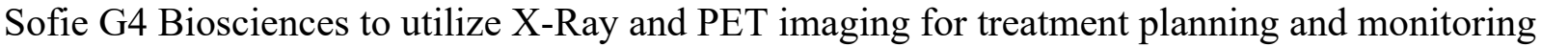

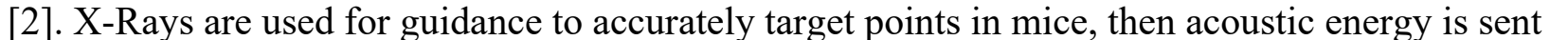

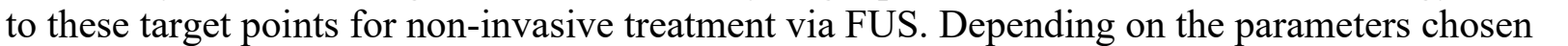

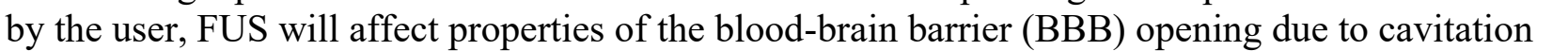

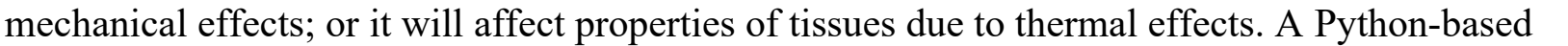

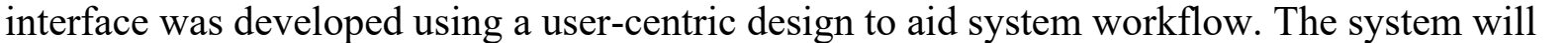

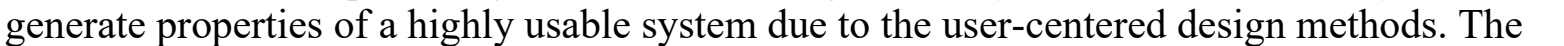

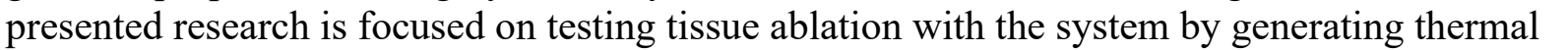

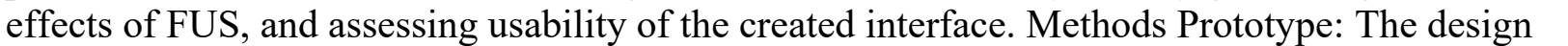

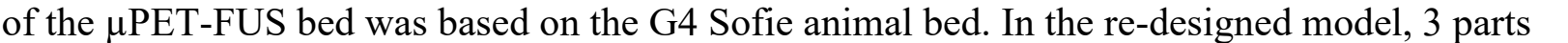

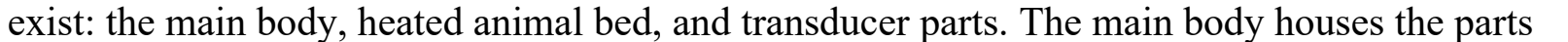

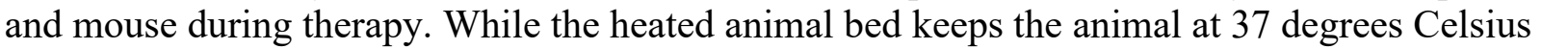

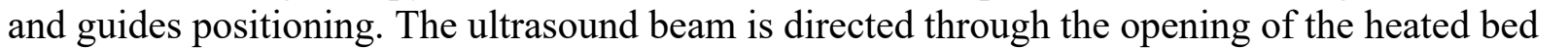

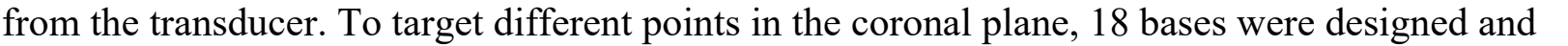

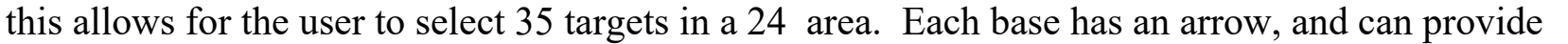

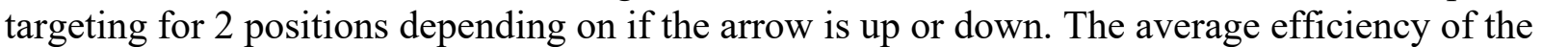

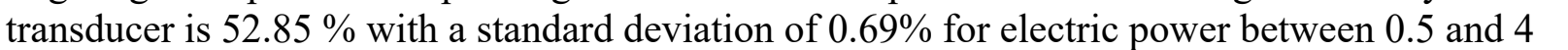

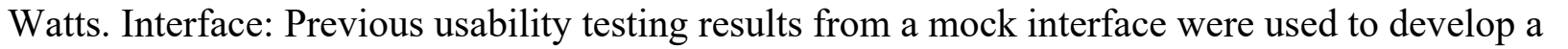

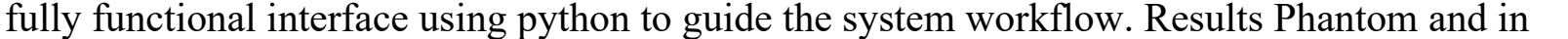

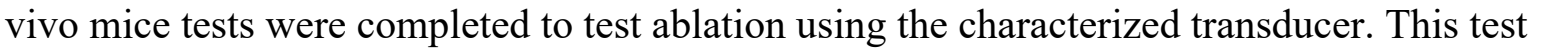
ए

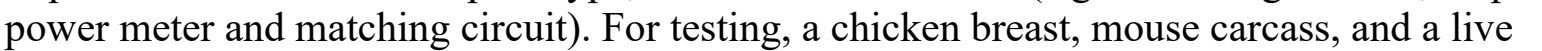

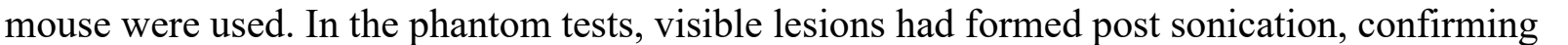

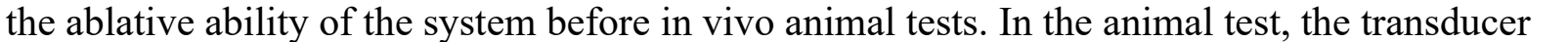

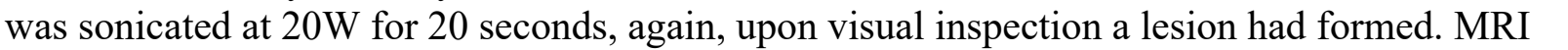
ए

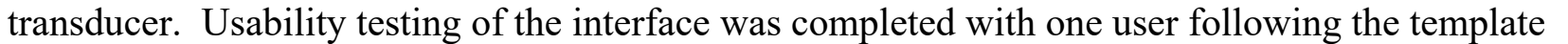

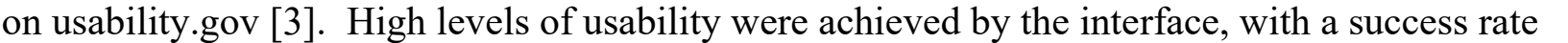

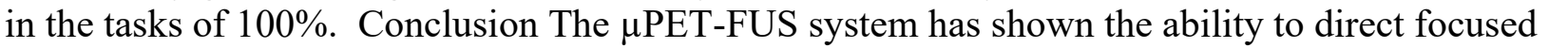

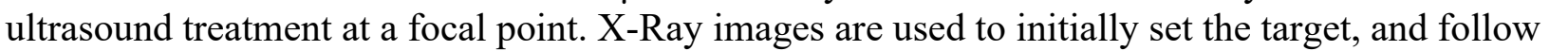

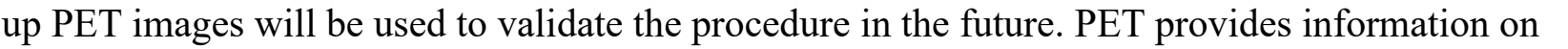

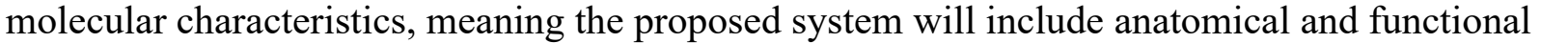


References: [1] W. J. Fry and F. J. Fry, "Fundamental Neurological Research and Human

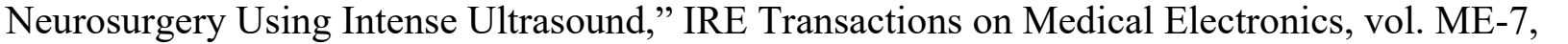

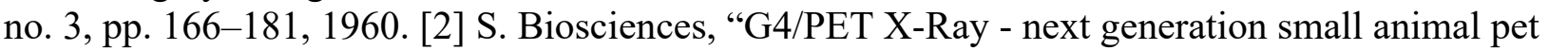
technology," 2014. [3] D. of Health and H. Services, "Usability.gov," Jul 2013. [Online].

\section{Image/Figure:}

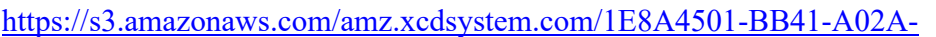

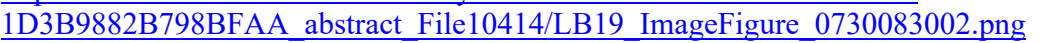

Image/Figure Caption:

First Name: $\square[\|\square\| 1 \|$

Last Name: $\square \square \square \square \square m$

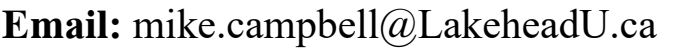

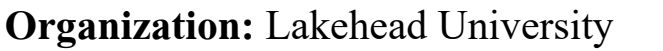

Country: $\square \square \square \square \square \|$ 


\title{
ID: LB20 \\ Quantitative MR imaging of liver fibrogenesis in nonalcoholic steatohepatitis (NASH)
}

\author{
Iris Zhou, Massachusetts General Hospital and Harvard Medical School, \\ iris.zhou@mgh.harvard.edu
}

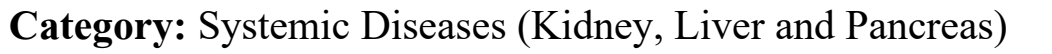

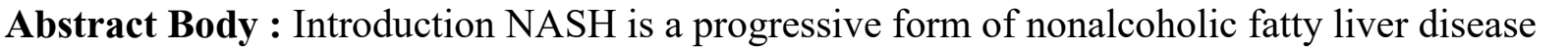

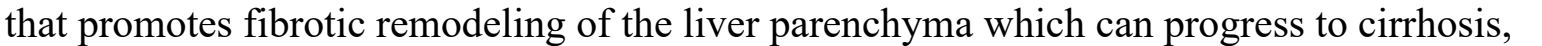

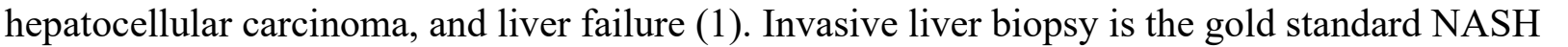

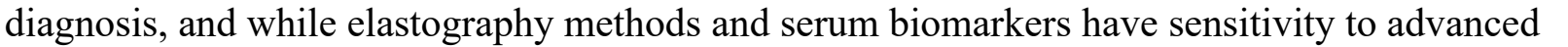

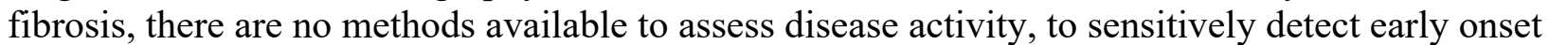

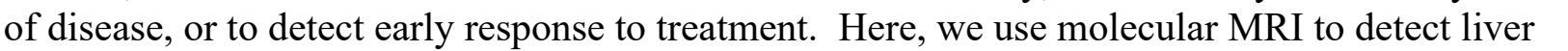

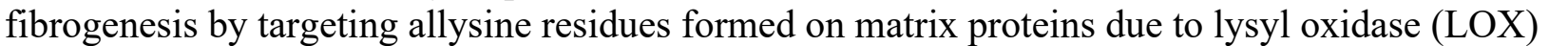

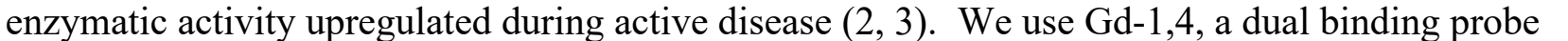

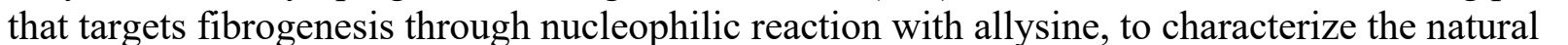

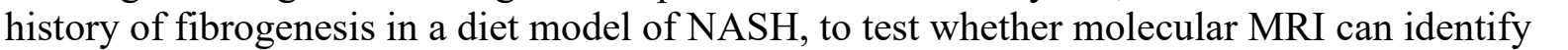

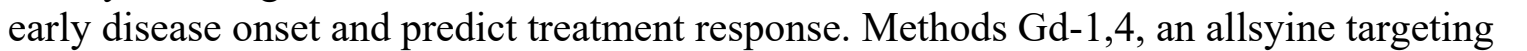

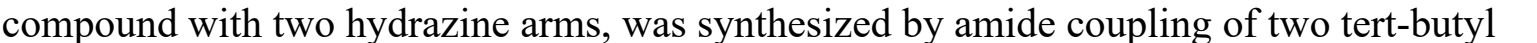

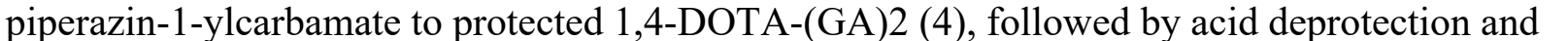

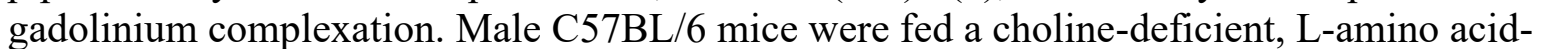

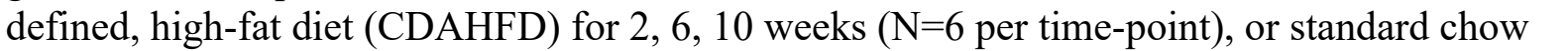

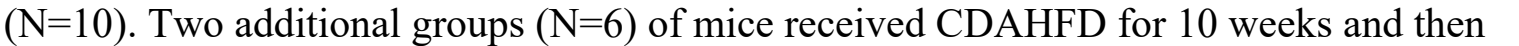

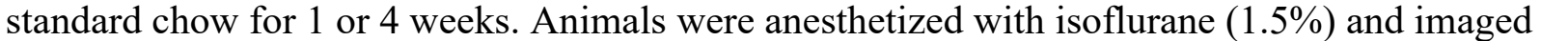

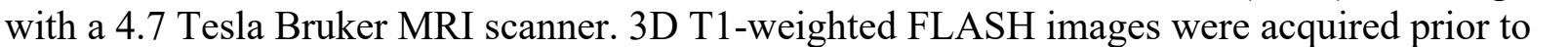

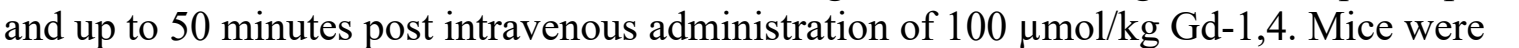

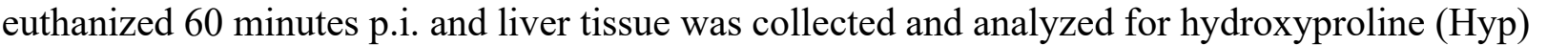

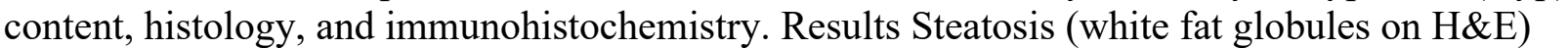

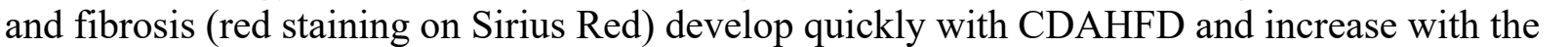

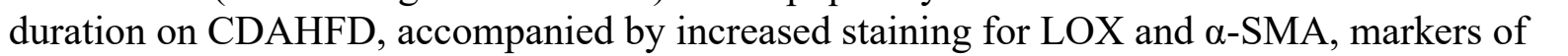

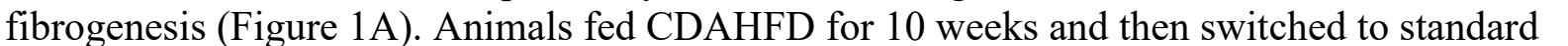

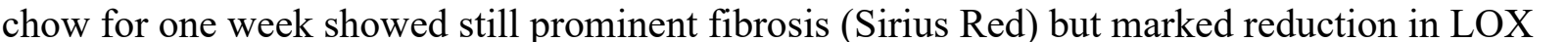

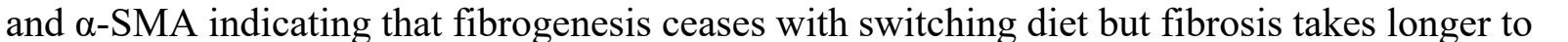

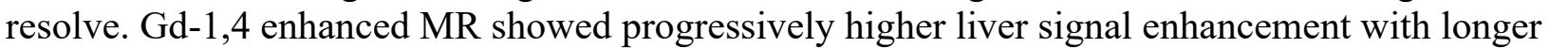

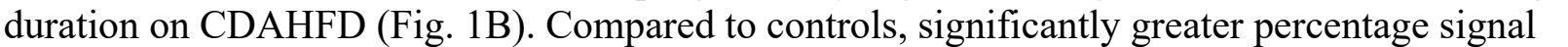

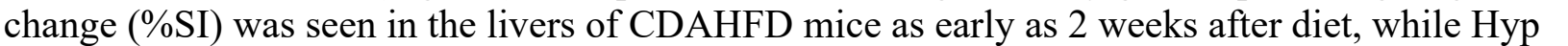

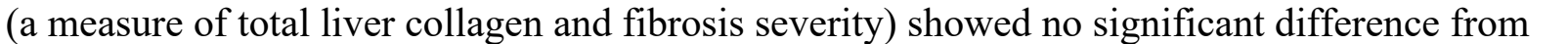

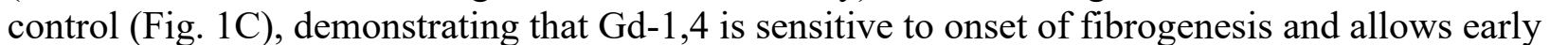

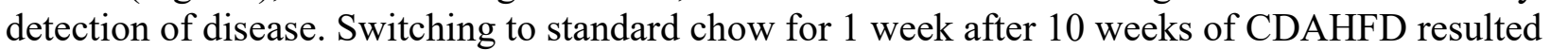

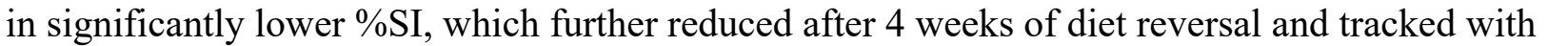

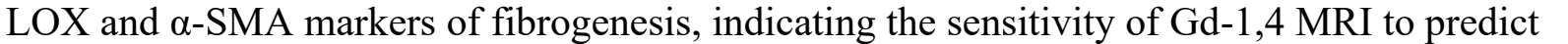

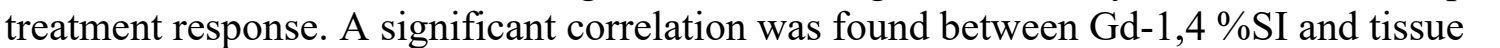




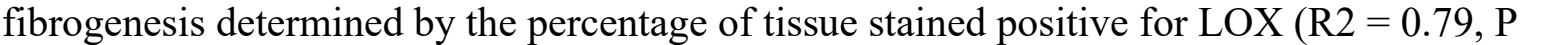

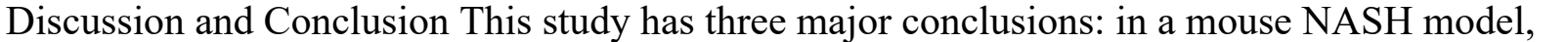

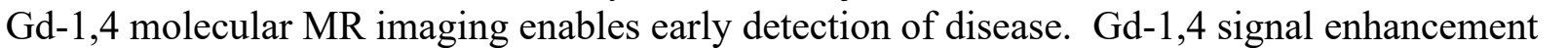

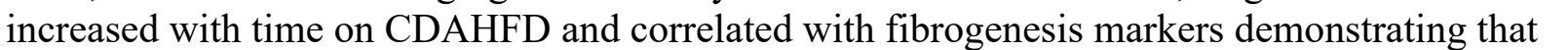

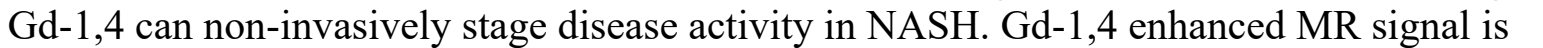

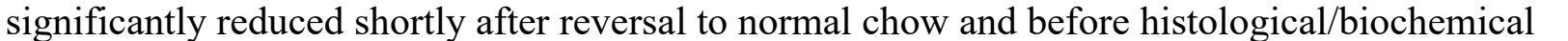

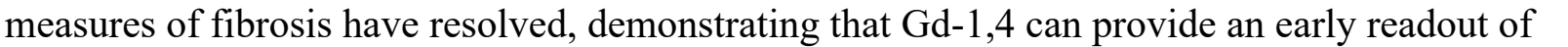

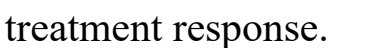

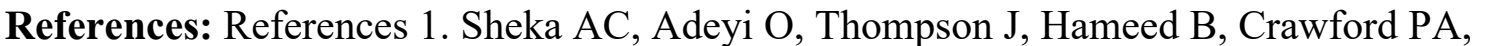

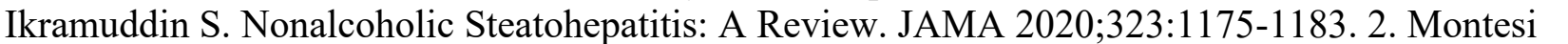

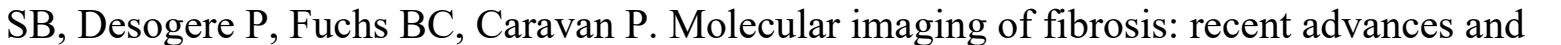

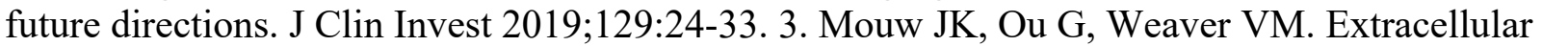

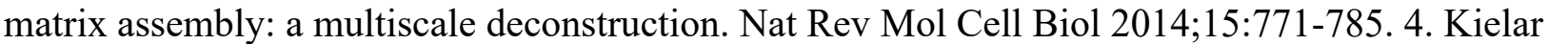

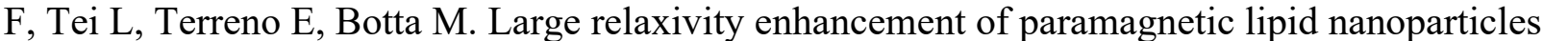

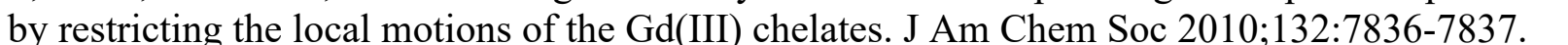

\section{Image/Figure:}

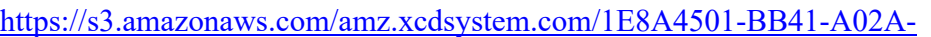

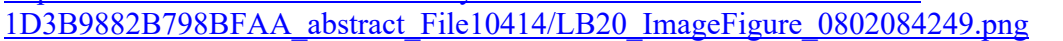

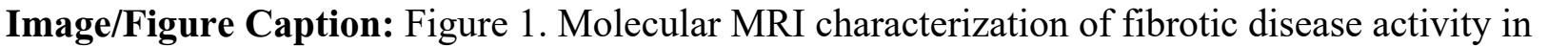

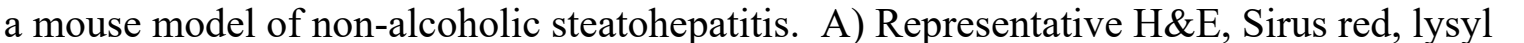

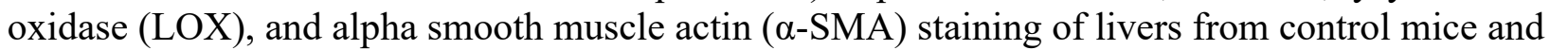

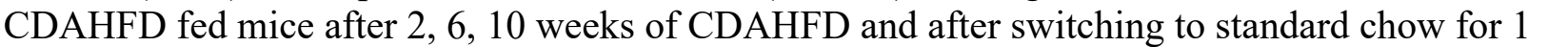

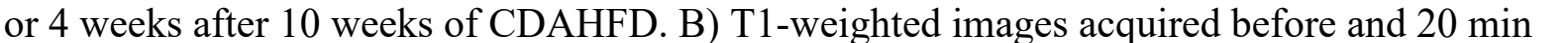

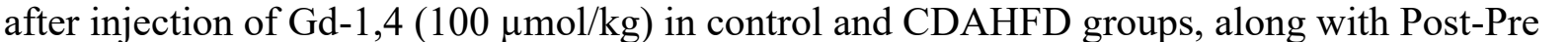
पाm

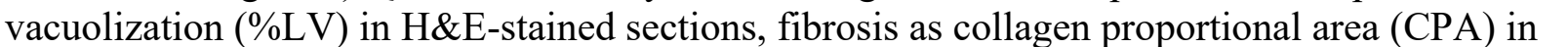
Sirius Red stained slides, percentage of tissue stained positive for LOX and for $\alpha \square \square \square \square \square$

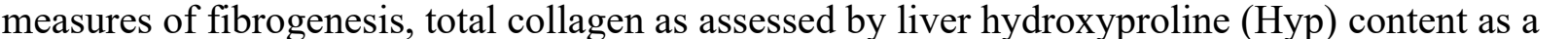

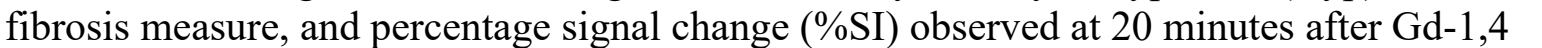
ए

Full Name of Abstract's 1st Author : $\square\|m\| \square \square \square \square$

First Name:

Last Name: $\square \square \square \square$

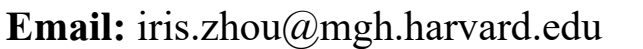

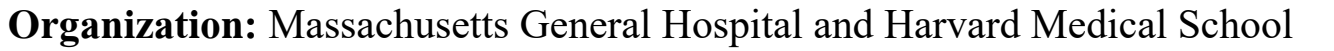

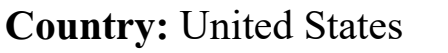




\title{
ID: LB21 \\ $89 \mathrm{Zr}-\alpha$ Tox immunotracer for the in vivo detection of $\mathrm{S}$. aureus infection in osteoarthritis model
}

\author{
Mario González-Arjona, Fundación para la Investigación Biomédica del Hospital Gregorio \\ Marañón, mgarjona@hggm.es
}

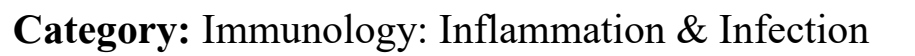

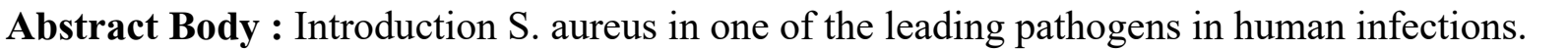

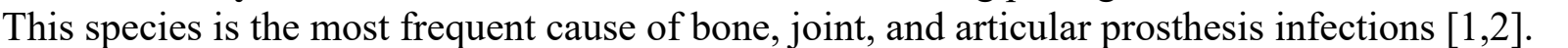
One of the main virulent factors in the staphylococcal infection is the release of $\alpha \square \square|\square| \square \square$

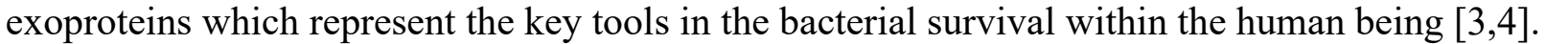

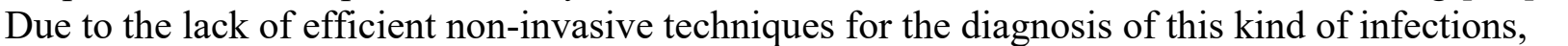

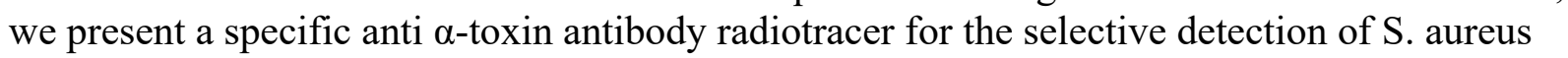
infection by PET/CT in an infective osteoarthritis mice model. Methods S. aureus $\alpha \square \square \square \square$

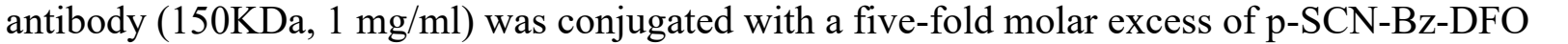

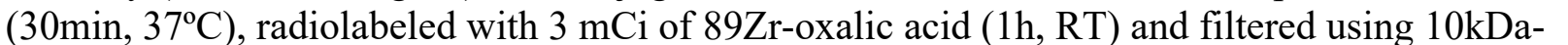

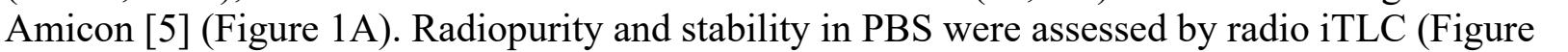

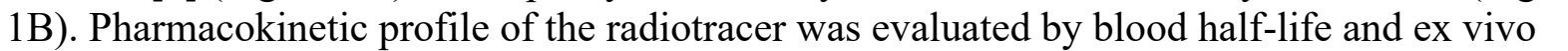

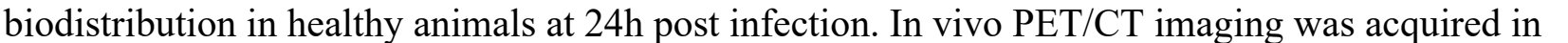

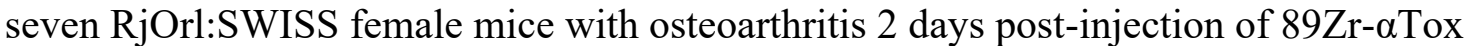

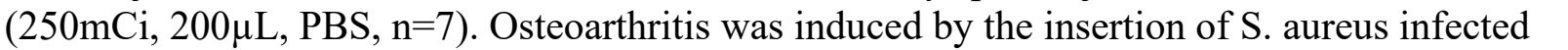

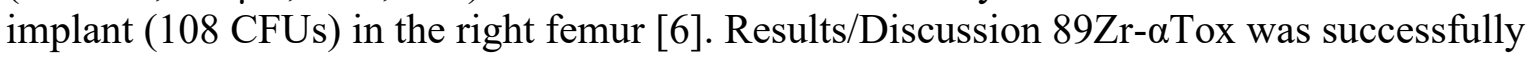

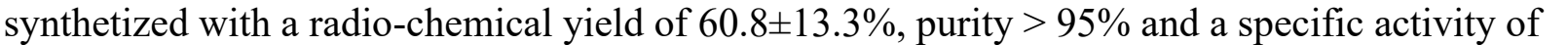

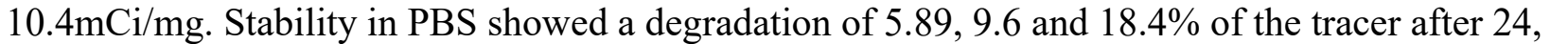

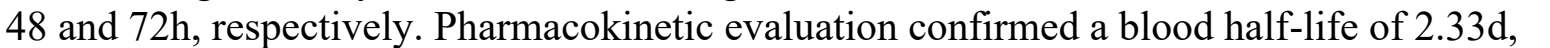

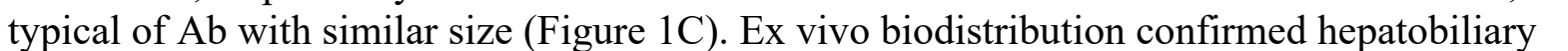

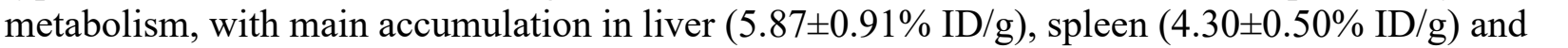

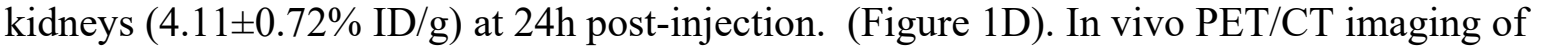

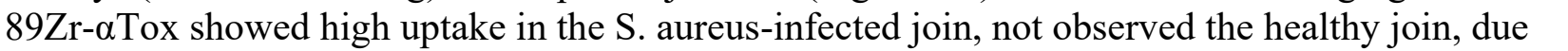

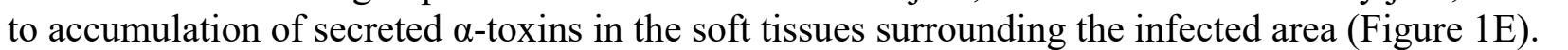

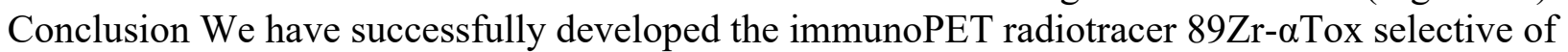

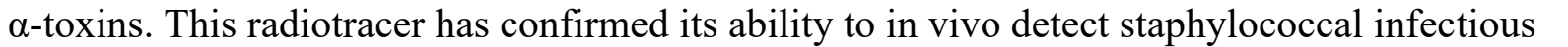

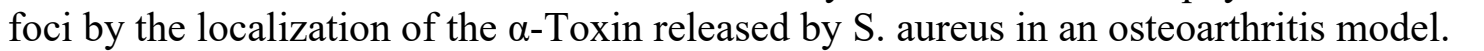

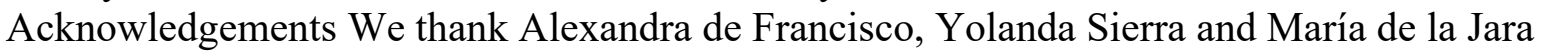

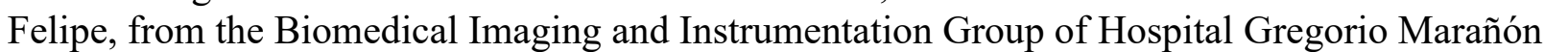

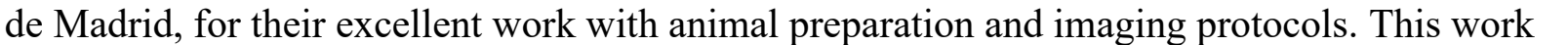
has been supported by "Diagnosis and treatment follow-up of severe Staphylococcal Infections

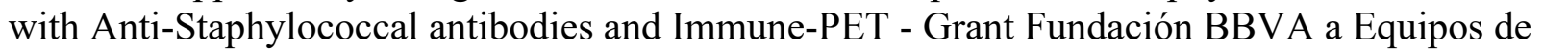
Investigación Científica 2018”. This work has been also supported by Ministerio de Ciencia e Innovación, Instituto de Salud Carlos III, project "PI20/01632”, co-funded by European Union,

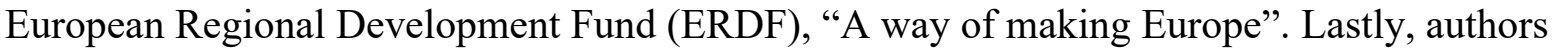

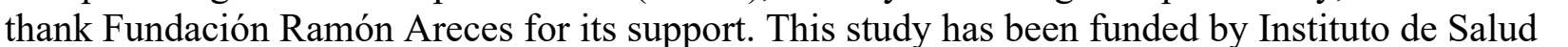




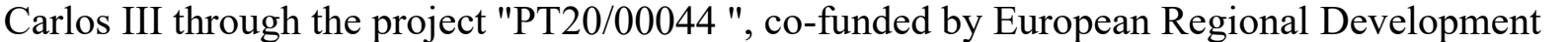

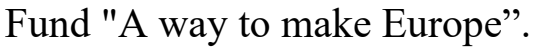

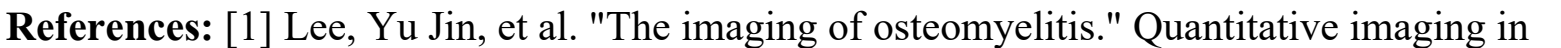

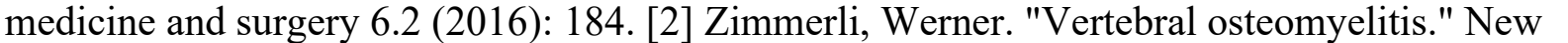

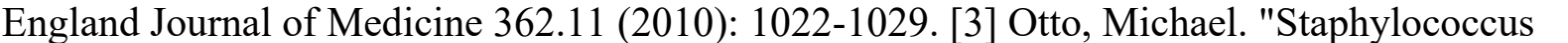

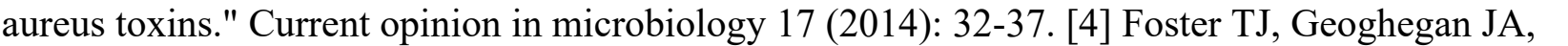

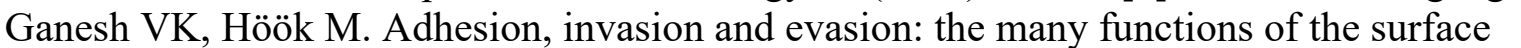

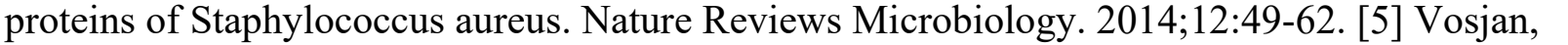

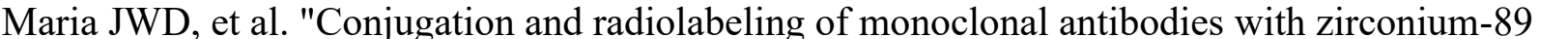

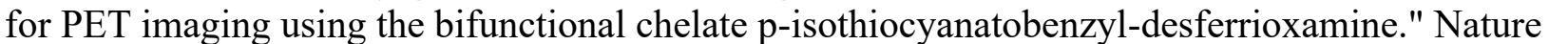

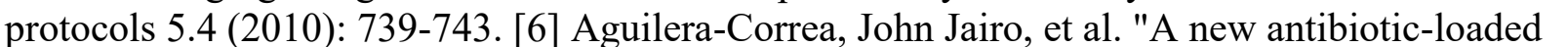

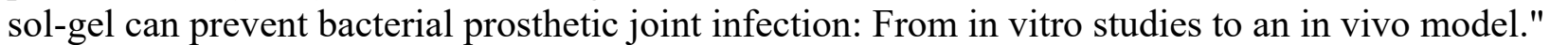

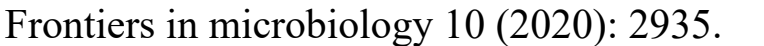

\section{Image/Figure Caption:}

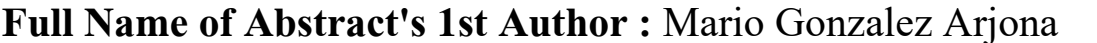

First Name: $\square \square \square \square$

Last Name: $\square \square \square\|\|\|\|\|\square\|\|\| \|$

Email: $\square \square \square\|\Pi\| \square \square \square \square \square \square \square$

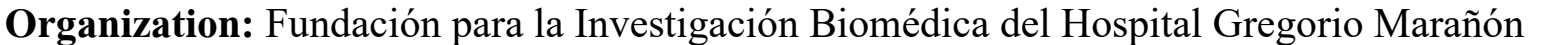

Country: $\square \square \| m \mid \square$ 


\title{
ID: LB22 \\ Smart hybrid "nanoparticle-immune cell" systems for improving $T$ cell accumulation in solid tumors
}

\author{
Javier Hernandez Gil, Memorial Sloan Kettering Cancer Center, \\ javier.hernandezgil@kuleuven.be
}

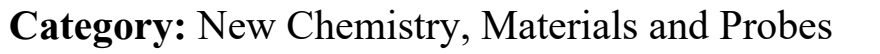

\begin{abstract}
Body : Cancer immunotherapy, which works by activating the body's own immune

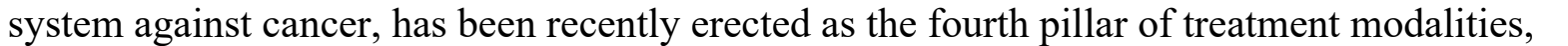

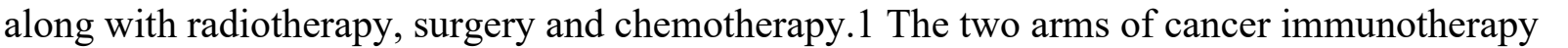

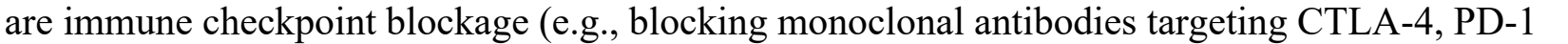

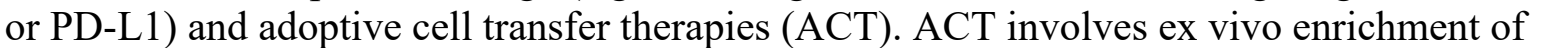

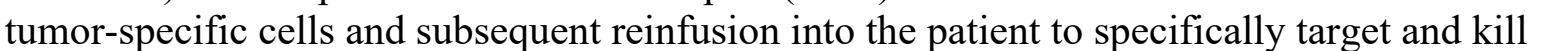

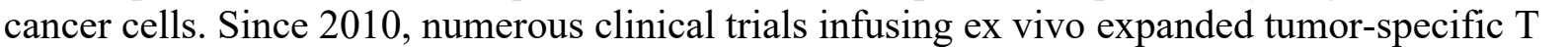

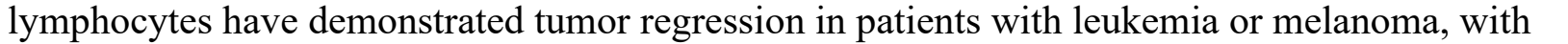

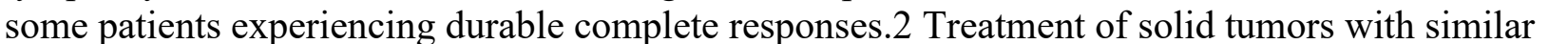

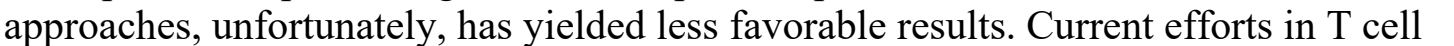

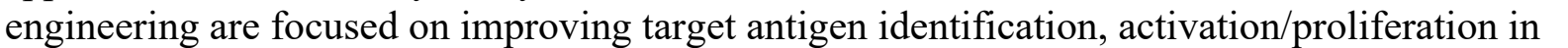

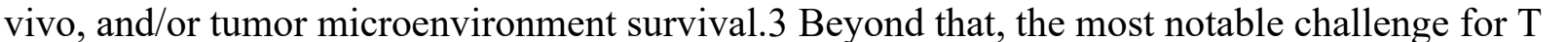

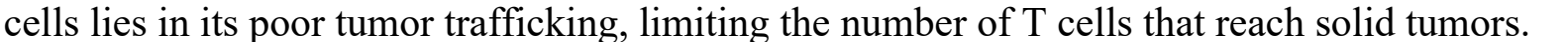

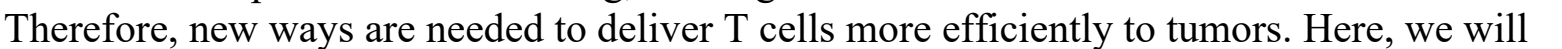

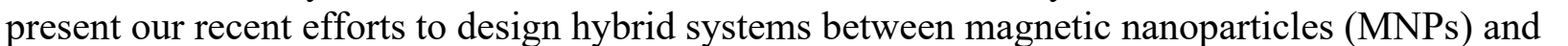

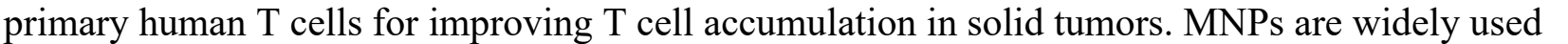

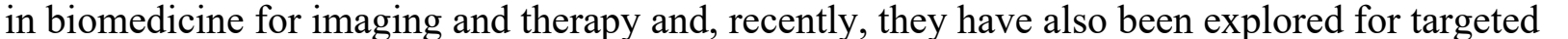

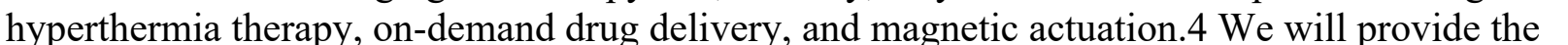

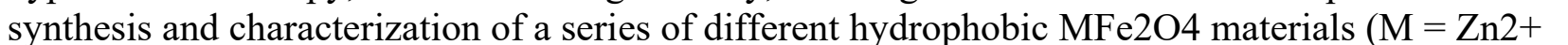

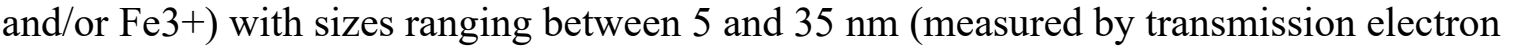

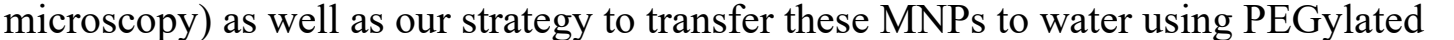

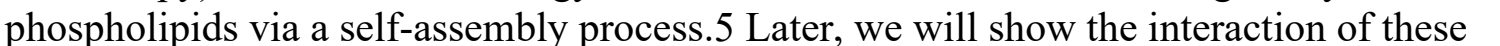

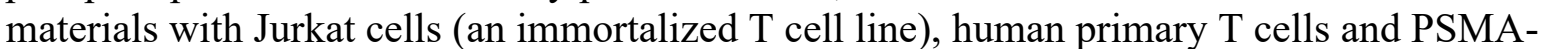

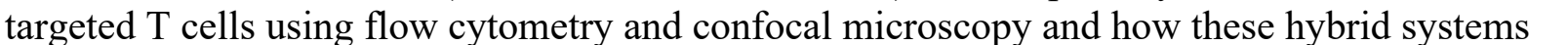

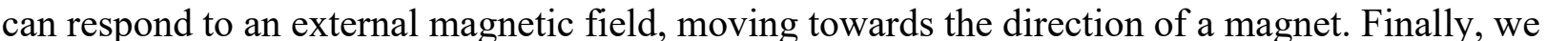

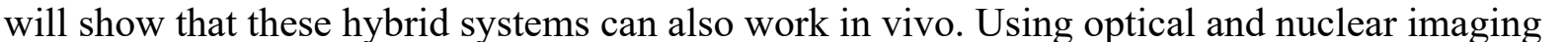

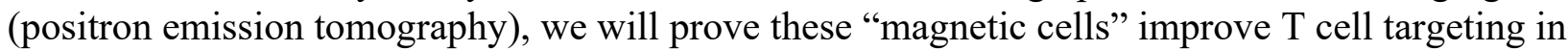

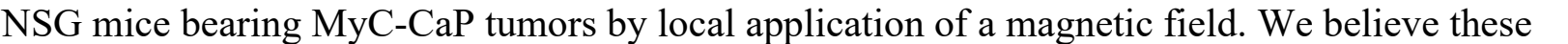

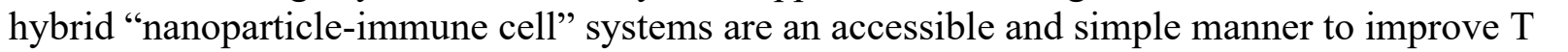

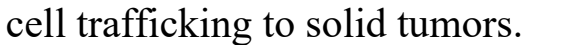

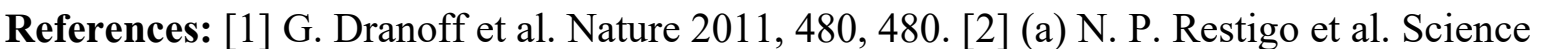

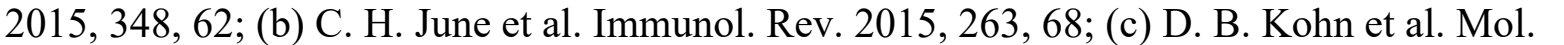

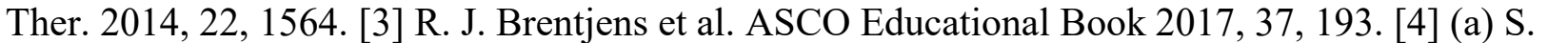


Image/Figure:

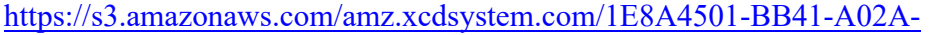

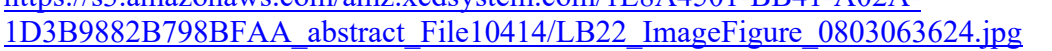

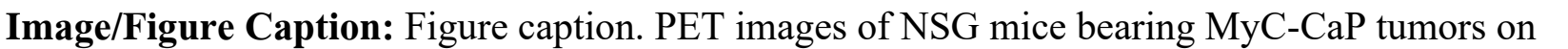

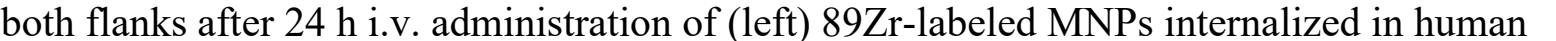

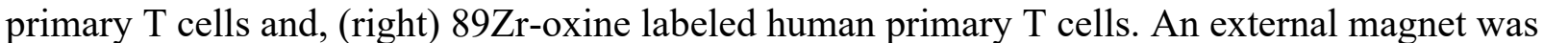

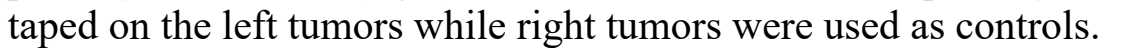

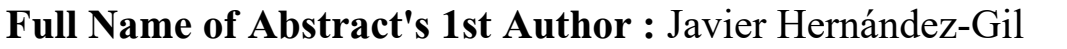

First Name: $\square \square\|m\| \|$

Last Name: $\square \square\|\|\|\|\|\| \square \|$

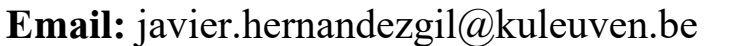

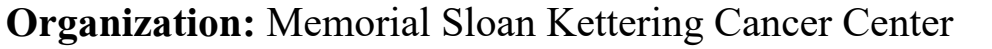

Country: $\square \square \square \square \square \square$ 


\title{
ID: LB23 \\ Zr-89 Labeled Liposome for Bone Marrow PET Imaging \\ Sang Gyu Lee, Memorial Sloan Kettering Cancer Center, lees3@mskcc.org
}

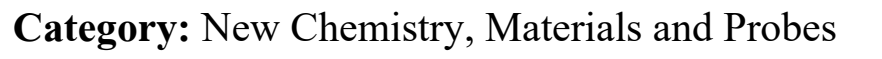

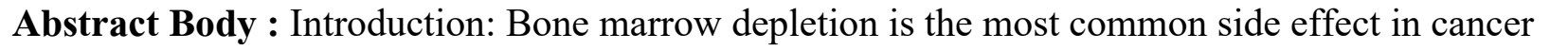

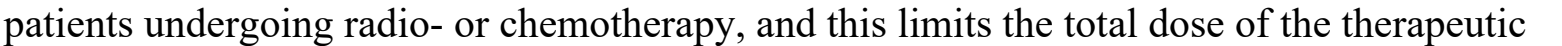

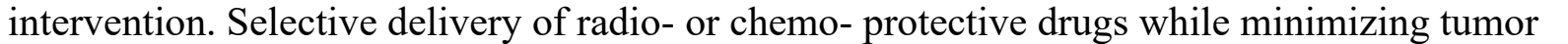

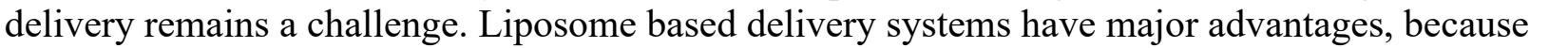

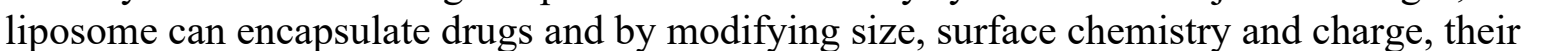

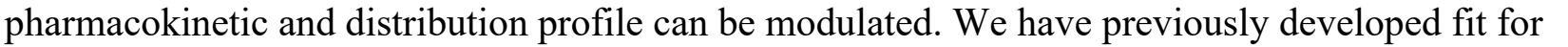

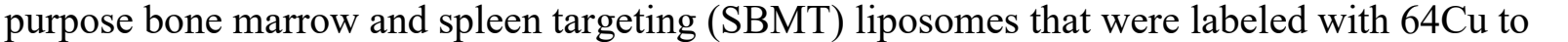

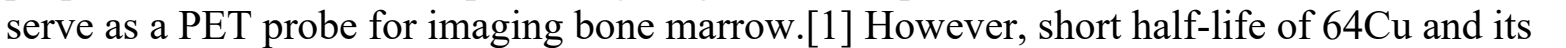

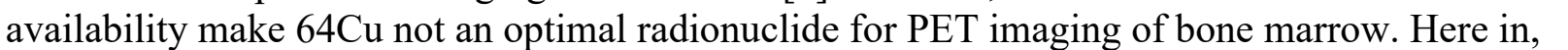

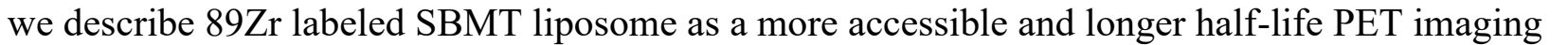

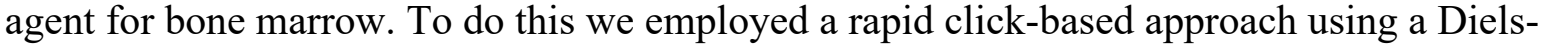

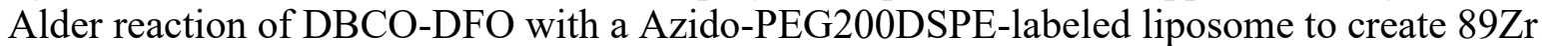

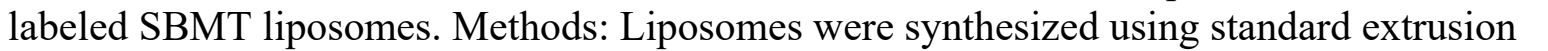

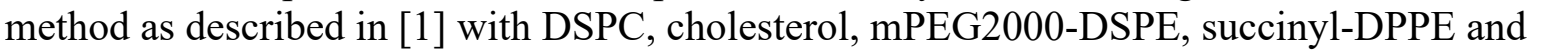

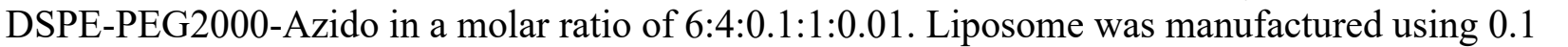

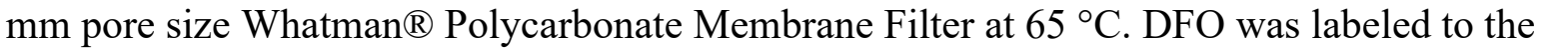

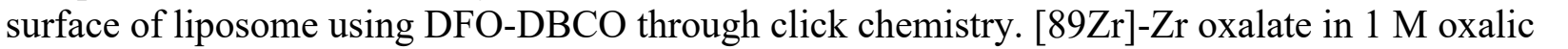

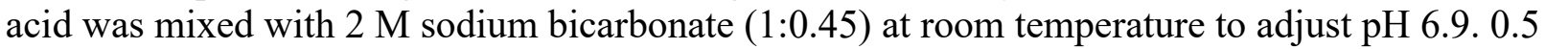

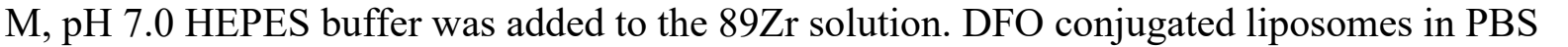

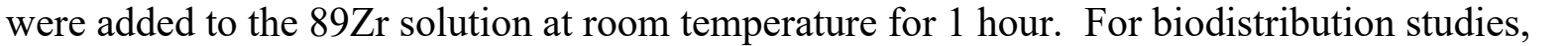

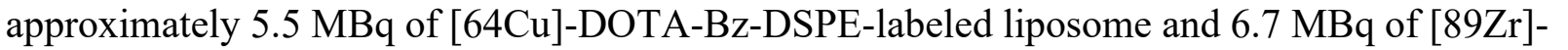

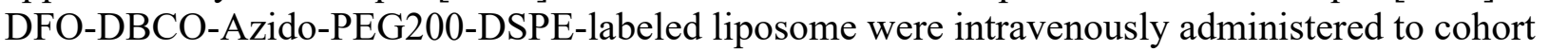

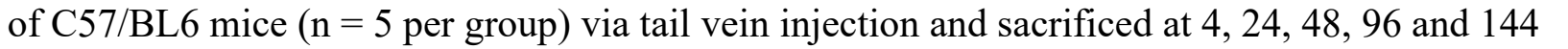

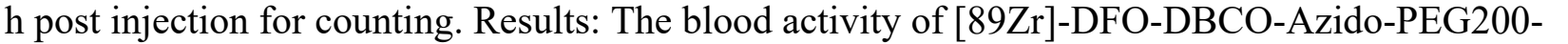

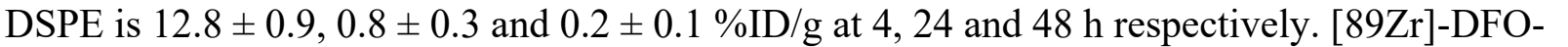

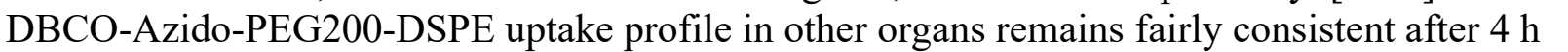

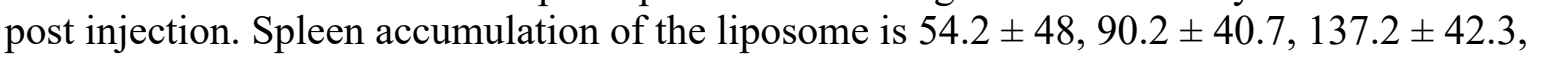

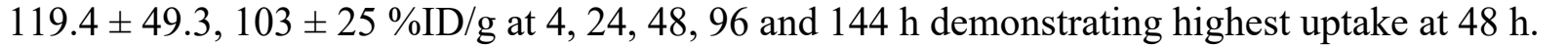

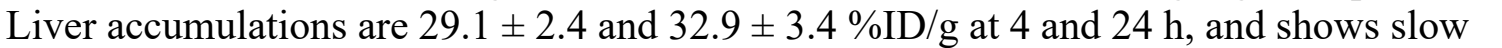

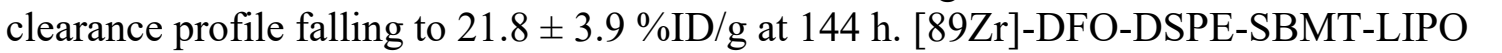

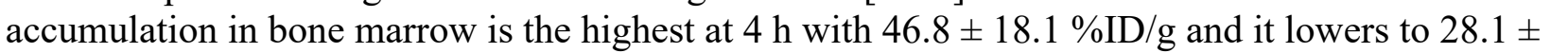

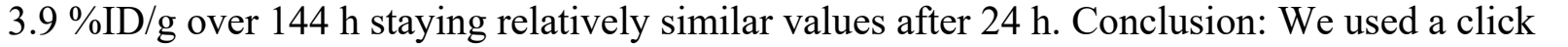

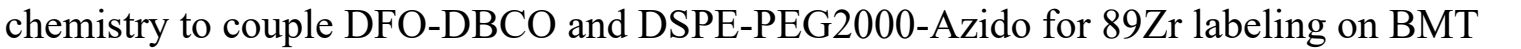

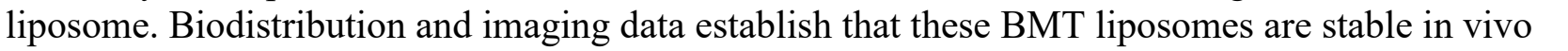

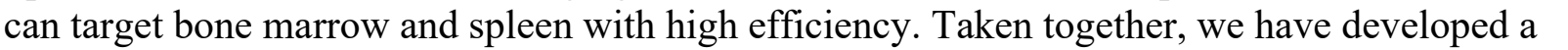

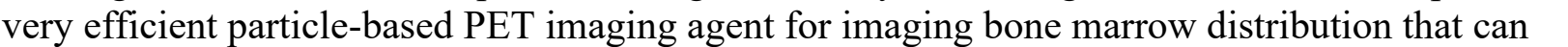




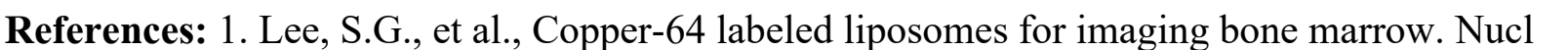

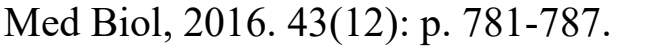

Image/Figure:

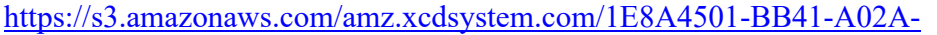

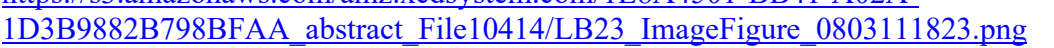

First Name: $\square \square \square \square \square \square \square \square$

Last Name: $\square \square\|\|$

Email: $\square\|\| \square \square \square \square\|m\| \square$

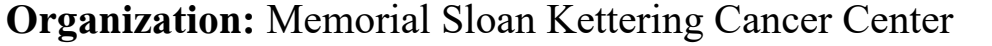

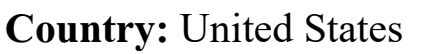




\title{
ID: LB24 \\ Imaging pharmacokinetics of an 18F-labelled ATM inhibitor \\ Claudia Fraser, University of Oxford, claudia.fraser@oncology.ox.ac.uk
}

Category: $\square \square \square \square \square \square$

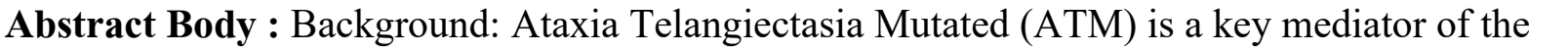

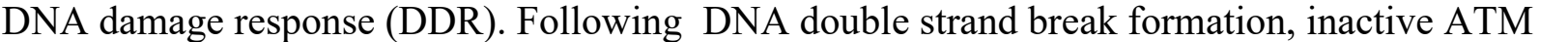

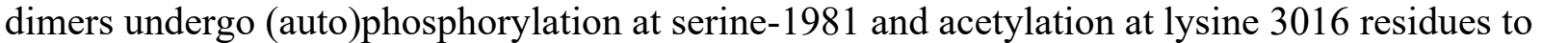

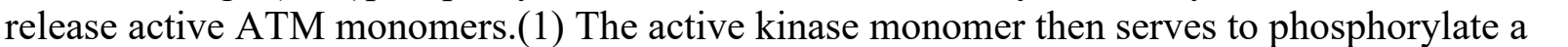

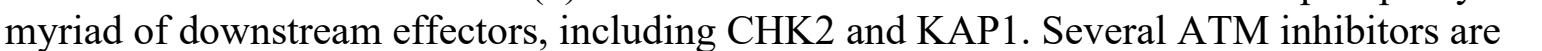

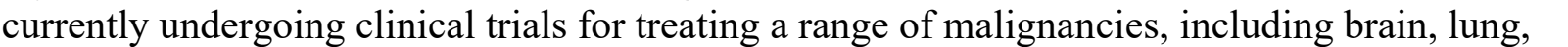

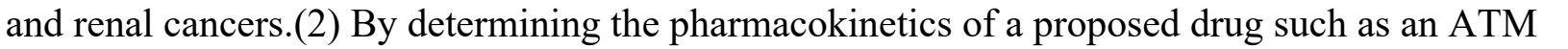

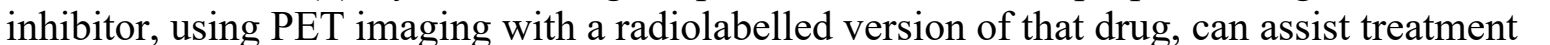

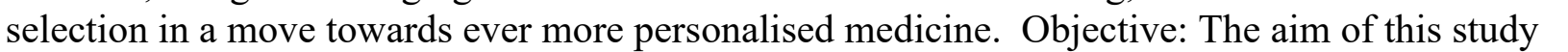

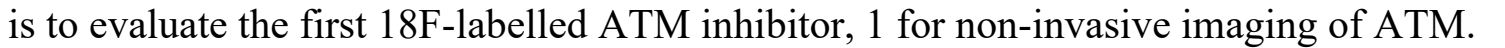

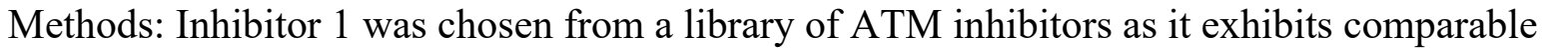

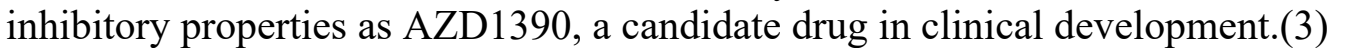

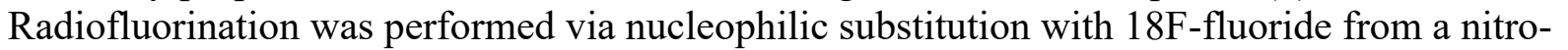

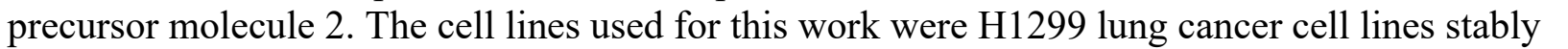

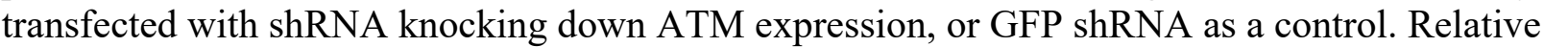

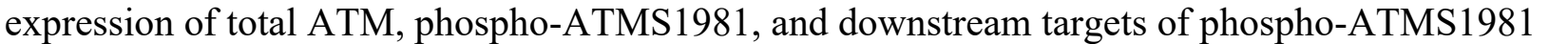

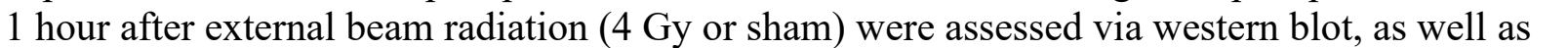
ए ए

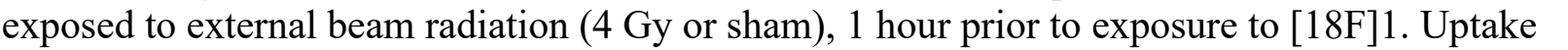

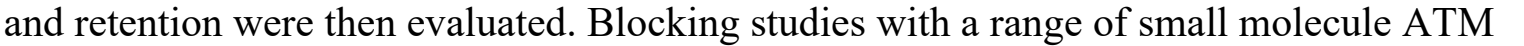

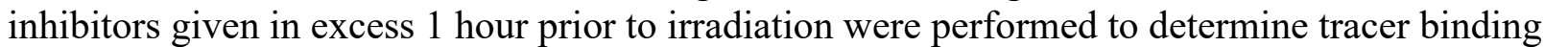
घण

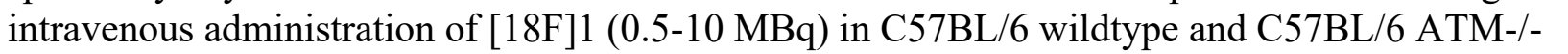

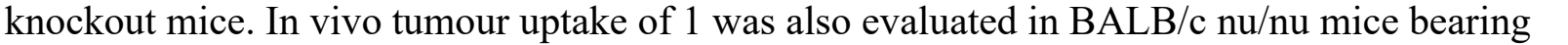

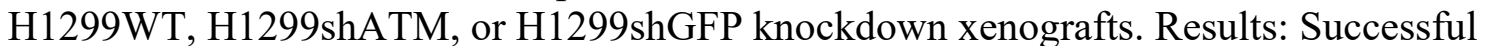

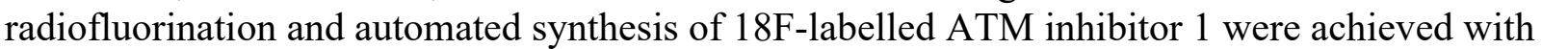

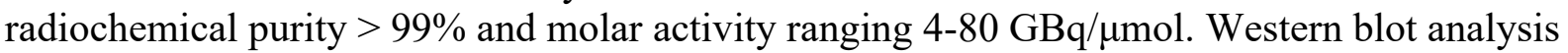

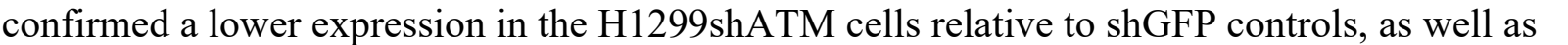

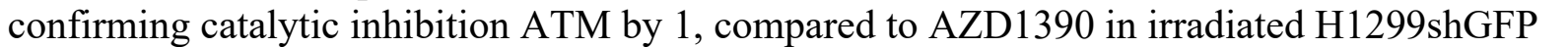

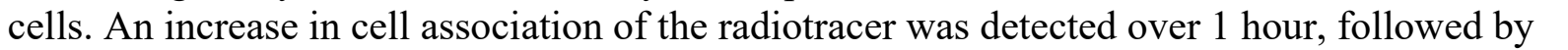

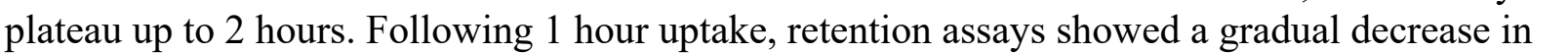

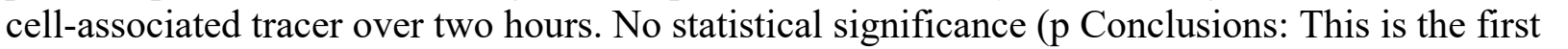

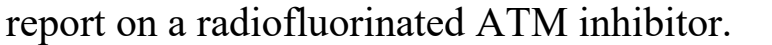

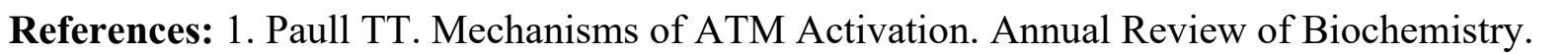

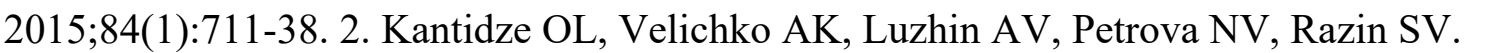

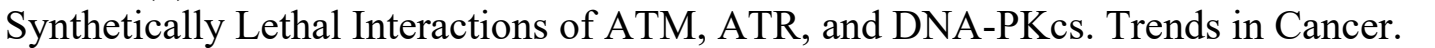




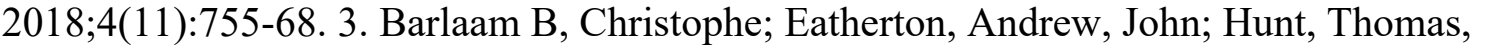

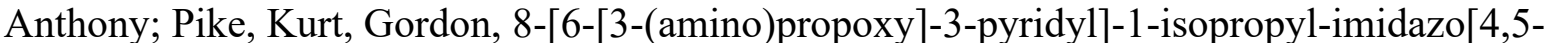

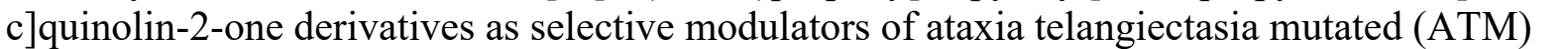

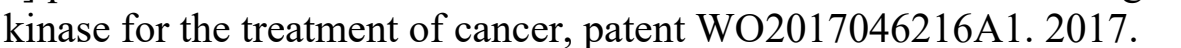

\section{Image/Figure:}

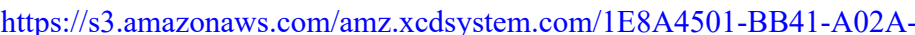

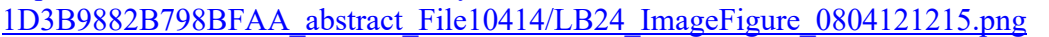

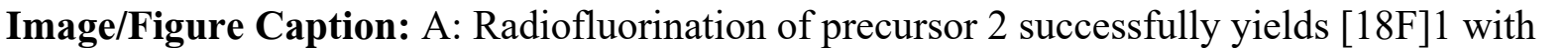

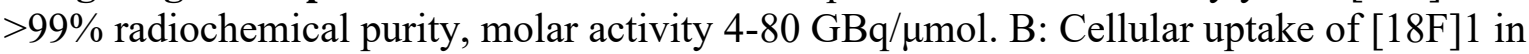

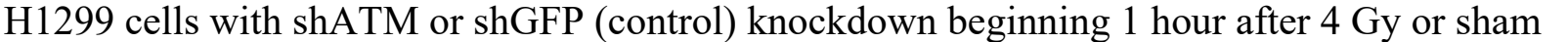

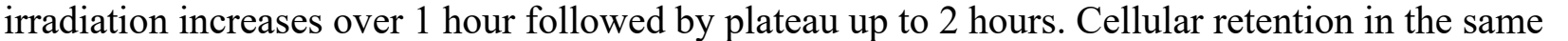

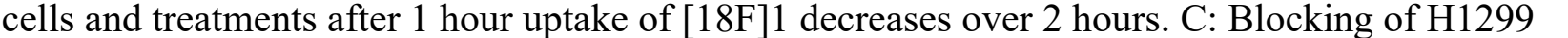

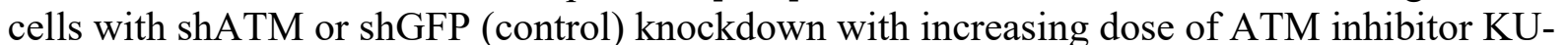

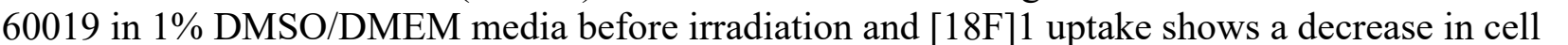

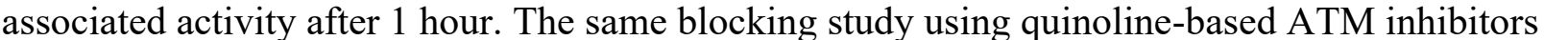

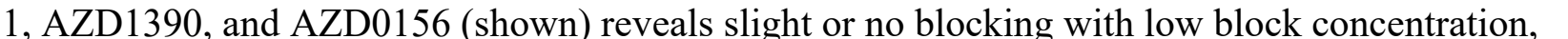

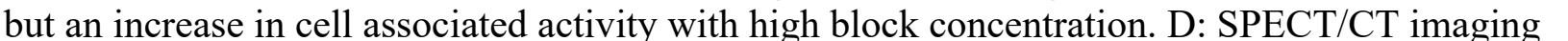

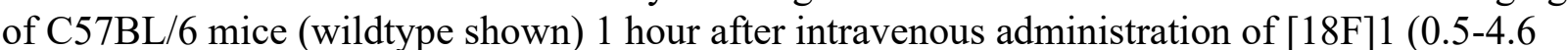

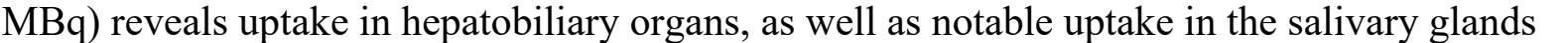

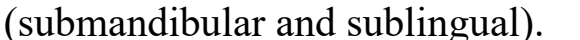

First Name: $\square \square \square \square \square \square$

Last Name: $\square\|\square\|\|\| \|$

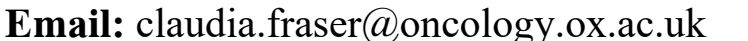

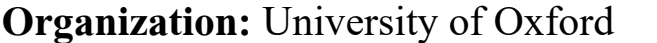

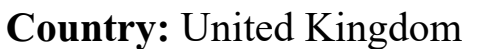




\title{
ID: LB25 \\ FLUORESCENCE IMAGE GUIDED SURGICAL RESECTION OF SOLID \\ TUMORS USING THE PH-RESPONSIVE MICELLAR IMAGING AGENT PEGSITACIANINE: A SUMMARY OF AN ONGOING PHASE 2 STUDY
}

\author{
Brian Madajewski, OncoNano Medicine Inc, bmadajewski@onconanomed.com
}

\section{Category: $\square \square\|\| \square$}

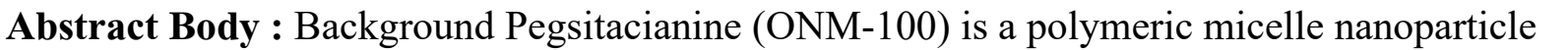

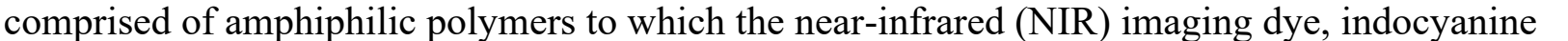

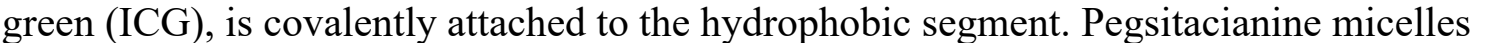

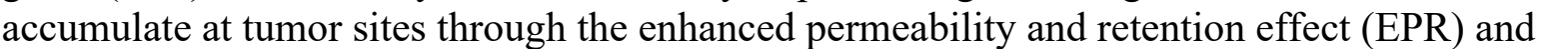

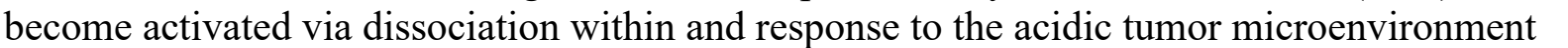

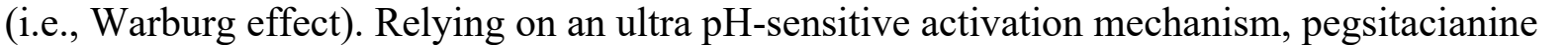
exists in a fluorescently dark ("Off") state at physiological pH but transitions rapidly to a

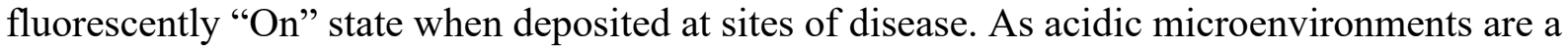

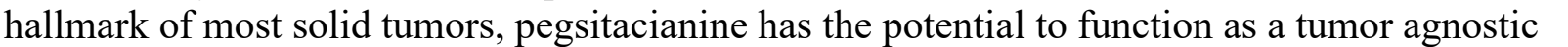

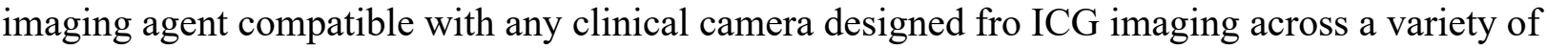

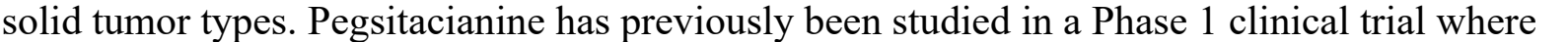

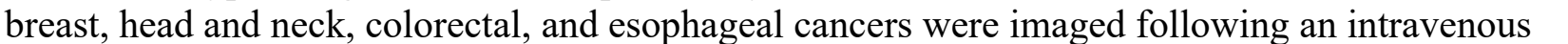

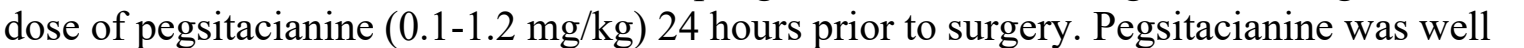

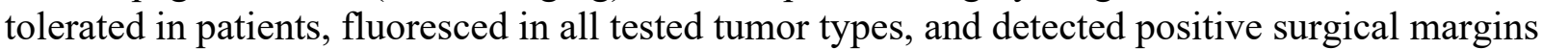

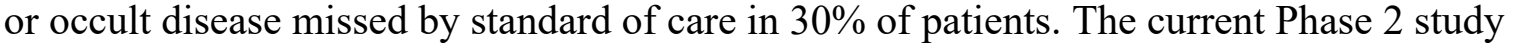

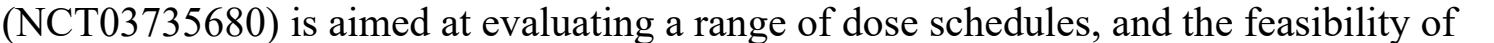

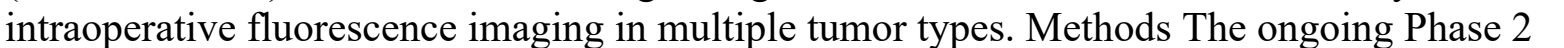

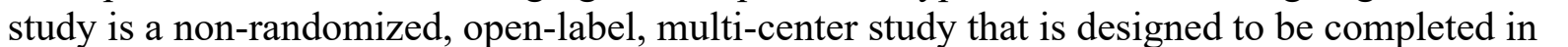
ए

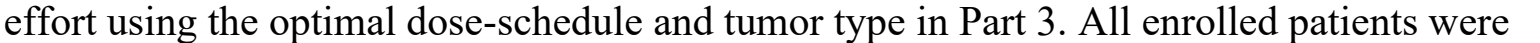

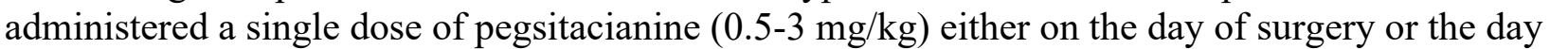

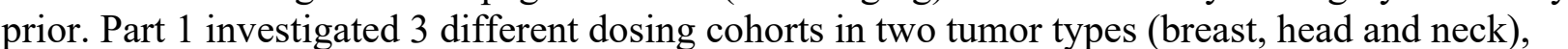

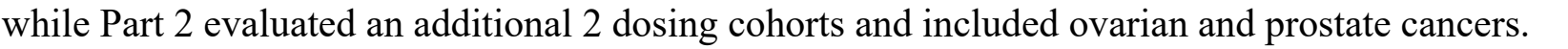

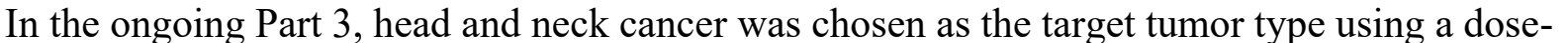

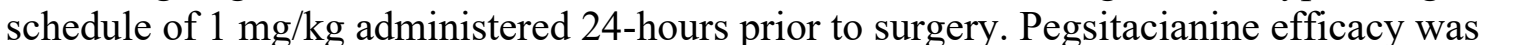

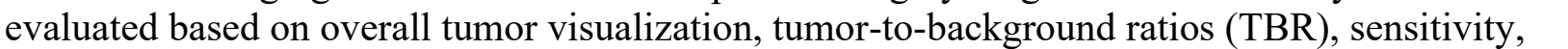

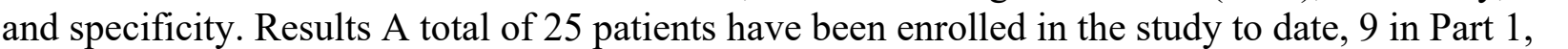

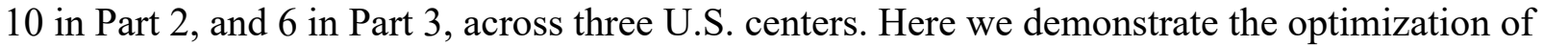

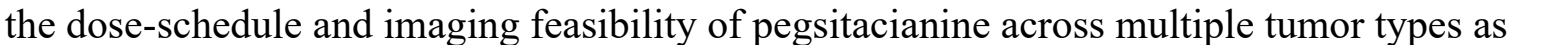

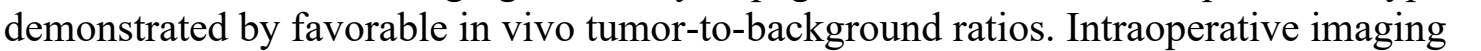

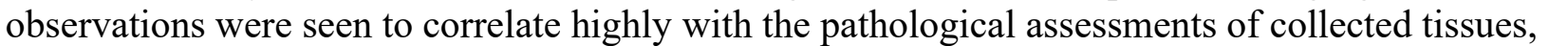

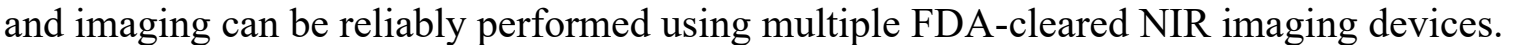

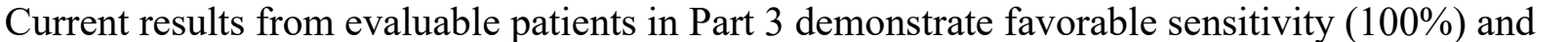

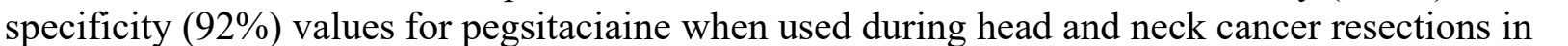

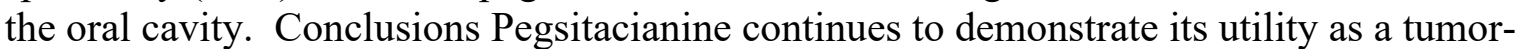




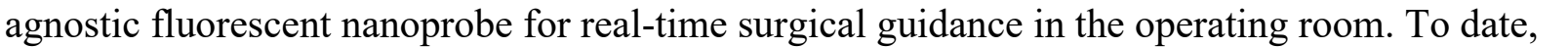

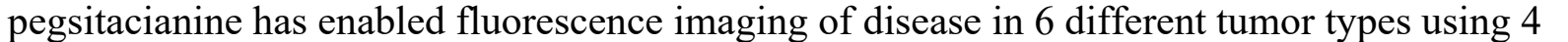

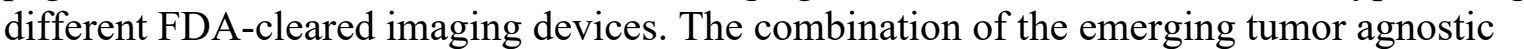

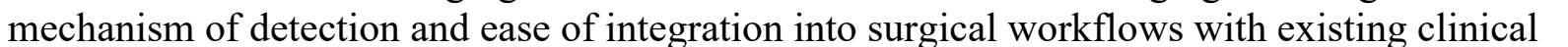

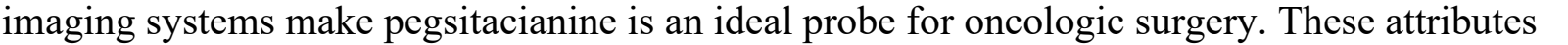

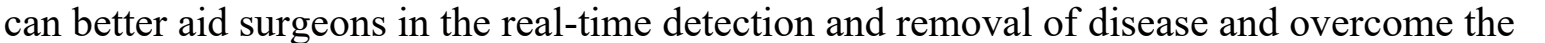

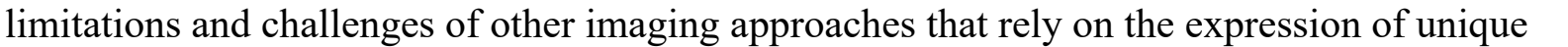

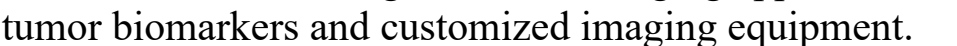

\section{Image/Figure:}

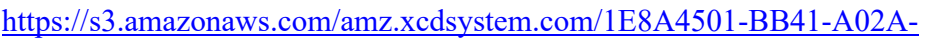

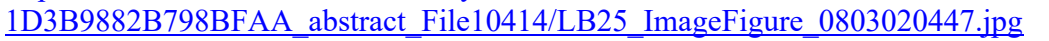

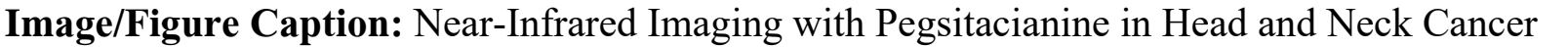

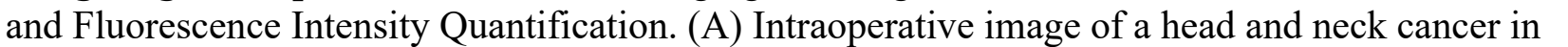

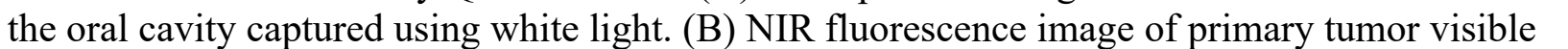

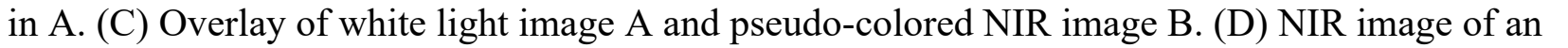
एव

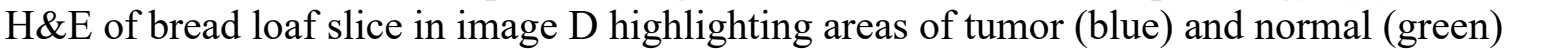

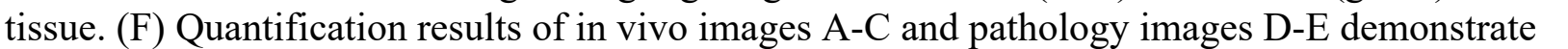

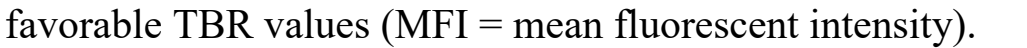

First Name: $\square \square[\mid \square$

Last Name: $\square \square \square \| \square \square \square \square$

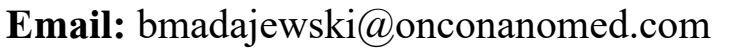

Organization: $\square \square\|\square \square\| \square \square \square \square m\|\Pi\| \square$

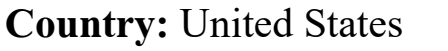




\title{
ID: LB26 \\ Exploration of the advantages of host-guest pretargeted PET with novel copper- 64-labeled radioligands
}

\author{
Vilma Jallinoja, Vanderbilt University, vilma.i.jallinoja@vanderbilt.edu
}

\section{Category: $\square \square\|\| \| \square$}

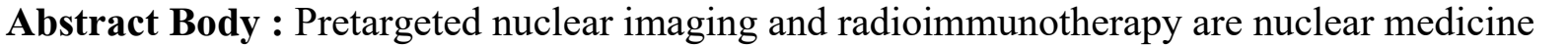

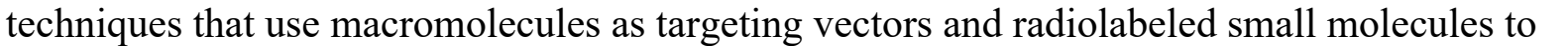

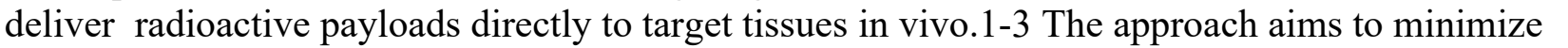
प

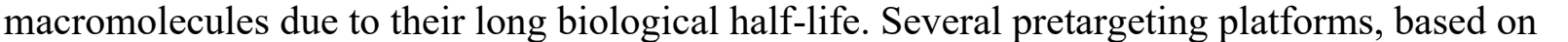

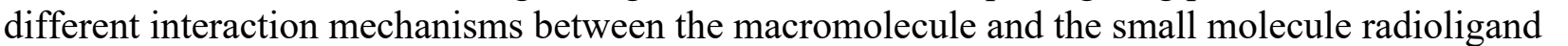

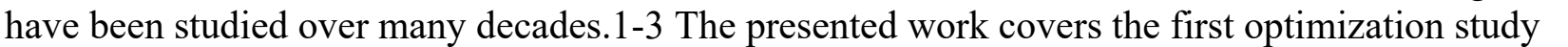

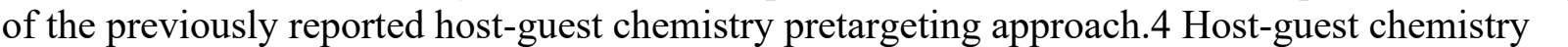

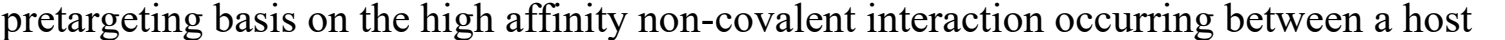

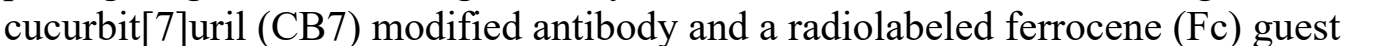

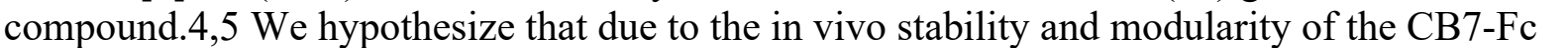

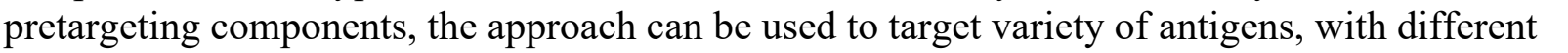

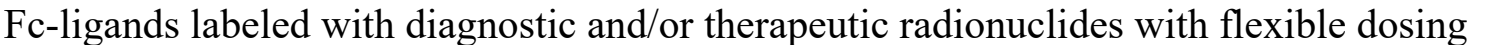

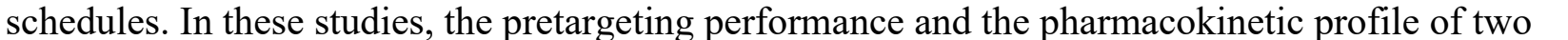

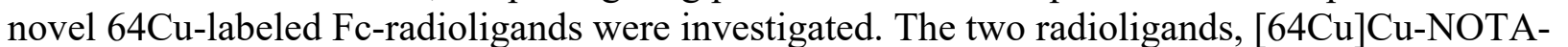

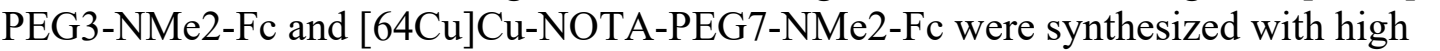
एवा

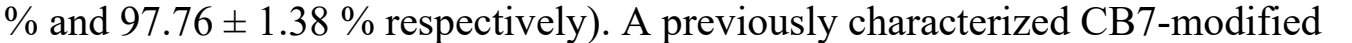

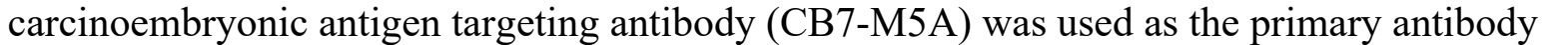

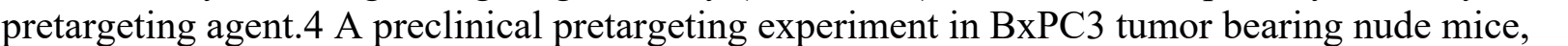

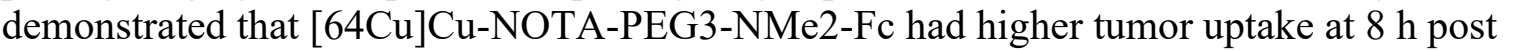
घण

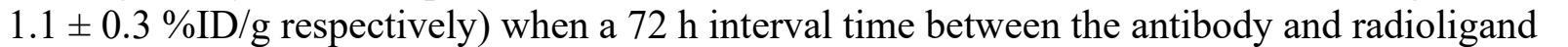
ए

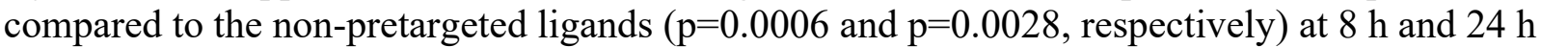

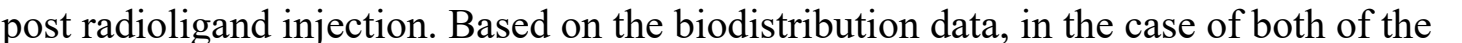

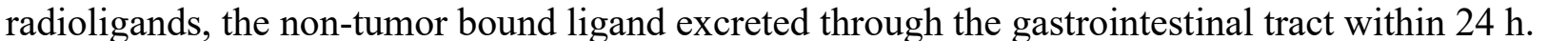

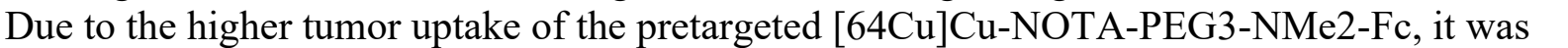

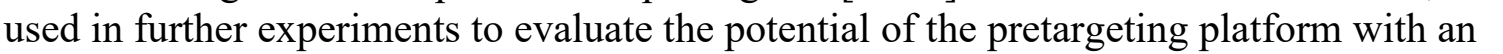

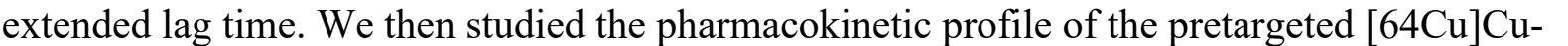

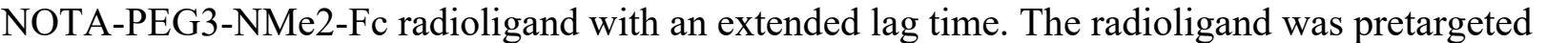

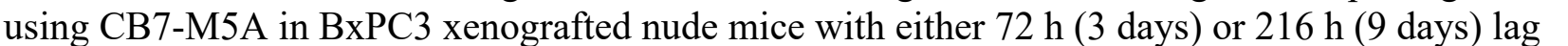

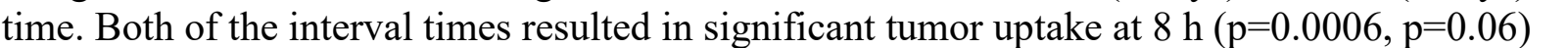

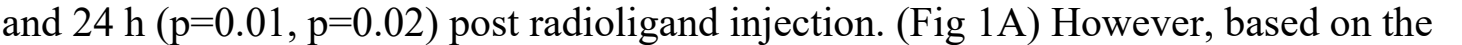

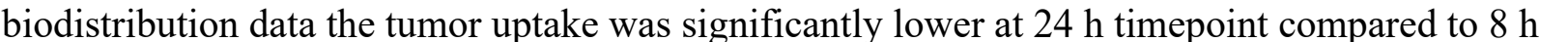

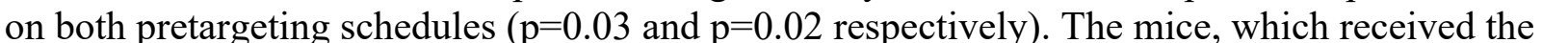




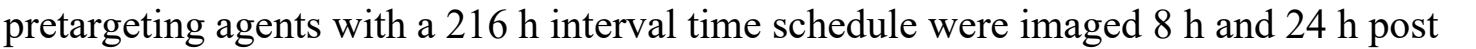
ए

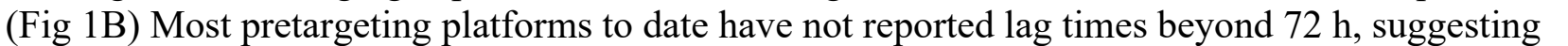

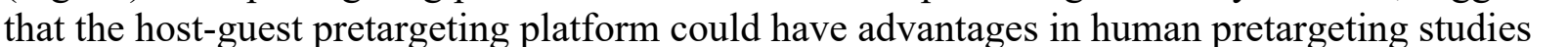

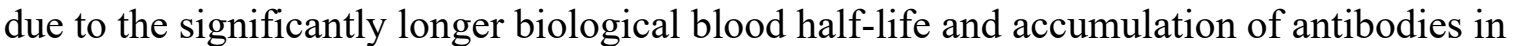

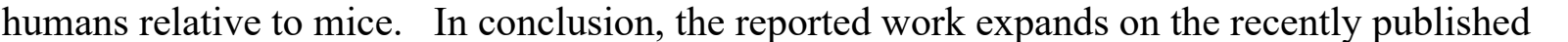

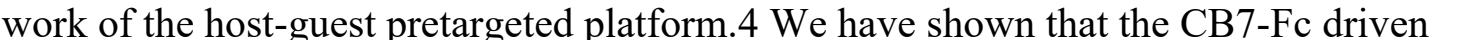

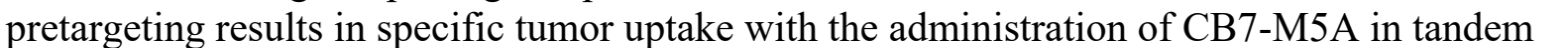

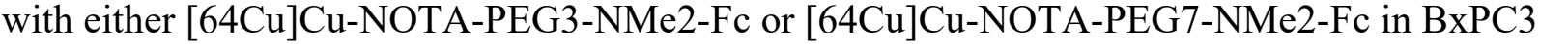

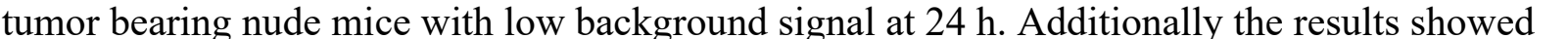

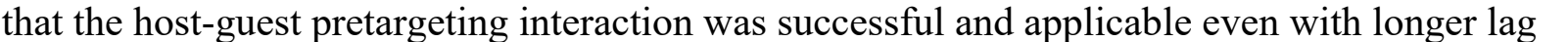

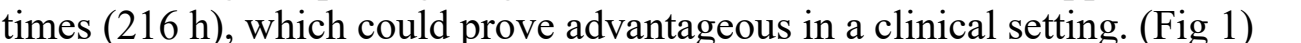

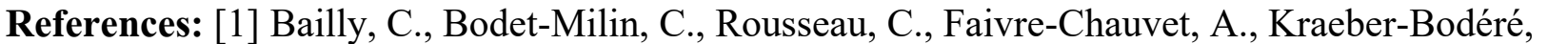

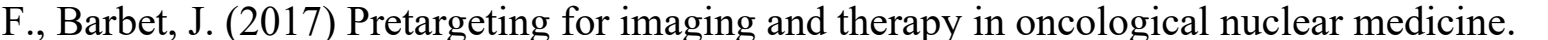

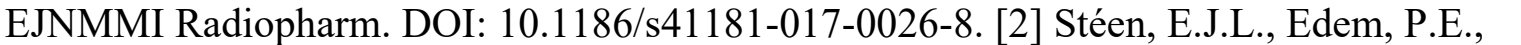

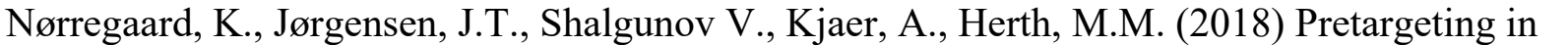

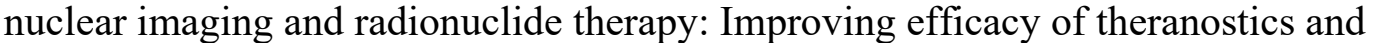

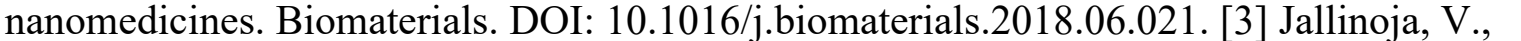

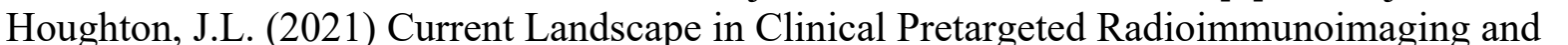

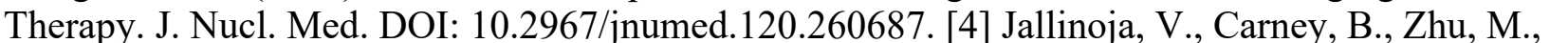

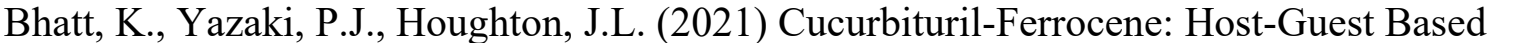

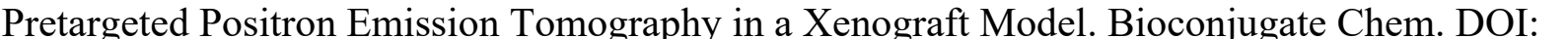

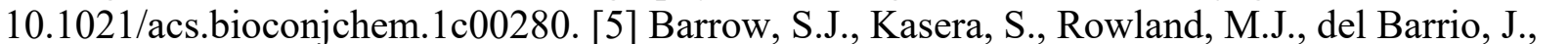

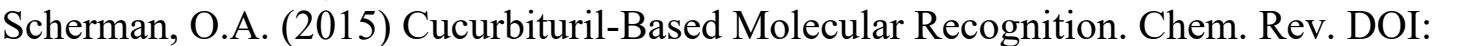

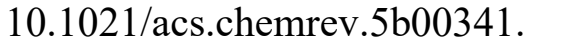

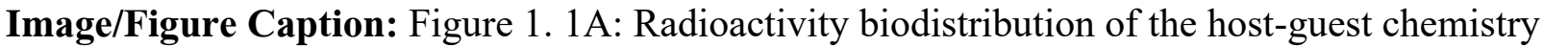

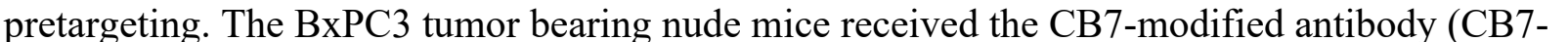

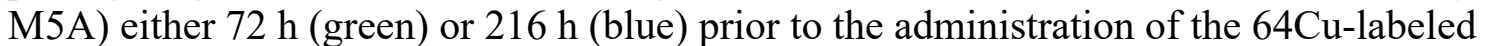

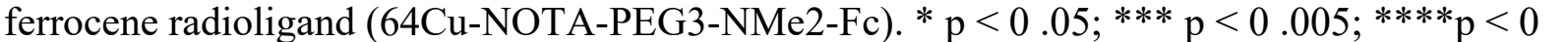

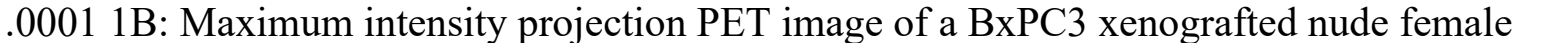

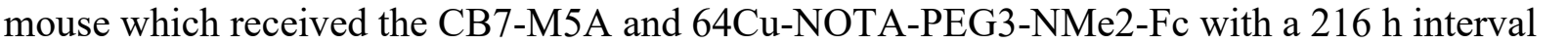

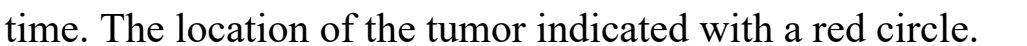

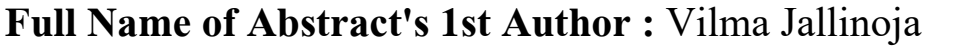

First Name: $\square \square \square \square \square$

Last Name: $\square\|\|\|\| \|$

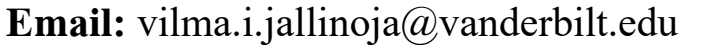

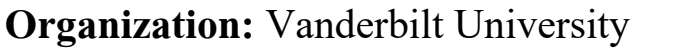

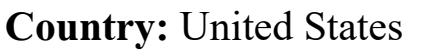




\title{
ID: LB27 \\ Targeting Extra Cellular Matrix disruption by PET imaging as early in vivo marker for pulmonary fibrosis in a mouse model
}

\author{
Simon Freisinger, Eberhard Karls University Tübingen, simon.freisinger@med.uni- \\ tuebingen.de
}

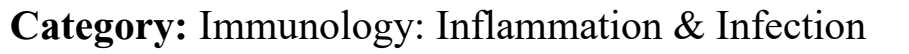

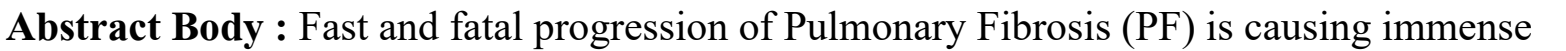

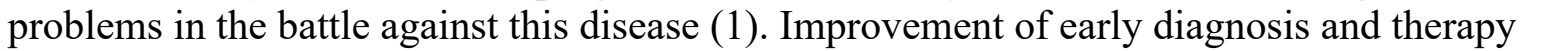

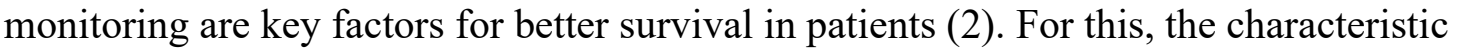

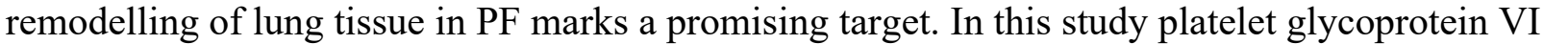

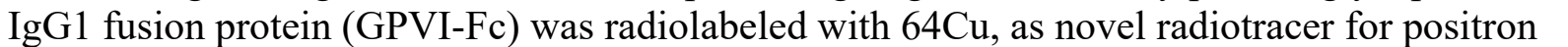

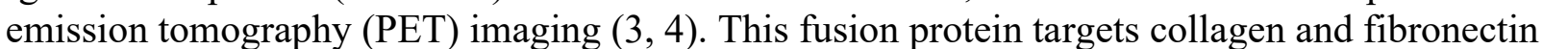

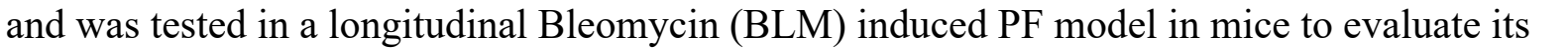

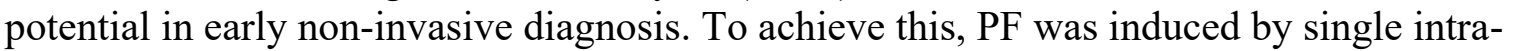

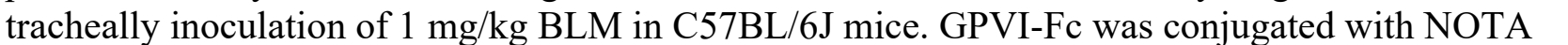

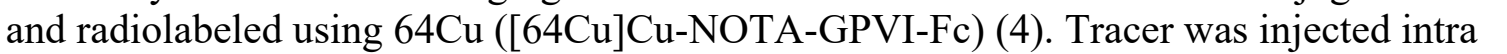

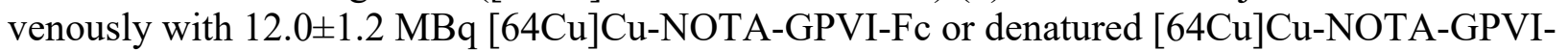

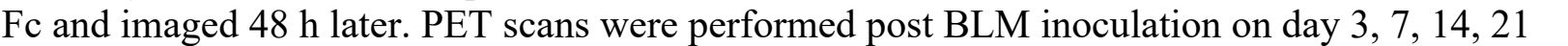

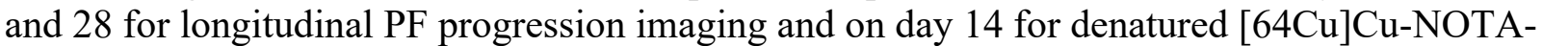

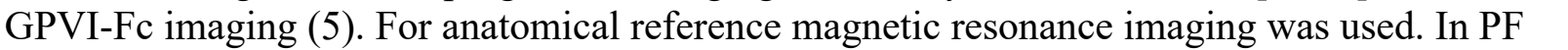

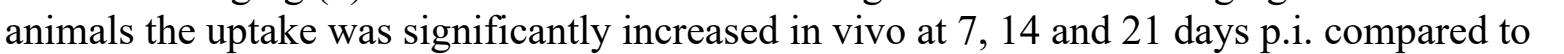

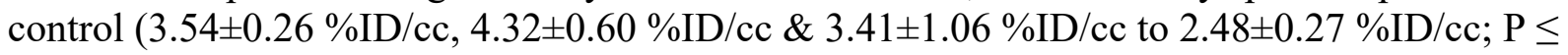
$0.01, \mathrm{P} \leq 0.0001 \& \mathrm{P} \leq 0.05$ respectively). Specific tracer uptake was shown by imaging of

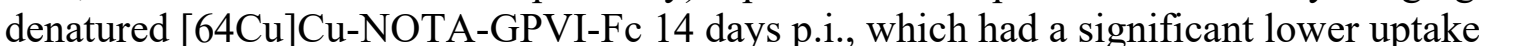

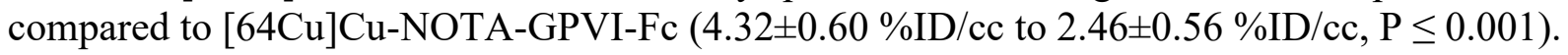

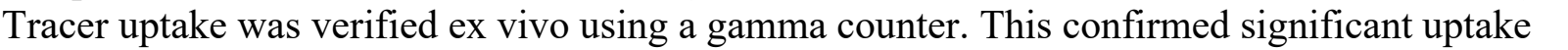

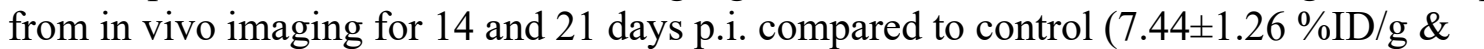

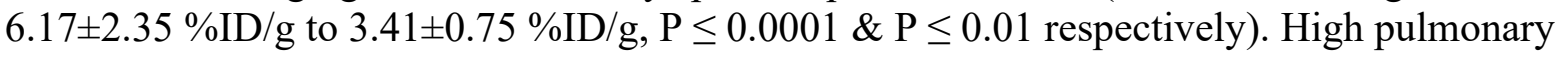

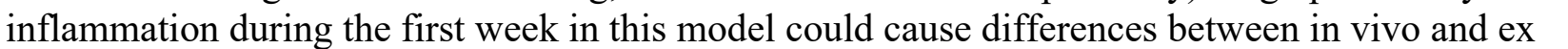

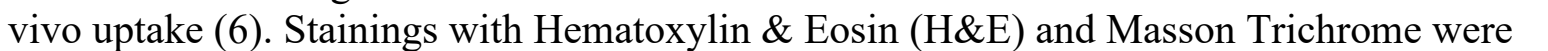

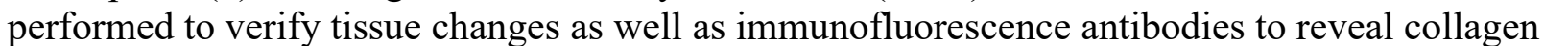

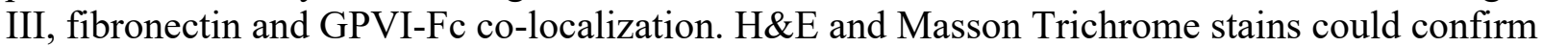
एव

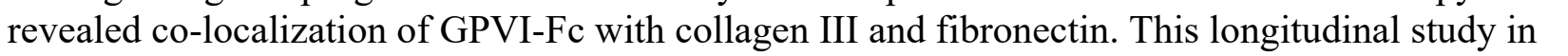

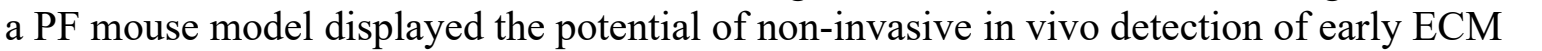

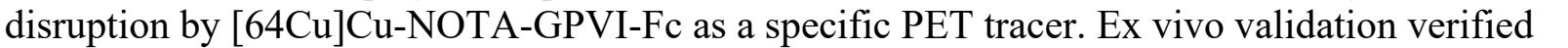

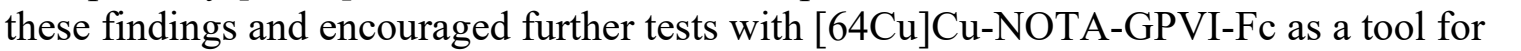

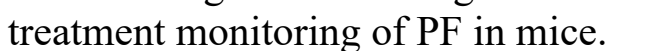

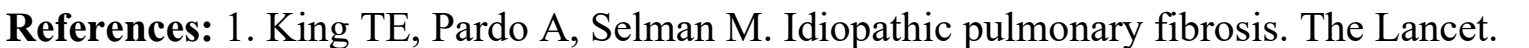

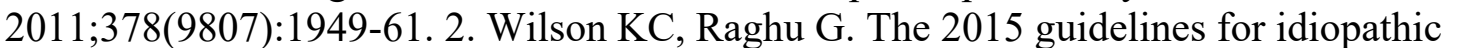




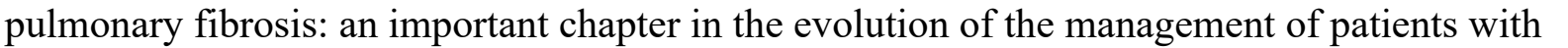

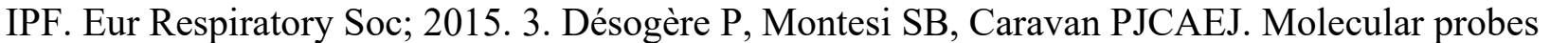

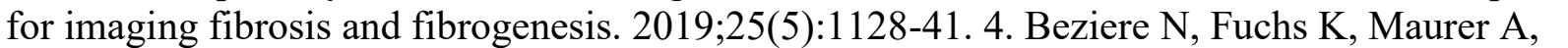

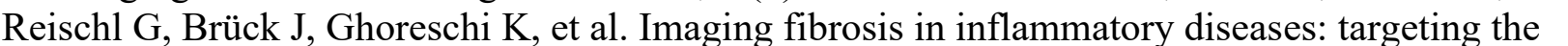

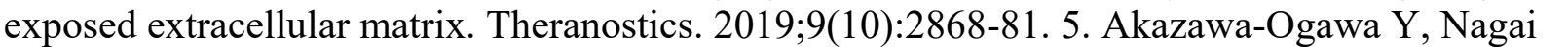

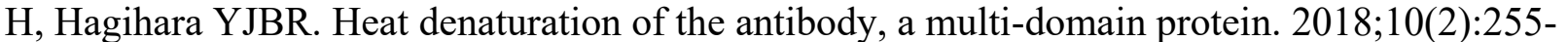

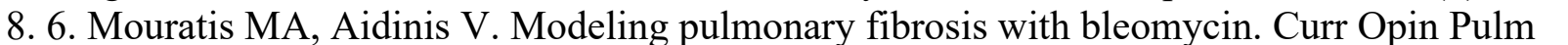

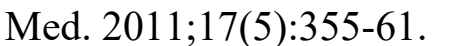

\section{Image/Figure:}

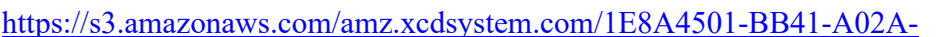

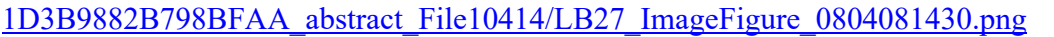

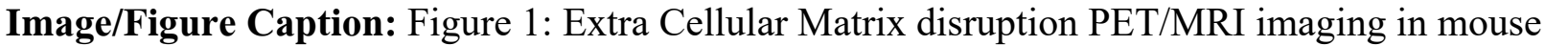

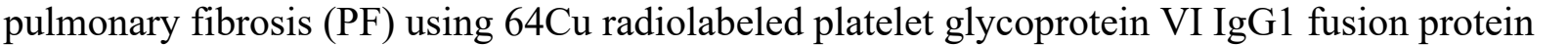
ए

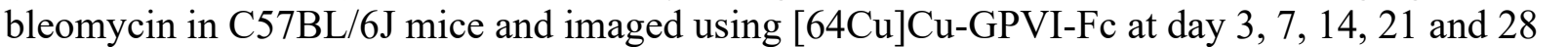

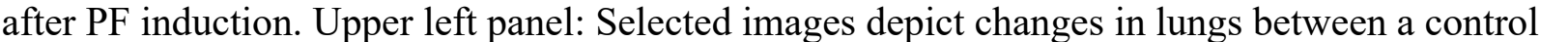

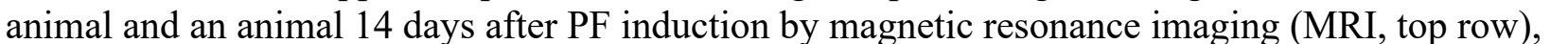

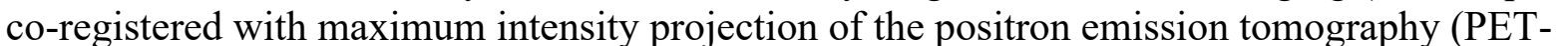

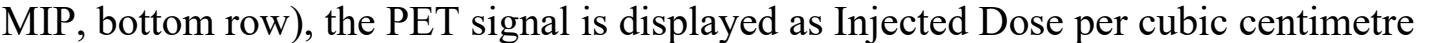

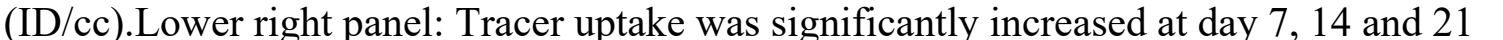

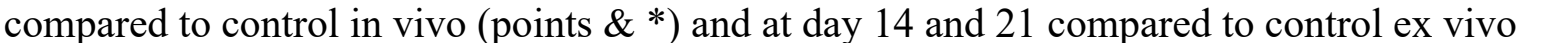

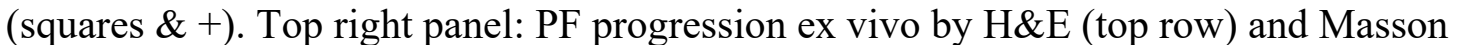

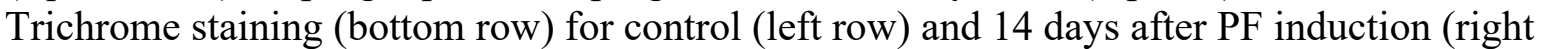

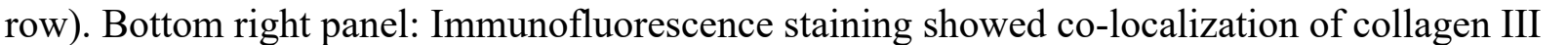

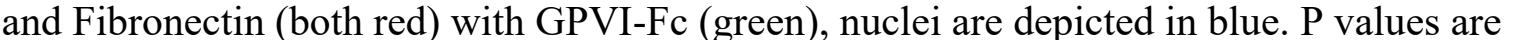

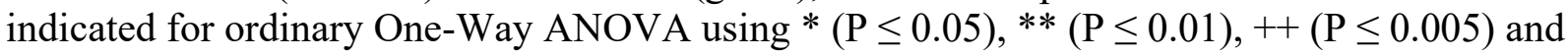
$* * * /++++(\mathrm{P} \leq 0.0001)$.

First Name: $\square \square \square \square$

Last Name: $\square\|\|\|\|\|\| \| \square$

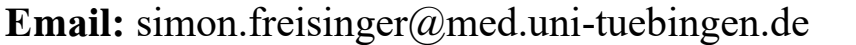

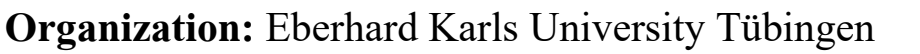

Country: $\square \square \square \square \square \square$ 


\title{
ID: LB28 \\ Elucidating super-resolution Magnetic Particle Imaging: superferromagnetic remanence decay through MPI signal evolution informs super-resolution MPI scan strategies
}

\author{
K. L. Barry Fung, University of California, Berkeley, barry.kl.fung@berkeley.edu
}

Category: $\square \square\|m \square \square \square\| m \mid \square$

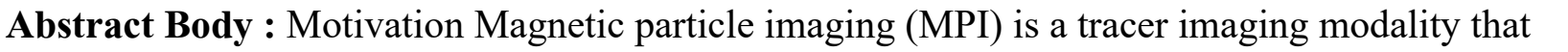

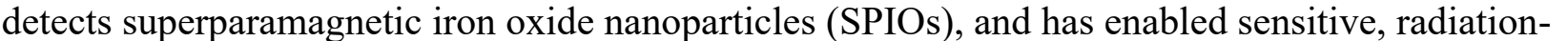

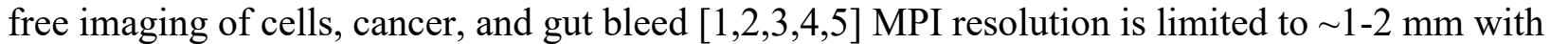

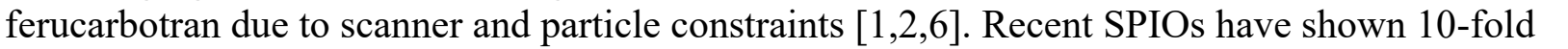

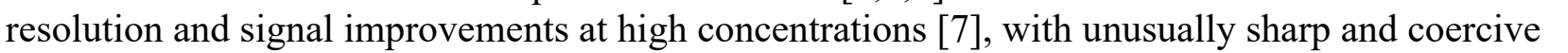

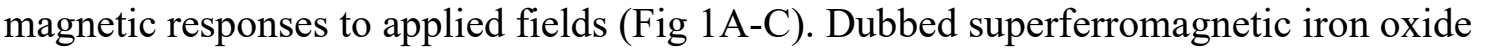

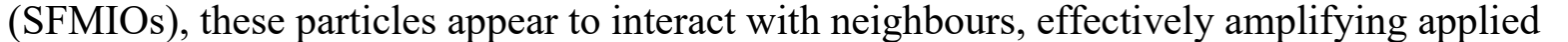

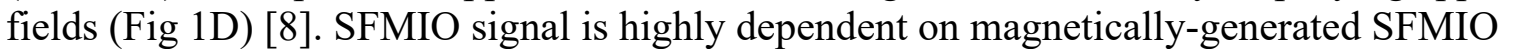

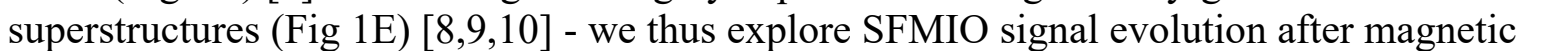

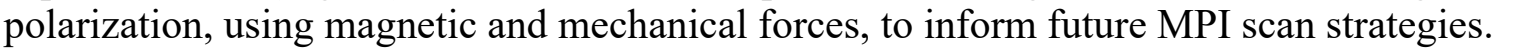

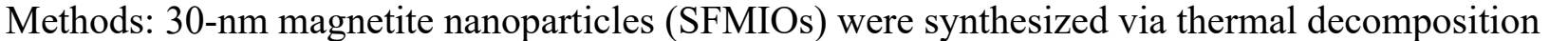

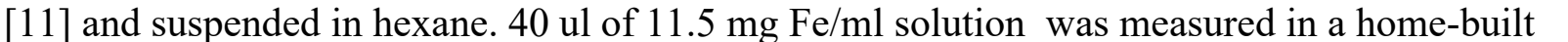

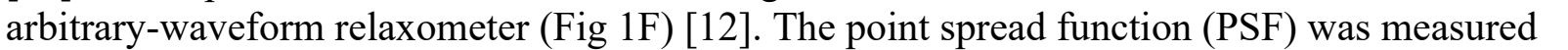

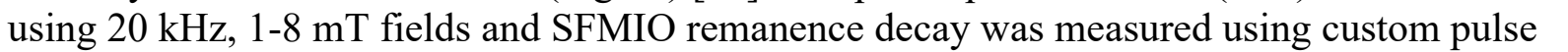

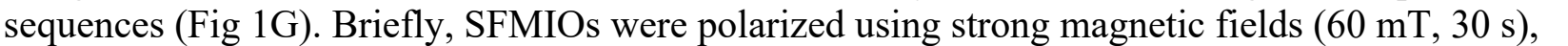

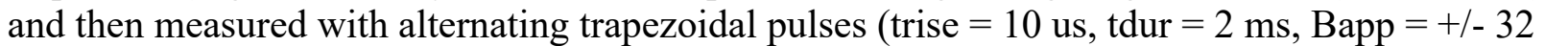

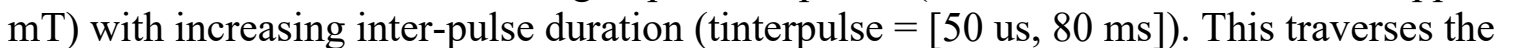

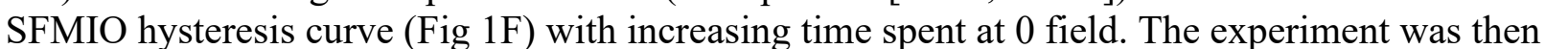

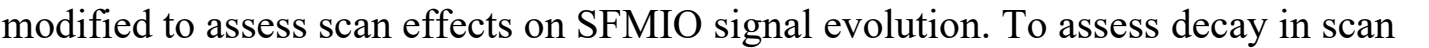

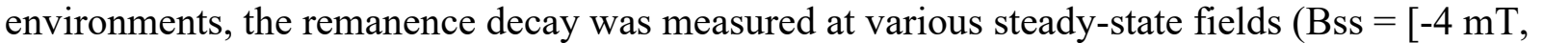

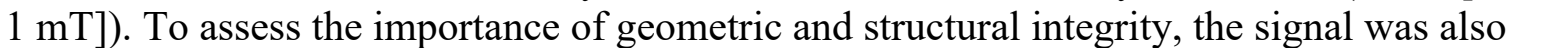

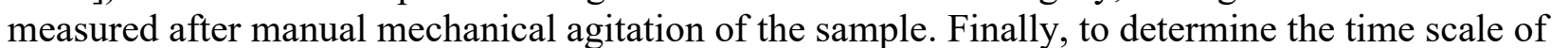

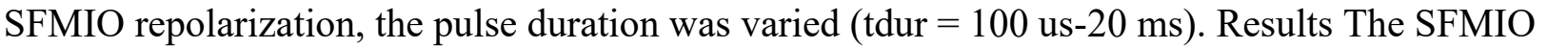

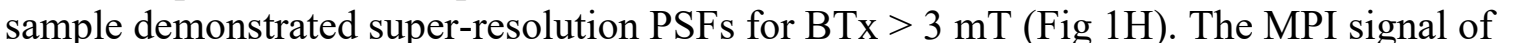

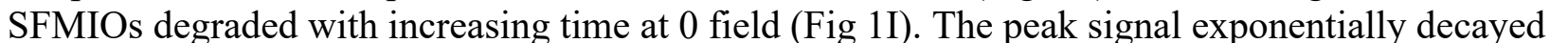

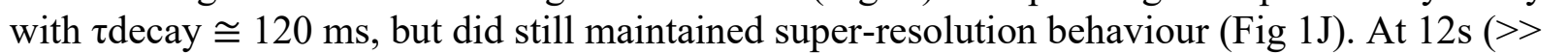

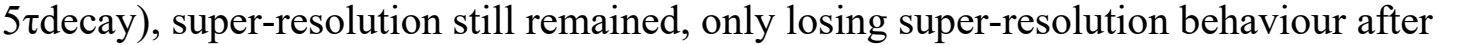

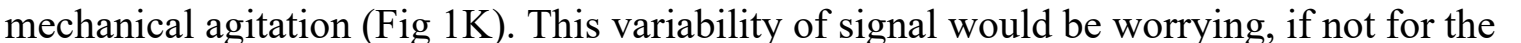

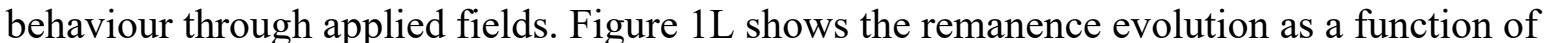

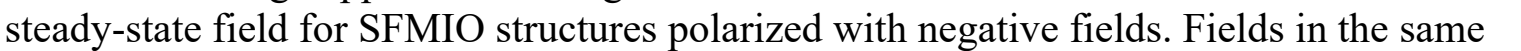

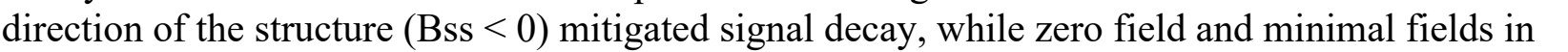

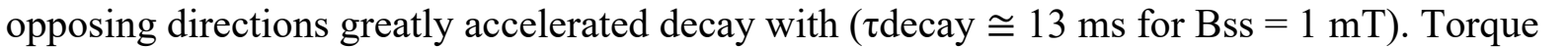

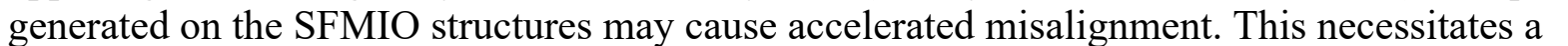

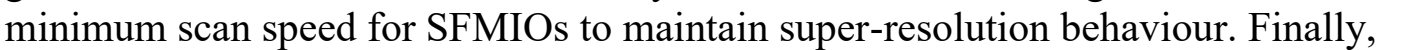

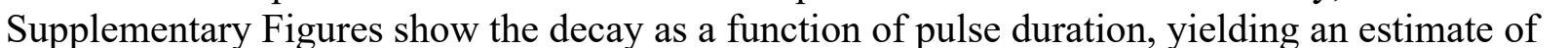




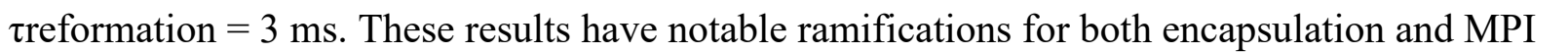

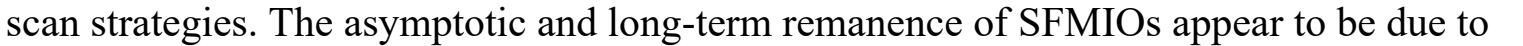

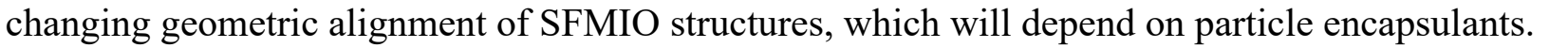

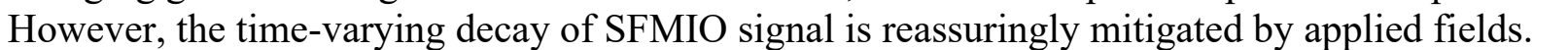

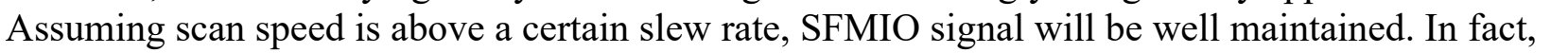

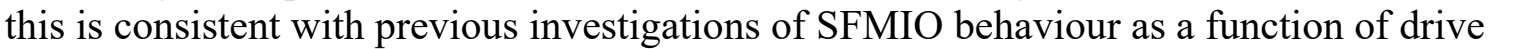

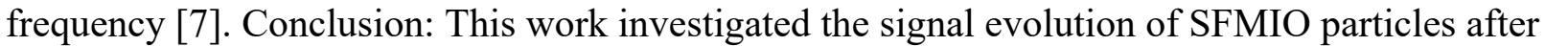

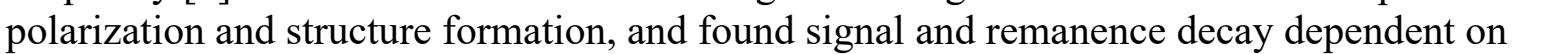

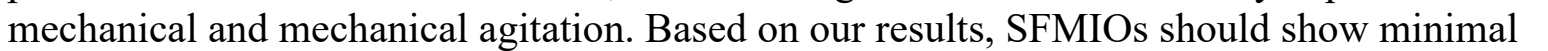

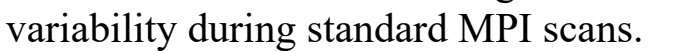

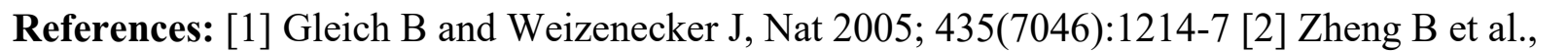

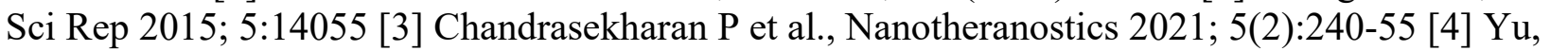

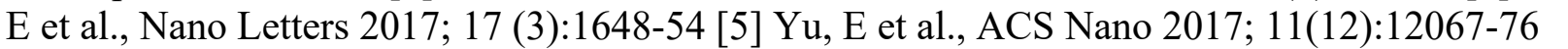

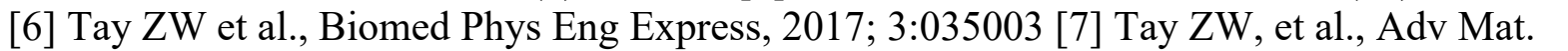

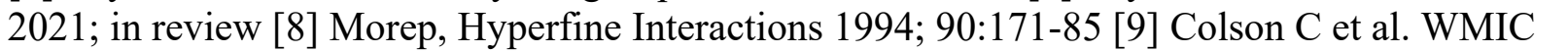

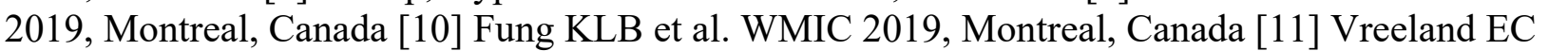

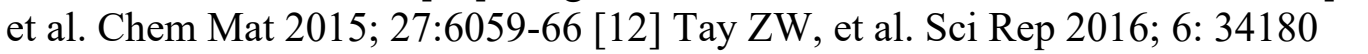

\section{Image/Figure:}

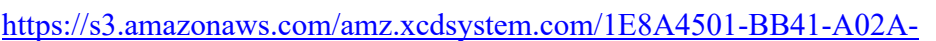

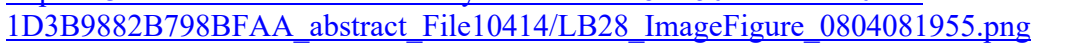

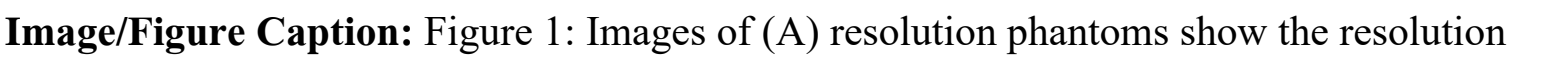

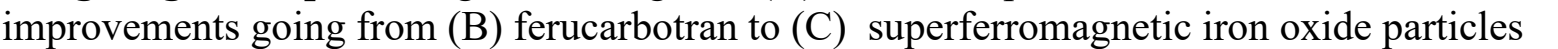

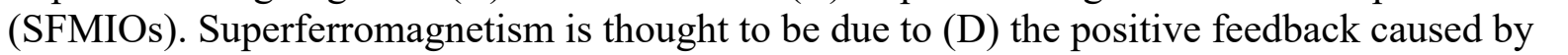

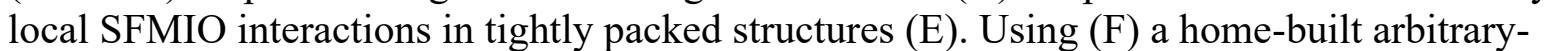

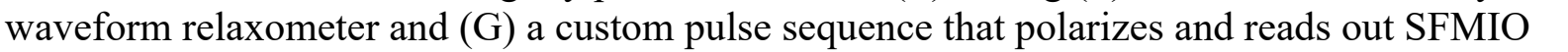

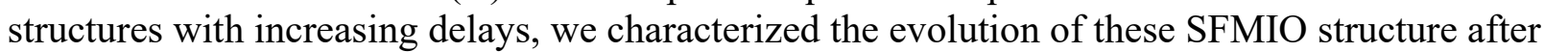

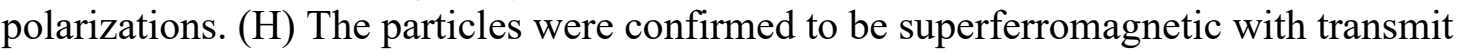

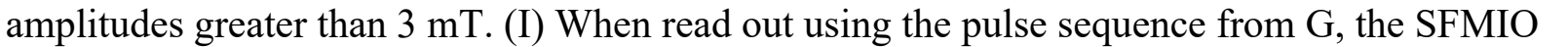

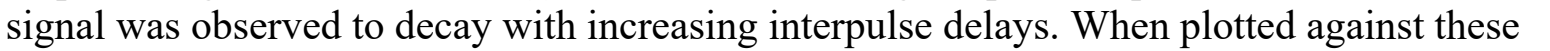

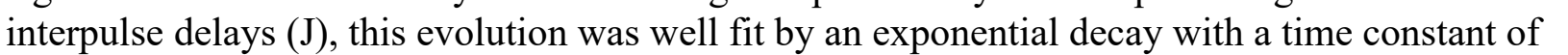

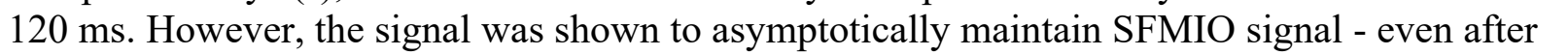

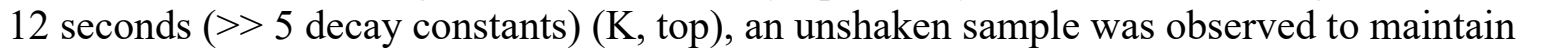

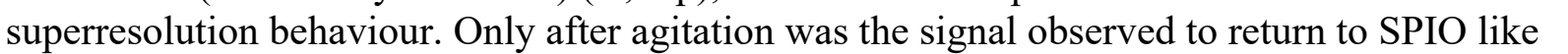

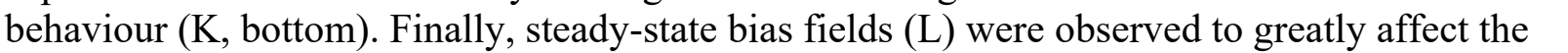

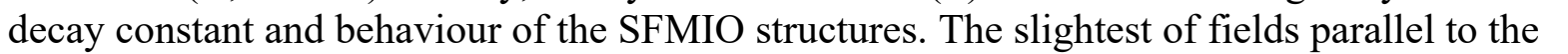

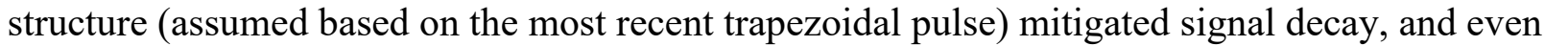

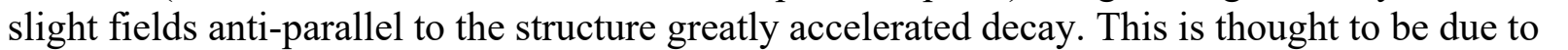

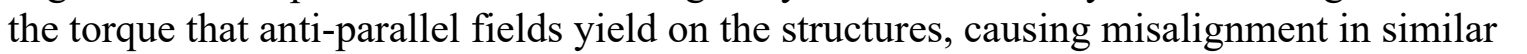

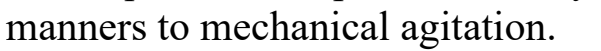

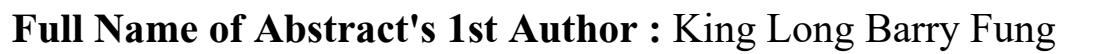

First Name: $\square \square m \square \square \| m$ 
Last Name:

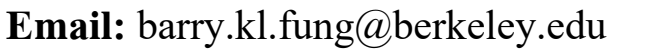

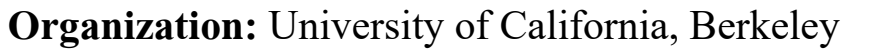

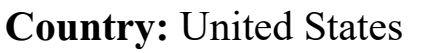




\title{
ID: LB29
}

Effect of Phosphatase and Tensin homolog (PTEN) on the regulation of ProstateSpecific Membrane Antigen (PSMA) in Prostate Cancer.

Teja Muralidhar Kalidindi, Memorial Sloan Kettering Cancer Center, kalidint@mskcc.org

Category: $\square \square \| \square \square \square$

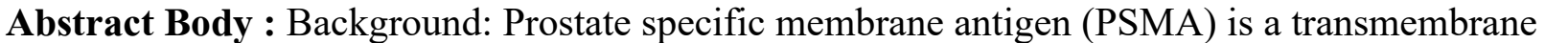

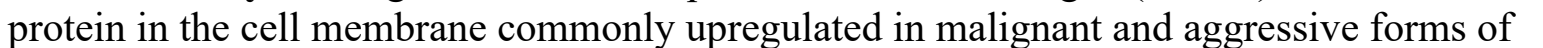

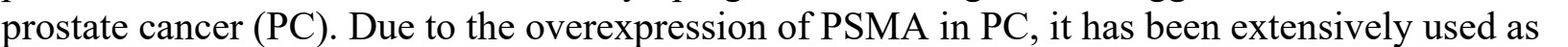

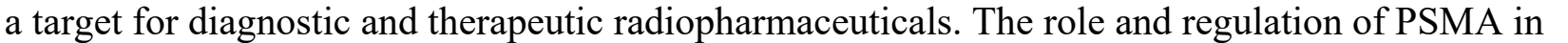

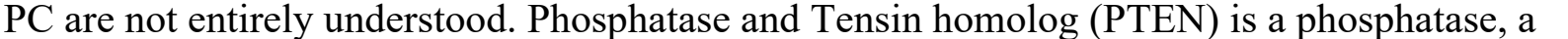

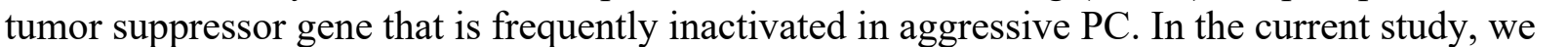

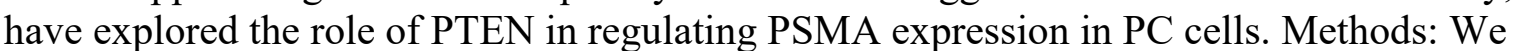
ए

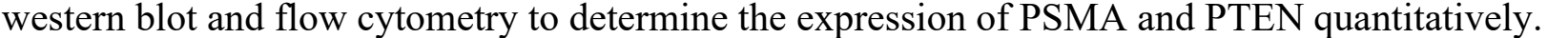

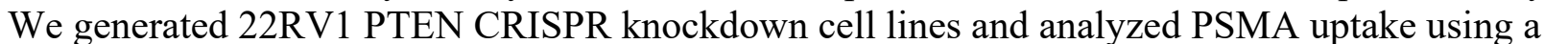
ए ए

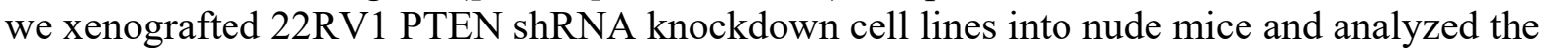

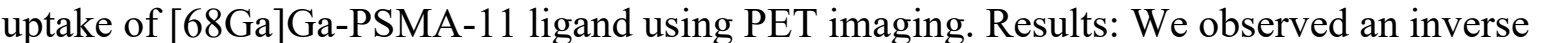

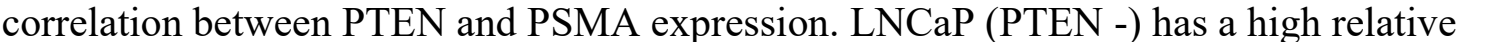

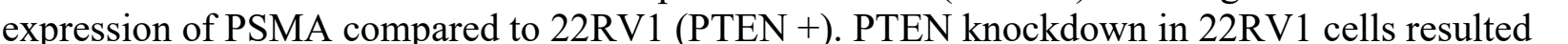
एரm

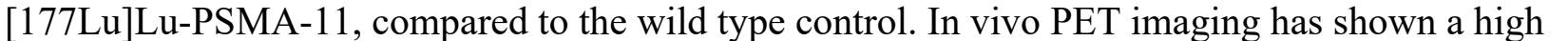

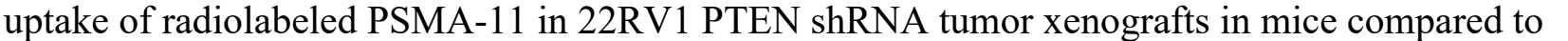

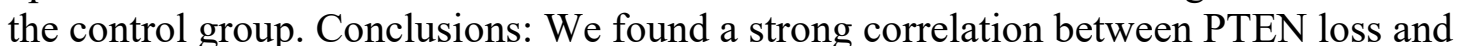

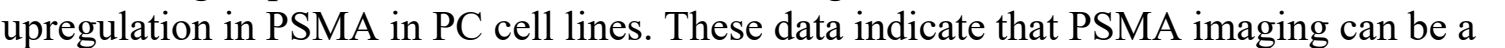

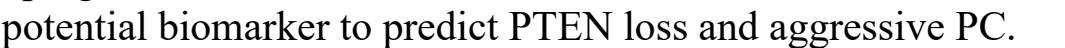

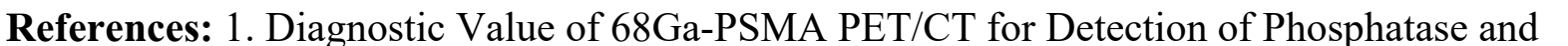

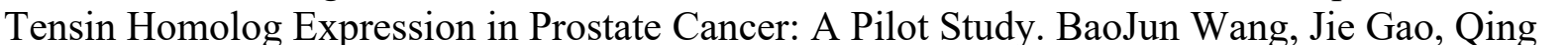

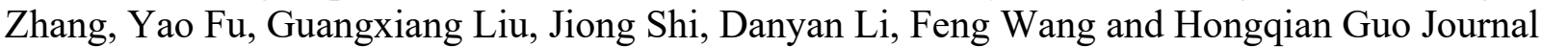

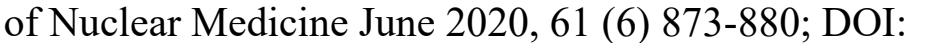

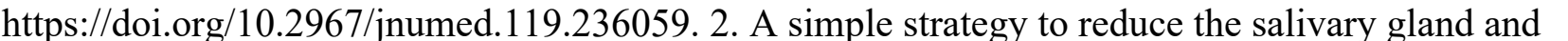

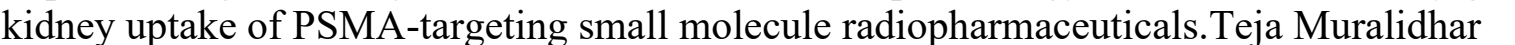

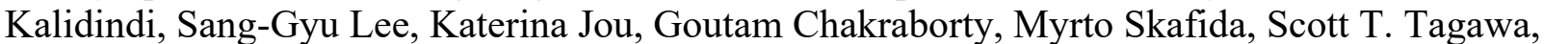

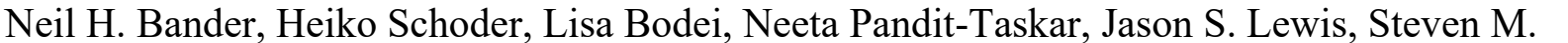

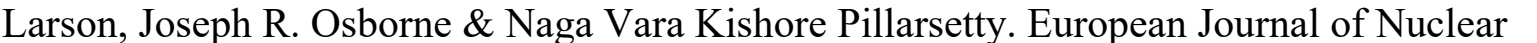

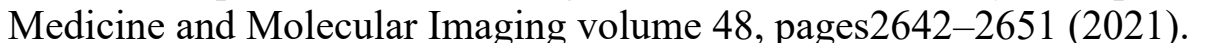

\section{Image/Figure:}




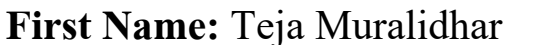

Last Name: $\square \square\|m\| \square$

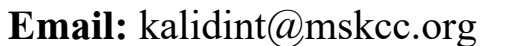

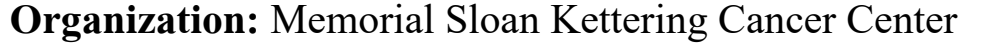

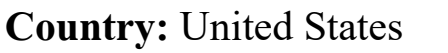




\title{
ID: LB30 \\ 99mTc-MIBI SPECT/CT as a probe for multi-drug resistance in locally advanced breast cancer: histopathological validation study
}

Yaser Abdelhafez, South Egypt Cancer Institute, Assiut University, yga1979@gmail.com

\section{Category: $\square \square\|\| \| \square$}

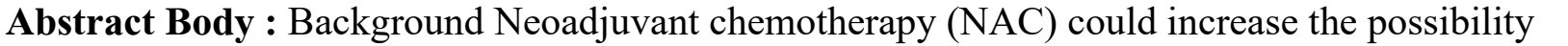

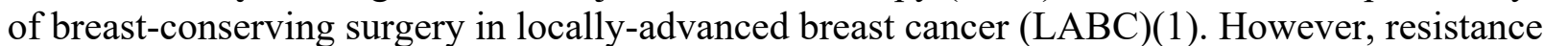

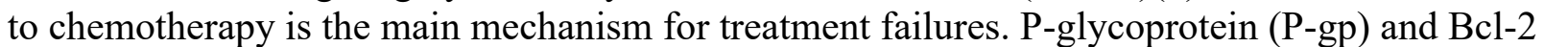

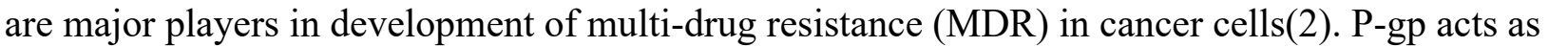

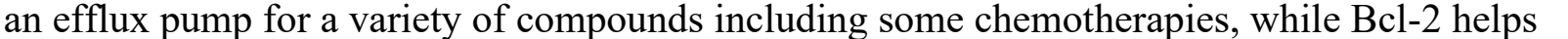

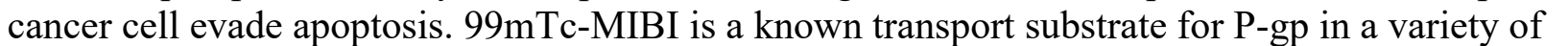

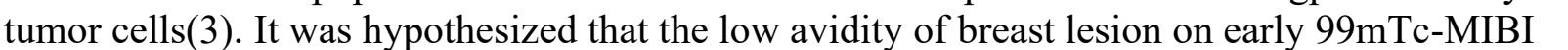

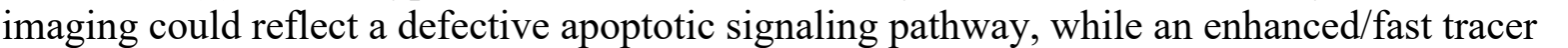

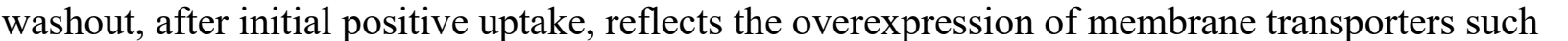

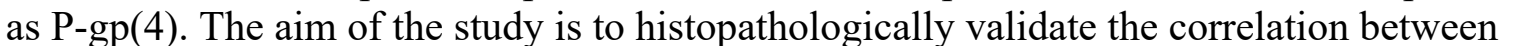

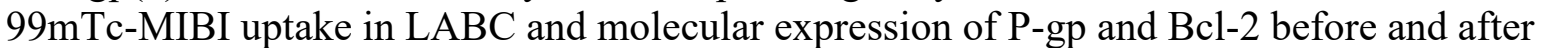

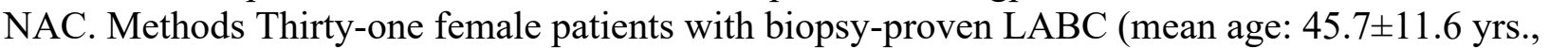

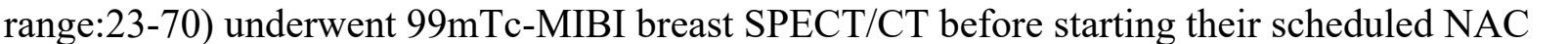
ए

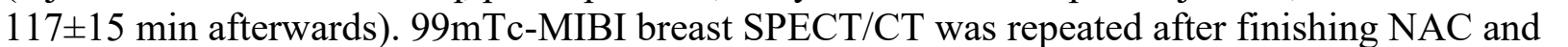

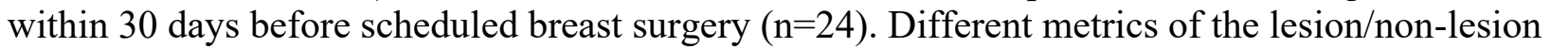

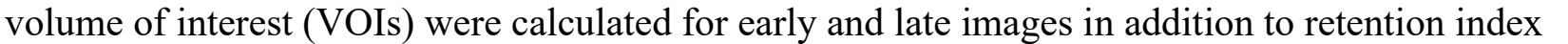
ए ए

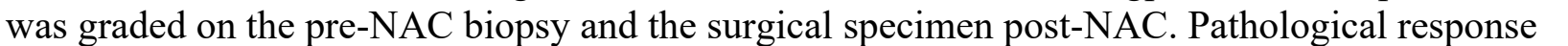

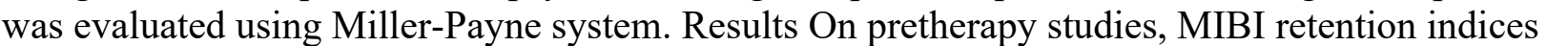

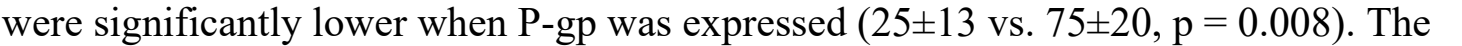

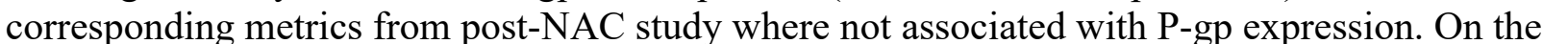

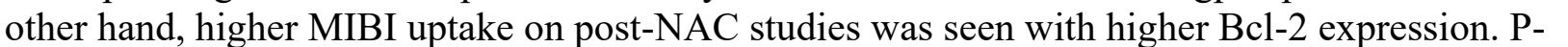

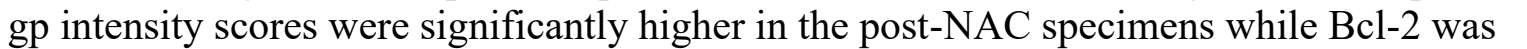

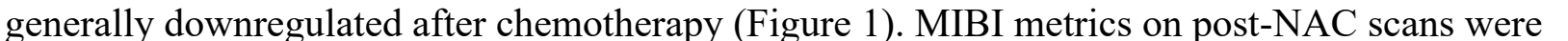

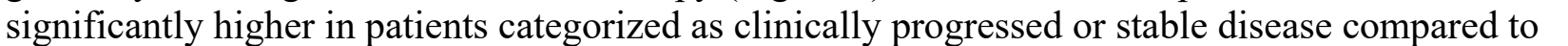

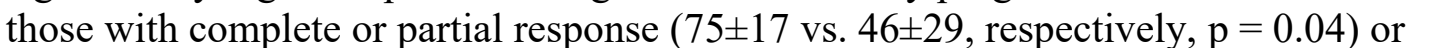

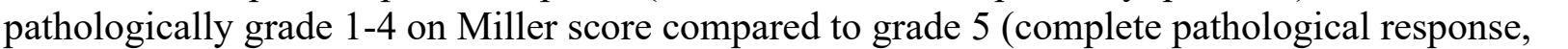

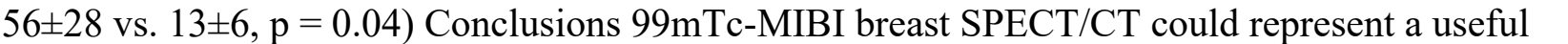

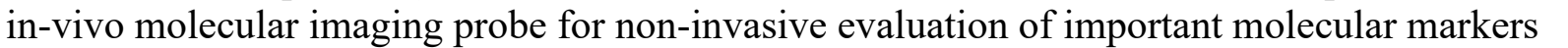

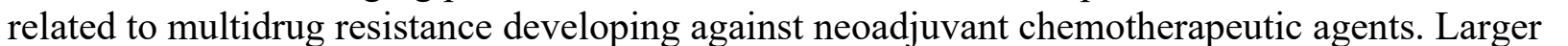

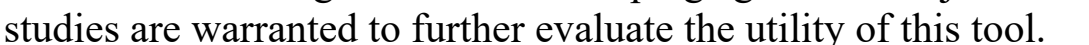

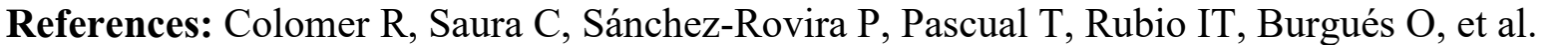

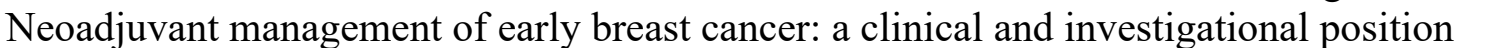




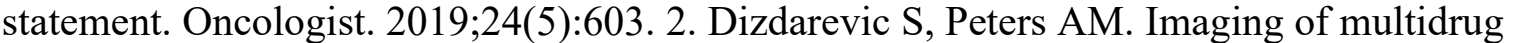

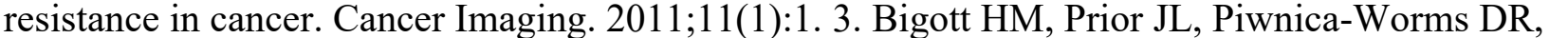

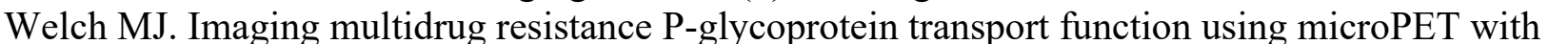

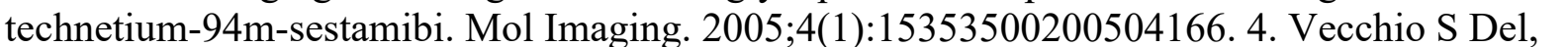

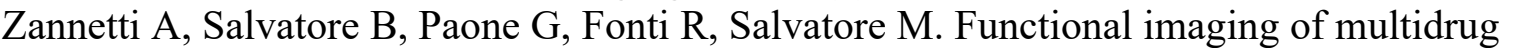

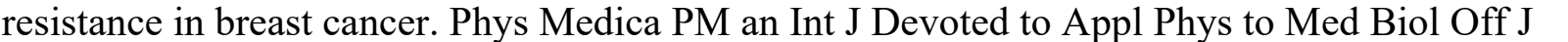

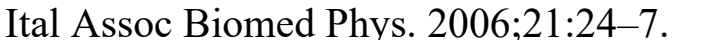

\section{Image/Figure:}

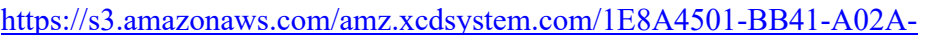

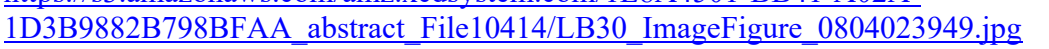

First Name: $\square \square\|\|\|\|$

Last Name: $\square \square \square\|\|\|\|\|\| \|$

Email: $\square\|\|\|\square \square \square \square\| \square \square$

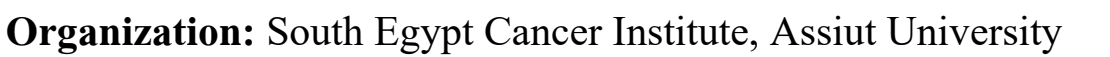

Country: $\square \square \square \square \square$ 


\title{
ID: LB31 \\ Multimodal in vivo tracking of CAR T-cells in preclinical glioblastoma models
}

\author{
Wei Wu, Stanford University, vvwu@stanford.edu
}

Category: $\square \square\|\| \square \square$

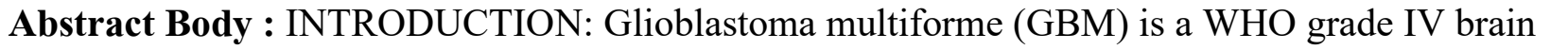

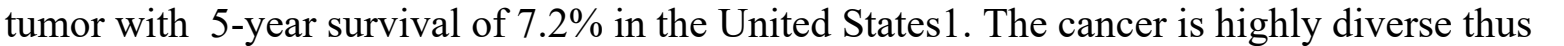

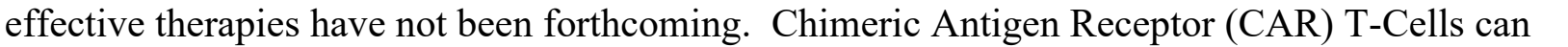

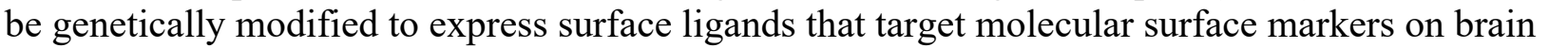

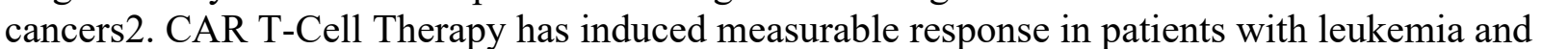

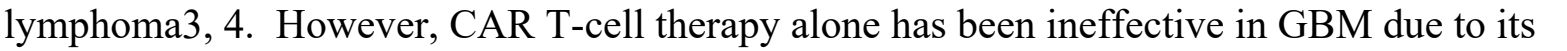

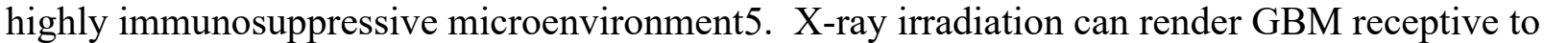

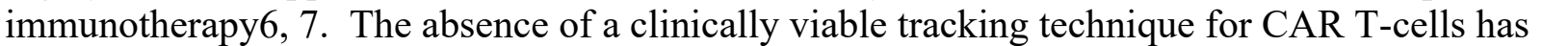

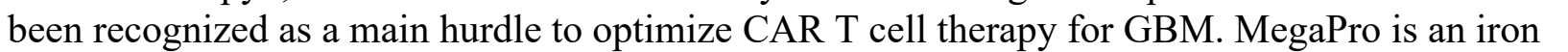

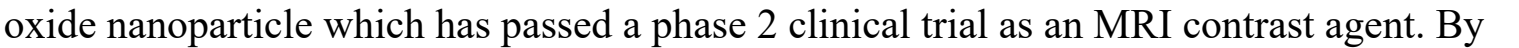

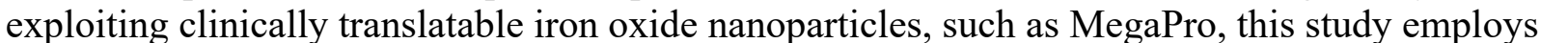
ए ए

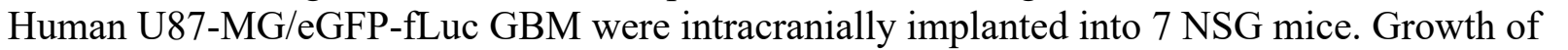

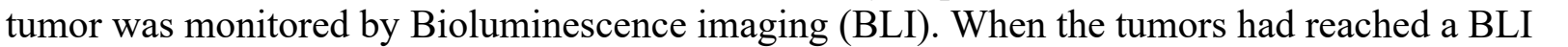

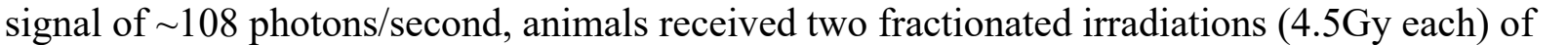

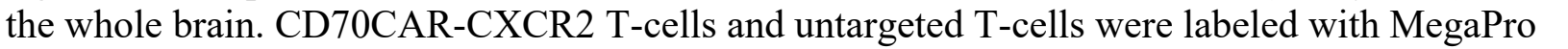

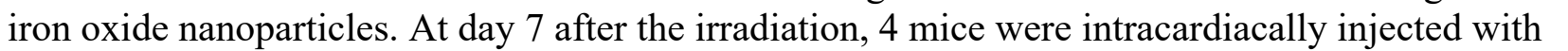

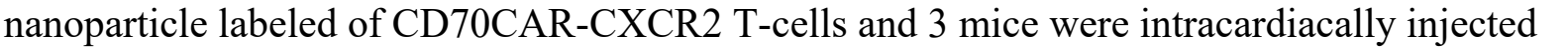

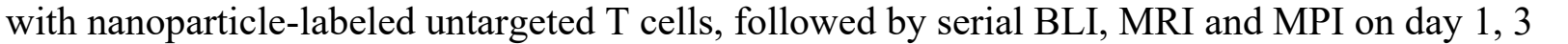

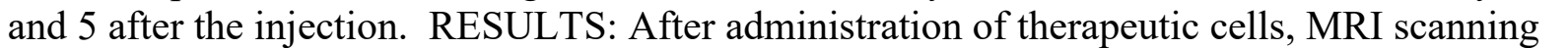

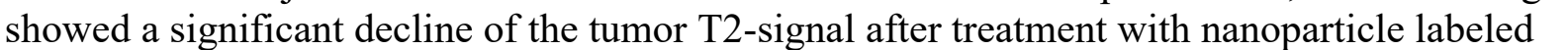

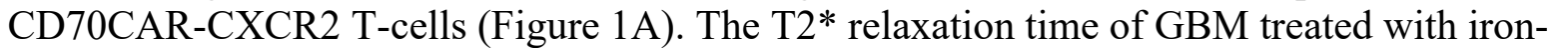

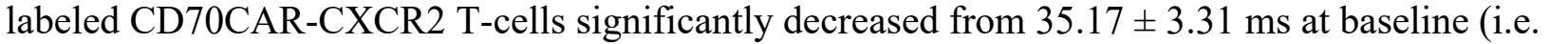

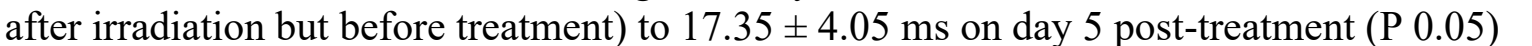

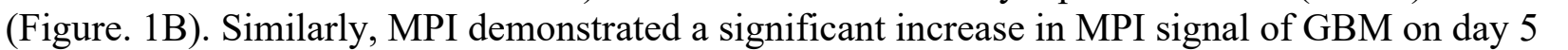

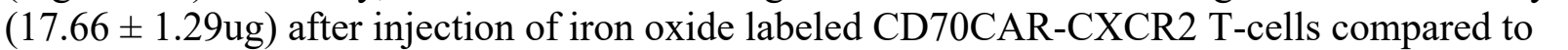

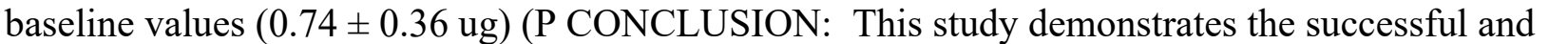

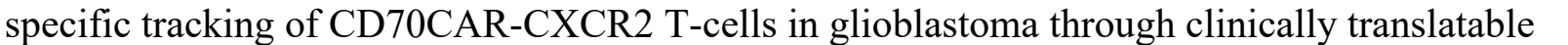

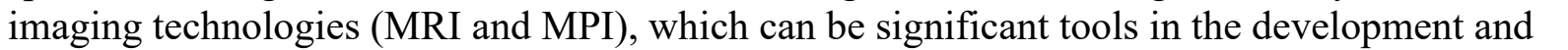

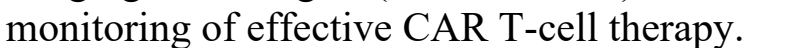

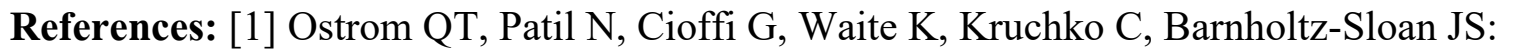

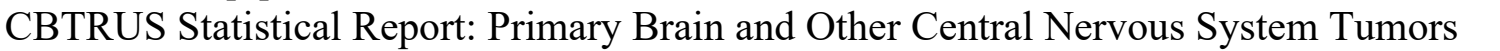

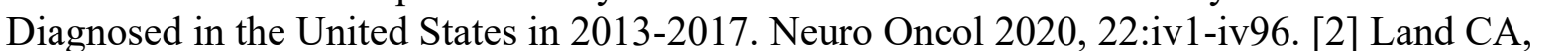

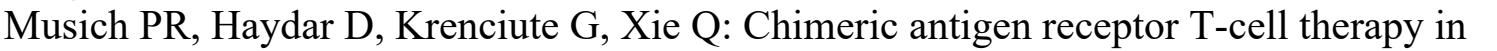

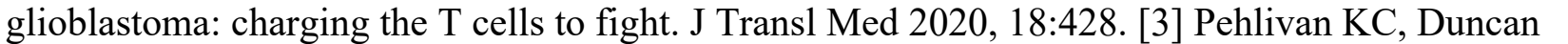




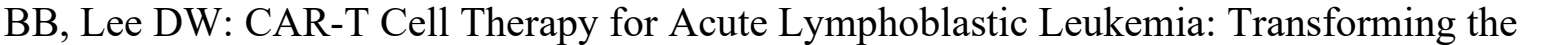

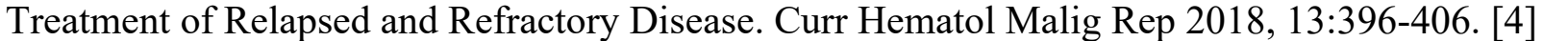

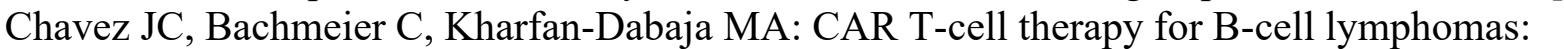

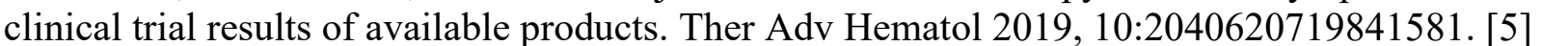

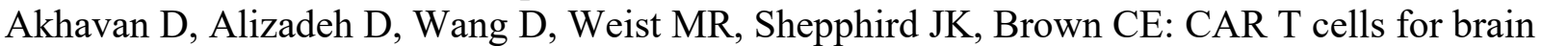
ए

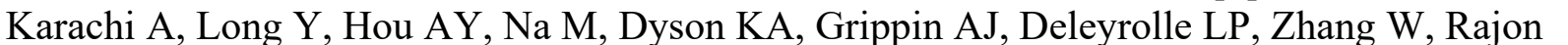

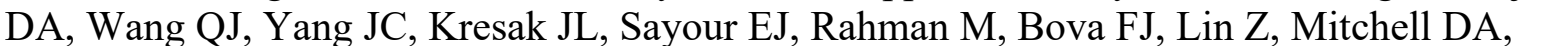

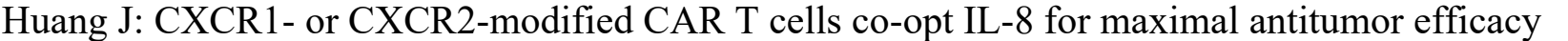

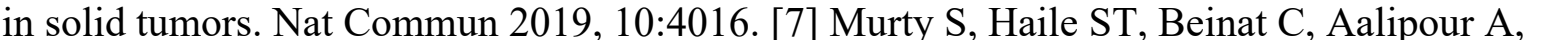

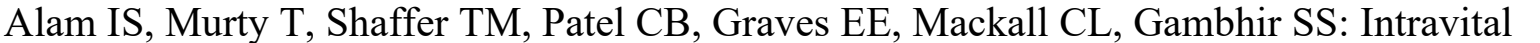

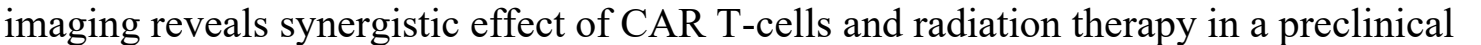

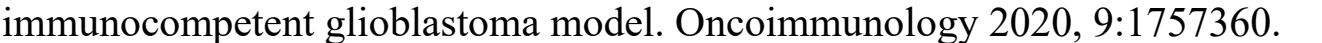

\section{Image/Figure:}

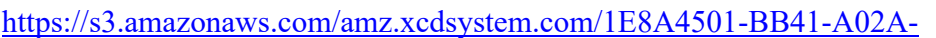

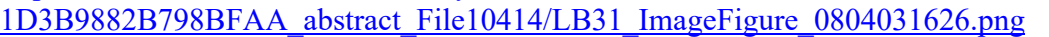

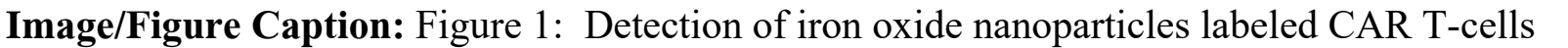

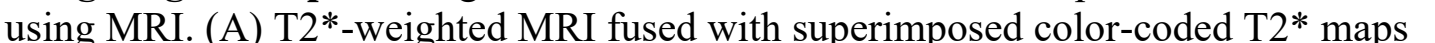

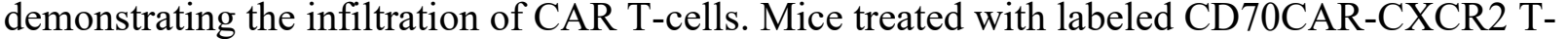

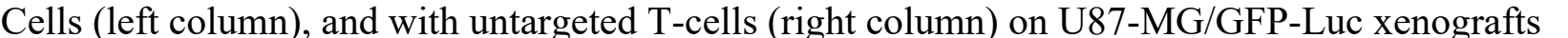

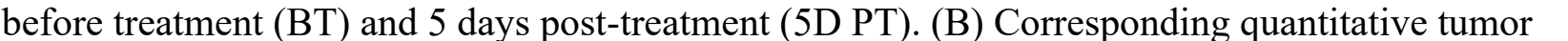

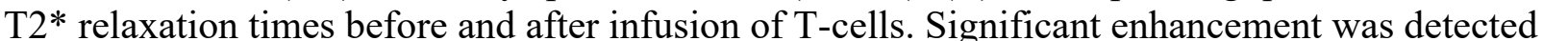
ए

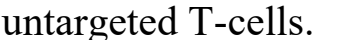

Full Name of Abstract's 1st Author : $\square \square \square \square \square$

First Name: $\square \square m$

Last Name: $\square \square \square$

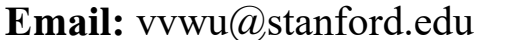

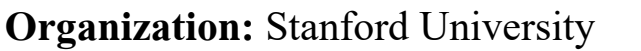

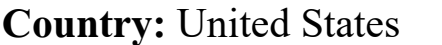




\title{
ID: LB32 \\ VEGF overexpression in breast cancer xenograft significantly increases nanoparticle-mediated siRNA delivery and target gene downregulation
}

\author{
Shanshan Tan, Johns Hopkins University, tanshanshan0425@gmail.com
}

\section{Category: $\square \square\|\| \sqcap \|$}

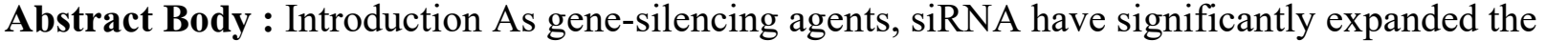
specificity and range of 'druggable' targets making them promising agents for precision

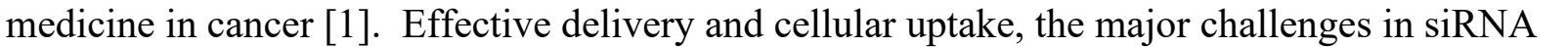

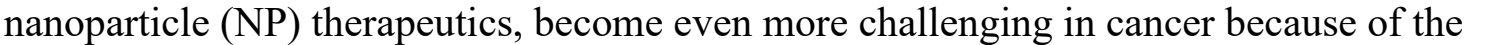

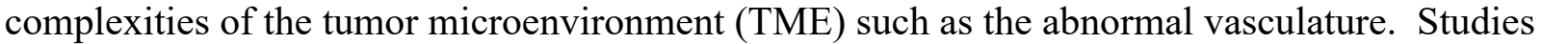

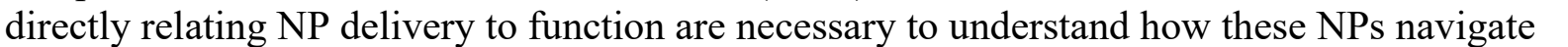

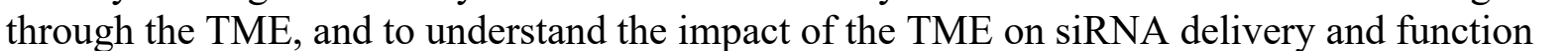

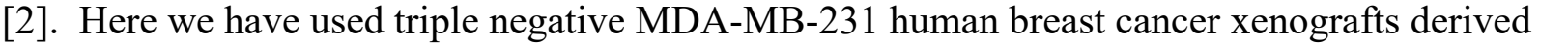

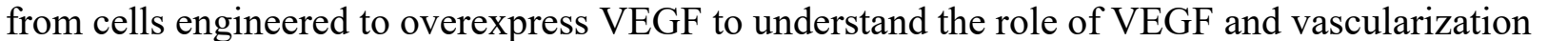

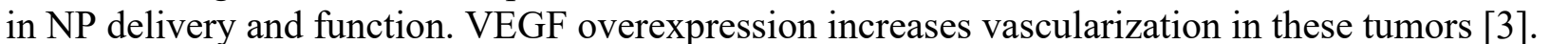

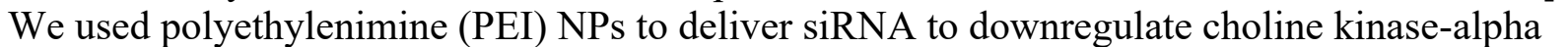

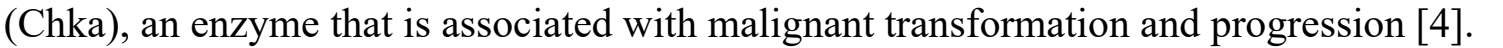

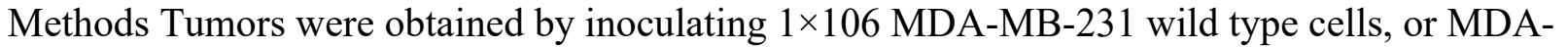

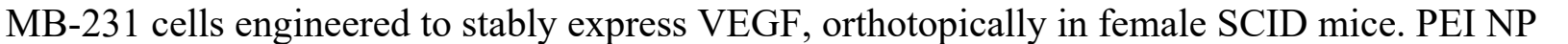

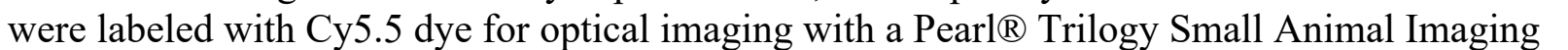

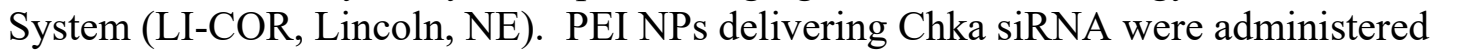
ए

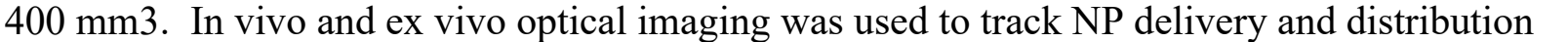

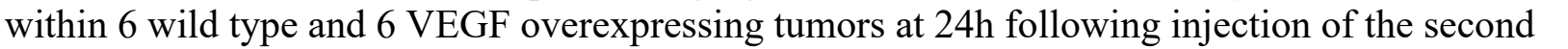

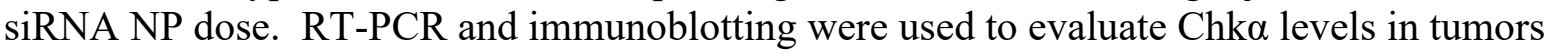

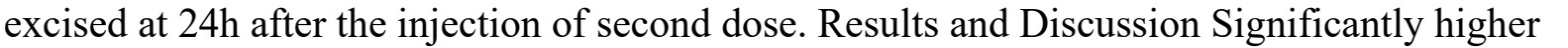

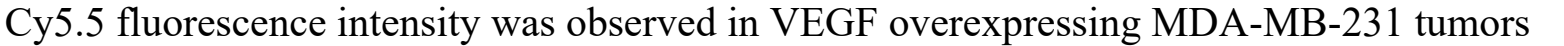

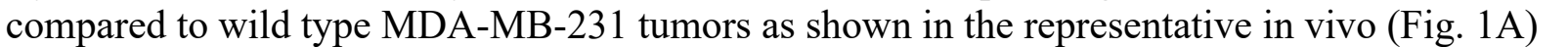

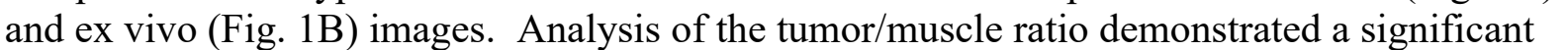
ए

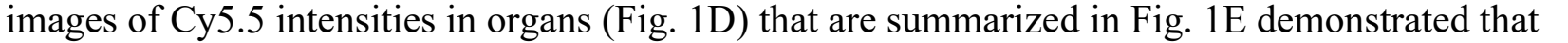

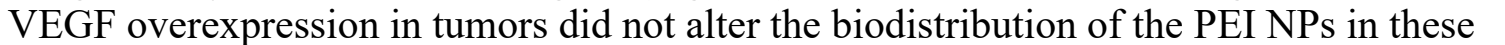

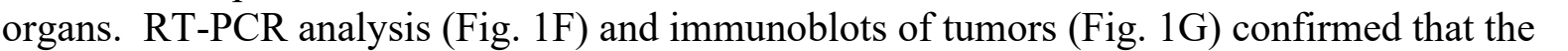

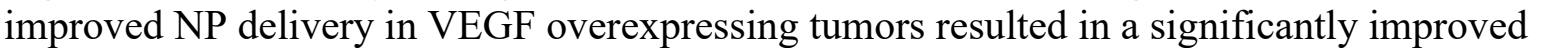

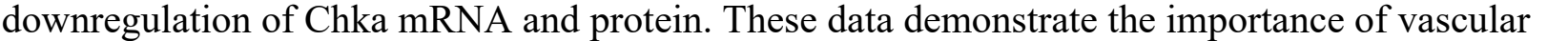

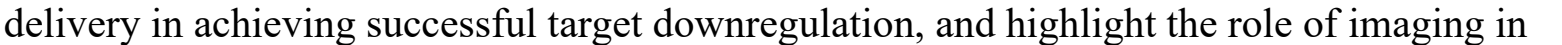

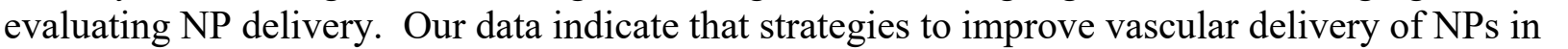
ए
\end{abstract}

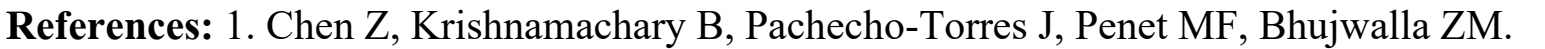

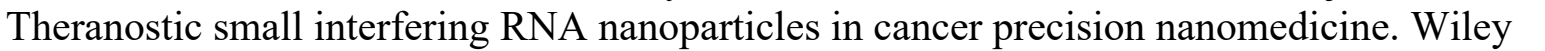




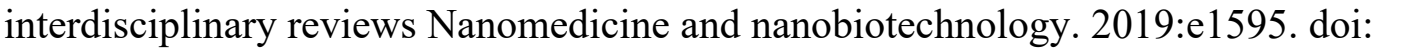

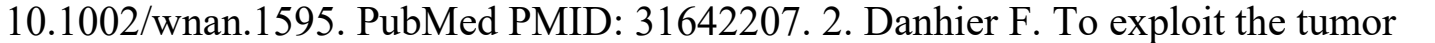

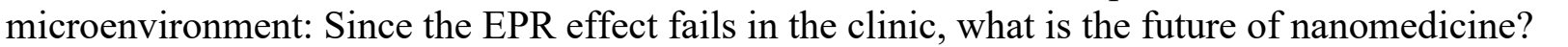

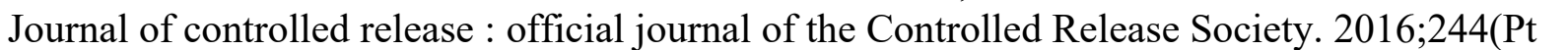

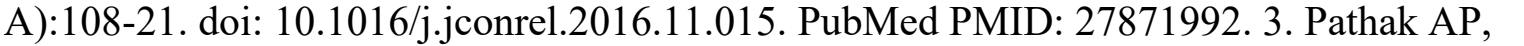

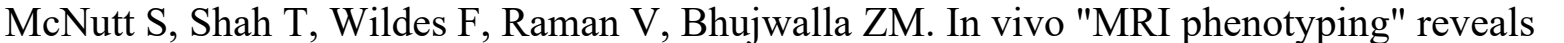

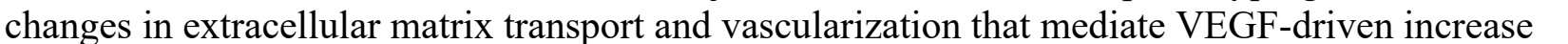

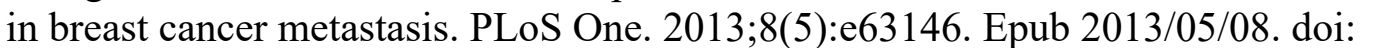

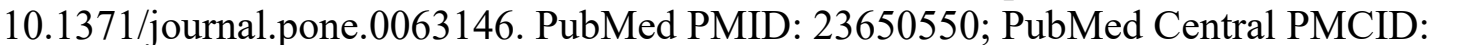

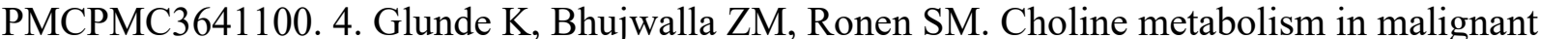

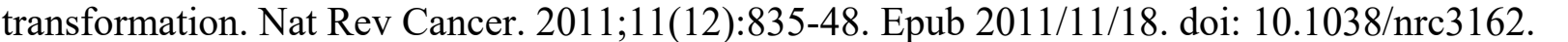

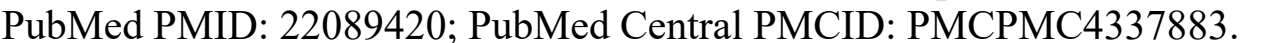

\title{
Image/Figure:
}

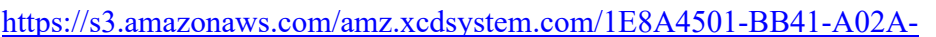

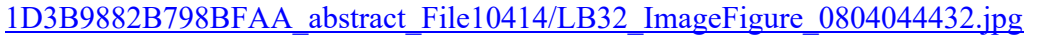

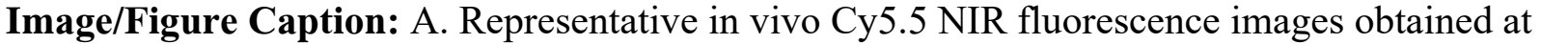

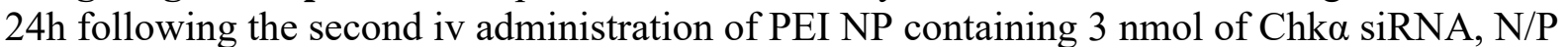

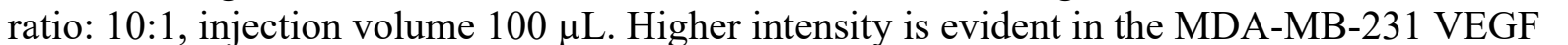

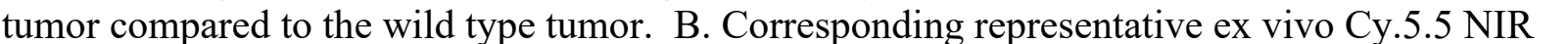

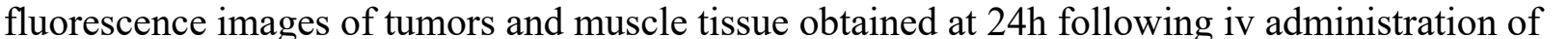

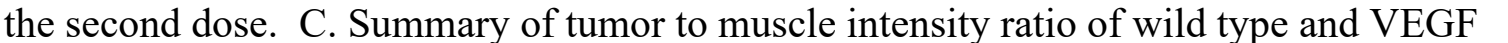

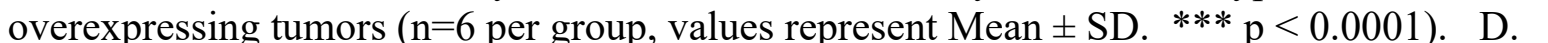

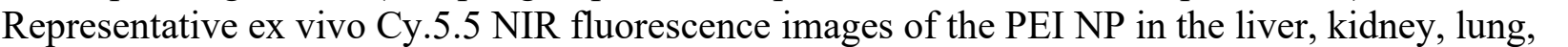

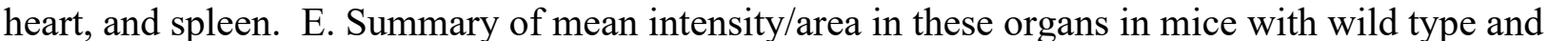

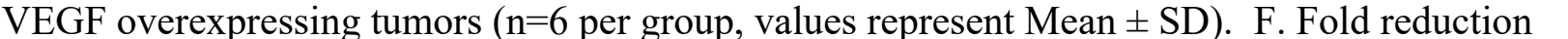

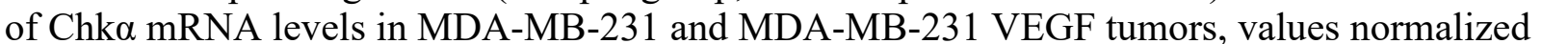

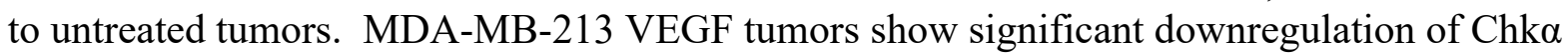

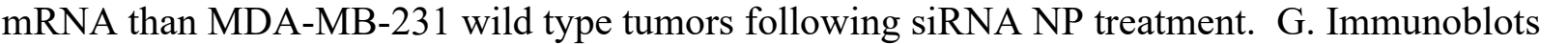

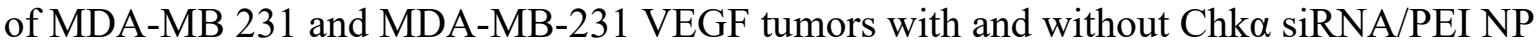

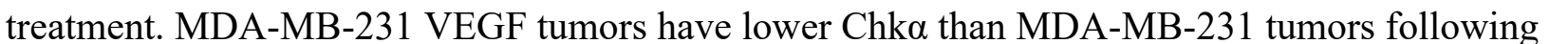
Chk $\alpha$ siRNA/PEI NP treatment.

Full Name of Abstract's 1st Author : $\square \square \square \square \square \square \square(1 \square]$

First Name: $\square \square \square\|\| \square \| \square$

Last Name: $\square \square \square$

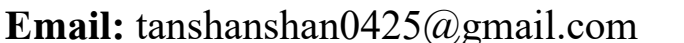

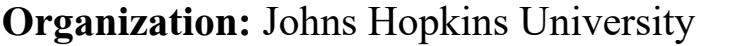

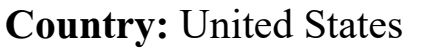




\title{
ID: LB33 \\ 18F-FLT - PET Imaging as a Pharmacodynamic Biomarker for PF-07104091, a CDK2 Selective Inhibitor
}

\author{
Bing Yang, Pfizer Inc, Bing.Yang@pfizer.com
}

\section{Category: $\square \square\|\| \square[1]$}

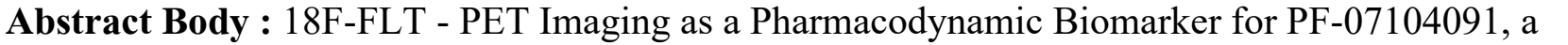

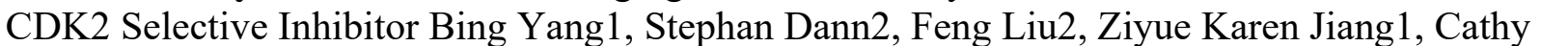

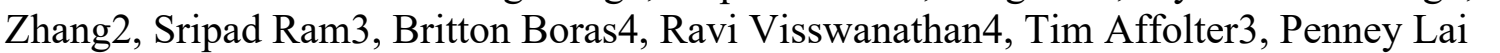

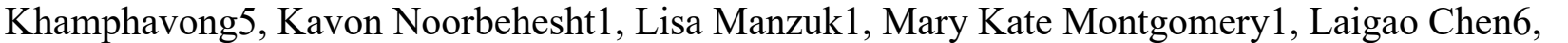

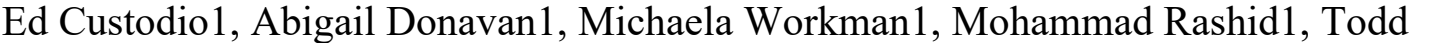

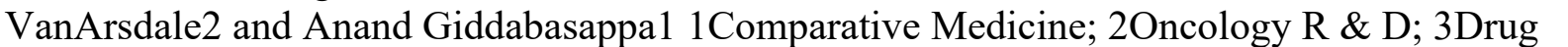

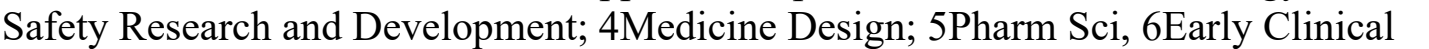

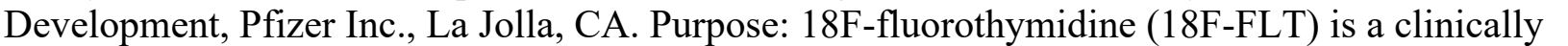

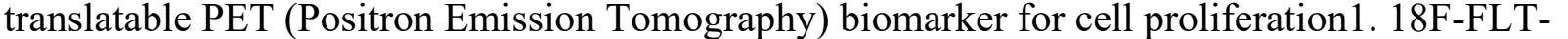

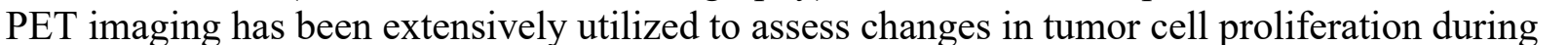

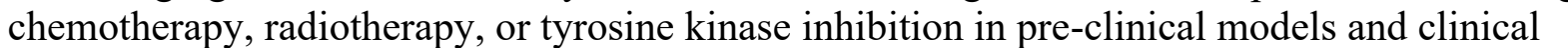

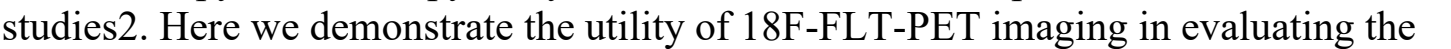

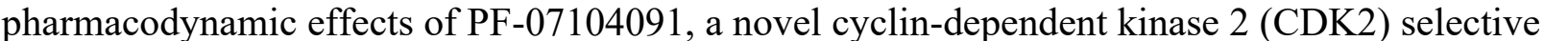
ए

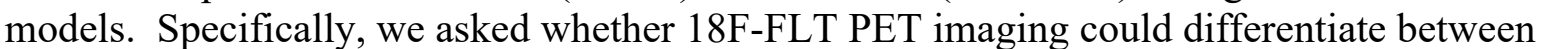

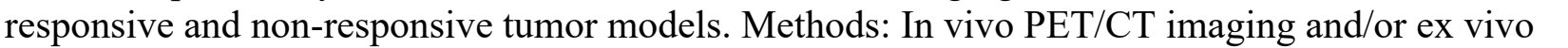

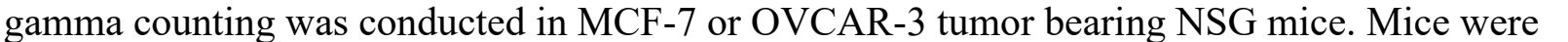

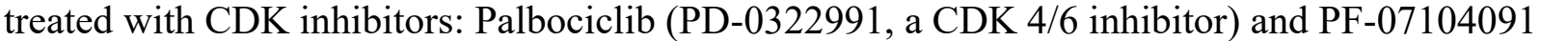

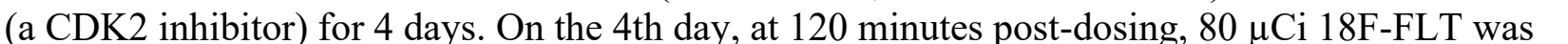

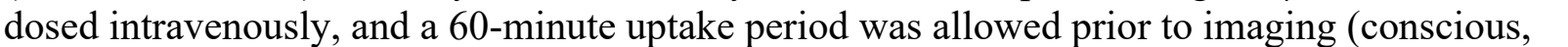

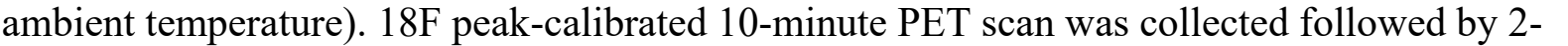

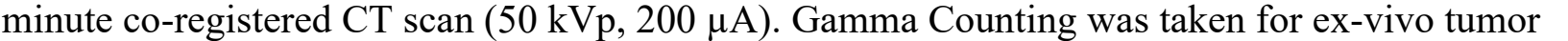

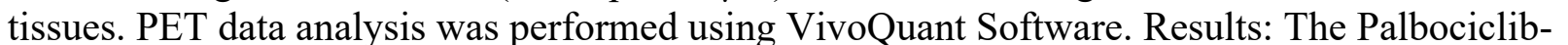

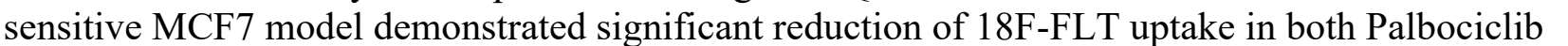

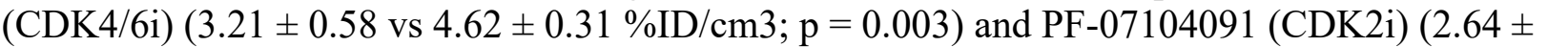

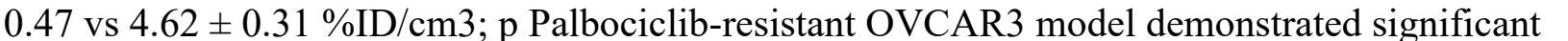

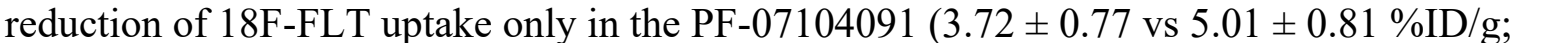

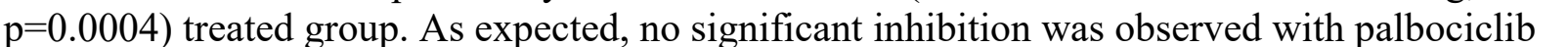

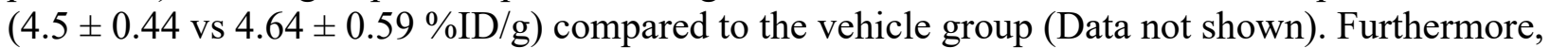

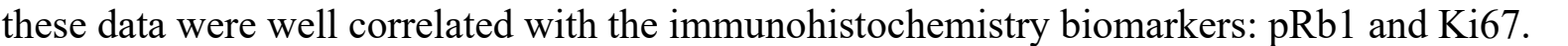

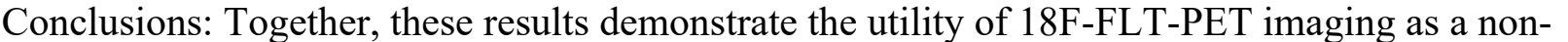

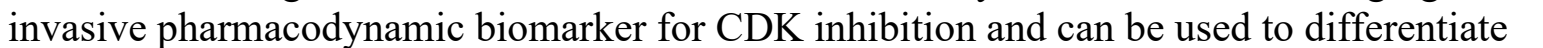

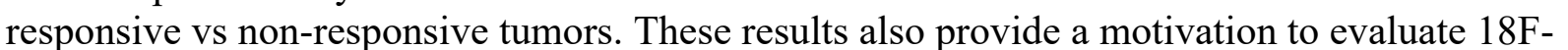

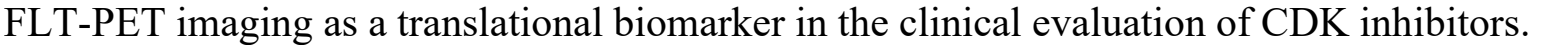

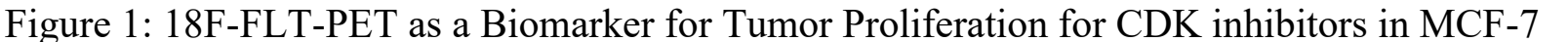

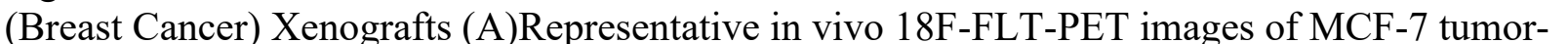




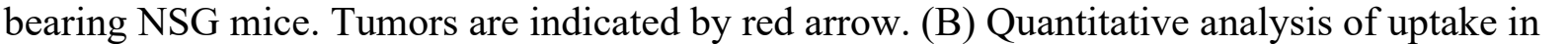

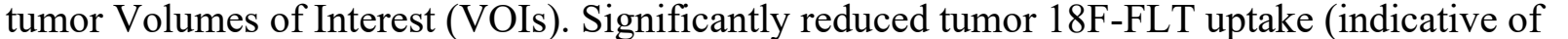

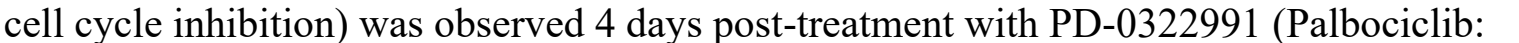

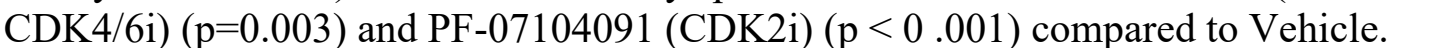

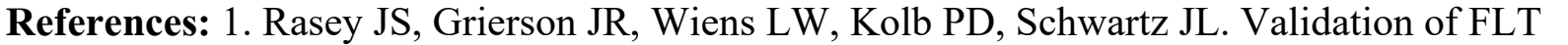

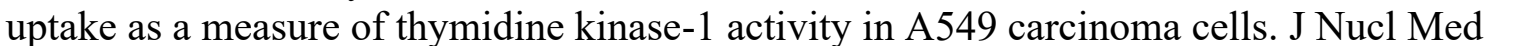

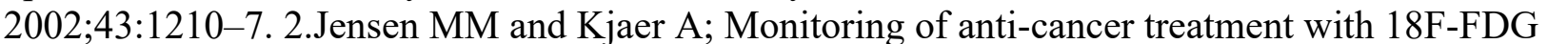

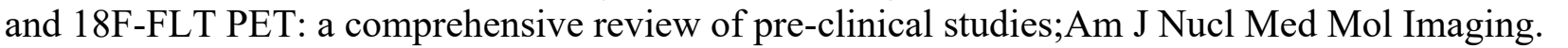

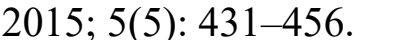

\section{Image/Figure:}

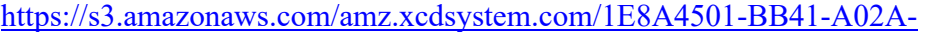

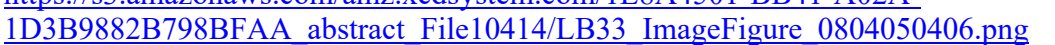

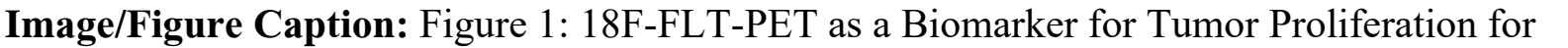

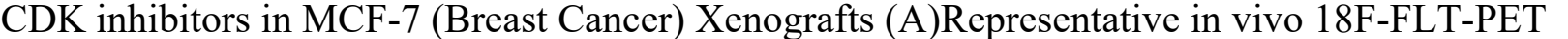

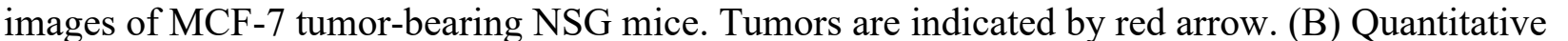

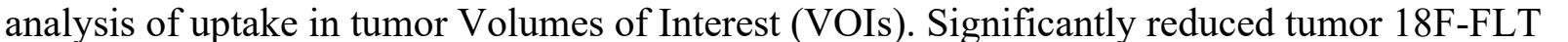

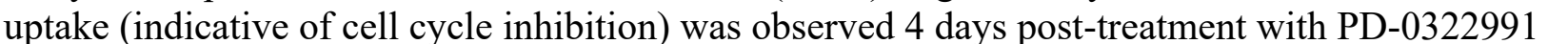

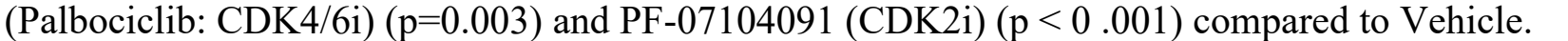

First Name:

Last Name: $\square \square \square \square$

Email: $\square \square \square \square \square \square \square \square\|m\| \| \square \square$

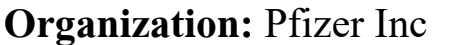

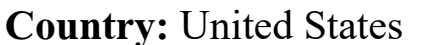




\title{
ID: LB34 \\ Integrating PET and MALDI-2 Imaging for Discovery of a Theranostic Probe for Brain Endocannabinoid $\alpha / \beta$-Hydrolase Domain 6 (ABHD6)
}

Muneer Ahamed, The University of Queensland, muneer.ahamed@uq.edu.au

Category: $\square \square \square\|\square\| \| \square \square \square$

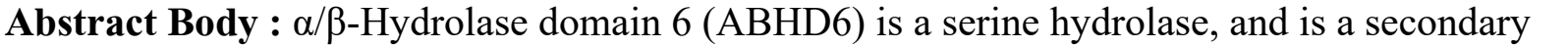

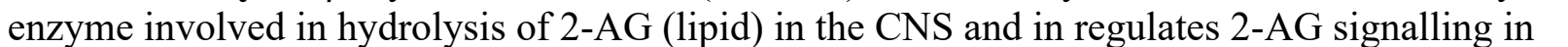

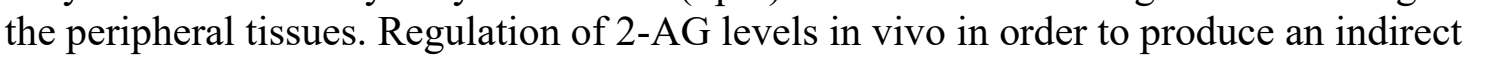

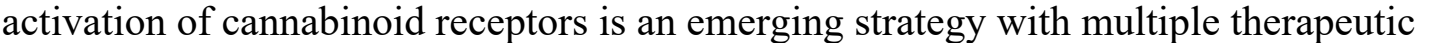

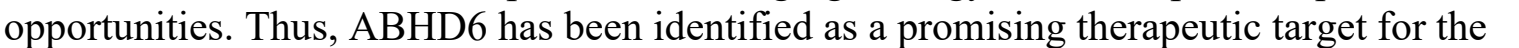

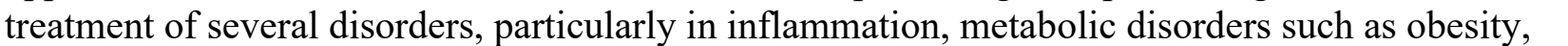

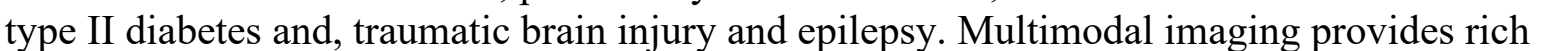

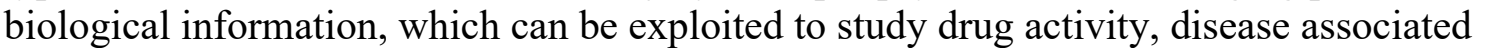

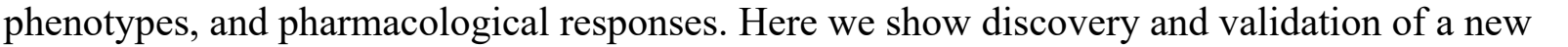

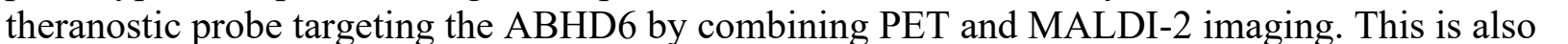

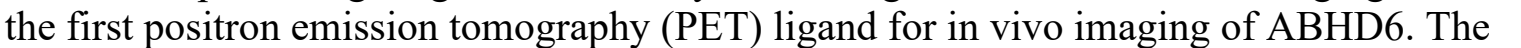

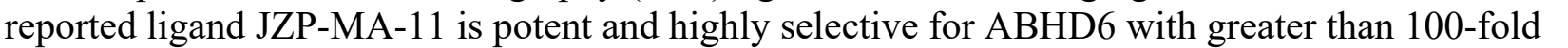

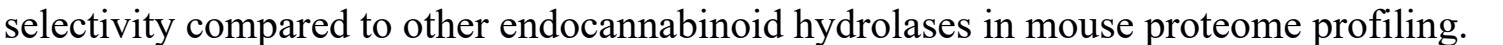

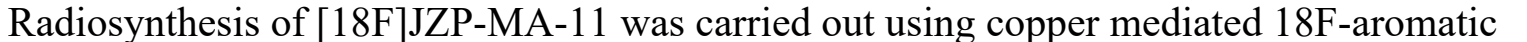

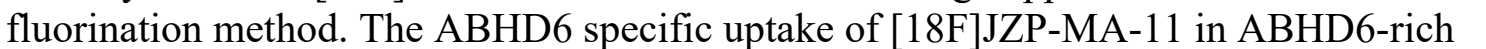

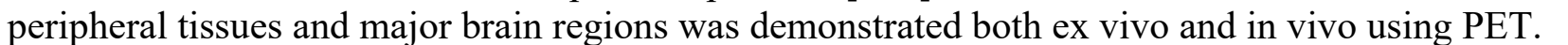

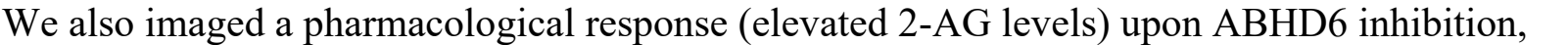

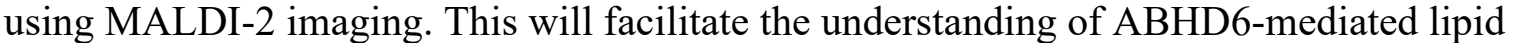

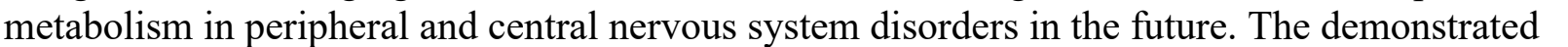

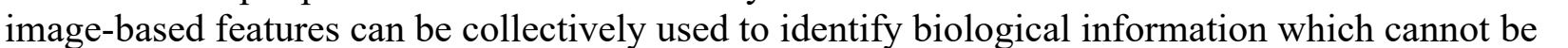

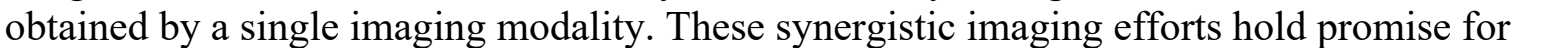

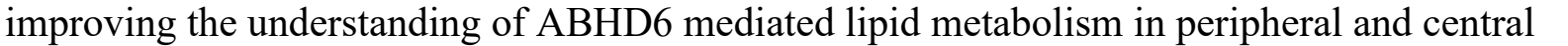

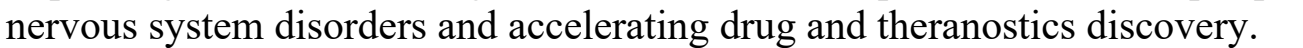

References: Key References: • Positron Emission Tomography Imaging of the Endocannabinoid

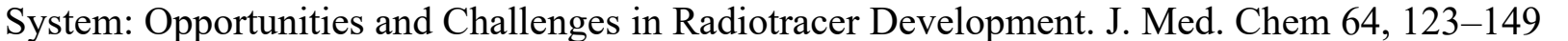

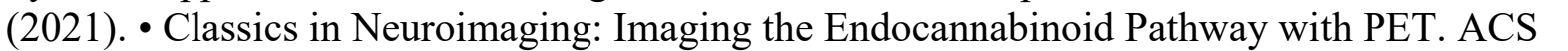
0

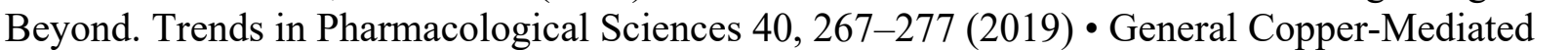

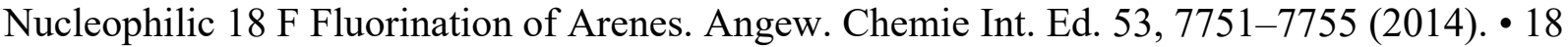

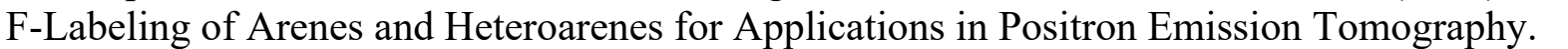

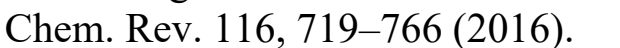

\section{Image/Figure:}


First Name:

Last Name:

Email: $\square \square \square\|\|\|\square \square \square \square \square \square\| \square \square \square$

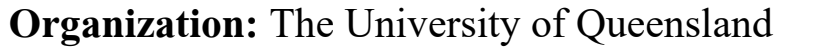

Country: $\square \square\|\|\|\|\|\| \square$ 


\title{
ID: LB35
}

A rare case of papillary thyroid carcinoma with a breast metastasis

kadhafi Syammach, Universitas Padjadjaran, kadhafi2015@gmail.com

Category: $\square \square\|\| \square \square$

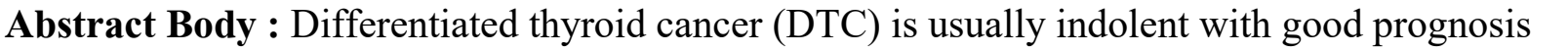

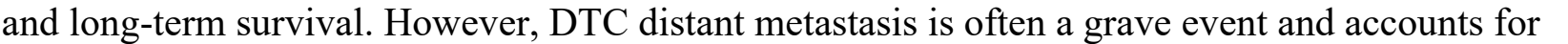

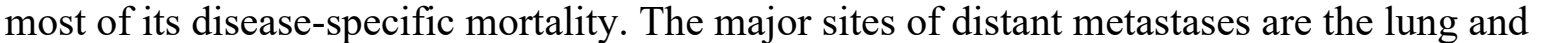

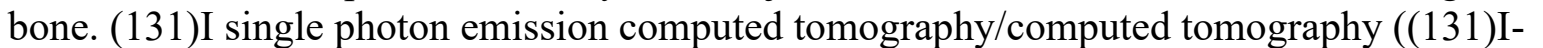

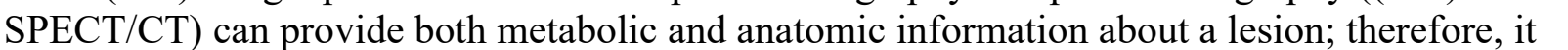

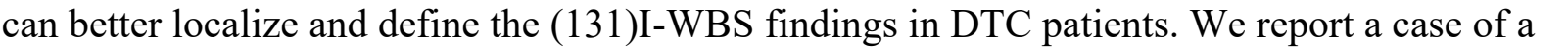

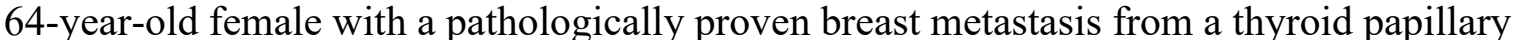

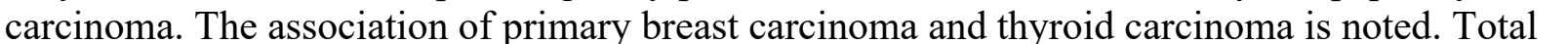

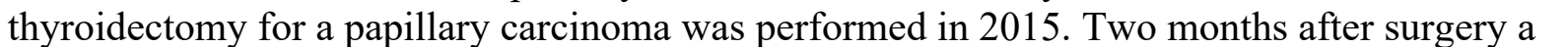

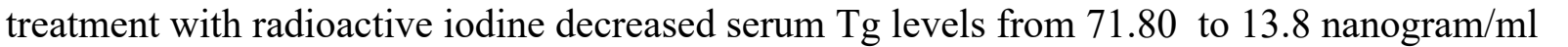
口

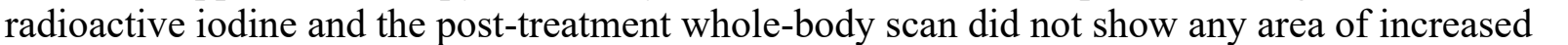

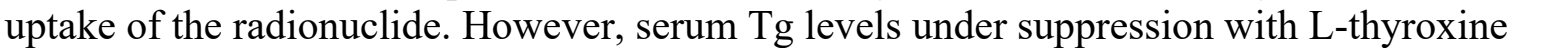

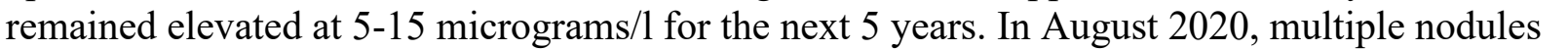

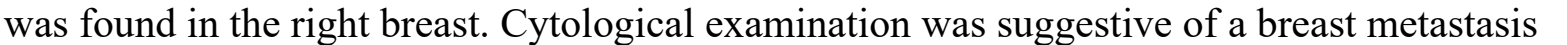

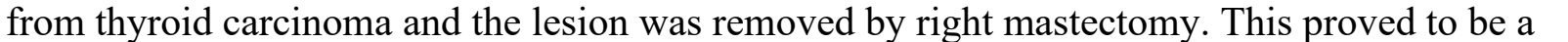

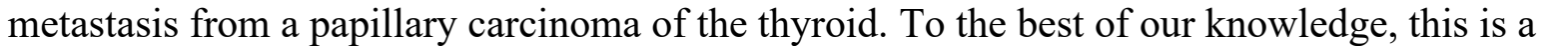

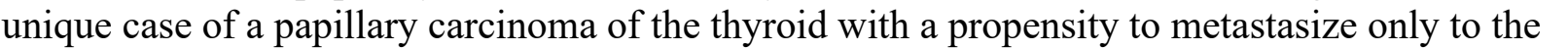

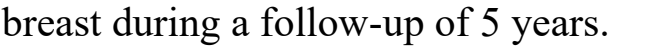

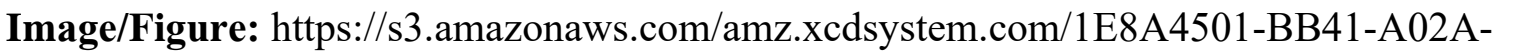

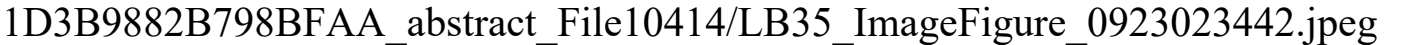

First Name: $\square\|\sqcap\| \Pi$

Last Name: $\square \square \square \square \square \| m$

Email: $\square \square \square\|\Pi \sqcap \square \square \square\| m \square \square$

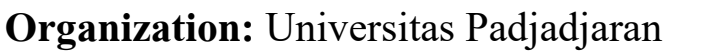

Country: $\square \square\|\sqcap\| \| m$ 


\title{
ID: LB36 \\ In vitro and in vivo validation of a novel humanized scFv antibody-based reporter gene for in vivo tracking of CAR-T cell-based immunotherapies
}

\author{
Alessia Volpe, Memorial Sloan Kettering Cancer Center, VolpeA1@mskcc.org
}

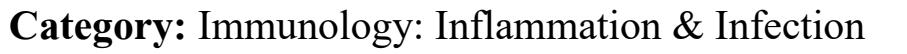

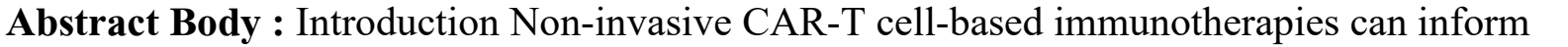

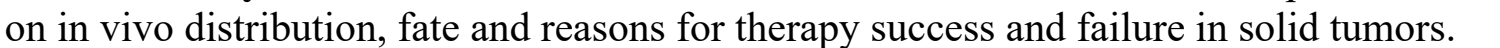

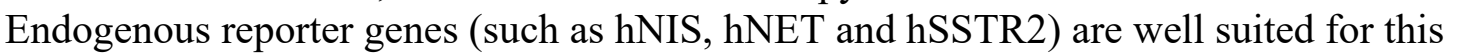

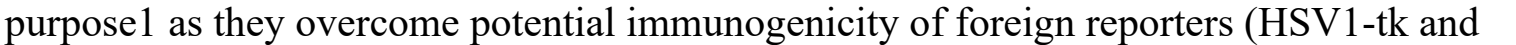

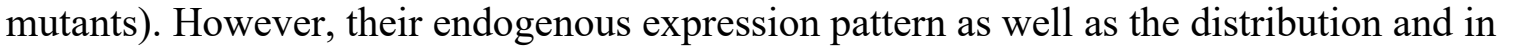

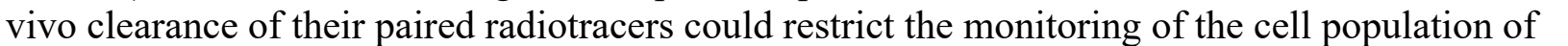

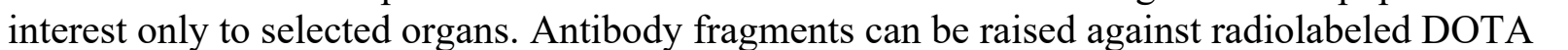

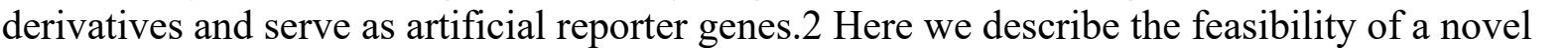

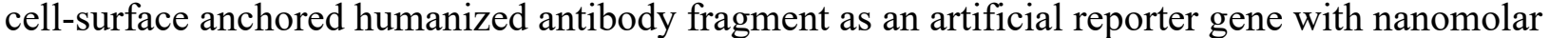

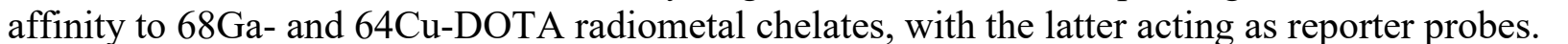

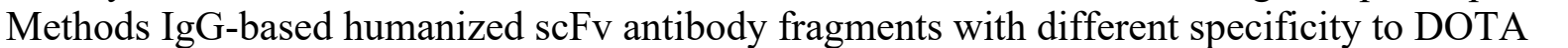

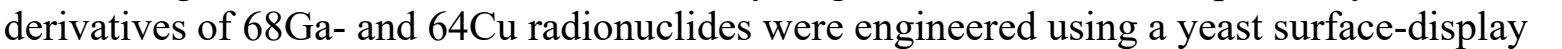

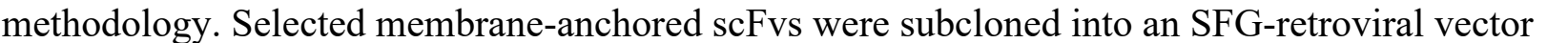

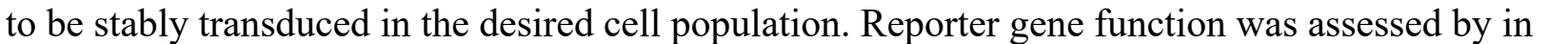

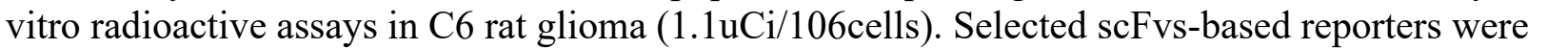

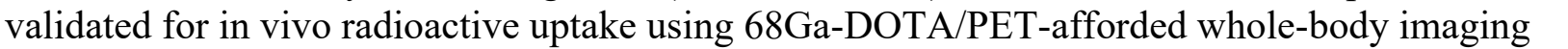
ए

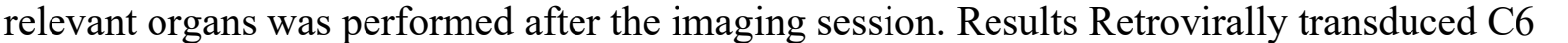

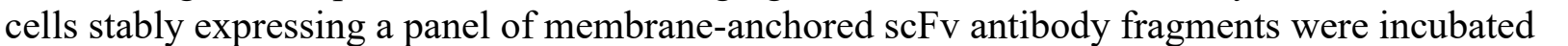

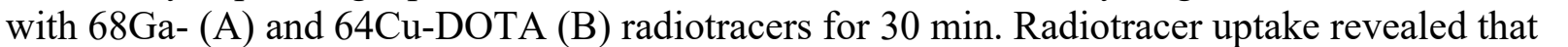

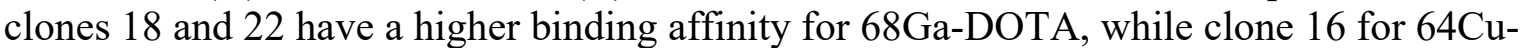

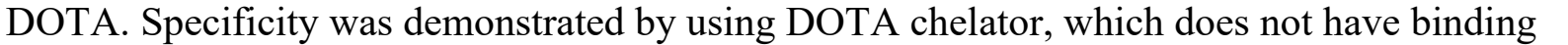

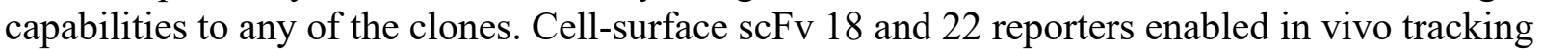

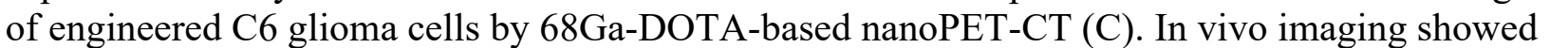

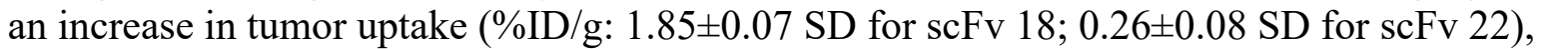

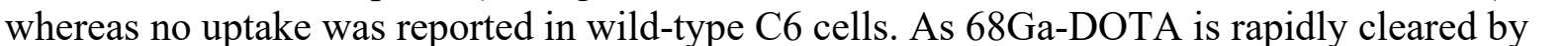

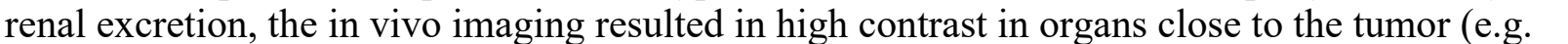

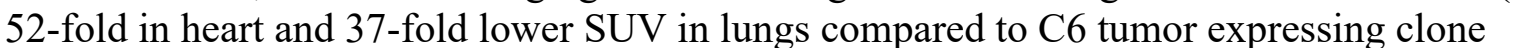

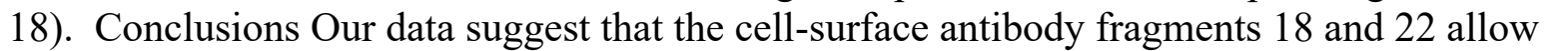

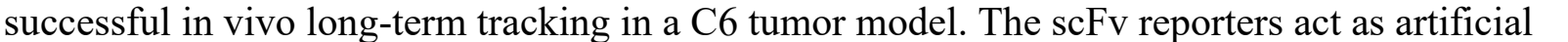

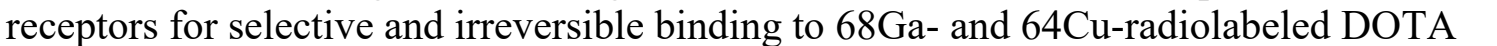

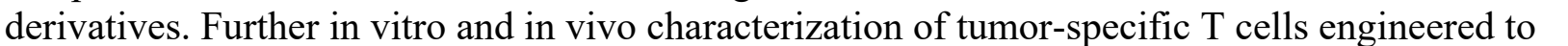

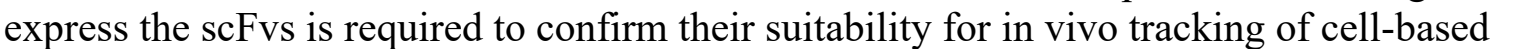

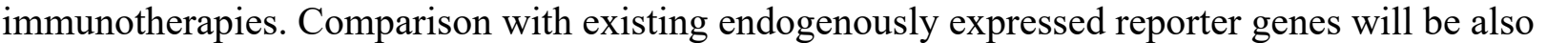

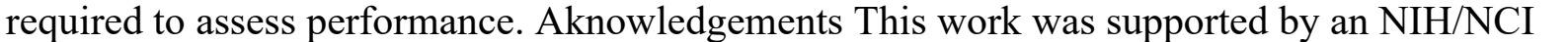
$\square|l| W$
\end{abstract}




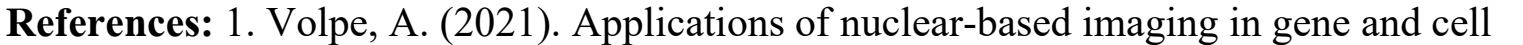

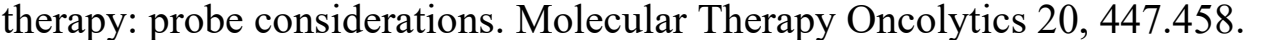

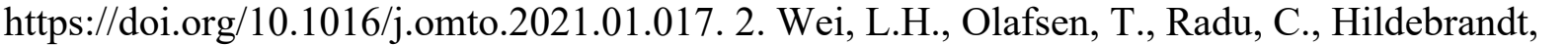

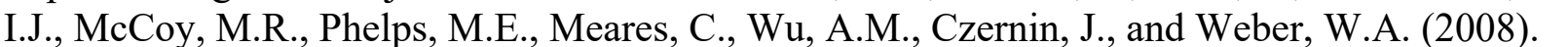

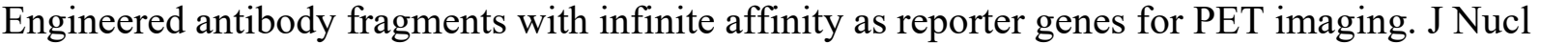

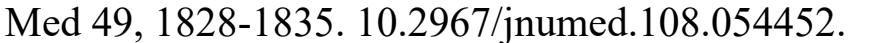

\section{Image/Figure:}

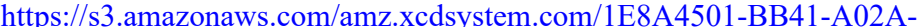

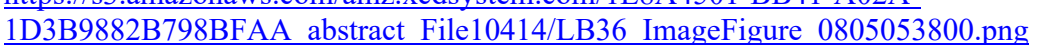

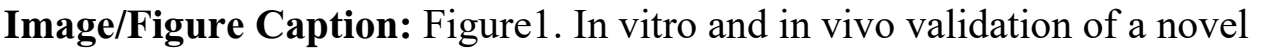

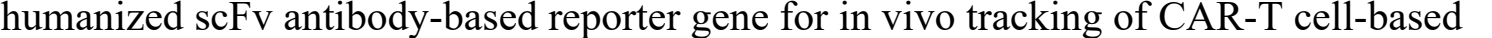

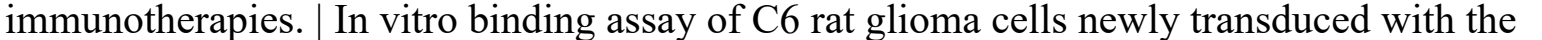

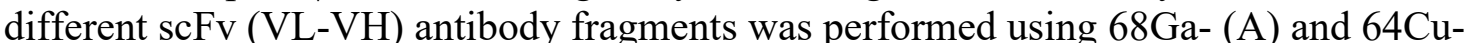

$\square \square \square \square(\mathrm{B})$ (DOTA concentration was $5 \mu \mathrm{M}$ ). Specificity was demonstrated by usin $\square \square \square \square \square \square$

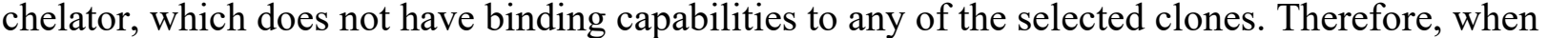

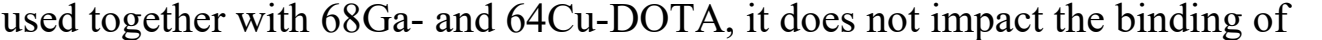

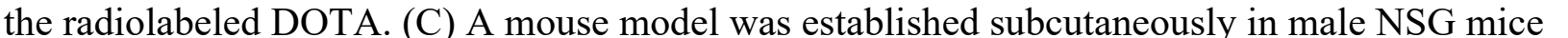

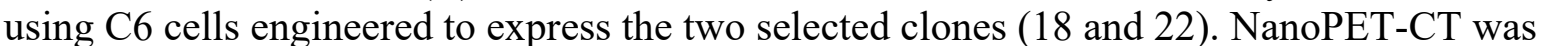

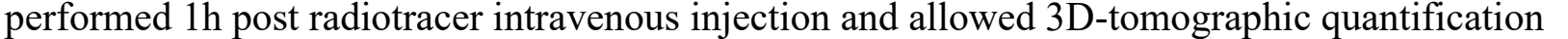

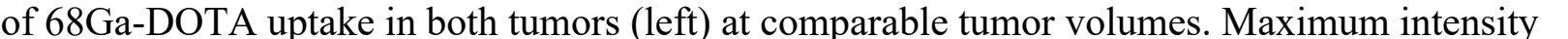

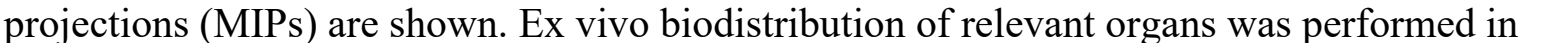

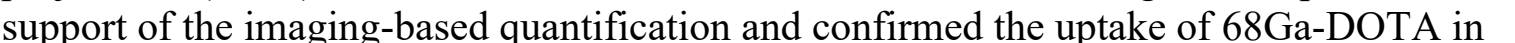

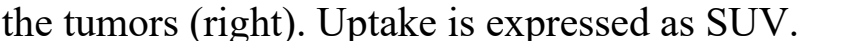

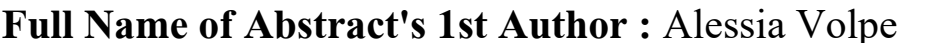

First Name: $\square \square\|\|\|\| \|$

Last Name: $\square \square \square \square \square$

Email: $\square \square\|\| \square \square \square \square \square\|\|\|\| \|$

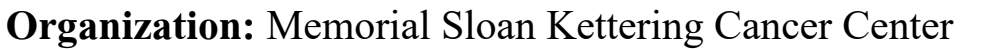

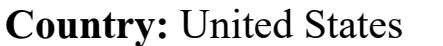




\title{
ID: LB37 \\ First-in-Human PET Imaging of a Blood-Brain Barrier Permeable Beta Tubulin Tracer
}

\author{
Akiva Mintz, Columbia University, am4754@cumc.columbia.edu
}

Category: $\square \square \square\|\|\|\|\|\square\| m$

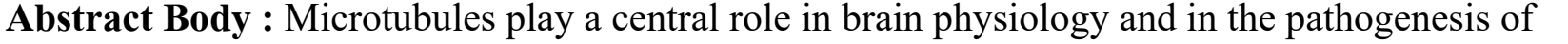
various central nervous system (CNS) disorders, including ALS, Alzheimer's Disease and

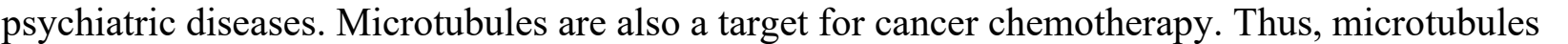

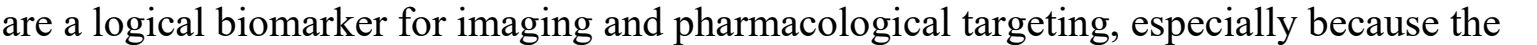

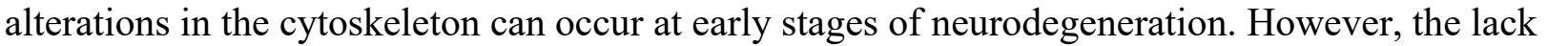

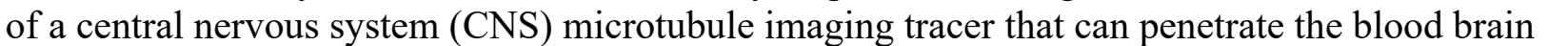

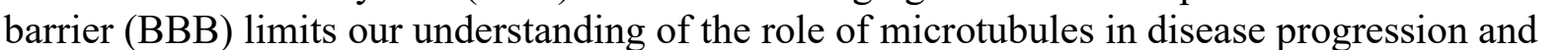

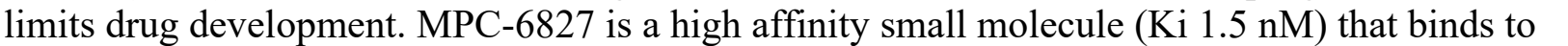
the colchicine site of tubulin $\alpha \beta$ heterodimers and is not a substrate for Pgp transporters. In

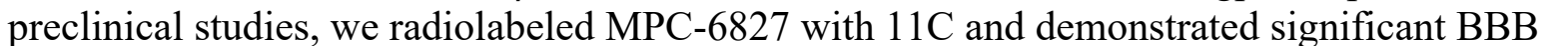

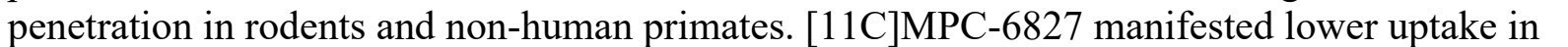

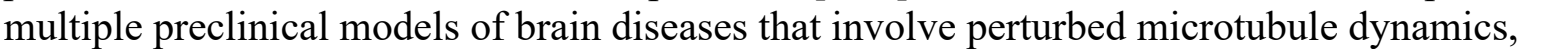
including ALS, Alzheimer's disease and alcohol use disorder. We hypothesized that [11C]MPC $\square$

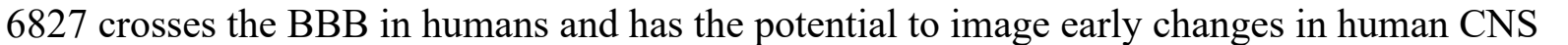

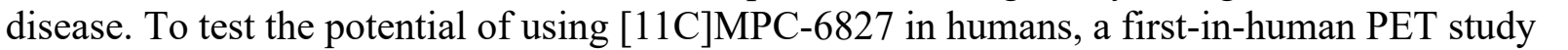

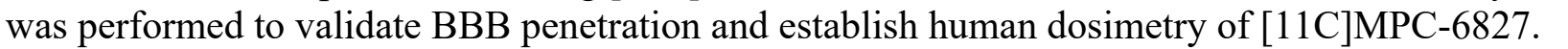

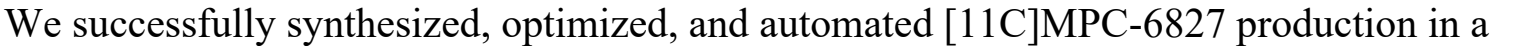

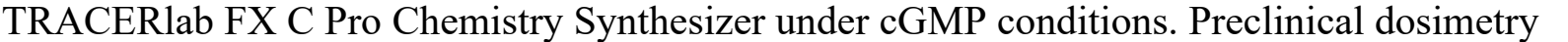

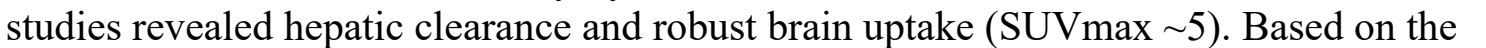

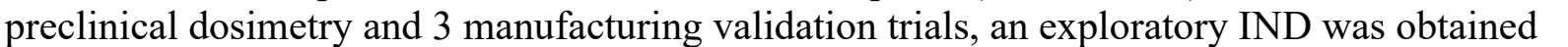

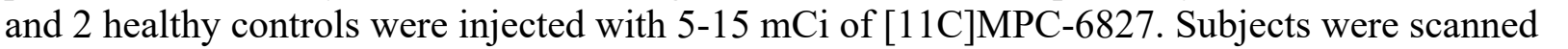

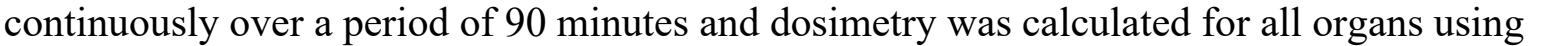

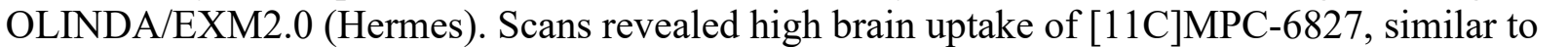

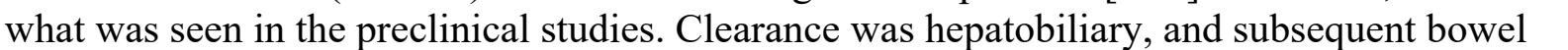

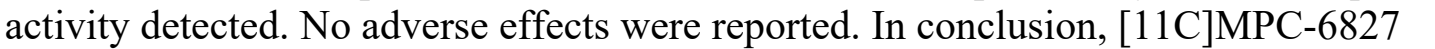

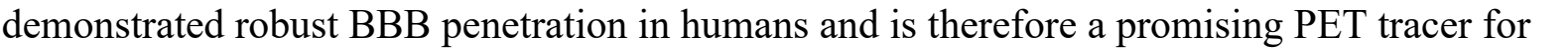

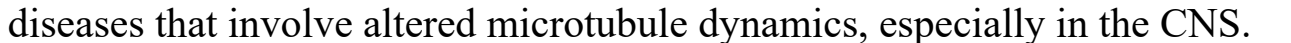

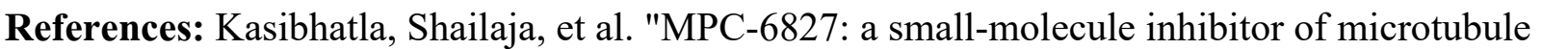

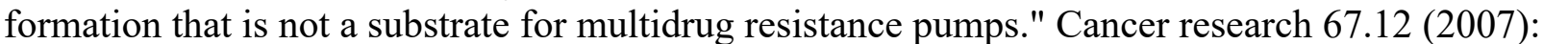

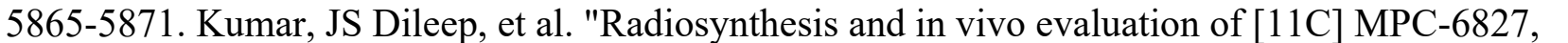

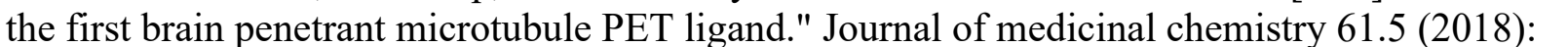

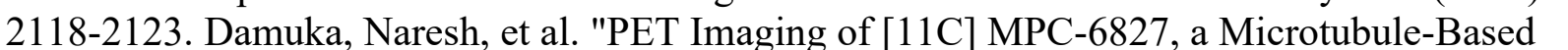

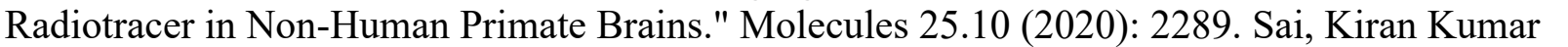

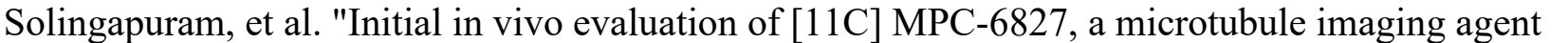

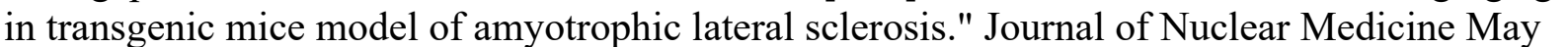


2019, 60 (supplement 1) 181; Macauley-Rambach, Shannon, et al. "[11C] MPC-6827: A microtubule-based pet imaging tracer for $\mathrm{AD}$ and related dementias: Neuroimaging/animal

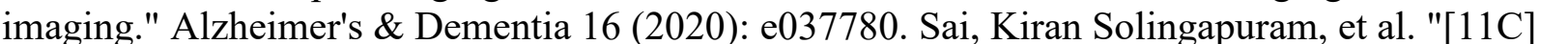
MPC-6827, a microtubule-based PET imaging tracer: A potential early imaging biomarker for

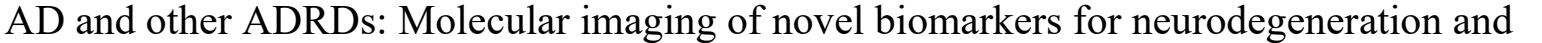

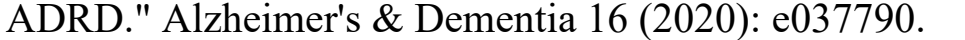

\section{Image/Figure:}

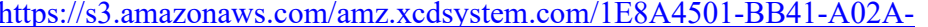

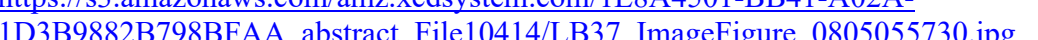

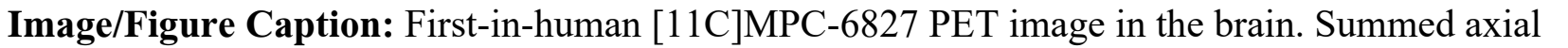

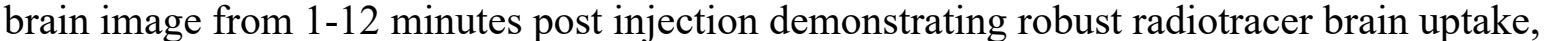

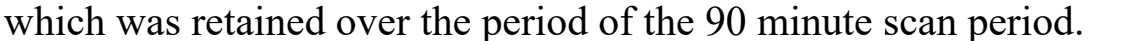

First Name:

Last Name: $\square \square m \square$

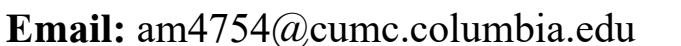

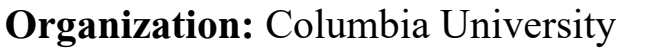

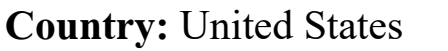




\title{
ID: LB38 \\ Optimization of radiolabelling of (68)Ga with DOTA[c(RGD)2] peptide and preclinical assessment of its in-vitro characteristics.
}

\author{
Meryl Maria Vilangattil, Tata Memorial Hospital, merylmaria7@gmail.com
}

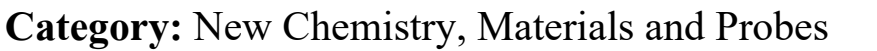

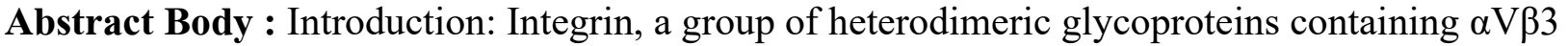

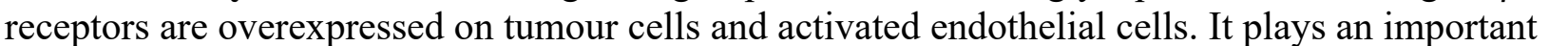

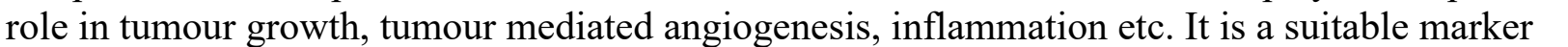

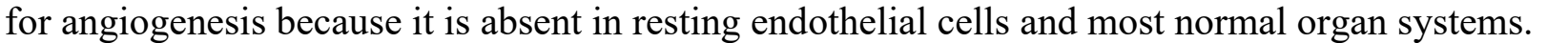

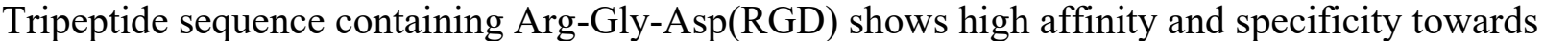
$\alpha \mathrm{V} \beta 3$ receptors $[1,2,3]$. It has been shown that cyclization of linear RGD peptides and

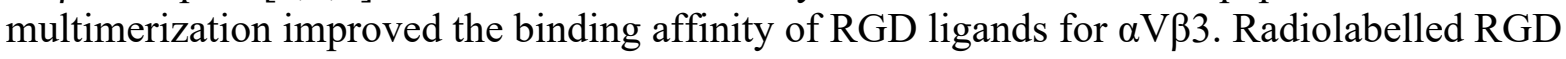

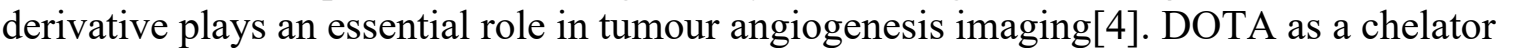

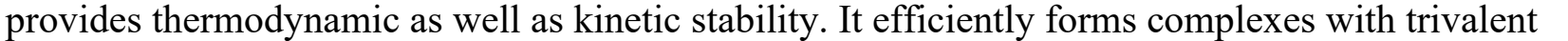

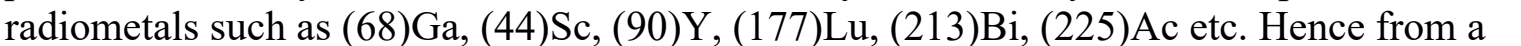

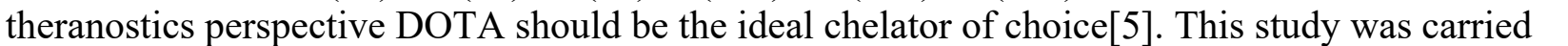

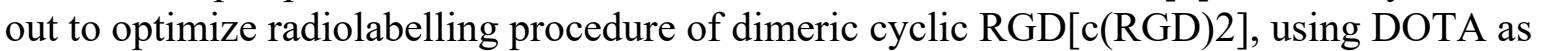

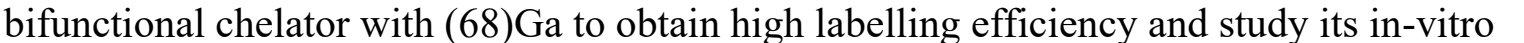

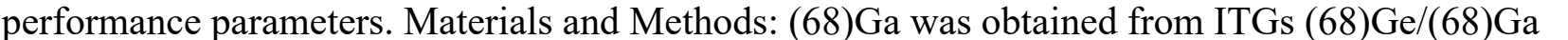

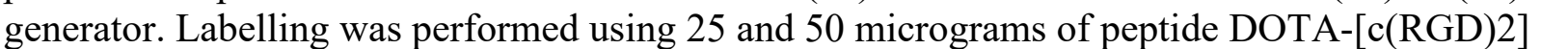

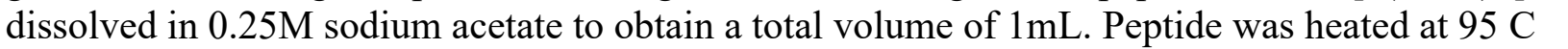

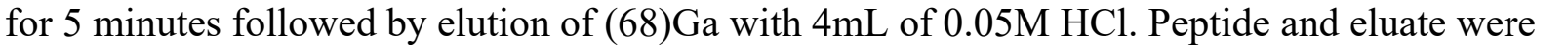

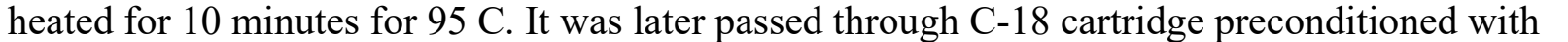

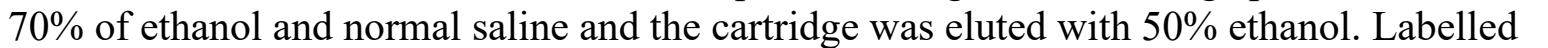

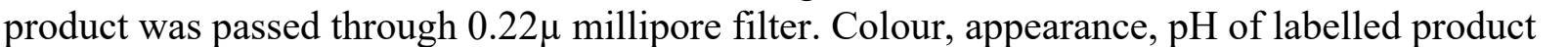

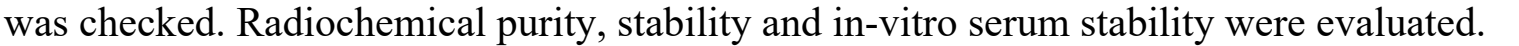

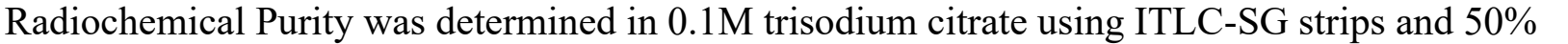

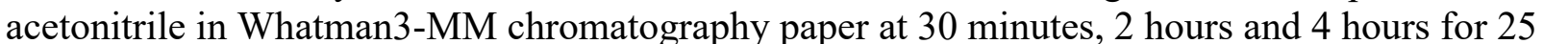

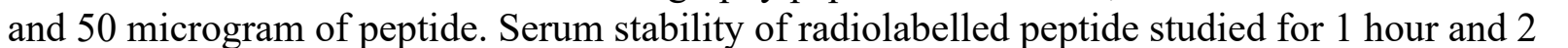

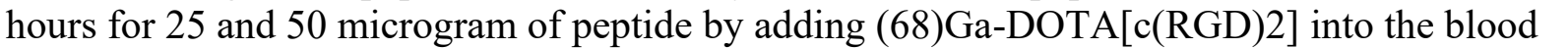

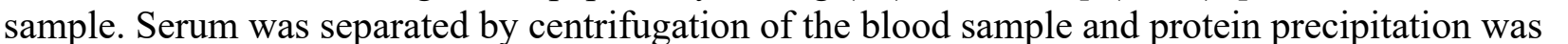

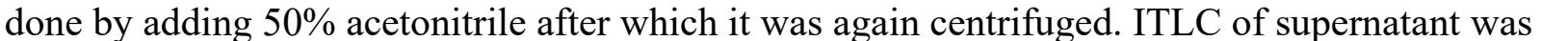

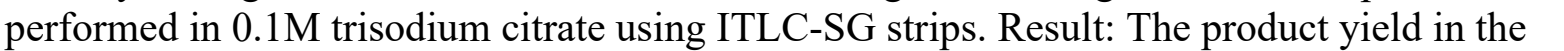

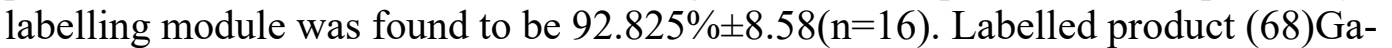

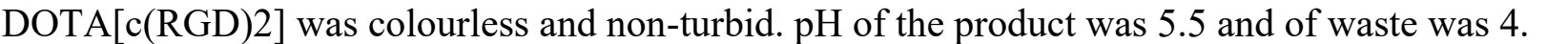

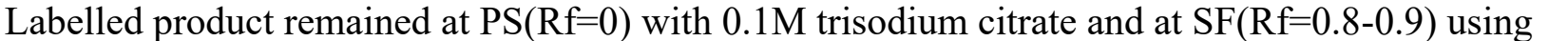

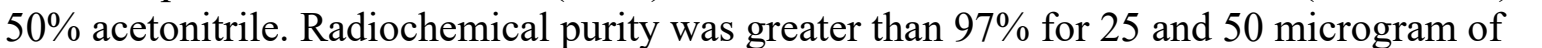

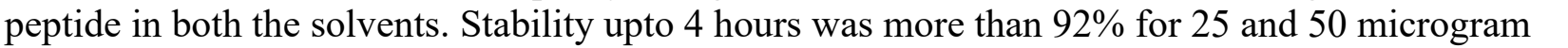

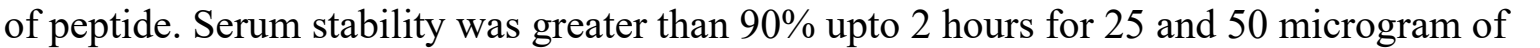

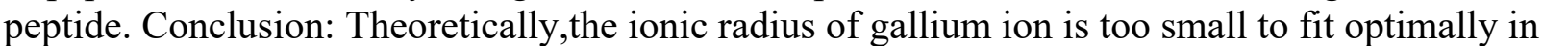
ए
\end{abstract}




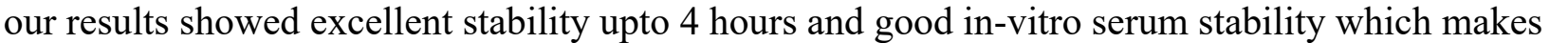

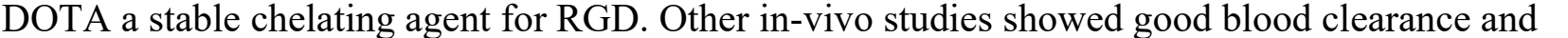

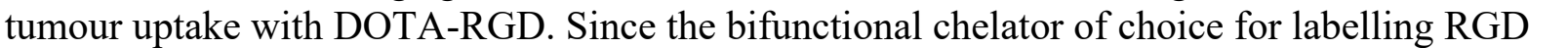

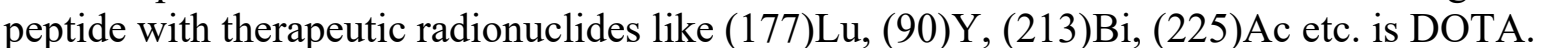

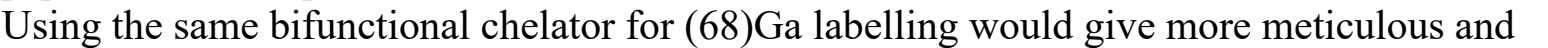

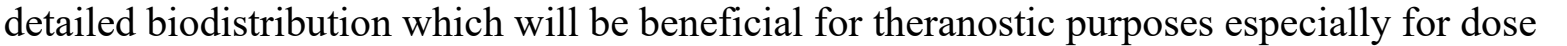

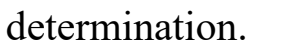

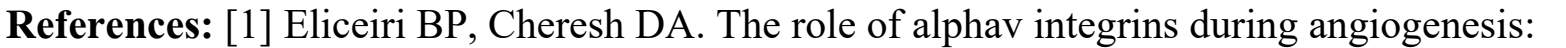

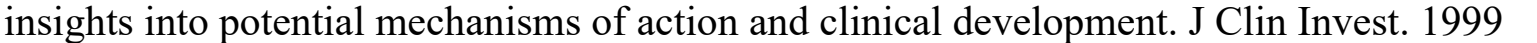

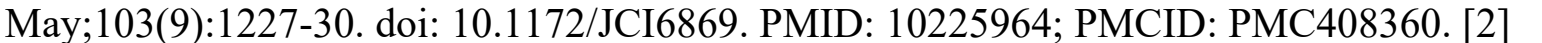

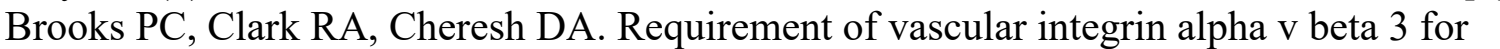

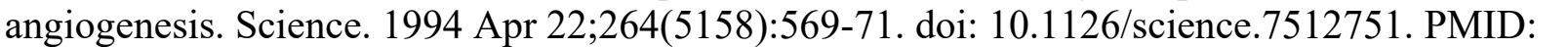

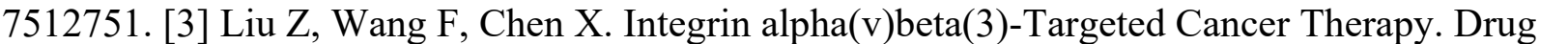

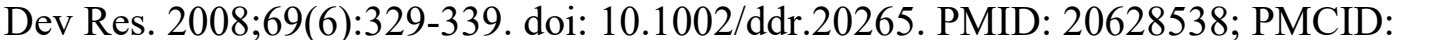

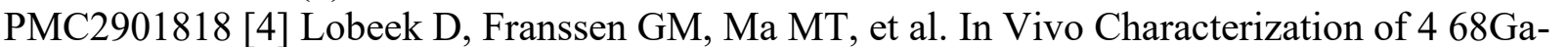
Labeled Multimeric RGD Peptides to Image $\alpha v \beta 3$ Integrin Expression in 2 Human Tumor

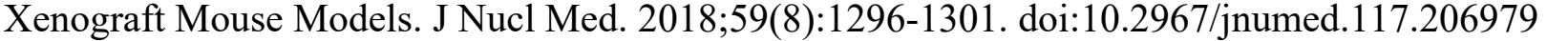

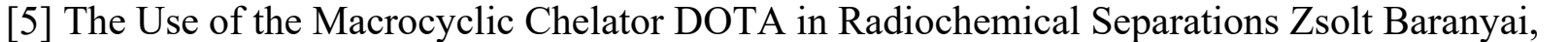

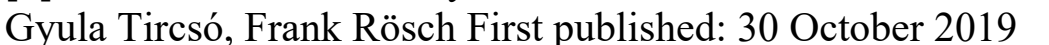

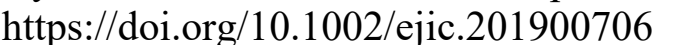

\section{Image/Figure:}

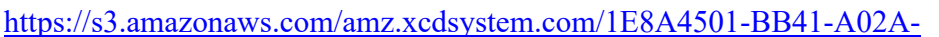

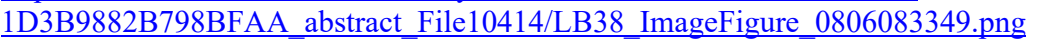

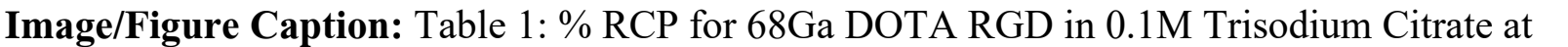

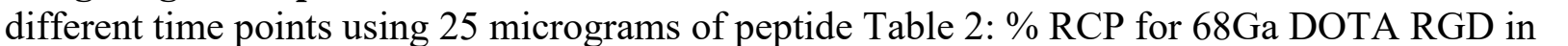

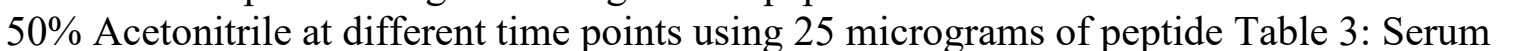

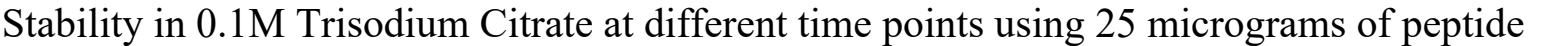

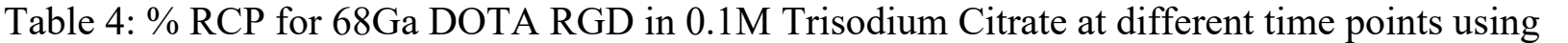

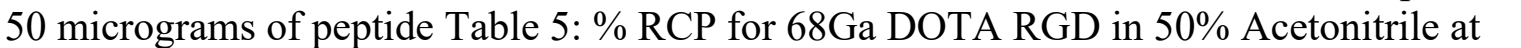

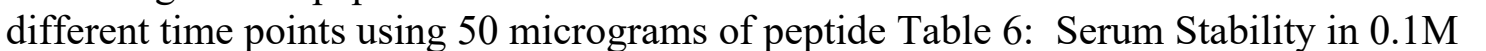

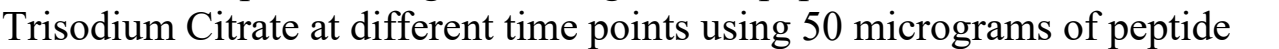

First Name: $\square \square \square \square \square \square \square \square \square$

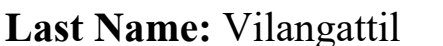

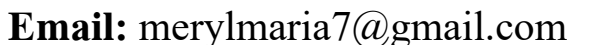

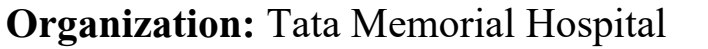

Country: $\square \square \square \square$ 


\title{
ID: LB39 \\ In vivo interstitial and cerebrospinal fluid exchanging process detected by non- invasive magnetization transfer indirect spin labeling MRI
}

\author{
Anna Li, Kennedy Krieger Institute, annamlilehigh@gmail.com
}

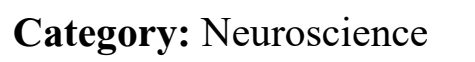

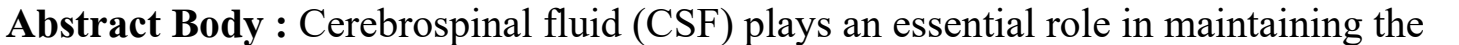

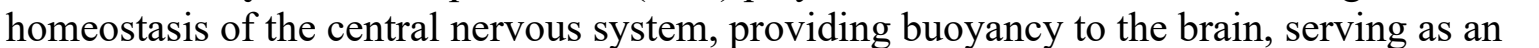

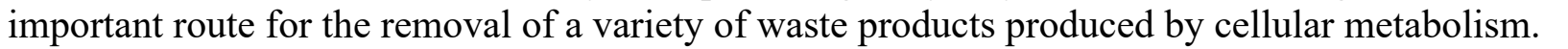

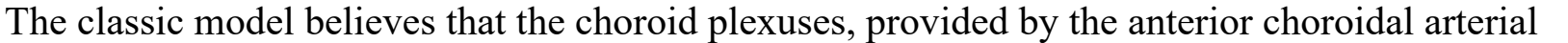

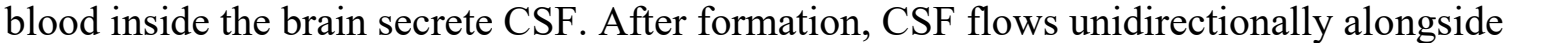

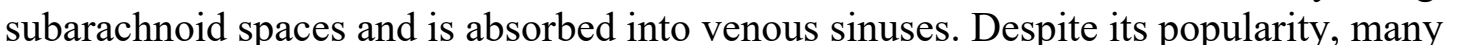

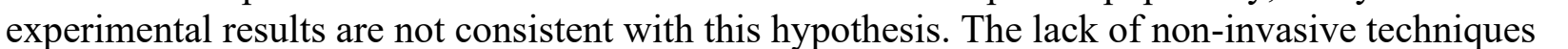

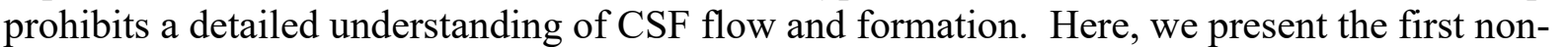

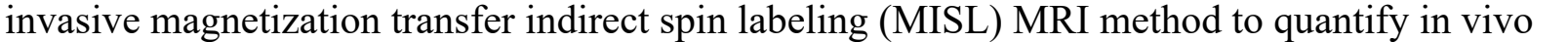

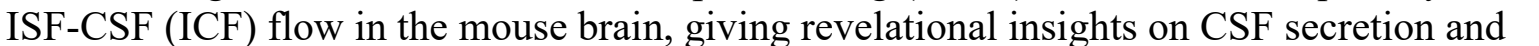

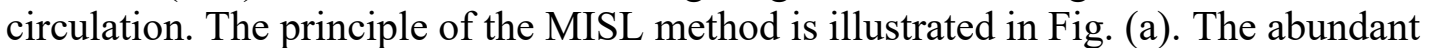

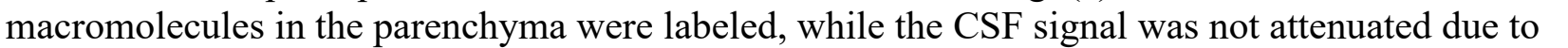

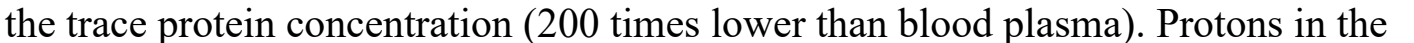

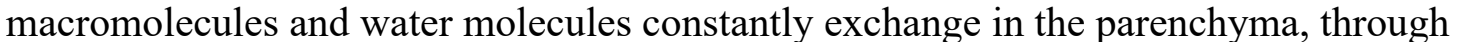

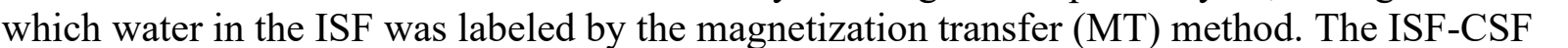

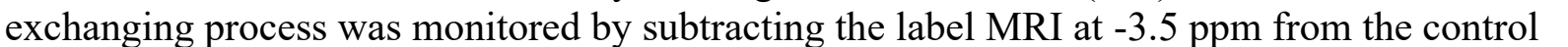

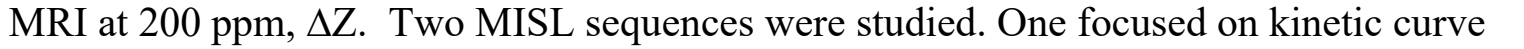

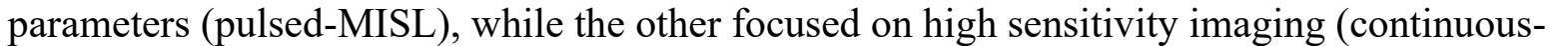

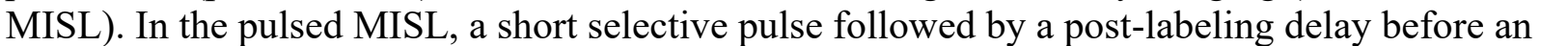

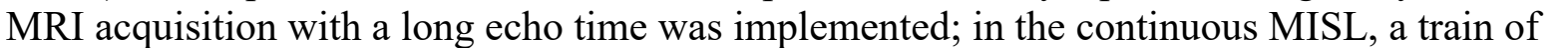

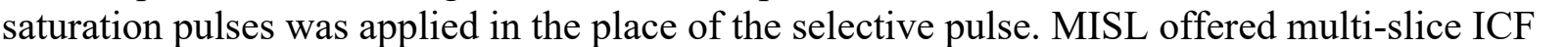

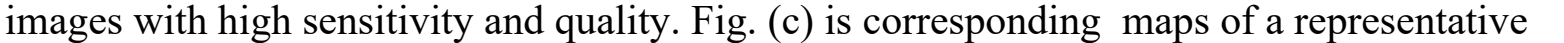

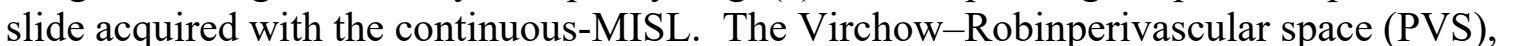

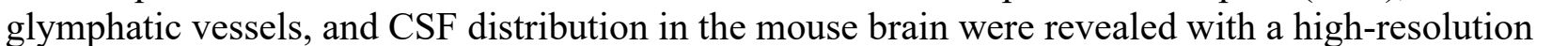

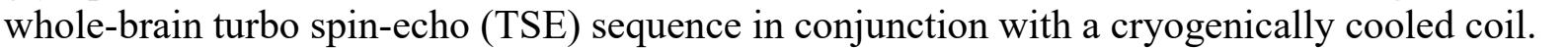

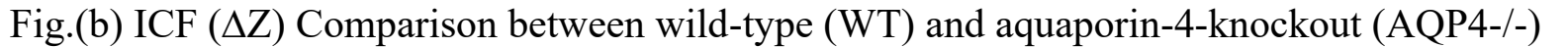

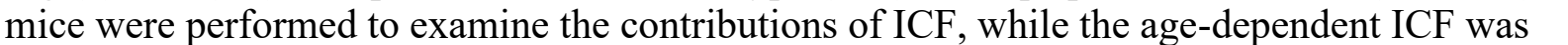

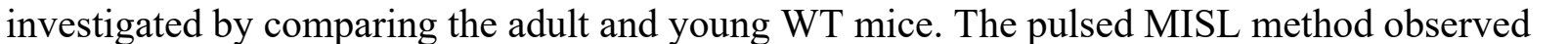

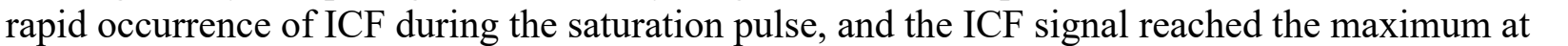

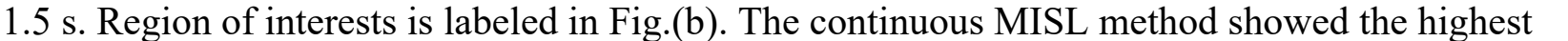

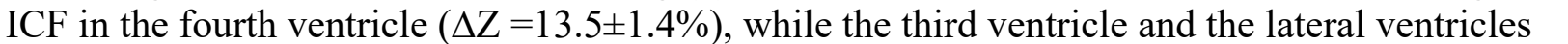
had similar ICF $\Delta \mathrm{Z}$ values $(\Delta \mathrm{Z}=12.0 \pm 1.8 \%)$. The current study showed an evident reduction of $\mathrm{CSF}$ recirculation related to aged brain ventricles. A significantly lower $\Delta \mathrm{Z}(9.3 \sqcap \square \square \square$

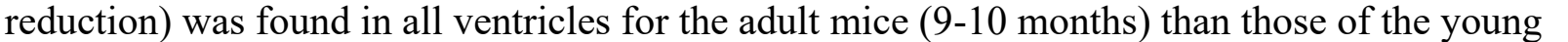

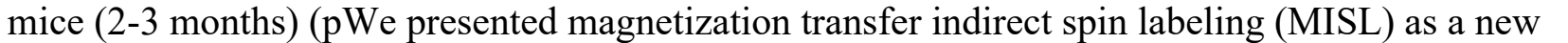

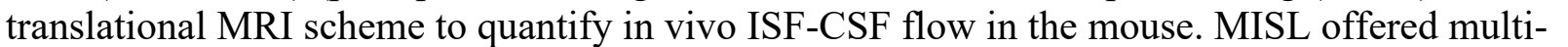




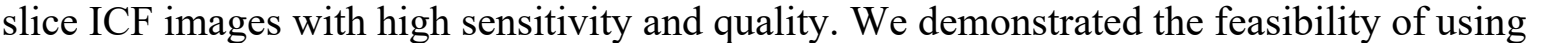

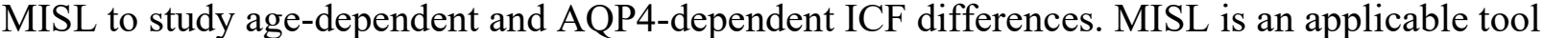

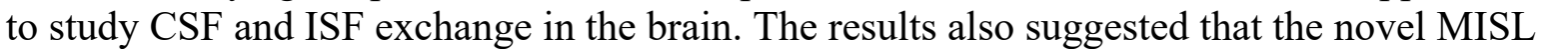

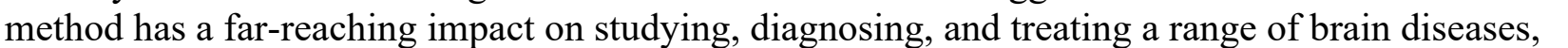

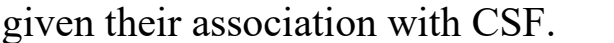

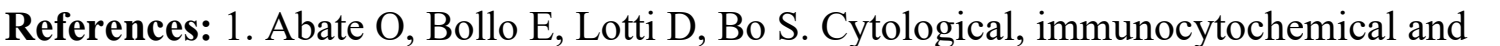

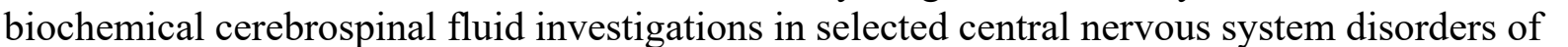

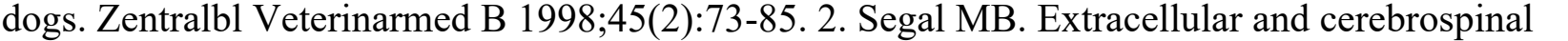

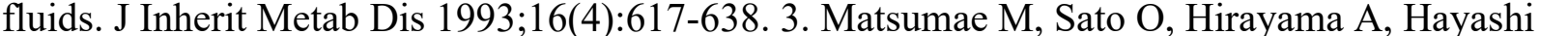

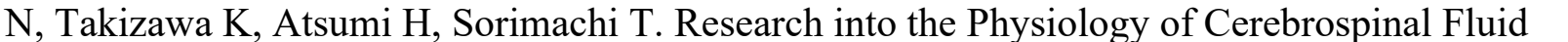

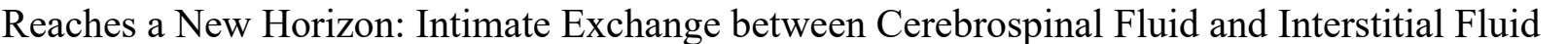

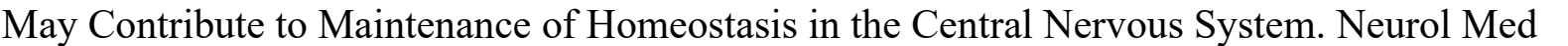

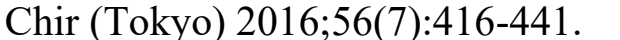

\section{Image/Figure:}

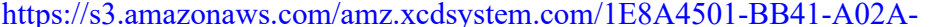

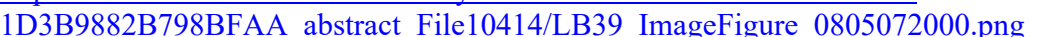

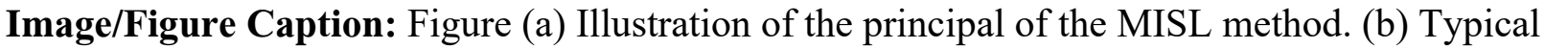

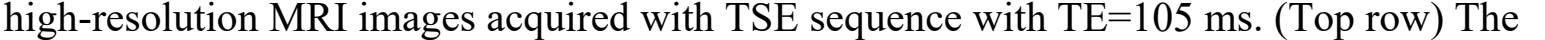
पा

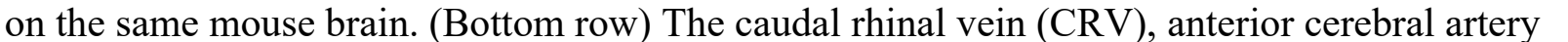

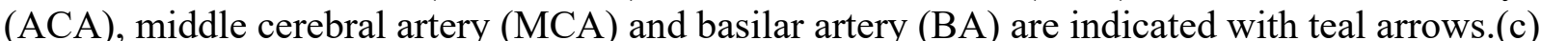

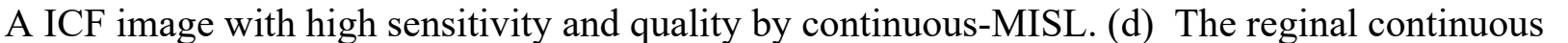

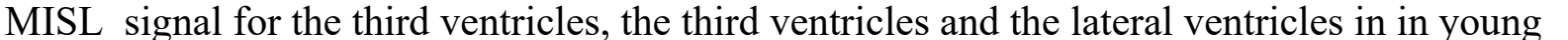

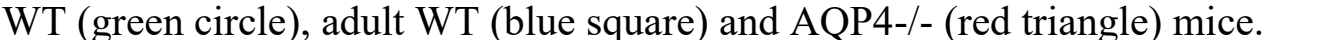

Full Name of Abstract's 1st Author : $\square \square \square \square \square \square$

First Name: $\square \square \square\|\|$

Last Name: $\square \square$

Email: $\square \square\|\square \square\| m\|\square \square \square\| m \square \square$

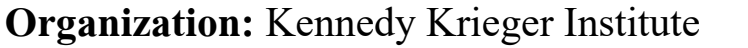

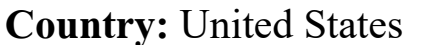




\title{
ID: LB40 \\ Investigating the efficacy of TRAIL-enhanced CAR-T cells to augment cancer immunotherapy in malignant pleural mesothelioma
}

\author{
Alessia Volpe, Memorial Sloan Kettering Cancer Center, VolpeA1@mskcc.org
}

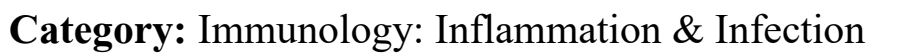

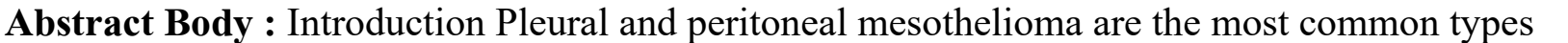

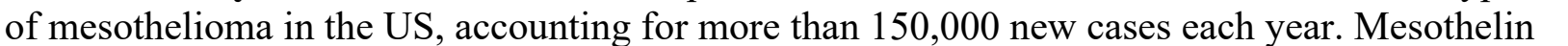

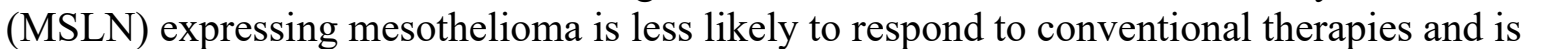

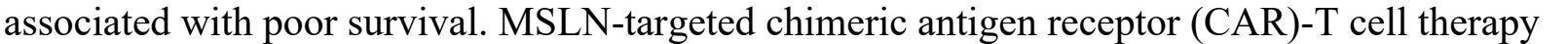

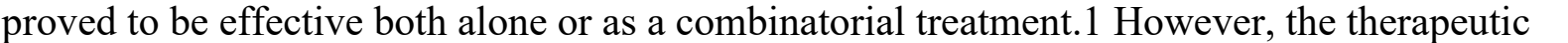

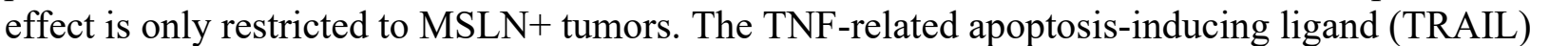

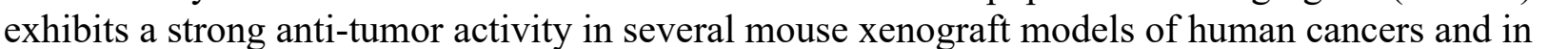

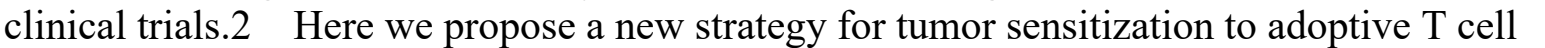

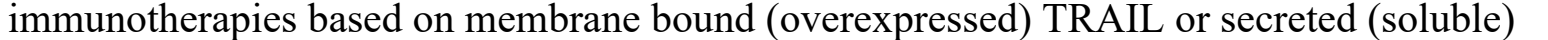

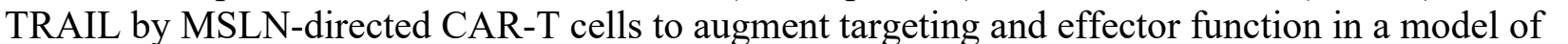

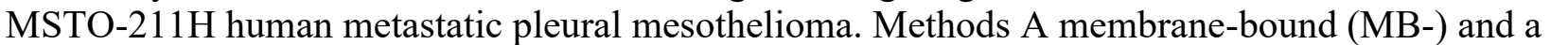

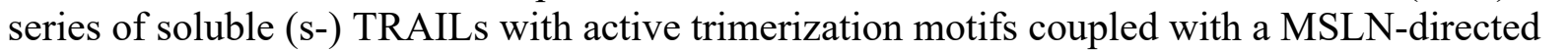

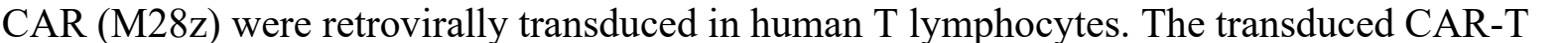

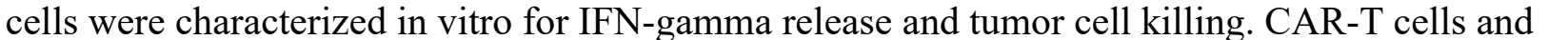

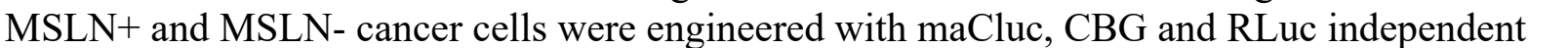

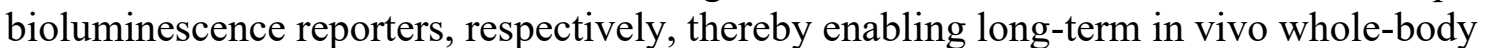

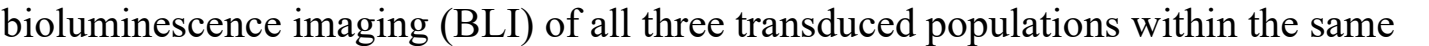

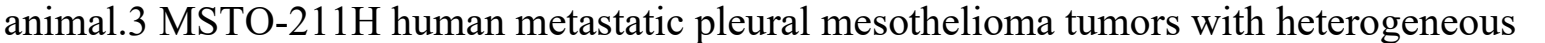

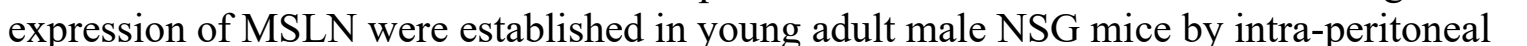

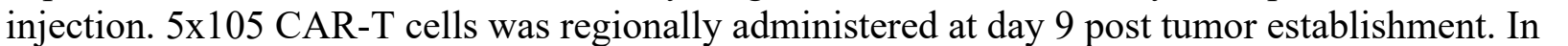

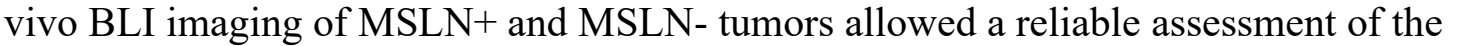
ए

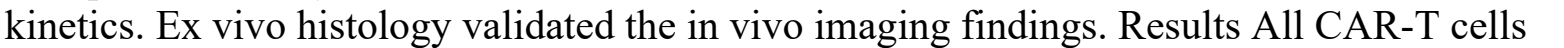

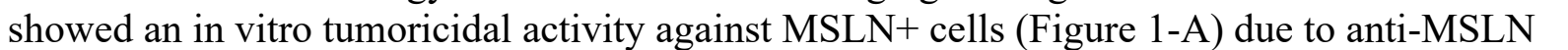

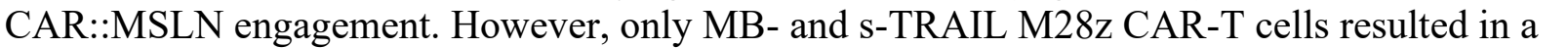

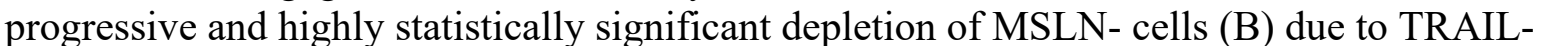

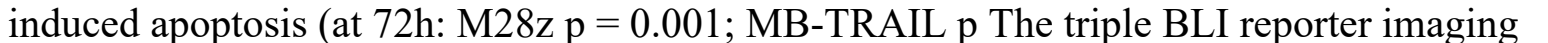

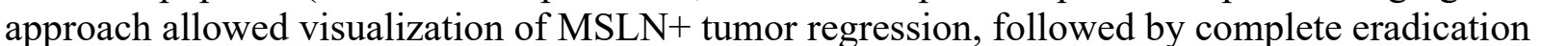

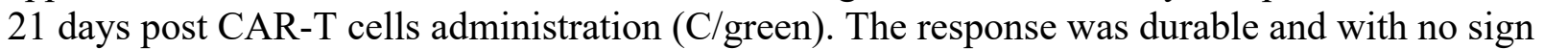

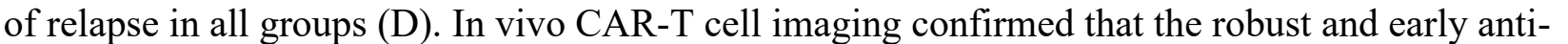

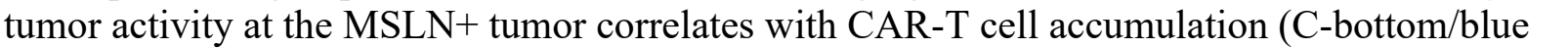

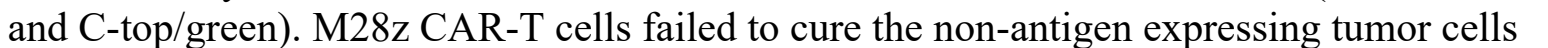

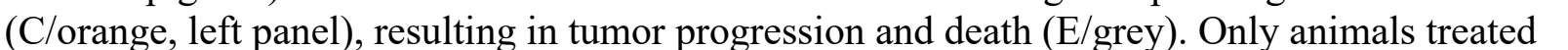

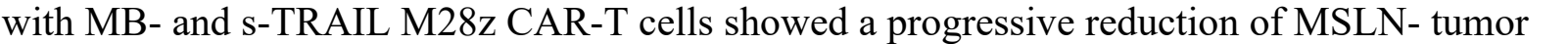

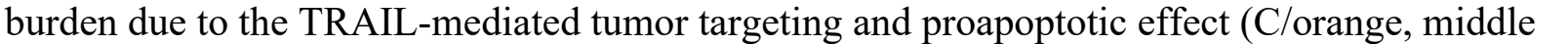

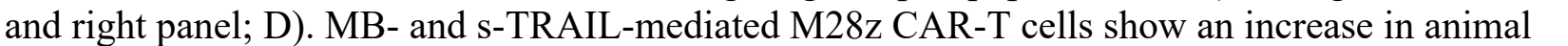




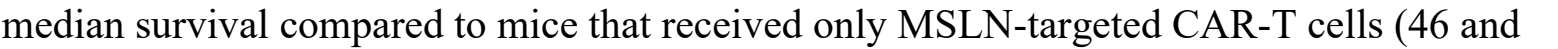

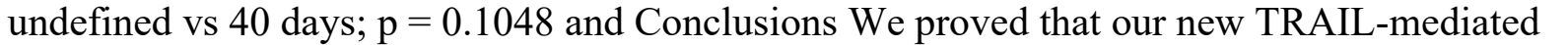

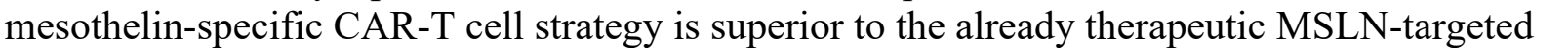

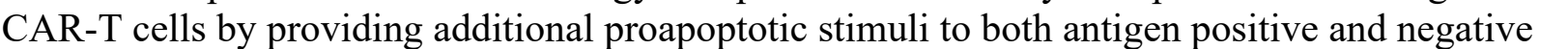
ए b पाm |

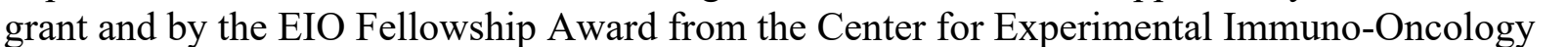

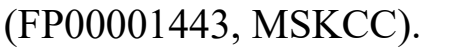

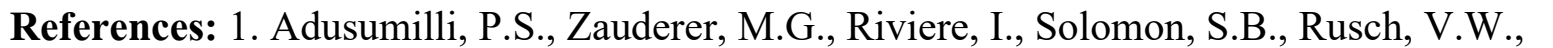

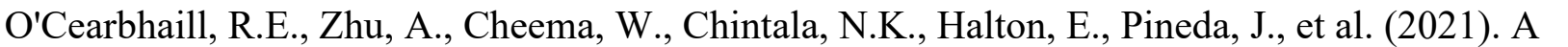

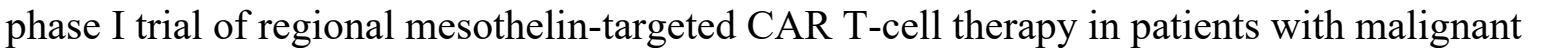

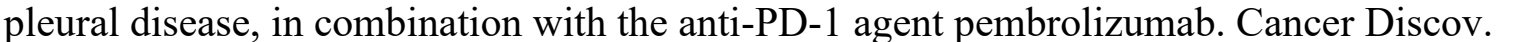

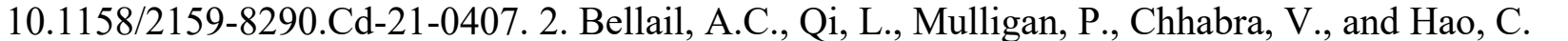
ए

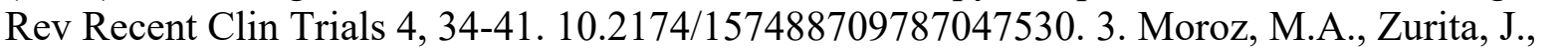

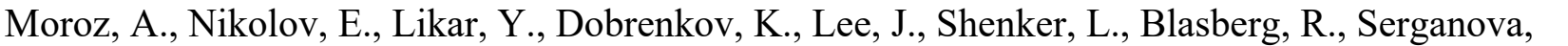

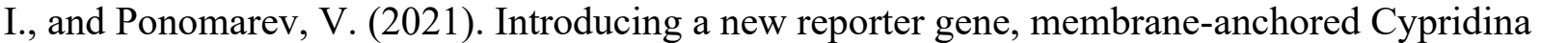

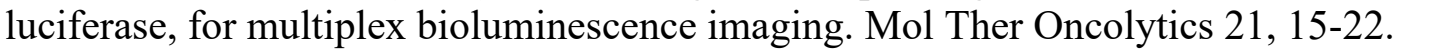

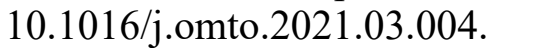

\section{Image/Figure:}

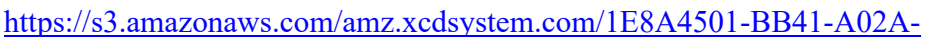

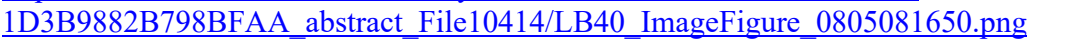

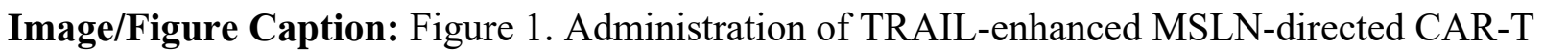

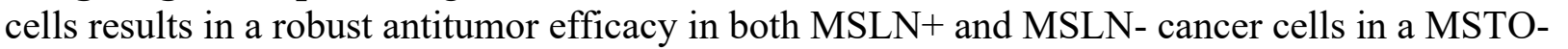

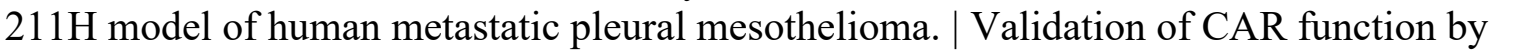

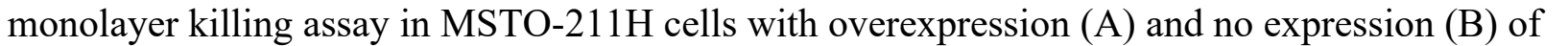

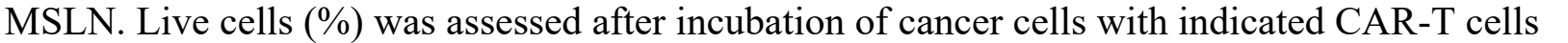

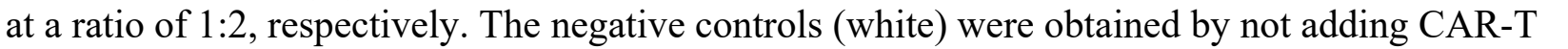

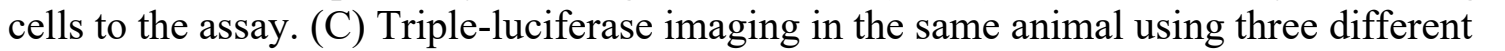

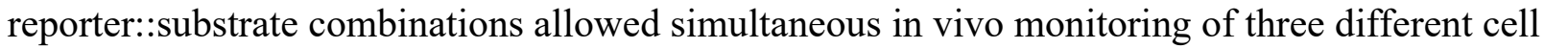

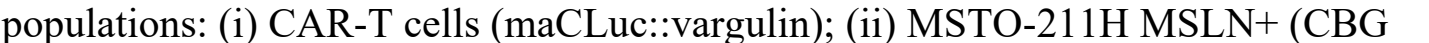

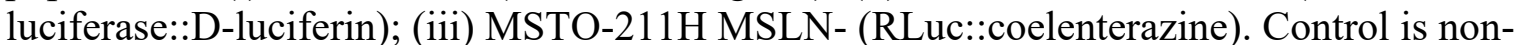

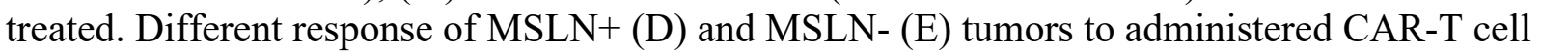

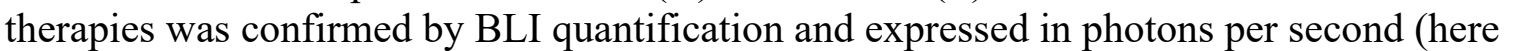

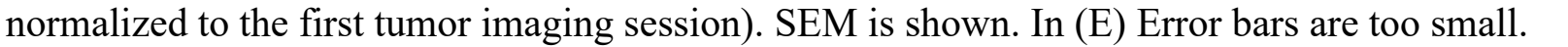

Full Name of Abstract's 1st Author : $\square \square \square\|\square\| \square \square \square \square$

First Name: $\square \square\|\|\|\| \square$

Last Name: $\square \square \square \square \mid \square$ 
Email:

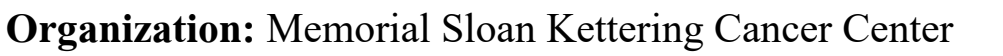

Country: 


\title{
ID: LB41 \\ The Synthesis and In Vivo Evaluation of a Cadherin-17 Targeted Radioimmunoconjugate for the PET Imaging of Pancreatic Adenocarcinoma
}

\author{
Samantha Delaney, Hunter College, sdelaney@gradcenter.cuny.edu
}

Category: $\square \square\|\| \| \square$

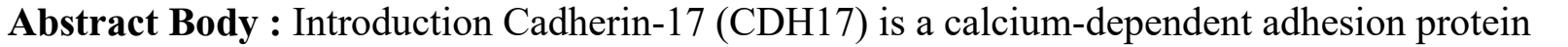

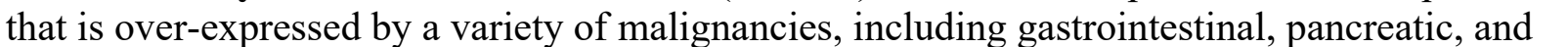

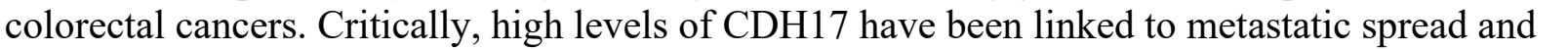

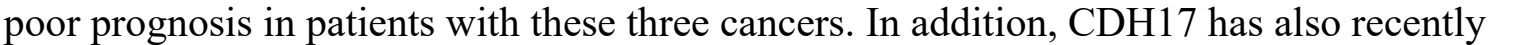

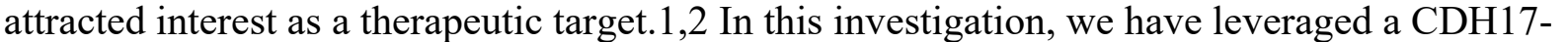

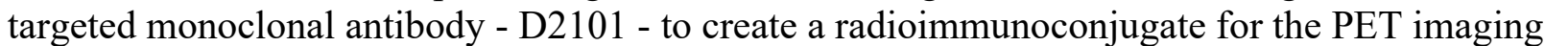

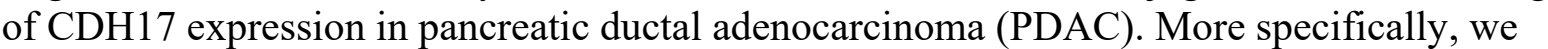

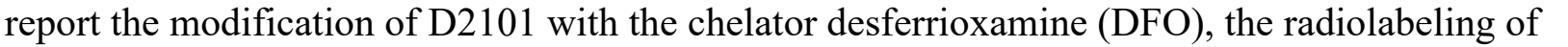

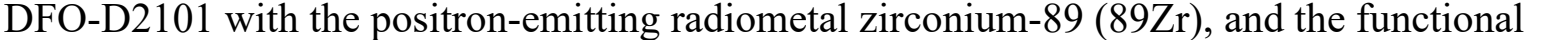

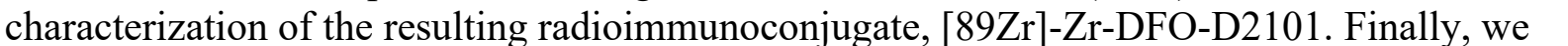

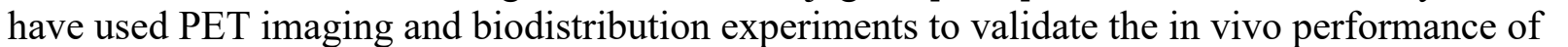
एம

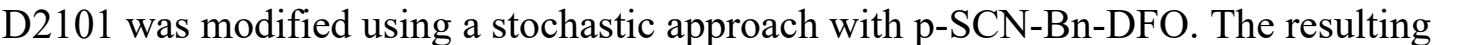

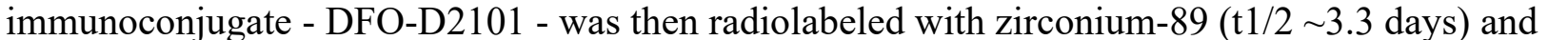

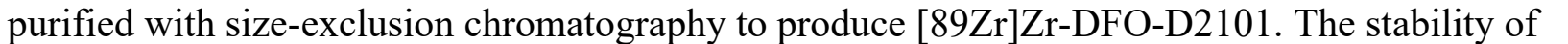
ए

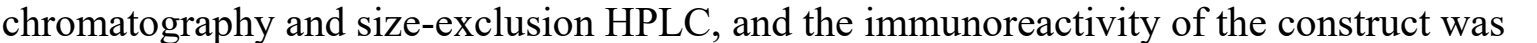

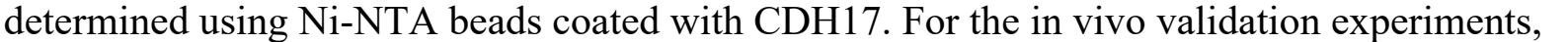
घम

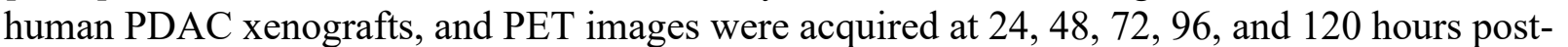

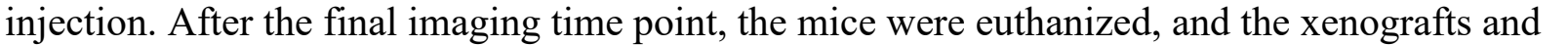

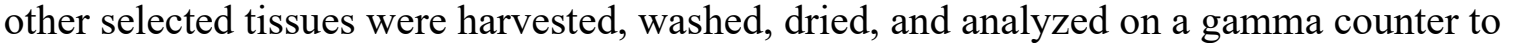

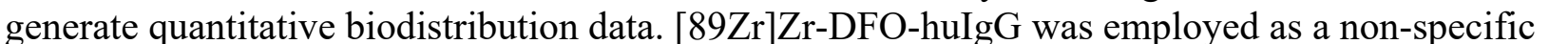

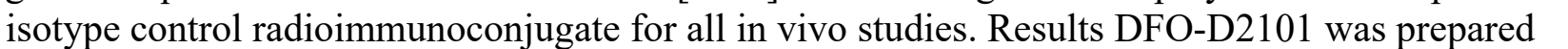

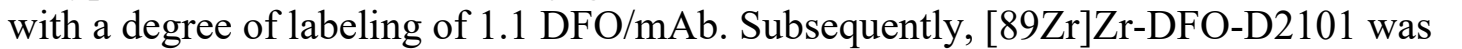

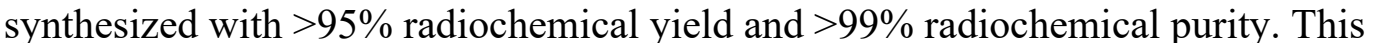

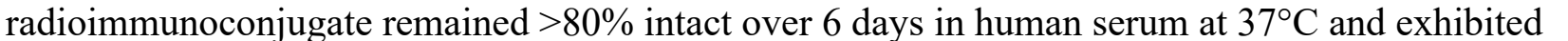

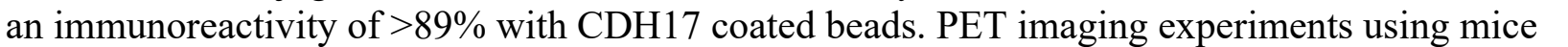

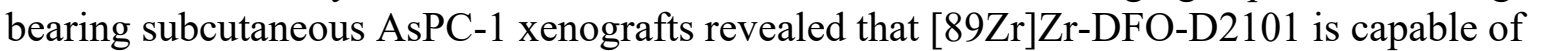

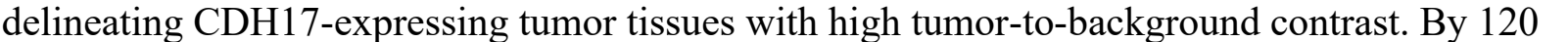

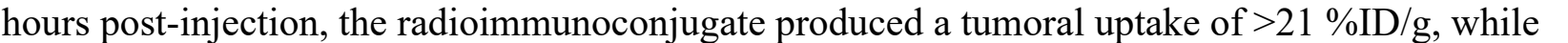

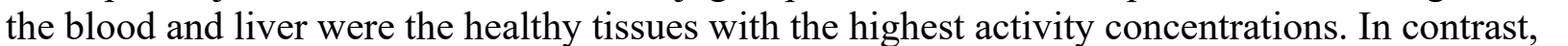
पम

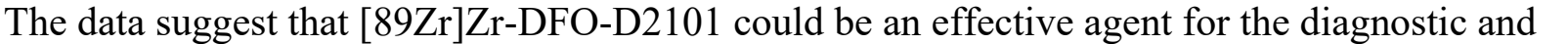

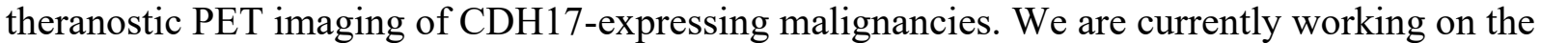




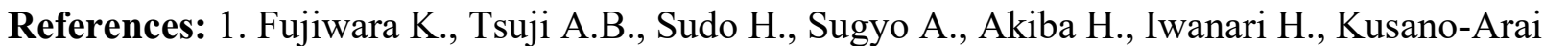

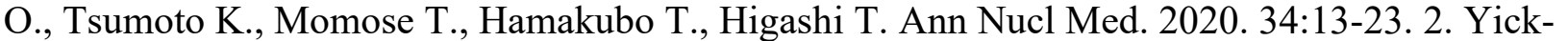

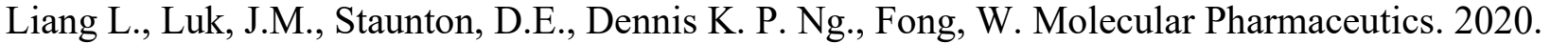

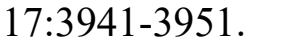

\section{Image/Figure:}

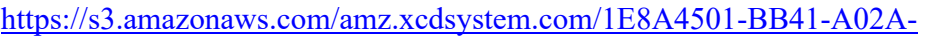

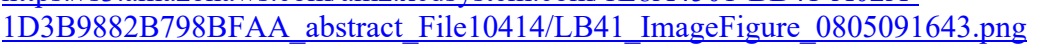

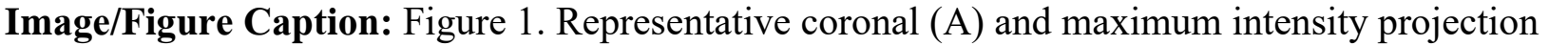

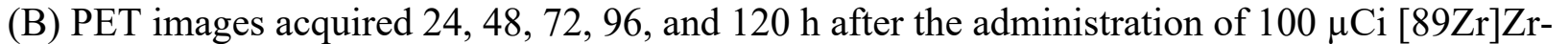

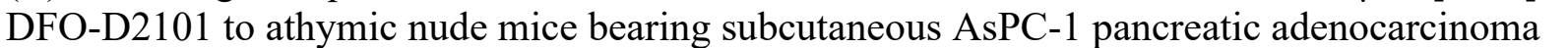

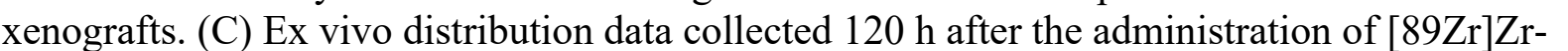

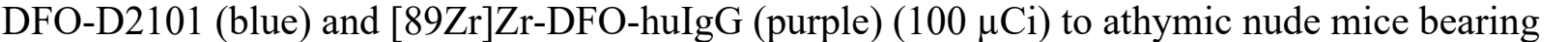

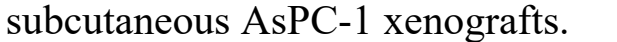

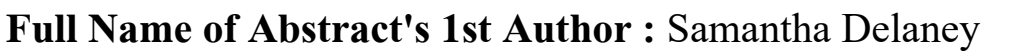

First Name: $\square \square \square \square \square \square \square$

Last Name: $\square \square \square \square \| \square$

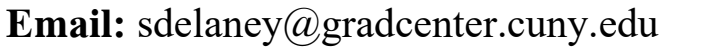

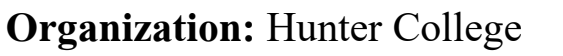

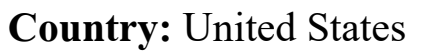




\title{
ID: LB42 \\ A pilot PET imaging study with [18F]SynVesT-1 to identify synaptic loss in the spinal cord and brain regions in a rat model of spinal cord injury
}

\author{
Baosheng Chen, Yale School of Medicine - New Haven, CT, baosheng.chen@yale.edu
}

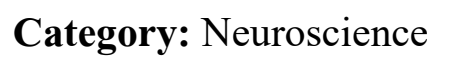

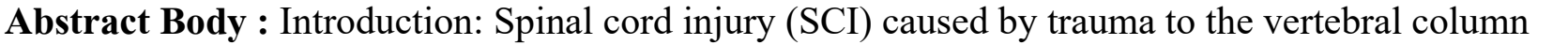

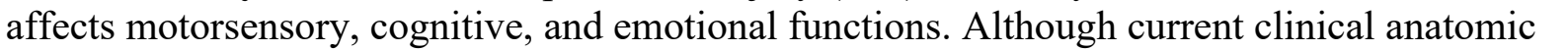

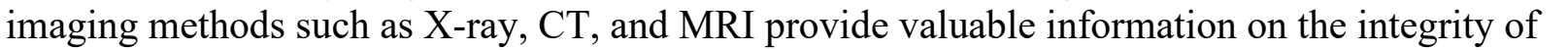

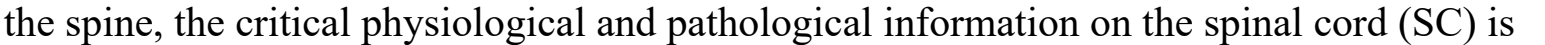

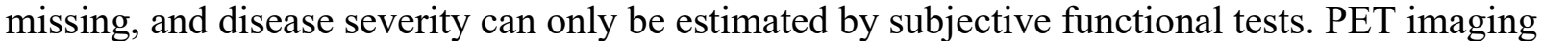

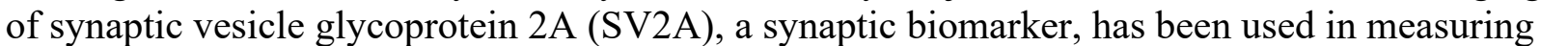

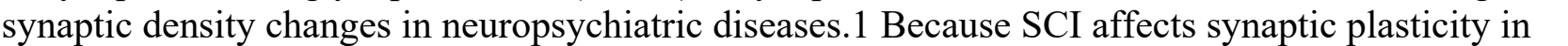

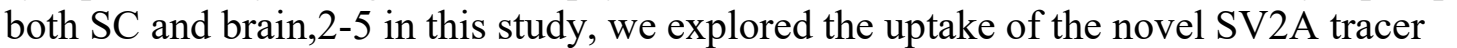

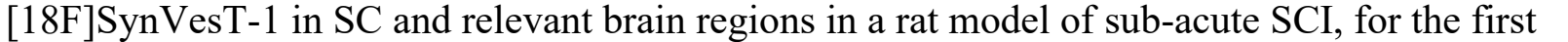

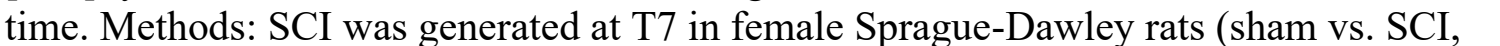

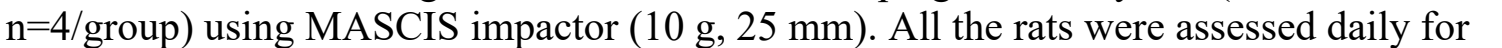

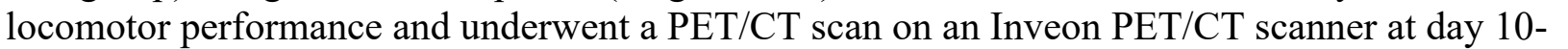

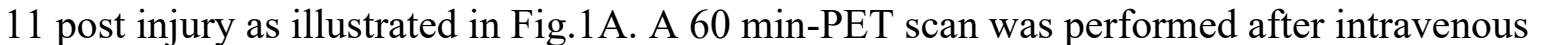

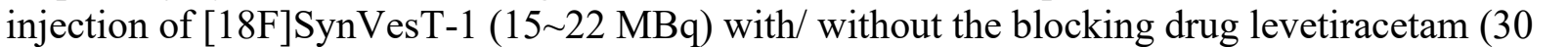

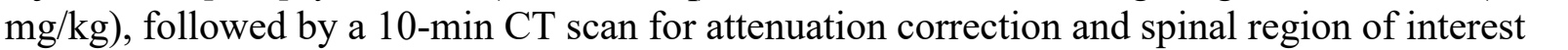

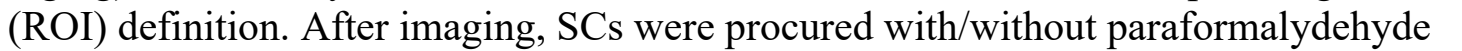

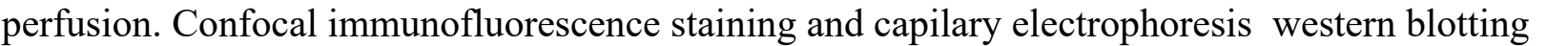

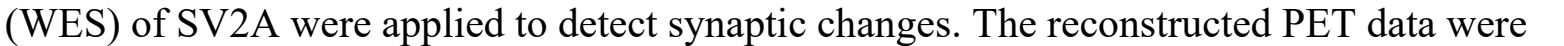

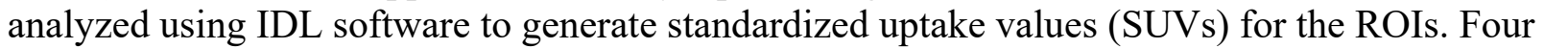

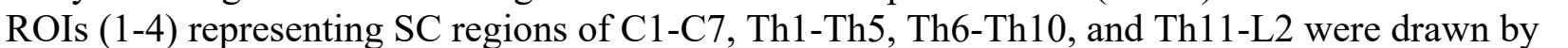

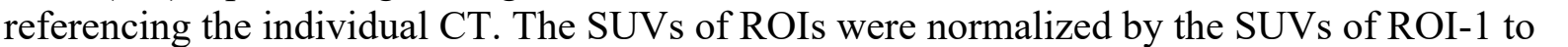

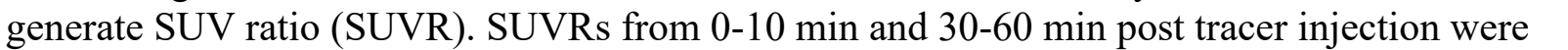

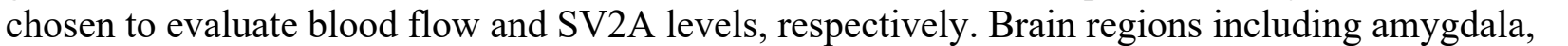

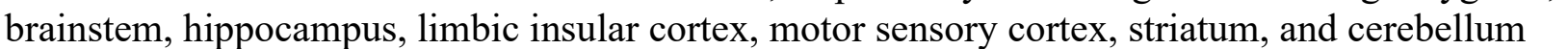

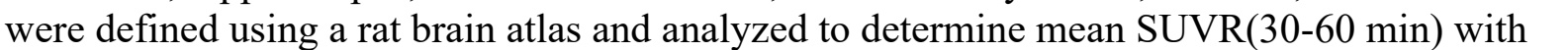

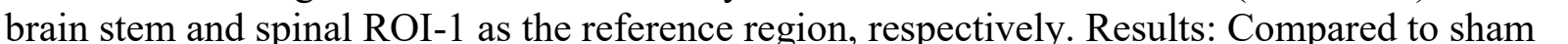

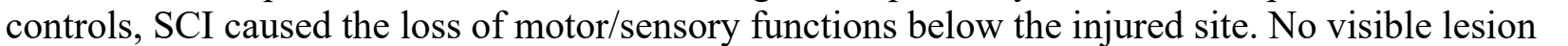

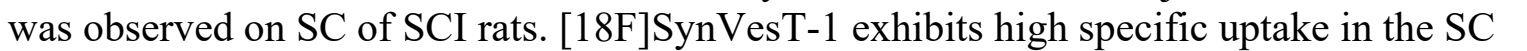

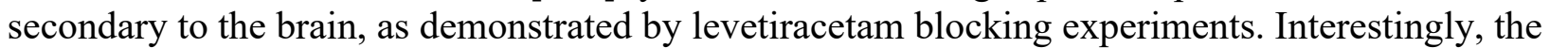

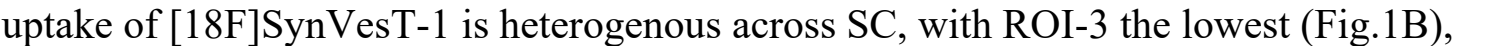

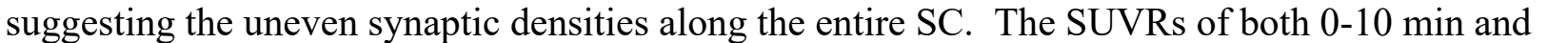

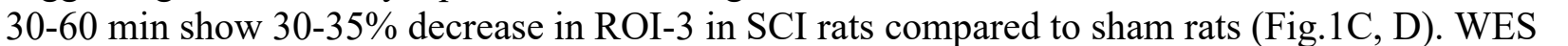

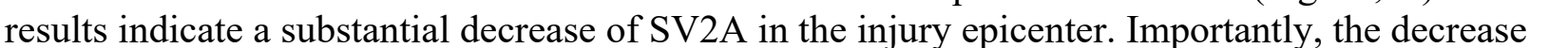

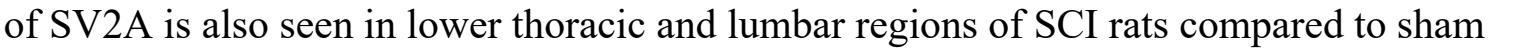

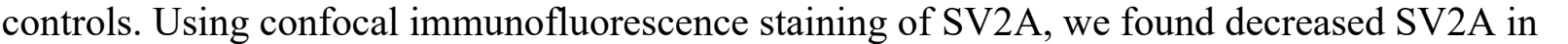

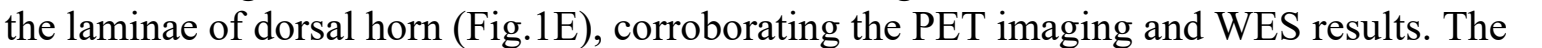




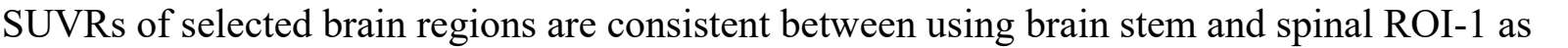
ए

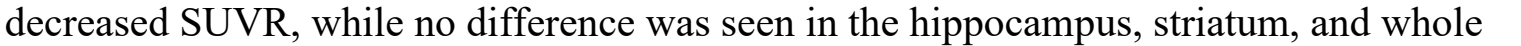

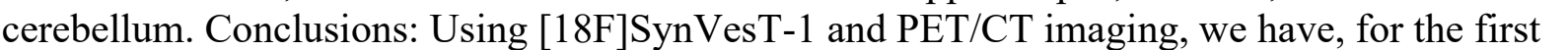

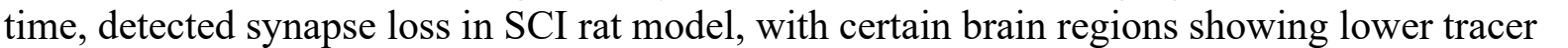

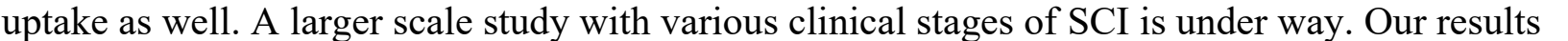
पा

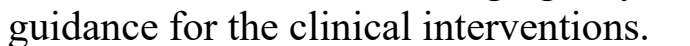

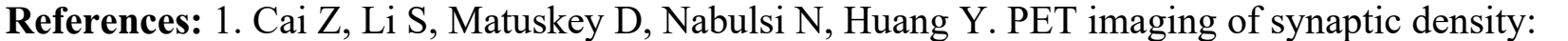

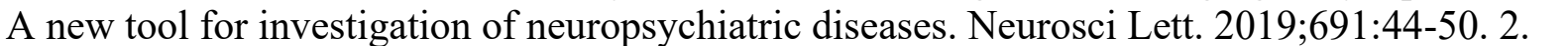

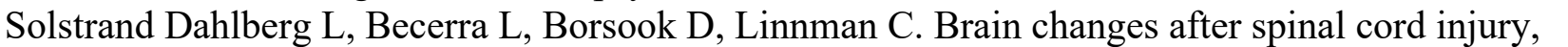

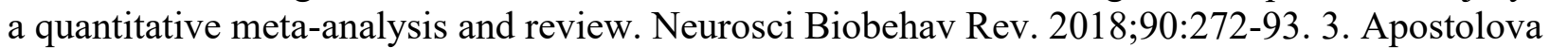

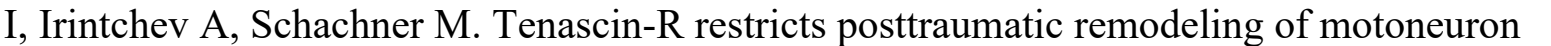

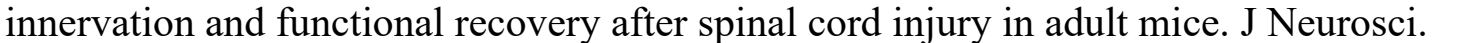

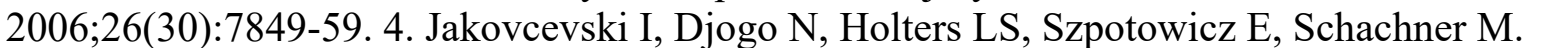

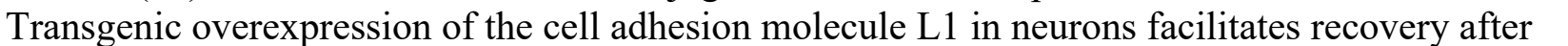

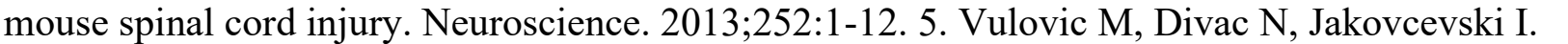

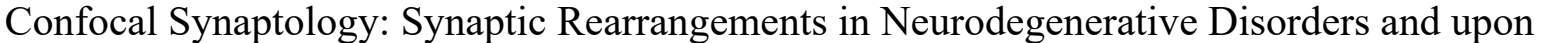

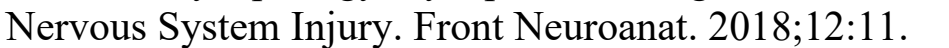

\section{Image/Figure:}

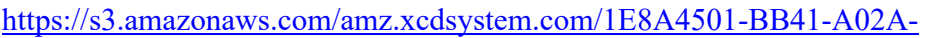

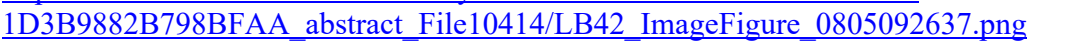

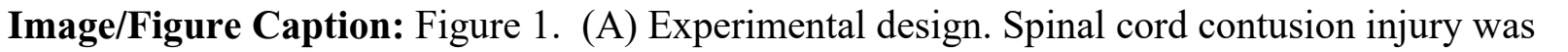

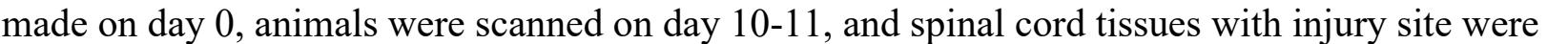

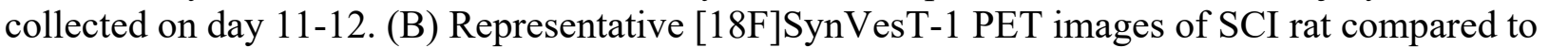

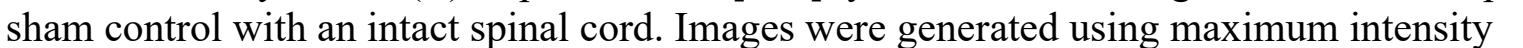

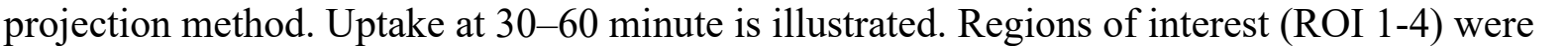

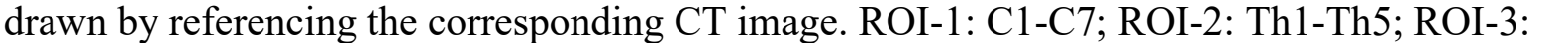

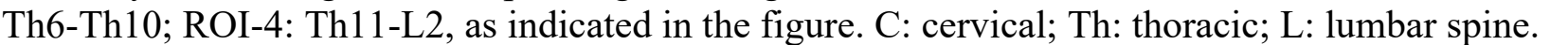

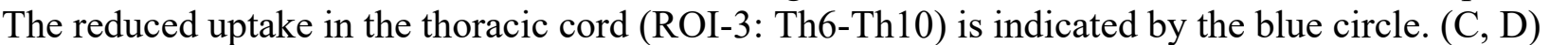

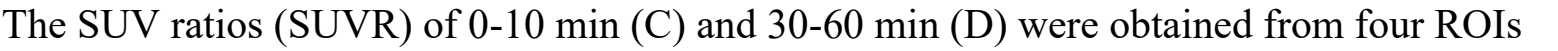

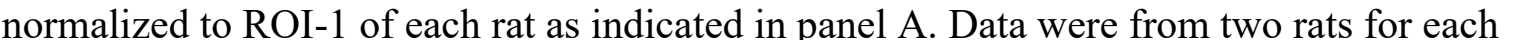

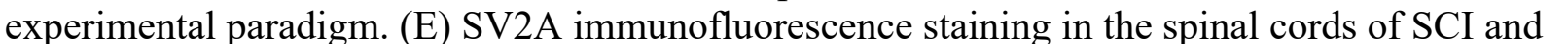

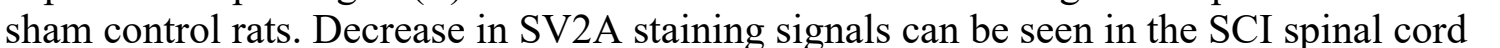

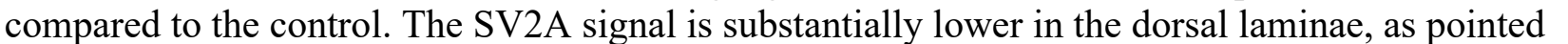

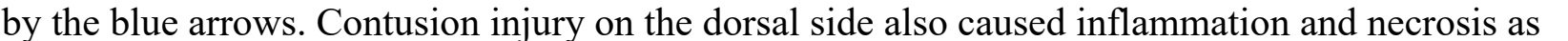

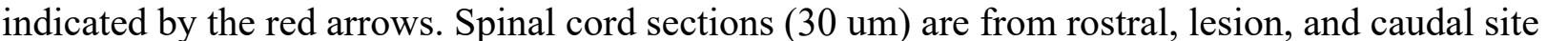

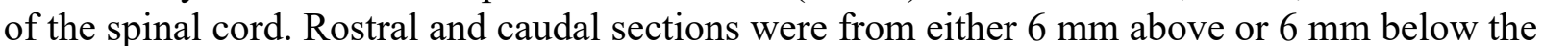

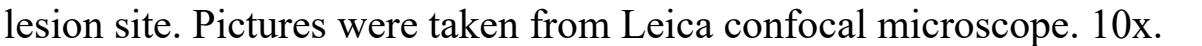

Full Name of Abstract's 1st Author : $\square \square\|\|\|\| \Pi \square \| \square$

First Name: $\square \square \square\|\square\| \| \square$ 
Last Name:

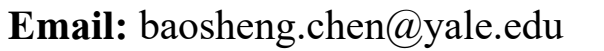

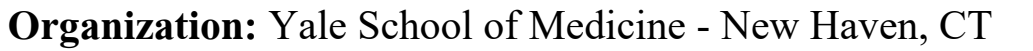

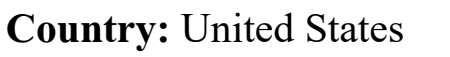




\title{
ID: LB43 \\ Preclinical imaging studies in rats and nonhuman primate reveals [11C]PyBic as a novel brain penetrant radiotracer for PARP
}

\author{
Baosheng Chen, Yale School of Medicine - New Haven, CT, baosheng.chen@yale.edu
}

\section{Category: $\square \square\|\| \| \square$}

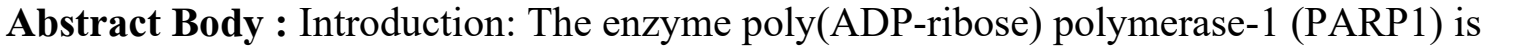

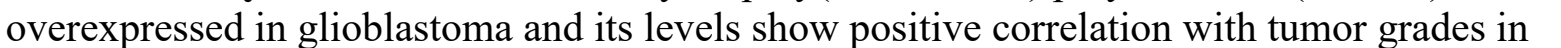

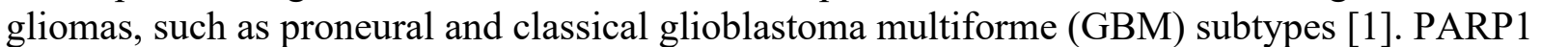
पा

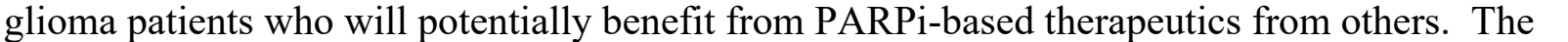

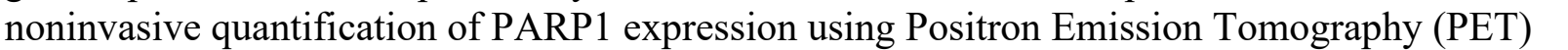

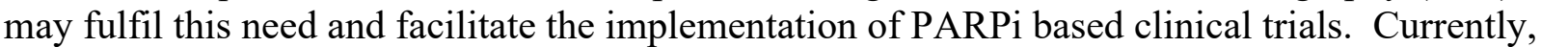

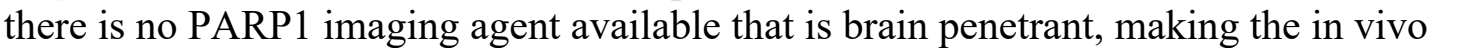

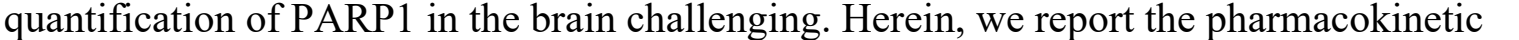

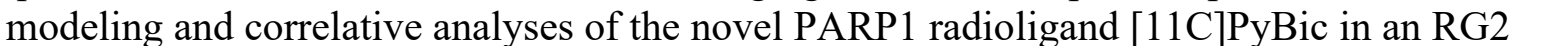

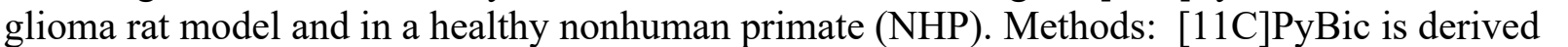

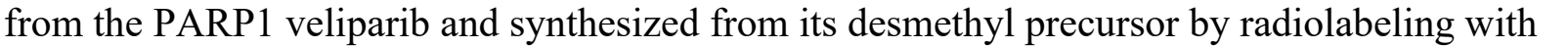
ए

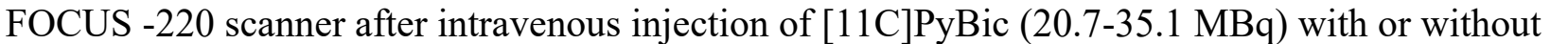

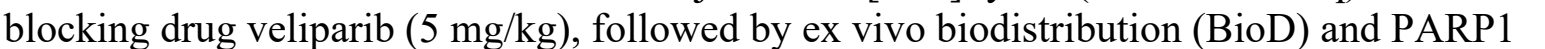

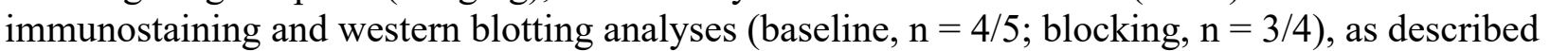

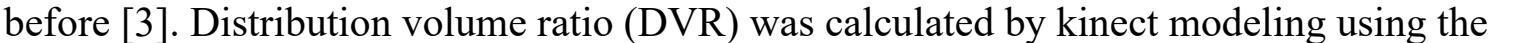

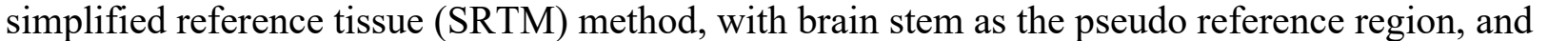

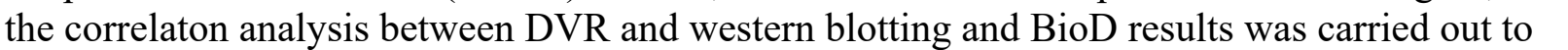

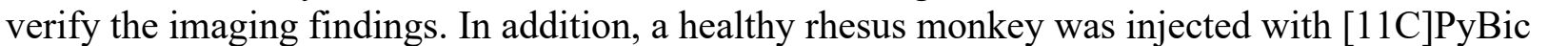

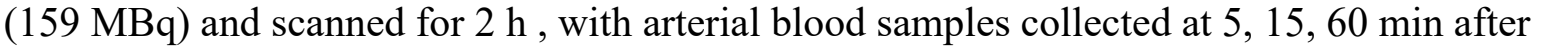
ए

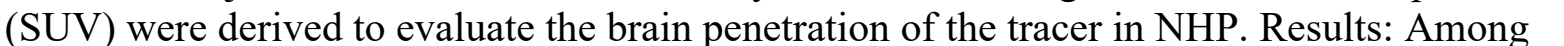

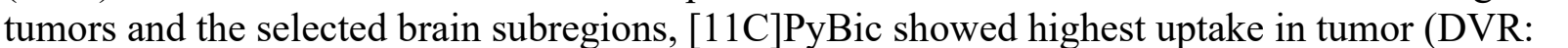

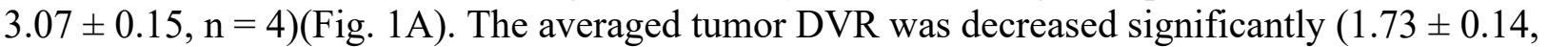

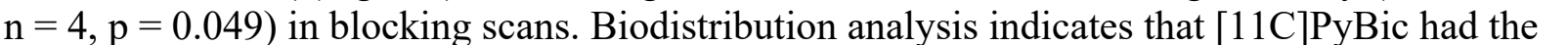

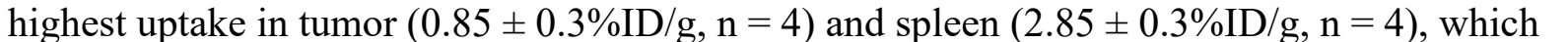

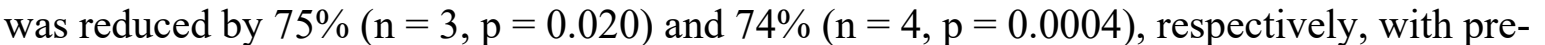

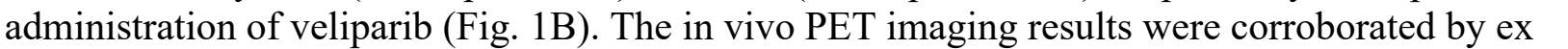

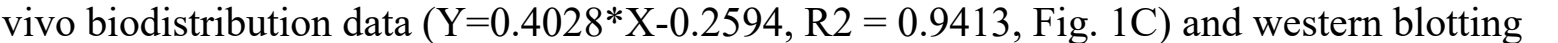

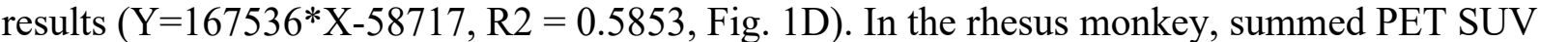

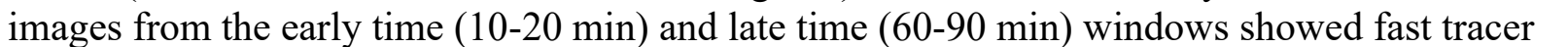

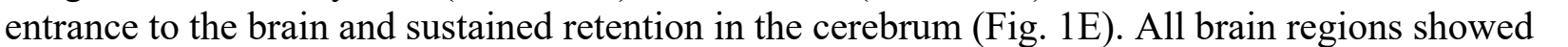

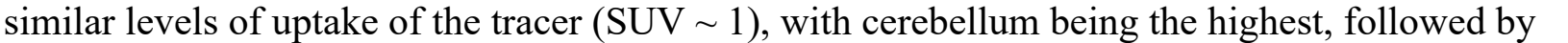

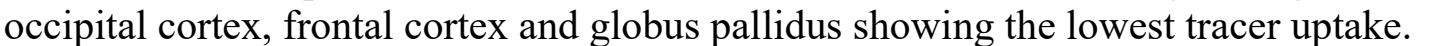

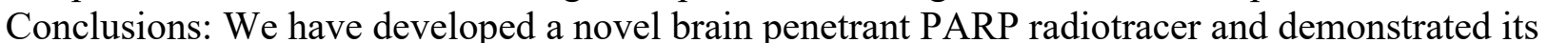




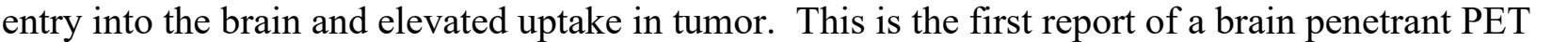

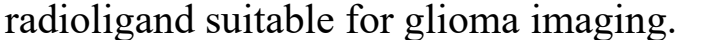

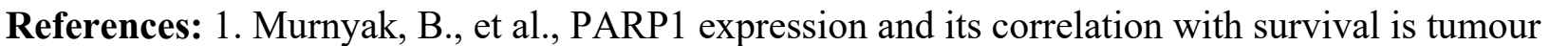

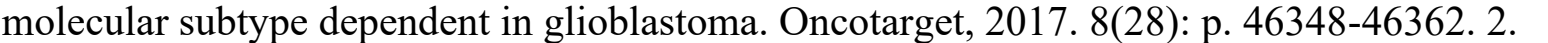

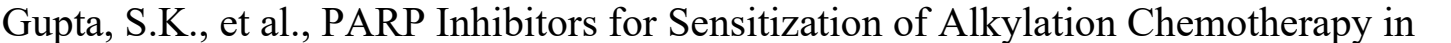

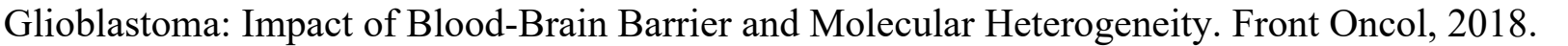

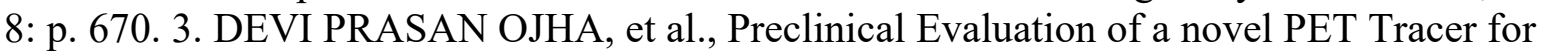

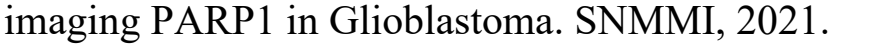

\section{Image/Figure:}

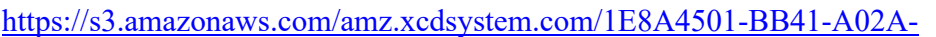

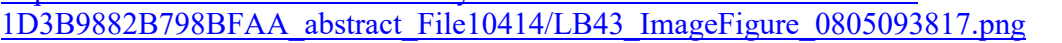

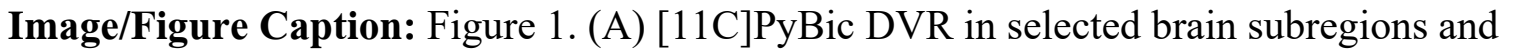

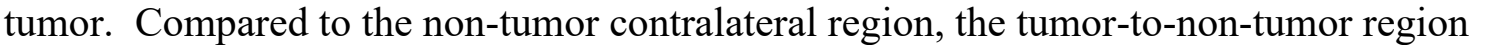

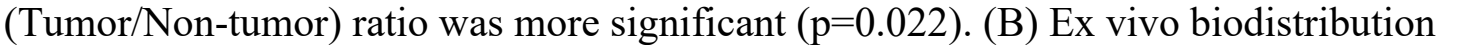

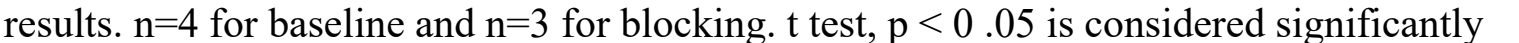

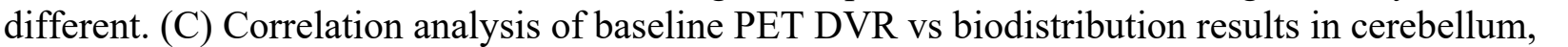

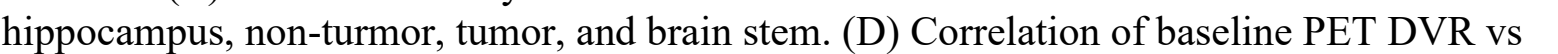

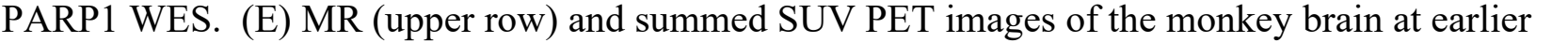

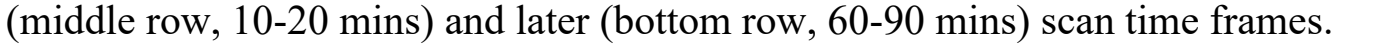

Full Name of Abstract's 1st Author : $\square \square \square\|\square\| \square \square \square \square \square$

First Name: $\square \square \square\|\square\|\|\|$

Last Name: $\square \square \square \square$

Email: $\square \square \square\|\square \square\|\|\square \square \square\| \square \| \square \square \square$

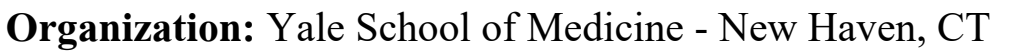

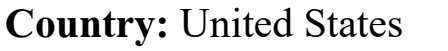




\title{
ID: LB44 \\ Gold Nanoparticle-loaded Macrophages for Magnified Tumor-specific Delivery and Radiosensitization of Oral Cancer
}

Stella Tsao, National Yang Ming Chiao Tung University, s10031824@gmail.com

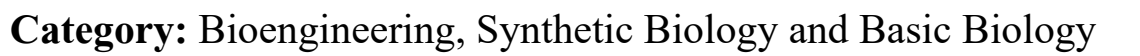

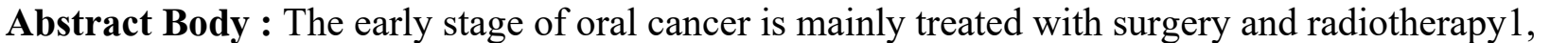

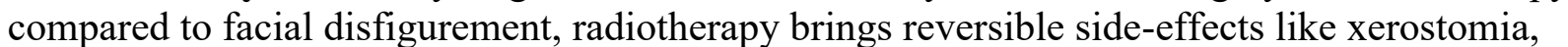

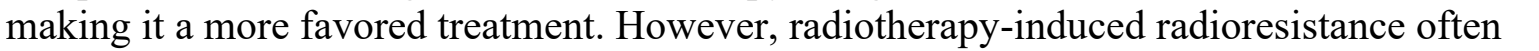

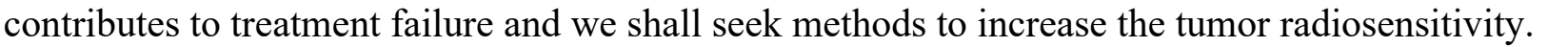

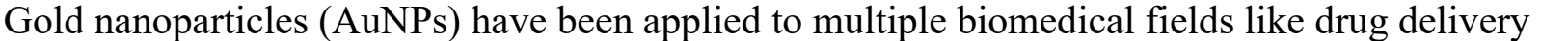

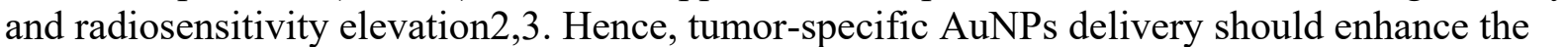

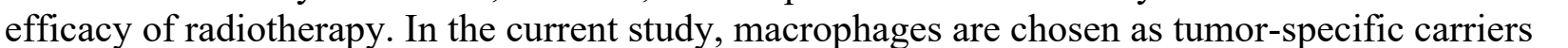
ए

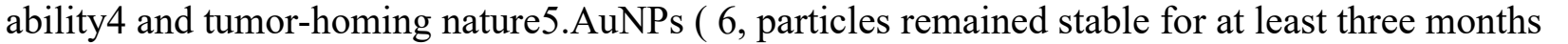

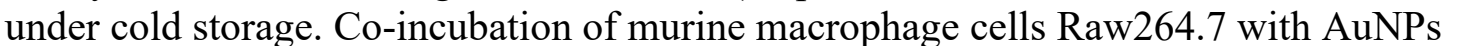

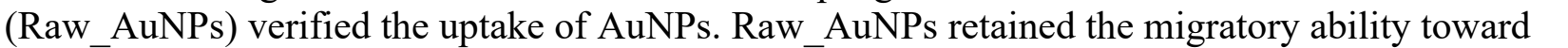

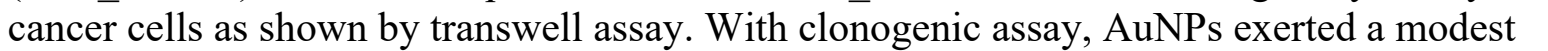

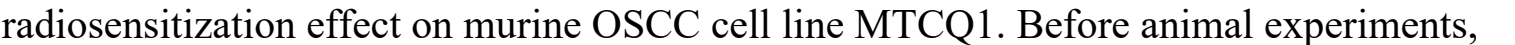

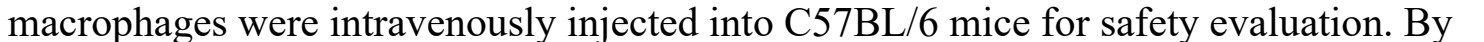

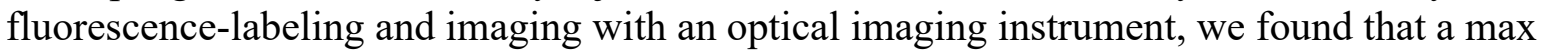

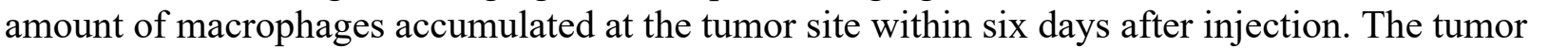

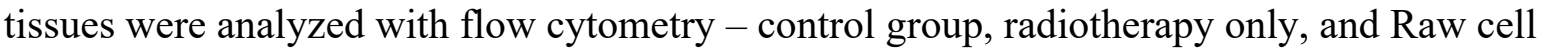

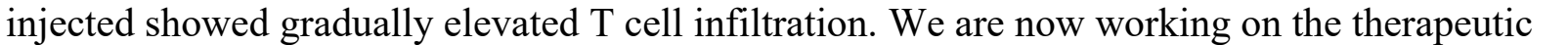

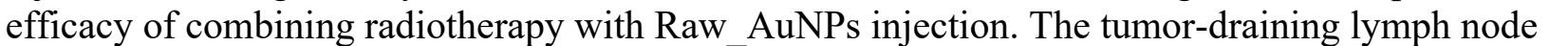

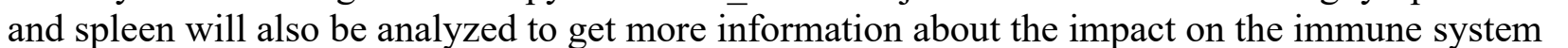

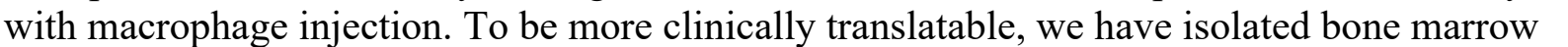

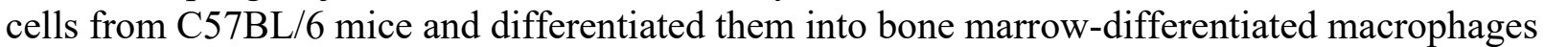

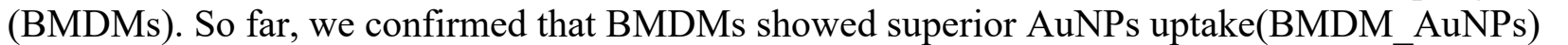

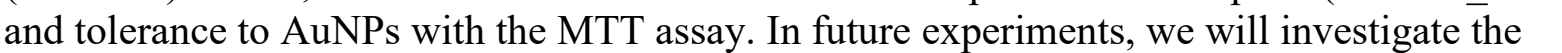

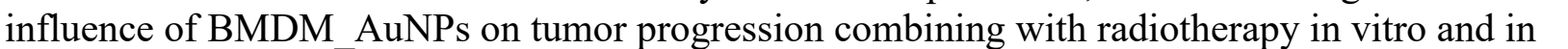
$\square[1 \mathrm{Wl}$
\end{abstract}

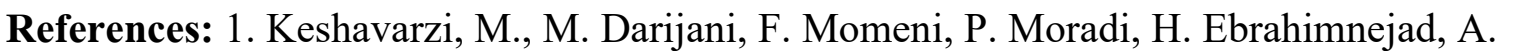

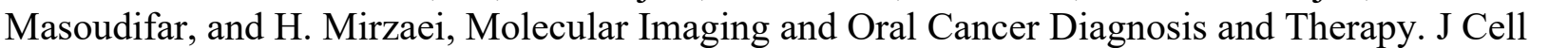

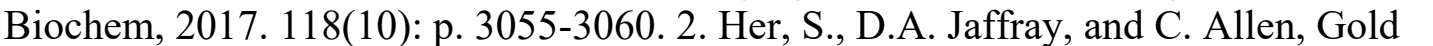

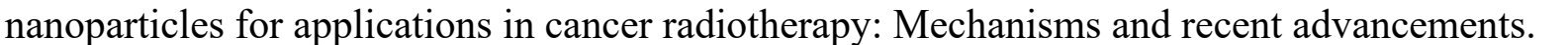

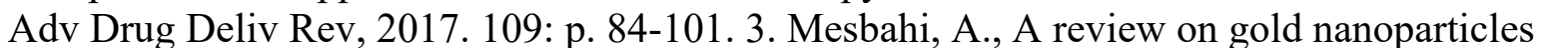

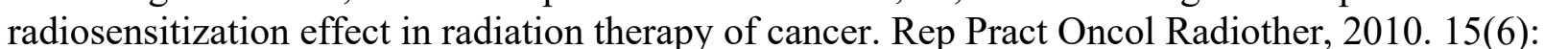

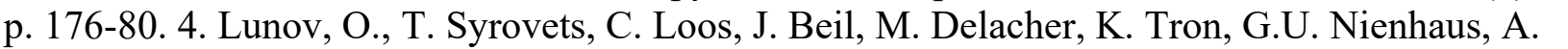

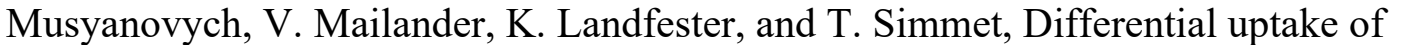

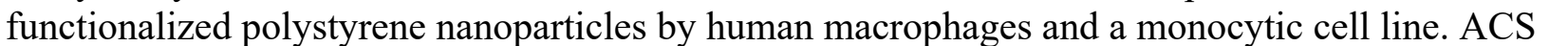


First Name: $\square[\|\|\|\| \square$

Last Name: $\square \square\|\| \square$

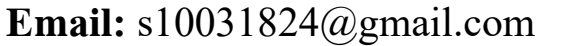

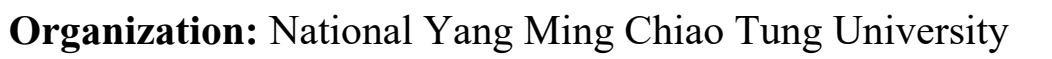

Country: $\square \square \| \square \square \square$ 


\title{
ID: LB46 \\ Radiolabeled dendrimer PET biodistribution in a rabbit model of neuroinflammation
}

\author{
Elizabeth Winans Tucker, Johns Hopkins University School of Medicine, \\ etucker9@jhmi.edu
}

Category: $\square \square \square\|\square\|\|\square\| \square$

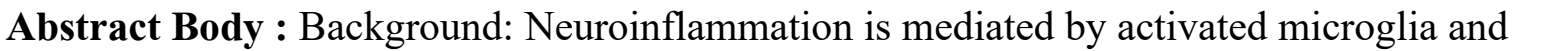

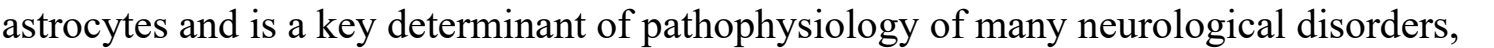
ए

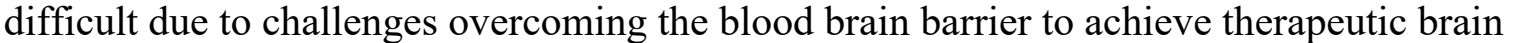

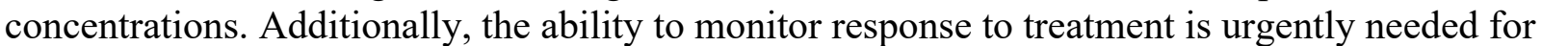

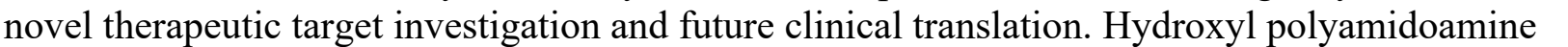

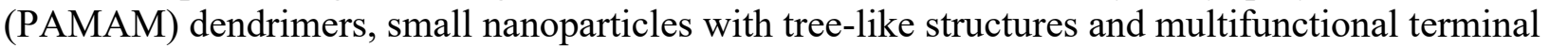

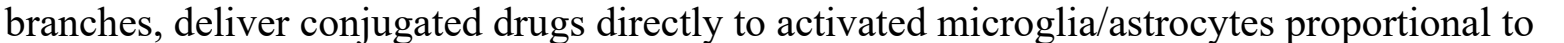

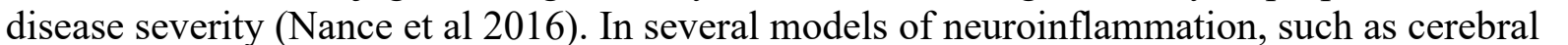

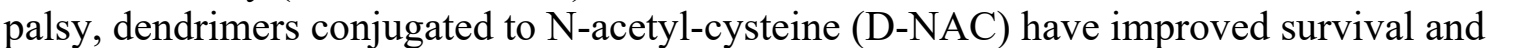
neurological outcomes (Kannan et al 2012). Additionally, dendrimer's flexible terminal groups

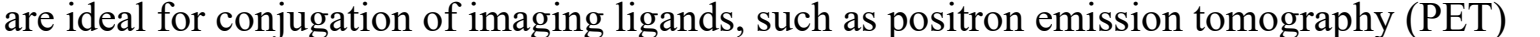

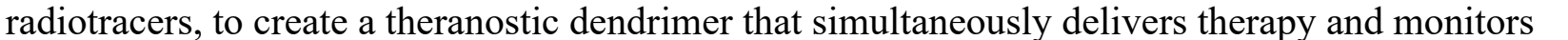

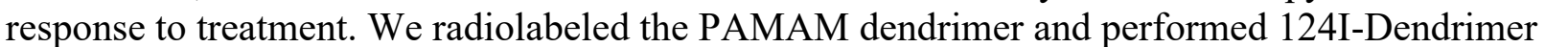

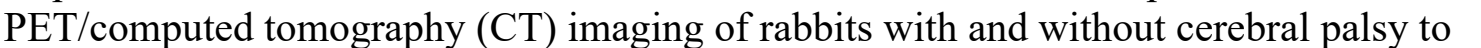

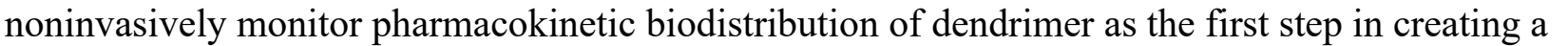
ए

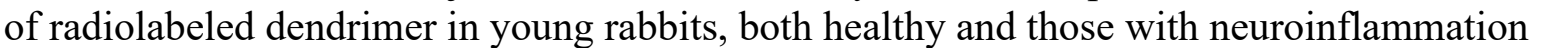

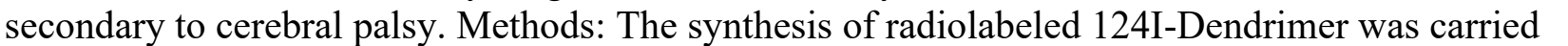

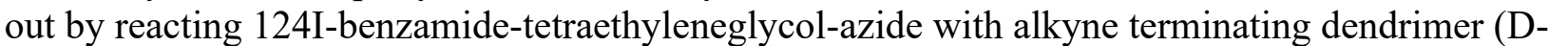

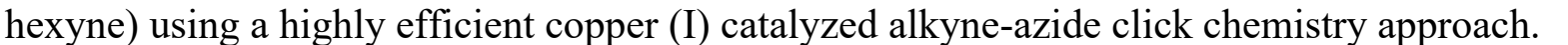

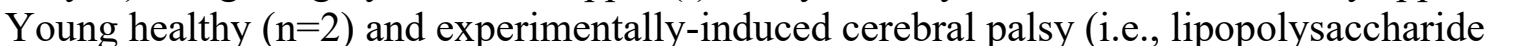

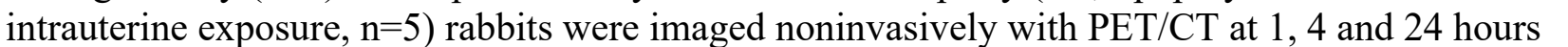

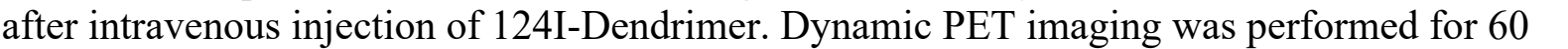

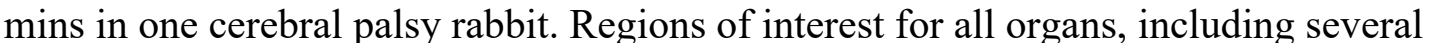

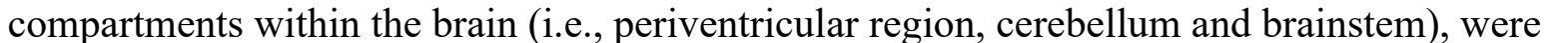

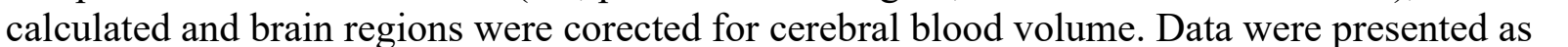

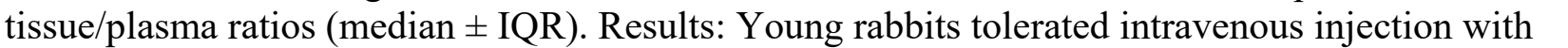

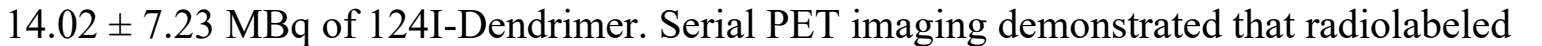

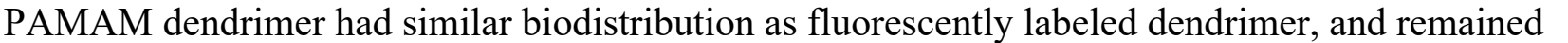

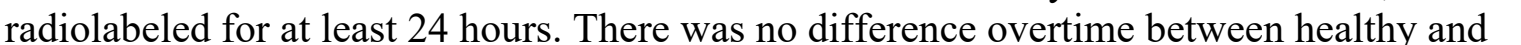

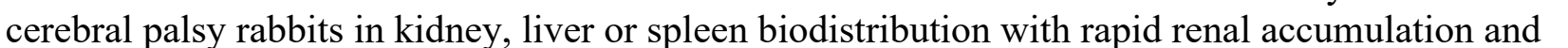

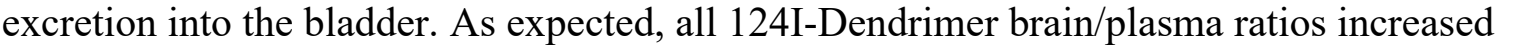

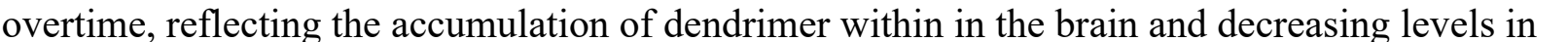

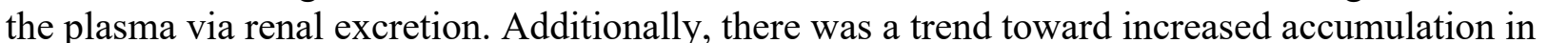




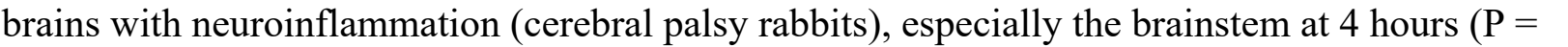

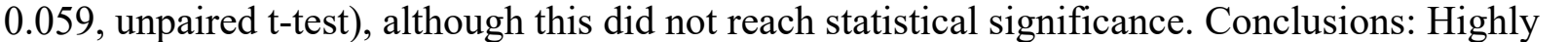

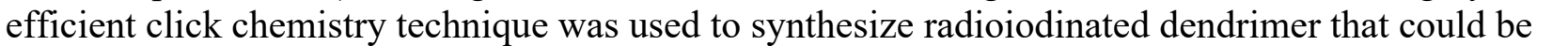

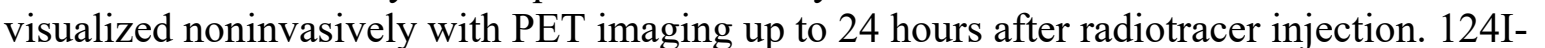

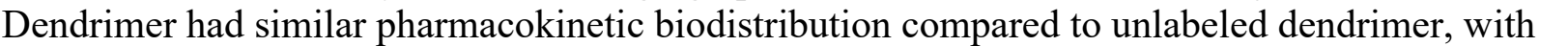

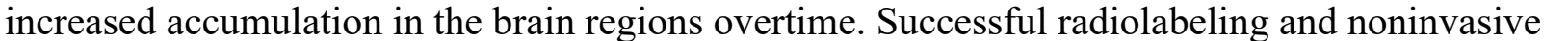

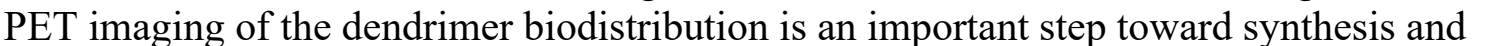

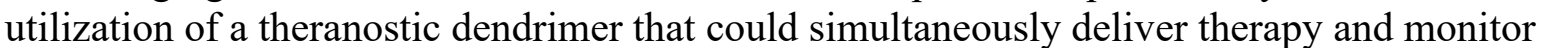

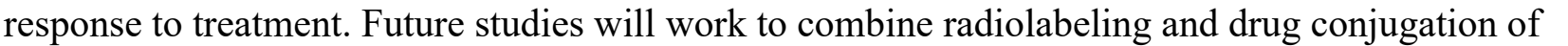

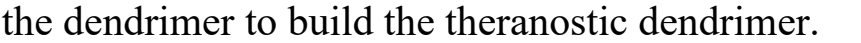

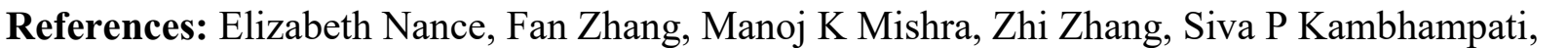

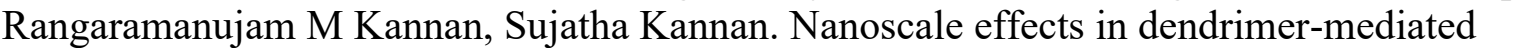

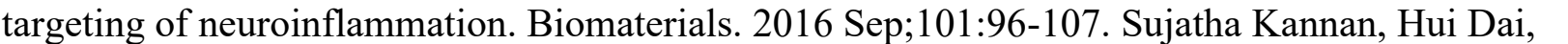

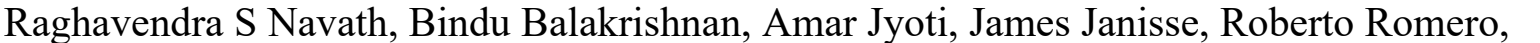

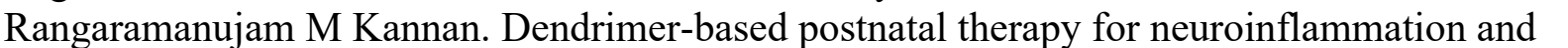

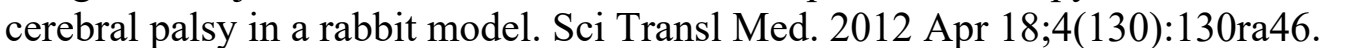

\section{Image/Figure:}

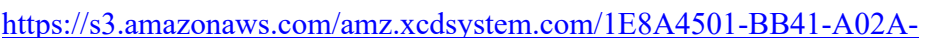

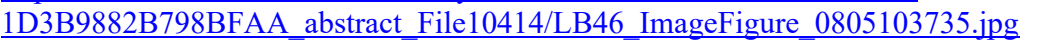

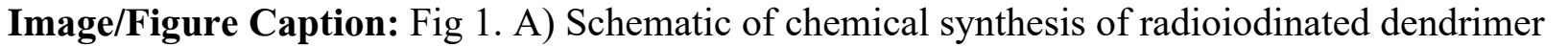

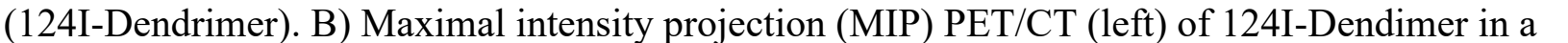

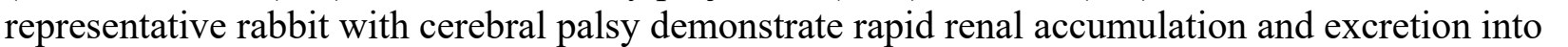
ए

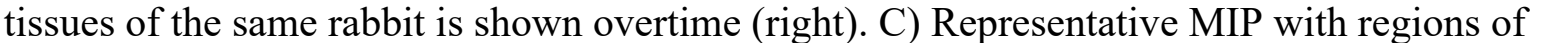

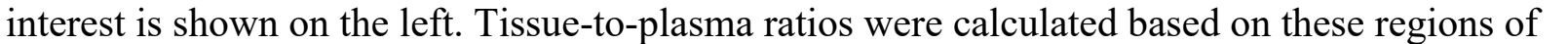

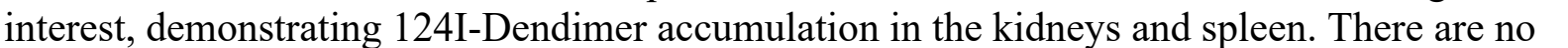

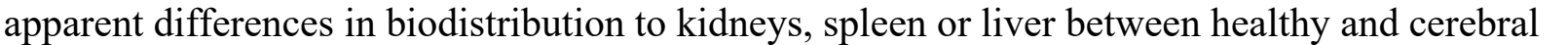

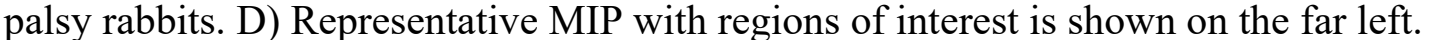

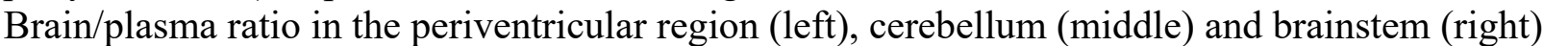

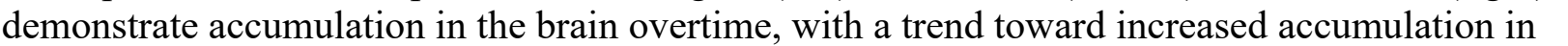

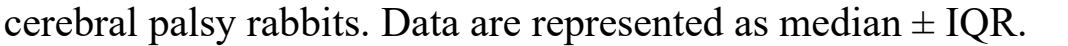

First Name: $\square \square\|\|\|\|\|\|$

Last Name: $\square \square \square\|\|\|\square \square\|\|\|$

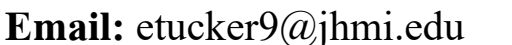

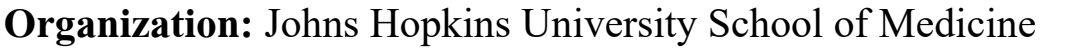

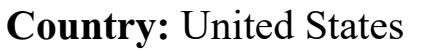




\title{
ID: LB47 \\ Age-specific 18F-FDG PET template for epileptogenic focus localization in pediatric patients who underwent epilepsy surgery
}

Teng Zhang, Second Affiliated Hospital Of Zhejiang University School Of Medicine, zhangteng630@zju.edu.cn

Category: $\square \square \square\|\square\| \| \square \square \square$

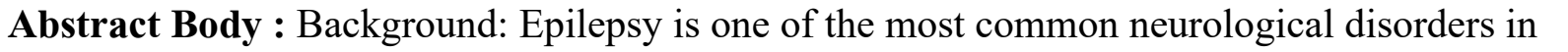

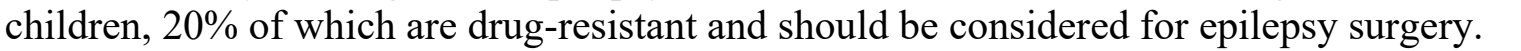

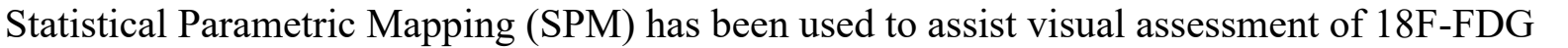

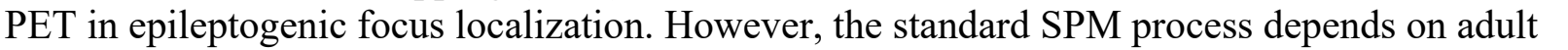

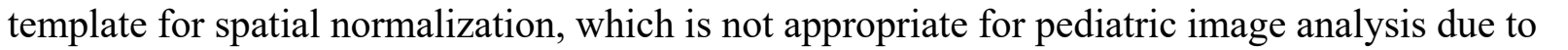

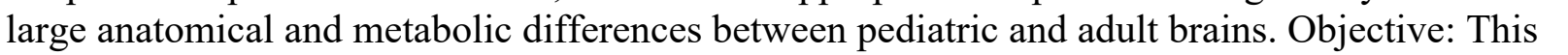

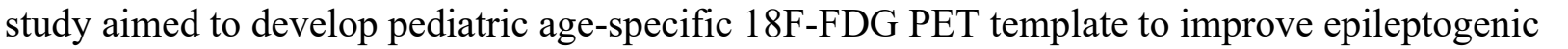

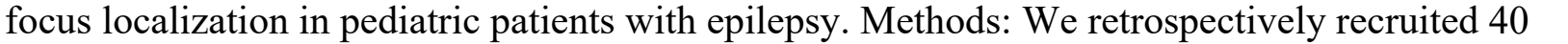

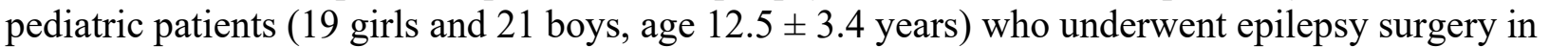

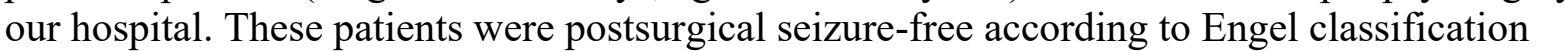

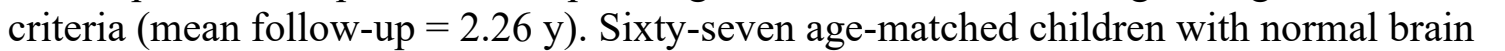

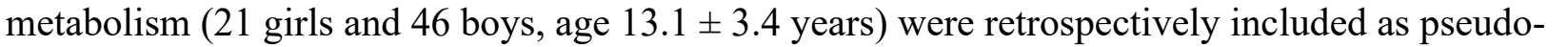

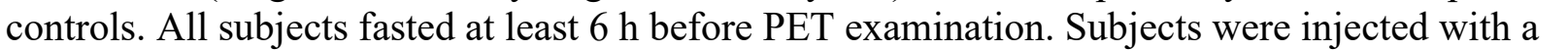

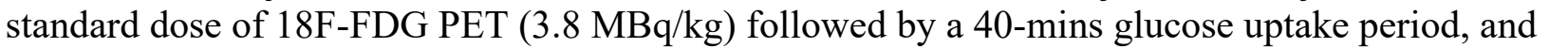

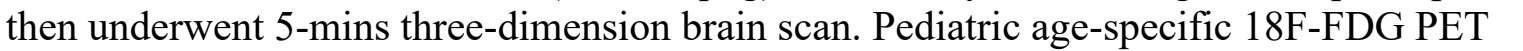
ए

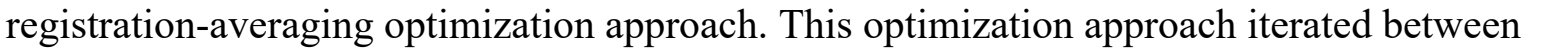

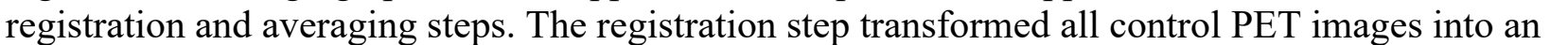

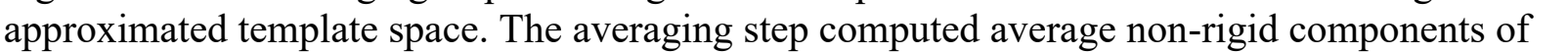

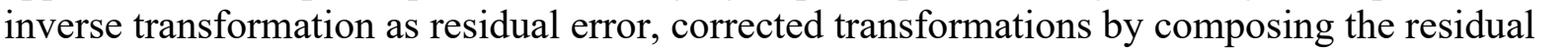

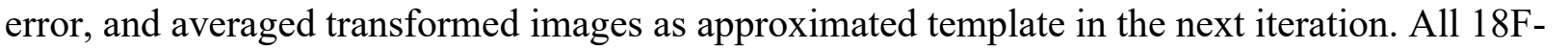

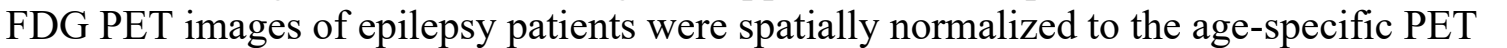

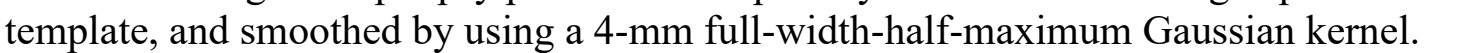

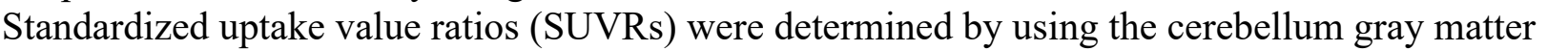

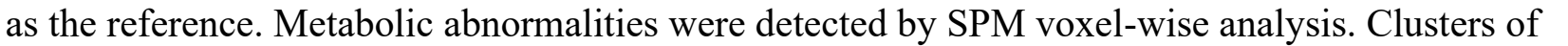

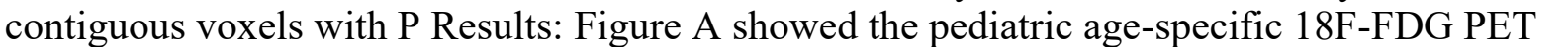

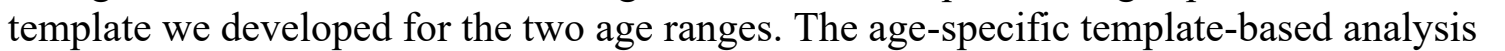

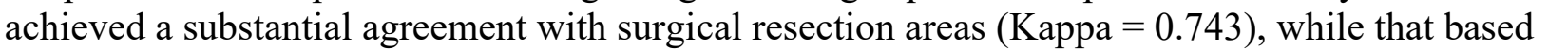

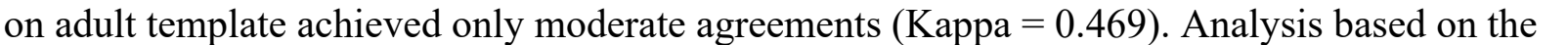

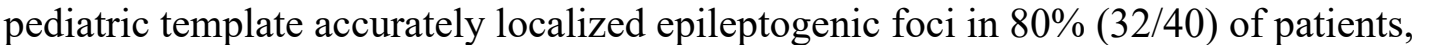

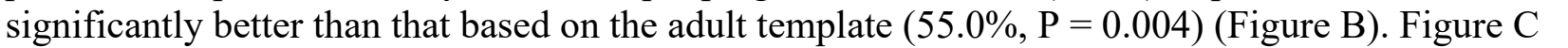

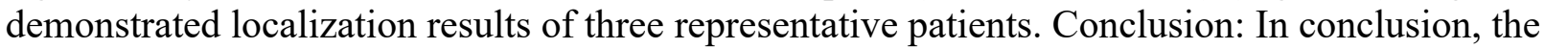

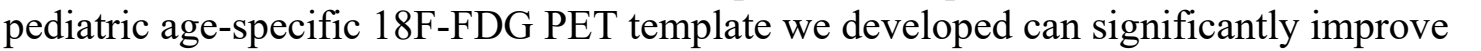

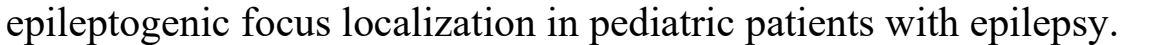


Image/Figure:

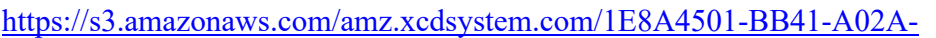

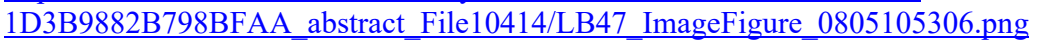

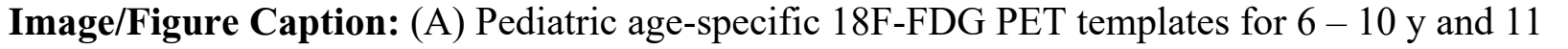

-

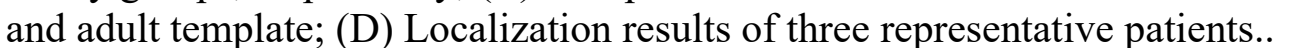

Full Name of Abstract's 1st Author :

First Name:

Last Name:

Email: $\square\|\|\|\|\|\|\|\square \square\| m\|\square\| \square$

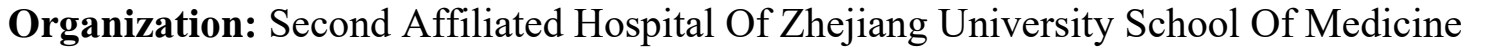

Country: $\square \square \square \square \square$ 


\section{ID: LB48}

The expanding toolbox for bioluminescence imaging

Jennifer Prescher, UC Irvine, jpresche@uci.edu

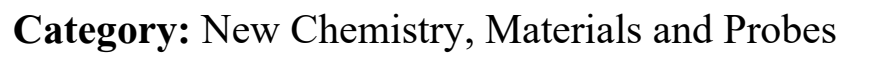

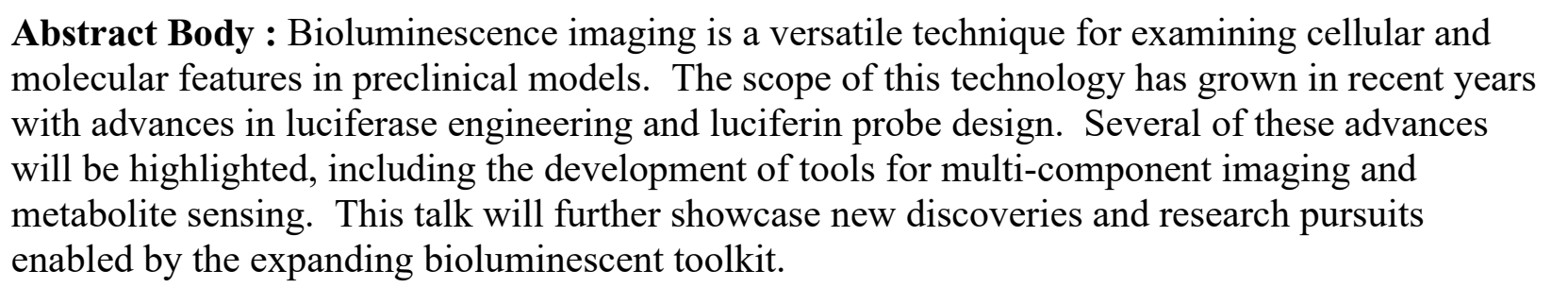

First Name: $\square \square|| m|n| m$

Last Name: $\square[\|\|\|\square \mid\| \square$

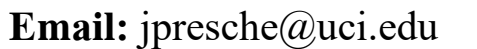

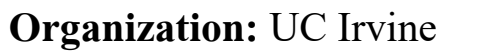

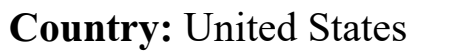




\title{
ID: LB49 \\ Evaluation of 18F-FEPPA as a PET Radiotracer for Cardiac Inflammation in a Canine Model of Myocardial Infarction
}

\author{
Haris Smailovic, Lawson Health Research Institute, hsmailov@uwo.ca
}

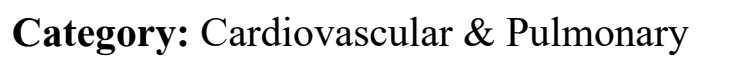

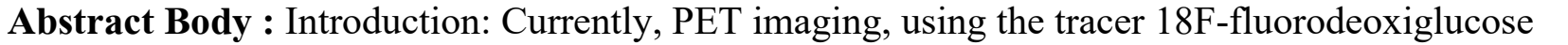

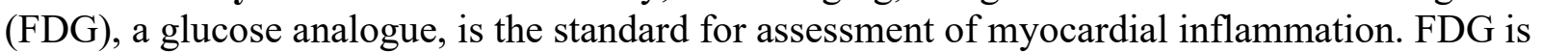

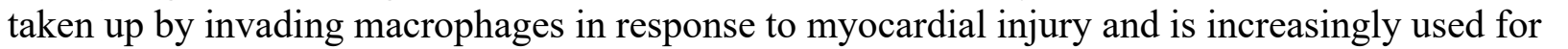

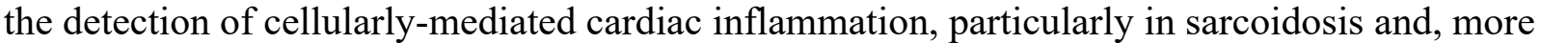

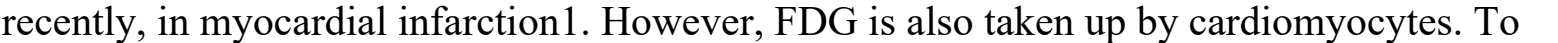

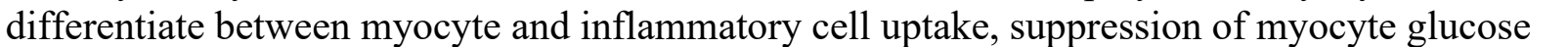

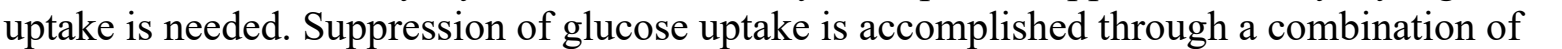

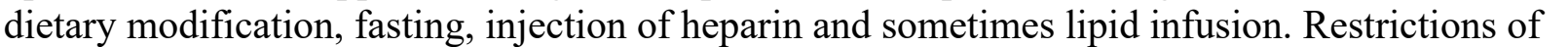

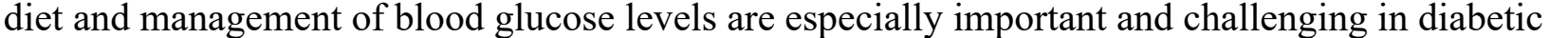

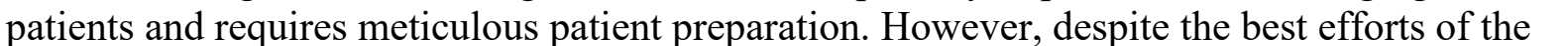

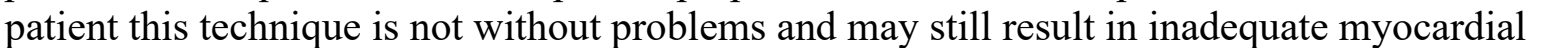

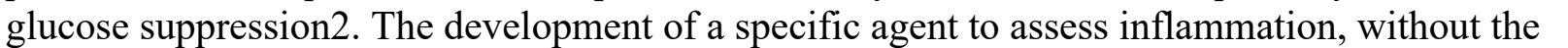

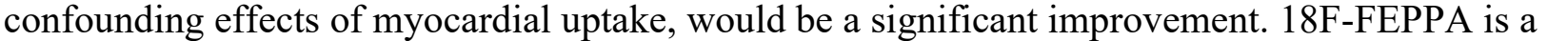

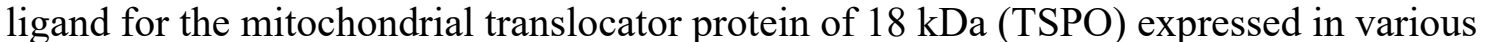

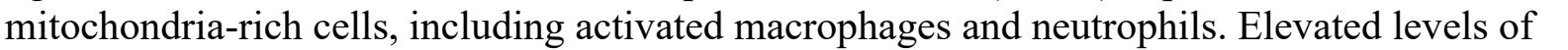

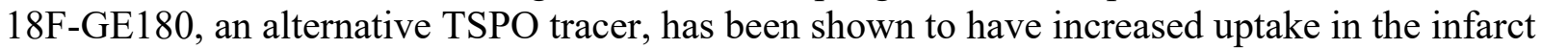

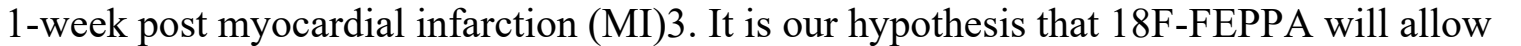

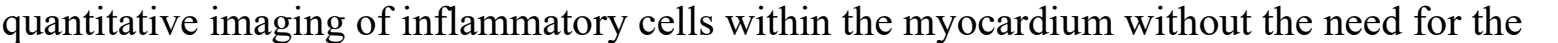

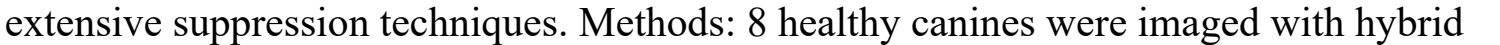

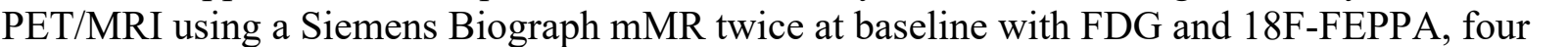

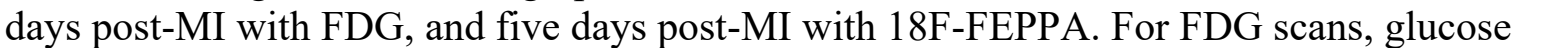

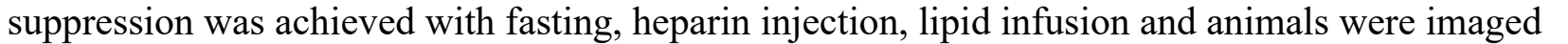

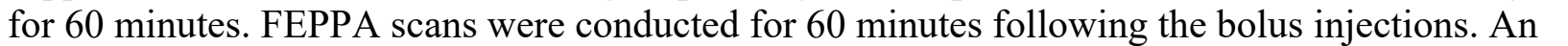

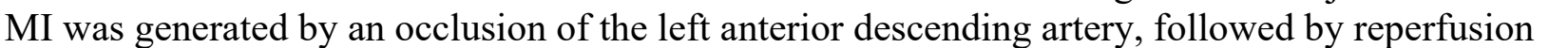

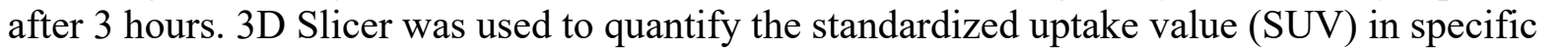

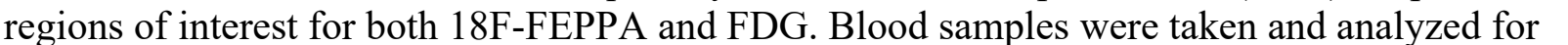

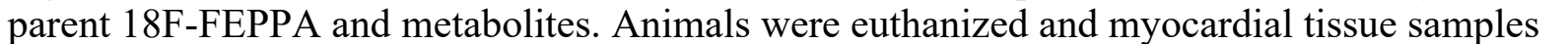

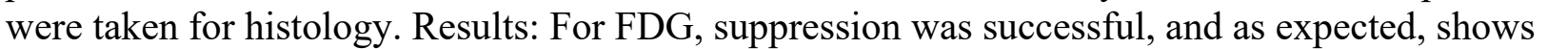

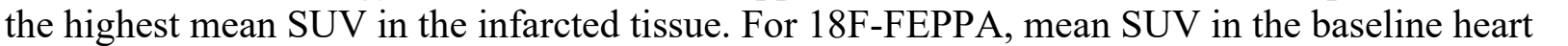

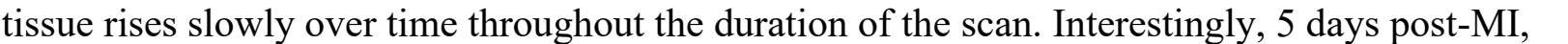

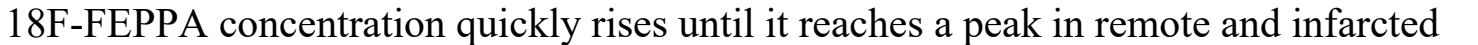

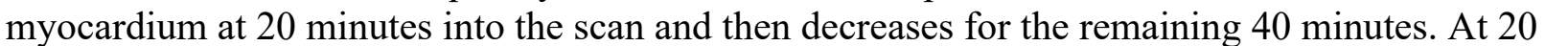

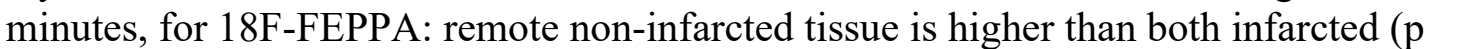

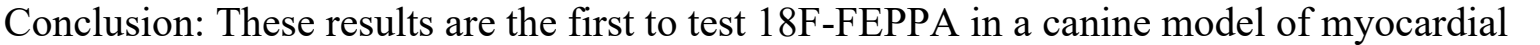

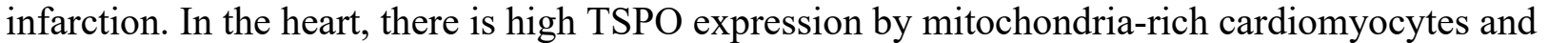

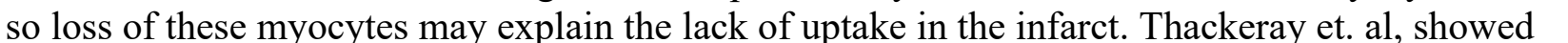


ए

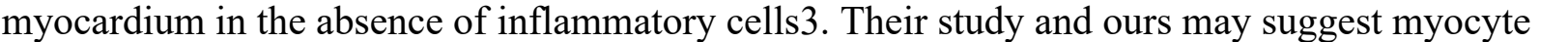

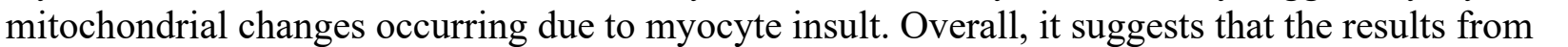

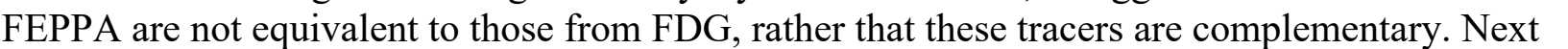

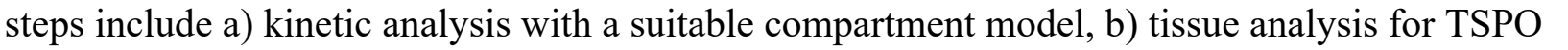

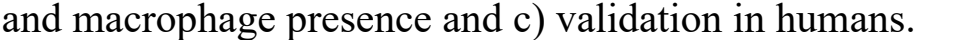

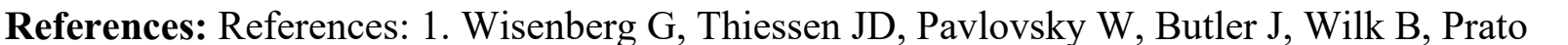

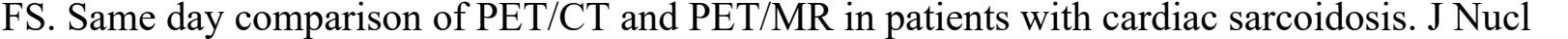

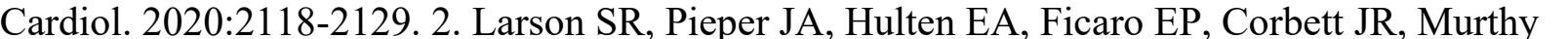

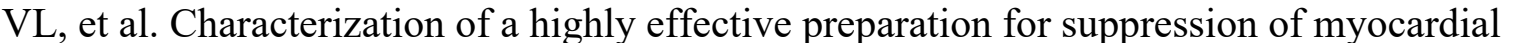

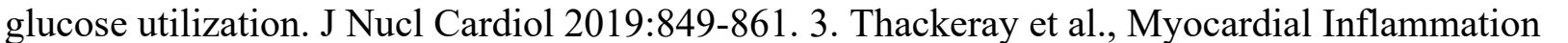

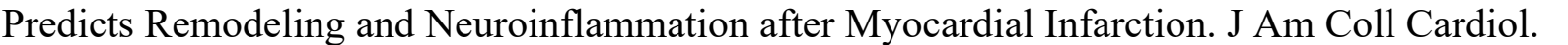

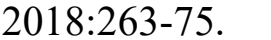

\section{Image/Figure:}

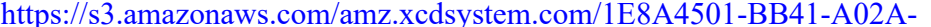

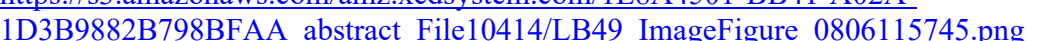

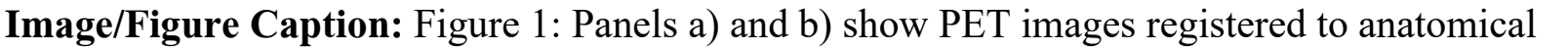

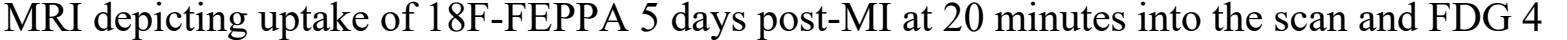

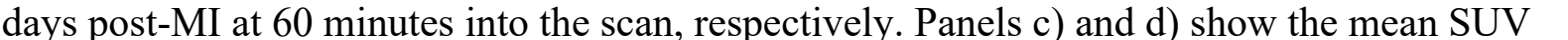

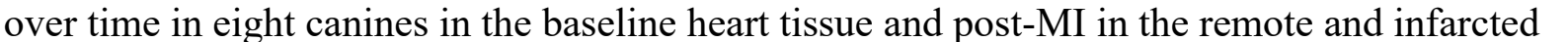

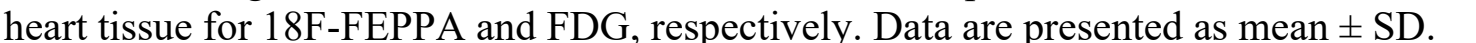

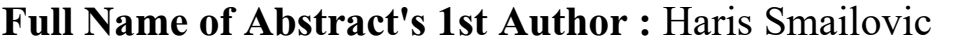

First Name: $\square \square \square \| m$

Last Name: $\square \square \square \| m|\square| m$

Email: $\square \square \square \square \| \square \square \square \square \square \square \square$

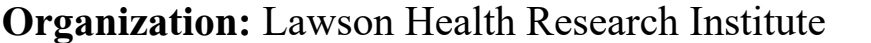

Country: $\square \square\|\|\|\|$ 


\title{
ID: LB50 \\ Intraperitoneal delivery of iopamidol to assess extracellular pH of orthotopic pancreatic tumor model by CEST MRI
}

Bruna Victorasso Jardim Perassi, Moffitt Cancer Center, Bruna.Perassi@moffitt.org

Category: $\square \square\|\| \| \square \mid \square$

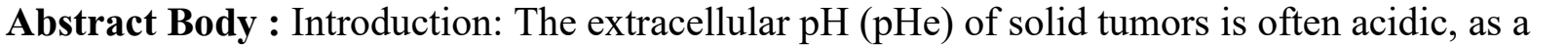

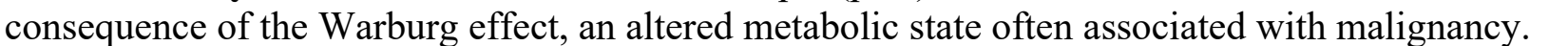

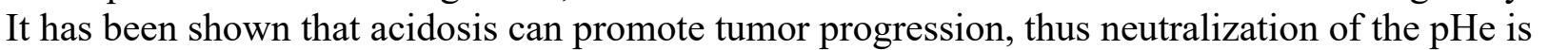

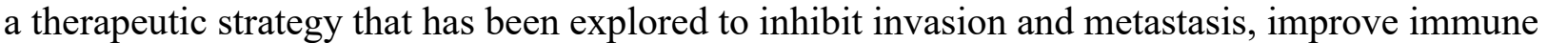

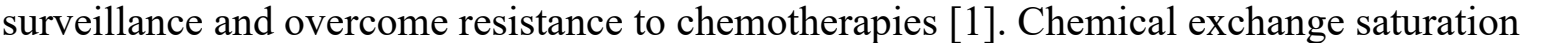

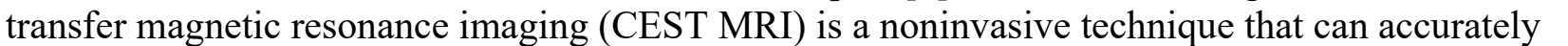

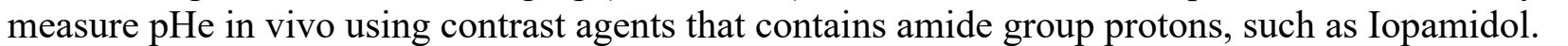

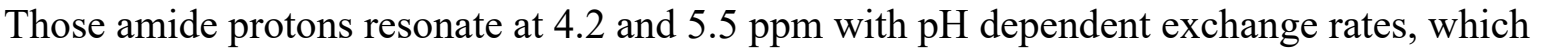

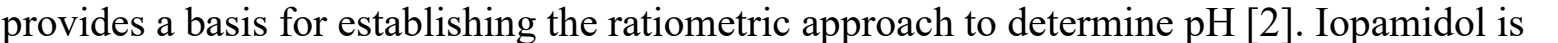

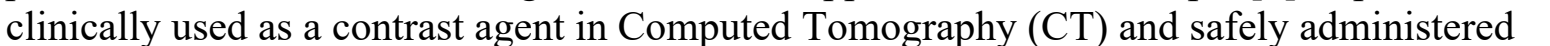

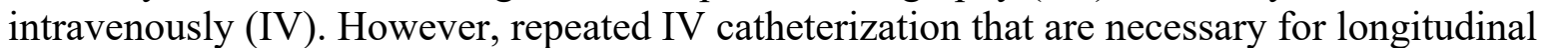

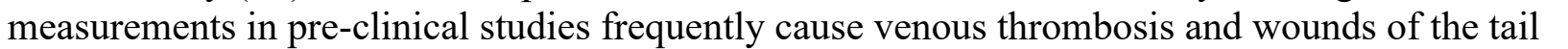

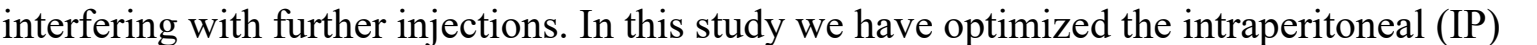

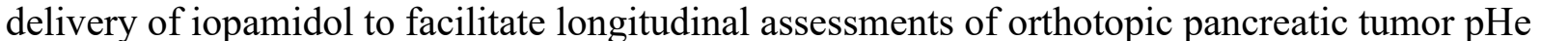

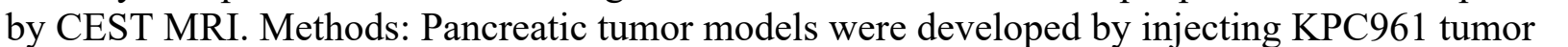

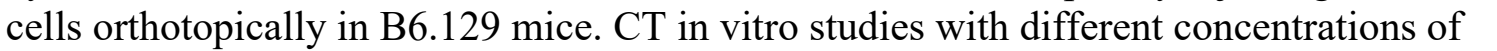

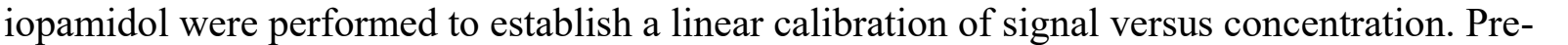

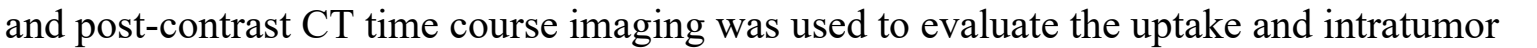

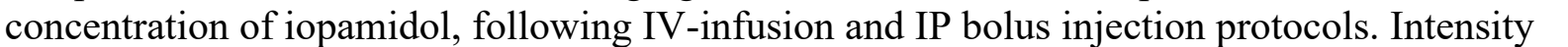

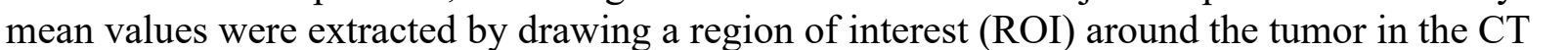

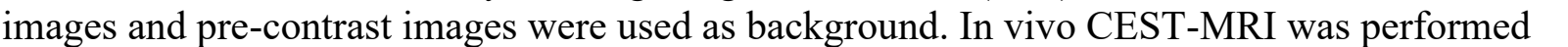

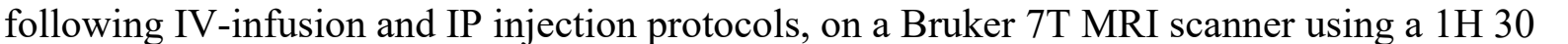

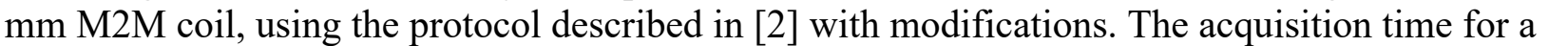

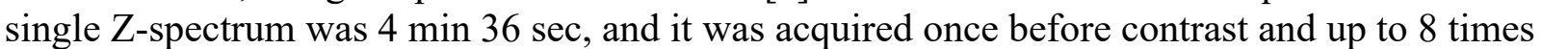

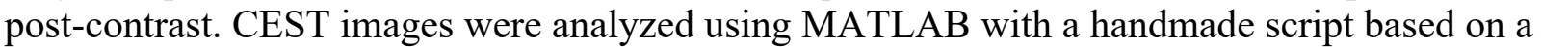

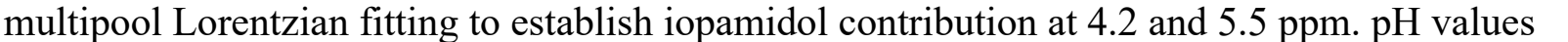

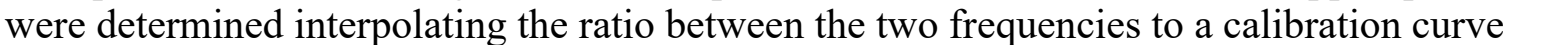

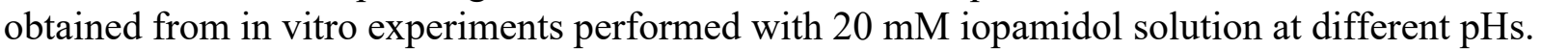

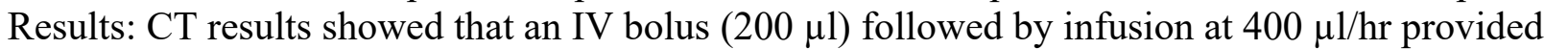

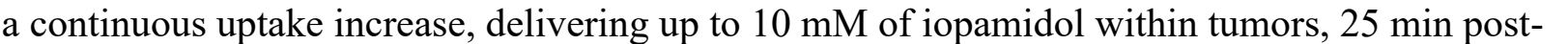

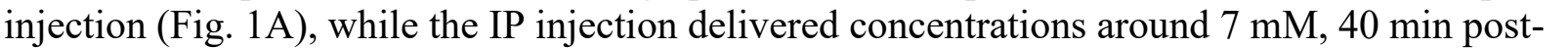

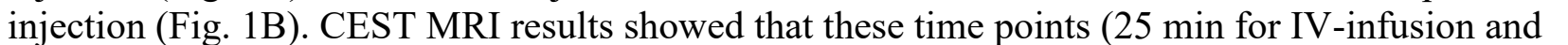

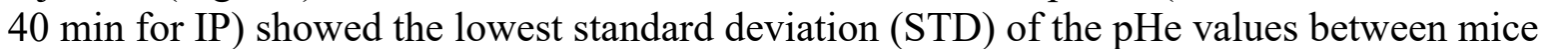

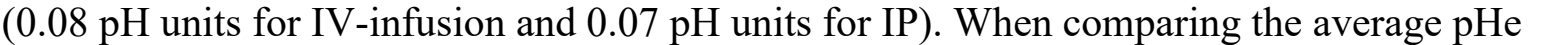

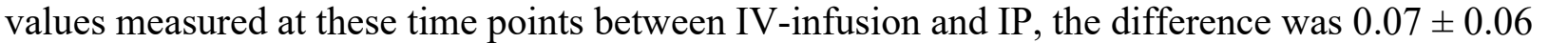

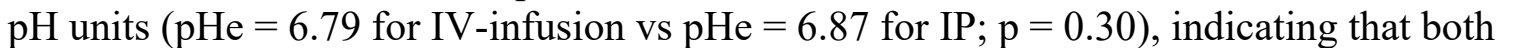




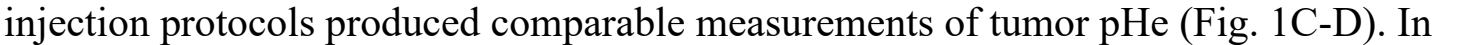

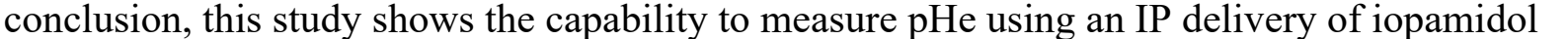

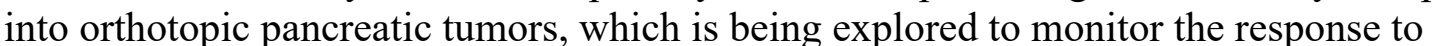

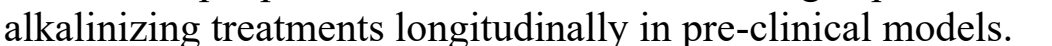

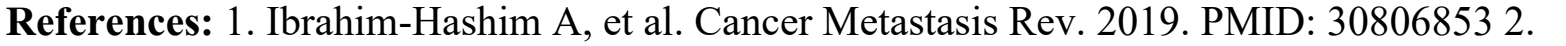

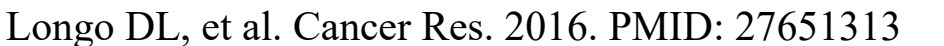

\section{Image/Figure:}

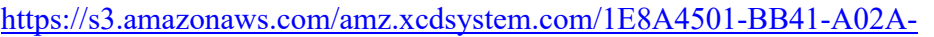

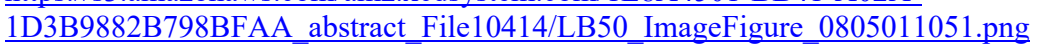

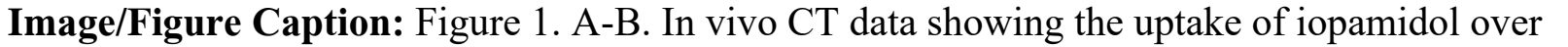

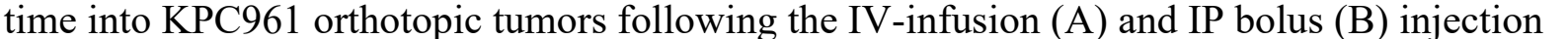

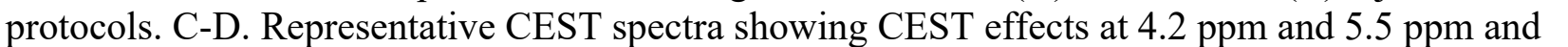

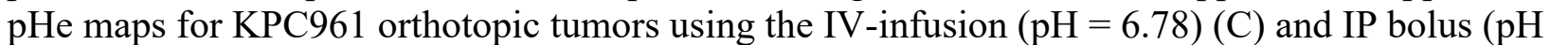

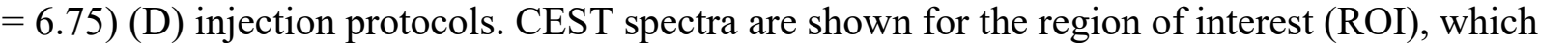

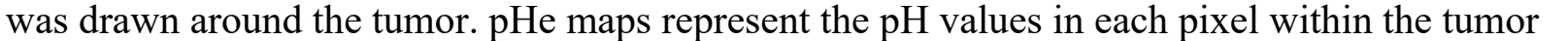

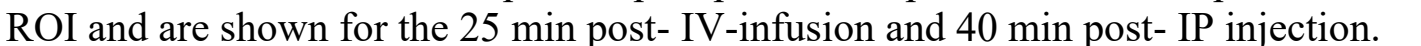

First Name: $\square \square \square|\||$

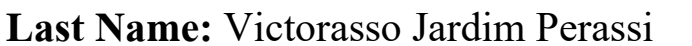

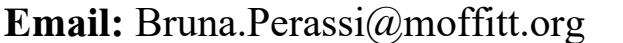

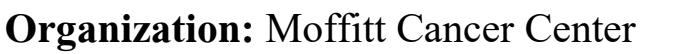

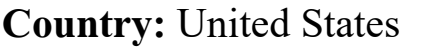




\title{
ID: LB51 \\ Imaging Antibody Uptake in vivo Using a pH Responsive SWIR Fluorescent Probe
}

\author{
Michael Luciano, National Cancer Institute, lucianomp@nih.gov
}

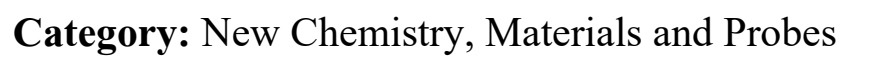

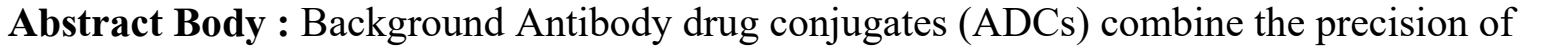

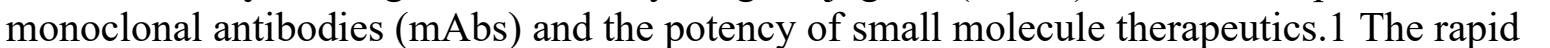

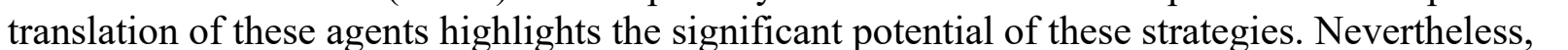
प

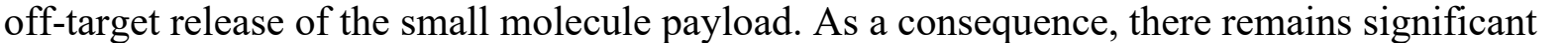

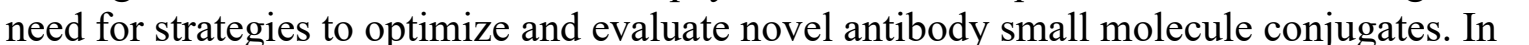

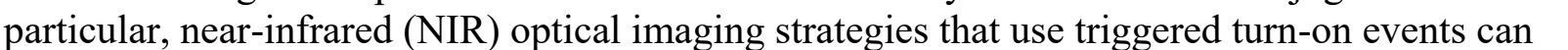

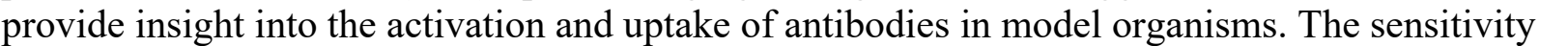

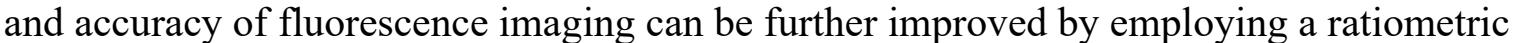

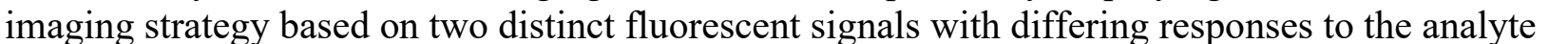

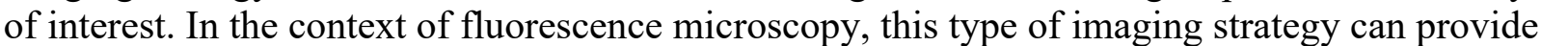

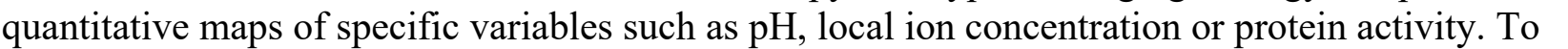

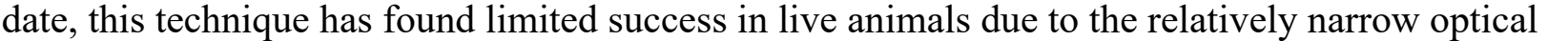

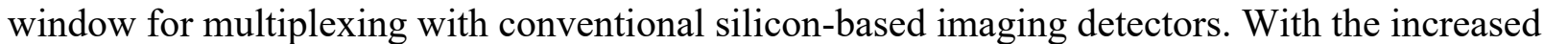

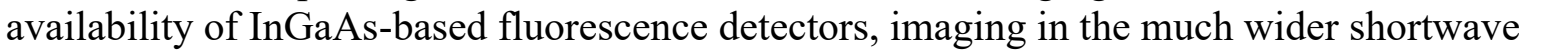

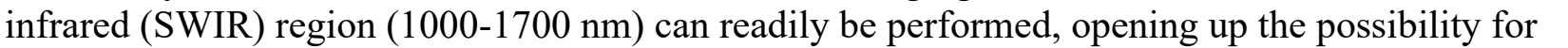

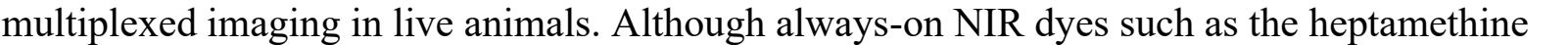

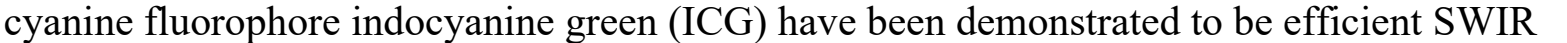

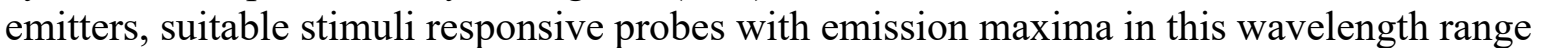

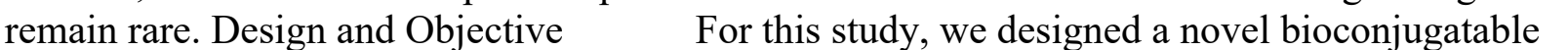

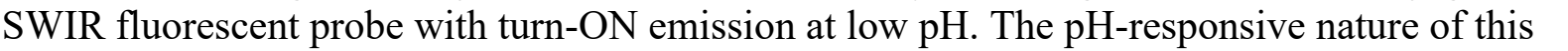

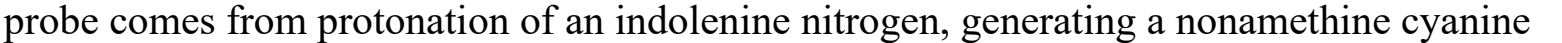

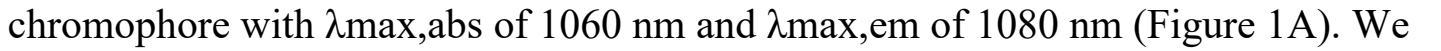

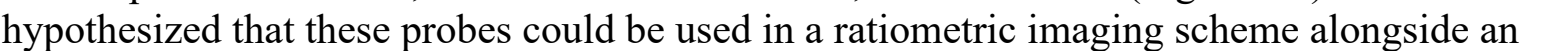

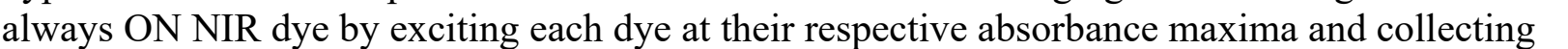

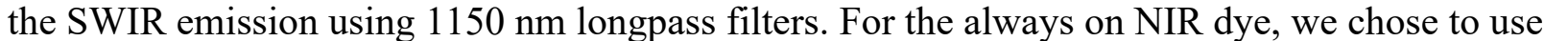

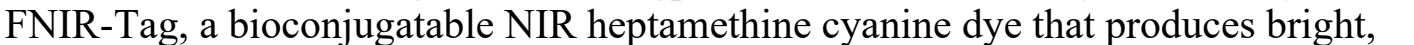

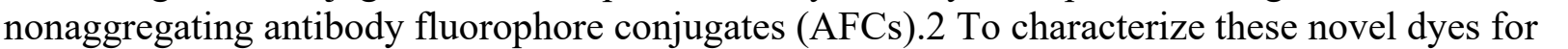

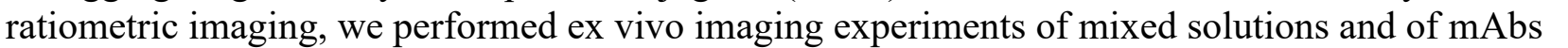

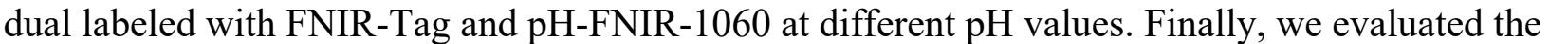

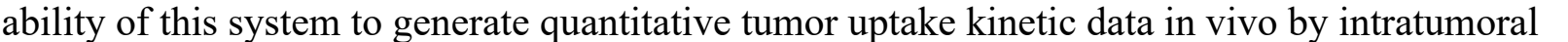
ए ए

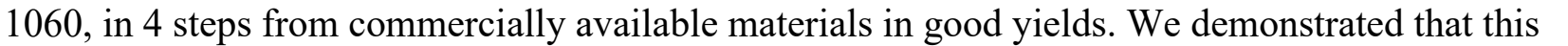

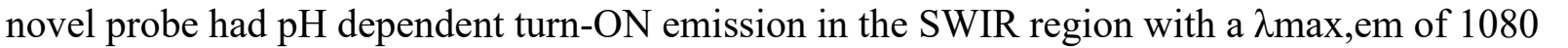

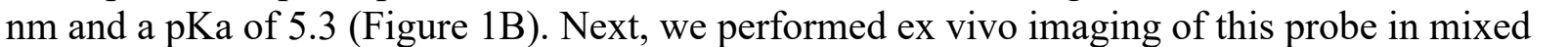




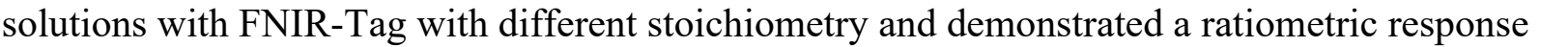

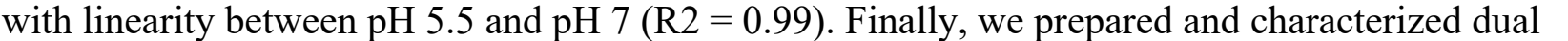

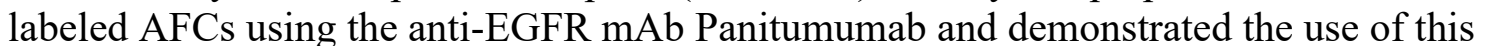

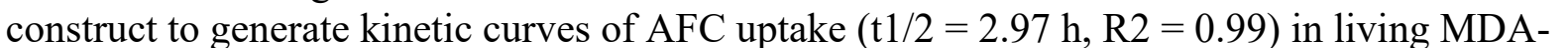

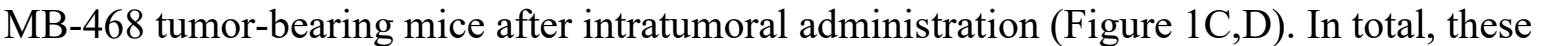

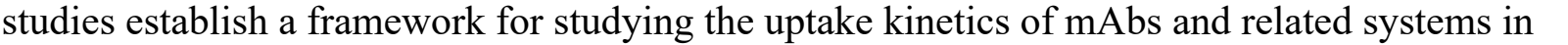

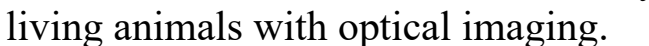

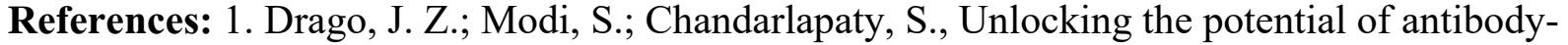

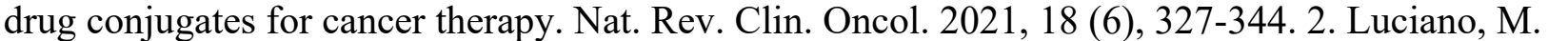

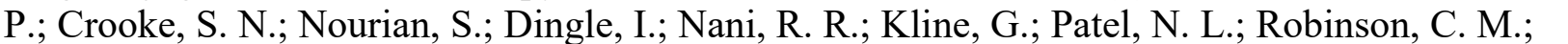

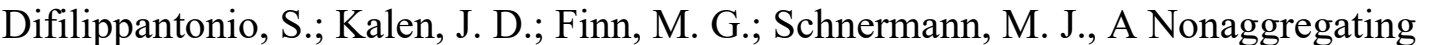

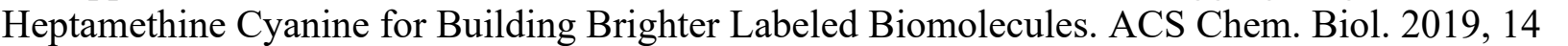

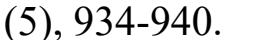

\section{Image/Figure:}

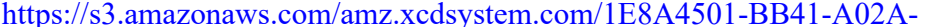

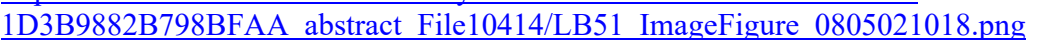

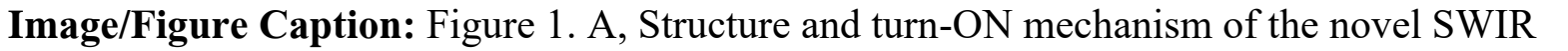

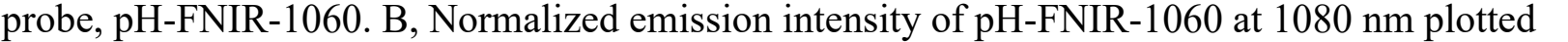
ए ए

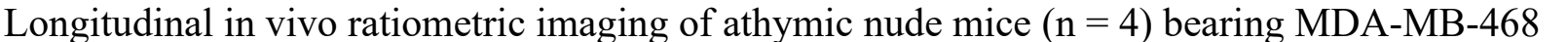

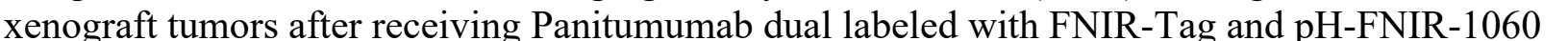

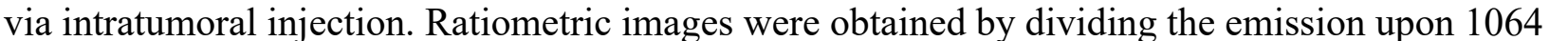

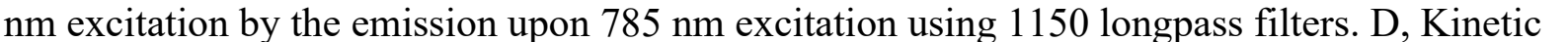

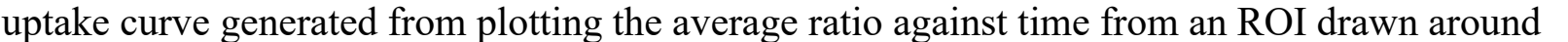

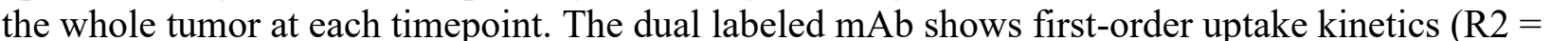

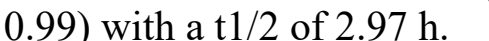

First Name: $\square \square\|\sqcap\| \|$

Last Name: $\square \square\|\| \square \mid \Pi$

Email: $\square \square\|\| \square \square \square\|ा\| \square$

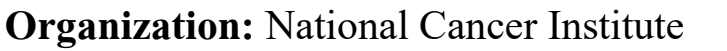

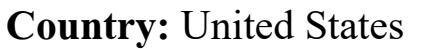




\title{
ID: LB52 \\ Automated Segmentation and Quantitation of PET/CT Images Using 124I-p5+14 (AT-01) in Patients with AL and ATTR Amyloidosis
}

\author{
Jonathan Wall, University of Tennessee Graduate School of Medicine, jwall@utmck.edu
}

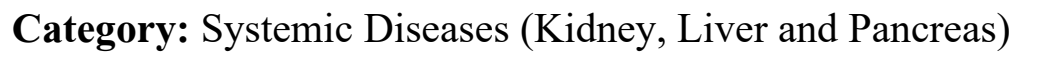

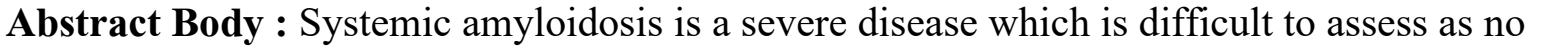

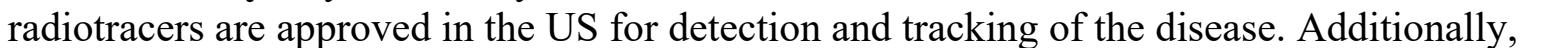

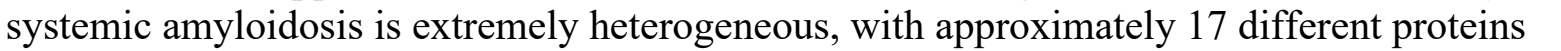

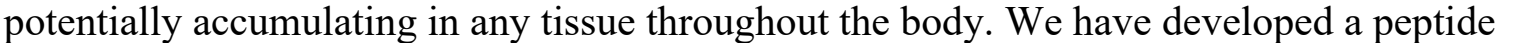

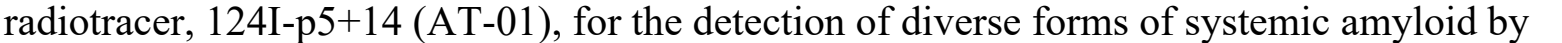

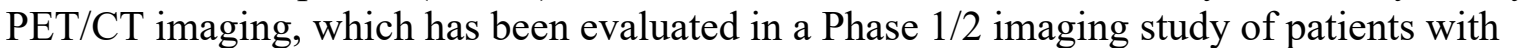

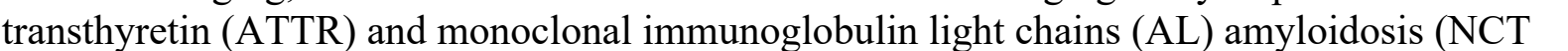

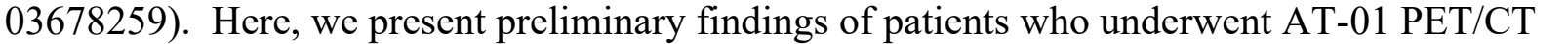
imaging and were evaluated using a recently developed application of AIQ Solutions'

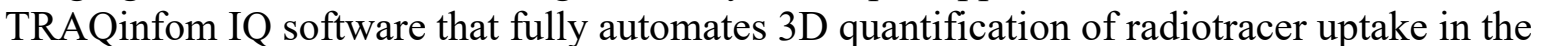

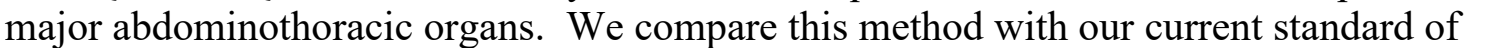

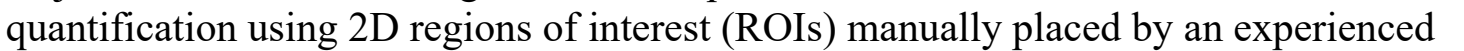

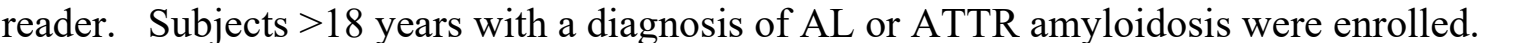

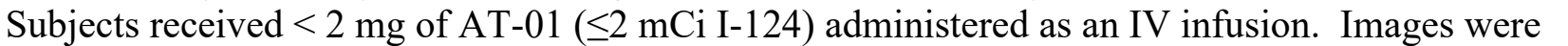

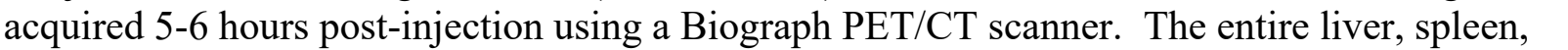

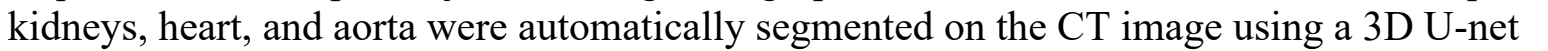

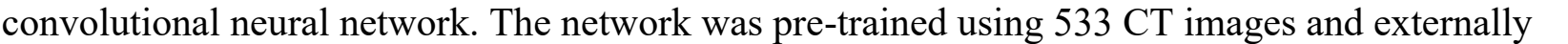

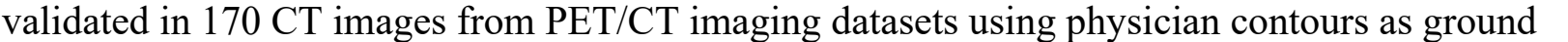

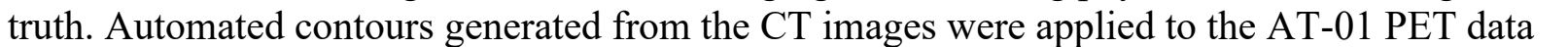

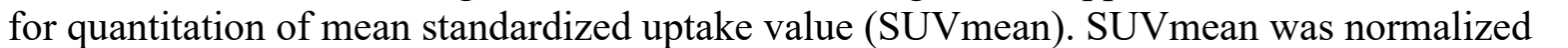

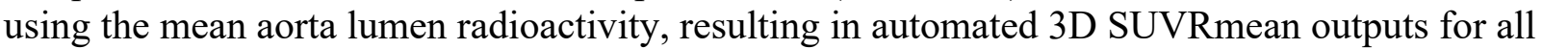

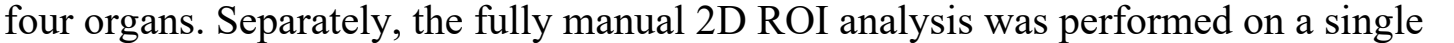

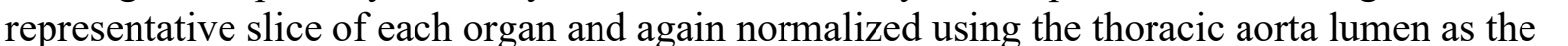

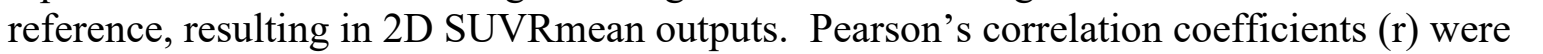

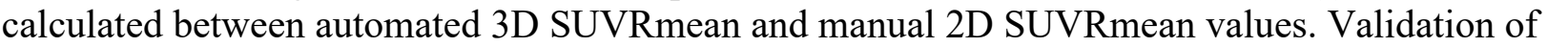

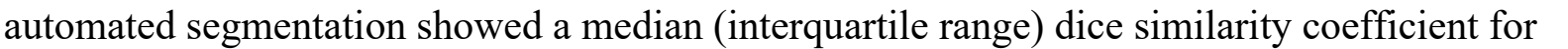

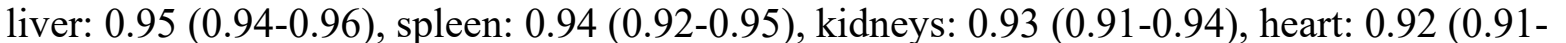

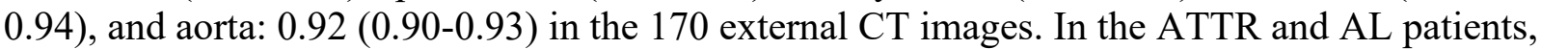

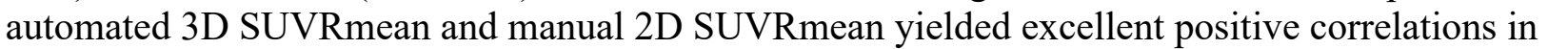

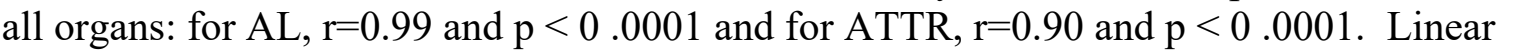

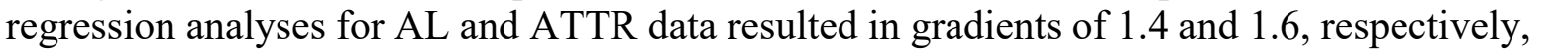

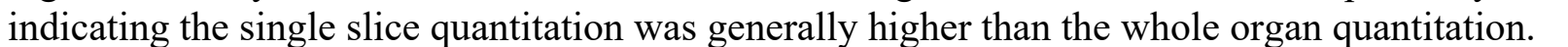

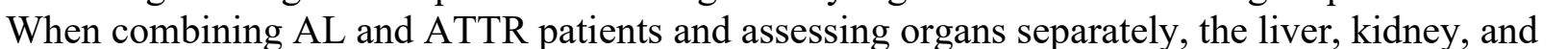

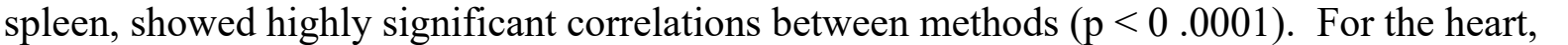

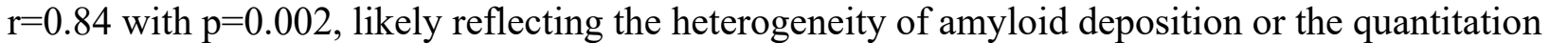

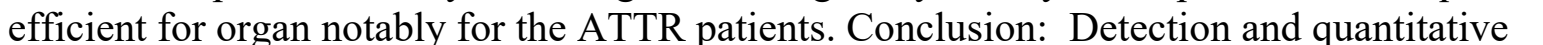




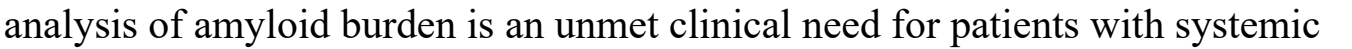

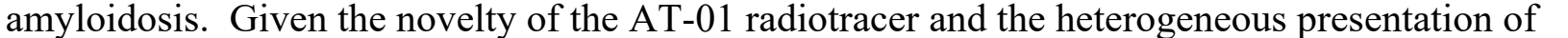

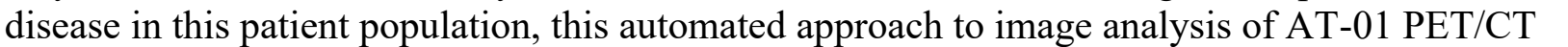

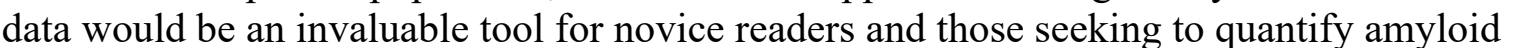

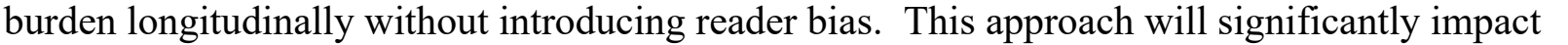

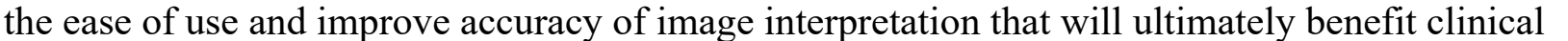

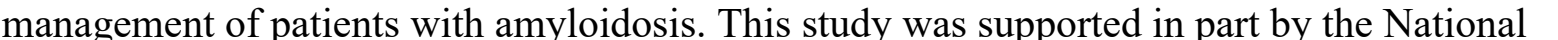

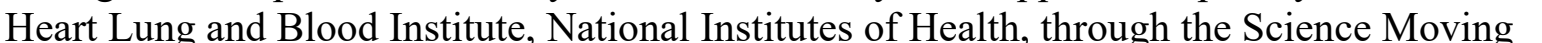

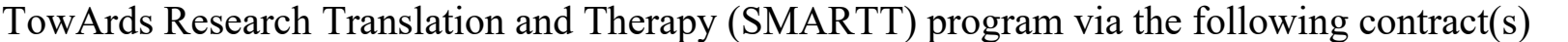

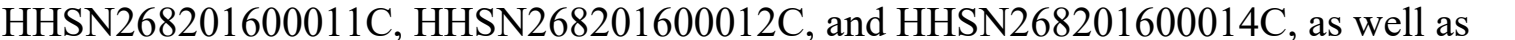

\section{Image/Figure:}

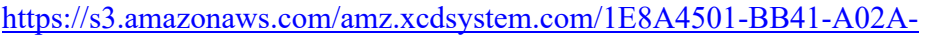

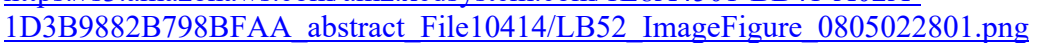

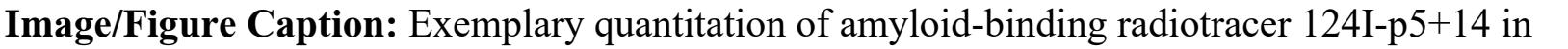

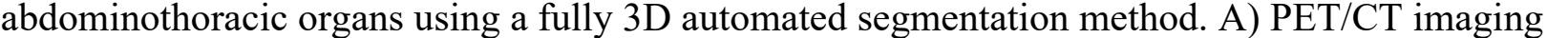

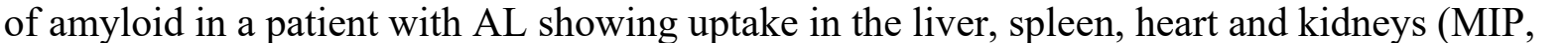

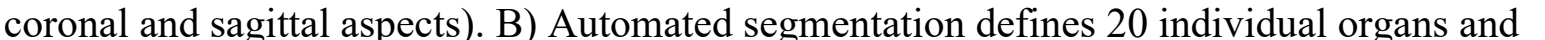

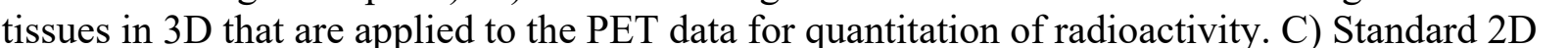
analysis by positioning ROIs on "representative" single slices (arrows). D) Correlation between

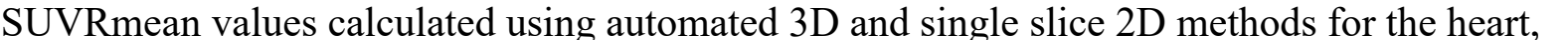

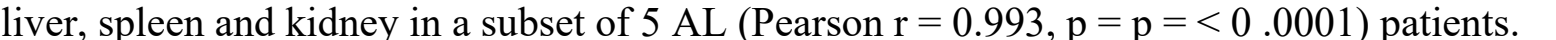

First Name: $\square \square\|\|\|\| \|$

Last Name: $\square \square \square$

Email: $\square \square \square \square \square \square \square \| \square$

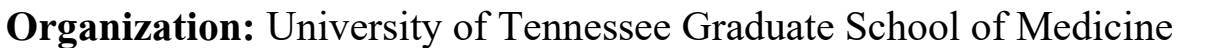

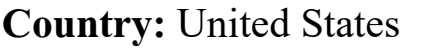




\title{
ID: LB53 \\ Fully Automated Purification of Radiolabeled Macromolecules by Gravity-based Size Exclusion Chromatography.
}

\author{
Serge Lyashchenko, Memorial Sloan Kettering Cancer Center, lyashchs@mskcc.org
}

\section{Category: $\square \square\|\| \| \square$}

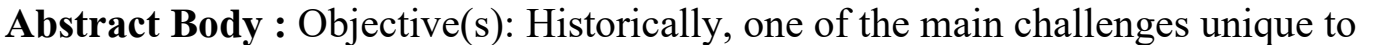

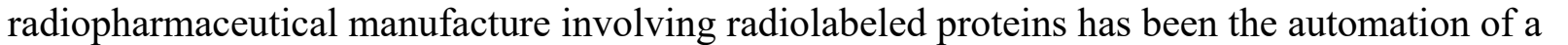
ए

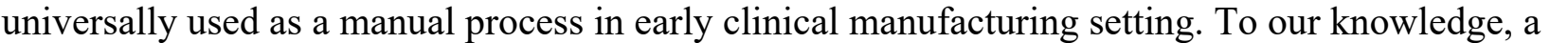
प

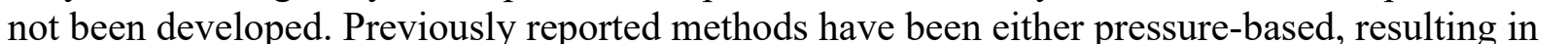

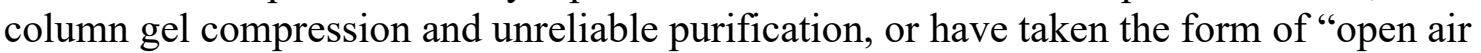
purification column" systems that preclude the collection of isolated eluted product fraction,

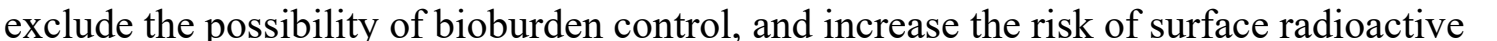

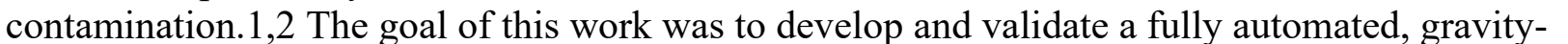

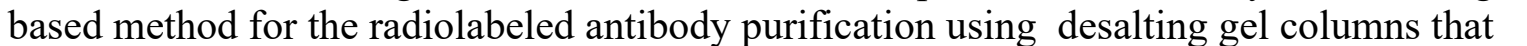

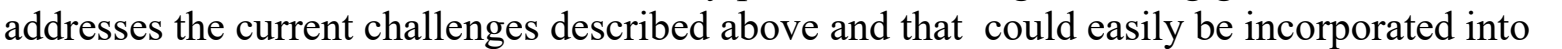

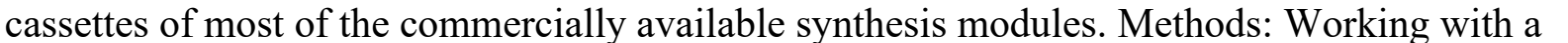

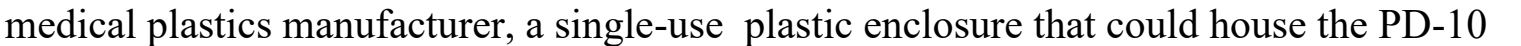

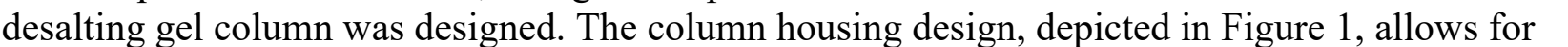

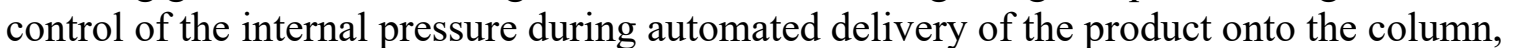

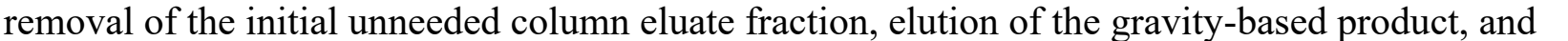

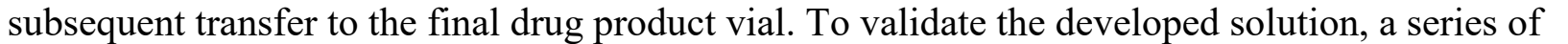

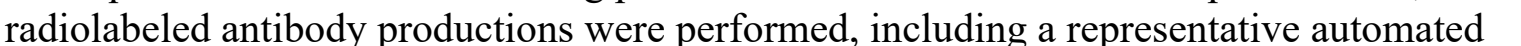

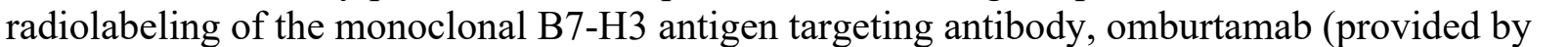

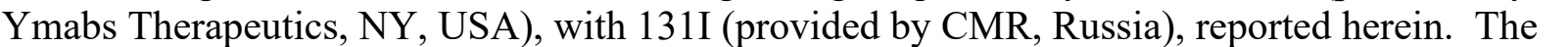

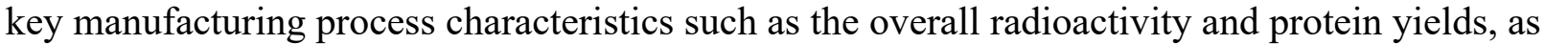

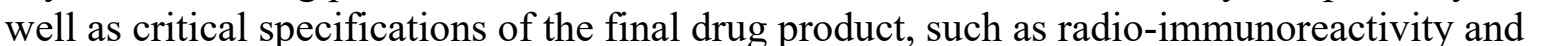

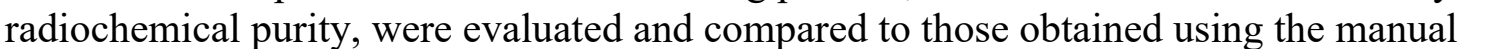

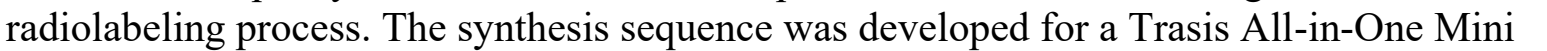

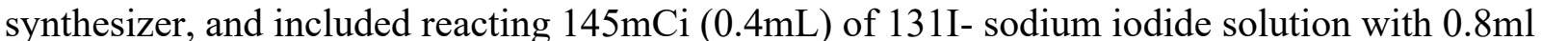

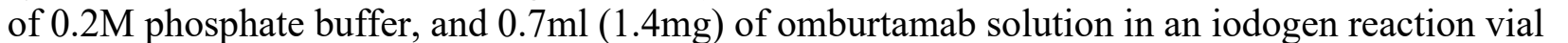

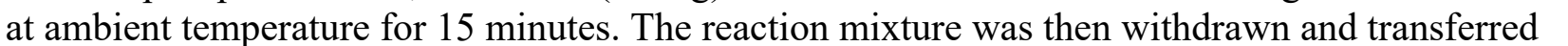

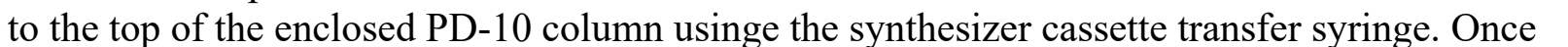

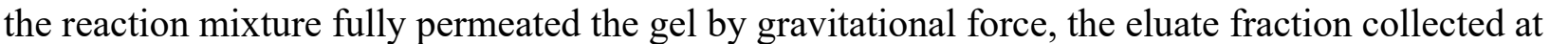
the enclosure's bottom reservoir was pushed into waste by applying air pressure from the transfer

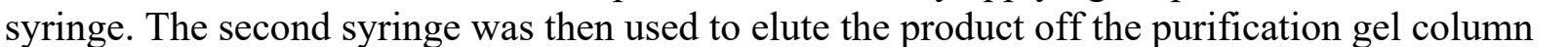

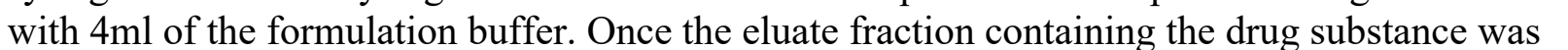
collected at the enclosure's bottom reservoir, syringe pressure was applied to transfer the final

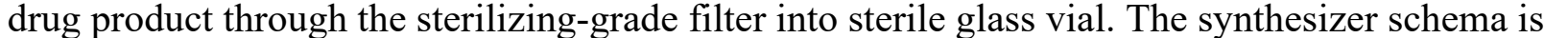

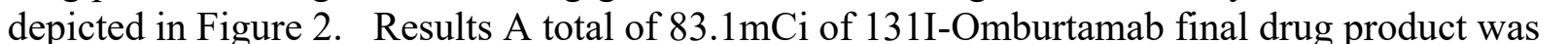




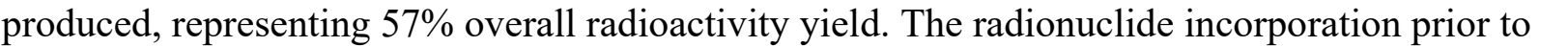

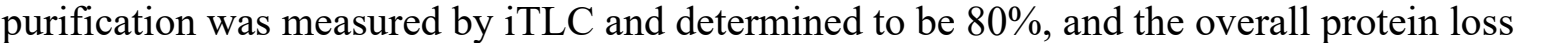

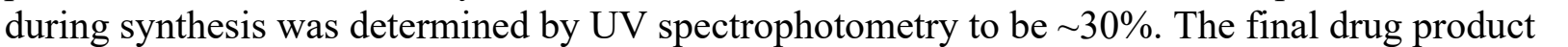

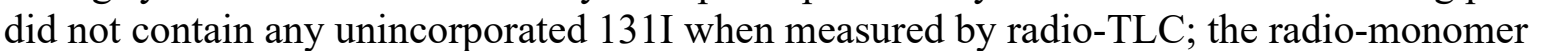

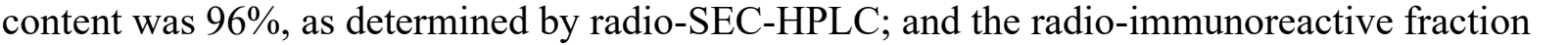

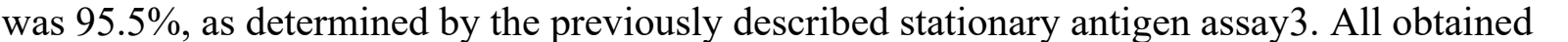

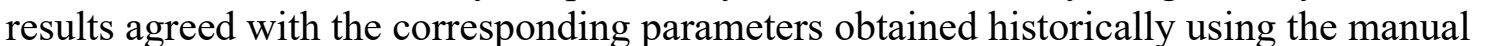

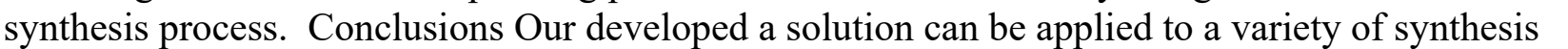

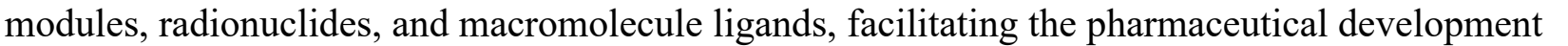

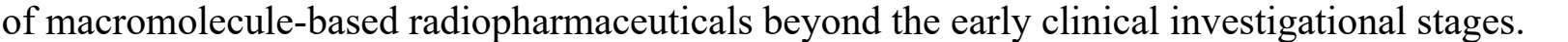

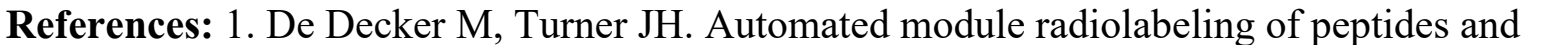

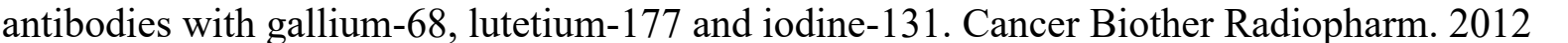

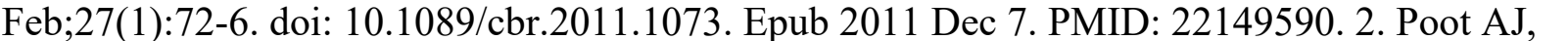

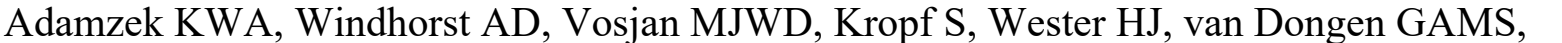

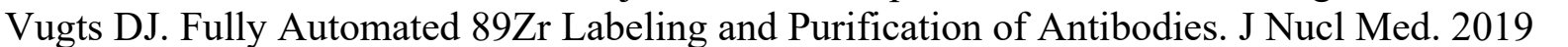

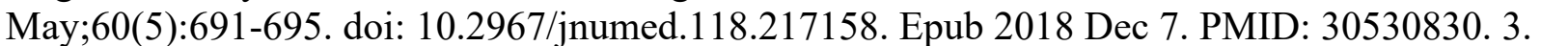

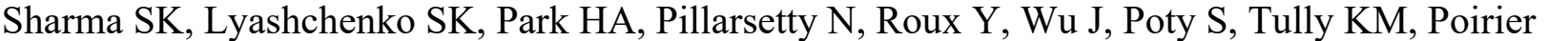

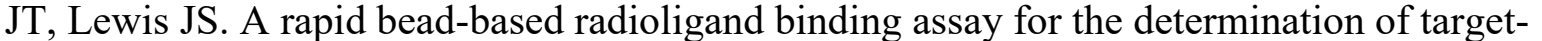

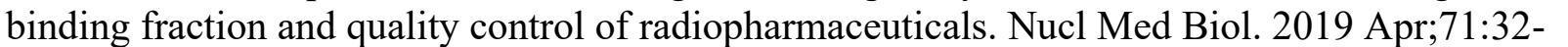

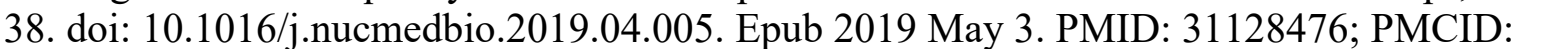

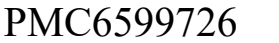

\section{Image/Figure:}

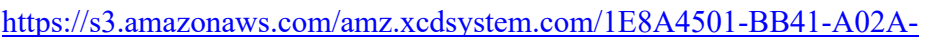

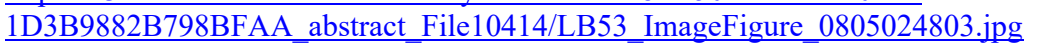

First Name: $\square \square \square|\square| \square$

Last Name: $\square \square \mid\|\|\|\|\|\| \| \square$

Email: $\|\square\|\|\|\|\square \square\|\|\| \| \square$

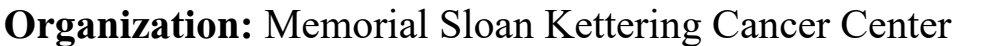

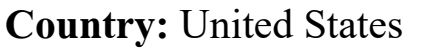




\title{
ID: LB54 \\ Characterization of Human Prostate Cancer using Non-Invasive Molecular Imaging
}

Josephine Tan, University of Western Ontario, jodibtan@gmail.com

Category: $\square \square \| \square \square ा \square$

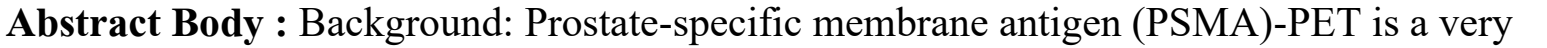

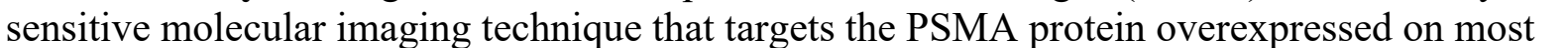

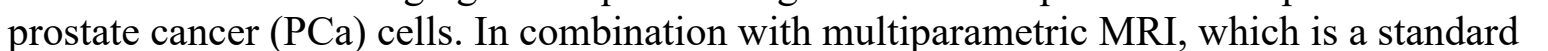

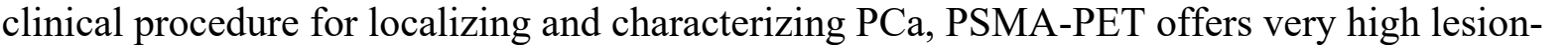

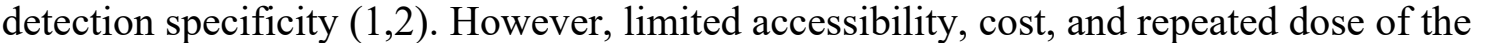

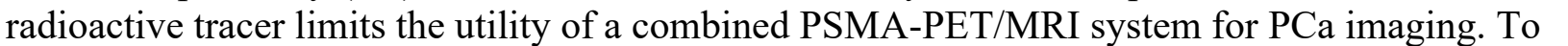

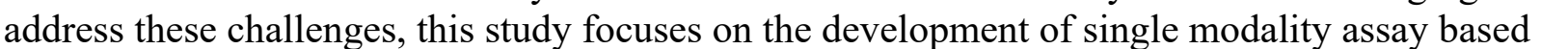

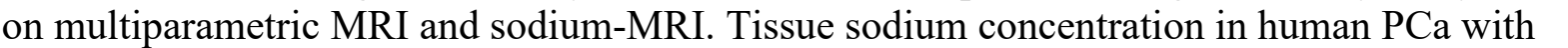

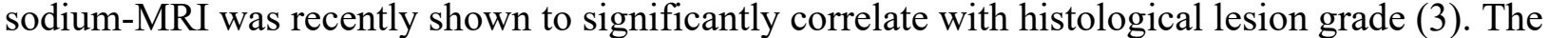

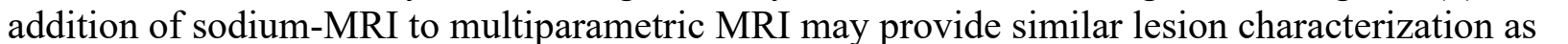

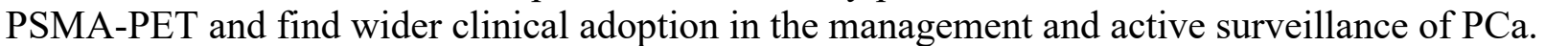

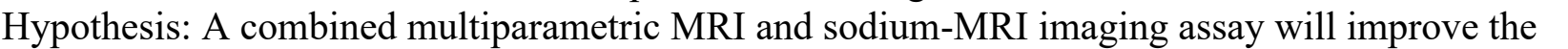

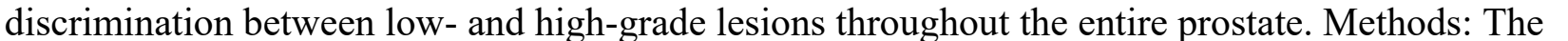

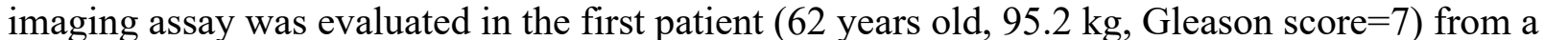

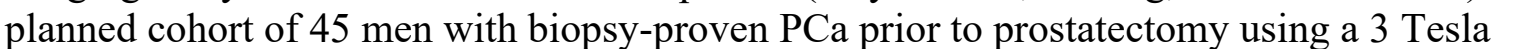

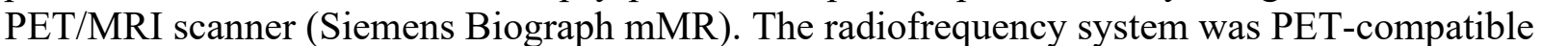

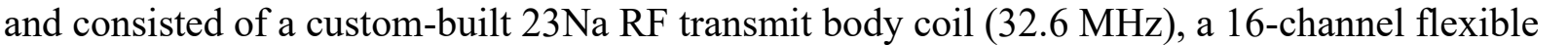

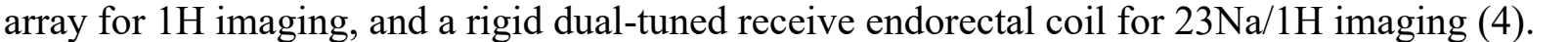

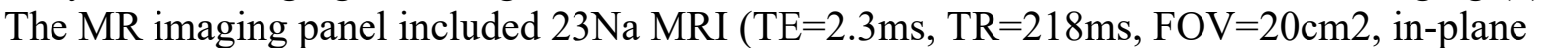
resolution $=3 \mathrm{~mm} 2$, flip angle $=50^{\circ}$, scan time $=32 \mathrm{~min}$ ), followed by $\mathrm{T} 2 \square \square \square \mathrm{Wl} \square \square \square$

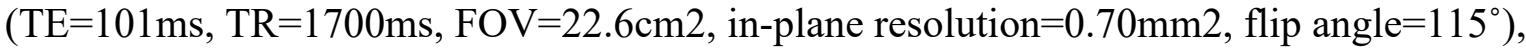

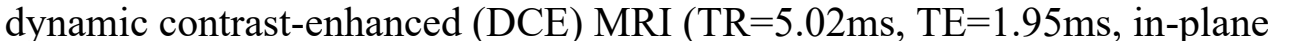

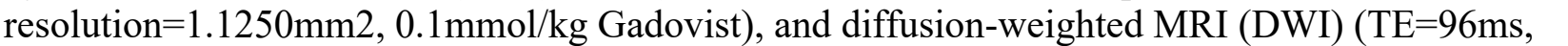

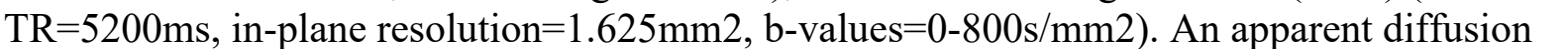

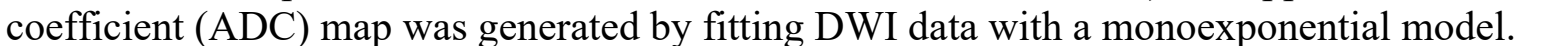
$\square \square \square \square$ PET (3D mode, resolution=4.1725 $\times 4.1725 \times 2.0313 \mathrm{~mm} 3$, frame duration=20min) was

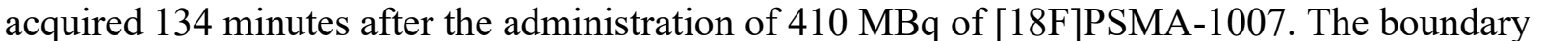

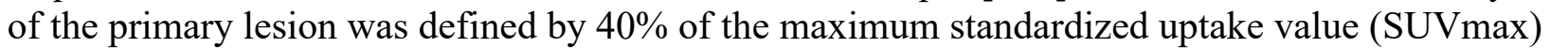

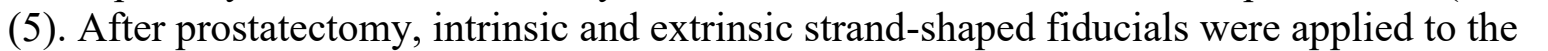

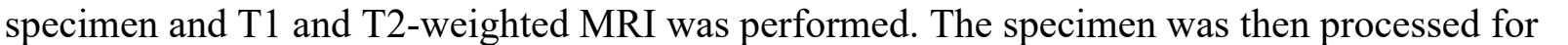

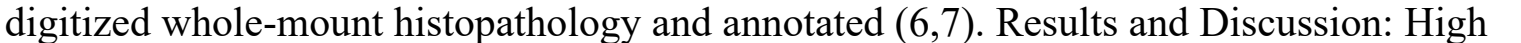

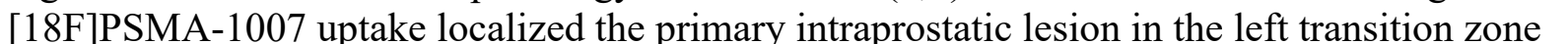

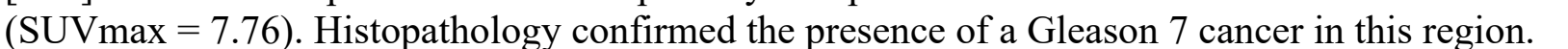

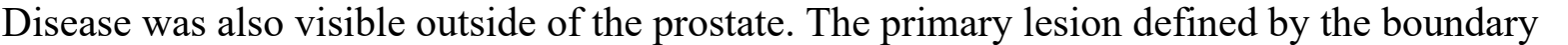

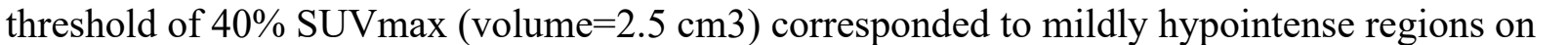
एा
\end{abstract}




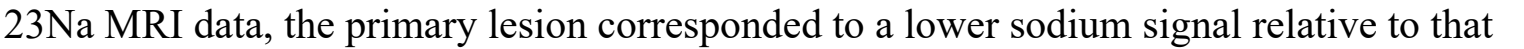

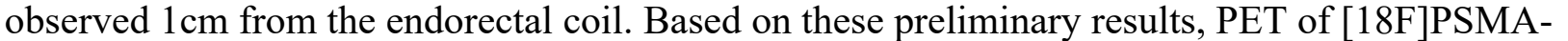

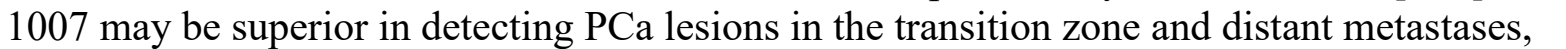

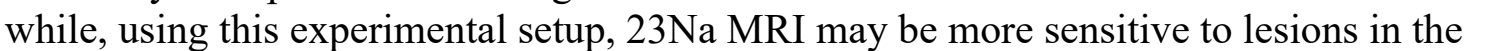

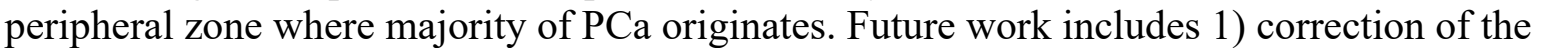

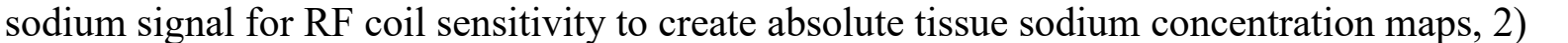

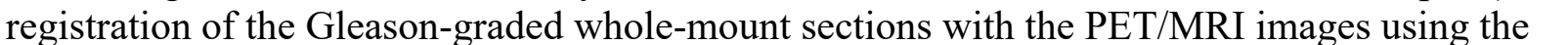

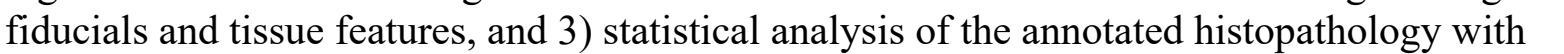

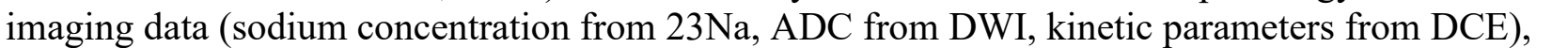

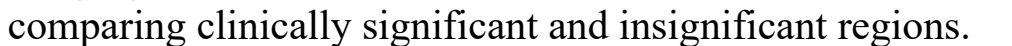

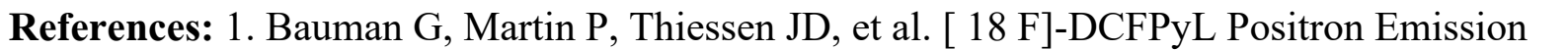

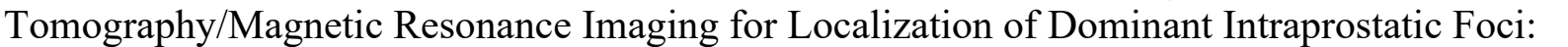

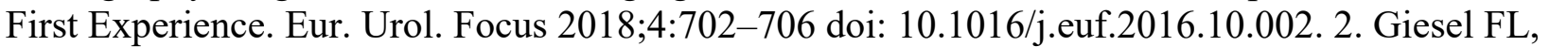

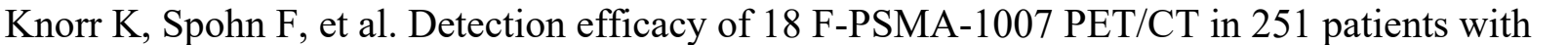

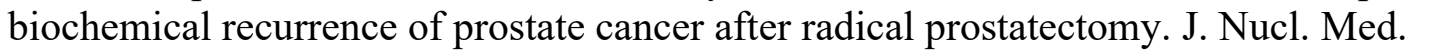

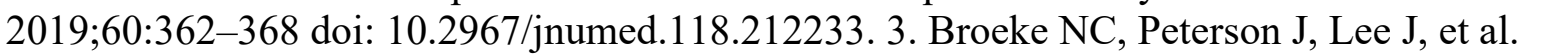

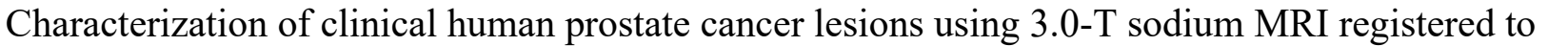

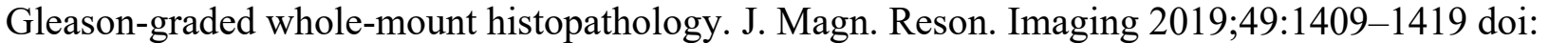

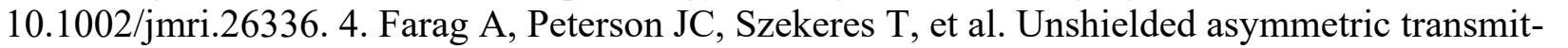

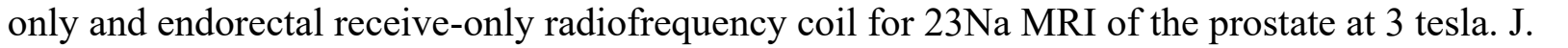

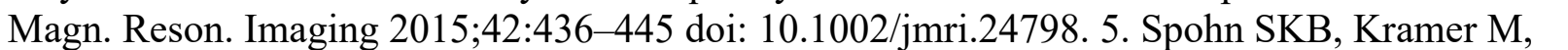

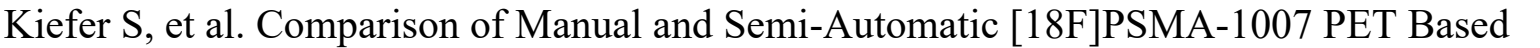

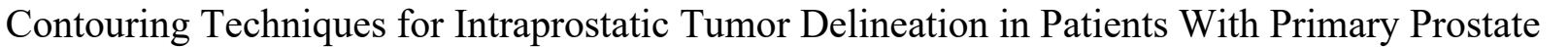

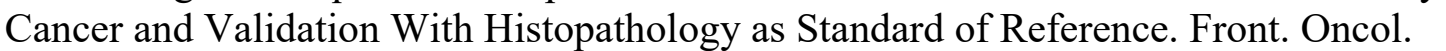

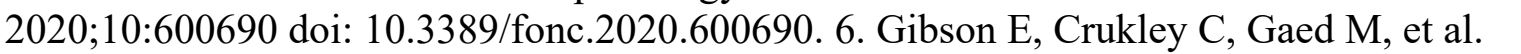

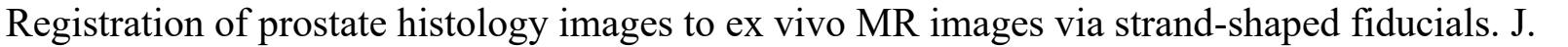

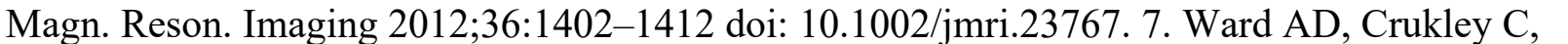

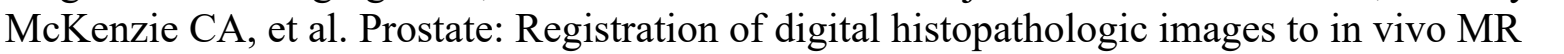

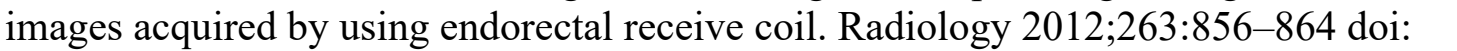

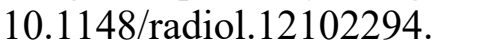

\section{Image/Figure:}

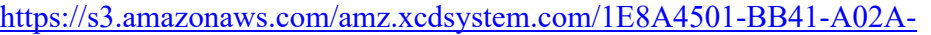

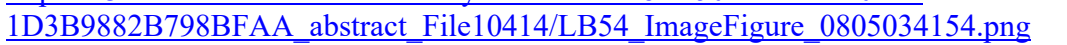

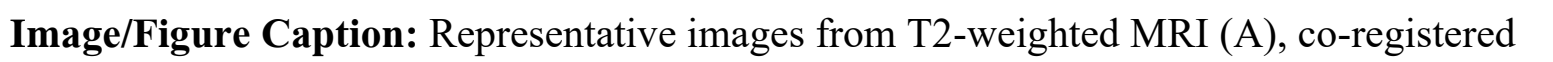

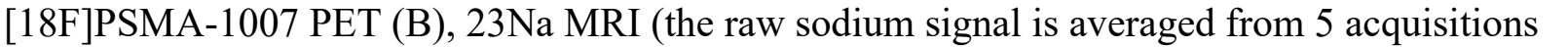

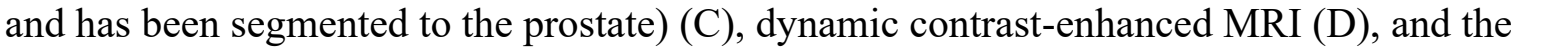

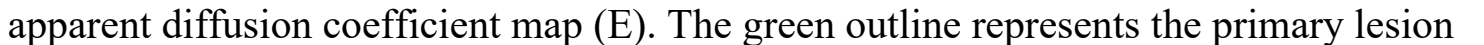

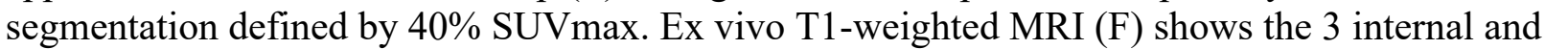

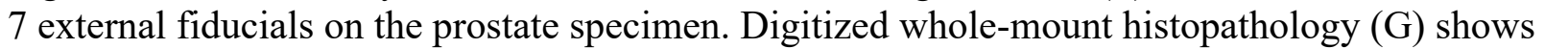

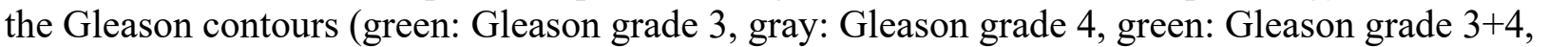
एणी

First Name: $\square \square\|\|\|\| \square \| \square$ 
Last Name:

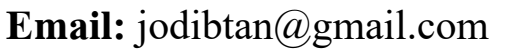

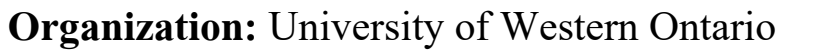

Country: $\square \square \square\|\| \square$ 


\title{
ID: LB55 \\ Evaluation of gender differences in the uptake of PET probes 2-[18F]A85380 and [18F]nifene for nicotinic acetylcholine receptors in vivo
}

\author{
Matthew Zammit, University of Chicago, mzammit@uchicago.edu
}

\section{Category: $\square \square \square\|\square\| ा\|\square\| ा \square$}

\begin{abstract}
Body :
radioligands that bind with high affinity to the $\alpha 4 \beta 2$ subtype of nicotinic acetylcholine receptors

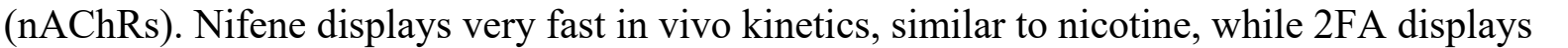

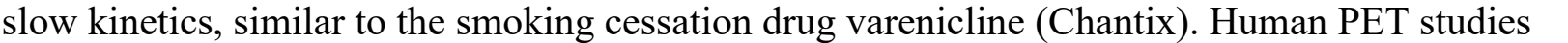

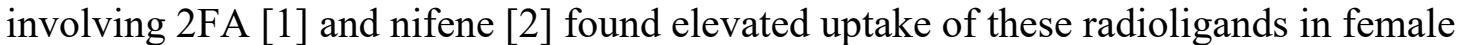

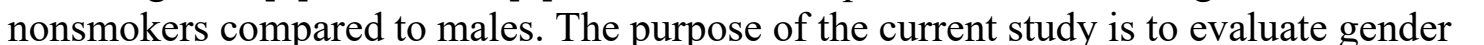

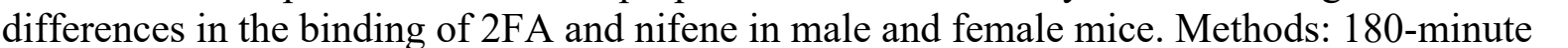
dynamic 2FA PET scans were performed on wild type (5M/5F), $\beta 2$ nAChR knockout (8M/3F),

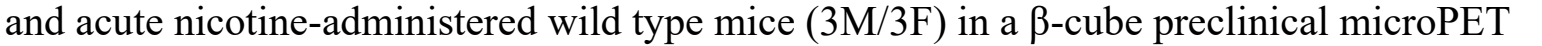

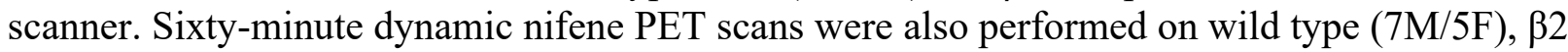

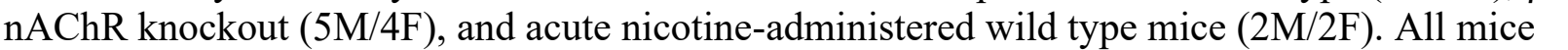

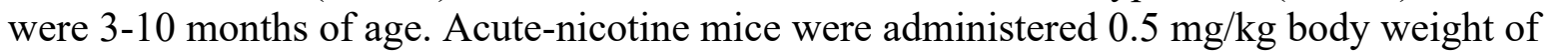

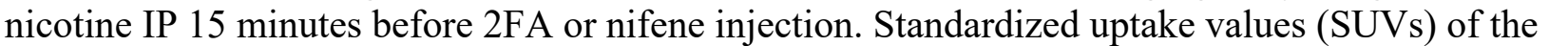

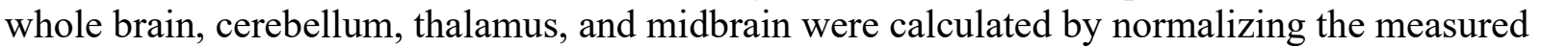
प

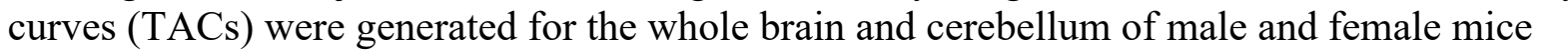

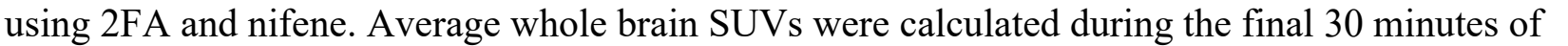

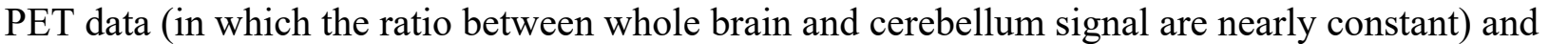

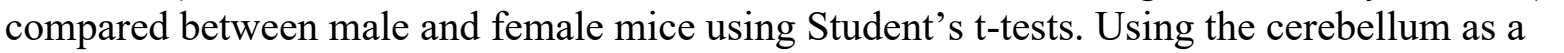

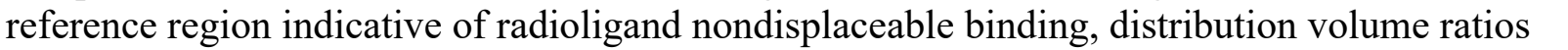

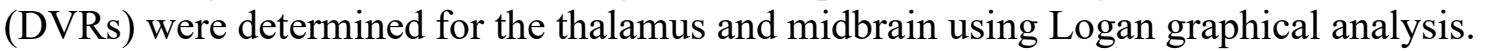

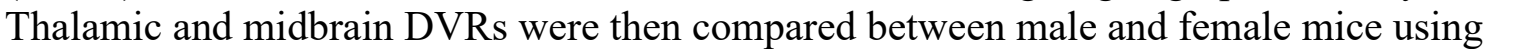

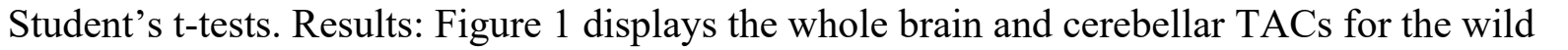

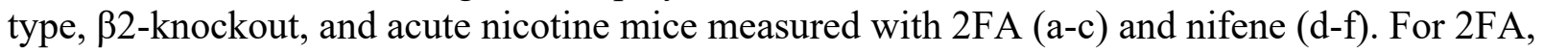

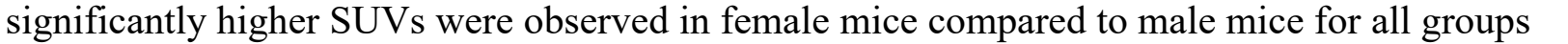

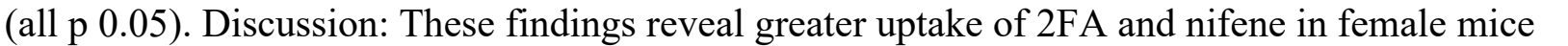
compared to males. Uptake of 2FA and nifene were completely abolished in the $\beta 2 \square \square \square \square \square$

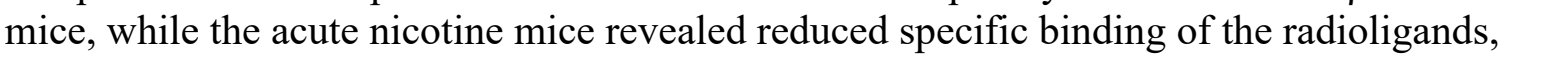
पा

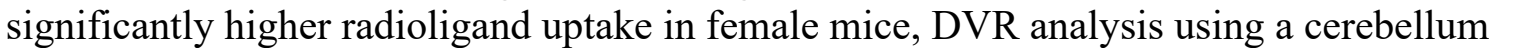

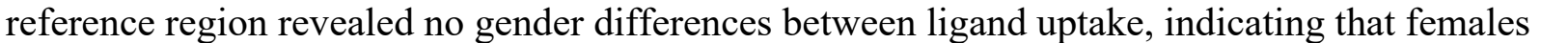

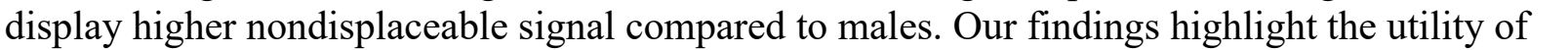
PET to monitor $\alpha 4 \beta 2$ ligand trapping in vivo for the application of identifying gender differences

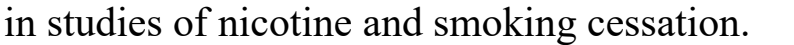




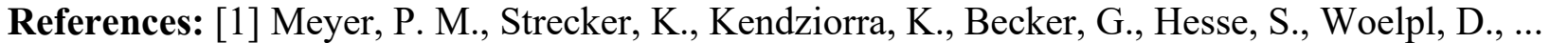

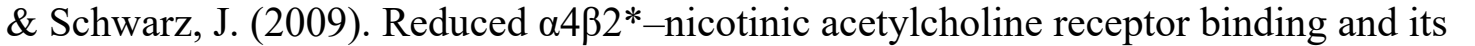

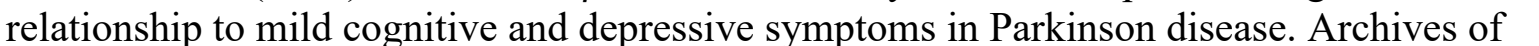
प

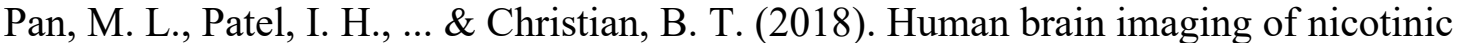
acetylcholine $\alpha 4 \beta 2 *$ receptors using [18F]Nifene: Selectivity, functional activity, toxicity, aging

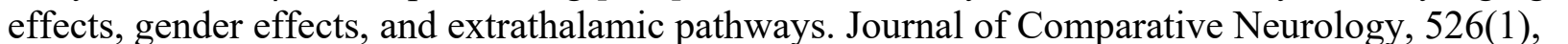
$\square \mid\|1\|$

\section{Image/Figure:}

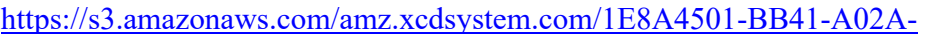

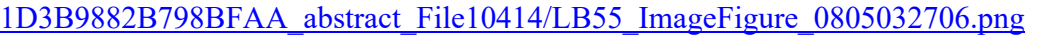

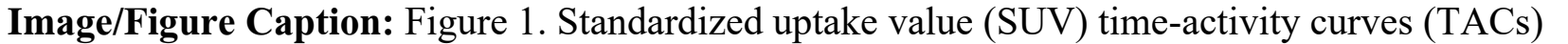

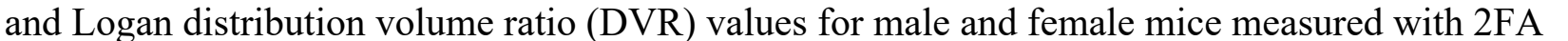

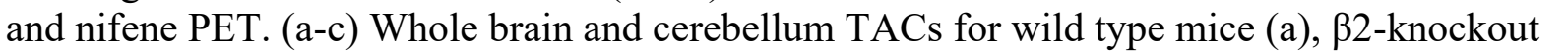

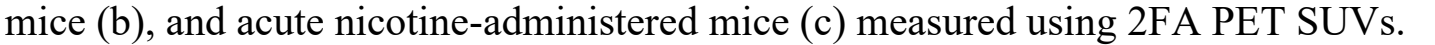

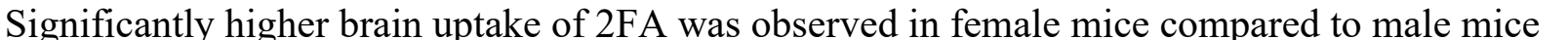

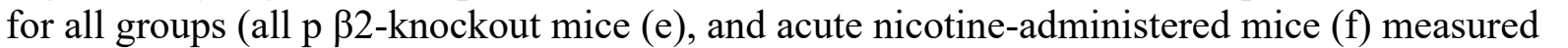

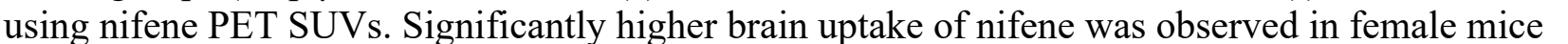

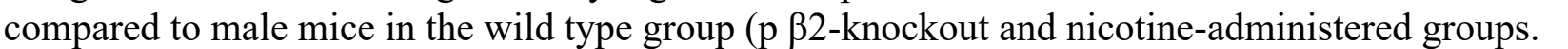

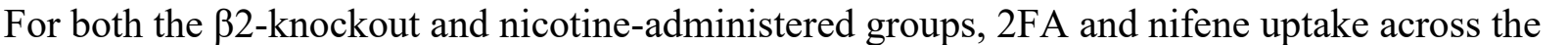

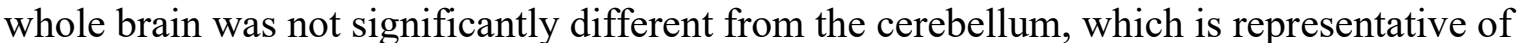

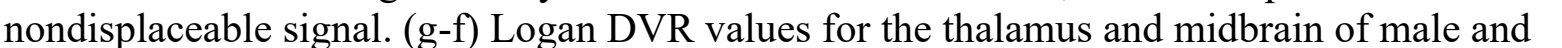

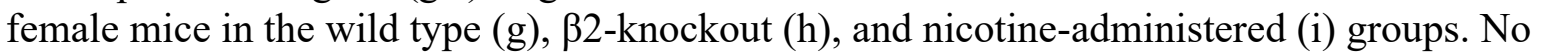

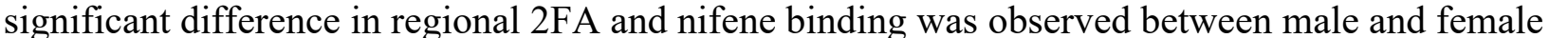

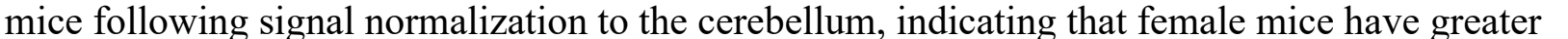

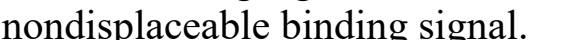

First Name: $\square \square \| \square \square \square$

Last Name: $\square \square \square \square(m$

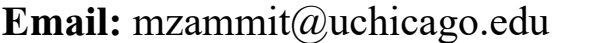

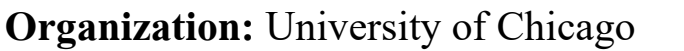

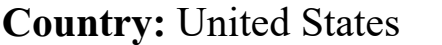




\title{
ID: LB56 \\ Identification of a panel of aberrantly expressed microRNAs in Triple Negative Breast Cancer and its therapeutic evaluation in combination chemotherapy
}

Negar Sadeghipour, Stanford University, sadeghi@stanford.edu

\section{Category: $\square \square\|\| \| \square$}

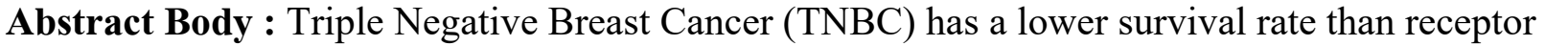

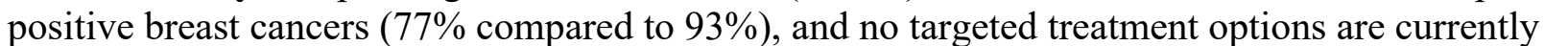

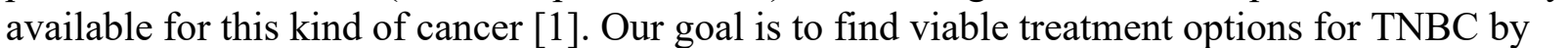

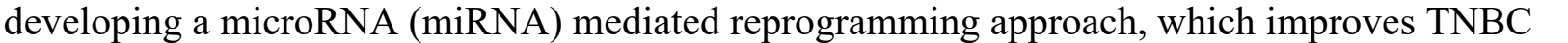

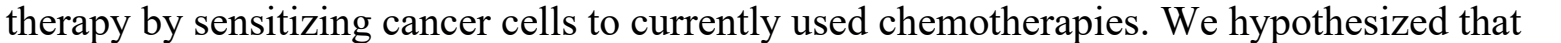

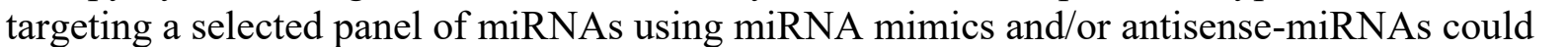

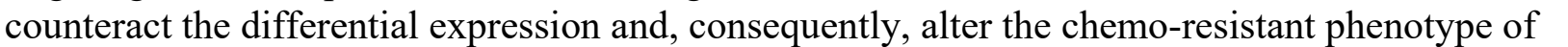

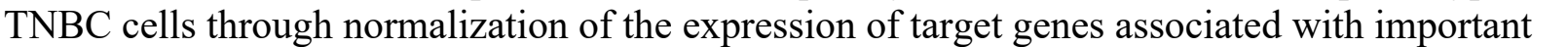

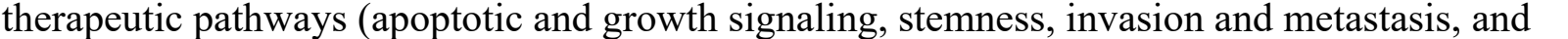

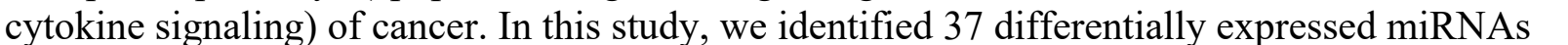

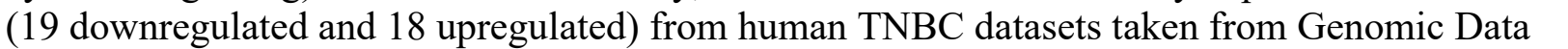

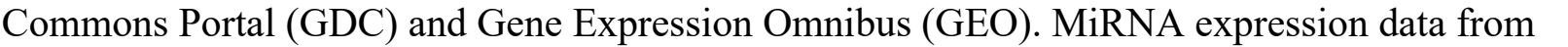

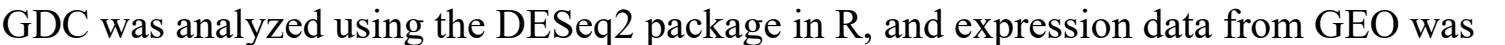

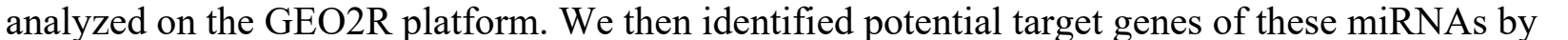

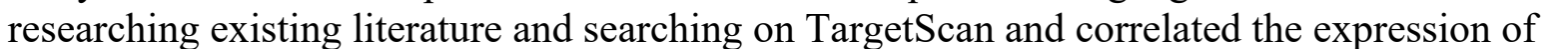

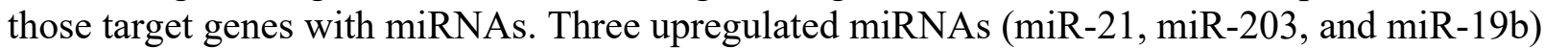

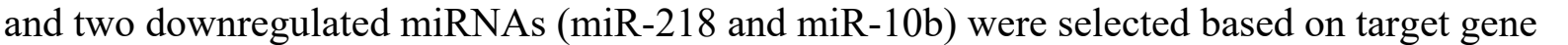

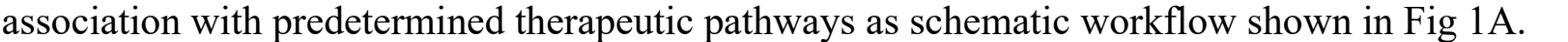

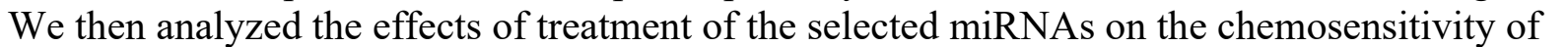

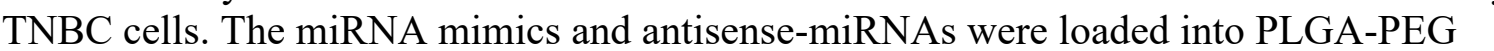

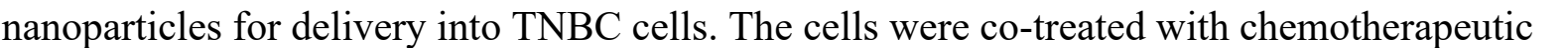

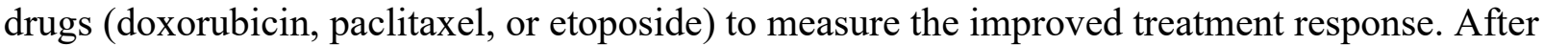

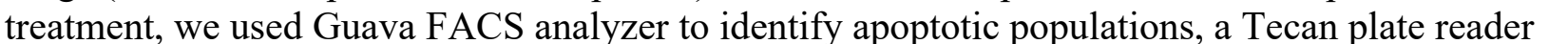

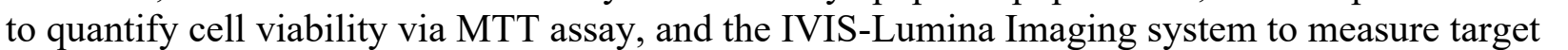

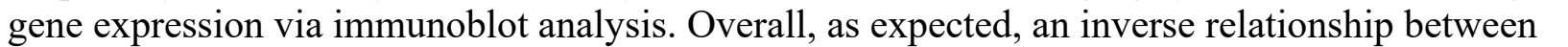

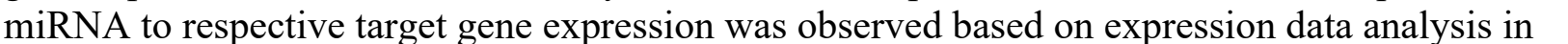

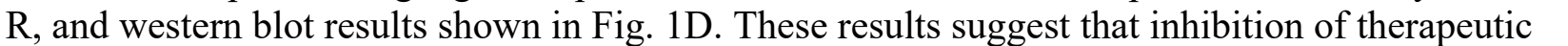

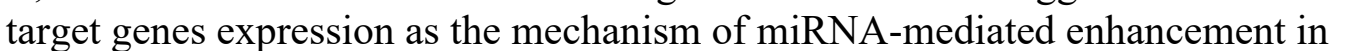

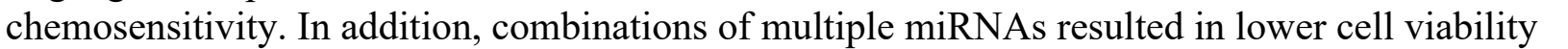

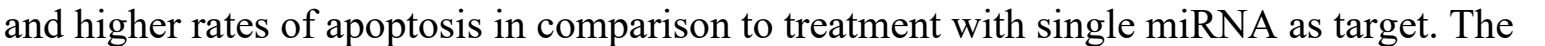

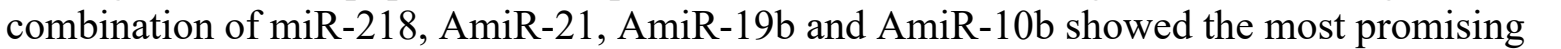

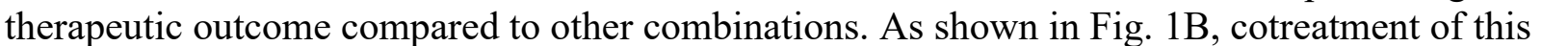

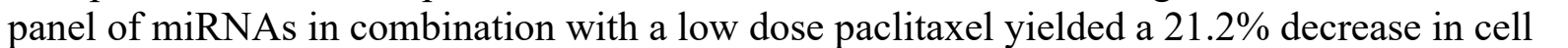

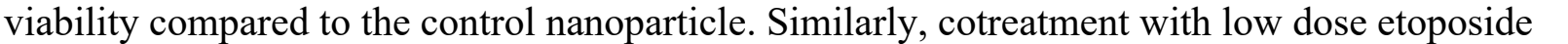
yielded a $19.9 \%$ decrease in cell viability. Cotreatment of this combination with $0.5 \mu \mathrm{M}$ of Do $\square$

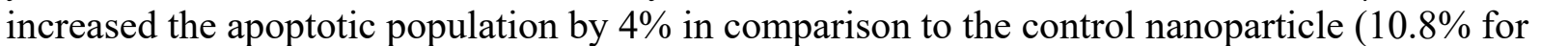




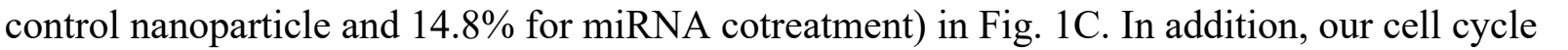

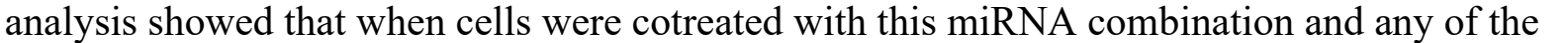

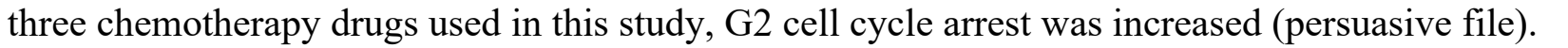

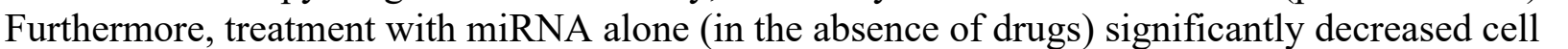

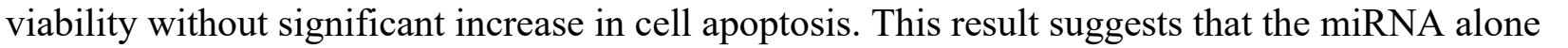
ए

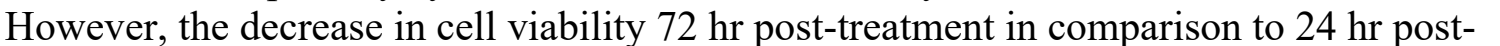

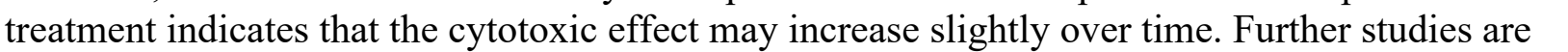

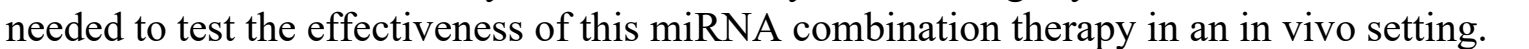

References: 1. Lehmann BD, Jovanović B, Chen X, Estrada MV, Johnson KN, Shyr Y, $\square \square \| \square$

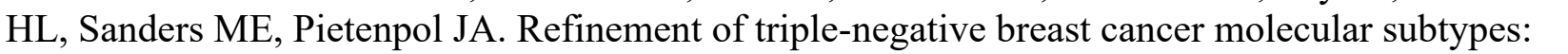

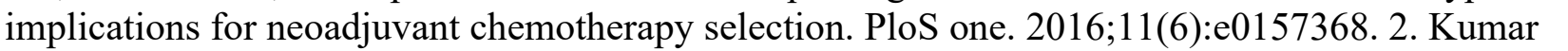

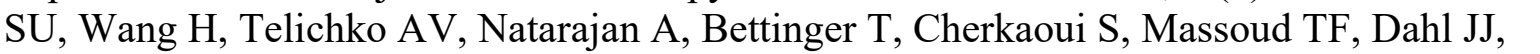

Paulmurugan R. Ultrasound Triggered Co-Delivery of Therapeutic MicroRNAs and a Triple

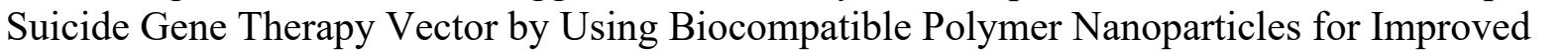

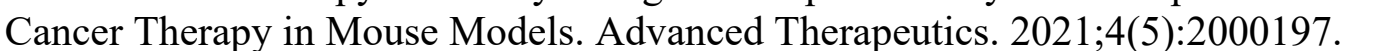

\section{Image/Figure: $\square$}

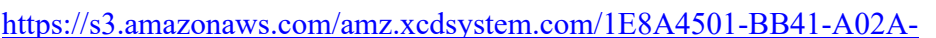

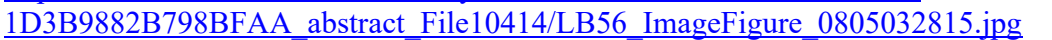

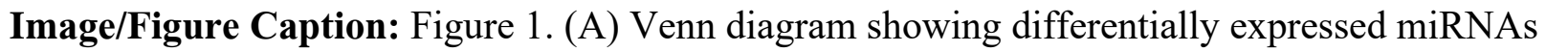

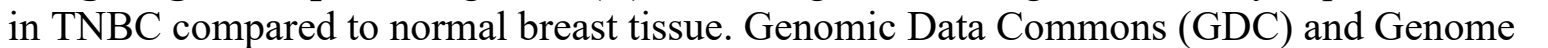

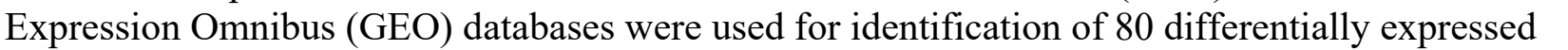

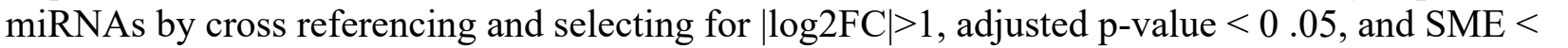

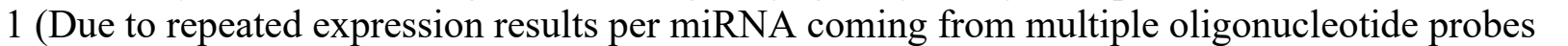

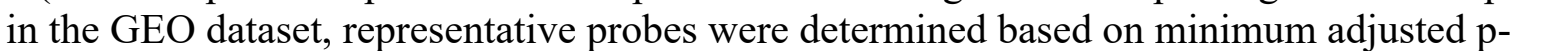

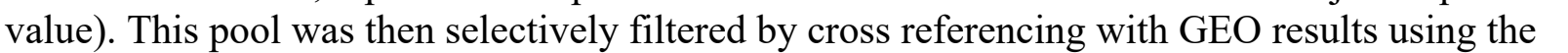

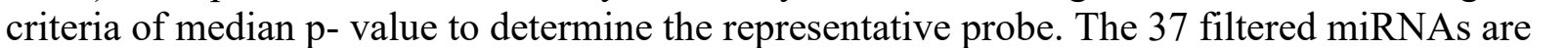
presented in a Venn diagram of various therapeutic pathways. The miRNAs labeled "Not Identified" lacked sufficient studies and could not be successfully categorized into any of the

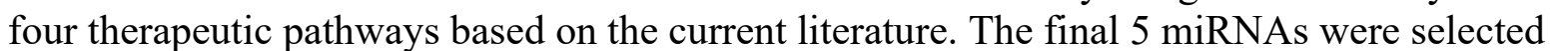

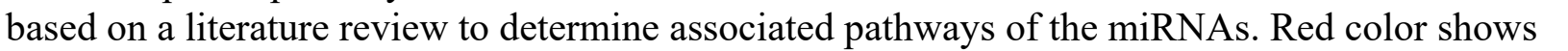
ए

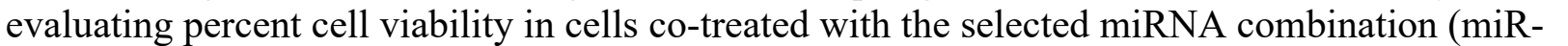

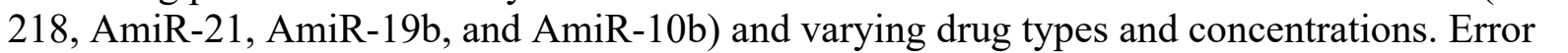

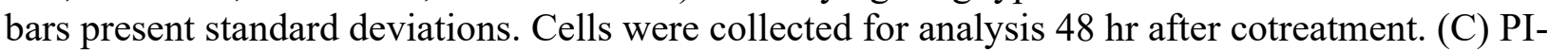

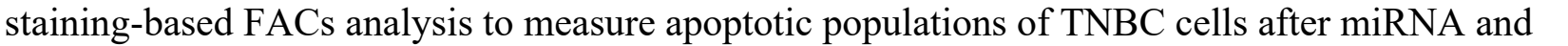

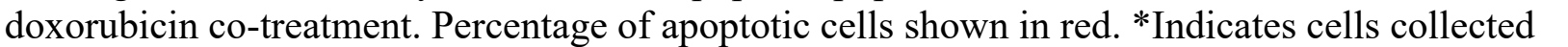

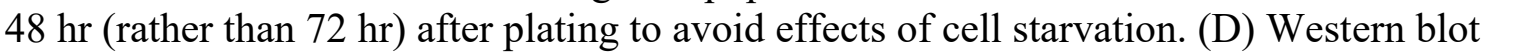

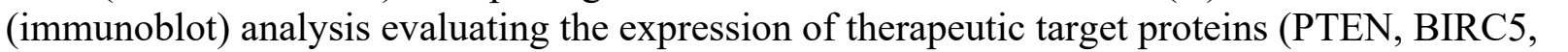

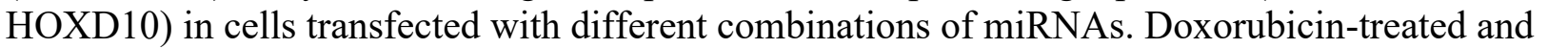

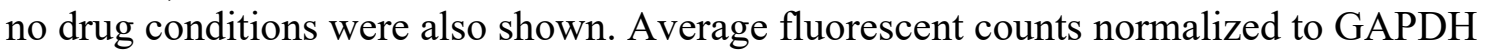

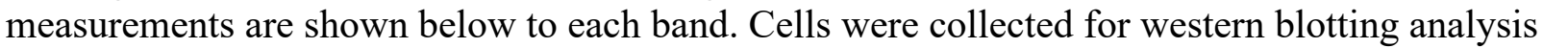

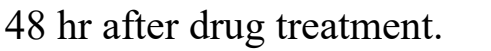


First Name:

Last Name:

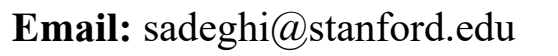

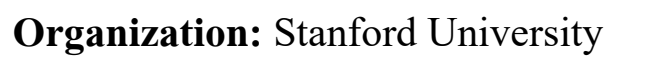

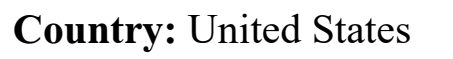




\title{
ID: LB57 \\ Preclinical evaluation of a series of PSMA-617-based inhibitors for astatine-211 targeted radionuclide therapy of prostate cancer
}

\author{
Mohamed El Fakiri, Department of Nuclear Medicine, University Medical Center \\ Freiburg, Faculty of Medicine, University of Freiburg, mohamed.el.fakiri@uniklinik- \\ freiburg.de
}

\section{Category: $\square \square\|\| \sqcap \|$}

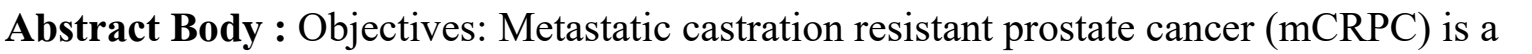

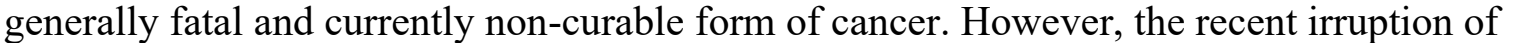

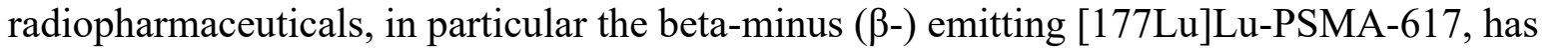

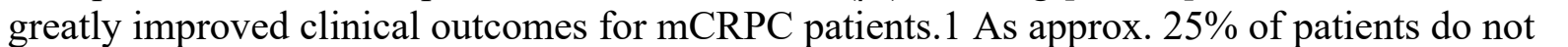

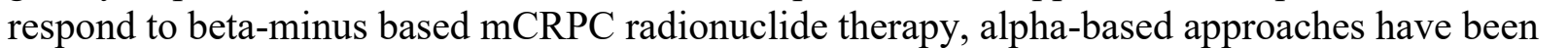

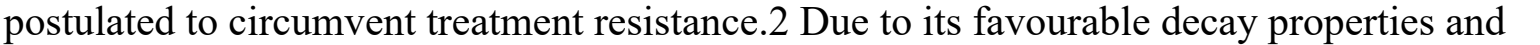

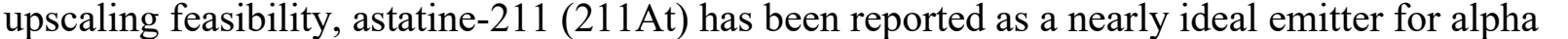

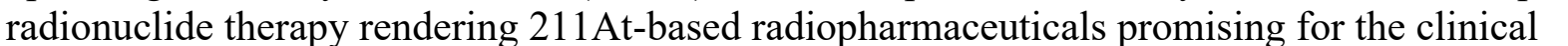

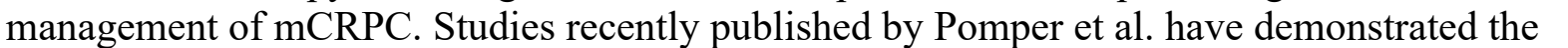

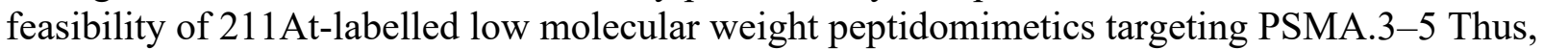
ए

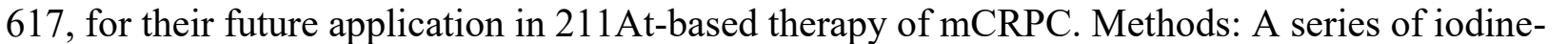

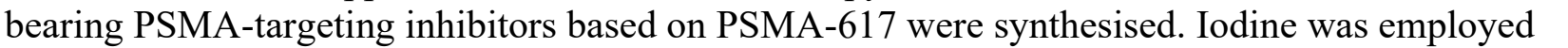

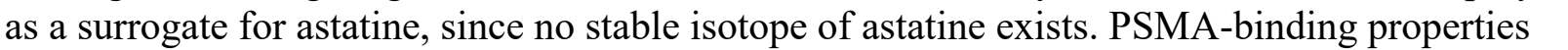

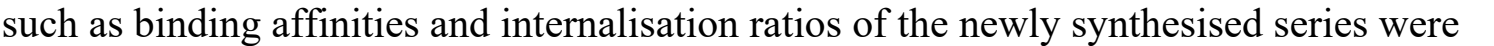

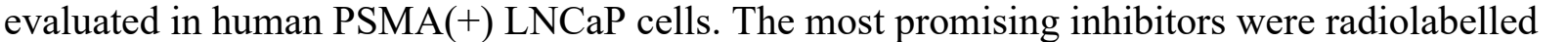

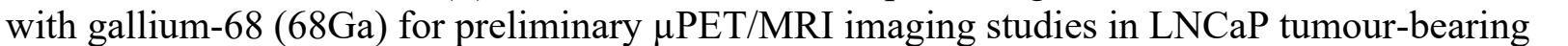

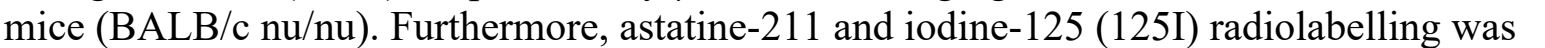

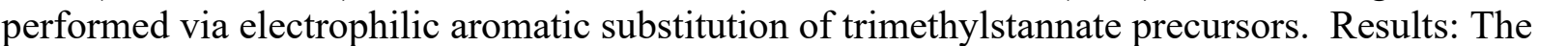

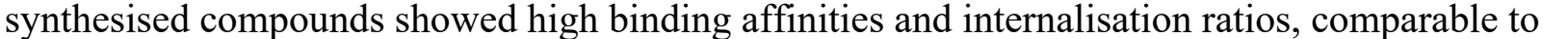

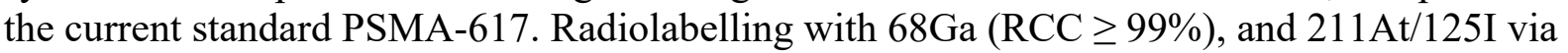

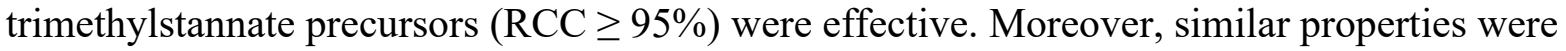

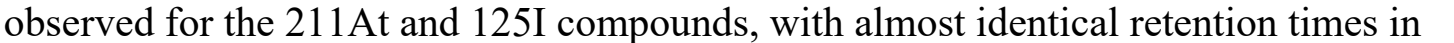

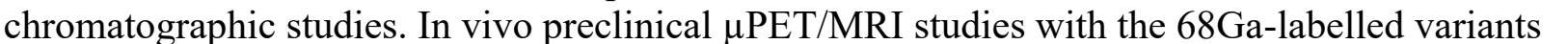

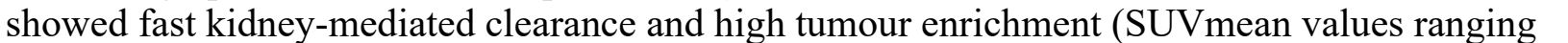

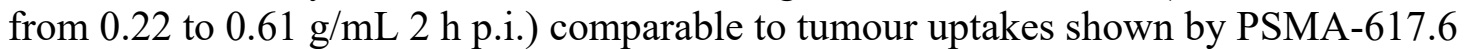

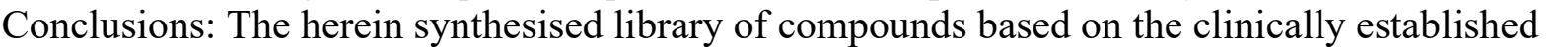

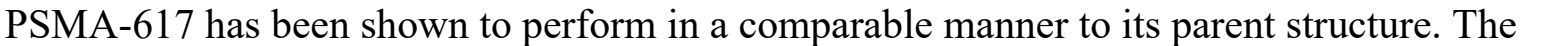

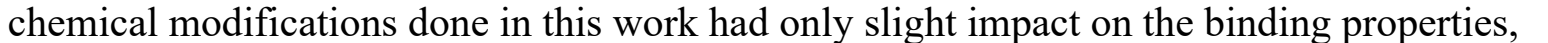

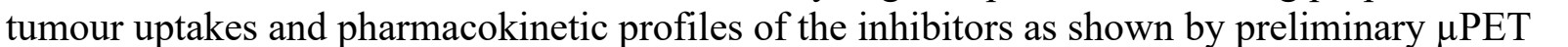

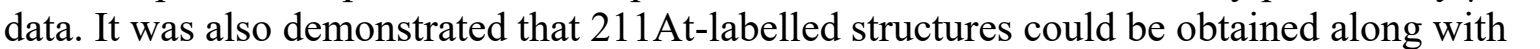

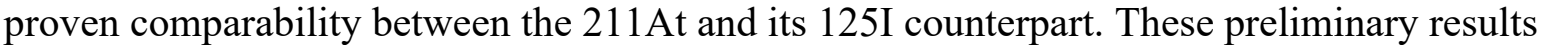

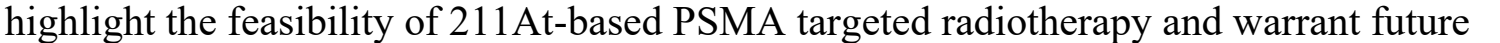

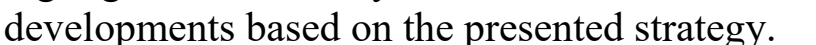




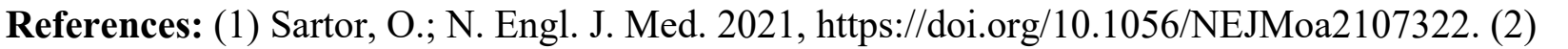

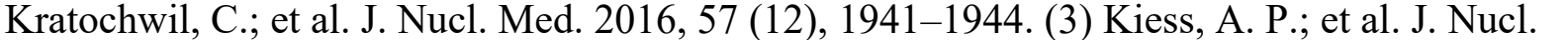

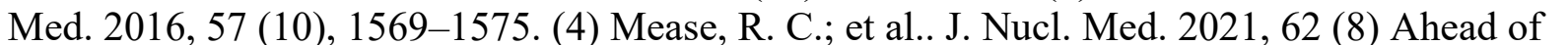

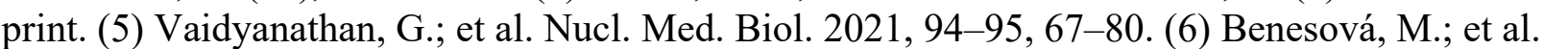

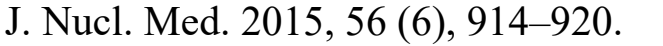

First Name: $\square \square \square \square \square \square \square$

Last Name: $\square\|m \square \square\| m$

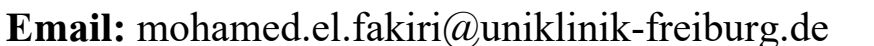

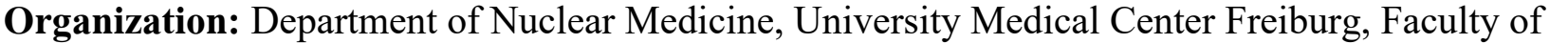

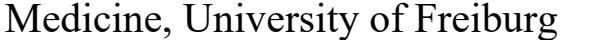

Country: $\square \square \square \square \square \square$ 


\title{
ID: LB58 \\ Imaging brain neuroinflammation in a mouse model of multiple sclerosis using 18F-labeled S1PR1 tracer
}

\author{
Caroline Guglielmetti, University of California San Francisco, \\ caroline.guglielmetti@ucsf.edu
}

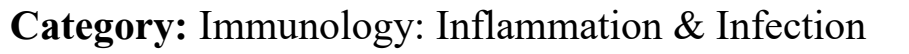

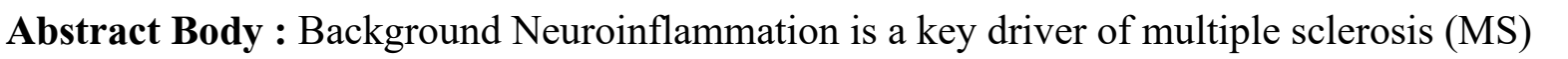

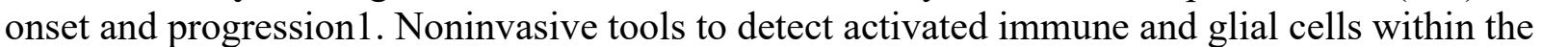

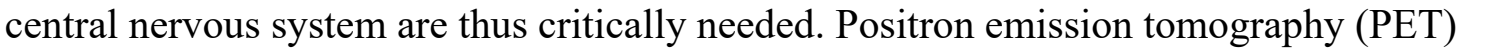

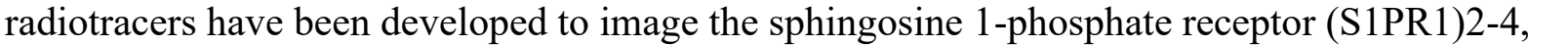

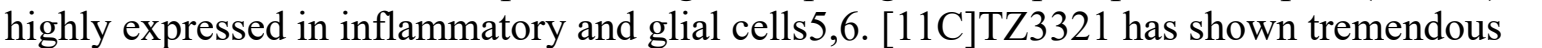

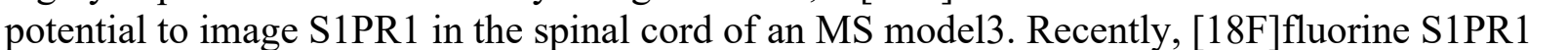

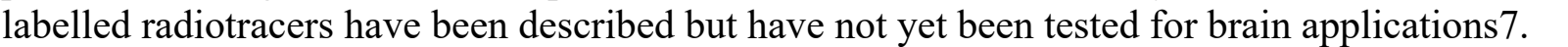

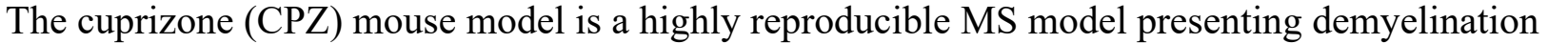

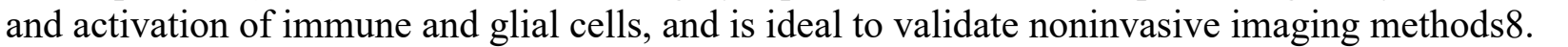

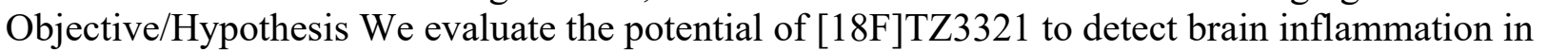

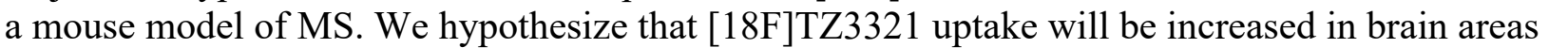

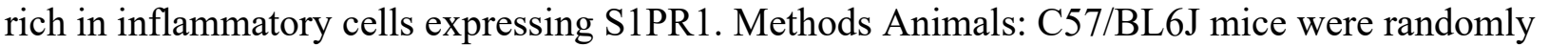

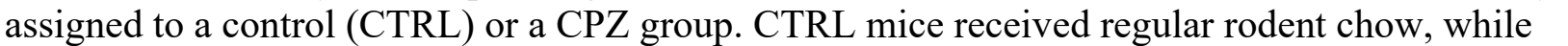

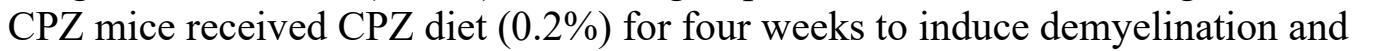

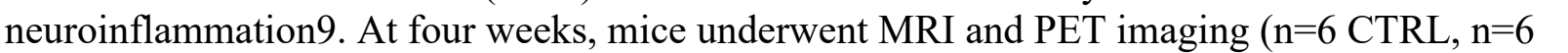

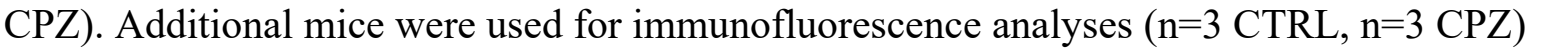

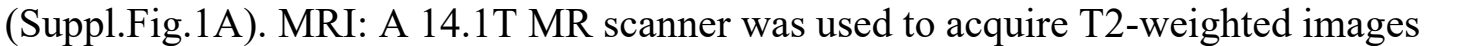

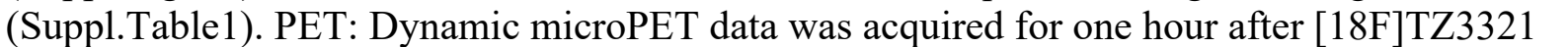

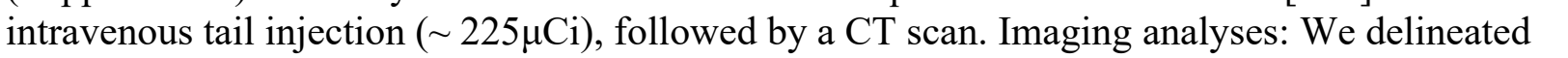

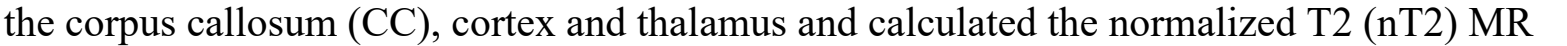

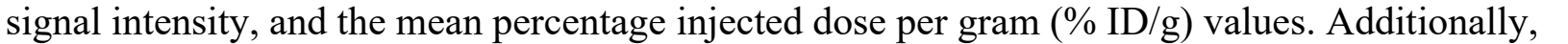

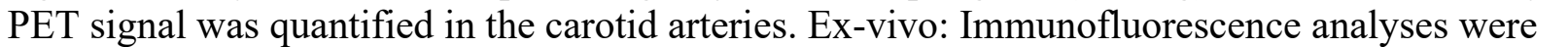

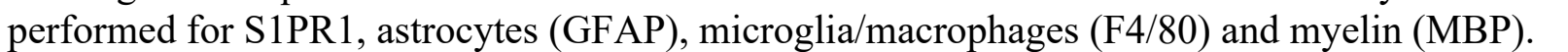

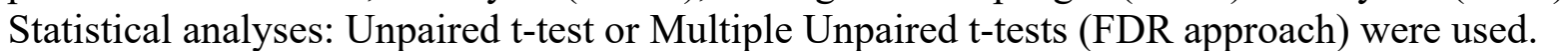

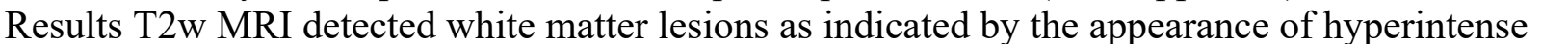

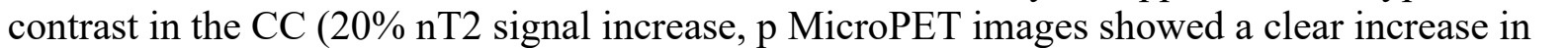

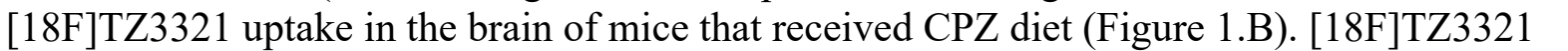

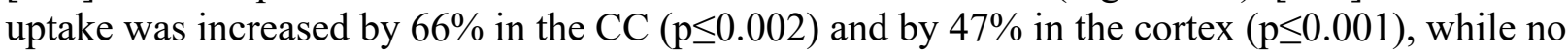

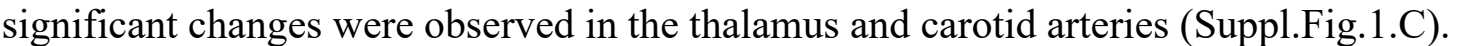

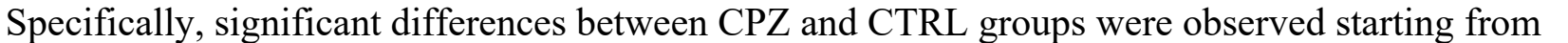

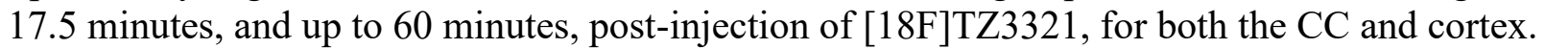

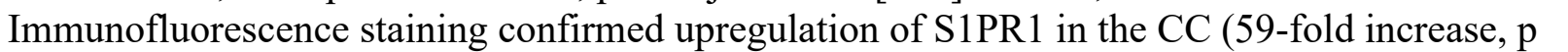

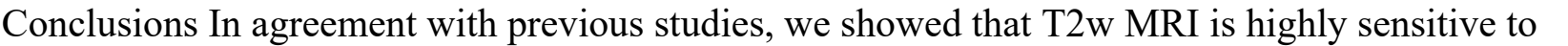

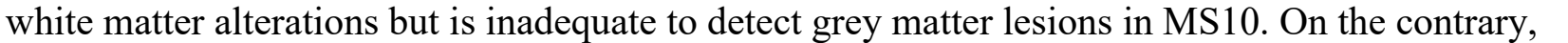

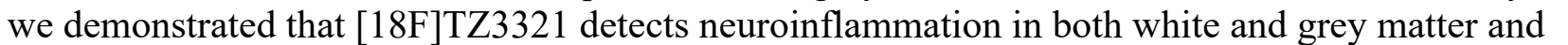




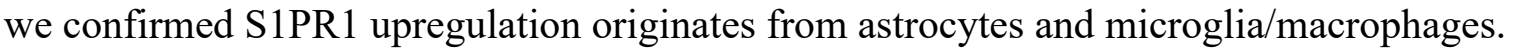

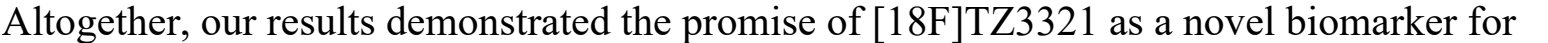

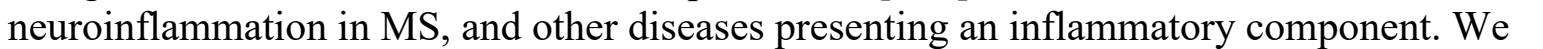

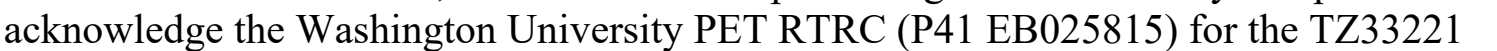

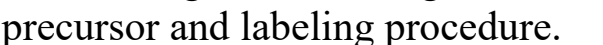

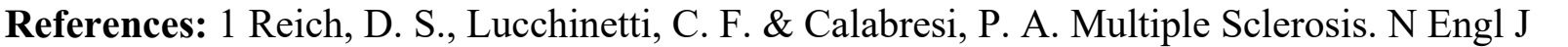

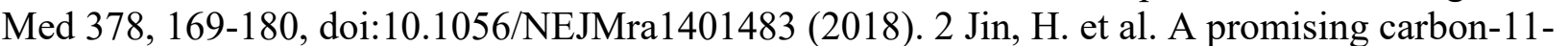
ए

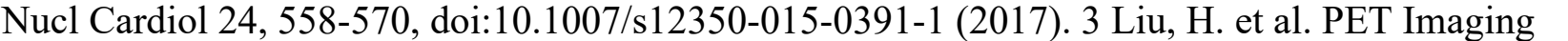

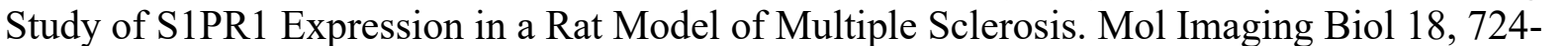

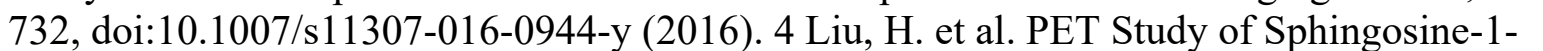

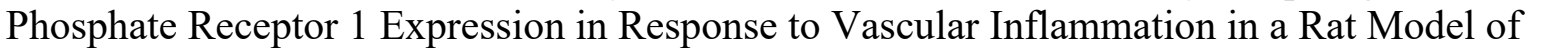

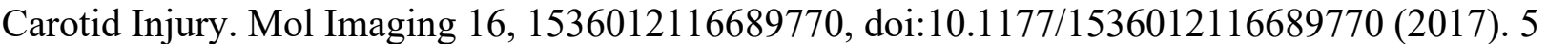

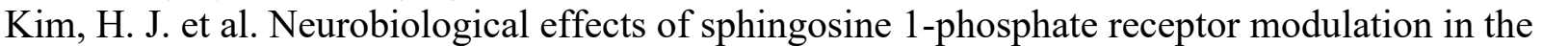

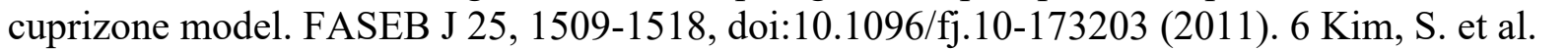

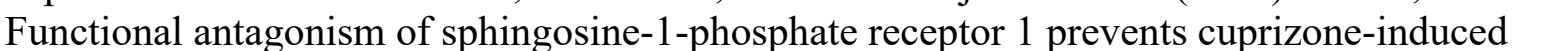

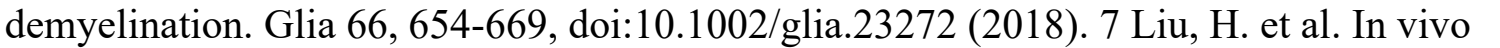

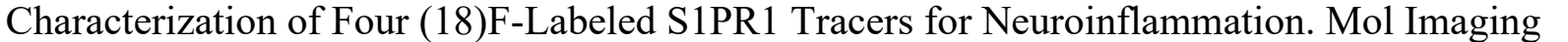

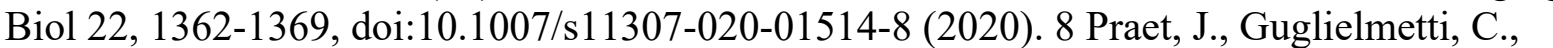

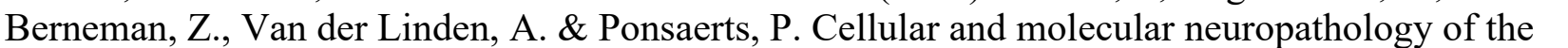

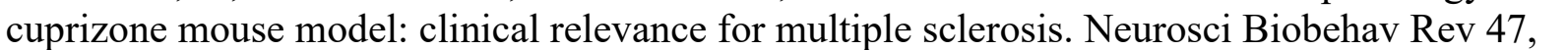

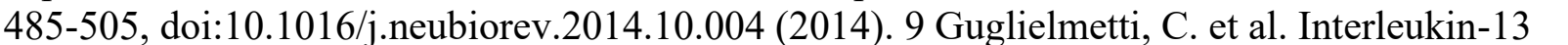

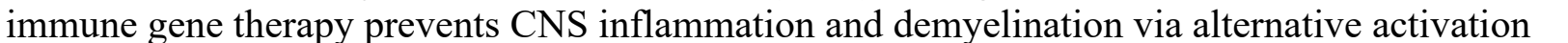

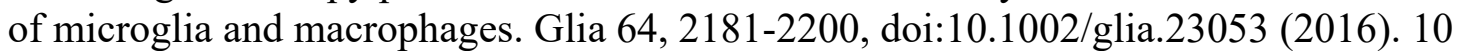

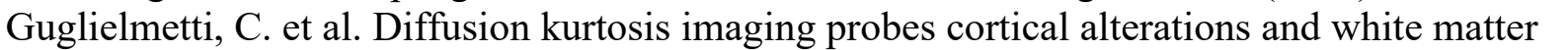

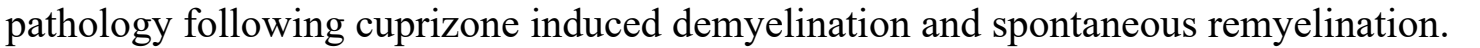

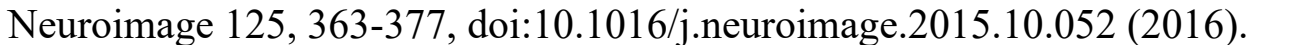

\section{Image/Figure:}

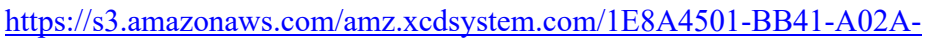

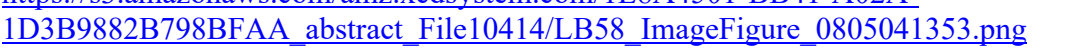

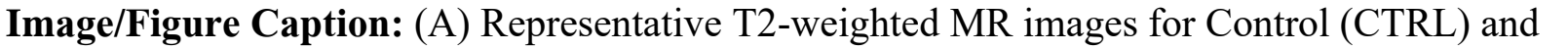

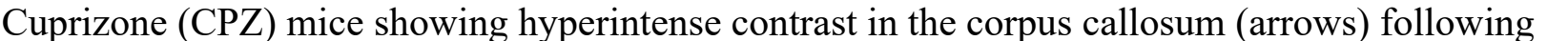

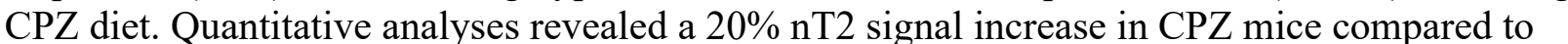

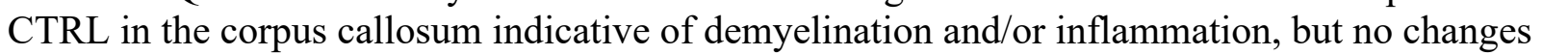

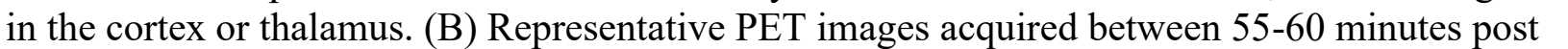
ए

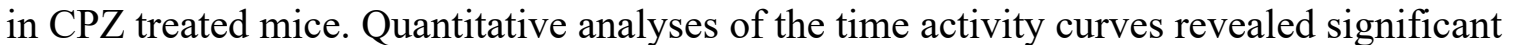
ए

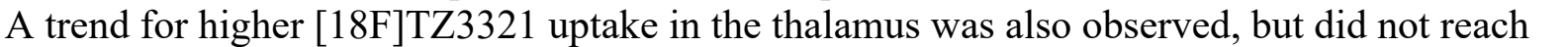

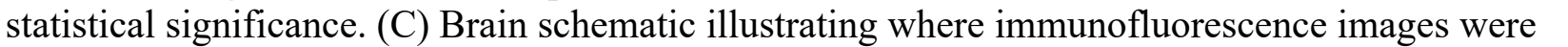

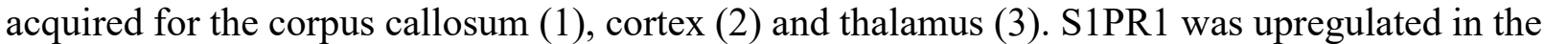

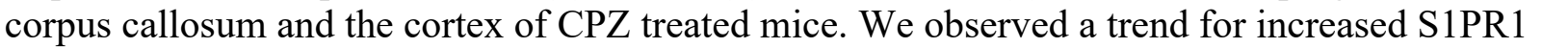

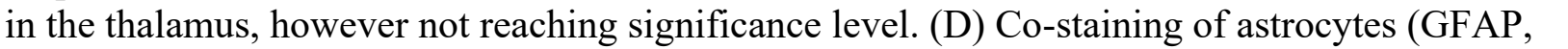




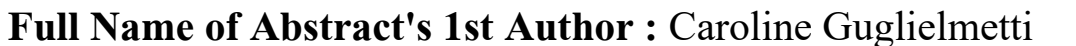

First Name: $\square \square\|\|\|\| \square$

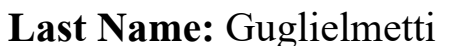

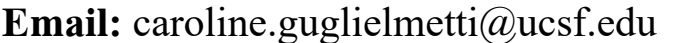

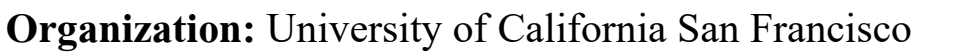

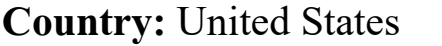




\title{
ID: LB59 \\ Discovery of injection site dynamics of rapid-acting insulin formulations through photoacoustic microscopy
}

\author{
Anjul Khadria, California Institute of Technology, anjul.nitrkl@gmail.com
}

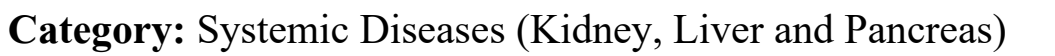

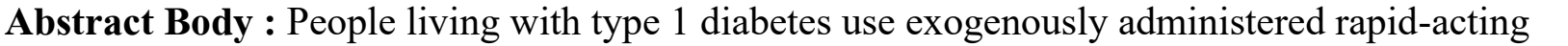

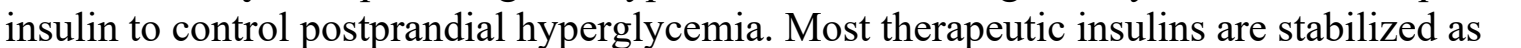

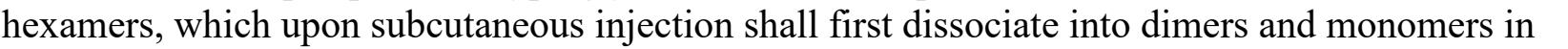

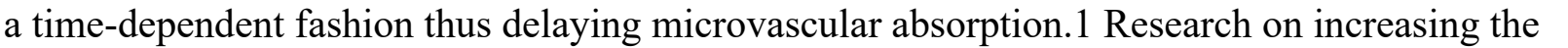

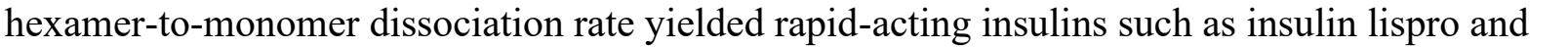

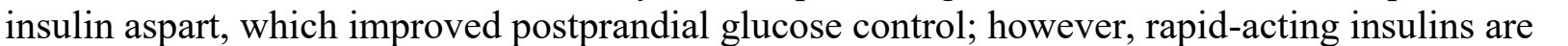

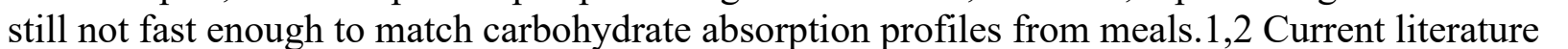

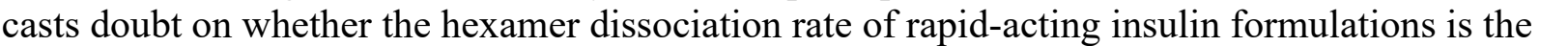

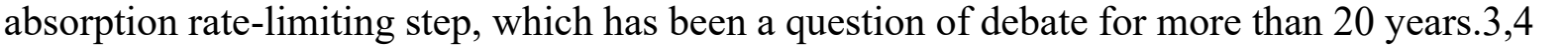

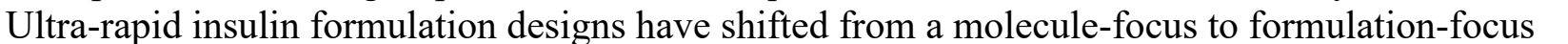
ए

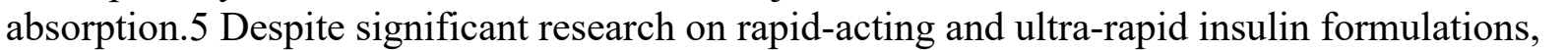

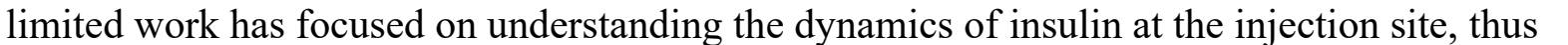

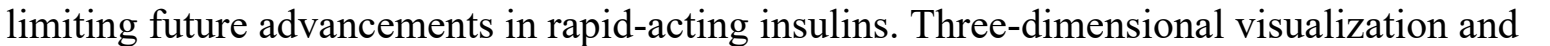

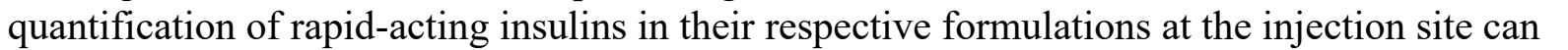

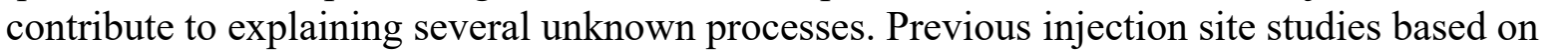

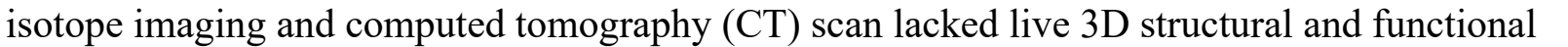

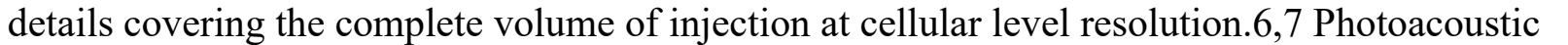

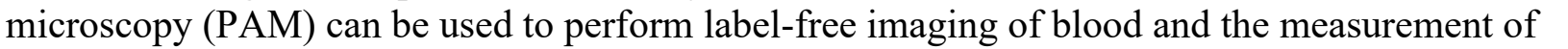

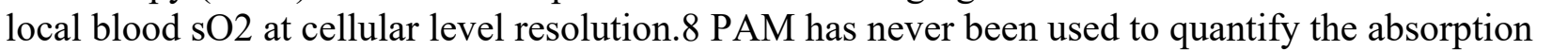

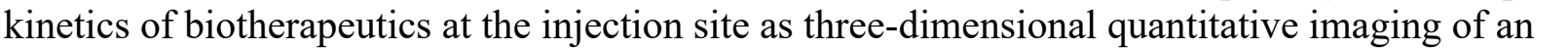

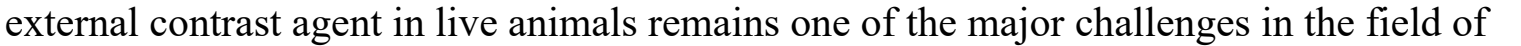

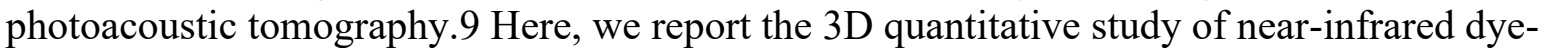
पा

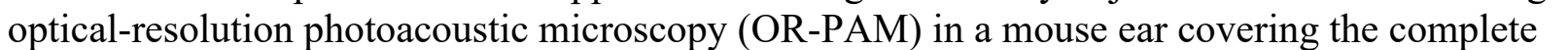

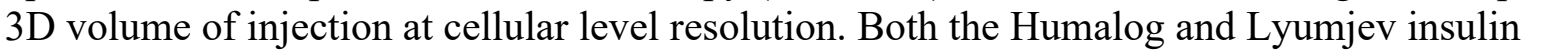

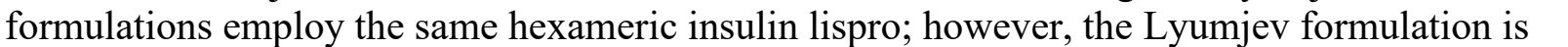

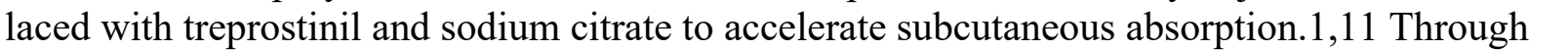

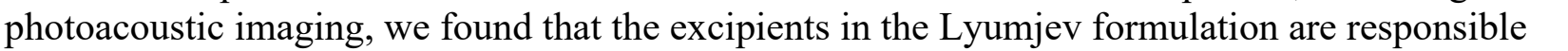

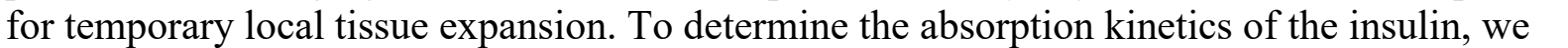

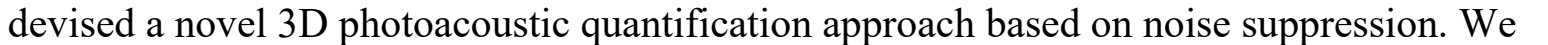

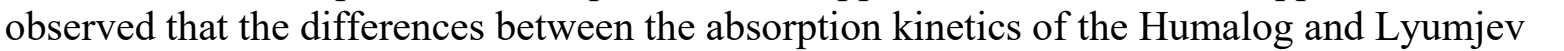

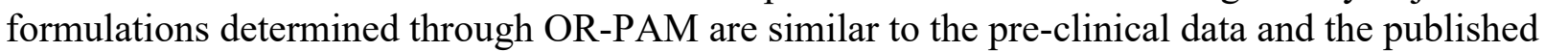

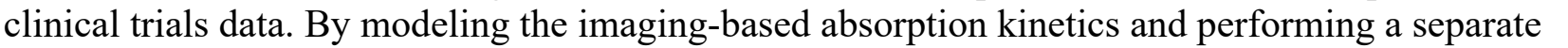

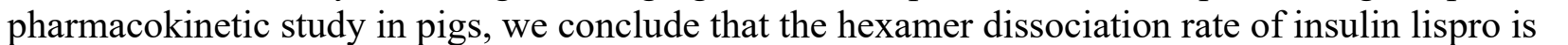

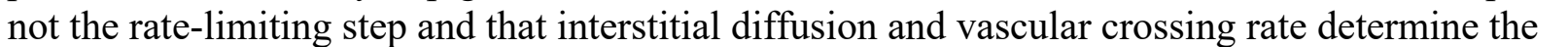




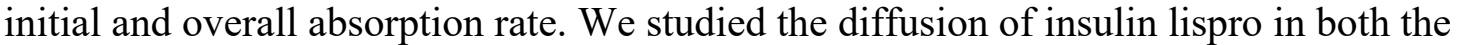

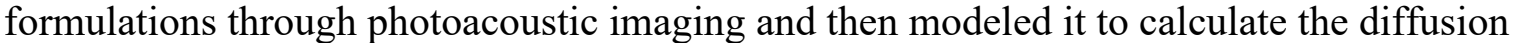

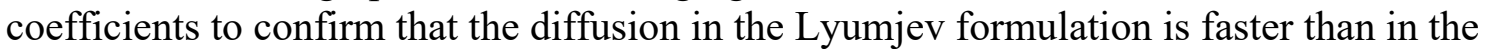

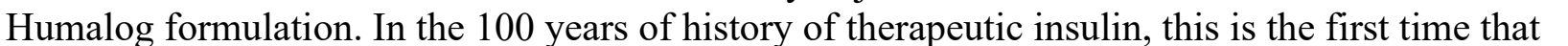

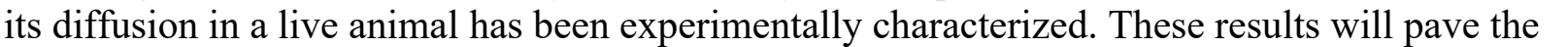

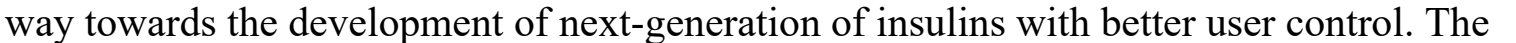

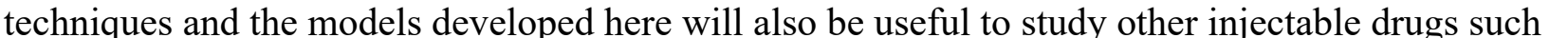

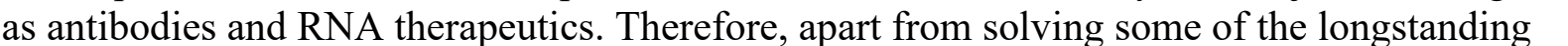

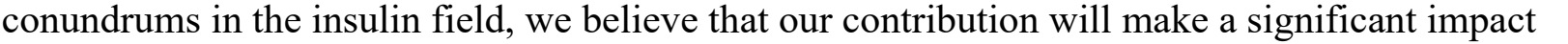

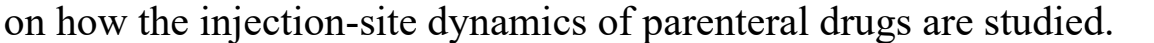

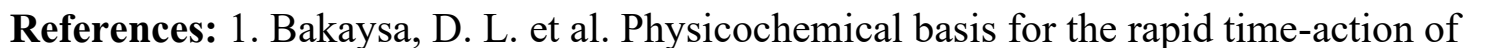

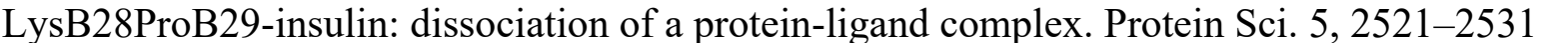

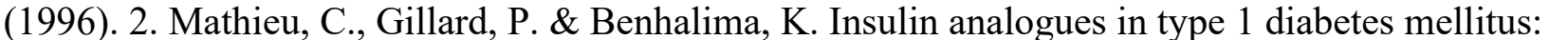

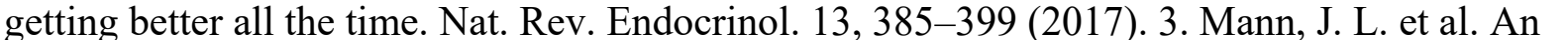

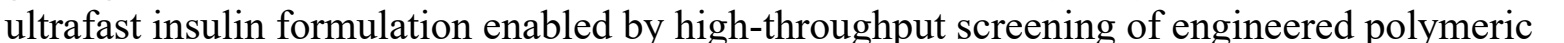

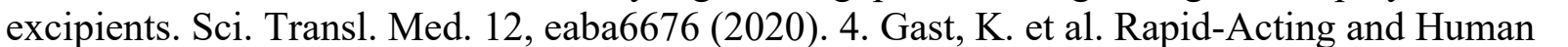

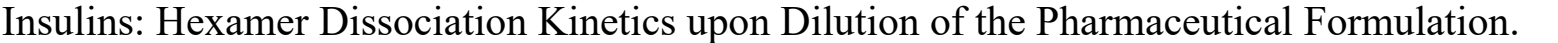

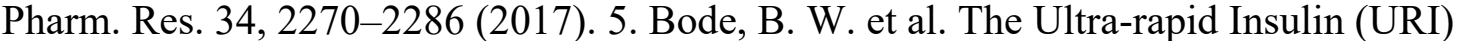

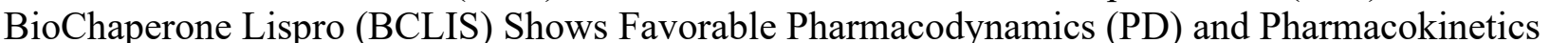

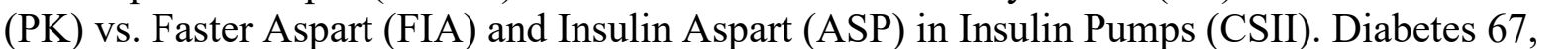

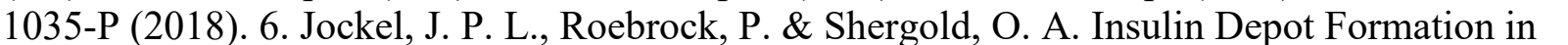

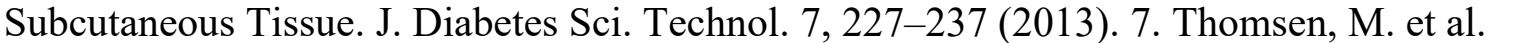

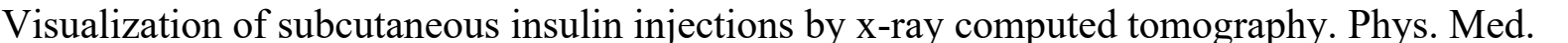

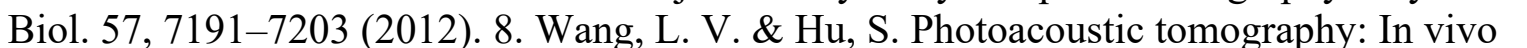

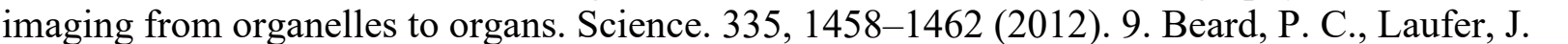

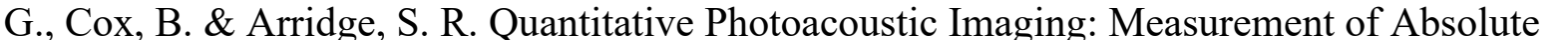

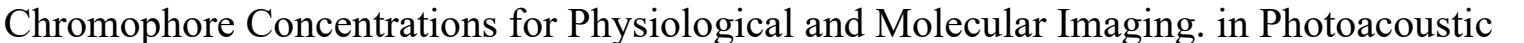

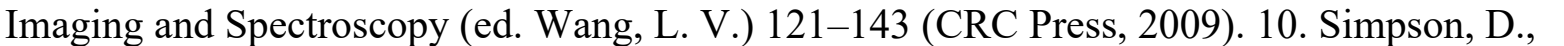

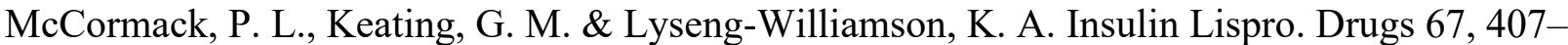

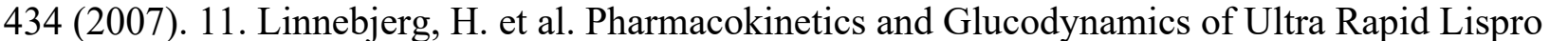

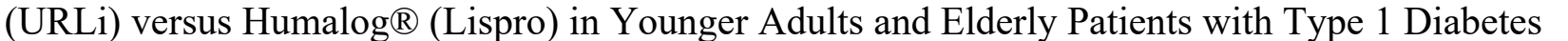

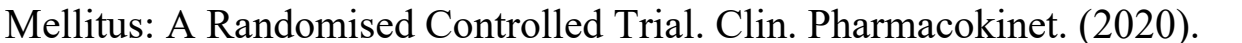

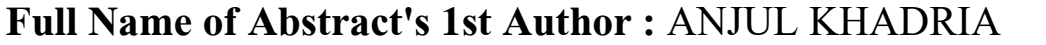

First Name: $\square \square \square|\Pi| \square$

Last Name: $\square \square\|\square\|\|\|$

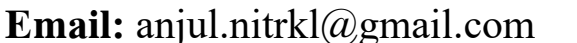

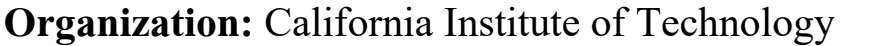

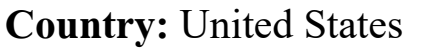




\title{
ID: LB60 \\ Monitoring linezolid treatment with FDG and 64Cu-LLP2A in a macaque model of tuberculosis
}

Michael Bellavia, University of Pittsburgh, mcb131@pitt.edu

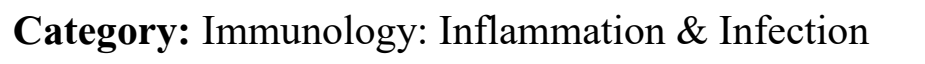

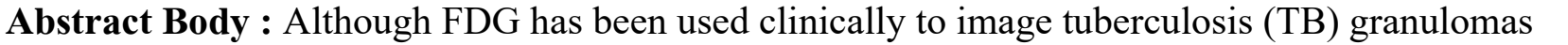

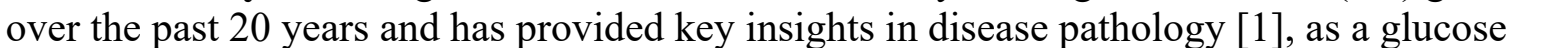

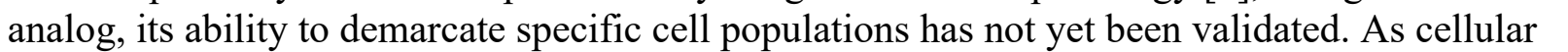

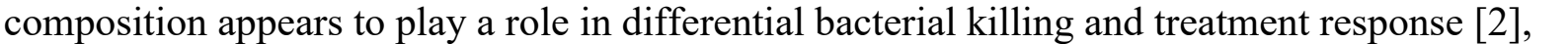

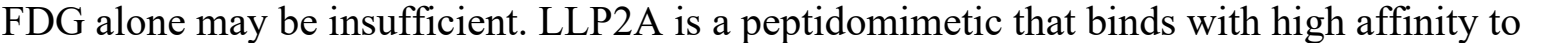
$\square \square \square 4$ (integrin $\alpha 4 \beta 1$ ) [3], a surface protein highly expressed by immune cells that mediates

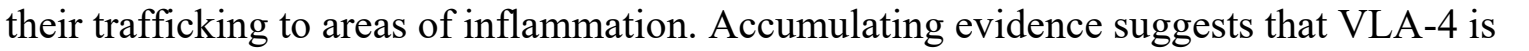

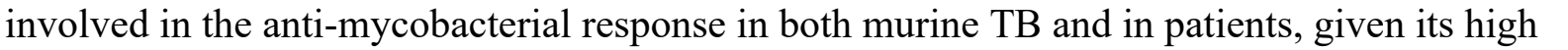

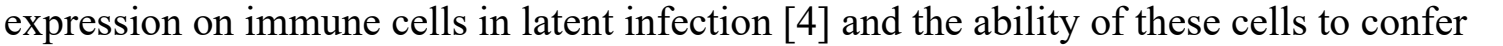

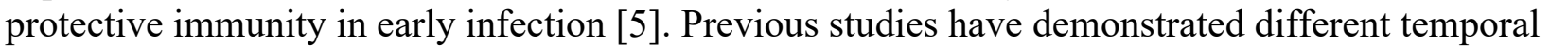

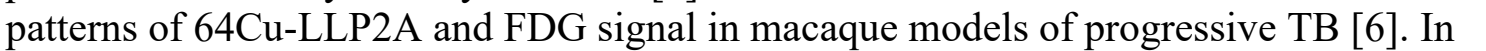

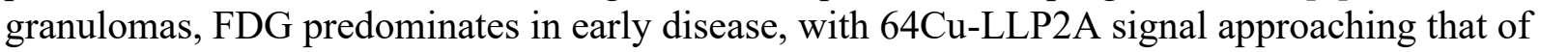

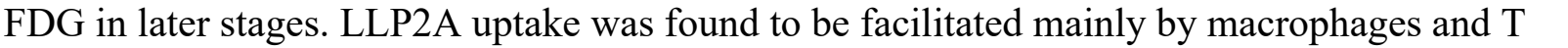

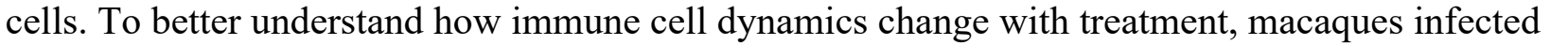

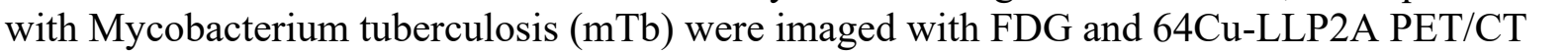

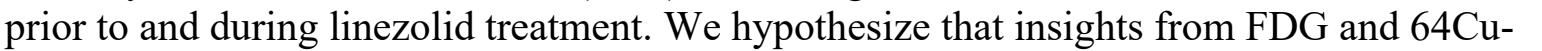

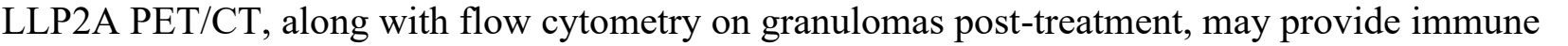

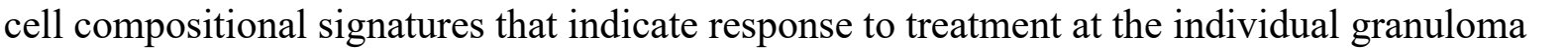
ए

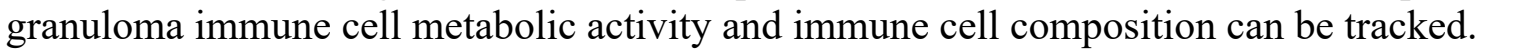

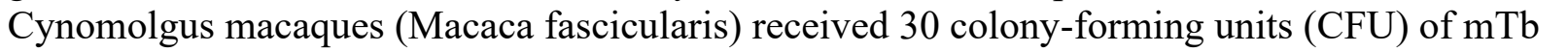

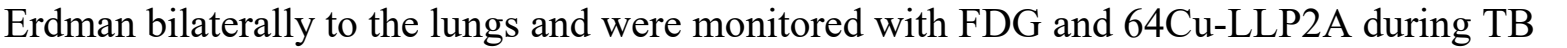

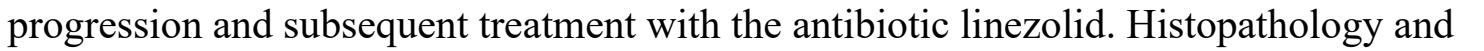

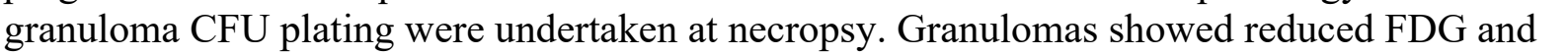

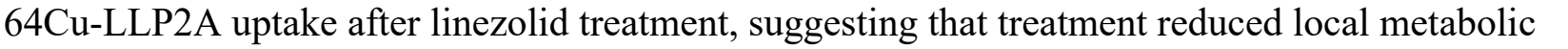

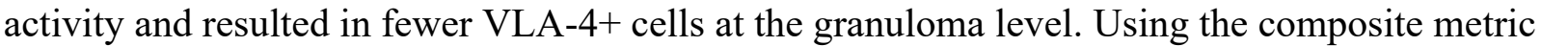
ए

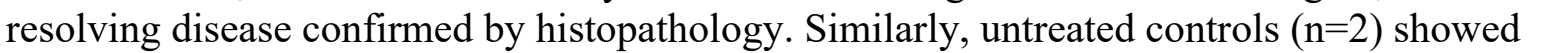
ए

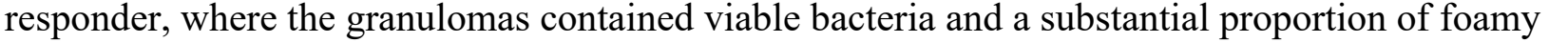

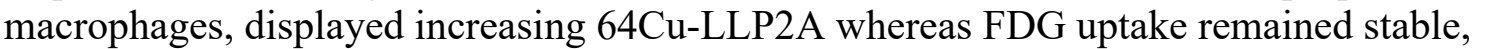

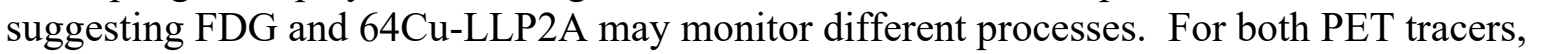
ए ए

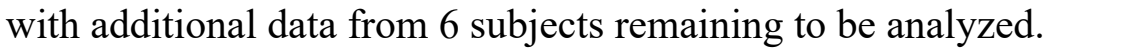




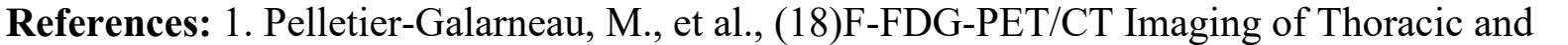

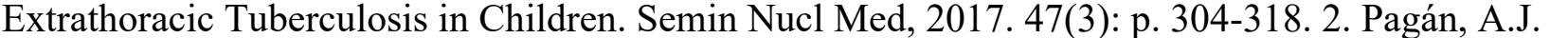

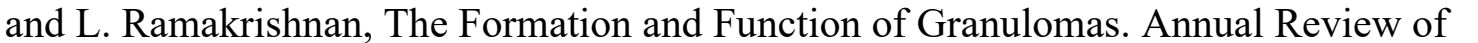

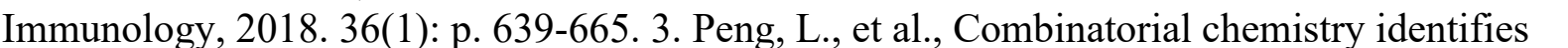

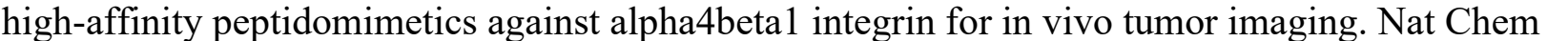

\begin{tabular}{ll|l|l|l|l|l|l|l|} 
&
\end{tabular}

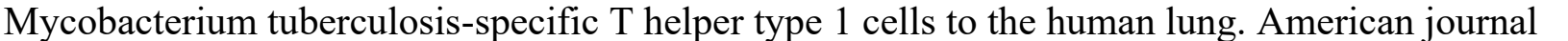

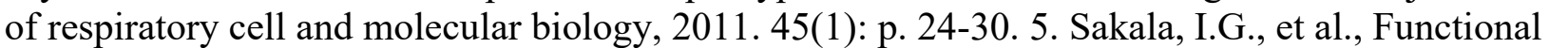

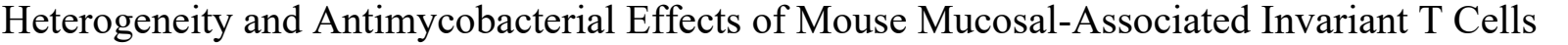

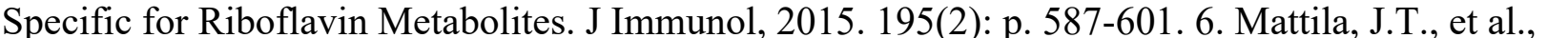

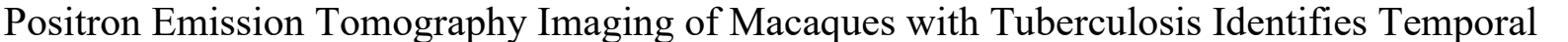

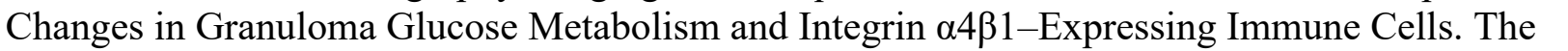

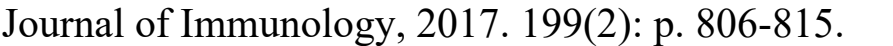

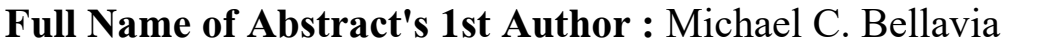

First Name: $\square \square\|\|\|\|$

Last Name: $\square \square\|\| \| \square \mid m$

Email: $\square \square\|\| \square \square \square\|\Pi\| \square$

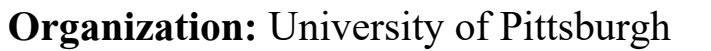

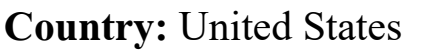




\title{
ID: LB61 \\ Correlation of 99mTc-pertechntate thyroid scanning with ablation outcome of radioactive iodine.
}

\author{
Aya Abdelnaim, Assiut university, aayahkhaled@gmail.com
}

Category: $\square \square\|\| ा \| \square$

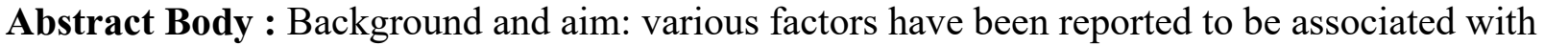

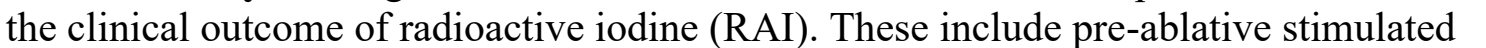

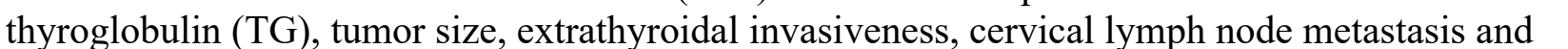

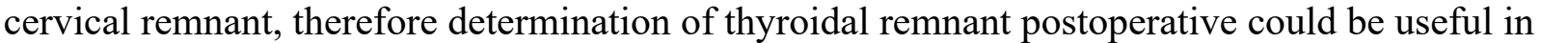

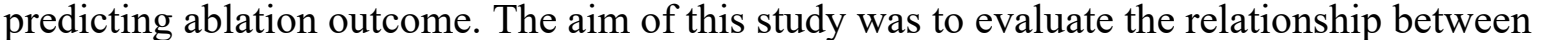

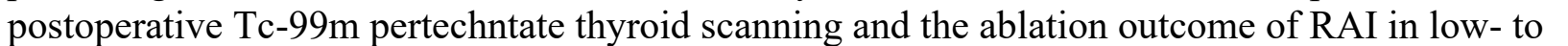

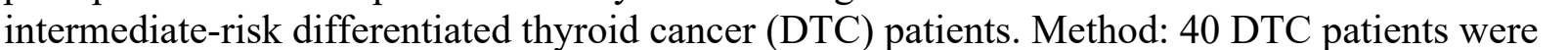

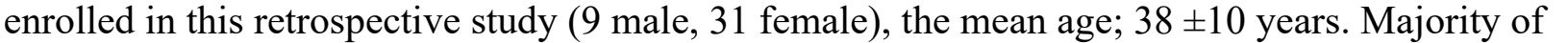

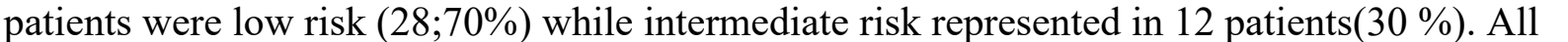

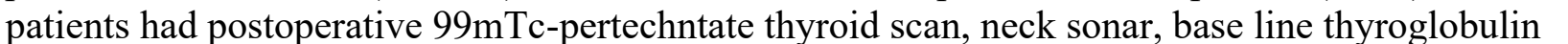

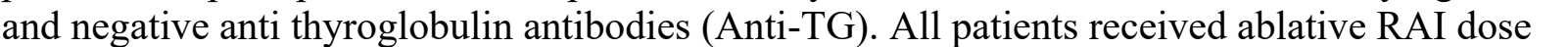

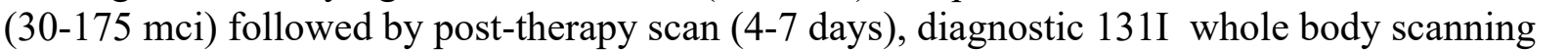

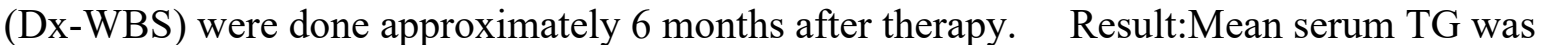

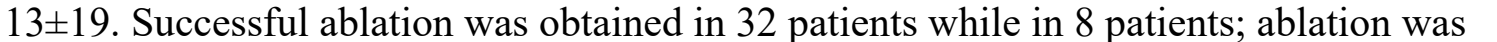

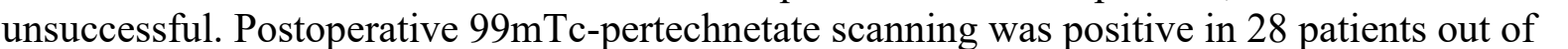

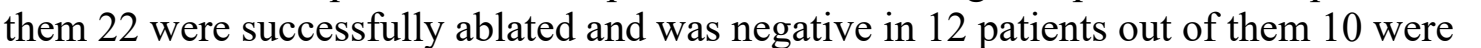

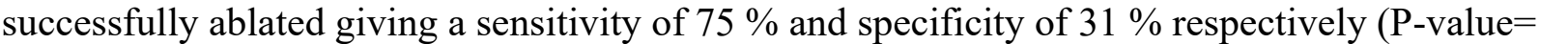

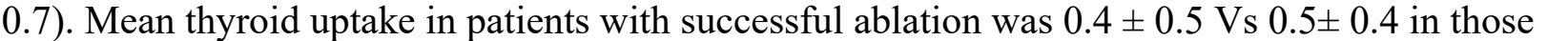

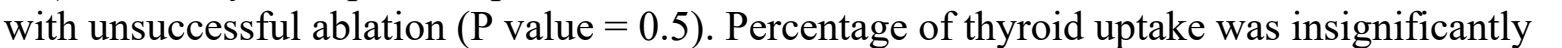

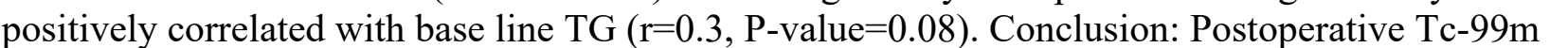

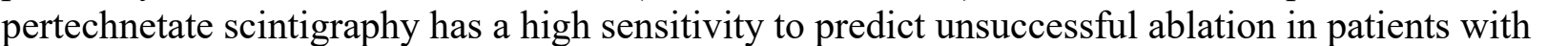

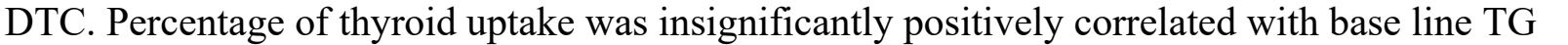

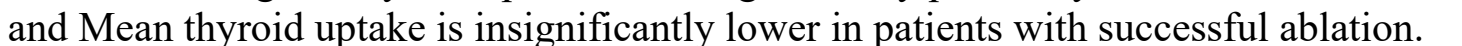

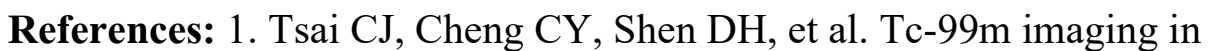

ए ए

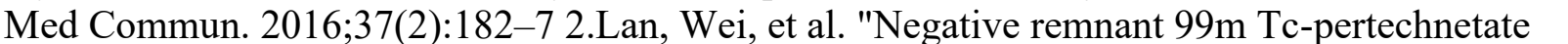

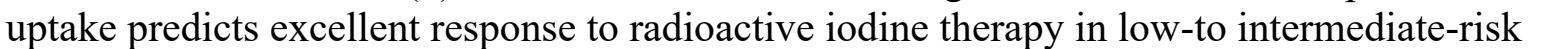

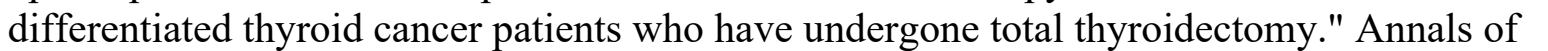

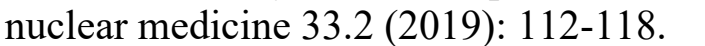

First Name:

Last Name: $\square \square \square \| \square \square \square \square$

Email: $\square\|\|\|\| \| \square \square \square m \square \square$ 


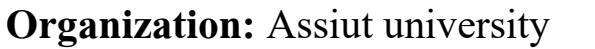

Country: $\square \square \square \| \square$ 


\title{
ID: LB62 \\ Bioluminescent Imaging of SARS-CoV2-NanoLuc Luciferase in hACE2- transgenic Mice with Fluorofurimazine
}

Joel Walker, Promega, joel.walker@promega.com

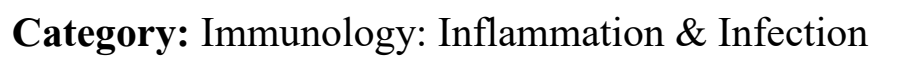

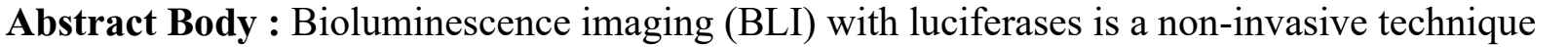

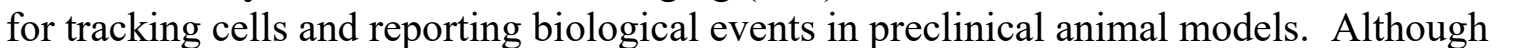

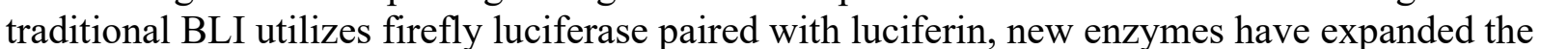

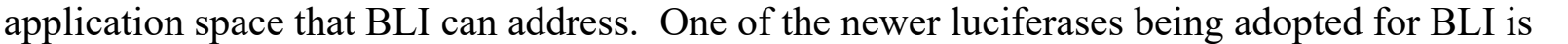

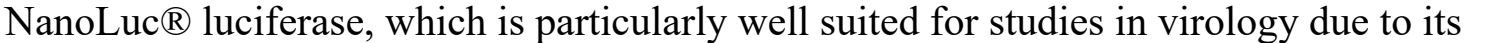

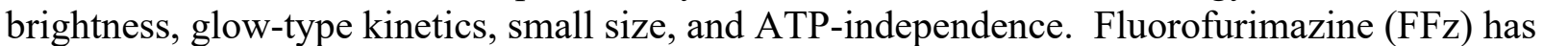

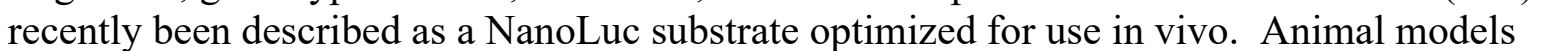

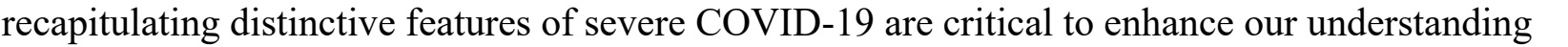

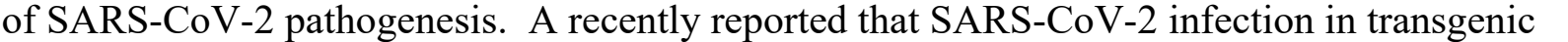

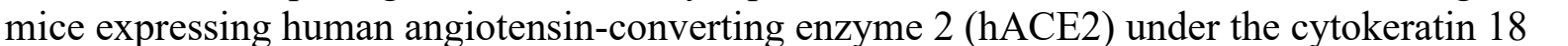

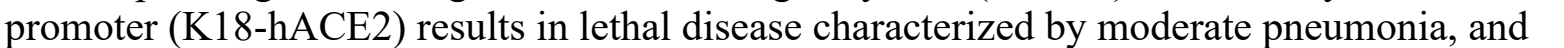

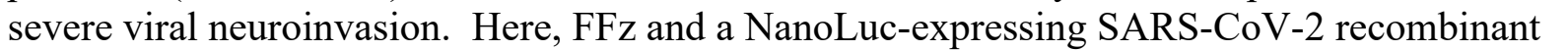

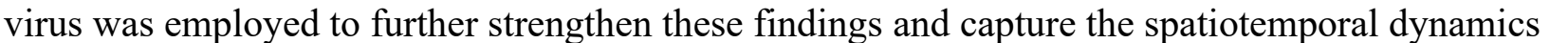

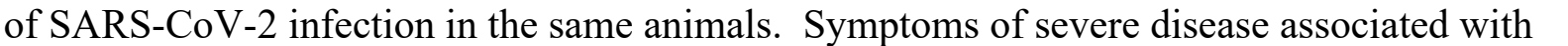

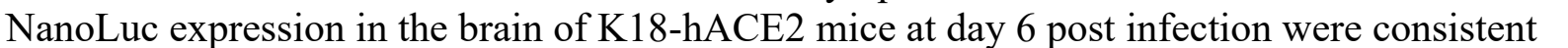

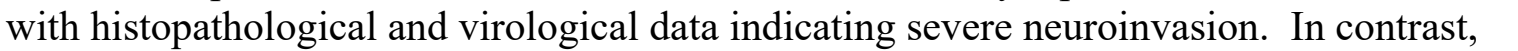

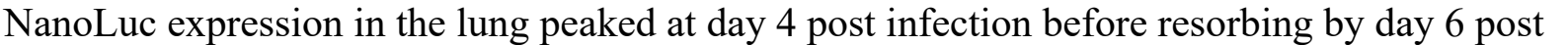
ए

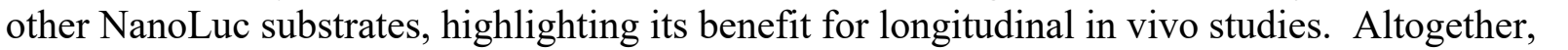
ए

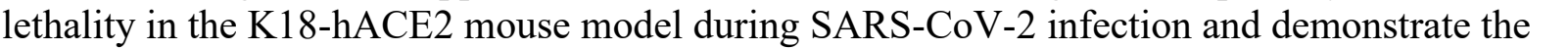

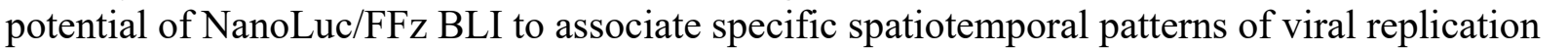

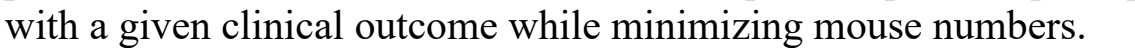

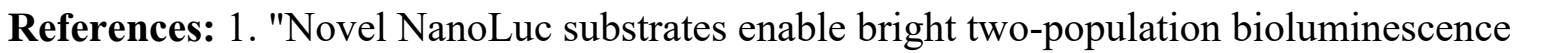

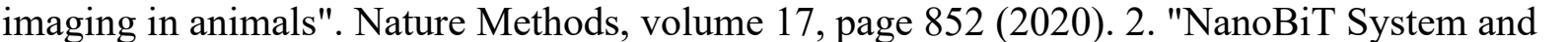

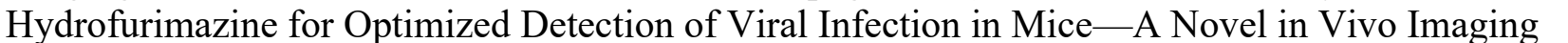

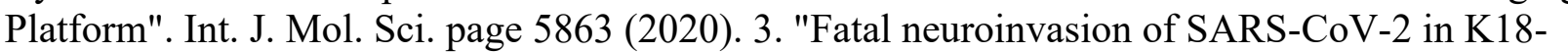

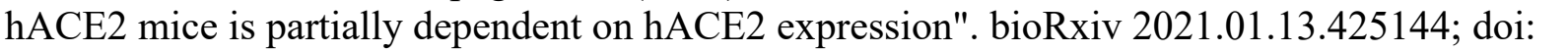

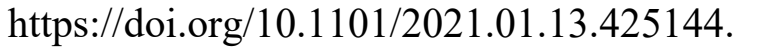

First Name: $\square 1 \|$

Last Name: $\square \square \| \square \mid m$

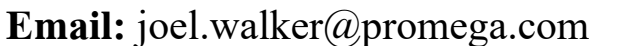


Organization:

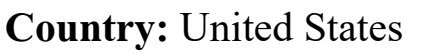




\title{
ID: LB63 \\ Difference in Targeting Effects of U-87 Derived Microvesicle and Exosome Coated Prussian Blue Nanoparticles and their Phototheranostic Application for Glioblastoma Treatment
}

\author{
Meghan Hill, Michigan State University, hillmeg7@msu.edu
}

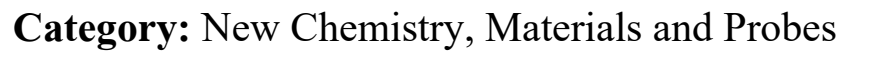

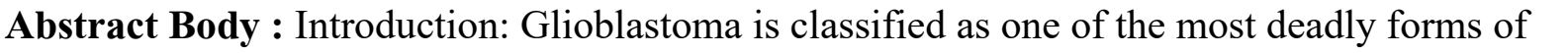

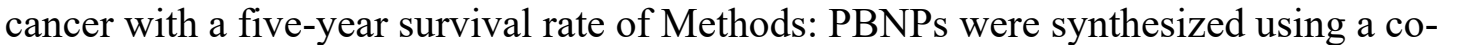

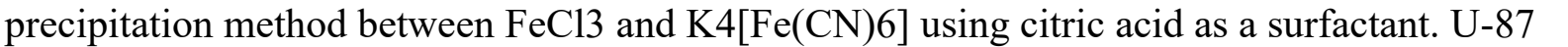

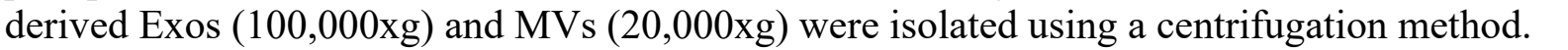

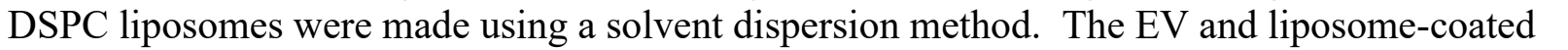

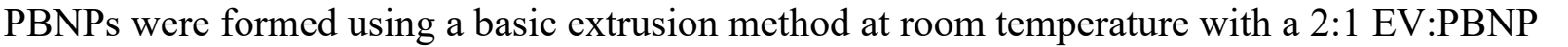

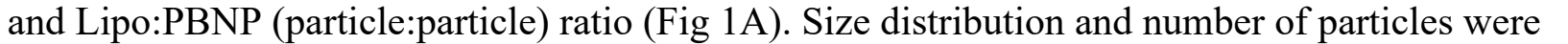

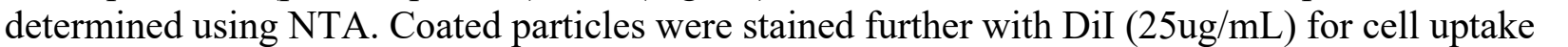

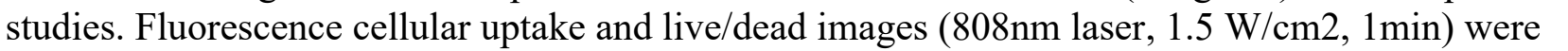

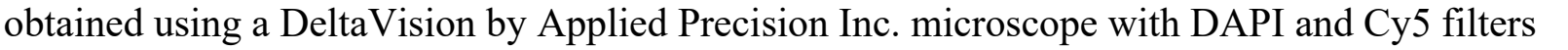

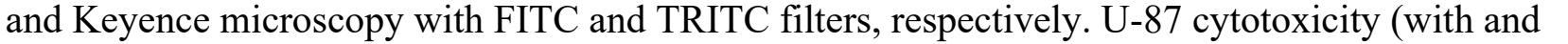

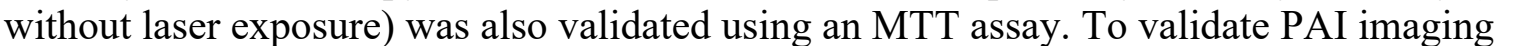

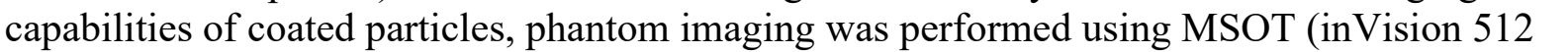

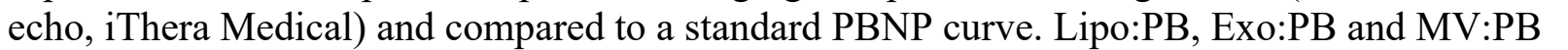

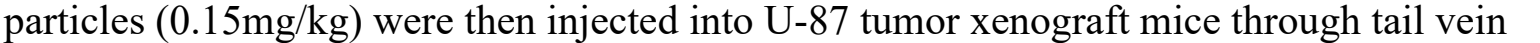

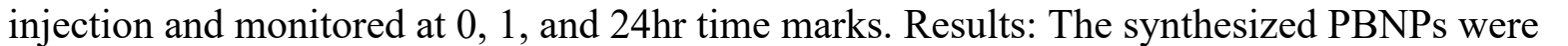

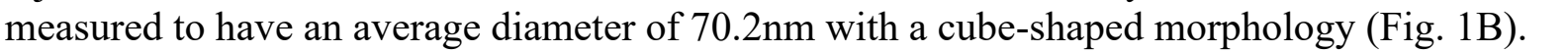

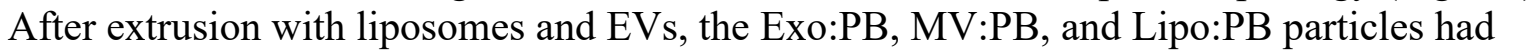

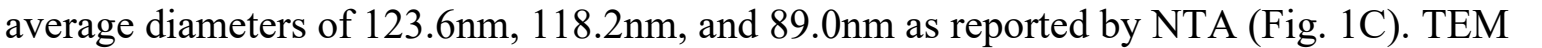

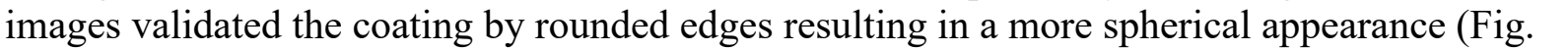

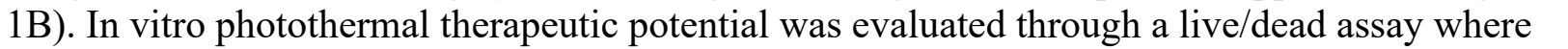

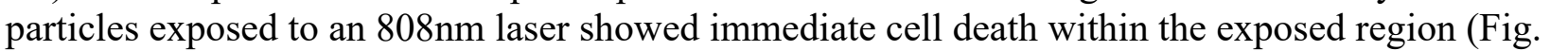

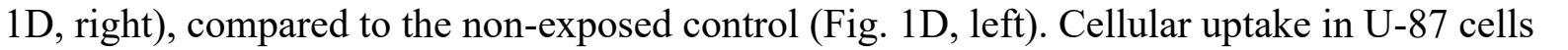

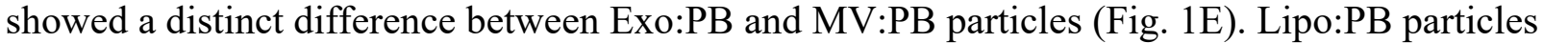

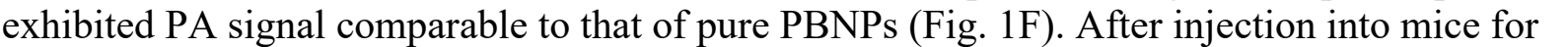
PBNPs and Lipo:PB, there wasn't much difference in targeting

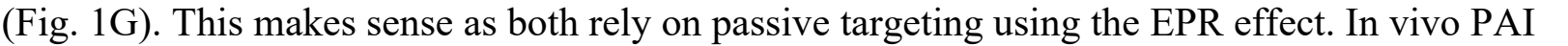

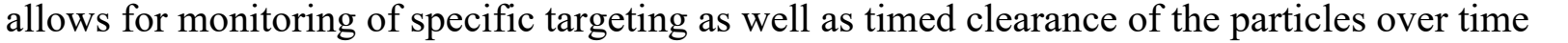

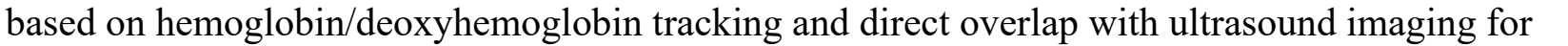

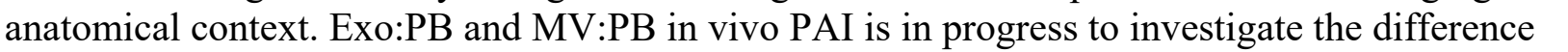
ए

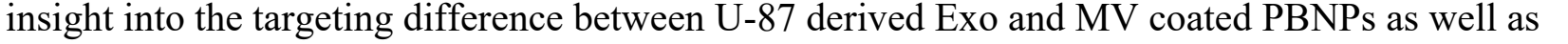

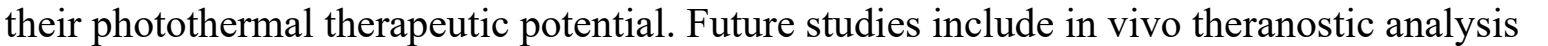

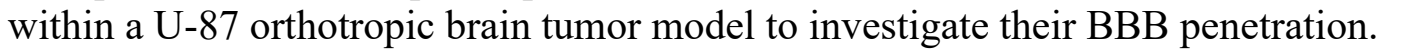




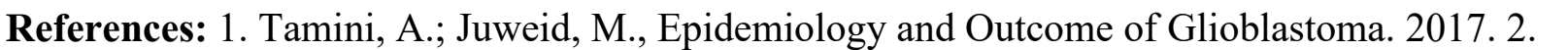

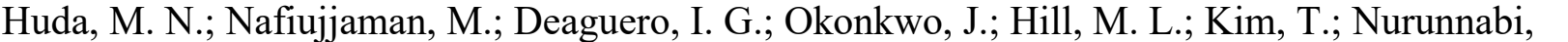

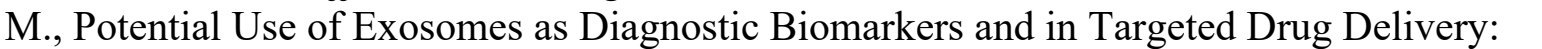

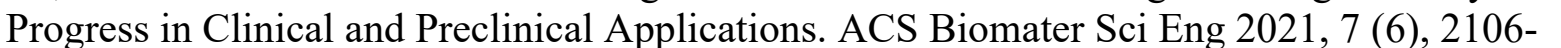

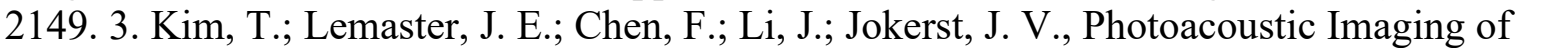

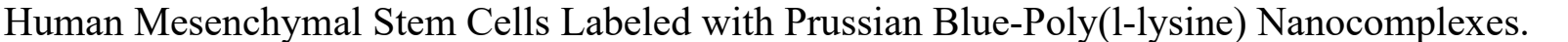

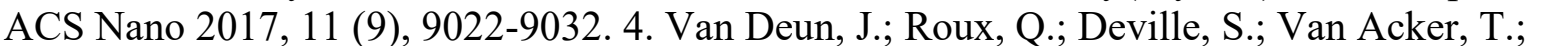

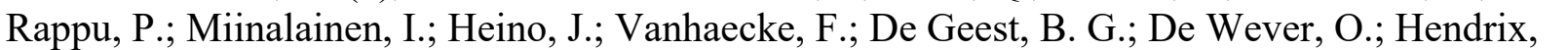

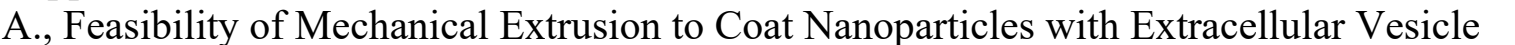

Membranes. Cells 2020, 9 (8). 5. Fathi, P.; Rao, L.; Chen, X., Extracellular vesicle-coated

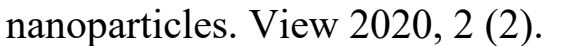

\section{Image/Figure:}

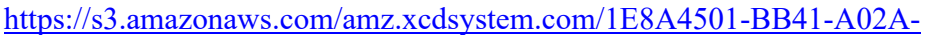

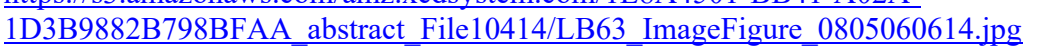

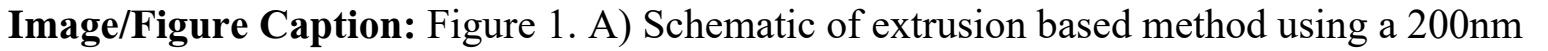

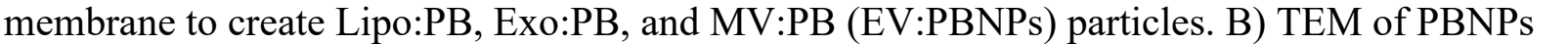
口ण

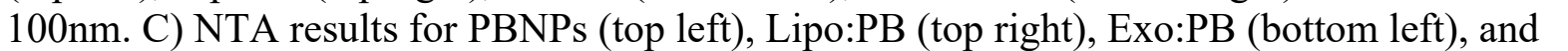

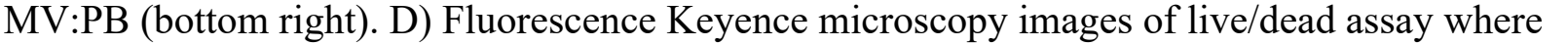

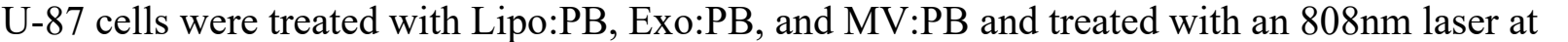

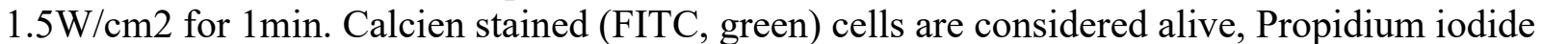

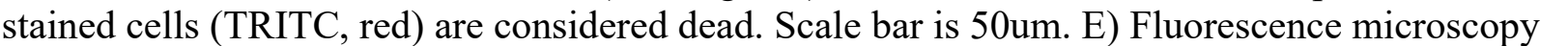

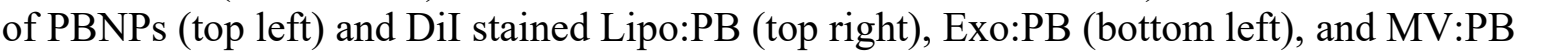
ए ए

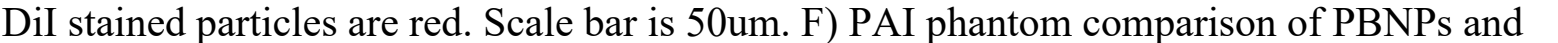

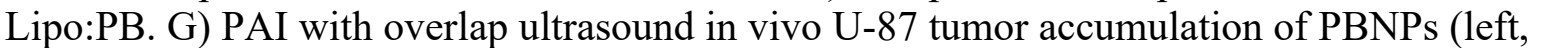

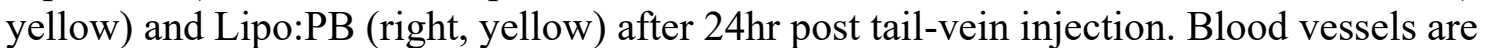

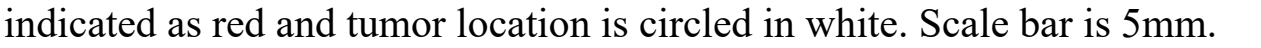

First Name: $\square \square\|\| \Pi$

Last Name: $\square \square$ 而菂

Email: $\square \square \square \square \square \square \square ा \| \square$

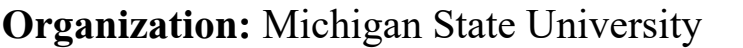

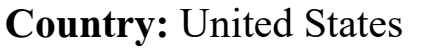




\title{
ID: LB65 \\ In vivo evaluation of COX-2 PET radiotracer [11C]BRD1158 in the Thy-1-COX- 2 transgenic mouse model of human-COX-2 overexpression
}

\author{
Jessica Wang, MGH/Martinos Center for Biomedical Imaging, \\ jwang122@mgh.harvard.edu
}

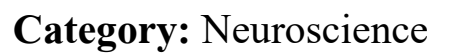

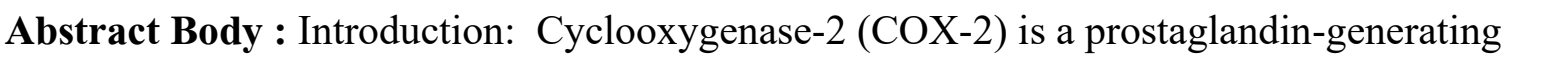

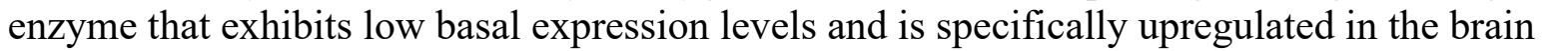

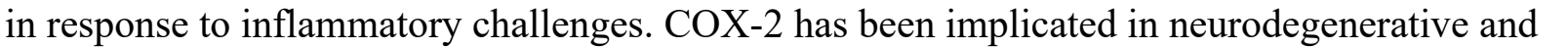

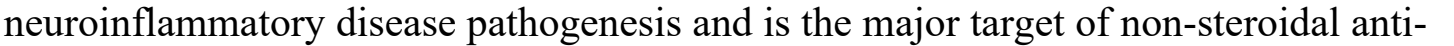
ए

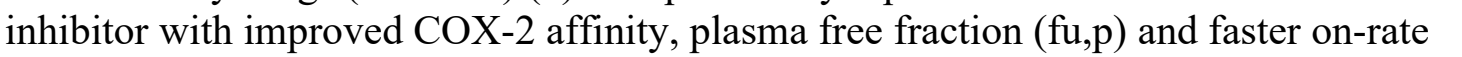

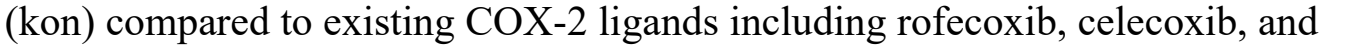

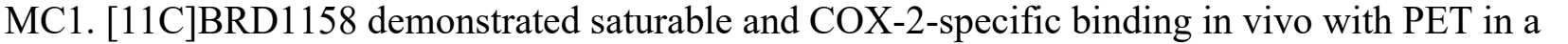
एण

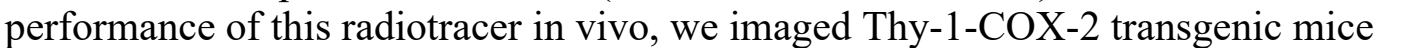

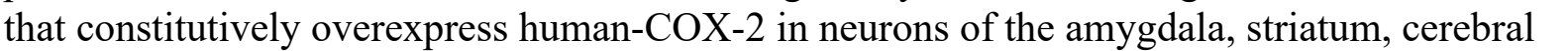

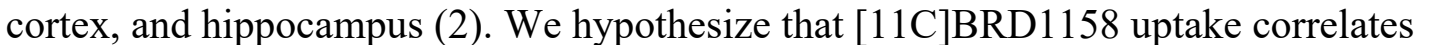

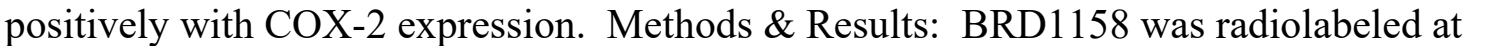

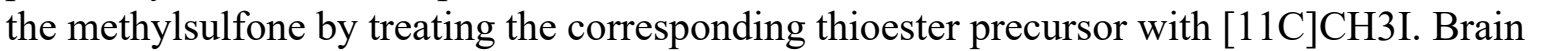

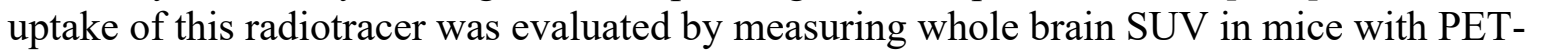

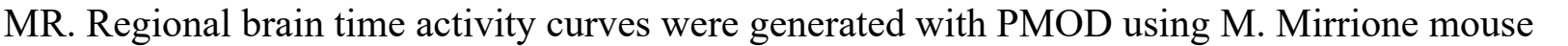

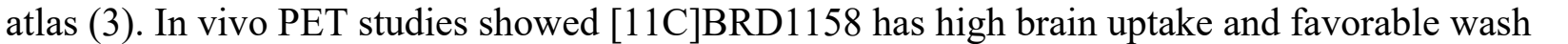

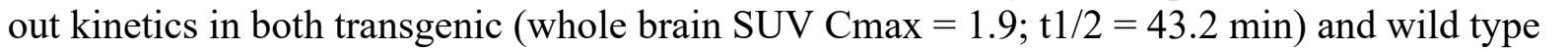

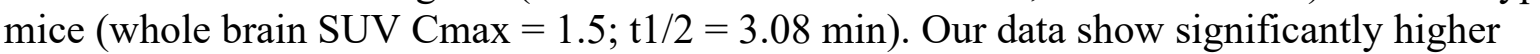
ए ए

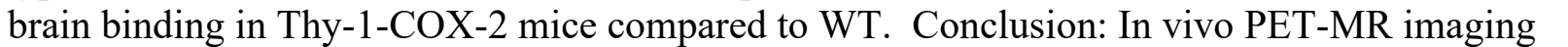

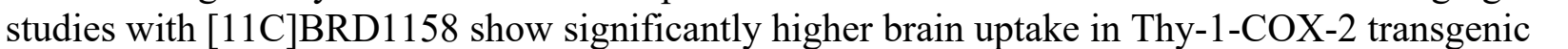

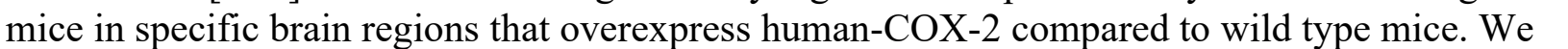

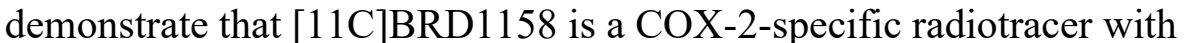

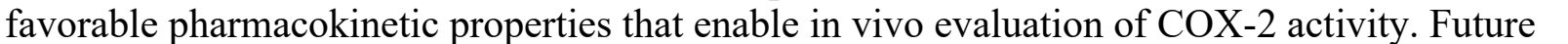

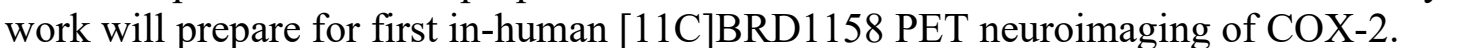

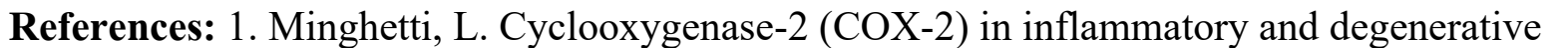

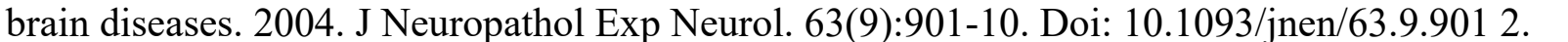

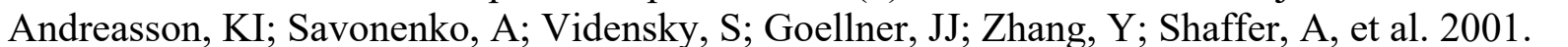

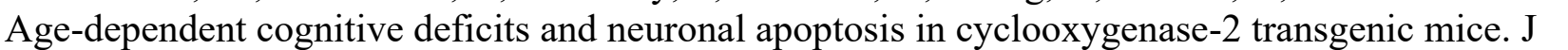

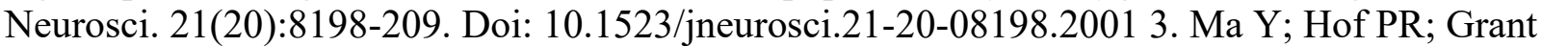

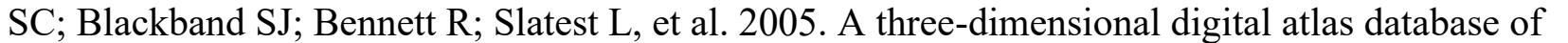


Image/Figure:

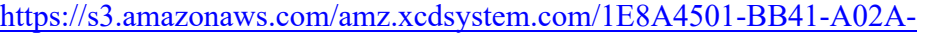

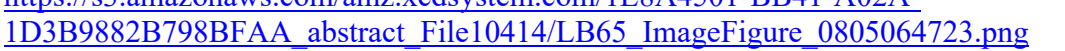

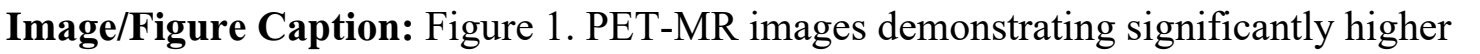

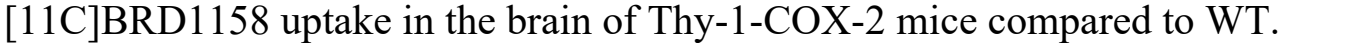

First Name: $\square \square\|\| \| \square$

Last Name: $\square \square \square \square$

Email: $\square \square\|\|\|\square \square \square\|\|\| \square \| m \square \square$

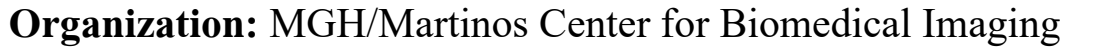

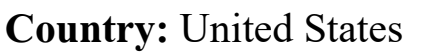




\title{
ID: LB66 \\ Automated Classification Of Lung Shunt Fraction With Deep Learning Using Selective Internal Radiation Therapy Virtual Phantom Data
}

\author{
Shanmukha Srinivas, University of California San Diego School of Medicine, \\ s6sriniv@health.ucsd.edu
}

Category: $\square \square\|\| \square \square$

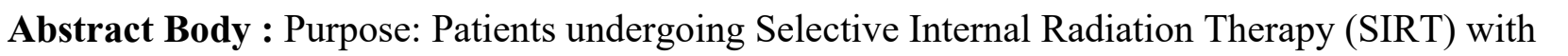

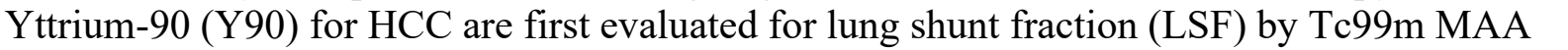

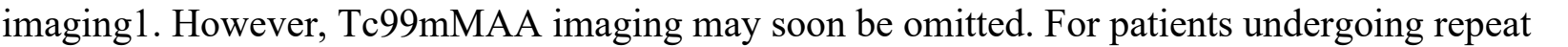

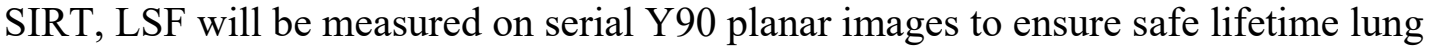

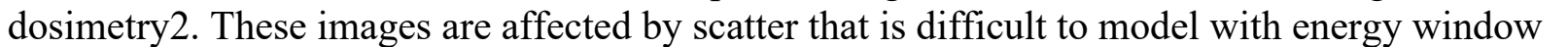

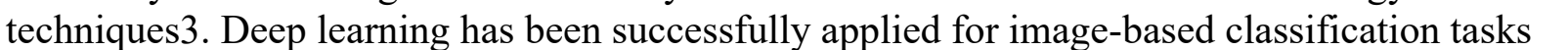

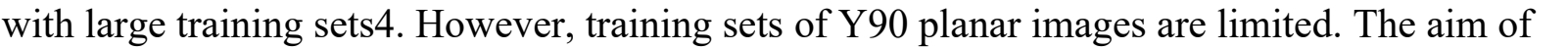
ए

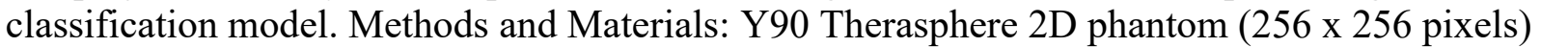

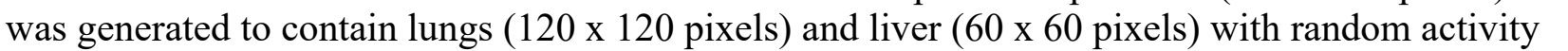

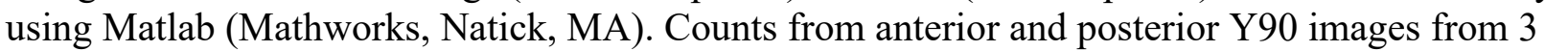

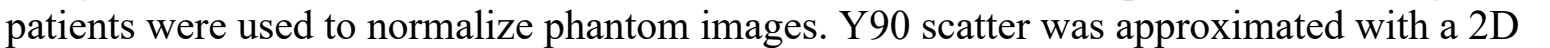

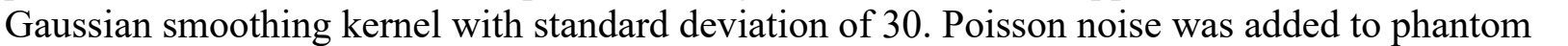

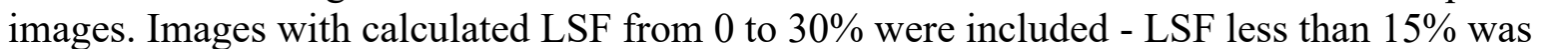

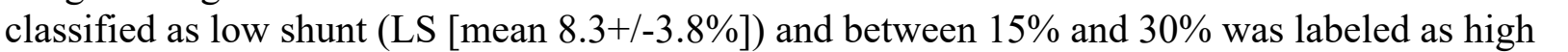

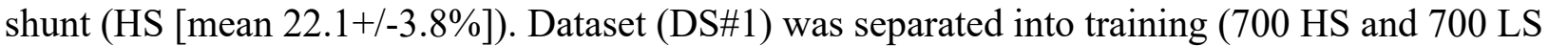

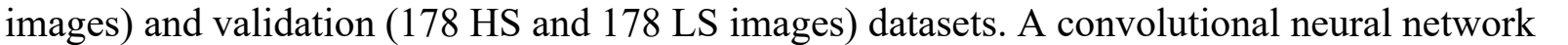

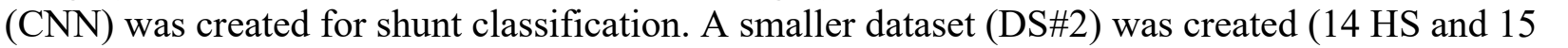

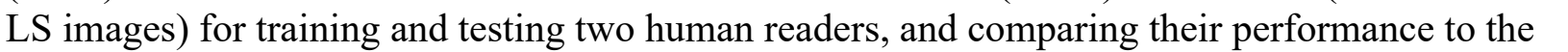

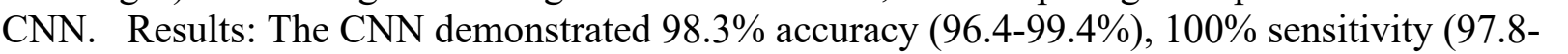

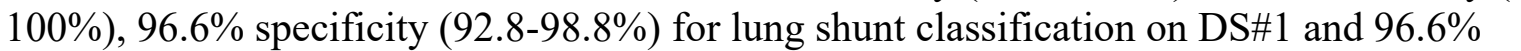

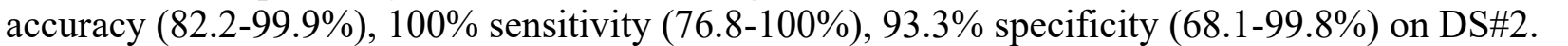

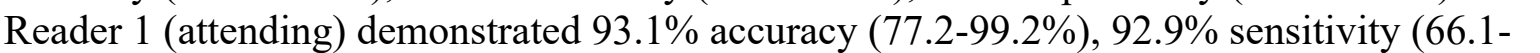

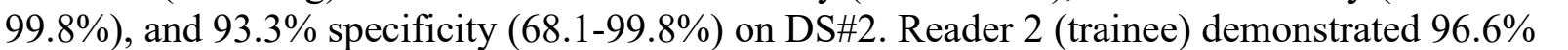

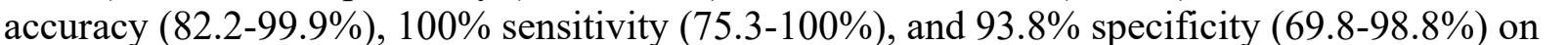

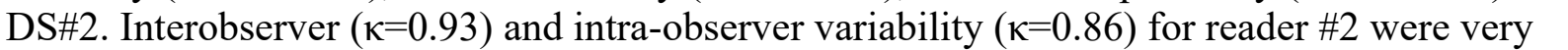

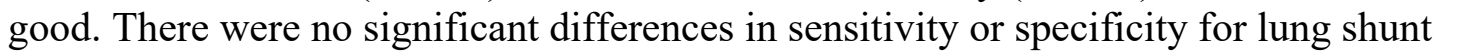

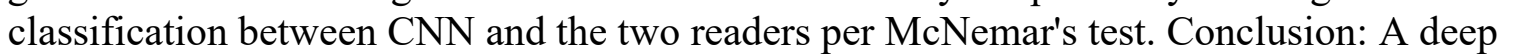

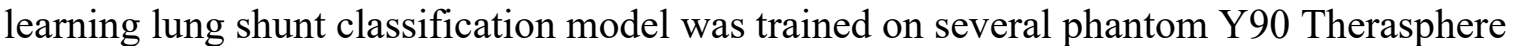

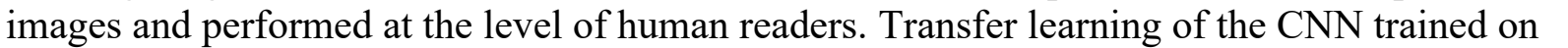

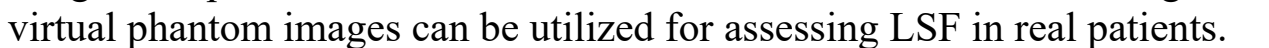

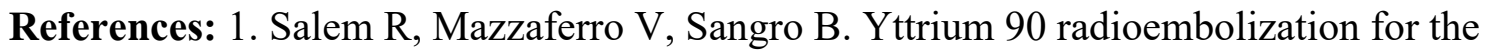

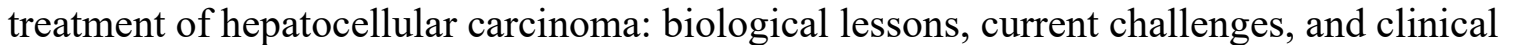

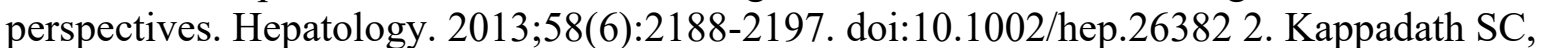




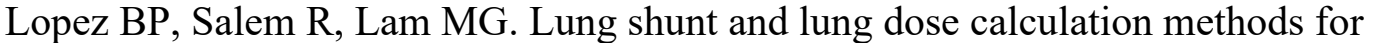

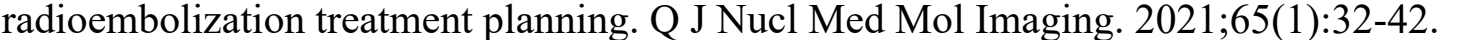

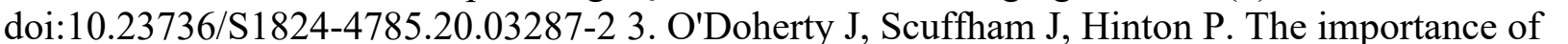

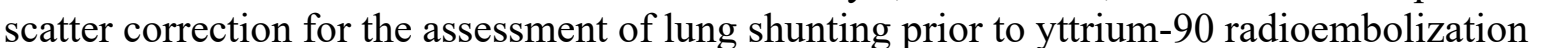

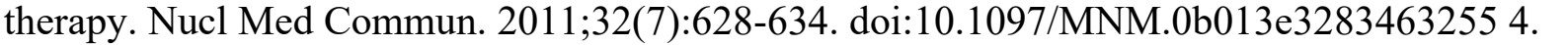

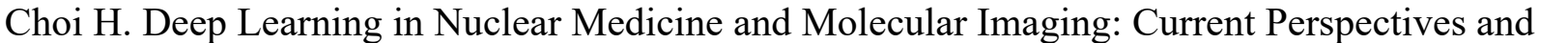

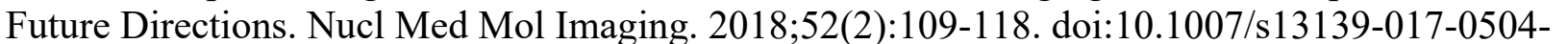

$\square$

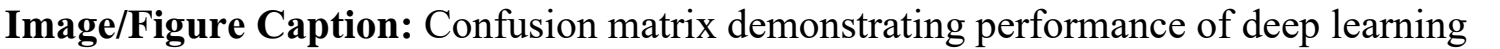

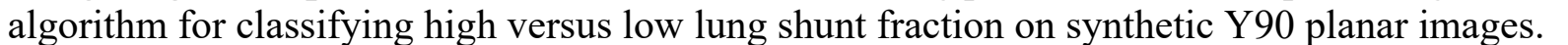

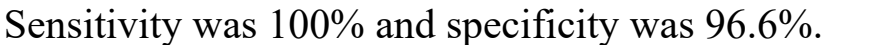

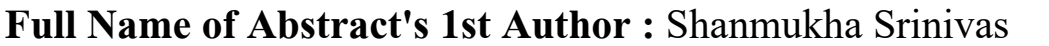

First Name: $\square \square\|\square \square \square\| \square$

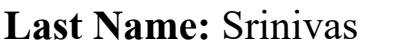

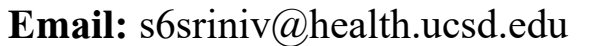

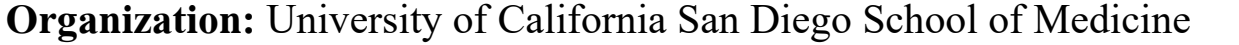

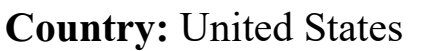




\title{
ID: LB67
}

Polypyrrole coated Magnetite Vortex Nanoring for Photoacoustic/Magnetic Resonance Imaging-Guided Hyperthermia-boosted Photothermal/Magnetothermal Tumor Ablation

Jianfeng Bao, The First Affiliated Hospital of Zhengzhou University, baoguojianfeng@gmail.com

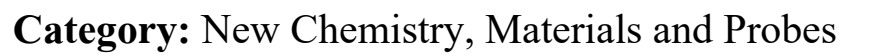

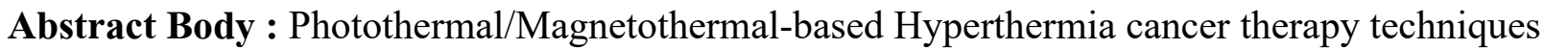

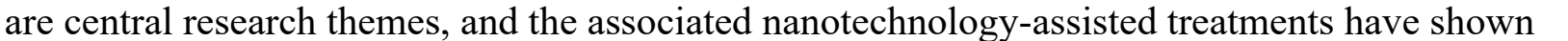

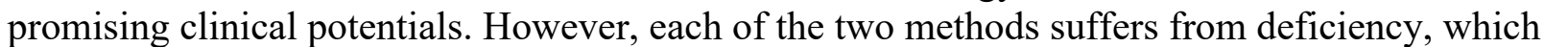

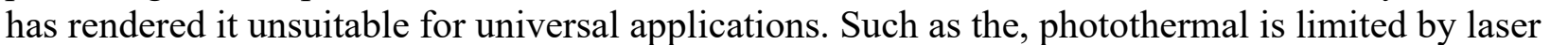

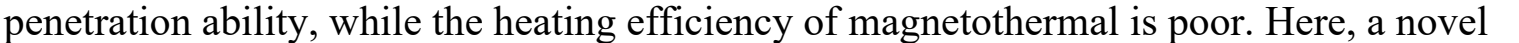

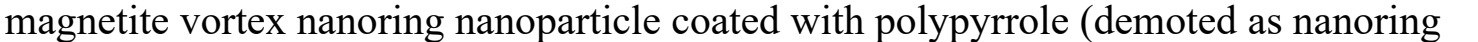

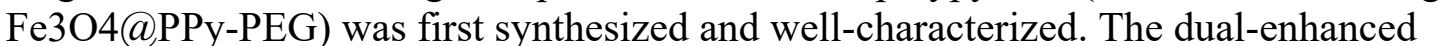

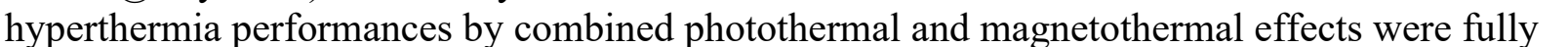

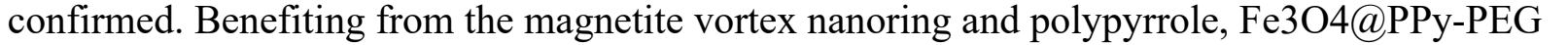

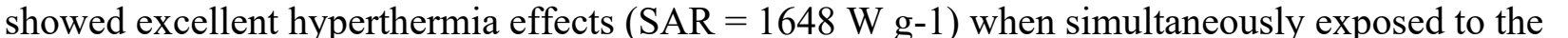

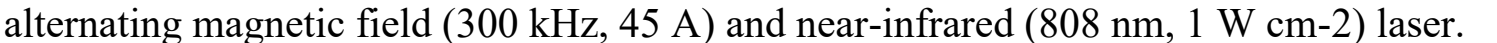

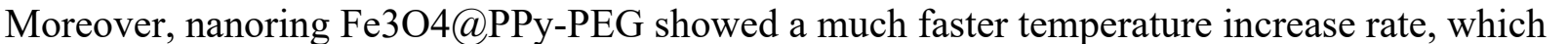

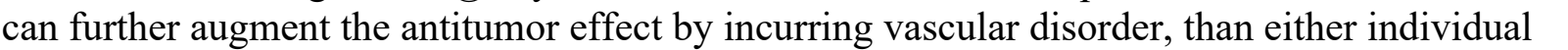
method. What's more, Fe3O4@PPy

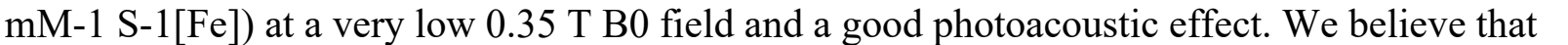

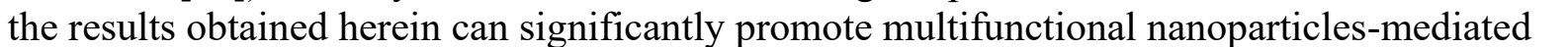

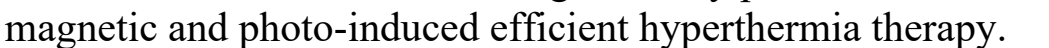

Image/Figure:

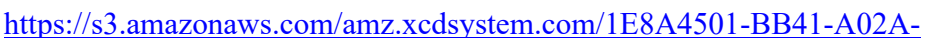

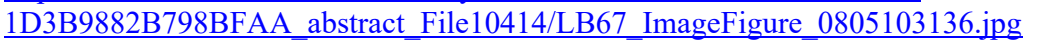

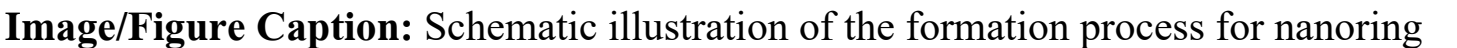

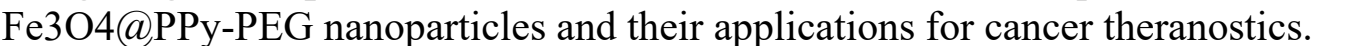

Full Name of Abstract's 1st Author : $\square$

First Name: $\square[|\|||| l$

Last Name: $\square \square \square$

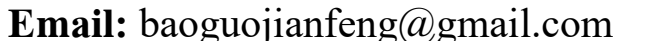




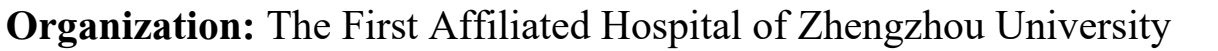

Country: $\square \square \square \square \square$ 


\title{
ID: LB68 \\ Development of Iron Oxide Nanochains as Sensitive Magnetic Particle Imaging Agents
}

\author{
Kay Hadrick, Michigan State University, hadrickk@msu.edu
}

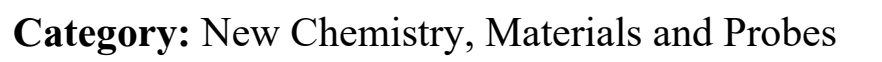

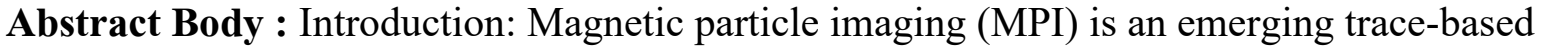

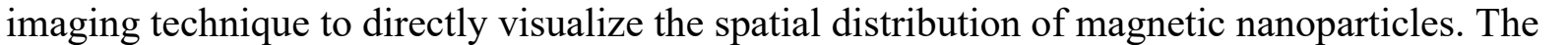

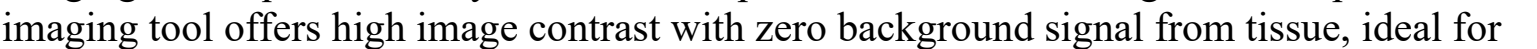

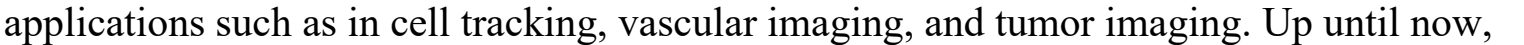

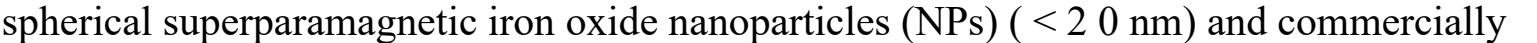

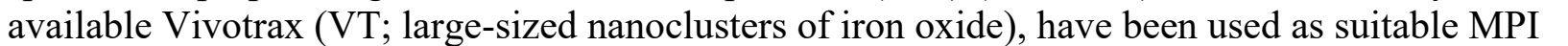

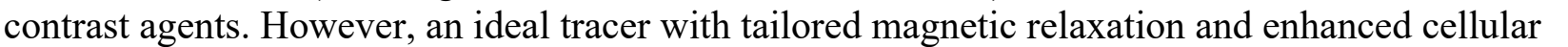
प

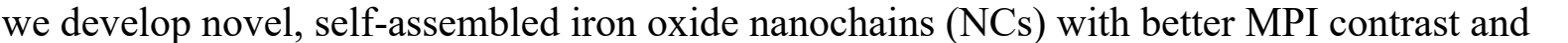

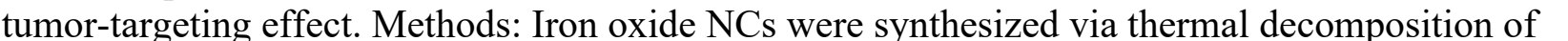

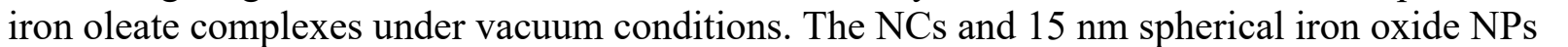

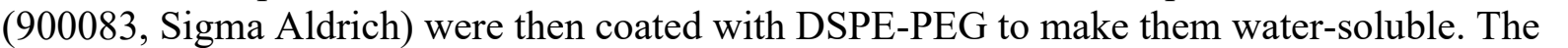

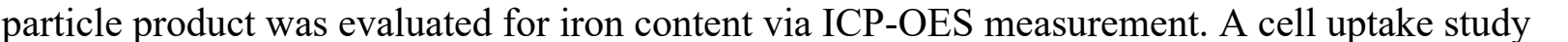

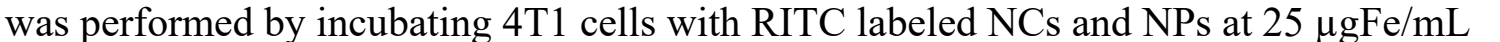

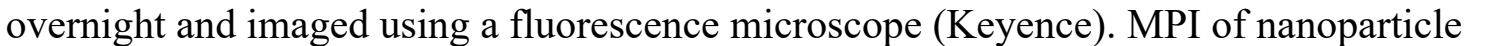

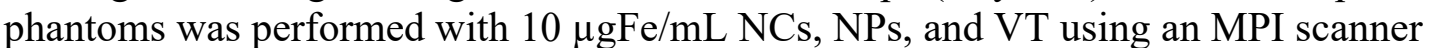

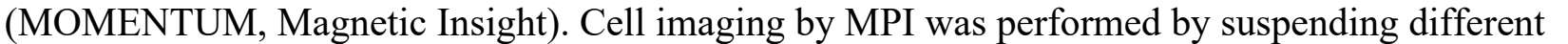

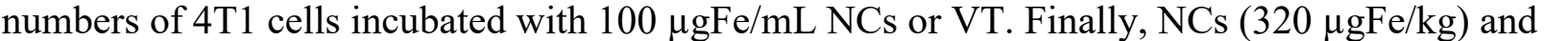

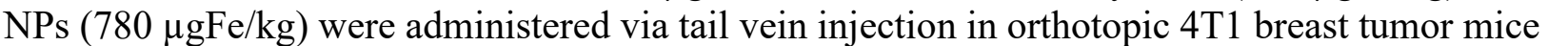

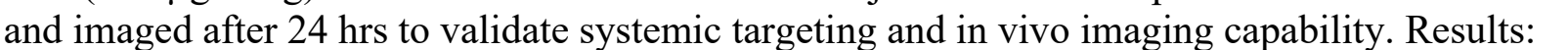

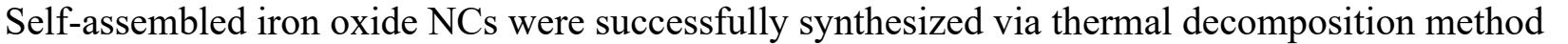
प

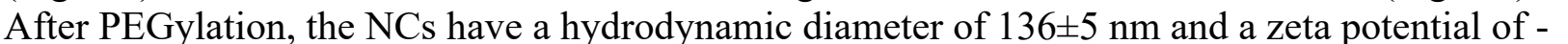

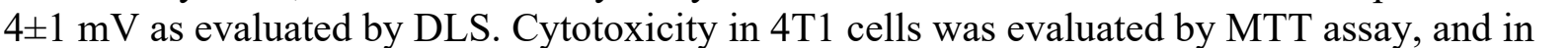

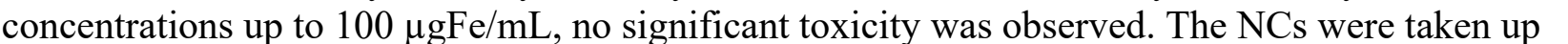

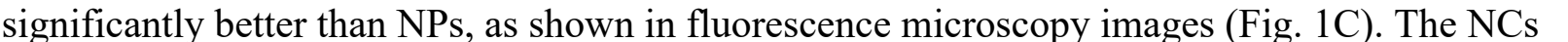

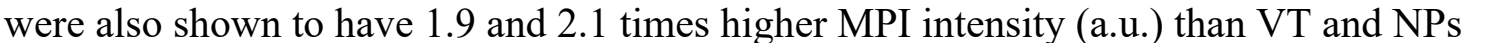

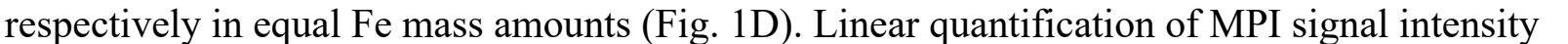

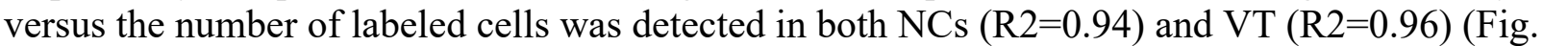

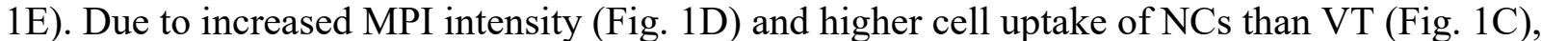
ए

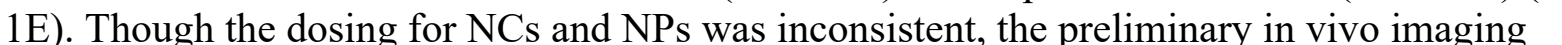

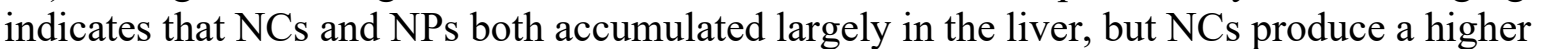

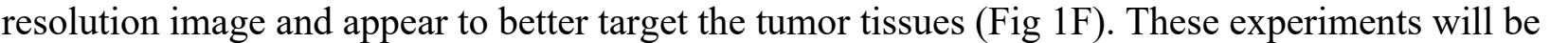

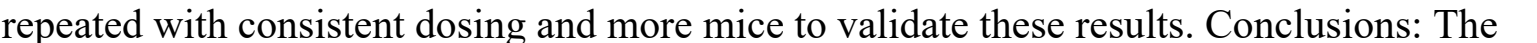

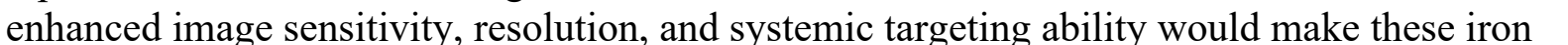




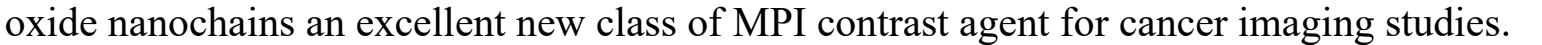

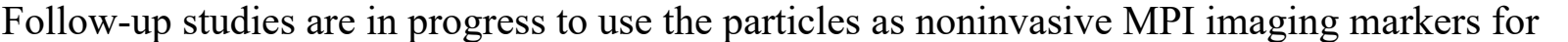

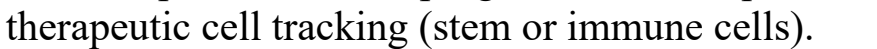

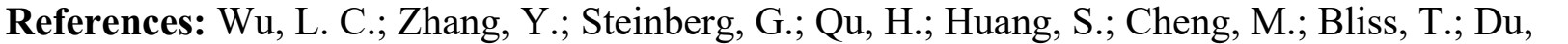

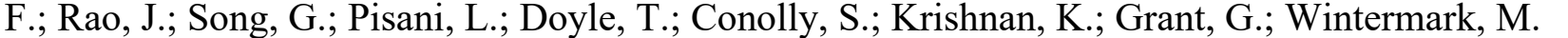

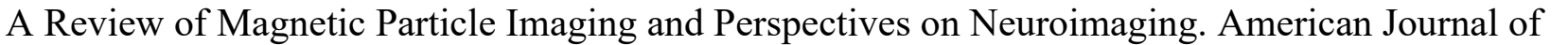

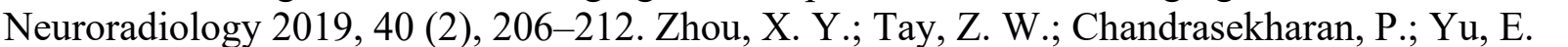

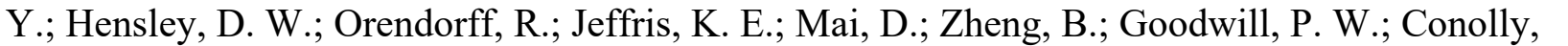

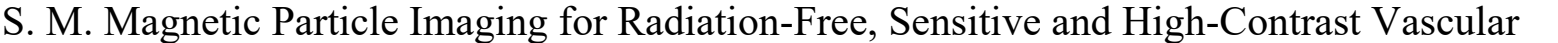

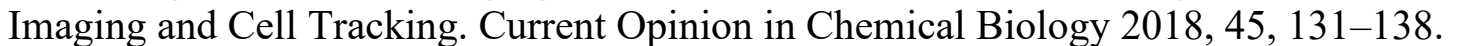

\section{Image/Figure:}

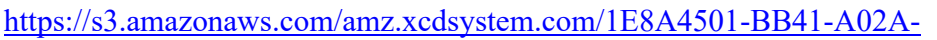

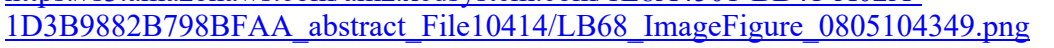

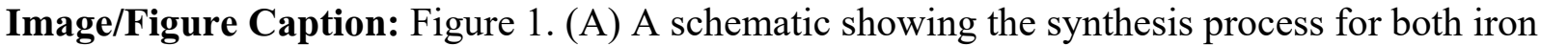

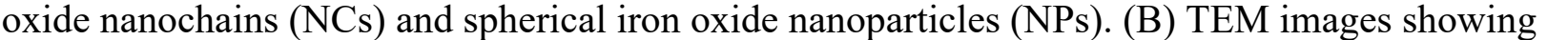

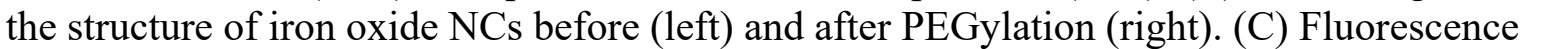

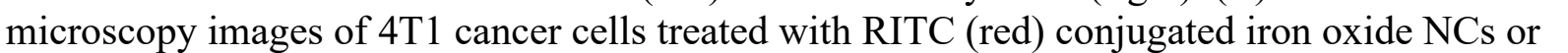

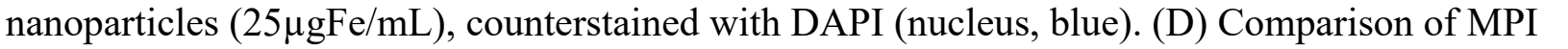

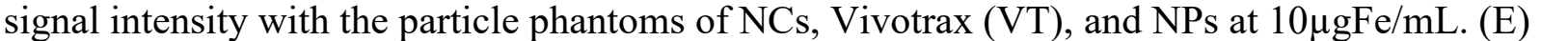

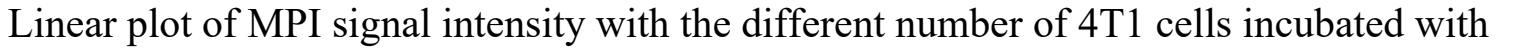

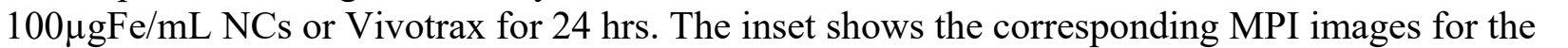

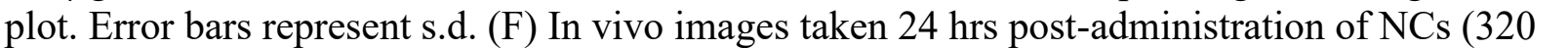

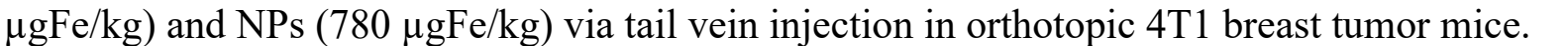

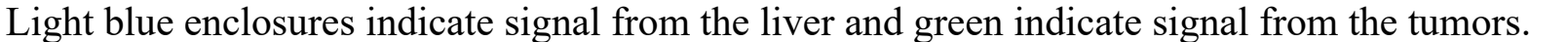

First Name: $\square \square \square \square$

Last Name: $\square \square \square\|\| \| \square$

Email: $\square\|\|\|\| \square \square \square\|\| \square \square$

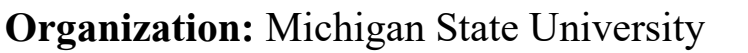

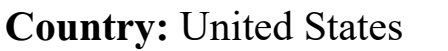




\title{
ID: LB70 \\ Combination Near-Infrared Photoimmunotherapy using Trastuzumab and small protein mimetic for HER2 Overexpressing Cancer
}

Haruka Yamaguchi Takezawa, Nippon Dental University, harukay@ngt.ndu.ac.jp

\section{Category: $\square \square\|\| \sqcap \|$}

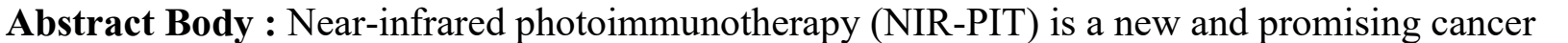

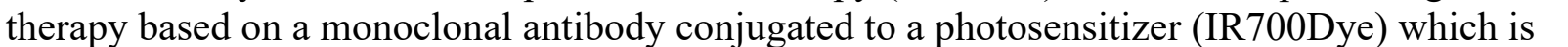

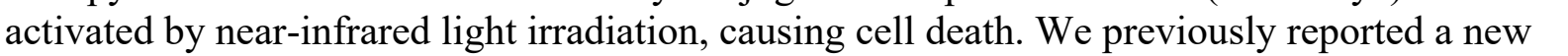

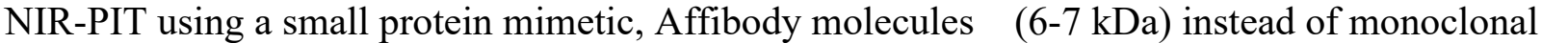

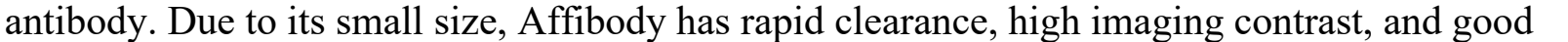

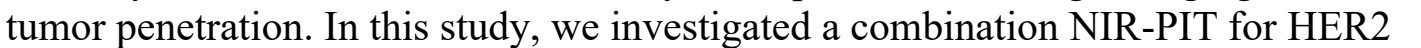

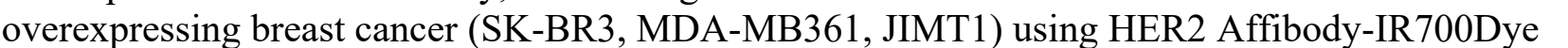

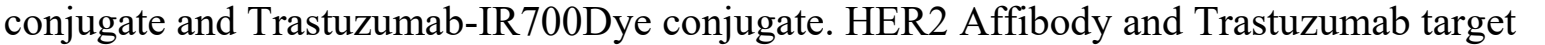

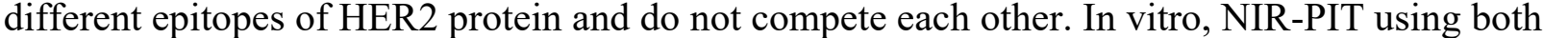

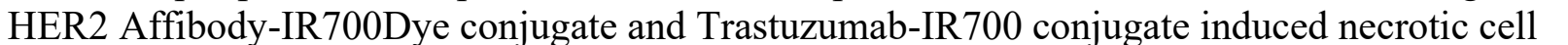
पा山ाm

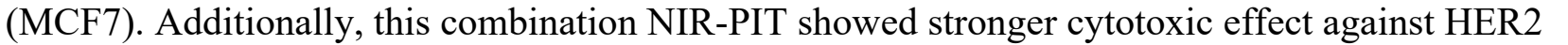

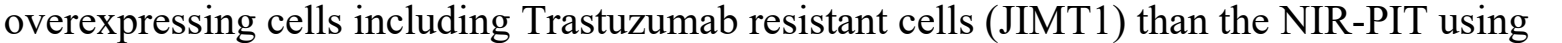

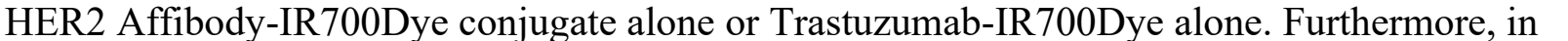

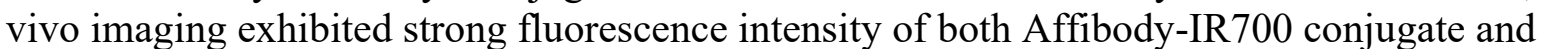

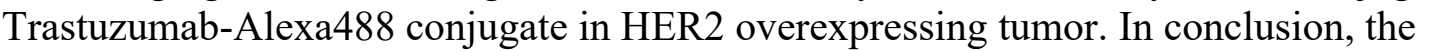

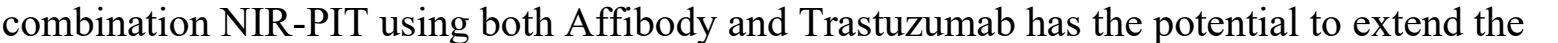

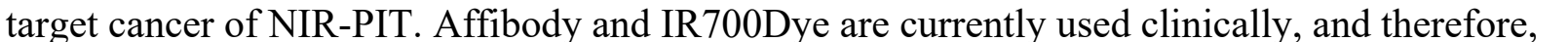

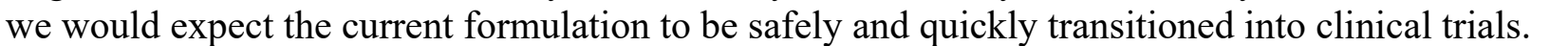

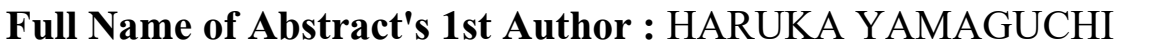

First Name: $\square \square\|1\| m$

Last Name: $\square \square \square\|\|\|\| \square\|\| \square \square \|$

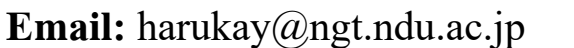

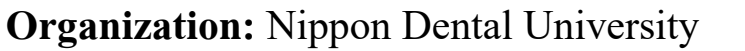

Country: $\square 11110$ 


\title{
ID: LB72 \\ Development of Prostate-Specific Membrane Antigen Targeted Theranostic Nanoparticles to Treat Prostate Cancer Using Boron Neutron Capture Therapy
}

\author{
Niranjan Meher, University of California, San Francisco, niranjan.meher@ucsf.edu
}

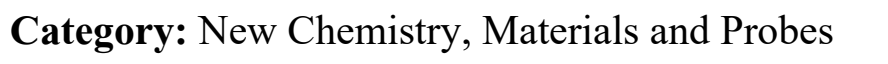

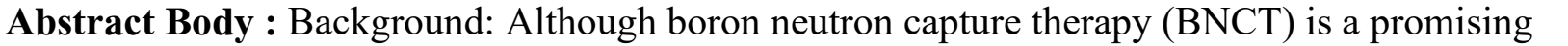

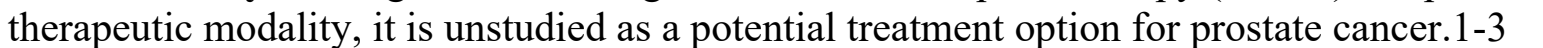

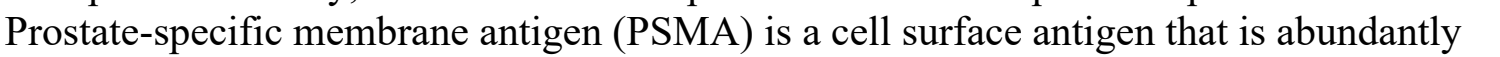

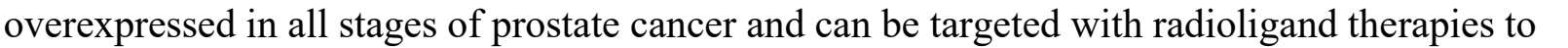
एव

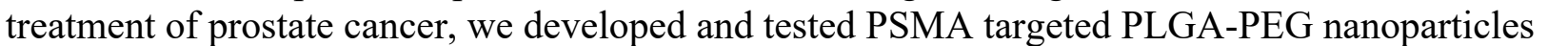

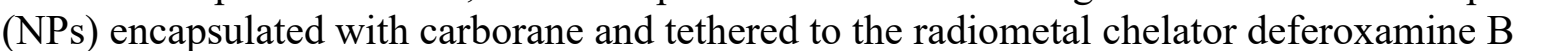

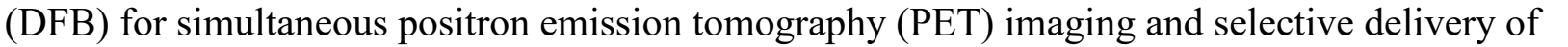

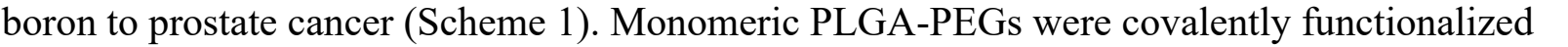

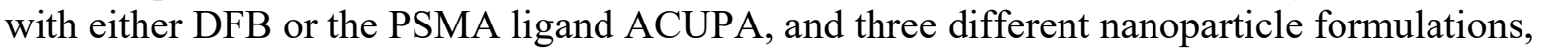

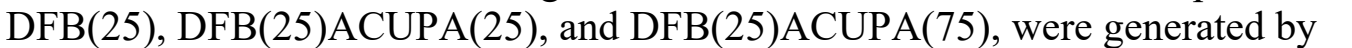

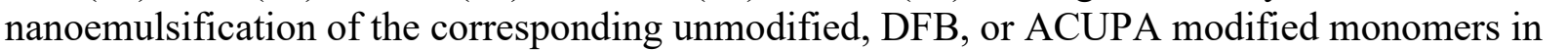

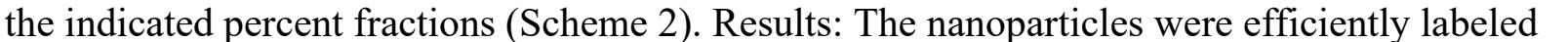

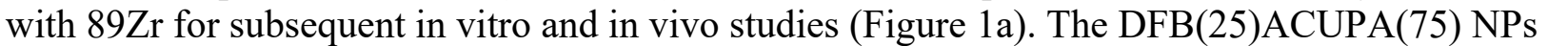

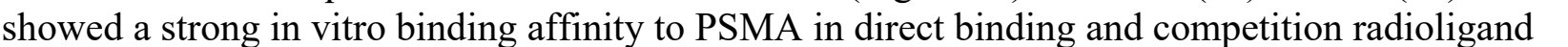

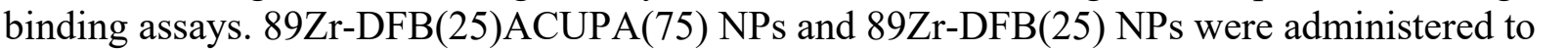

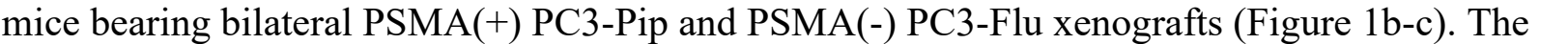

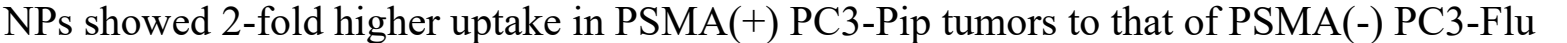
ए

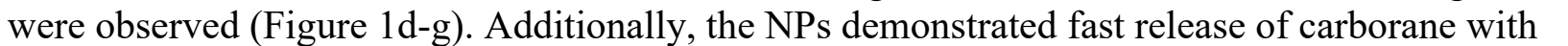
ए ए

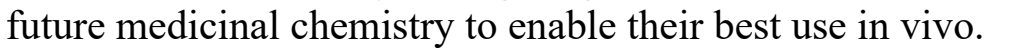

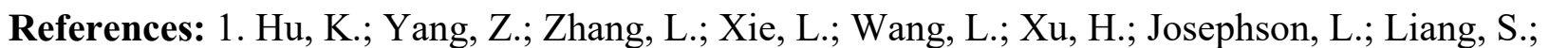

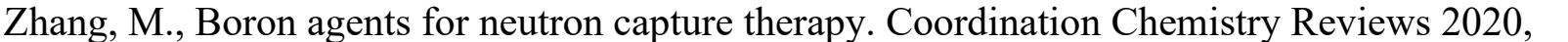

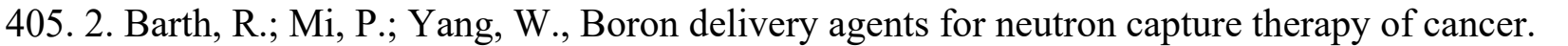

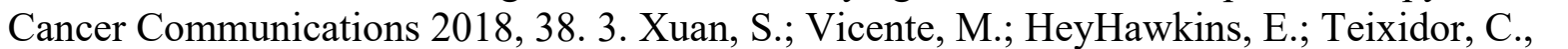

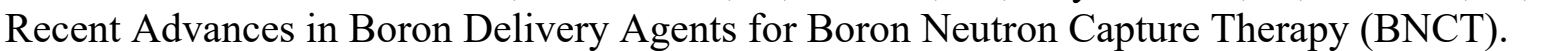

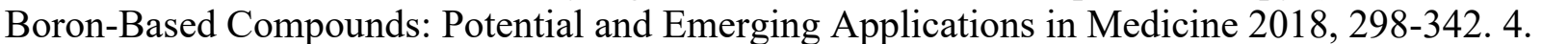

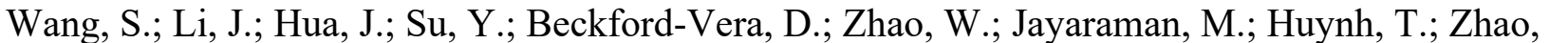

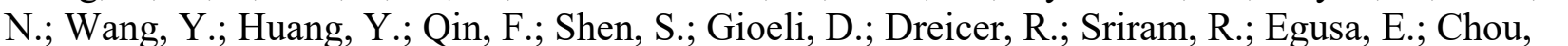
घण

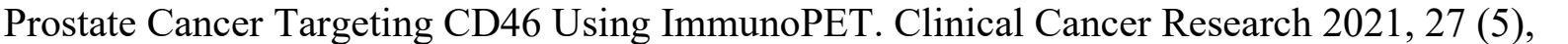

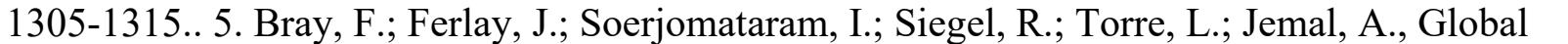

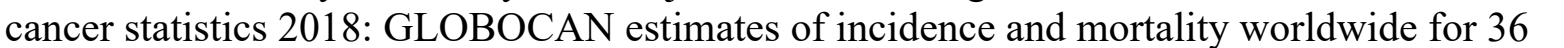




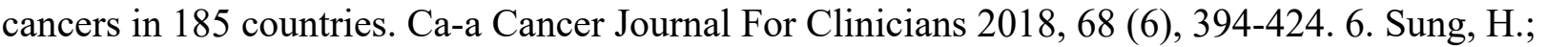

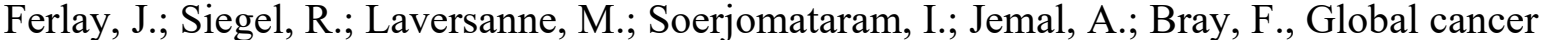

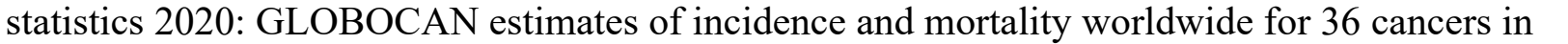

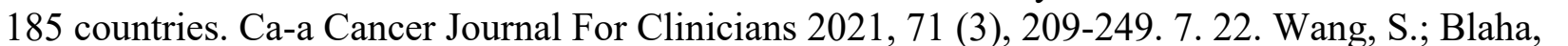

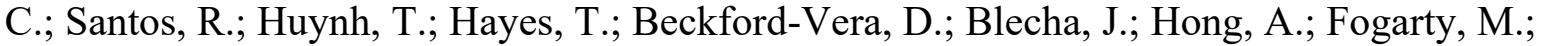

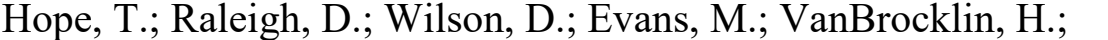

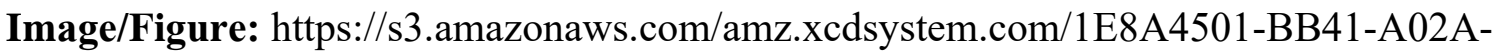

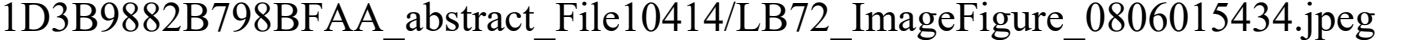

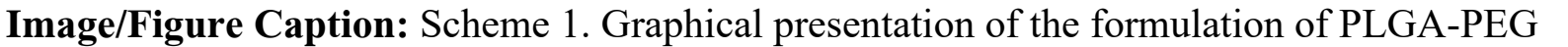

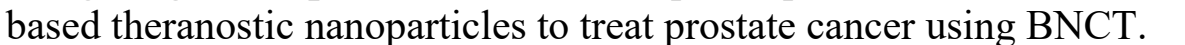

First Name: $\square \square\|\|\|\| \square$

Last Name: $\square \square \square \square \| \square$

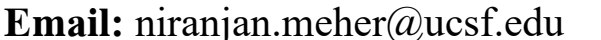

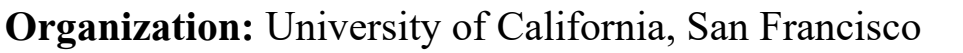

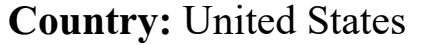




\title{
ID: LB73
}

New MRI contrast agents for high-resolution angiography

Sunghwi Kang, Center for NanoMedicine, Institute for Basic Science (IBS), kangsh931221@yonsei.ac.kr

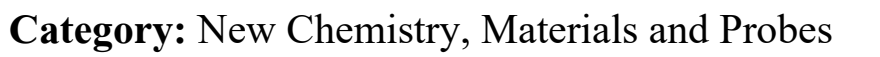

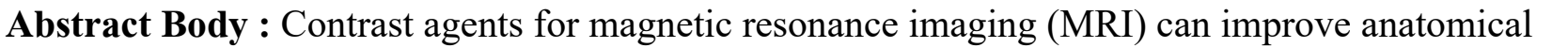

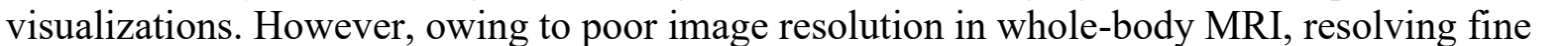

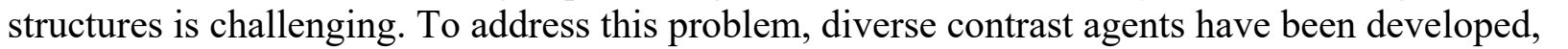

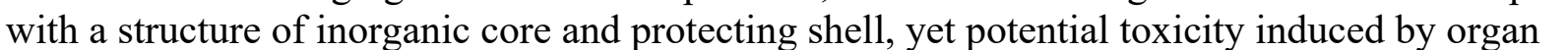

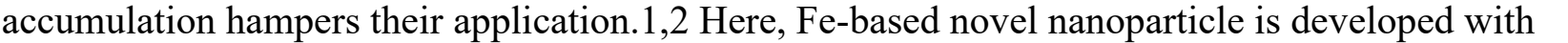

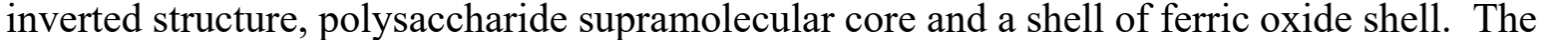

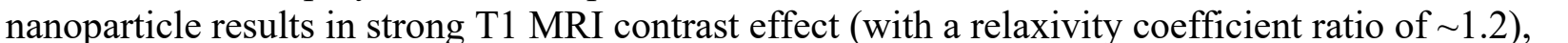

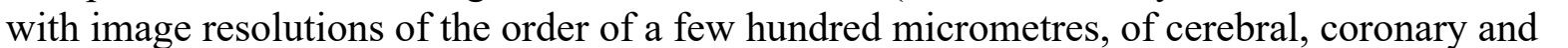

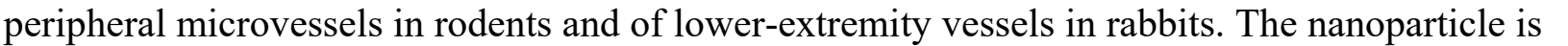

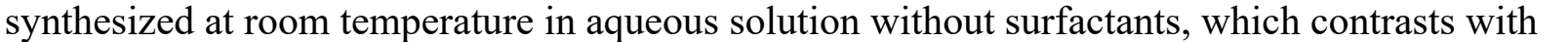

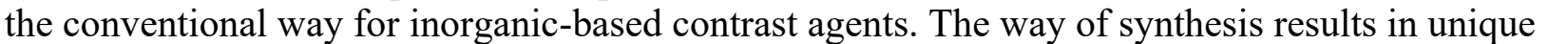

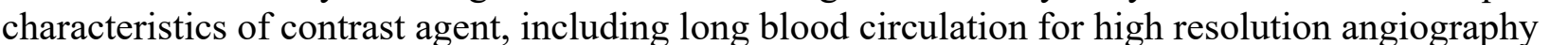

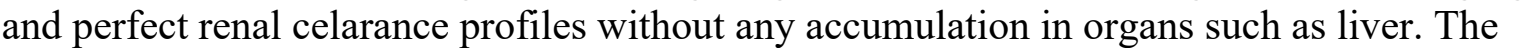

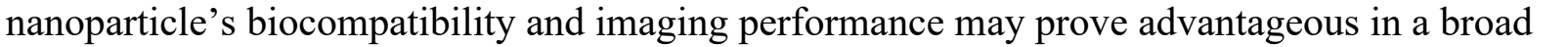

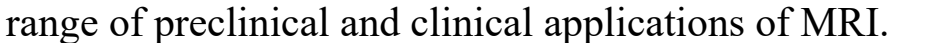

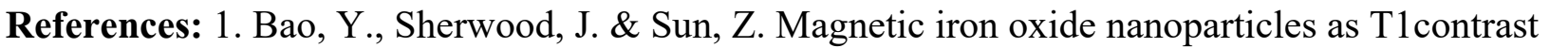

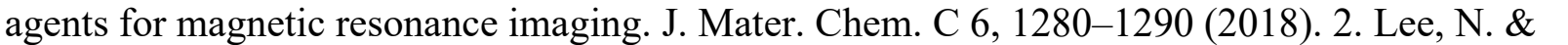

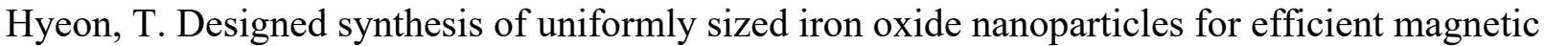

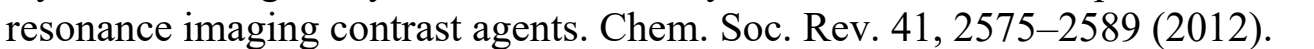

First Name: $\square \square \square \square \square \square$

Last Name: $\square \square \square \square$

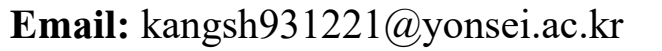

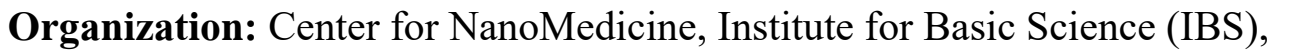

Country: $\square \square|\square| m \square \square \| m$ 


\title{
ID: LB74 \\ Evaluation by intravital fluorescent imaging of the angiogenic potential of endothelial cells derived from human pluripotent stem cells (hPSC-ECs) after ischemic injury in mice
}

\author{
Valerie Rouffiac, Gustave Roussy, Université Paris-Saclay, UMS AMMICa INSERM \\ US23-CNRS 3655., valerie.rouffiac@gustaveroussy.fr
}

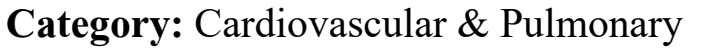

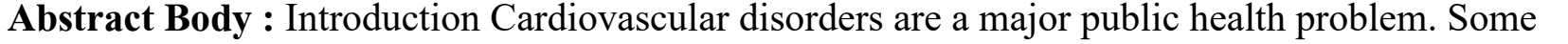

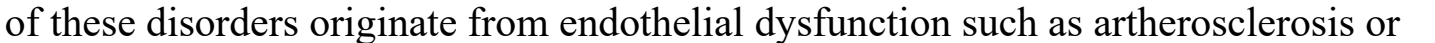

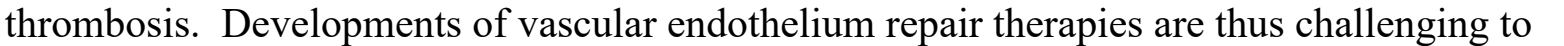

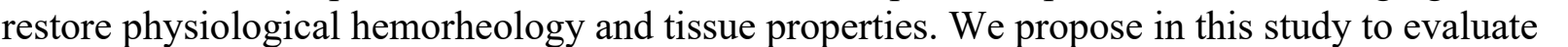

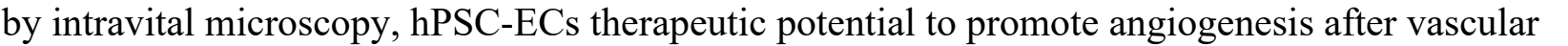

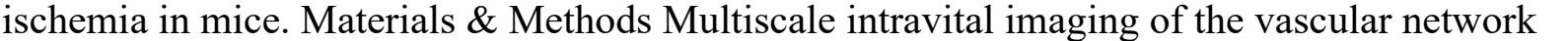

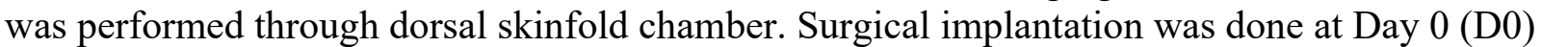

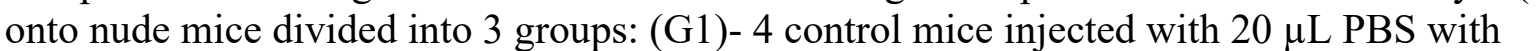

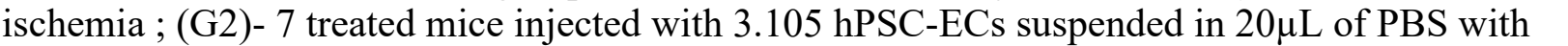

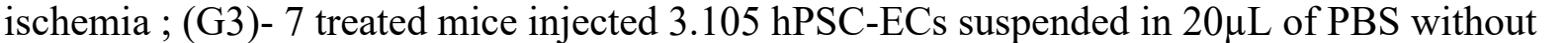
ए

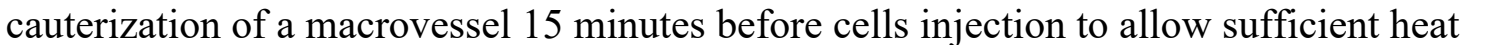

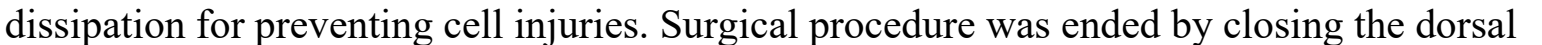

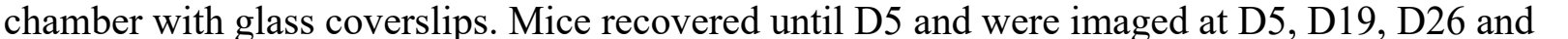

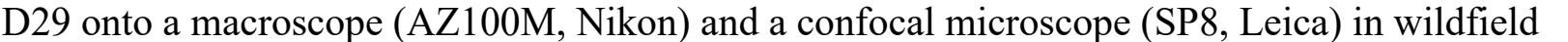

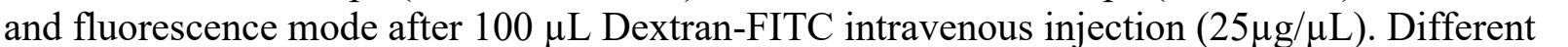

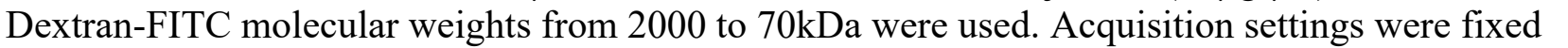

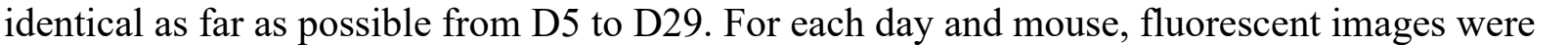

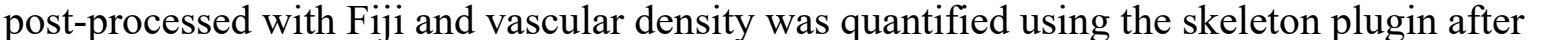

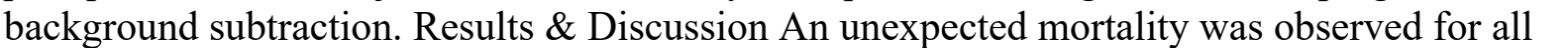

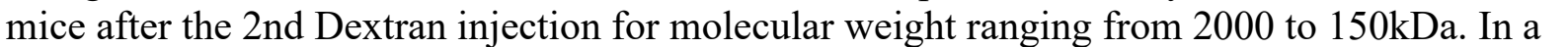

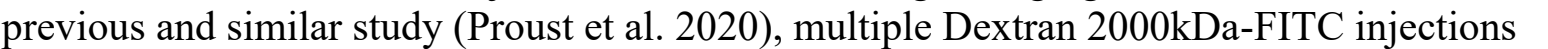

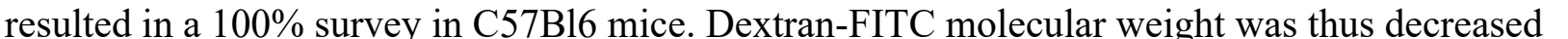

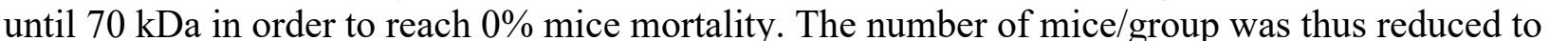

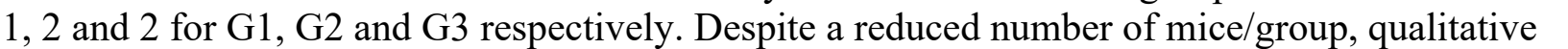

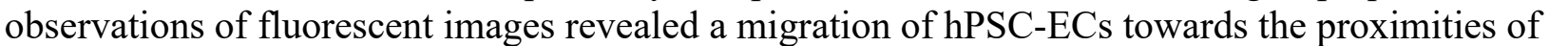

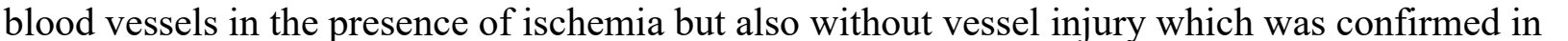

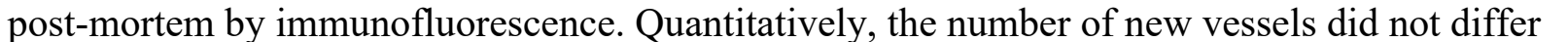

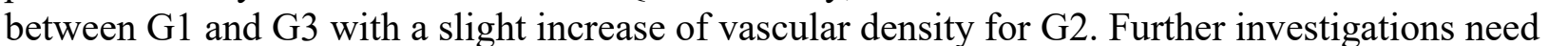

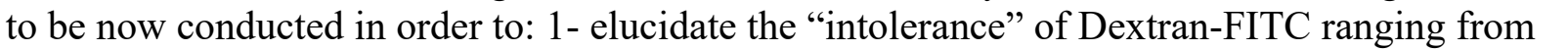

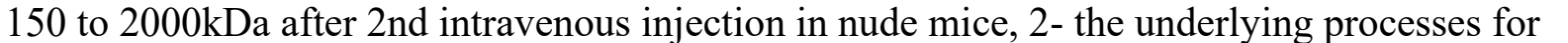

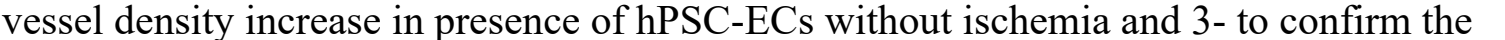

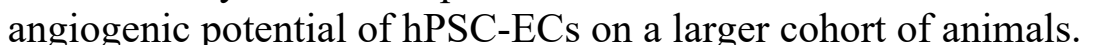


First Name: $\square \square \square m \mid m$

Last Name: $\square \square \square\|\| \| \square$

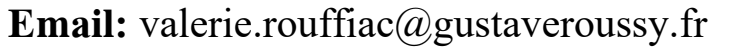

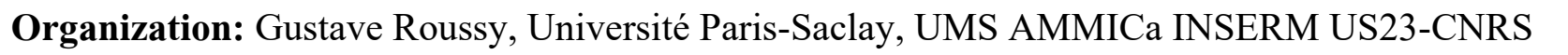

Country: $\square \square\|\| \| \square$ 


\title{
ID: LB75 \\ Purified PHIP hyperpolarized pyruvate and fumarate for in vivo metabolic MRI \\ Stephan Knecht, NVision Imaging GMBH, stephan@nvision-imaging.com
}

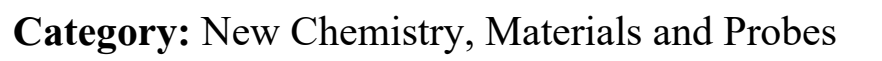

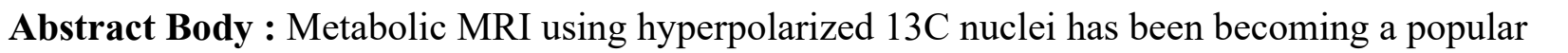

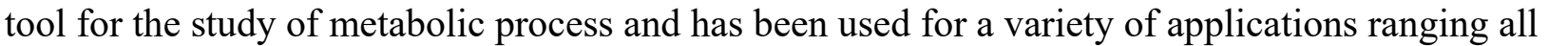

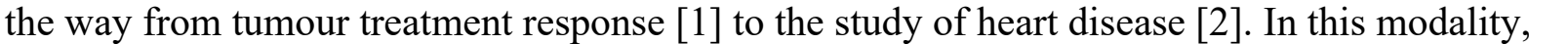

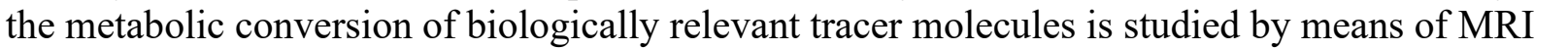

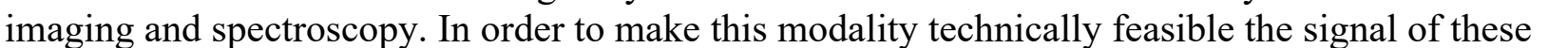

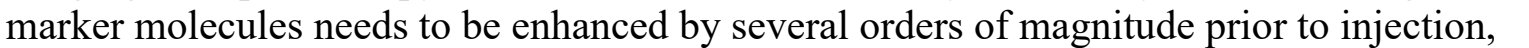

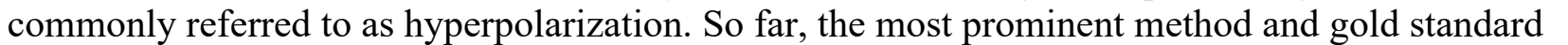

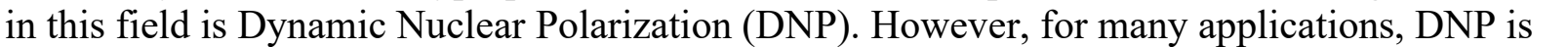

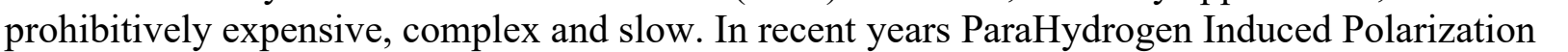

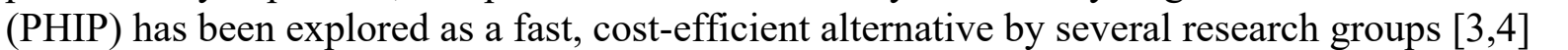

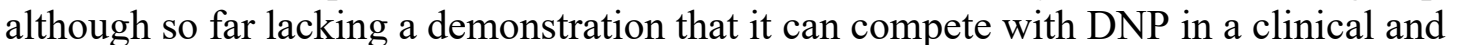

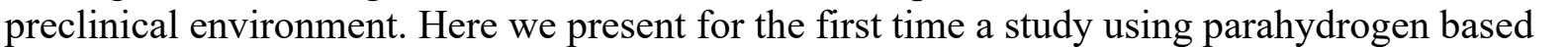

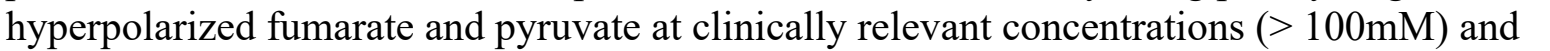

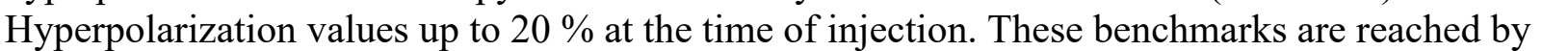

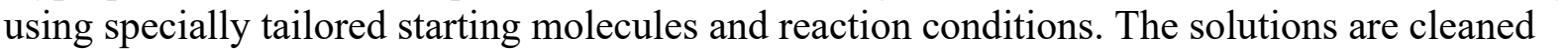

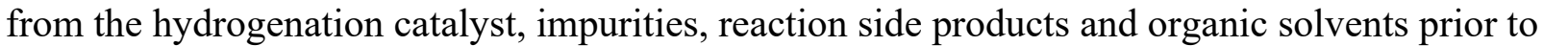

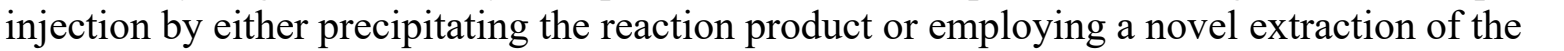

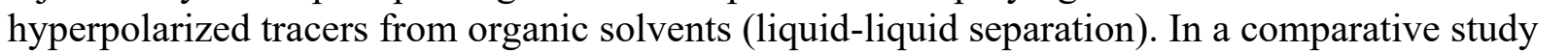

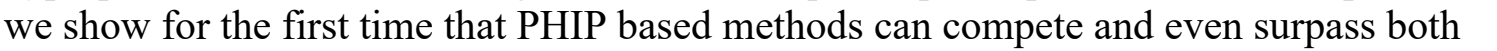

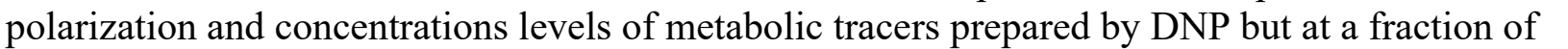

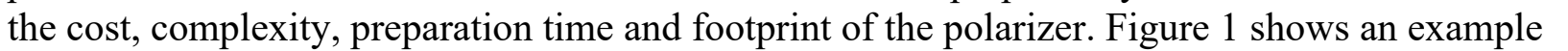

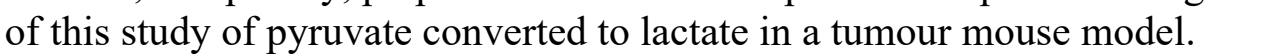

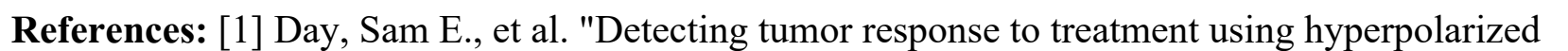

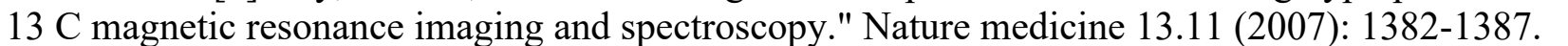

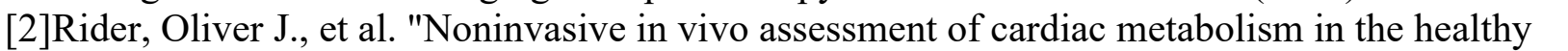

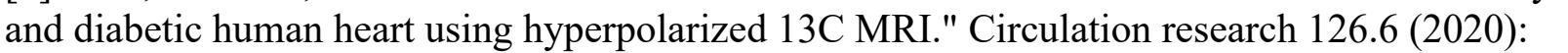

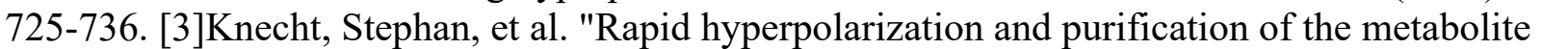

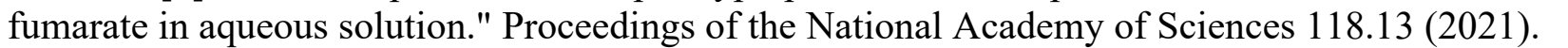

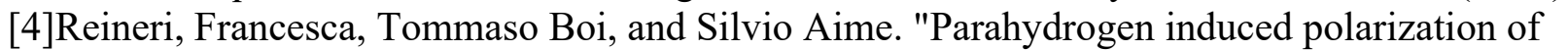

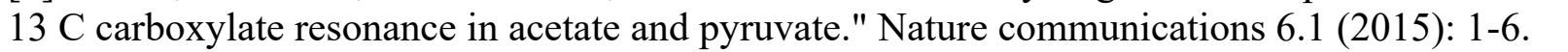

\section{Image/Figure:}

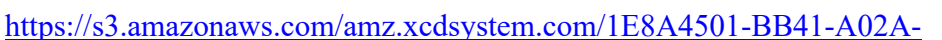

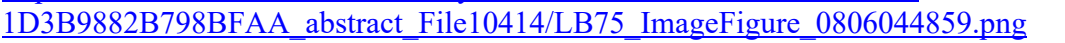




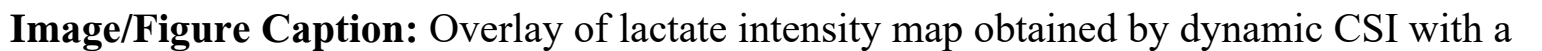

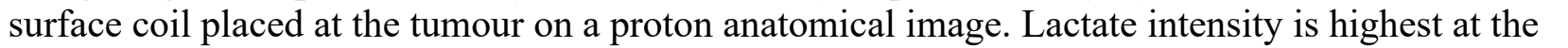
एणमाण

First Name: $\square \square\|\square\| \|$

Last Name: $\square \square \square \square \square \square$

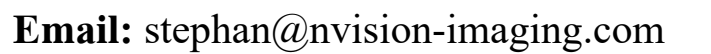

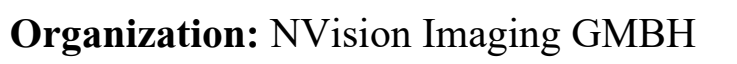

Country: 


\title{
ID: LB76 \\ Feasibility of the Noninvasive Gene Delivery to Large Brain Areas
}

\section{Shirin Nouraein, Rice University, sn54@rice.edu}

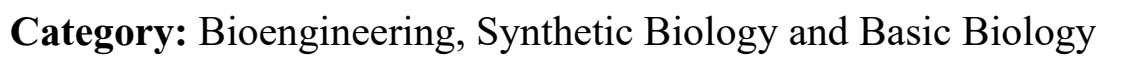

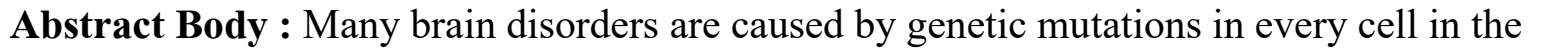

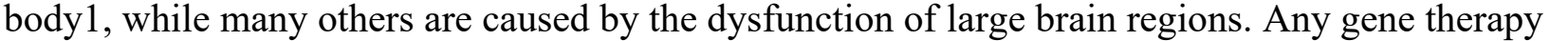

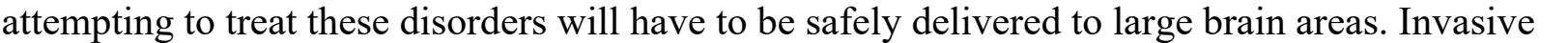

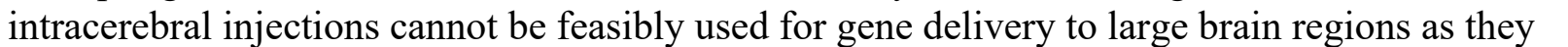

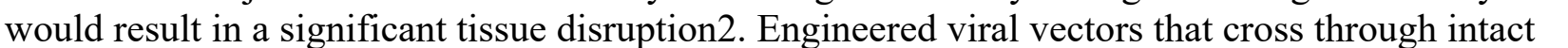

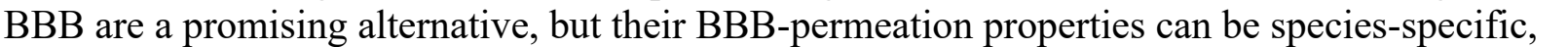

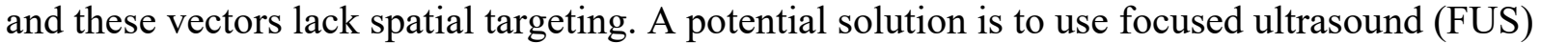
ए

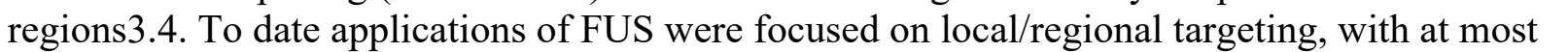

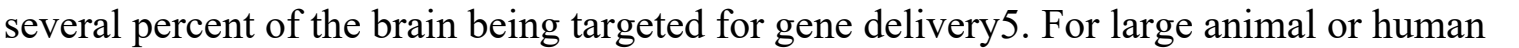

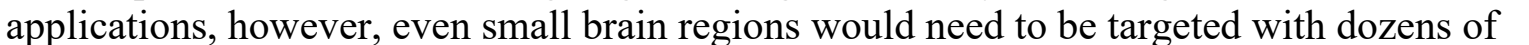

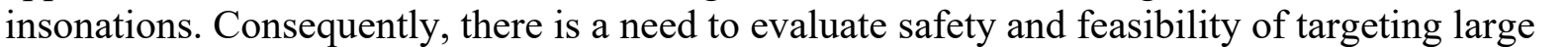

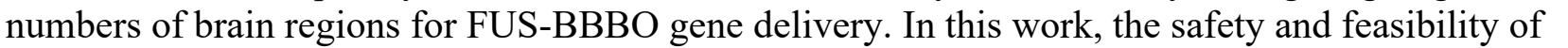

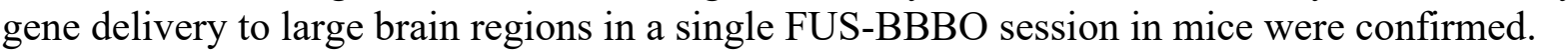
ए

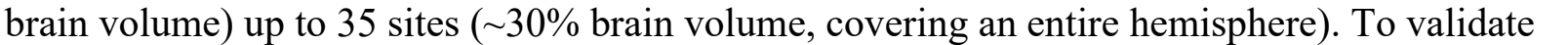

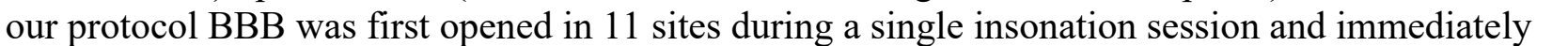

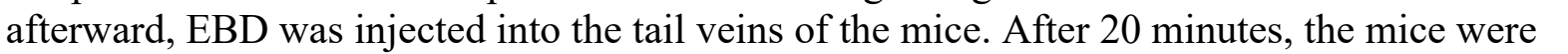

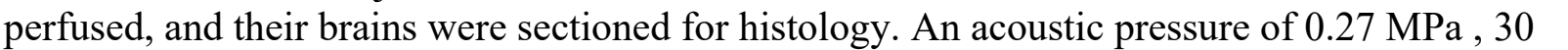

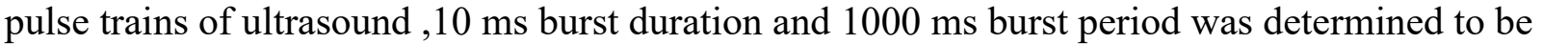

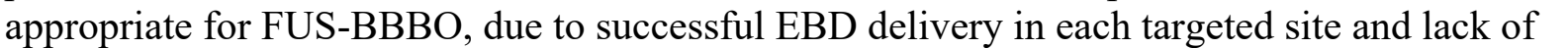

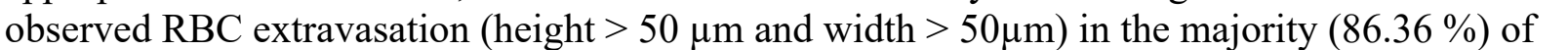

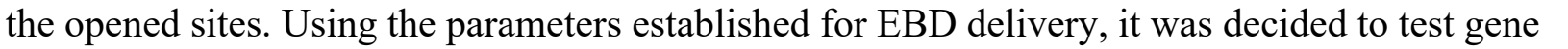

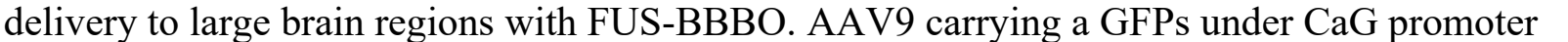

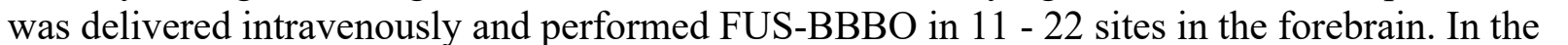

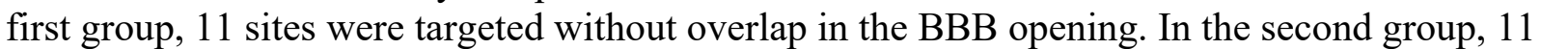

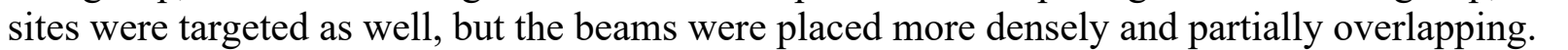

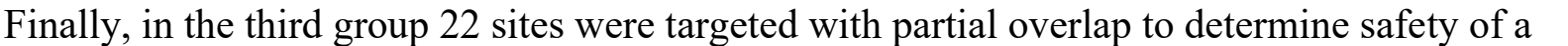

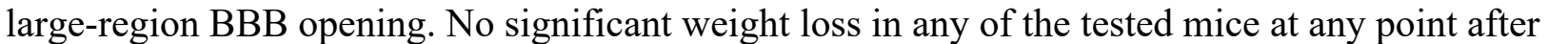
ए

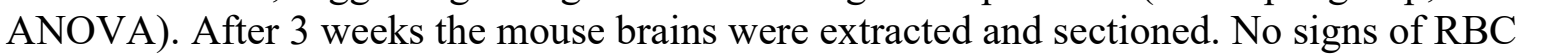

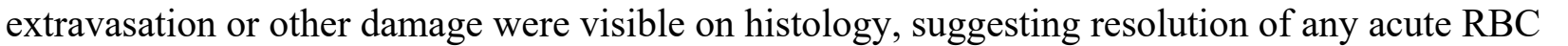

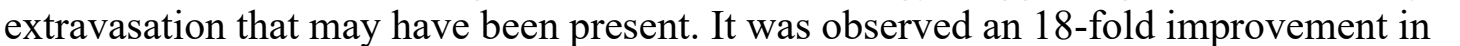

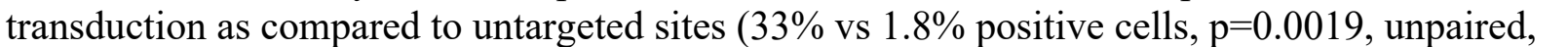

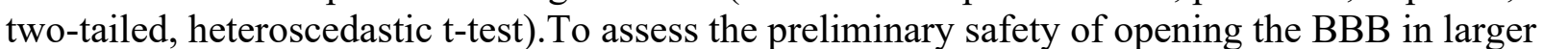

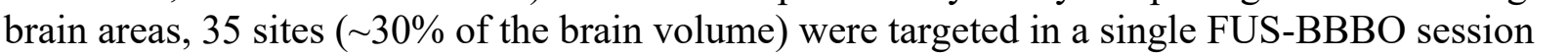

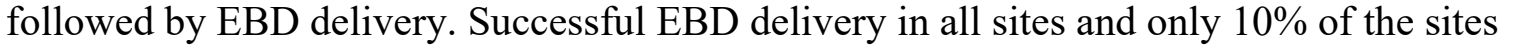




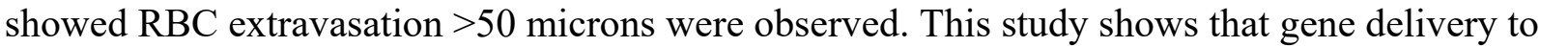

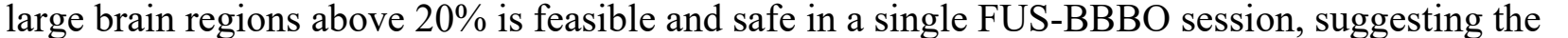

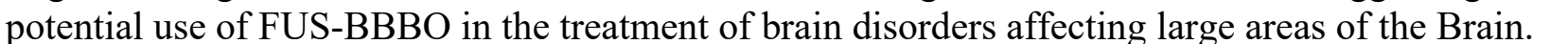

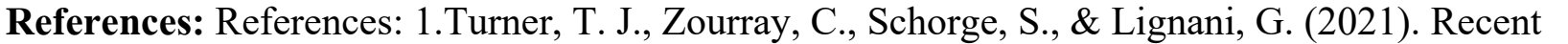

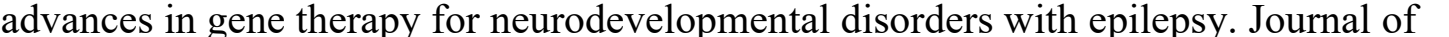

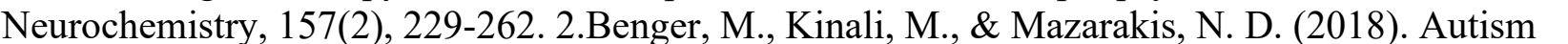

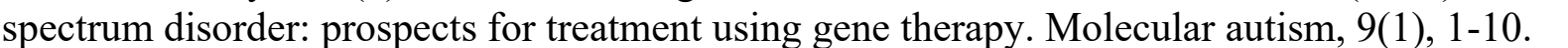

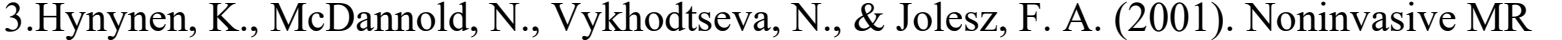

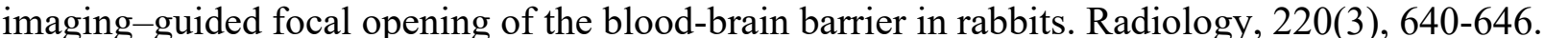

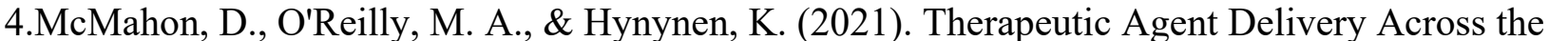

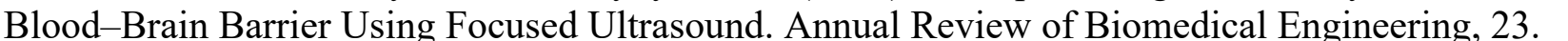

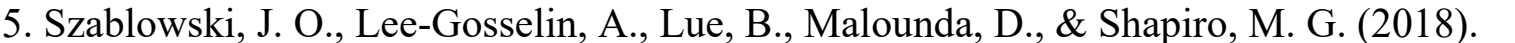

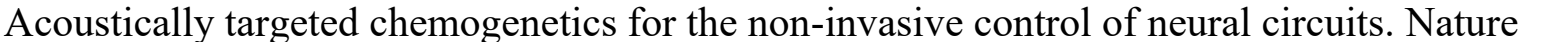

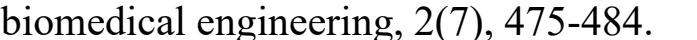

\section{Image/Figure:}

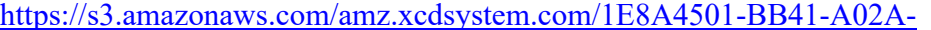

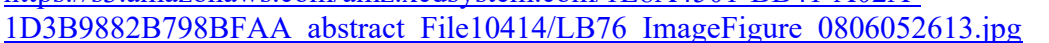

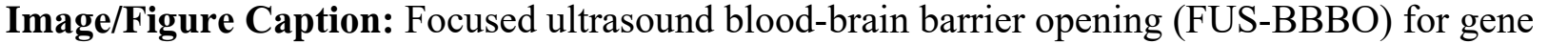

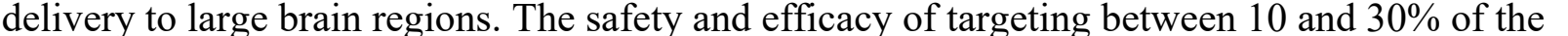

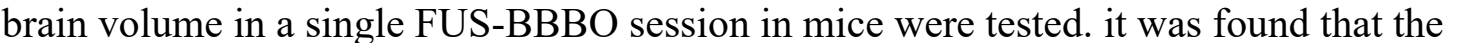

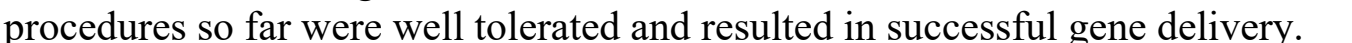

First Name: $\square \square \square \| m||$

Last Name: $\square \square \square\|\| \| m$

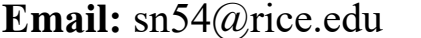

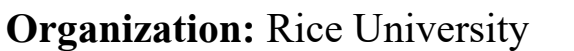

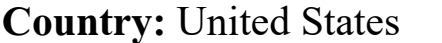




\title{
ID: LB77 \\ Evaluation of novel anti-CD8 immuno-PET agent for non-invasive imaging of solid organ rejection in a non-human primate kidney transplant study
}

Dangshe Ma, Regeneron Pharmaceuticals, dangshe.ma@regeneron.com

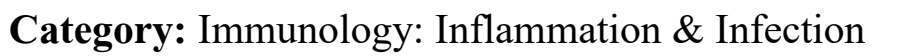

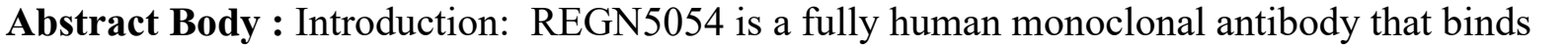

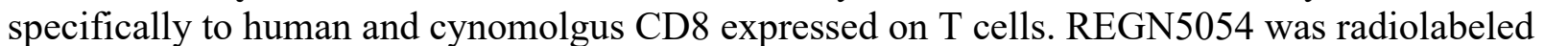

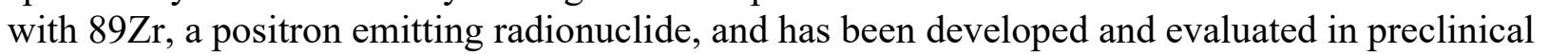

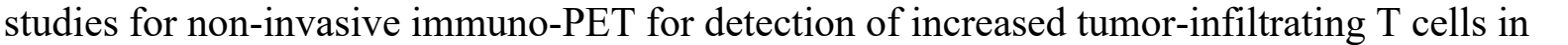

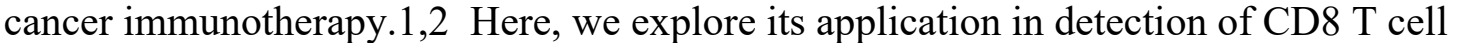

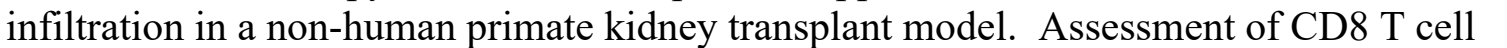
ए

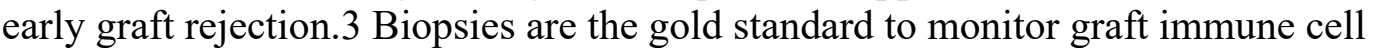

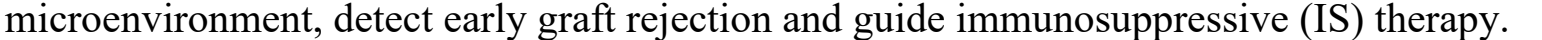

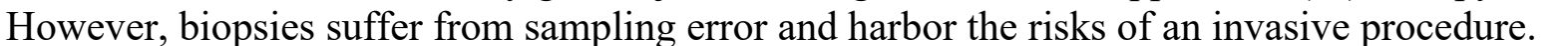

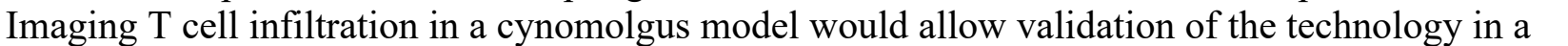

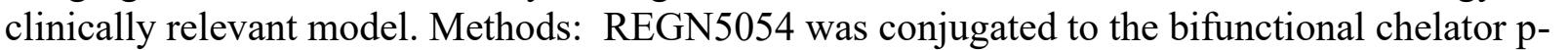

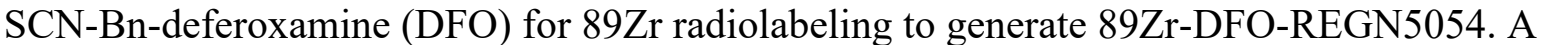

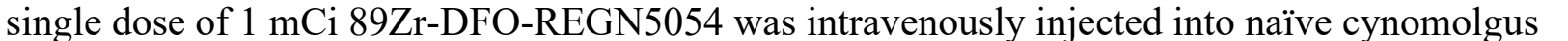

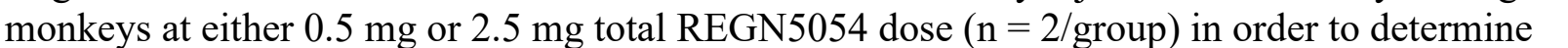

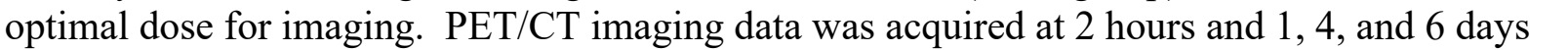

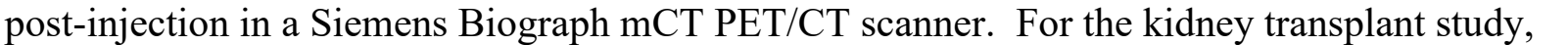

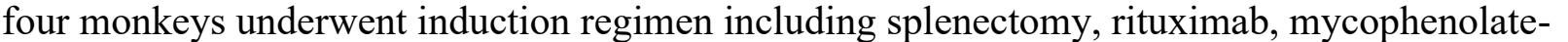

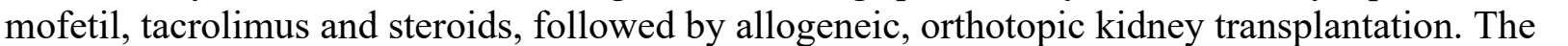

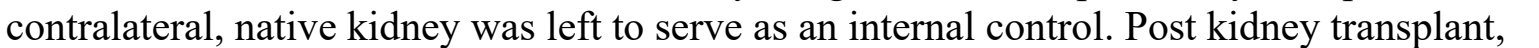

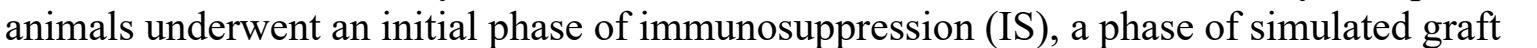
प पाmए

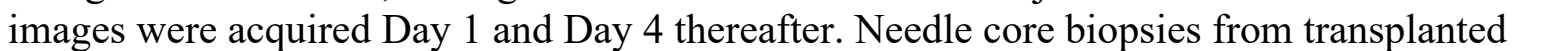

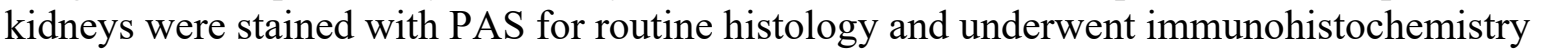

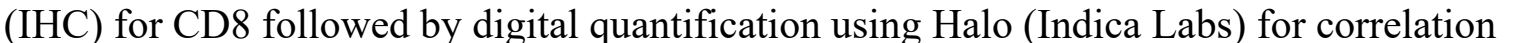

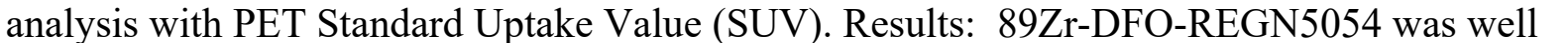
पाm

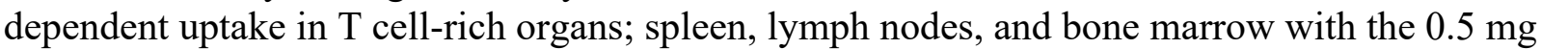

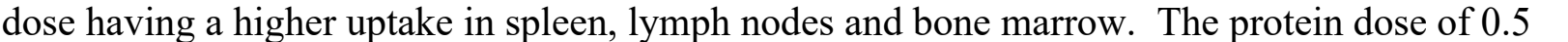

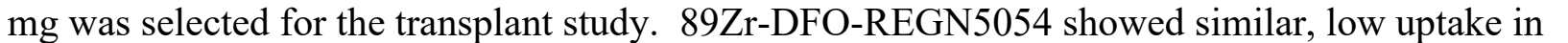

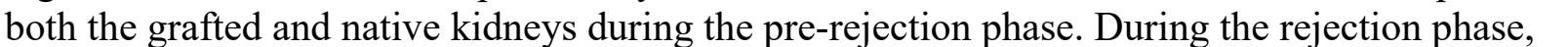

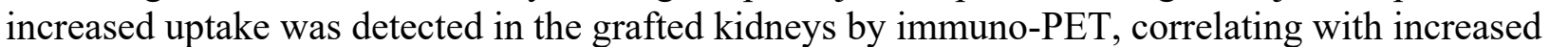
ए

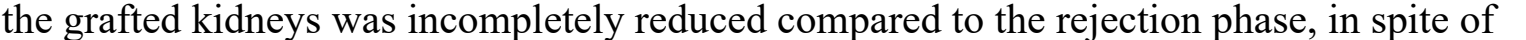

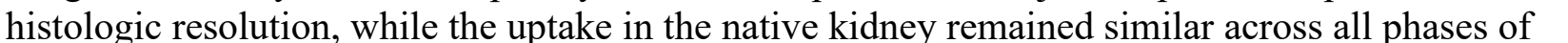




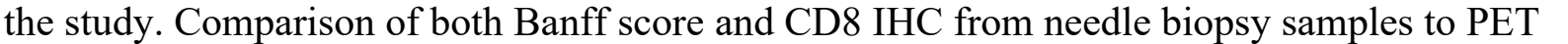

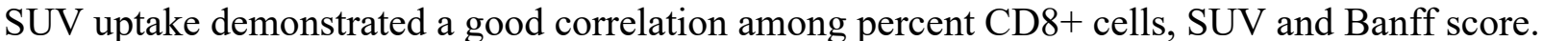

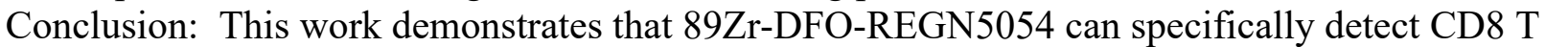

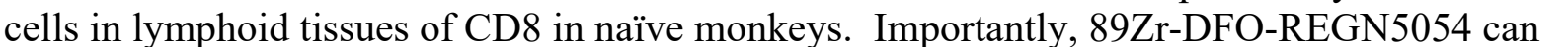

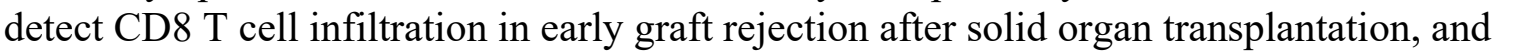

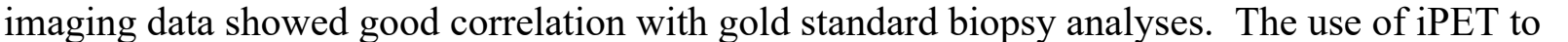
ए

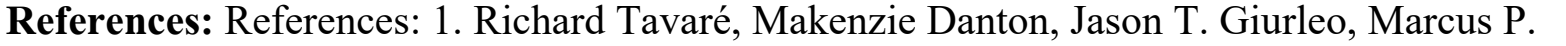

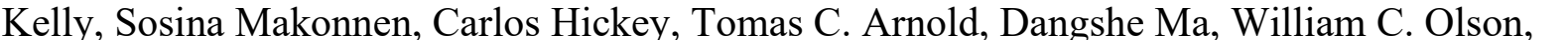

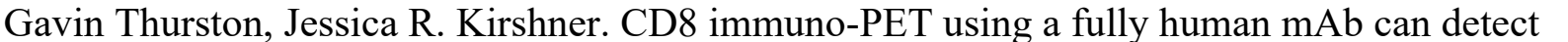

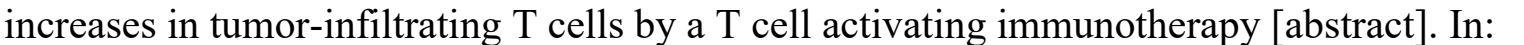

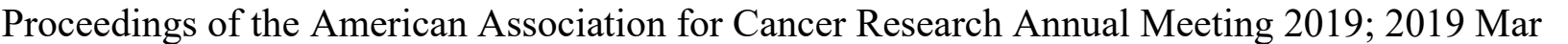

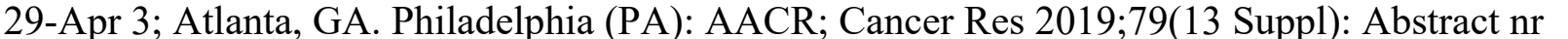

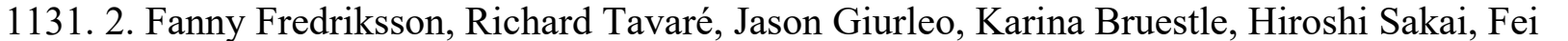

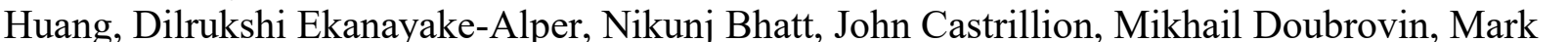

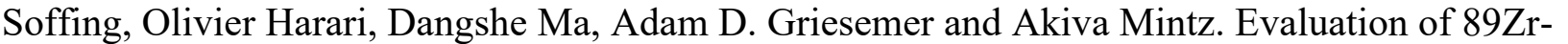

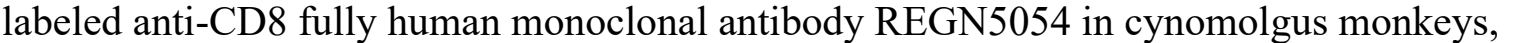

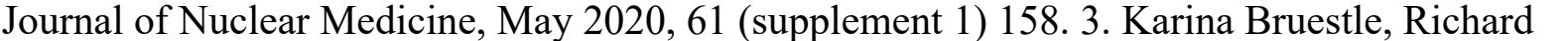

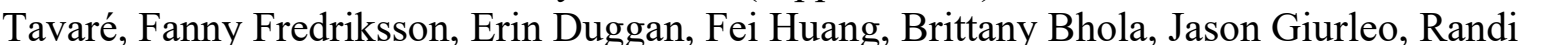

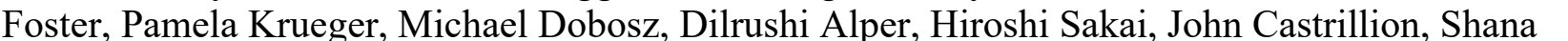

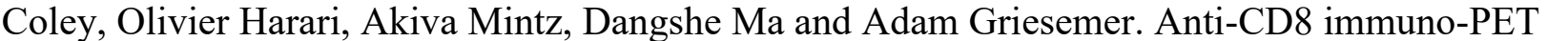

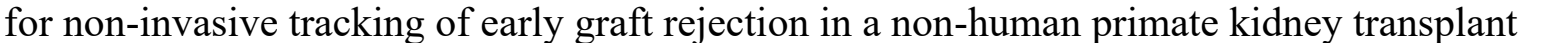

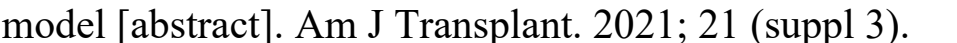

First Name: $\square \square\|\|\|\square\|$

Last Name: $\square \square \square$

Email: $\square\|\|\|\square \square \square \square \square\|\|\| \square \| \square \square$

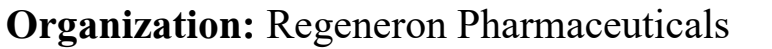

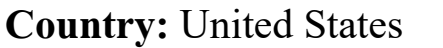




\title{
ID: LB78 \\ Atherothrombosis-on-chip: a site-specific in vitro microfluidic model for observing real-time disease progression and drug discovery
}

Fahima Akther, The university of Queensland, s4553538@student.uq.edu.au

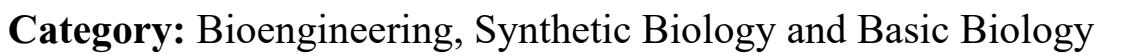

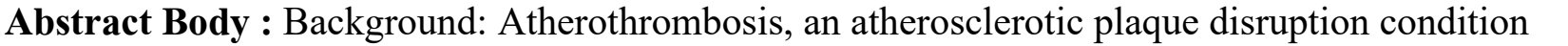

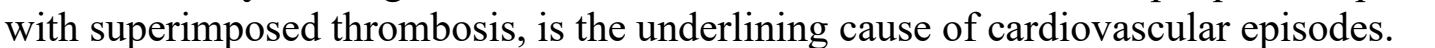

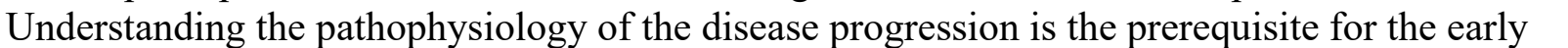

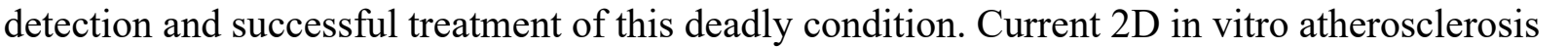

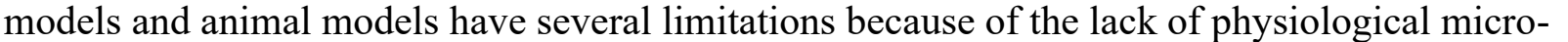

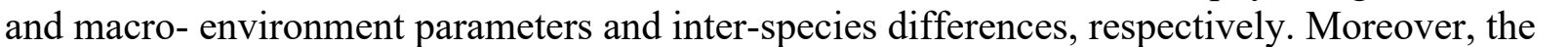

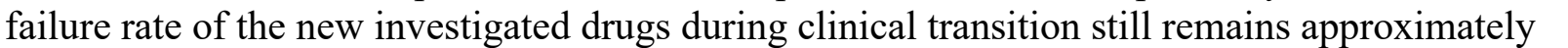

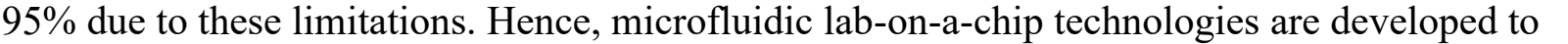
प

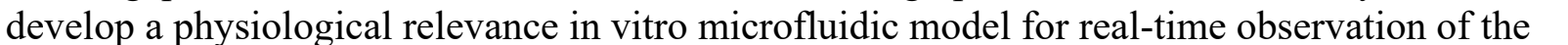

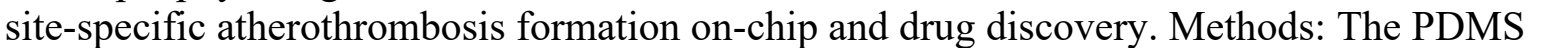

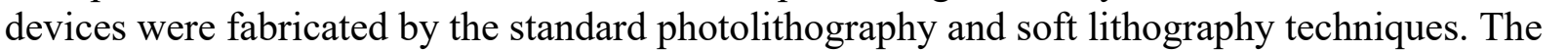

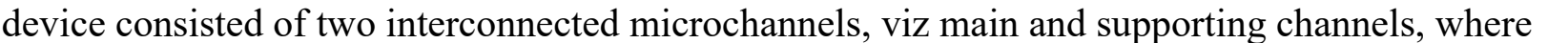

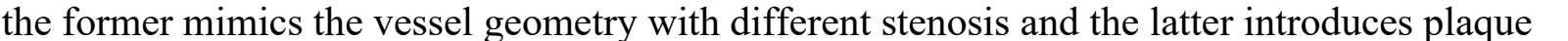

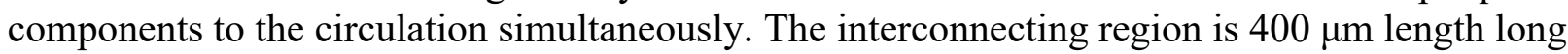
with a pore size of $2 \mu \mathrm{m}$. Devices with different stenosis were fabricated by reducing the width

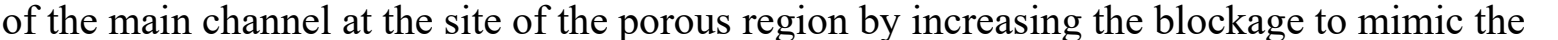

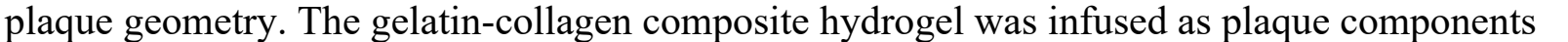

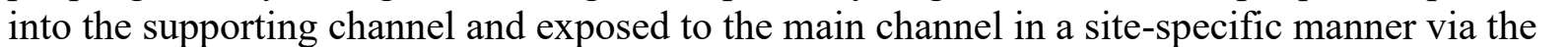

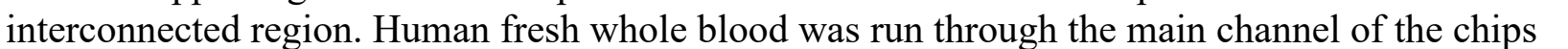

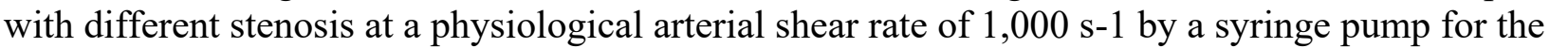

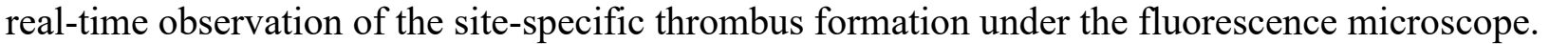

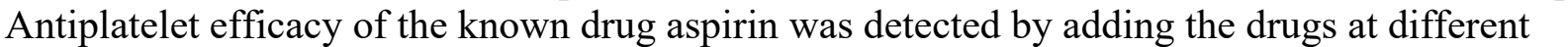

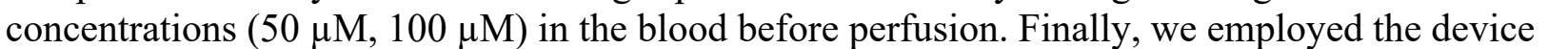

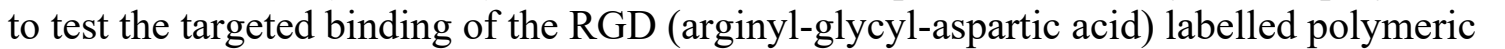

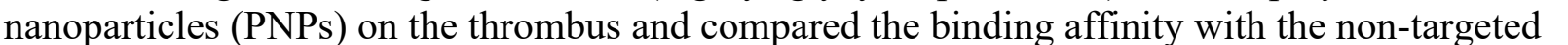

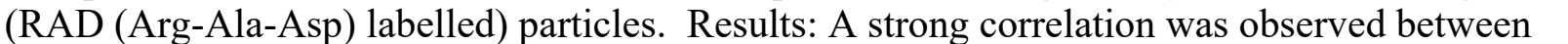

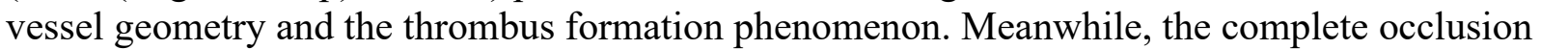

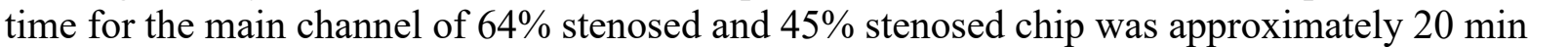

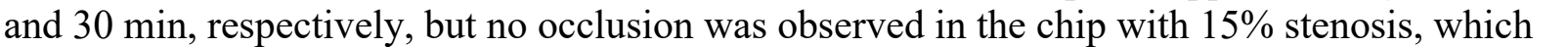

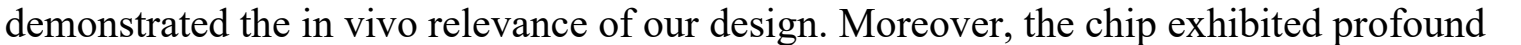

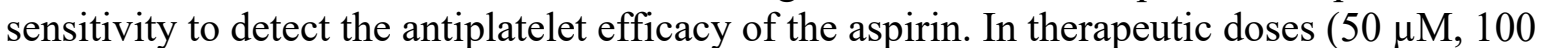

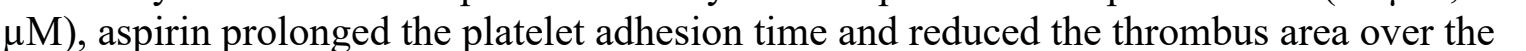

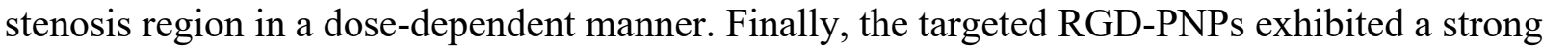

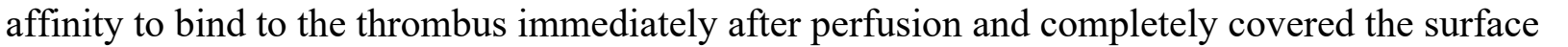

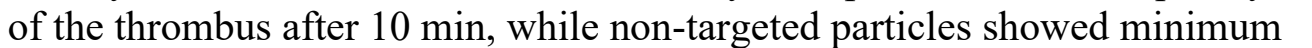




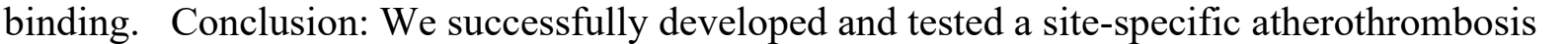

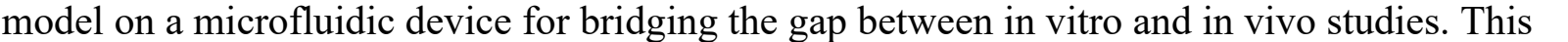

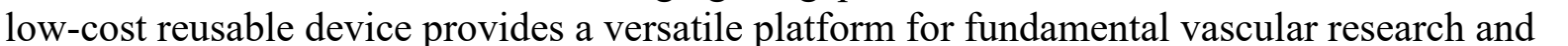

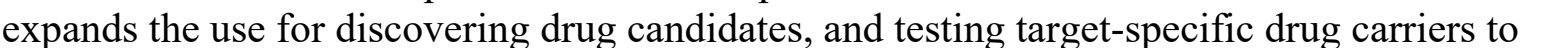

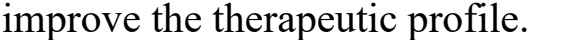

\section{Image/Figure:}

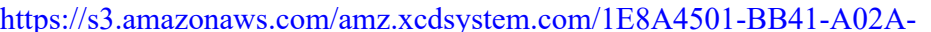

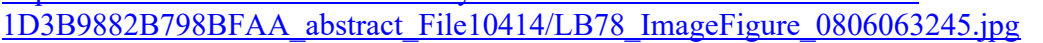

Image/Figure Caption:

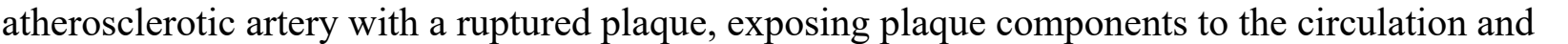

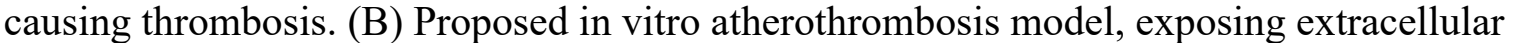

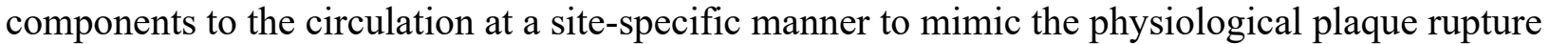

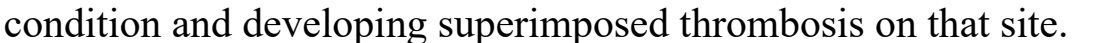

Full Name of Abstract's 1st Author : $\square \square\|\square \square \square \square \square \square\| \|$

First Name: $\square \square \square \square \square \square$

Last Name: $\square \square\|\|\|\|$

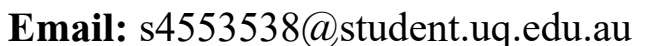

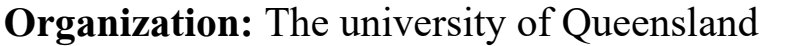

Country: $\square \square\|m\| n \| m \mid \square$ 


\title{
ID: LB79 \\ Gold-iron oxide nanoparticle: A multi-modality theranostic agent for site-specific thrombus imaging and enhanced thrombolysis
}

Najma Fithri, Monash University, najma.fithri@monash.edu

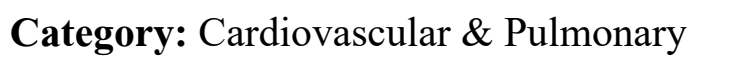

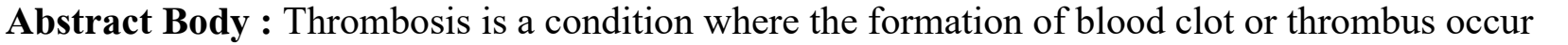
ए

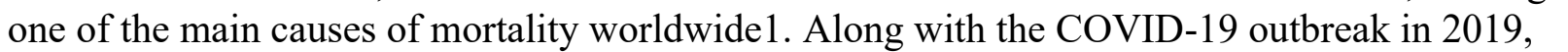

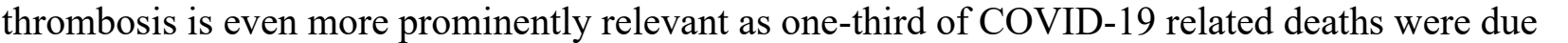

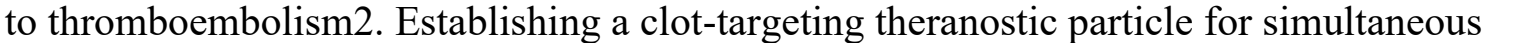

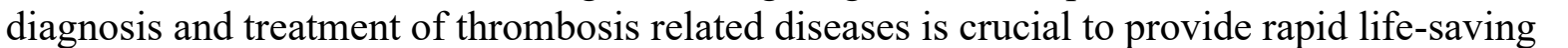

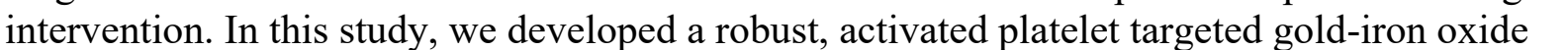

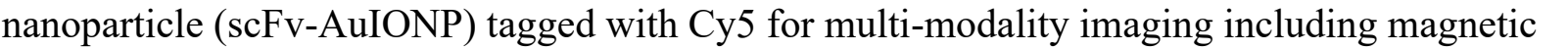

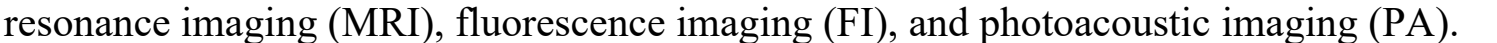

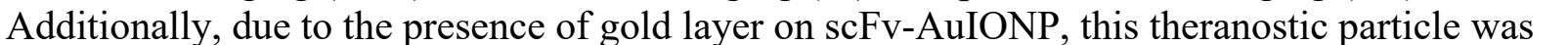

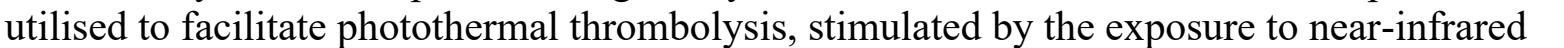

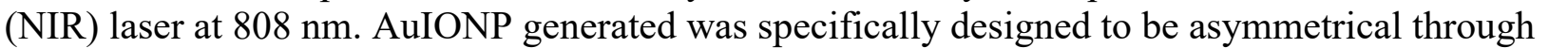

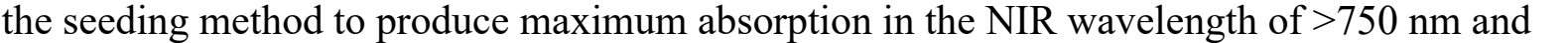

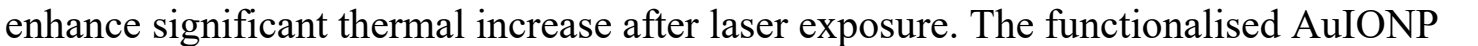

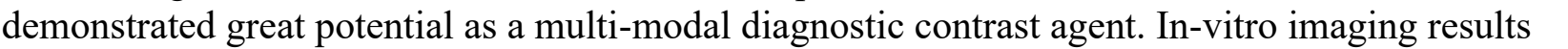

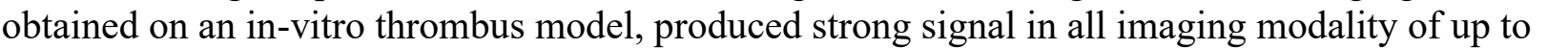

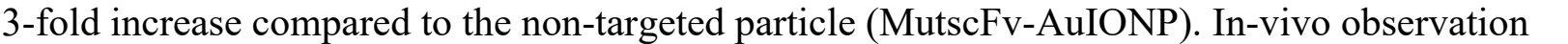

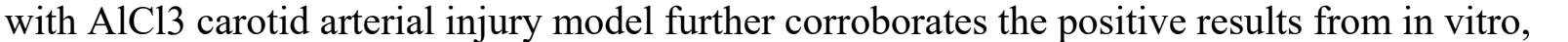

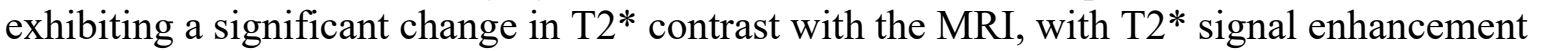

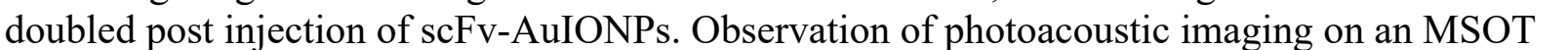

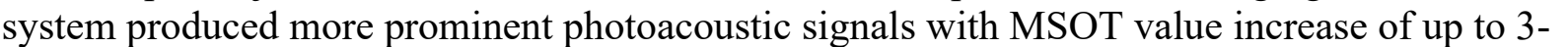
ए

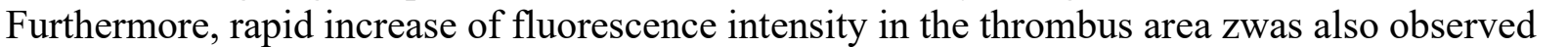

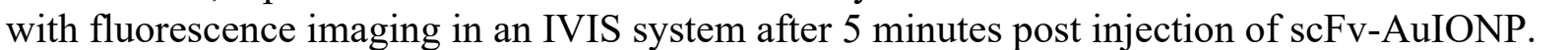

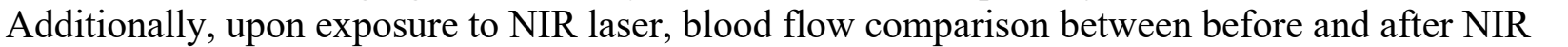

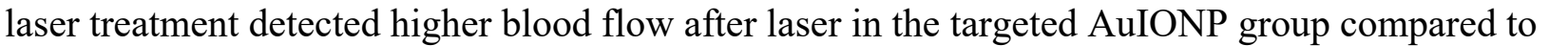

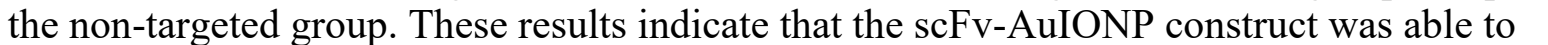

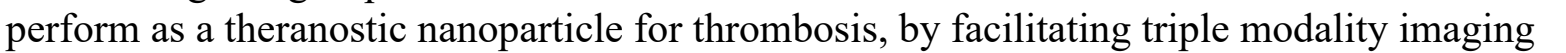

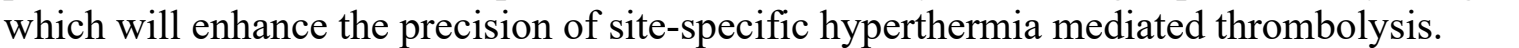

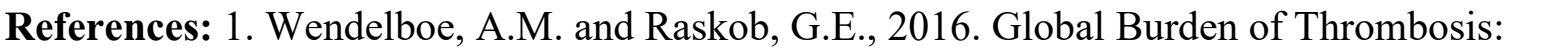

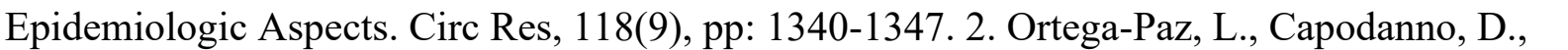

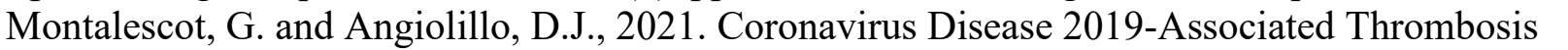

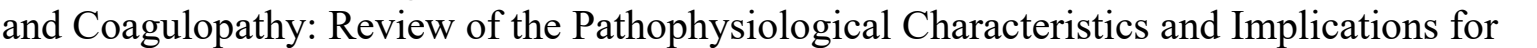

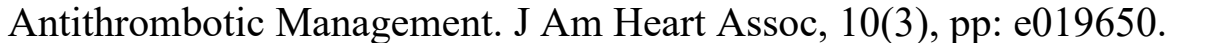


Image/Figure:

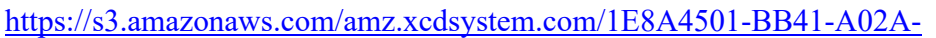

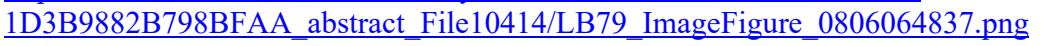

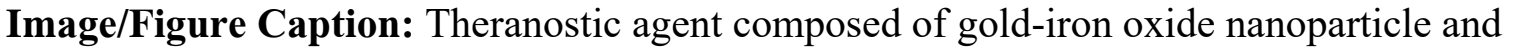

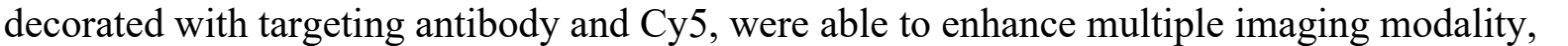

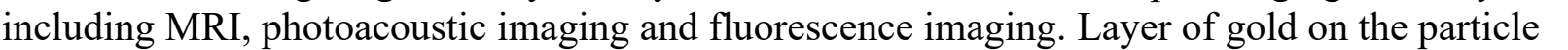

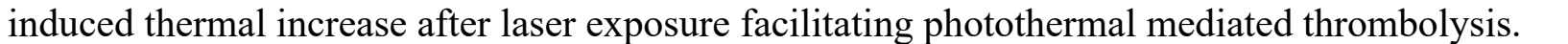

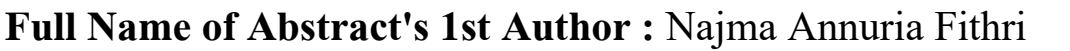

First Name: $\square \square \square \square \square$

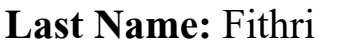

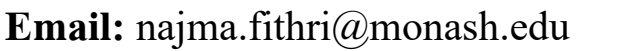

Organization: $\square \square \square\|\|\|\square \square\|\|\|\|\| \square$

Country: $\square \square\|\square\|\|\| \square$ 


\title{
ID: LB80 \\ Metal oxide-chitosan compounds as theranostic modular nano-cocktails for ROS and inflammatory related diseases
}

\author{
Yuao Wu, Griffith University - Queensland Micro Nanotechnology Centre, \\ yuao.wu@griffith.edu.au
}

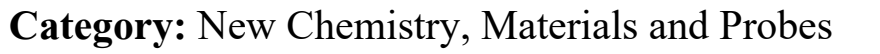

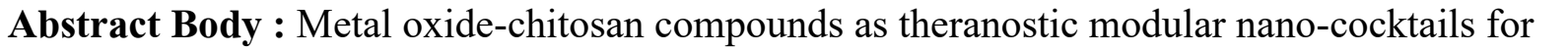

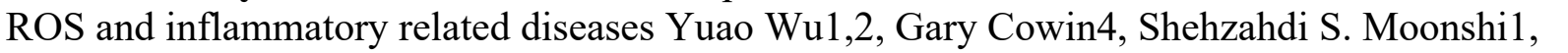

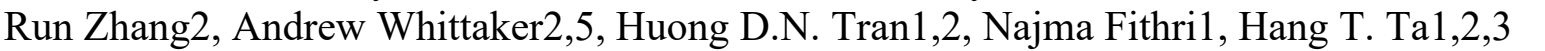

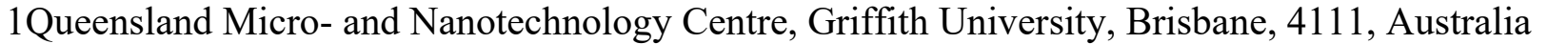

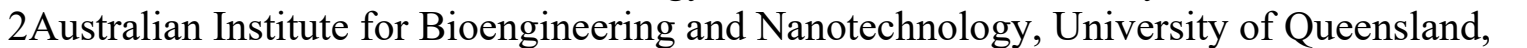

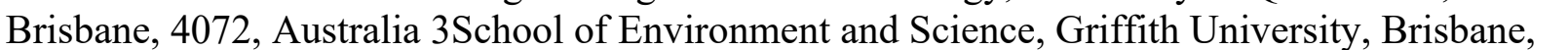

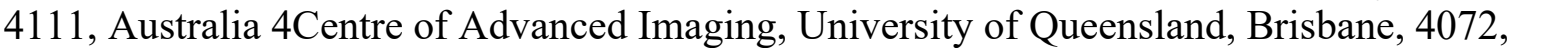

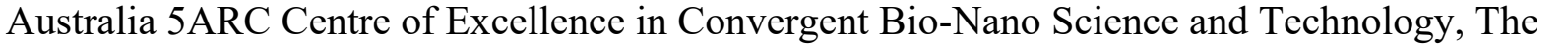

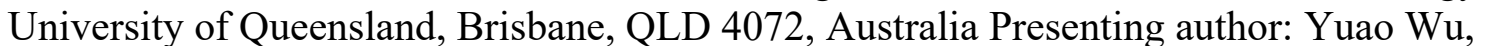

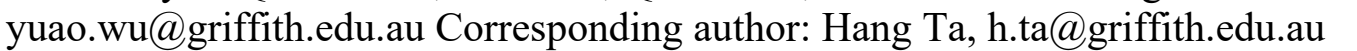

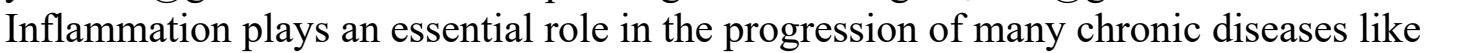

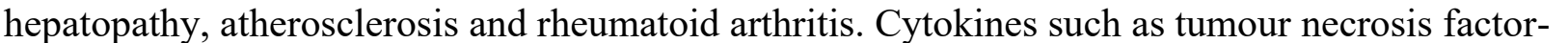

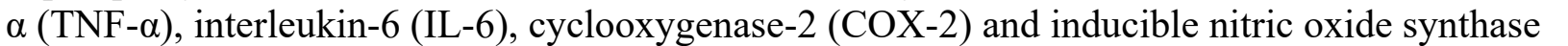

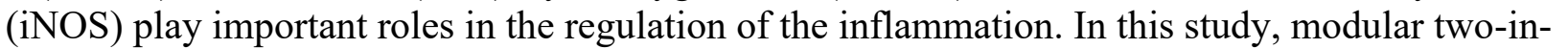

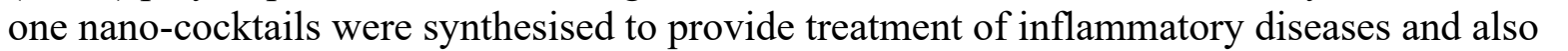

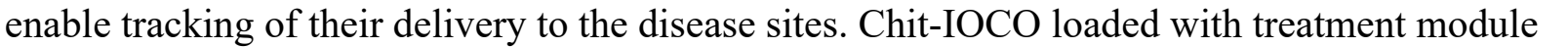
ए

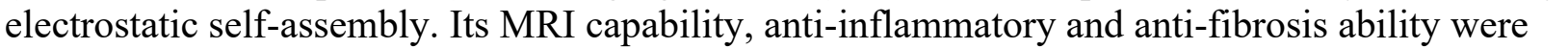
ए

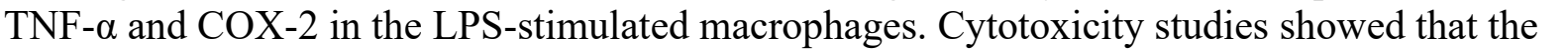

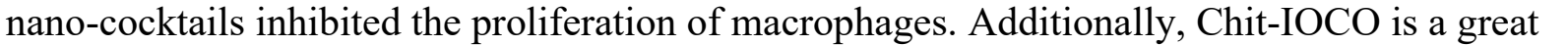

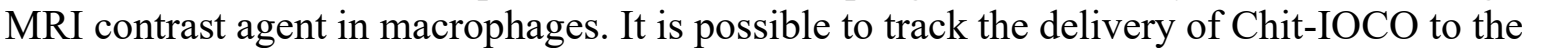
ए

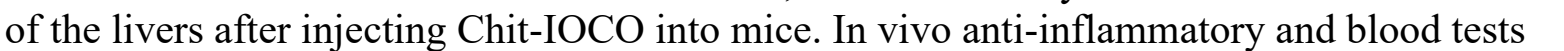

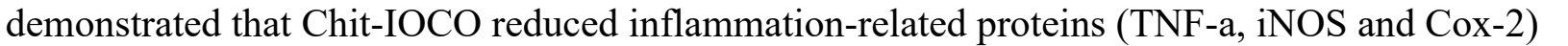

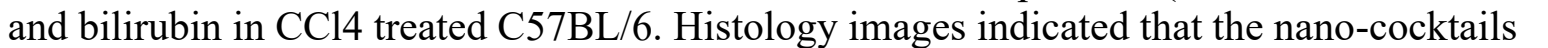

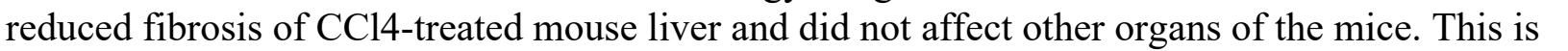

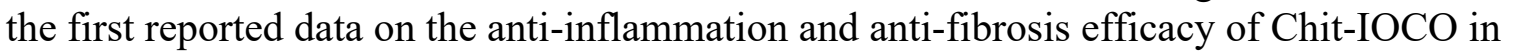

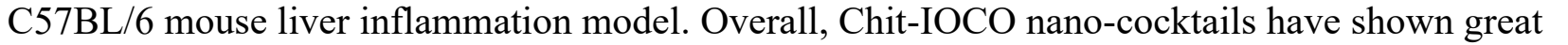

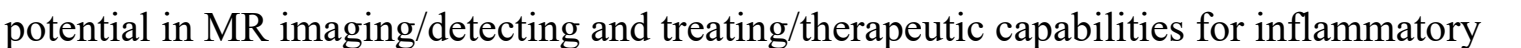

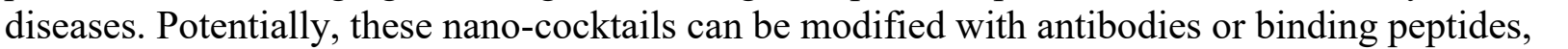

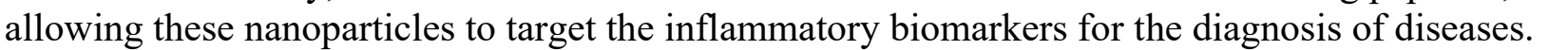

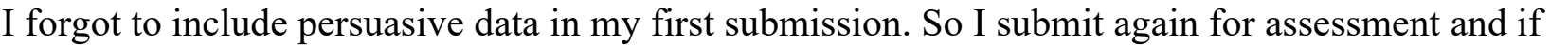

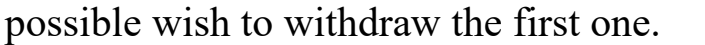


Full Name of Abstract's 1st Author : $\square \square \square \square \square \square$

First Name:

Last Name:

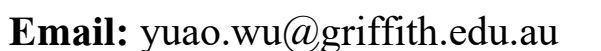

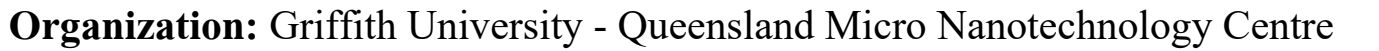

Country: $\square \square\|\|\|\|\|\| \square$ 


\title{
ID: LB81 \\ Longitudinal monitoring of CD8 $\mathrm{T}$ cell migration dynamics in response to cancer immunotherapy
}

Stefania Pezzana, University Hospital Tübingen, stefania.pezzana@med.uni-tuebingen.de

\section{Category: $\square \square\|\| \| \square$}

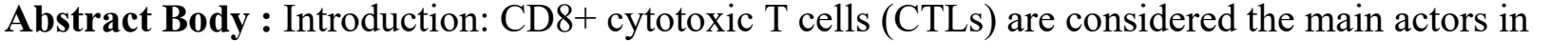

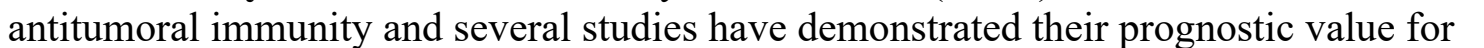

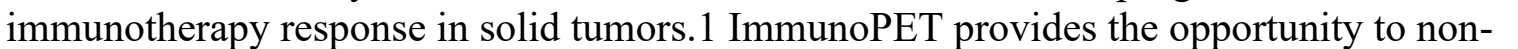

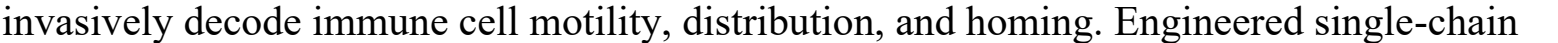

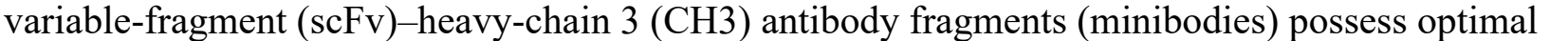

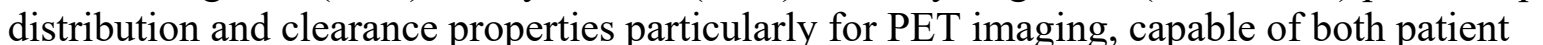

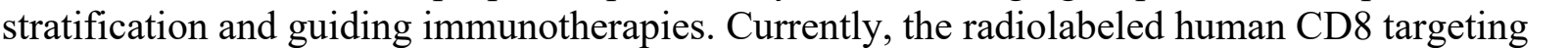

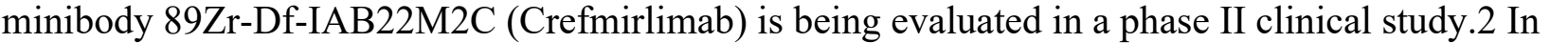
प ए

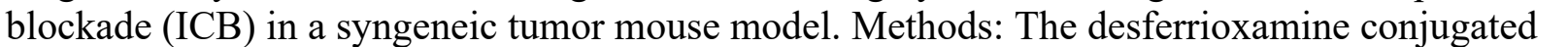

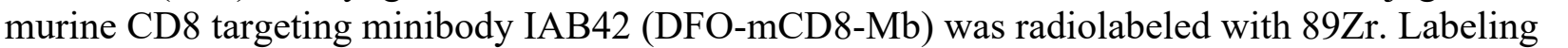

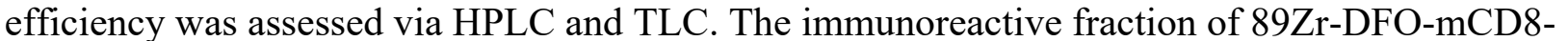

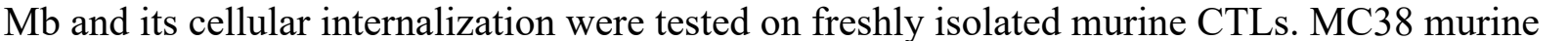

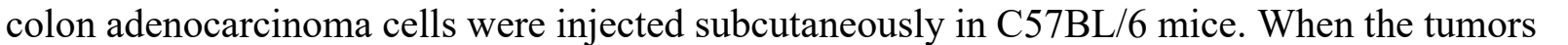

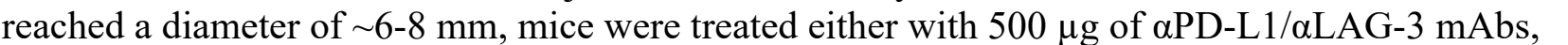

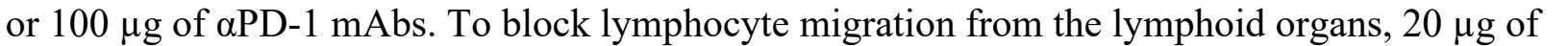

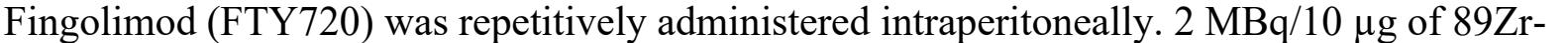

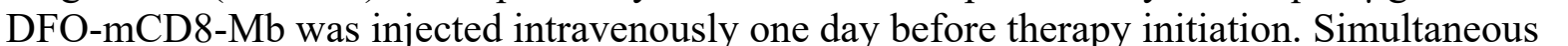

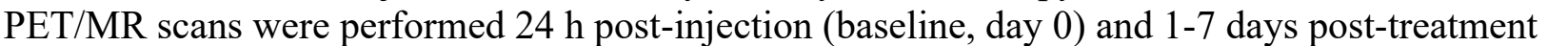

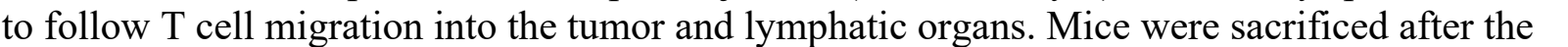
ए

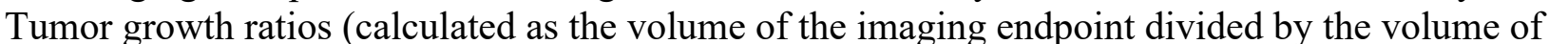
ए

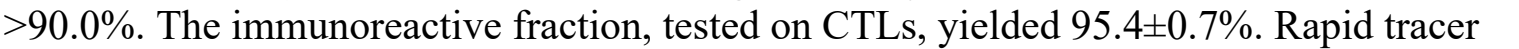

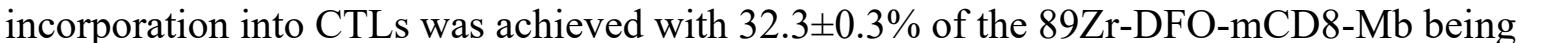

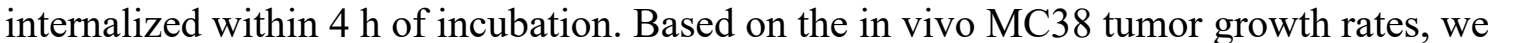

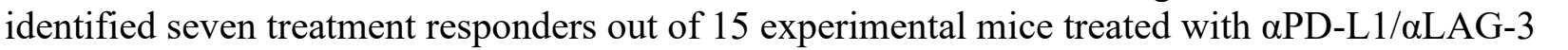

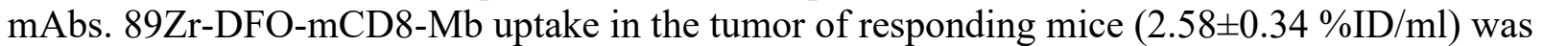

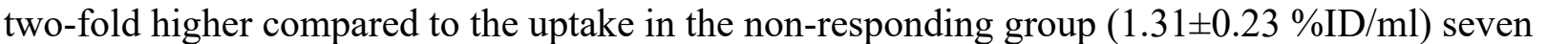

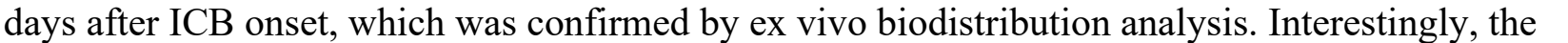

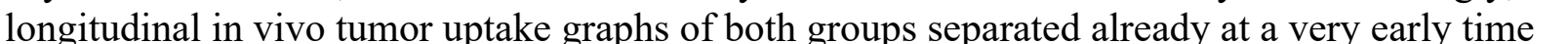

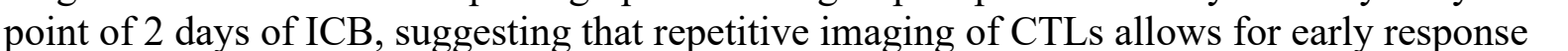

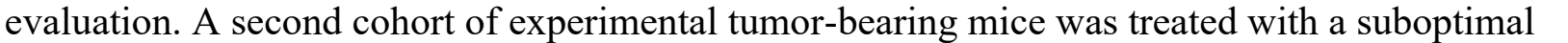

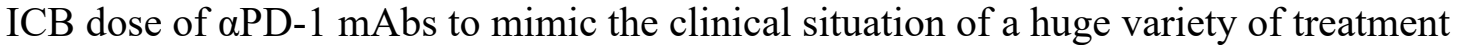

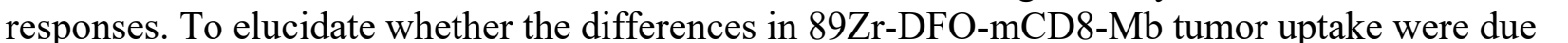




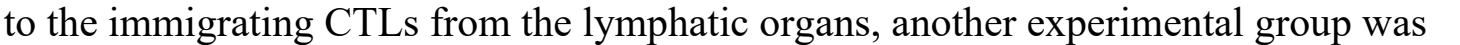

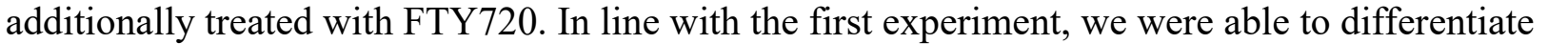

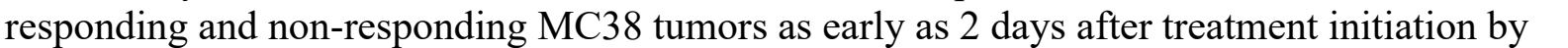

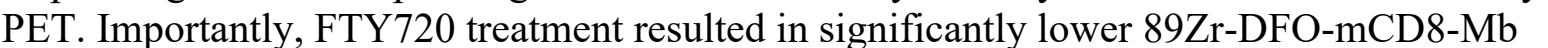

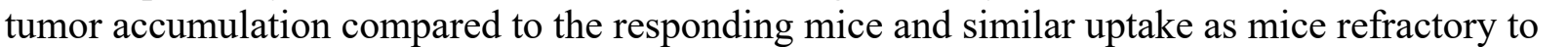

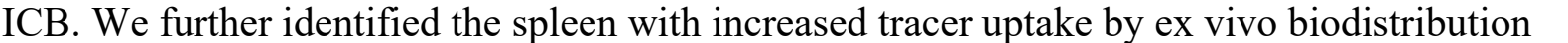

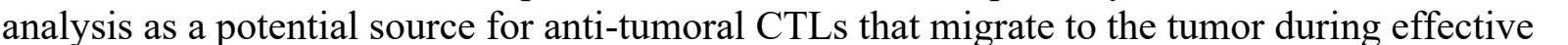

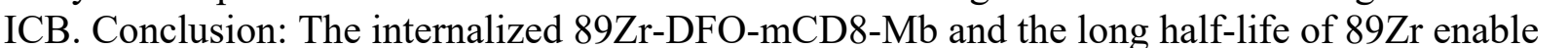
प ए

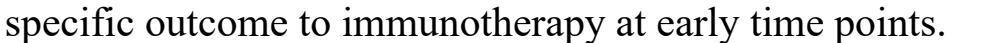

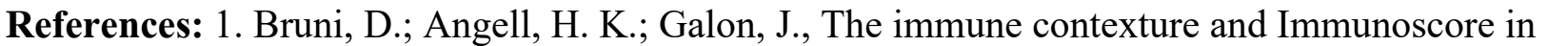

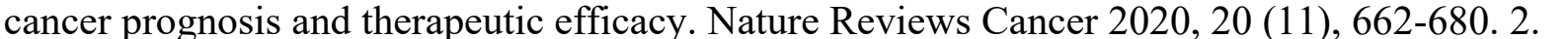

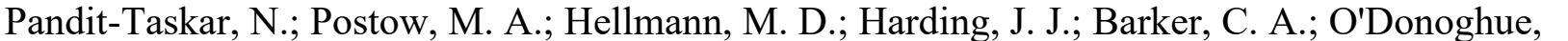

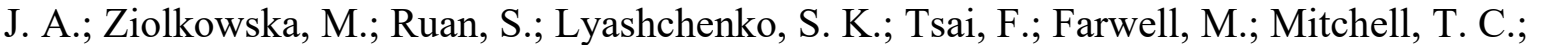

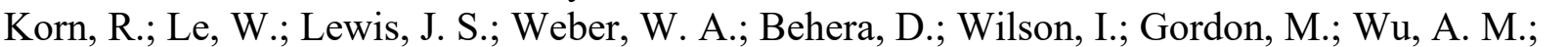

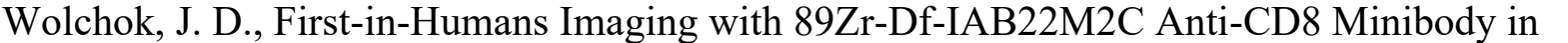

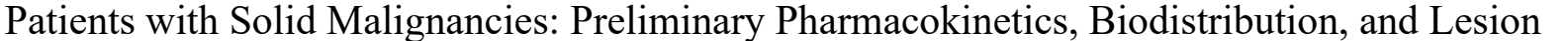

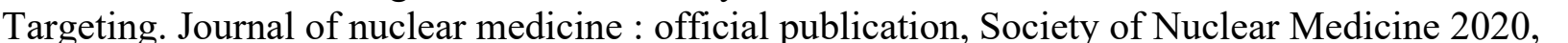

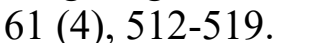

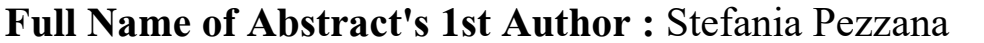

First Name: $\square \square\|\| \square \square$

Last Name: $\square \square\|\| \| \square \square$

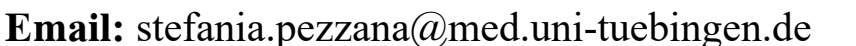

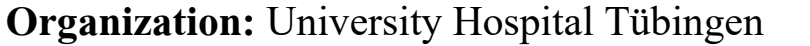

Country: $\square \square \square \square \square \square$ 


\title{
ID: LB82 \\ Dynamic cardiac FDG PET and CMR imaging in vivo assess the effect of metformin in failing spontaneously hypertensive rat hearts
}

\author{
Bijoy Kundu, University of Virginia, bkk5a@virginia.edu
}

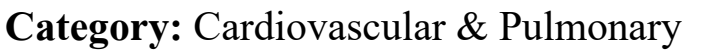

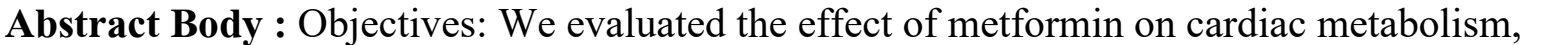

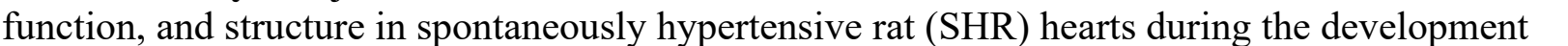
of diastolic and systolic heart failure (HF), using dynamic 2-[18F] fluoro-2-deoxy-D-gluco $\square \square$

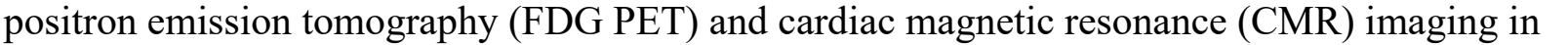

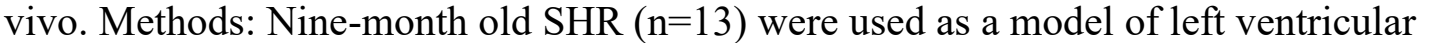

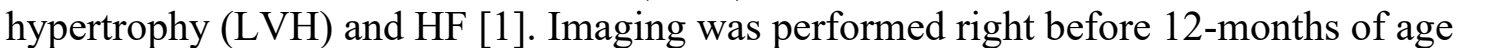

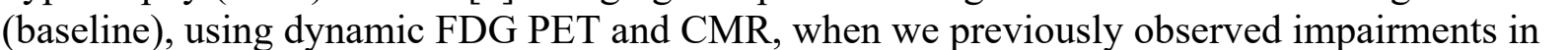

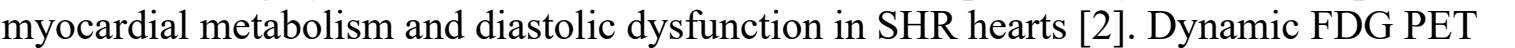

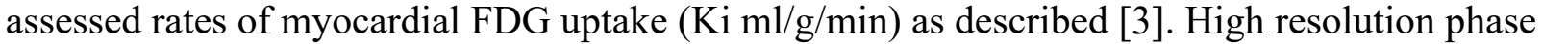

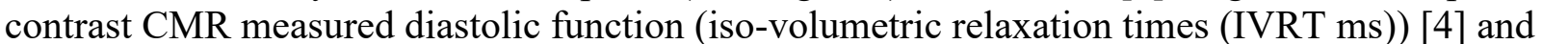

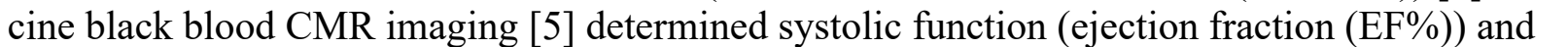

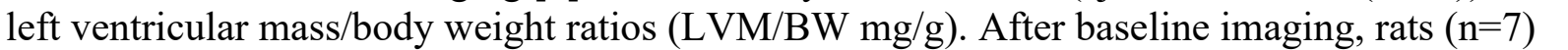

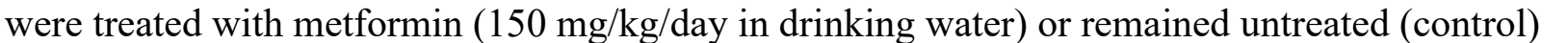

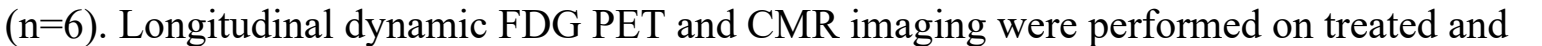

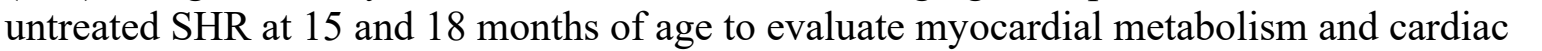

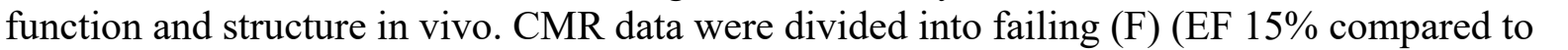

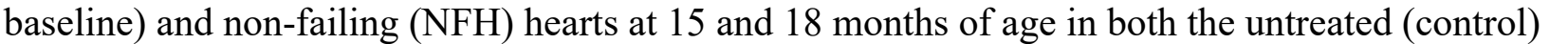

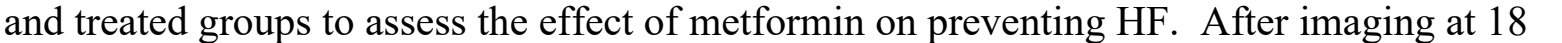

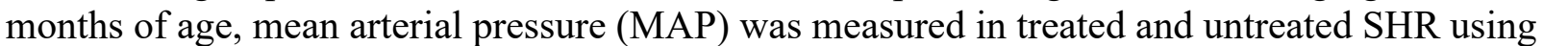
ए

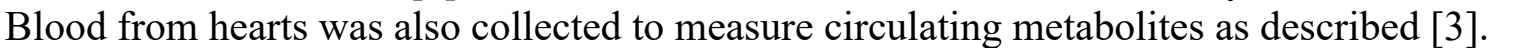

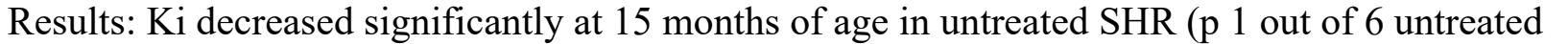

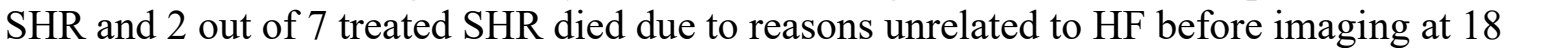

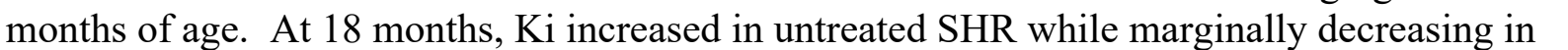
ए

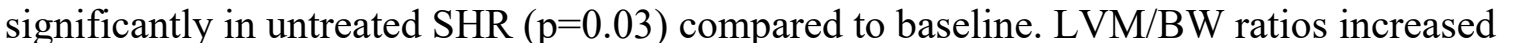

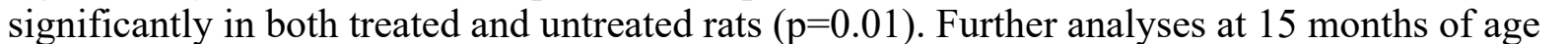

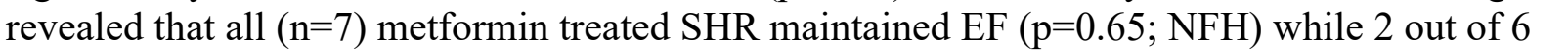

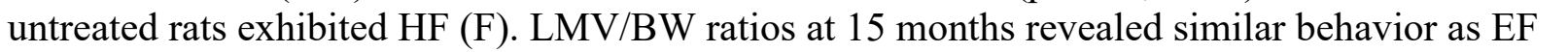

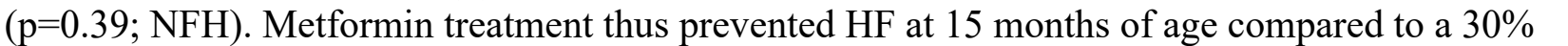

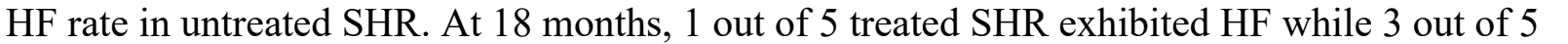

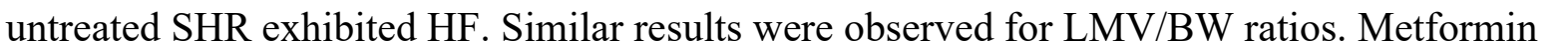

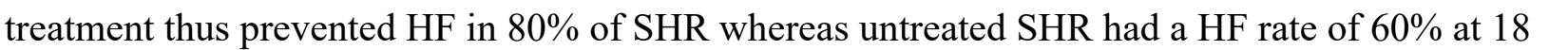

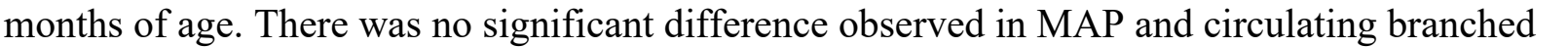

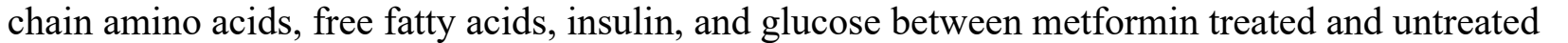

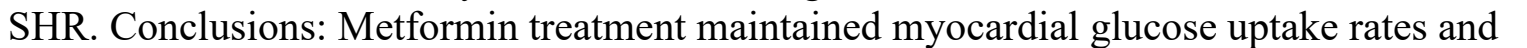




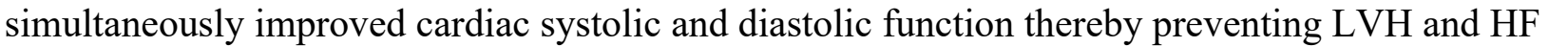

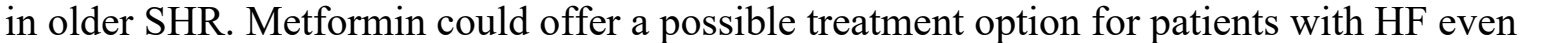

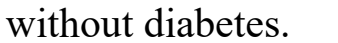

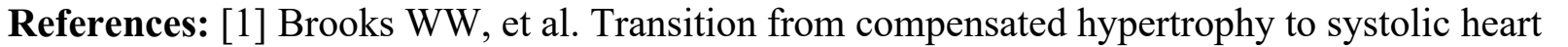

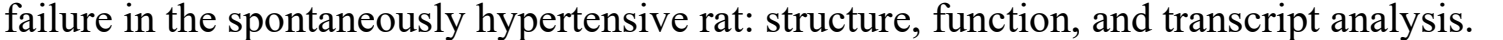

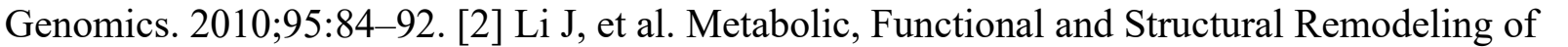

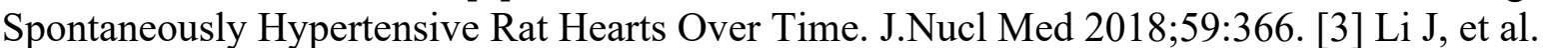

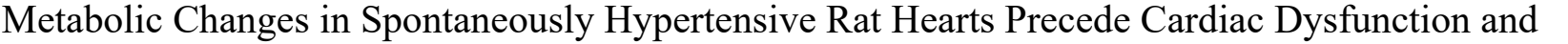

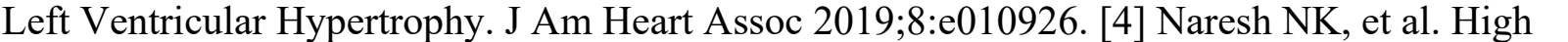

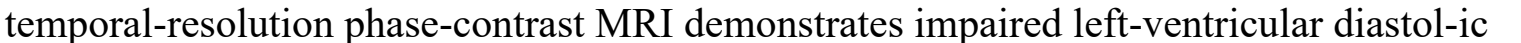

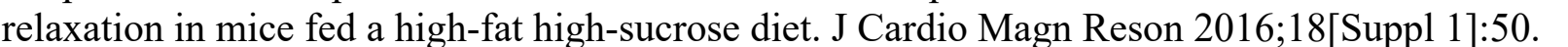

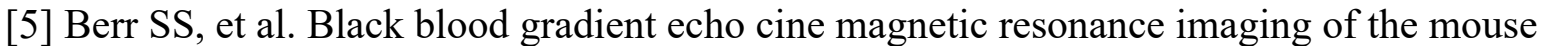

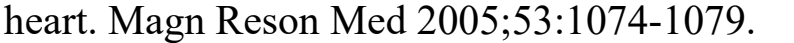

\section{Image/Figure:}

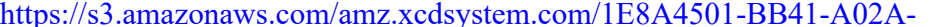

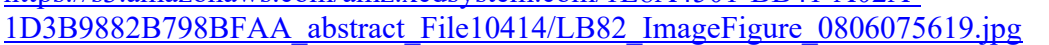

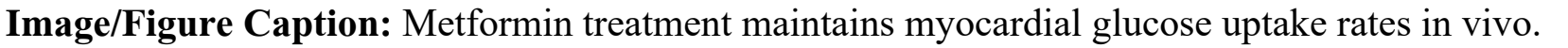

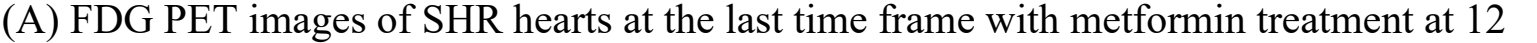

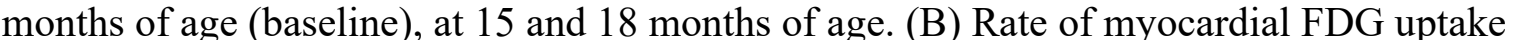

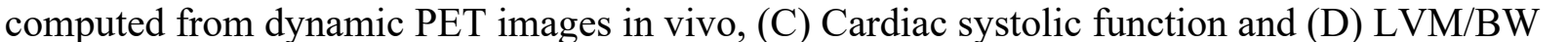

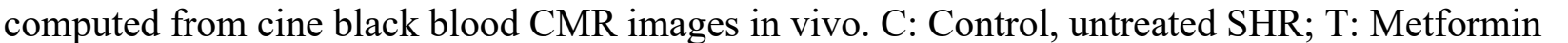
ए

First Name: $\square \square \| \square$

Last Name: $\square \square \square \| \square$

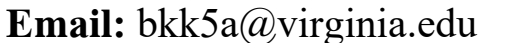

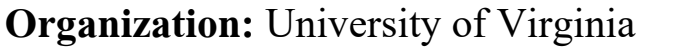

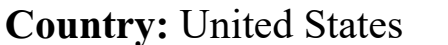




\title{
ID: LB83 \\ Noninvasive in vivo assessment of the hepatic lipid composition and the infiltrating CD8+ $\mathrm{T}$ cells in a diet-induced NASH mouse model
}

\author{
Vera Jörke, Werner Siemens Imaging Center, Eberhard Karls University Tübingen, \\ vera.joerke@med.uni-tuebingen.de
}

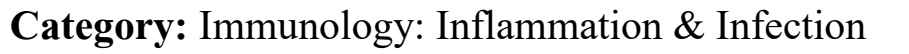

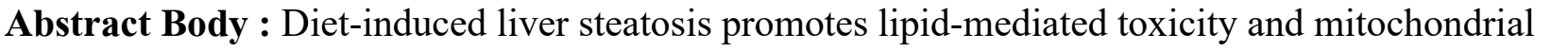

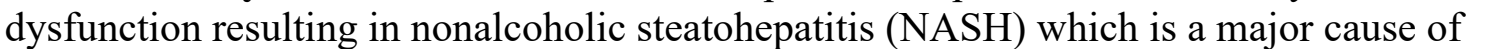

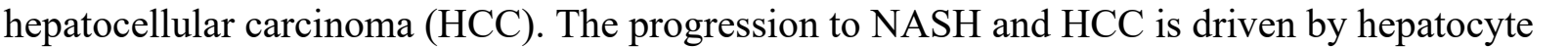

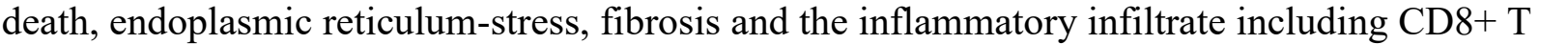

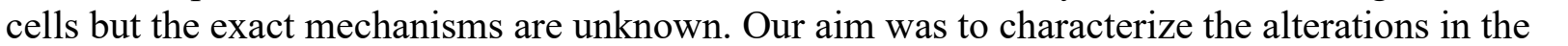

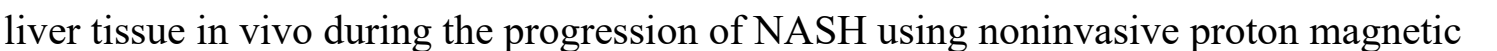

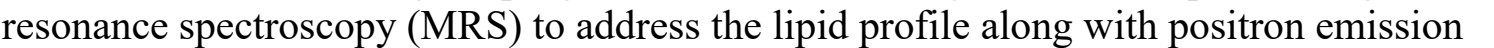

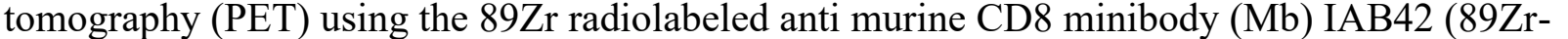

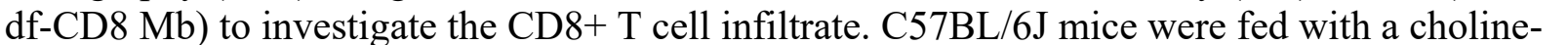

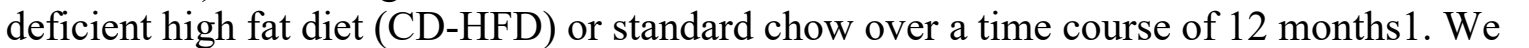

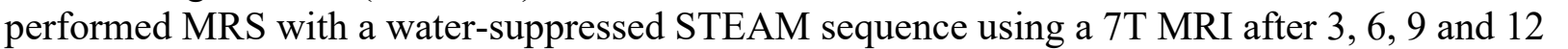

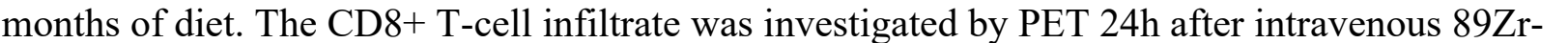

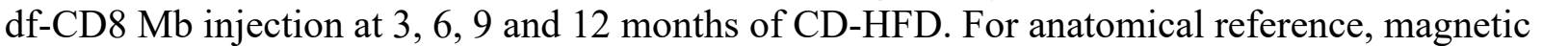

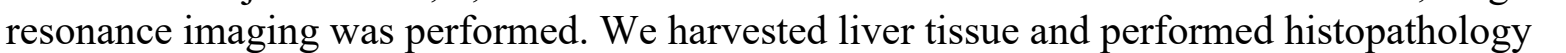
ए प

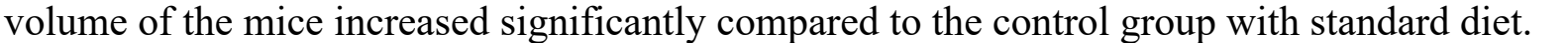

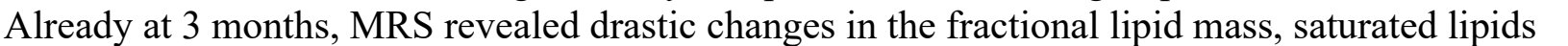

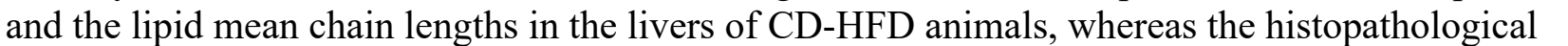

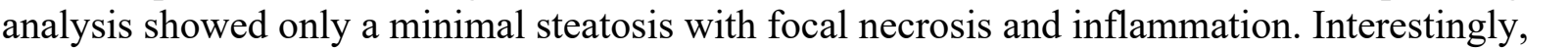

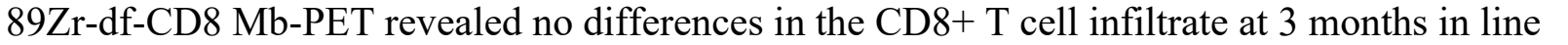

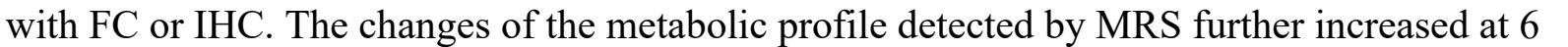

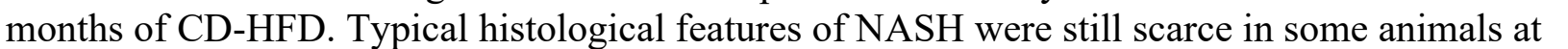

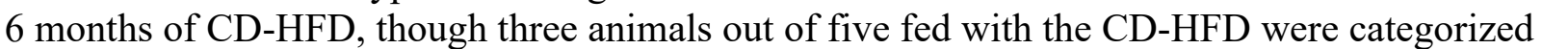

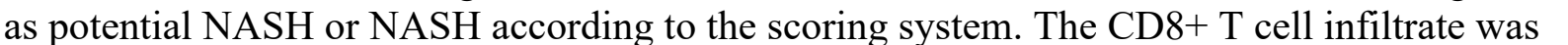

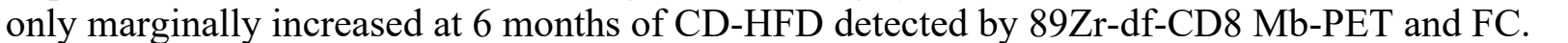

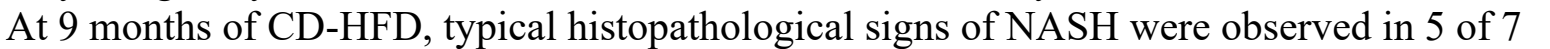

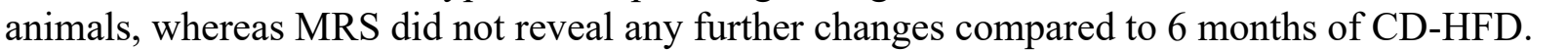

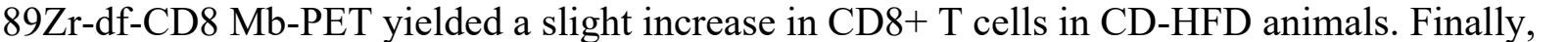

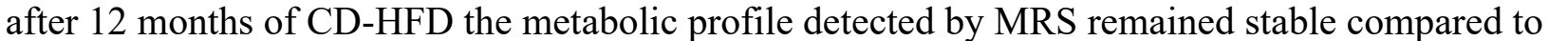

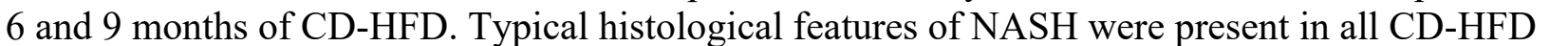

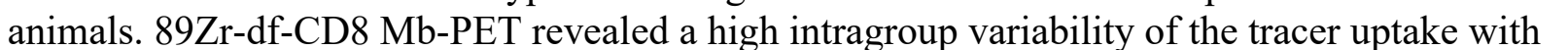

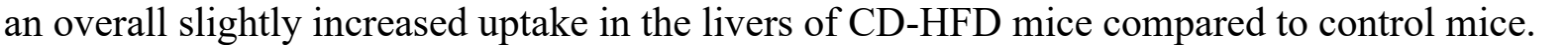

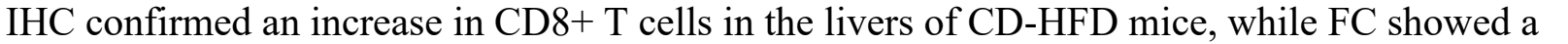

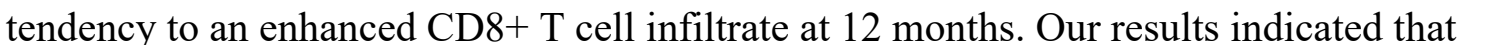


References: 1 Wolf, Monika Julia, et al., ... Heikenwalder M. "Metabolic activation of

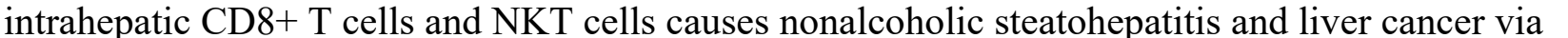

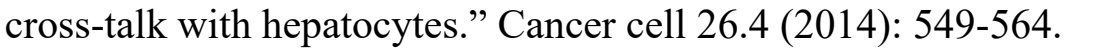

Full Name of Abstract's 1st Author : $\square \square\|\|\|\| \| \mid \square$

First Name: $\square \square \square \square$

Last Name: $\square \square\|\| \|$

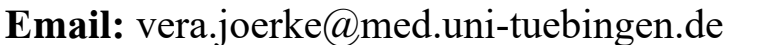

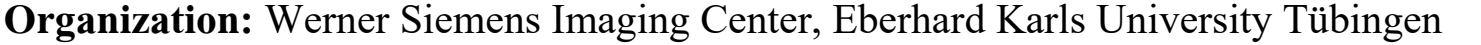

Country: $\square \square \square \square \square \square$ 


\title{
ID: LB84 \\ Addition of Peptide Receptor Radiotherapy to Immune Check Point Inhibition Therapy Improves Treatment Outcome in Neuroendocrine Tumors
}

Shadi Esfahani, Massachusetts General Hospital, esfahani.shadi@mgh.harvard.edu

\section{Category: $\square \square\|\| \| \square$}

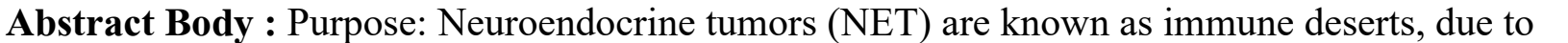

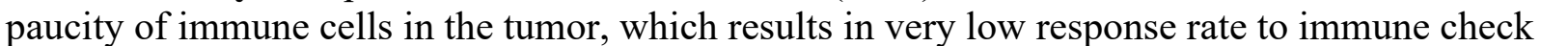

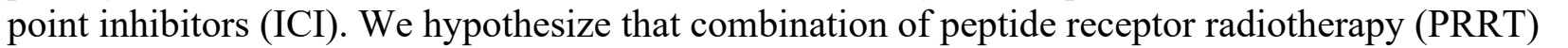

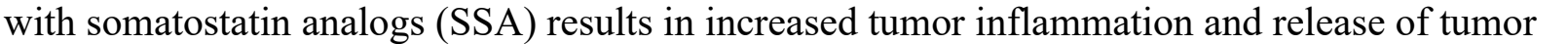

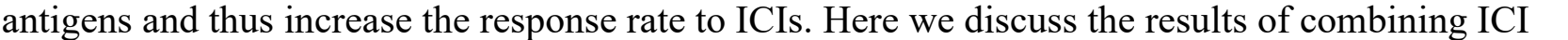

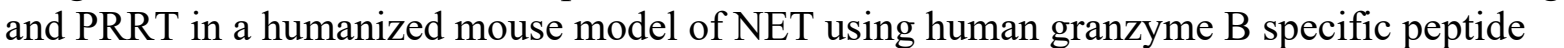

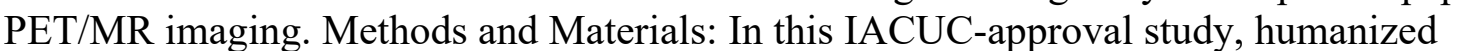

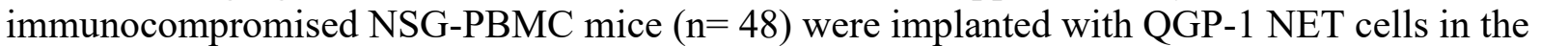

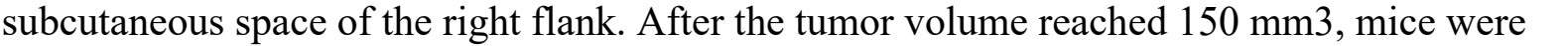

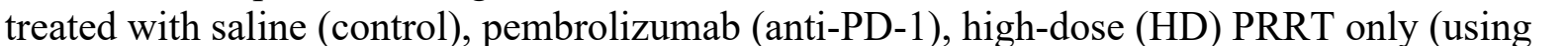

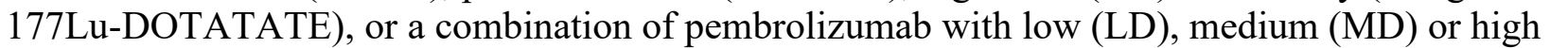

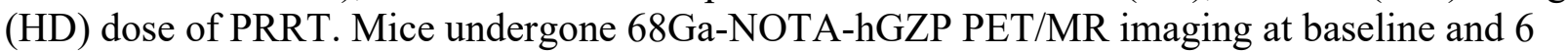

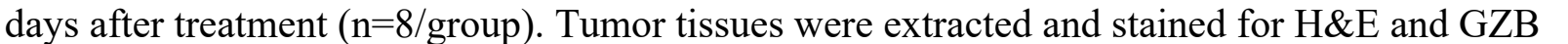

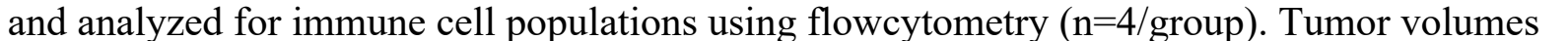

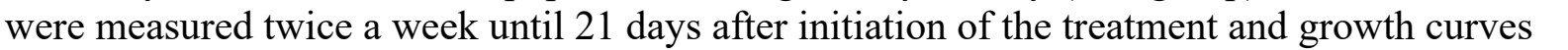

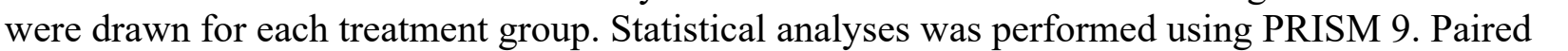

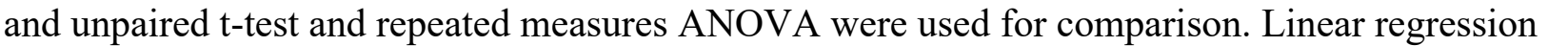

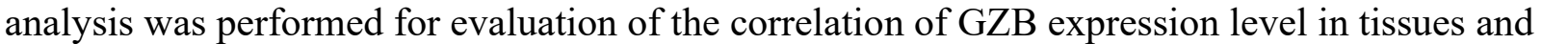

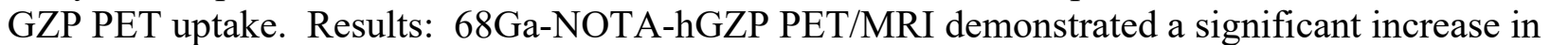

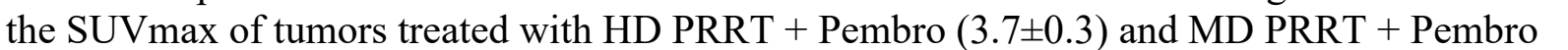
ए

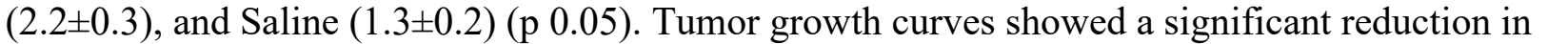

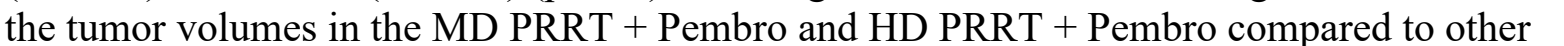

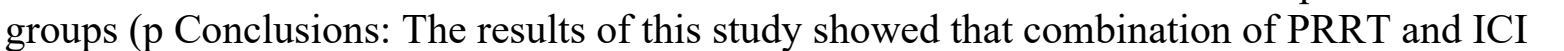

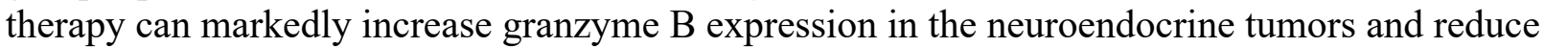
ए

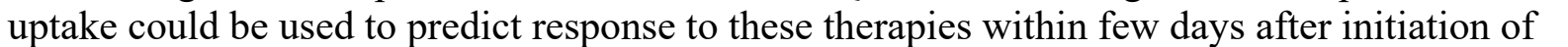

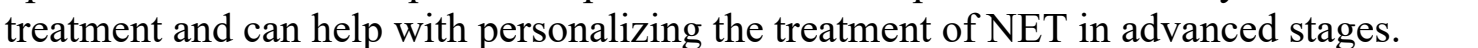

\section{Image/Figure:}

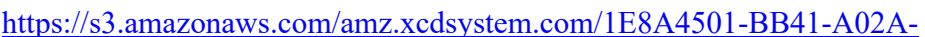

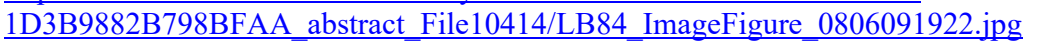

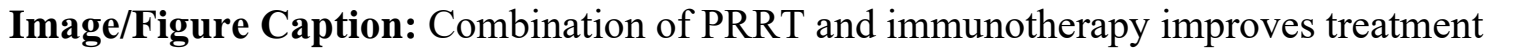

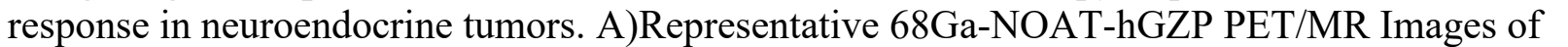




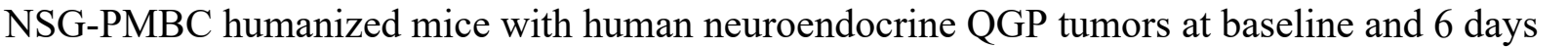

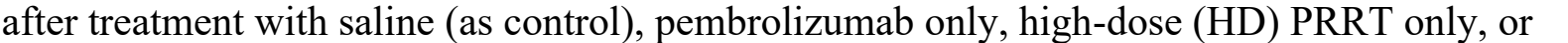

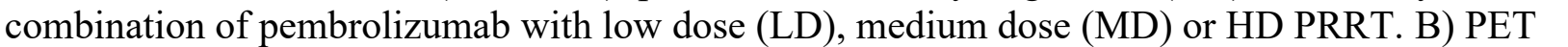

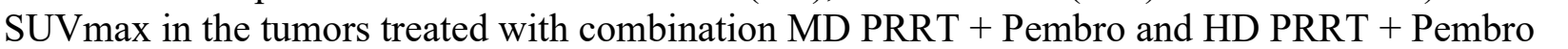

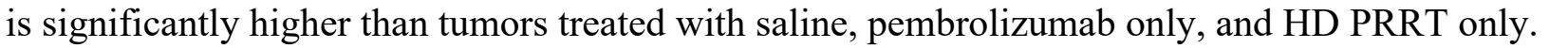

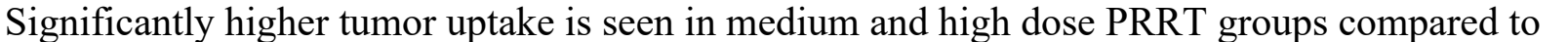
ए

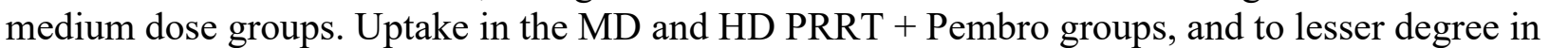

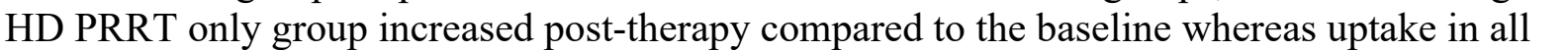

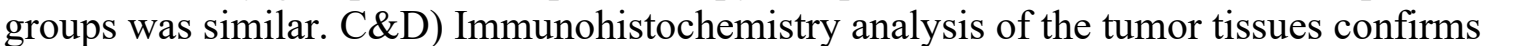

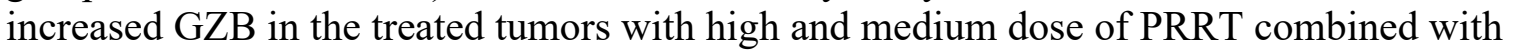

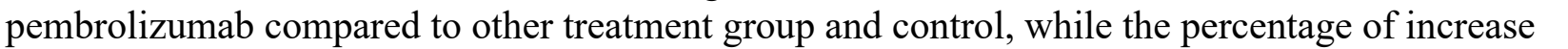

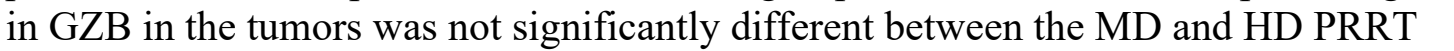

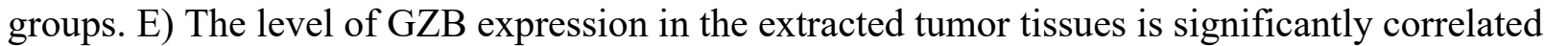

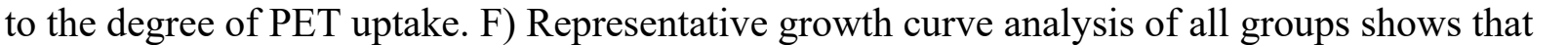

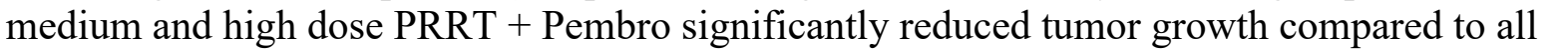

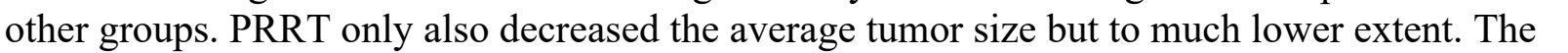
पा

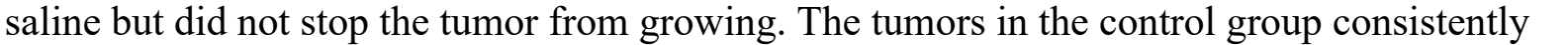
$\square \square \square \square$

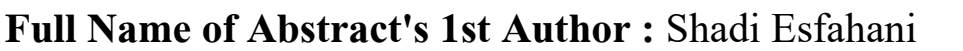

First Name: $\square \square \square|\Pi| \square$

Last Name: $\square\|\|\|\|\|\| \|$

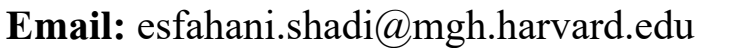

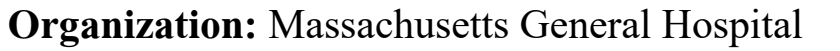

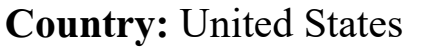




\title{
ID: LB85 \\ Investigating the Theranostic Potential of a Novel MUC16-targeted Antibody for Ovarian Cancer
}

Kyeara Mack, Memorial Sloan Kettering, mackk1@mskcc.org

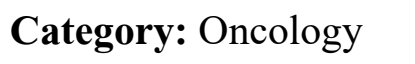

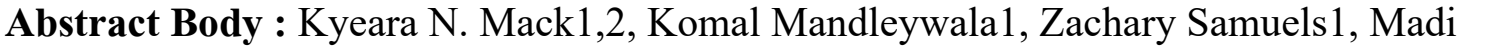

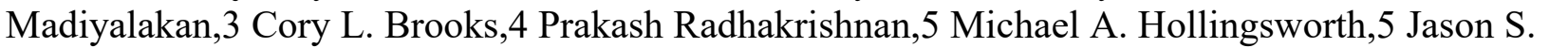

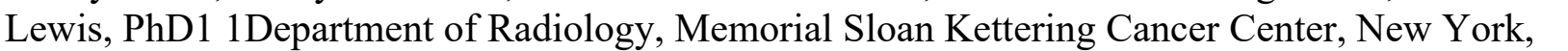

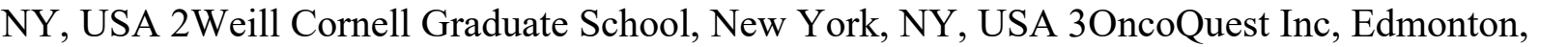

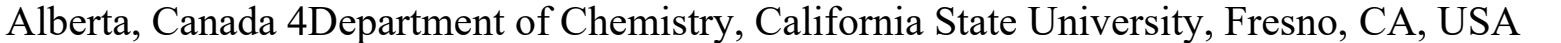

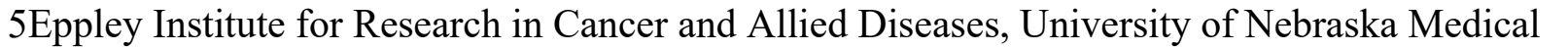

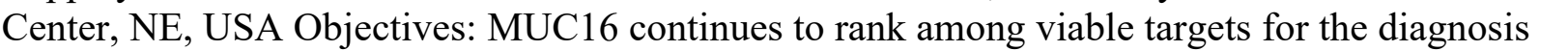

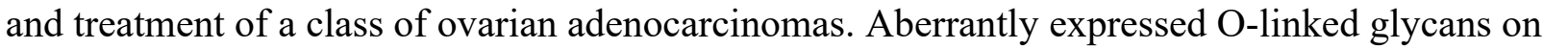

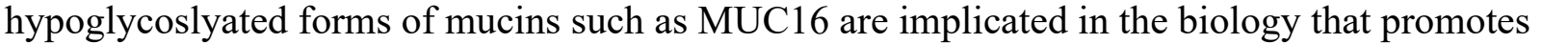
ए

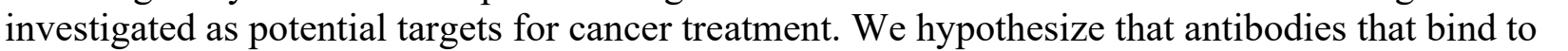

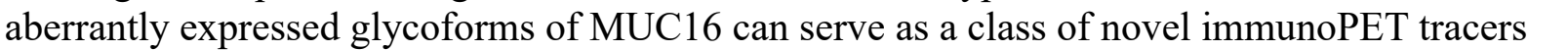

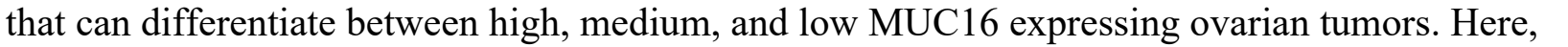

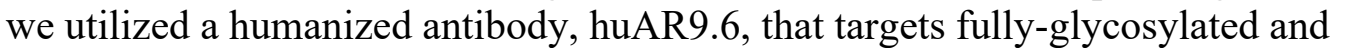

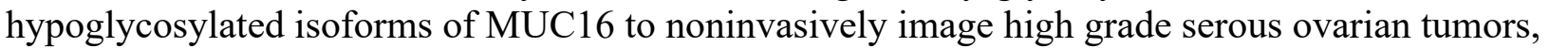

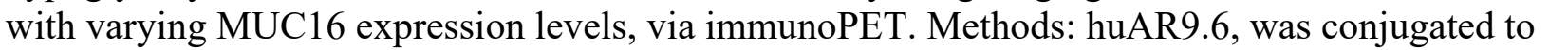

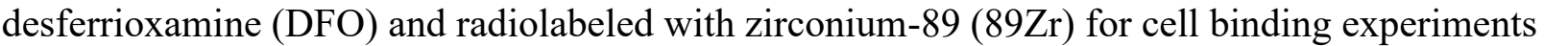

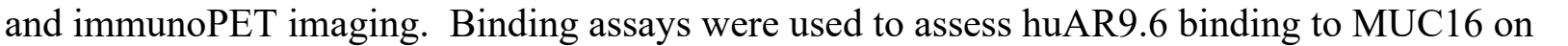

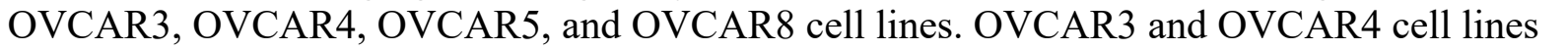

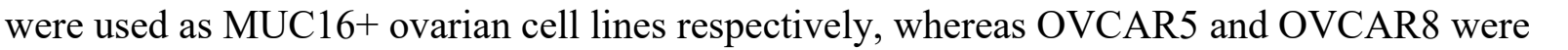

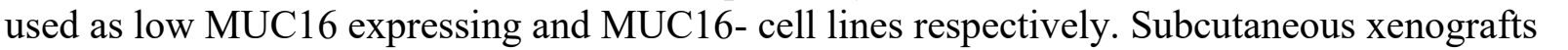

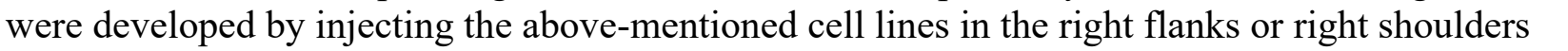

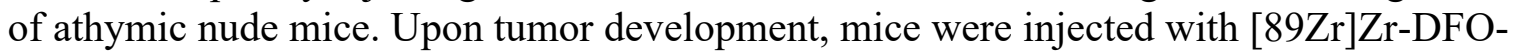

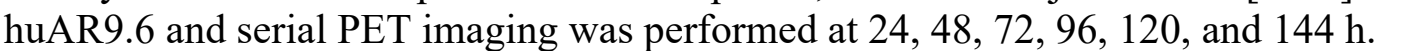

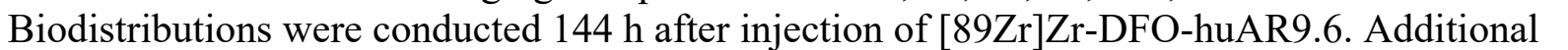
ए

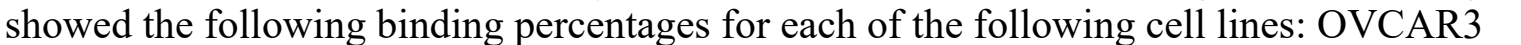

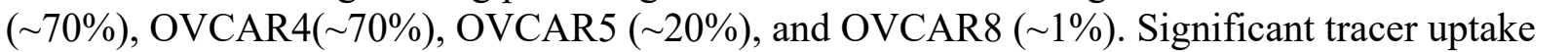

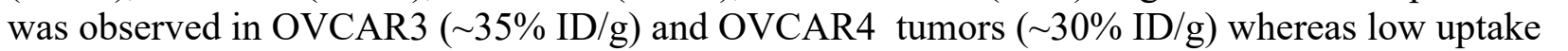

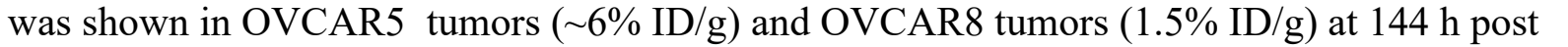

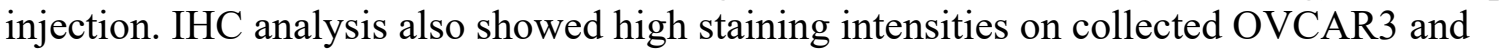

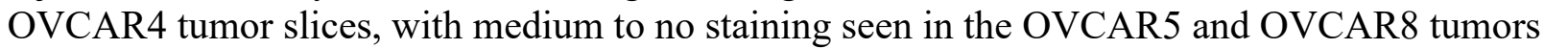

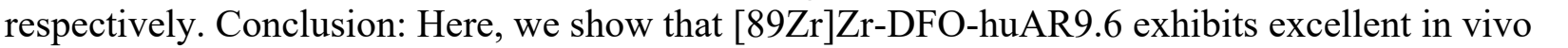

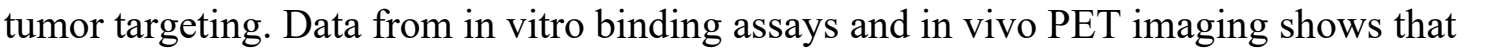

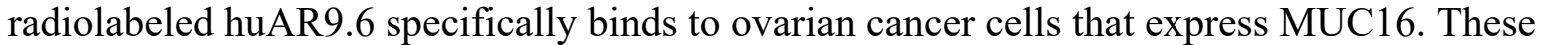

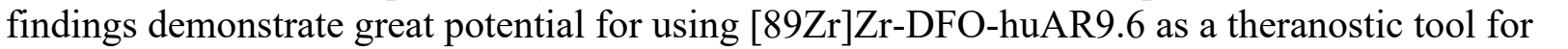


Image/Figure:

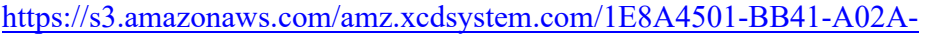

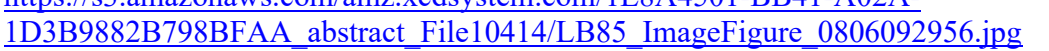

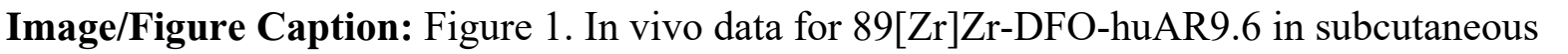

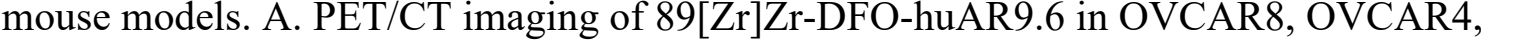

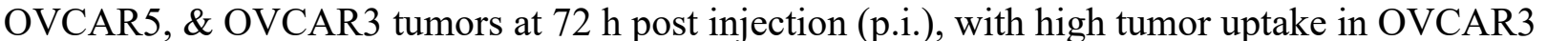

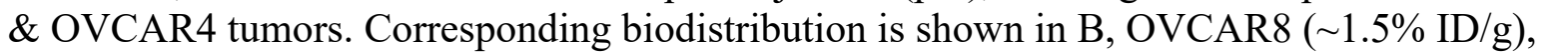

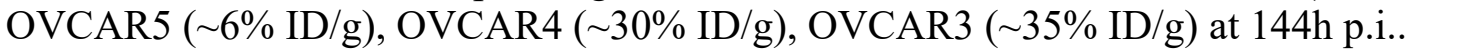

First Name: $\square \square \square\|\| \|$

Last Name: $\square \square \| \square$

Email: $\square \square\|\| \square \square \square\|\|\|\| \| \square$

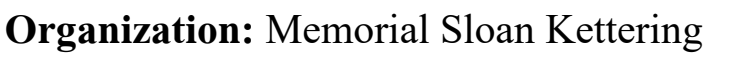

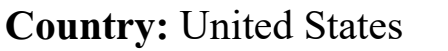




\title{
ID: LB86 \\ Detection of invaded nerve for pain-relieving targeting nerve using MRI/MPI in pancreatic cancer
}

\author{
kunxiong guo, Zhujiang Hospital, Southern Medical University, gkx0810@163.com
}

\section{Category: $\square \square\|\| \| \square$}

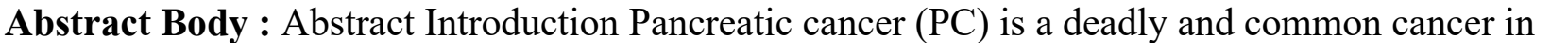

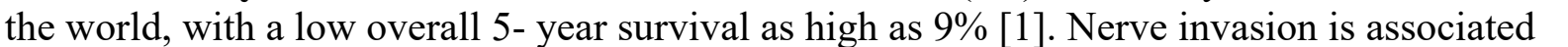

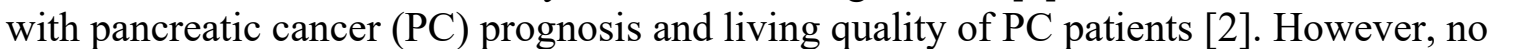

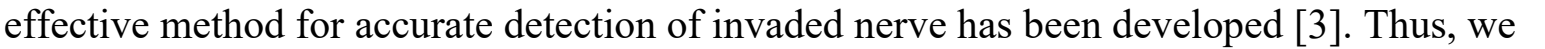

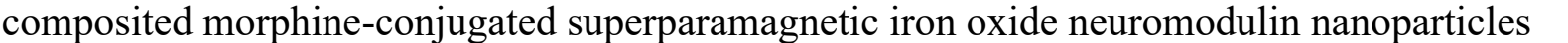

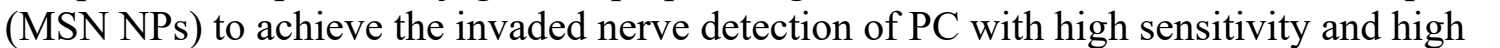

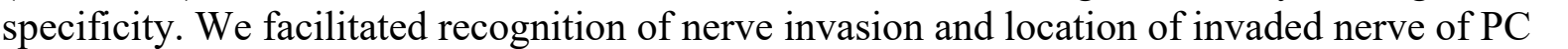

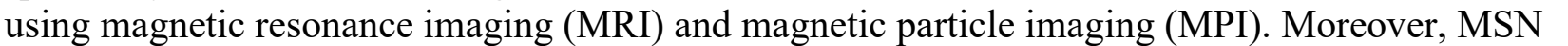

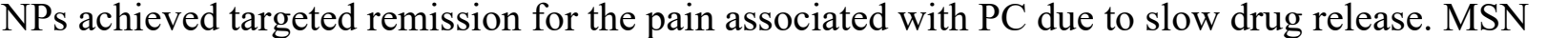

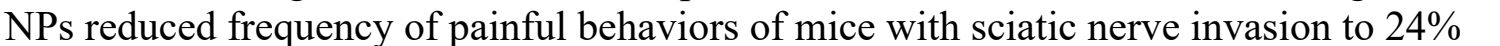

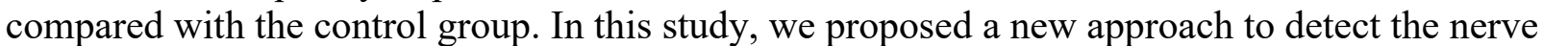

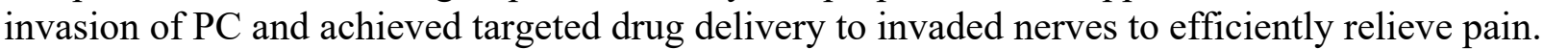

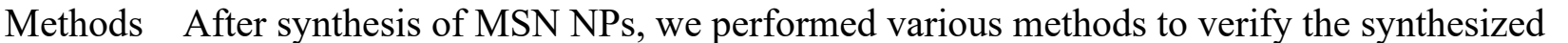

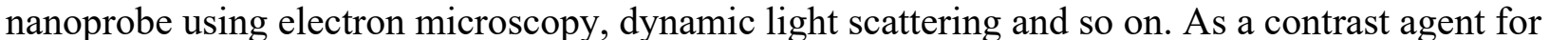

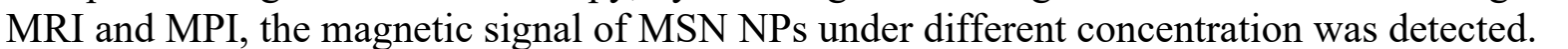

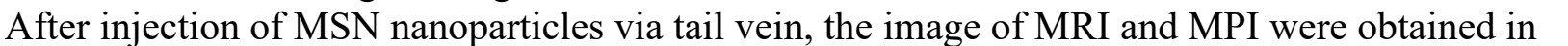

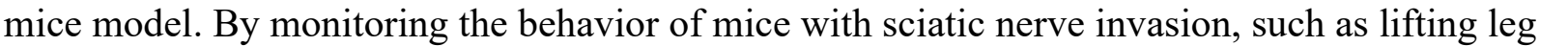

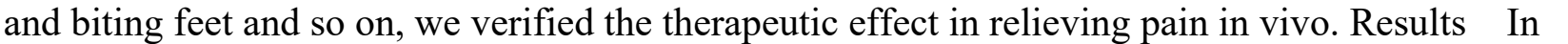
ए

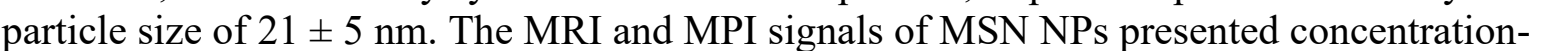

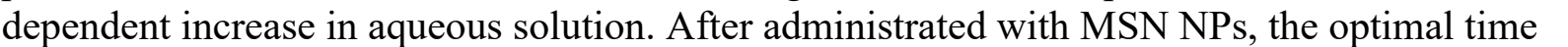

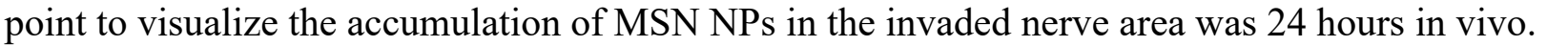

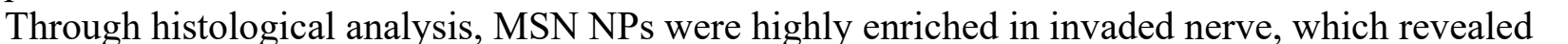

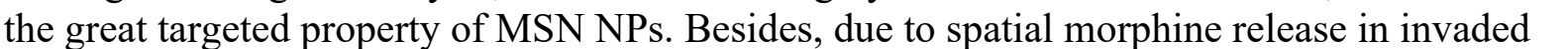

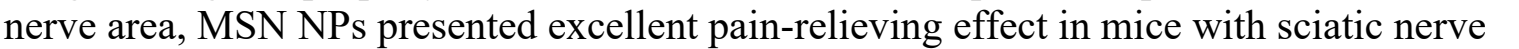

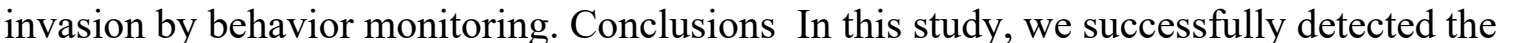

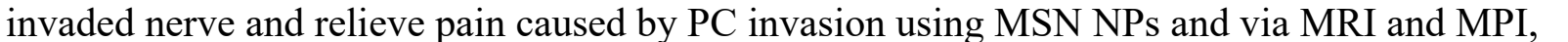

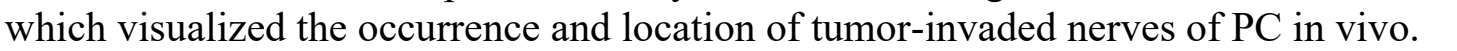

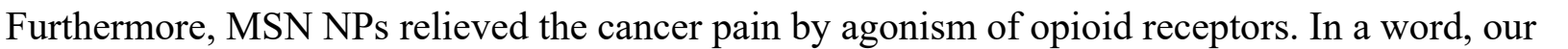

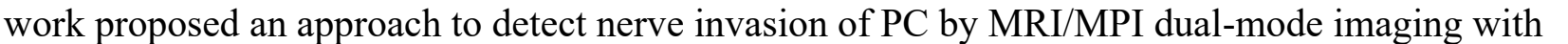

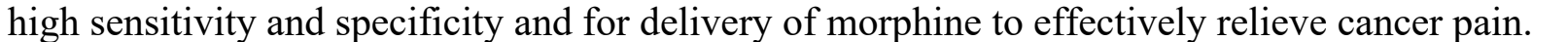

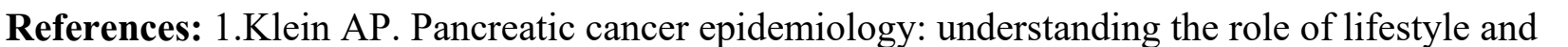

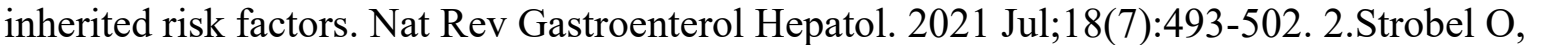

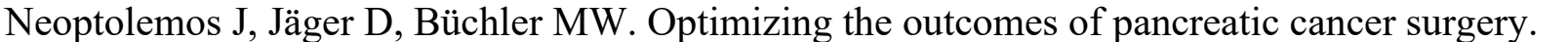

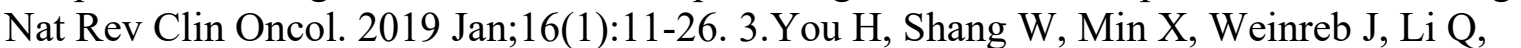


First Name:

Last Name:

Email:

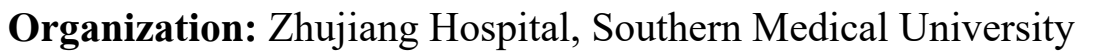

Country: $\square \square \square \square \square$ 


\title{
ID: LB87 \\ Colorful fluorescence-based carbon dots for imaging-guided nanosurgery of cancer
}

pengli gao, Beijing University, plgao@ciac.ac.cn

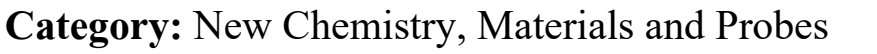

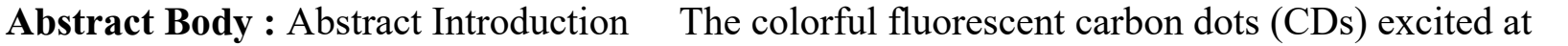

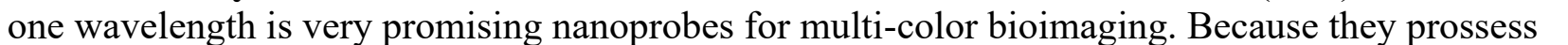

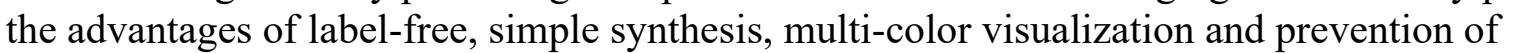

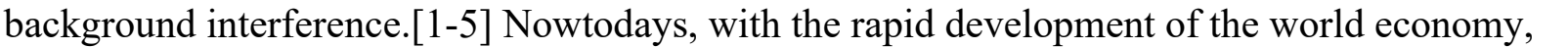

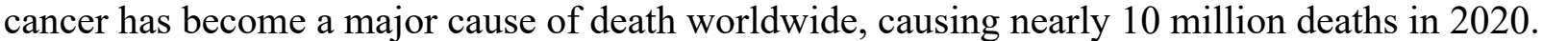

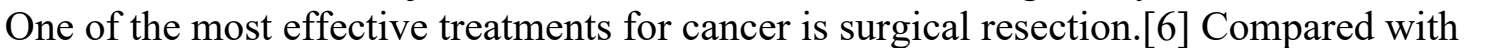

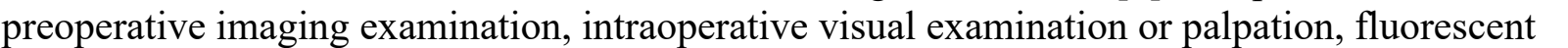

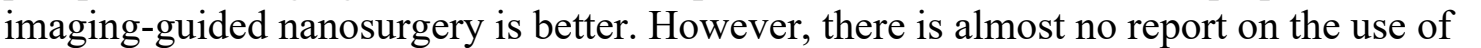

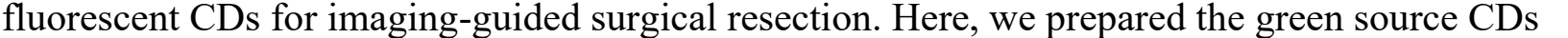

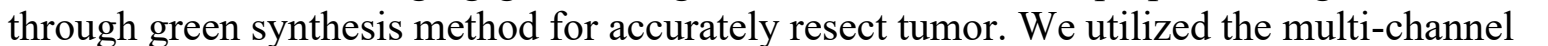

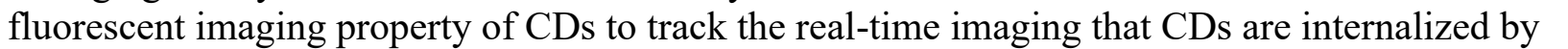

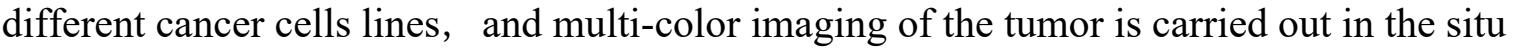

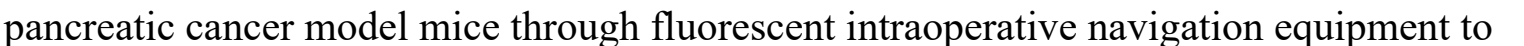

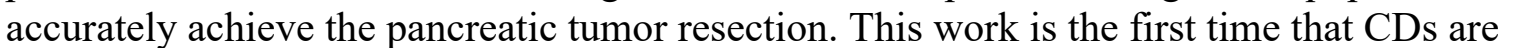

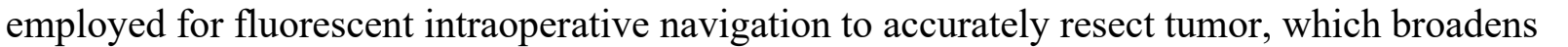

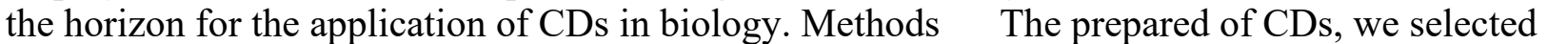

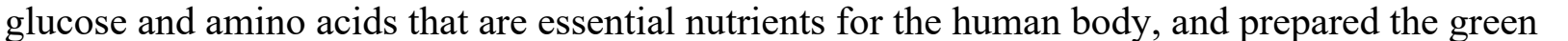

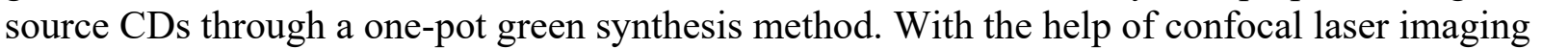

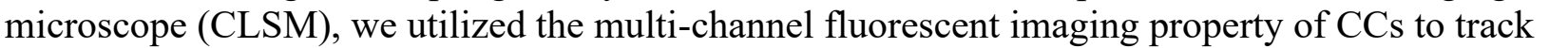

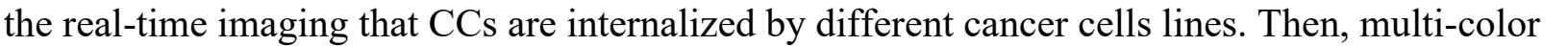

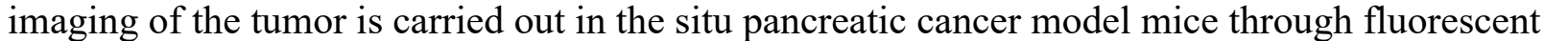

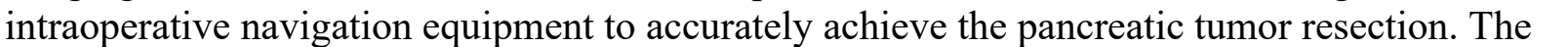

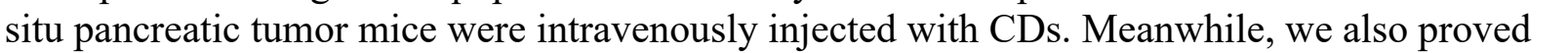

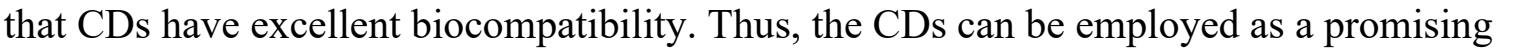

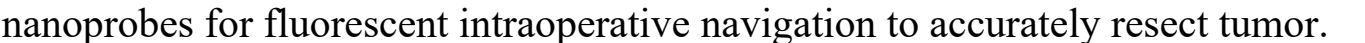

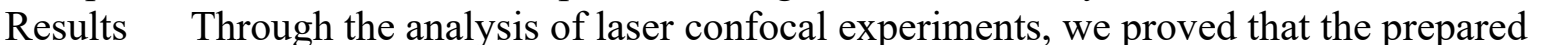

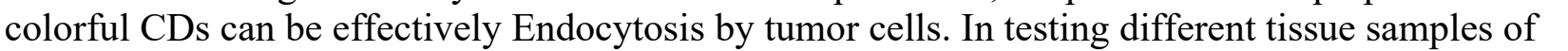

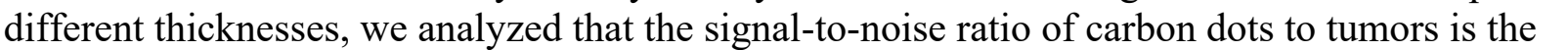

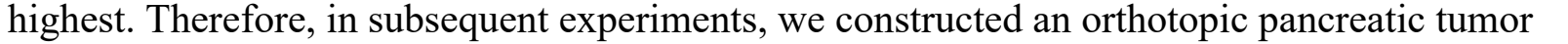

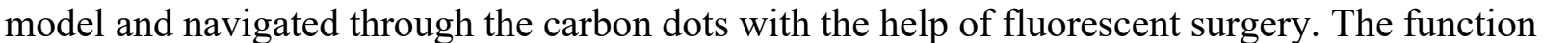

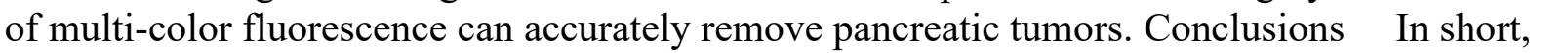

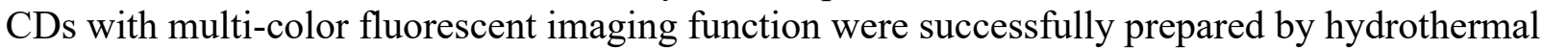

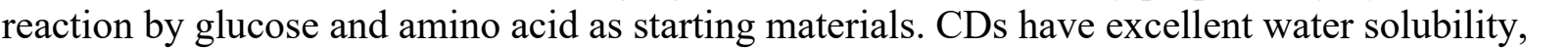

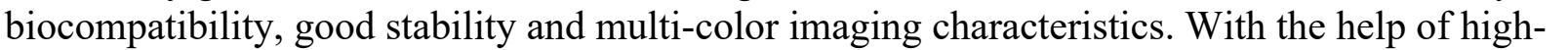

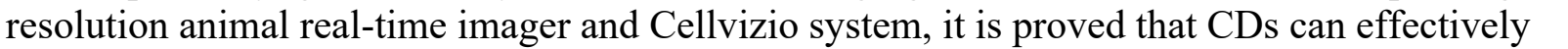

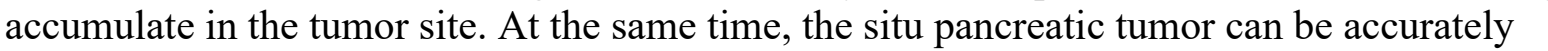




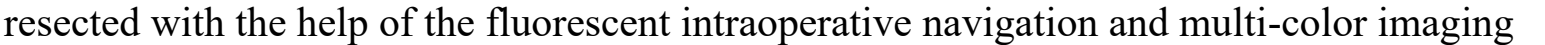

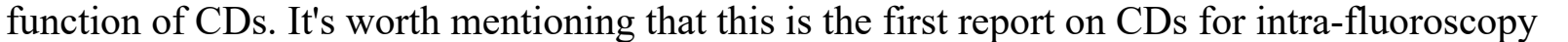

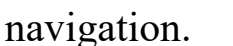

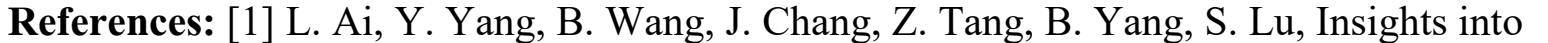

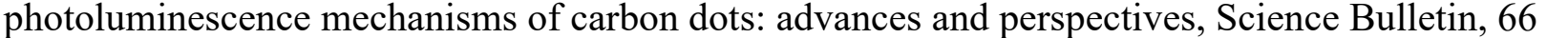

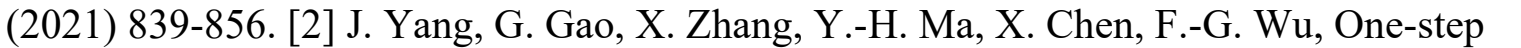

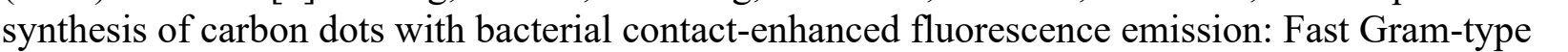

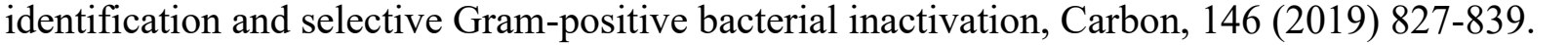

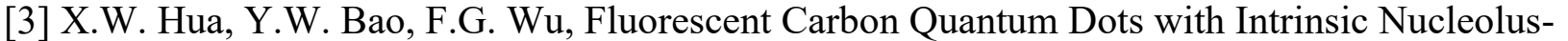

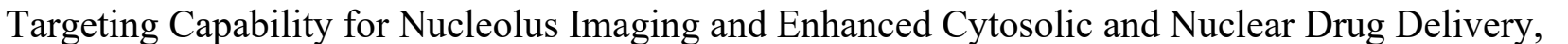

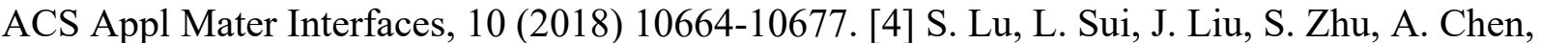

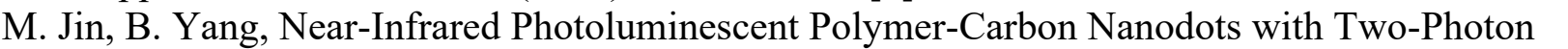

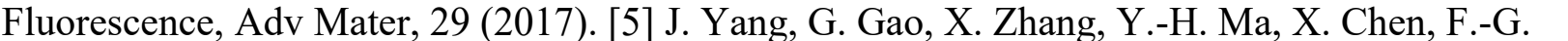

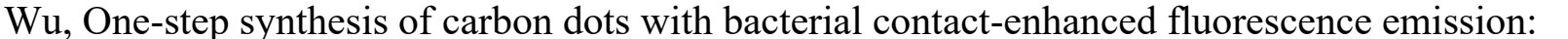

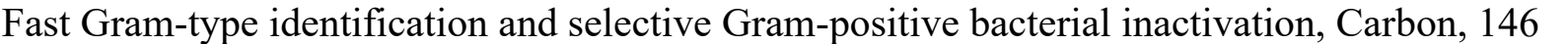

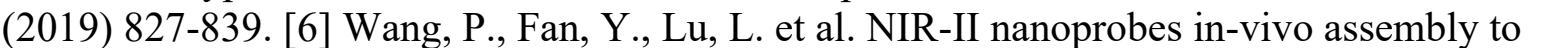

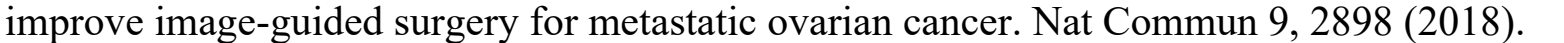

First Name:

Last Name: $\square \| \square$

Email: $\square\|\|\|\square \square\|\|\|\|\| \square$

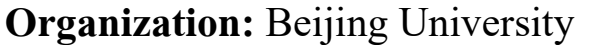

Country: $\square \square \| \square \square$ 


\title{
ID: $\mathbf{L B 8 8}$ \\ Aptamer-functionalized microbubbles: ultrasound molecular imaging using a fluorescent anti-P-selectin aptamer for imaging inflammation in murine models of acute bowel inflammation.
}

\author{
Una Goncin, University of Saskatchewan, ung039@usask.ca
}

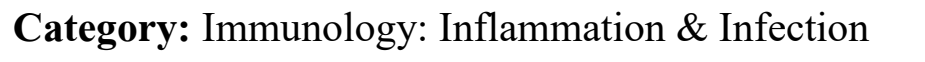

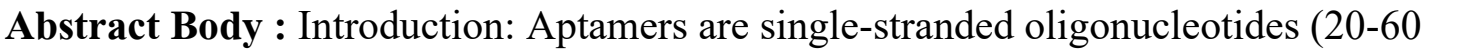

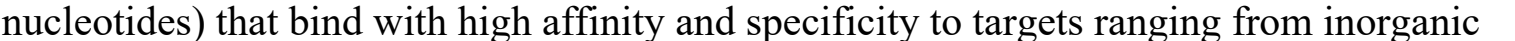

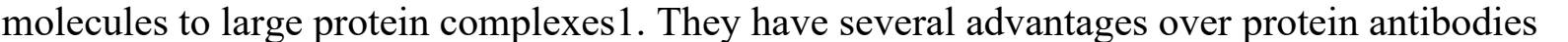

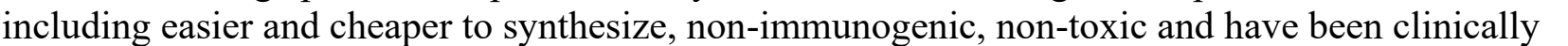

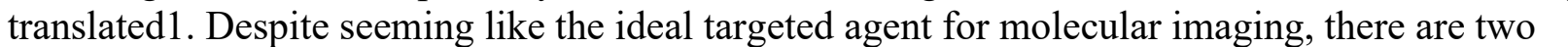

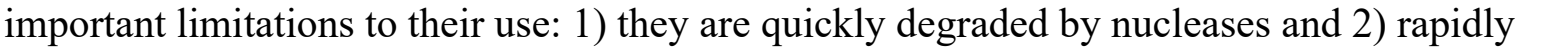

ए

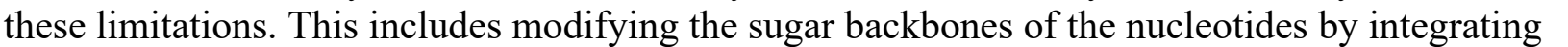

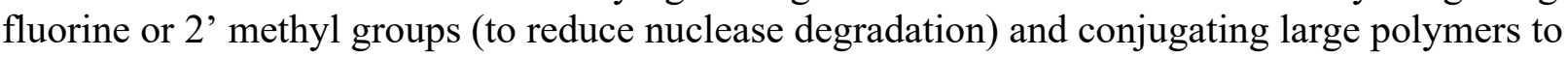

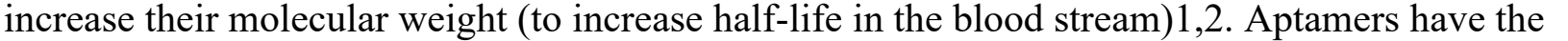

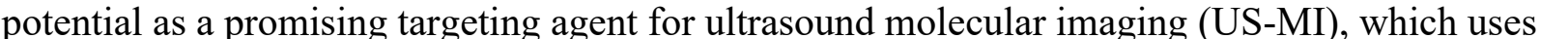

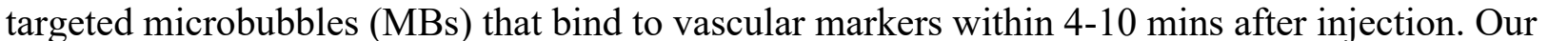

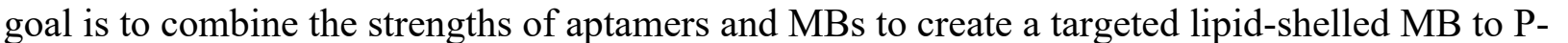

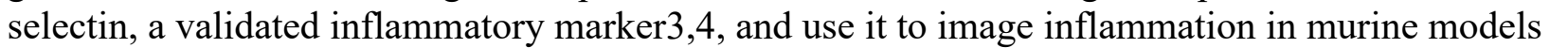

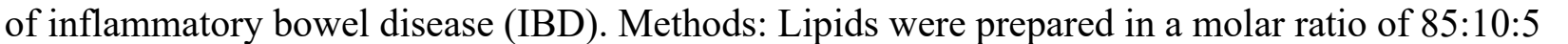

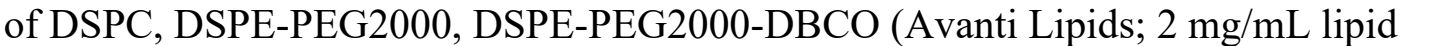

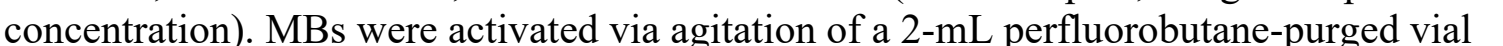

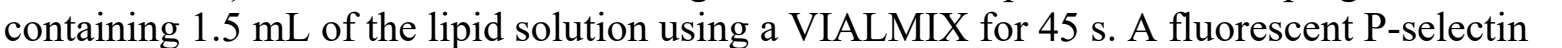

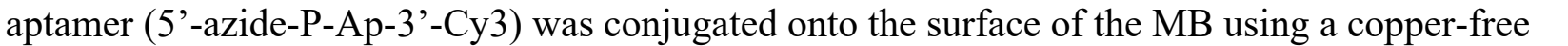

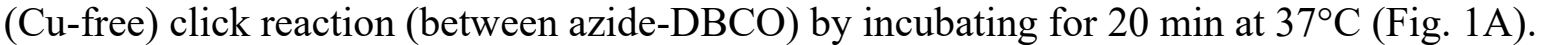

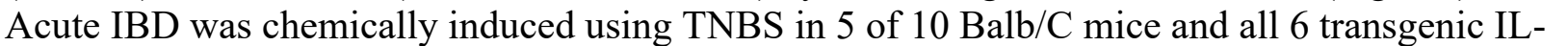

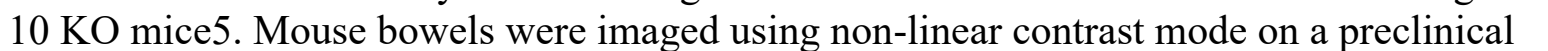

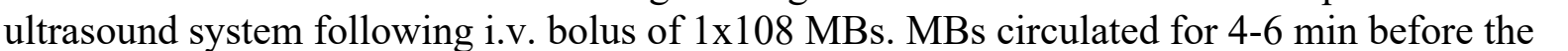

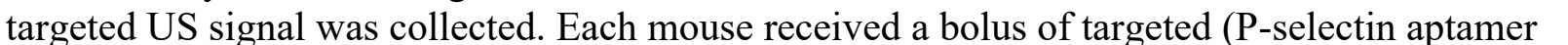
ए

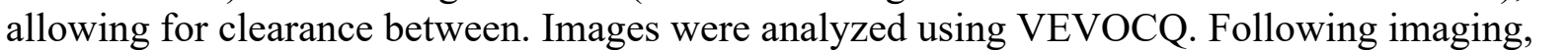

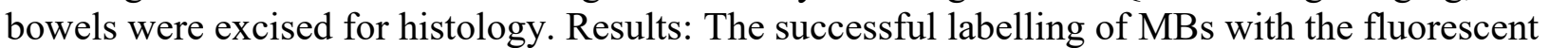

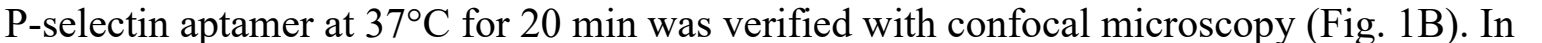

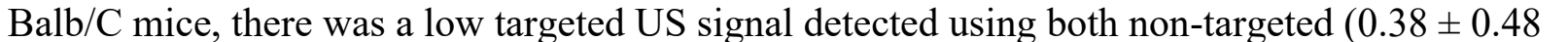

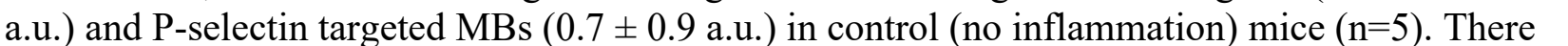

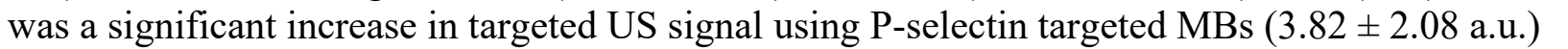

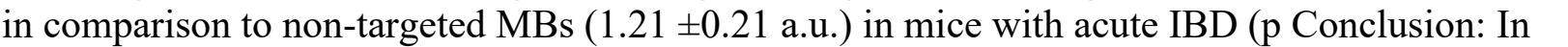

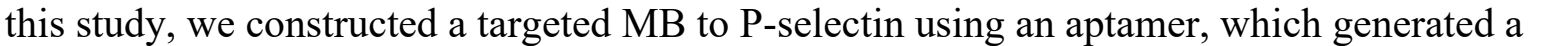

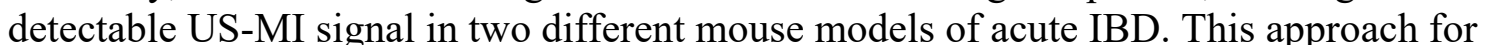




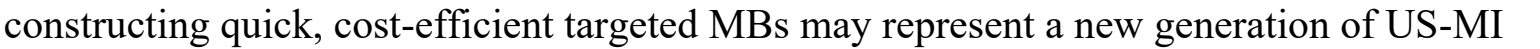

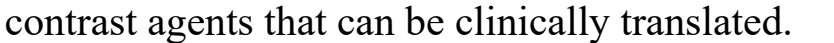

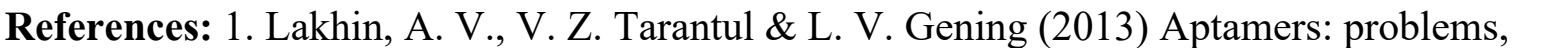

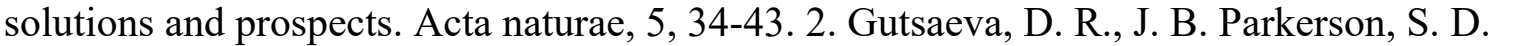

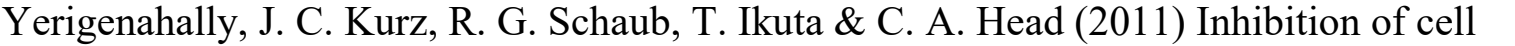

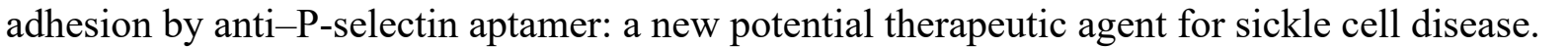

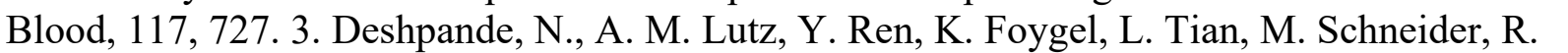

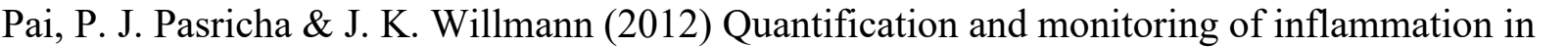

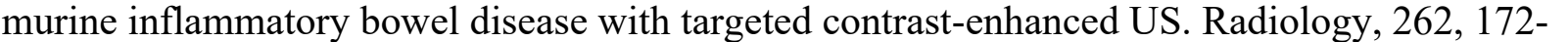

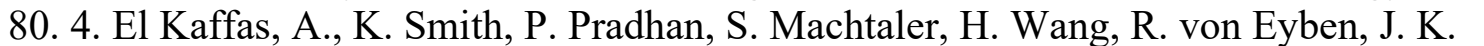

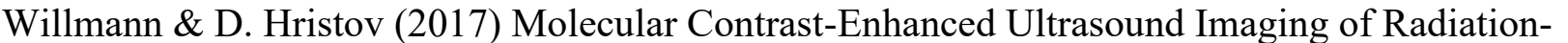

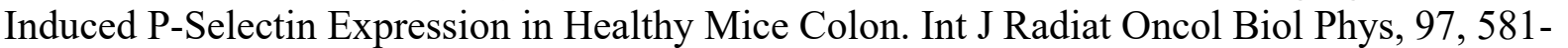

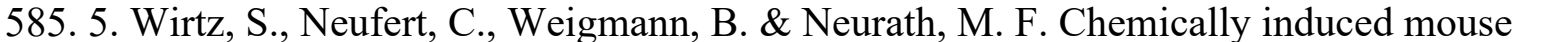

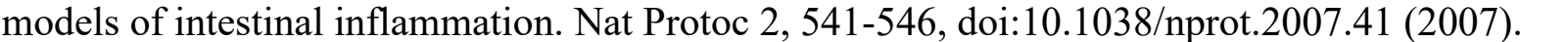

\section{Image/Figure:}

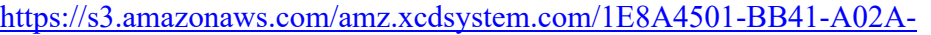

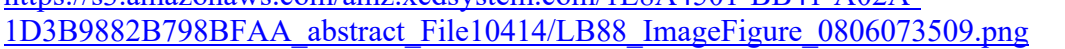

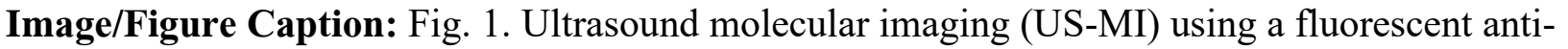

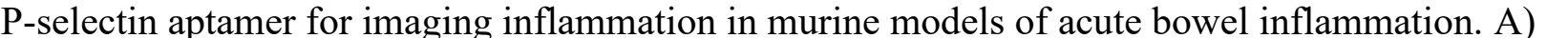

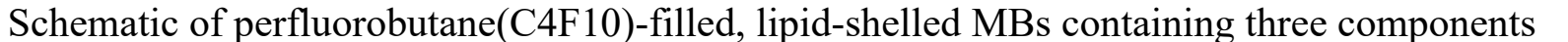

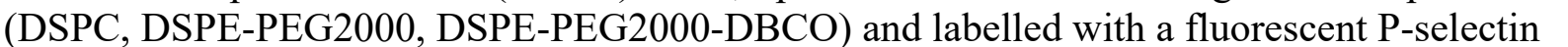

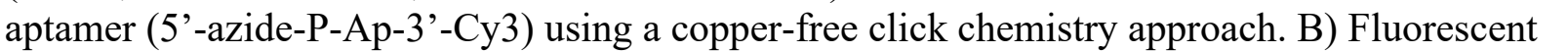

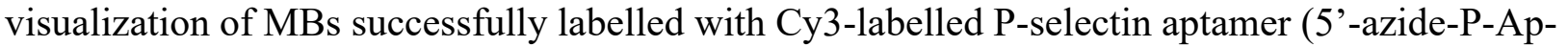

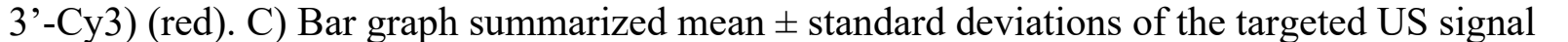

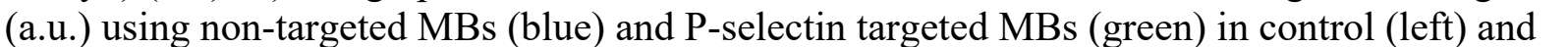

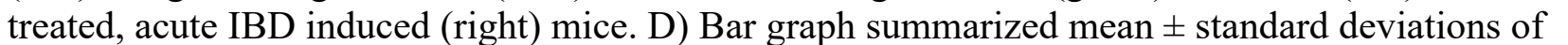
ए

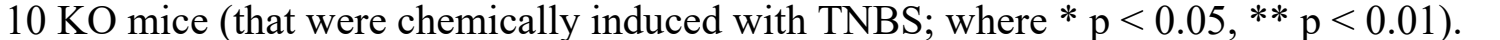

First Name:

Last Name: $\square \square \square\|\| \|$

Email: $\square\|\|\|\square \square\|\|\|\|\| \square$

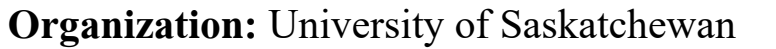

Country: $\square \square \square\|\Pi\|$ 


\title{
ID: LB89 \\ Detection of small hepatocellular carcinoma in cirrhosis using PAI/MPI with targeted novel nanoparticles binding GPC3 in vivo
}

Han Deng, The southern medical university,zhujiang hospital, d_hann@163.com

\section{Category: $\square \square\|\| \| \square$}

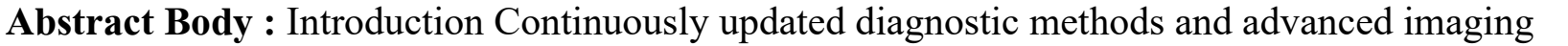

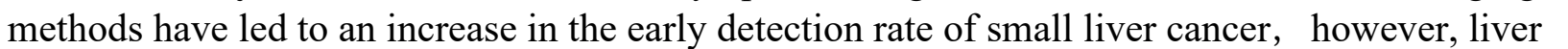

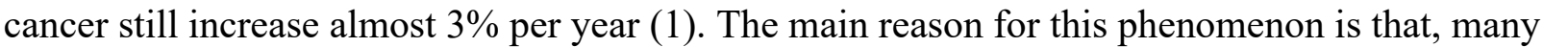

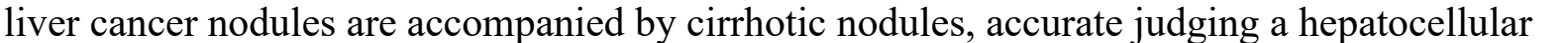

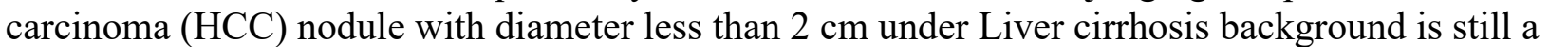

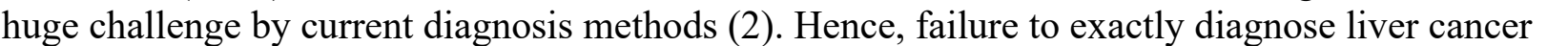

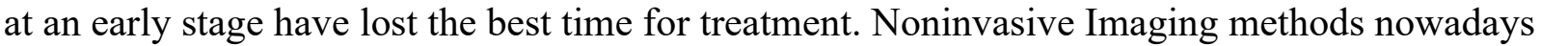

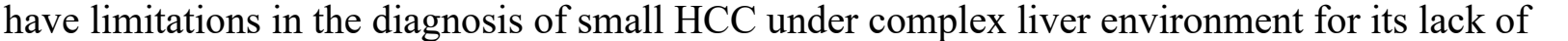

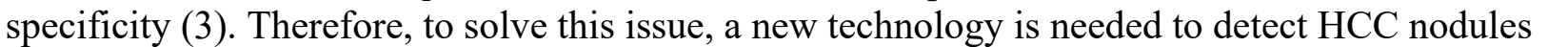

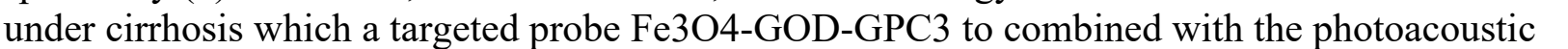

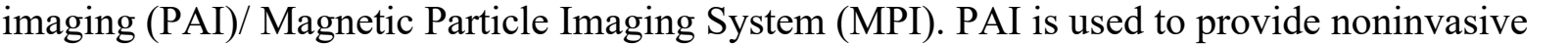

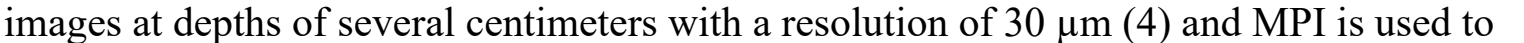

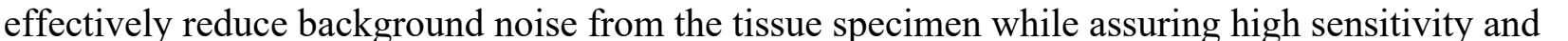

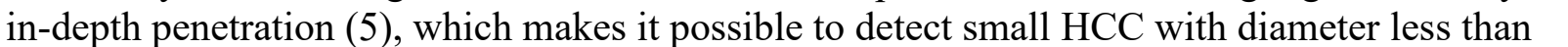

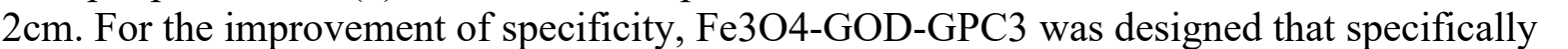
एणी

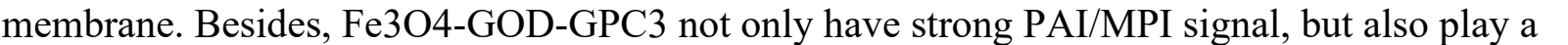

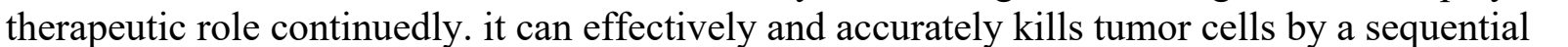

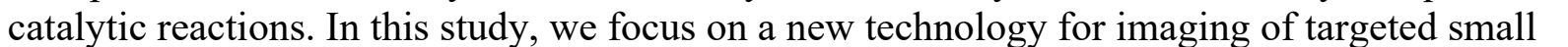

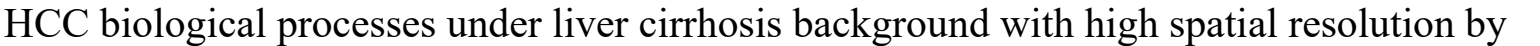

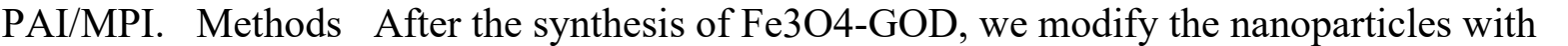

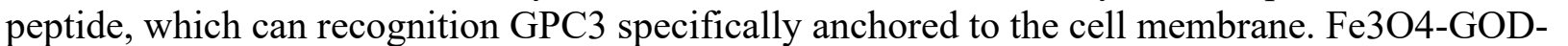

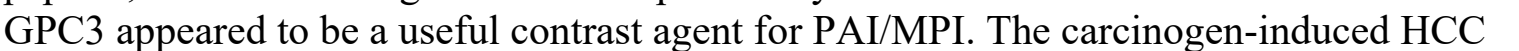

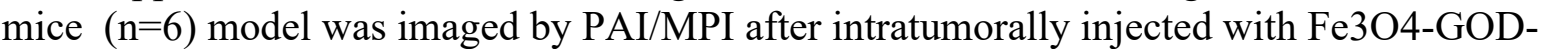

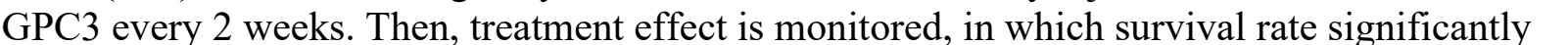

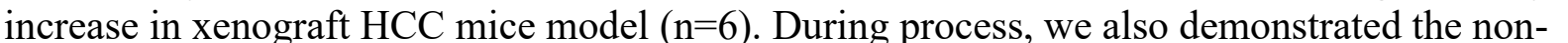

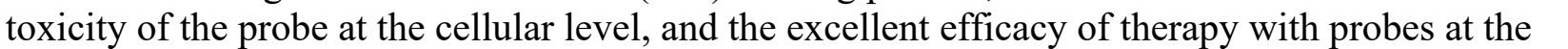

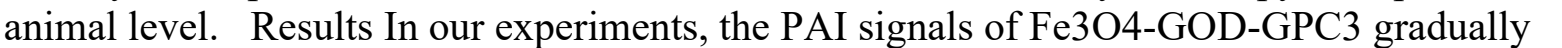

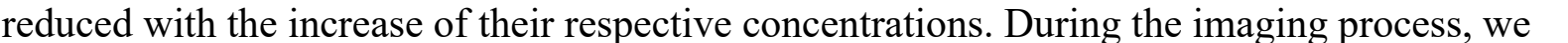

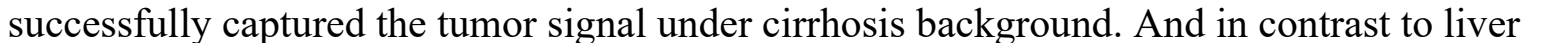

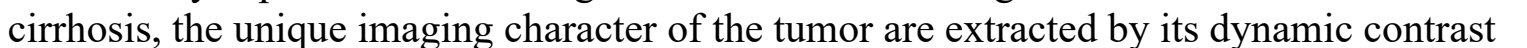

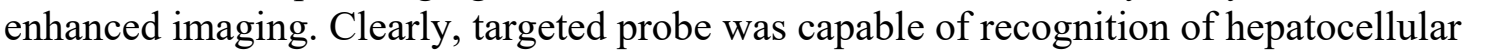

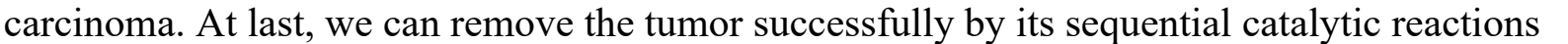

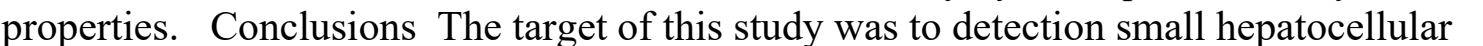

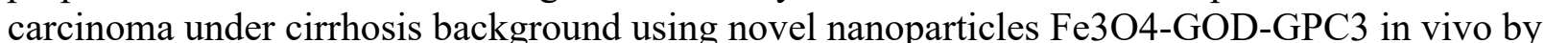




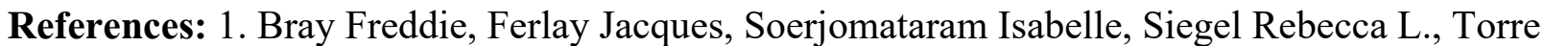

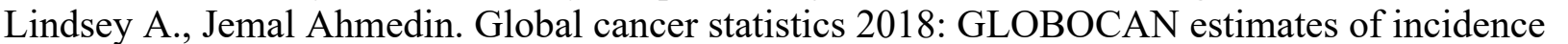

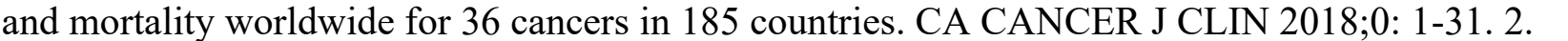

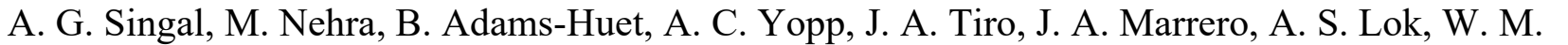

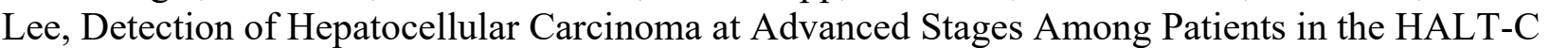

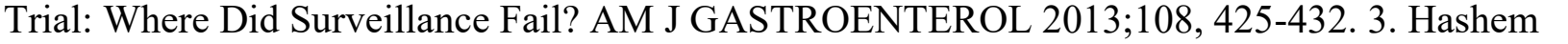

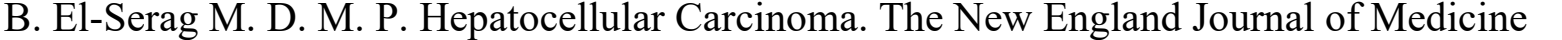

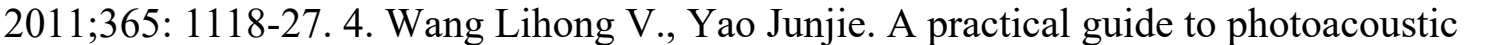

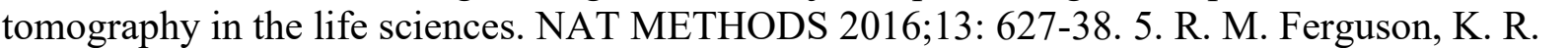

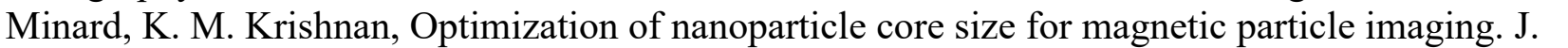

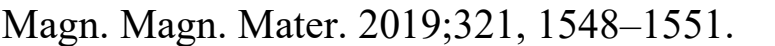

First Name:

Last Name: $\square \square \square \square$

Email: $\square \square \| \square \square \square \square \square \square \square$

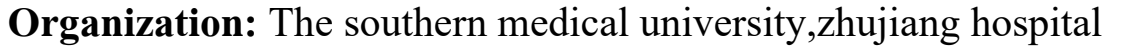

Country: $\square \square \square \mid \square$ 


\title{
ID: LB90 \\ Changes in the microenviroment matrix of the pancreatic cancer reflected by the magnetic partical imaging
}

\author{
chanjuan guo, Southern Medical University, gcj151221@163.com
}

\section{Category: $\square \square\|\| \| \square$}

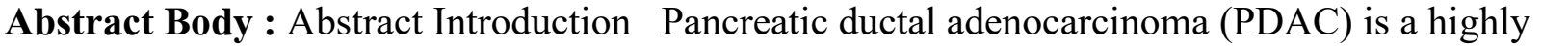

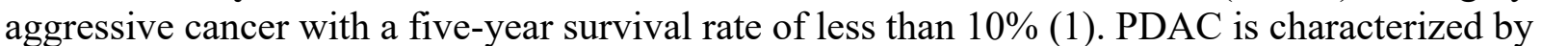

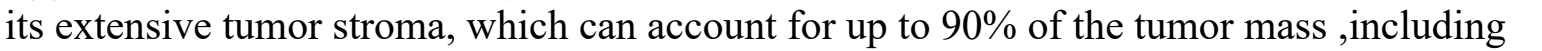

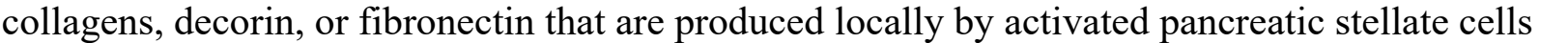

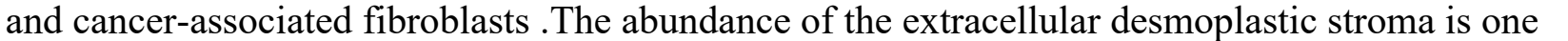

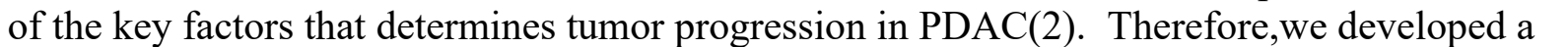

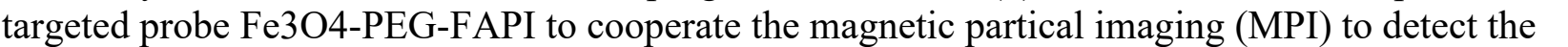

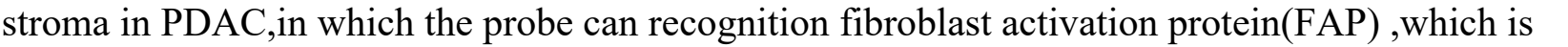

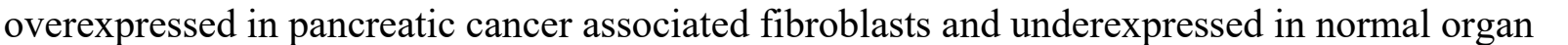

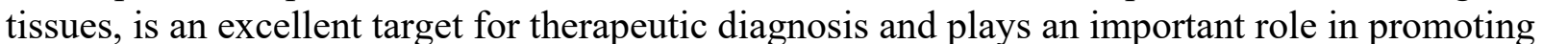

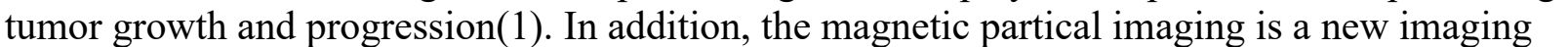

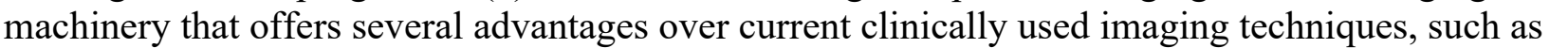

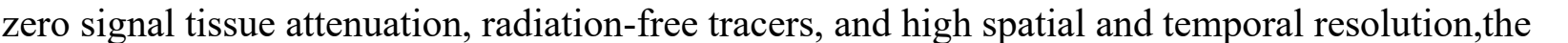

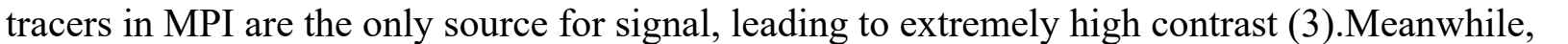

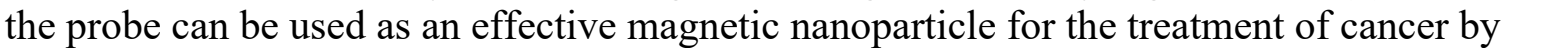

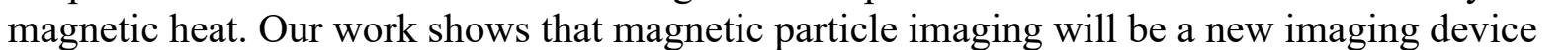
ए

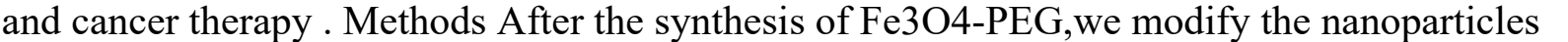

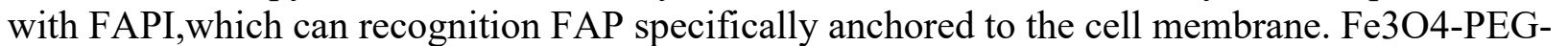

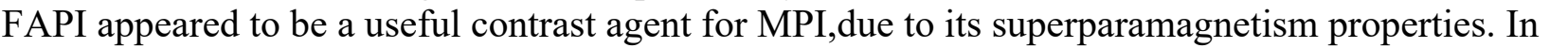

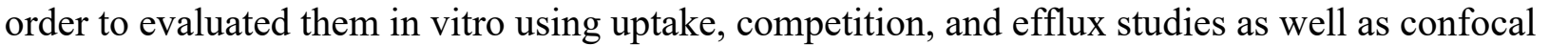

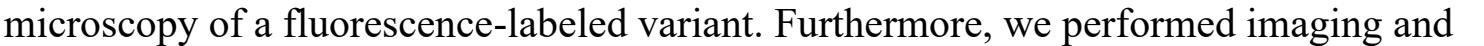

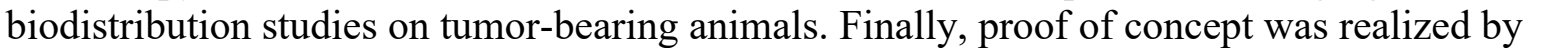
प

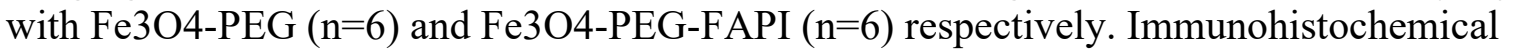

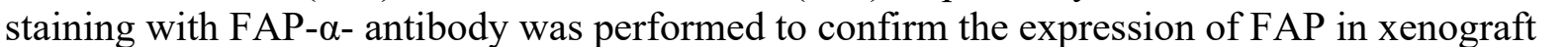

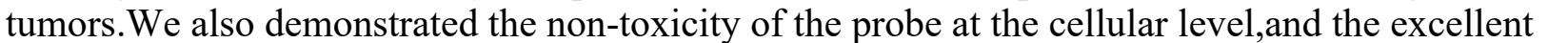

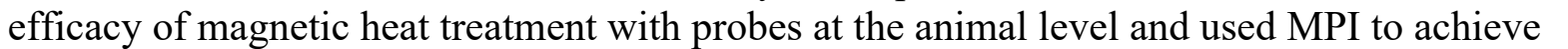

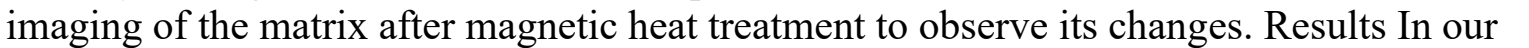

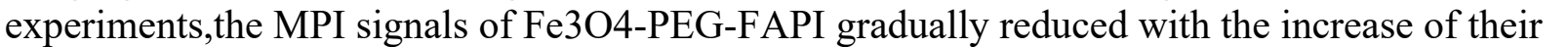

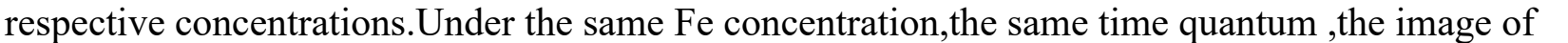

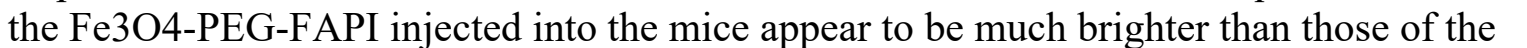

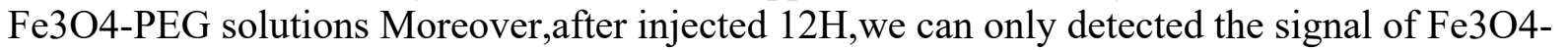

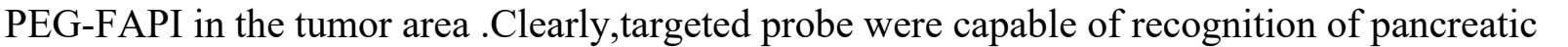

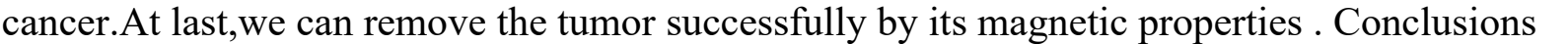

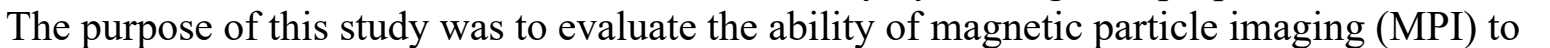




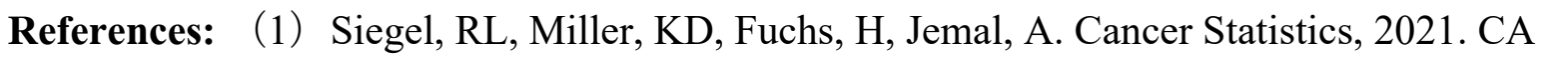

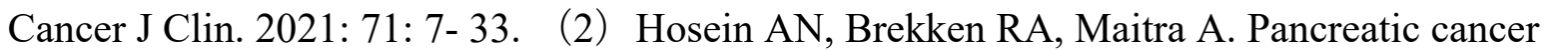

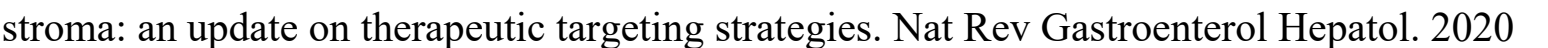

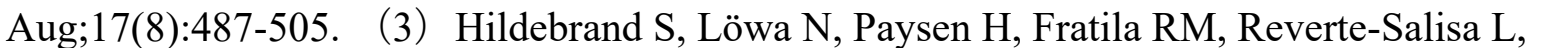

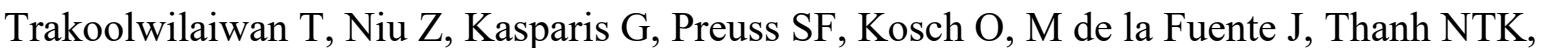

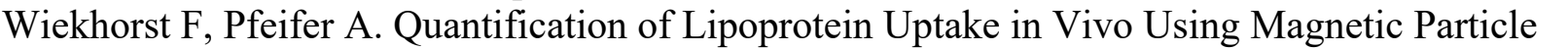

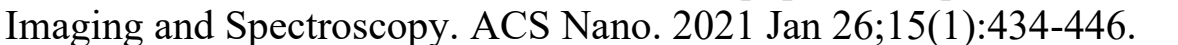

First Name: $\square|||||| \mid \square$

Last Name: $\square \square \|$

Email: $\square\|\|\|\|\|\square \square \square\| \square \square \square$

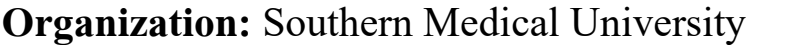

Country: $\square \square \square \mid \square$ 


\title{
ID: LB91 \\ Cerenkov luminescence imaging (CLI) with [18F]fm-PBR28-d2 targeting TSPO for visualizing interscapular brown adipose tissues (iBAT) in UCP1 Thermomouse
}

Seok-Yong Lee, Seoul National University Hospital, iamlsy25451@snu.ac.kr

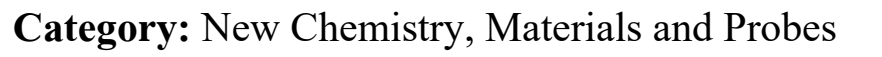

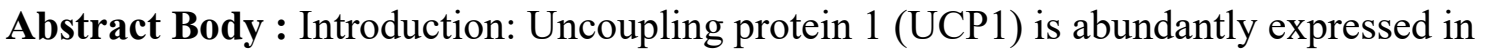

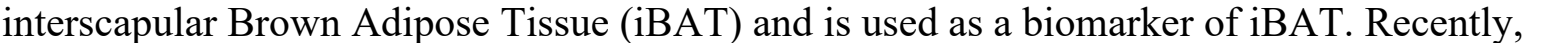

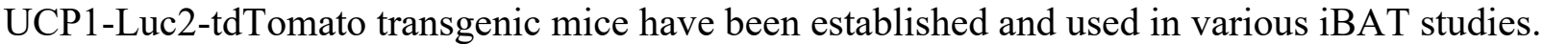

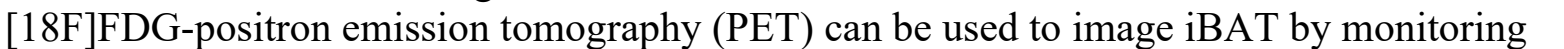

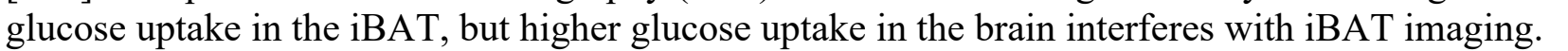

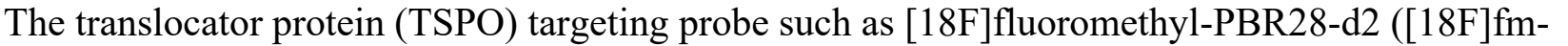

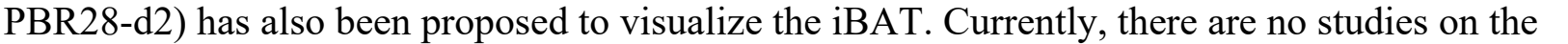

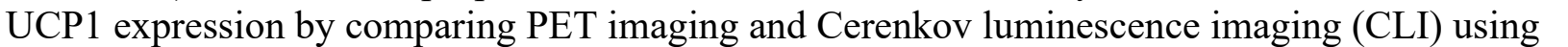

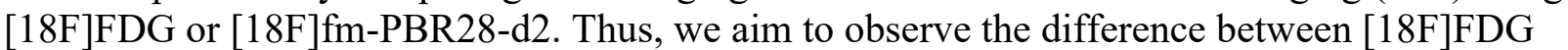

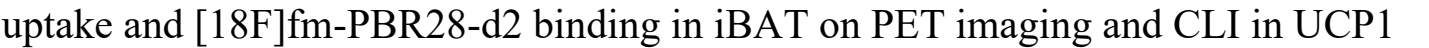

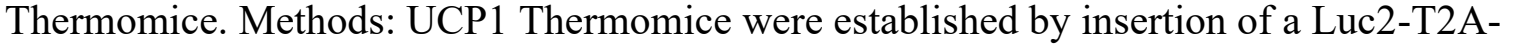
ए

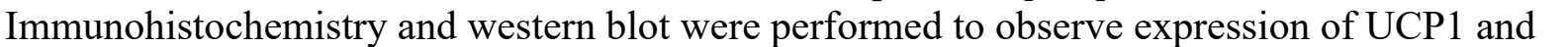

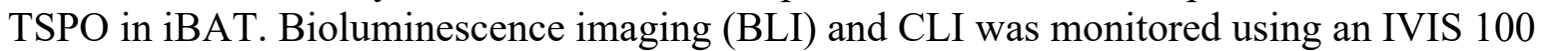

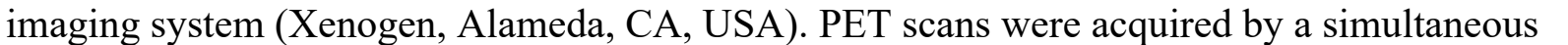

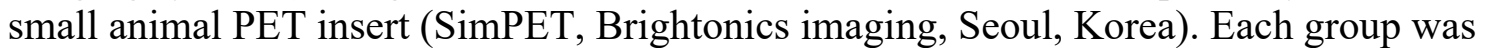

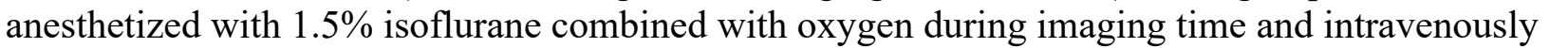

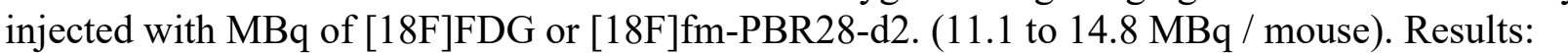

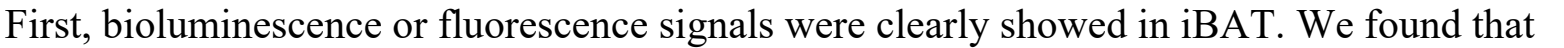

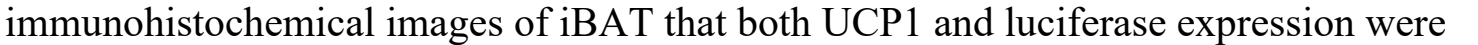

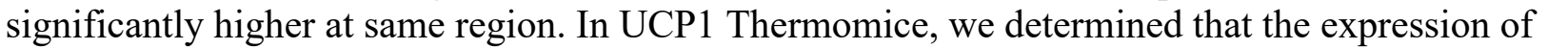

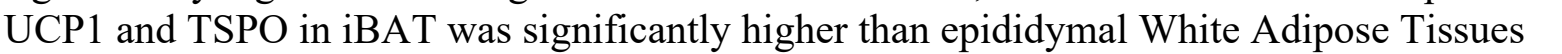

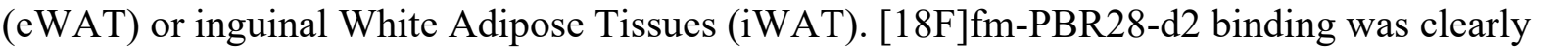

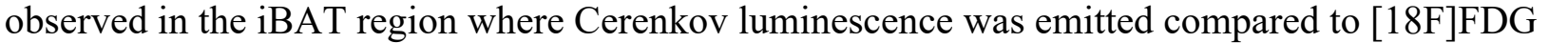

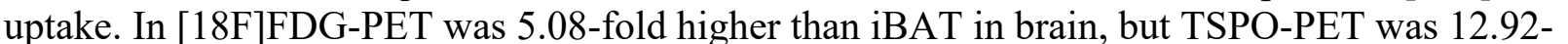

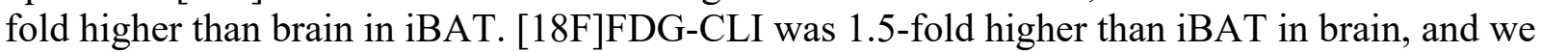

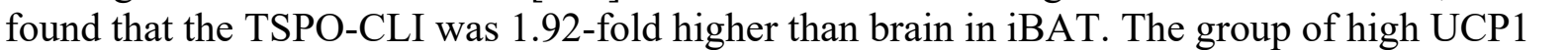

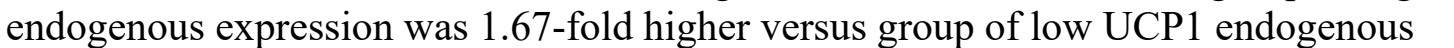

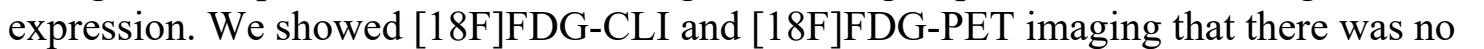

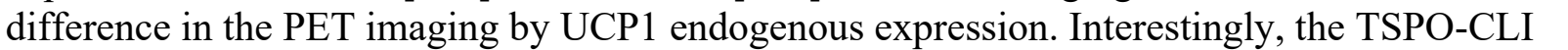

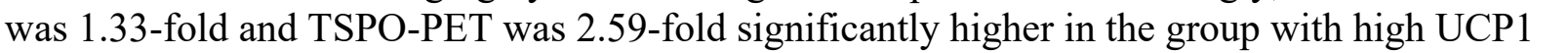

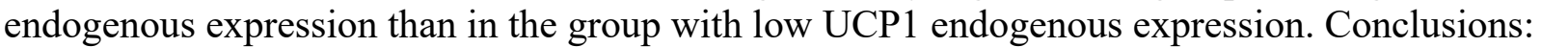

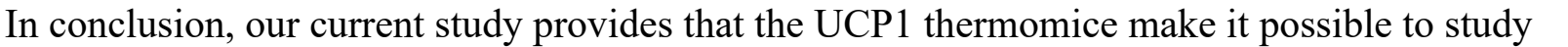

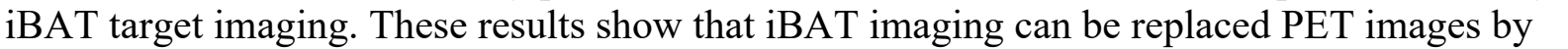

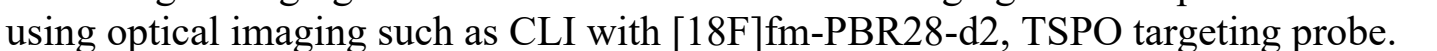


Full Name of Abstract's 1st Author : $\square \square \square \square \square \square \square \square \square \square$

First Name:

Last Name:

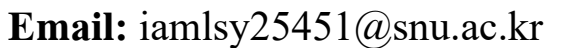

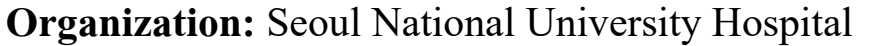

Country: $\square \square \square \square \square \square \square \| \square \square$ 


\title{
ID: LB92 \\ Copper-free click conjugation of P-selectin antibody to targeted microbubbles for ultrasound molecular imaging of inflammation in a murine model of inflammatory bowel disease
}

\author{
Una Goncin, University of Saskatchewan, ung039@usask.ca
}

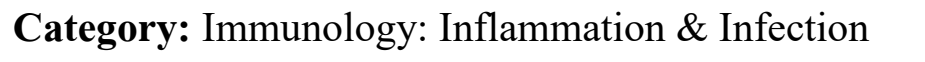

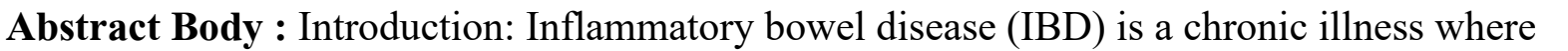

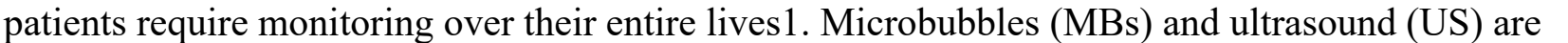

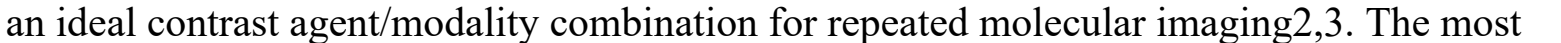

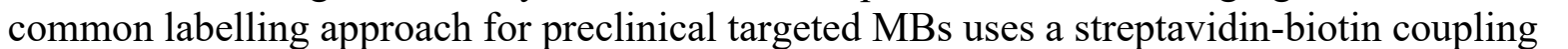

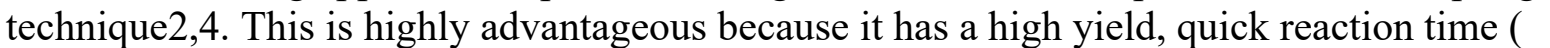

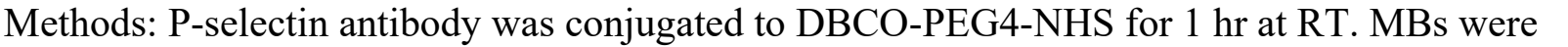

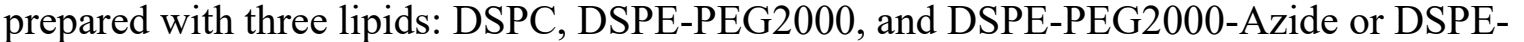

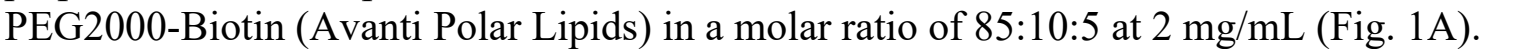

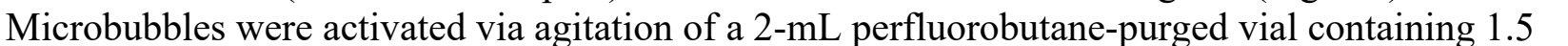

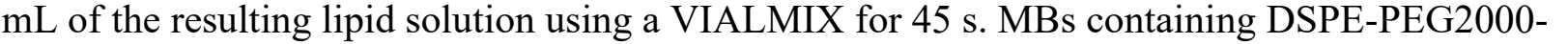

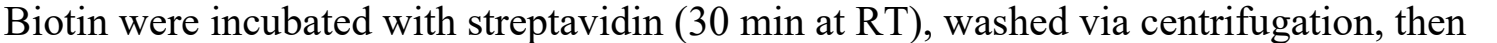

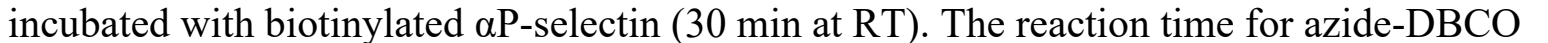

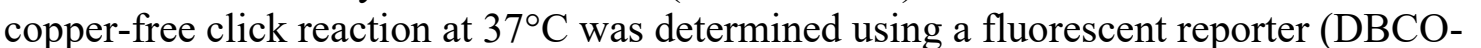

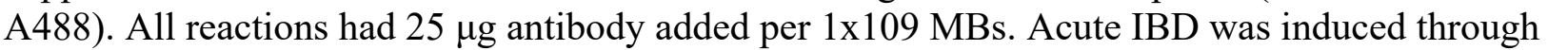

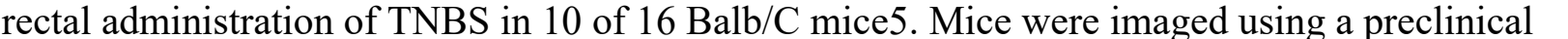

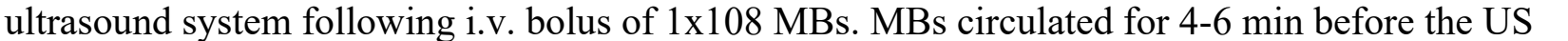

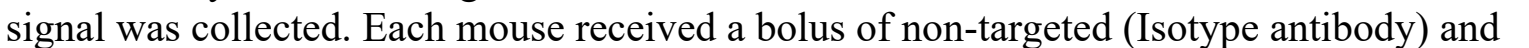

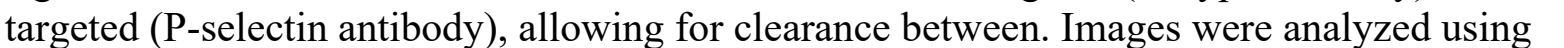

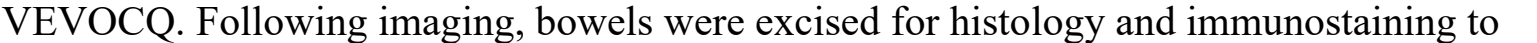

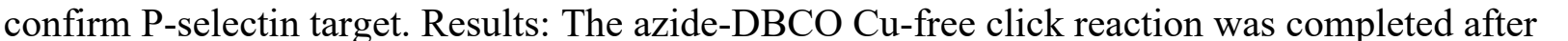

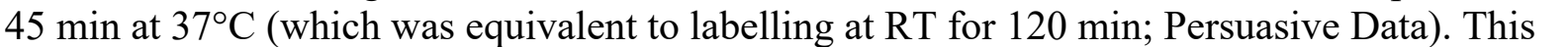

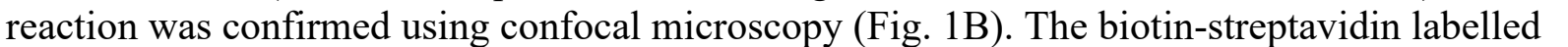

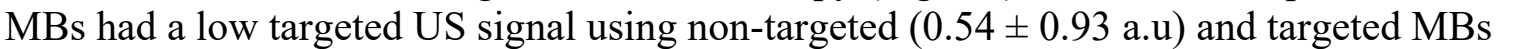

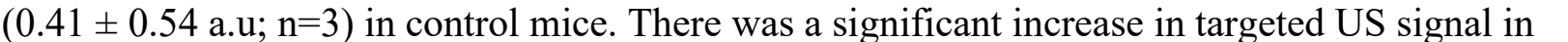

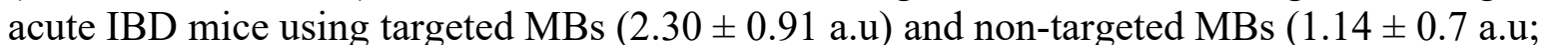

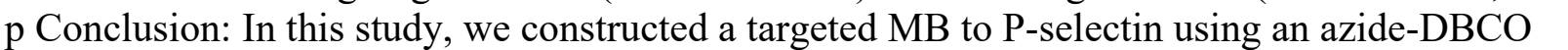

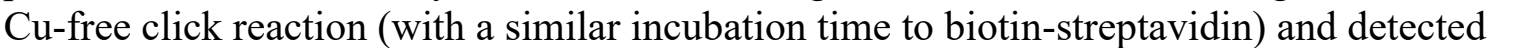
ए

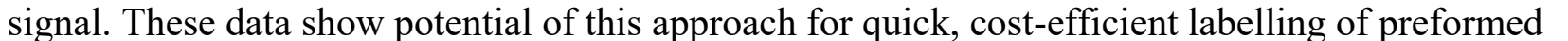

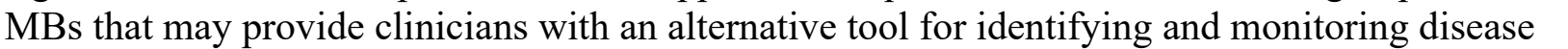

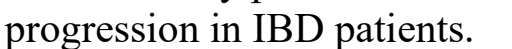

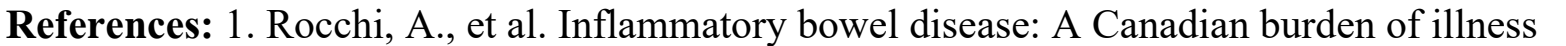

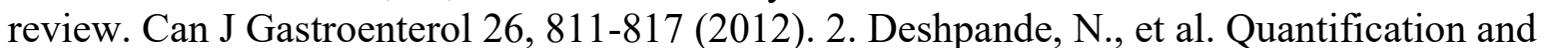

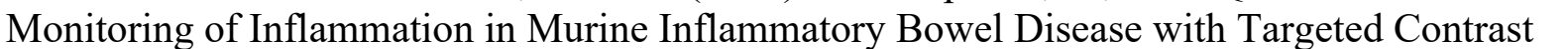




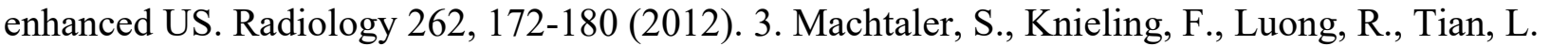

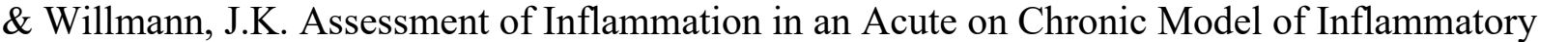

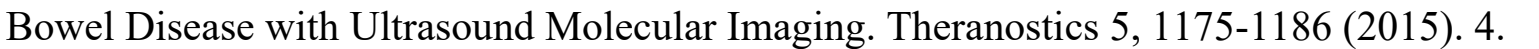

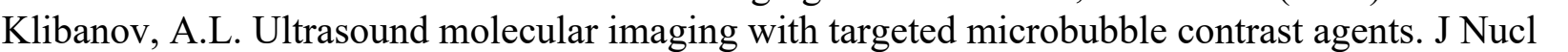

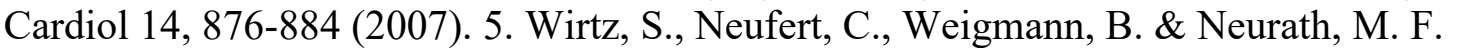

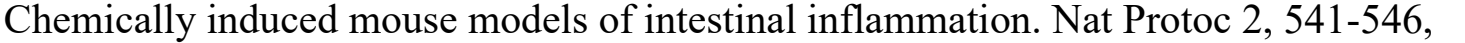

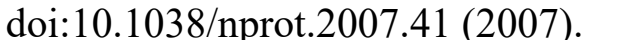

\section{Image/Figure:}

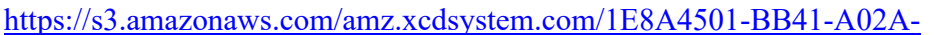

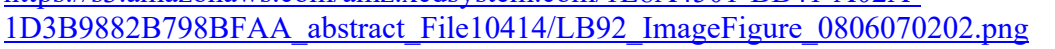

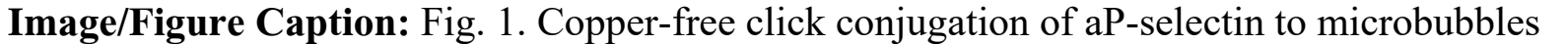

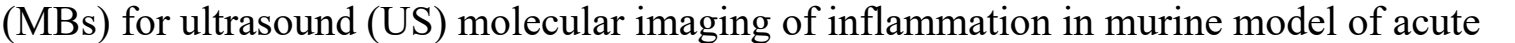
ए

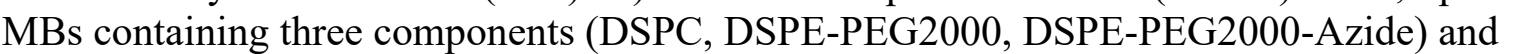

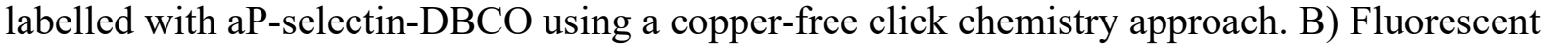

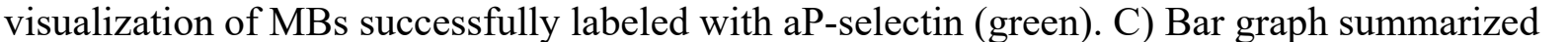

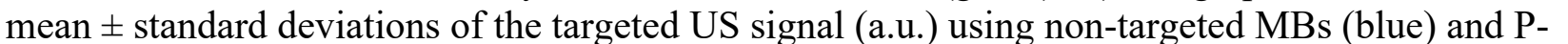

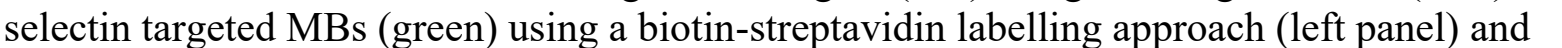

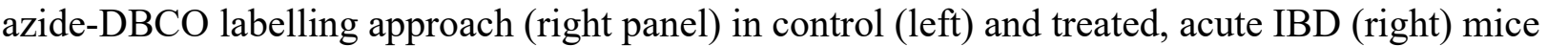

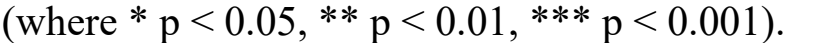

First Name:

Last Name: $\square \square \square\|\| \| \square$

Email: $\square \square\|\|\|\square \square\|\|\| \square\|\| \square$

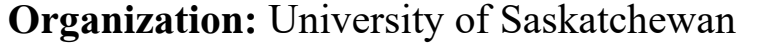

Country: $\square \square \square \square \square \square$ 


\title{
ID: LB93 \\ Optimizing Quantum Dot Probes for Multiplexed Preclinical Imaging in the NIR-II Optical Windows
}

\author{
Maria Moreno, National Research Council, maria.moreno@nrc-cnrc.gc.ca
}

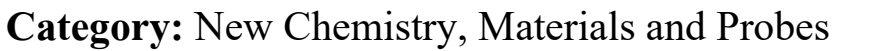

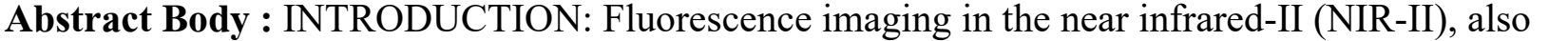

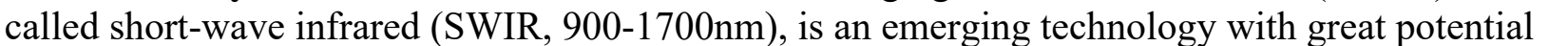

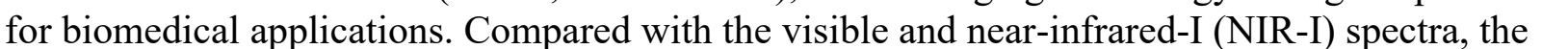

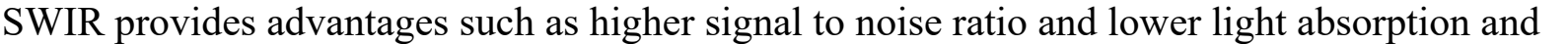

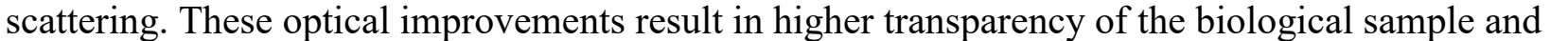

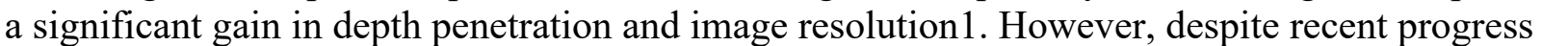

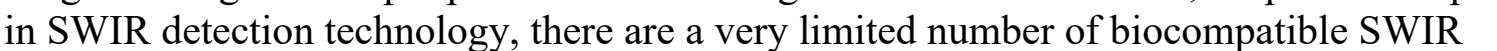

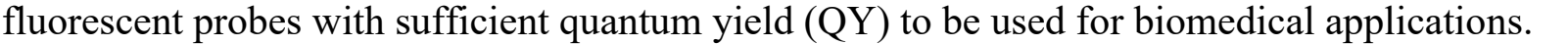

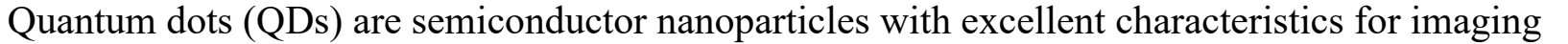

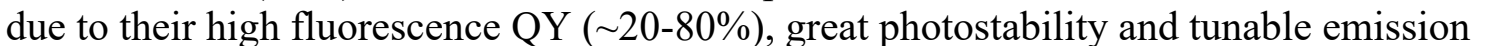

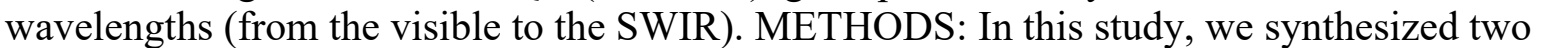

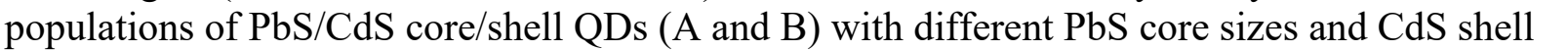

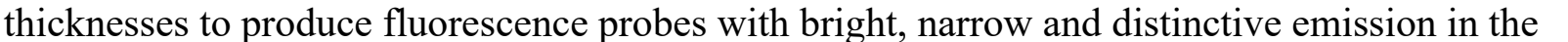

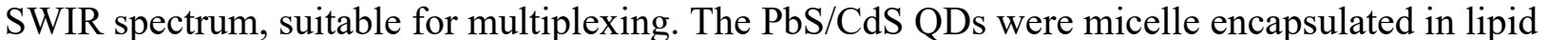

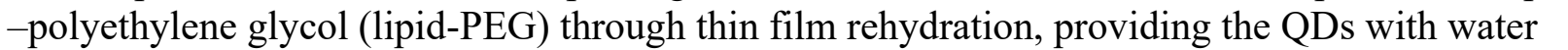
solubility and terminal azide functional groups available to 'click react' with any moiety

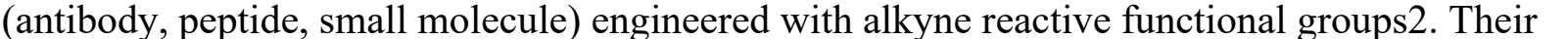

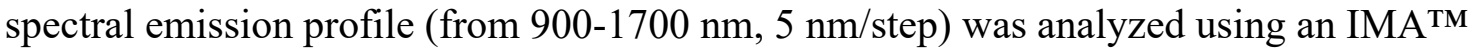

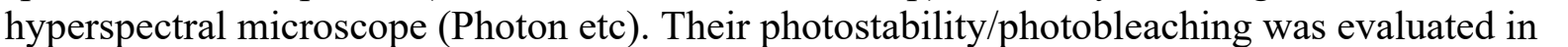

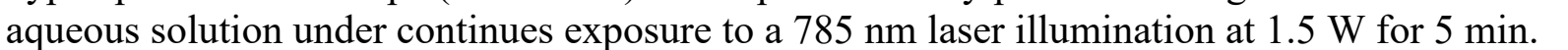

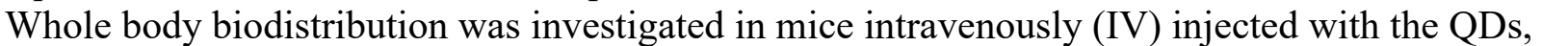

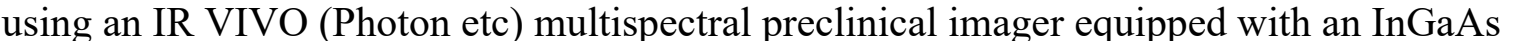

ZephIR $^{\text {TM }}$ 1.7) detector. RESULTS: The spectral profile of the two populations of PbS/CdS

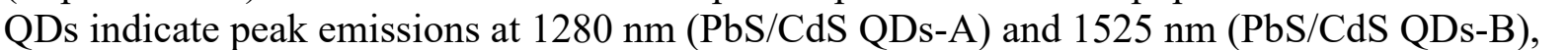

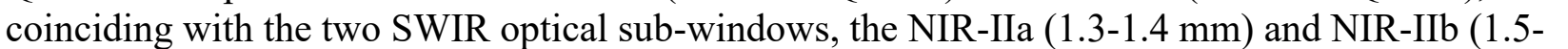

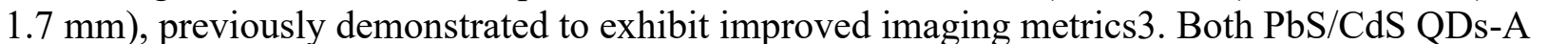

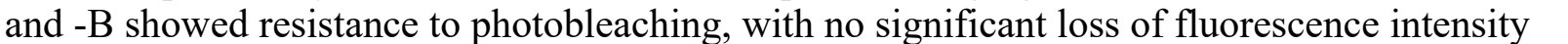

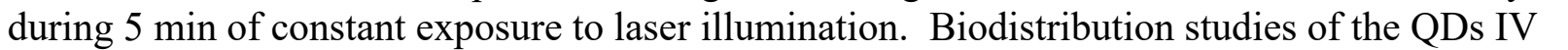

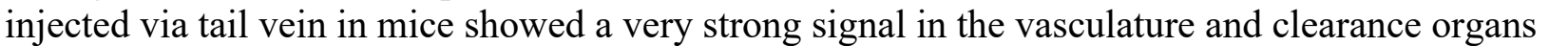

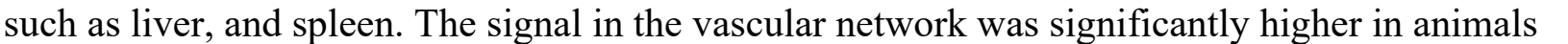

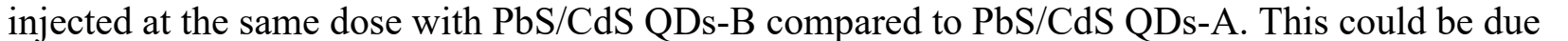
ए

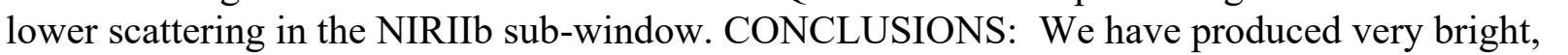

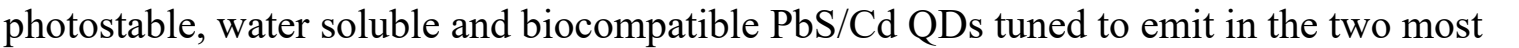

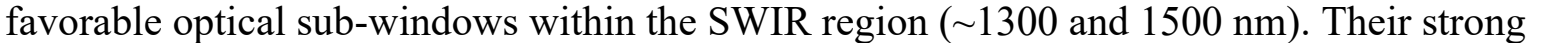

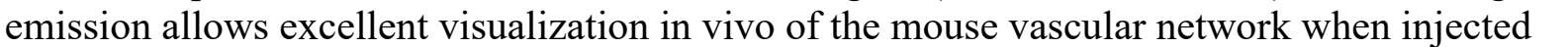




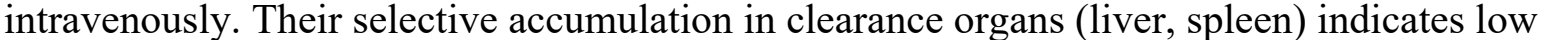

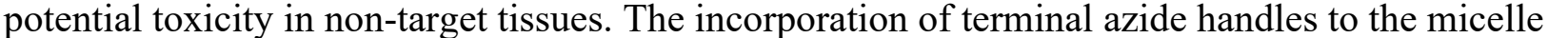

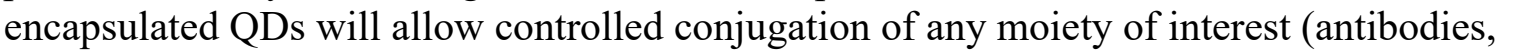
proteins, peptides, etc) by ' copperffree click chemistry'. This functionality can be exploited to

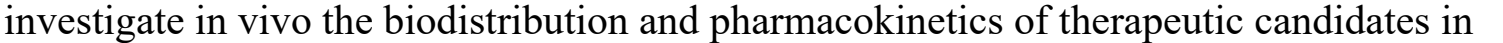

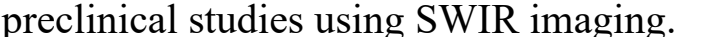

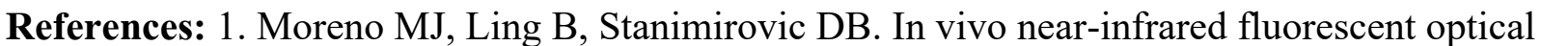

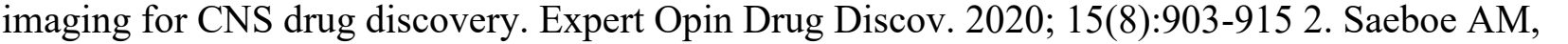

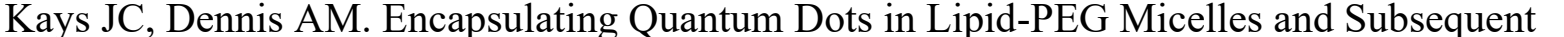

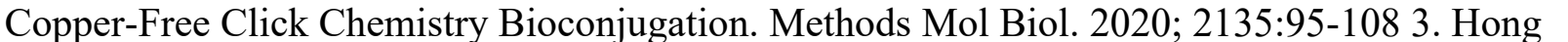

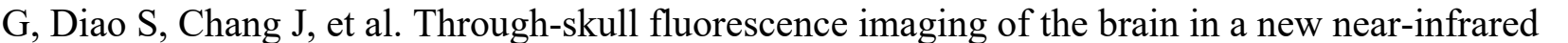

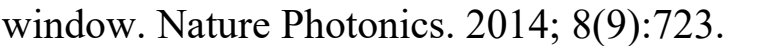

First Name: $\square \square \square \square \square$

Last Name: $\square \square \square \square \square \square$

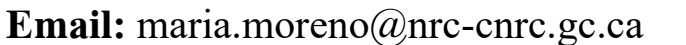

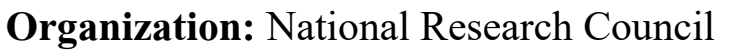

Country: $\square \square \square \square \| \square$ 


\title{
ID: LB94
}

Human Claudin-3 monoclonal antibody as a potential multimodal theranostic probe in ovarian cancer

Sera Oh, Seoul National University, ohseraaaa@snu.ac.kr

\section{Category: $\square \square\|\| \| \square$}

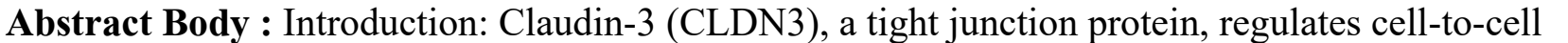

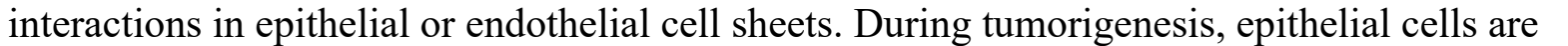

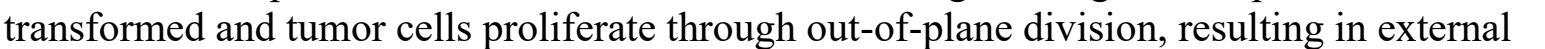
प

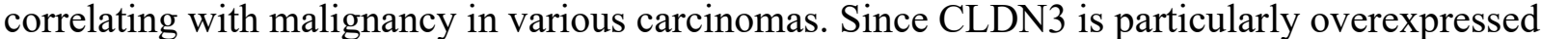

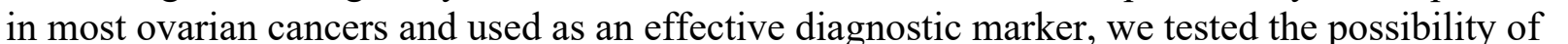

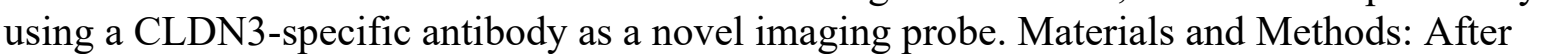

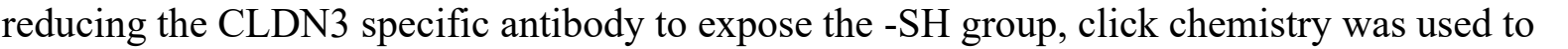

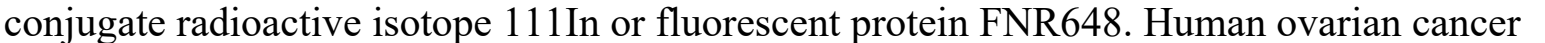

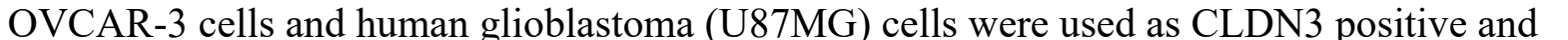

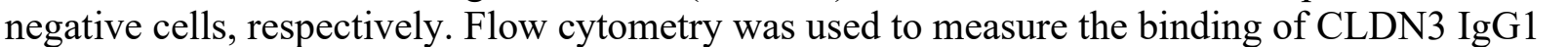

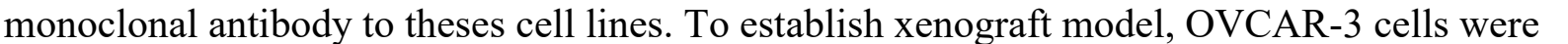

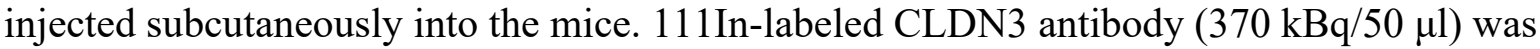

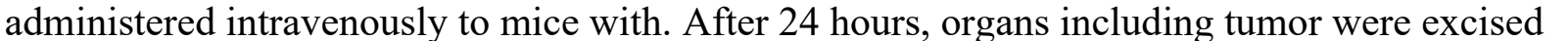

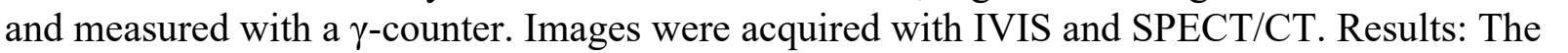

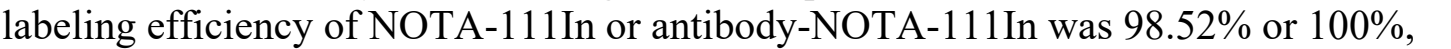

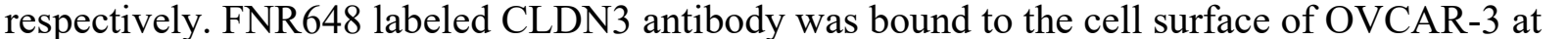

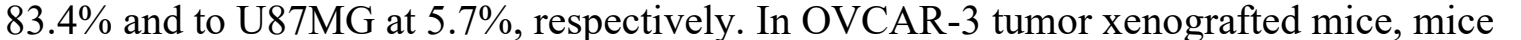
ए

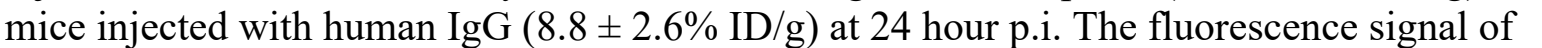

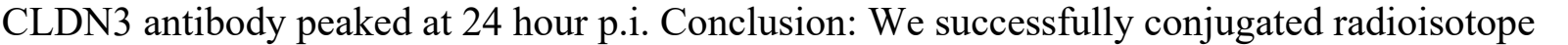

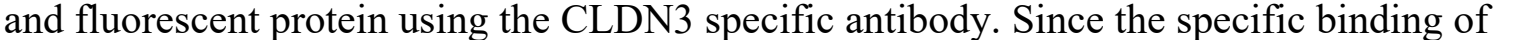

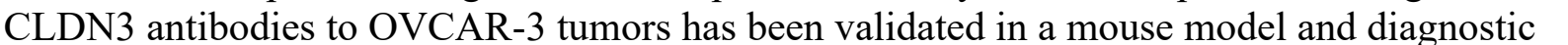

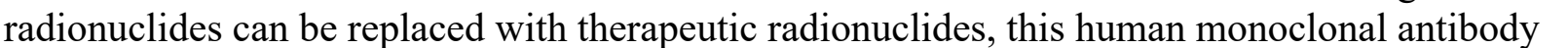

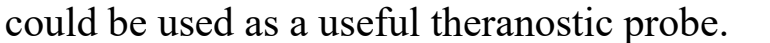

Full Name of Abstract's 1st Author : $\square \square\|\| m \square \square$

First Name:

Last Name: $\square \square \square$

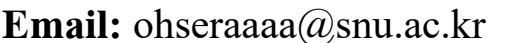

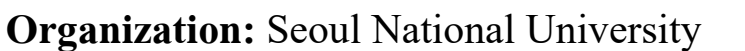


Country: 


\title{
ID: LB95 \\ Evaluation of an GPC3 and PSMA Dual-receptor Targeting Tracer for Hepatocellular carcinoma Imaging
}

li xing Chen, Tongji Hospital, Tongji Medical College, Huazhong University of Science and Technology, lixing-ch@foxmail.com

Category: $\square \square\|ा\| ा \|$

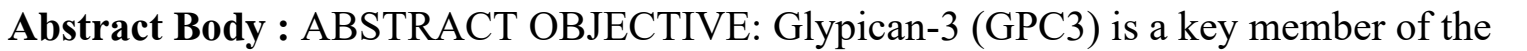

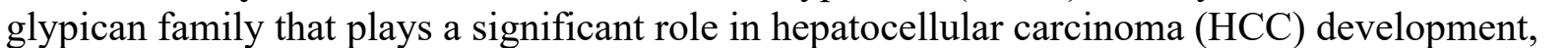

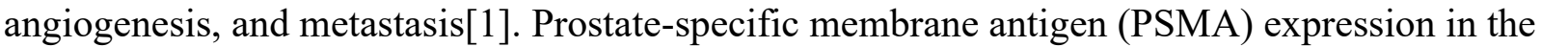
ए

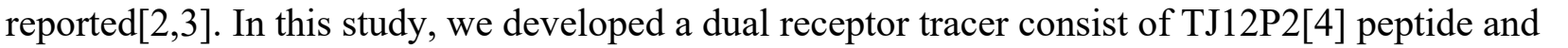

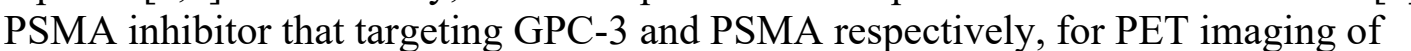

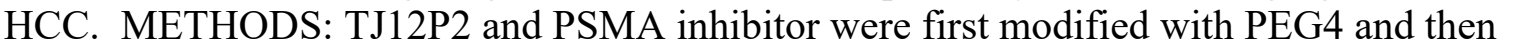

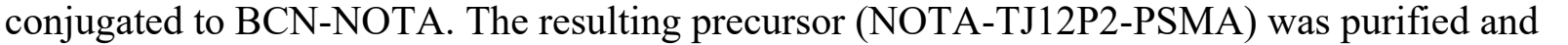

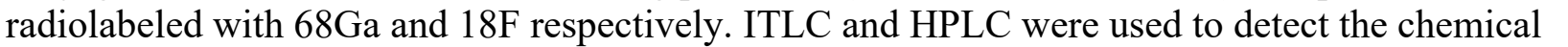

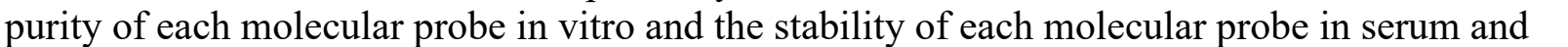

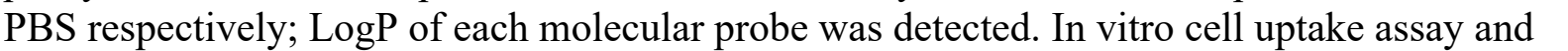

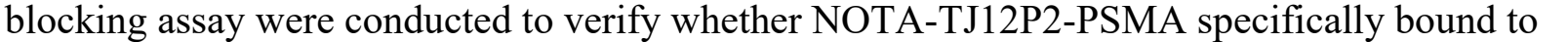

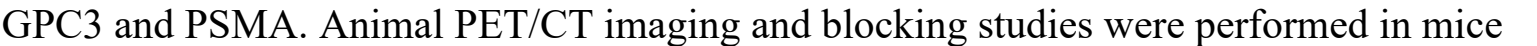

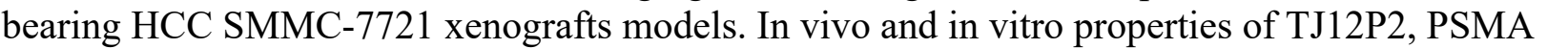

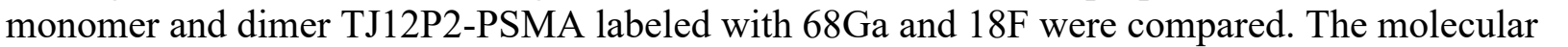

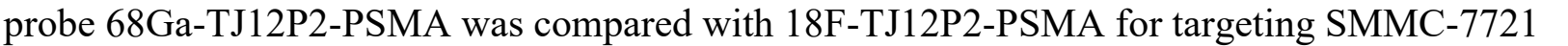
ए

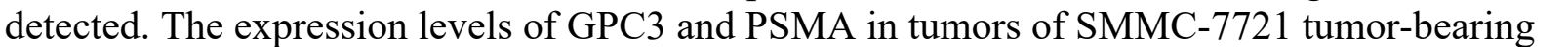

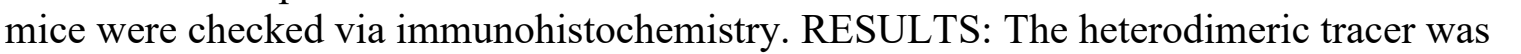

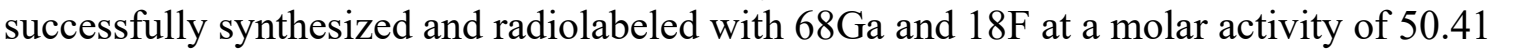
घणm

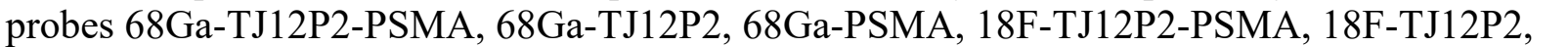

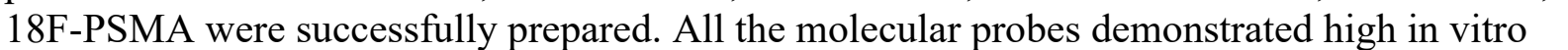

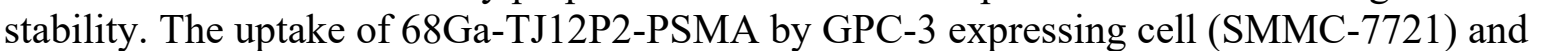

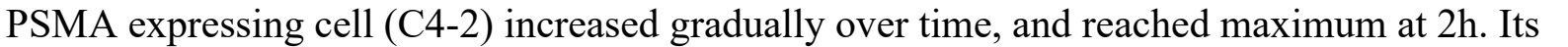

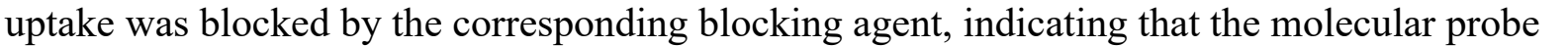

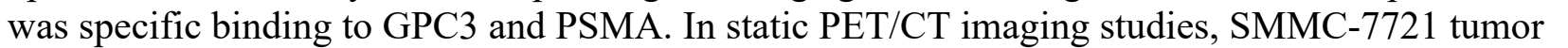

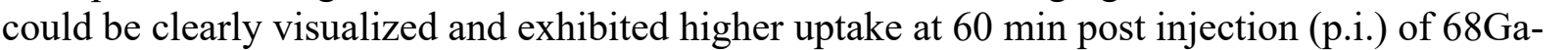

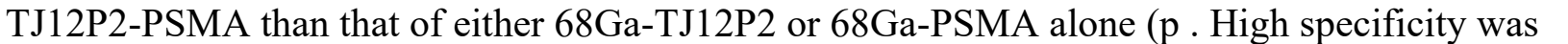

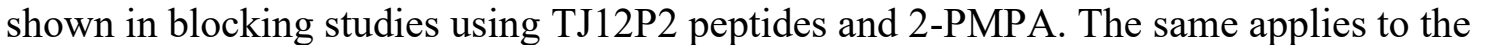

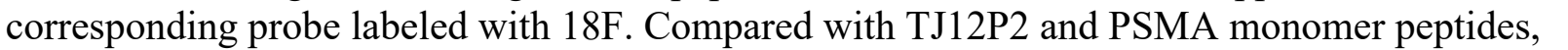

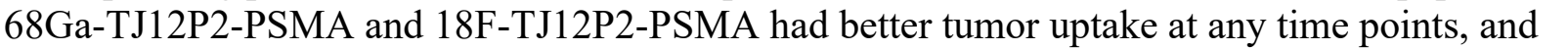

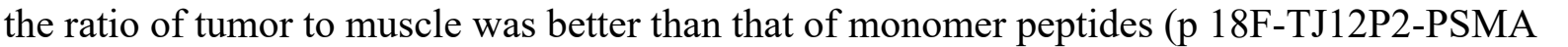

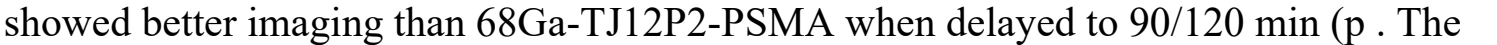

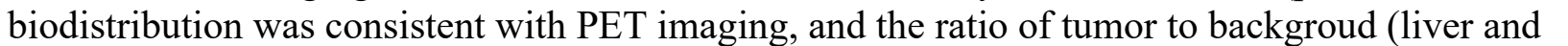




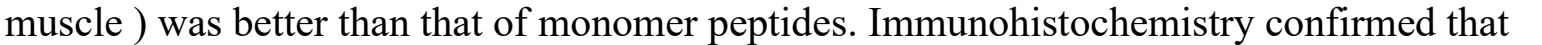

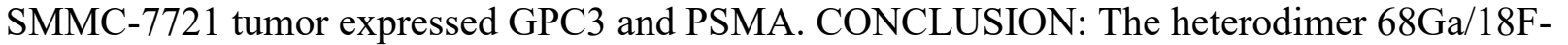

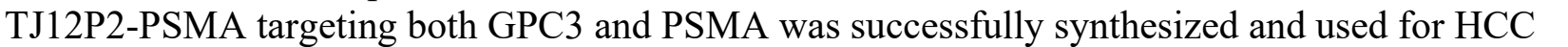

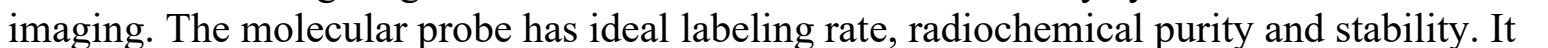

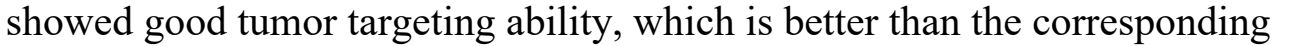

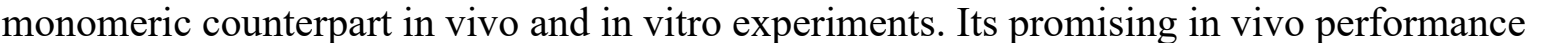

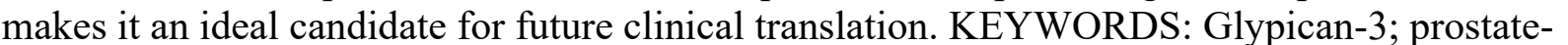

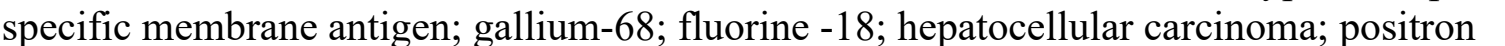

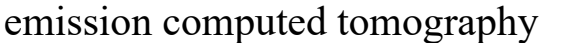

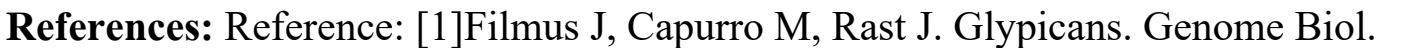

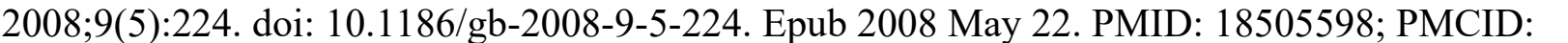

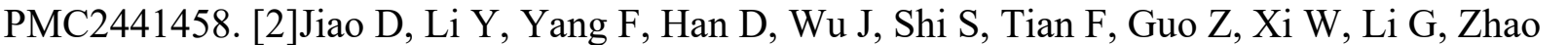

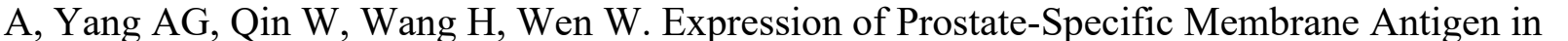

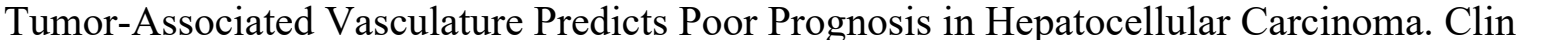

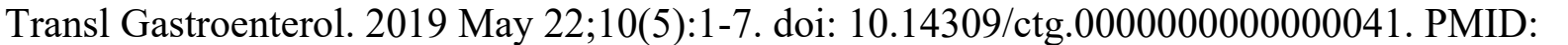

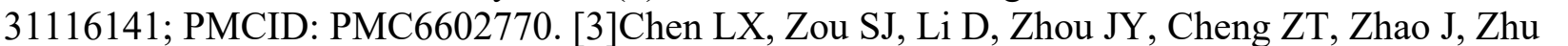

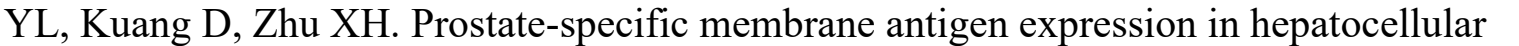

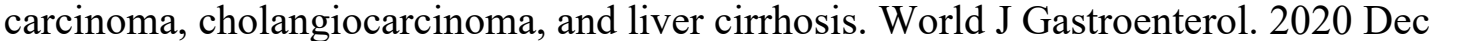

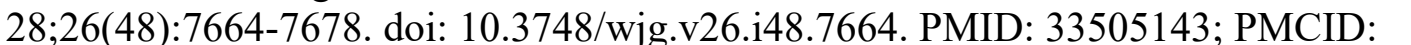

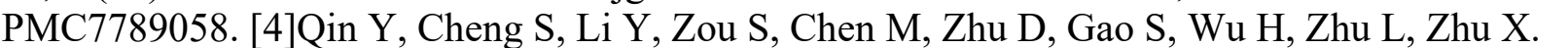

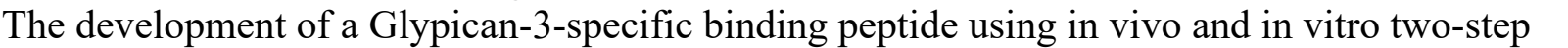

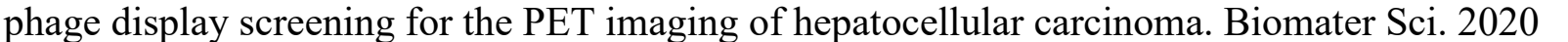

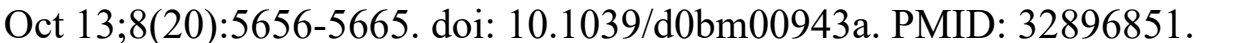

Full Name of Abstract's 1st Author : $\square \square \square \square \square \square \square \square$

First Name: $\square m \| m$

Last Name: $\square \square \square \square$

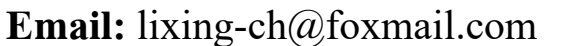

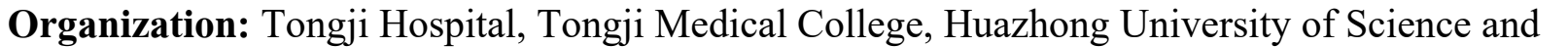
$\square[|||||||| \mid$

Country: $\square \square \square \square \square$ 


\title{
ID: LB96 \\ Novel conjugation protocols for fluorescently labeled adeno-associated virus (AAV1 and AAV8) to maintain cellular uptake and transduction efficiency
}

Travis Shaffer, Regeneron Pharmaceuticals, Inc, travis.shaffer@regeneron.com

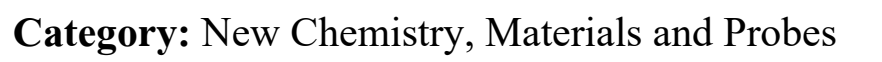

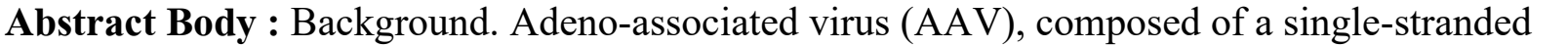

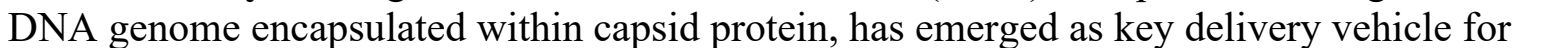

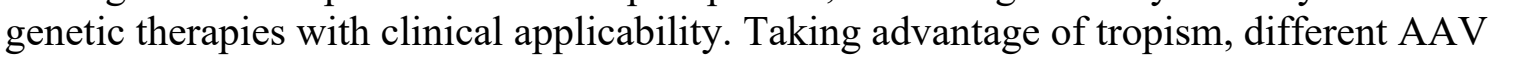

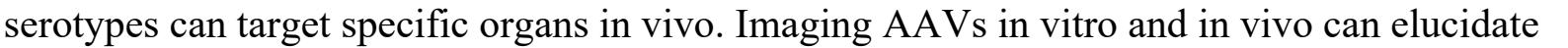

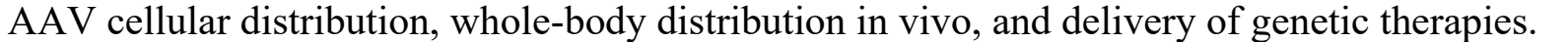

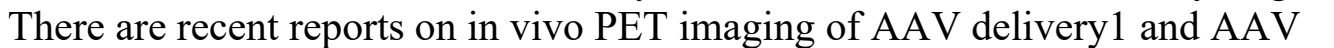

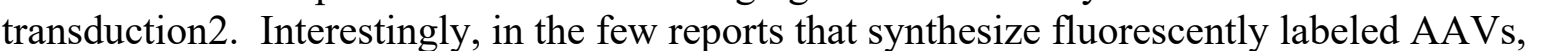

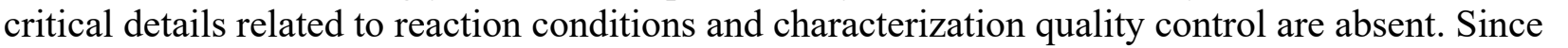

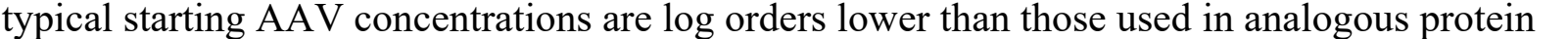

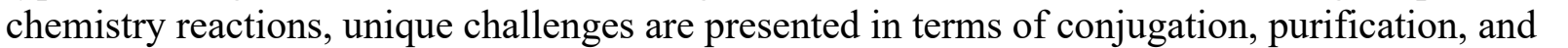

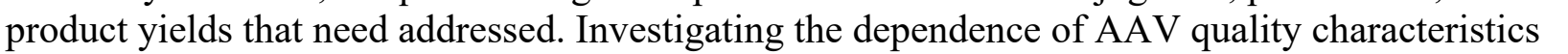

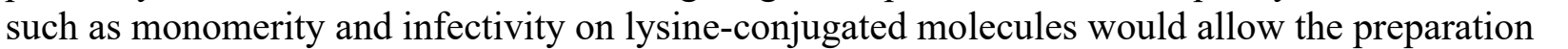

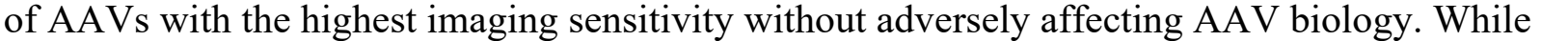

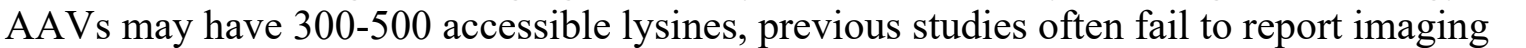

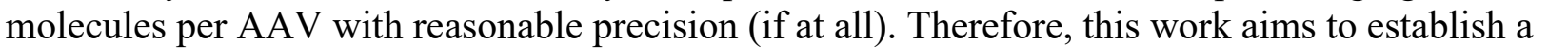

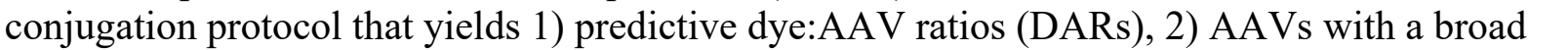

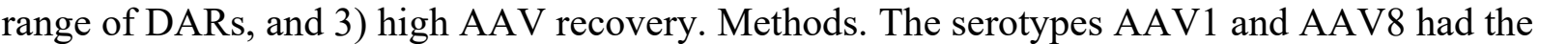

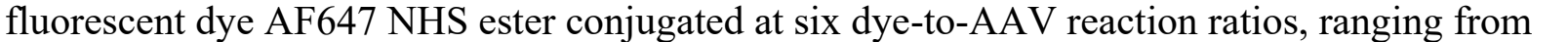

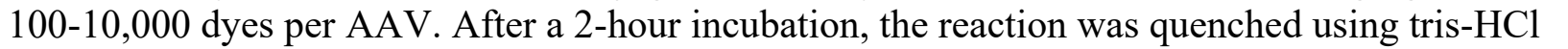

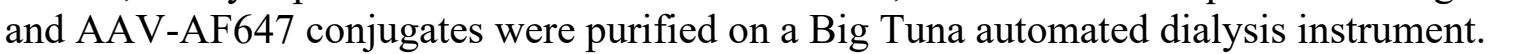

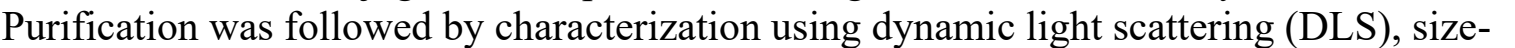

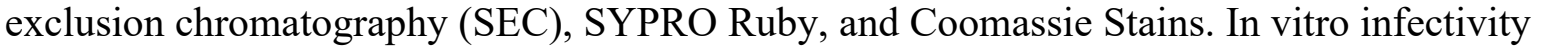

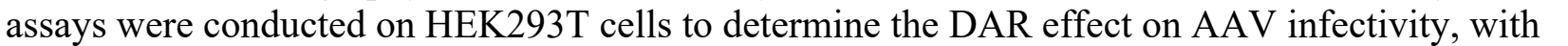

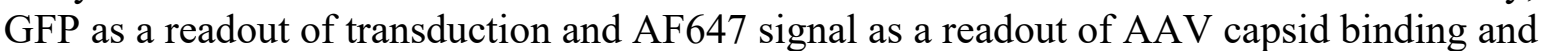

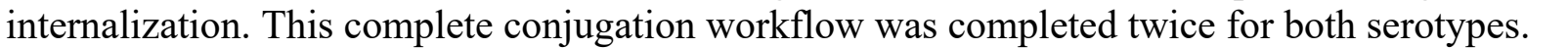

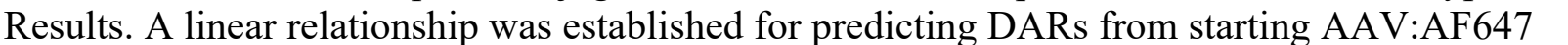

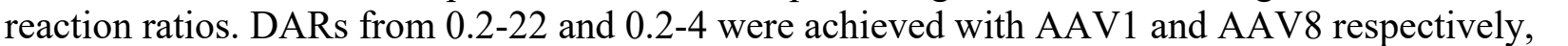

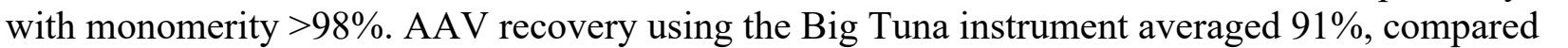

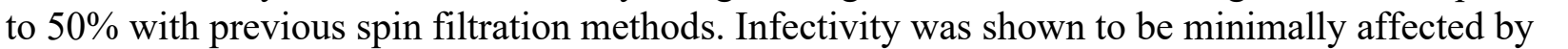

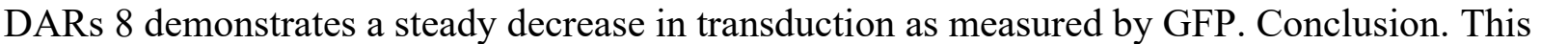

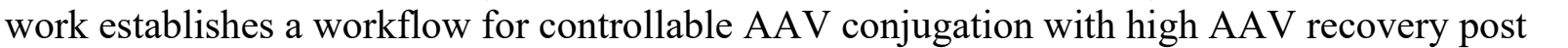

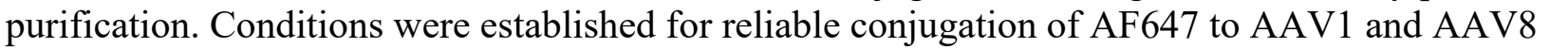

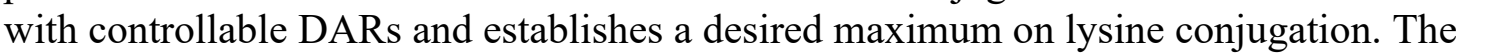

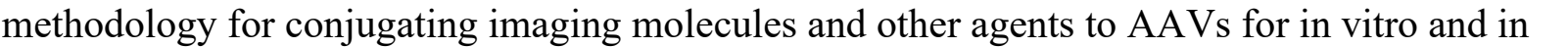

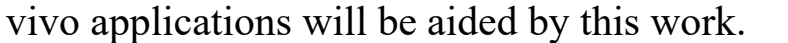




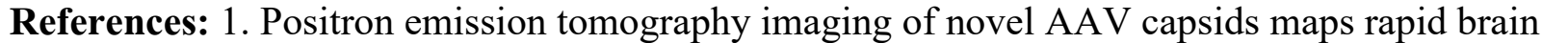

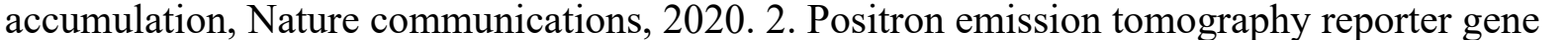

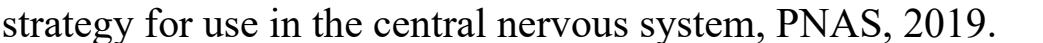

First Name: $\square \square\|\square\| \square$

Last Name: $\square \square|\|\|| \| \square$

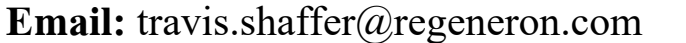

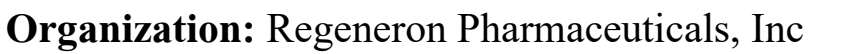

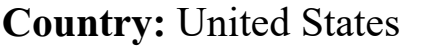




\title{
ID: LB97 \\ Metabolic Profiling for Determining Therapeutic Efficacy in Pancreatic Cancer \\ Trinity Brigham, MD Anderson Cancer Center, trinityb375@gmail.com
}

Category: $\square \square \square \square \square \square$

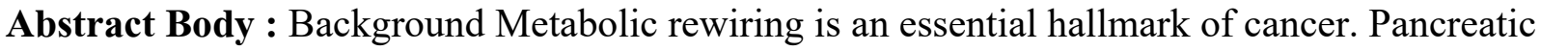

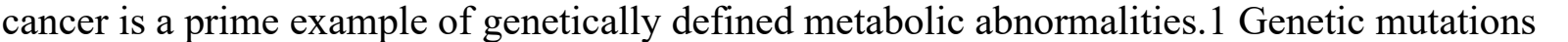

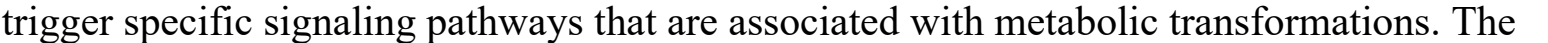

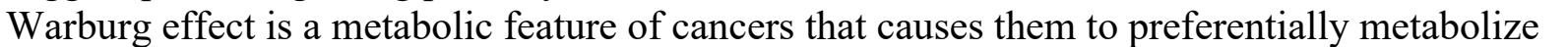

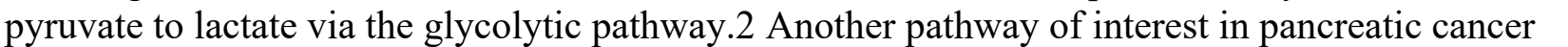

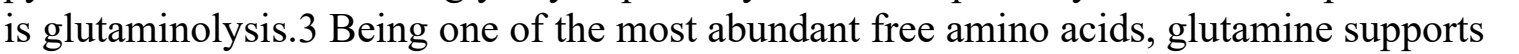

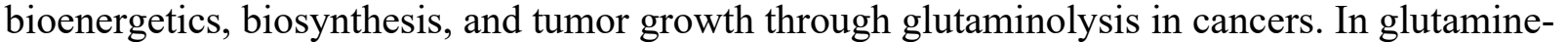

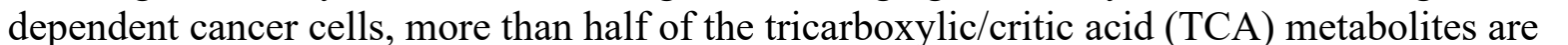

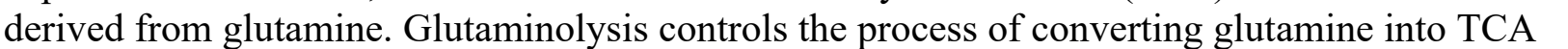

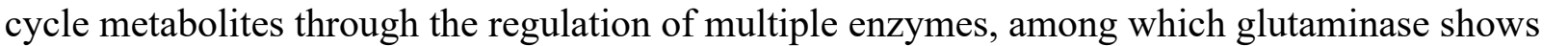

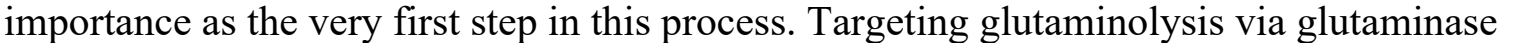

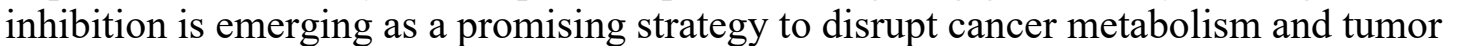

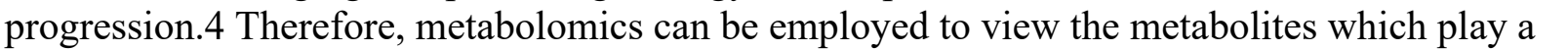

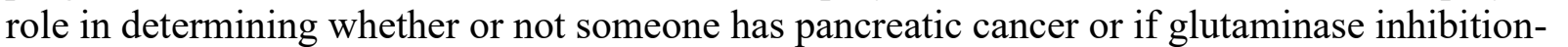

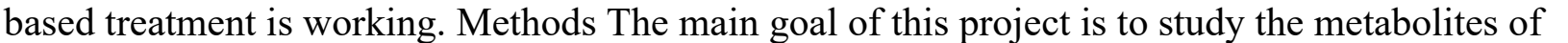

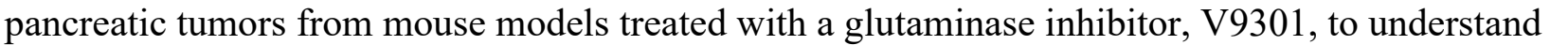
ए

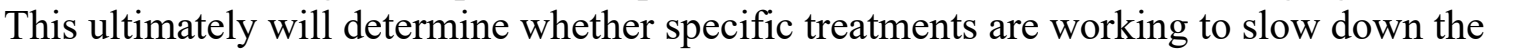

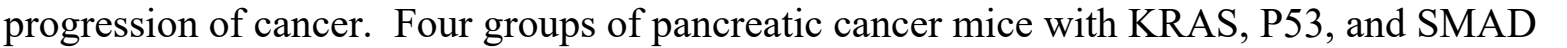

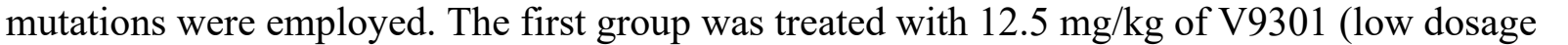
ए

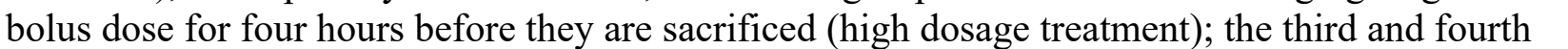

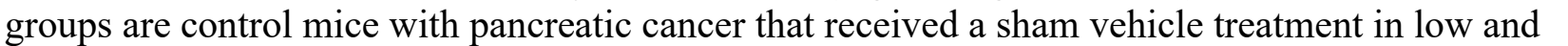

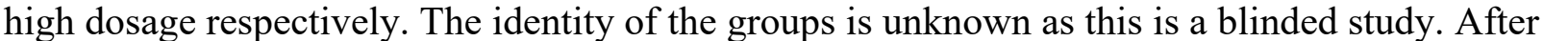

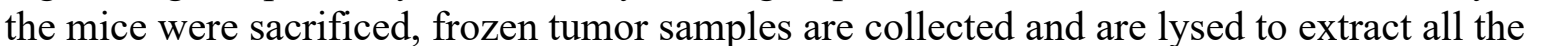

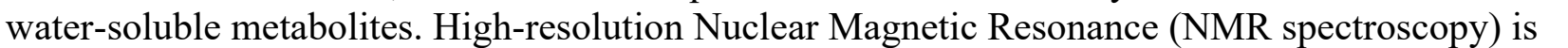
ए एம

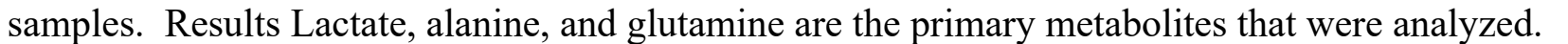

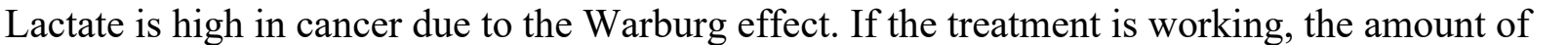

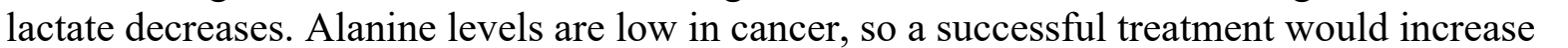

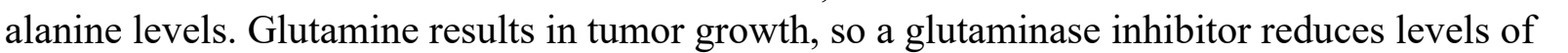

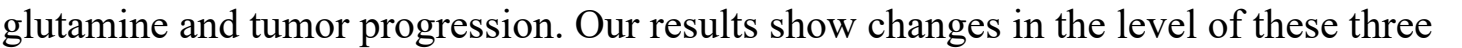

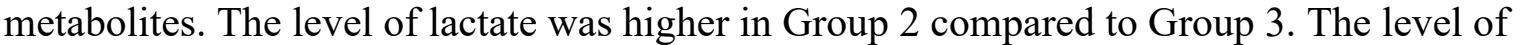

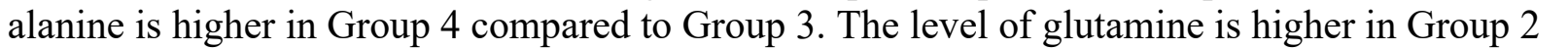

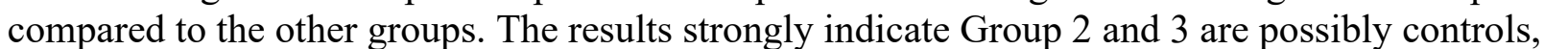

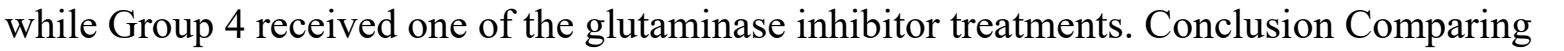

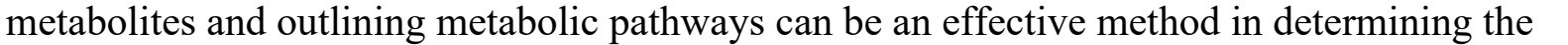




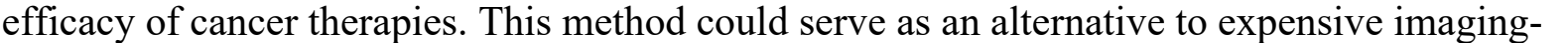

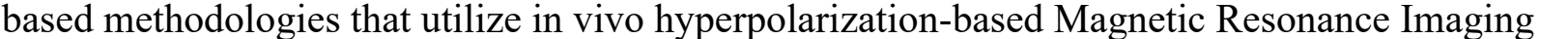

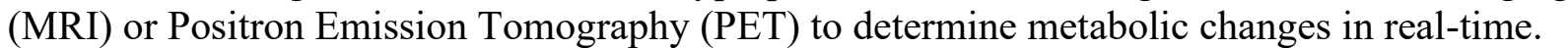

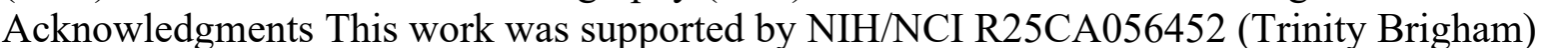

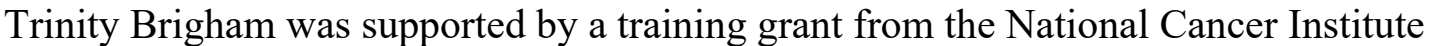

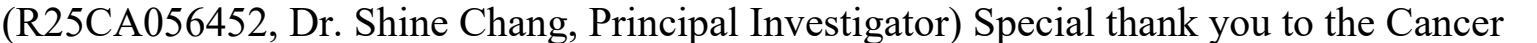

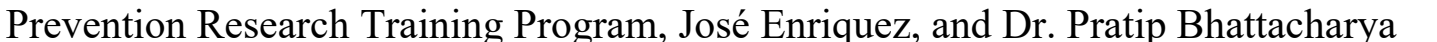

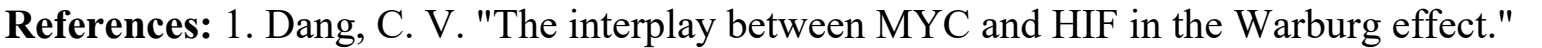

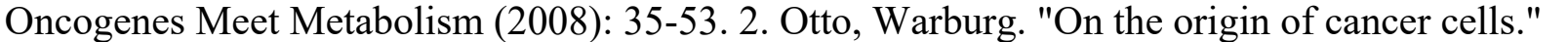

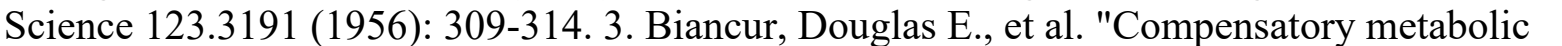

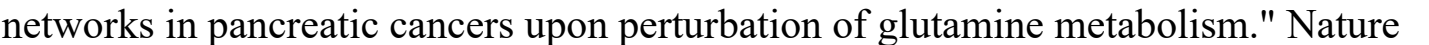

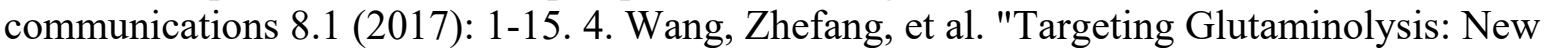

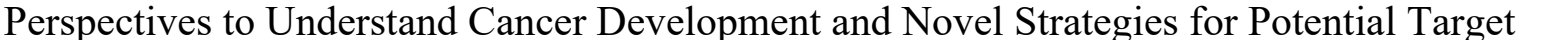

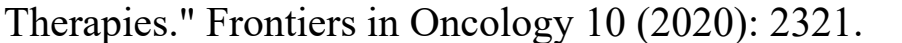

\section{Image/Figure:}

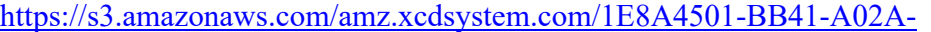

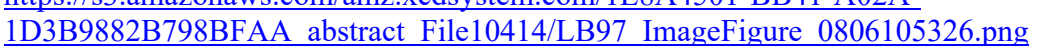

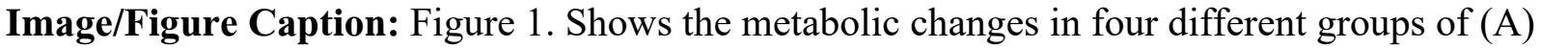
ए

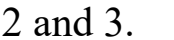

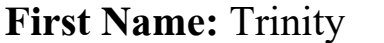

Last Name: $\square \square \| \square \square \square$

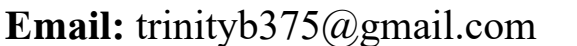

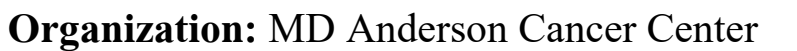

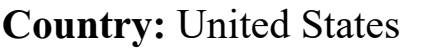




\title{
ID: LB98 \\ Molecular MRI with Hyperpolarized Gas at Ultra Low Field Strengths
}

Nicholas Bryden, University of North Carolina at Chapel Hill, nbryden@live.unc.edu

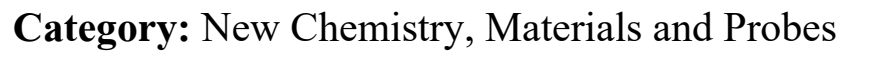

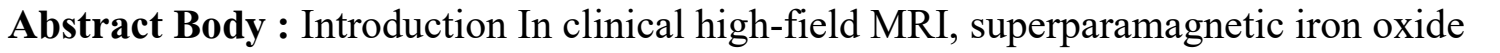

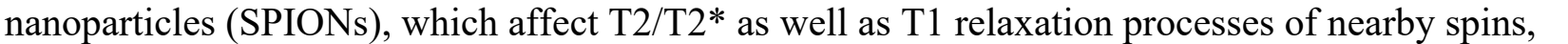

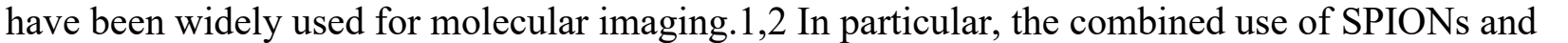

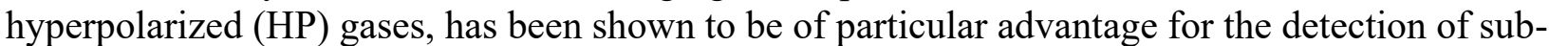

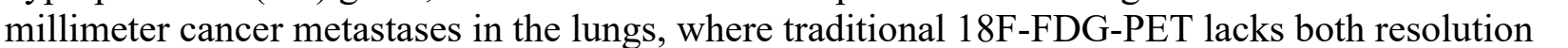

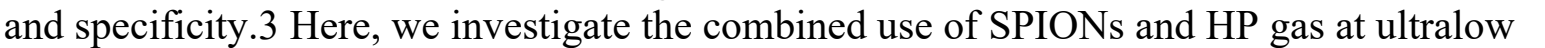

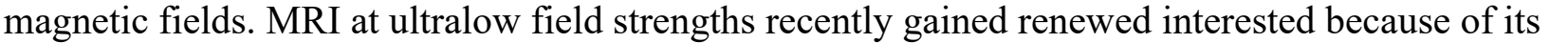

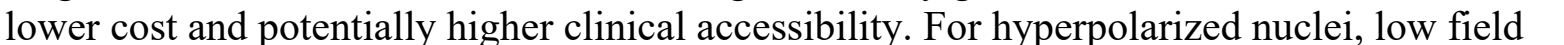

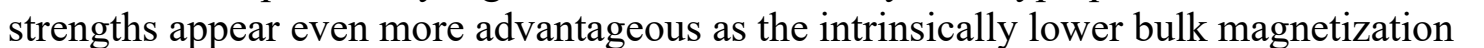

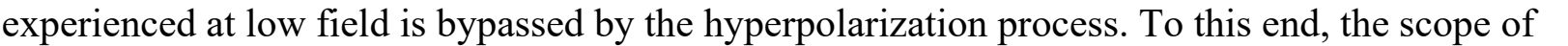

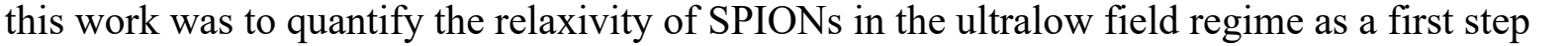

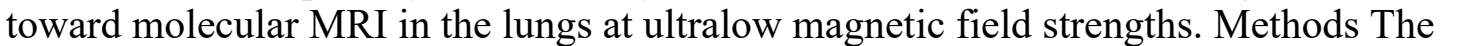
ए

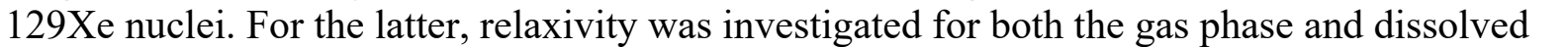

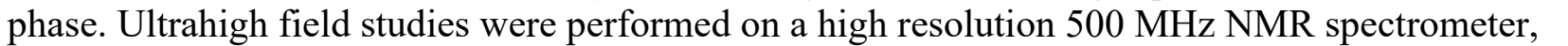

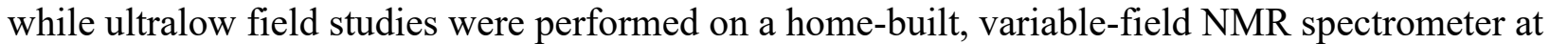

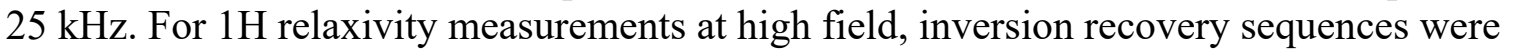

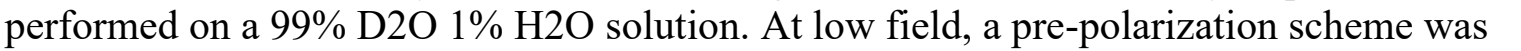

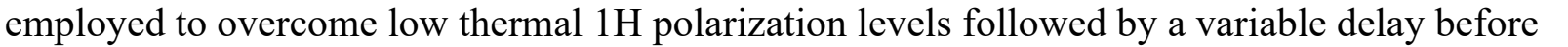

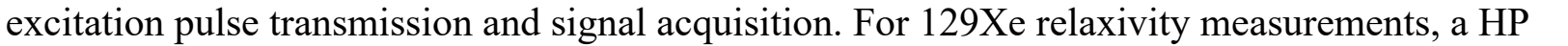

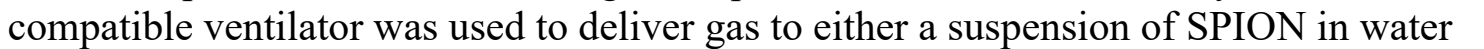

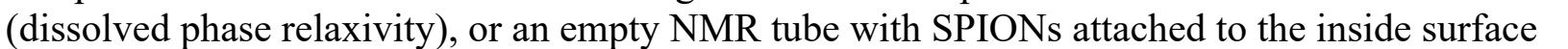

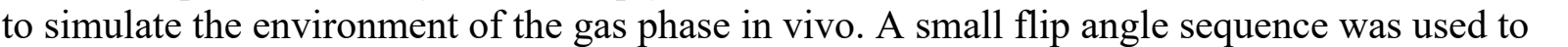

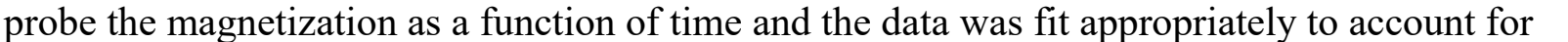

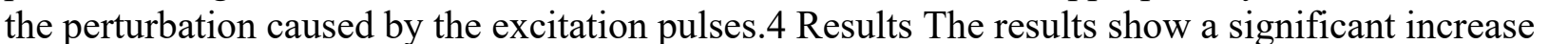
ए

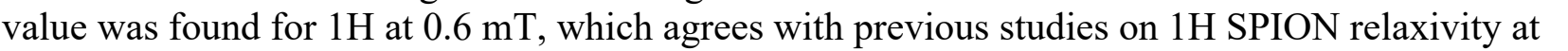

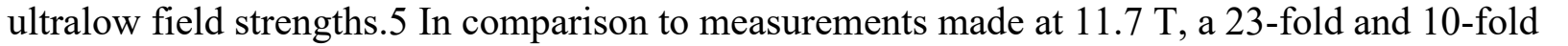

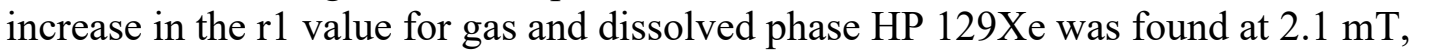

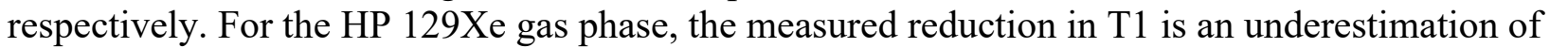

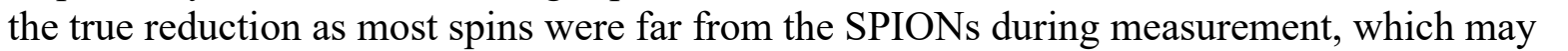

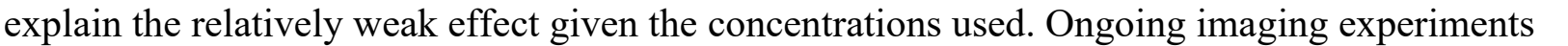

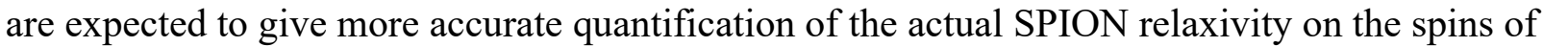

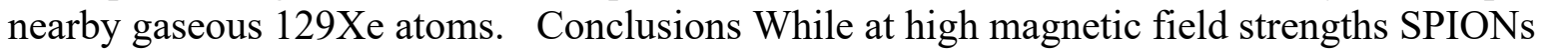

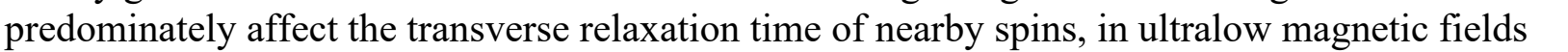
ए

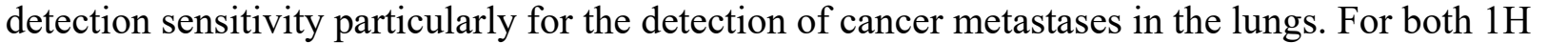




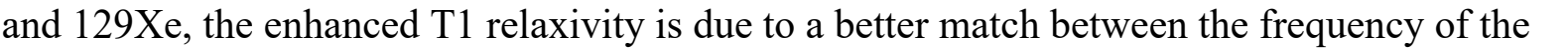

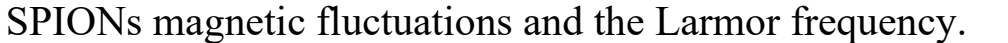

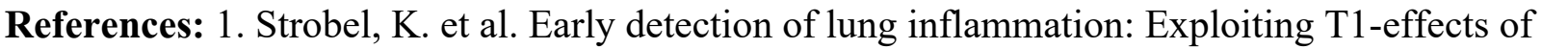

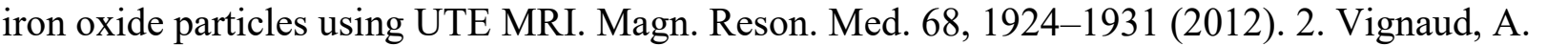

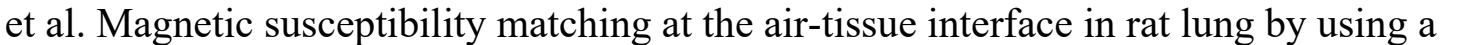

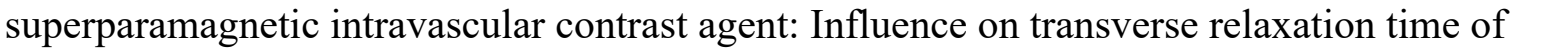

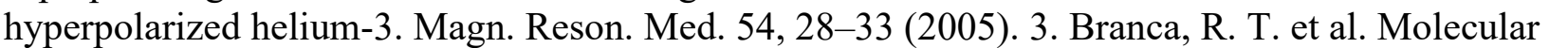

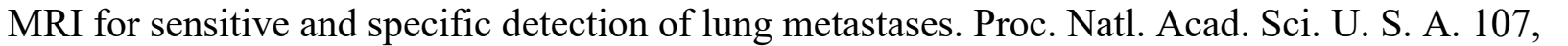

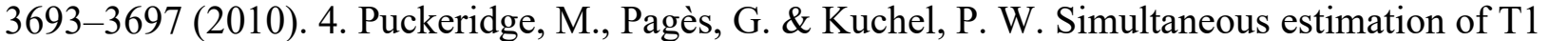

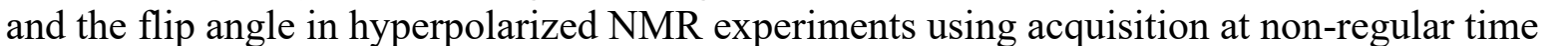

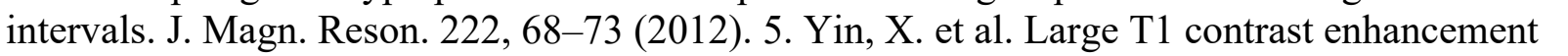

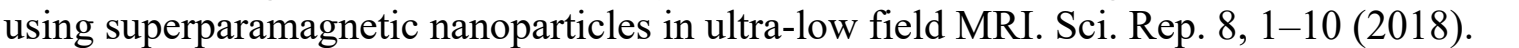

\section{Image/Figure:}

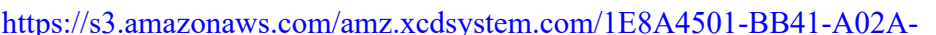

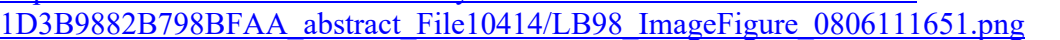

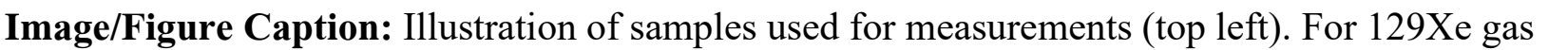

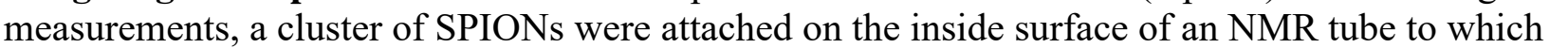

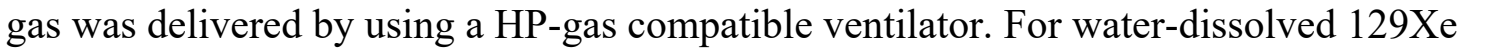

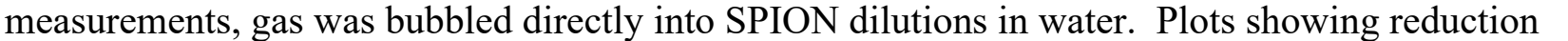

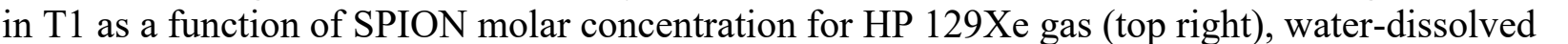

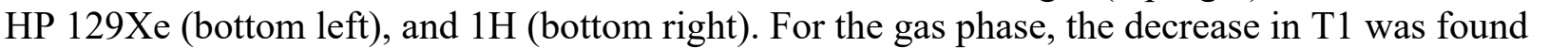
पाm

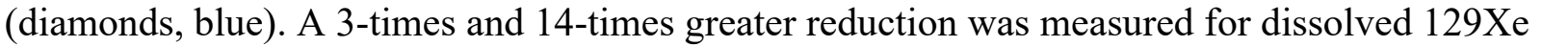

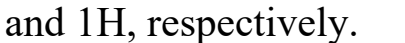

First Name: $\square \square\|\square\|\|\|$

Last Name: $\square \square\|\| \| \square$

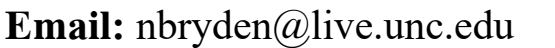

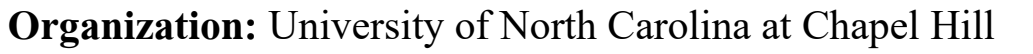

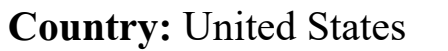




\title{
ID: LB99 \\ Screening Novel Anti-DLL3 Antibodies for Pretargeted ImmunoPET imaging and Radioimmunotherapy
}

Kathryn Tully, Memorial Sloan Kettering Cancer Center, tullyk@mskcc.org

\section{Category: $\square \square\|\| \| \square$}

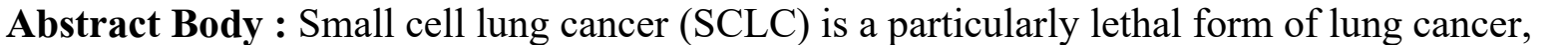

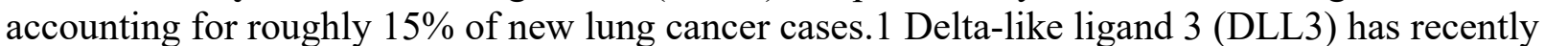

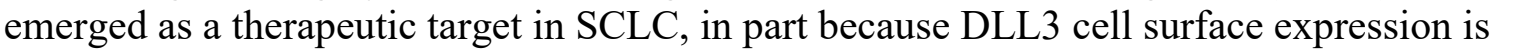
ए

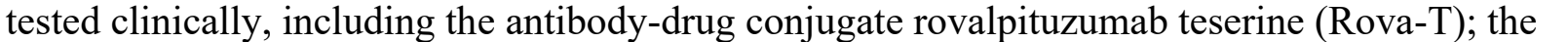

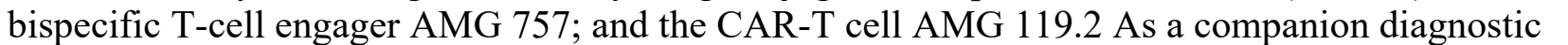

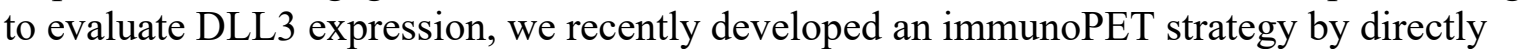

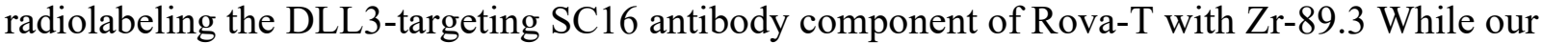

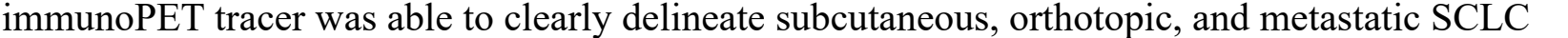

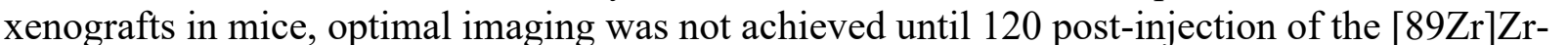

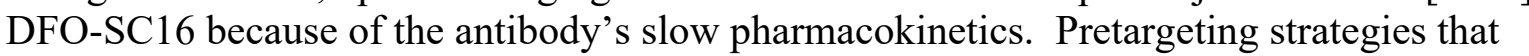

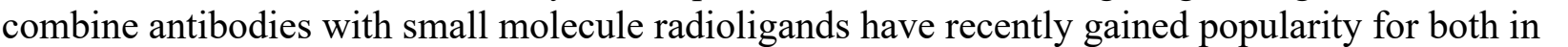

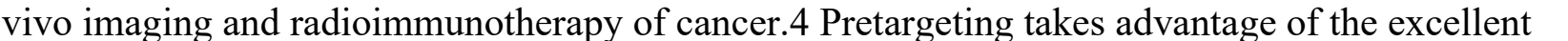

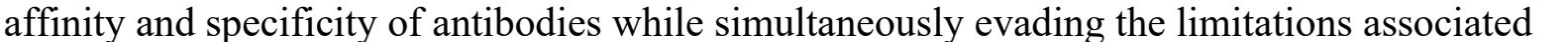

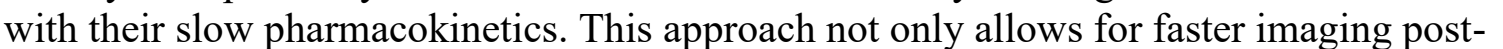

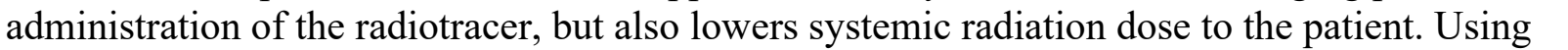

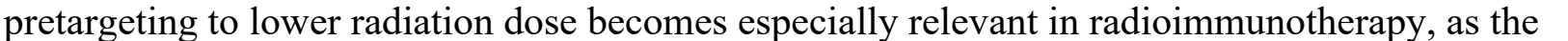

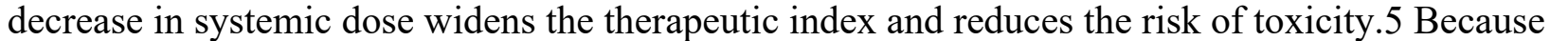

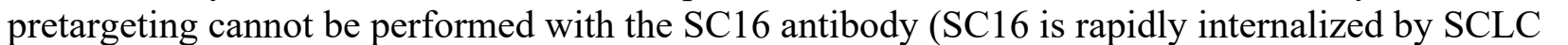

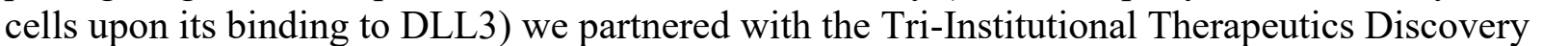
ए

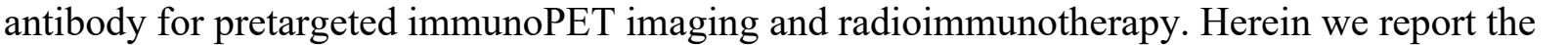

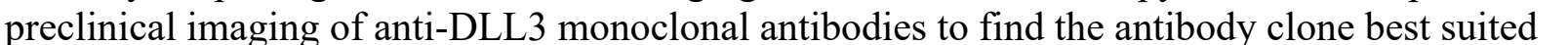

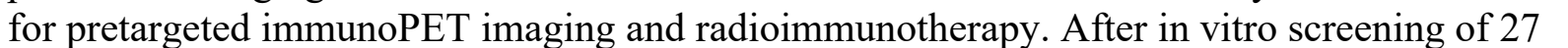

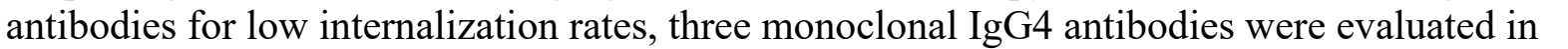

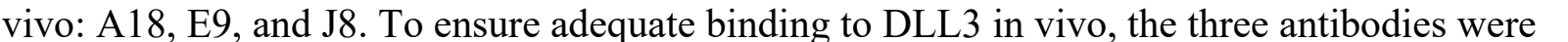
ए

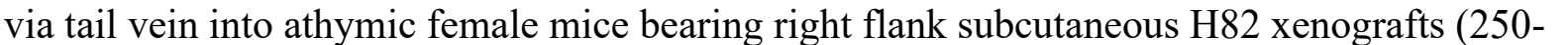
$\square \mu \mathrm{Ci}, 30 \mu \mathrm{g})$. ImmunoPET imaging was performed every $24 \mathrm{~h}$ until $120 \mathrm{~h}$, when a terminal ex

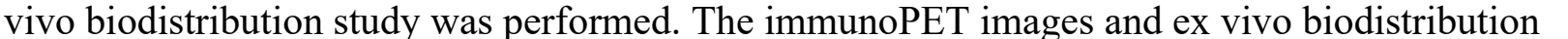

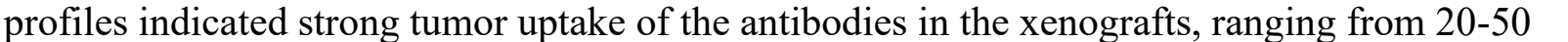

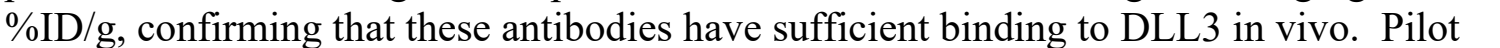

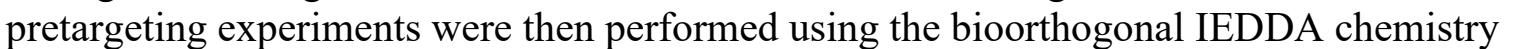

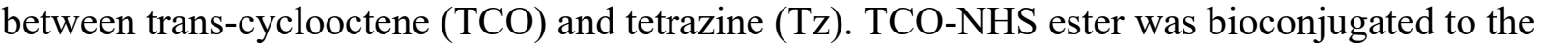

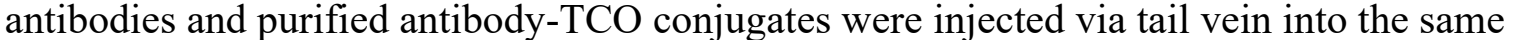
xenograft model as previously described $(100 \mu \mathrm{g}, 0.67 \mathrm{nmol})$. After allowing the antibody $\square \square \square \square$ 


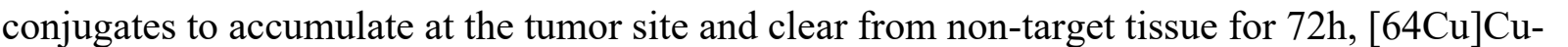

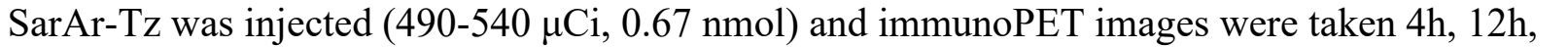

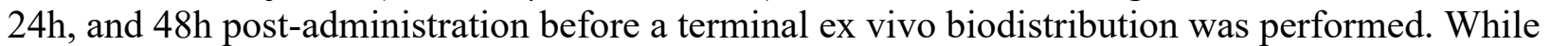

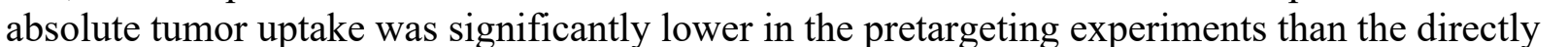

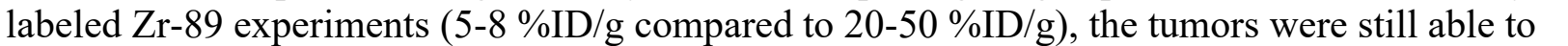

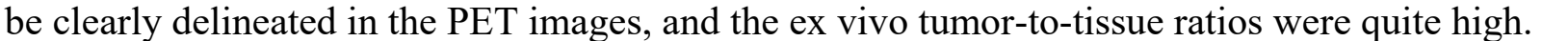

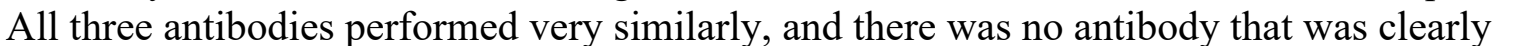

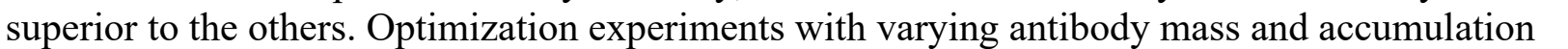

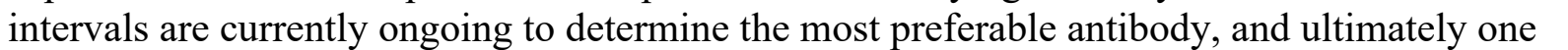

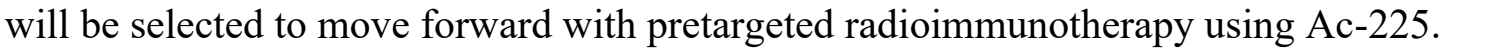

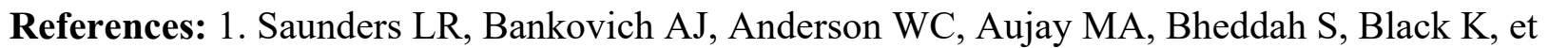

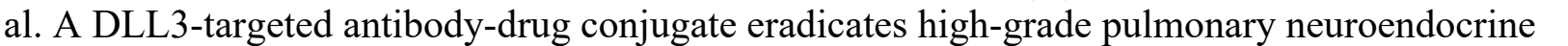

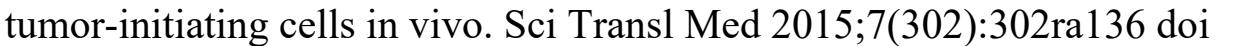

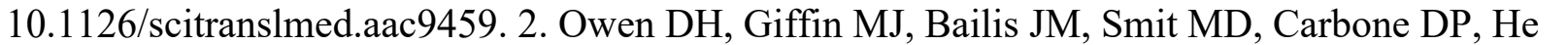

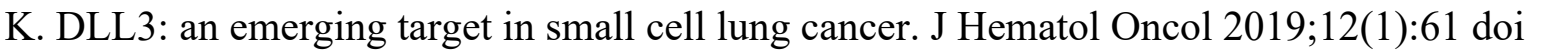

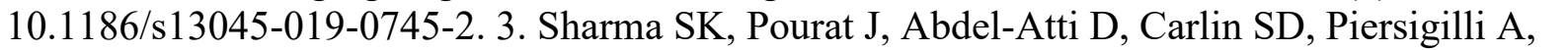

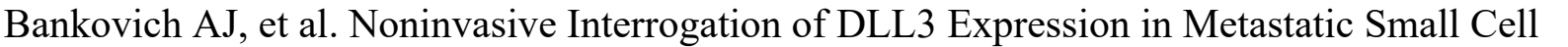

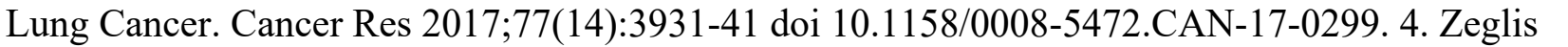

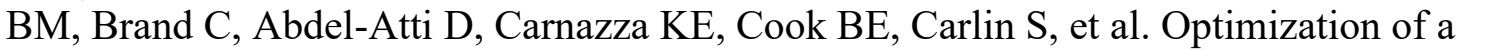

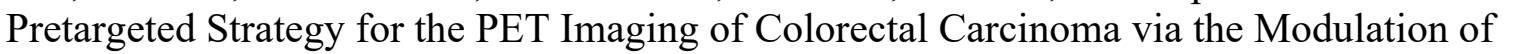

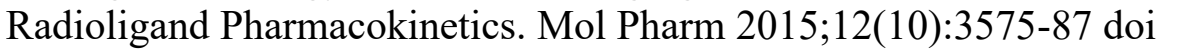

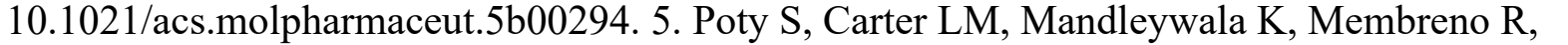

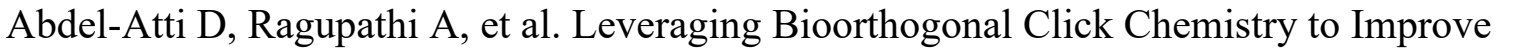

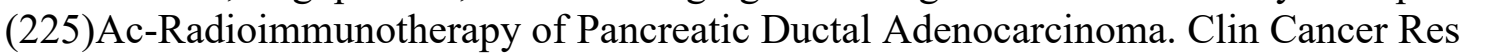

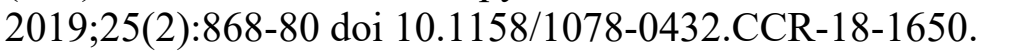

\section{Image/Figure: $\square$}

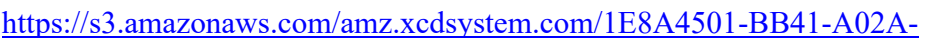

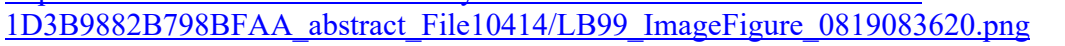

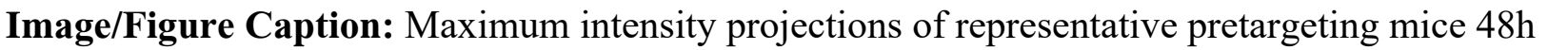

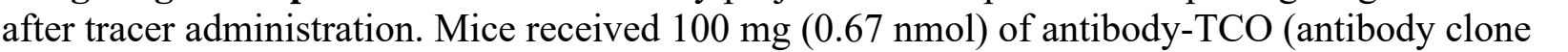
घण

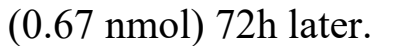

First Name: $\square \square \| m \mid \square$

Last Name: $\square \square \| m$

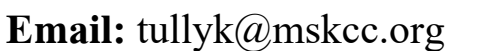

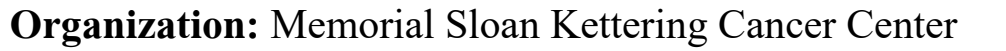

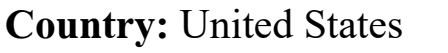




\title{
ID: LB100 \\ Biomimetic Manganese-eumelanin Nanocomposites for Combined Hyperthermia-immunotherapy against Prostate Cancer
}

Yu Liu, 1Beijing Advanced Innovation Center for Big Data-Based Precision Medicine, School of Biological Science and Medical Engineering, Beihang University, yuliu66880@163.com

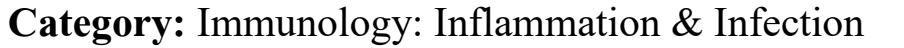

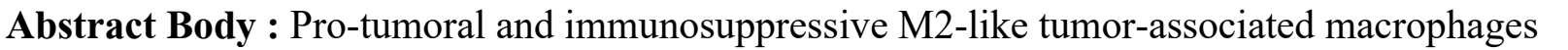

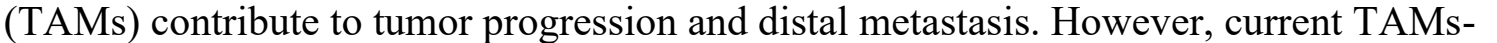

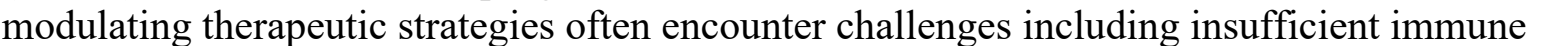

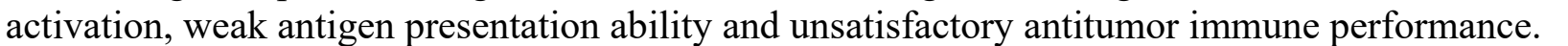

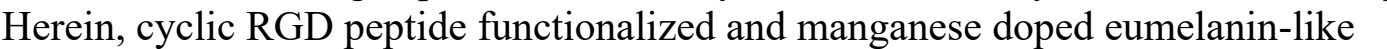

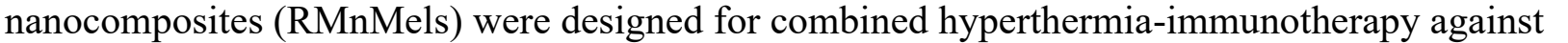

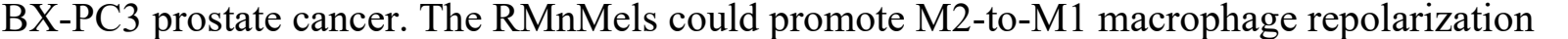

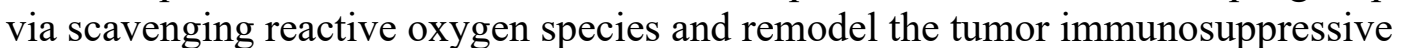

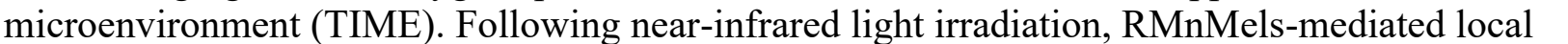

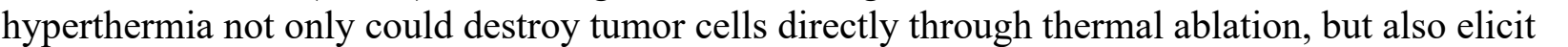
ए ए

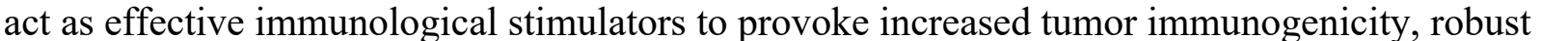
ए

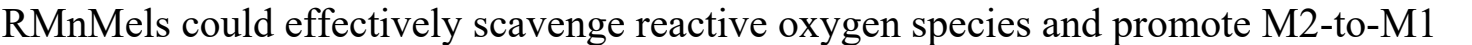

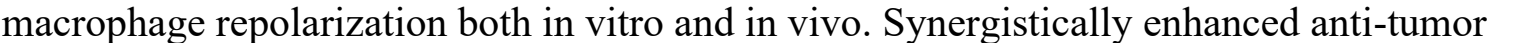

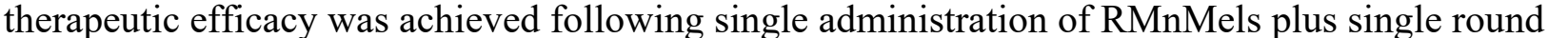

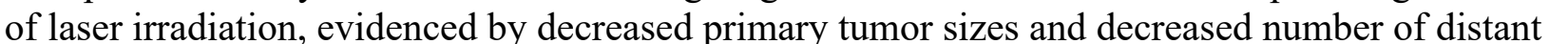

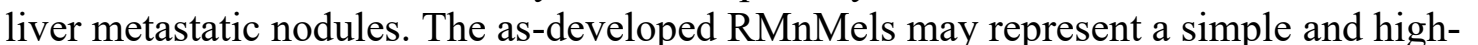

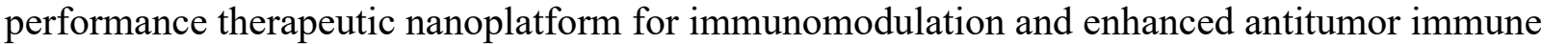

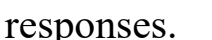

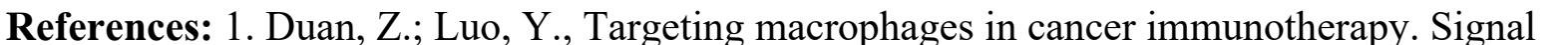

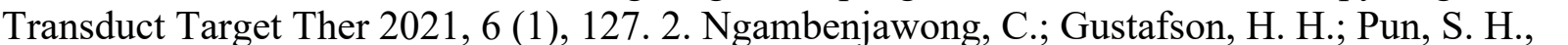

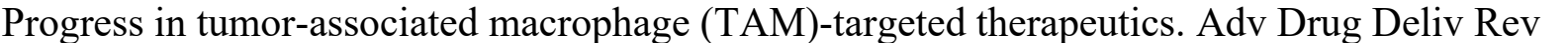

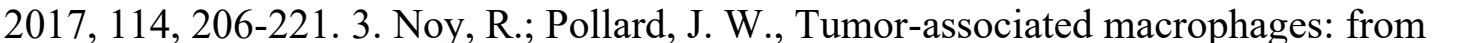

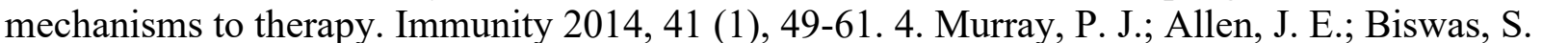

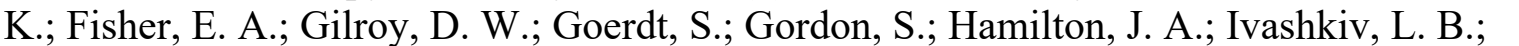

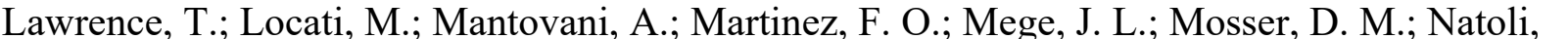

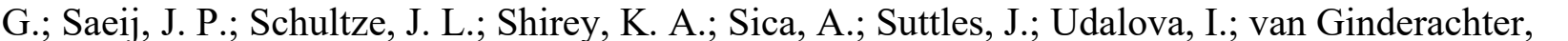

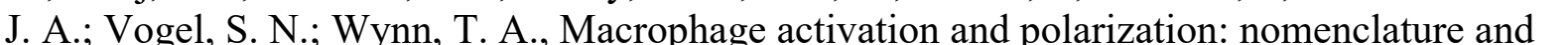

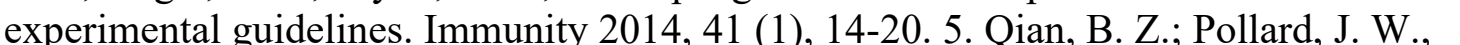

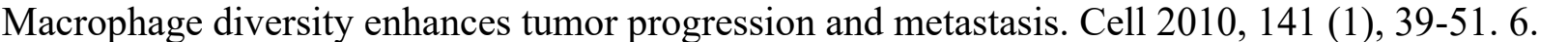

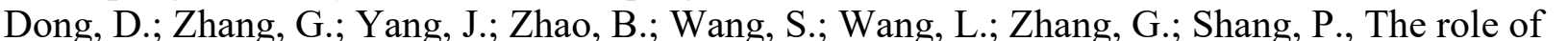

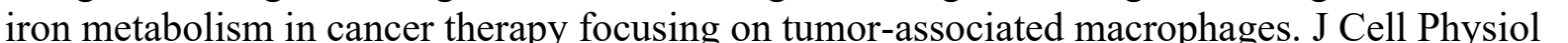




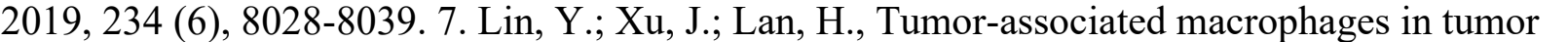

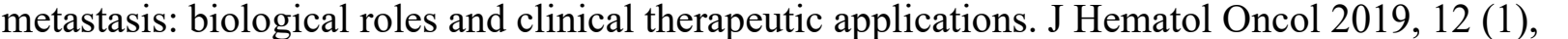

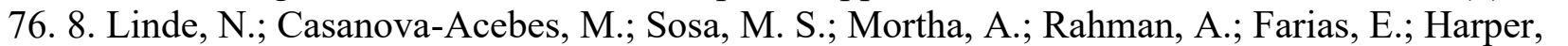

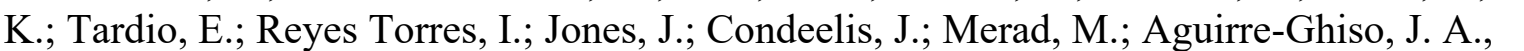

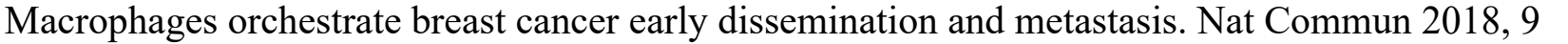

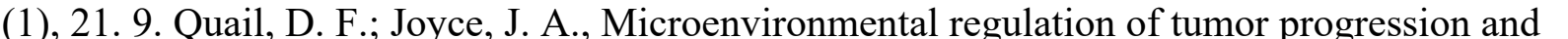

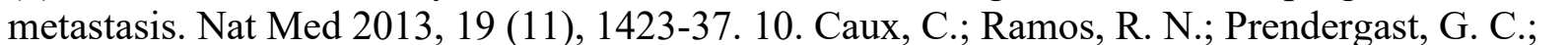

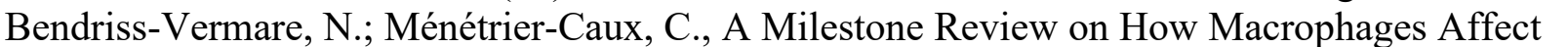

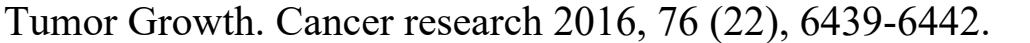

First Name: $\square \square \square$

Last Name: $\square \square \square$

Email: $\square \square\|\square \square\| \square \square \square \square \square \square \square$

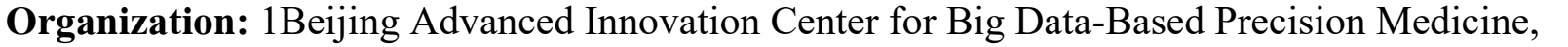

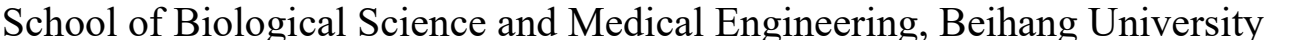

Country: $\square \square \square \square \square$ 


\title{
ID: LB101 \\ Tracking Gut Permeability and Microbial Therapeutics in the Pig: PET/MRI of Ingested 89Zr-labeled Probiotic Escherichia coli Nissle 1917
}

\author{
Donna Goldhawk, Lawson Health Research Institute, dgoldhawk@lawsonimaging.ca
}

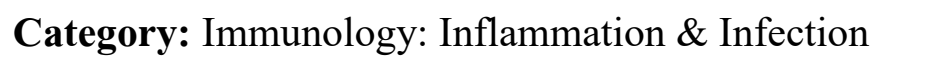

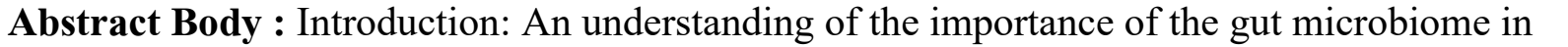

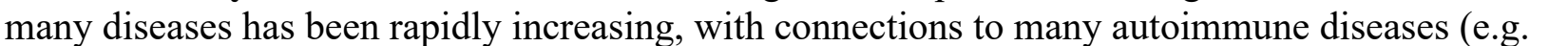

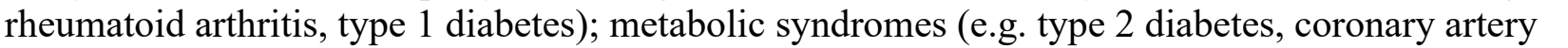

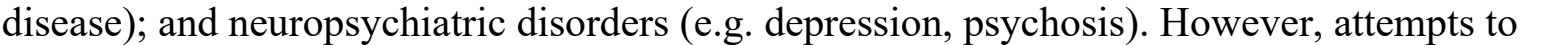

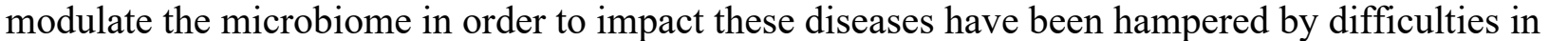

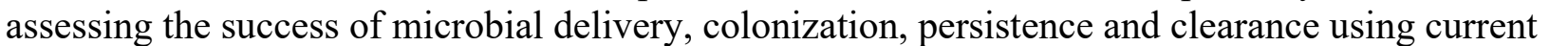

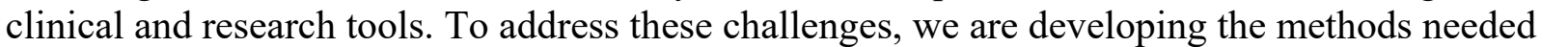

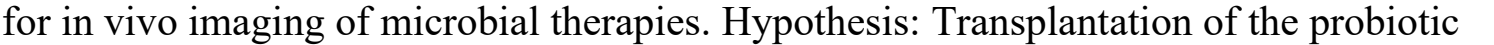

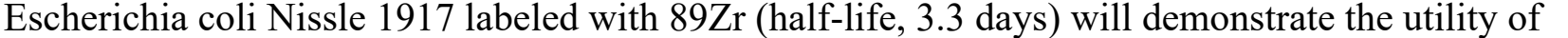

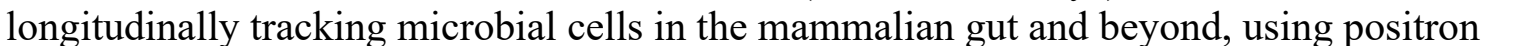

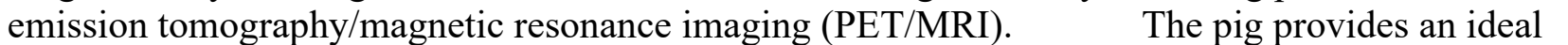

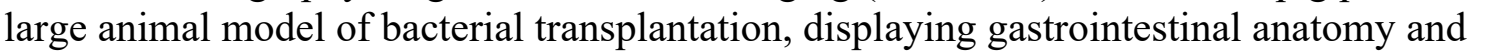

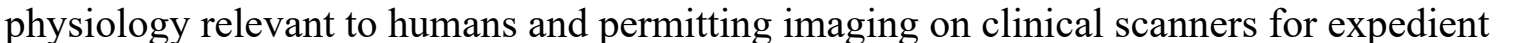

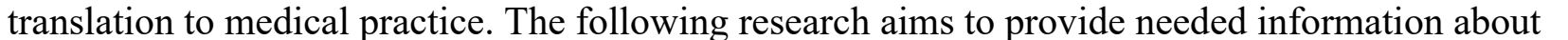

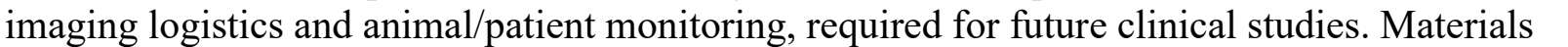

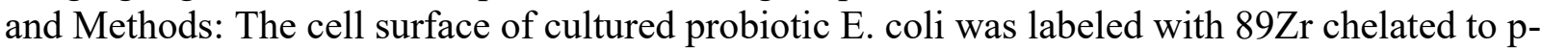

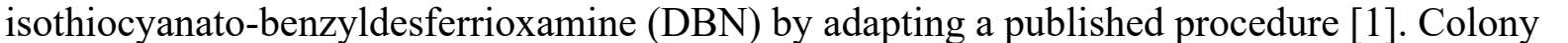

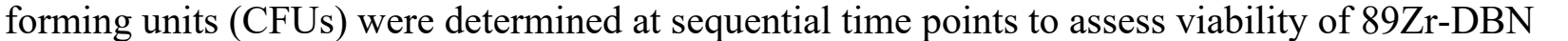
ए

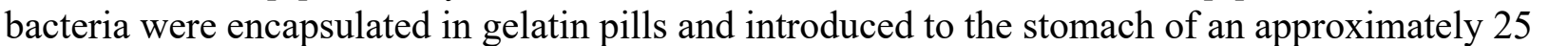

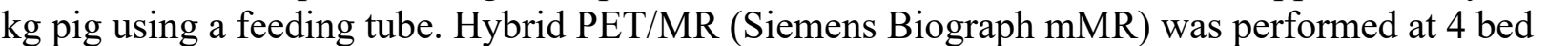

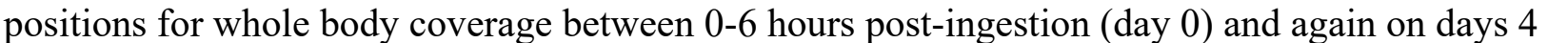

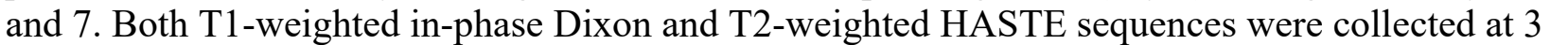

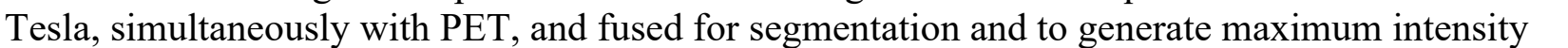

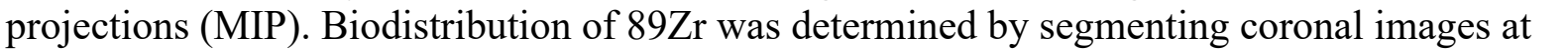

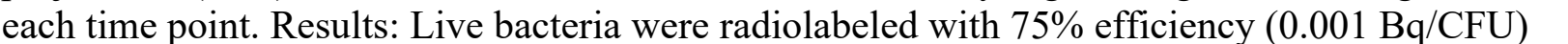

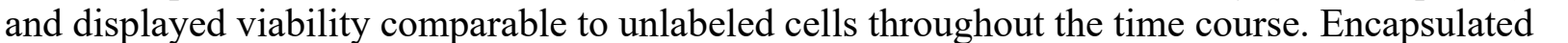

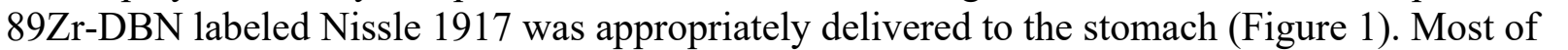

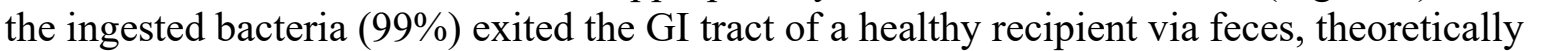

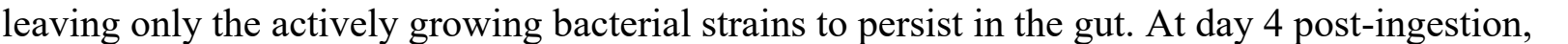

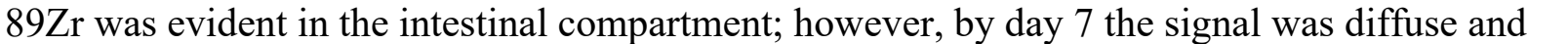

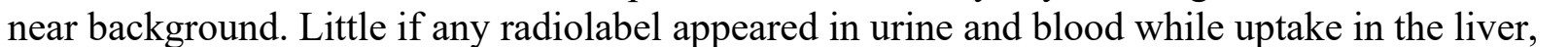

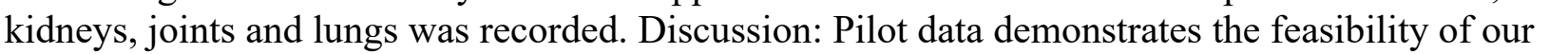

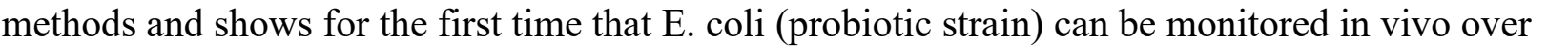

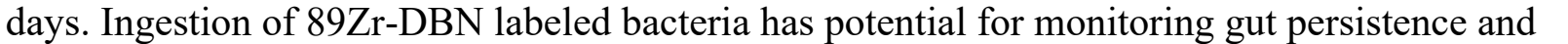

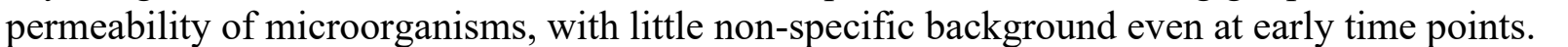




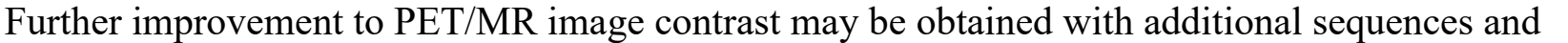

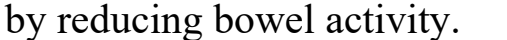

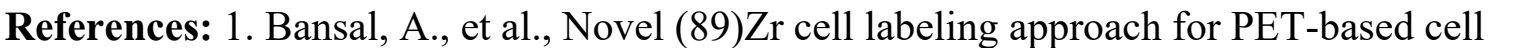

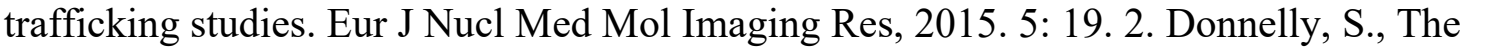

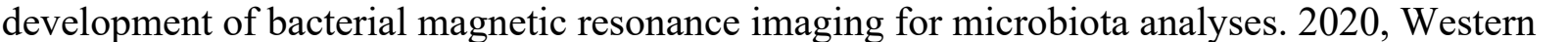

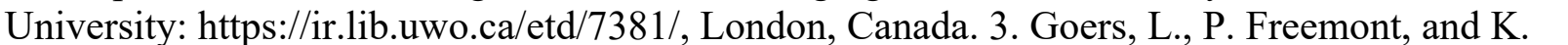

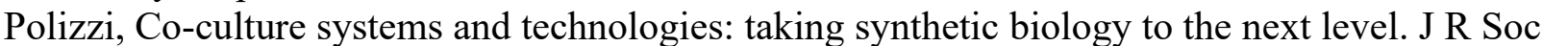

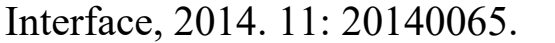

\section{Image/Figure:}

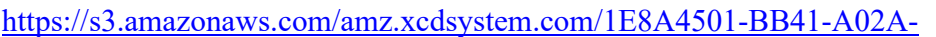

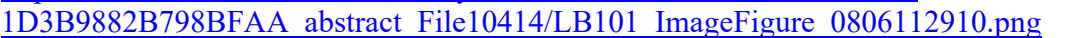

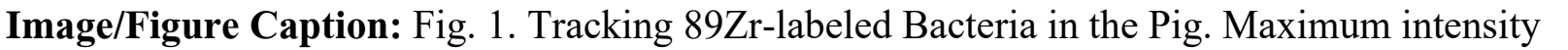

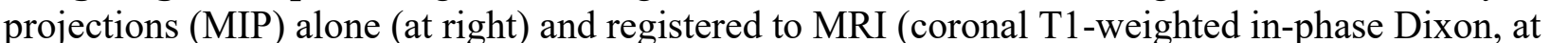

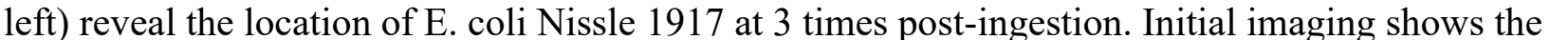

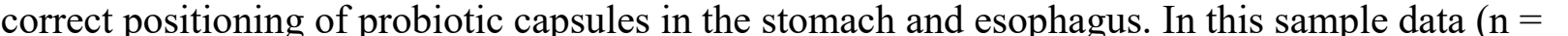

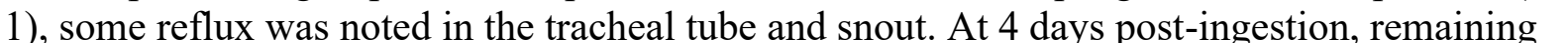

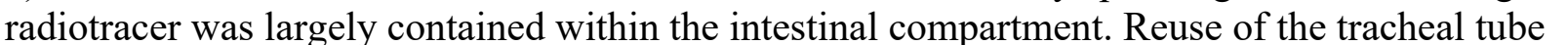

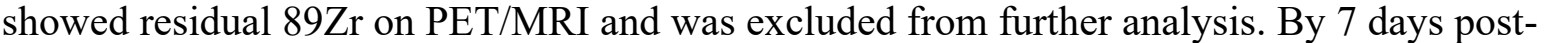

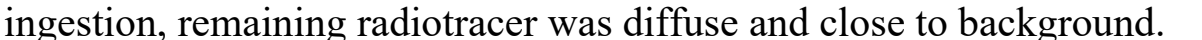

First Name: $\square \square \square \square \| \square$

Last Name: $\square \square \square \square \square \square \square$

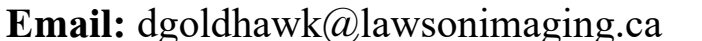

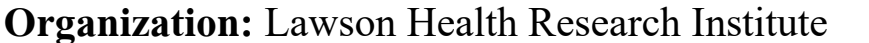

Country: $\square \square \square \square \square \|$ 


\title{
ID: LB102 \\ In-Vitro Validation of Senescence via SASP targets \\ Spencer Kaminsky, Sloan Kettering, kaminsks@mskcc.org
}

Category: $\square \square\|\| \square \square$

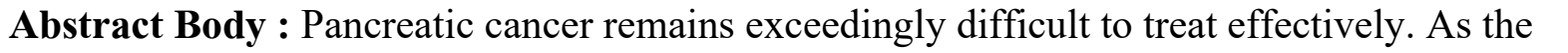

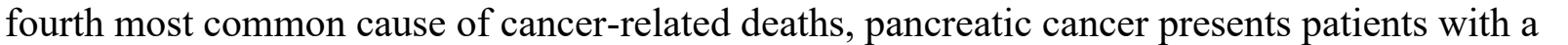

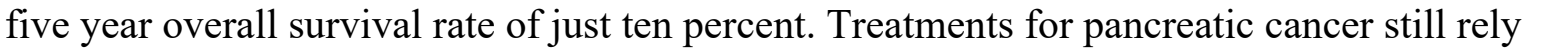

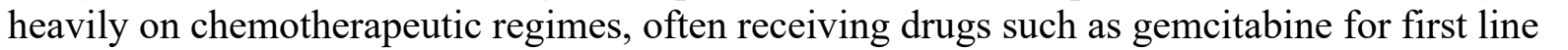

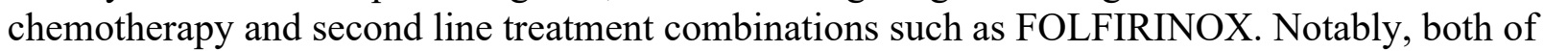

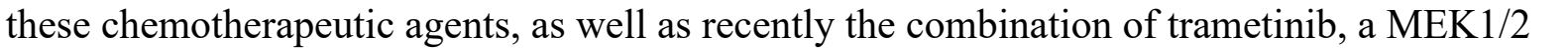

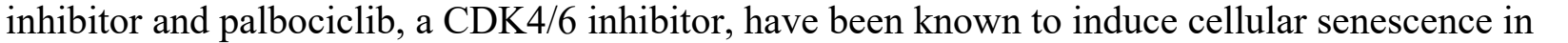

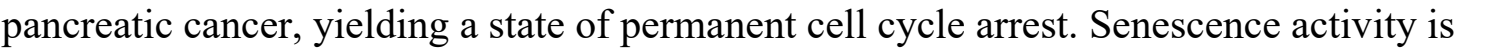
associated with activation of beta galactosidase ( $\beta$ gal) functioning as a surrogate marker where

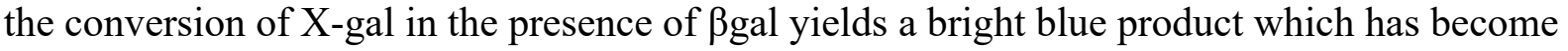

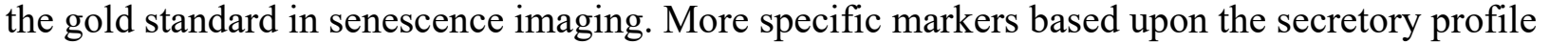

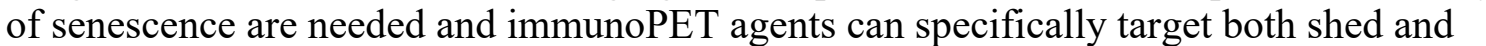

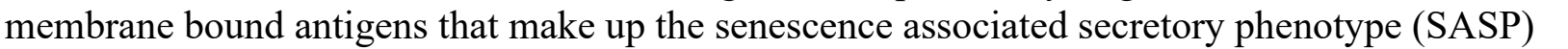

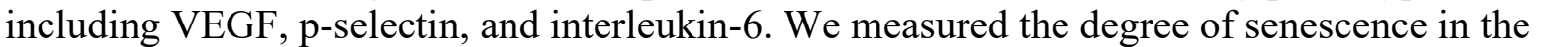

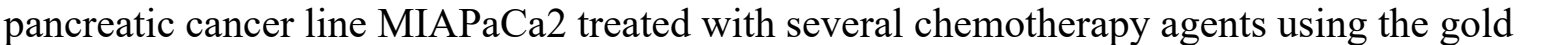

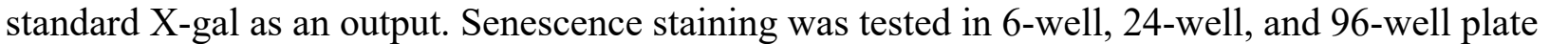
ए

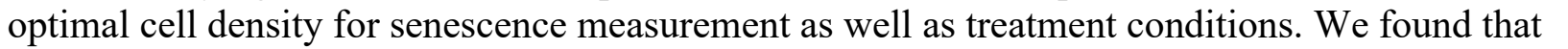

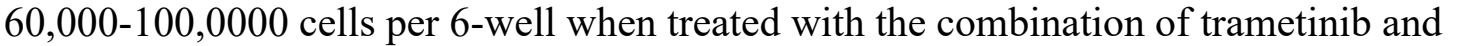

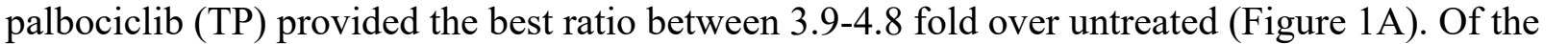

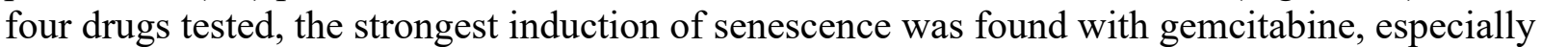

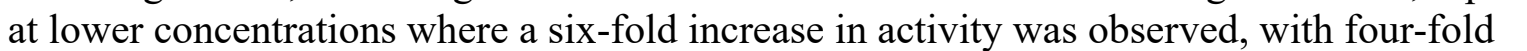
ए

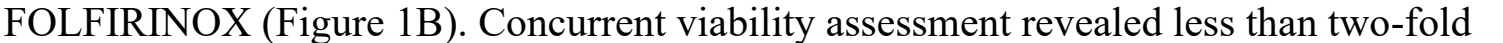
ए ए

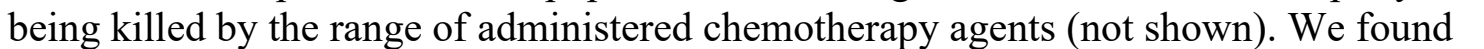

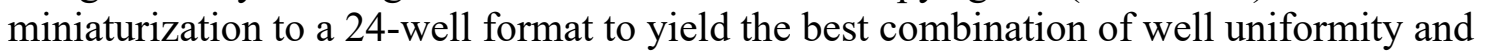

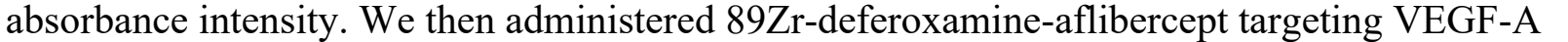

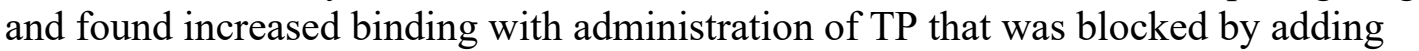

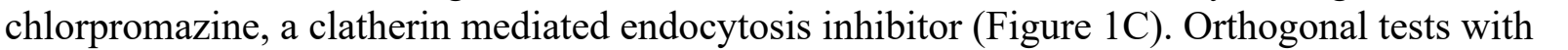

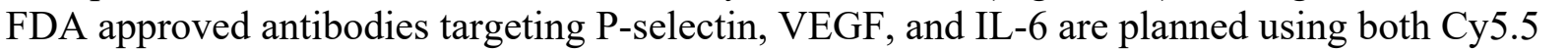

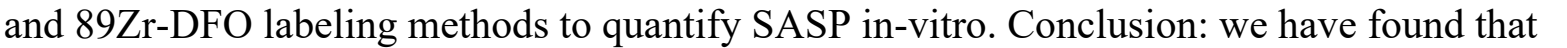

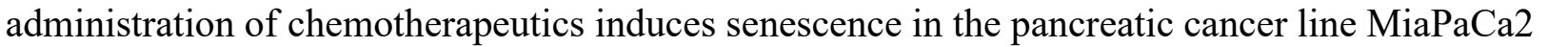

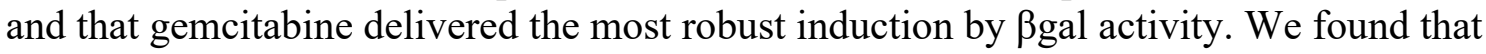

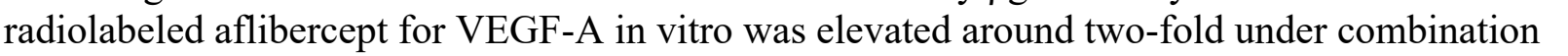

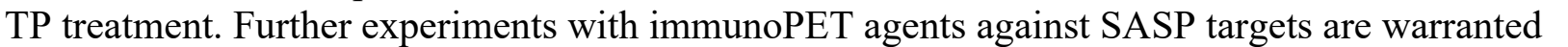


First Name: $\square \square \square\|\|\|\| \square$

Last Name: $\square \square \square \square \| \square \square$

Email: $\square \square \square \mid\|1\| \square \square \square\|\|\|\| \square$

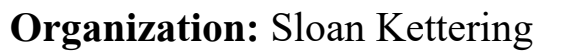

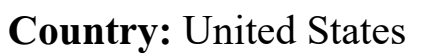




\title{
ID: LB103 \\ [124I]-ATRi VE-821 analogue as a new PET tracer in mouse models for prostate cancer
}

Raik Artschwager, Memorial Sloan Kettering Cancer Center, artschwr@mskcc.org

Category: $\square \square\|\| \sqcap \|$

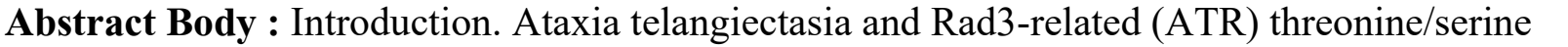

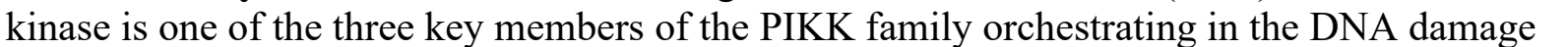

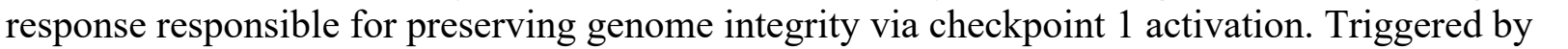

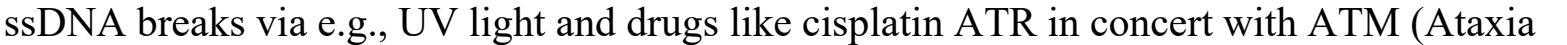

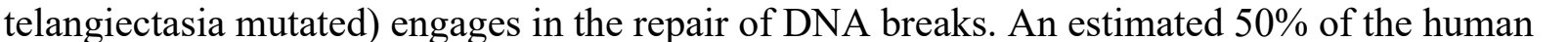

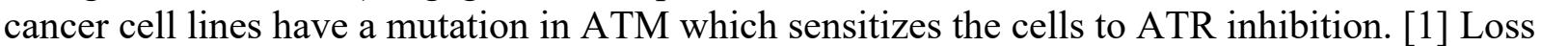
or mutation of the ATM function renders cancer cells sensitive to ATR inhibitors (ATRi's). Our

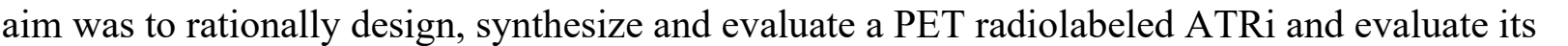
प

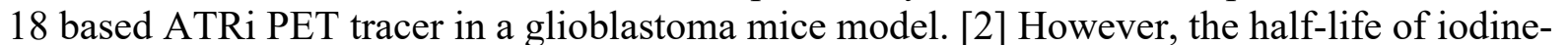

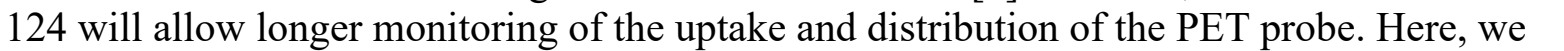

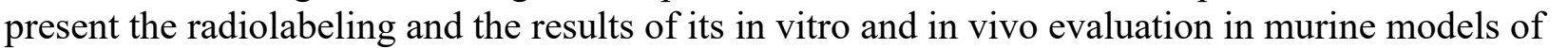

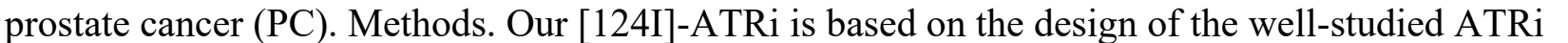

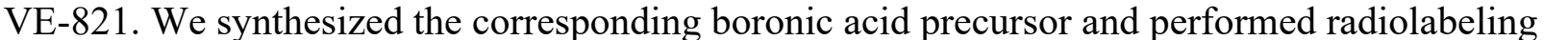

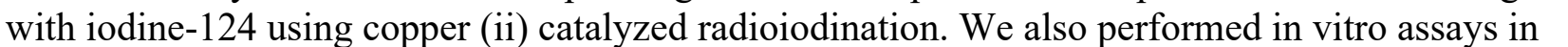

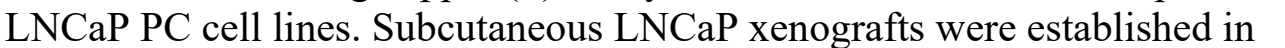

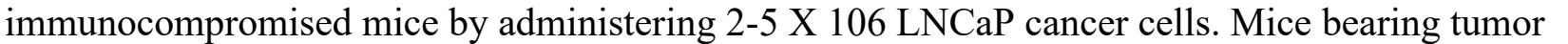

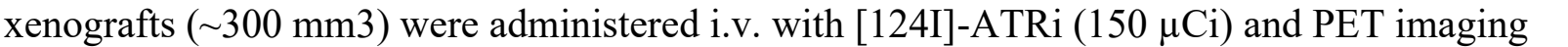

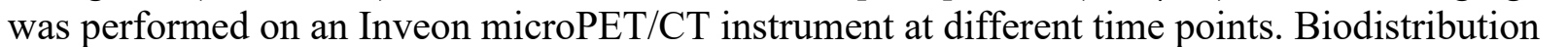

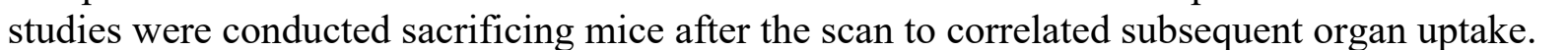

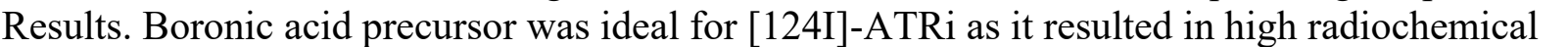

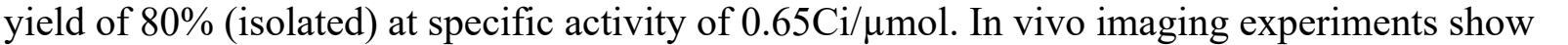

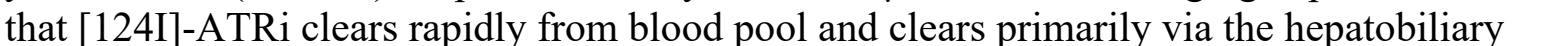

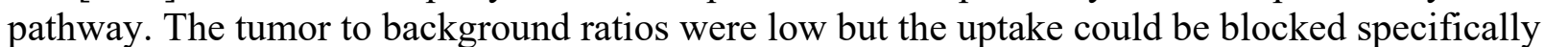

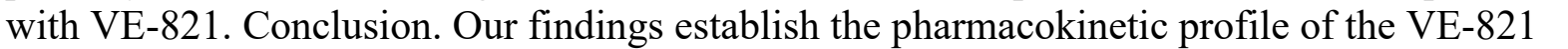

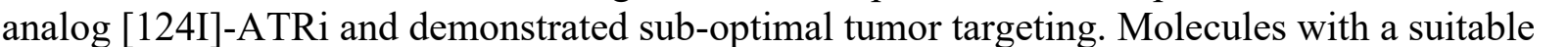

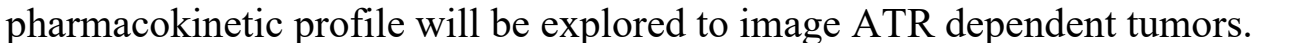

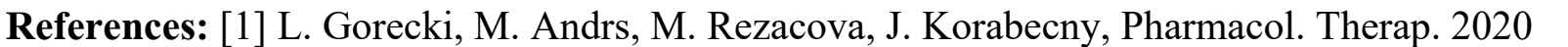

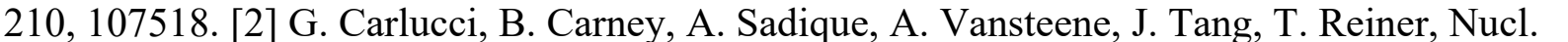

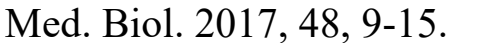

\section{Image/Figure:}

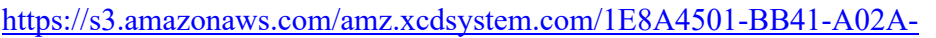

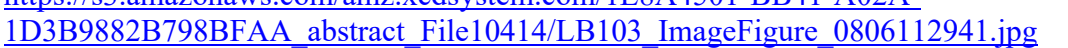




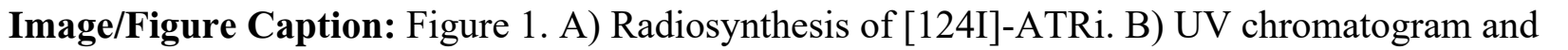

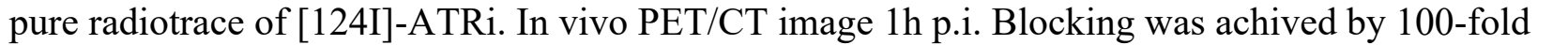

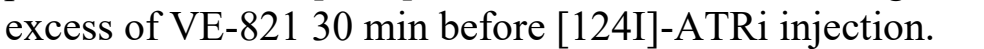

First Name: $\square \square \| \square$

Last Name: $\square \square\|\|\|\square \square\| \| \square$

Email: $\square\|\|\|\square \square \square \square \square\|\|\square\| \square$

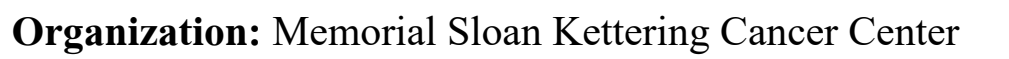

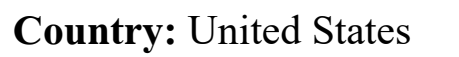




\title{
ID: LB104 \\ 89Zr-ImmunoPET to guide the development of anti-prostate stem cell antigen (PSCA) antibody fragment based radioimmunotherapy for prostate and pancreatic cancer
}

\section{Kirstin Zettlitz, City of Hope, kzettlitz@coh.org}

\section{Category: $\square \square \| \Pi \square \square$}

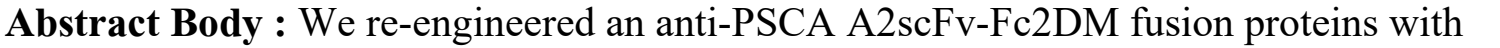

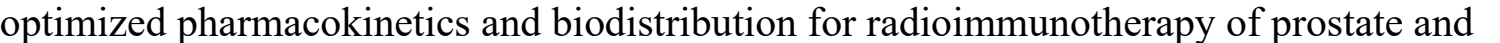

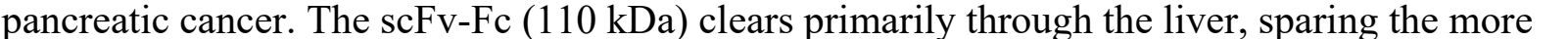

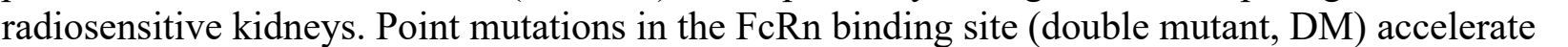

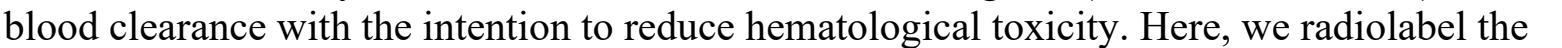

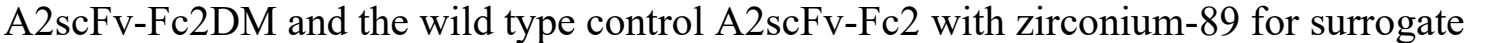

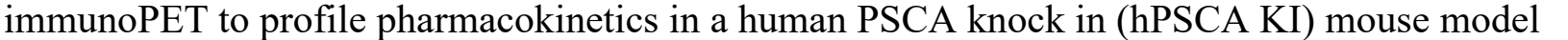

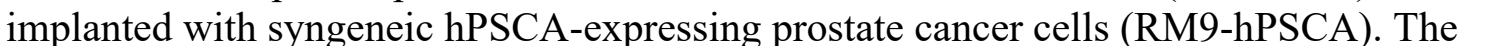

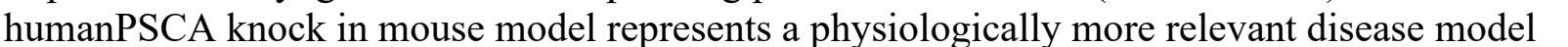

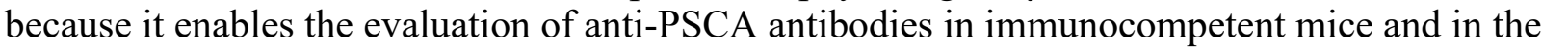

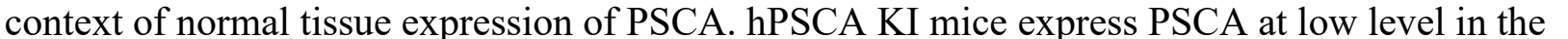

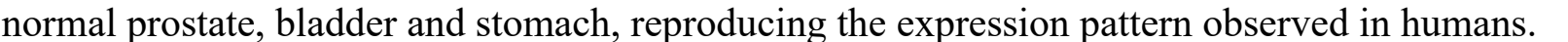

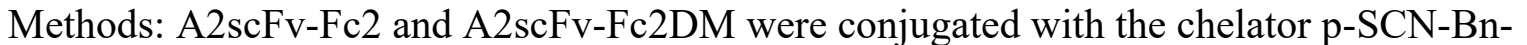

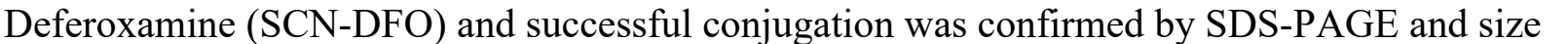

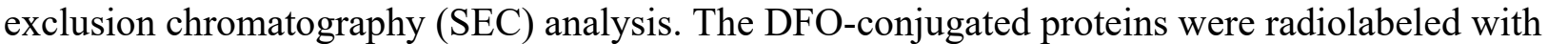

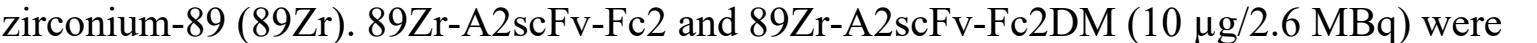

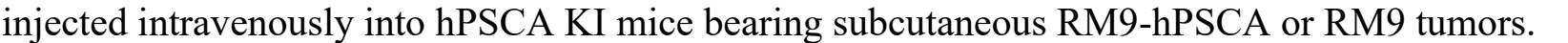

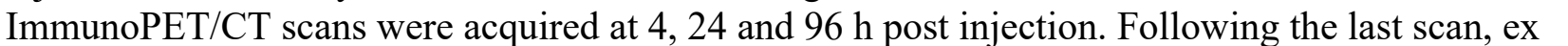

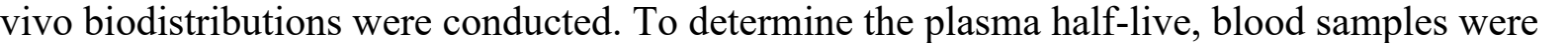
ए

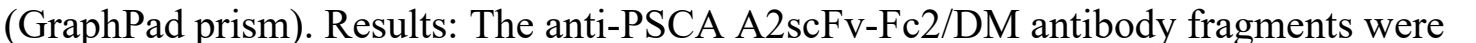

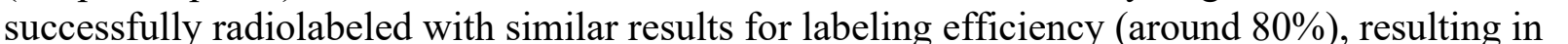

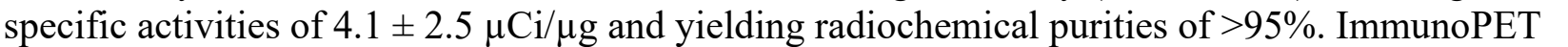

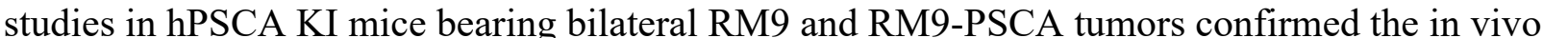

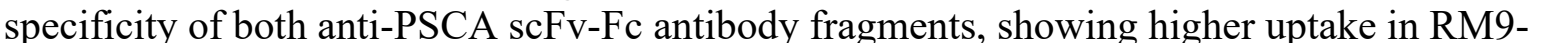

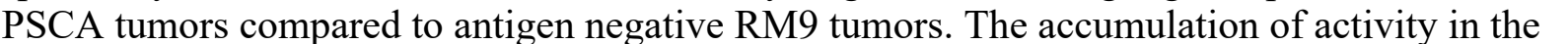

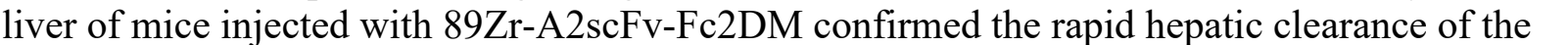

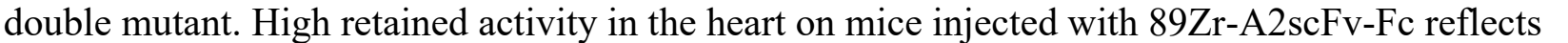
ए

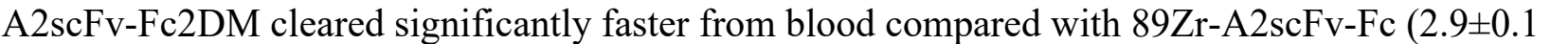

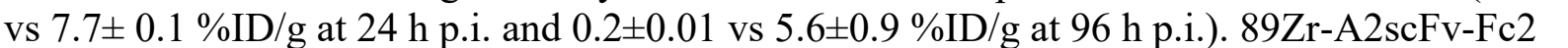

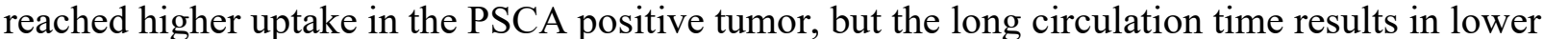

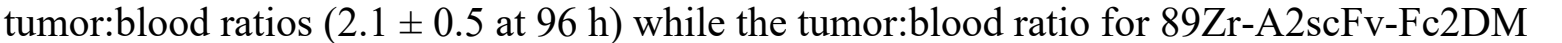

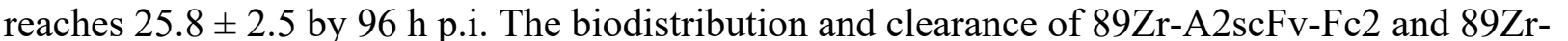

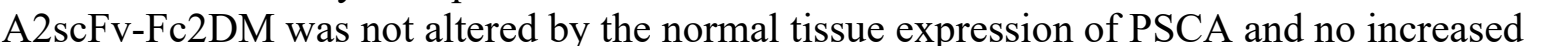




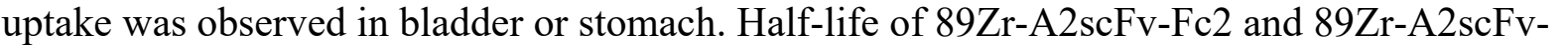

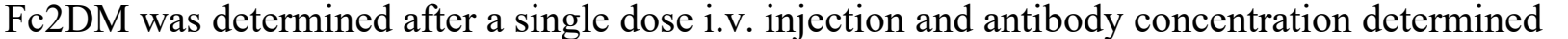

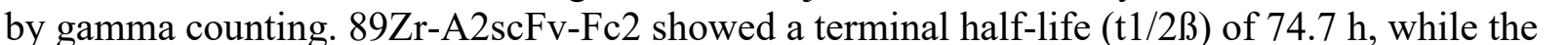

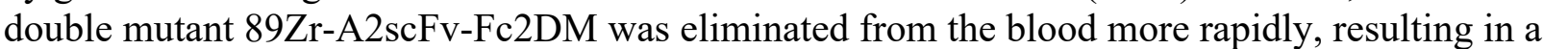
ए

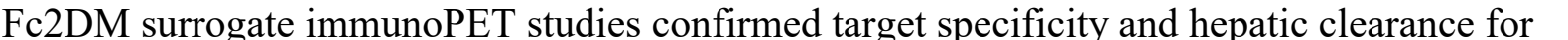

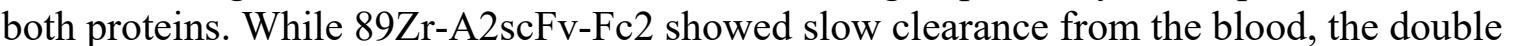

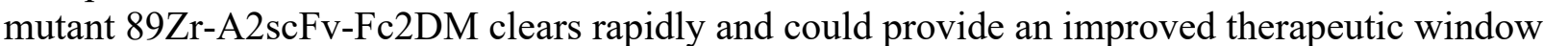

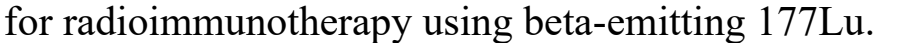

First Name: $\square \square\|m\| m$

Last Name: $\square \square \square m|n| m \mid \square$

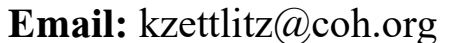

Organization: $\square \square\|m\|\|\square \square \square\|$

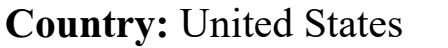




\title{
ID: LB105 \\ Contrast-enhanced perfusion imaging of inflammation the bowels of rainbow trout (Oncorhynchus mykiss)
}

\author{
Marianna Horn, University of Saskatchewan, marianna.horn@usask.ca
}

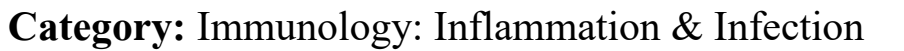

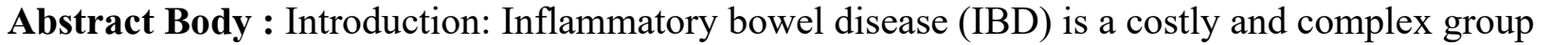

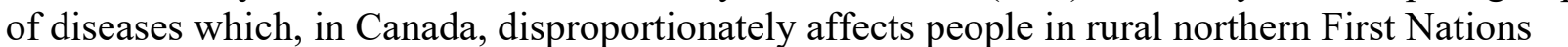

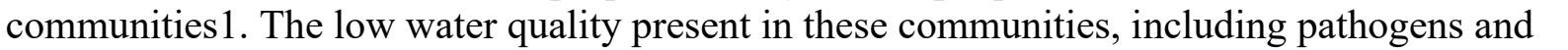

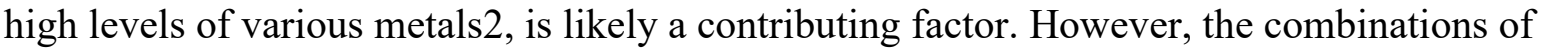

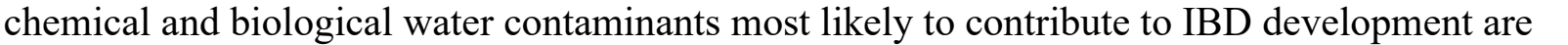

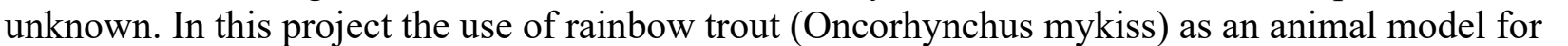
ए

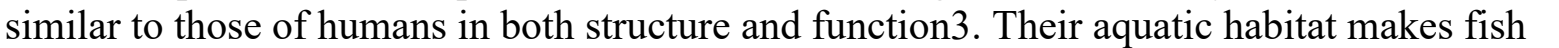

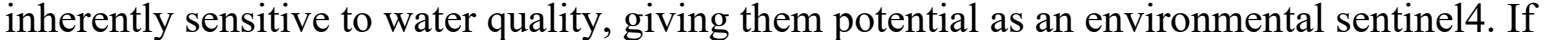

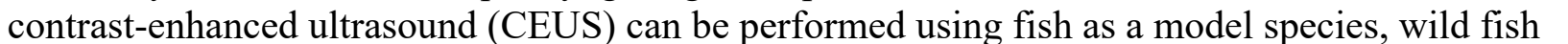

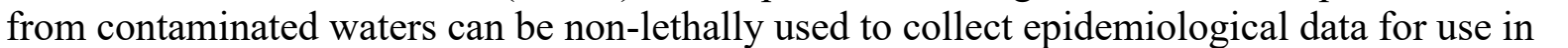

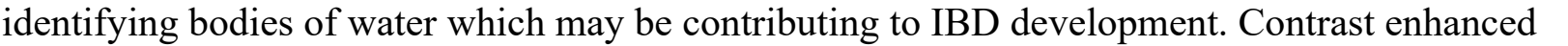

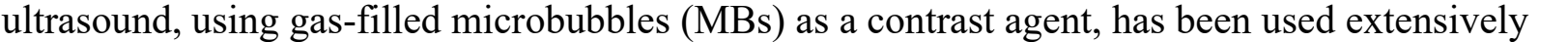

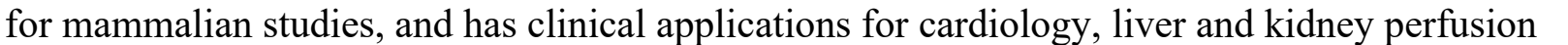

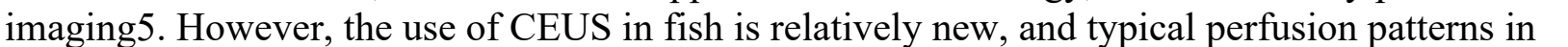

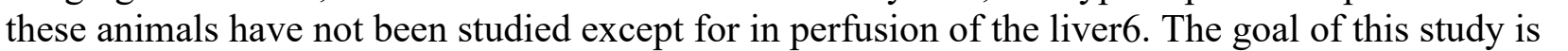
ए

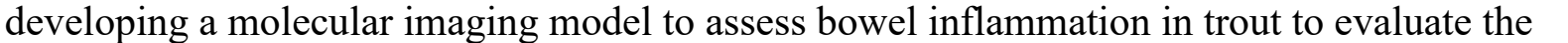
ए

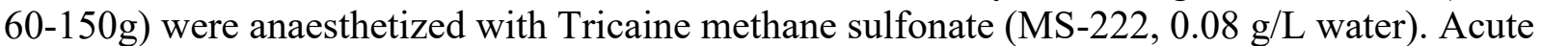

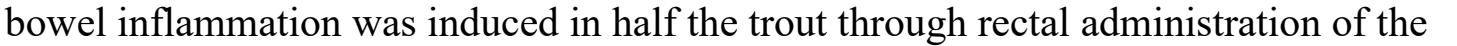

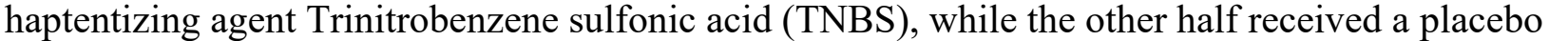

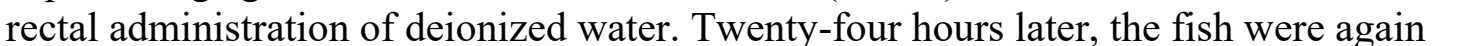

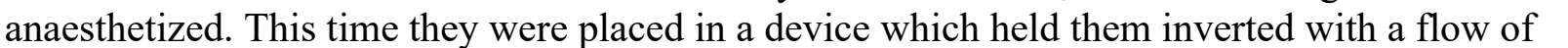

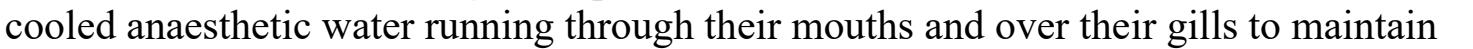

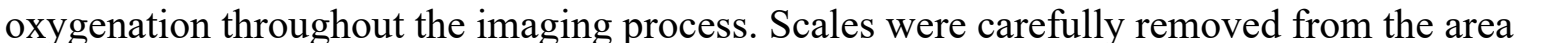

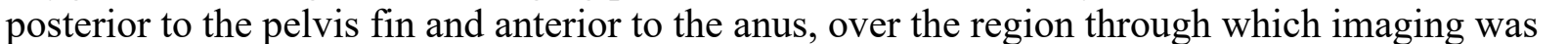

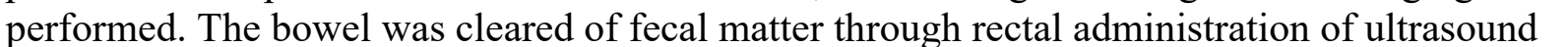

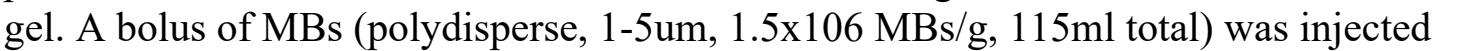

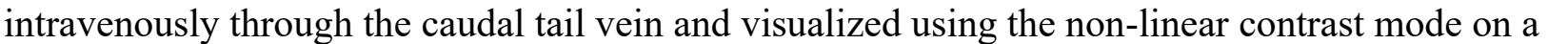

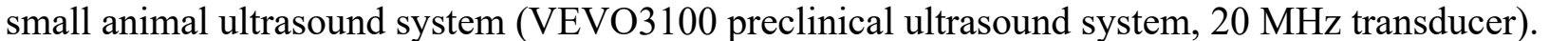

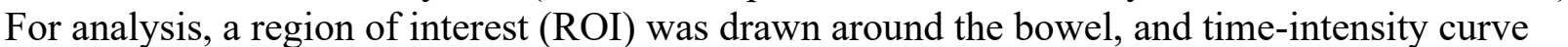

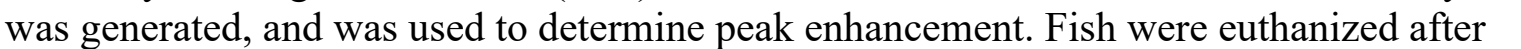

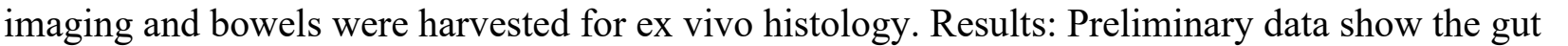

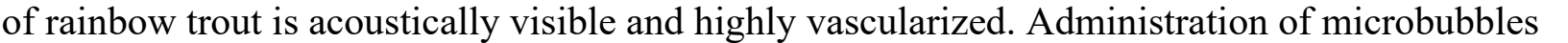

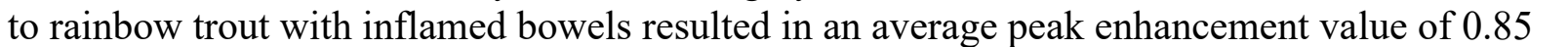




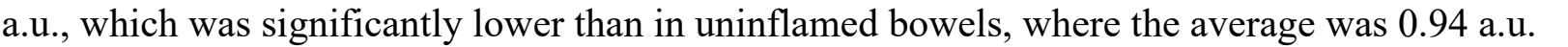

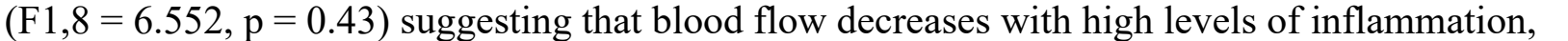

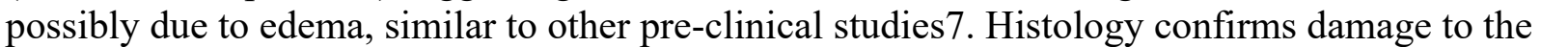

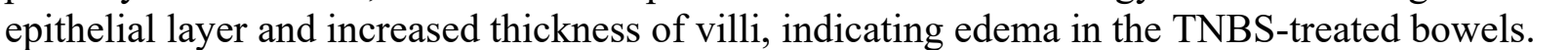

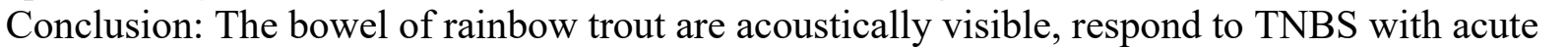

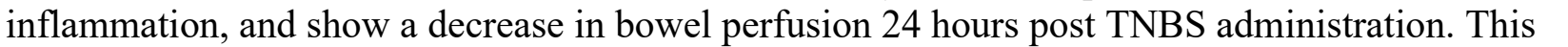

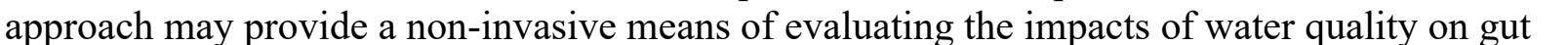

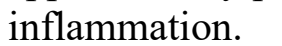

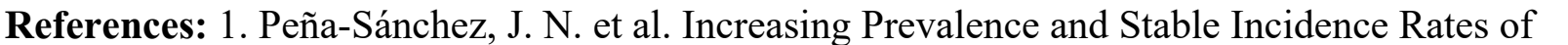

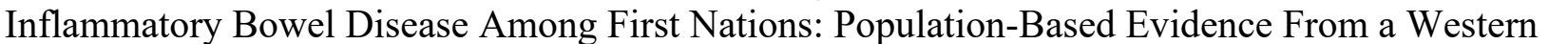

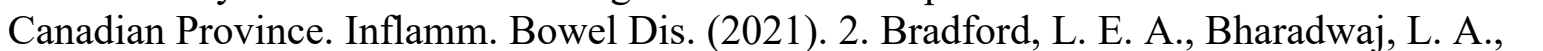

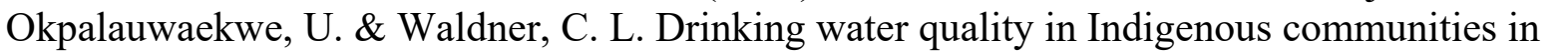

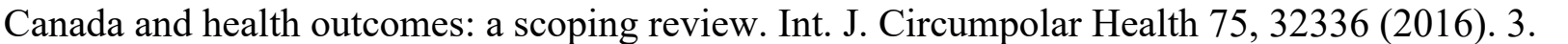

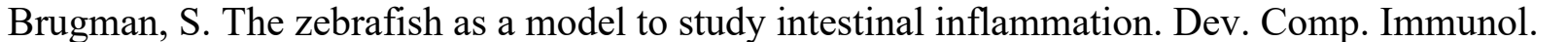

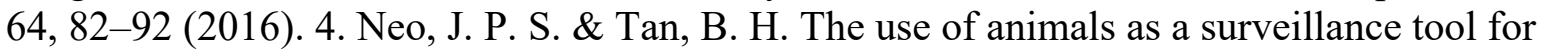

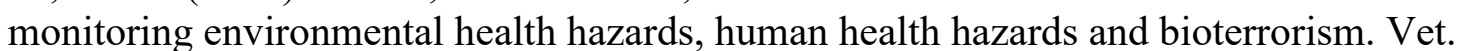

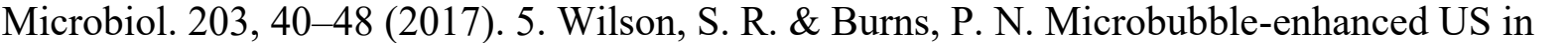

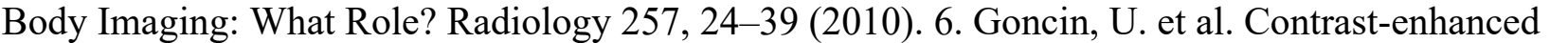

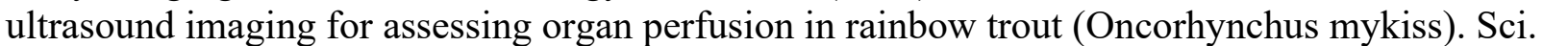

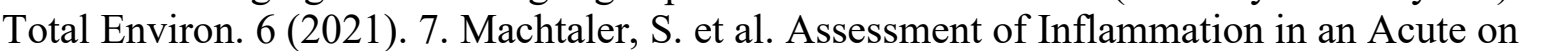

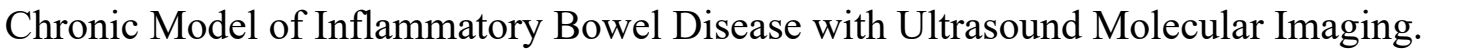

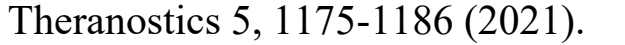

\section{Image/Figure:}

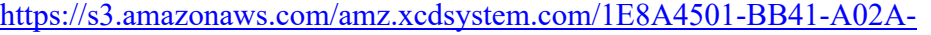

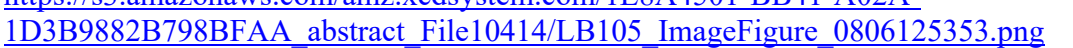

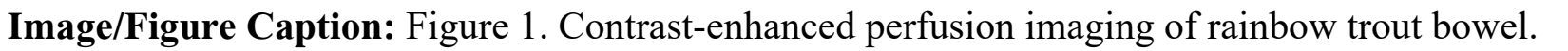

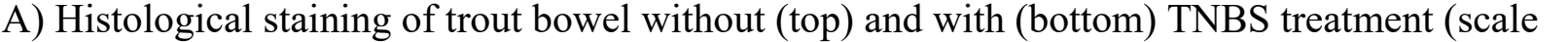

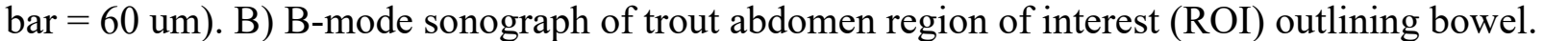

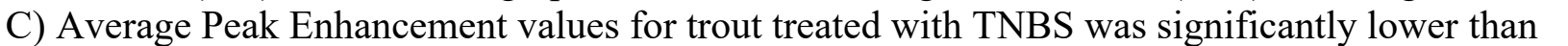

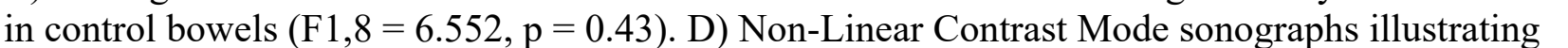

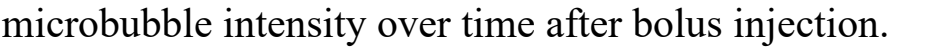

First Name: $\square \square\|\|\|\| m$

Last Name: $\square \square \square \| \square$

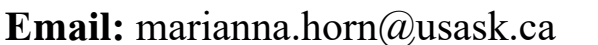

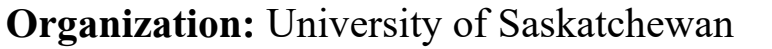

Country: $\square \square \square \square\|\|$ 


\title{
ID: LB106 \\ Developing a Magnetic Resonance Reporter Gene: Analysis of the C-terminal tail of the magnetosome gene mamL
}

\author{
Qin (Daisy) Sun, Lawson Health Research Institute, qsun@lawsonimaging.ca
}

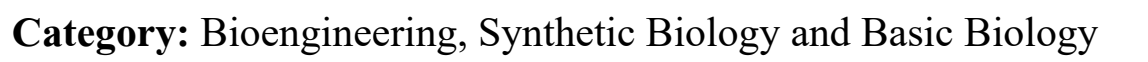

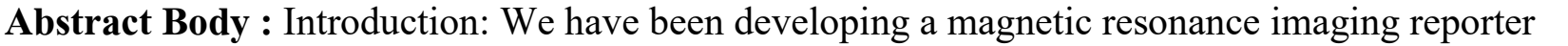

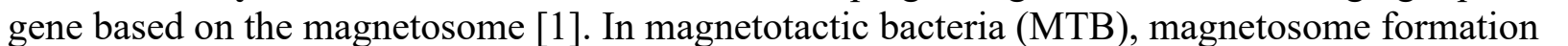

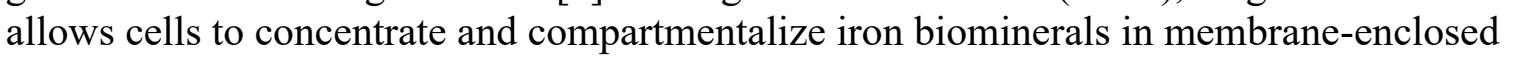

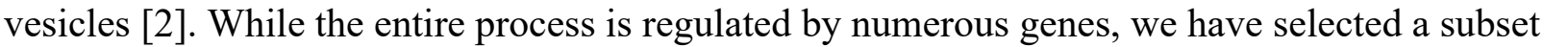

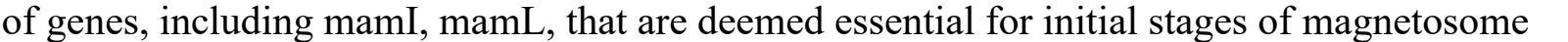

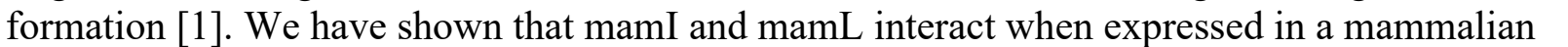

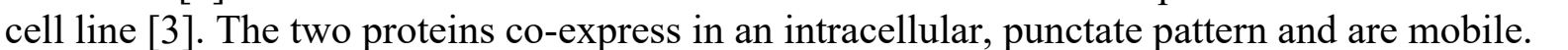

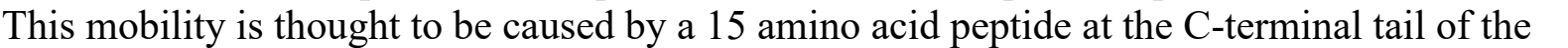

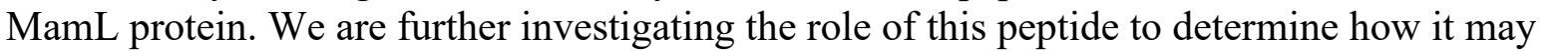

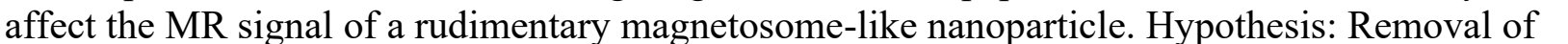

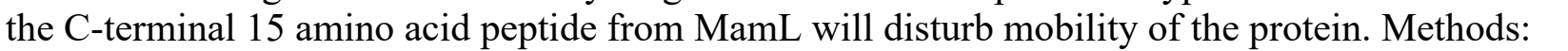

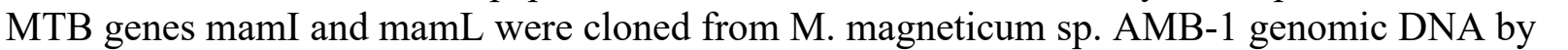

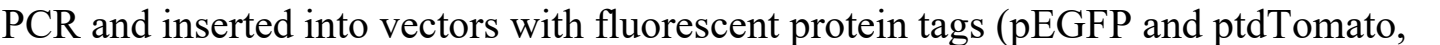

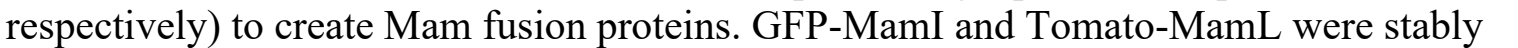

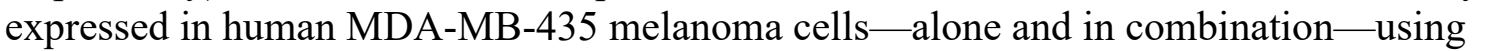

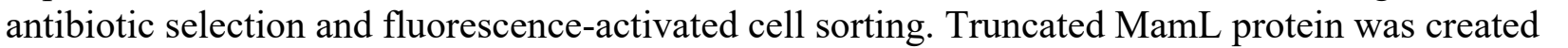

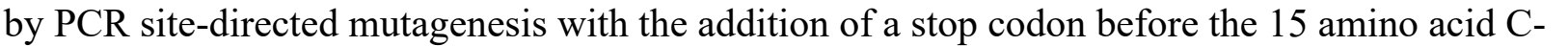

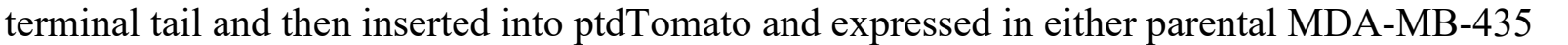

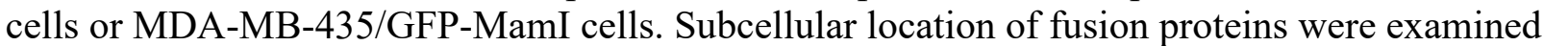

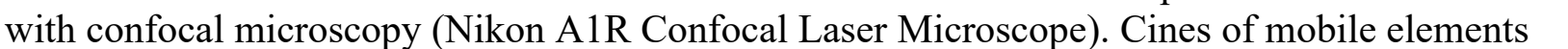

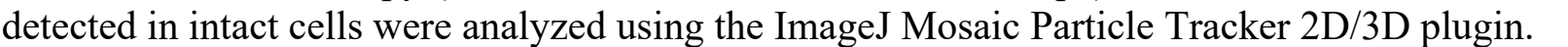

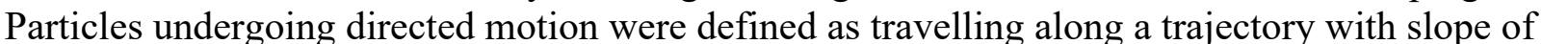
the mean squared displacement (MSD) above $1.2 \mu \mathrm{m}^{2} / \mathrm{s}$; whereas particles undergoing Brownian $\square \square \square \square \square \square$ defined by a slope of the MSD between 0.7 and $1.1 \mu \mathrm{m}^{2} / \mathrm{s}$. Results: Compared to

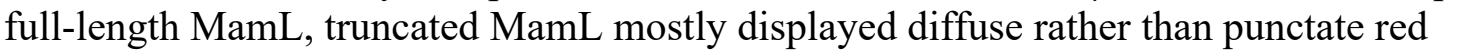

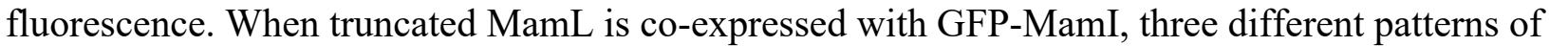
ए

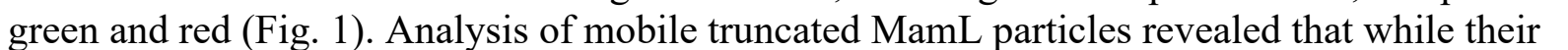

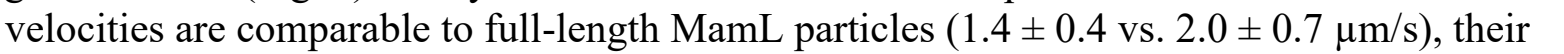

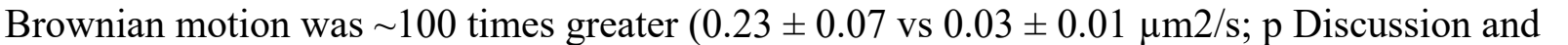

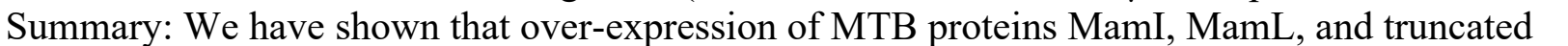

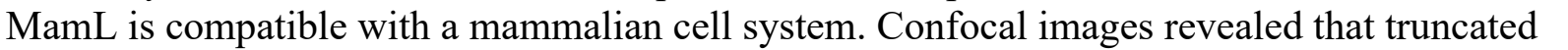

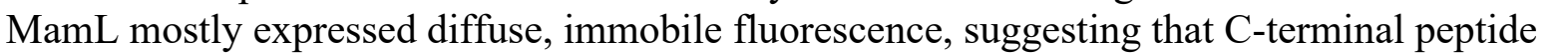

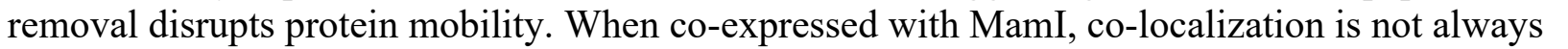

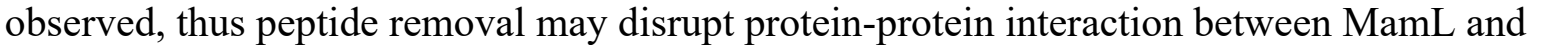

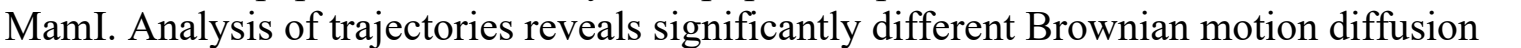




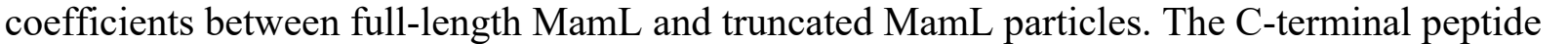

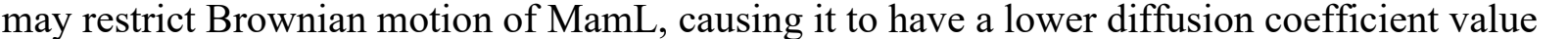

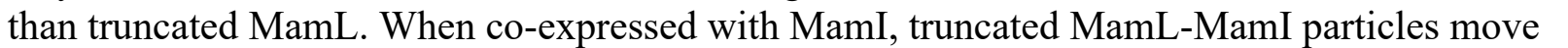

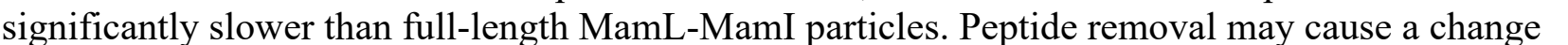

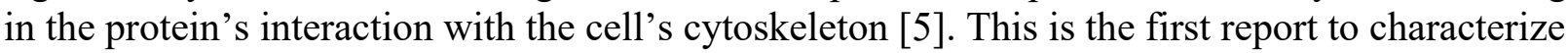

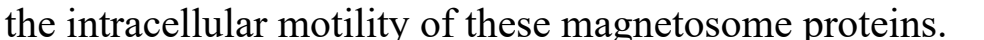

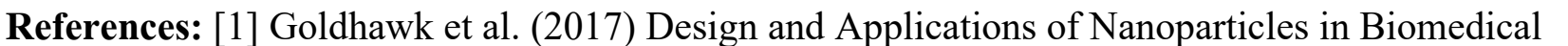

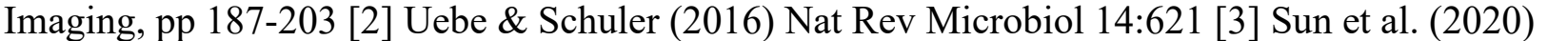

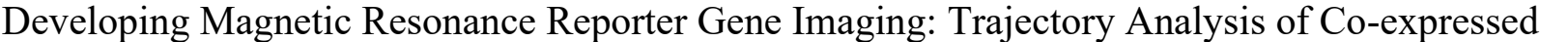

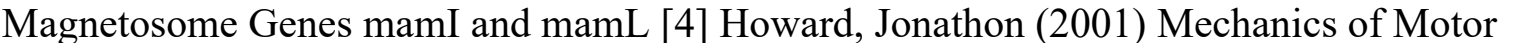

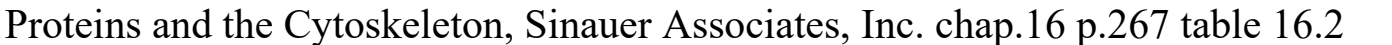

\section{Image/Figure:}

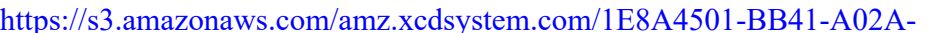

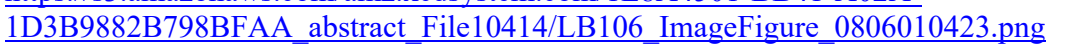

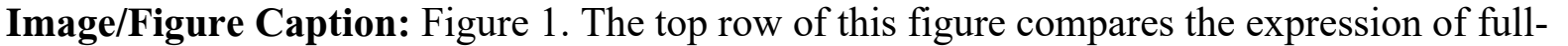

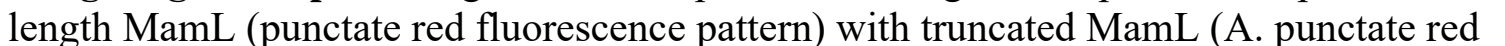

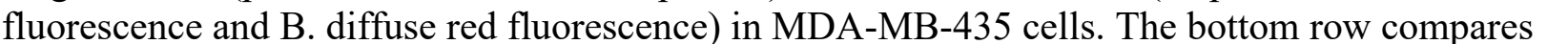
ए

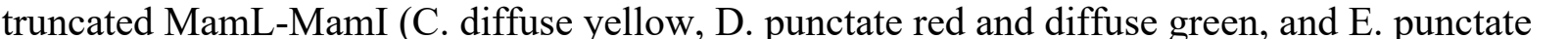

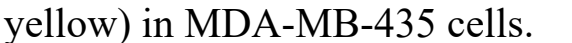

First Name: $\square \square \square \square \square \square \square \square \square$

Last Name:

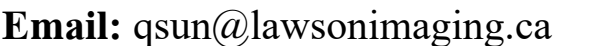

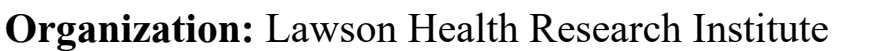

Country: $\square \square \square \square \square \| \square$ 


\title{
ID: LB107 \\ Leveraging a Bivalent Phenyloxadiazolyl Methyl Sulfone Bioconjugation Reagent to Create Highly Immunoreactive Fab Fragment for HER2-Targeted PET
}

\author{
GUILLAUME DEWAELE-LE ROI, Memorial Sloan Kettering Cancer Center, \\ dewaeleg@mskcc.org
}

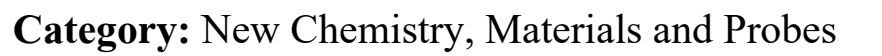

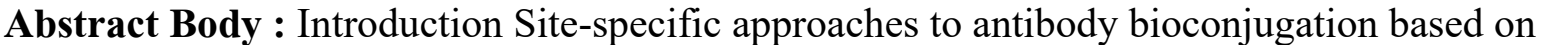

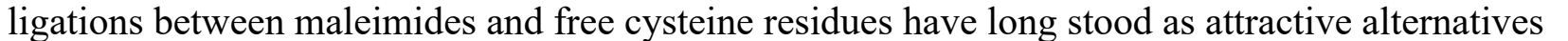

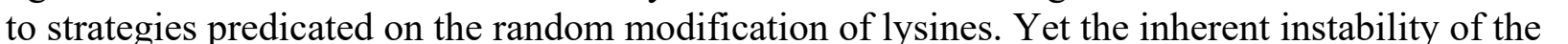

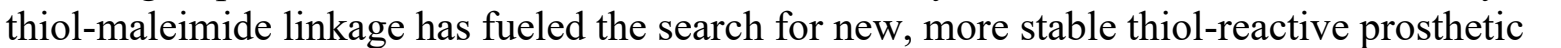

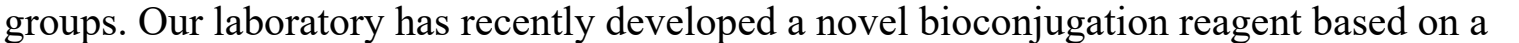

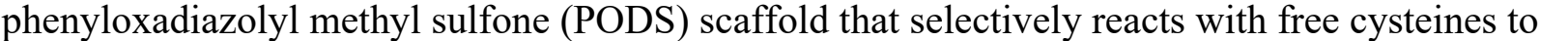

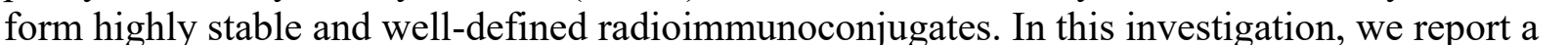

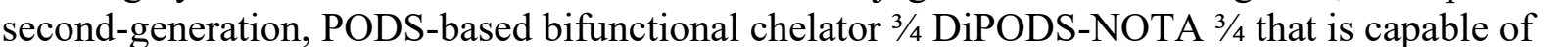

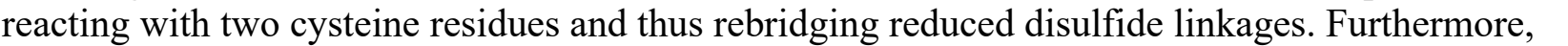

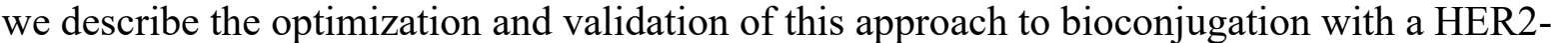

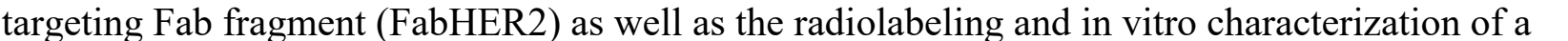

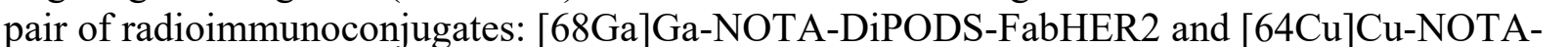

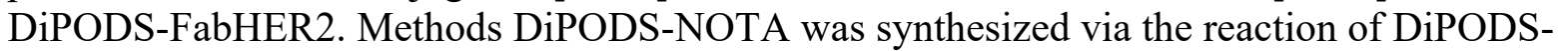

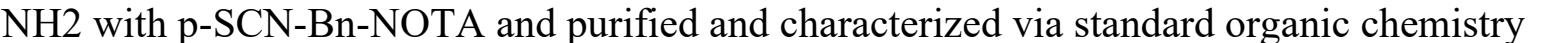

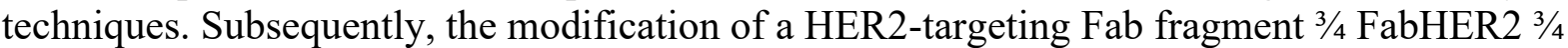

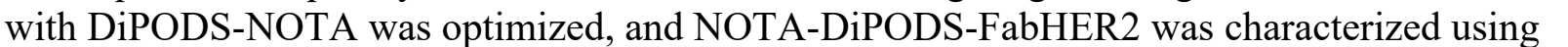

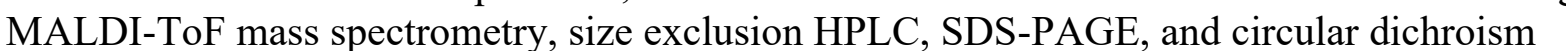

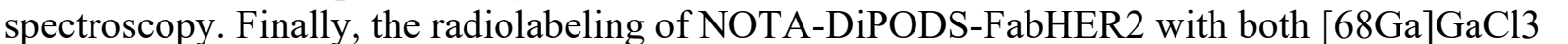

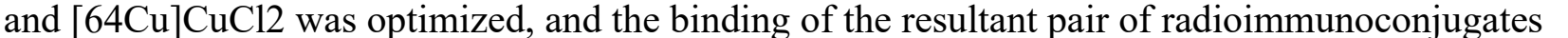

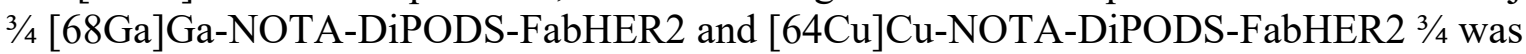

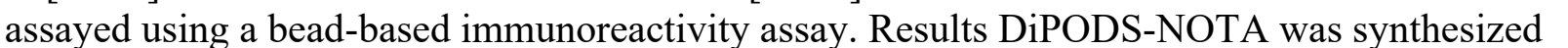

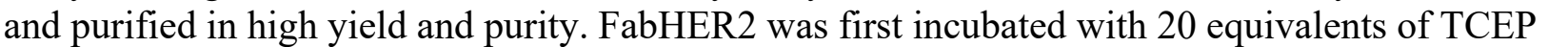

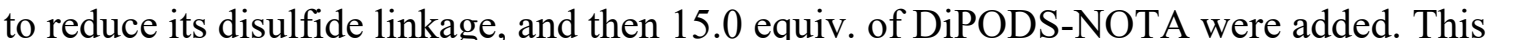

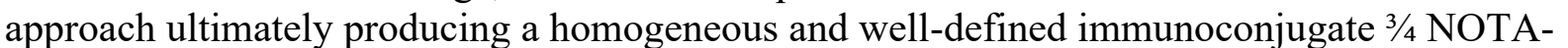

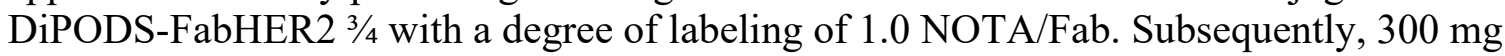

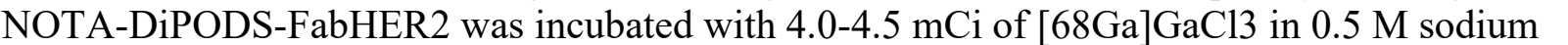

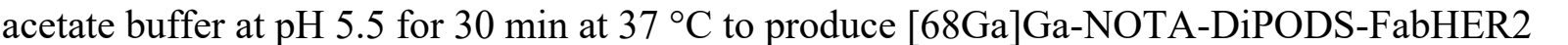

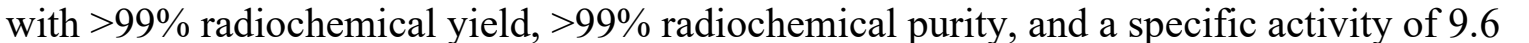

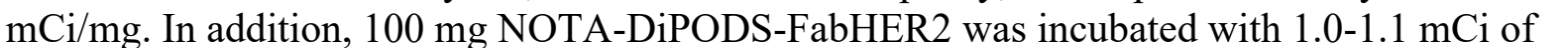
एम ए

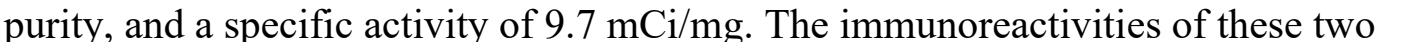

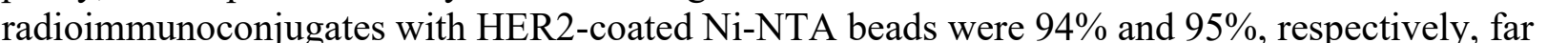
higher than those exhibited by analogous radioimmunoconjugates $3 / 4[68 \mathrm{Ga}] \mathrm{Ga} \square \square \square \square \square \square \square$
\end{abstract}




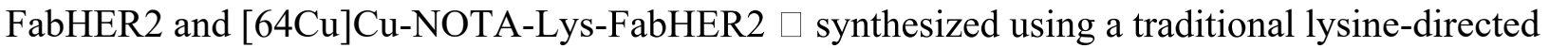

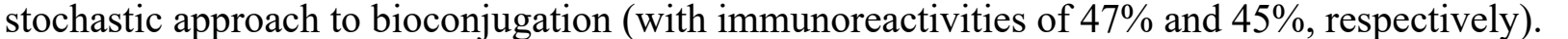

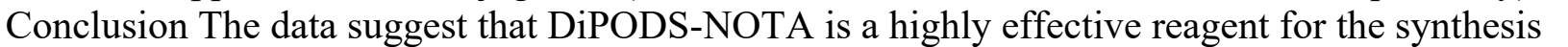

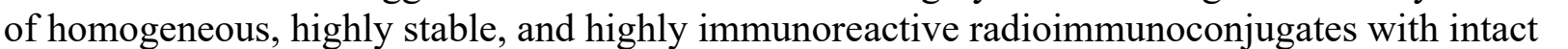

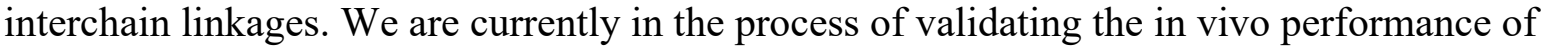
घण

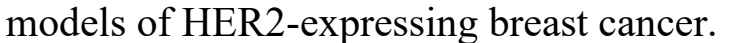

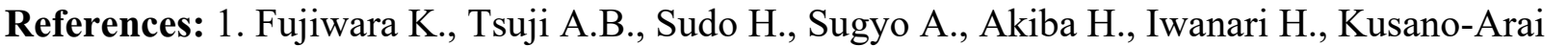

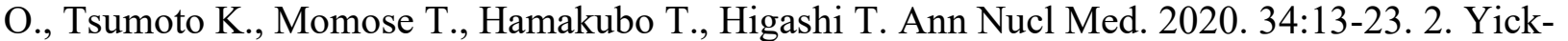

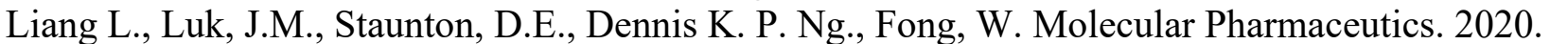

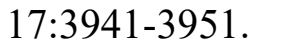

\section{Image/Figure:}

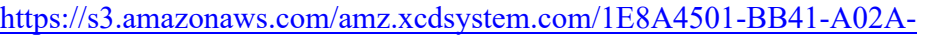

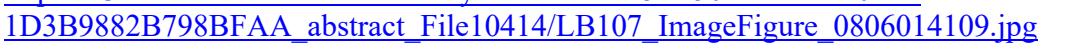

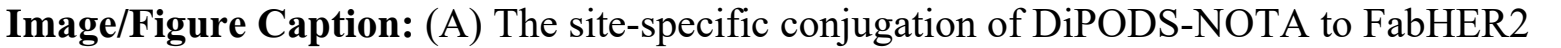

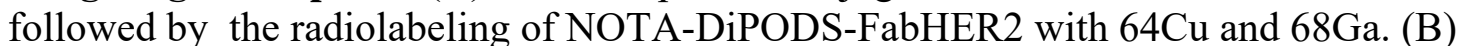

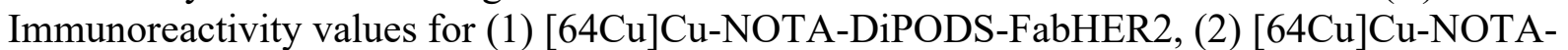

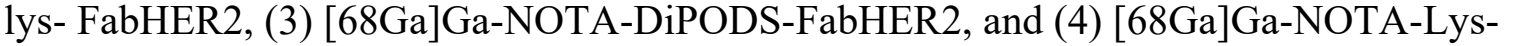

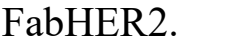

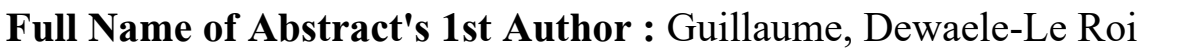

First Name: $\square \square \square \square \square \square \square \square \square$

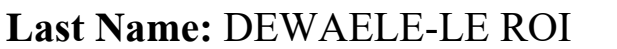

Email: $\square \square \square\|m \square \square \square\|\|\| \| \square$

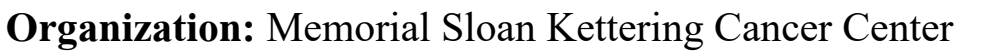

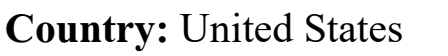




\title{
ID: LB108 \\ Effect of Z-spectral Noise on CEST-MRI Detectable pH Range of Iodinated Contrast Agents Iopamidol and Iopromide
}

Pietro Irrera, Moffitt Cancer Center, pietro.irrera@moffitt.org

\section{Category: $\square \square\|\| \| \square$}

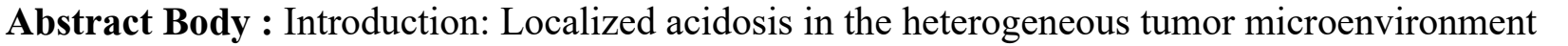

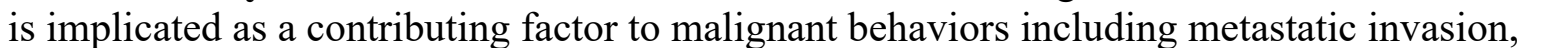

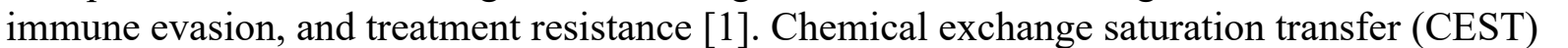

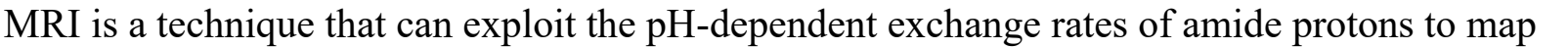
ए

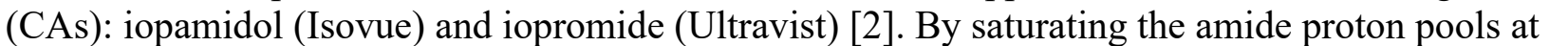

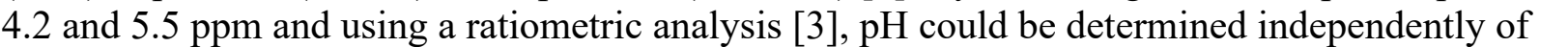

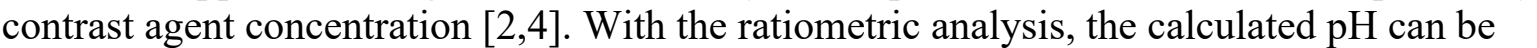

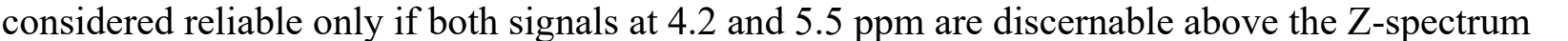

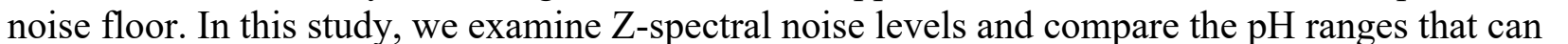

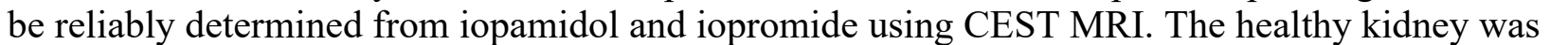

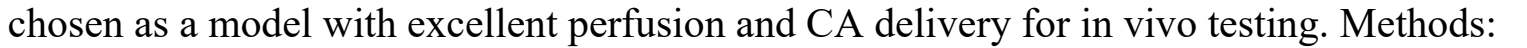

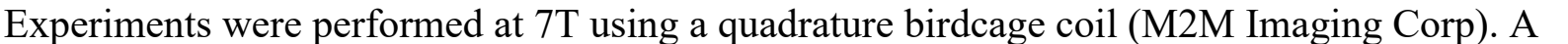

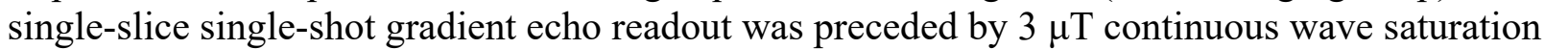

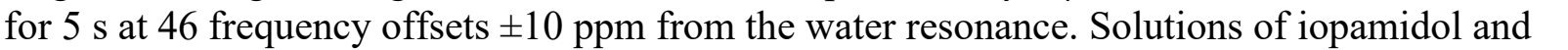
ए।

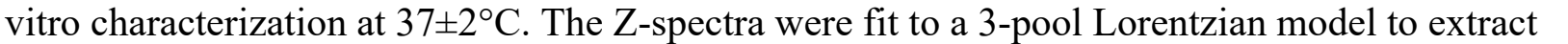

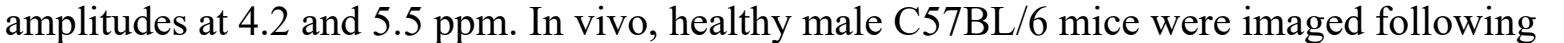
bolus injection of $200 \mu \mathrm{L} \mathrm{CA}$ via tail vein catheter. Regions of interest over the kidneys were

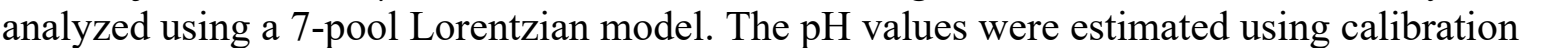
प

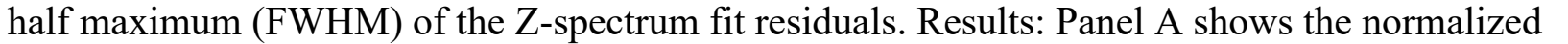

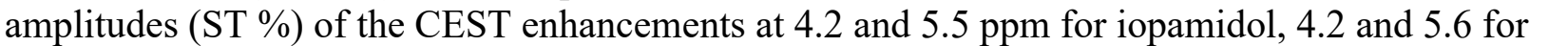

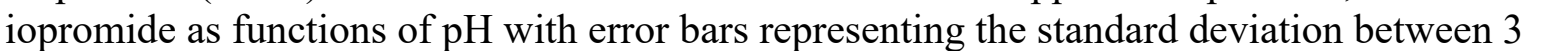
घh

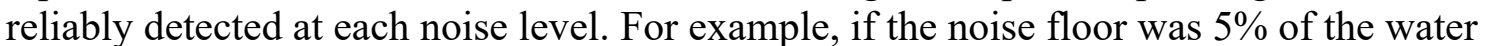

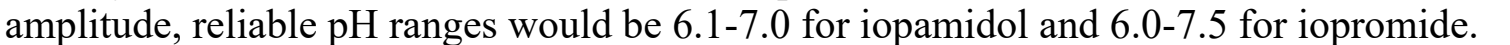

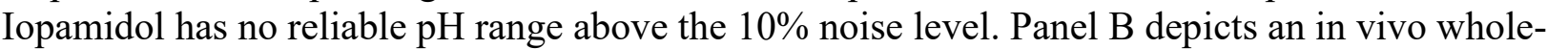

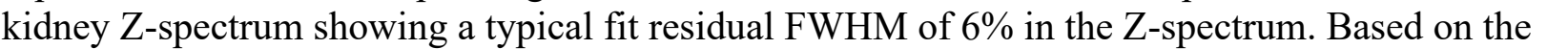

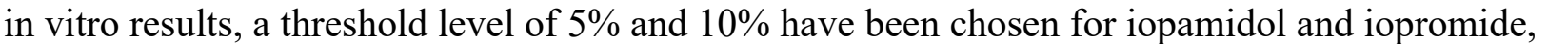

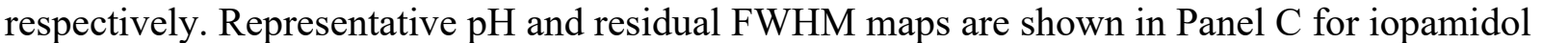

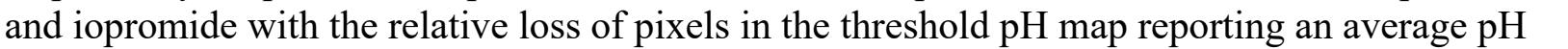

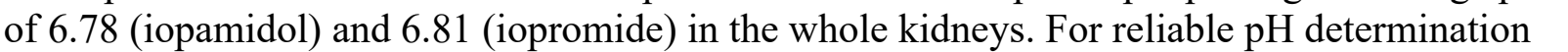

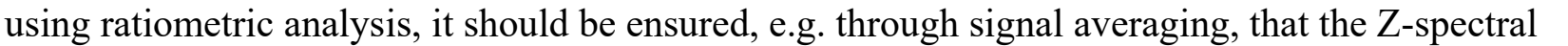

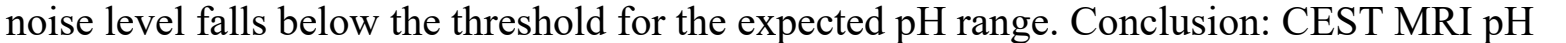




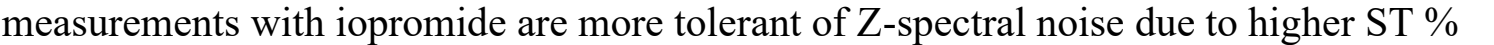

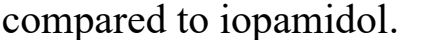

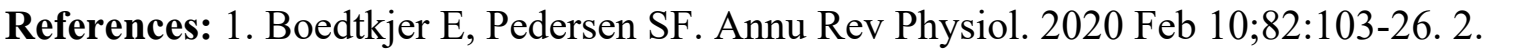

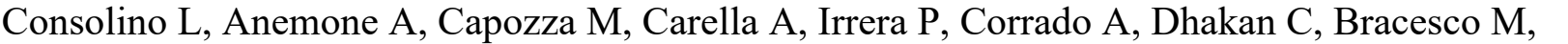

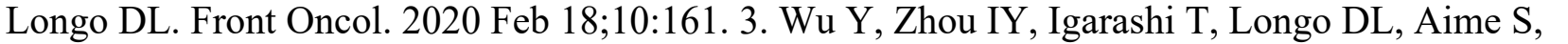

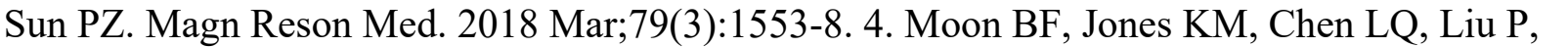

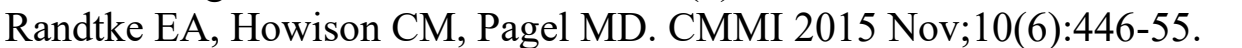

\section{Image/Figure:}

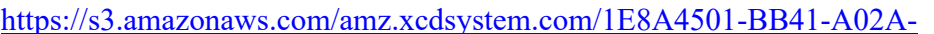

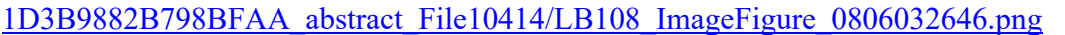

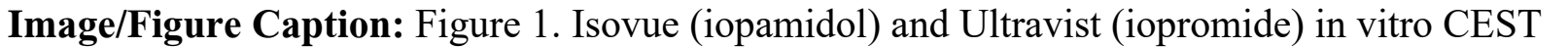

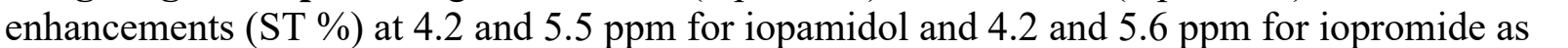
ए।

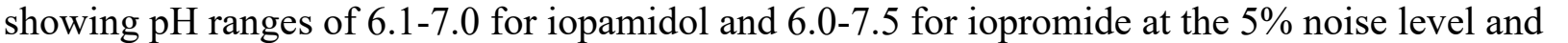

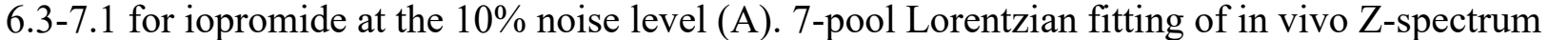

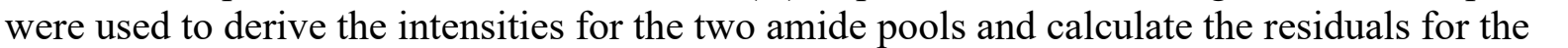

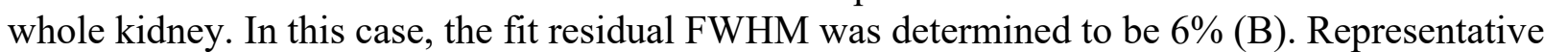

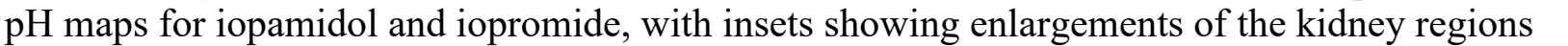

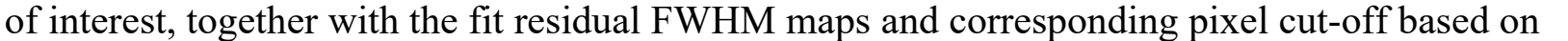

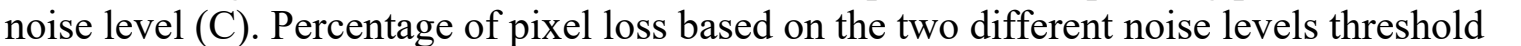

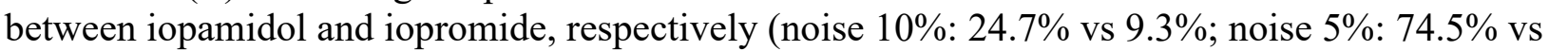

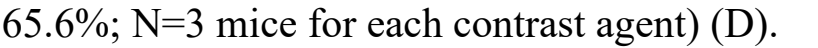

First Name: $\square \square\|m\|$

Last Name: $\square\|\| \| m$

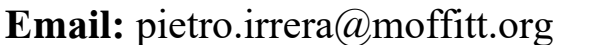

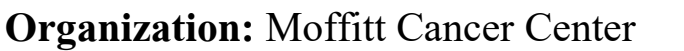

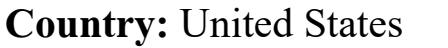




\title{
ID: LB109 \\ PODS-based site-specific labeling for host:guest pretargeting \\ Jacob Houghton, Stony Brook University, jacob.houghton@stonybrookmedicine.edu
}

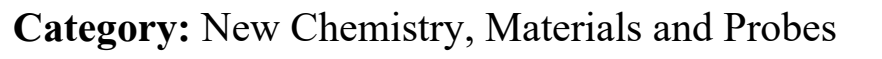

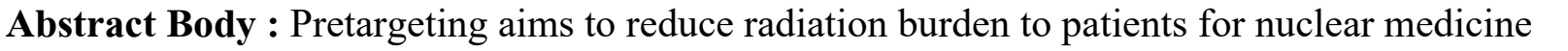

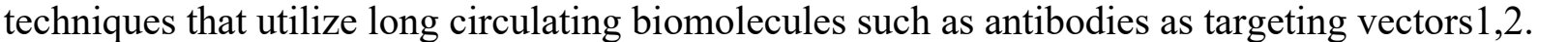

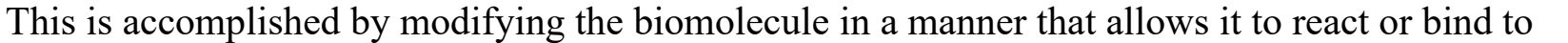

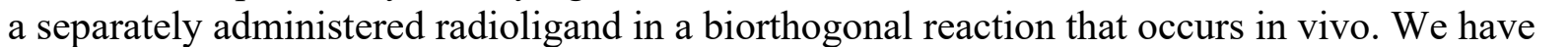

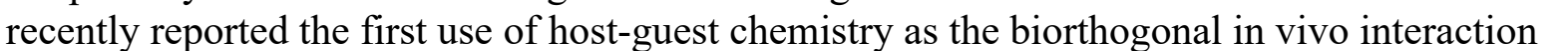

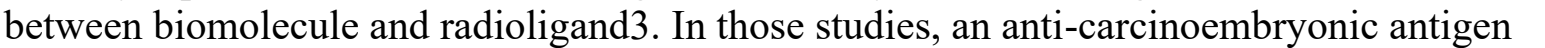

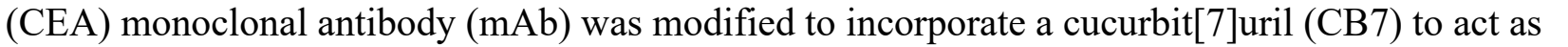

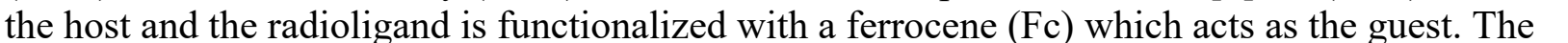

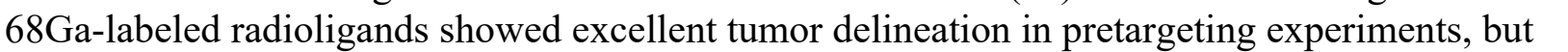

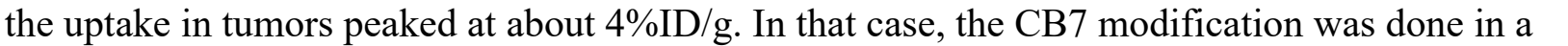

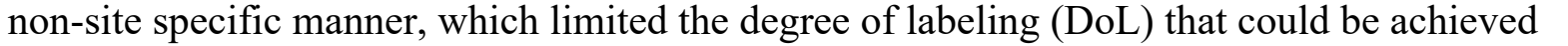

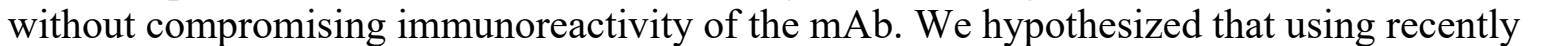

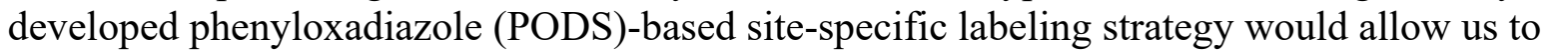
improve the degree of CB7 conjugation, thereby increasing the number of "host" sites and

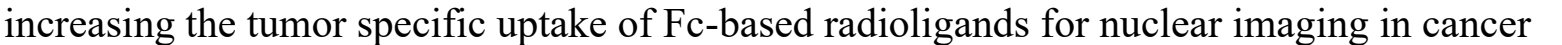

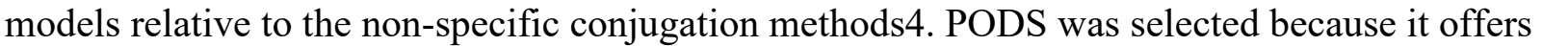

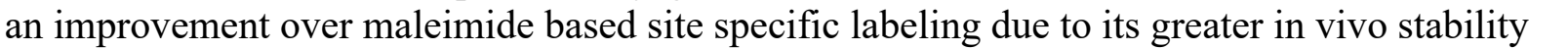

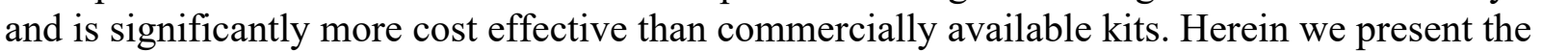

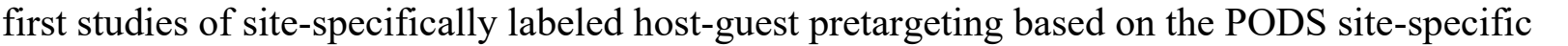

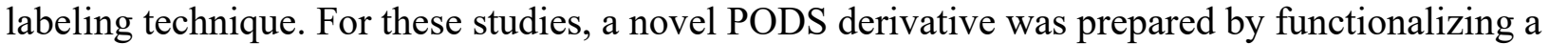

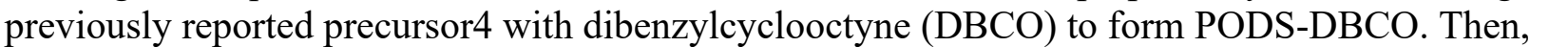

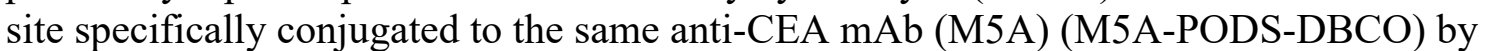

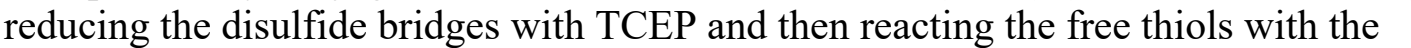

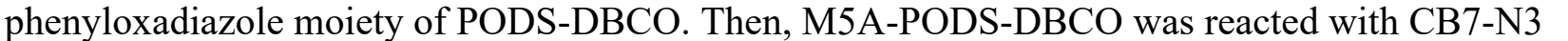

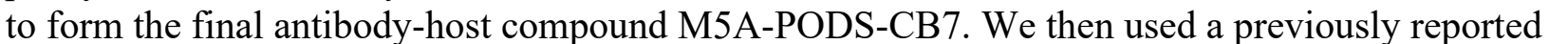

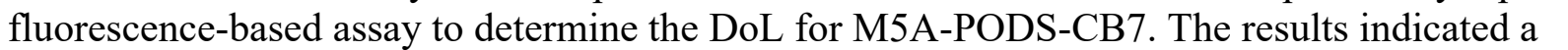

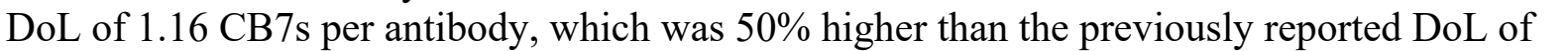

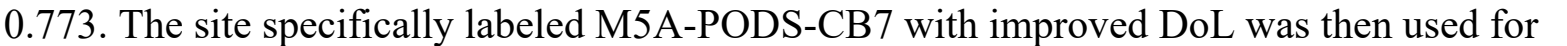

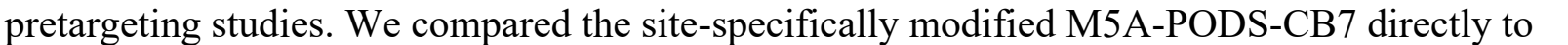

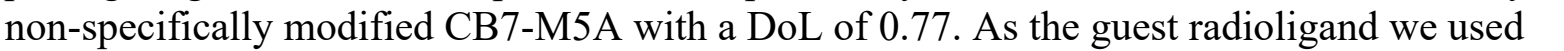
एम ए

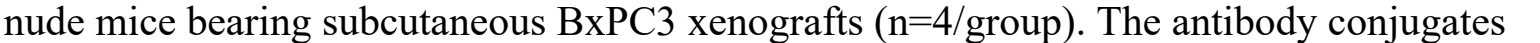

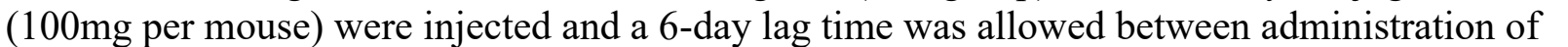

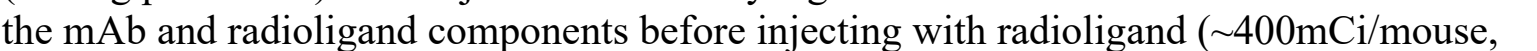

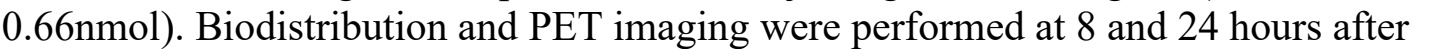

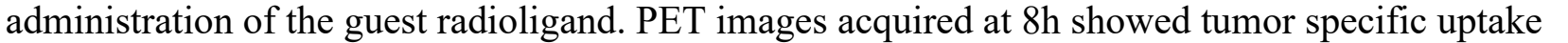




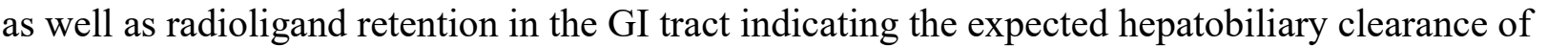

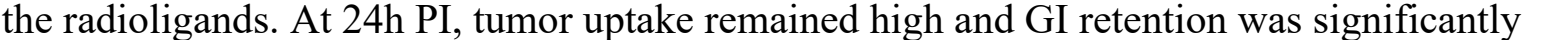
घम

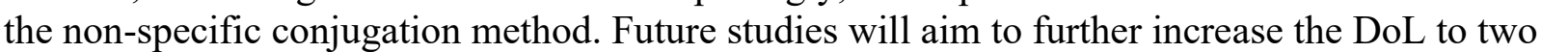

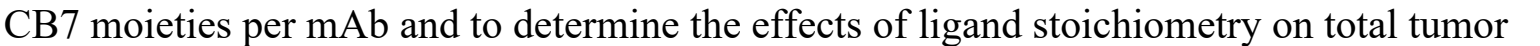

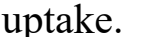

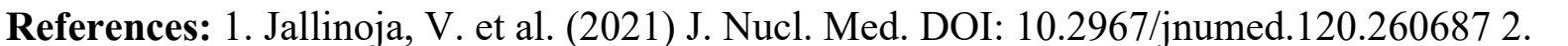

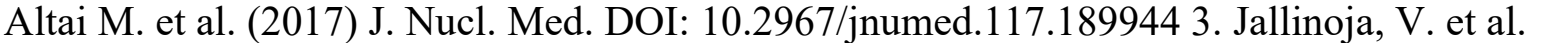

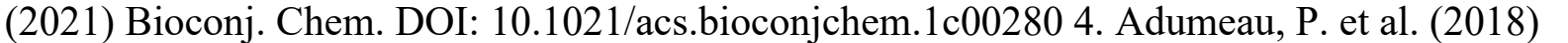

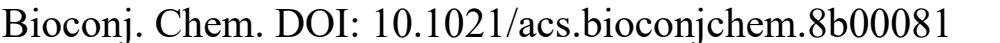

\section{Image/Figure:}

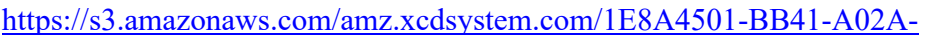

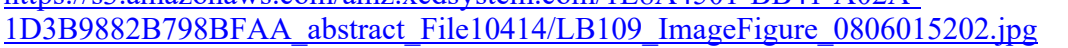

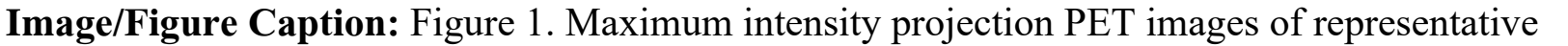

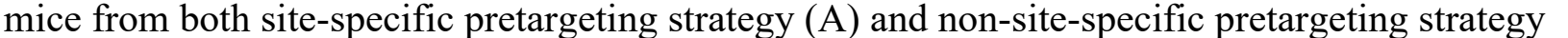

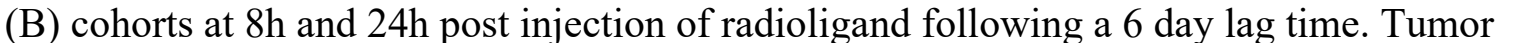

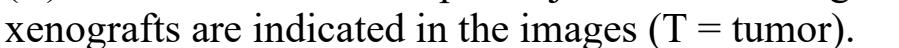

First Name: $\square \square \square \|$

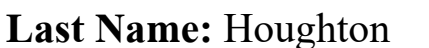

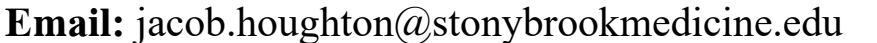

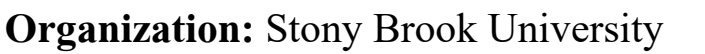

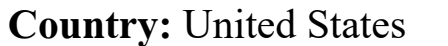




\title{
ID: LB110 \\ Bacillus subtilis coated with superparamagnetic iron for in vivo imaging and to guide development of bacteriotherapies
}

Emily Greeson, Michigan State University, greesone@msu.edu

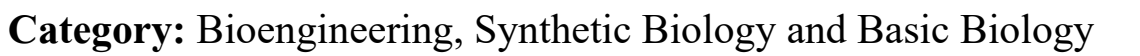

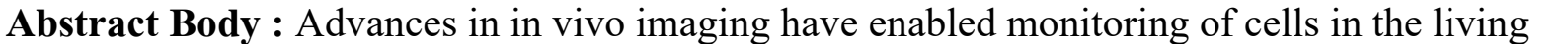

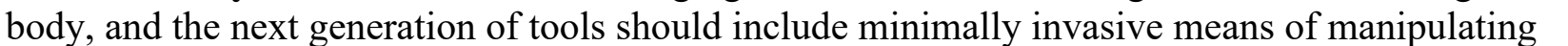

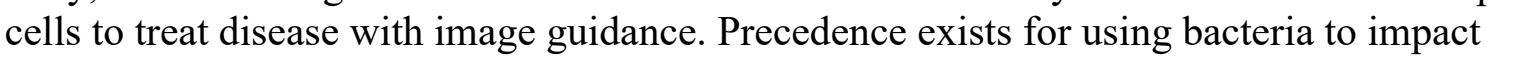

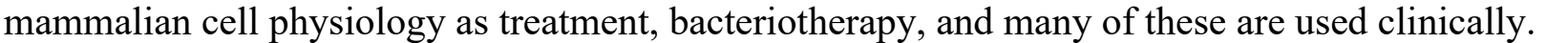

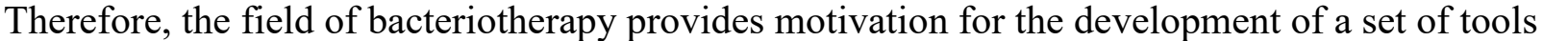

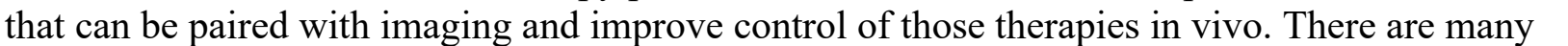

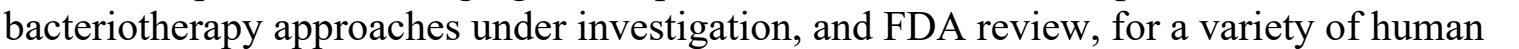

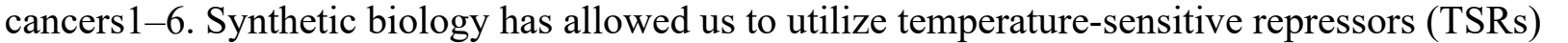
ए

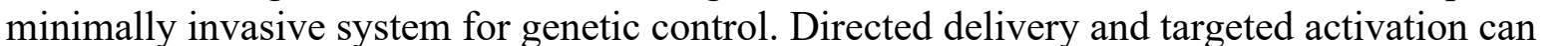

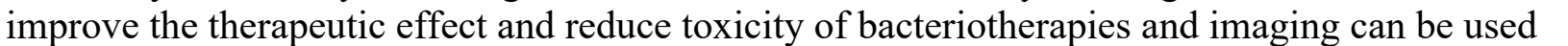

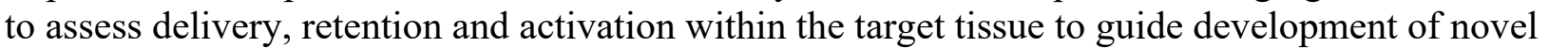

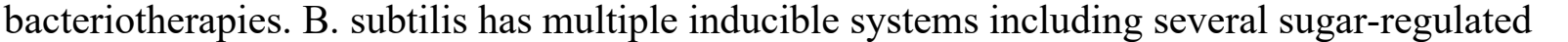
ए

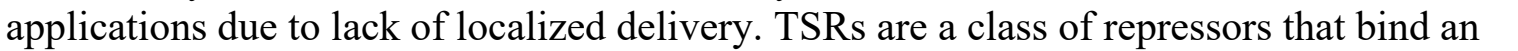

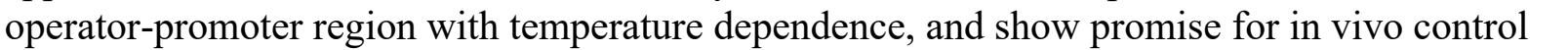

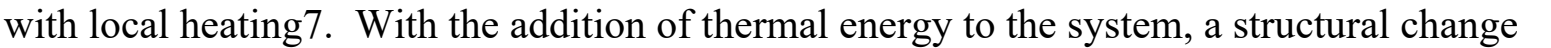

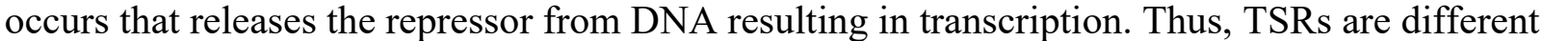
from heat shock promoters (HSP) and rely on housekeeping sigma factors such as $\sigma \mathrm{A}$ in B.

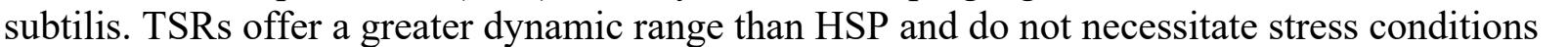

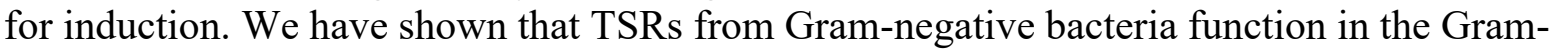

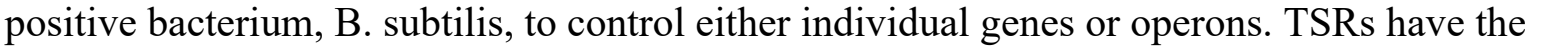

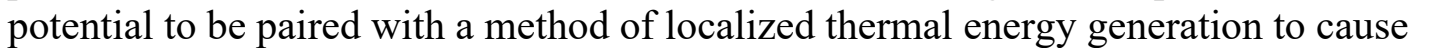

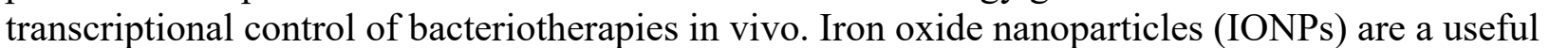

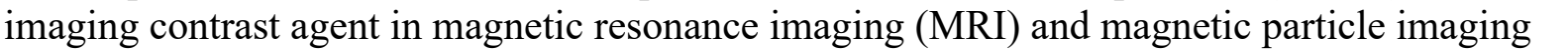

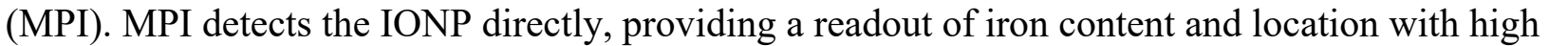

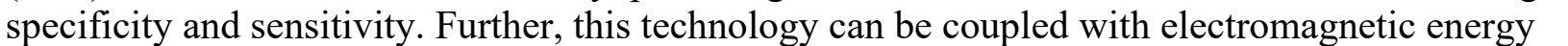

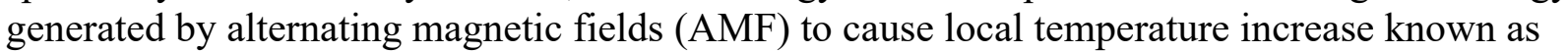

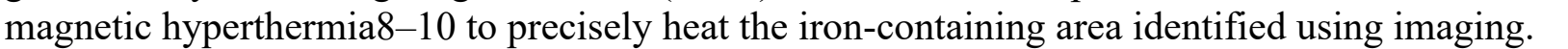

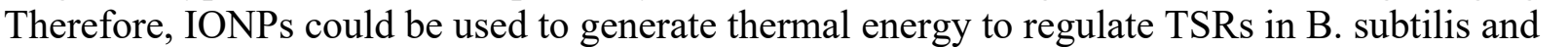

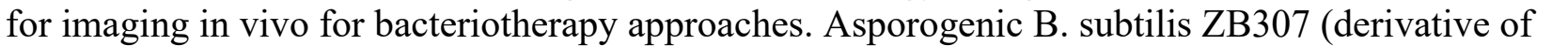

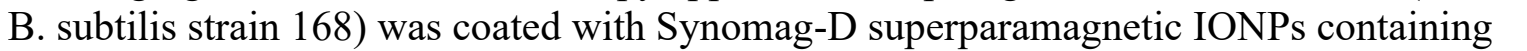

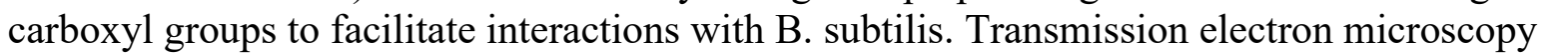

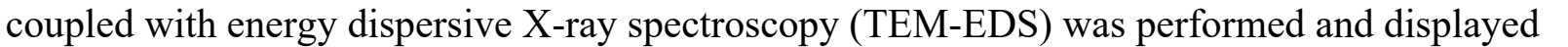

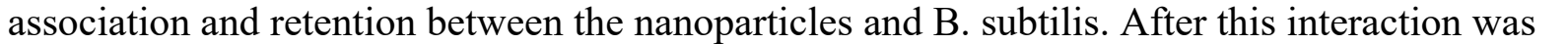

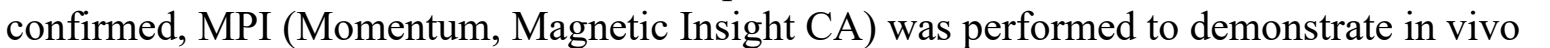




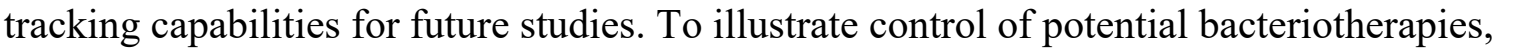

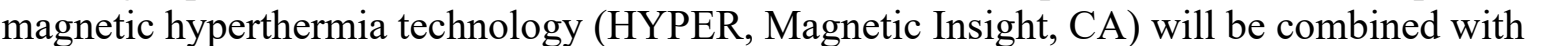

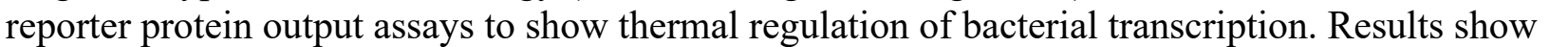

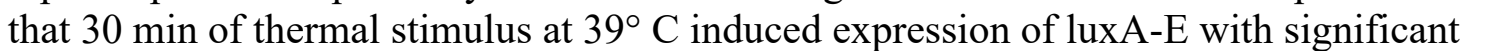

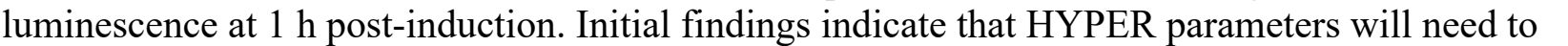

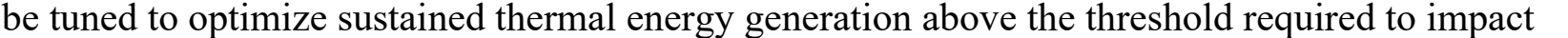

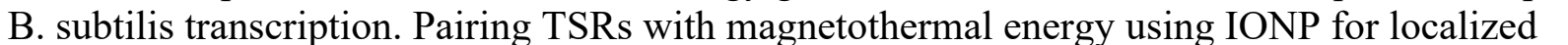

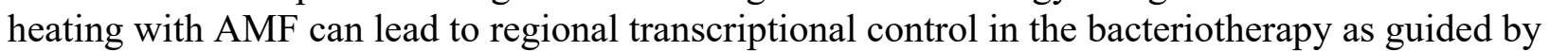

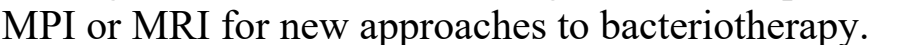

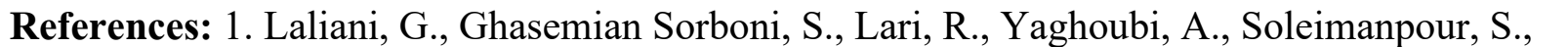

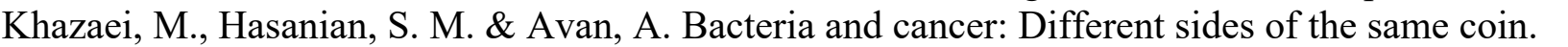

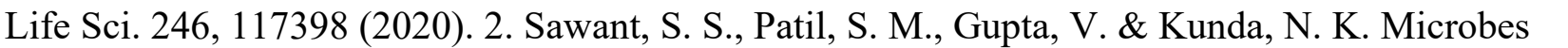

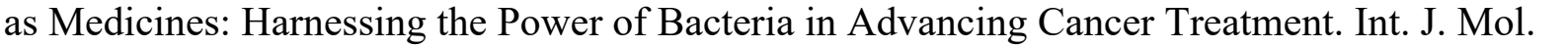

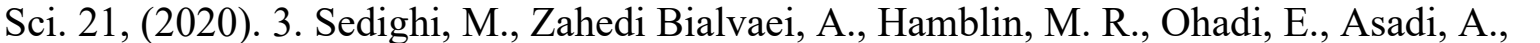

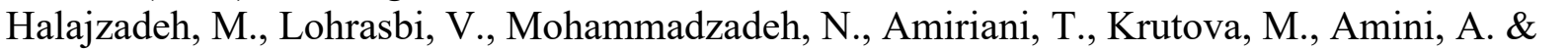

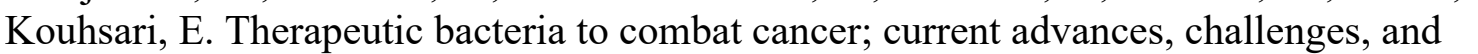

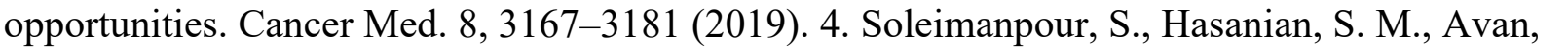

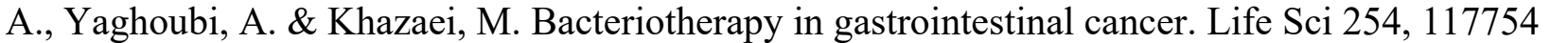

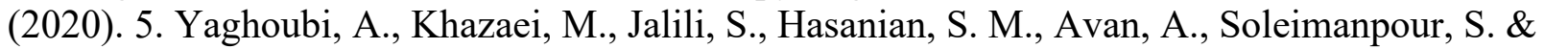

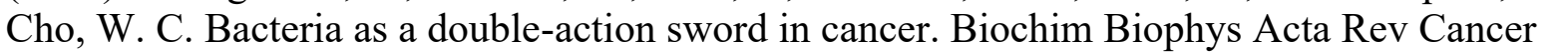

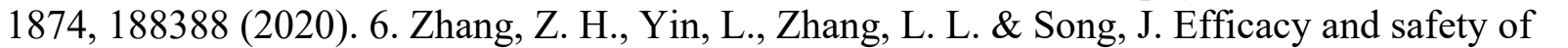

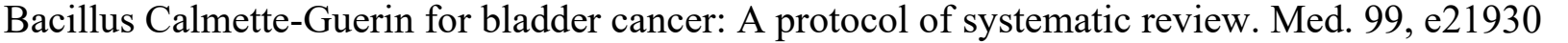

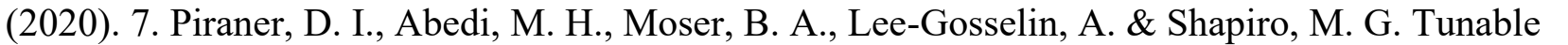

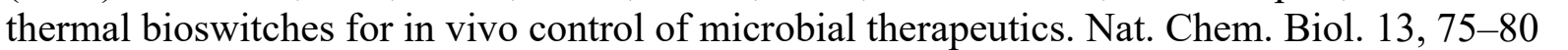

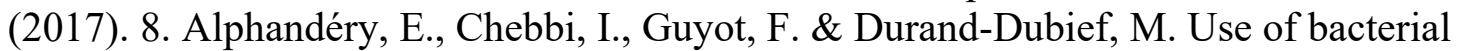

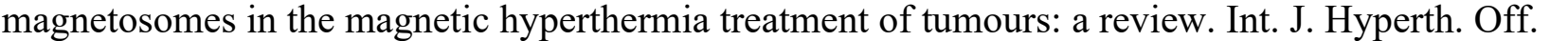

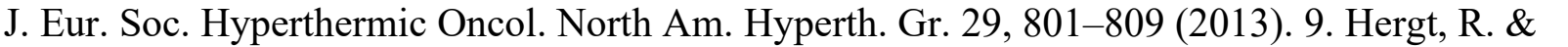

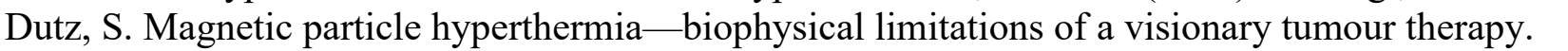

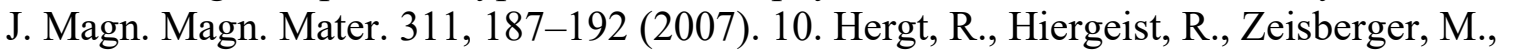

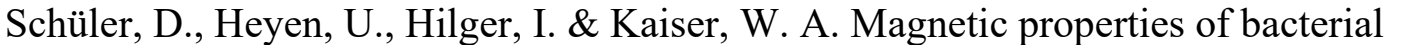

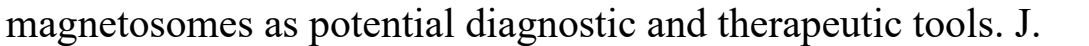

First Name: $\square \square \square \square \square$

Last Name: $\square \square\|\|\|\mid\|$

Email: $\square\|\|\|\| \square\|\square \square \square\| \square \square$

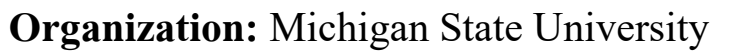

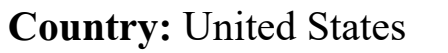




\title{
ID: LB111 \\ Imaging of heterogeneic tumors using an anti-CEA (carcinoembryonic antigen) SNAP-tag fusion protein
}

Stefanie Maurer, Icahn School of Medicine at Mount Sinai, stefanie.maurer@mssm.edu

Category: $\square \square \| \square \square ा \square$

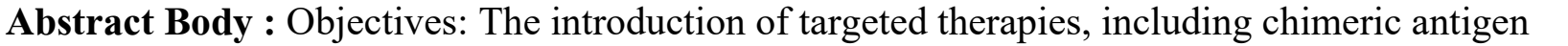

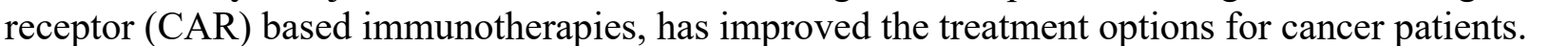

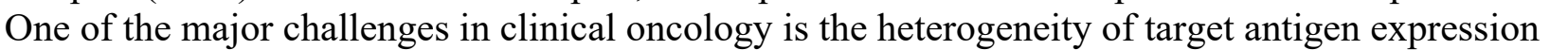

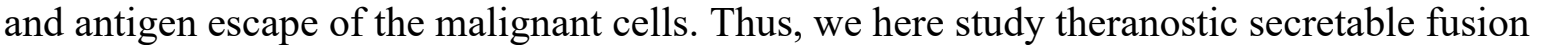

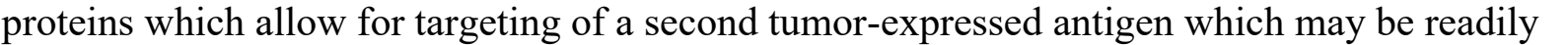

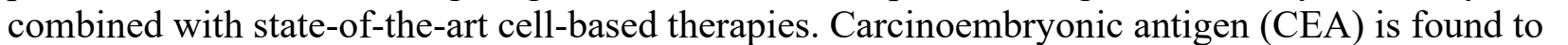

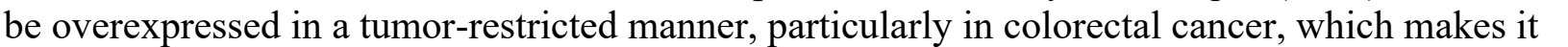

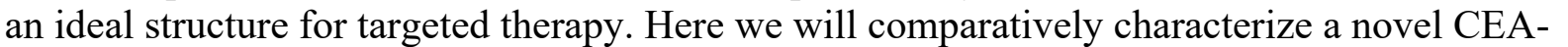
ए

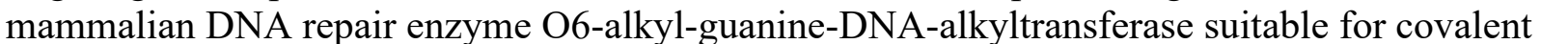

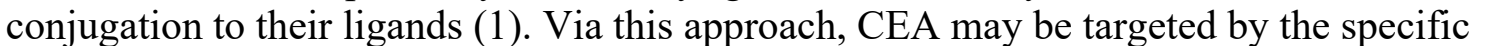

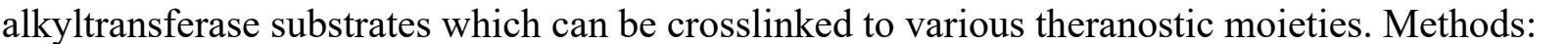

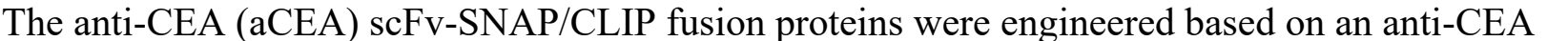

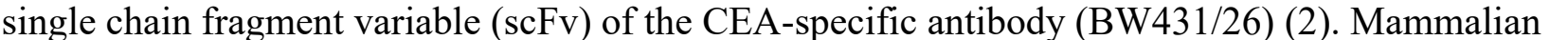

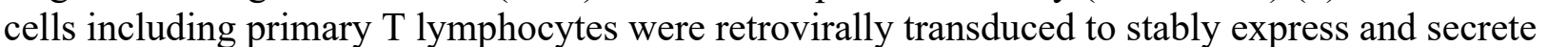

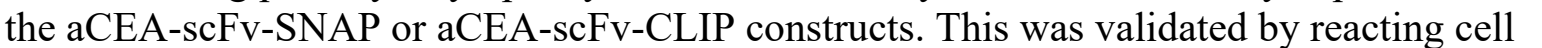
ए

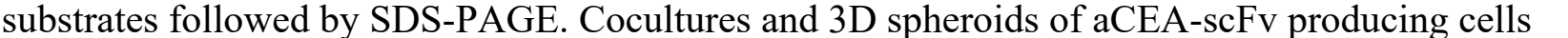

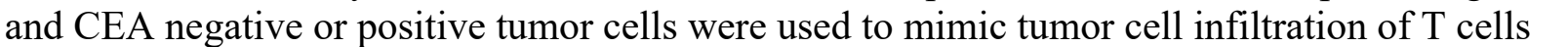

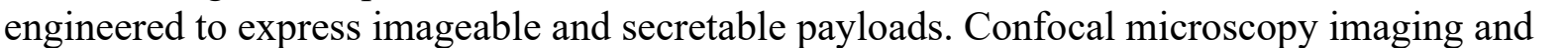

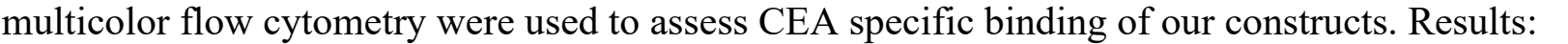

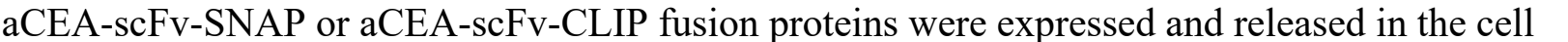

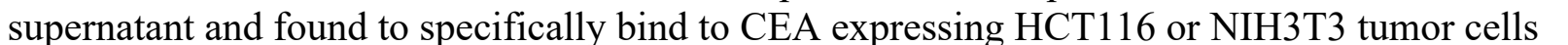

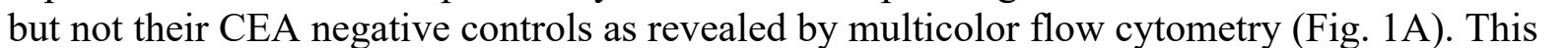

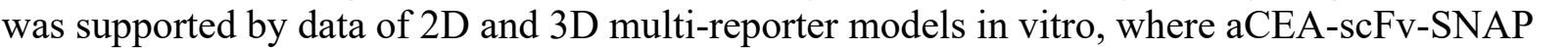

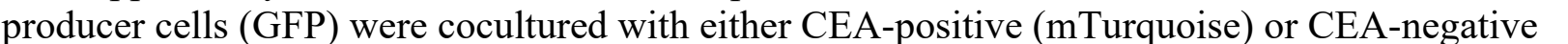
$\mathrm{m}$

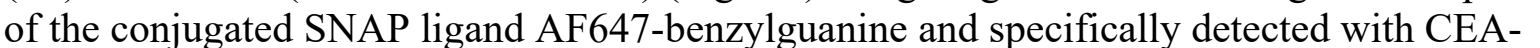

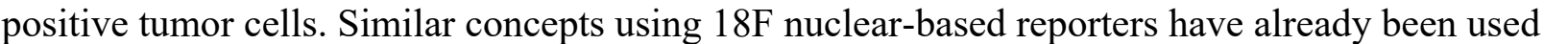

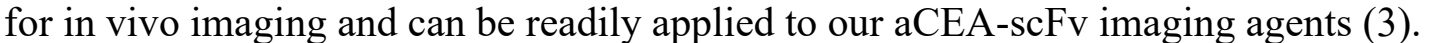

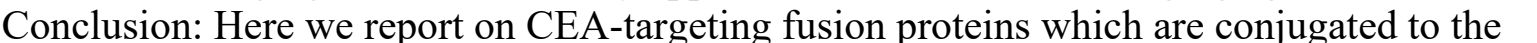

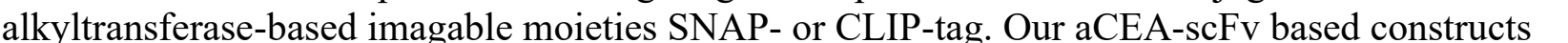

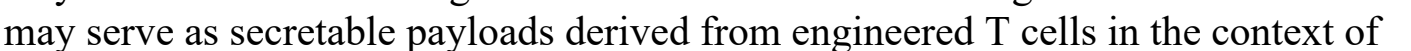

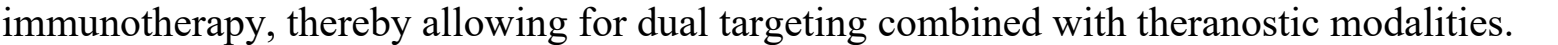




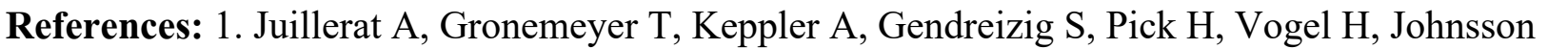

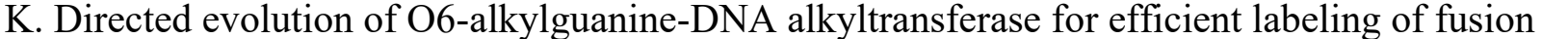

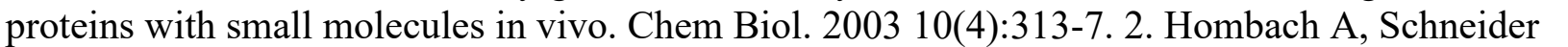

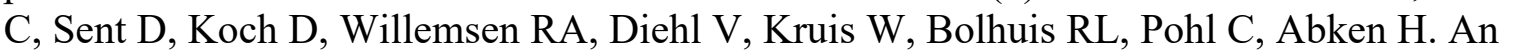

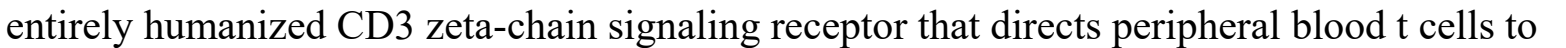

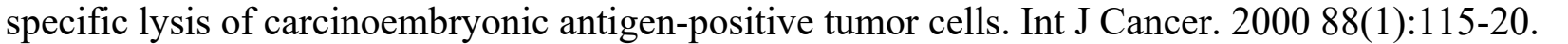

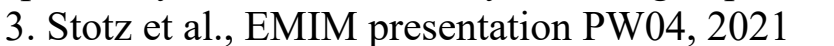

\section{Image/Figure:}

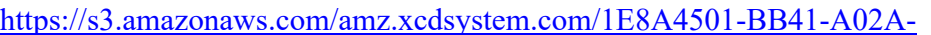

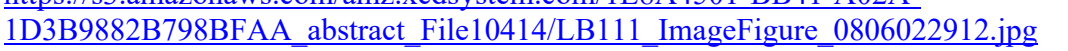

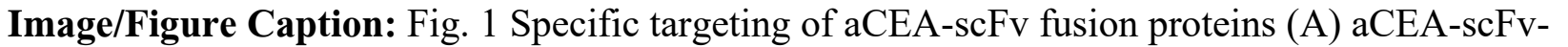

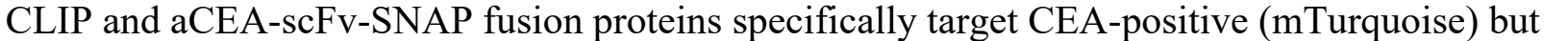

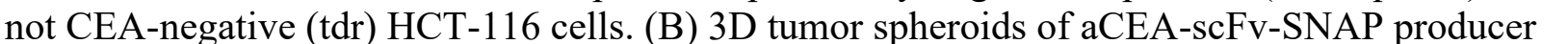

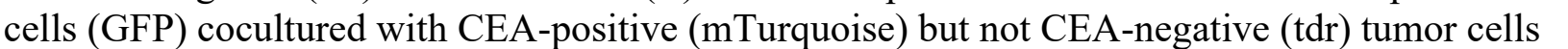

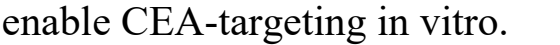

First Name: $\square \square\|\| \cdots \mid \square$

Last Name: $\square \square|\|\mid\| \square$

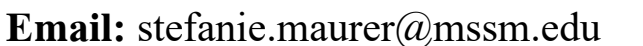

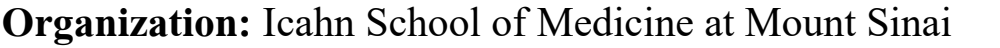

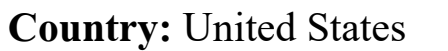




\title{
ID: LB112
}

Validation Against Magnetic Resonance of A Combination UltrasoundBioluminescence Imaging System In A Murine Orthotopic Pancreatic Tumor Model

Juan Rojas, SonoVol, Inc., jrojas@sonovol.com

Category: $\square \square \square \square \square \square \square$

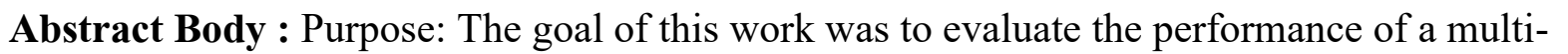

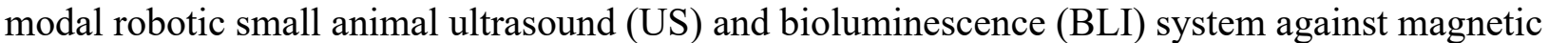

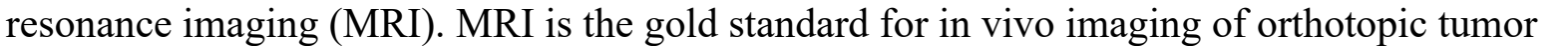

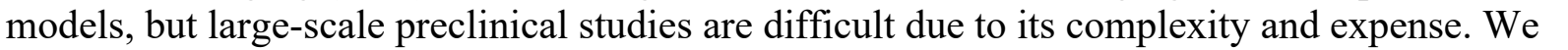

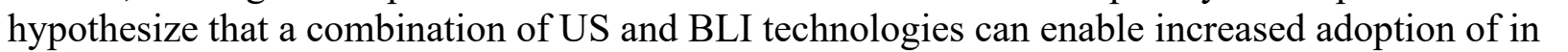

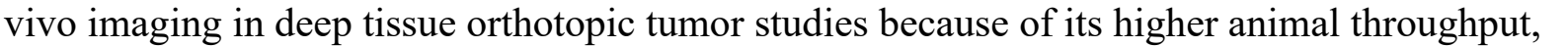

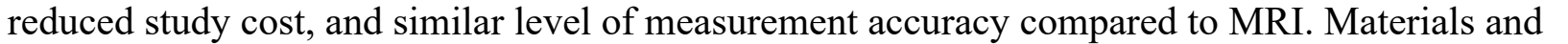

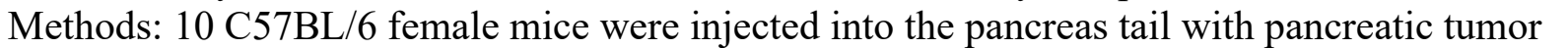

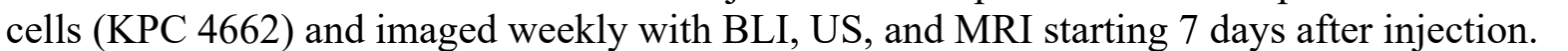

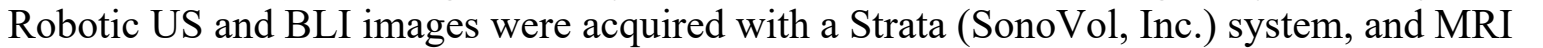
प

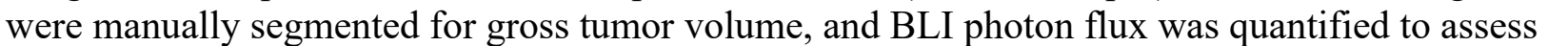
ए

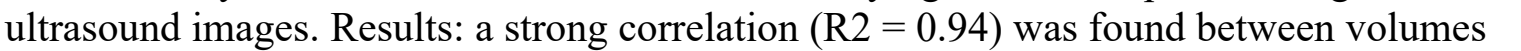

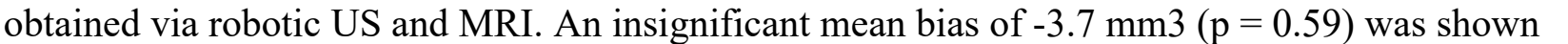

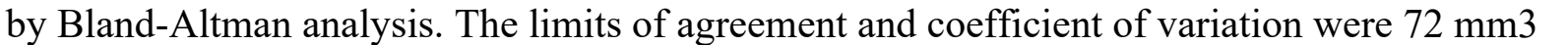

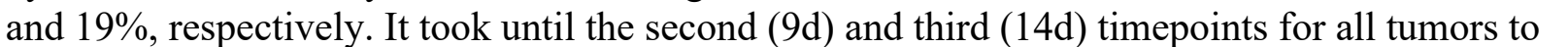

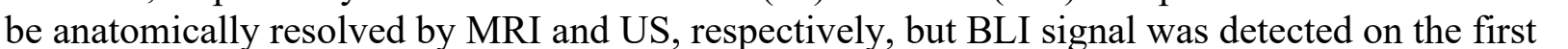

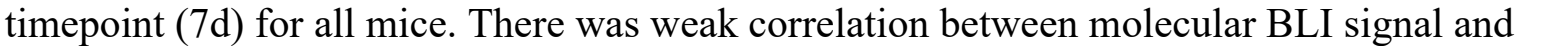

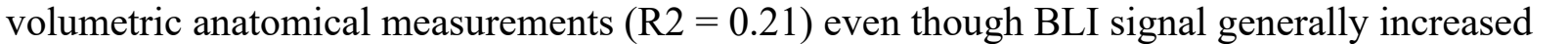

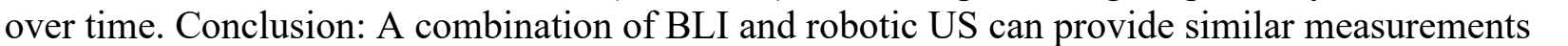

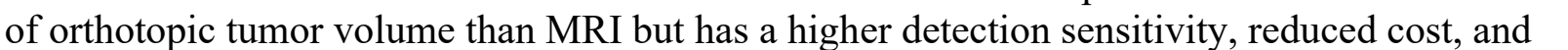

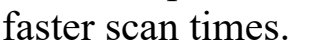

\section{Image/Figure:}

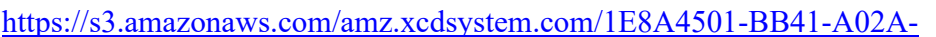

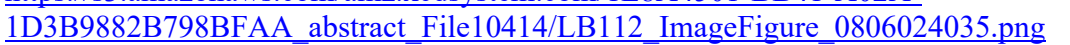

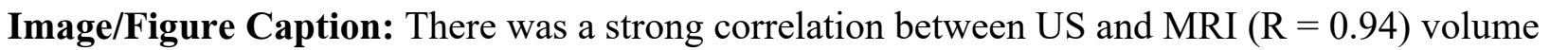

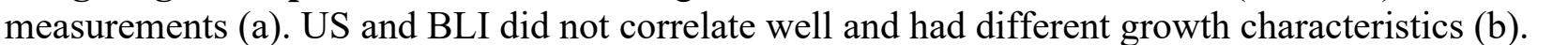

First Name: $\square \square\|\|$

Last Name: $\square \square m|m| \square$ 


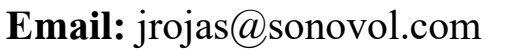

Organization: $\square \square \square \square \square \square m \square \| m$

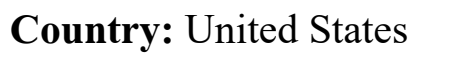




\title{
ID: LB113 \\ New Methods for Improving Multiparametric MR-TRUS Guided Fusion \\ Prostate Biopsies With Hyperpolarized C-13 Pyruvate Molecular Imaging \\ Daniel Gebrezgiabhier, University of California, San Francisco, \\ Daniel.gebrezgiabhier@ucsf.edu
}

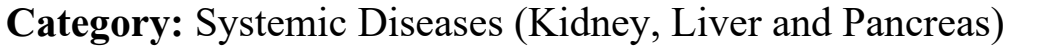

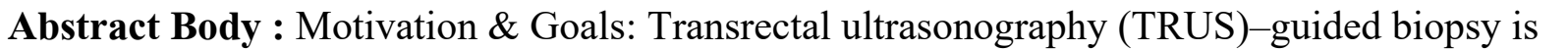

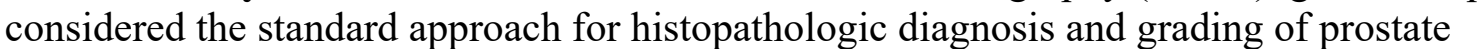

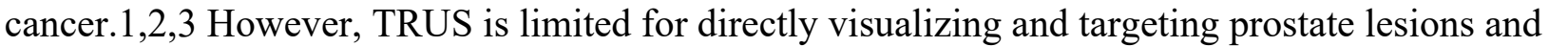

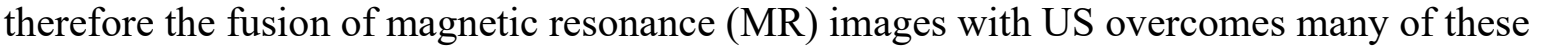
ए

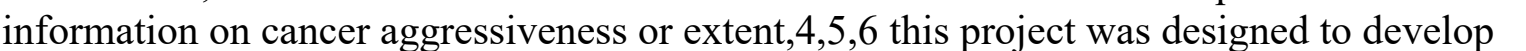

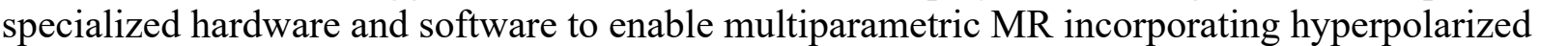
प

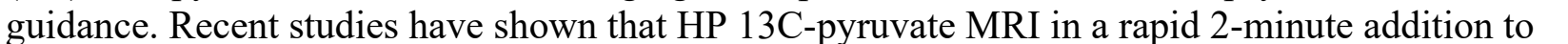

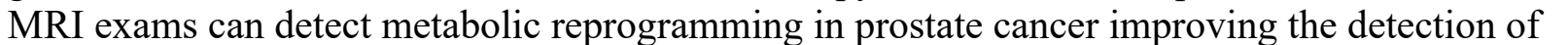

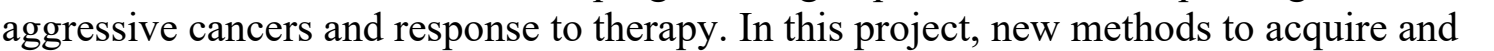

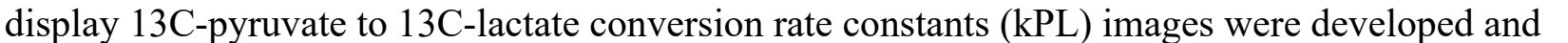

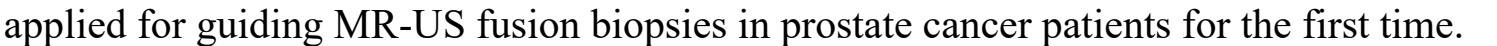

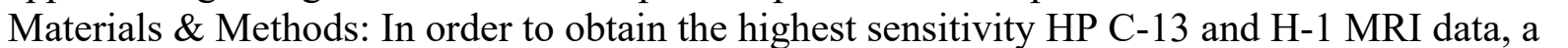

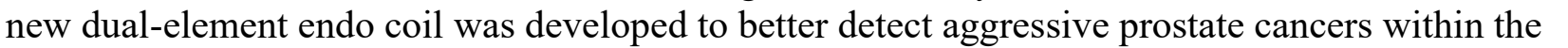

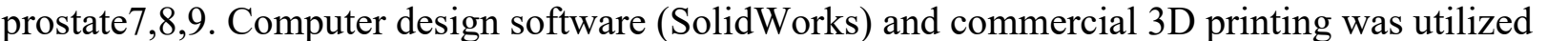

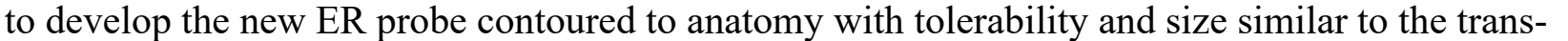

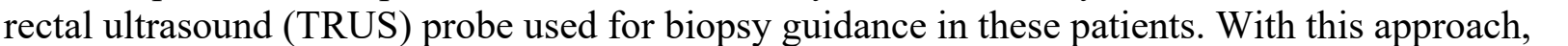

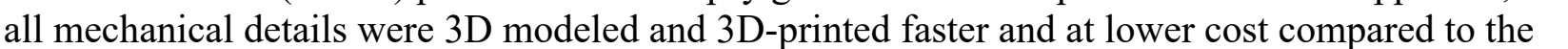

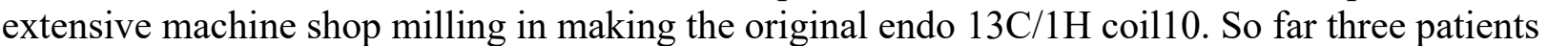

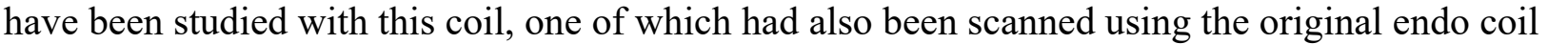

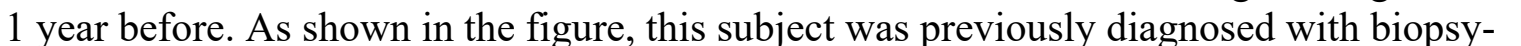

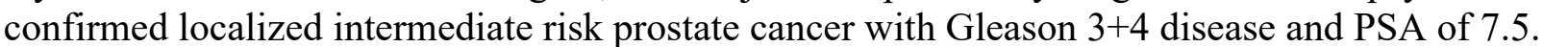

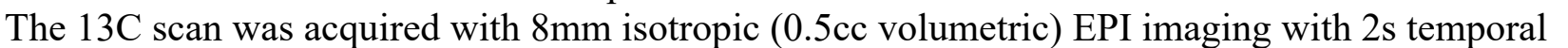

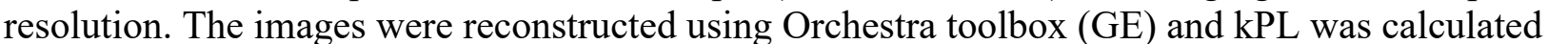

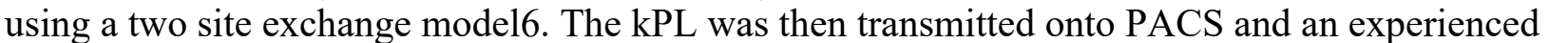
ए ए

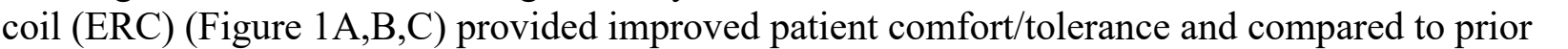

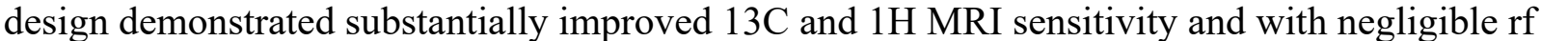

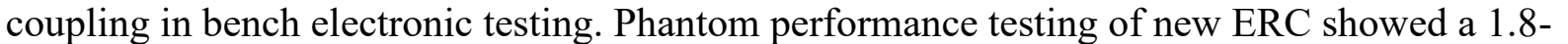
ए ए

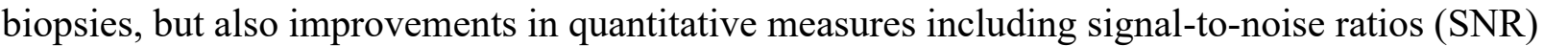

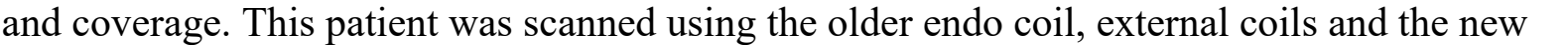

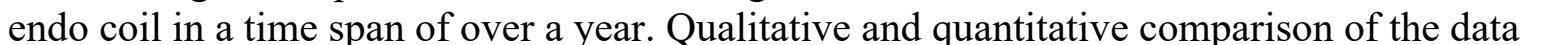




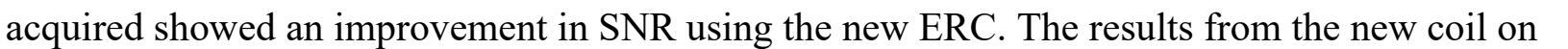
ए

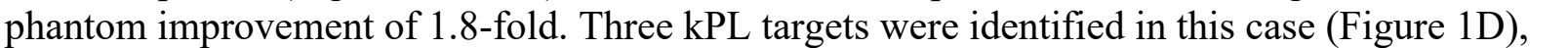

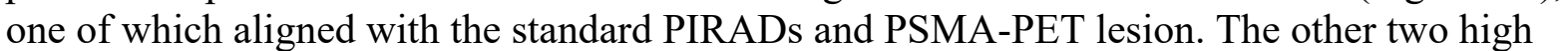

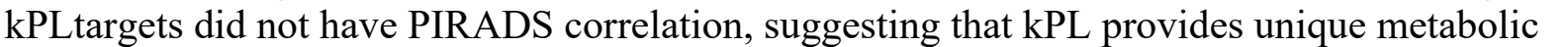

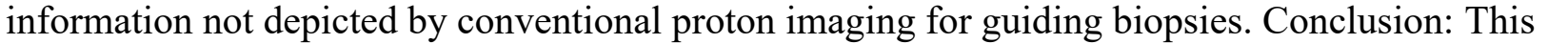

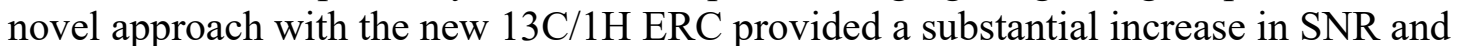

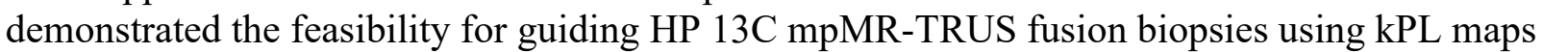

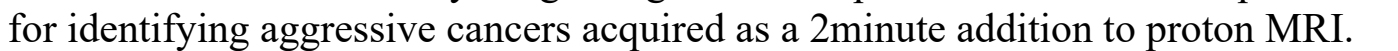

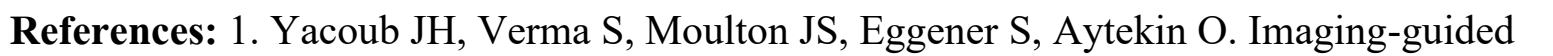

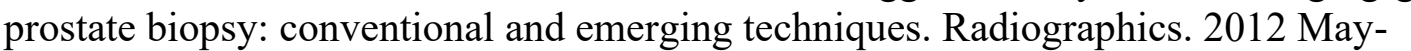

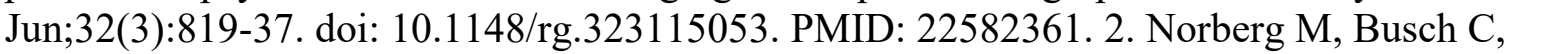

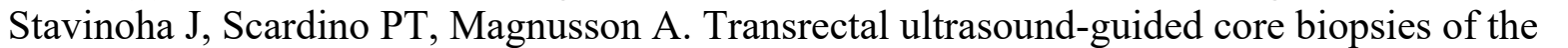

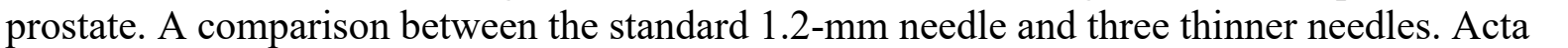

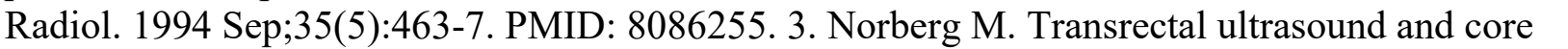

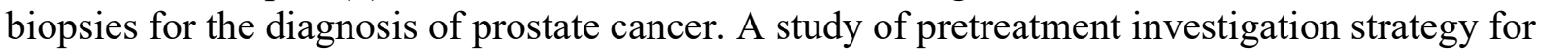

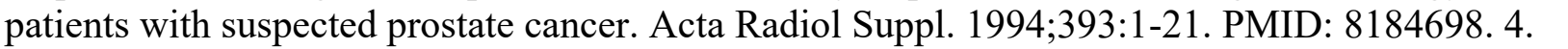

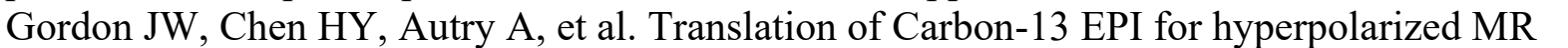

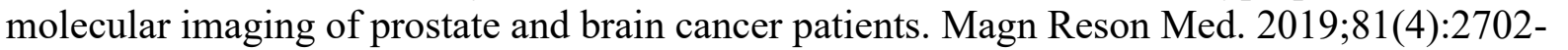

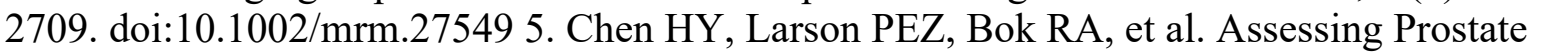

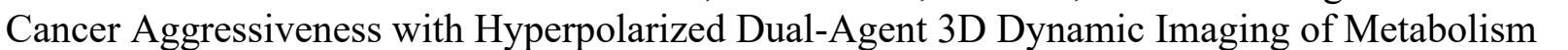

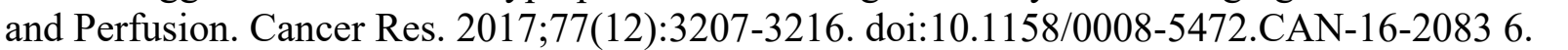

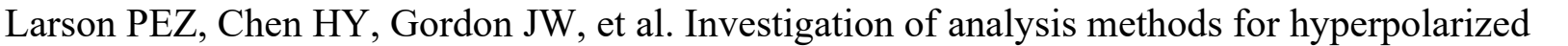

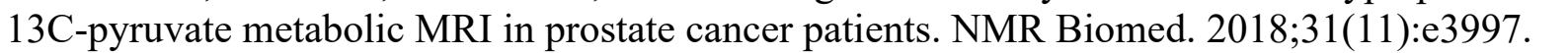

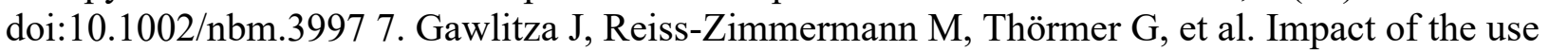

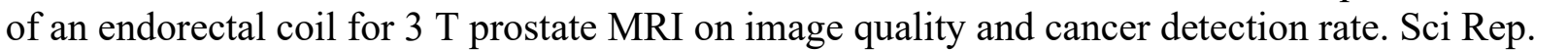

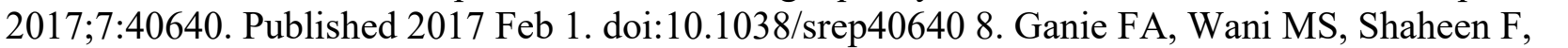

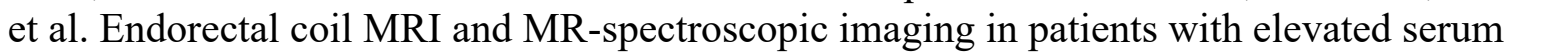

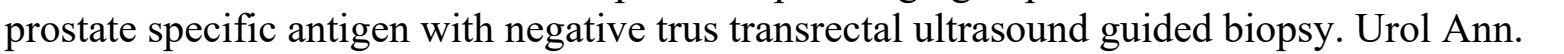

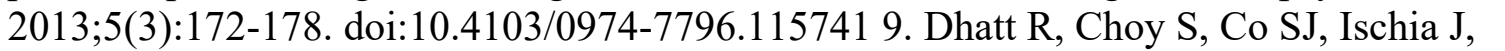

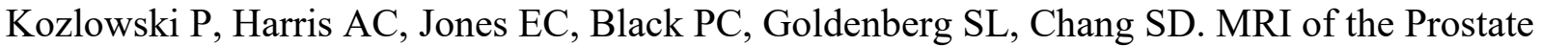

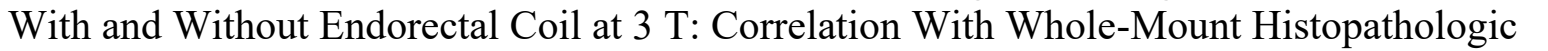

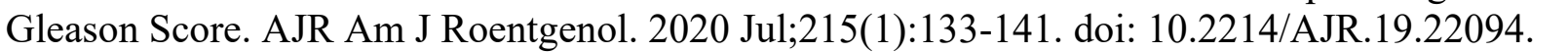

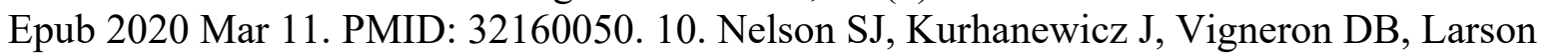

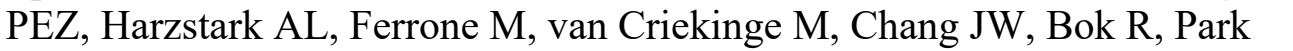

\section{Image/Figure: $\square$}

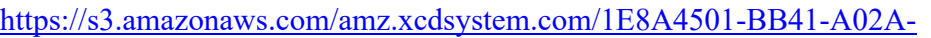

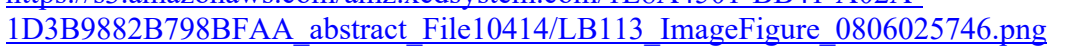

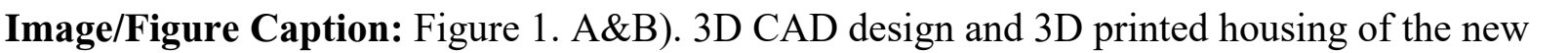

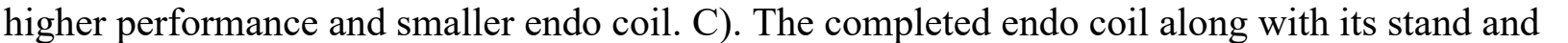

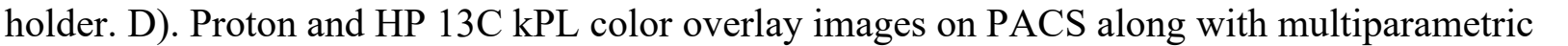

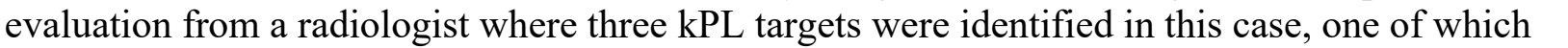

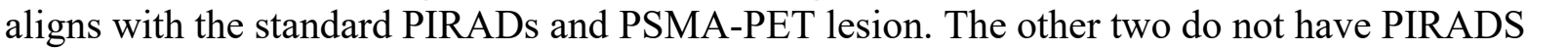




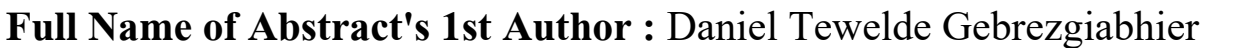

First Name: $\square \square \square m \square$

Last Name: $\square \square \square\|\|\|\|\|\|\|\| \|$

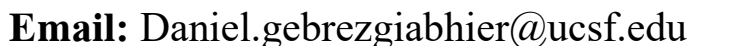

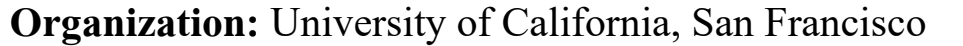

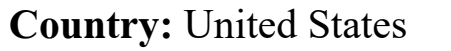




\title{
ID: LB114 \\ PARPi-FL in polyethylene glycol 3350 (PEG3350) as a practical vehicle for clinical use
}

Tara Viray, Memorial Sloan-Kettering Cancer Center, virayt@mskcc.org

\section{Category: $\square \square\|\| \square \square$}

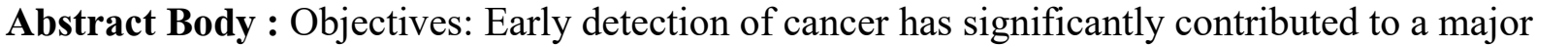

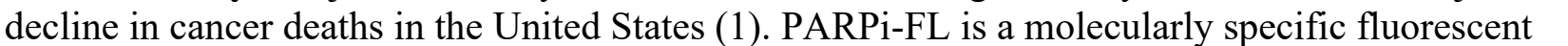

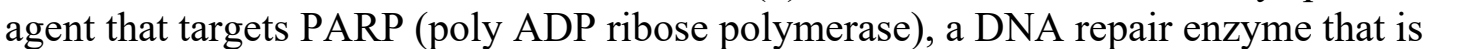

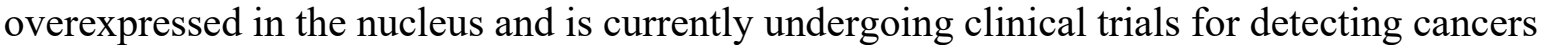
ए

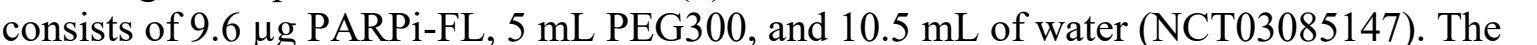
mouthwash is swished in the patient's mouth for 1 minute and spit out, followed by rinsing with

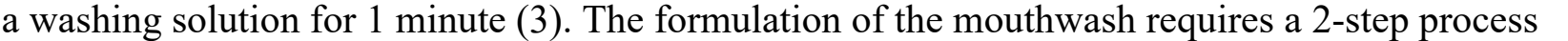

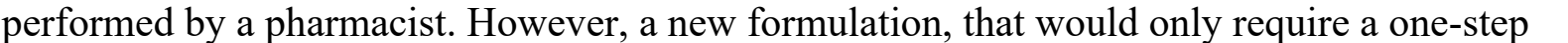

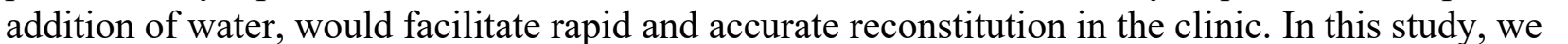

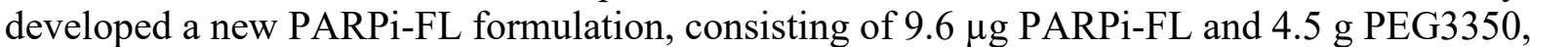

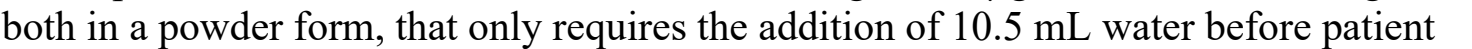

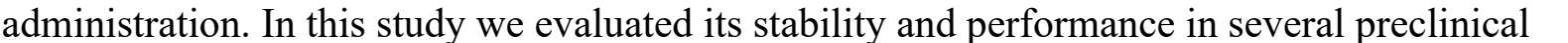

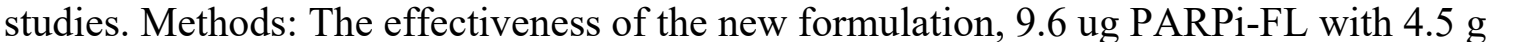

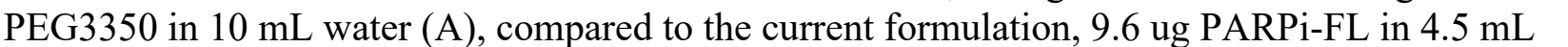

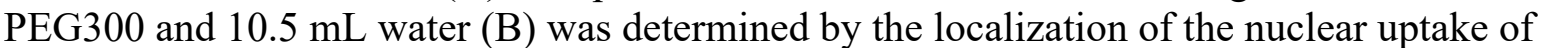

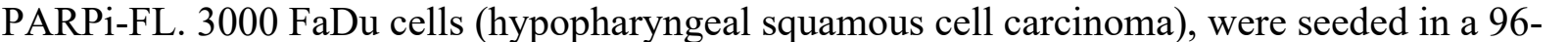

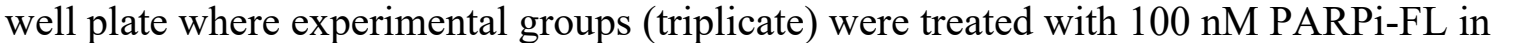

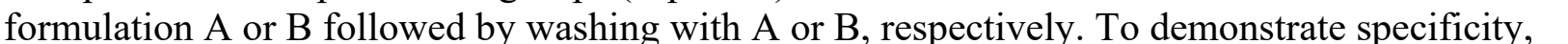

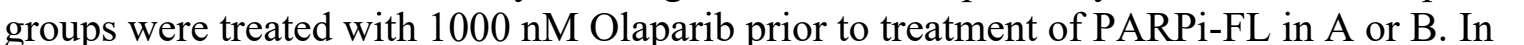

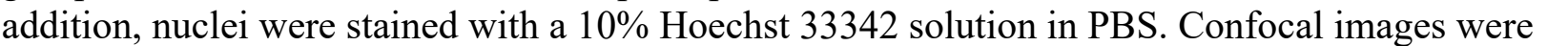

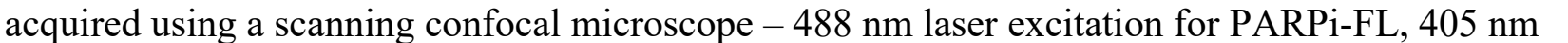

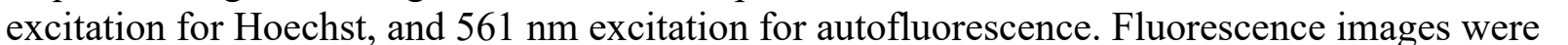

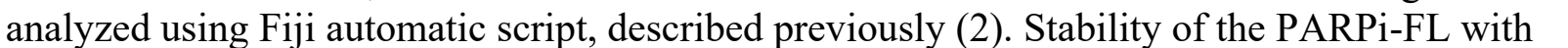

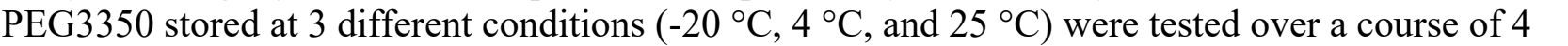

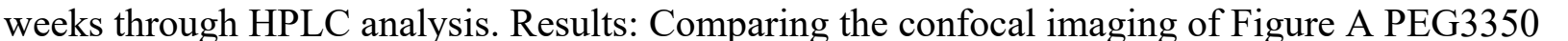

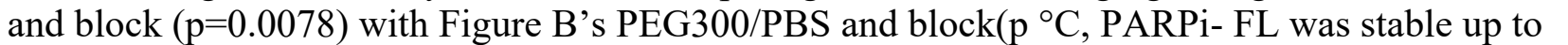

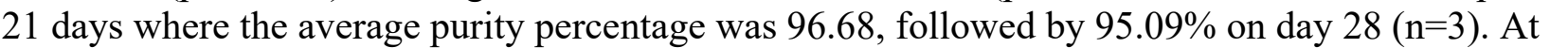

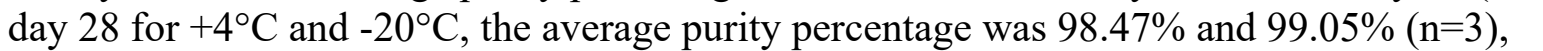

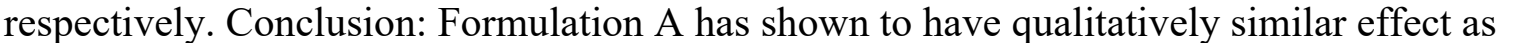

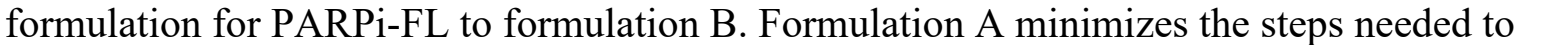

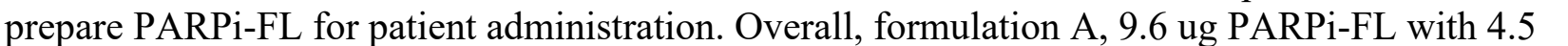

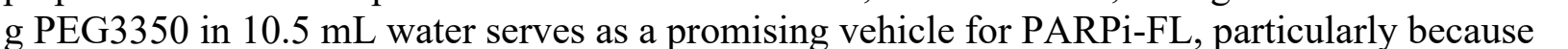
ए
\end{abstract}




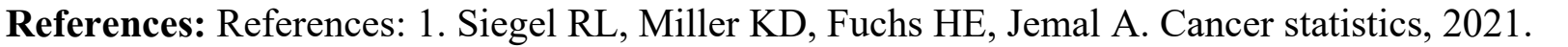

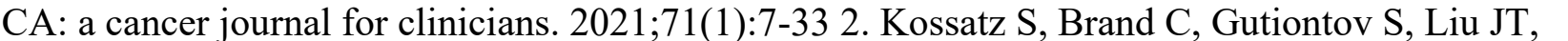

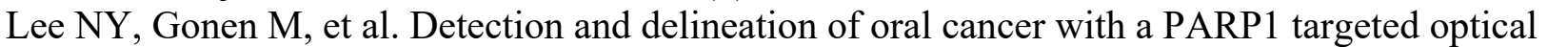

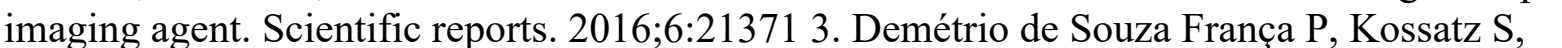

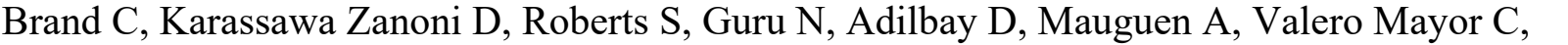

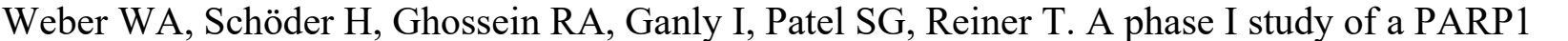

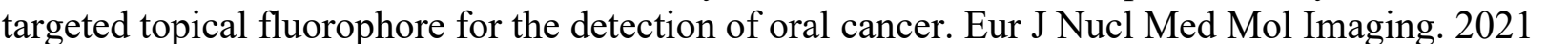

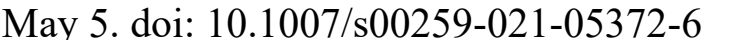

\section{Image/Figure:}

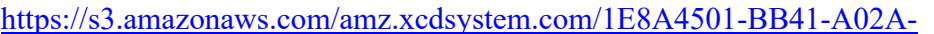

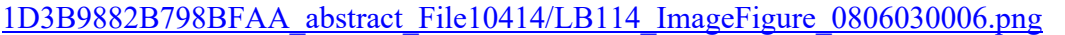

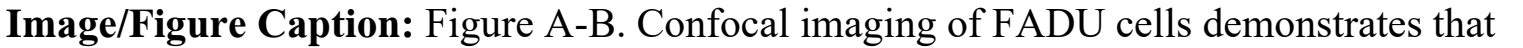

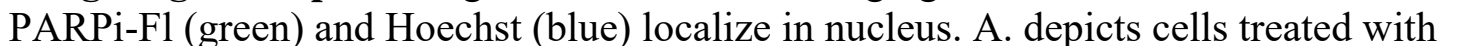

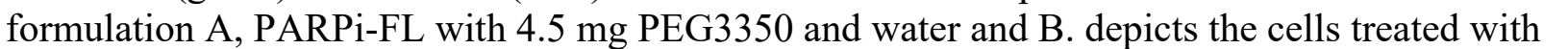

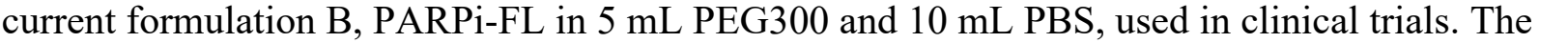

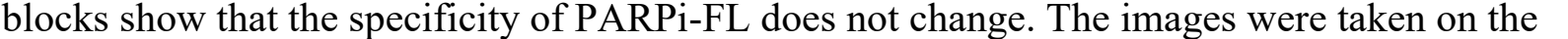

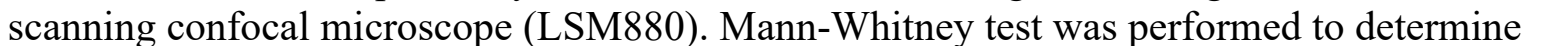

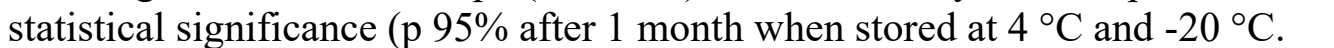

First Name: $\square \square\|\| \|$

Last Name: $\square \square\|\| \square$

Email: $\square\|\|\|\square \square \square \square\|\|\| \| \square$

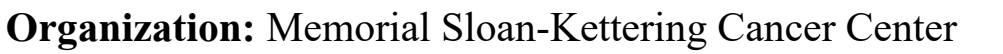

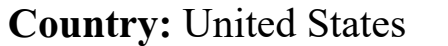




\title{
ID: LB115 \\ Evaluation of precision and sensitivity in photoacoustic mesoscopy
}

\author{
Lina Hacker, University of Cambridge, Ih592@cam.ac.uk
}

\author{
Category: $\square \square\|m\| m$
}

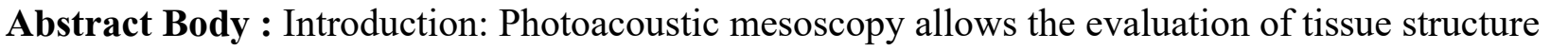

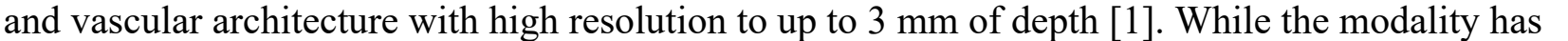

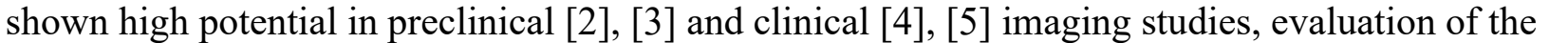

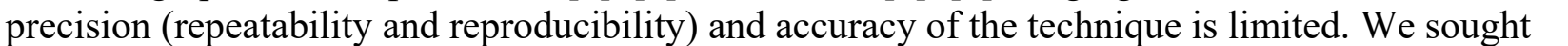

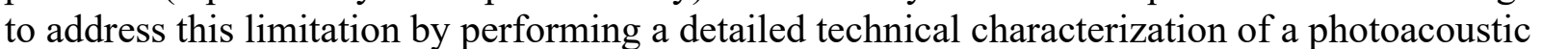

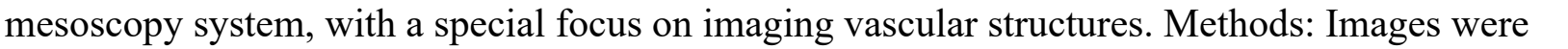

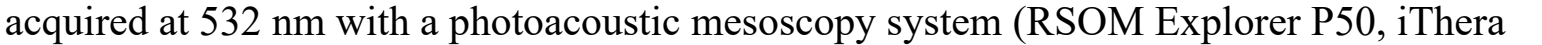

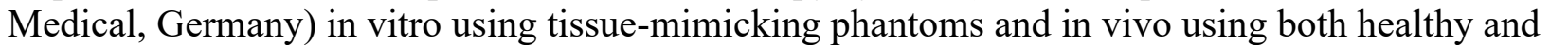

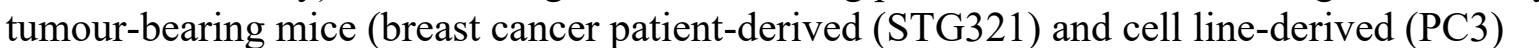

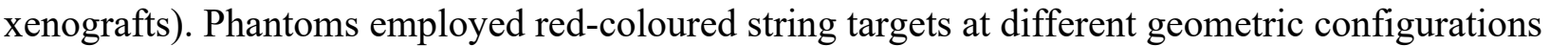

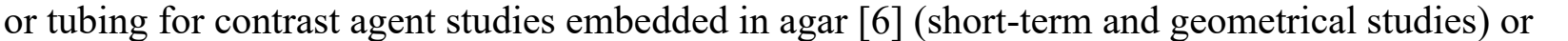

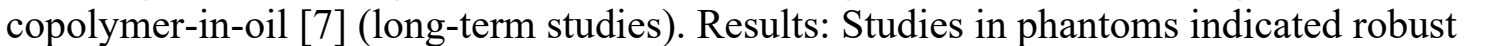

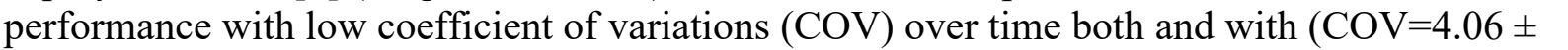

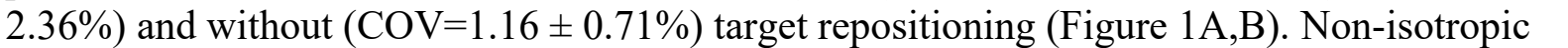

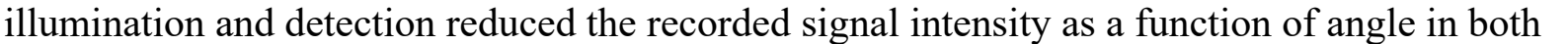

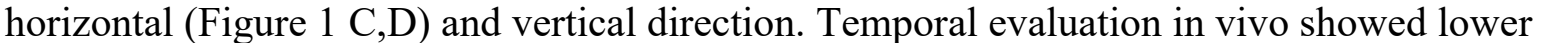

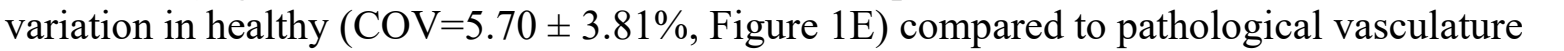

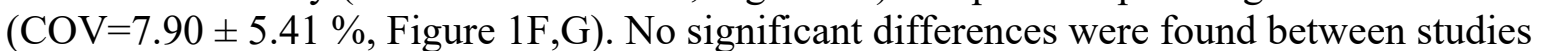

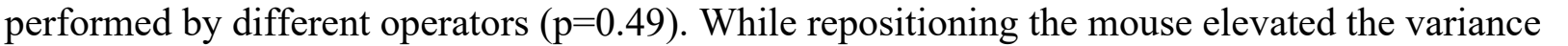

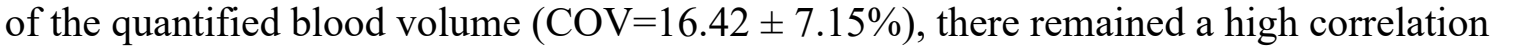

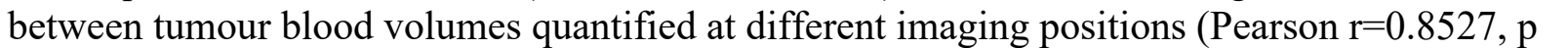

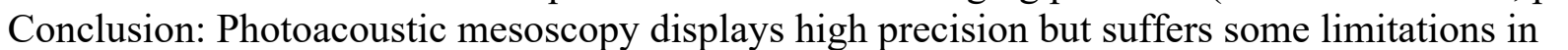

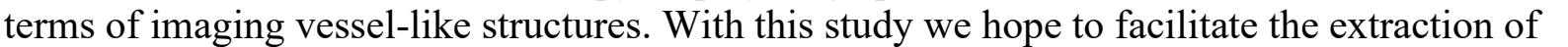

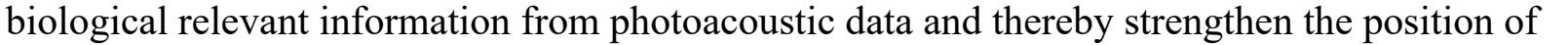

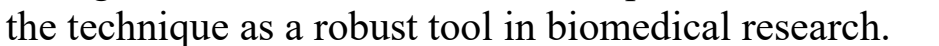

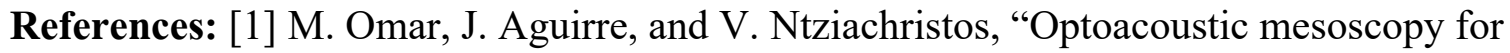

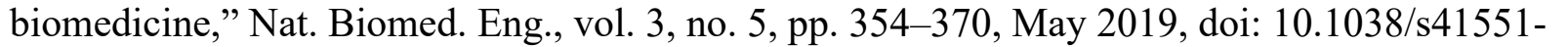
पाणमा14. [2] F. Knieling et al., "Raster

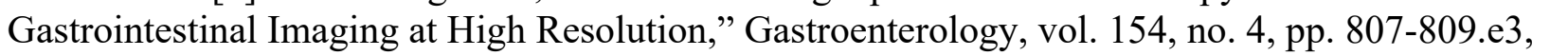

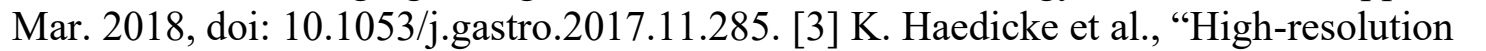

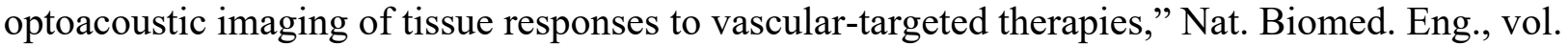

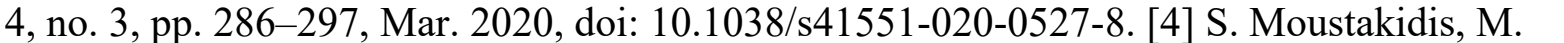
Omar, J. Aguirre, P. Mohajerani, and V. Ntziachristos, "Fully automated identification of skin

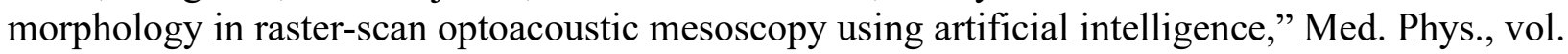

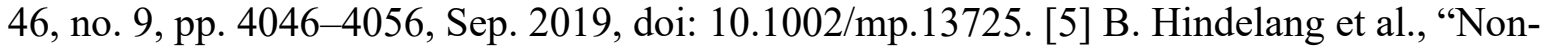
invasive imaging in dermatology and the unique potential of raster-scan optoacoustic 


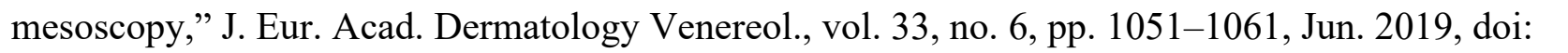

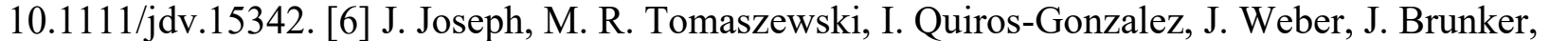
and S. E. Bohndiek, "Evaluation of Precision in Optoacoustic Tomography for Preclinical

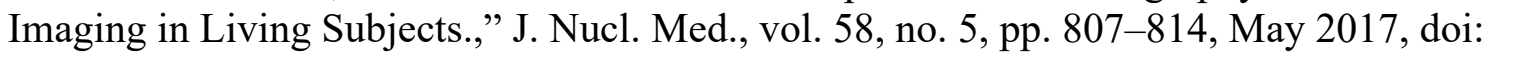
10.2967/jnumed.116.182311. [7] L. Hacker et al., “A copolymer material with tuneable acoustic and optical characteristics for photoacoustic imaging phantoms,"

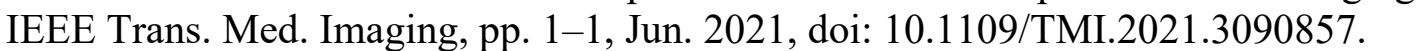

\section{Image/Figure:}

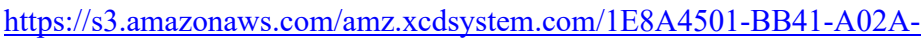

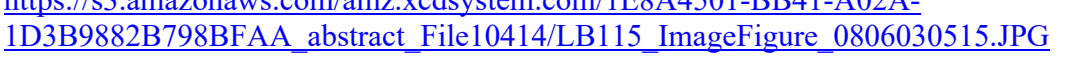

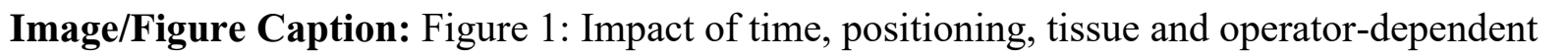

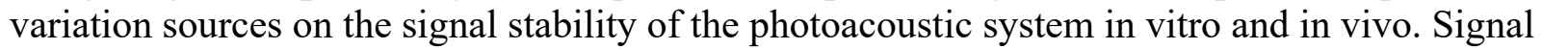

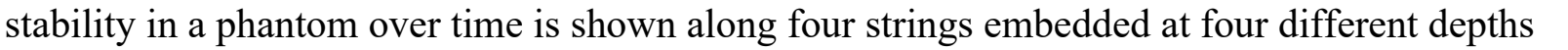

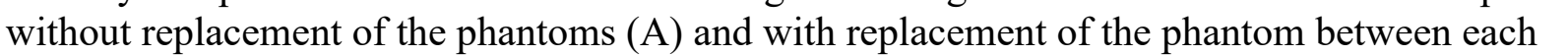
प

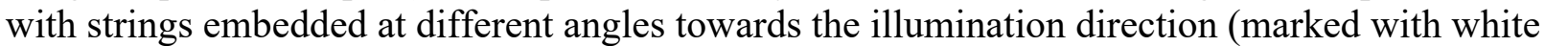

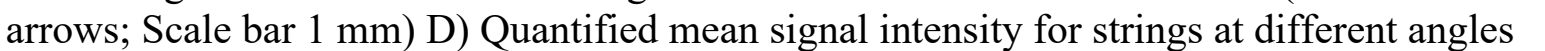
ए

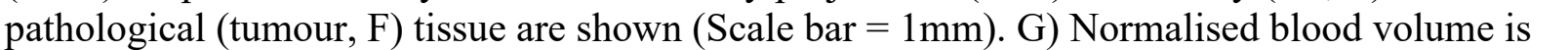

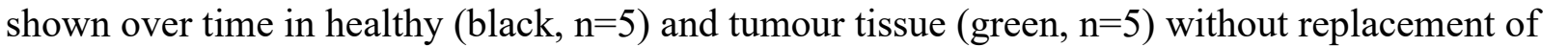

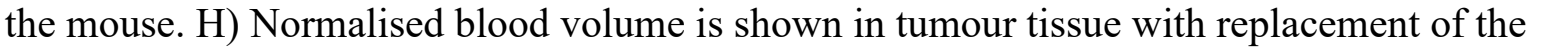
mouse by two different operators (each $n=5$, operator $1=$ black, operator $2=$ green). I) The "belly" (left) and "leg" (right) positioning of the mouse in the system a

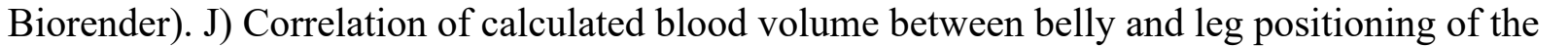

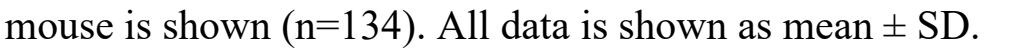

First Name: $\square \square \| m$

Last Name: $\square \square\|\| \| \square$

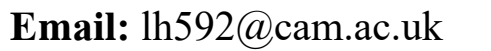

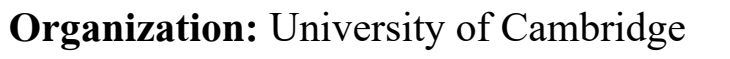

Country: $\square \square \| m \square \square \square \square$ 


\title{
ID: LB116 \\ Assessing the impact of structural differences in CAR T Cell reporter gene design on performance
}

Keifer Kurtz, Memorial Sloan Kettering, kurtzk@mskcc.org

\section{Category: $\square \square\|\| \square \square$}

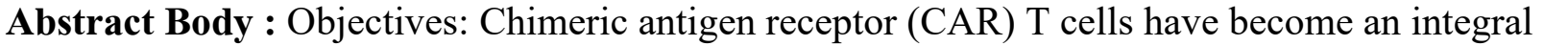

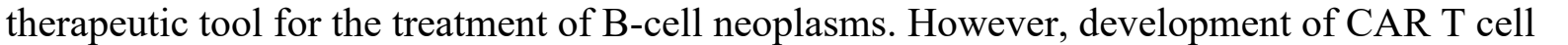

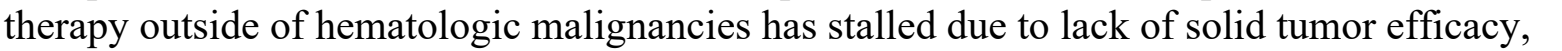

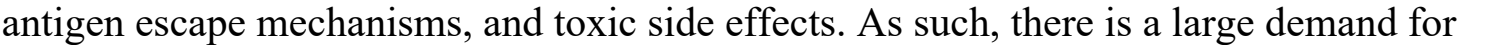

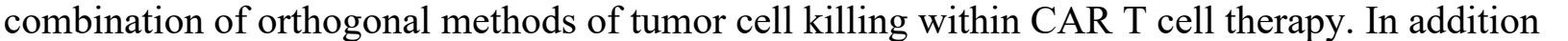

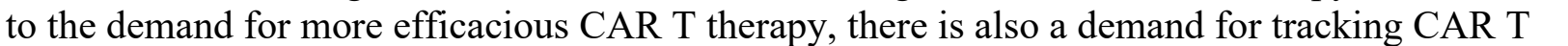

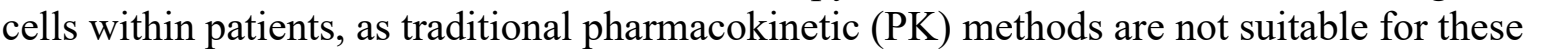

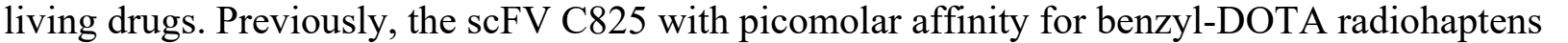

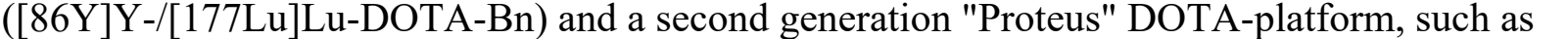

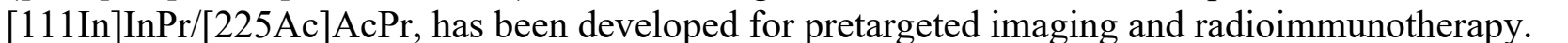

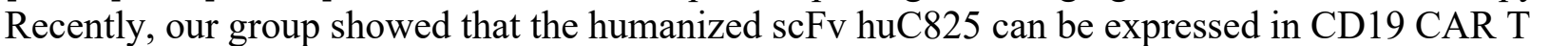

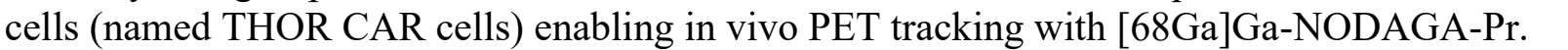

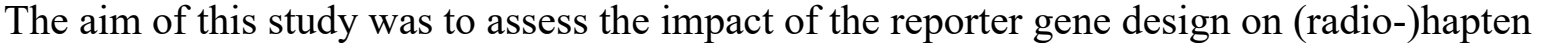

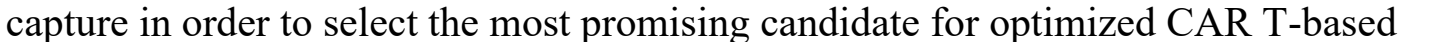

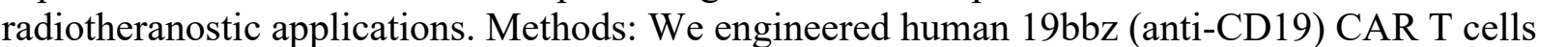

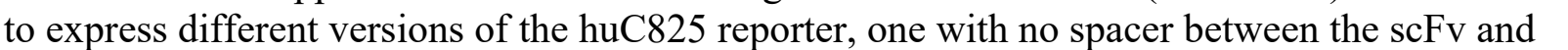
ए

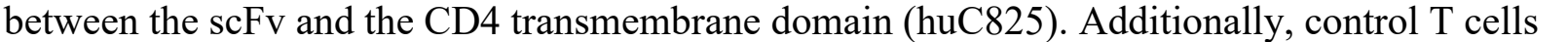

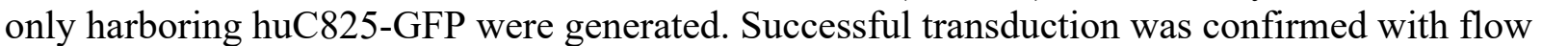

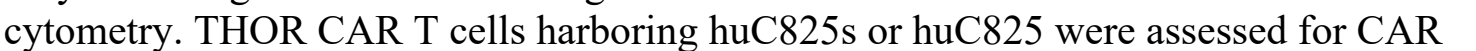

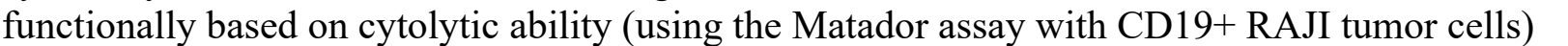

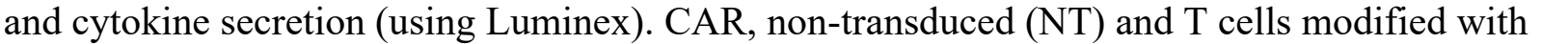

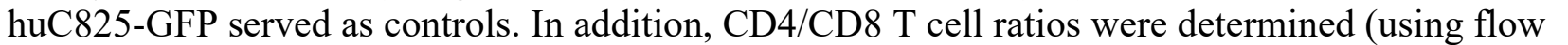

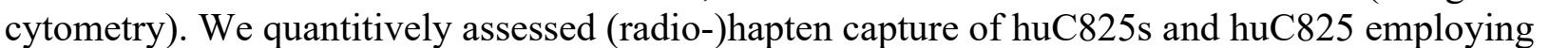

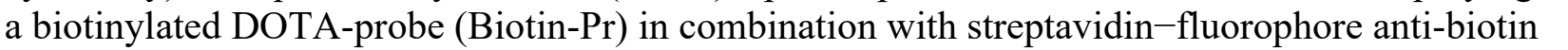

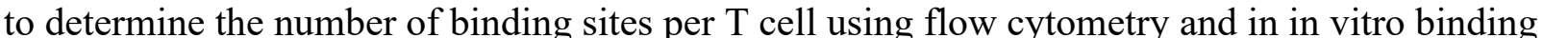

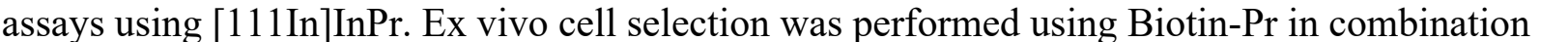

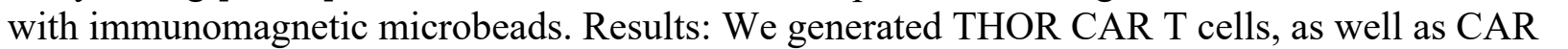

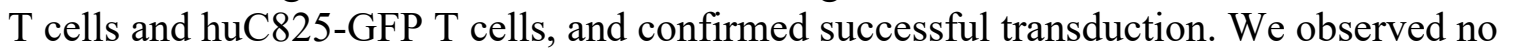

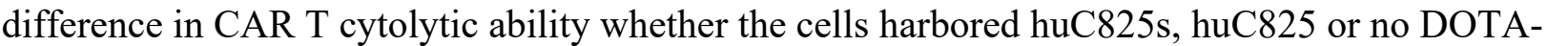

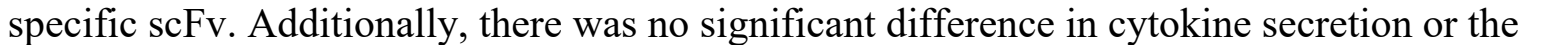

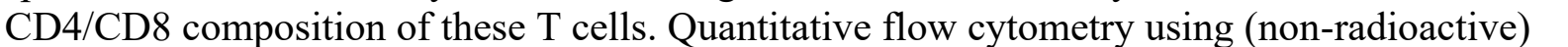

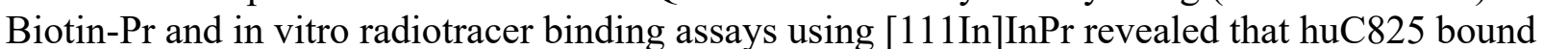

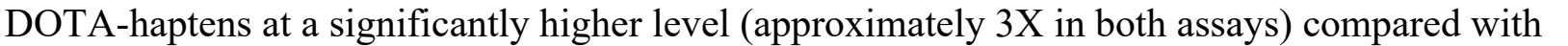

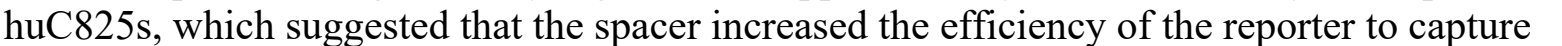

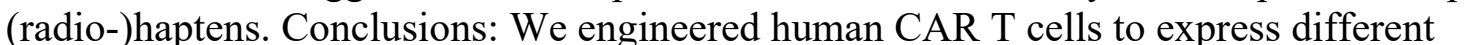




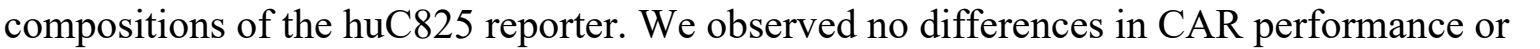

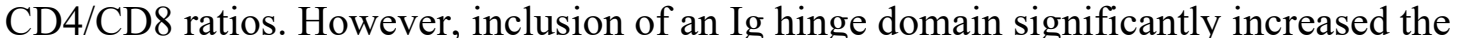

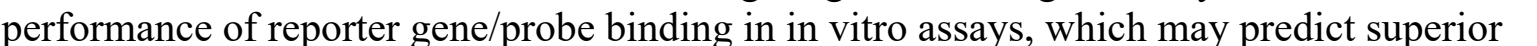

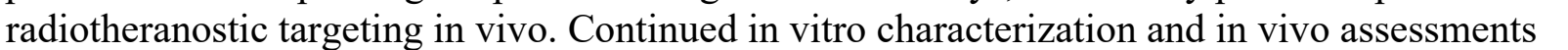

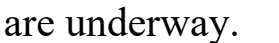

First Name: $\square \square \square \square \square \square$

Last Name: $\square \square \| m \mid \square$

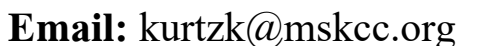

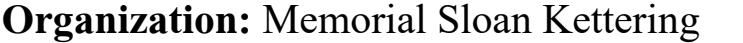

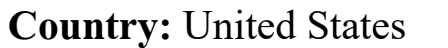




\title{
ID: LB117 \\ SESORRS: Balancing Sampling Frequency and Measurement Speed for High Contrast Pre-Clinical Cancer Raman Imaging at Depth
}

Fay Nicolson, Dana-Farber Cancer Institute, fay_nicolson@dfci.harvard.edu

\section{Category: $\square \square\|\| \| \square$}

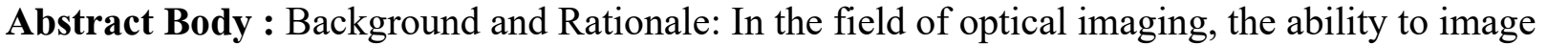

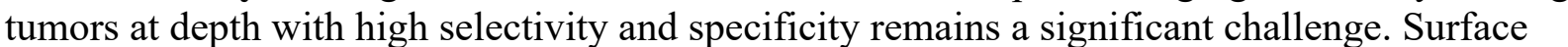

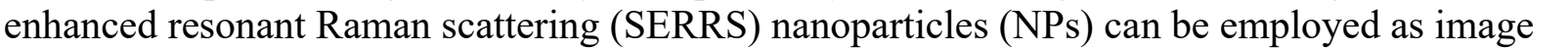

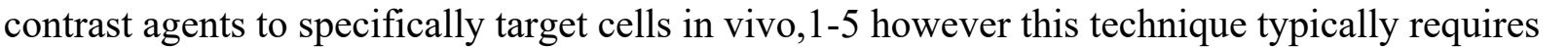

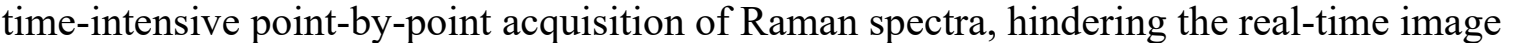

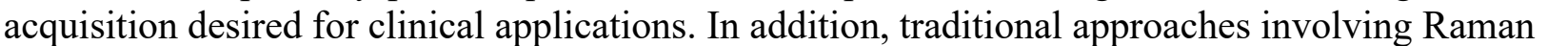

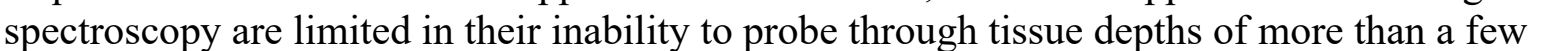

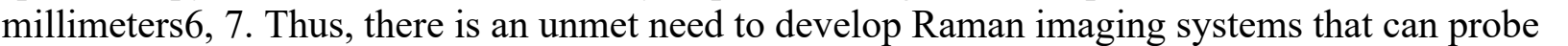

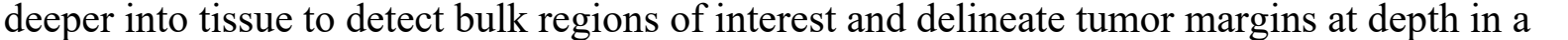

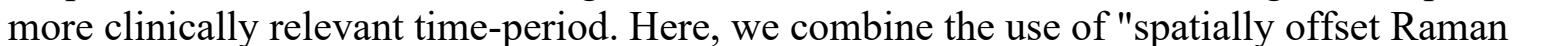

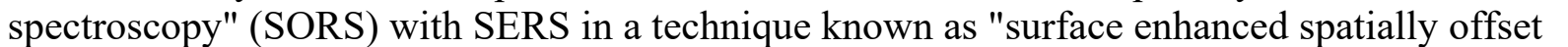

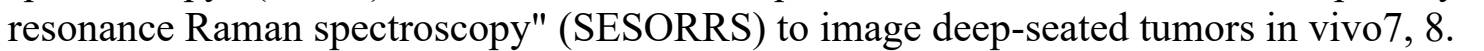

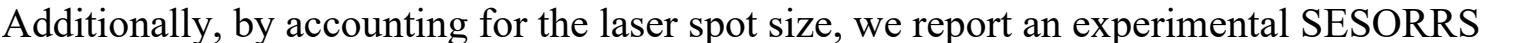

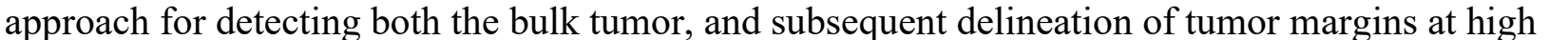

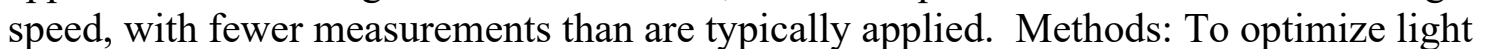

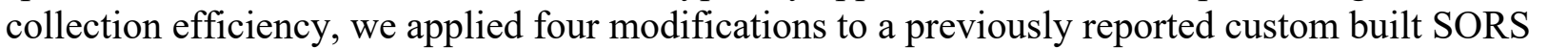

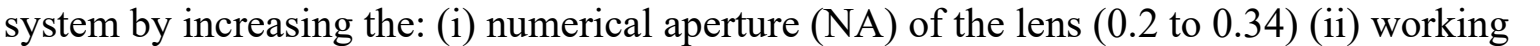

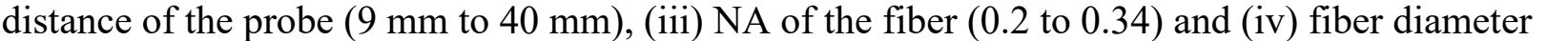

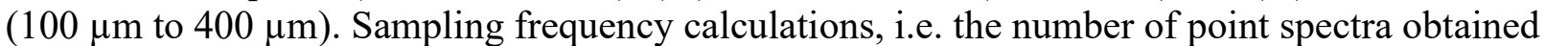

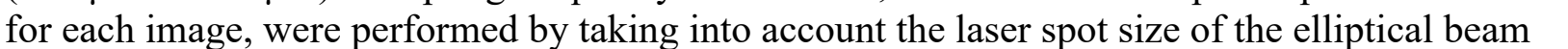

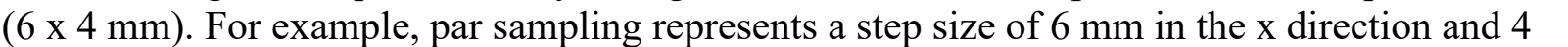

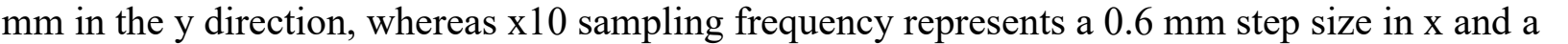

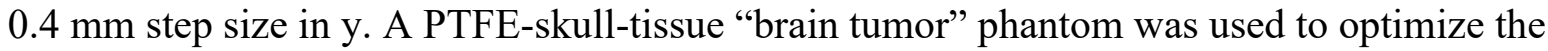

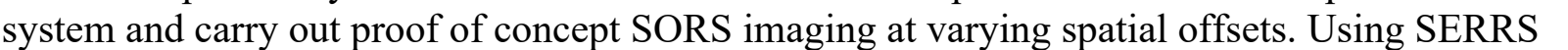

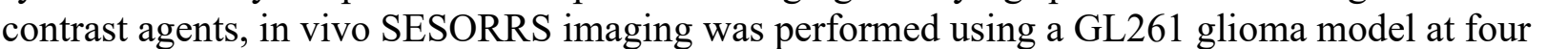

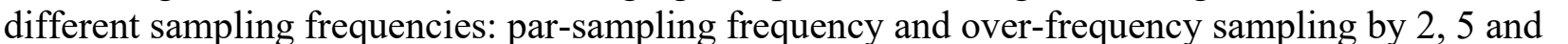

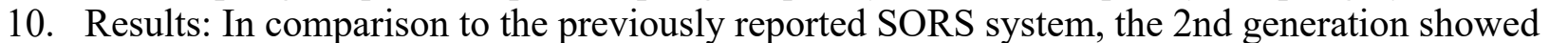

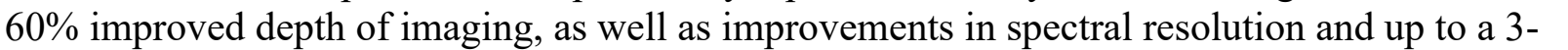

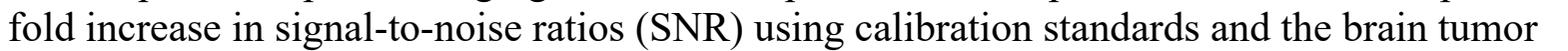

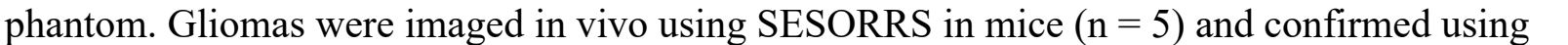

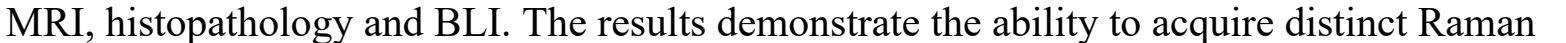

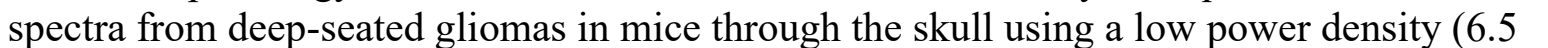

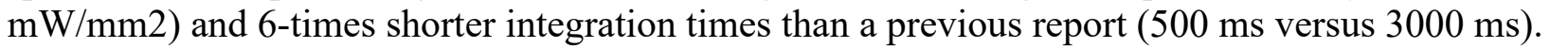

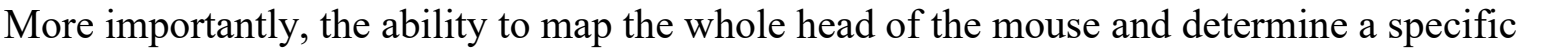

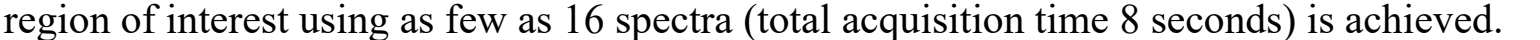

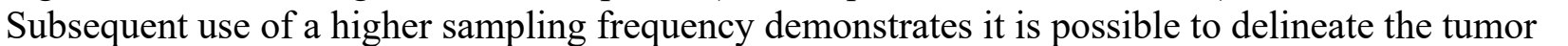




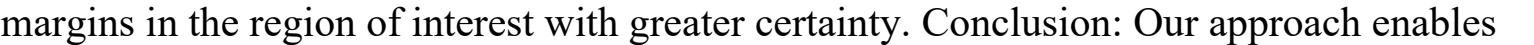

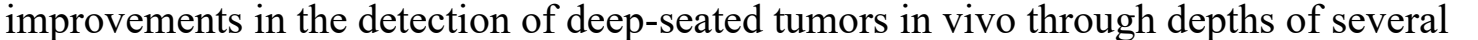

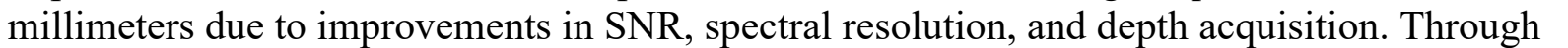

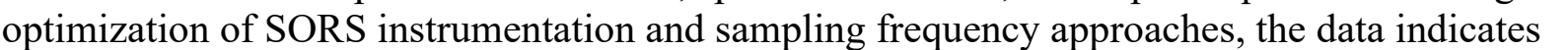

ए

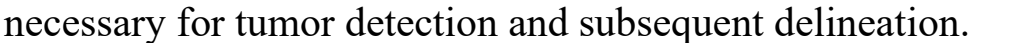

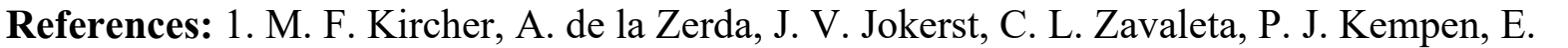

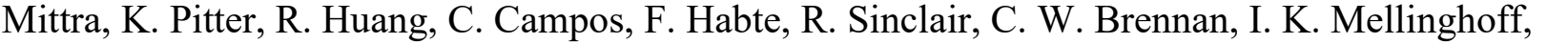

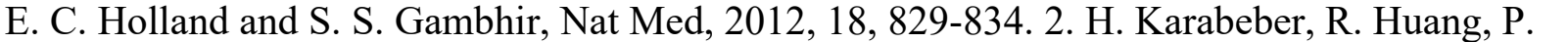

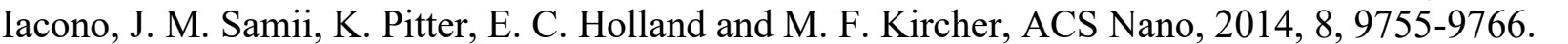

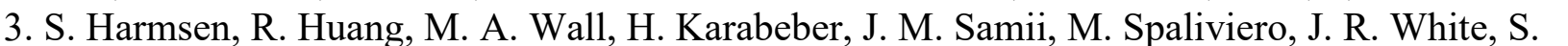

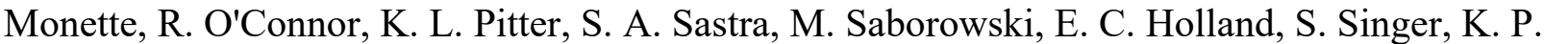

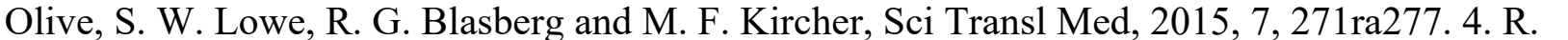

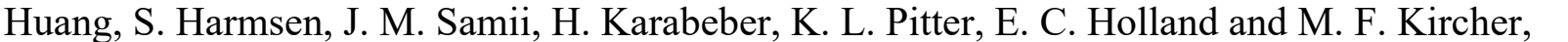

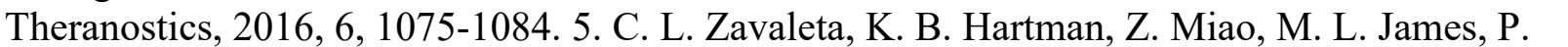

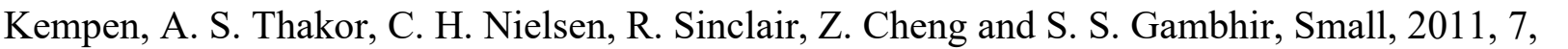

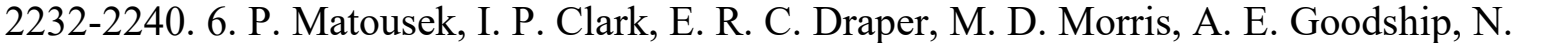

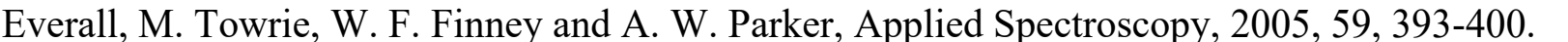

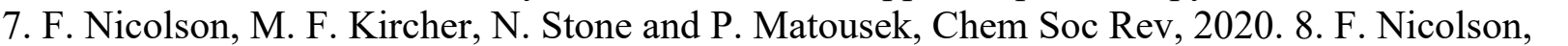

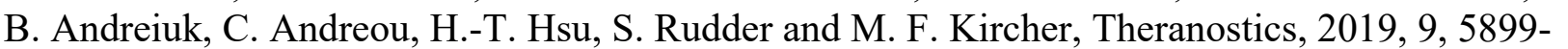

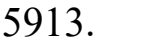

First Name:

Last Name: $\square \square\|\|\|\| \| \square$

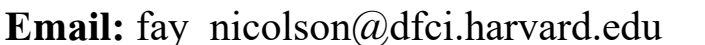

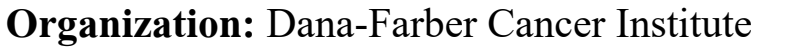

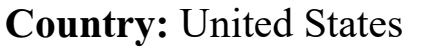




\title{
ID: LB118 \\ Optimizations of PARPi-FL Imaging with the Clinical Quest System
}

Alexa Michel, Memorial Sloan Kettering Cancer Center, michela1@mskcc.org

Category: $\square \square\|\| \square \square$

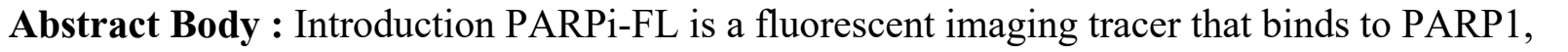

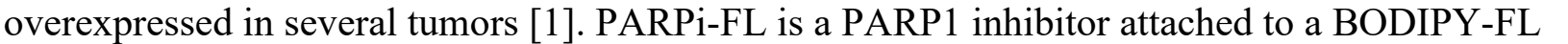

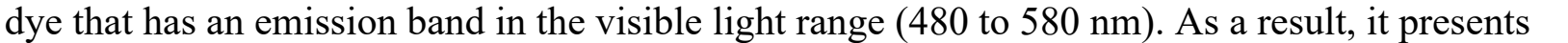

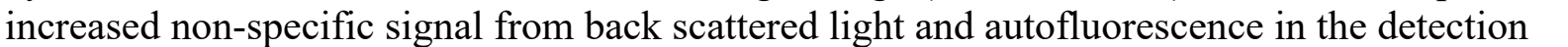

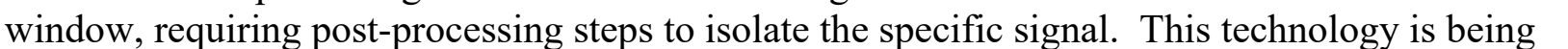

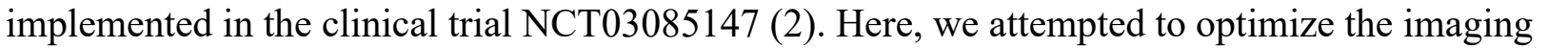

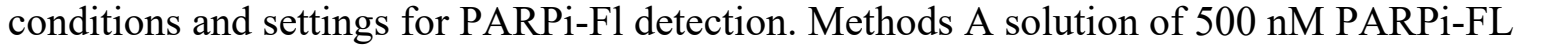

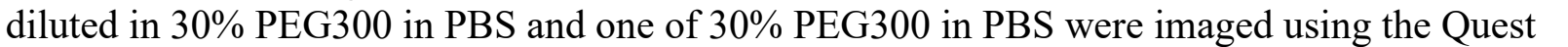

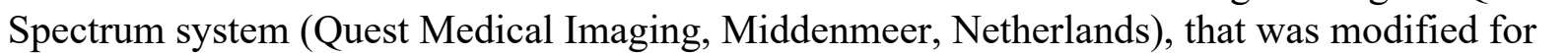

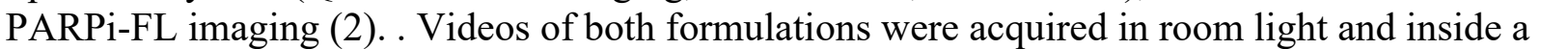

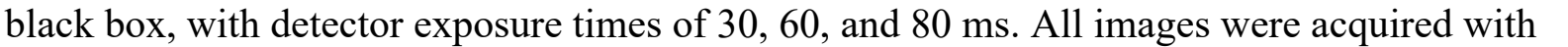

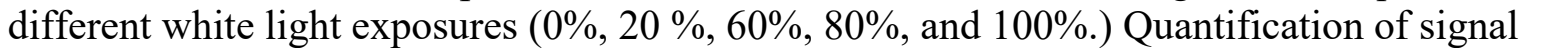
ए

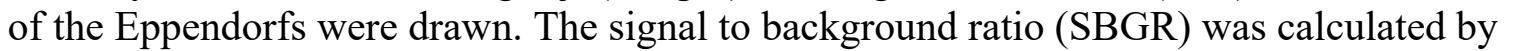

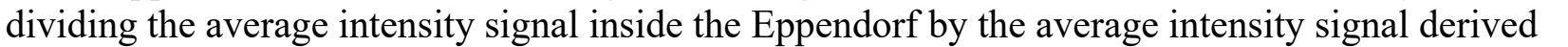

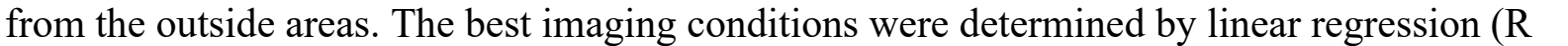

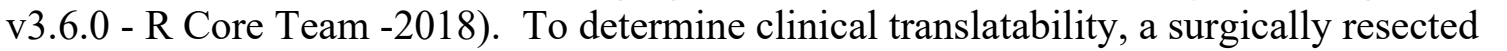

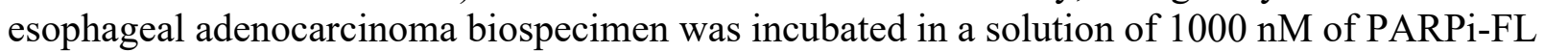

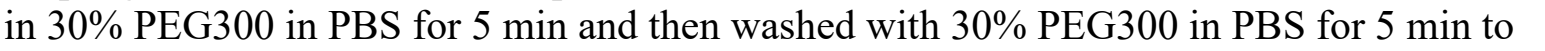

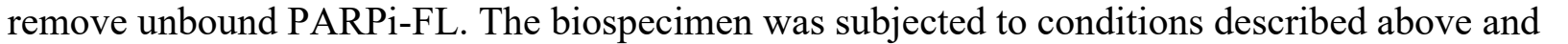

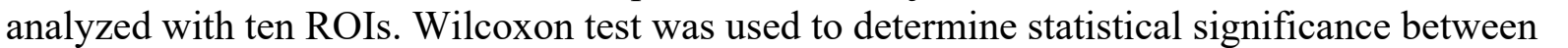

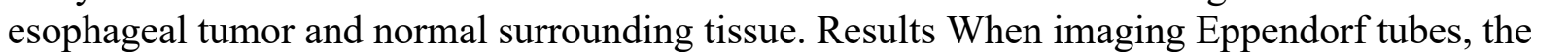

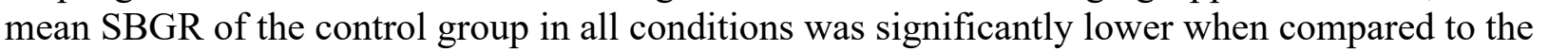

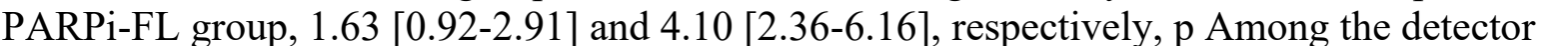

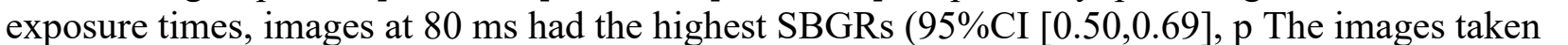

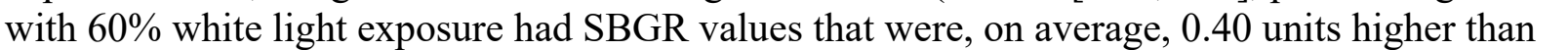

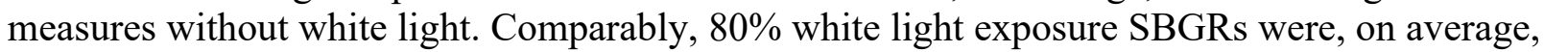

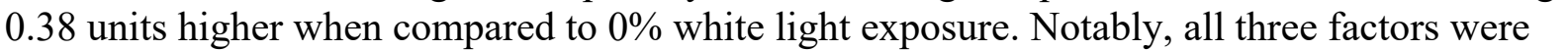

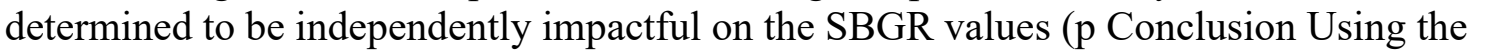

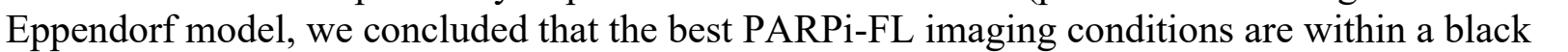

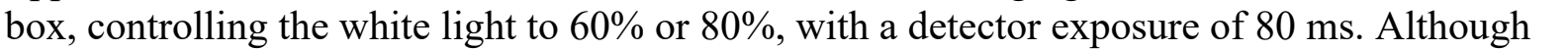

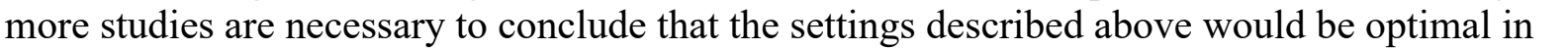

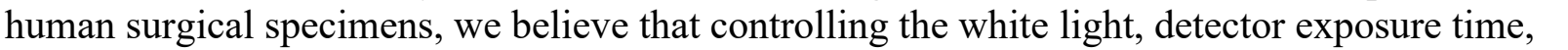

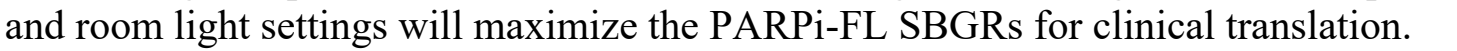

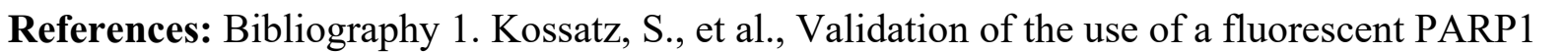

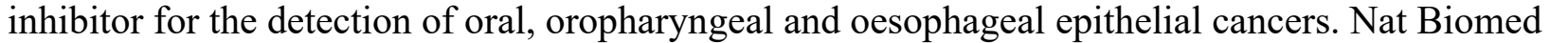




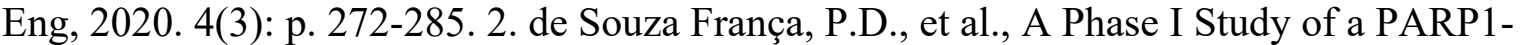
ए

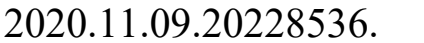

\section{Image/Figure:}

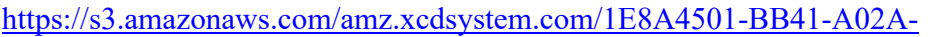

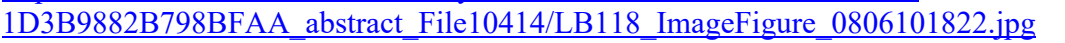

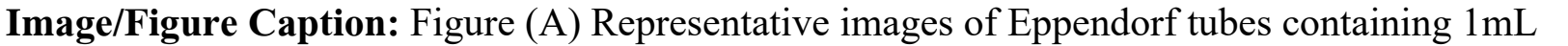

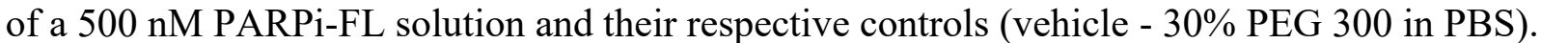

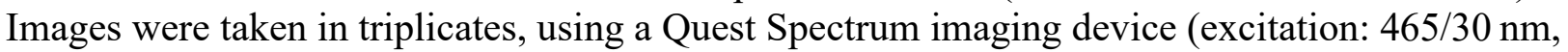
$\square \square \square \square \square \square-580 \mathrm{~nm}$ ), at $1 \mathrm{~cm}$ distance from the Eppendorf. Figure demonstrates tubes imaged

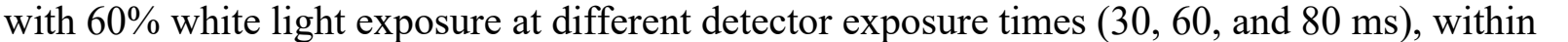

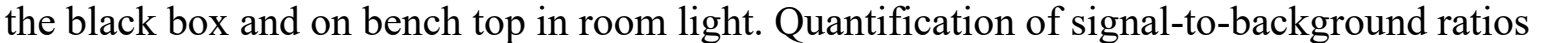
प प

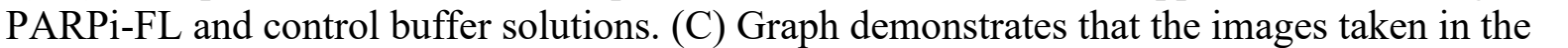

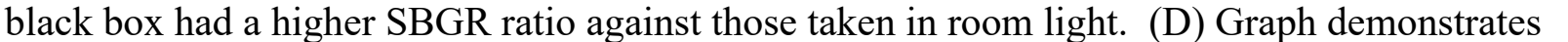

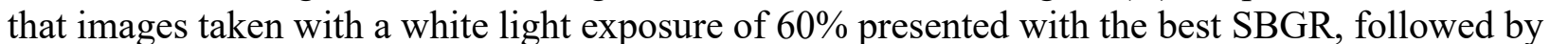

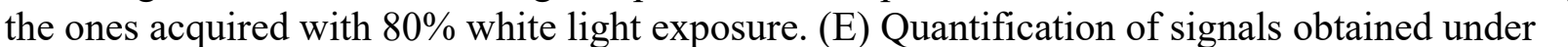

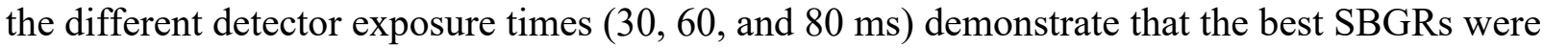

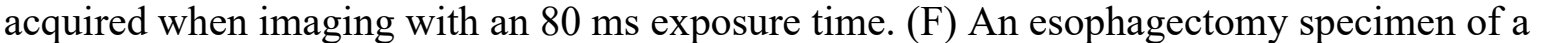

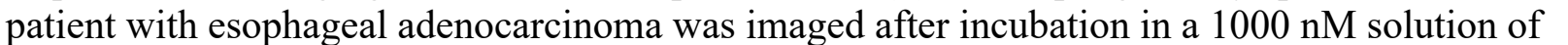

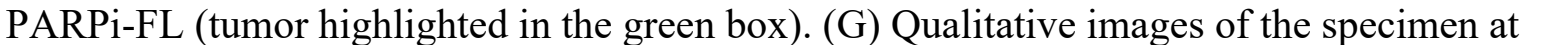

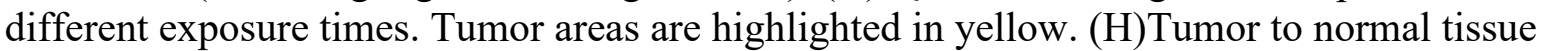

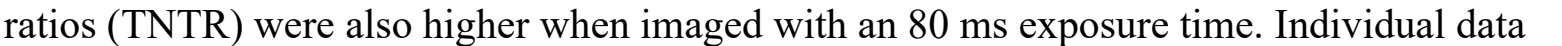

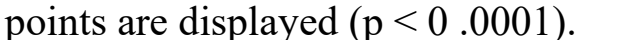

First Name: $\square \square \| 11]$

Last Name: $\square \square \square\|\|$

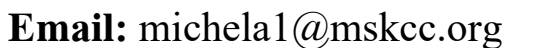

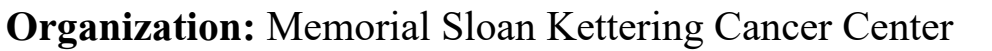

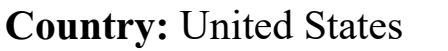




\title{
ID: LB119 \\ Initial curative therapy and its relationship to detection by a PSMA-targeting PET agent (18F-DCFPyL) and multiparametric MRI in men with biochemically recurrent prostate cancer
}

\author{
Liza Lindenberg, Molecular Imaging Branch, CCR, NCI, NIH, liza.lindenberg@nih.gov
}

Category: $\square \square\|\| 1 \| m$

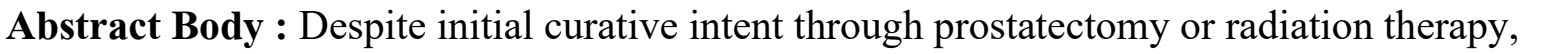

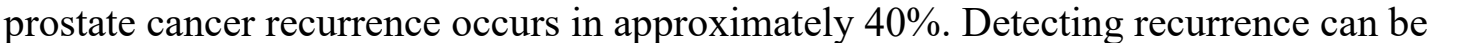

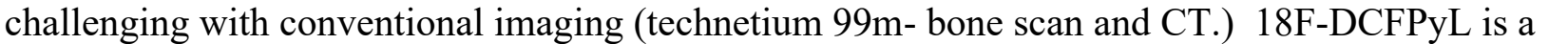

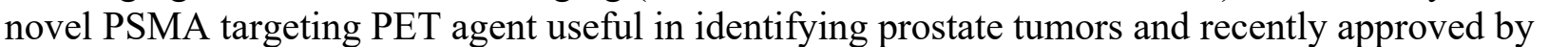

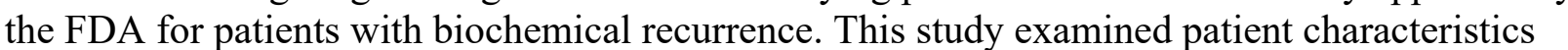

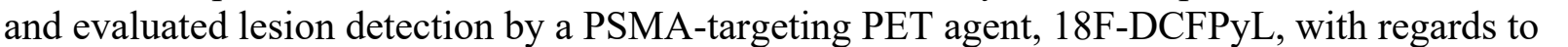

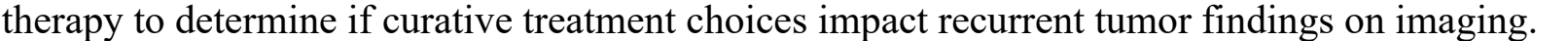

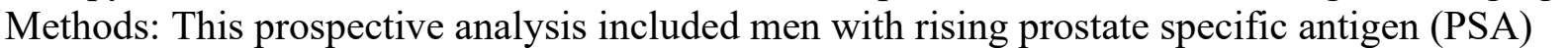

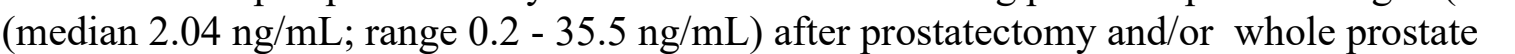

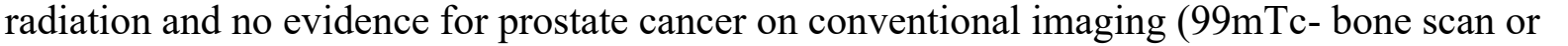

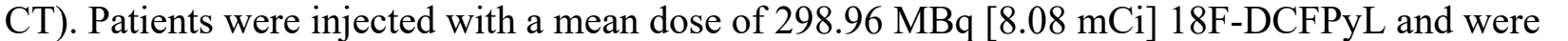

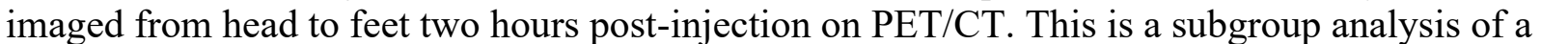

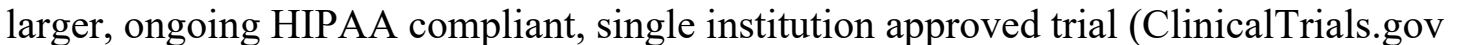

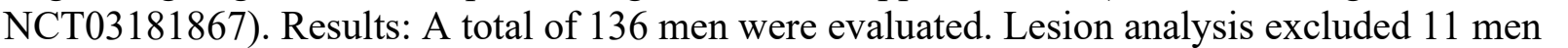

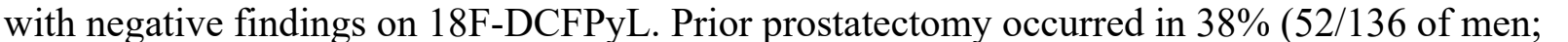

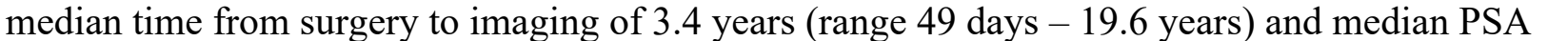

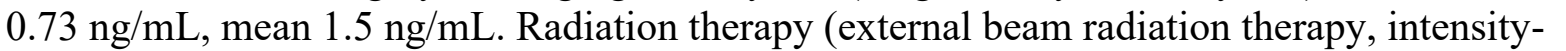

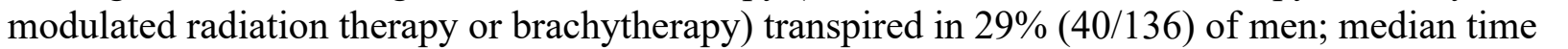

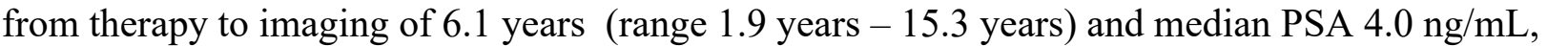

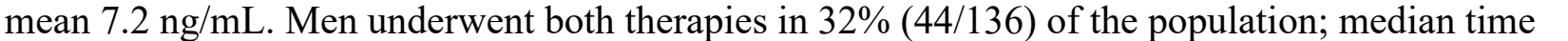

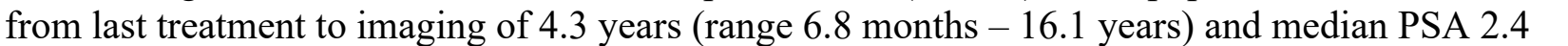

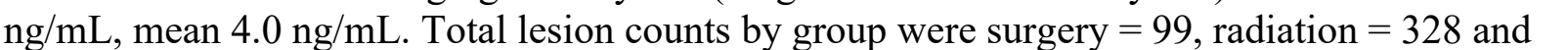

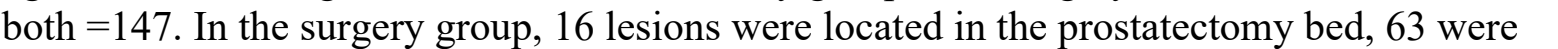

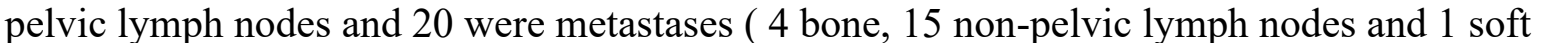

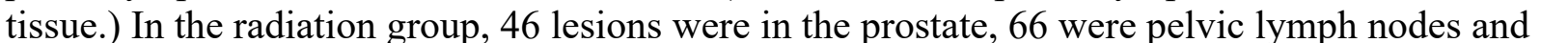

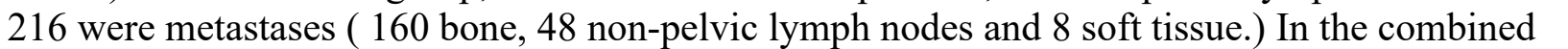

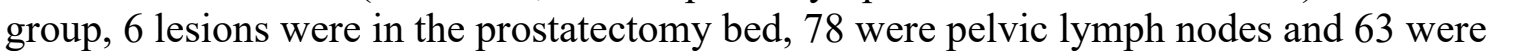

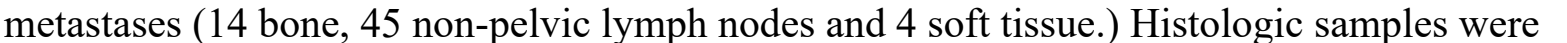

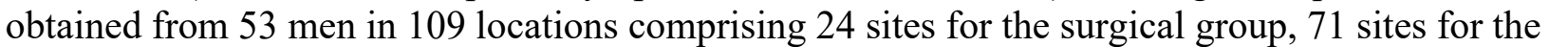

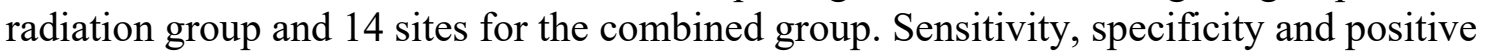

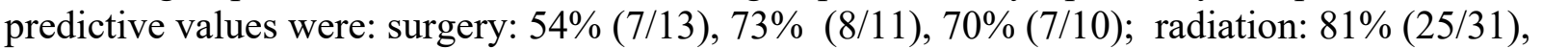

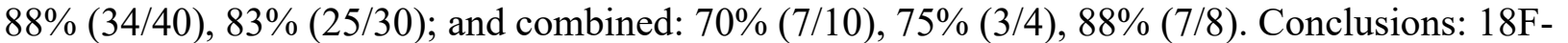

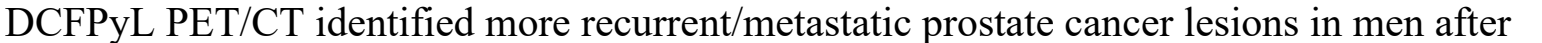

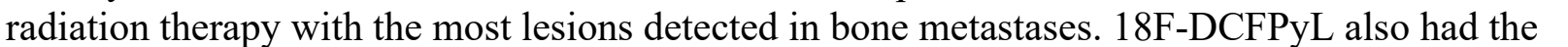

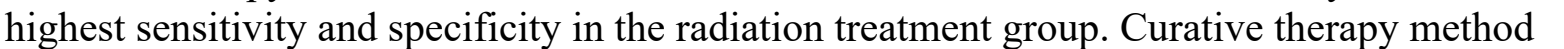




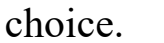

First Name: $\square \square\|\|$

Last Name: $\square \square\|\|\|\|\|\|$

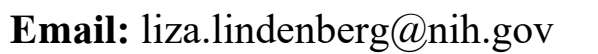

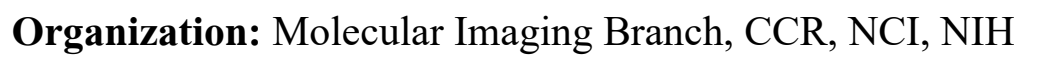

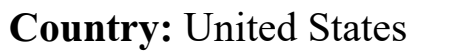




\title{
ID: LB120 \\ Wide-field depth ranging of sub-surface fluorescence margins with novel time-of- flight LiDAR
}

Petr Bruza, Dartmouth College, petr.bruza@dartmouth.edu

Category: $\square \square \| \square \square \square \square \square \square$

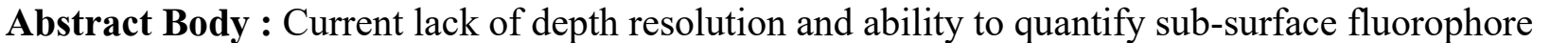

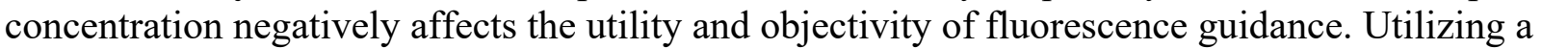

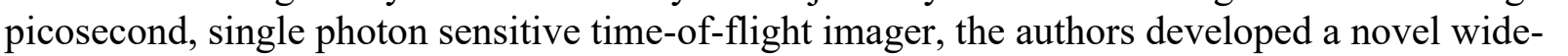

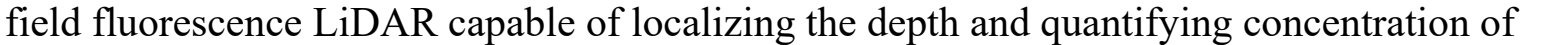

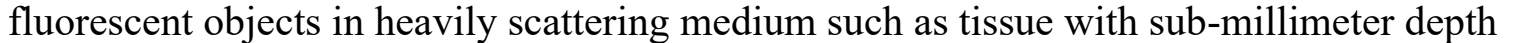

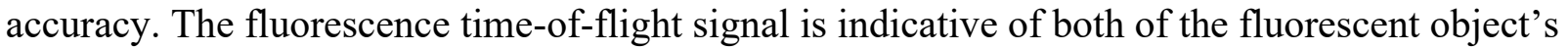

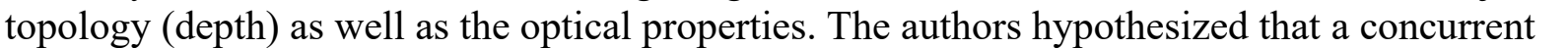

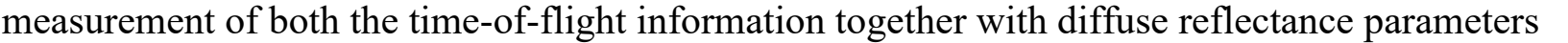

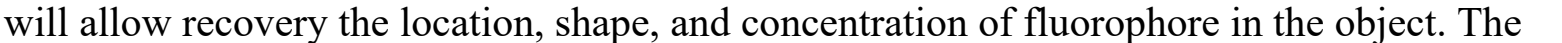

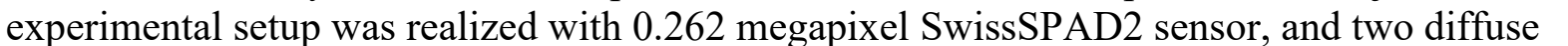
ए

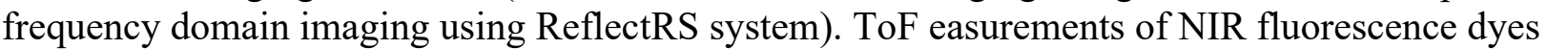

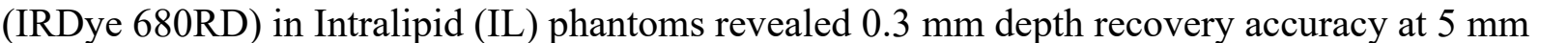

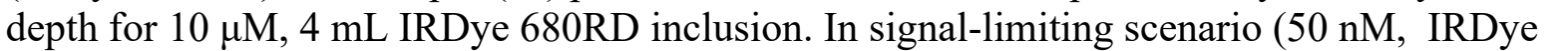

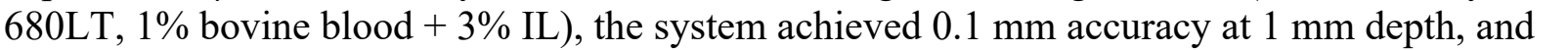

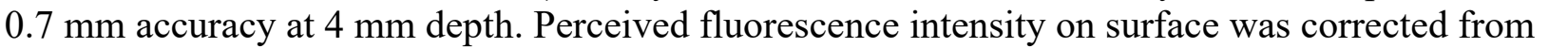

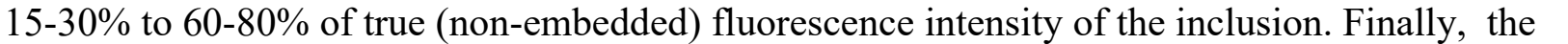

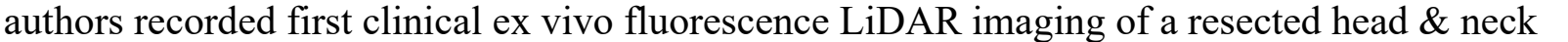

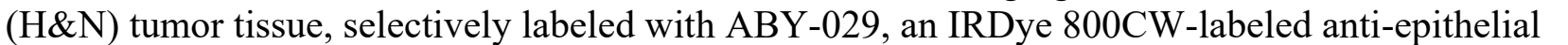

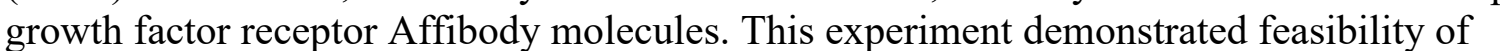
ए

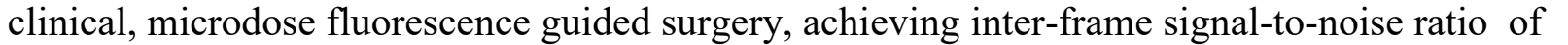

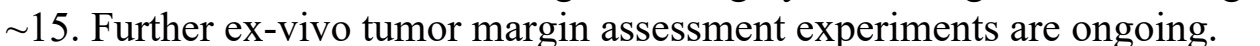

\section{Image/Figure:}

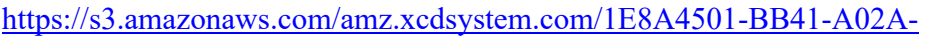

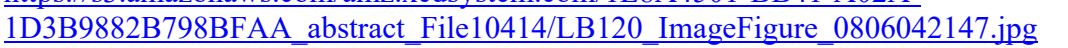

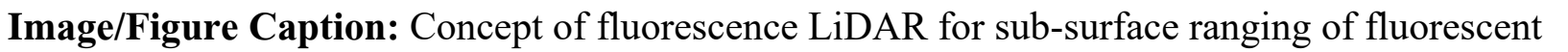

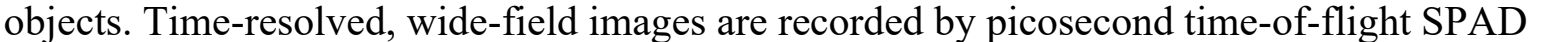

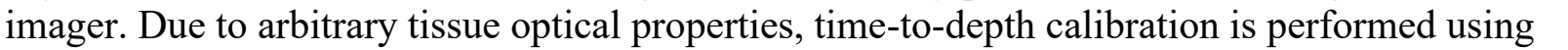

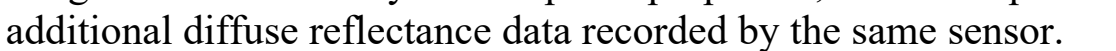

First Name: 
Last Name:

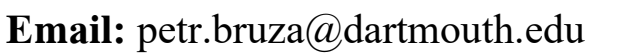

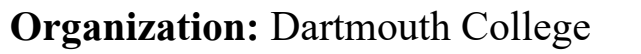

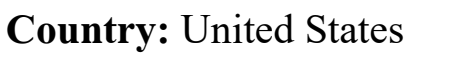




\title{
ID: LB121 \\ Development of Real-time Metabolic Imaging Biomarkers for Detection of Pancreatic Premalignant Lesions
}

\author{
Jose Enriquez, The University of Texas MD Anderson Cancer Center, \\ Jose.EnriquezOrtiz@uth.tmc.edu
}

Category: $\square \square\|ा\| ा \|$

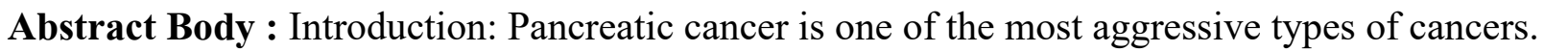

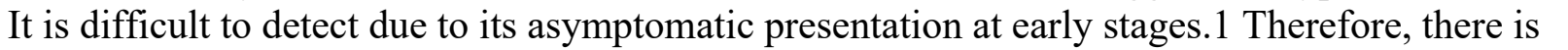

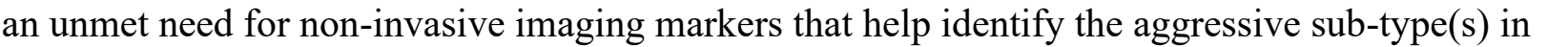

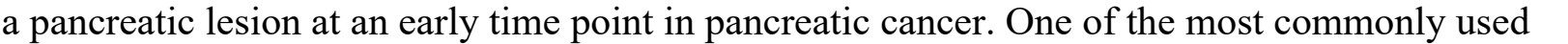

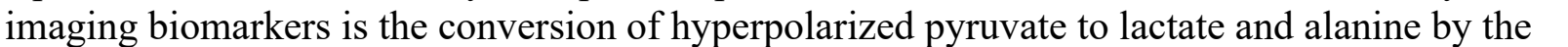

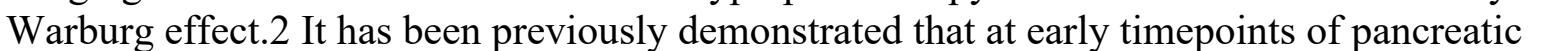

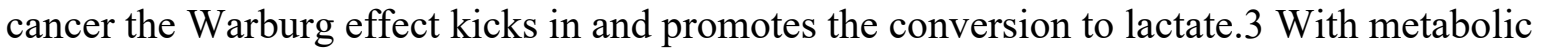

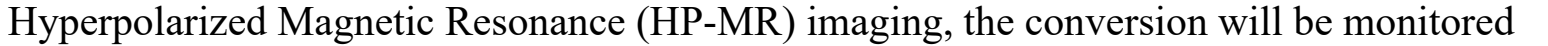

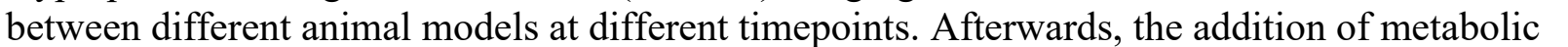

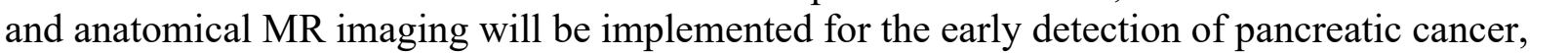

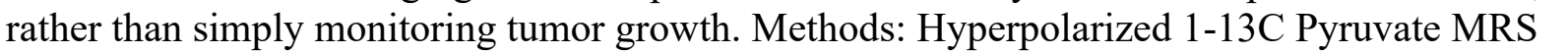

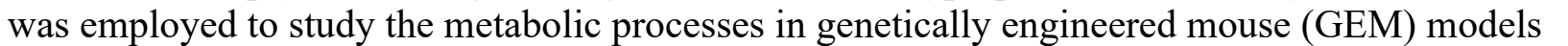

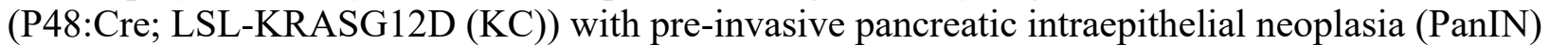

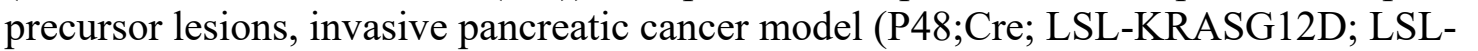

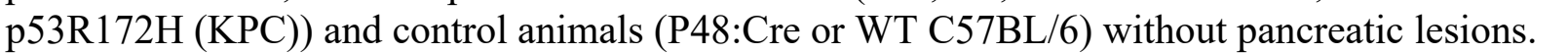

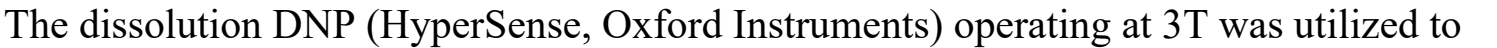

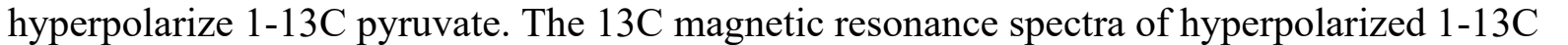

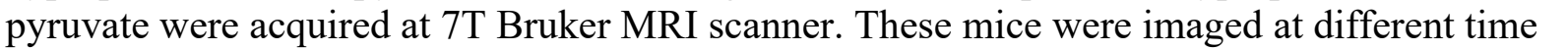

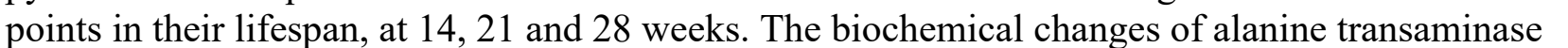

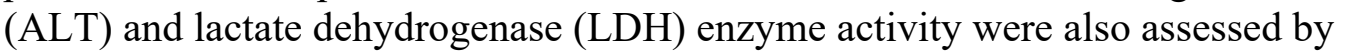

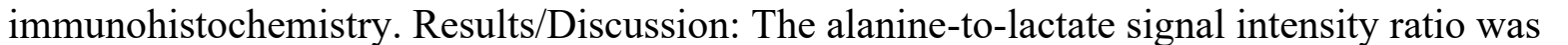

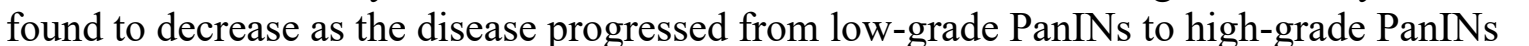
प

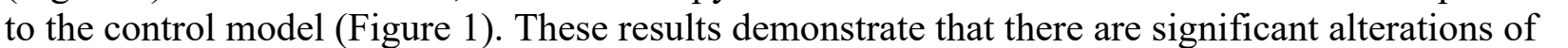

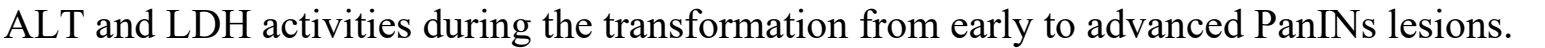

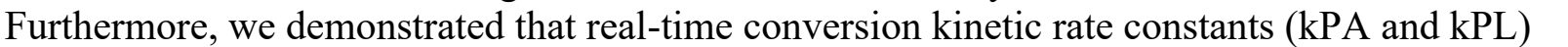

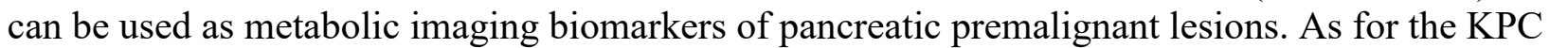

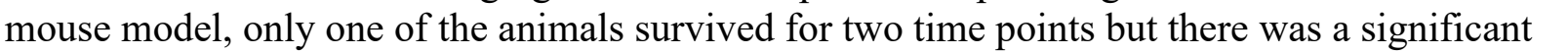

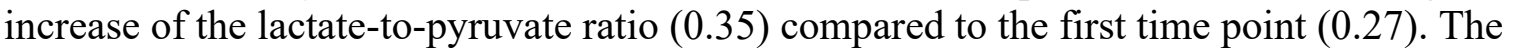

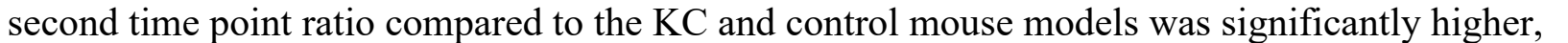

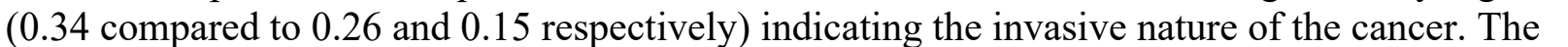

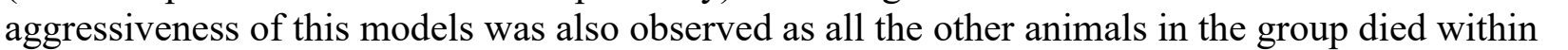

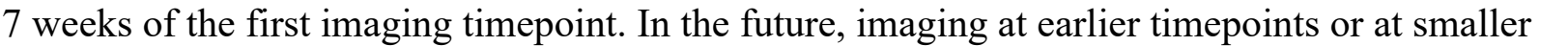

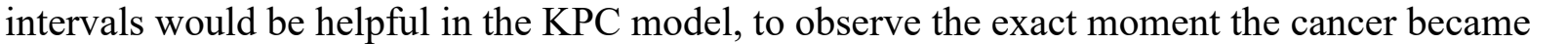

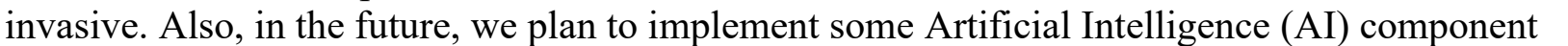




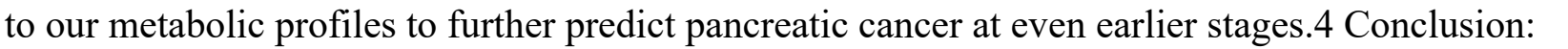

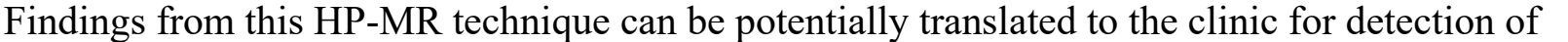

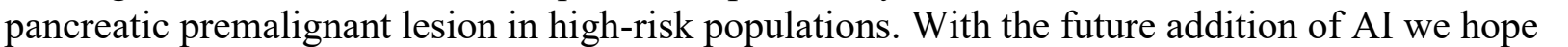

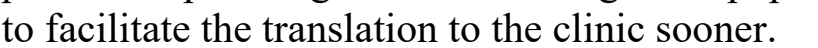

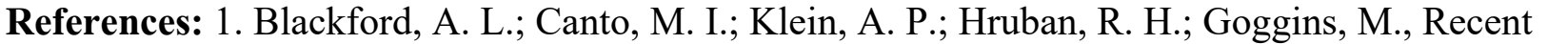

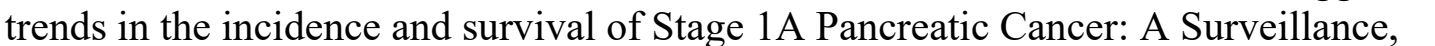

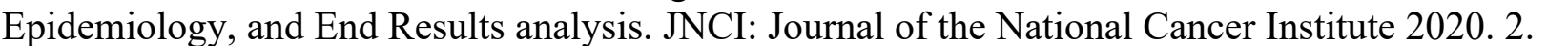

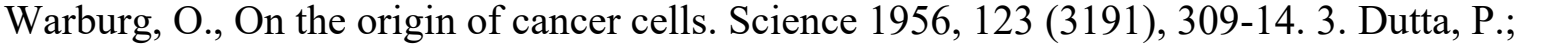

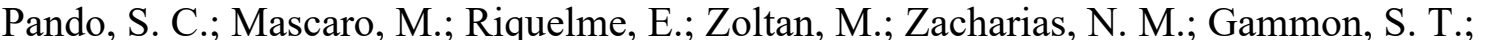

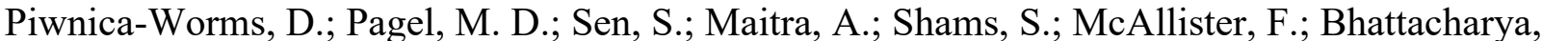

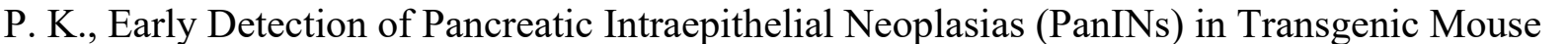

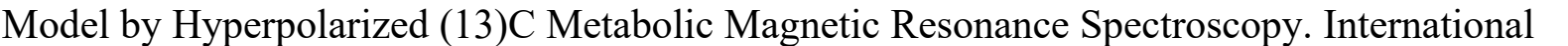
ए

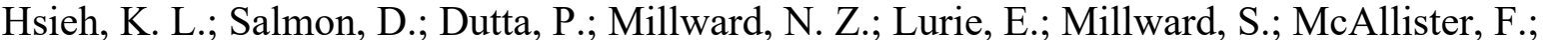

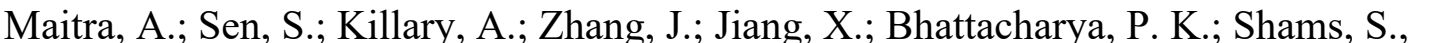

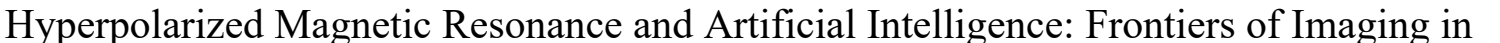

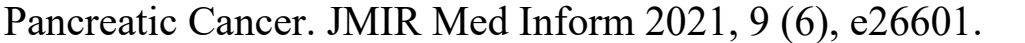

\section{Image/Figure:}

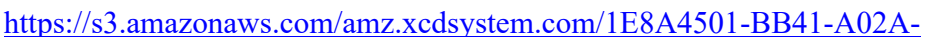

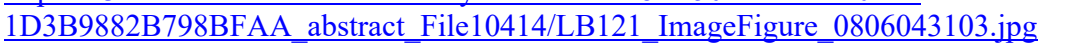

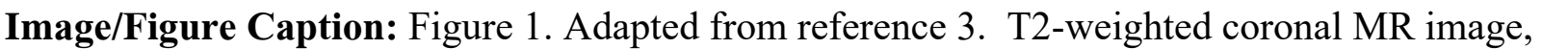

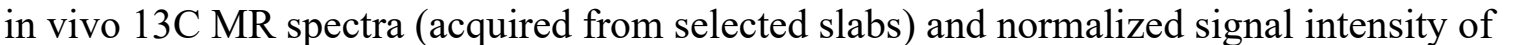

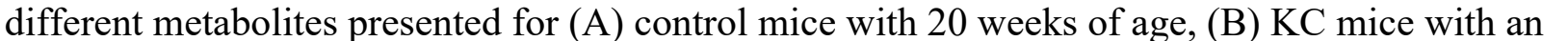

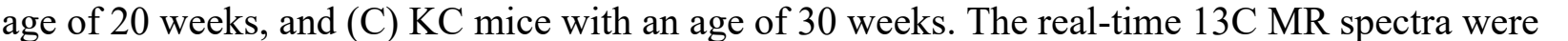

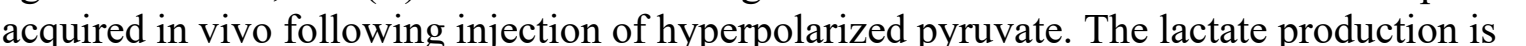

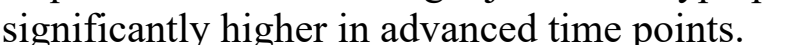

First Name: $\square \square\|\| \square$

Last Name: $\square \square\|\|\|\| \| \square$

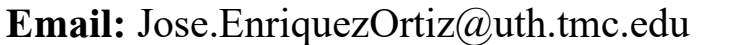

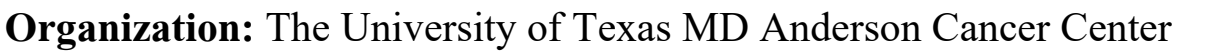

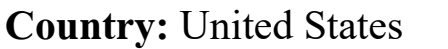




\title{
ID: LB122 \\ Automated detection of Esophageal Cancer using PARPi-FL, a step to clinical translation
}

Jayasree Chakraborty, Memorial Sloan Kettering Cancer Center, chakrabj@mskcc.org

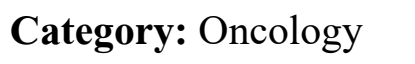

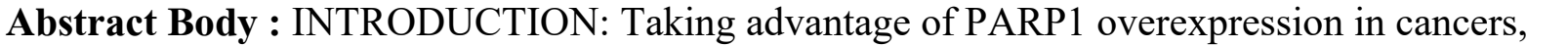

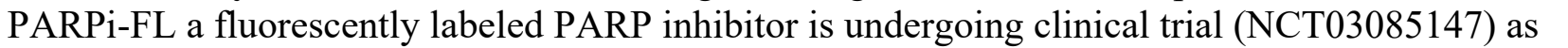

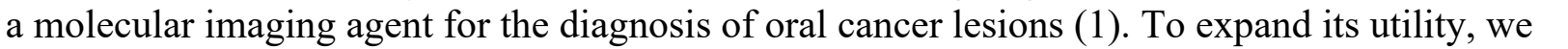

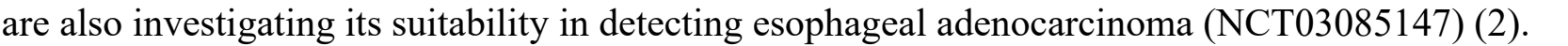

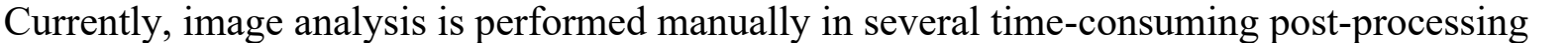

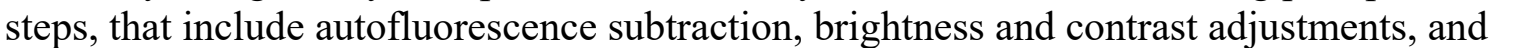

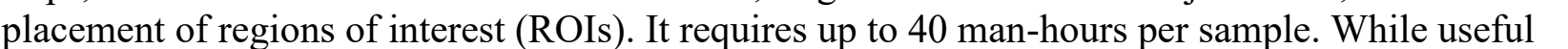
ए

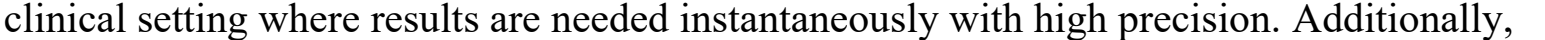

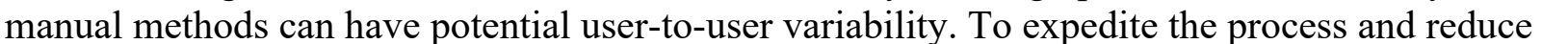

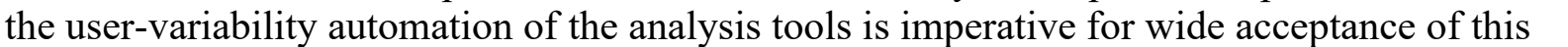

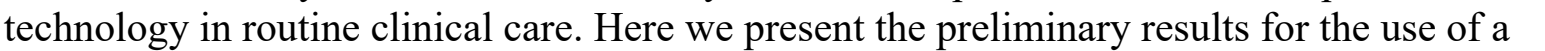

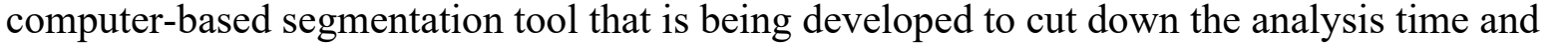

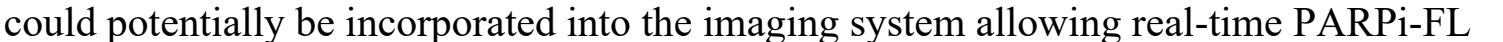

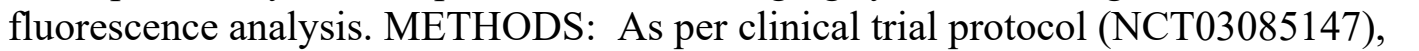

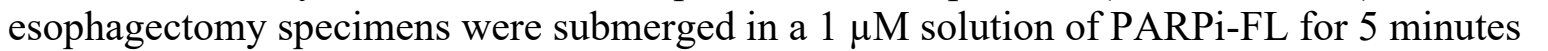
ए

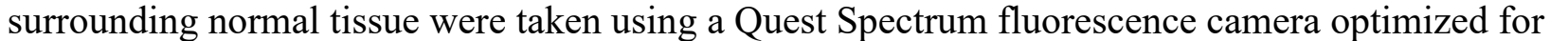

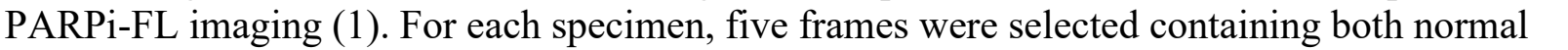

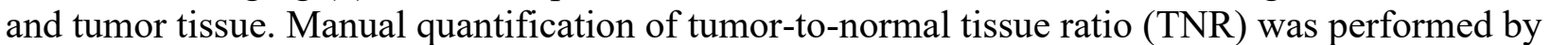

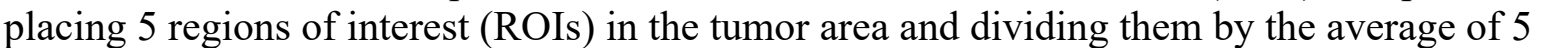

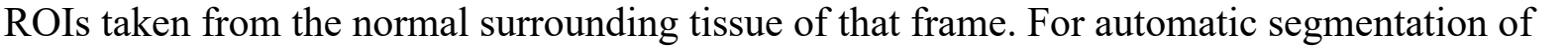

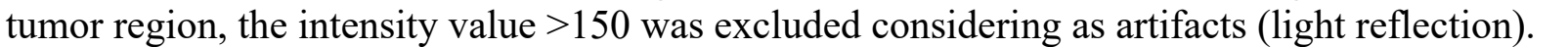

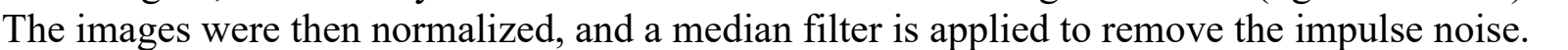

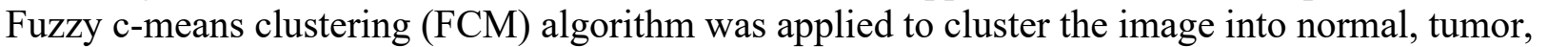

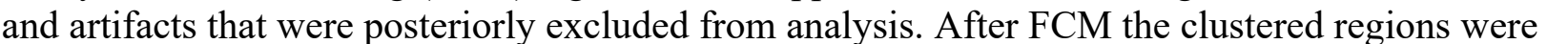

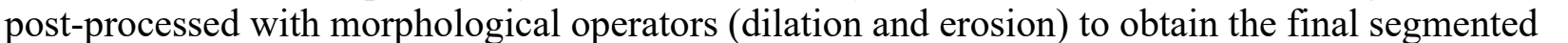

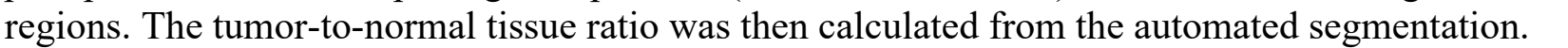

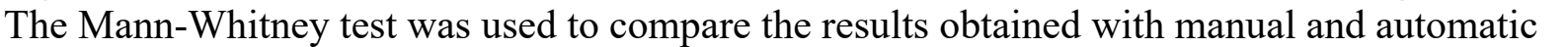

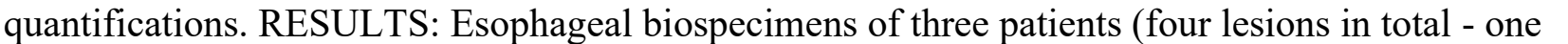

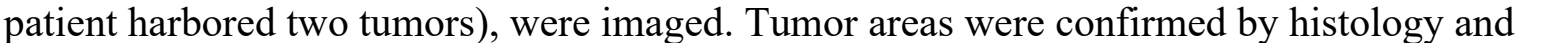

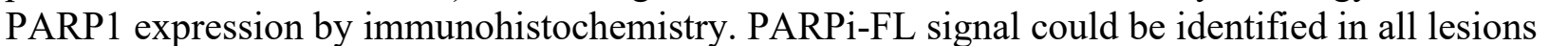

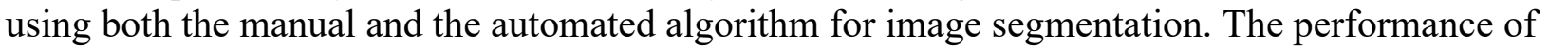

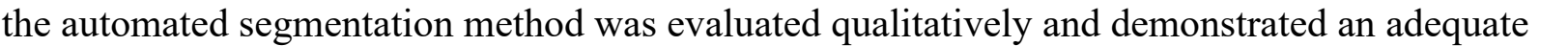

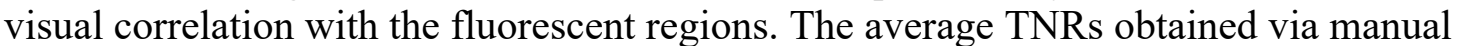

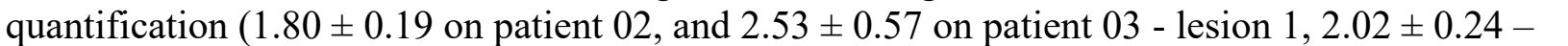




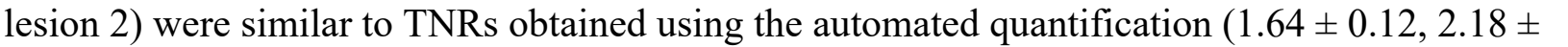

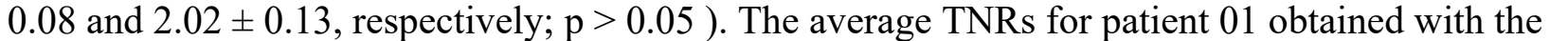

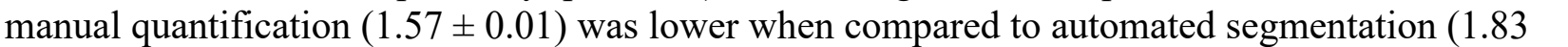

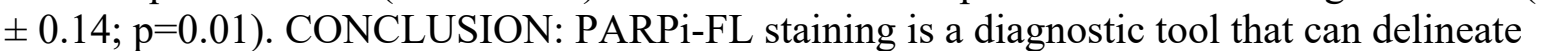

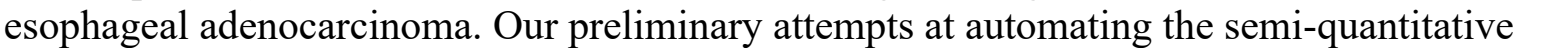

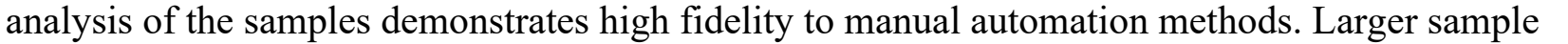

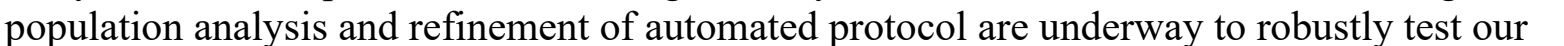

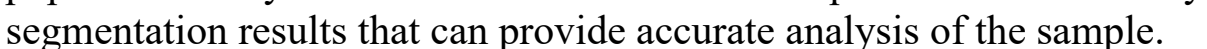

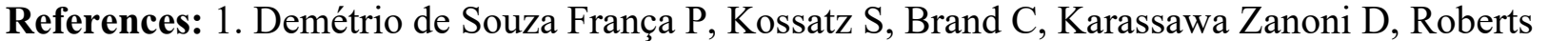

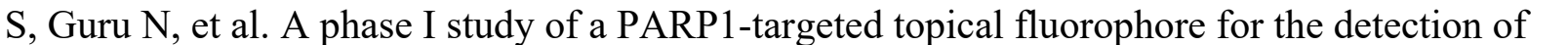

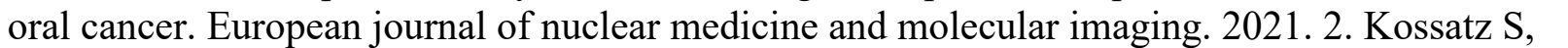

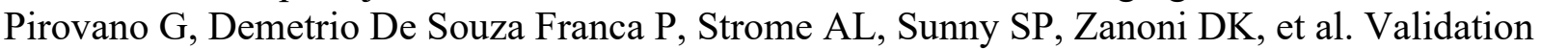

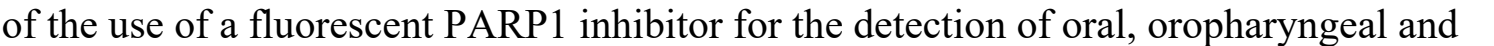

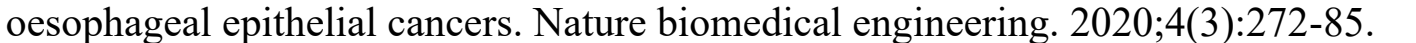

\section{Image/Figure:}

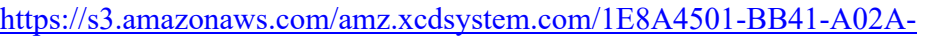

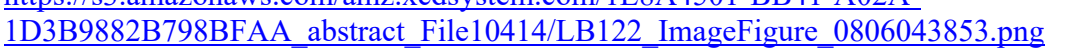

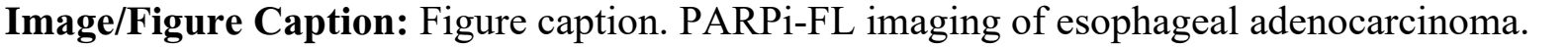

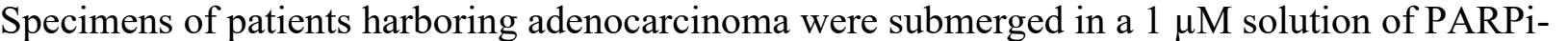

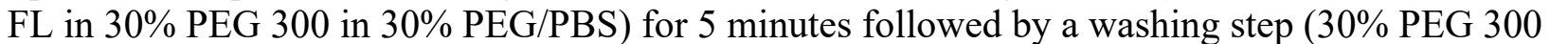

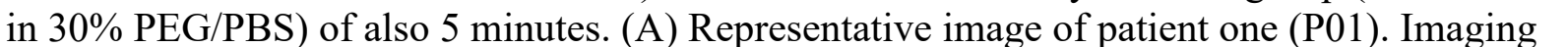

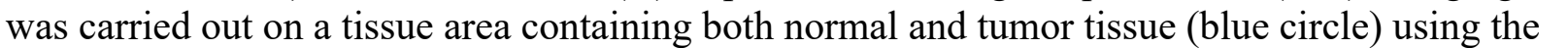

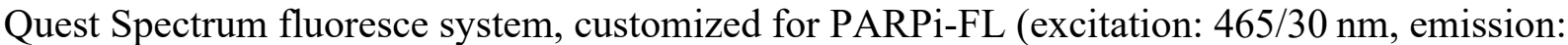
$\square \square-80 \mathrm{~nm}$ ). (B) Representative image of P01 histology and PARP1 immunohistochemistry

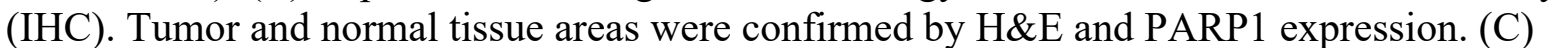

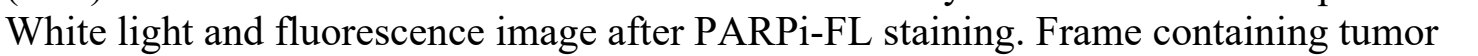
पा

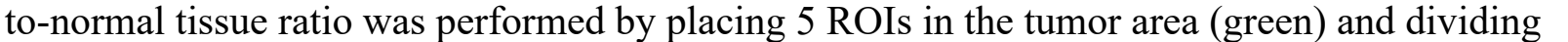

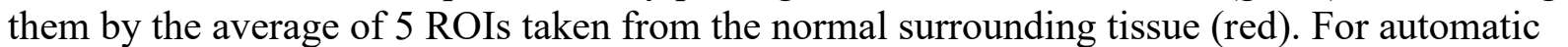

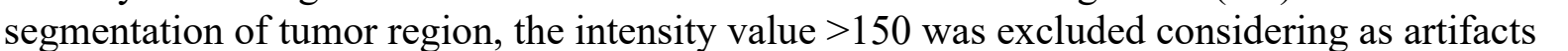

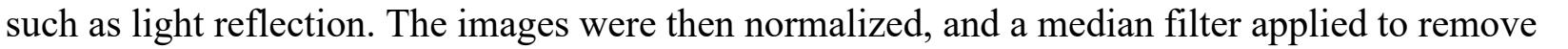
ए

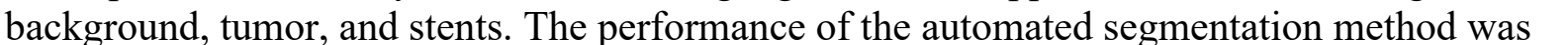

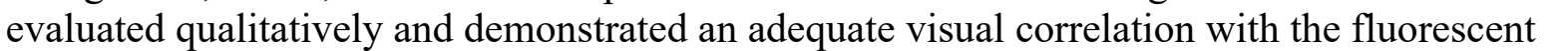

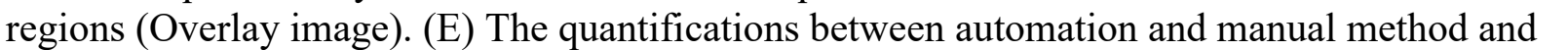

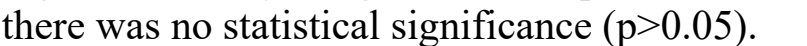

First Name: $\square \square\|\|\|\| \| \square$

Last Name: $\square \square \square \square\|\| \square \| m \mid \square$

Email: $\square\|\|\|\| \square \square \square\|\|\|\| \|$ 


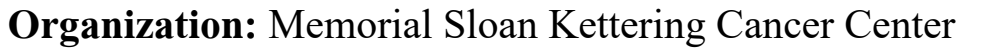

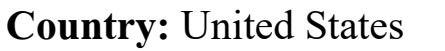




\title{
ID: LB123 \\ Variability of physiological 18F- FDG uptake in the spinal cord of cancer patients
}

\author{
Nsreen RA Mohamadien, Assiut University Hospital, nsreen@aun.edu.eg
}

Category: $\square \square \| \square \square \square$

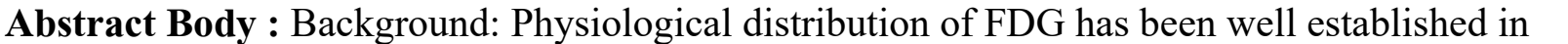

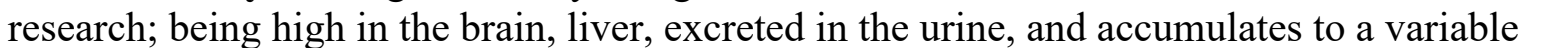

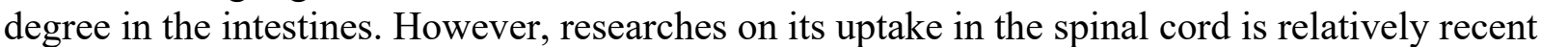

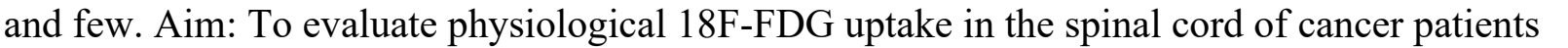

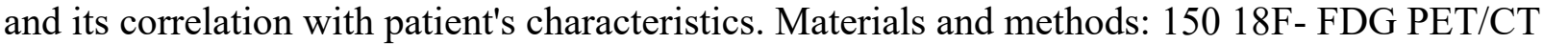

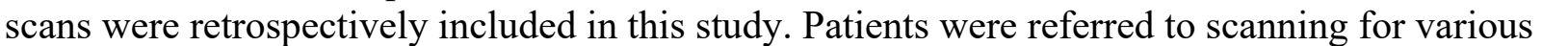

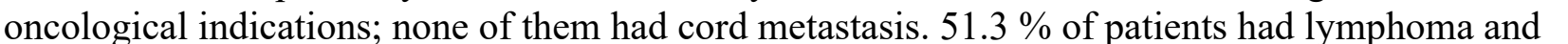

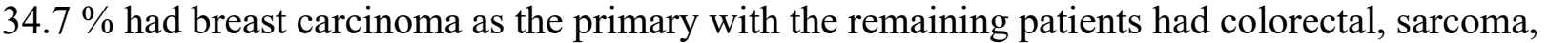

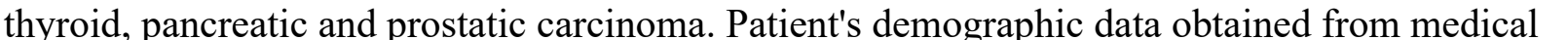

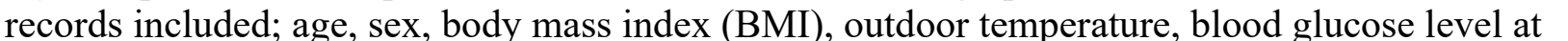
प

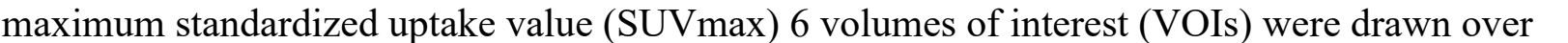

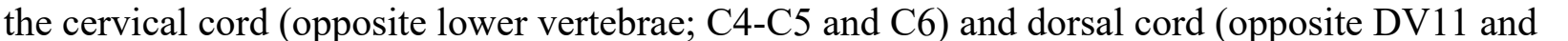

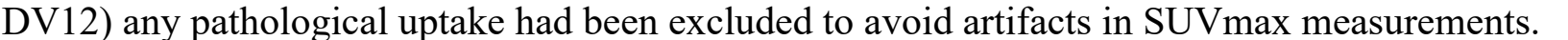

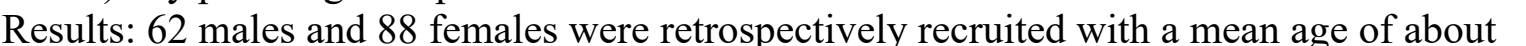

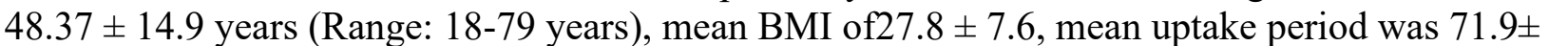

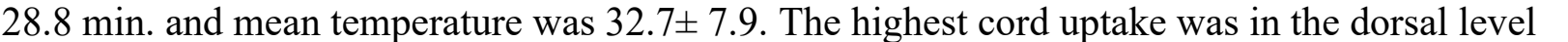

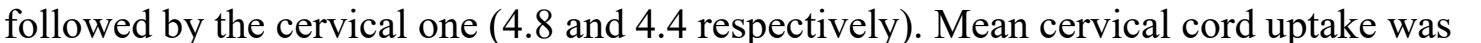

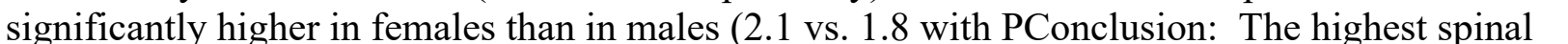

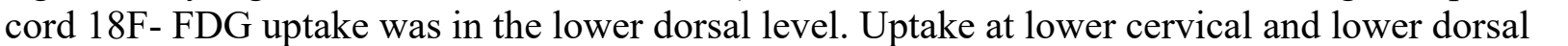

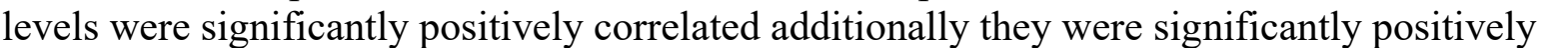

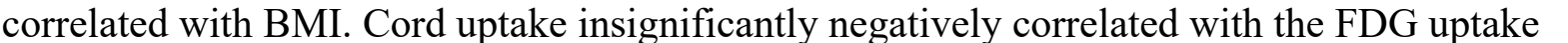

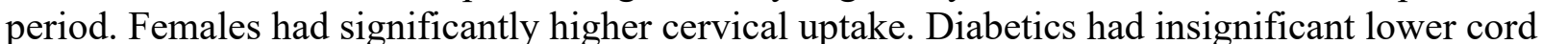

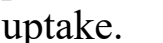

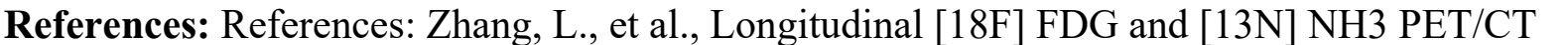

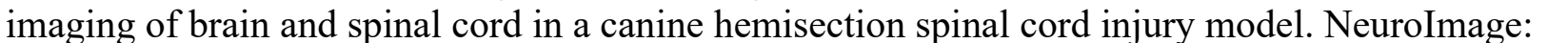
म

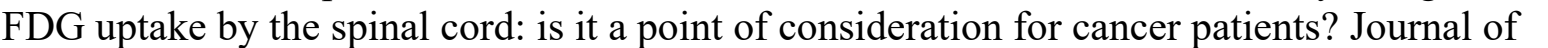

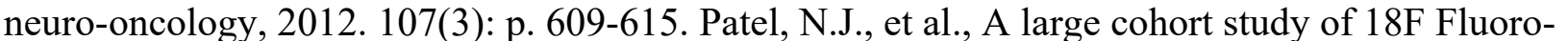

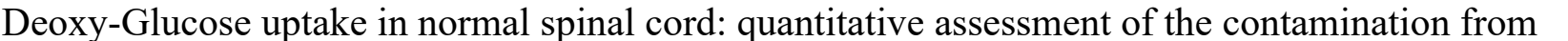

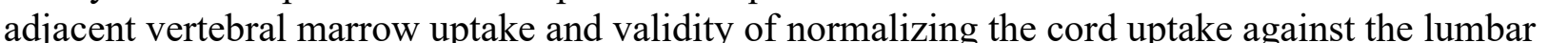

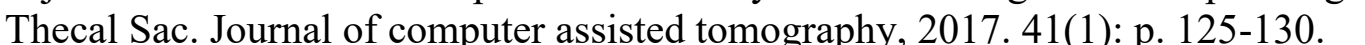

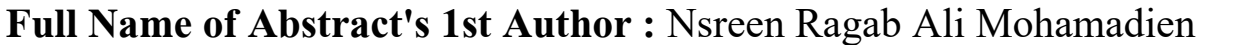


First Name: $\square \square\|\| \| \square \square \square$

Last Name: $\square \square \square \square \square \square \| \square$

Email: $\square\|\| \square \square \square \square \square \square \square \square \square \square$

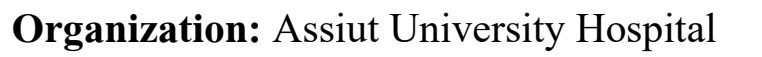

Country: 


\title{
ID: LB124 \\ In vitro and in vivo assessment of docetaxel-loaded theranostic nanobubbles for imaging and treatment of locally advanced breast cancer
}

\author{
Patrick Dong Min Chang, University of Toronto, patrickdongmin.chang@mail.utoronto.ca
}

\author{
Category: $\square \square\|\| \square \square$
}

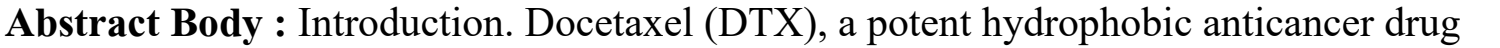

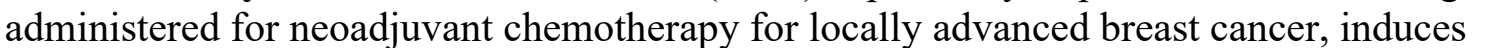

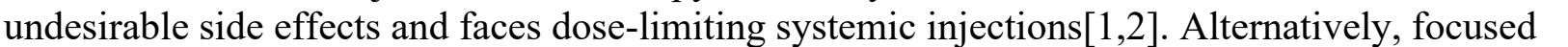

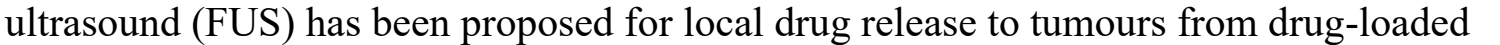

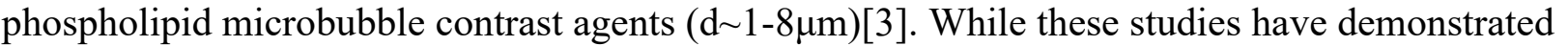

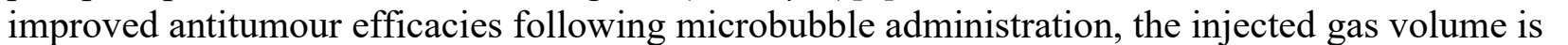

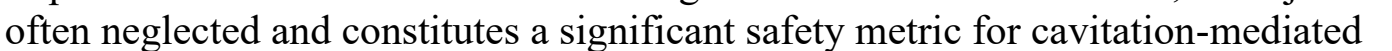

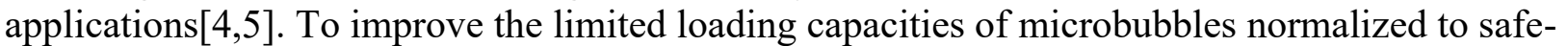

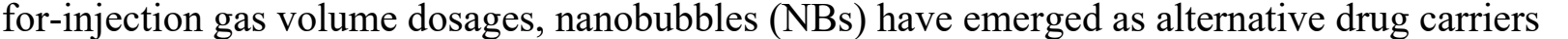

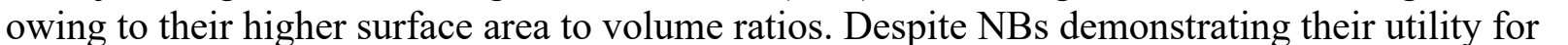

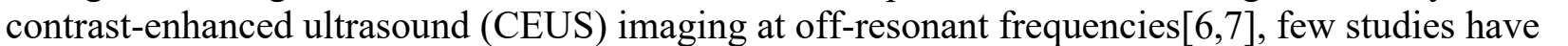

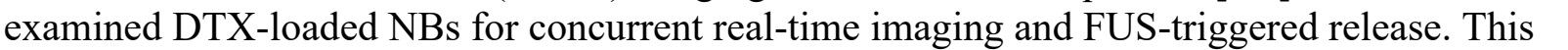

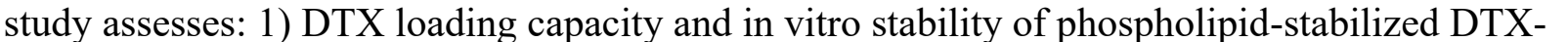
ए

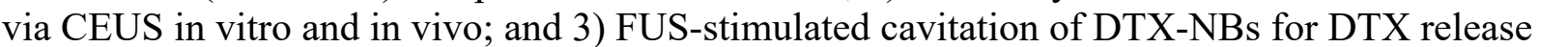

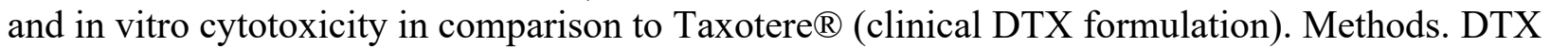

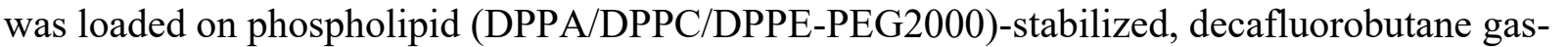
m

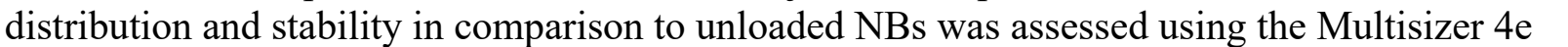

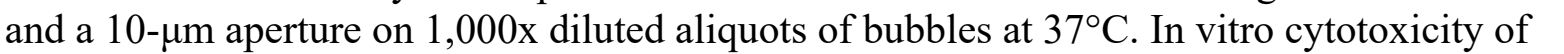

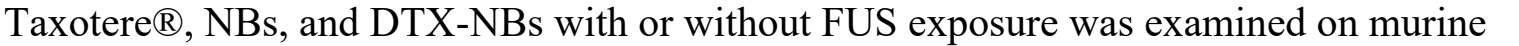

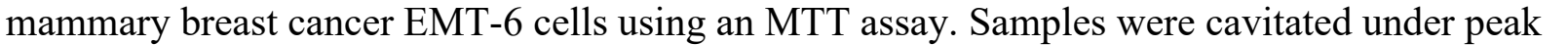

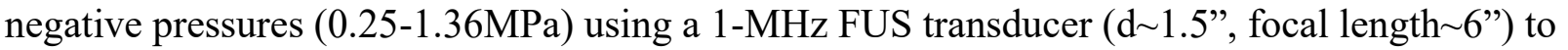

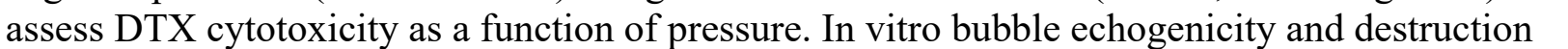

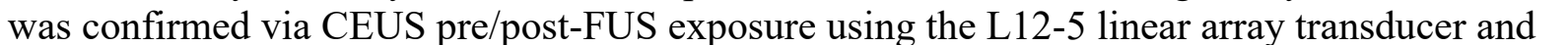

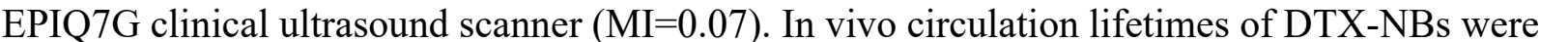

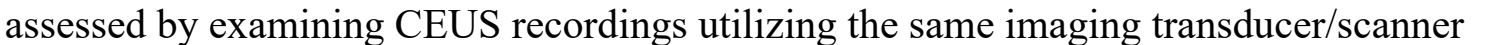

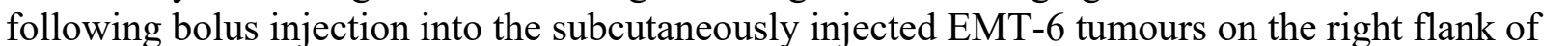

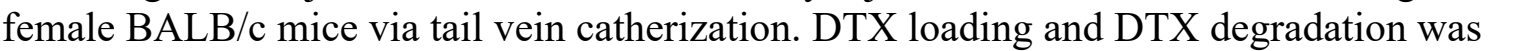

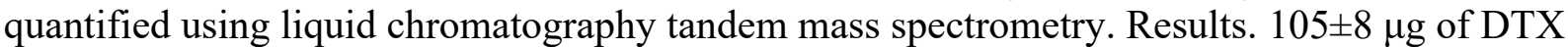
was loaded onto $\sim 1010$ bubbles with a mode diameter of $\sim 230 \mathrm{~nm}$ per $\sim 0.4 \mu \mathrm{L}$ of gas $(\mathrm{n}>6)$ and

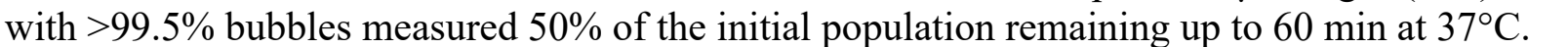

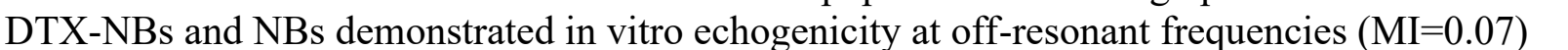

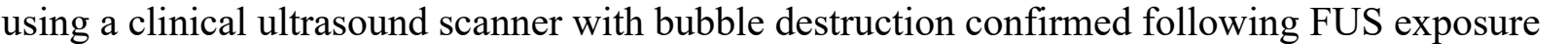

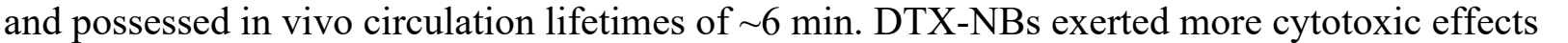

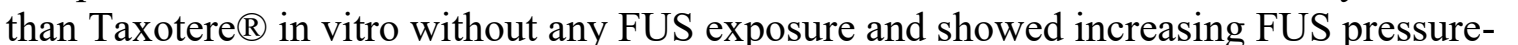




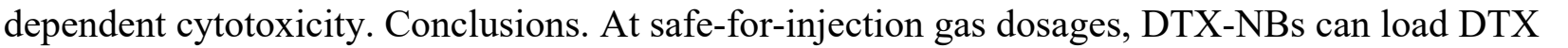

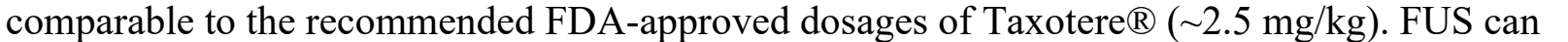

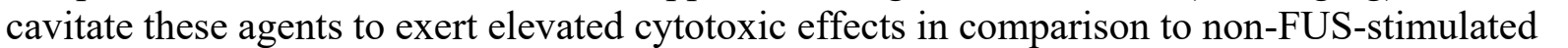

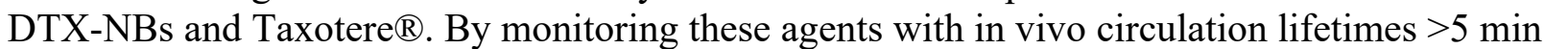

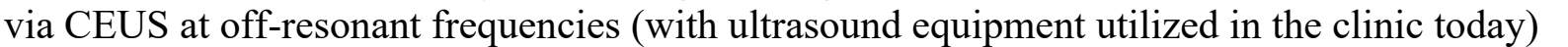

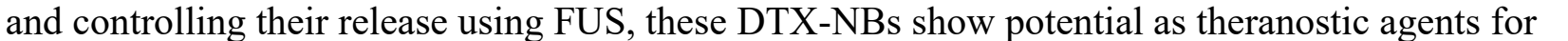

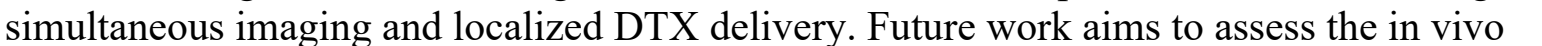

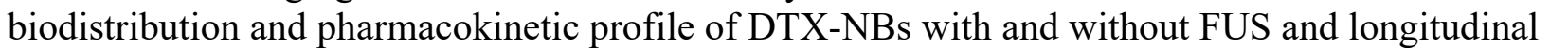

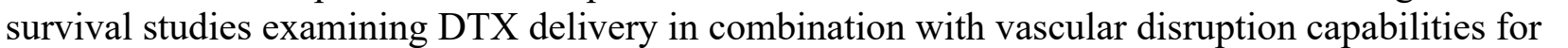

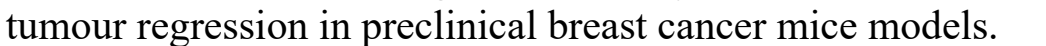

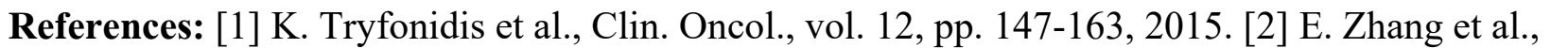

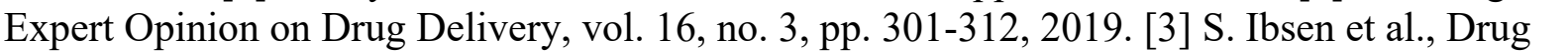

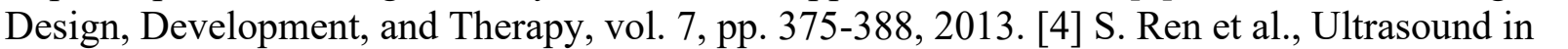

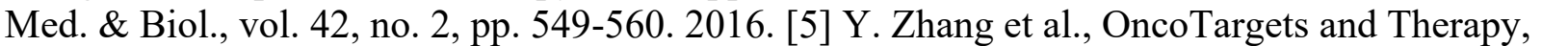

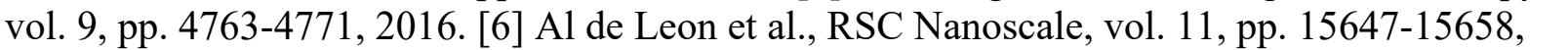

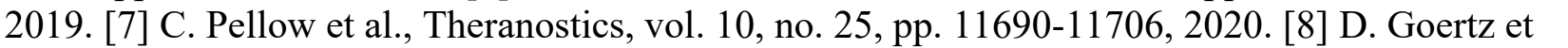

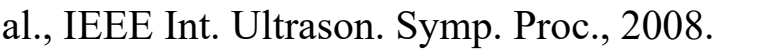

\section{Image/Figure: $\square$}

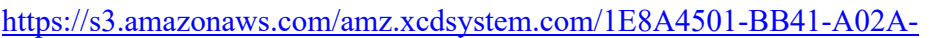

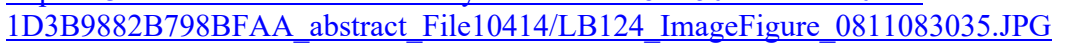

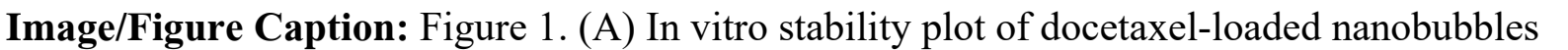
$\square \square \square(\mathrm{NBs})$ and unloaded nanobubbles (NB) at physiological temperature $\left(37^{\circ} \mathrm{C}\right)(\mathrm{n} \geq 3)$.

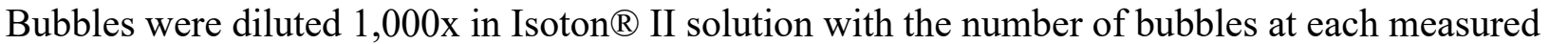

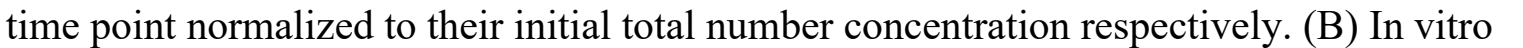

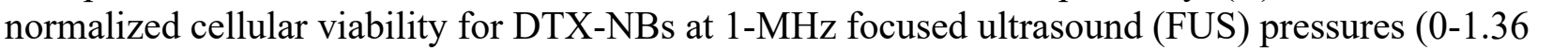

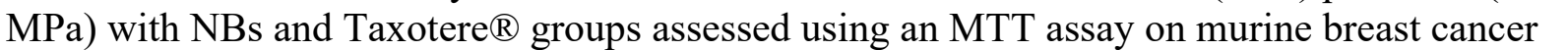

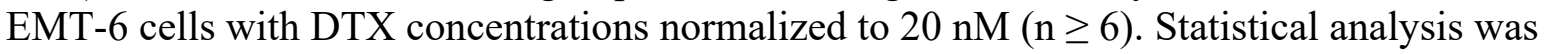

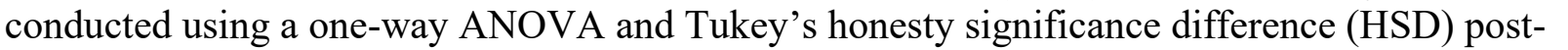

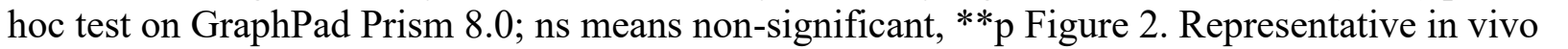

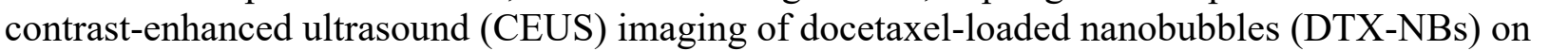
ए

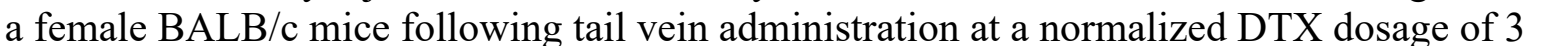

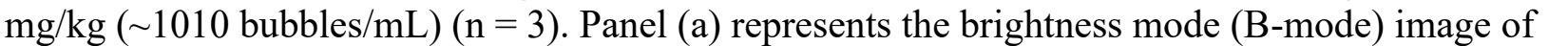

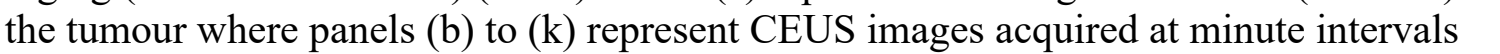

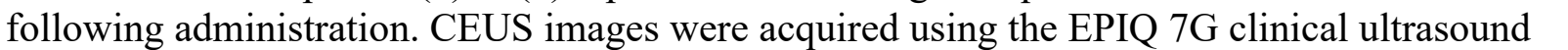

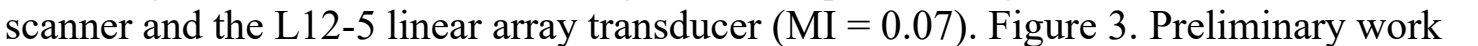

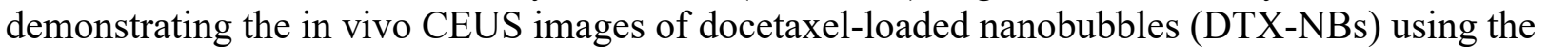

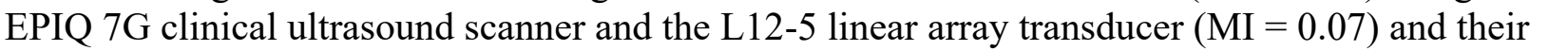

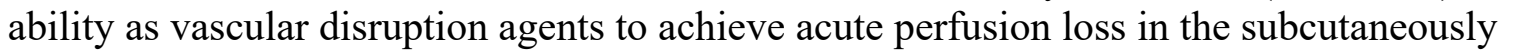
ए

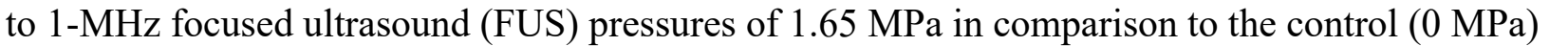

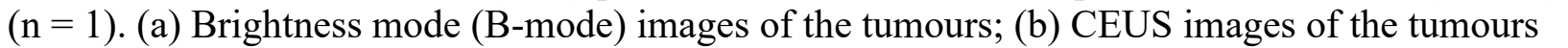

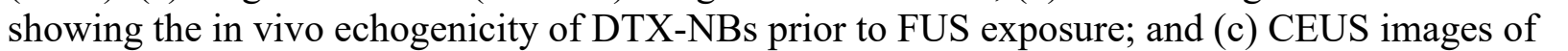


First Name: $\square \square \square\|\square\| \square \square \square \square \square \square$

Last Name:

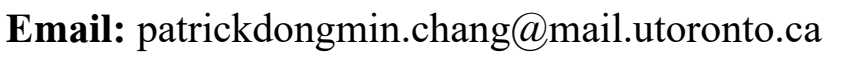

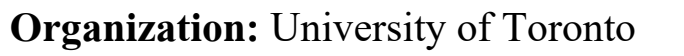

Country: $\square \square \square \square\|\|$ 


\title{
ID: LB125 \\ First human study with [18F]3F4AP: biodistribution, safety and radiation dosimetry
}

Pedro Brugarolas, Massachusetts General Hospital, pbrugarolas@mgh.harvard.edu

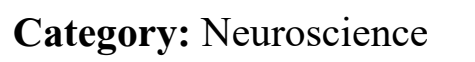

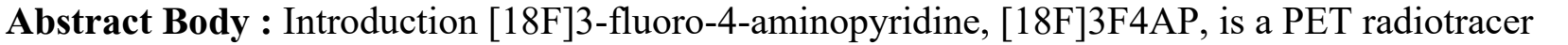

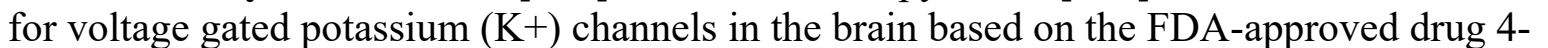

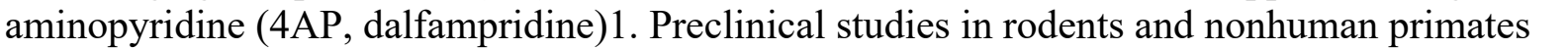

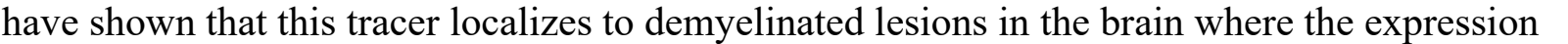

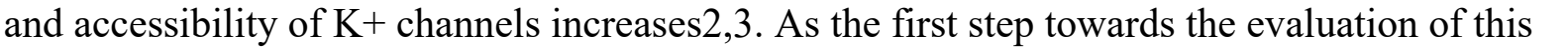

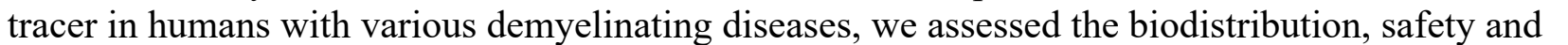

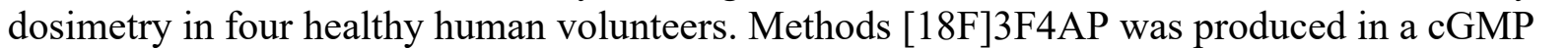

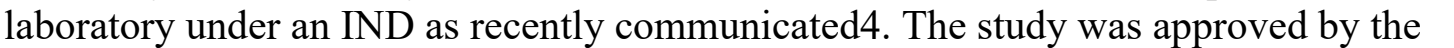

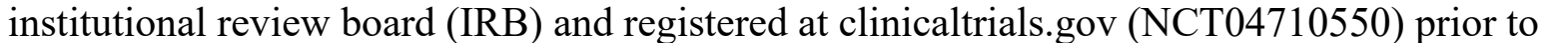

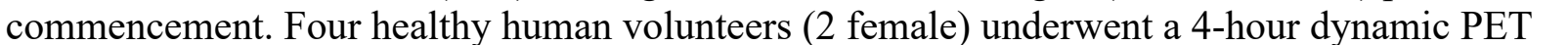

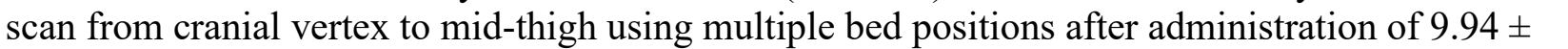

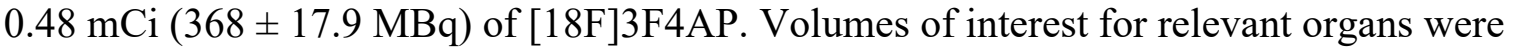

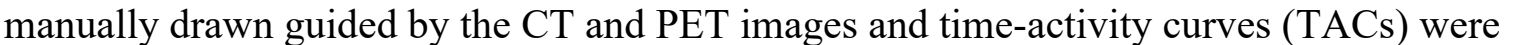

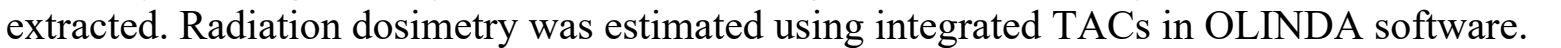

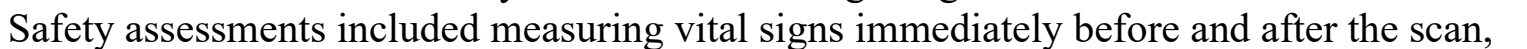

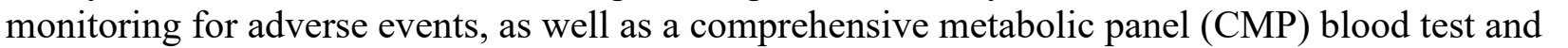

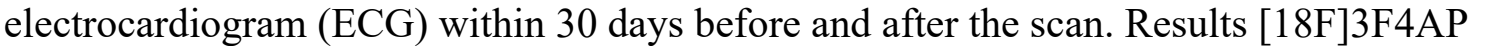

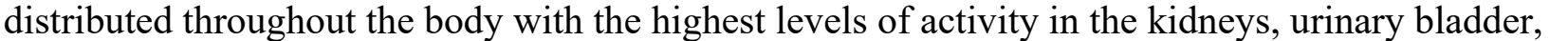

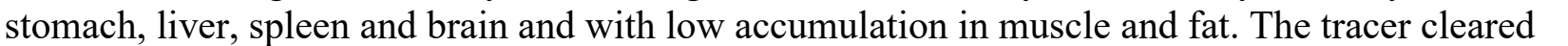

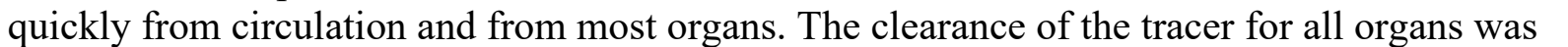

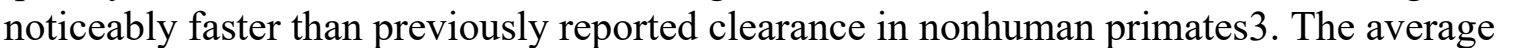
effective dose (ED) across all subjects was $11.4 \pm 1.6 \mu \mathrm{Sv} / \mathrm{MBq}$ which is lower than the estimated ED from the nonhuman primate studies $(21.6 \pm 0.6 \mu \mathrm{Sv} / \mathrm{MBq})$ as well as the ED of

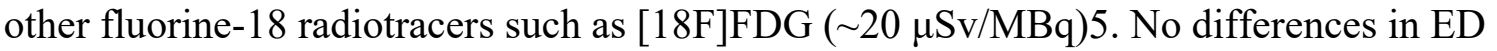

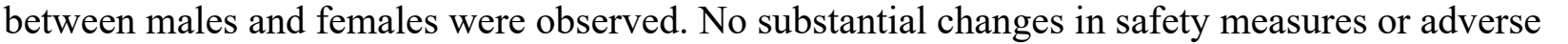

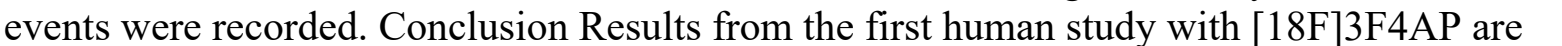

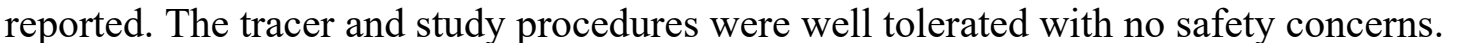

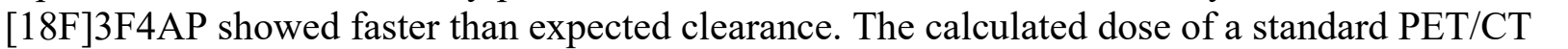

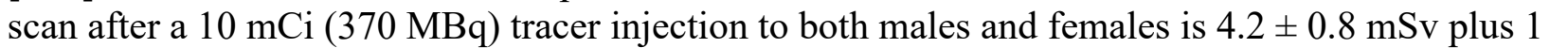

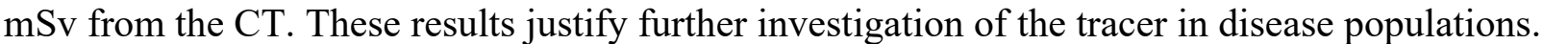

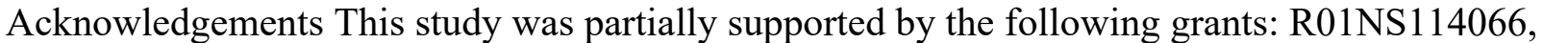

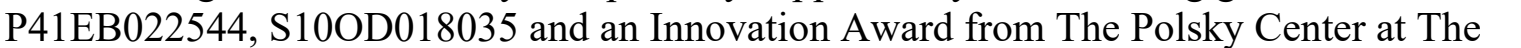

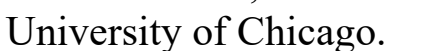

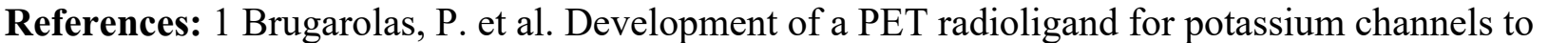

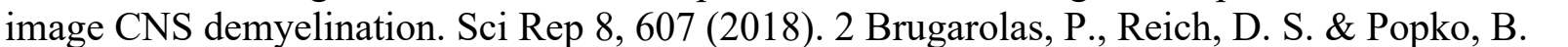




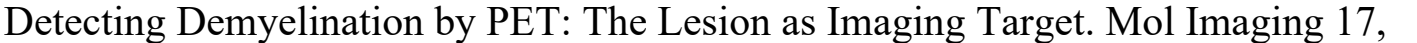

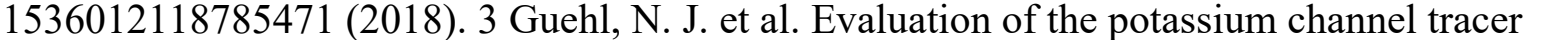

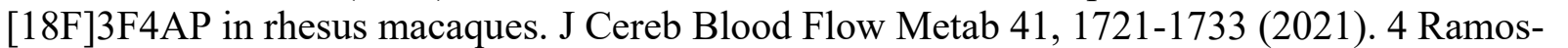

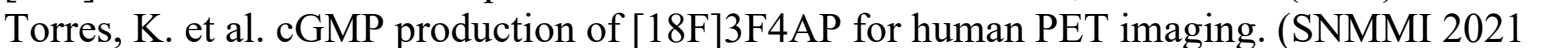

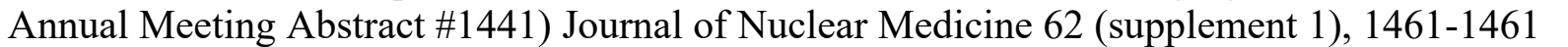

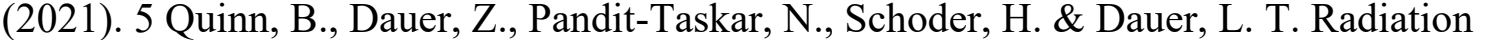

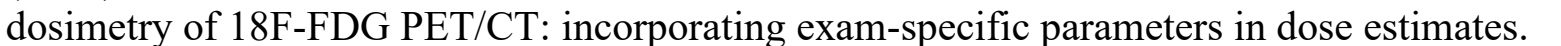

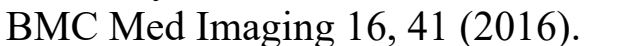

\section{Image/Figure:}

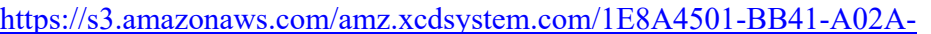

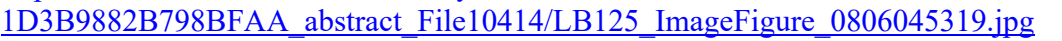

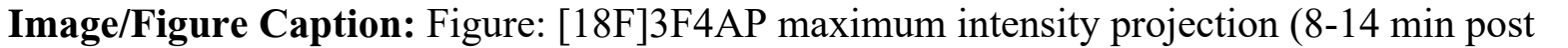

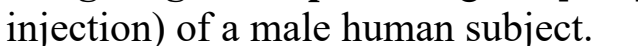

First Name: $\square \square||\|\mid\|$

Last Name: $\square \square \square|| \||\omega| \square$

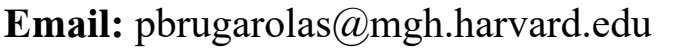

Organization: $\square \square\|\|\|\|\|\|\|\|\|\square \square\|\|\|\|\square \square\|\|\| \|$

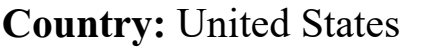




\title{
ID: LB127 \\ Deep learning generation of high count preclinical PET images from low count PET images
}

\author{
Kaushik Dutta, Washington University in St. Louis, kaushik.dutta@wustl.edu
}

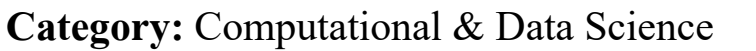

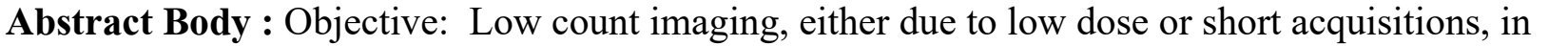

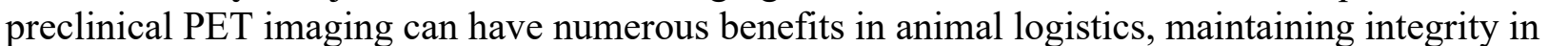

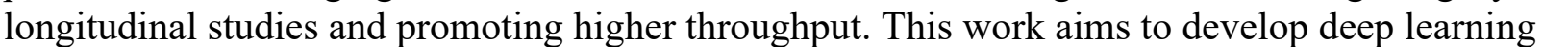

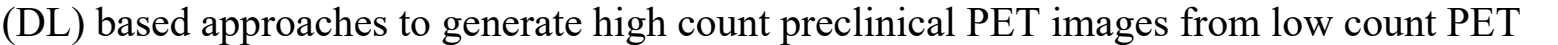

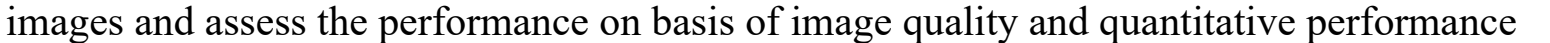

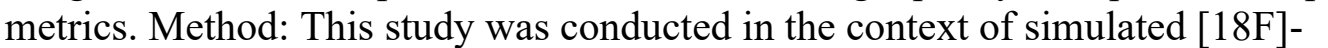

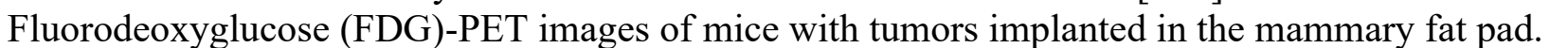

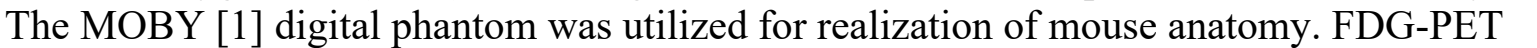

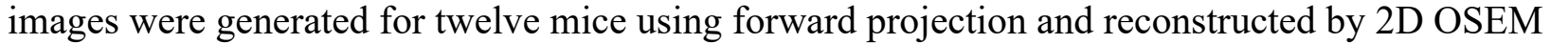

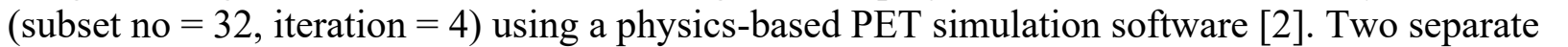

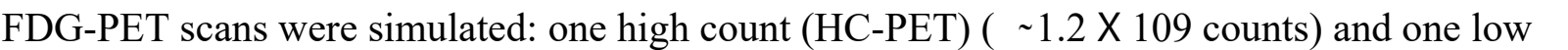

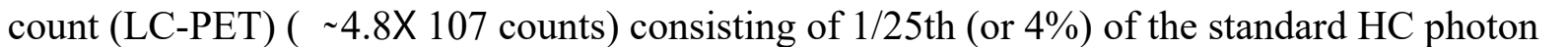

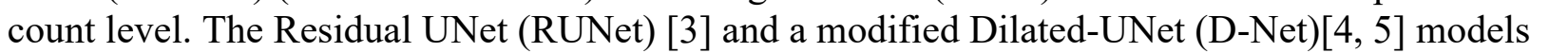

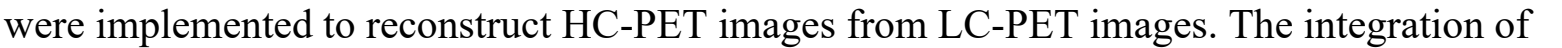

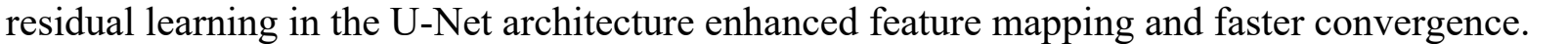

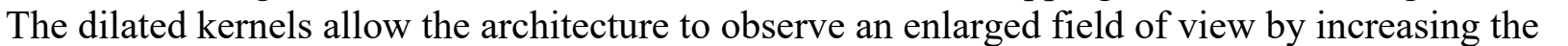

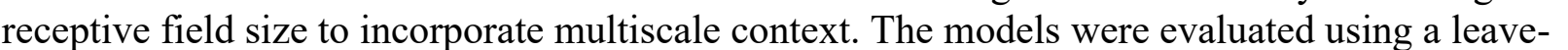

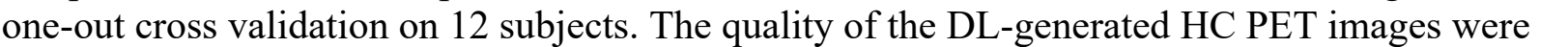

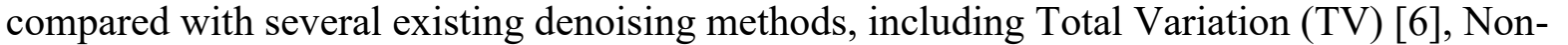

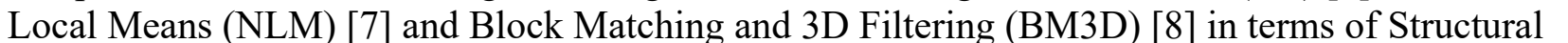

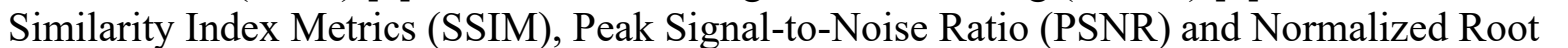

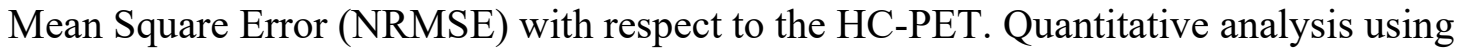

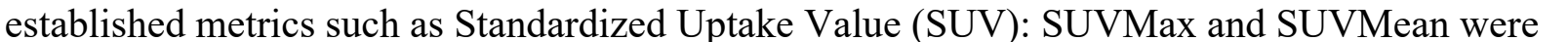

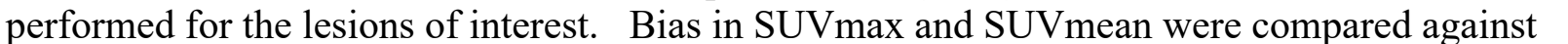

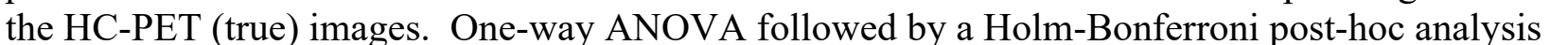

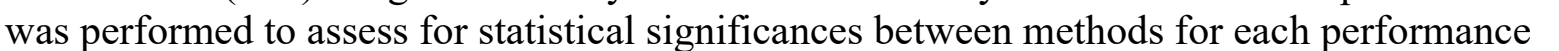
metric with a P Results: Both DL models exhibited significantly better performance $(\mathrm{P} \leq 0.05$, Fig

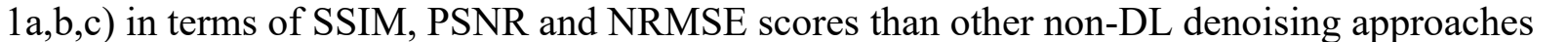

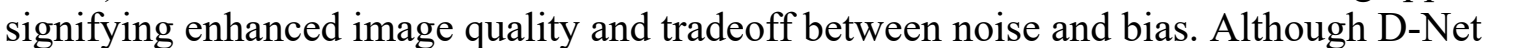

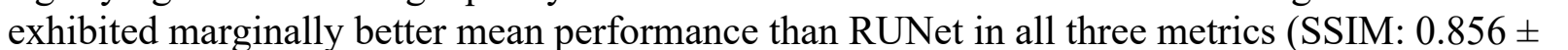

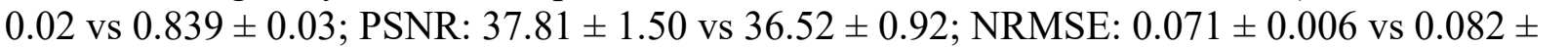

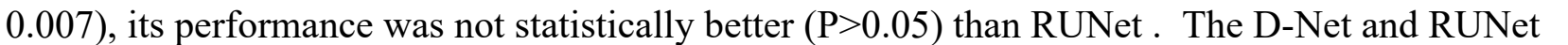

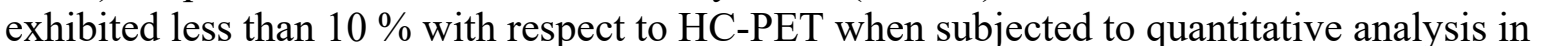

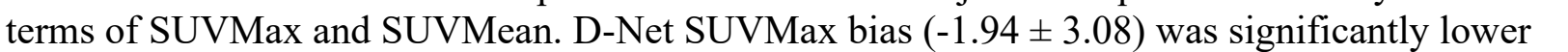

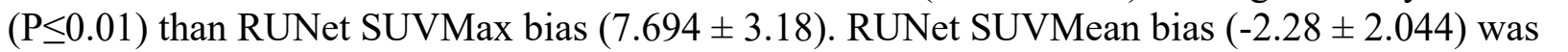

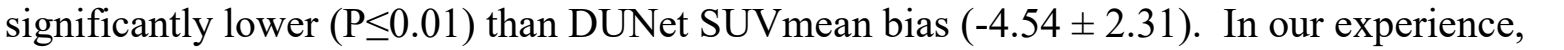




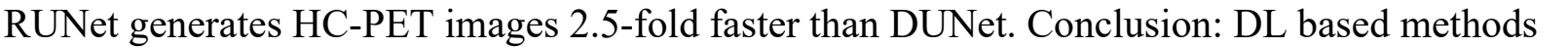

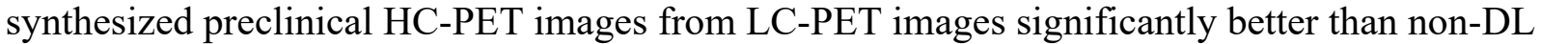

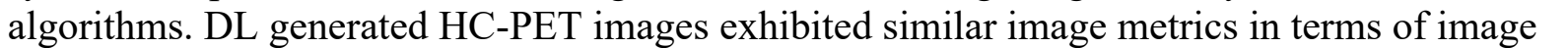

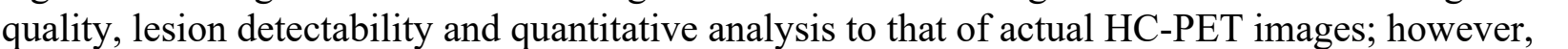

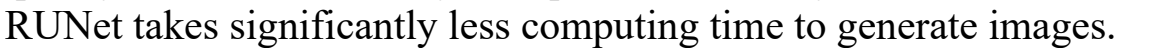

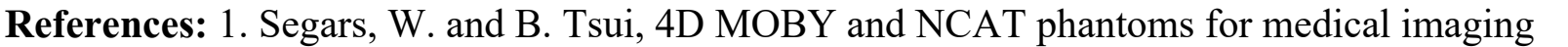

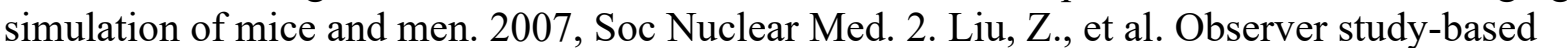

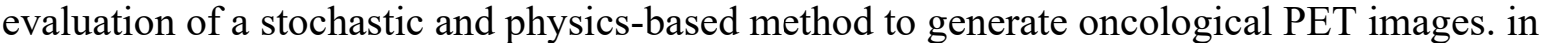

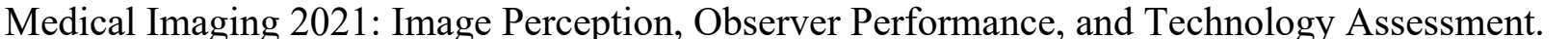

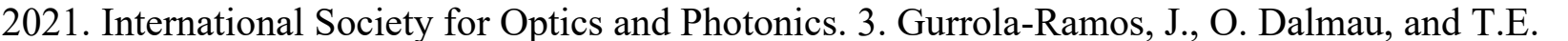

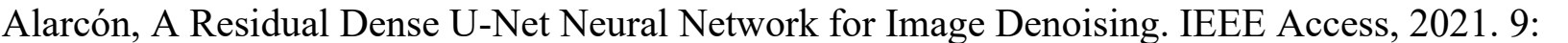

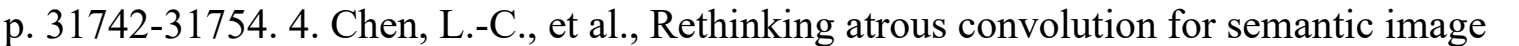

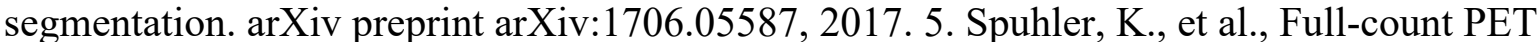
recovery from low-count image using a dilated convolutional neural network. Medical Physics,

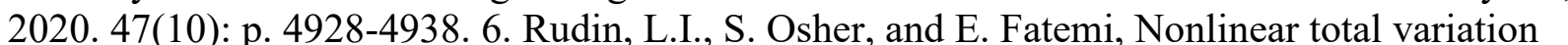

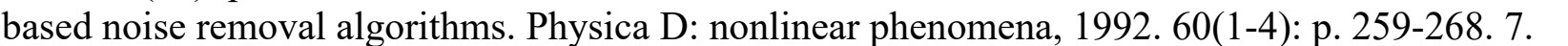

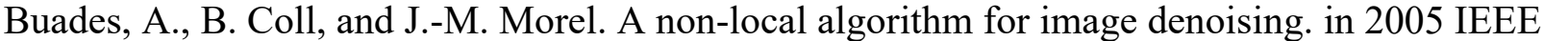

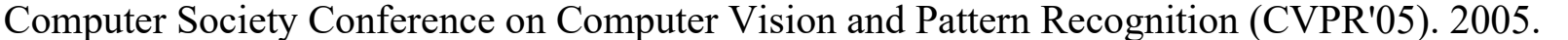

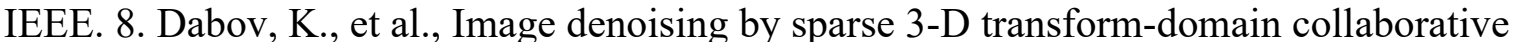

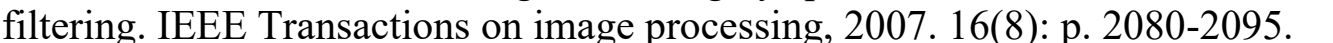

\section{Image/Figure:}

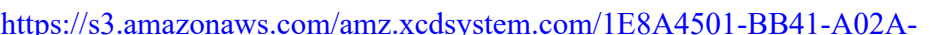

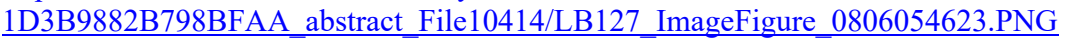

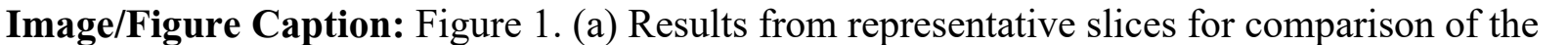

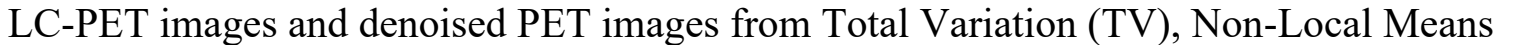

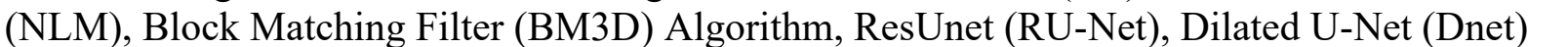

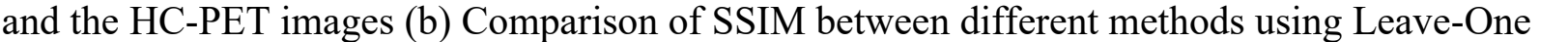

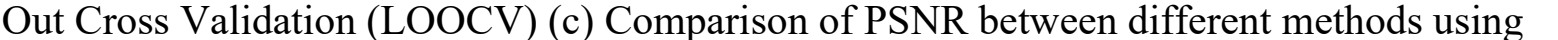

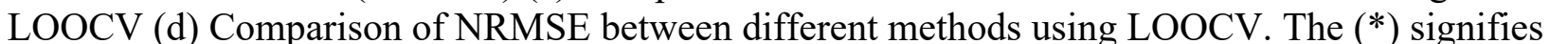
पा एणा

First Name: $\square \square \square\|\| m \mid m$

Last Name: $\square \square \| m$

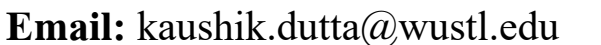

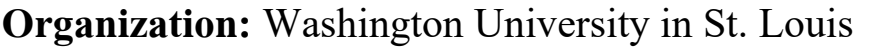

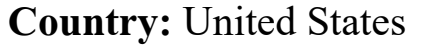




\title{
ID: LB128 \\ Changing Paradigms: Towards More Impactful Discovery of Novel Neuroimmune PET Imaging Biomarkers for Multiple Sclerosis
}

\author{
Poorva Jain, University of Miami Miller School of Medicine, poorva.pj.jain@gmail.com
}

\section{Category: $\square \square \square\|\square\| ा\|\square\| ा \square$}

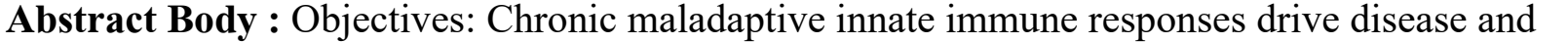

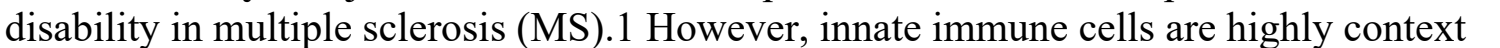

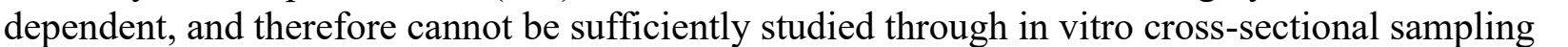

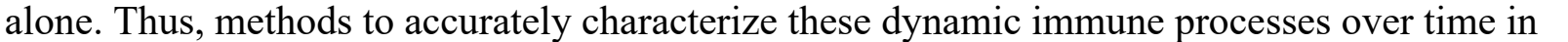

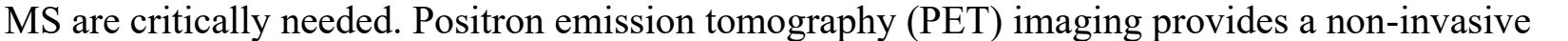

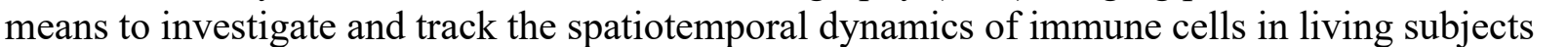

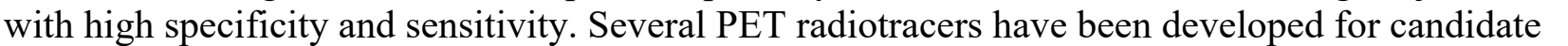

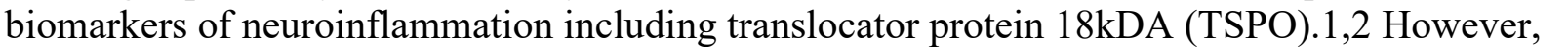

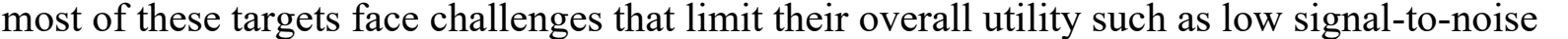

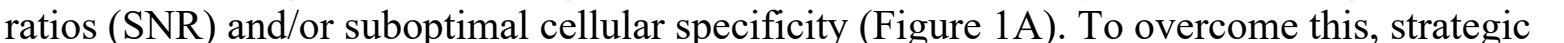

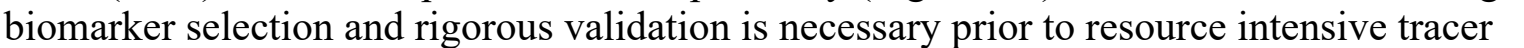

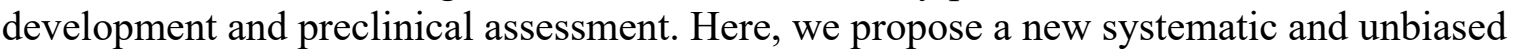

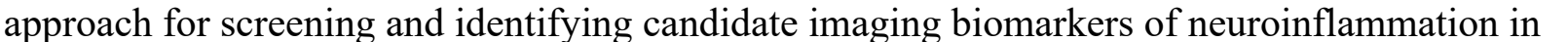

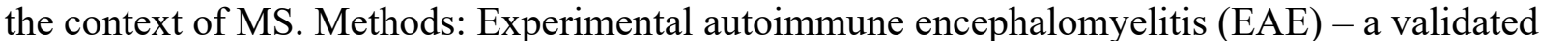

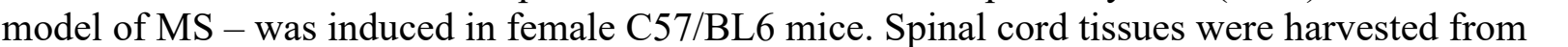

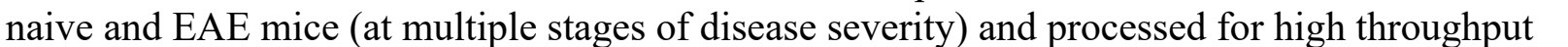

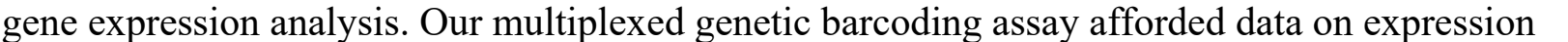

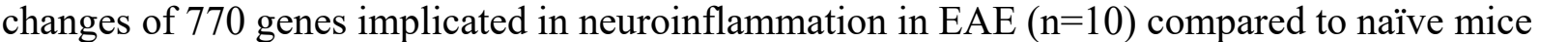

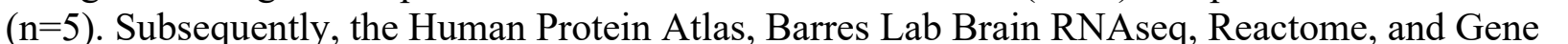

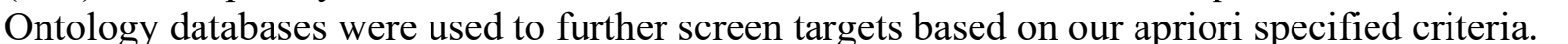

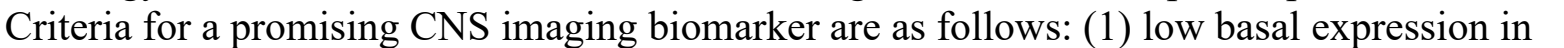

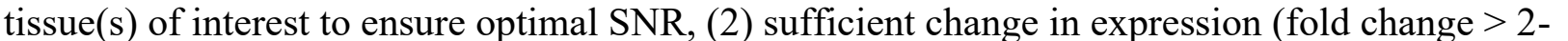

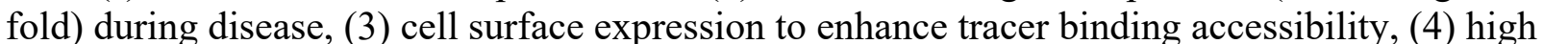

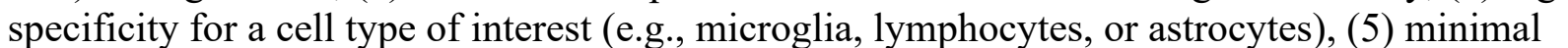

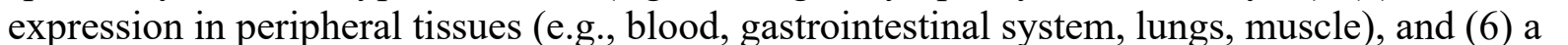

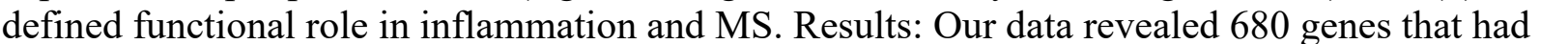

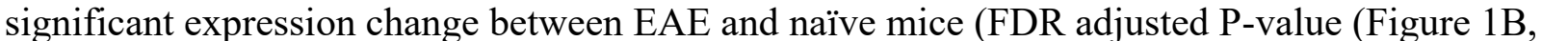

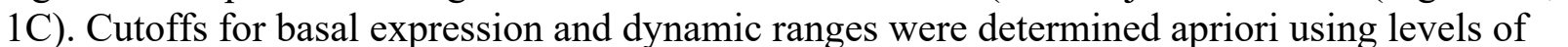

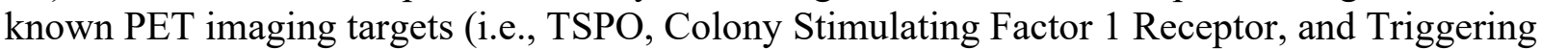

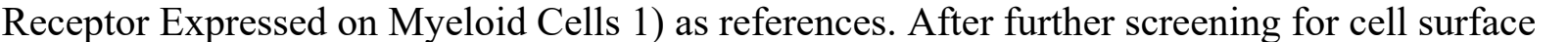

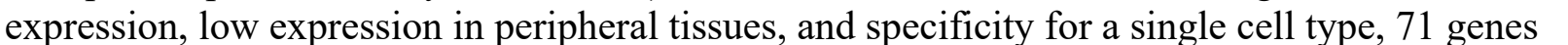

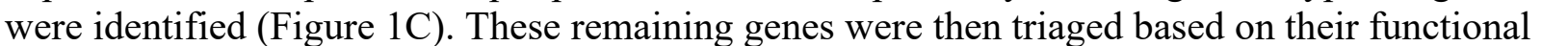

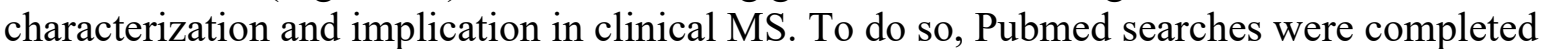
using target name and the following terms: "Neuroinflammation", "Inflammation" $\square \square \square \square C N S "$,

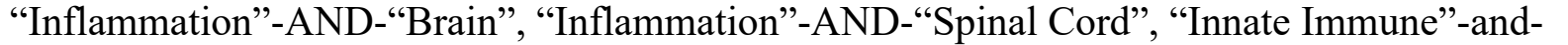

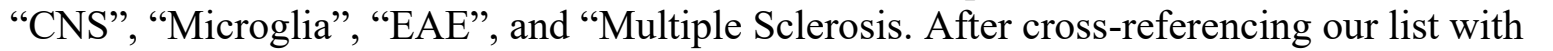




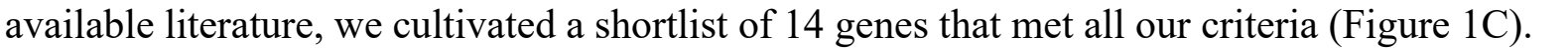

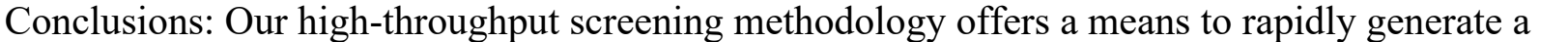

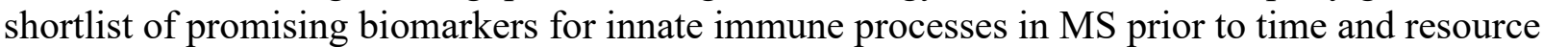

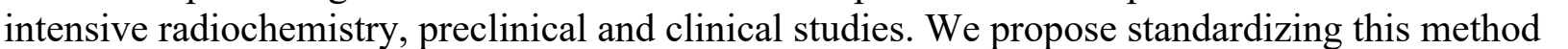

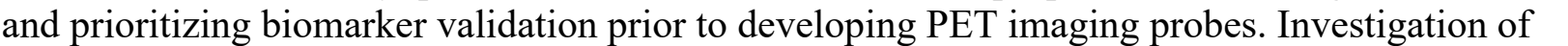

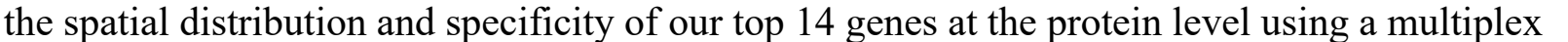

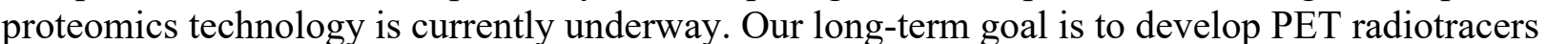

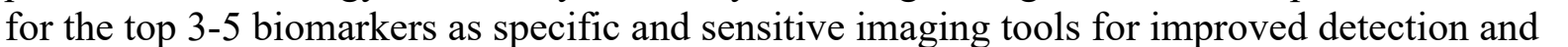

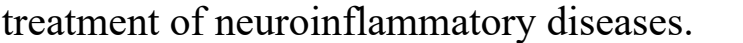

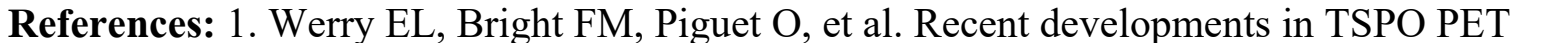

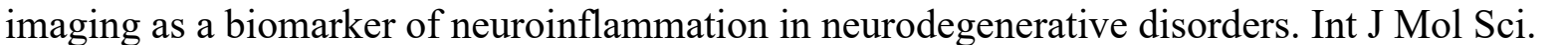

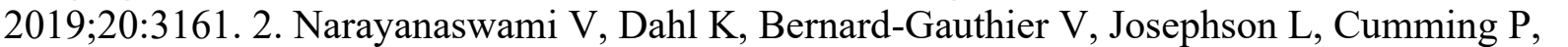

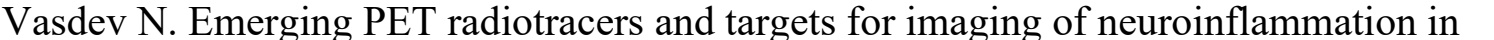

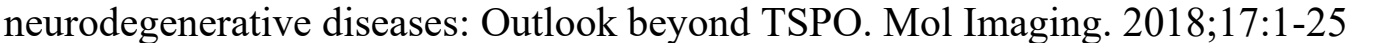

\section{Image/Figure:}

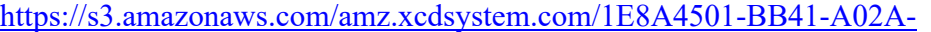

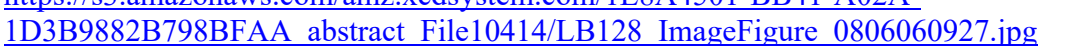

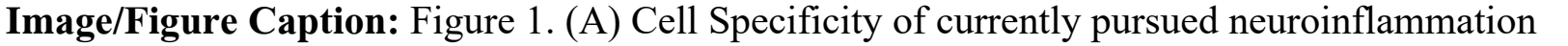

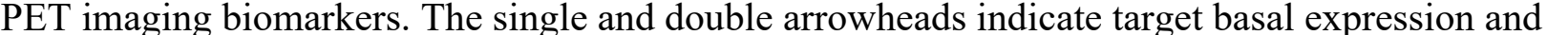

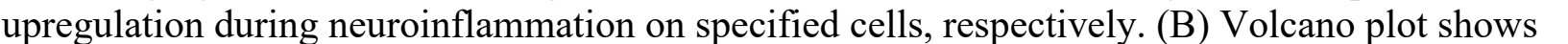

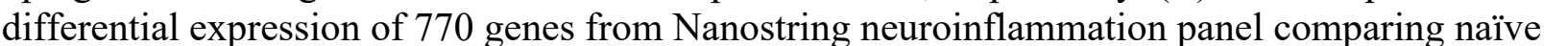

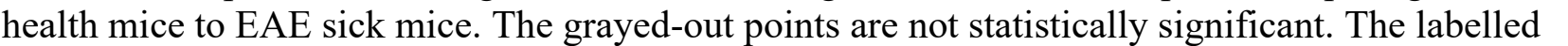

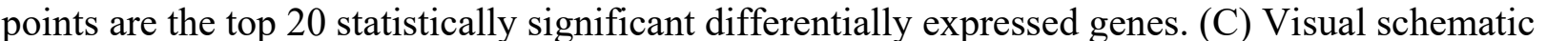

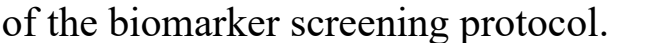

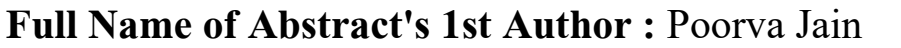

First Name: $\square \square \mid\|\| \| \square$

Last Name: $\square \square \| \square$

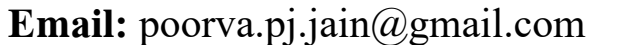

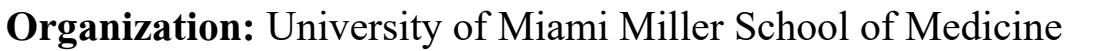

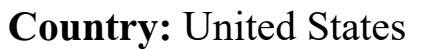




\title{
ID: LB129 \\ Deuterium Metabolic Imaging of [6,6'-2H2] Fructose Metabolism in Mouse Liver at $9.4 \mathrm{~T}$
}

\author{
Guannan Zhang, Memorial Sloan Kettering Cancer Center, ZhangG1@mskcc.org
}

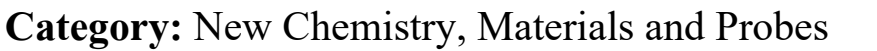

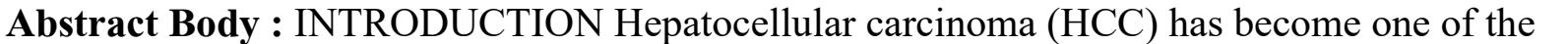

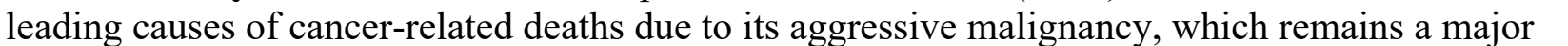

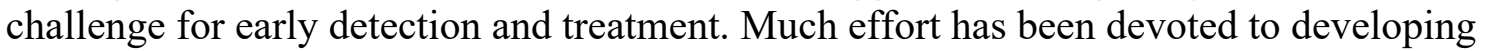

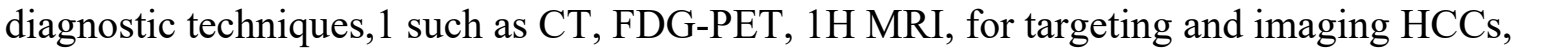

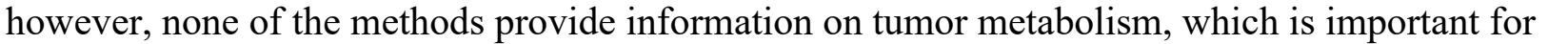

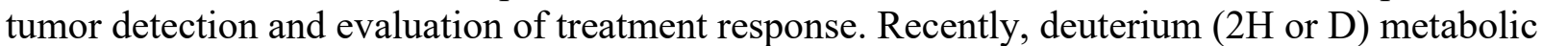

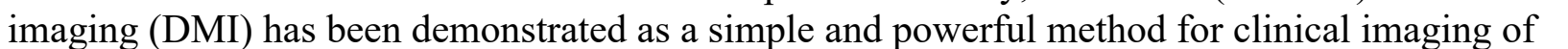

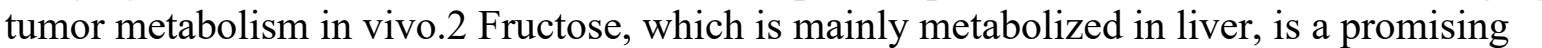
reporter of metabolism in liver tumors. Here, we show dynamics of $[6,6, \square \square \square \square \| \square \square \square \square \square$

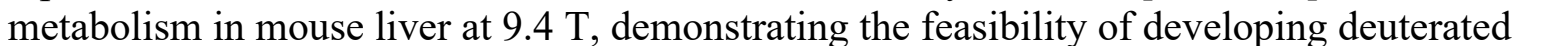

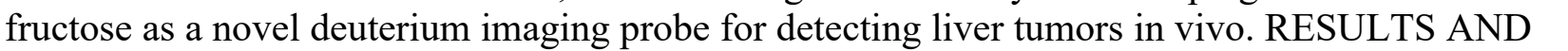

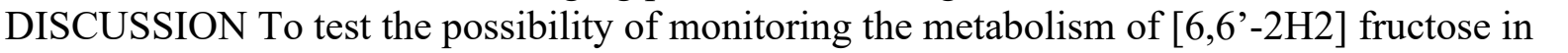

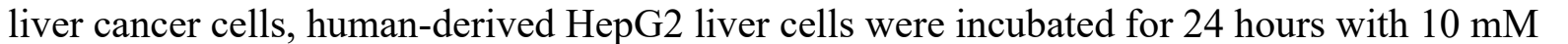

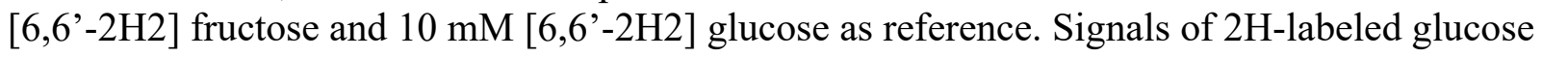

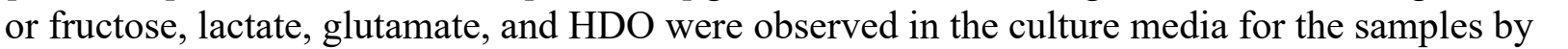

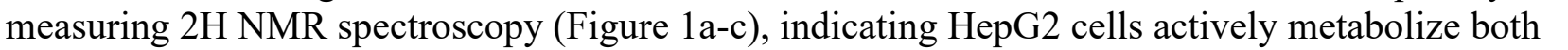

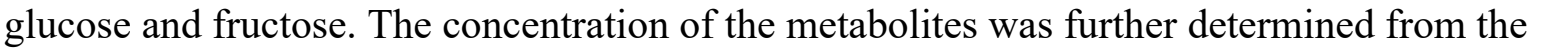

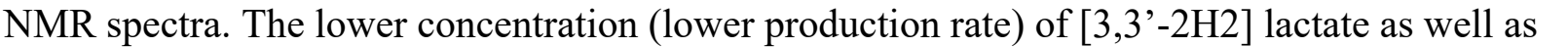

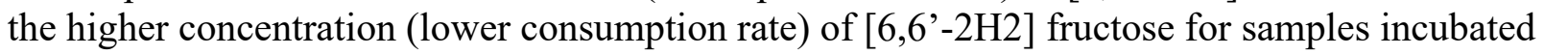

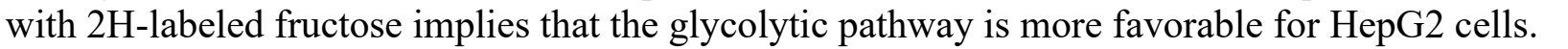

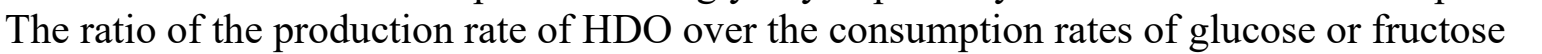

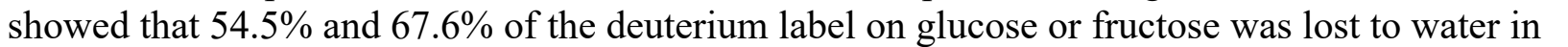

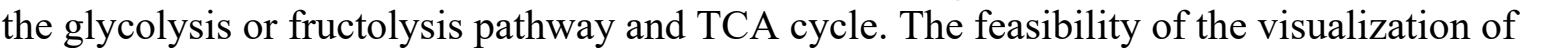
fructose metabolism in vitro allows further study of its metabolism in vivo. Dynamics of [6,6' $\square$

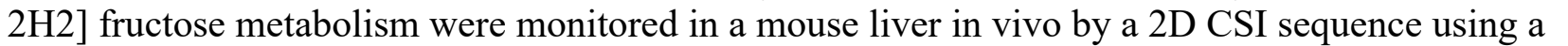

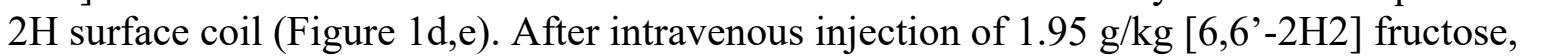

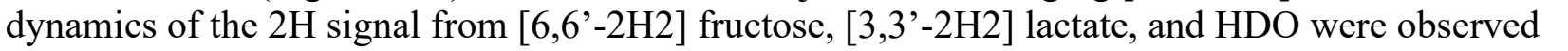

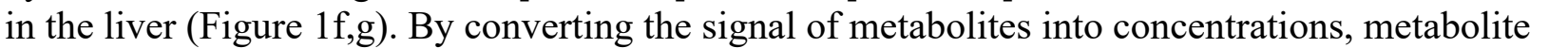

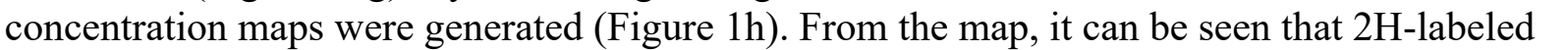

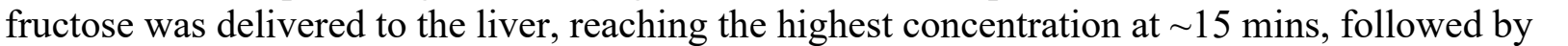

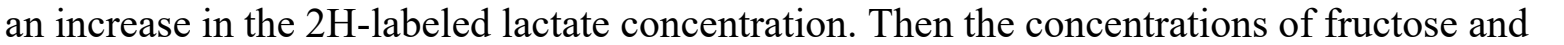

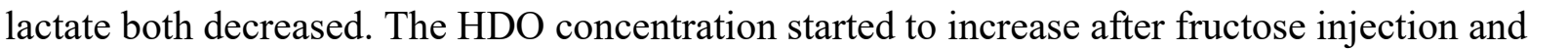

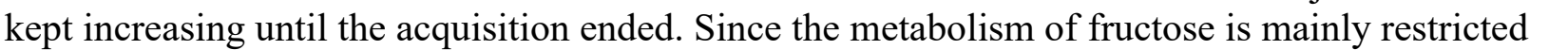

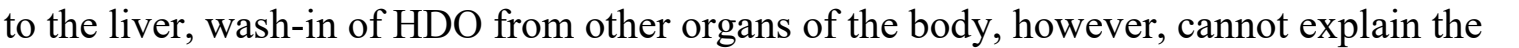

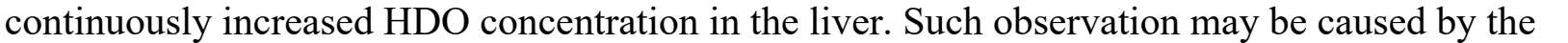

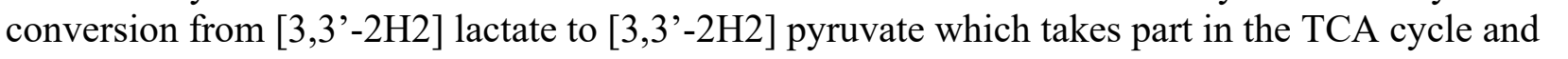


पा

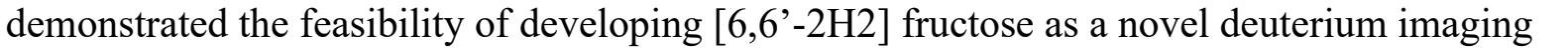

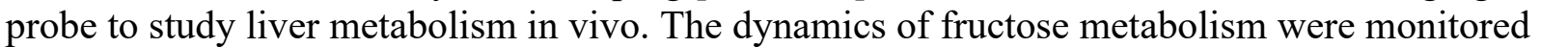

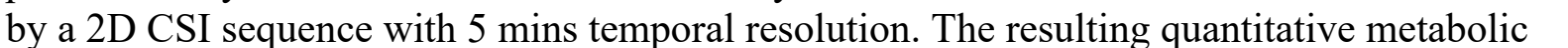

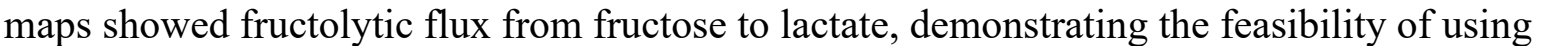

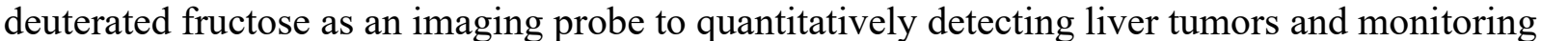

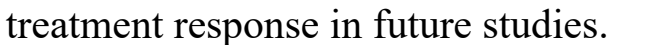

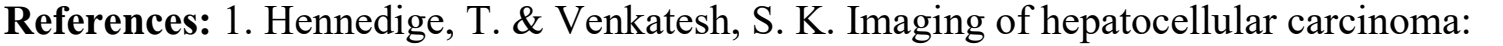

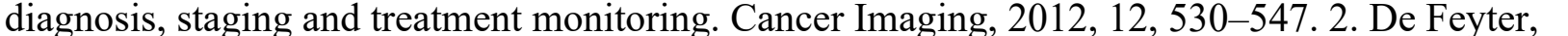

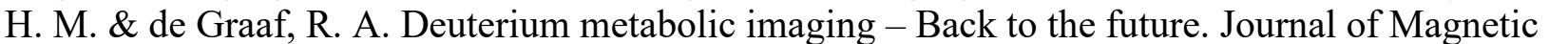

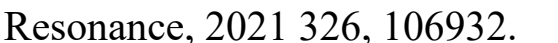

\section{Image/Figure:}

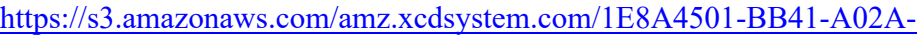

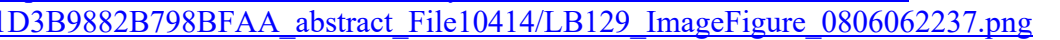

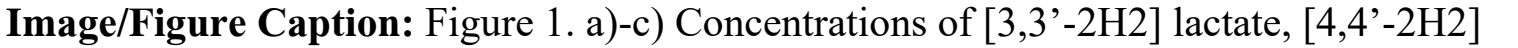
glutamate, HDO, [6, $6^{\prime} \square \square \square$ fructose, and $\left[6,6^{\prime} \square \square \square\right.$ incubating HepG2 cell suspensions with $10 \mathrm{mM}\left[6,6^{\prime} 2 \mathrm{H} 2\right]$ fructose and $10 \mathrm{mM}\left[6,6^{\prime} \mathrm{W} \square \mathrm{D}\right.$

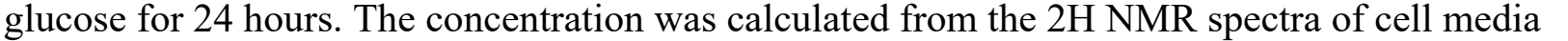

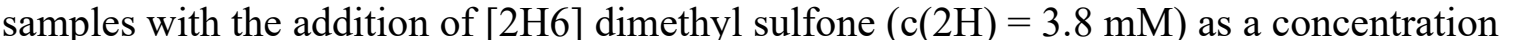
政 $2 \mathrm{H} 2]$ lactate, $\mathrm{HDO}$, and $\left[4,4^{\prime}, 2 \mathrm{H} 2\right]$ glutamate as well as the consumption rates of $\left[6,6^{\prime} \mathrm{W}\right.$ fructose and $\left[6,6^{\prime}, 2 \mathrm{H} 2\right]$ glucose. c) The ratio of $\mathrm{HDO}$ production to $\left[6,6^{\prime} 2 \mathrm{H} 2\right]$ fructose or $\left[6,6^{\prime} \square\right.$

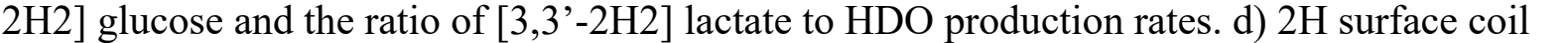

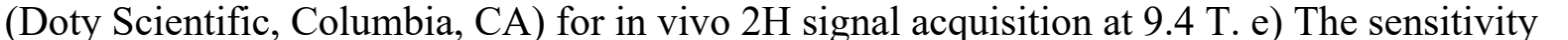
region of the surface coil is $24 \square 24 \square 10 \mathrm{~mm} 3$ which is determined by the acquisition of a spin $\square$

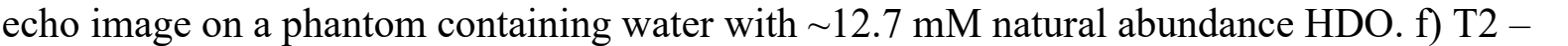

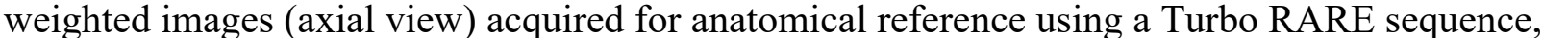
with $35 \times 25 \mathrm{~mm} 2$ field of view, $0.18 \times 0.17 \mathrm{~mm} 2$ spatial resolution. g) Dynamics of the $2 \mathrm{H}$

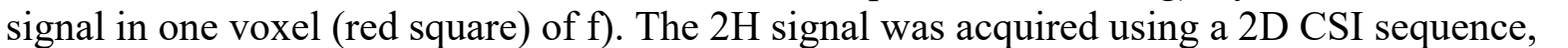
axial, $40 \times 40 \mathrm{~mm} 2$ field of view, $5 \times 5 \mathrm{~mm} 2$ in $\square 101 \mathrm{~m}$

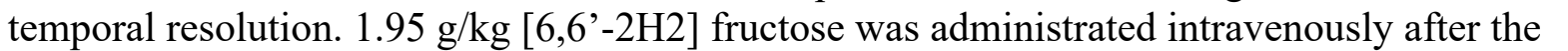

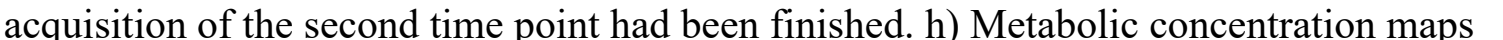

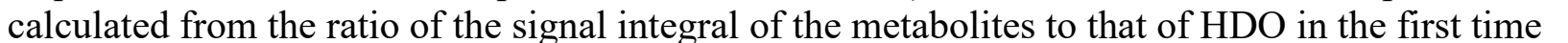

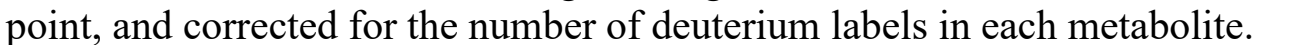

First Name: $\square \square \square\|\| \|$

Last Name: $\square \square \square \mid \Pi$

Email: $\square \square\|\square \square \square \square\|\|\|\|\|$

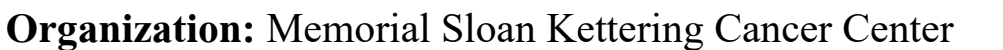




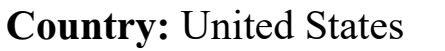




\title{
ID: LB130 \\ Predicting glioblastoma recurrence with hyperpolarized magnetic resonance \\ Travis Salzillo, The University of Texas MD Anderson Cancer Center, tcsalzillo@mdanderson.org
}

\section{Category: $\square \square\|\|\|\| \|$}

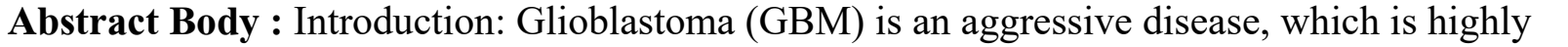

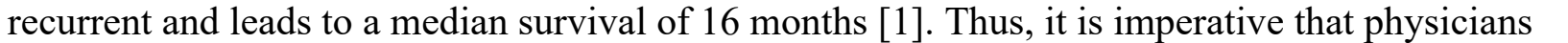

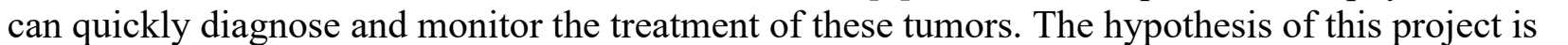

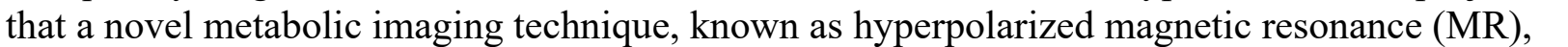

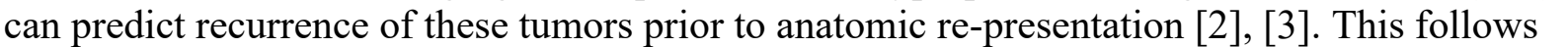

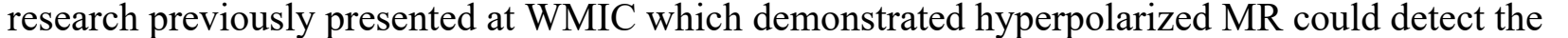

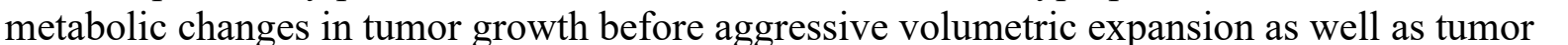
ए

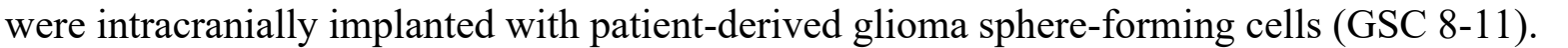

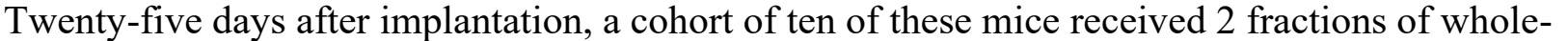

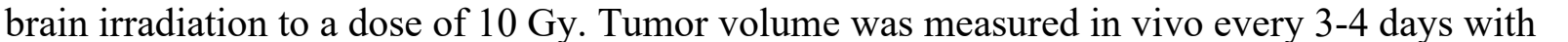

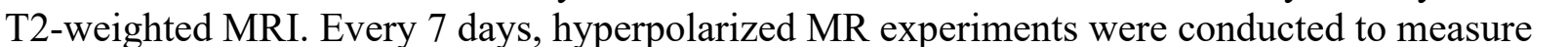

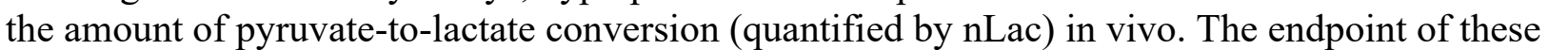

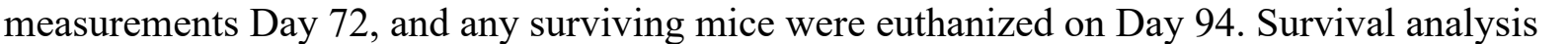

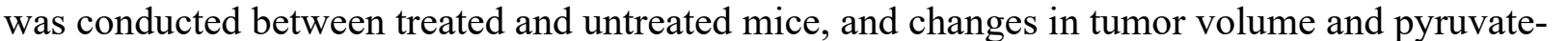

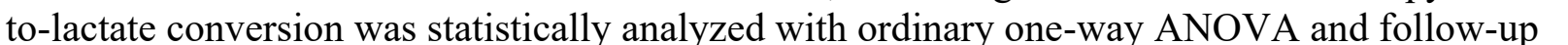

Fisher's Least Significant Difference tests. Tests that produced $\mathrm{p}$ Results: The median survival of

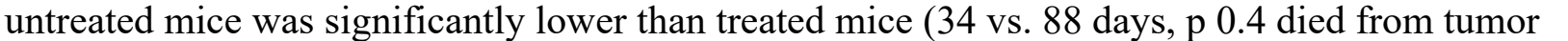

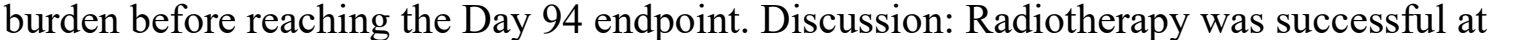

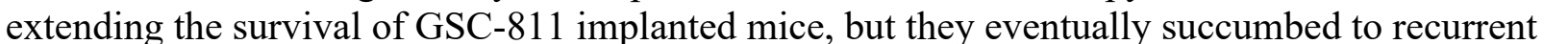

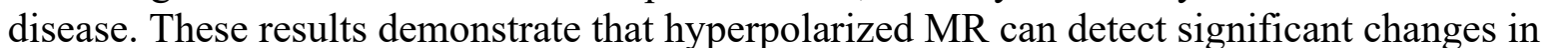

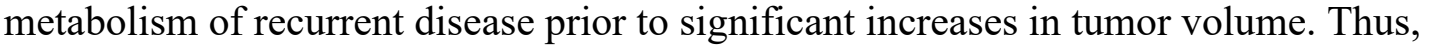

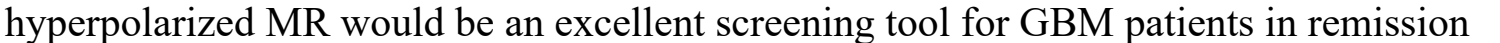

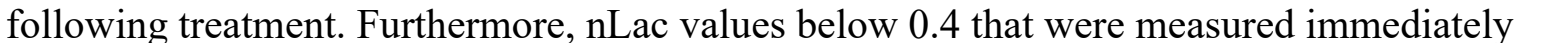

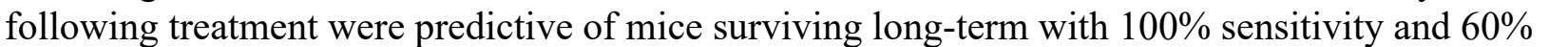

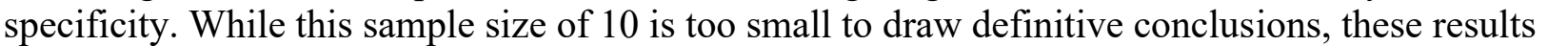

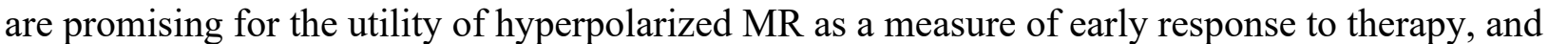

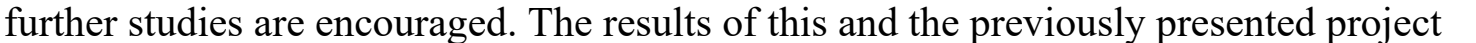

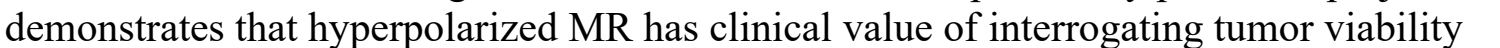

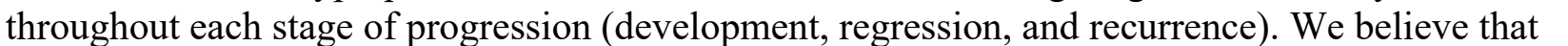

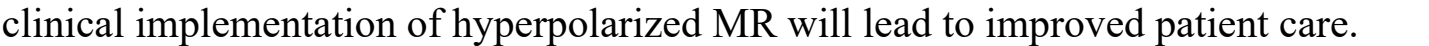

\section{Image/Figure:}




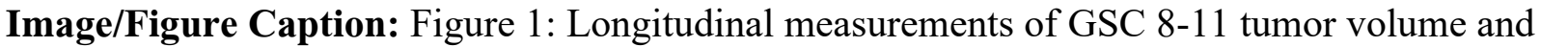

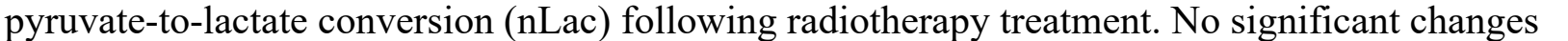

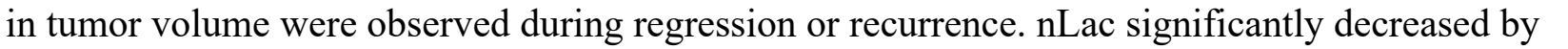

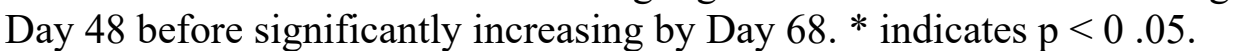

First Name: $\square \square \square \mid \| \square \square$

Last Name: $\square \square\|m\| m$

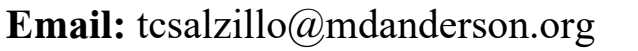

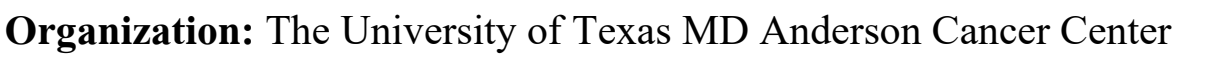

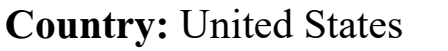




\title{
ID: LB132 \\ ICOS-ImmunoPET Enables Early Diagnosis of Murine Acute Graft-versus-Host Disease
}

\author{
Israt Alam, Stanford University, israt@stanford.edu
}

Category: $\square \square \| m \mid \square$

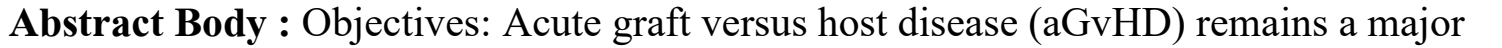

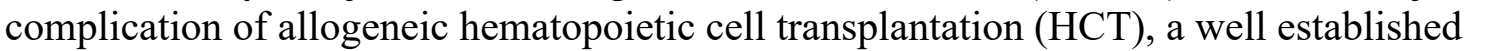

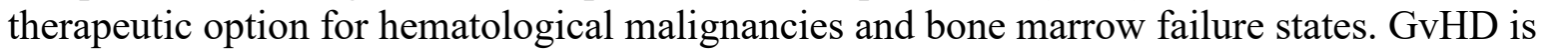

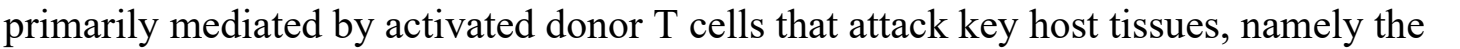

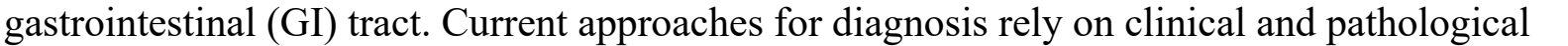

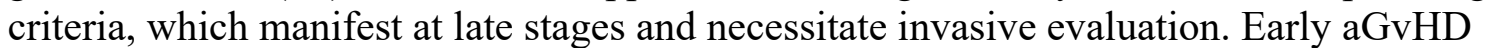

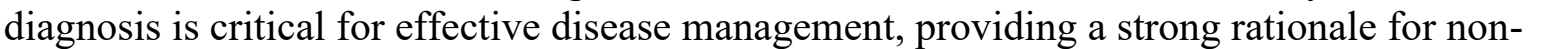

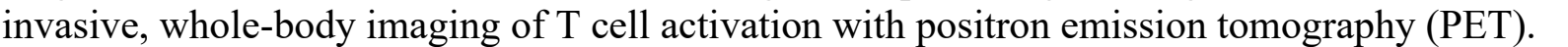

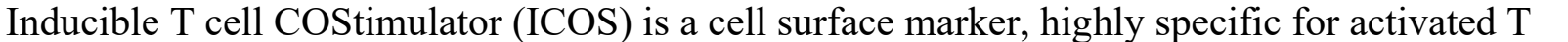

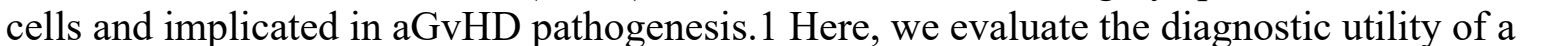
एण प

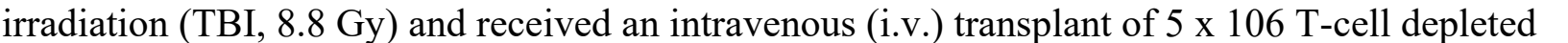

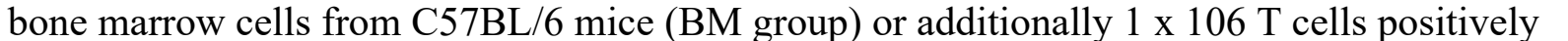

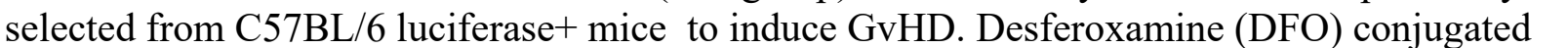

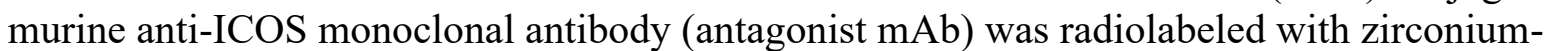
$\square($ mean $1.4 \mathrm{DFOs} / \mathrm{mAb}$, molar activity; $7 \mu \mathrm{Ci} / \mu \mathrm{g}$, radiochemical purity; $>99 \%)$. On day 4 , mice

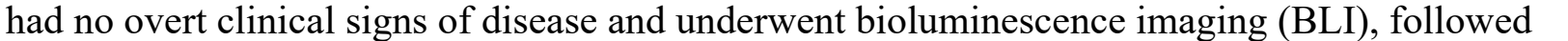

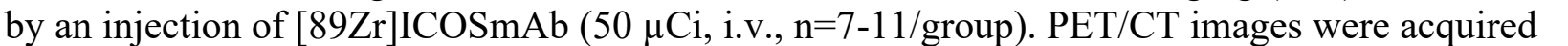

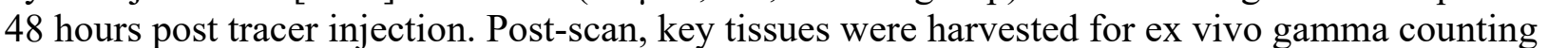

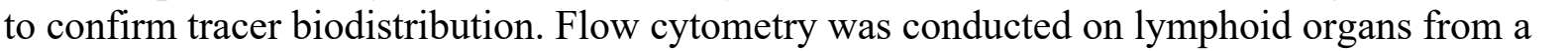

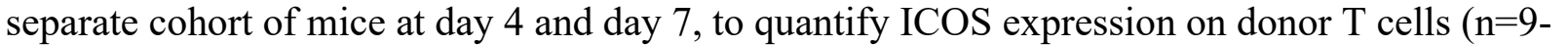

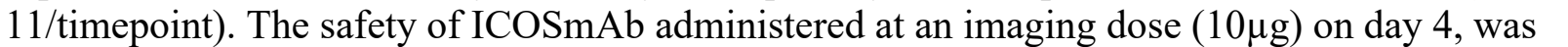

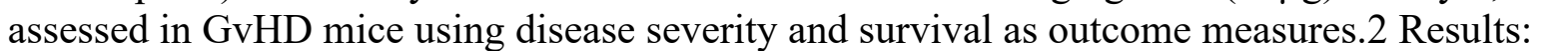

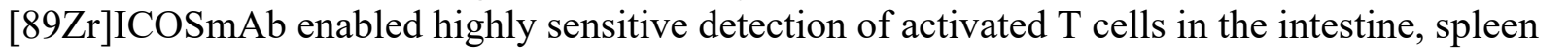

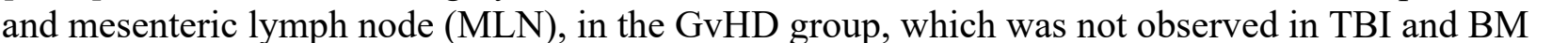
ए

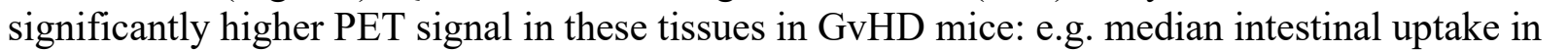

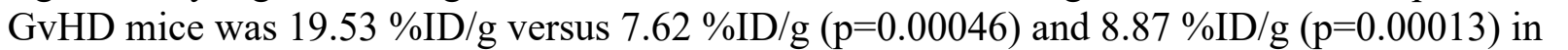

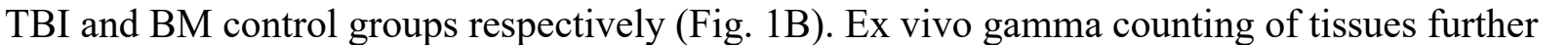

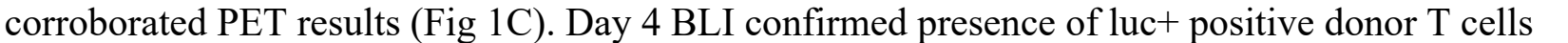

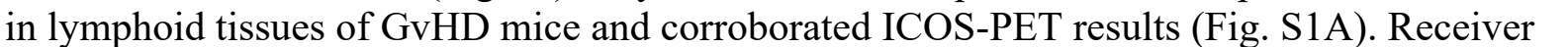

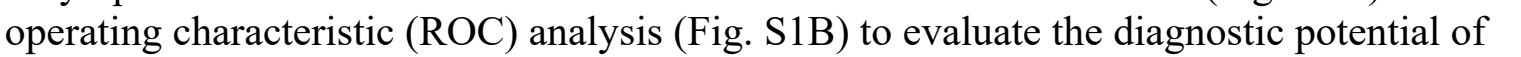

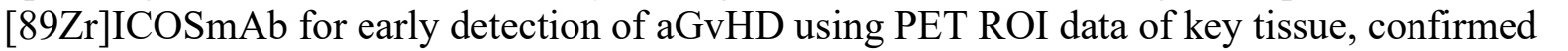

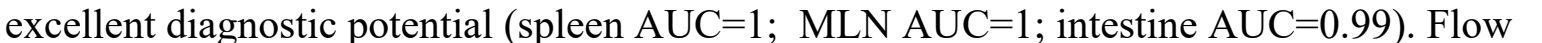

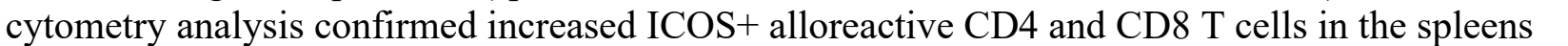




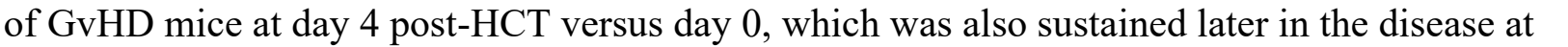

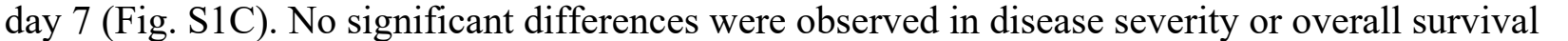
ए पाm

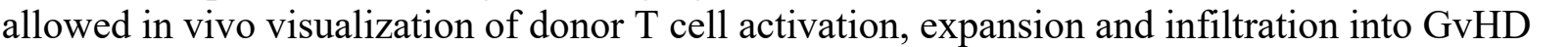

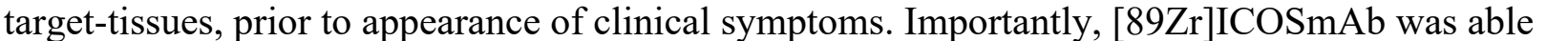

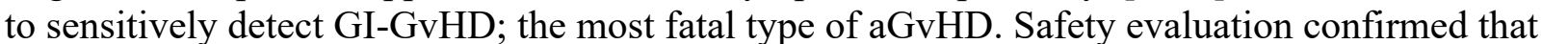

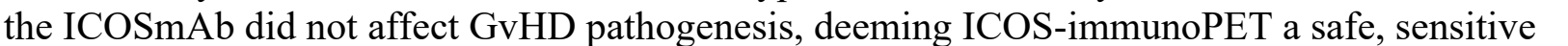
पा

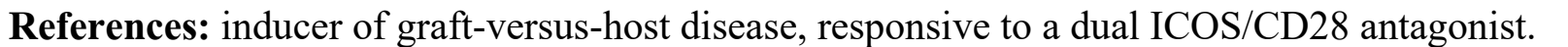

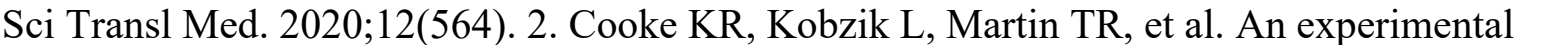

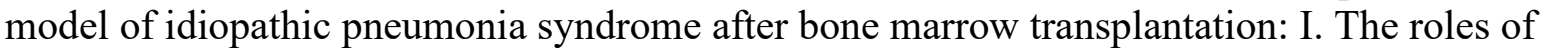

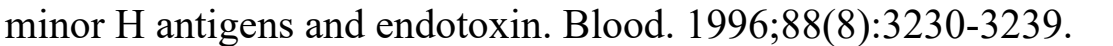

\section{Image/Figure:}

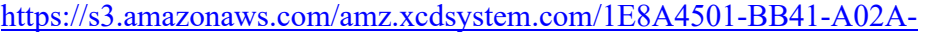

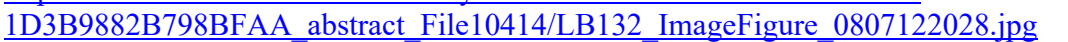

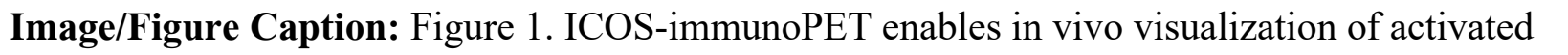

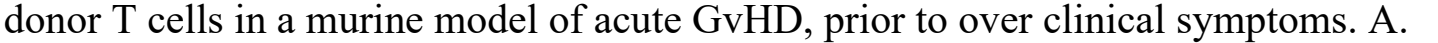

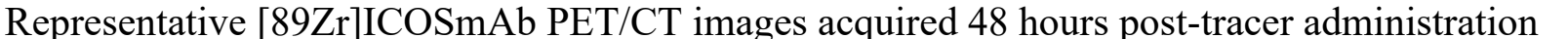

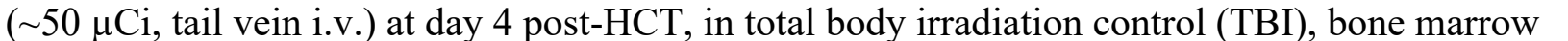

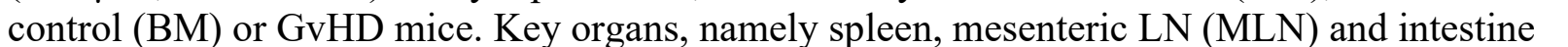

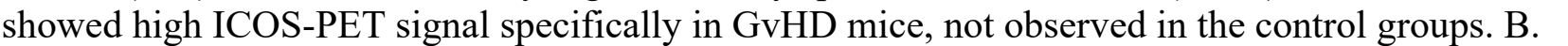

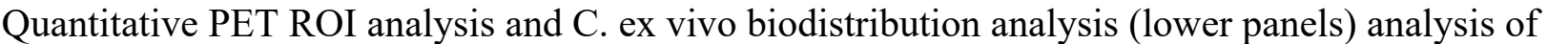

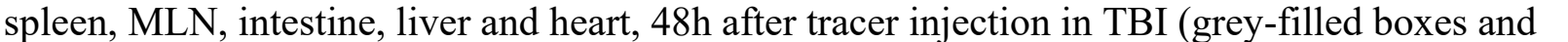

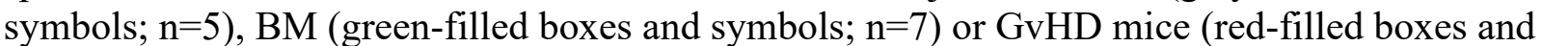

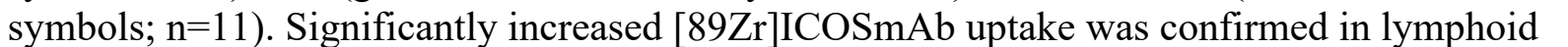

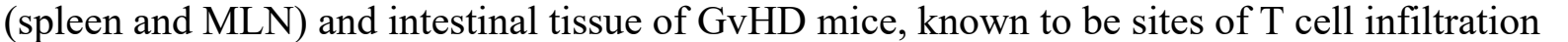

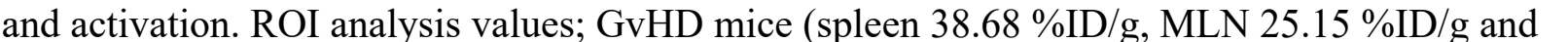
पाm

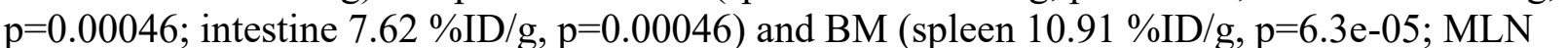

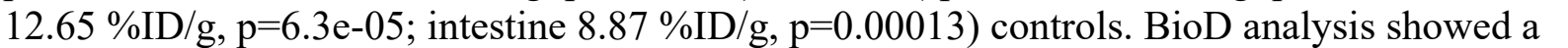

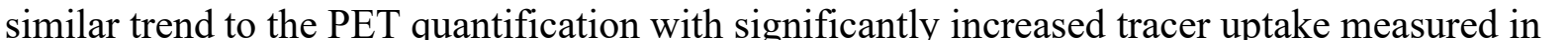

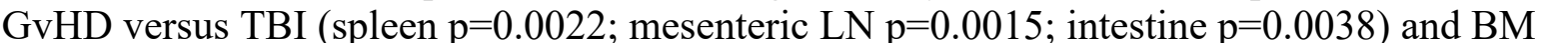

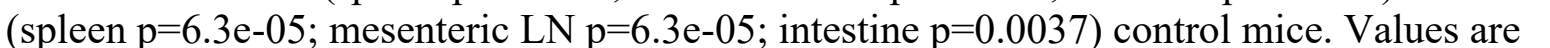

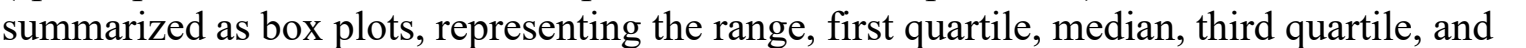

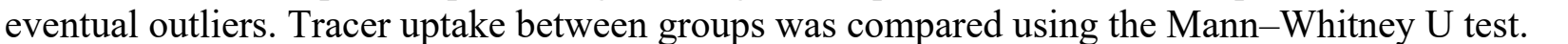

First Name: $\square \| m$

Last Name: $\square \square \square \square$

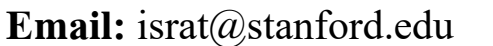




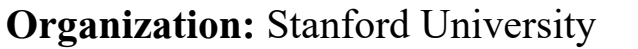

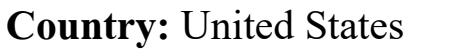




\title{
ID: LB133 \\ Development of a preclinical imaging XNAT-enabled informatics (PIXI) platform for co-clinical imaging research
}

Kooresh Shoghi, Washington University School of Medicine, shoghik@wustl.edu

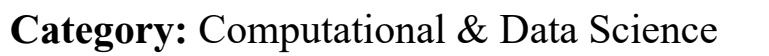

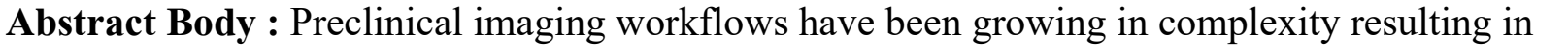

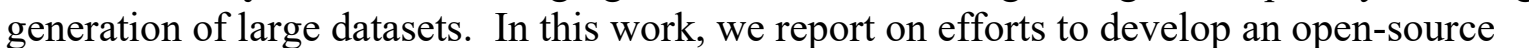

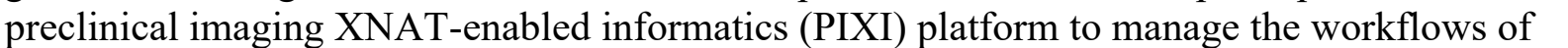

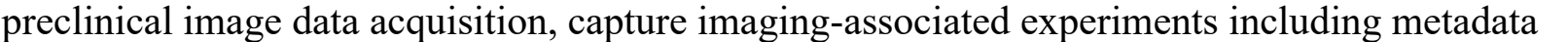

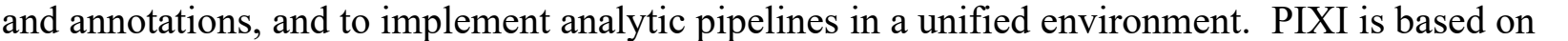

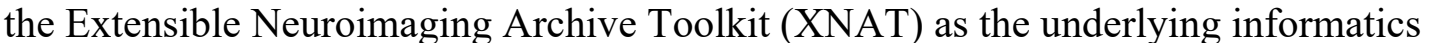

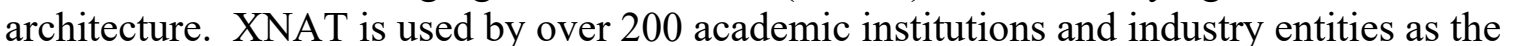

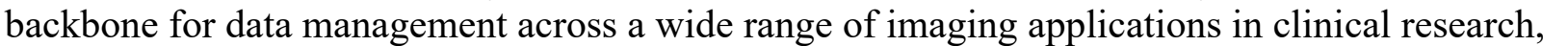

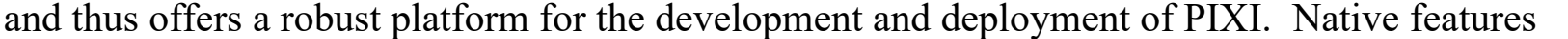
ए

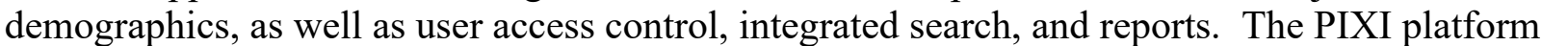
ए

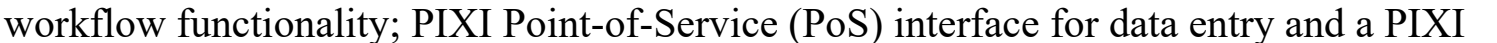

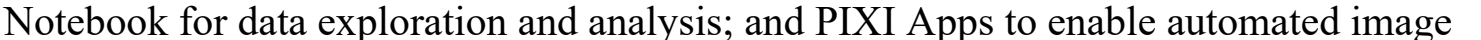

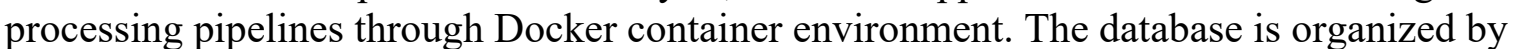

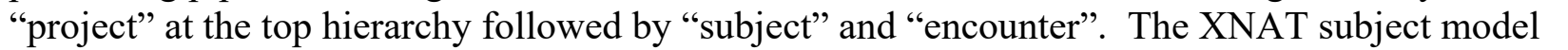

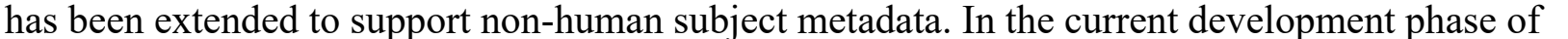

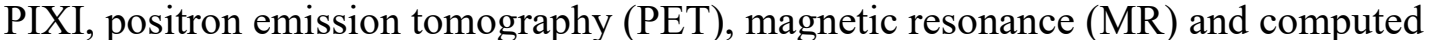

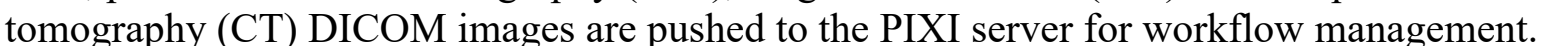

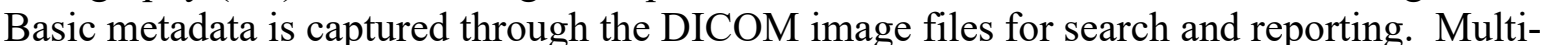

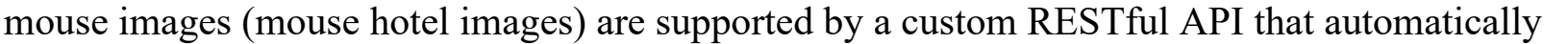

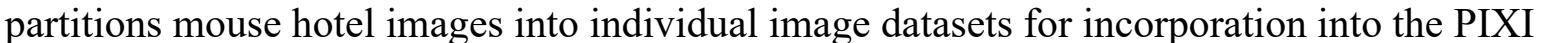

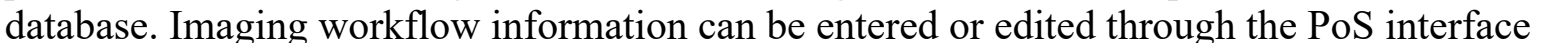

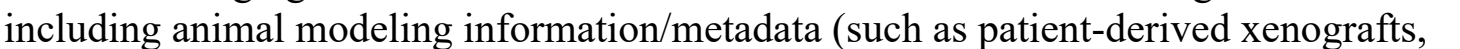

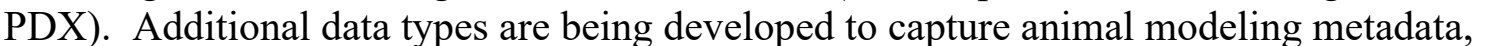

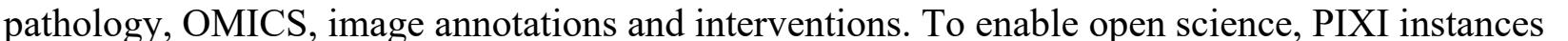

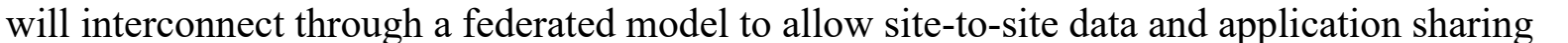

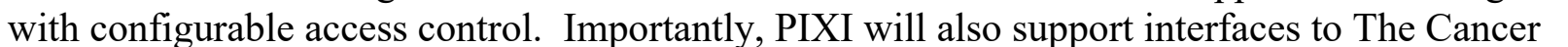

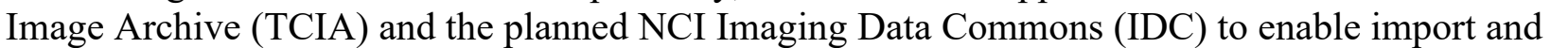

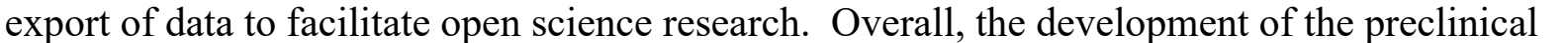

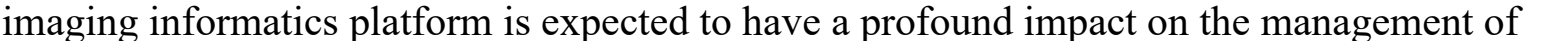

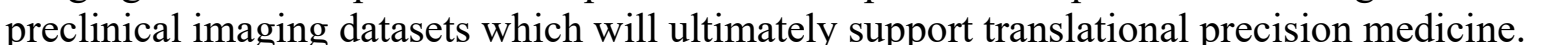

\section{Image/Figure:}




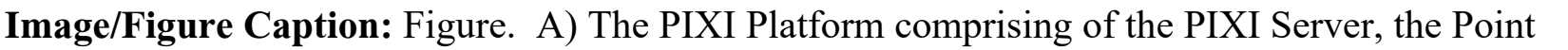

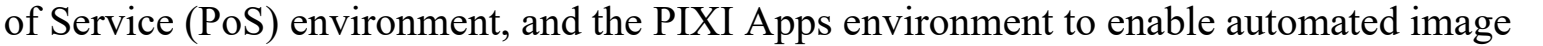

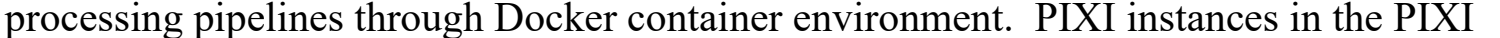

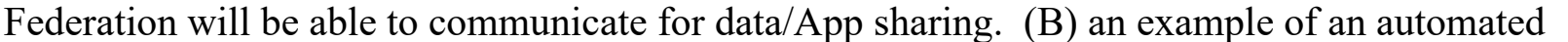

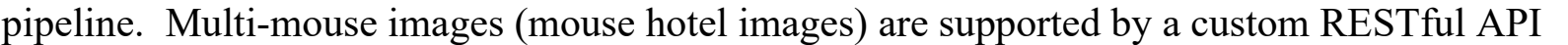

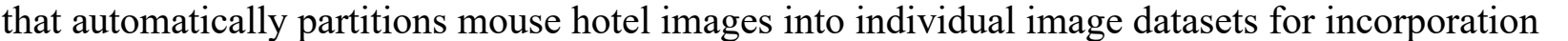

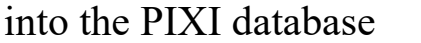

First Name: $\square \square \square\|\|\|\| \|$

Last Name: $\square \square \square \square \| \square$

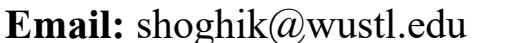

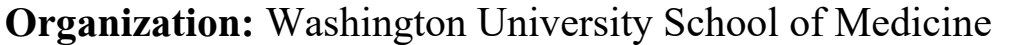

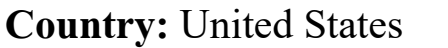




\title{
ID: LB134 \\ Human $\mathbf{T}$ cell iron-oxide nanoparticle labeling for magnetic particle imaging
}

\author{
Maxim Moroz, Stanford University, m.a.moroz@gmail.com
}

Category: $\square \square\|\| \square \square$

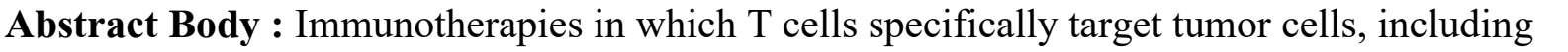

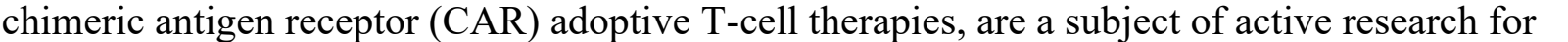

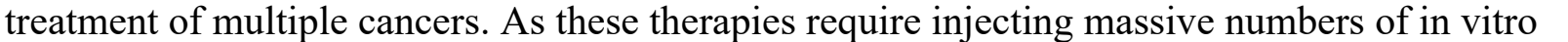

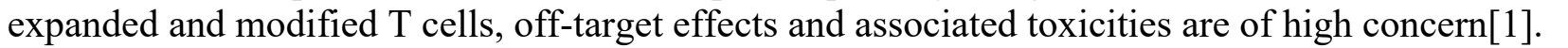

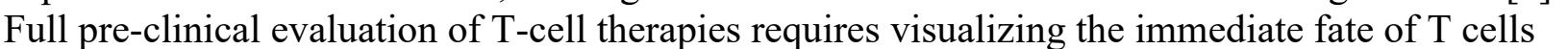

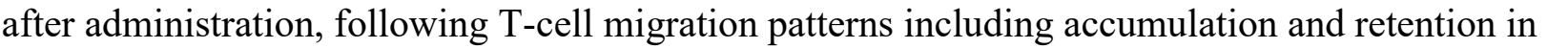

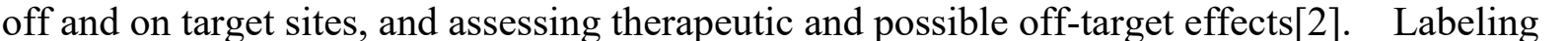

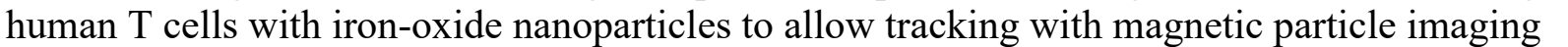

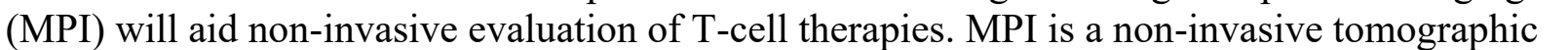

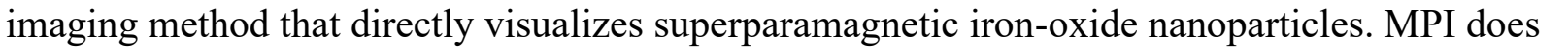

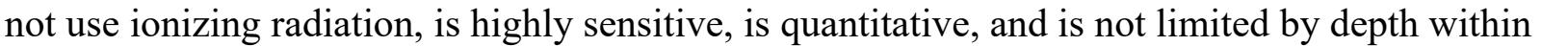
ए

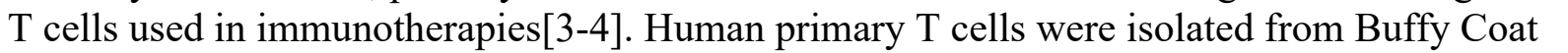
samples obtained from our institution's blood bank using the Ficoll separation method. Cells

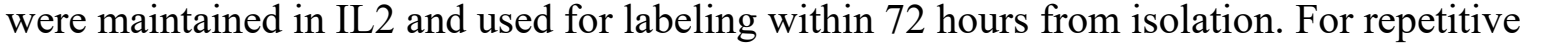

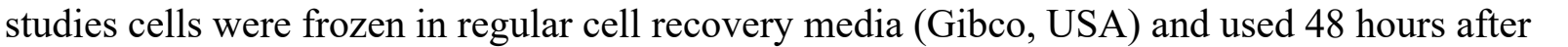
एण

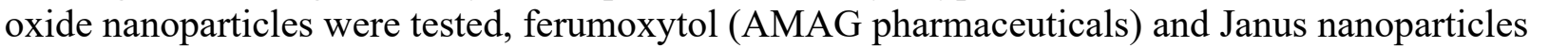

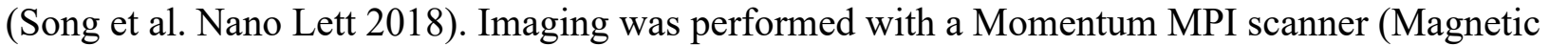

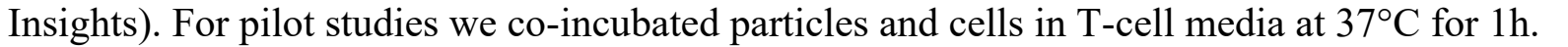

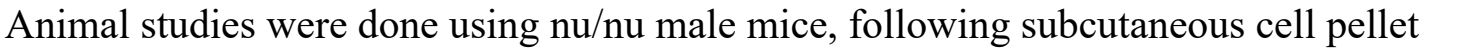

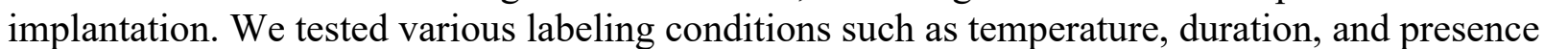

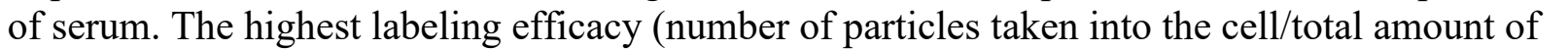

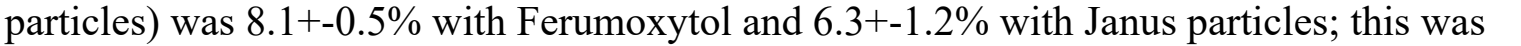

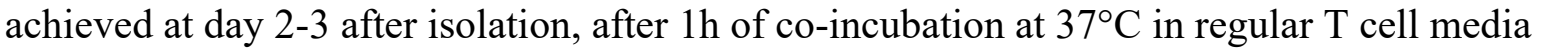

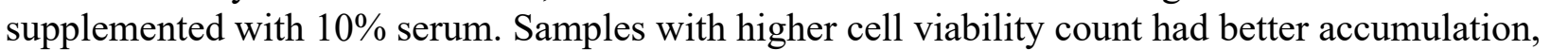

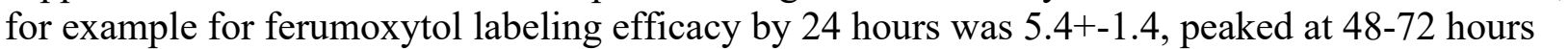

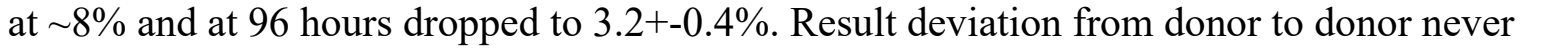

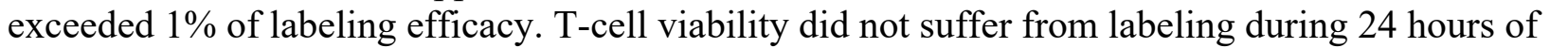

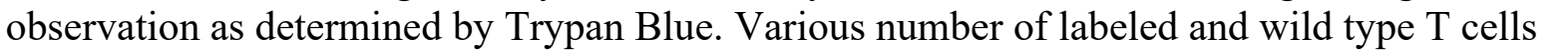

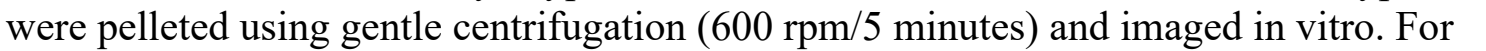

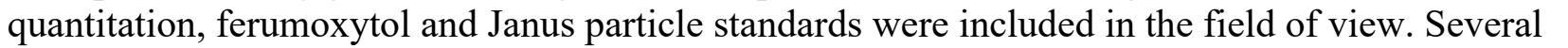

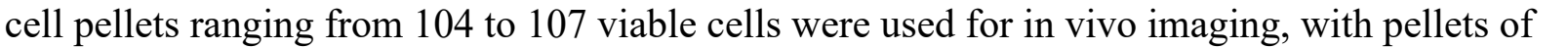

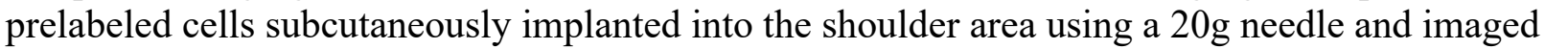

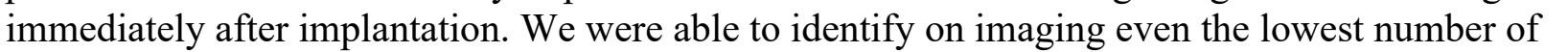

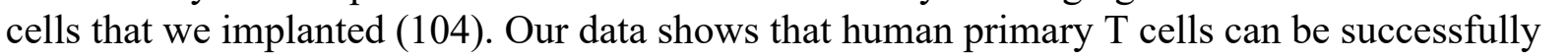

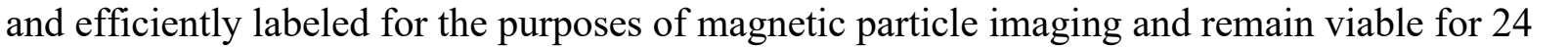




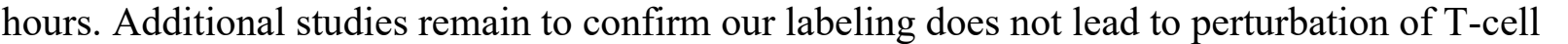
ए

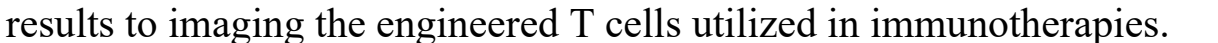

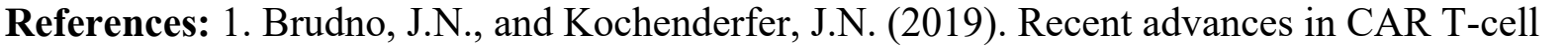

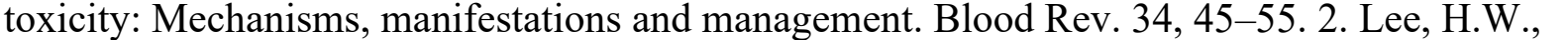

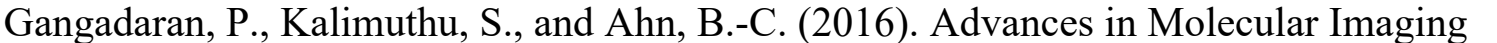

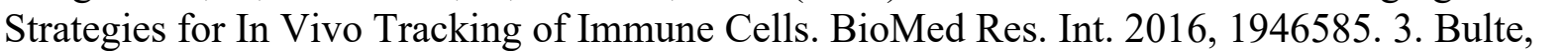

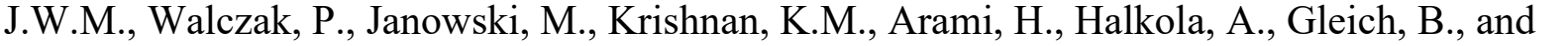

Rahmer, J. (2015). Quantitative "Hot Spot" Imaging of Transplanted Stem Cells u $\square \square \square$

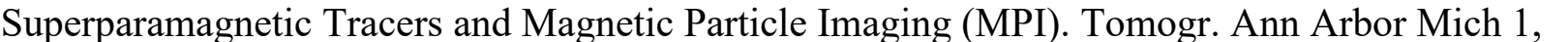
I-97. 4. Newick, K., O’Brien, S., Moon, E., and Albelda, S.M. (2017). CAR T Cell Therapy for

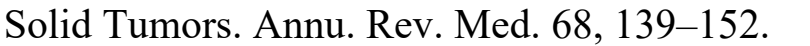

\section{Image/Figure:}

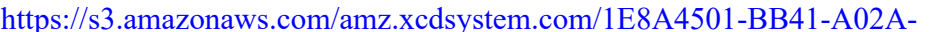

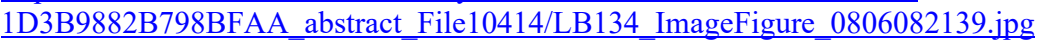

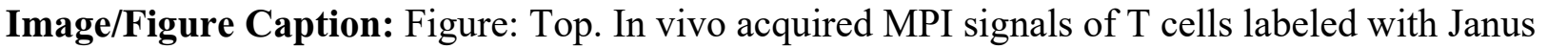

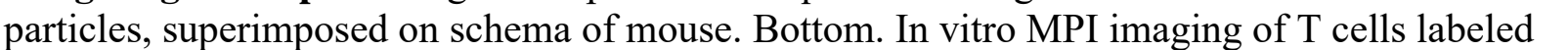

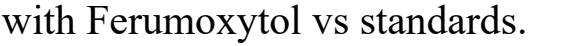

First Name: $\square \square \square \square \square$

Last Name: $\square \square\|\square\| \square$

Email: $\square \square \square \square \square \square \square \square \square ा \Pi \square \square$

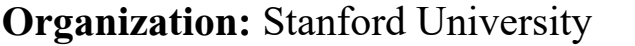

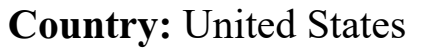




\title{
ID: LB135 \\ Four-Channel Multispectral Optoacoustic Tomography Imaging for the \\ Detection and Characterization of Murine Melanoma
}

\author{
Ammar Bahauddin, Auburn University, dr.ammar.b@hotmail.com
}

Category: $\square \square \| \square \square ा \square$

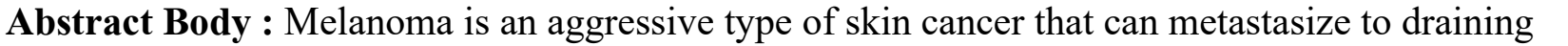

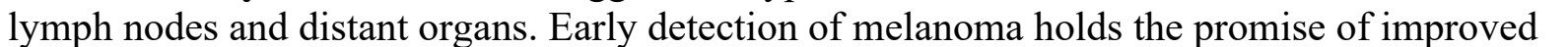

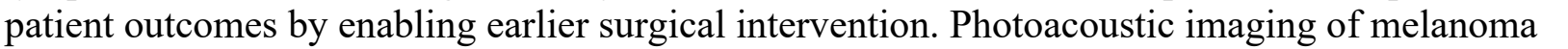

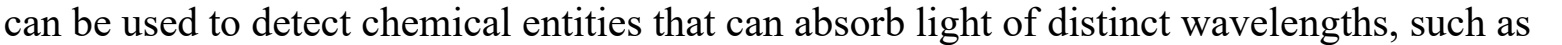

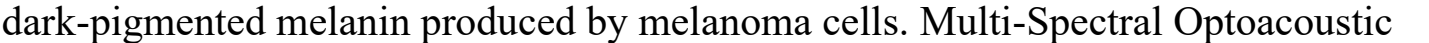

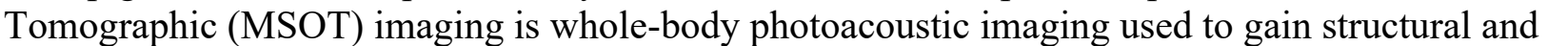

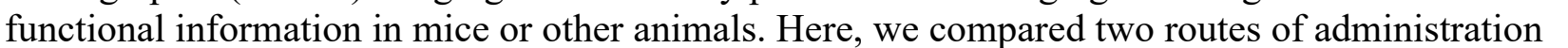

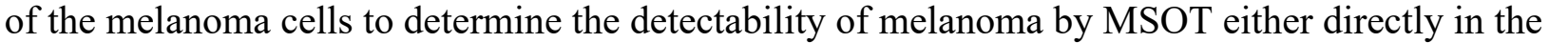

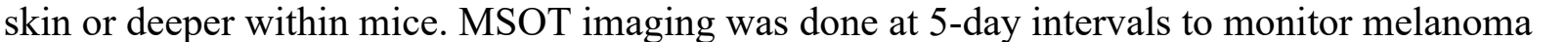

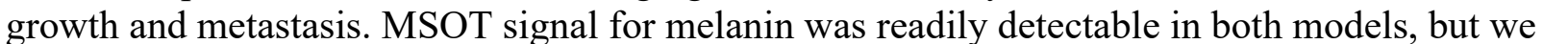

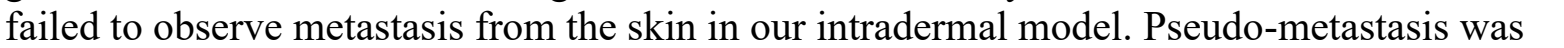

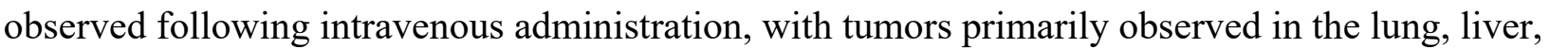

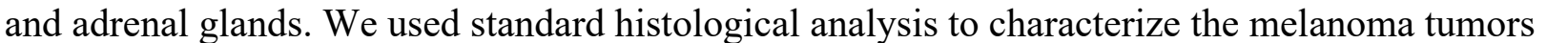

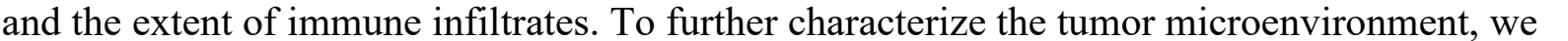

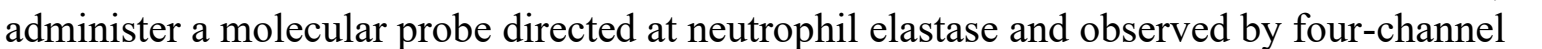

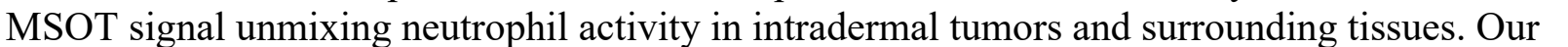

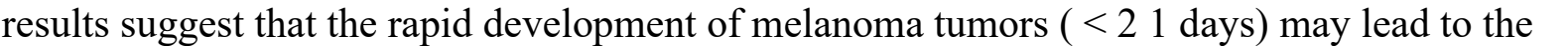

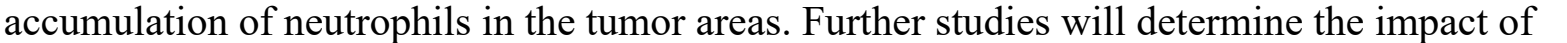

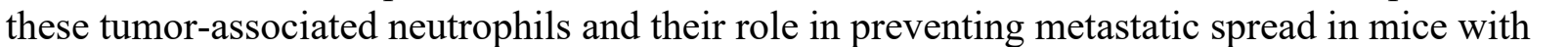

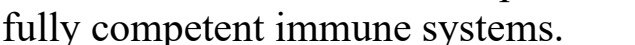

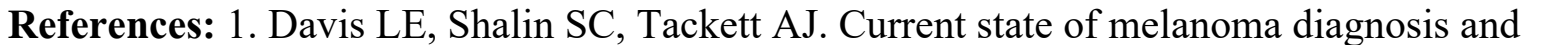

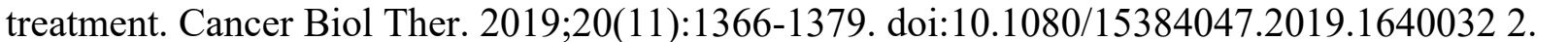

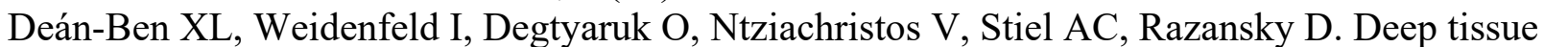

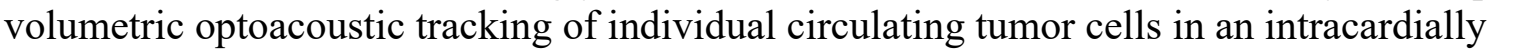

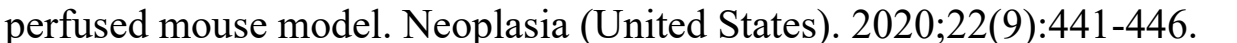

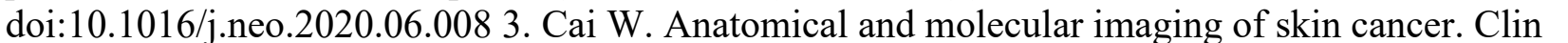

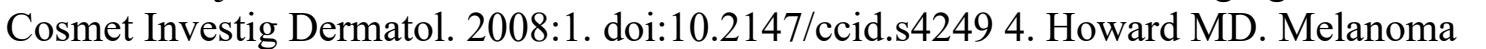

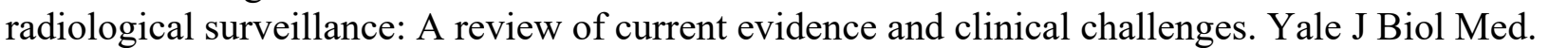

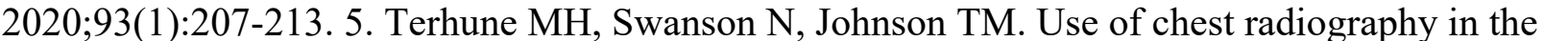
ए

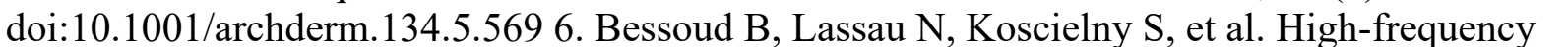

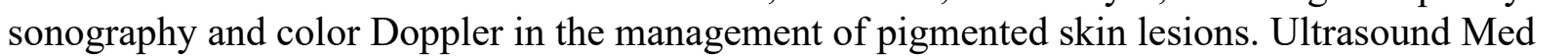

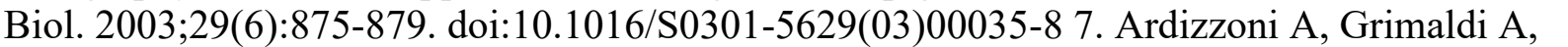

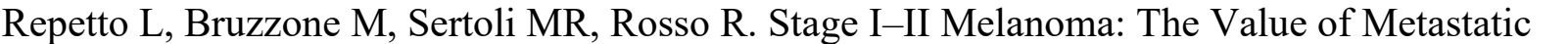

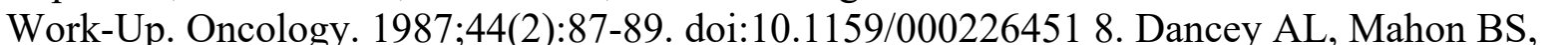




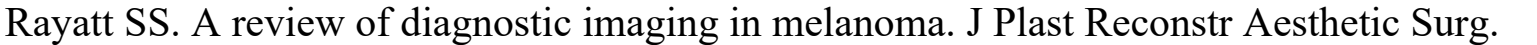

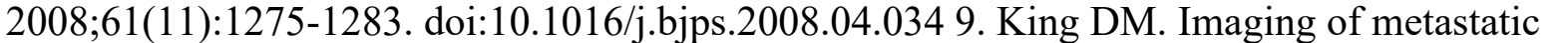

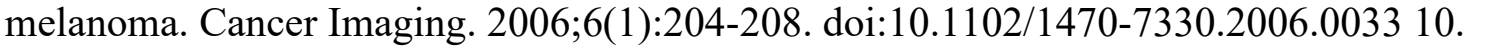

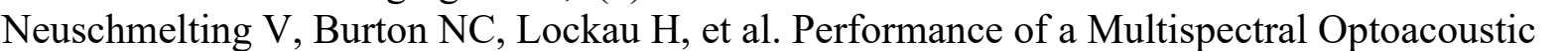

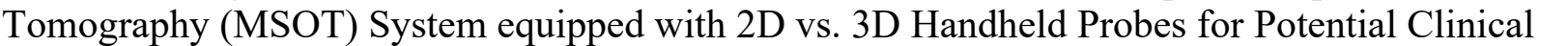

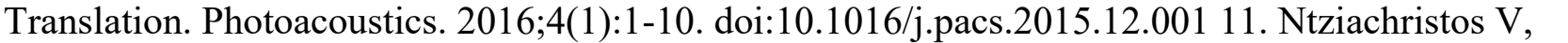

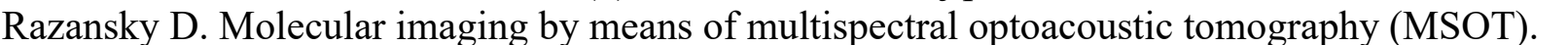

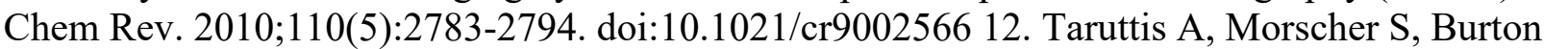

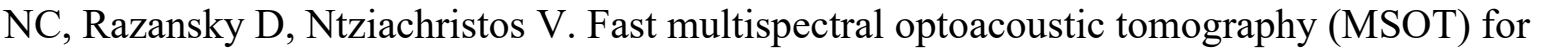

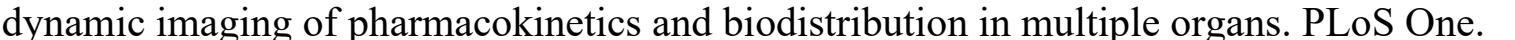

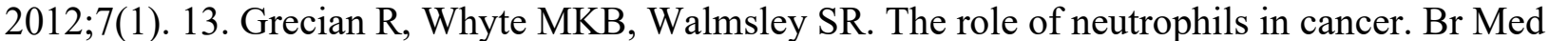

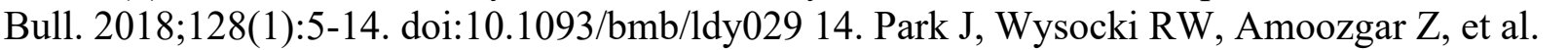

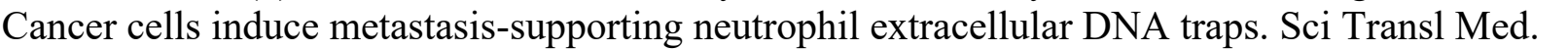

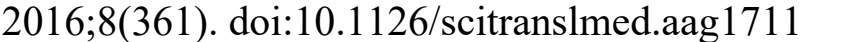

Full Name of Abstract's 1st Author : $\square \square \square \square \square \square \square \square \| \square|\square| \square$

First Name: $\square \square \square \square \square \square$

Last Name: $\square \square \square\|\|\|\| \|$

Email: $\square\|\| \square \square \square\|\square \square \square \square \square \square\| \square \square$

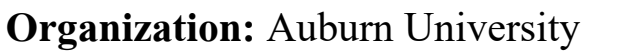

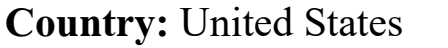




\title{
ID: LB136 \\ Development of 89Zr-DFO-16C4-TM-Fab as a new immunoPET tracer for probing CD19-positive B lymphocytes in a mouse model of multiple sclerosis
}

Carmen Azevedo, Stanford University, ecarmenazevedo@gmail.com

Category: $\square \square \square\|\square\| \| \square \square \square$

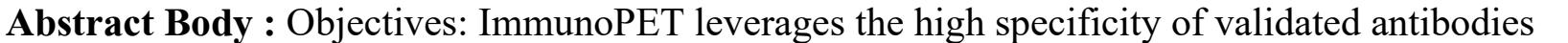

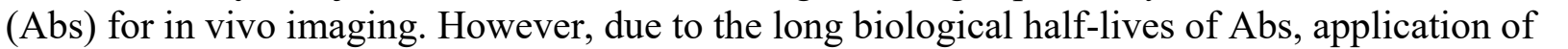

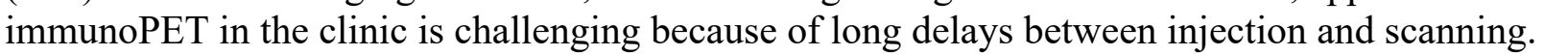

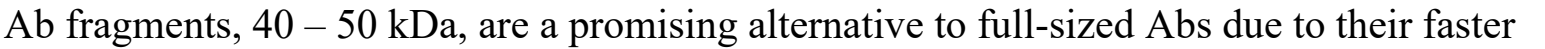

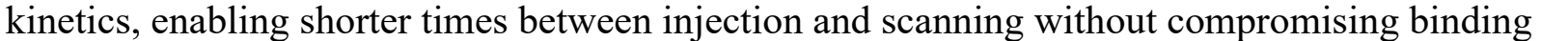

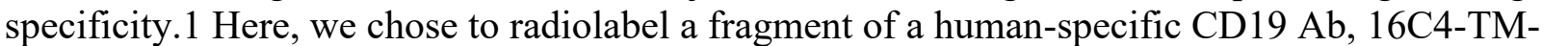

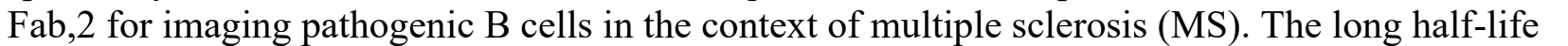

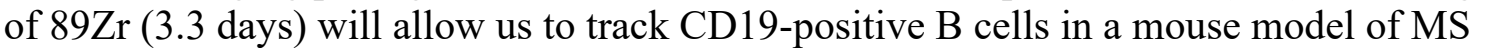
ए

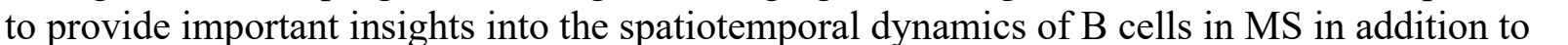

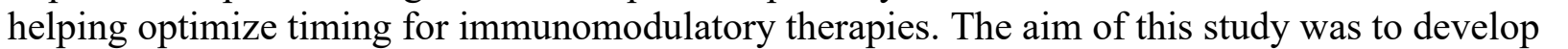

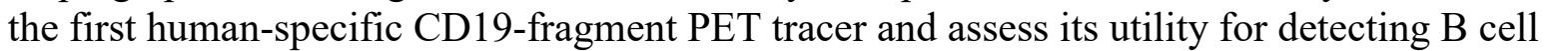

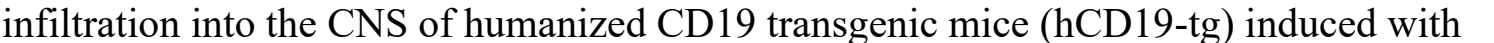

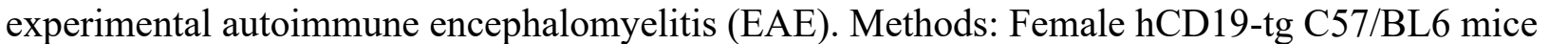

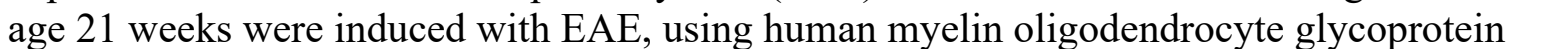

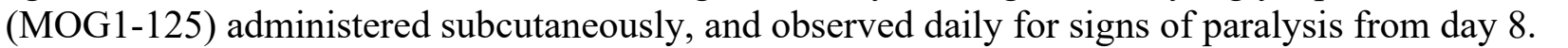

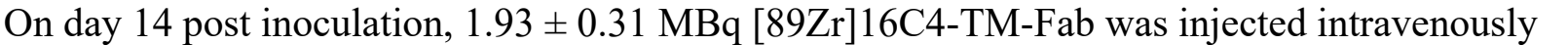

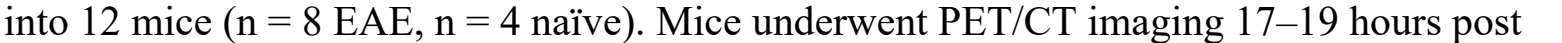
ए

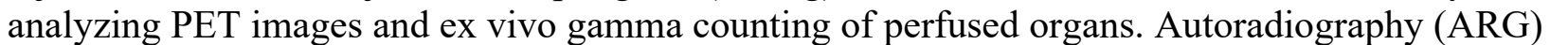

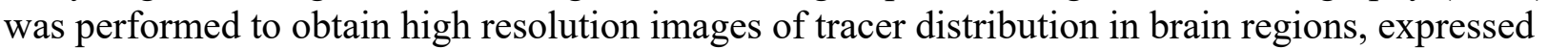

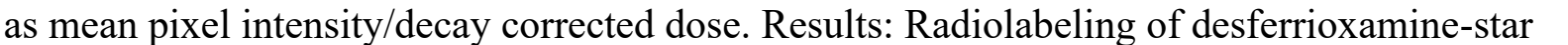

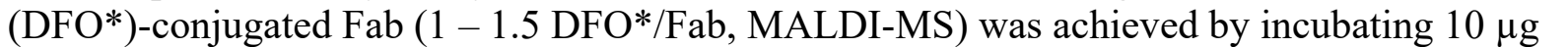

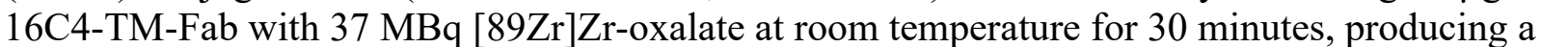

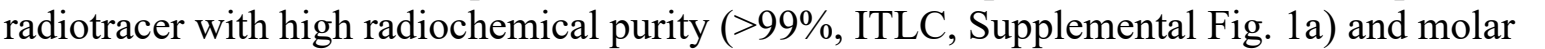
activity of $2 \mathrm{MBq} / \mu \mathrm{g}$. ARG and gamma counting data provide evidence that $[89 \mathrm{Zr}] \square \square \square \square \square \square \square$

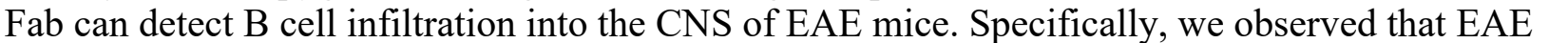

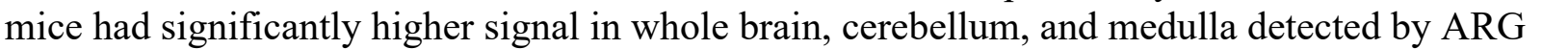

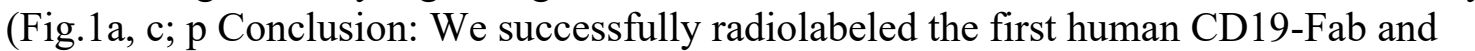

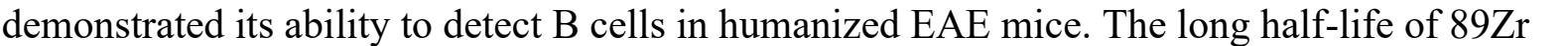

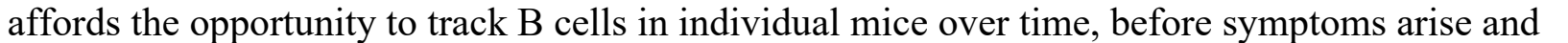

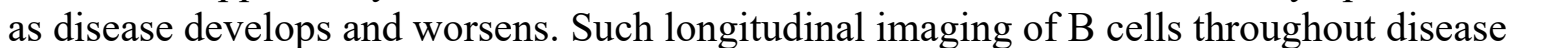

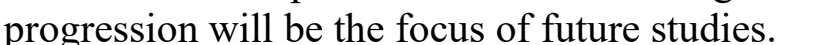

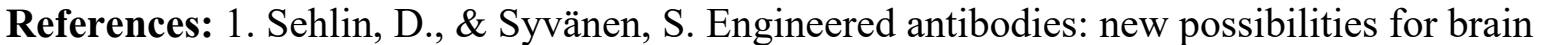

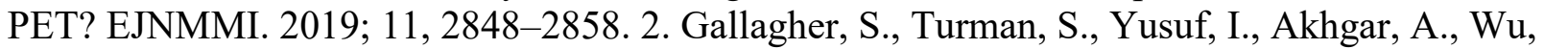




\section{Image/Figure:}

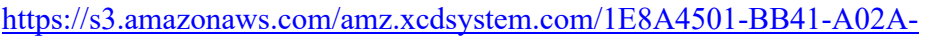

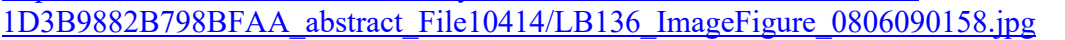

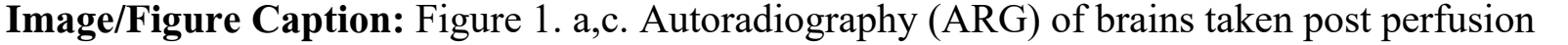

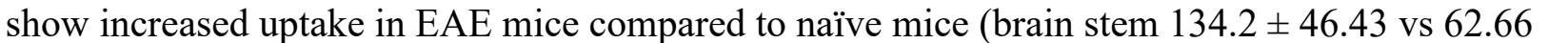

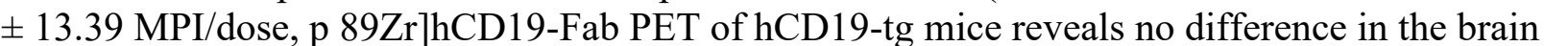

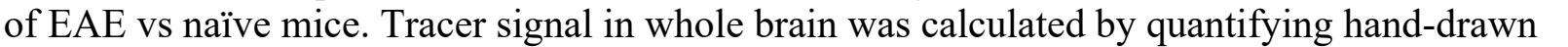

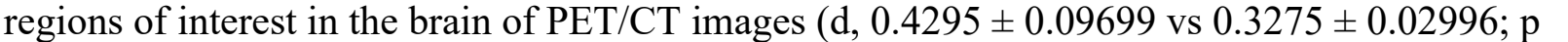

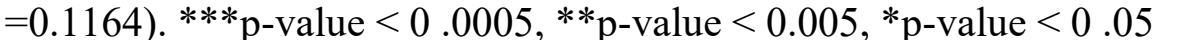

Full Name of Abstract's 1st Author : $\square \square \square \square \square \square \square \square \| ा \square$

First Name: $\square \square \| \square \square \square$

Last Name: $\square \square\|\|\|\| \|$

Email: $\square\|\| \square \square\|\|\|\| \square \square \square\|\Pi\| \square \square$

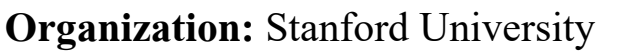

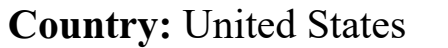




\title{
ID: LB137 \\ PSMA Ligand-Decorated Microbubbles, a Molecular Ultrasound Imaging Tool to Probe for (Lack of) Mosaic Vessels in a PC3/PIP Tumor Rodent Model.
}

\author{
Alexander Klibanov, University of Virginia, sklib1@gmail.com
}

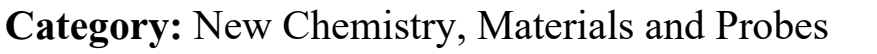

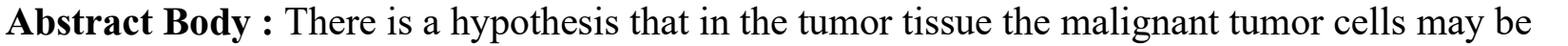

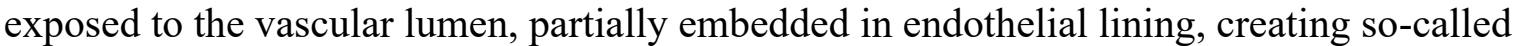

"mosaic" vessels: up to $4 \%$ of the surface of the vasculature may be tumor cells, not endothelium

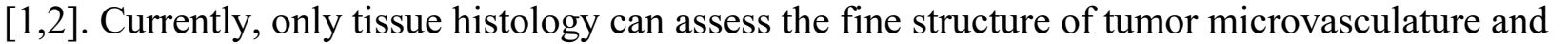
ए

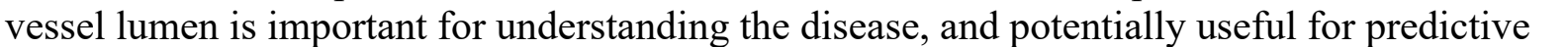

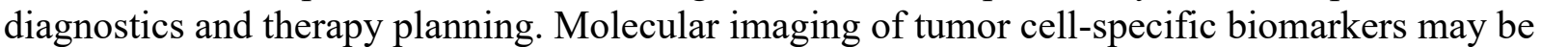

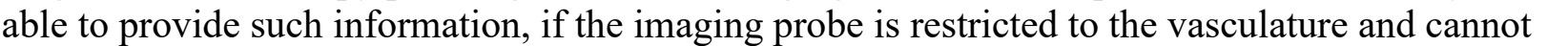

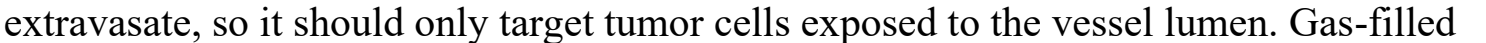

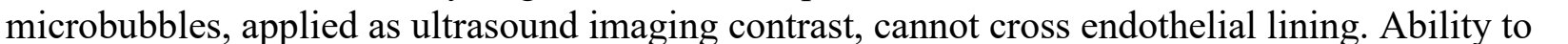

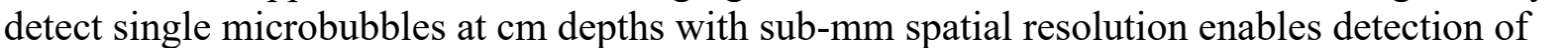

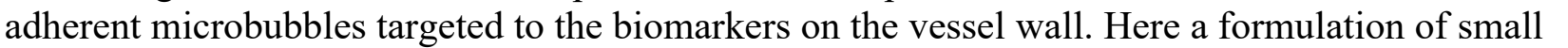

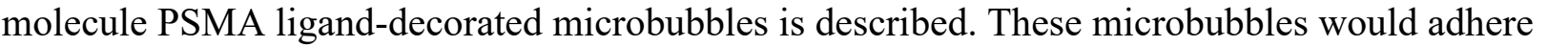

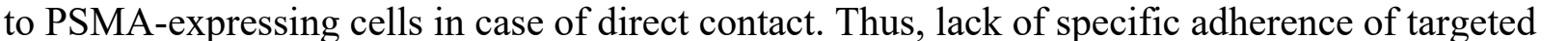

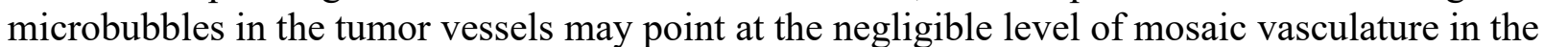

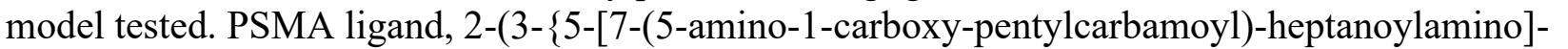

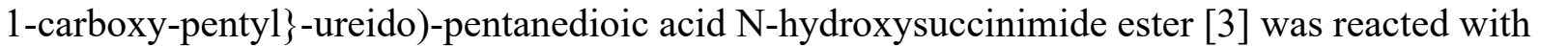
एव

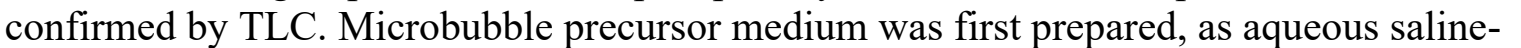

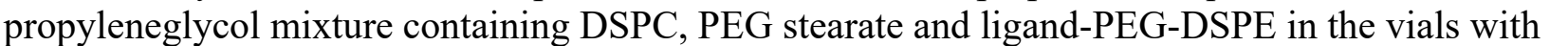

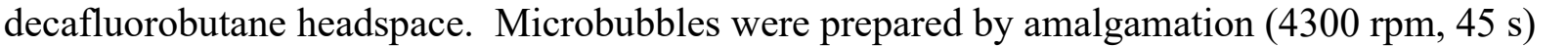
ए

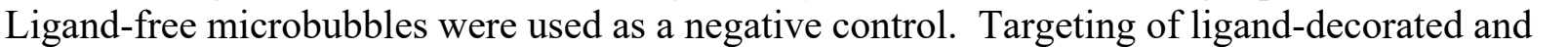

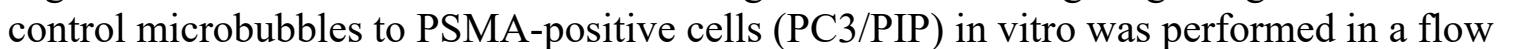

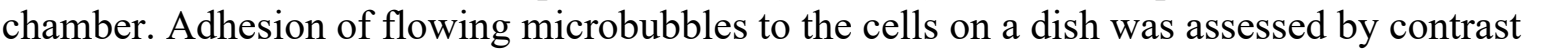

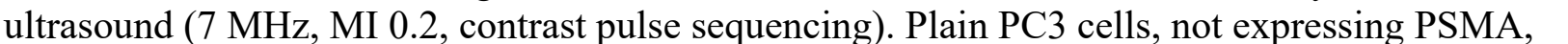

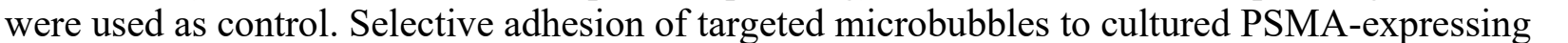

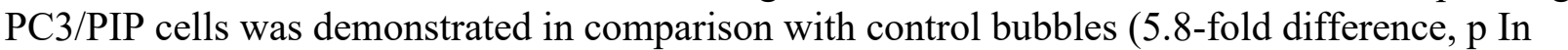

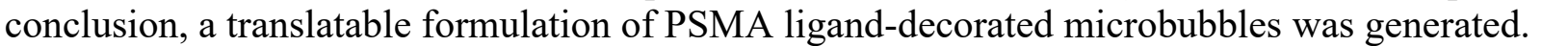

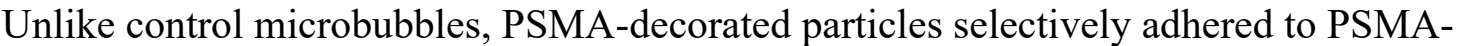

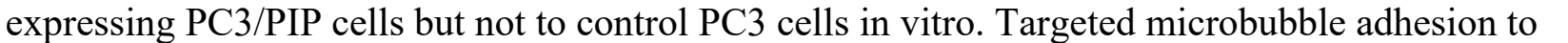

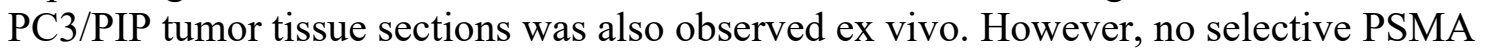

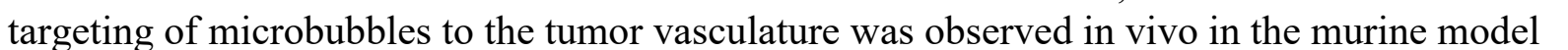
ए

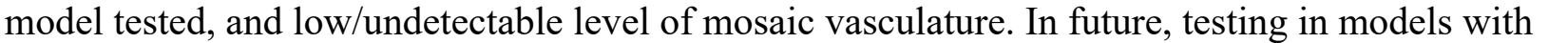

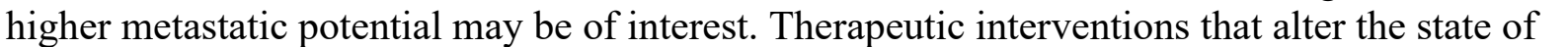




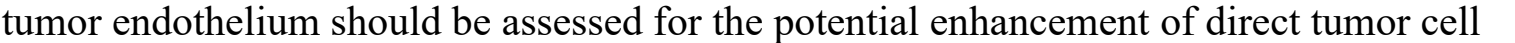

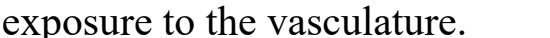

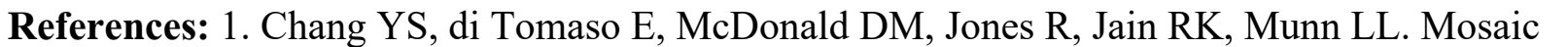

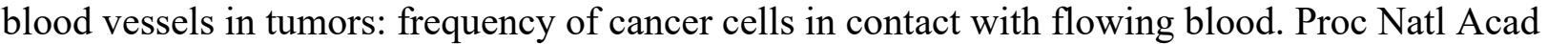

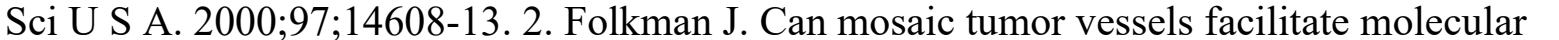

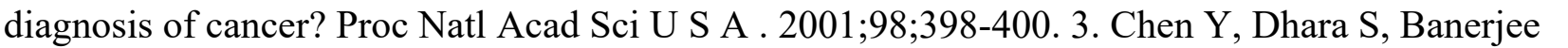

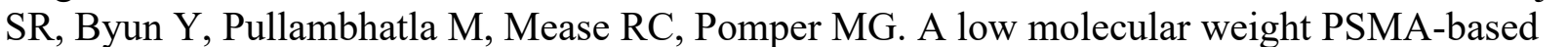

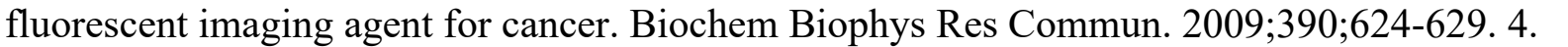

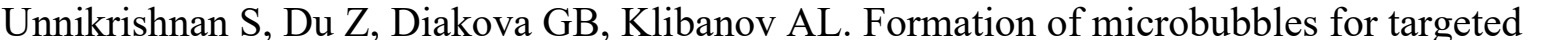

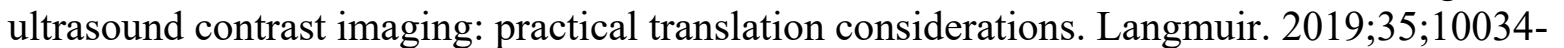

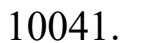

First Name: $\square \square\|\|\|\backslash\| \square$

Last Name: $\square \square\|\|\|\|$

Email: $\square \square\|\square \square \square \square\| \| \square \square$

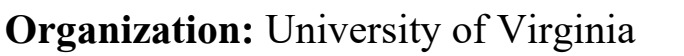

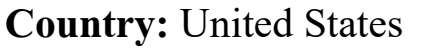




\title{
ID: LB138 \\ Label-free metabolic imaging for sensitive and robust monitoring of anti-CD47 immunotherapy response in triple negative breast cancer
}

\author{
Minfeng Yang, The HongKong Polytechnic University, 19110055r@connect.polyu.hk
}

\section{Category: $\square \square\|\| \| \square$}

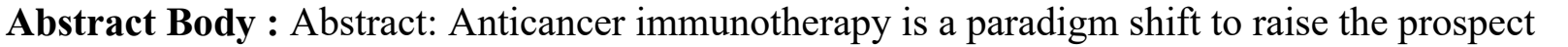

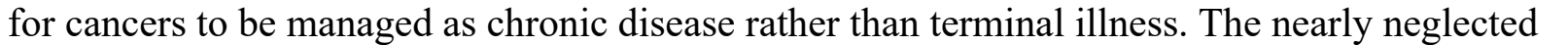
old idea of activating patients' own immune system to fight tumors has now resulted in an

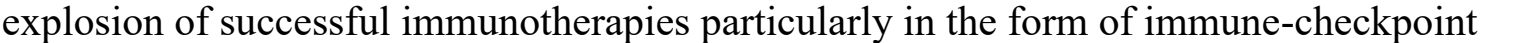

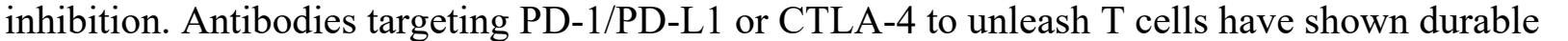

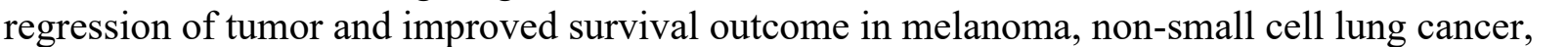

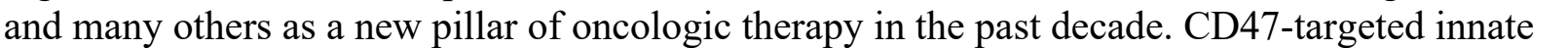

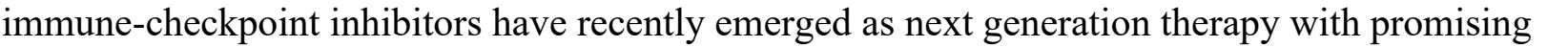

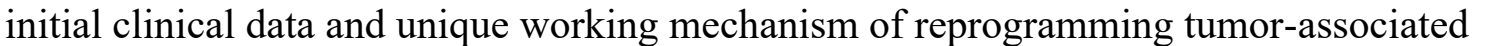

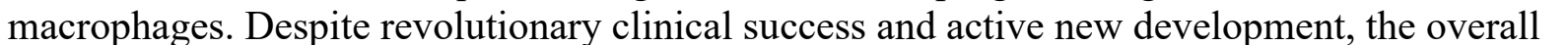
ए

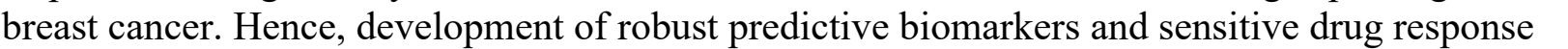

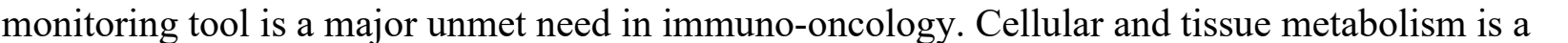

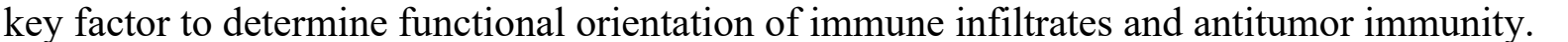

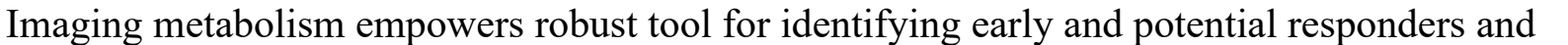

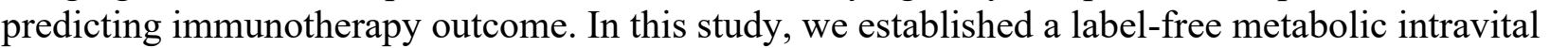

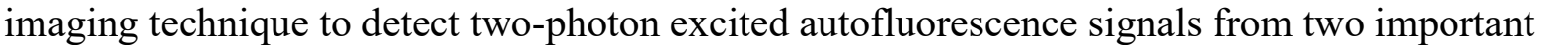

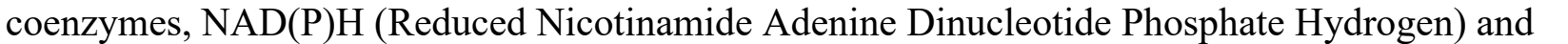

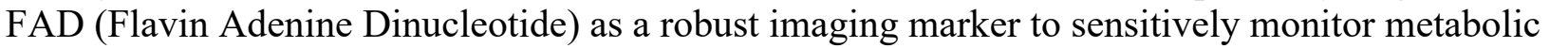

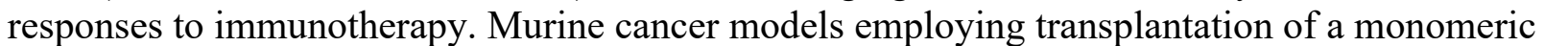
घh एம

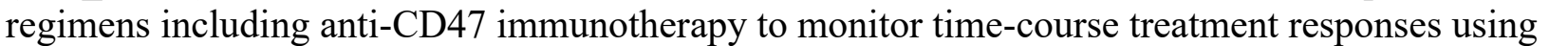

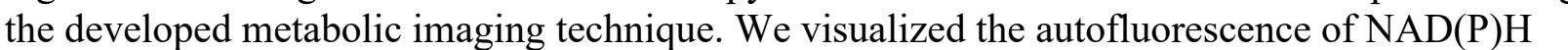

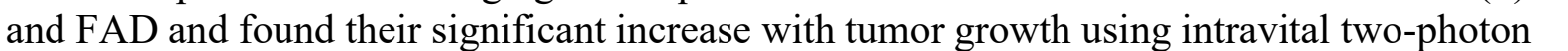

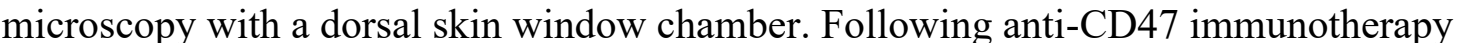

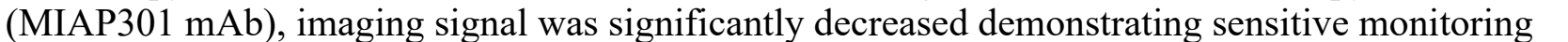

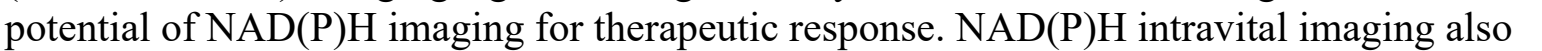

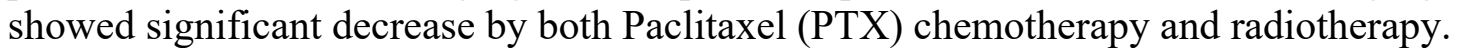

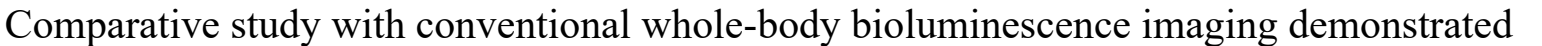

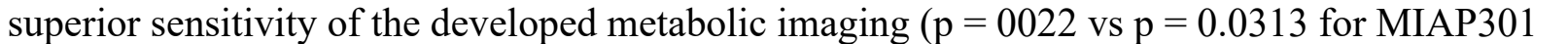

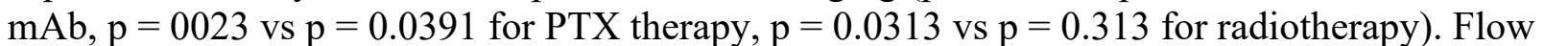

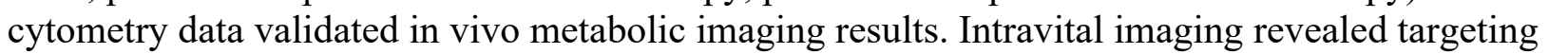

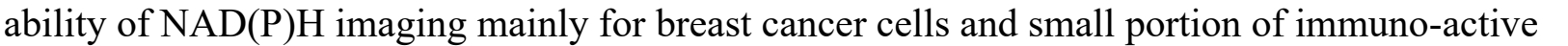

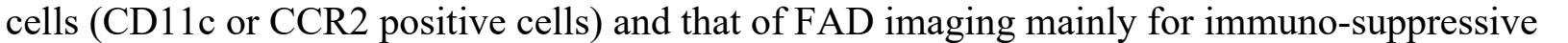

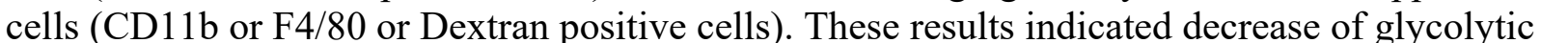




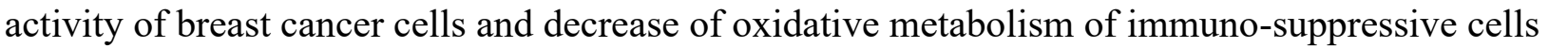

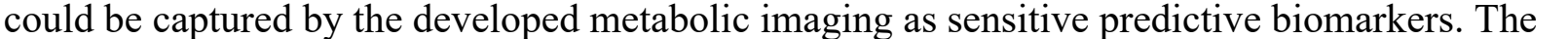

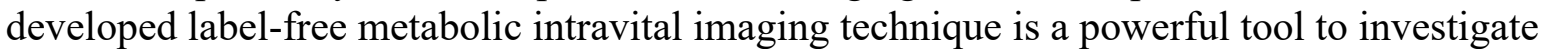

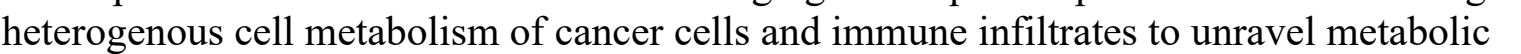

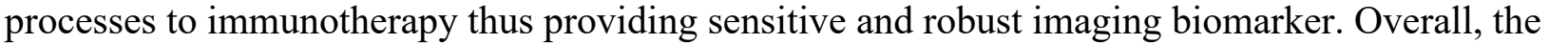

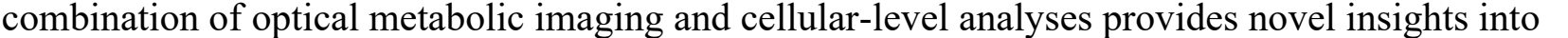

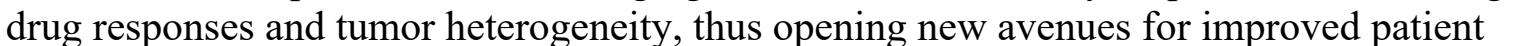

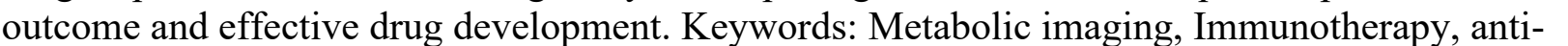

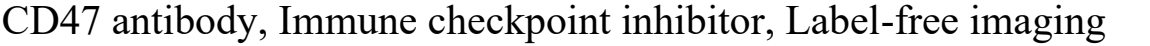

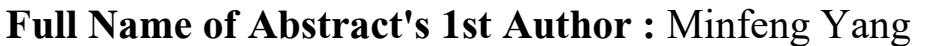

First Name: $\square \square \square \| \square \square$

Last Name: $\square \square \square \square \square$

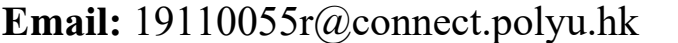

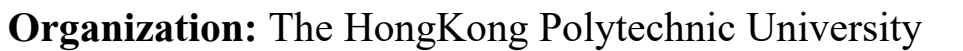

Country: $\square \square \square \square \square \square \square \square$ 


\title{
ID: LB139 \\ Engineered neural stem cell mimetics as nanocargos for glioblastoma specific delivery of different theranostics
}

Jagathesh Chandra Bose Rajendran, Masonic Medical research institute, rbose@mmri.edu

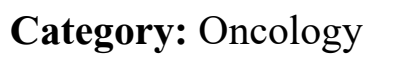

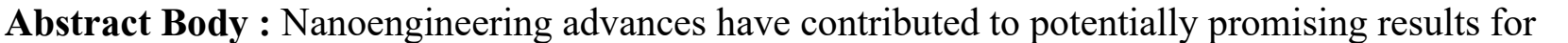

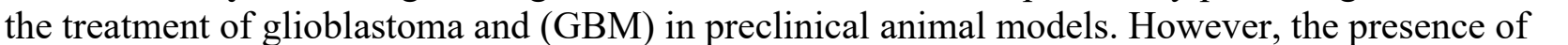

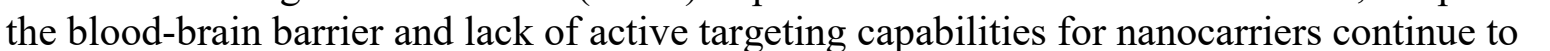

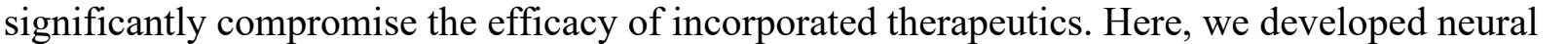

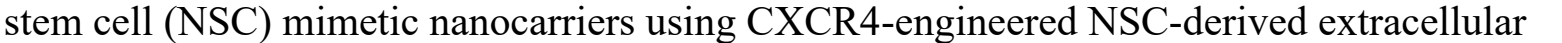

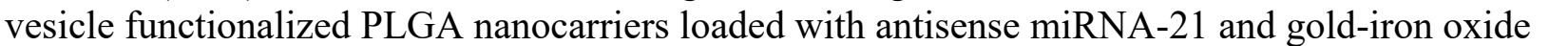

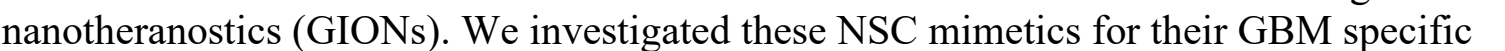

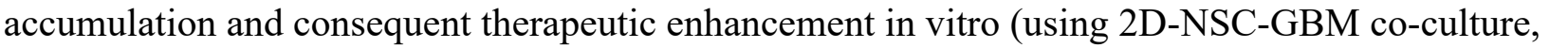

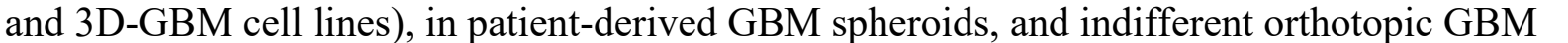

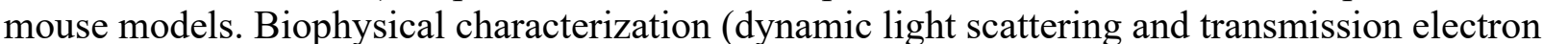

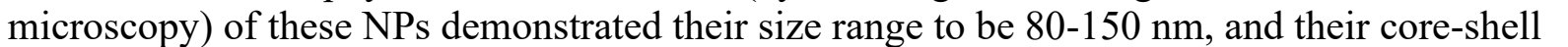

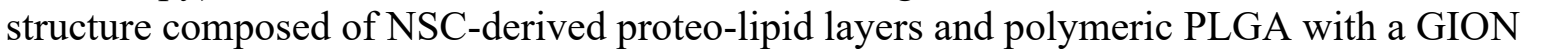

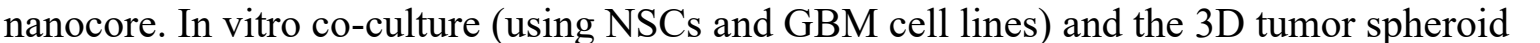

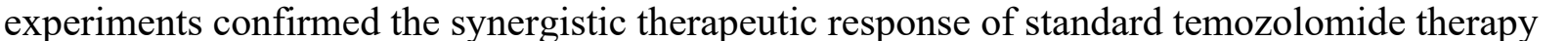

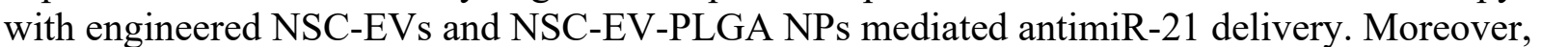

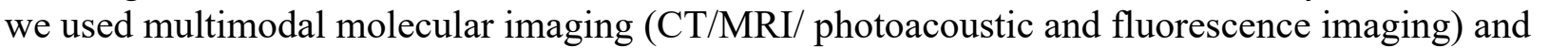

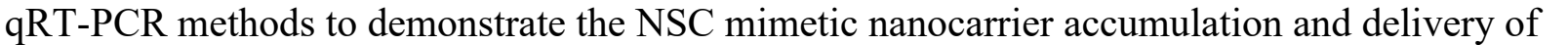

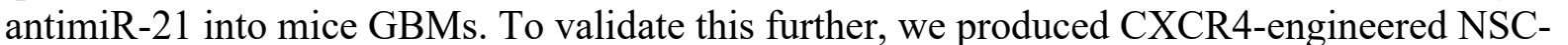

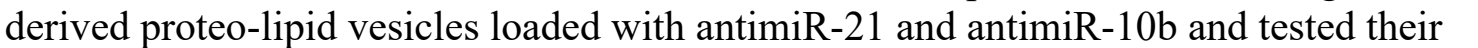

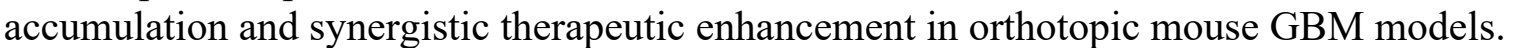

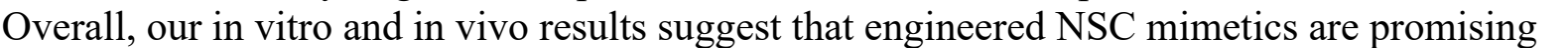

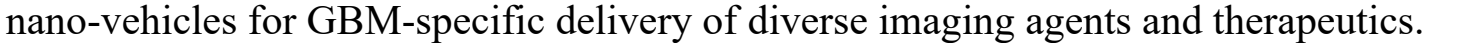

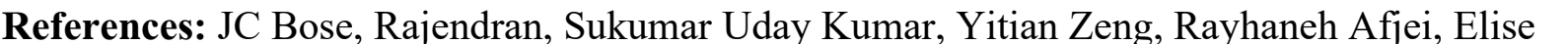

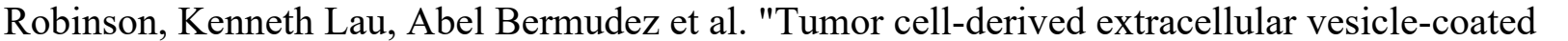

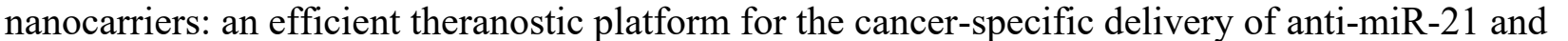

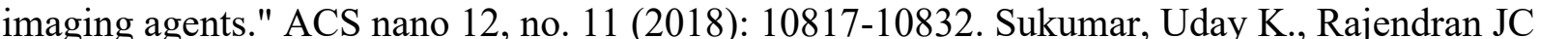

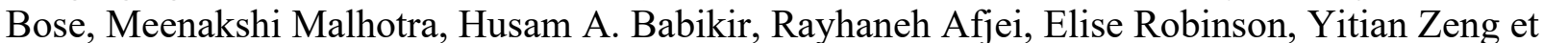

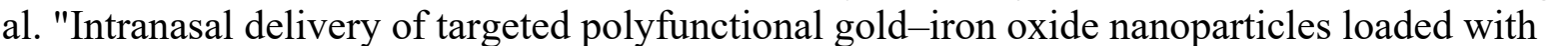
ए

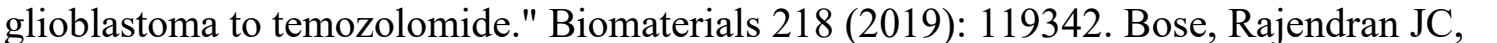

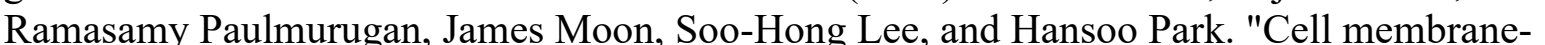

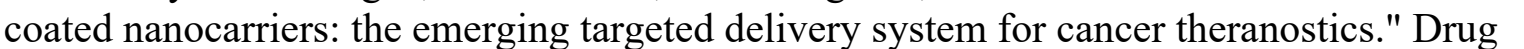

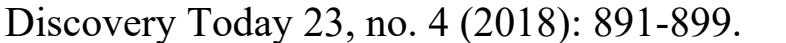

\section{Image/Figure:}




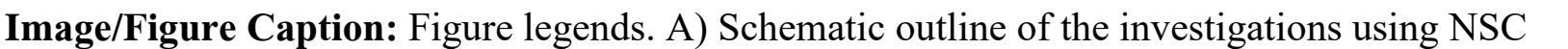

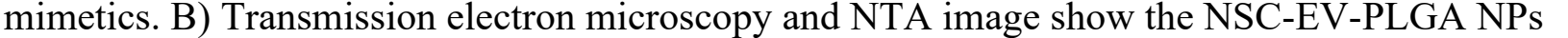

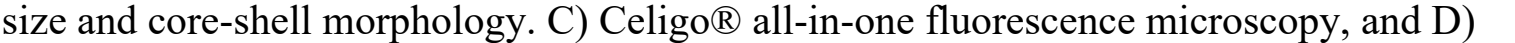

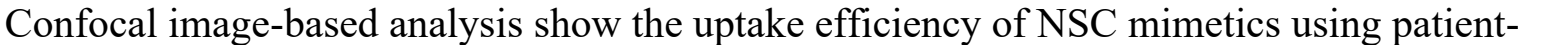

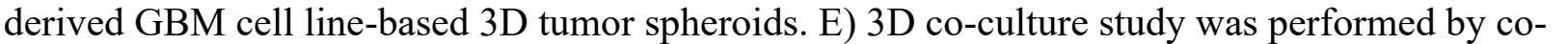

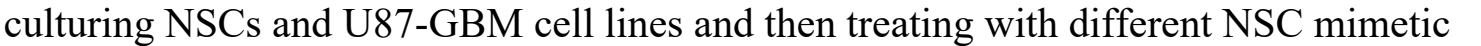

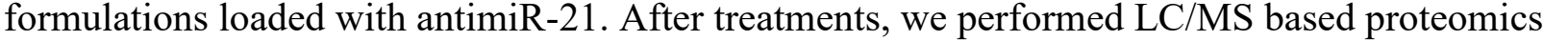

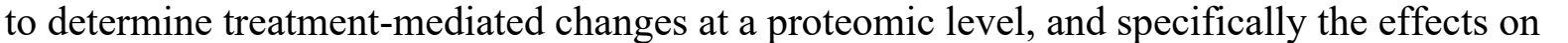

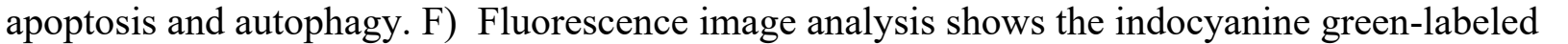

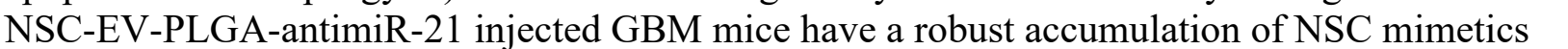

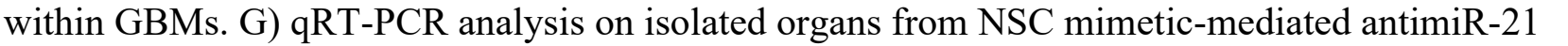

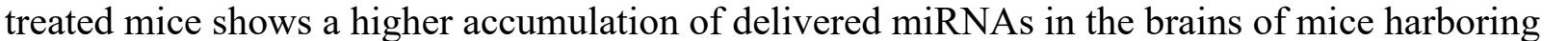

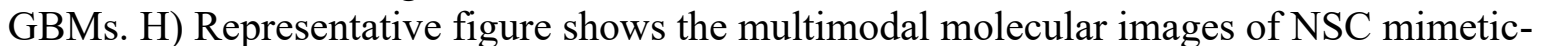

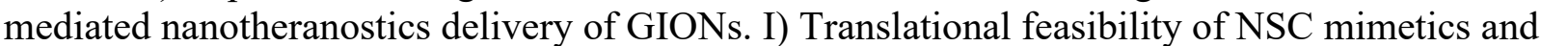

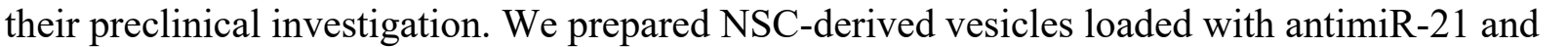

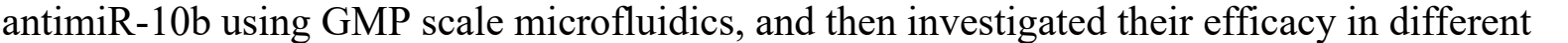

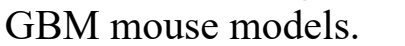

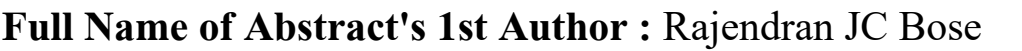

First Name: $\square \square\|\|\|\|\|\| \square \square \square\|m \square \square\| m$

Last Name: $\square \square\|\| \square|\| \square| \square$

Email: $\square \square\|\square \square \square \square\| \| \square$

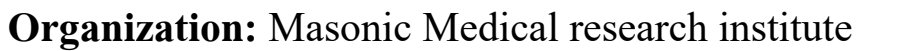

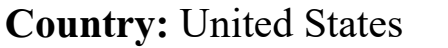




\title{
ID: LB140 \\ Comparing the performance of commercially available magnetic nanoparticles for magnetic particle imaging
}

Irati Rodrigo Arrizabalaga, University of California, Berkeley, irati.rodrigo@berkeley.edu

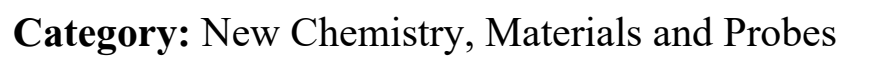

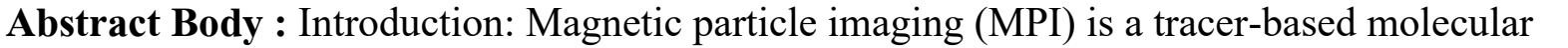

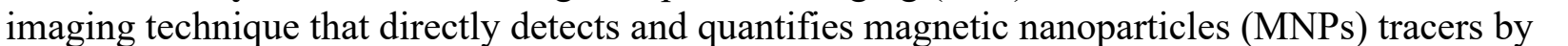

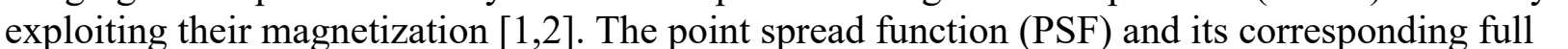

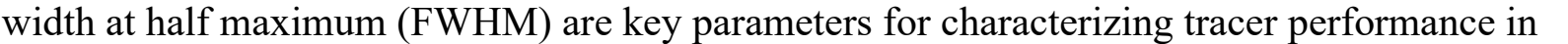

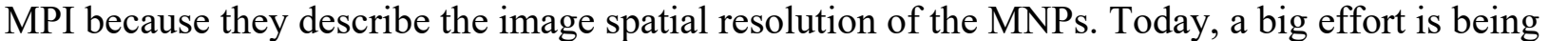

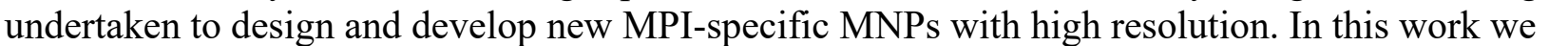

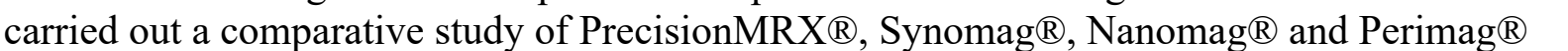

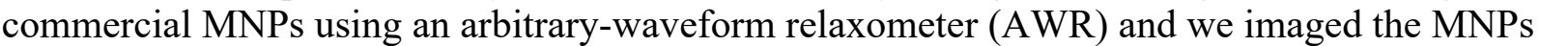

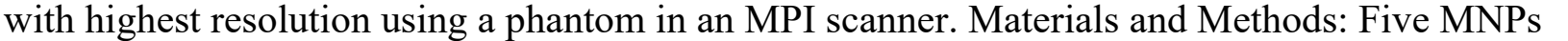

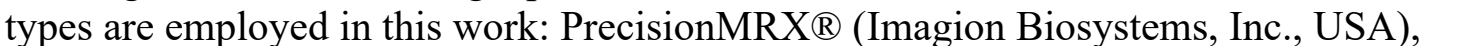

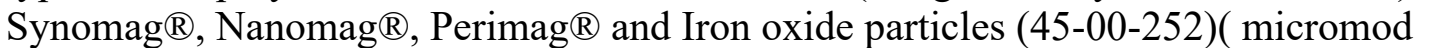

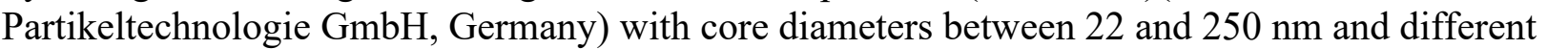

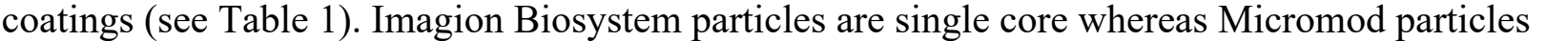

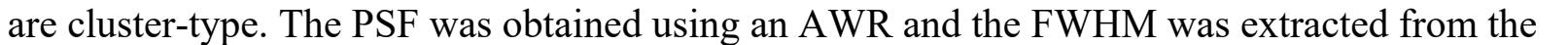

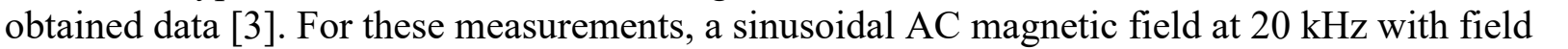

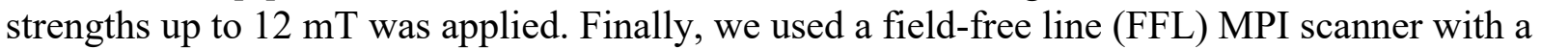

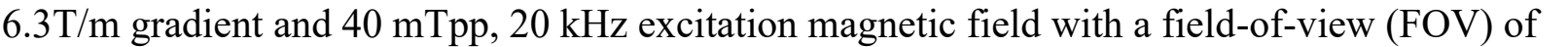

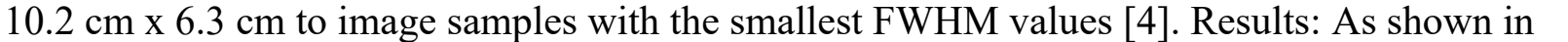

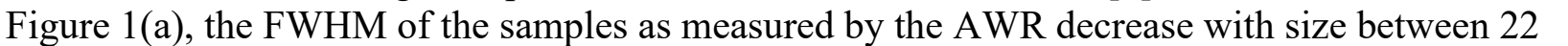

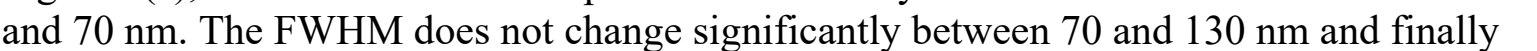

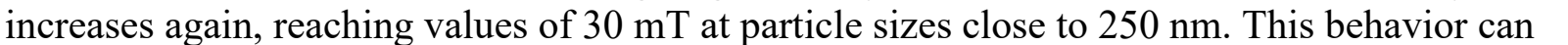

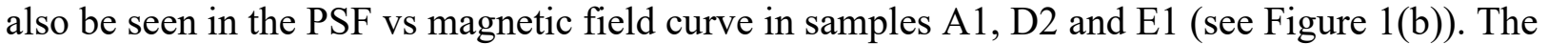

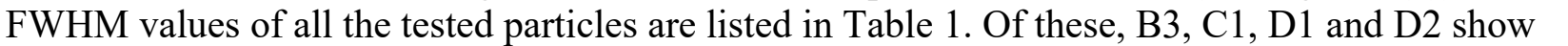

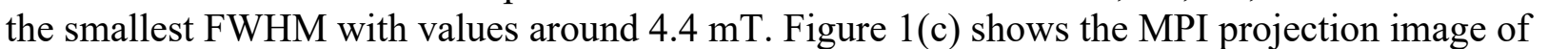

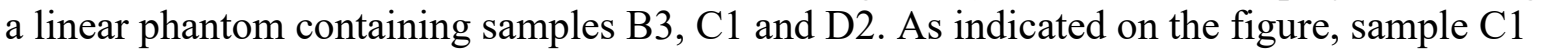

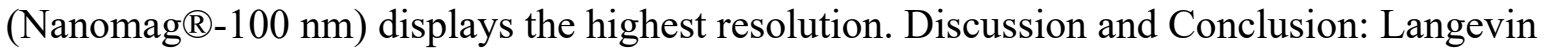

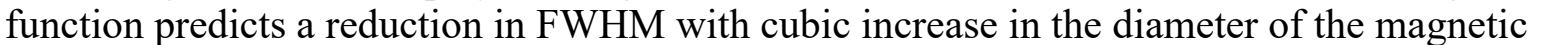

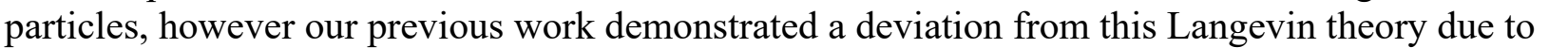

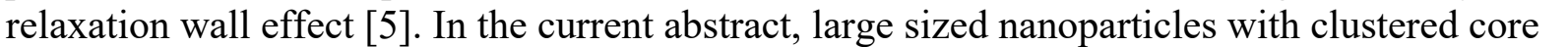

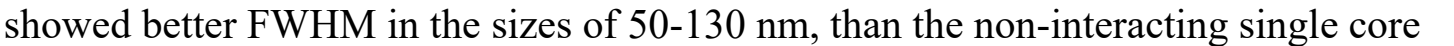

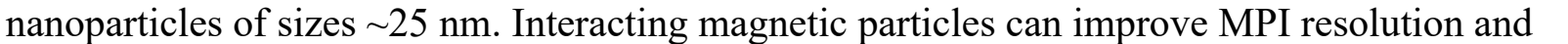

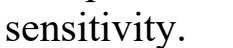

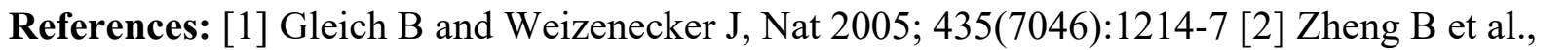

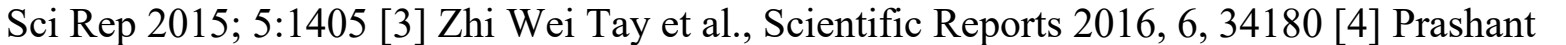




\section{Image/Figure:}

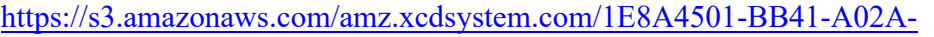

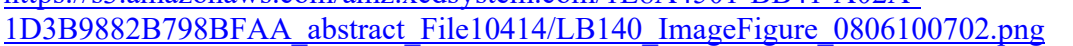

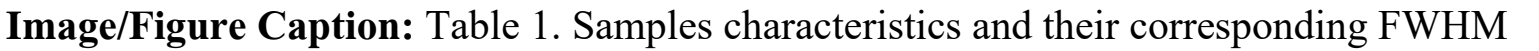

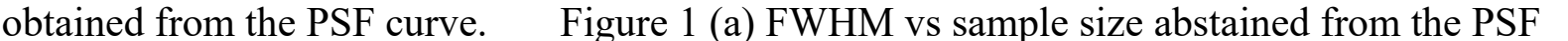

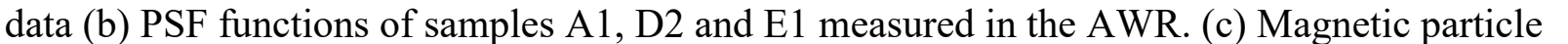

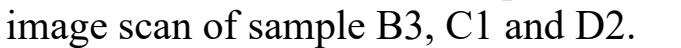

First Name: $\square\|\| m$

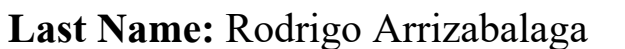

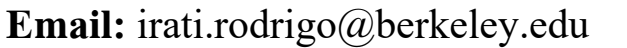

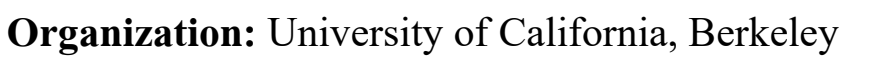

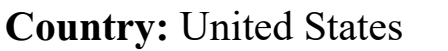




\title{
ID: LB141 \\ 18F-FAC PET images immune activation a B cell dependent multiple sclerosis mouse model
}

\author{
Bao Ying Chen, University of California, Los Angeles, BaoYingChen@mednet.ucla.edu
}

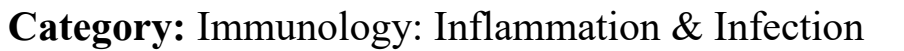

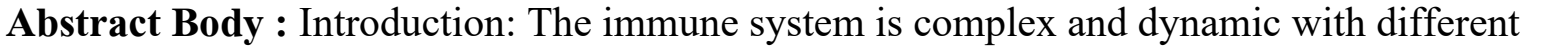

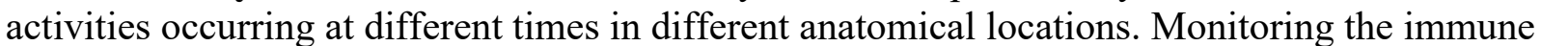

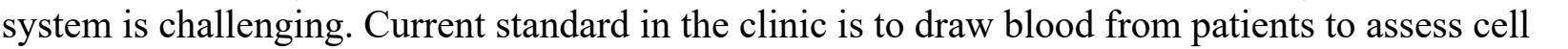

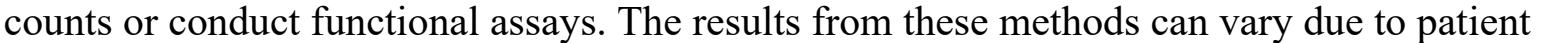

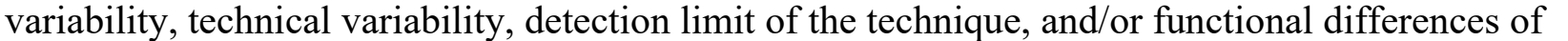

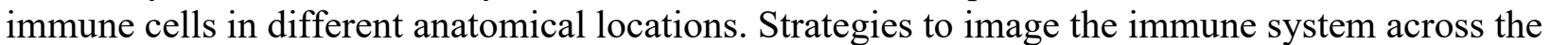

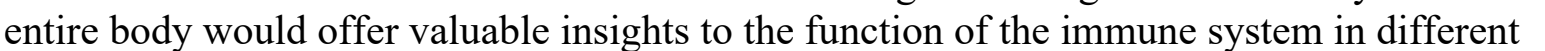

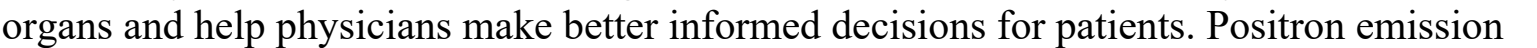
ए

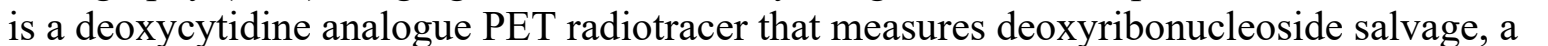

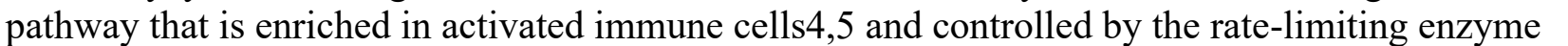

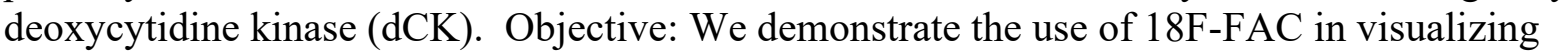

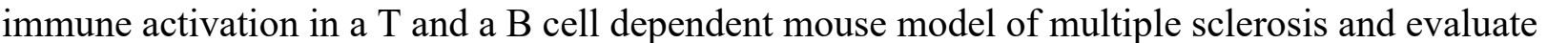

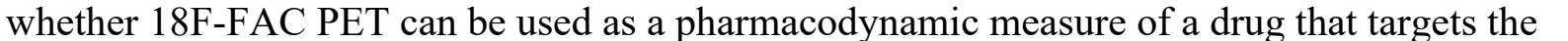

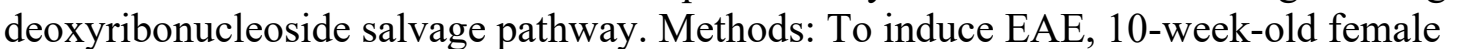

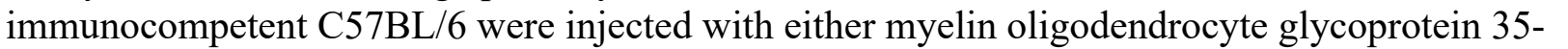

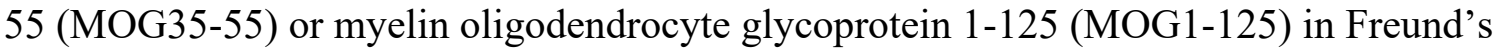

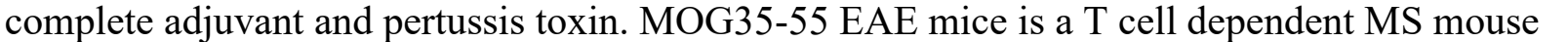

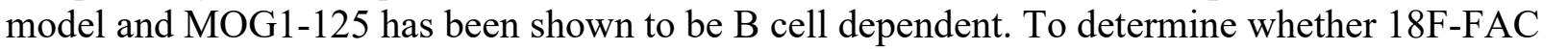

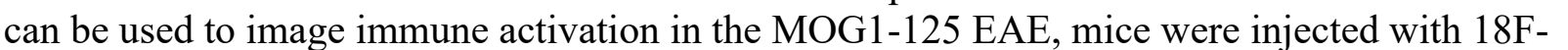

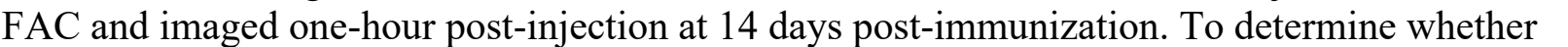

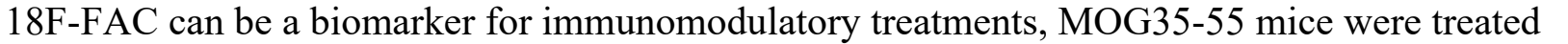

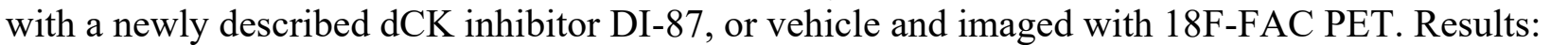

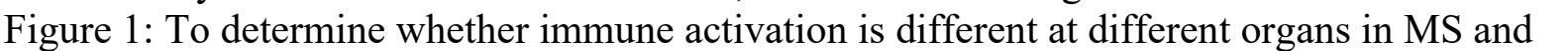

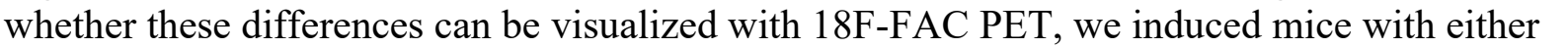

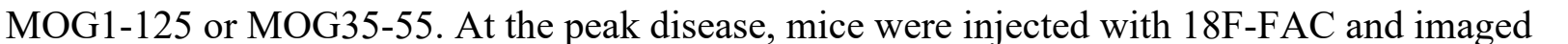

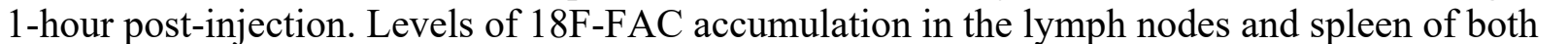

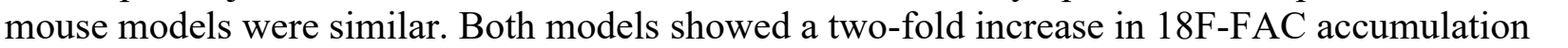

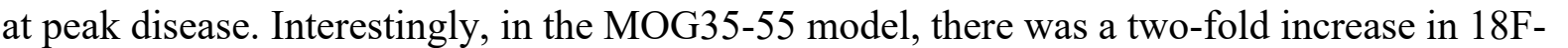

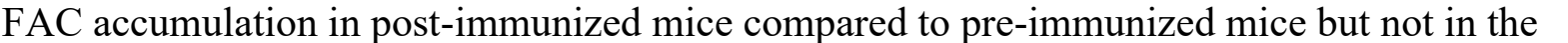

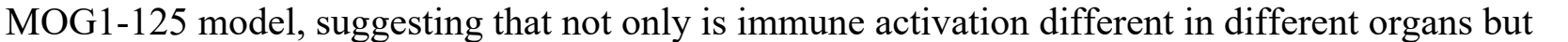

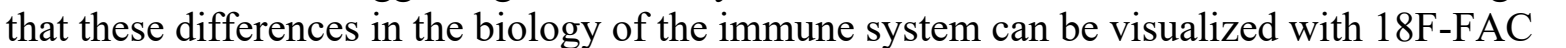

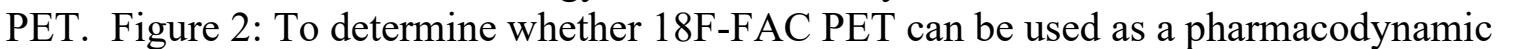

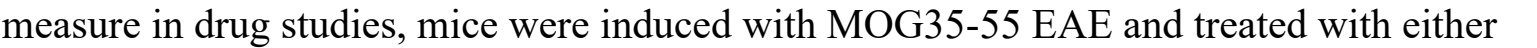

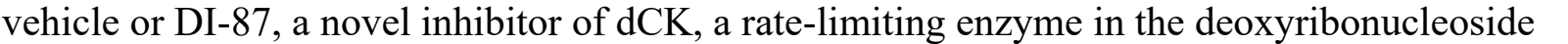

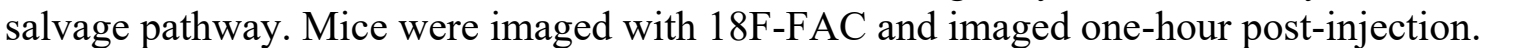




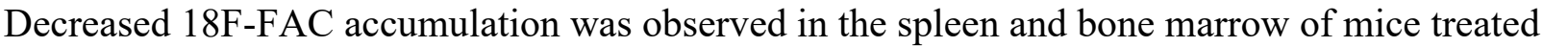
a ए

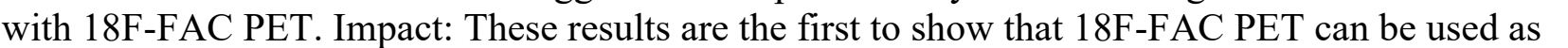

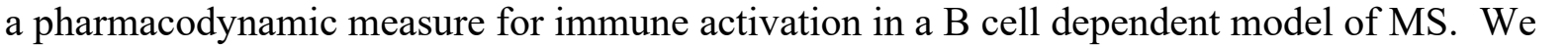

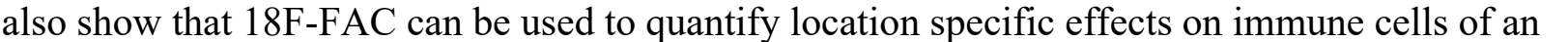

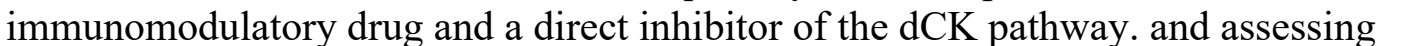

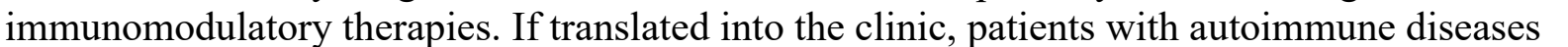

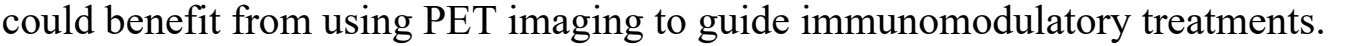

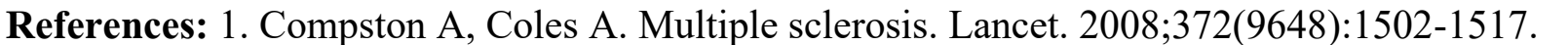

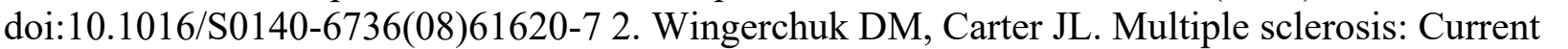

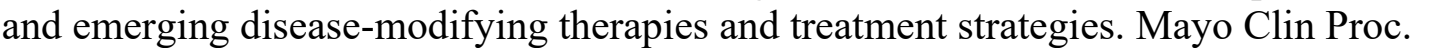

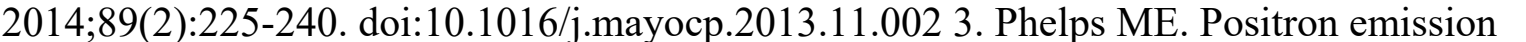
ए

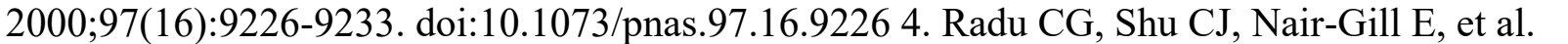

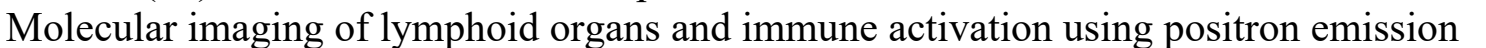

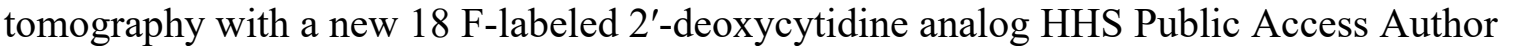

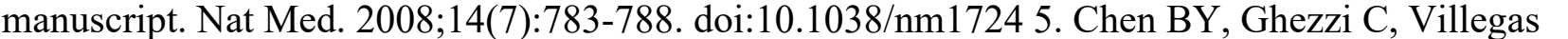

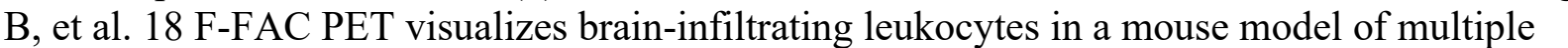

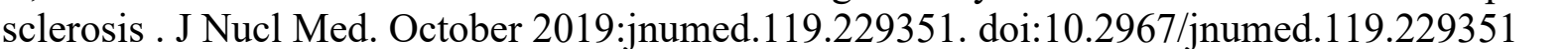

First Name: $\square \square \| m \square \square$

Last Name: $\square \square\|\| \square$

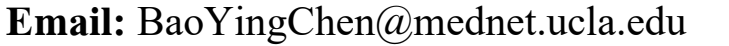

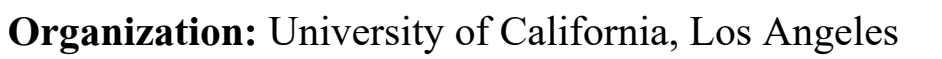

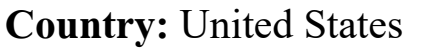




\title{
ID: LB142 \\ Simultaneous Dynamic FDG-PET and Gadolinium-Enhanced MRI for Tumor Characterization
}

\author{
Christiane Mallett, Michigan State University, christiane.mallett@gmail.com
}

Category: $\square \square\|\| ा \| \square$

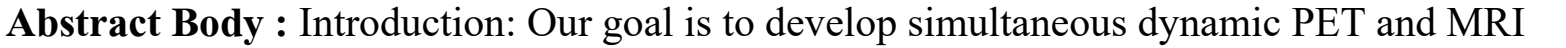

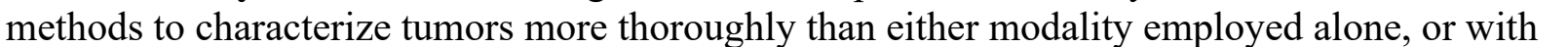

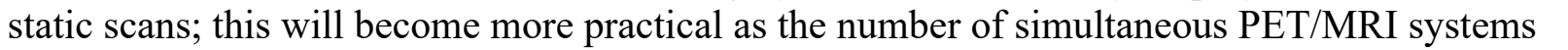

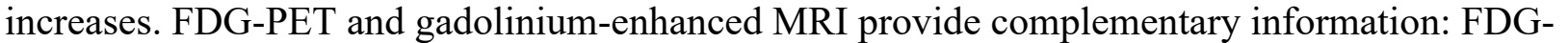

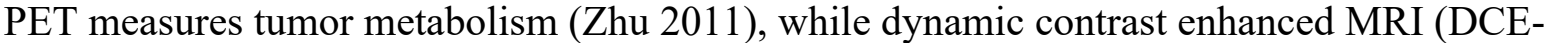

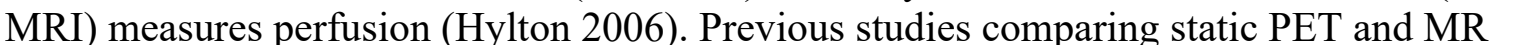

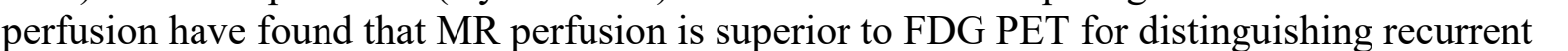

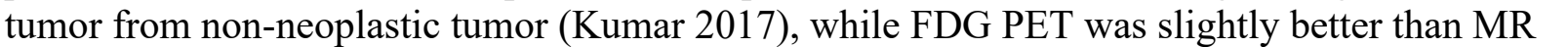

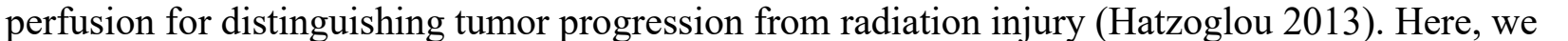

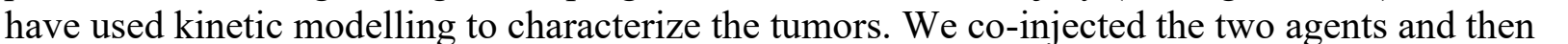

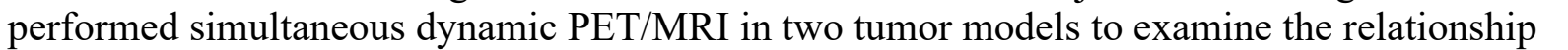

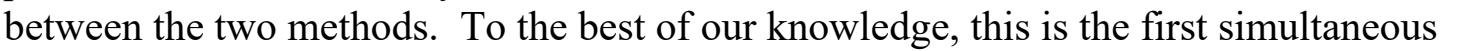

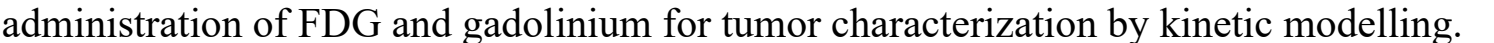

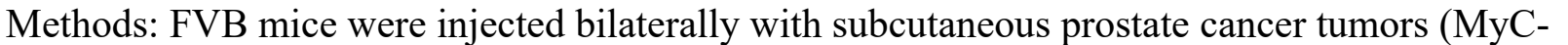

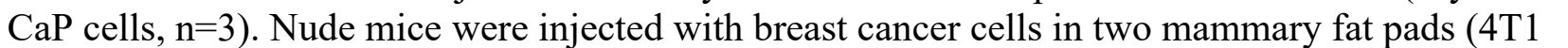

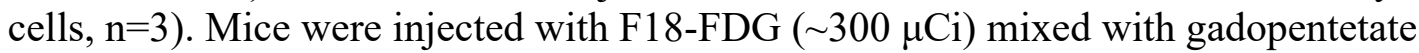

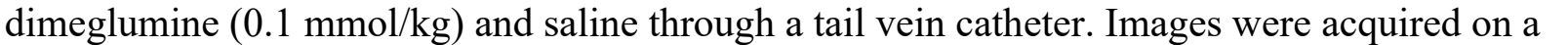
प

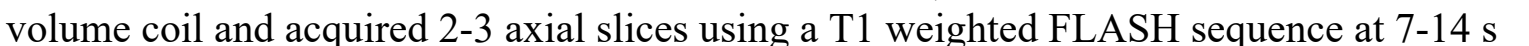

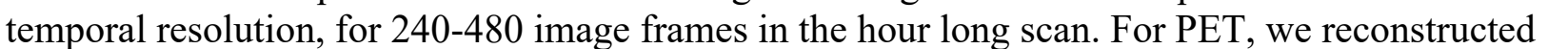

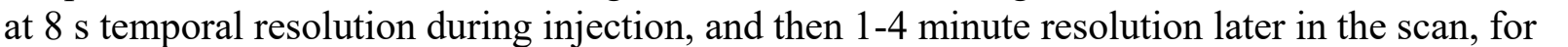

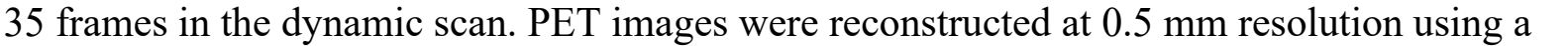

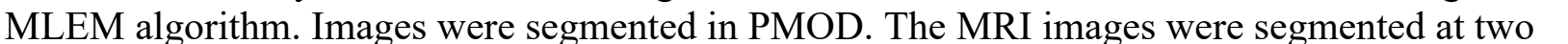
timepoints: 500 seconds after the start of the acquisition ("early") and at the end of the scan ("late"), and were segmented into regions with more than $40 \%$ enhancement over baseline ("enhancing regions") and regions with less than $40 \%$ enhacement over baseline ("non $\square$ enhancing regions"). Those ROIs were overlaid onto the PET images, and we used a Patlak

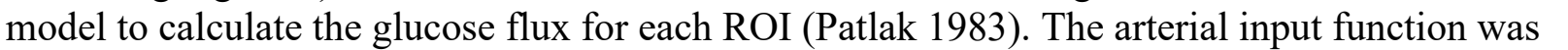

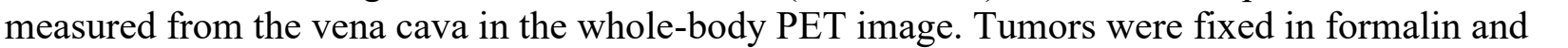

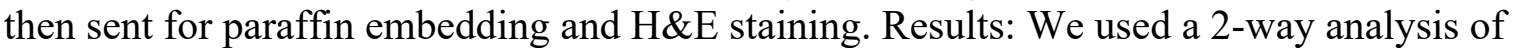

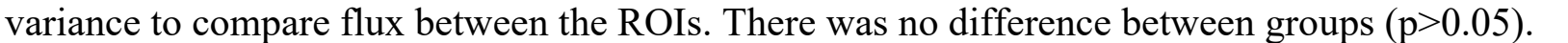

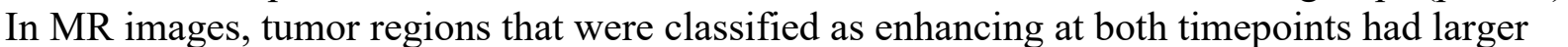

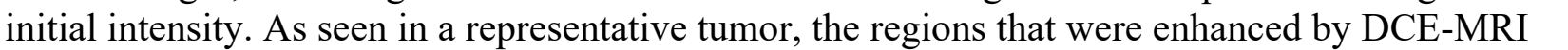

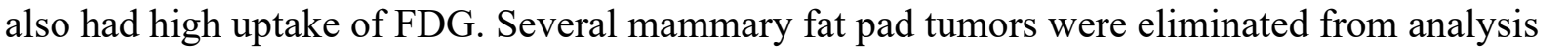

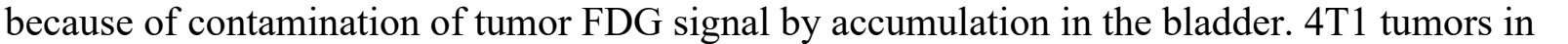

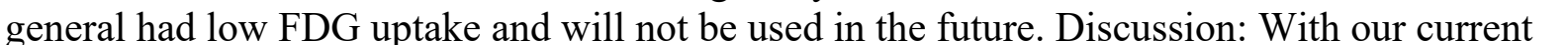




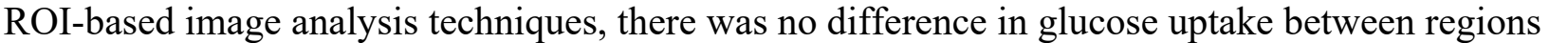

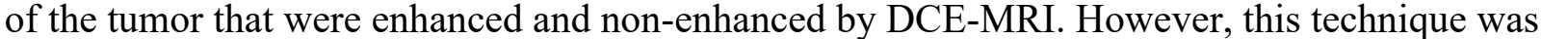
ए

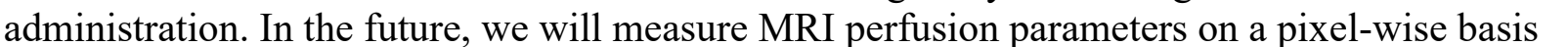

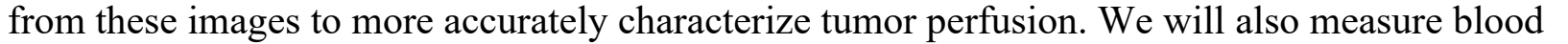

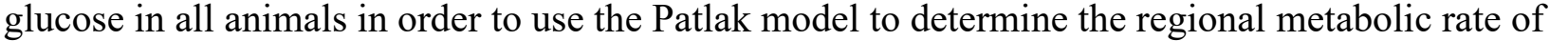

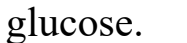

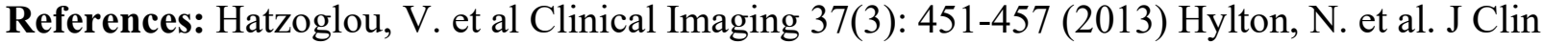

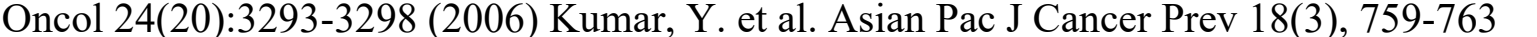

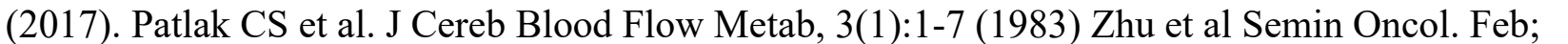

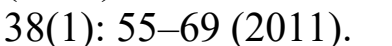

\section{Image/Figure:}

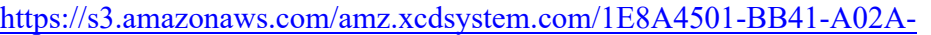

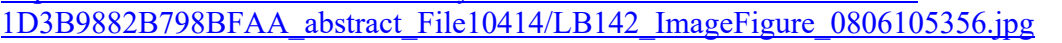

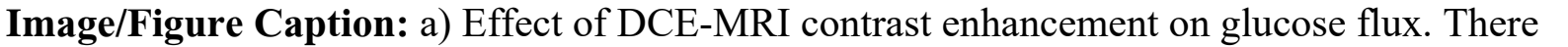

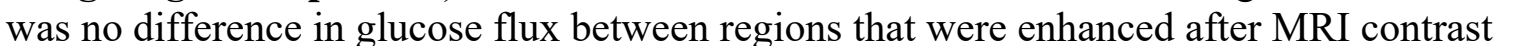

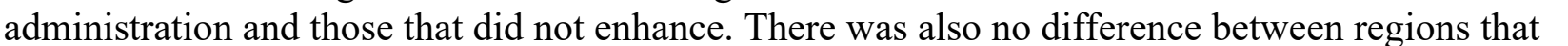

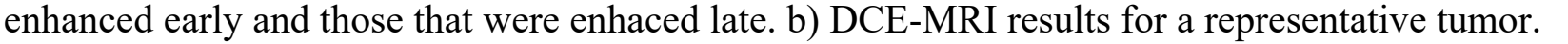

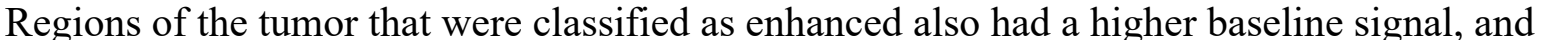

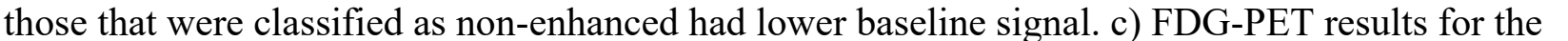

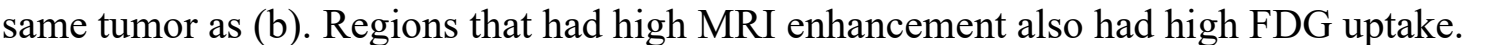

First Name: $\square \square \square\|\|\|\|\|\|$

Last Name: $\square \square \square\|m\| n$

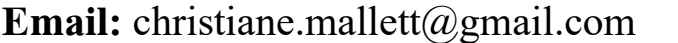

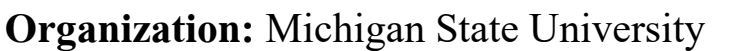

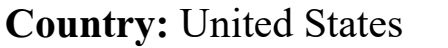




\title{
ID: LB143 \\ Viability of Calibrating Stereotaxic Coordinates Through Fringe Projection Profilometry
}

\author{
Kevin Gonzalez, Rice University, kag17@rice.edu
}

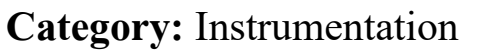

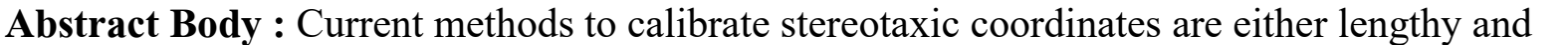

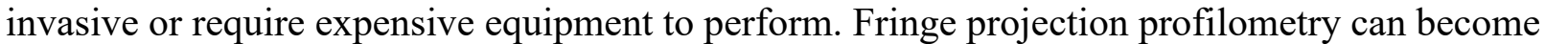

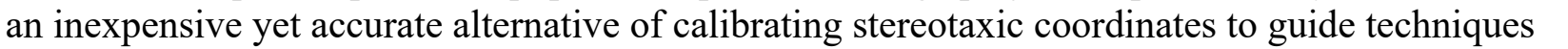

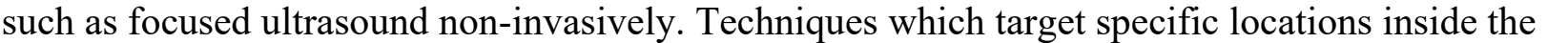

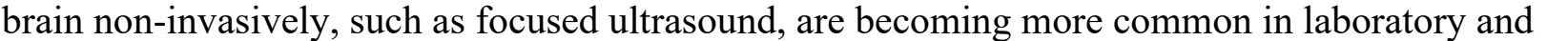

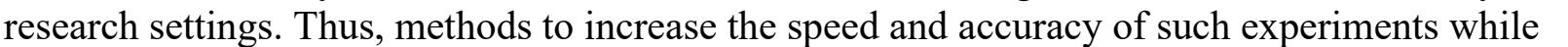

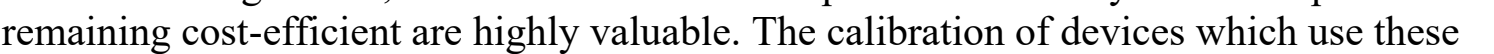

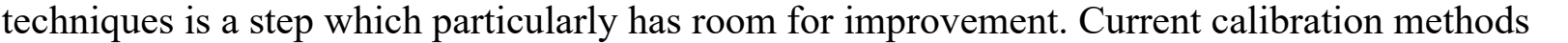

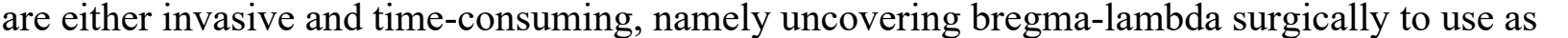

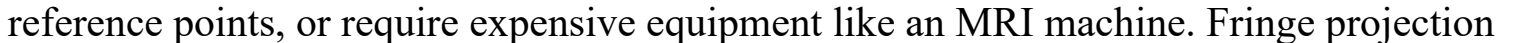

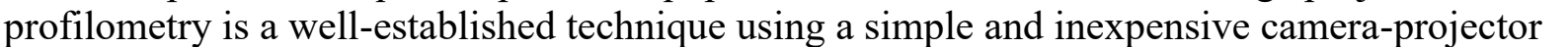

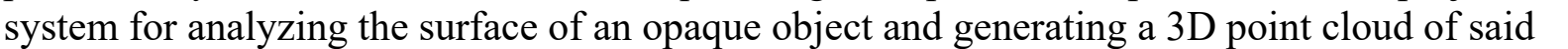

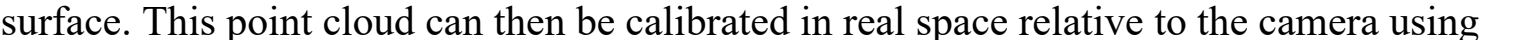
computer vision techniques. By using this technique on the surface of a mouse's shaved head,

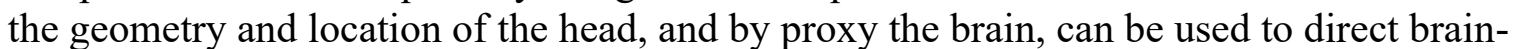

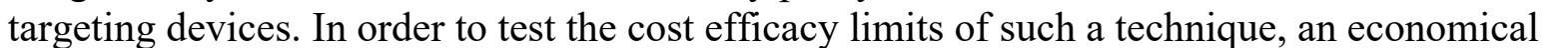

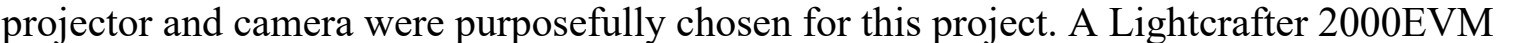

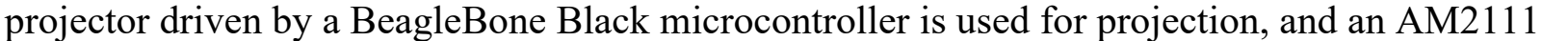

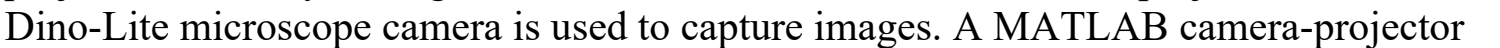

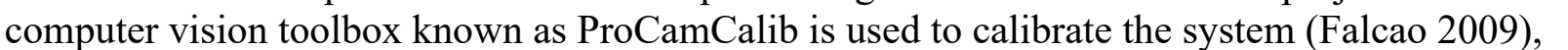

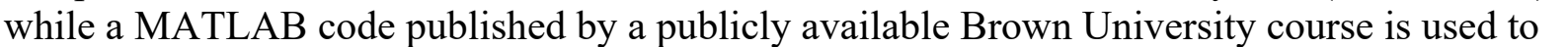

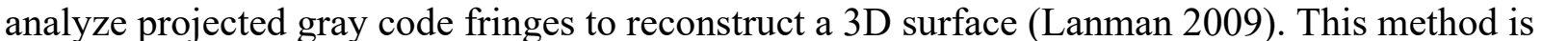

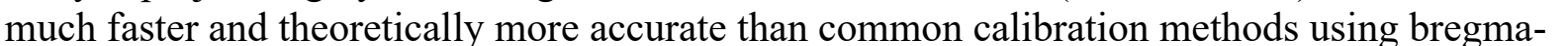

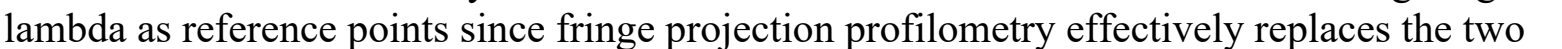

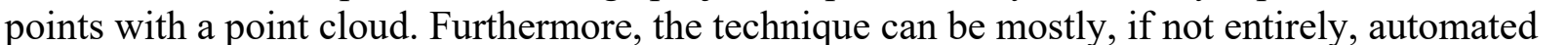

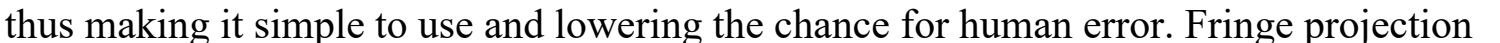

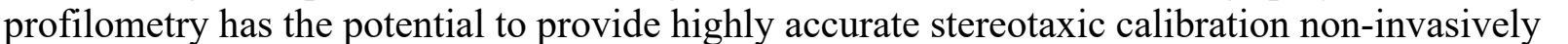

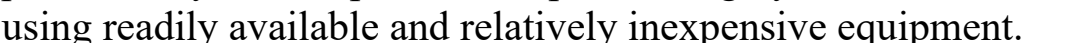

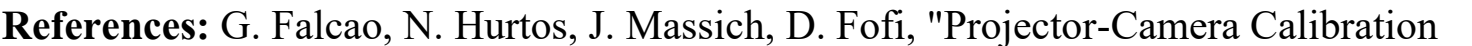

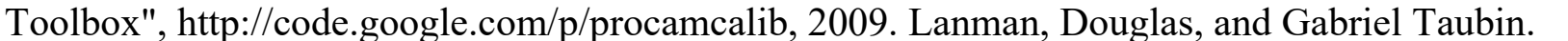

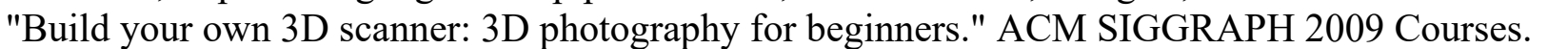
$\square|||||||||| \mid$

\section{Image/Figure:}




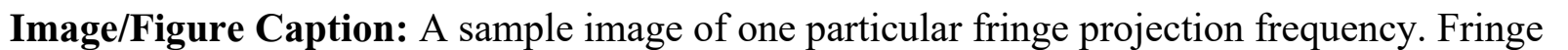

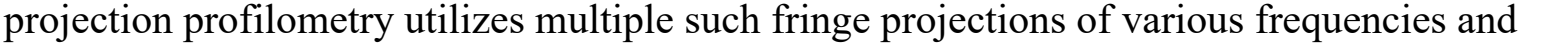

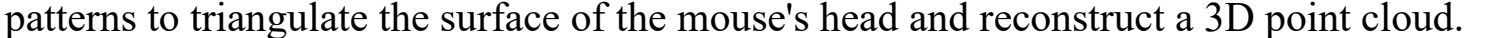

First Name: $\square \square \square \square \square$

Last Name: $\square \square \square\|\square\| \square$

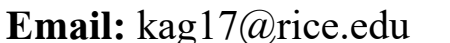

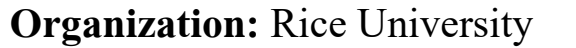

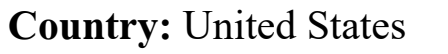




\title{
ID: LB144 \\ Comparison of Magnetic Resonance Imaging and Magnetic Particle Imaging of Islet transplantation using Machine Learning
}

\author{
Hanaan Hayat, Precision Health Program - College of Human Medicine, Michigan State \\ University, hayathan@msu.edu
}

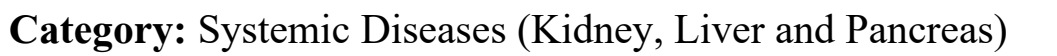

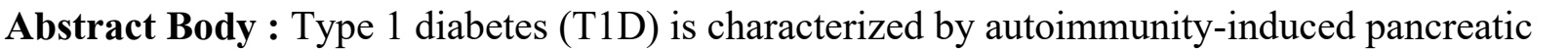

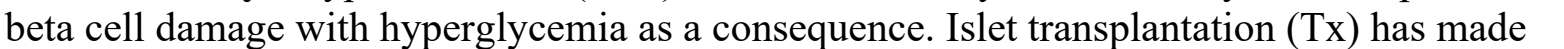

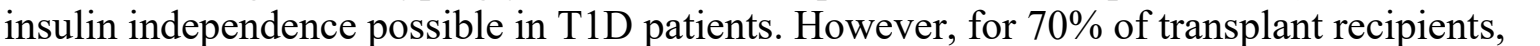

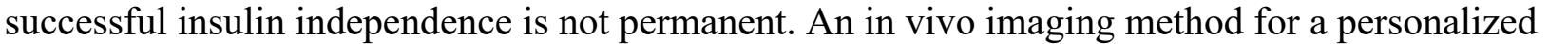

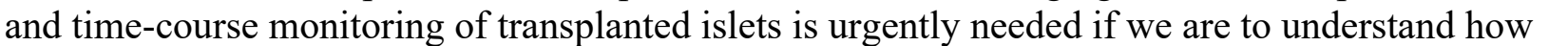

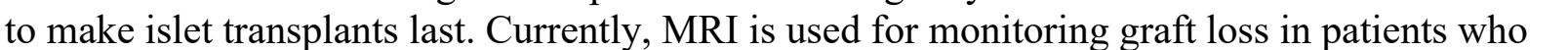

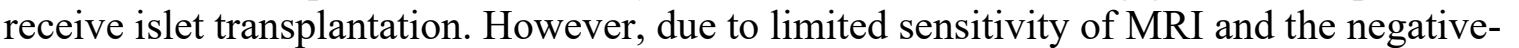

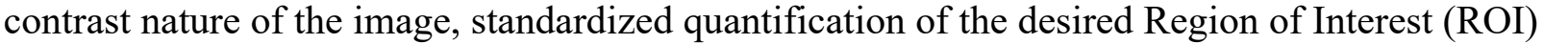

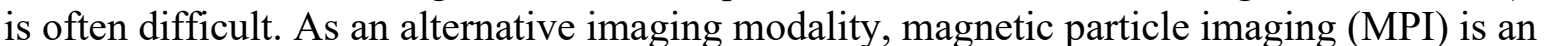

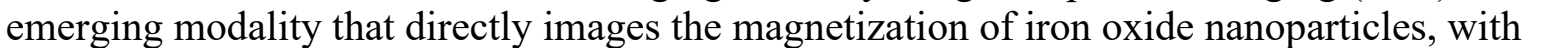

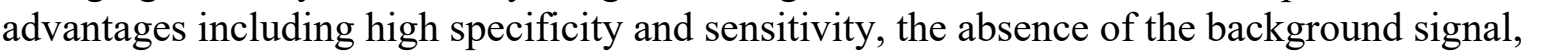

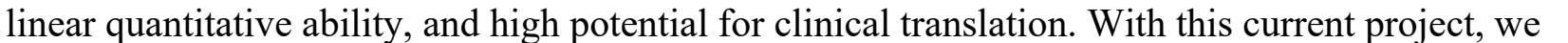

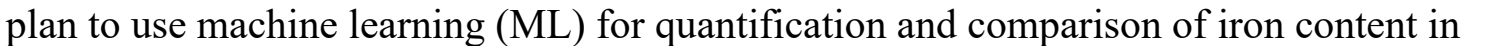

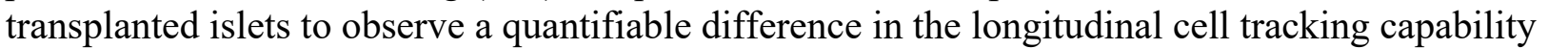

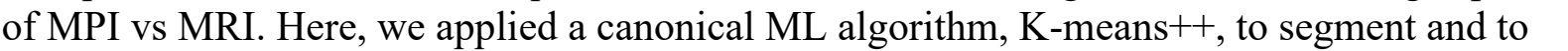

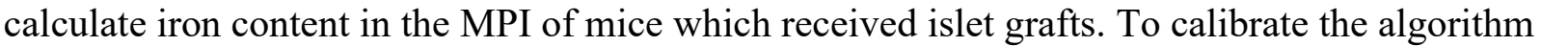

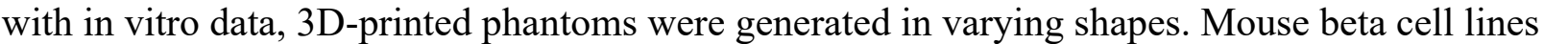

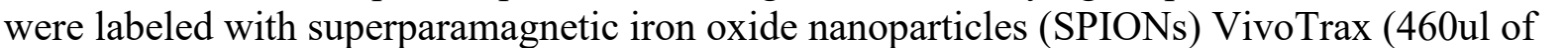
5.5ug/ul stock solution per $25 \mathrm{~cm}^{2}$ flask, $24 \mathrm{hr} @ 37^{\circ} \mathrm{C}$ ) prior to imaging and dispersed in 1\%

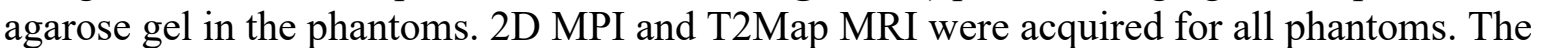

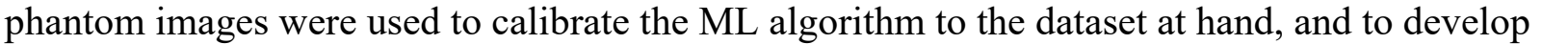

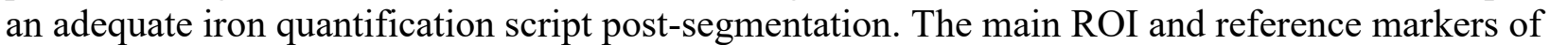

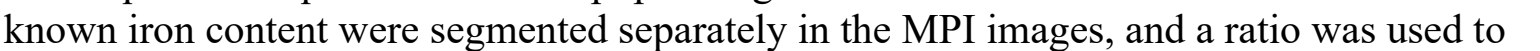

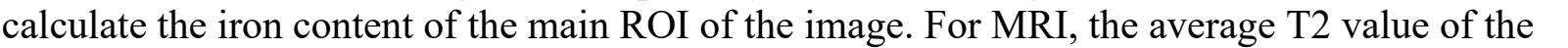

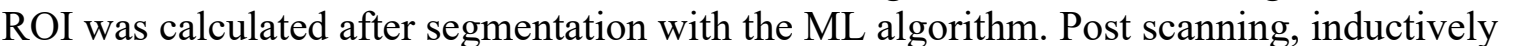

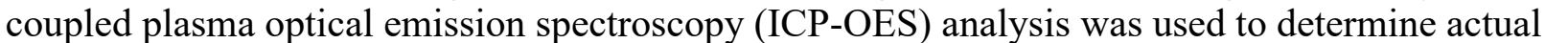

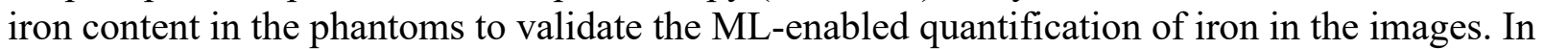

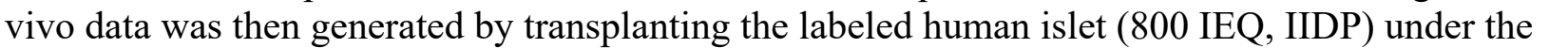

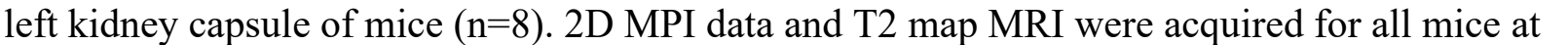
day $1,5,7,14,21$, and 30 post transplantation. The algorithm's performance was then tested on

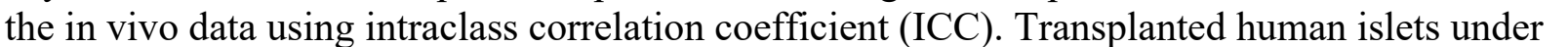

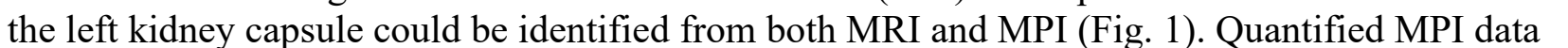

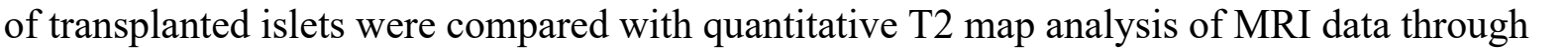

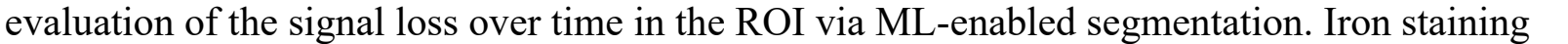

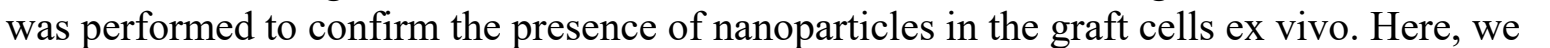




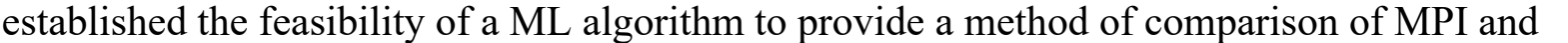

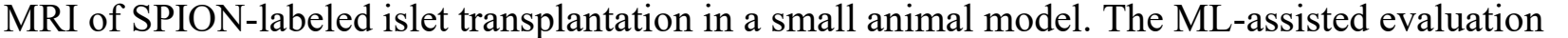

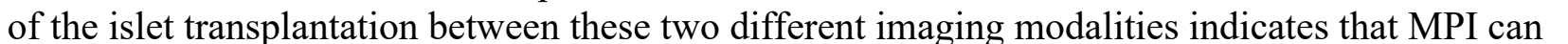

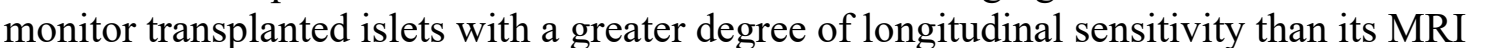

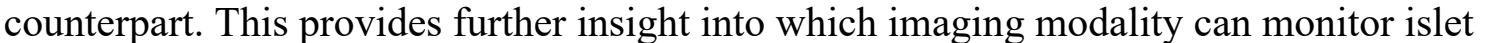

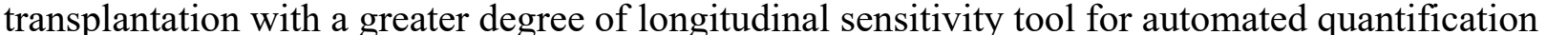

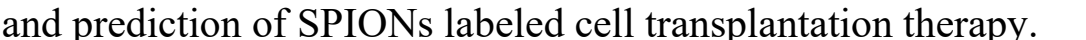

\section{Image/Figure:}

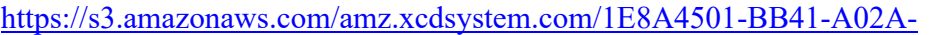

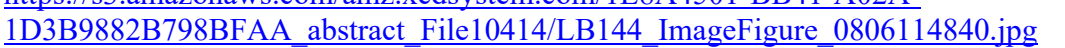

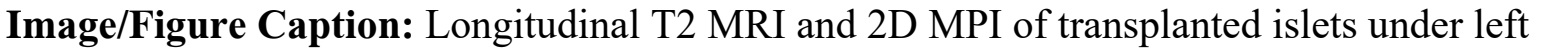

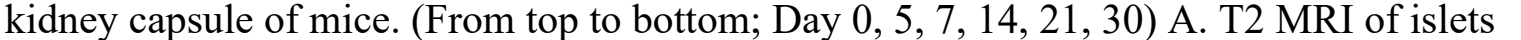

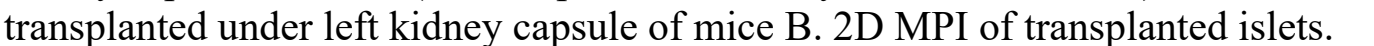

Full Name of Abstract's 1st Author : $\square \square \square\|\square\| \square \square \square \| m$

First Name: $\square \square \square\|\| \square$

Last Name: $\square \square \square\|\|$

Email: $\square\|\|\|\| \square \square \square\|\| \| \square$

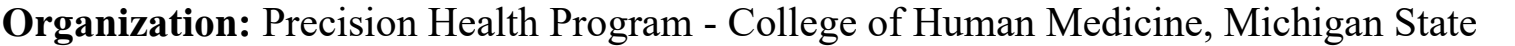

$\square \square\|\|\|\|\|\| \|$

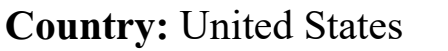




\title{
ID: LB145 \\ Magnetic Particle Imaging vs. Nuclear Medicine CAR-T Cell Imaging: A Comparison of Tracer Toxicity and Labeling Efficiency
}

\author{
Renesmee Kuo, University of California, Berkeley, renesmeek@berkeley.edu
}

\section{Category: $\square \square\|\| \| \square$}

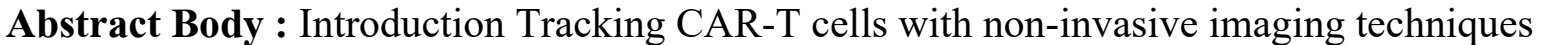

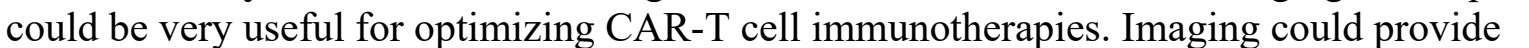

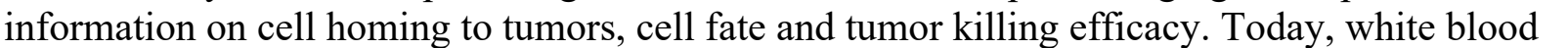

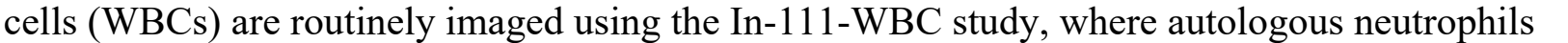

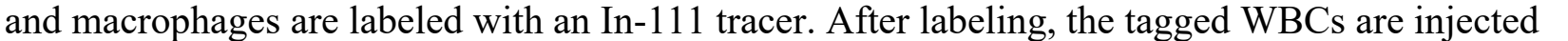

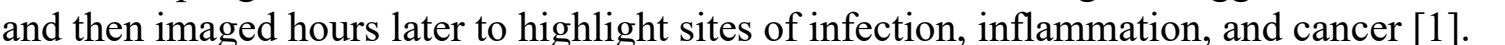

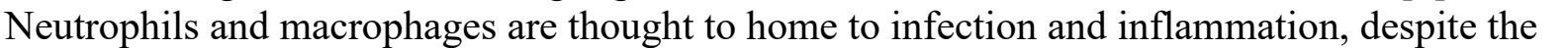

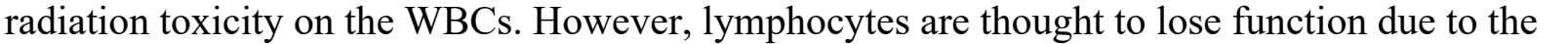

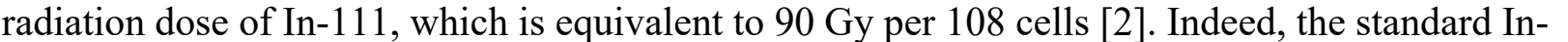

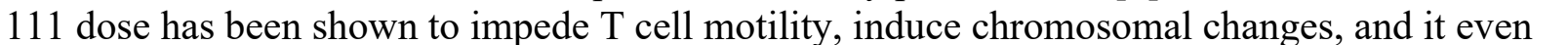

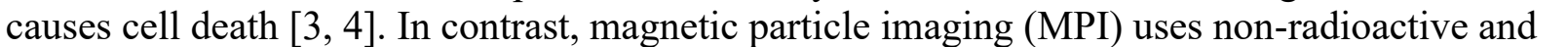

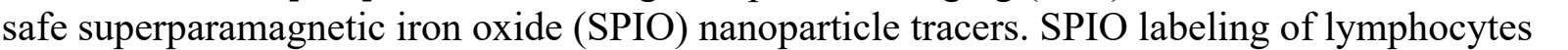

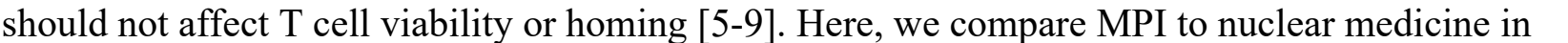

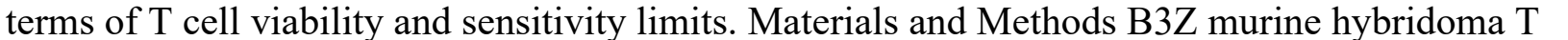

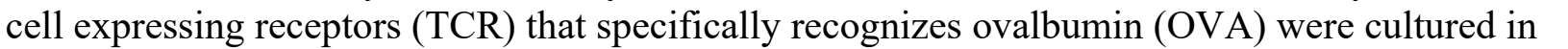

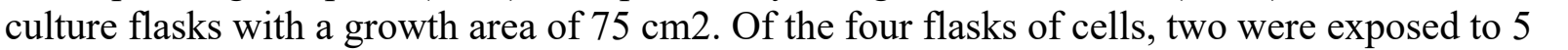

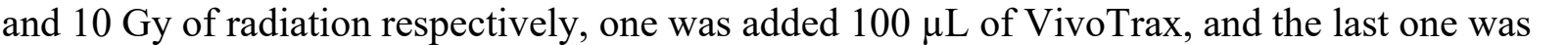

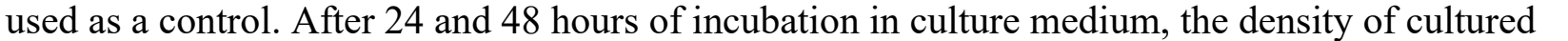

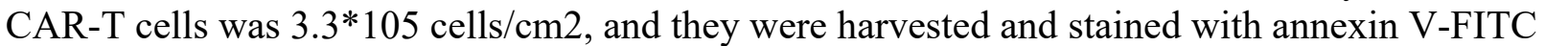

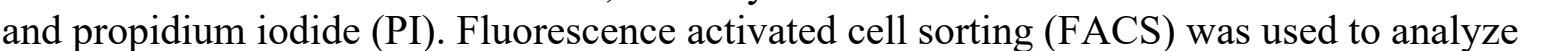

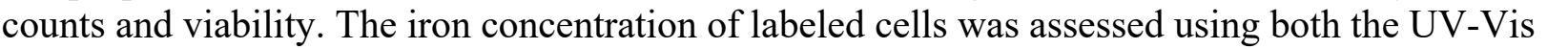

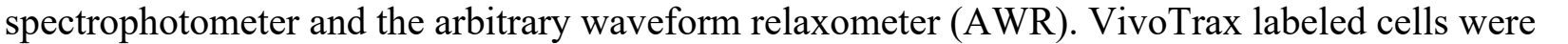

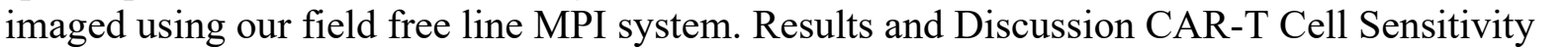

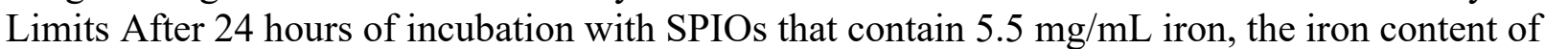

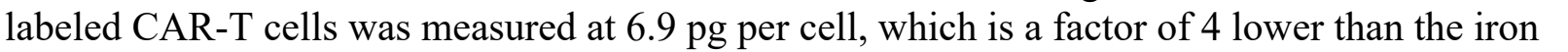

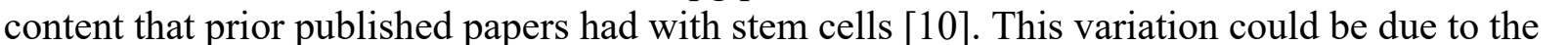

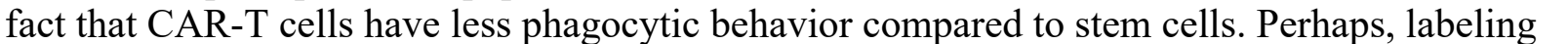

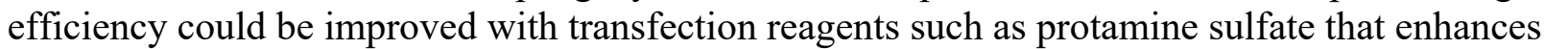

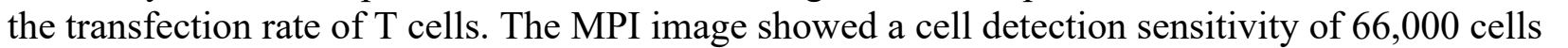

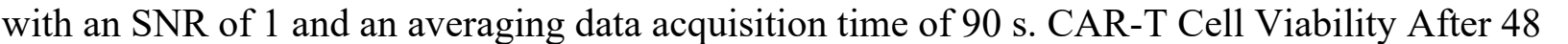

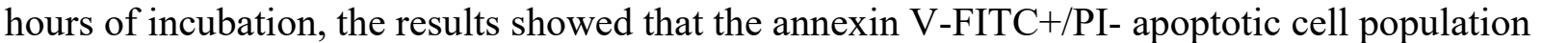

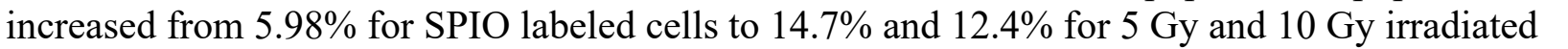

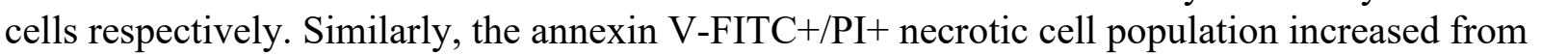

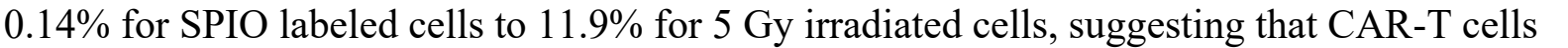

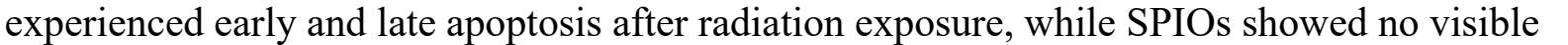

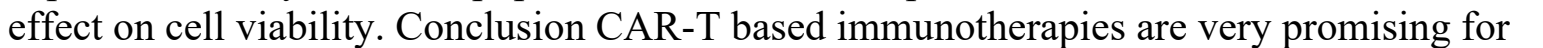




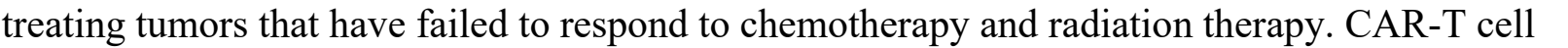

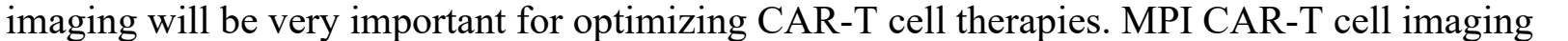

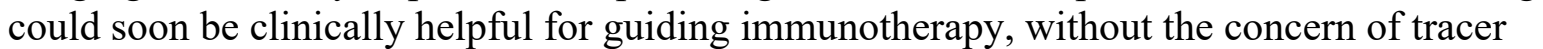

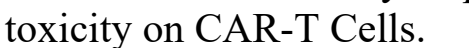

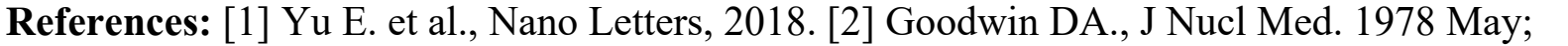

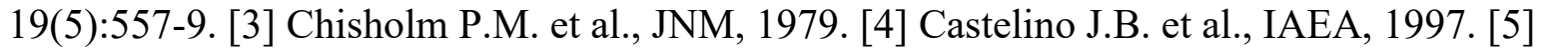

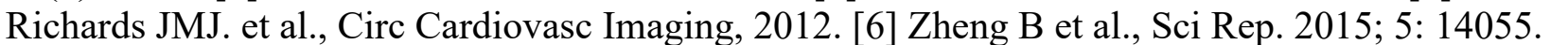

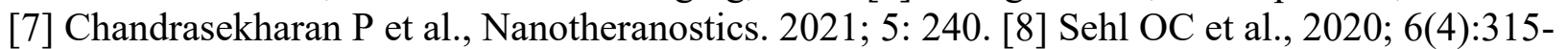

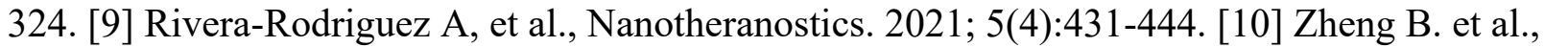

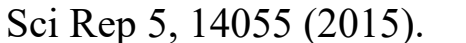

\section{Image/Figure:}

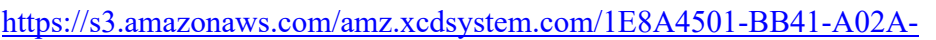

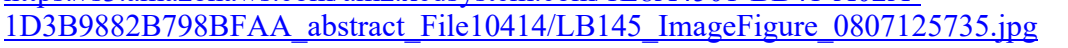

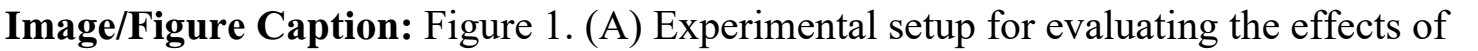

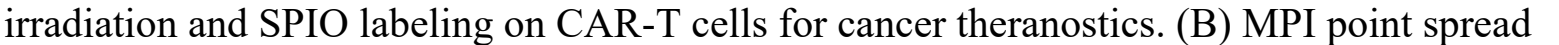

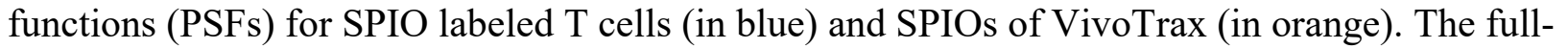

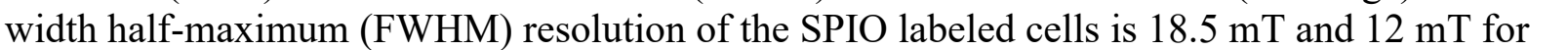

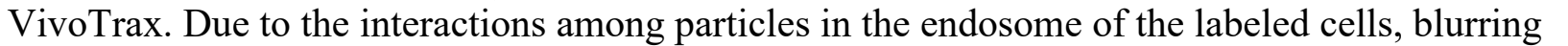

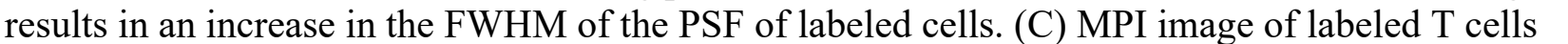

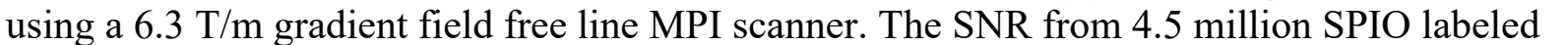

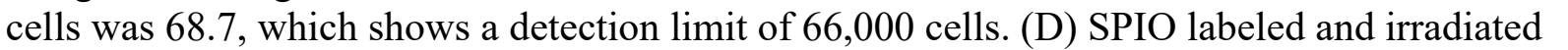

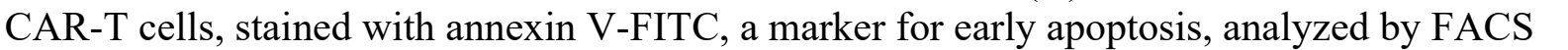

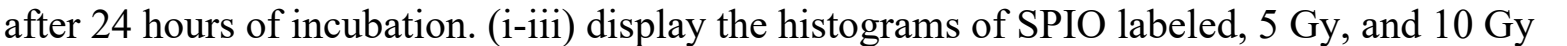

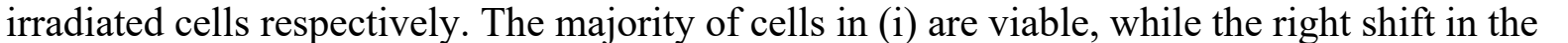
ए

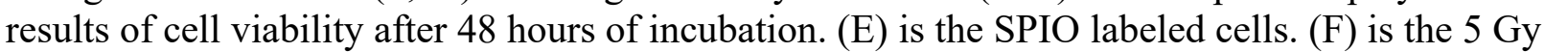

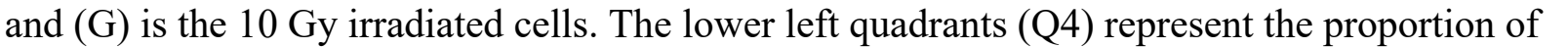

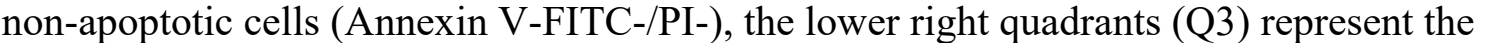

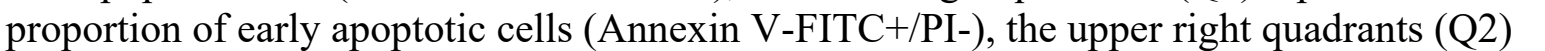

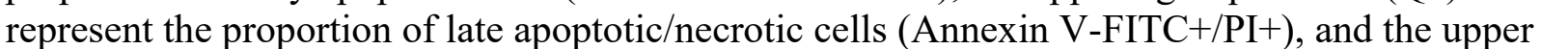

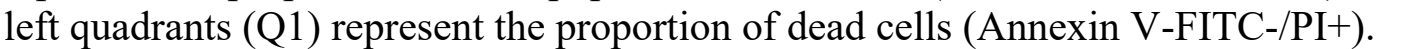

First Name: $\square \square\|\| \square \square$

Last Name: $\square \square \|$

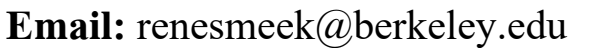

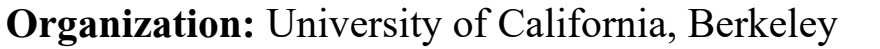

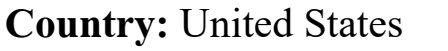




\title{
ID: LB146 \\ Inertial Measurement Unit-Based Rigid-Body Motion Tracking for Medical Imaging
}

\author{
Jonathan Fisher, Stanford University, jofisher@stanford.edu
}

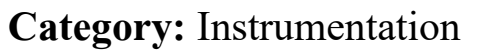

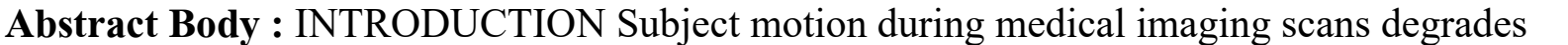

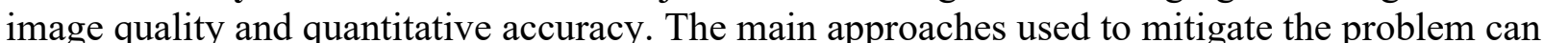

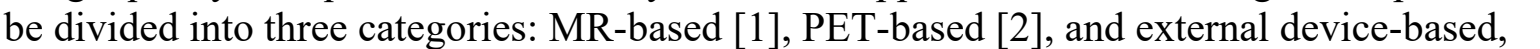

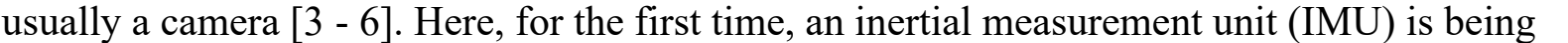

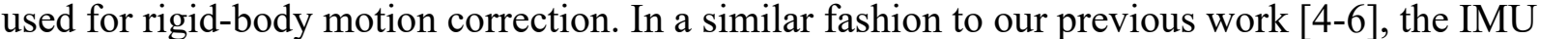
will be attached to the patient's head and track its motion in the IMU coordinate system, which

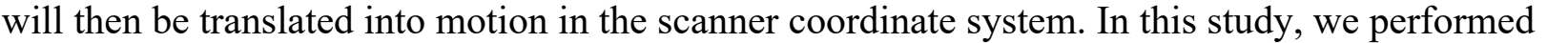

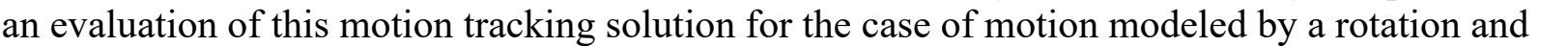

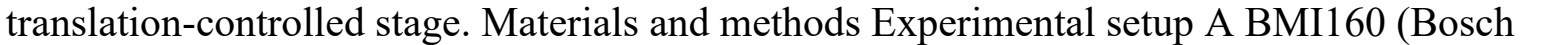

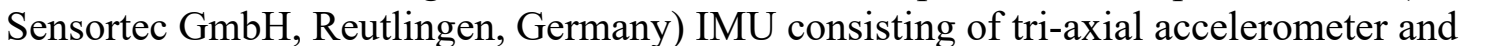

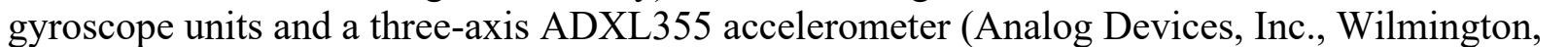

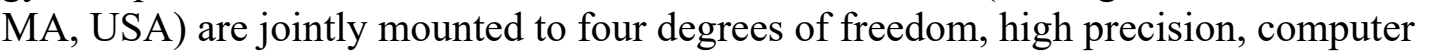

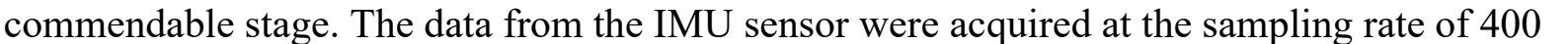

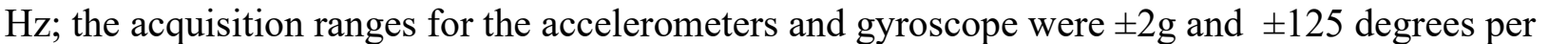

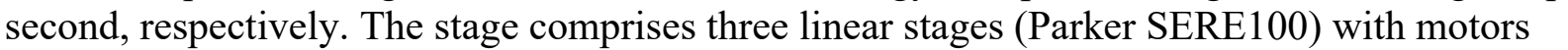

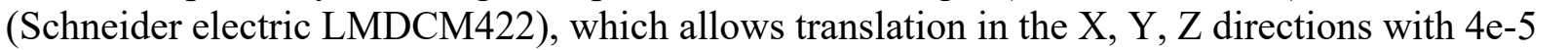

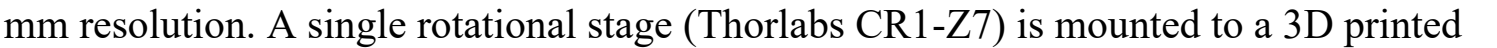

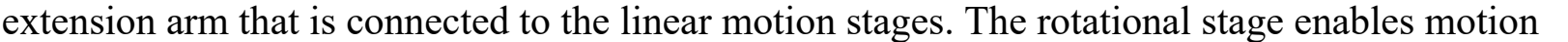

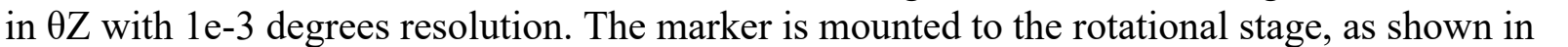

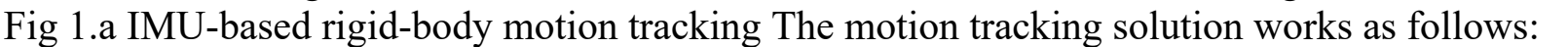

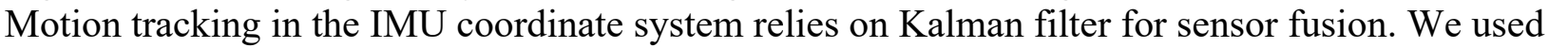

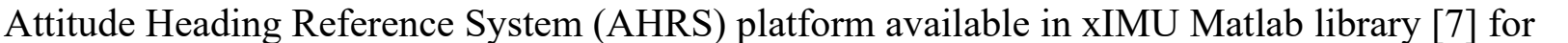

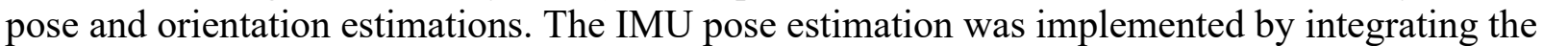

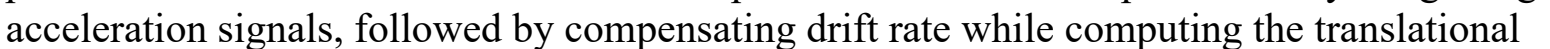

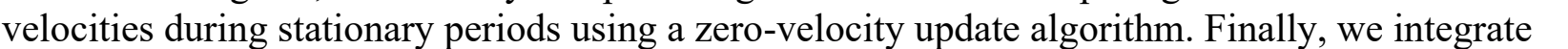

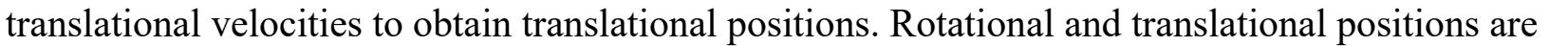

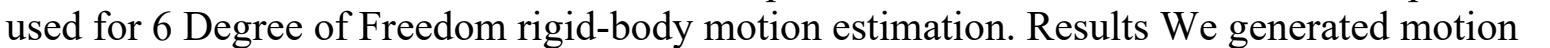

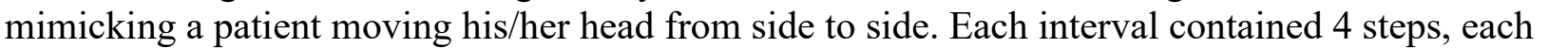

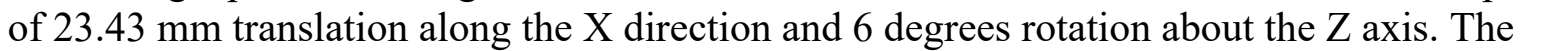

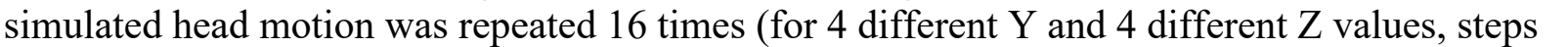

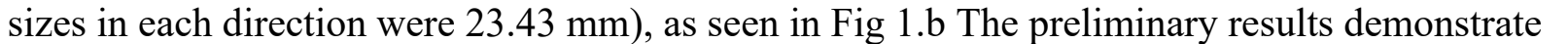

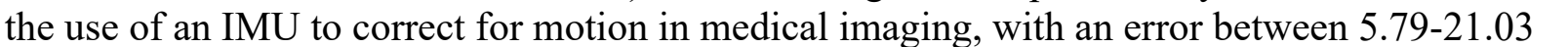

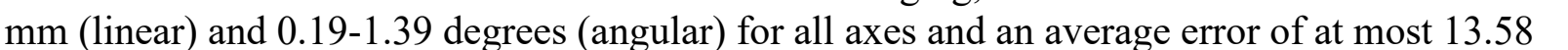

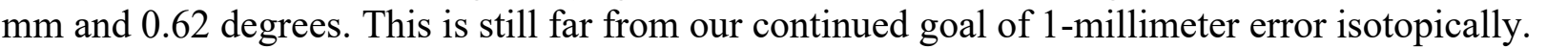

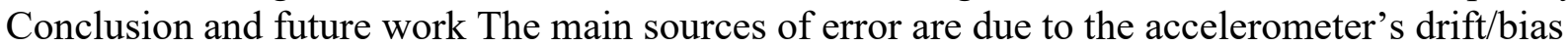

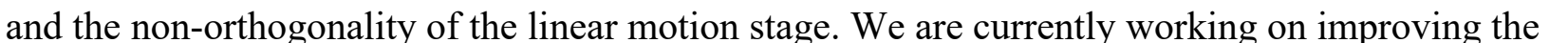




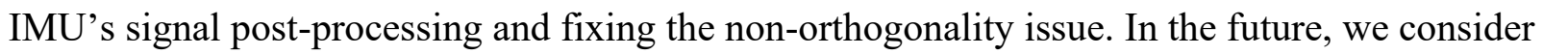

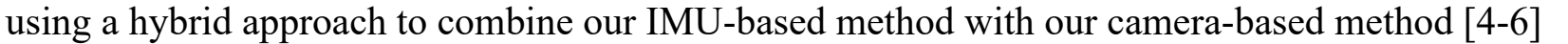
पाm

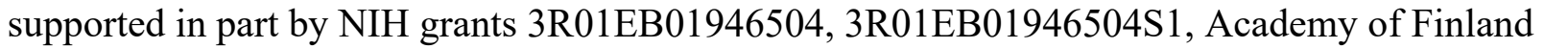

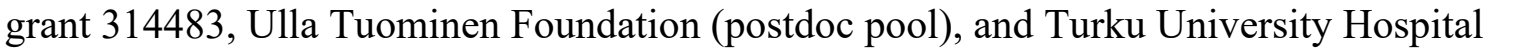

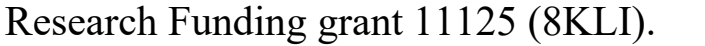

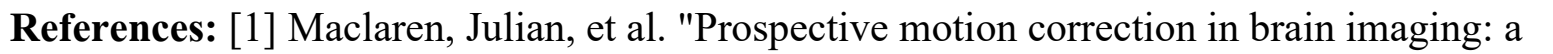
पण

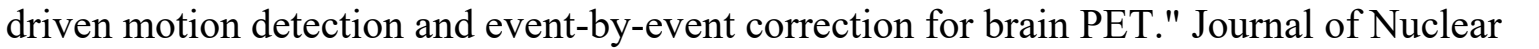

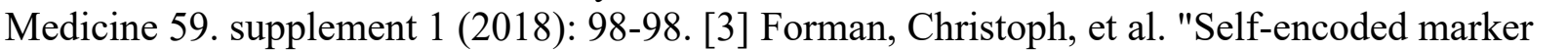
ए एव

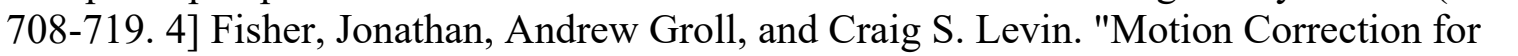
a

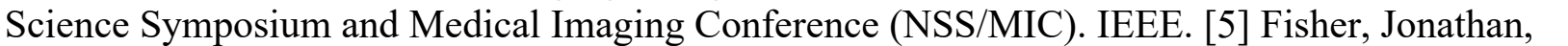

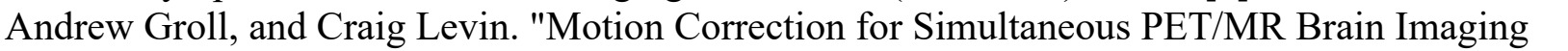

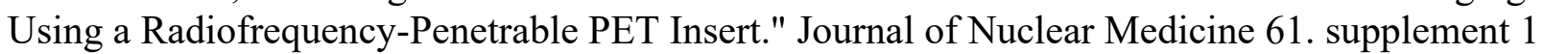

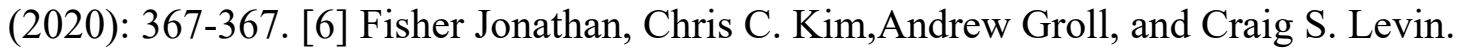

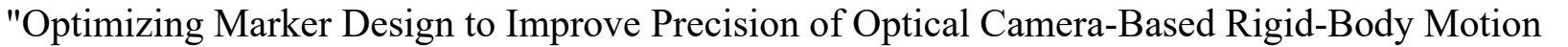

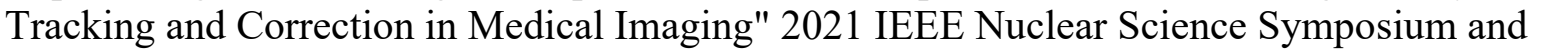

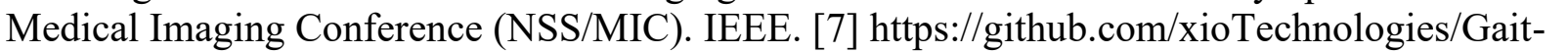

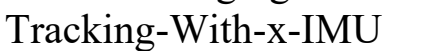

\section{Image/Figure:}

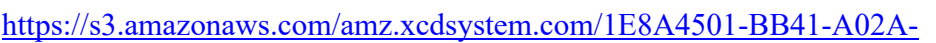

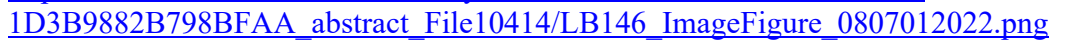

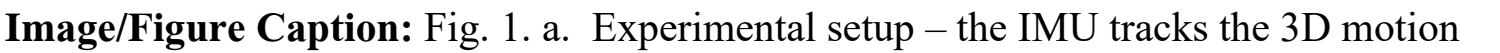

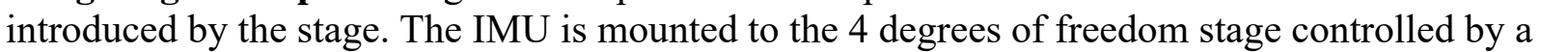

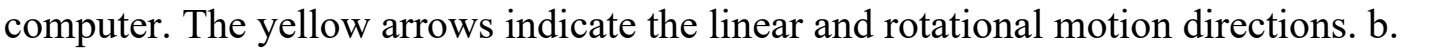

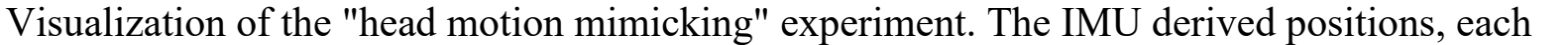

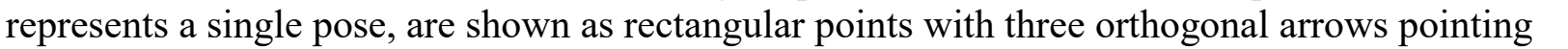
ए

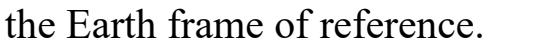

First Name: $\square \square|\||\|| \square$

Last Name: $\square\|\| \square\|\|$

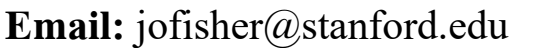

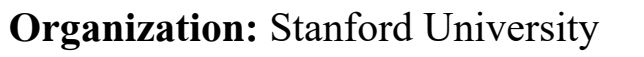

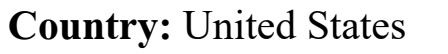




\title{
ID: LB147 \\ Gold quantum clusters for the imaging and therapy of gastrointestinal diseases \\ Dingxin Liu, State Key Laboratory of Oncology in South China, Sun Yat-sen University \\ Cancer Center, liudingx@sysucc.org.cn
}

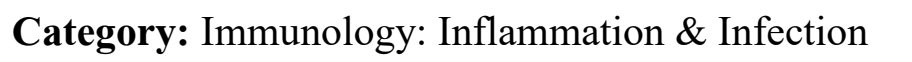

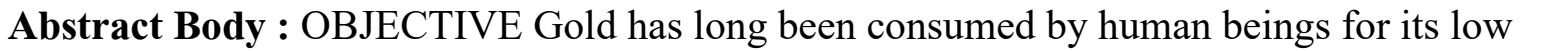

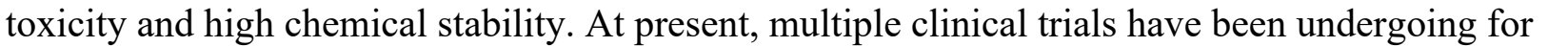

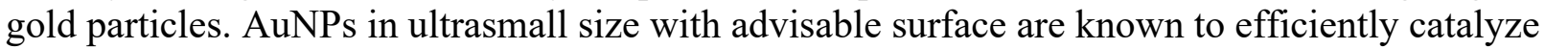

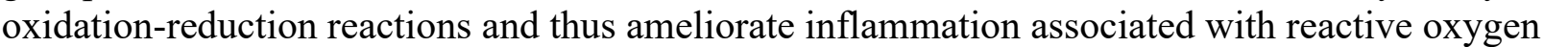
species (ROS) [1]. In this study, we aimed to synthesize AuQCs with alpha lactalbumin $(\alpha \square \square \square$

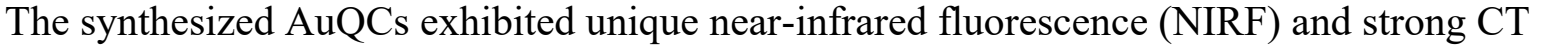

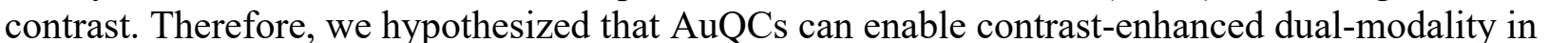

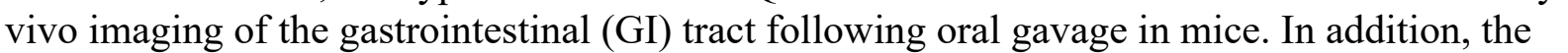

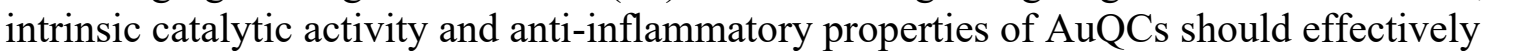

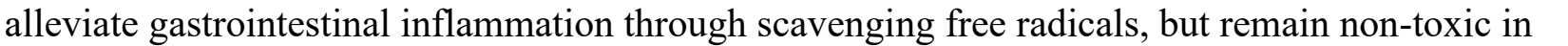

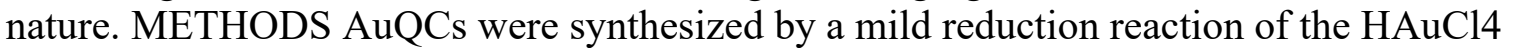

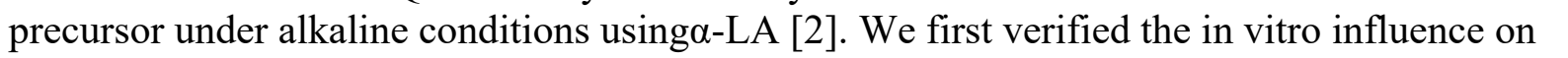

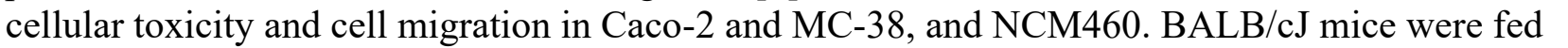

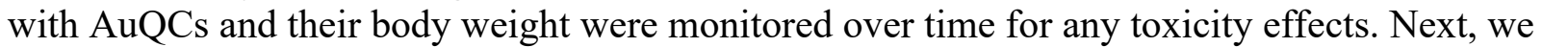

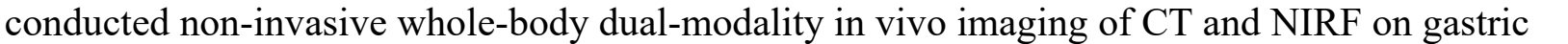

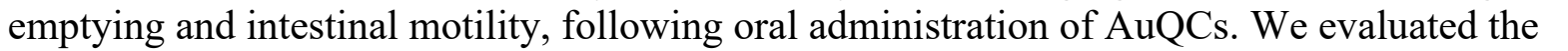

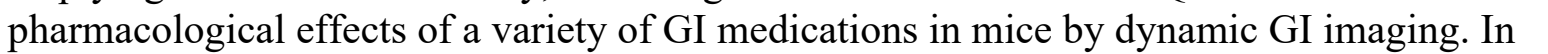

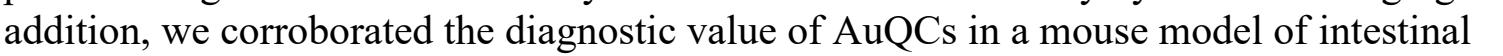

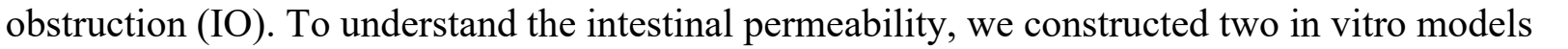

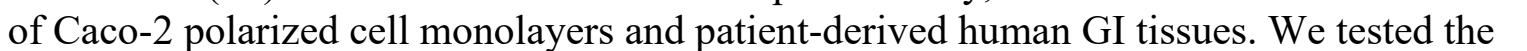

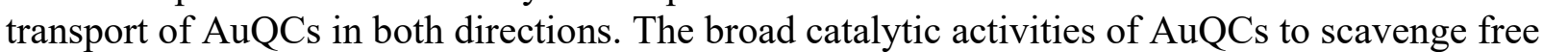

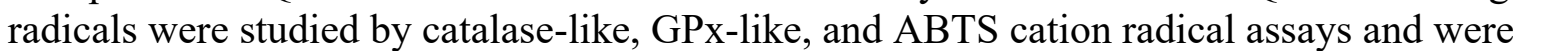

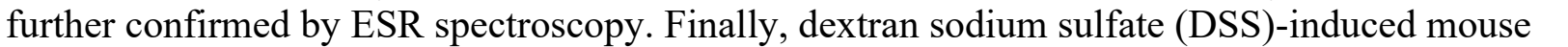

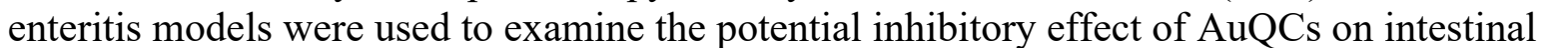

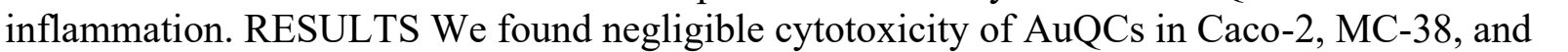

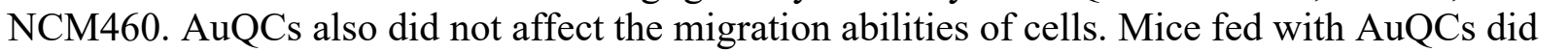

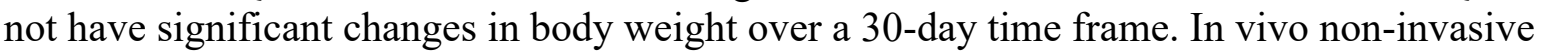

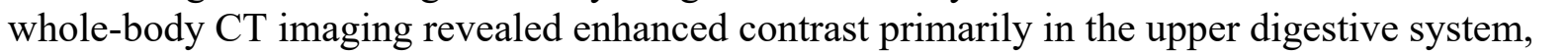

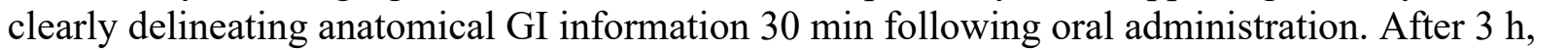

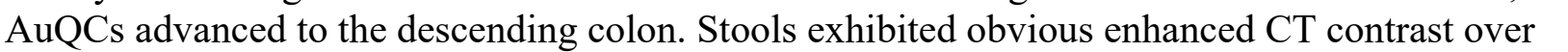

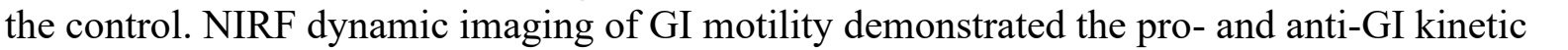

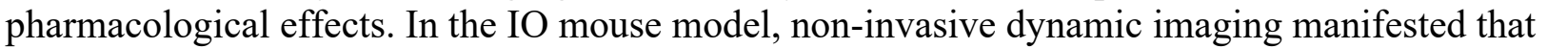

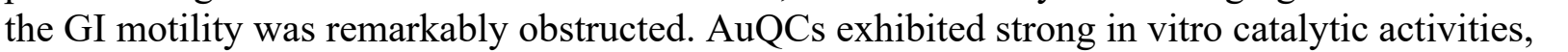

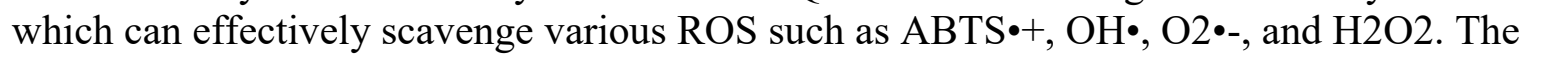

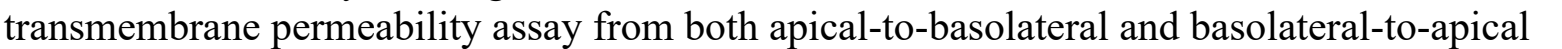

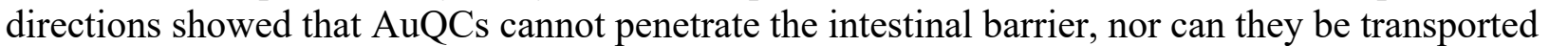




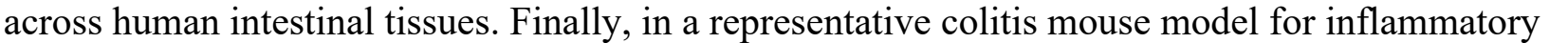

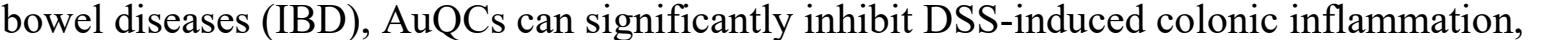

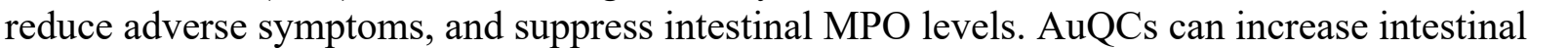

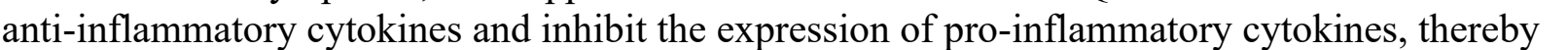

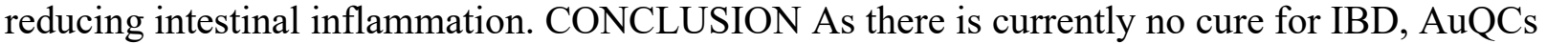

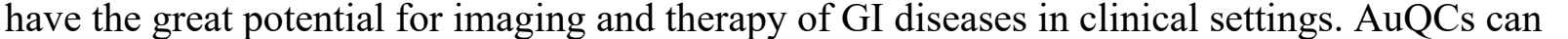

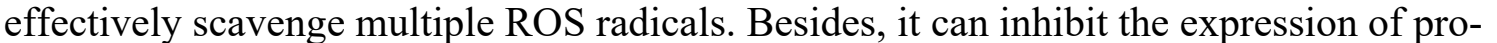

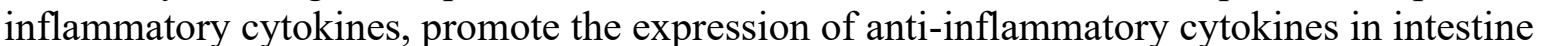

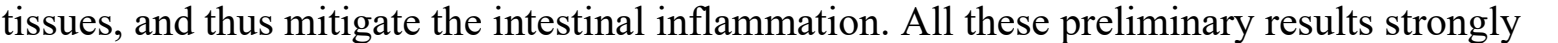

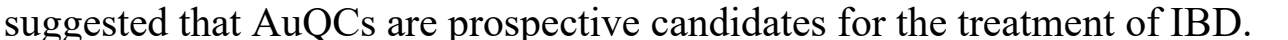

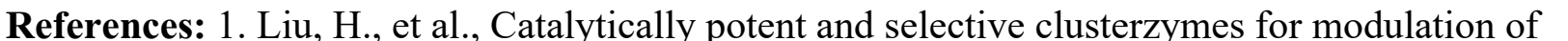

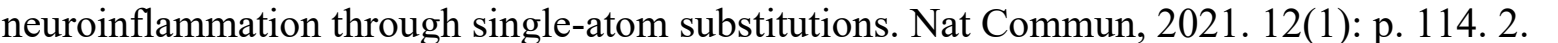

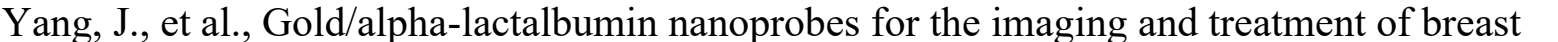

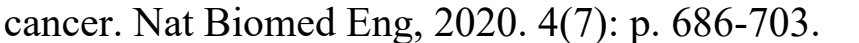

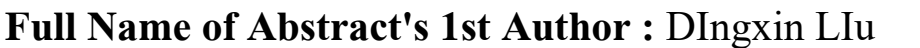

First Name: $\square \square\|\square\| \square$

Last Name: $\square \square \square$

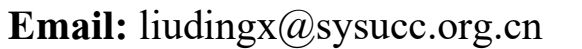

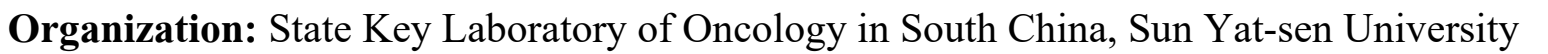

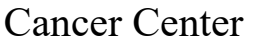

Country: $\square \square\|\| \square$ 


\title{
ID: LB148 \\ Development of a Modular readout design for a head and neck dedicated PET system based on CZT detectors
}

Shiva Abbaszadeh, University of California, Santa Cruz, sabbasza@ucsc.edu

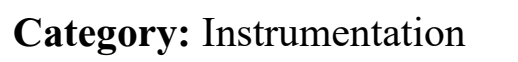

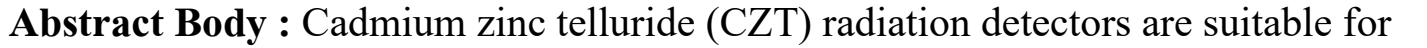

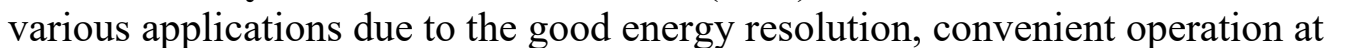

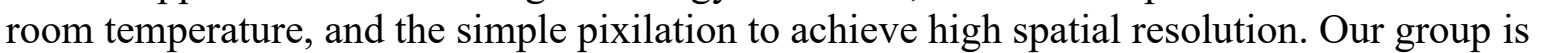

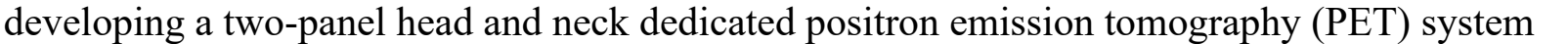

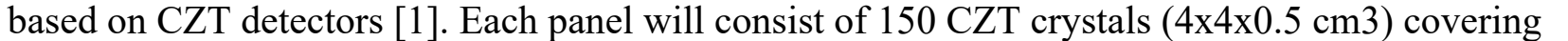

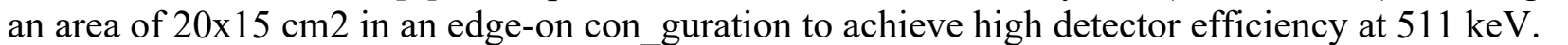

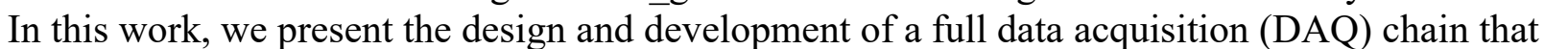

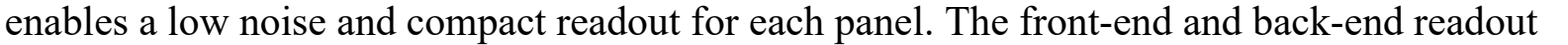

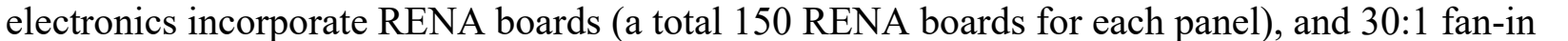

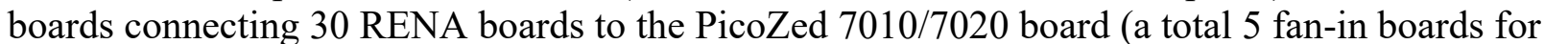

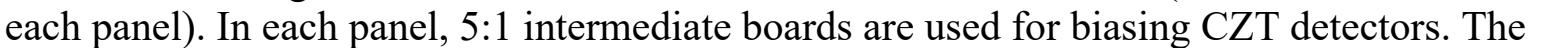

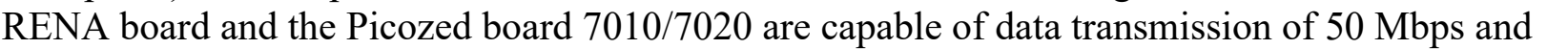

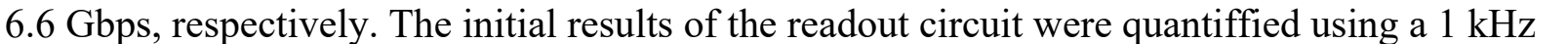

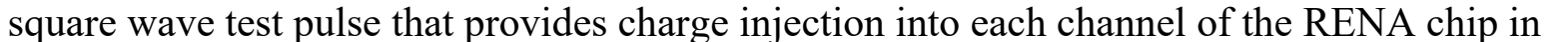

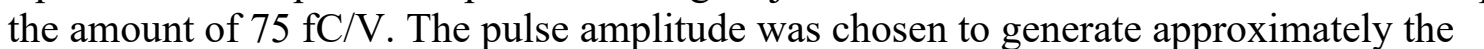

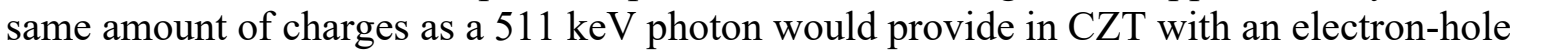

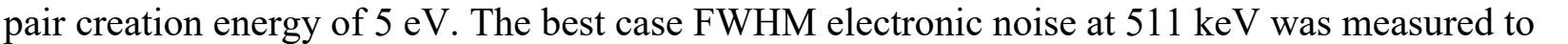

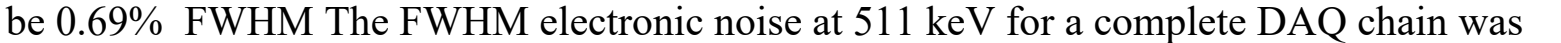

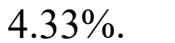

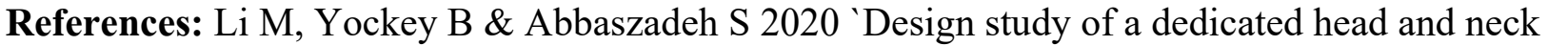

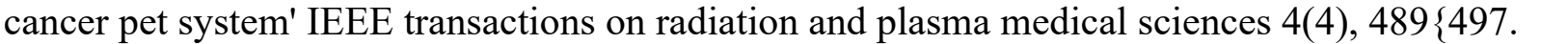

\section{Image/Figure:}

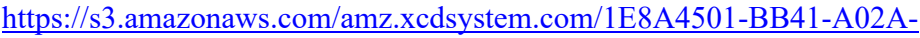

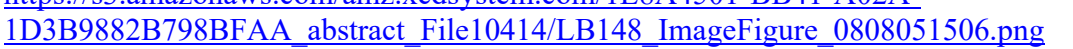

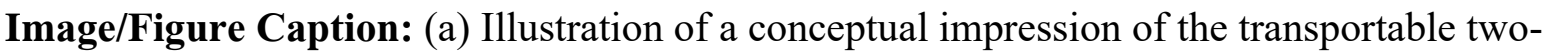

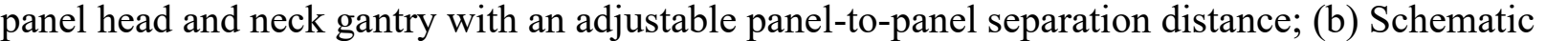

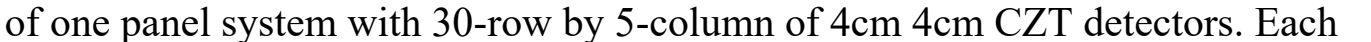

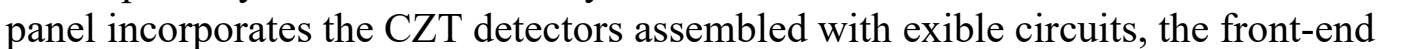

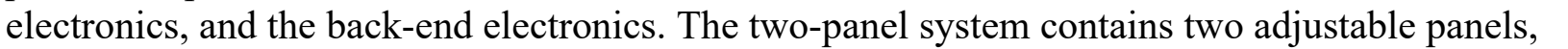

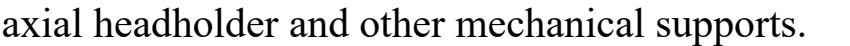

First Name: 
Last Name:

Email: $\square\|\square\|\|\square \square \square\| \| \square \square \square \square$

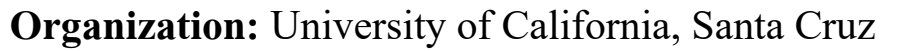

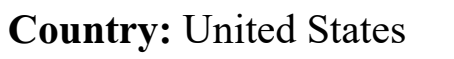




\title{
ID: LB149 \\ OregonFluor enables quantitative intracellular paired agent imaging (iPAI) to assess drug target availability in live cells and tissues
}

Lei Wang, Oregon Health \& Science University, wanglei@ohsu.edu

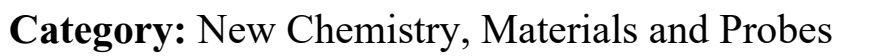

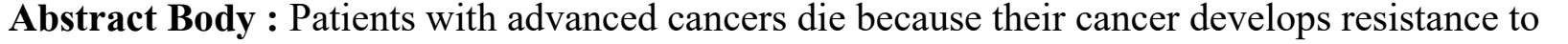
all available therapeutic strategies. "Personalized cancer medicine" strives to use biomarker $\square$

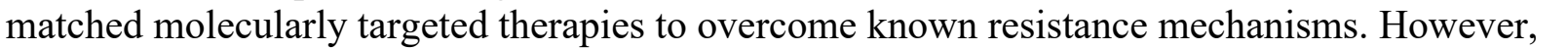

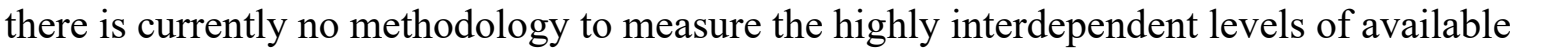

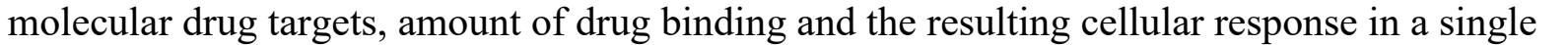

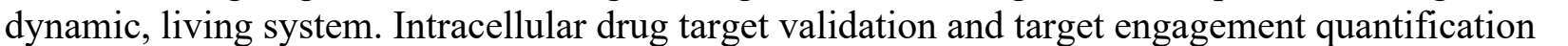

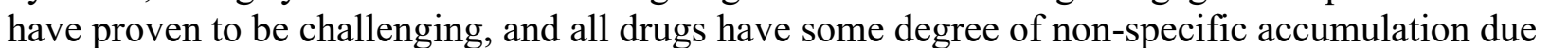
प

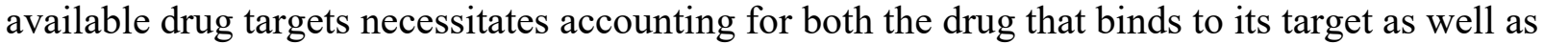

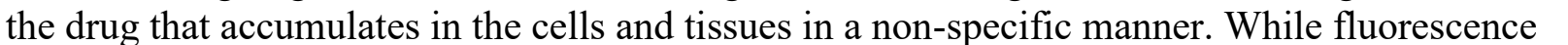

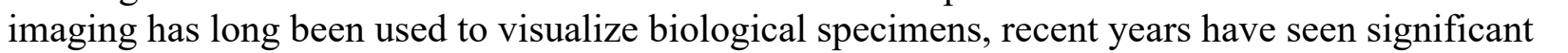

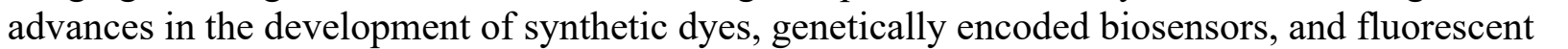

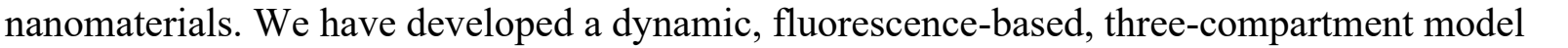

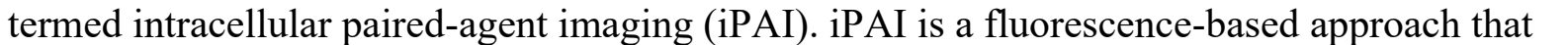

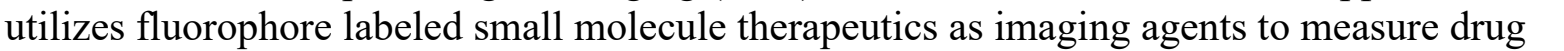

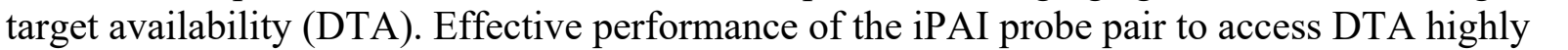

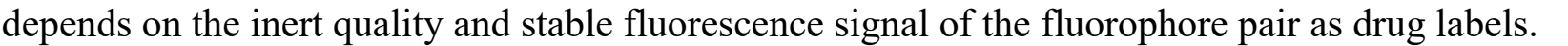

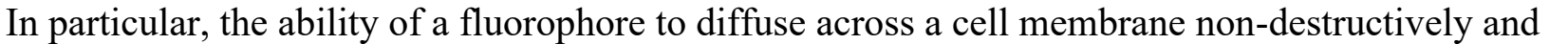

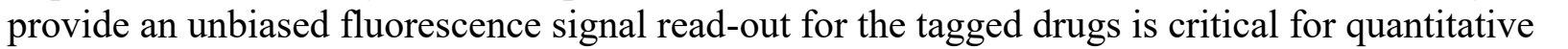

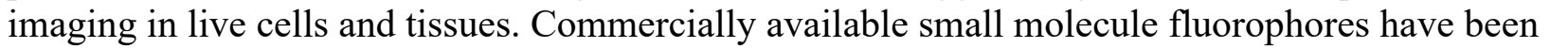

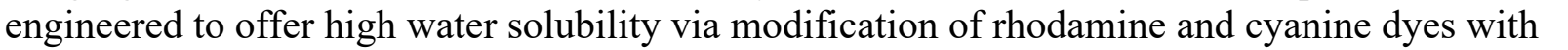

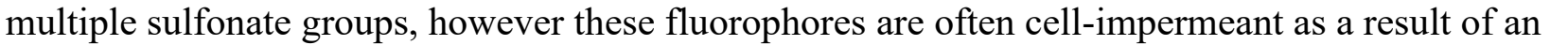

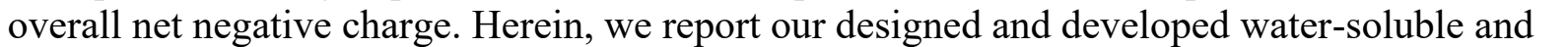

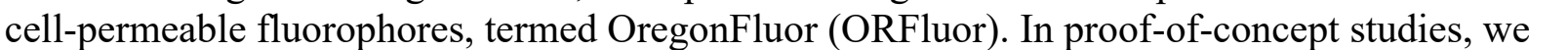

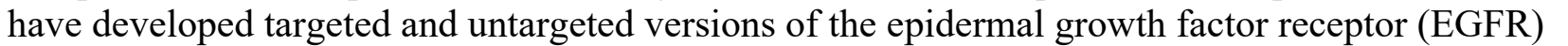

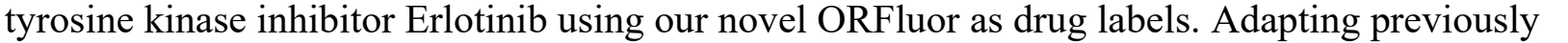

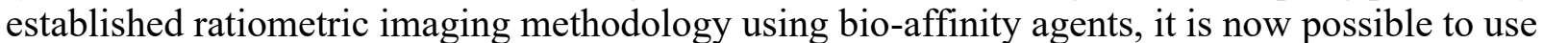

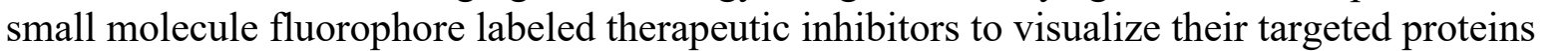
ए

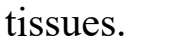

Full Name of Abstract's 1st Author : $\square \square \| m \square \square \square \square \square$

First Name: $\square \square \| m$

Last Name: $\square \square \square \square$ 
Email:

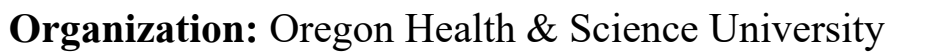

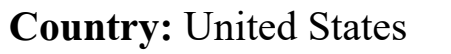




\title{
ID: LB150 \\ Machine Learning-Based Computational Magnetic Resonance Models for Rapid Diagnosis of Corona Virus Disease 2019 (COVID-19) Using Magnetic Resonance Fingerprinting Data
}

\author{
Michael Dada, Federal University of Technology, Minna, Nigeria, \\ dadamichael@futminna.edu.ng
}

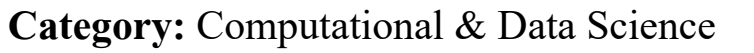

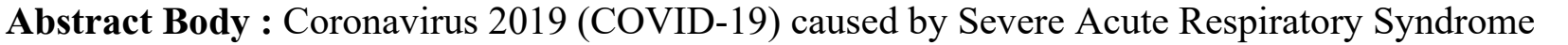

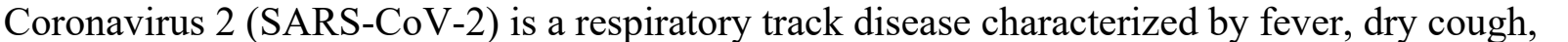

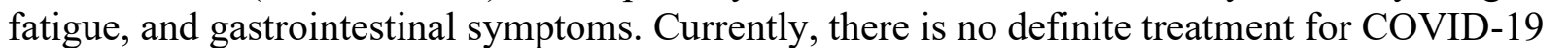

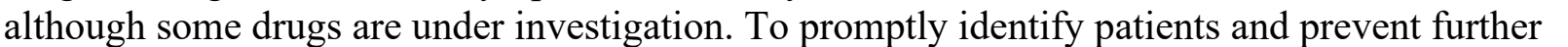

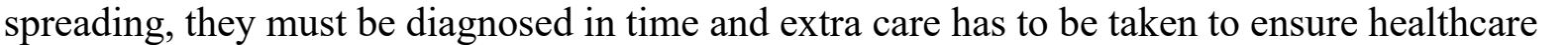

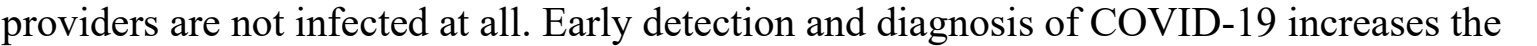

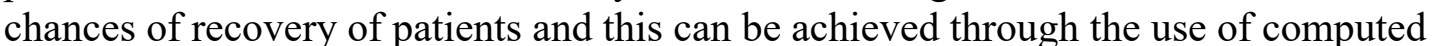

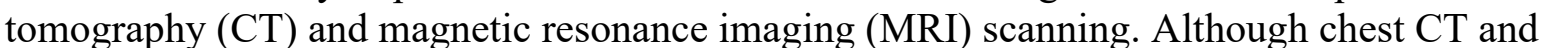

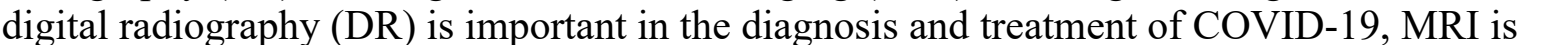

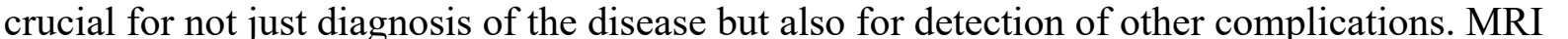

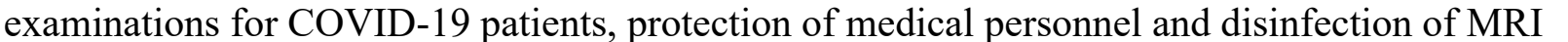

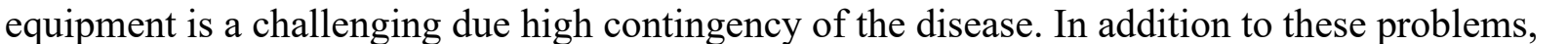

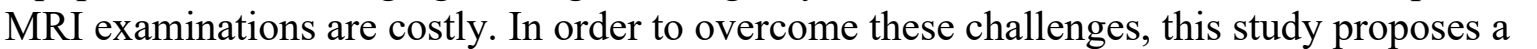

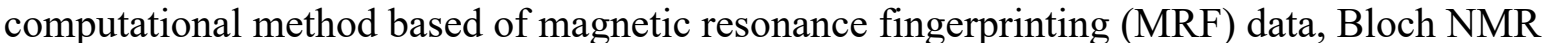

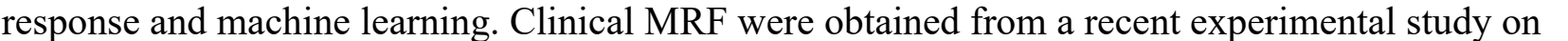

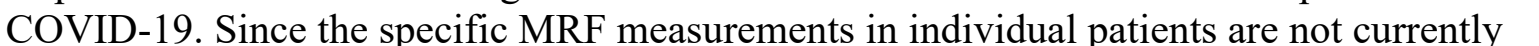

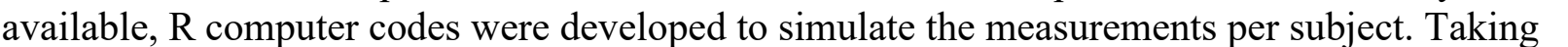
ए ए

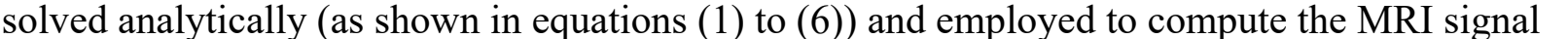

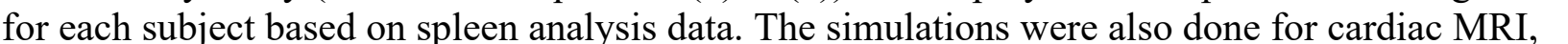

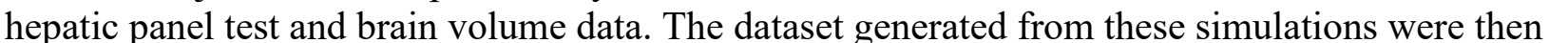

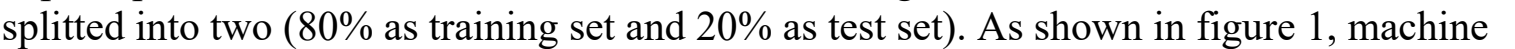

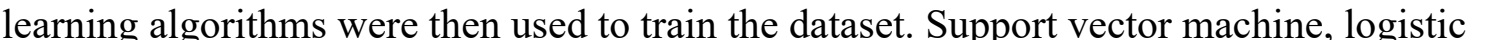

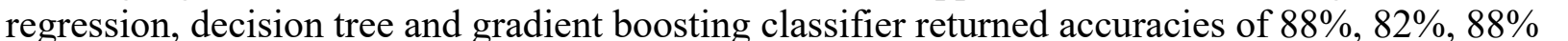

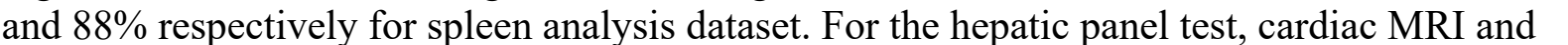

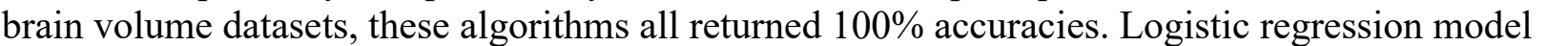

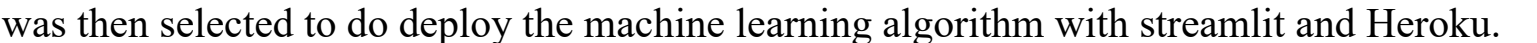

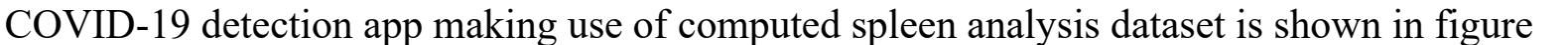

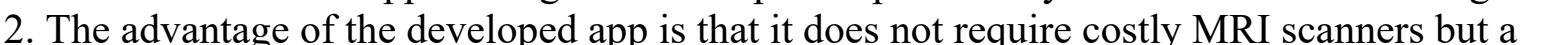

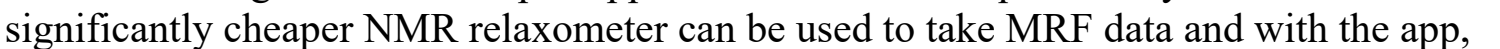

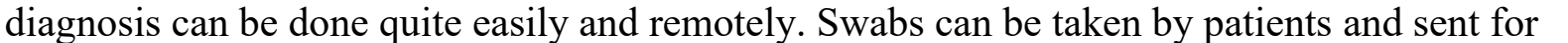

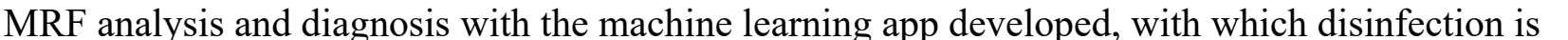

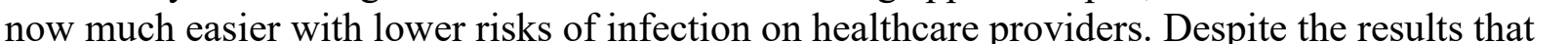

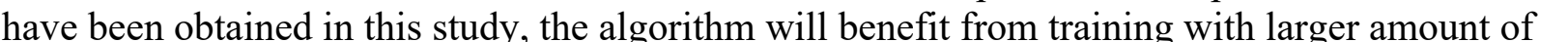




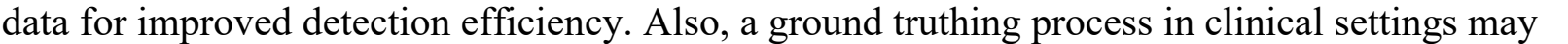

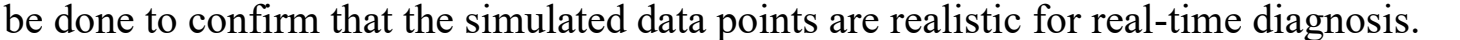

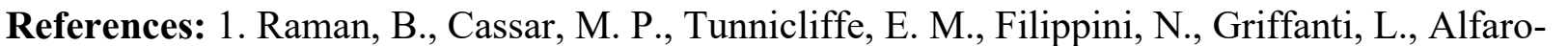

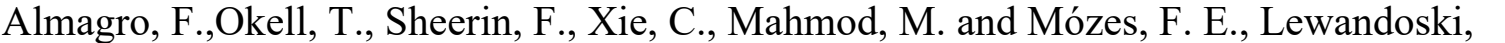

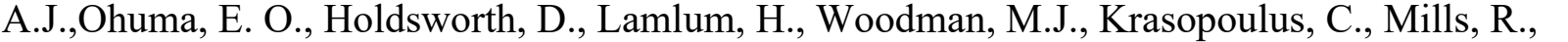

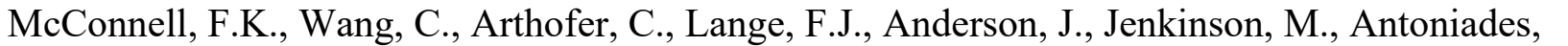

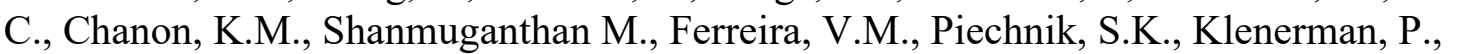

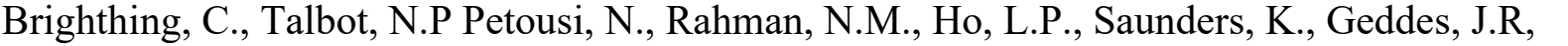

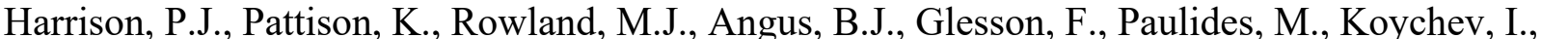

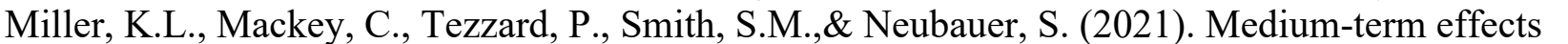

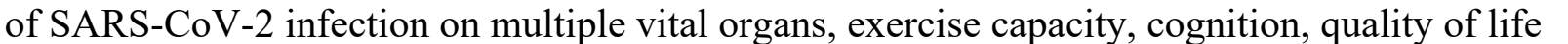

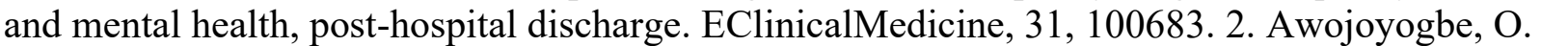

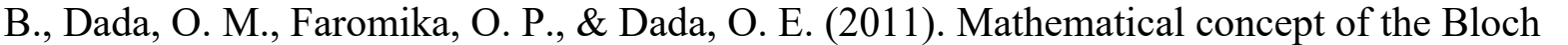

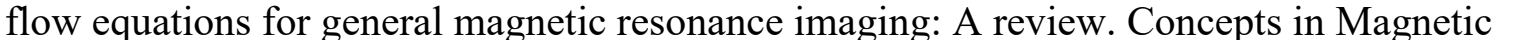

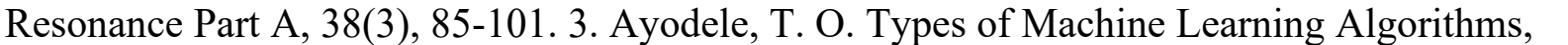

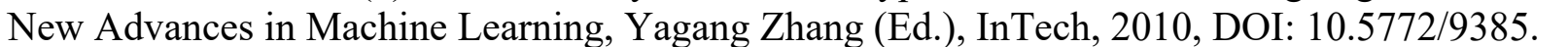

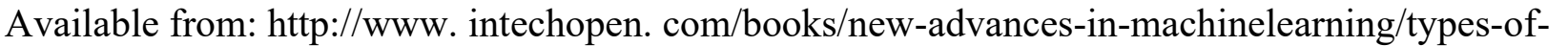

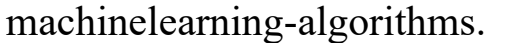

\section{Image/Figure:}

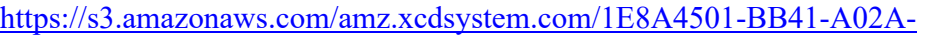

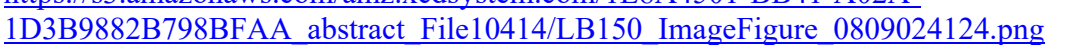

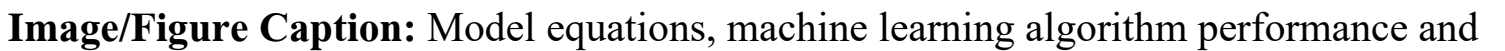
$\square\|\|\|\|\|\| \| \square$

First Name: $\square \square\|\|\|\|$

Last Name: $\square \square \square \| \square$

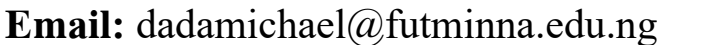

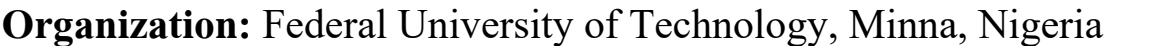

Country: $\square \square\|\|\|\|$ 


\title{
ID: LB151 \\ Development of Brain Atlas for Quantitative Comparison of Anatomical \\ Parcellations
}

Michael Dada, Federal University of Technology, Minna, Nigeria, dadamichael@futminna.edu.ng

Category: $\square \square \square\|\square\| \| \square \square \square$

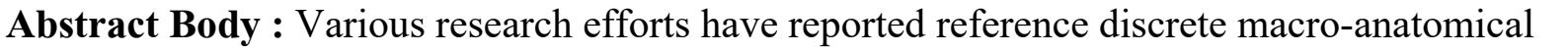

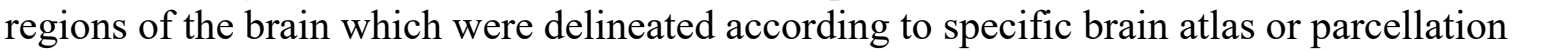

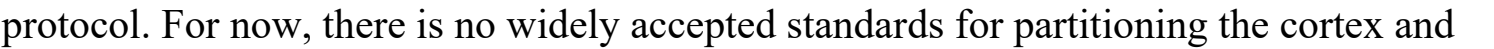

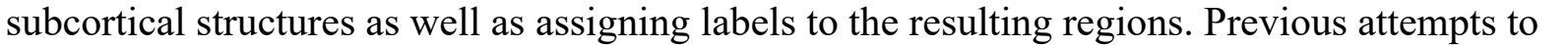
ए

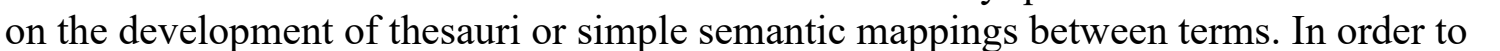

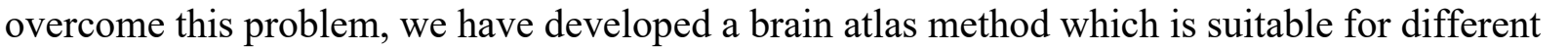

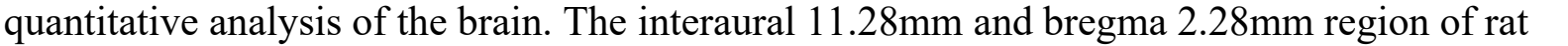

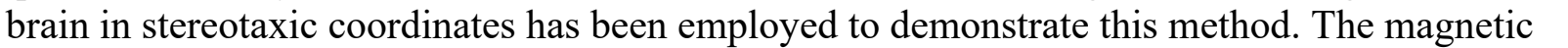

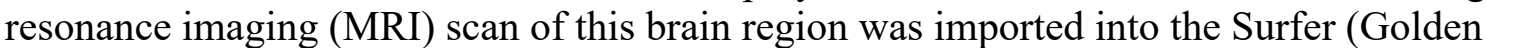

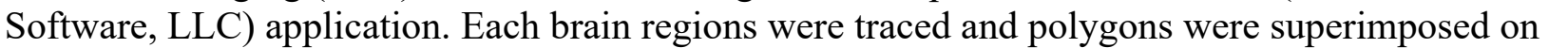
एण ए

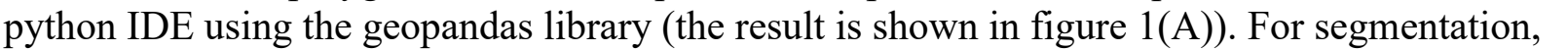

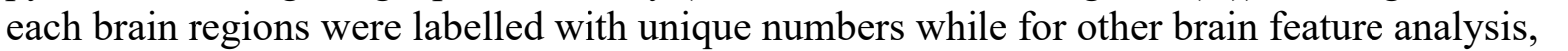

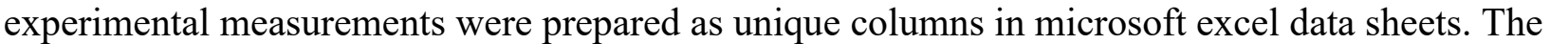

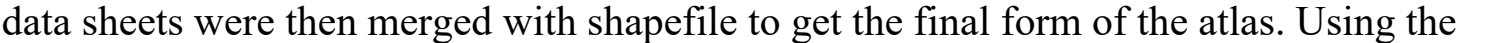

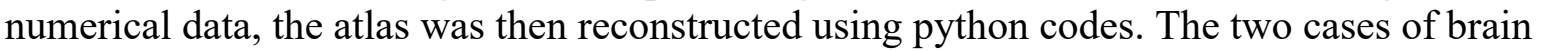

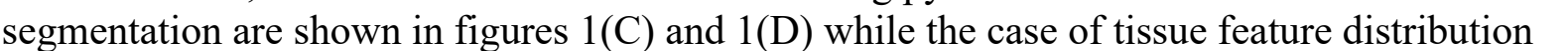

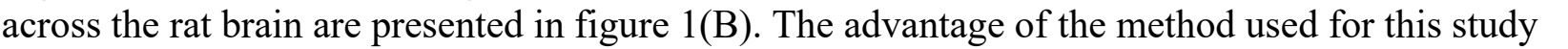
ए

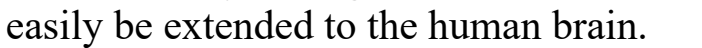

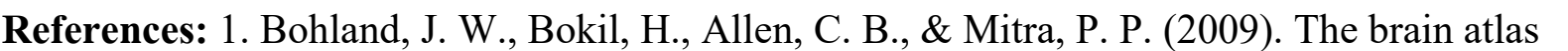

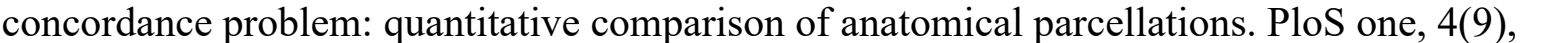

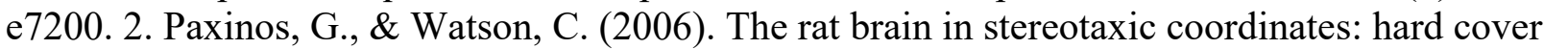

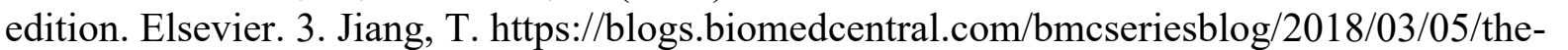

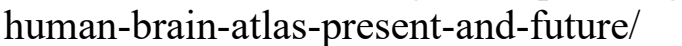

\section{Image/Figure:}

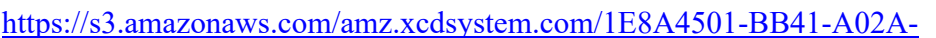

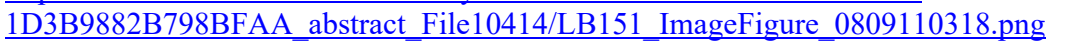




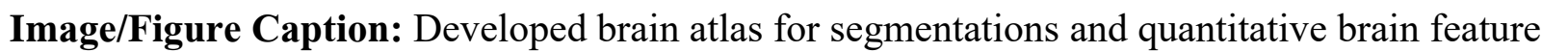

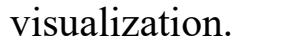

First Name: $\square \square\|\|\|\|$

Last Name: $\square \square \square \square$

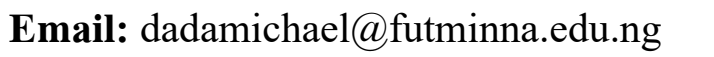

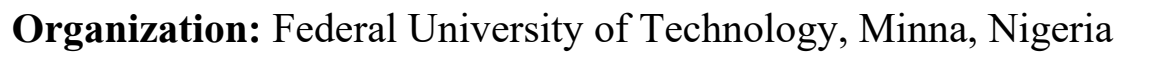

Country: $\square \square \square\|\| \Pi \|$ 